\title{
Plutonium Production Reactor Progression Problems: Magnox Neutronics Benchmarks
}

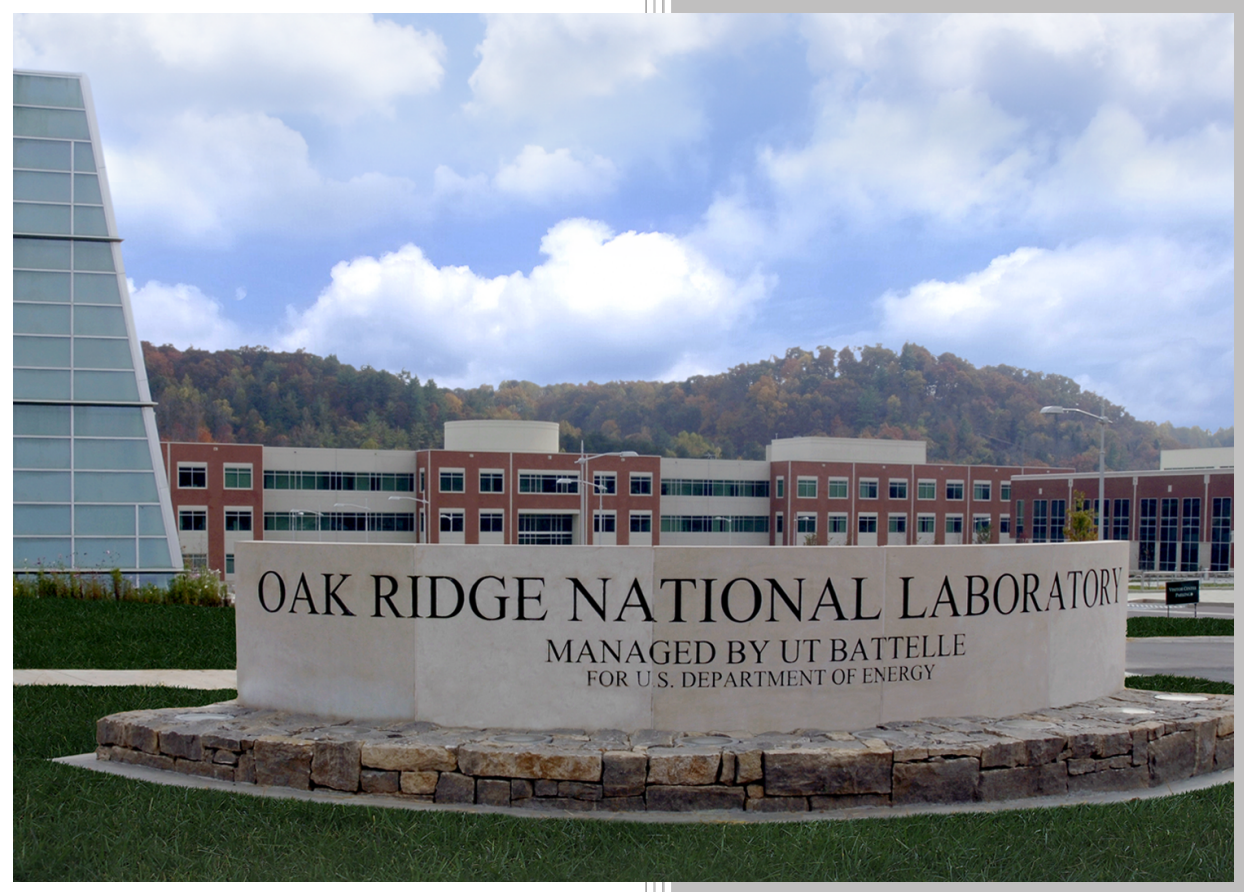

Andrew J. Conant Nicholas P. Luciano Brian J. Ade Cole Gentry Benjamin S. Collins

September 10, 2021 


\section{DOCUMENT AVAILABILITY}

Reports produced after January 1, 1996, are generally available free via US Department of Energy (DOE) SciTech Connect.

Website: www.osti.gov/

Reports produced before January 1, 1996, may be purchased by members of the public from the following source:

National Technical Information Service

5285 Port Royal Road

Springfield, VA 22161

Telephone: 703-605-6000 (1-800-553-6847)

TDD: $703-487-4639$

Fax: 703-605-6900

E-mail: info@ntis.gov

Website: http://classic.ntis.gov/

Reports are available to DOE employees, DOE contractors, Energy Technology Data Exchange representatives, and International Nuclear Information System representatives from the following source:

Office of Scientific and Technical Information

PO Box 62

Oak Ridge, TN 37831

Telephone: 865-576-8401

Fax: 865-576-5728

E-mail: report@osti.gov

Website: http://www.osti.gov/contact.html

This report was prepared as an account of work sponsored by an agency of the United States Government. Neither the United States Government nor any agency thereof, nor any of their employees, makes any warranty, express or implied, or assumes any legal liability or responsibility for the accuracy, completeness, or usefulness of any information, apparatus, product, or process disclosed, or represents that its use would not infringe privately owned rights. Reference herein to any specific commercial product, process, or service by trade name, trademark, manufacturer, or otherwise, does not necessarily constitute or imply its endorsement, recommendation, or favoring by the United States Government or any agency thereof. The views and opinions of authors expressed herein do not necessarily state or reflect those of the United States Government or any agency thereof. 
Nuclear Nonproliferation Division Nuclear Energy and Fuel Cycle Division

\title{
PLUTONIUM PRODUCTION REACTOR PROGRESSION PROBLEMS: MAGNOX NEUTRONICS BENCHMARKS
}

\author{
Andrew J. Conant \\ Nicholas P. Luciano \\ Brian J. Ade \\ Cole Gentry \\ Benjamin Collins
}

Date Published: September 10, 2021

Prepared by

OAK RIDGE NATIONAL LABORATORY

Oak Ridge, TN 37831-6283

managed by

UT-Battelle, LLC

for the

US DEPARTMENT OF ENERGY

under contract DE-AC05-00OR22725 



\section{CONTENTS}

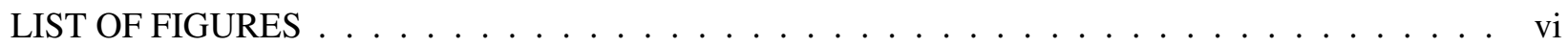

LIST OF TABLES $\ldots \ldots \ldots \ldots \ldots \ldots \ldots \ldots \ldots \ldots$

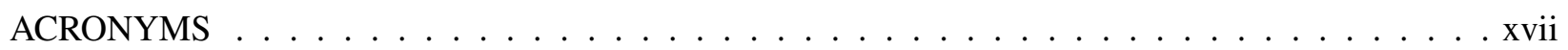

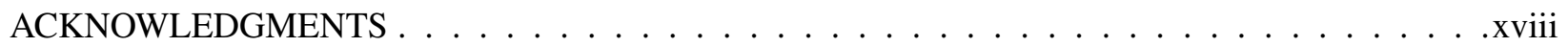

EXECUTIVE SUMMARY $\ldots \ldots \ldots \ldots \ldots \ldots \ldots \ldots$

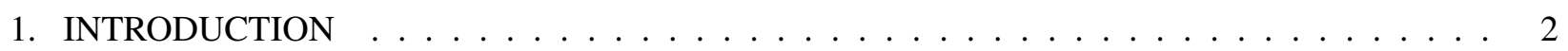

1.1 MODELING AND SIMULATION TOOLS $\ldots \ldots \ldots \ldots \ldots$

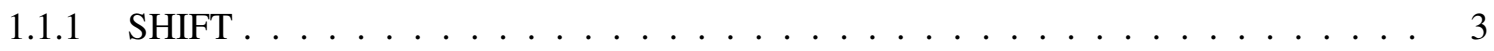

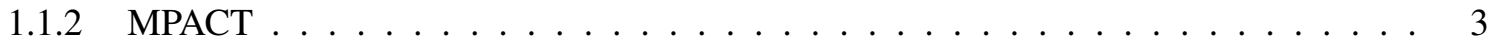

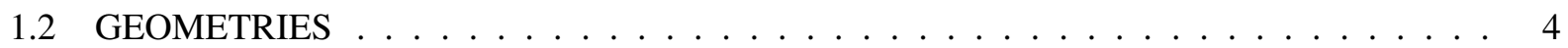

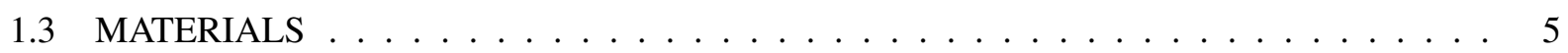

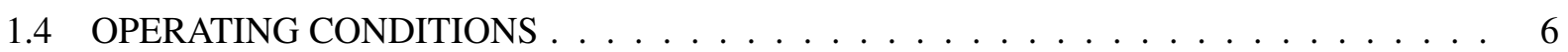

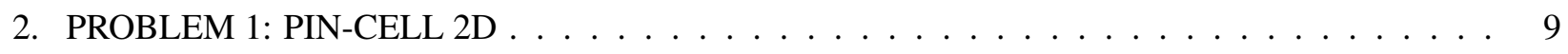

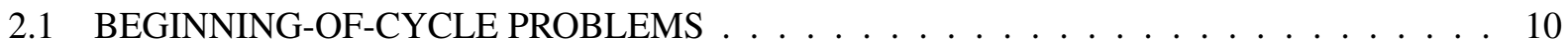

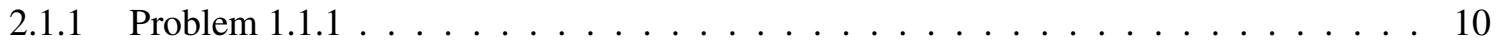

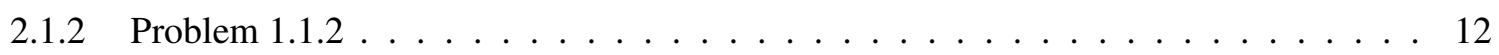

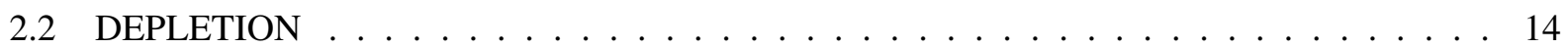

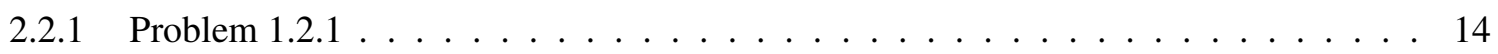

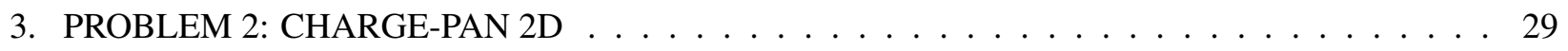

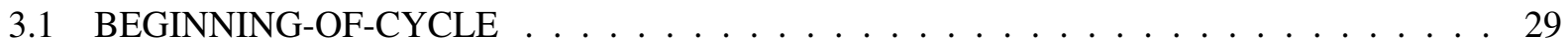

3.1 .1 Problem $2.1 .1 \ldots \ldots \ldots \ldots \ldots \ldots$

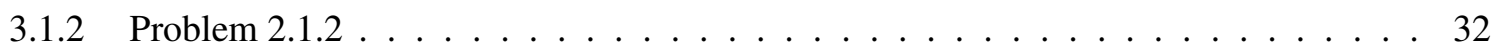

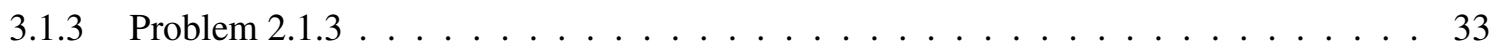

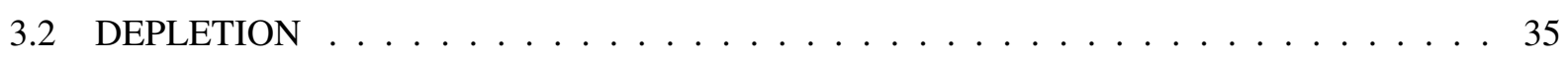

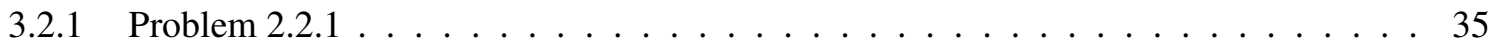

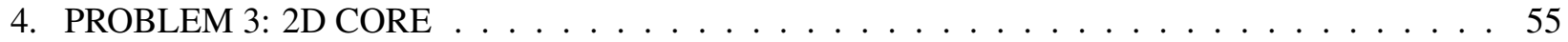

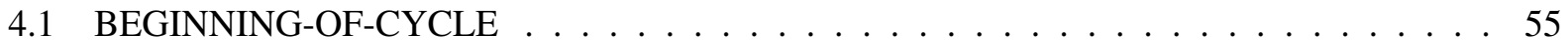

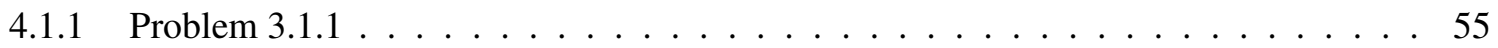

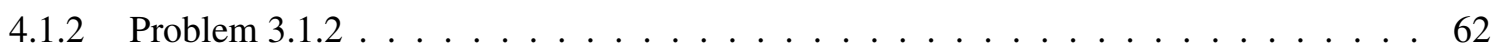

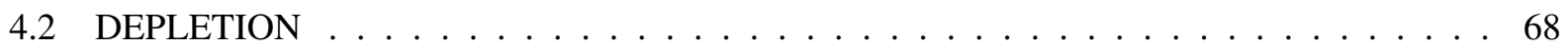

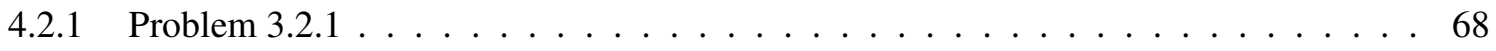

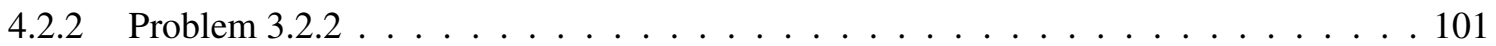

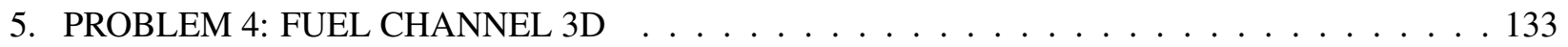

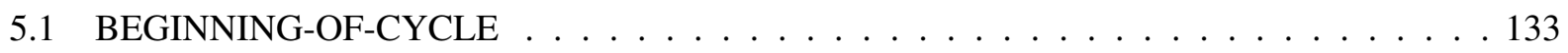

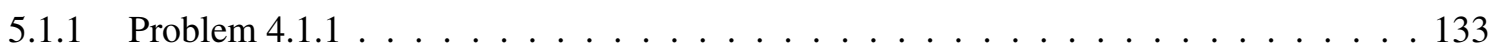

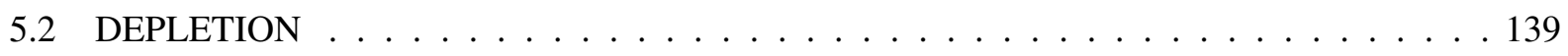

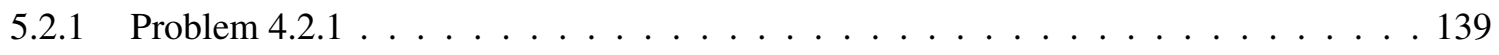

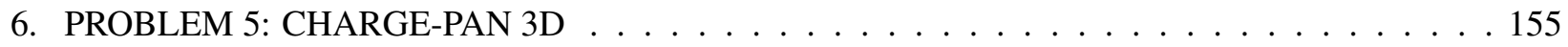

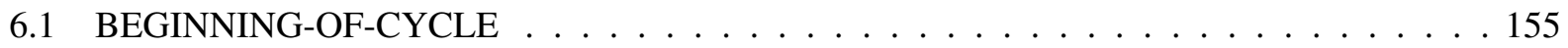

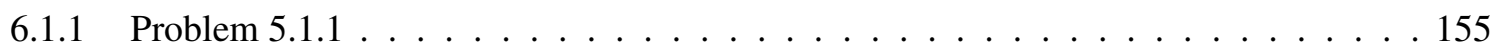

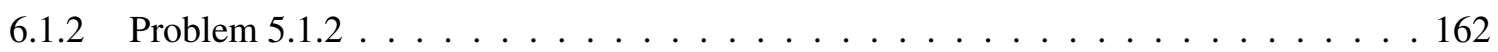

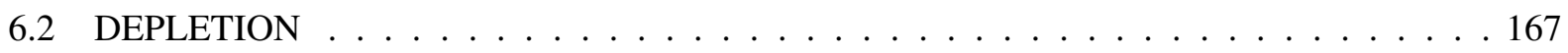

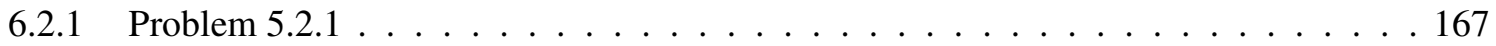

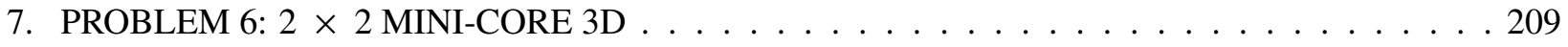


7.1 BEGINNING-OF-CYCLE . . . . . . . . . . . . . . . . . . . 209

7.1 .1 Problem $6.1 .1 \ldots \ldots \ldots \ldots \ldots$

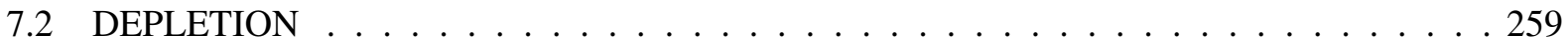

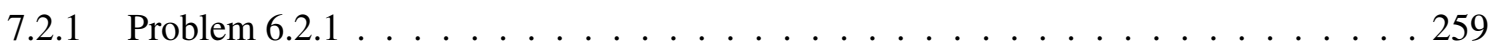

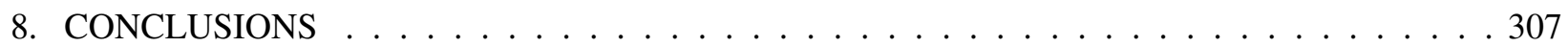

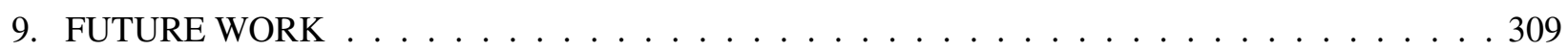

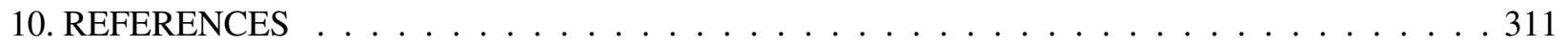





\section{LIST OF FIGURES}

1 Magnox pin-cell geometry with Zone B coolant channel. . . . . . . . . . . . . . . . 9

2 Percent difference in isotope concentrations between MPACT and Shift for actinides (top) and major fission products (bottom) $\ldots \ldots \ldots \ldots \ldots \ldots \ldots$

3 Magnox 2D charge-pan with Zone $\mathrm{C}$ coolant channel. . . . . . . . . . . . . . . . . . . . . 29

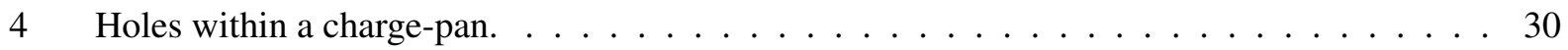

5 Magnox 2D charge-pan with Zone A coolant channel. . . . . . . . . . . . . . 32

6 Quarter core $2 \mathrm{D}$ with all rods out $(\mathrm{ARO}) . \ldots \ldots \ldots \ldots$

$7 \quad$ Shift (top) and MPACT (bottom) power peaking factors for Problem 3.1.1 . . . . . . . . . . 57

8 Absolute difference between Shift and MPACT power peaking factors for Problem 3.1.1 _ . 58

9 Quarter core 2D with all rods in (ARI), where red denotes the charge-pans with control rods inserted. . . . . . . . . . . . . . . . . . . . 63

10 Shift (top) and MPACT (bottom) power peaking factors for Problem 3.1.2 . . . . . . . . 64

11 Absolute difference between Shift and MPACT power peaking factors for Problem 3.1.2 . . 65

12 Quarter core 2D with all rods out (ARO) . . . . . . . . . . . . . . . . . . . 69

13 Shift power peaking factors for Problem 3.2.1 at BOC (top) and EOC (bottom) . . . . . . 71

14 MPACT power peaking factors for Problem 3.2.1 at BOC (top) and EOC (bottom) . . . . . 72

15 Quarter core 2D with main control bank inserted. . . . . . . . . . . . . . . . . . 103

16 3D fuel channel. . . . . . . . . . . . . . . . . . . . . . . . . . . . . . 134

17 Power peaking factors for Problem 4.1.1 for Shift and MPACT (top) and their absolute difference (bottom). . . . . . . . . . . . . . . . . . . . . 136

18 Power peaking factors for Problem 4.2.1 for Shift (top) and MPACT (bottom). Yellow represents time step 0 and red represents time step 15, with a gradient of colors in between. . 140

19 Power peaking factors absolute difference MPACT-Shift. Yellow represents time step 0 and red represents time step 15, with a gradient of colors in between. . . . . . . . . . . . . 141

203 3 charge-pan with six fuel element stacks. . . . . . . . . . . . . . 156

21 Power peaking factors for Problem 5.1.1 for Shift and MPACT (top) and their absolute difference (bottom). The three series correspond to pins ABCD (central), EFGHJKLM (edge), and PQRS (corner). . . . . . . . . . . . . . . . . . . . 157

22 Power peaking factors for Problem 5.1.2 for Shift and MPACT (top) and their absolute difference (bottom). The three series correspond to pins ABCD (central), EFGHJKLM (edge), and PQRS (corner). . . . . . . . . . . . . . . . . . . . 163

23 Power peaking factors for rods A-D in Problem 5.2.1 for Shift and MPACT with the latter having dashed lines (top). The absolute difference in the peaking factors MPACT-Shift (bottom). Yellow represents time step 0 and red represents time step 15, with a gradient of colors in between. . . . . . . . . . . . . . . . . . . . . . . 168

24 Power peaking factors for rods E-M in Problem 5.2.1 for Shift and MPACT with the latter having dashed lines (top). The absolute difference in the peaking factors MPACT-Shift (bottom). Yellow represents time step 0 and red represents time step 15, with a gradient of colors in between. . . . . . . . . . . . . . . . . . . . . . . . . . . . 169

25 Power peaking factors for rods P-S in Problem 5.2.1 for Shift and MPACT with the latter having dashed lines (top). The absolute difference in the peaking factors MPACT-Shift (bottom). Yellow represents time step 0 and red represents time step 15, with a gradient

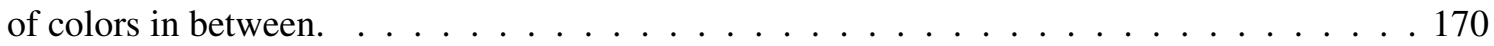

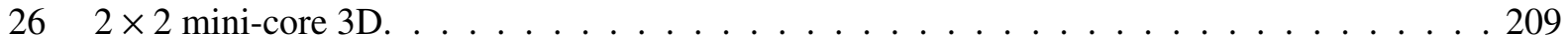


27 3D charge-pan with five fuel element stacks. . . . . . . . . . . . . . . . . 210

28 Power peaking factors for Problem 6.1.1 for Shift and MPACT (top) and their absolute difference (bottom). The three series correspond to charge-pans I-IV. Charge-pan I is the 5-element high stack, and charge-pans II and IV have the control rod inserted halfway. . . . 212 


\section{LIST OF TABLES}

1.1 Summary of naming convention for progression problems for geometry and solution method. 2

1.2 Geometrical parameters of gas-cooled, graphite-moderated reactor. . . . . . . . . . . . 4

1.3 Compositions of materials used in progression problems. . . . . . . . . . . . 5

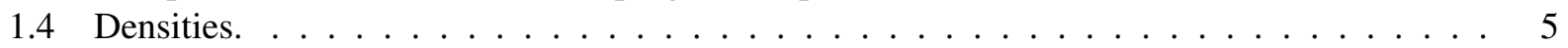

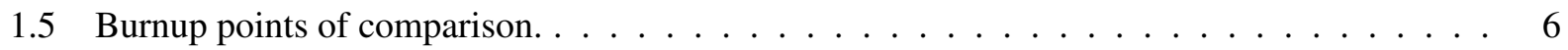

1.6 Power levels for various progression problems derived from total core power [7] $\ldots \ldots$. . 7

2.1 Temperatures for Problem 1.1.1. . . . . . . . . . . . . . . . . 10

2.2 Neutron multiplication for the reference solution of Problem 1.1.1 . . . . . . . . . . . 10

2.3 Timing variables for the reference solution of Problem 1.1.1 . . . . . . . . . . . . 11

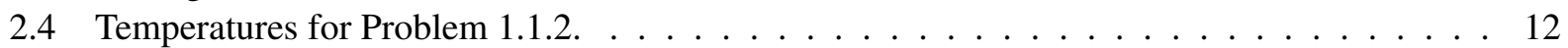

2.5 Neutron multiplication for the reference solution of Problem 1.1.2. . . . . . . . . . . . 12

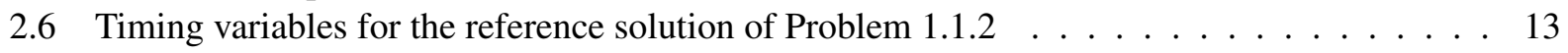

2.7 Neutron multiplication factor for the reference solution of Problem 1.2.1. . . . . . . . . 14

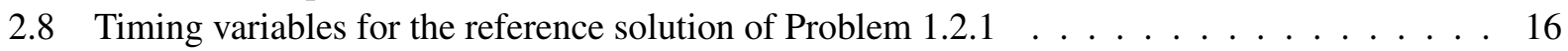

2.9 Uranium isotope concentrations for the reference solution of Problem 1.2.1 . . . . . . . 17

2.10 Plutonium isotope concentrations for the reference solution of Problem 1.2.1 . . . . . . . 18

2.11 Americium isotope concentrations for the reference solution of Problem 1.2.1 . . . . . . . 19

2.12 Curium isotope concentrations for the reference solution of Problem 1.2.1 . . . . . . . 20

2.13 Cesium isotope concentrations for the reference solution of Problem 1.2.1 . . . . . . . . 21

2.14 Neodymium isotope concentrations for the reference solution of Problem 1.2.1 . . . . . . 22

2.15 Strontium isotope concentrations for the reference solution of Problem 1.2.1. . . . . . . 23

2.16 Barium isotope concentrations for the reference solution of Problem 1.2.1 . . . . . . . . . 24

2.17 Technetium isotope concentrations for the reference solution of Problem 1.2.1. . . . . . . 25

2.18 Rubidium isotope concentrations for the reference solution of Problem 1.2.1. . . . . . . . 26

2.19 Relative difference between MPACT and Shift concentrations for uranium and plutonium isotopes . . . . . . . . . . . . . . . . . . . . . 27

2.20 Relative difference between MPACT and Shift concentrations for americium and curium

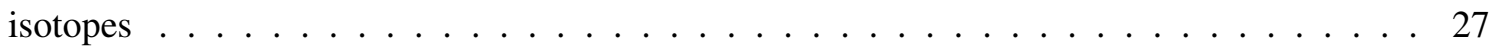

2.21 Relative difference between MPACT and Shift concentrations for cesium and neodymium

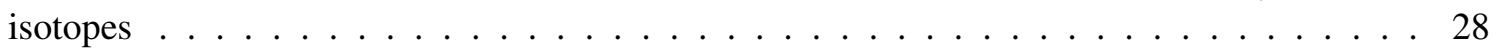

2.22 Relative difference between MPACT and Shift concentrations for miscellaneous fission

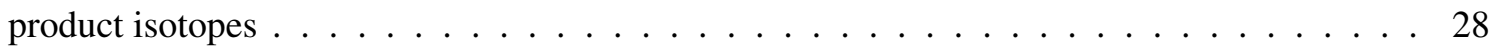

3.1 Neutron multiplication for the reference solution of Problem 2.1.1. . . . . . . . . . . 31

3.2 Power peaking factors for the reference solution of Problem 2.1.1 . . . . . . . . . . 31

3.3 Neutron multiplication for the reference solution of Problem 2.1.2. . . . . . . . . . 33

3.4 Power peaking factors for the reference solution of Problem 2.1.2 . . . . . . . . . . . 33

3.5 Neutron multiplication for the reference solution of Problem 2.1.3 . . . . . . . . . . . 34

3.6 Power peaking factors for the reference solution of Problem 2.1.3 . . . . . . . . . . . . 34

3.7 Neutron multiplication factor for the reference solution of Problem 2.2.1 . . . . . . . . . 35

3.8 Power peaking factors for fuel channels A-D of the reference solution of Problem 2.2.1. . . 36

3.9 Uranium isotope concentrations for fuel channels A-D of the reference solution of

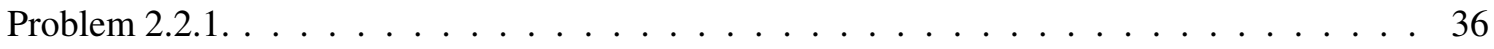

3.10 Plutonium isotope concentrations for fuel channels A through D of the reference solution

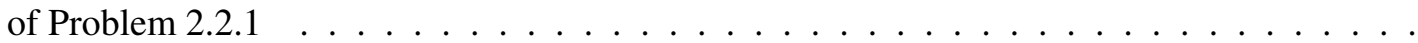


3.11 Americium isotope concentrations for fuel channels A-D of the reference solution of

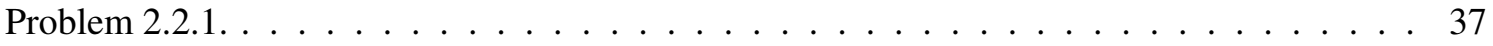

3.12 Curium isotope concentrations for fuel channels A-D of the reference solution of

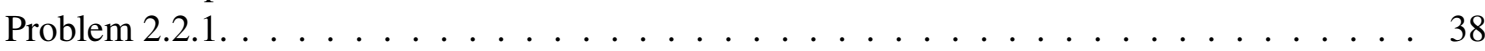

3.13 Cesium isotope concentrations for fuel channels A-D of the reference solution of

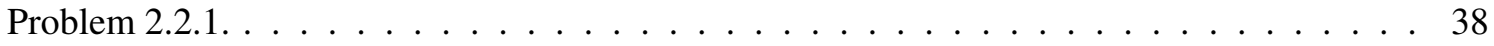

3.14 Neodymium isotope concentrations for fuel channels A-D of the reference solution of

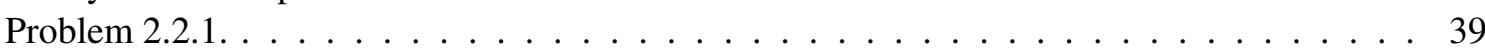

3.15 Strontium isotope concentrations for fuel channels A-D of the reference solution of Problem 2.2.1. . . . . . . . . . . . . . . . . . . . . . . . . . . . 39

3.16 Barium isotope concentrations for fuel channels A-D of the reference solution of

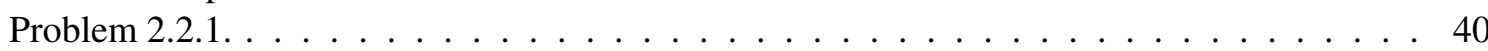

3.17 Technetium isotope concentrations for fuel channels A-D of the reference solution of

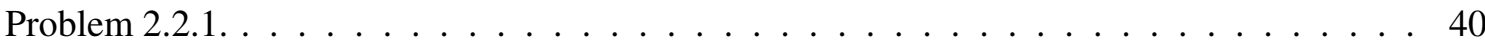

3.18 Rubidium isotope concentrations for fuel channels A-D of the reference solution of

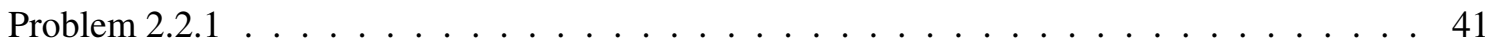

3.19 Power peaking factors for fuel channels E-M of the reference solution of Problem 2.2.1. . . 42

3.20 Uranium isotope concentrations for fuel channels $\mathrm{E}-\mathrm{M}$ of the reference solution of

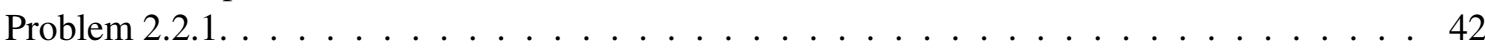

3.21 Plutonium isotope concentrations for fuel channels E-M of the reference solution of

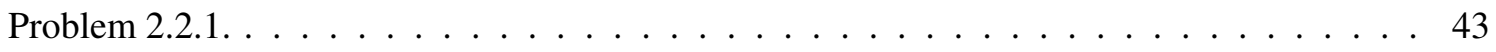

3.22 Americium isotope concentrations for fuel channels E-M of the reference solution of Problem 2.2.1. . . . . . . . . . . . . . . . . . . . . . . . 4 43

3.23 Curium isotope concentrations for fuel channels E-M of the reference solution of

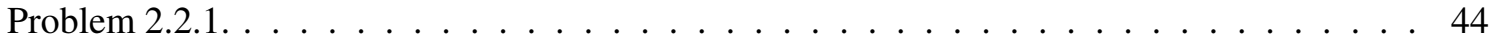

3.24 Cesium isotope concentrations for fuel channels E-M of the reference solution of Problem 2.2.1. . . . . . . . . . . . . . . . . . . . . . . 44

3.25 Neodymium isotope concentrations for fuel channels E-M of the reference solution of

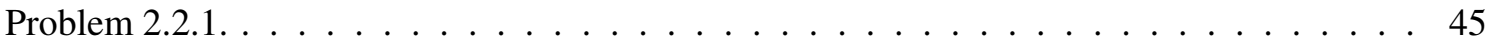

3.26 Strontium isotope concentrations for fuel channels E-M of the reference solution of Problem 2.2.1. . . . . . . . . . . . . . . . . . . . . . . 45

3.27 Barium isotope concentrations for fuel channels E-M of the reference solution of

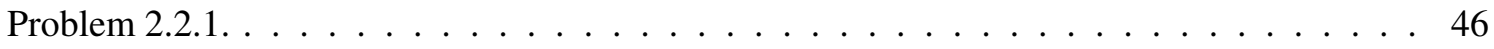

3.28 Technetium isotope concentrations for fuel channels E-M of the reference solution of

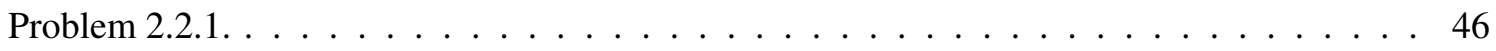

3.29 Rubidium isotope concentrations for fuel channels E-M of the reference solution of

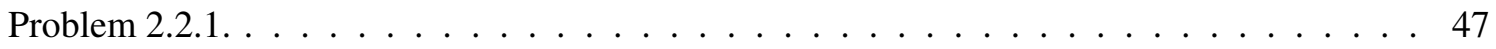

3.30 Power peaking factors for fuel channels P-S of the reference solution of Problem 2.2.1. . . . 48

3.31 Uranium isotope concentrations for fuel channels $\mathrm{P}-\mathrm{S}$ of the reference solution of

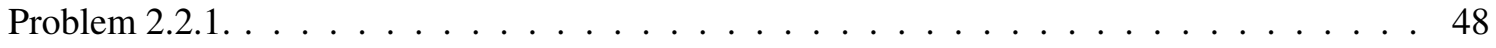

3.32 Plutonium isotope concentrations for fuel channels $\mathrm{P}-\mathrm{S}$ of the reference solution of Problem 2.2.1. . . . . . . . . . . . . . . . . . . . . . . . . . . . 49

3.33 Americium isotope concentrations for fuel channels $\mathrm{P}-\mathrm{S}$ of the reference solution of

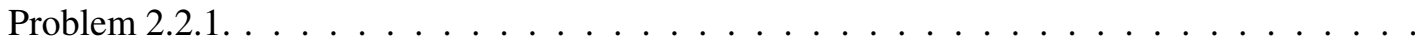


3.34 Curium isotope concentrations for fuel channels $\mathrm{P}-\mathrm{S}$ of the reference solution of Problem

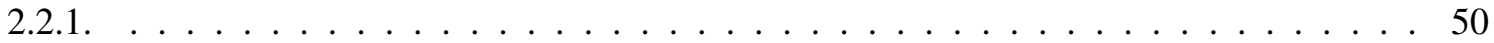

3.35 Cesium isotope concentrations for fuel channels $\mathrm{P}-\mathrm{S}$ of the reference solution of Problem

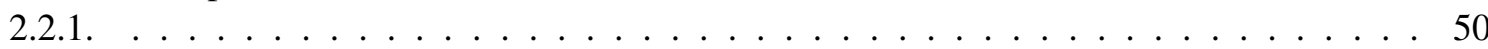

3.36 Neodymium isotope concentrations for fuel channels $\mathrm{P}-\mathrm{S}$ of the reference solution of

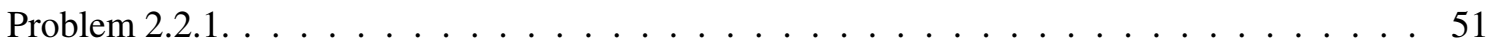

3.37 Strontium isotope concentrations for fuel channels $\mathrm{P}-\mathrm{S}$ of the reference solution of

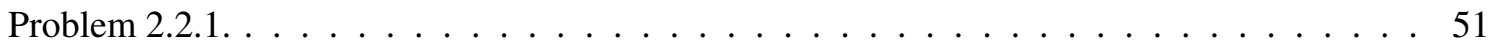

3.38 Barium isotope concentrations for fuel channels $\mathrm{P}-\mathrm{S}$ of the reference solution of Problem

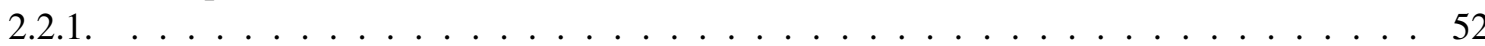

3.39 Technetium isotope concentrations for fuel channels $\mathrm{P}-\mathrm{S}$ of the reference solution of

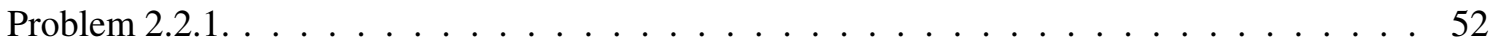

3.40 Rubidium isotope concentrations for fuel channels $\mathrm{P}-\mathrm{S}$ of the reference solution of

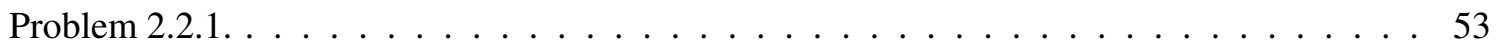

4.1 Neutron multiplication for the reference solution of Problem 3.1.1. . . . . . . . . . . 55

4.2 Timing variables for the reference solution of Problem 3.1.1 . . . . . . . . . . . . . . 59

4.3 Power peaking factors $\mathrm{A}-\mathrm{H}$ and $\mathrm{P}-\mathrm{S}$ of the reference solution of Problem 3.1.1. . . . . . . 60

4.4 Power peaking factors E-M of the reference solution of Problem 3.1.1 . . . . . . . . . 61

4.5 Neutron multiplication for the reference solution of Problem 3.1.2 . . . . . . . . . . . 62

4.6 Timing variables for the reference solution of Problem $3.1 .2 \ldots \ldots \ldots 2$

4.7 Power peaking factors $\mathrm{A}-\mathrm{H}$ and $\mathrm{P}-\mathrm{S}$ of the reference solution of Problem 3.1.2. . . . . . 66

4.8 Power peaking factors E-M of the reference solution of Problem 3.1.2 . . . . . . . . . . 67

4.9 Neutron multiplication factor for the reference solution of Problem 3.2.1 . . . . . . . . . 70

4.10 Timing variables for the reference solution of Problem 3.2.1 $\ldots \ldots \ldots$. . . . . 73

4.11 Timing variables for the reference solution of Problem 3.2.1 $\ldots \ldots \ldots$

4.12 Power peaking factors for charge-pan 0727 of the reference solution of Problem 3.2.1 . . . . 74

4.13 Problem 3.2.1 reference solution power peaking factors for charge-pans 0827 and 0728 . . . 75

4.14 Problem 3.2.1 reference solution power peaking factors for charge-pans 0827 and 0728 . . . 76

4.15 Problem 3.2.1 reference solution power peaking factors for charge-pans 0927 and 0729. . . . 77

4.16 Problem 3.2.1 reference solution power peaking factors for charge-pans 0927 and 0729 . . . 78

4.17 Problem 3.2.1 reference solution power peaking factors for charge-pans 1027 and 0730. . . . 79

4.18 Problem 3.2.1 reference solution power peaking factors for charge-pans 1027 and $0730 \ldots$. . 80

4.19 Problem 3.2.1 reference solution power peaking factors for charge-pans 1127 and $0731 \ldots$. . 81

4.20 Problem 3.2.1 reference solution power peaking factors for charge-pans 1127 and 0731 . . . 82

4.21 Problem 3.2.1 reference solution power peaking factors for charge-pans 1227 and 0732 . . . 83

4.22 Problem 3.2.1 reference solution power peaking factors for charge-pans 1227 and 0732 . . . 84

4.23 Problem 3.2.1 reference solution power peaking factors for charge-pan 0828. . . . . . . 85

4.24 Problem 3.2.1 reference solution power peaking factors for charge-pans 0928 and 0829 . . . 86

4.25 Problem 3.2.1 reference solution power peaking factors for charge-pans 0928 and 0829. . . 87

4.26 Problem 3.2.1 reference solution power peaking factors for charge-pans 1028 and $0830 \ldots$. . 88

4.27 Problem 3.2.1 reference solution power peaking factors for charge-pans 1028 and 0830. . . . 89

4.28 Problem 3.2.1 reference solution power peaking factors for charge-pans 1128 and 0831. . . 90

4.29 Problem 3.2.1 reference solution power peaking factors for charge-pans 1128 and 0831. . . 91

4.30 Problem 3.2.1 reference solution power peaking factors for charge-pans 1228 and 0832. . . . 92

4.31 Problem 3.2.1 reference solution power peaking factors for charge-pans 1228 and 0832 . . . 93 
4.32 Problem 3.2.1 reference solution power peaking factors for charge-pan 0929. . . . . . . . 94

4.33 Problem 3.2.1 reference solution power peaking factors for charge-pans 1029 and 0930. . . 95

4.34 Problem 3.2.1 reference solution power peaking factors for charge-pans 1029 and 0930. . . 96

4.35 Problem 3.2.1 reference solution power peaking factors for charge-pans 1129 and 0931. . . 97

4.36 Problem 3.2.1 reference solution power peaking factors for charge-pans 1129 and 0931. . . 98

4.37 Problem 3.2.1 reference solution power peaking factors for charge-pan 1030 . . . . . . . 99

4.38 Problem 3.2.1 reference solution power peaking factors for charge-pans 1130 and 1031 . . 100

4.39 Problem 3.2.1 reference solution power peaking factors for charge-pans 1130 and $1031 \ldots 101$

4.40 Neutron multiplication factor for the reference solution of Problem 3.2.2 . . . . . . . . . . 104

4.41 Timing variables for the reference solution of Problem 3.2.2 . . . . . . . . . . . 104

4.42 Power peaking factors for charge-pan 0727 of the reference solution of Problem 3.2.2 . . . . 105

4.43 Problem 3.2.2 reference solution power peaking factors for charge-pans 0827 and 0728 . . . 106

4.44 Problem 3.2.2 reference solution power peaking factors for charge-pans 0827 and 0728 . . . 107

4.45 Problem 3.2.2 reference solution power peaking factors for charge-pans 0927 and 0729 . . . 108

4.46 Problem 3.2.2 reference solution power peaking factors for charge-pans 0927 and 0729. . . . 109

4.47 Problem 3.2.2 reference solution power peaking factors for charge-pans 1027 and 0730 . . . 110

4.48 Problem 3.2.2 reference solution power peaking factors for charge-pans 1027 and 0730 . . . 111

4.49 Problem 3.2.2 reference solution power peaking factors for charge-pans 1127 and 0731 . . . 112

4.50 Problem 3.2.2 reference solution power peaking factors for charge-pans 1127 and 0731 . . . 113

4.51 Problem 3.2.2 reference solution power peaking factors for charge-pans 1227 and 0732 . . . 114

4.52 Problem 3.2.2 reference solution power peaking factors for charge-pans 1227 and 0732 . . . 115

4.53 Problem 3.2.2 reference solution power peaking factors for charge-pan 0828. . . . . . 116

4.54 Problem 3.2.2 reference solution power peaking factors for charge-pans 0928 and 0829. . . 117

4.55 Problem 3.2.2 reference solution power peaking factors for charge-pans 0928 and 0829. . . . 118

4.56 Problem 3.2.2 reference solution power peaking factors for charge-pans 1028 and 0830 . . . 119

4.57 Problem 3.2.2 reference solution power peaking factors for charge-pans 1028 and 0830 . . . 120

4.58 Problem 3.2.2 reference solution power peaking factors for charge-pans 1128 and $0831 \ldots$. . 121

4.59 Problem 3.2.2 reference solution power peaking factors for charge-pans 1128 and 0831 . . . 122

4.60 Problem 3.2.2 reference solution power peaking factors for charge-pans 1228 and 0832 . . . 123

4.61 Problem 3.2.2 reference solution power peaking factors for charge-pans 1228 and 0832. . . . 124

4.62 Problem 3.2.2 reference solution power peaking factors for charge-pan 0929. . . . . . . 125

4.63 Problem 3.2.2 reference solution power peaking factors for charge-pans 1029 and 0930 . . . 126

4.64 Problem 3.2.2 reference solution power peaking factors for charge-pans 1029 and 0930 . . . 127

4.65 Problem 3.2.2 reference solution power peaking factors for charge-pans 1129 and 0931. . . 128

4.66 Problem 3.2.2 reference solution power peaking factors for charge-pans 1129 and 0931. . . 129

4.67 Problem 3.2.2 reference solution power peaking factors for charge-pan 1030 . . . . . . . 130

4.68 Problem 3.2.2 reference solution power peaking factors for charge-pans 1130 and 1031 . . . 131

4.69 Problem 3.2.2 reference solution power peaking factors for charge-pans 1130 and 1031 . . . 132

5.1 Neutron multiplication factor for the reference solution of Problem 4.1.1 . . . . . . . . 135

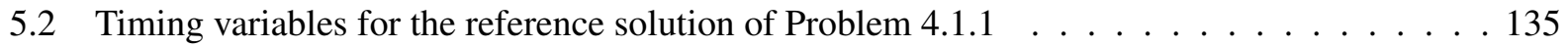

5.3 Problem 4.1.1 reference solution power peaking factors for axial segments in element 1. . . 135

5.4 Problem 4.1.1 reference solution power peaking factors for axial segments in element 2. . . . 137

5.5 Problem 4.1.1 reference solution power peaking factors for axial segments in element 3. . . . 137

5.6 Problem 4.1.1 reference solution power peaking factors for axial segments in element 4 . . . 137

5.7 Problem 4.1.1 reference solution power peaking factors for axial segments in element 5. . . 137 
5.8 Problem 4.1.1 reference solution power peaking factors for axial segments in element 6. . . 138

5.9 Neutron multiplication factor for the reference solution of Problem 4.2.1 . . . . . . . 139

5.10 Timing variables for the reference solution of Problem 4.2.1 . . . . . . . . . . . . 142

5.11 Problem 4.2.1 reference solution power peaking factors for axial segments 1-10 in element 1. 143

5.12 Problem 4.2.1 reference solution power peaking factors for axial segments 11-20 in element 1. . . . . . . . . . . . . . . . . . . . . 144

5.13 Problem 4.2.1 reference solution power peaking factors for axial segments 1-10 in element 2. 145

5.14 Problem 4.2.1 reference solution power peaking factors for axial segments 11-20 in element 2. . . . . . . . . . . . . . . . . . . . . . 146

5.15 Problem 4.2.1 reference solution power peaking factors for axial segments 1-10 in element 3. 147

5.16 Problem 4.2.1 reference solution power peaking factors for axial segments 11-20 in element 3. . . . . . . . . . . . . . . . . . . . . . . 148

5.17 Problem 4.2.1 reference solution power peaking factors for axial segments 1-10 in element 4. 149

5.18 Problem 4.2.1 reference solution power peaking factors for axial segments 11-20 in

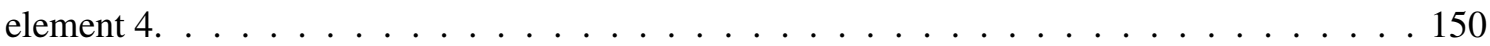

5.19 Problem 4.2.1 reference solution power peaking factors for axial segments 1-10 in element 5. 151

5.20 Problem 4.2.1 reference solution power peaking factors for axial segments 11-20 in

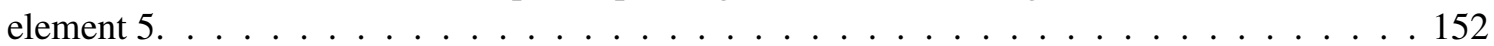

5.21 Problem 4.2.1 reference solution power peaking factors for axial segments 1-10 in element 6. 153

5.22 Problem 4.2.1 reference solution power peaking factors for axial segments 11-20 in

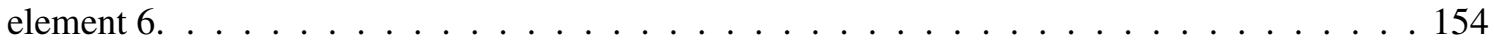

6.1 Neutron multiplication factor for the reference solution of Problem 5.1.1. . . . . . . . . 155

6.2 Timing variables for the reference solution of Problem 5.1.1 . . . . . . . . . . 158

6.3 Problem 5.1.1 reference solution power peaking factors for axial segments in element 1. . . 158

6.4 Problem 5.1.1 reference solution power peaking factors for axial segments in element 2. . . . 159

6.5 Problem 5.1.1 reference solution power peaking factors for axial segments in element 3. . . 159

6.6 Problem 5.1.1 reference solution power peaking factors for axial segments in element 4 . . . 160

6.7 Problem 5.1.1 reference solution power peaking factors for axial segments in element 5. . . . 160

6.8 Problem 5.1.1 reference solution power peaking factors for axial segments in element 6. . . 161

6.9 Neutron multiplication factor for the reference solution of Problem 5.1.2 . . . . . . . . 162

6.10 Timing variables for the reference solution of Problem 5.1.2 . . . . . . . . . . . 162

6.11 Problem 5.1.2 reference solution power peaking factors for axial segments in element 1. . . 164

6.12 Problem 5.1.2 reference solution power peaking factors for axial segments in element 2. . . . 164

6.13 Problem 5.1.2 reference solution power peaking factors for axial segments in element 3. . . 165

6.14 Problem 5.1.2 reference solution power peaking factors for axial segments in element 4. . . 165

6.15 Problem 5.1.2 reference solution power peaking factors for axial segments in element 5 . . . 166

6.16 Problem 5.1.2 reference solution power peaking factors for axial segments in element 6.166

6.17 Neutron multiplication factor for the reference solution of Problem 5.2.1 . . . . . . . 167

6.18 Timing variables for the reference solution of Problem 5.2.1 . . . . . . . . . 171

6.19 Problem 5.2.1 reference solution Shift power peaking factors for axial segments in fuel channels A, B, C, and D in element 1. . . . . . . . . . . . . . . . 172

6.20 Problem 5.2.1 reference solution MPACT power peaking factors for axial segments in fuel channels A, B, C, and D in element 1 . . . . . . . . . . . . . . . 173

6.21 Problem 5.2.1 reference solution Shift power peaking factors for axial segments in fuel channels A, B, C, and D in element $2 \ldots \ldots \ldots \ldots \ldots$ 
6.22 Problem 5.2.1 reference solution MPACT power peaking factors for axial segments in fuel channels A, B, C, and D in element 2 . . . . . . . . . . . . . . . 175

6.23 Problem 5.2.1 reference solution Shift power peaking factors for axial segments in fuel channels A, B, C, and D in element $3 . \ldots \ldots \ldots \ldots$. . . . . . . . . . . . . . . . .

6.24 Problem 5.2.1 reference solution MPACT power peaking factors for axial segments in fuel channels A, B, C, and D in element 3 . . . . . . . . . . . . . . . . 177

6.25 Problem 5.2.1 reference solution Shift power peaking factors for axial segments in fuel channels A, B, C, and D in element $4 \ldots \ldots \ldots \ldots$. . . . . . . . . . . . . . . . .

6.26 Problem 5.2.1 reference solution MPACT power peaking factors for axial segments in fuel channels A, B, C, and D in element 4 . . . . . . . . . . . . . . . 179

6.27 Problem 5.2.1 reference solution Shift power peaking factors for axial segments in fuel channels A, B, C, and D in element 5. . . . . . . . . . . . . . . 180

6.28 Problem 5.2.1 reference solution MPACT power peaking factors for axial segments in fuel channels A, B, C, and D in element 5 . . . . . . . . . . . . . . . 181

6.29 Problem 5.2.1 reference solution Shift power peaking factors for axial segments in fuel channels A, B, C, and D in element $6 \ldots \ldots \ldots \ldots$. . . . . . . . . . . . . . . . . . .

6.30 Problem 5.2.1 reference solution MPACT power peaking factors for axial segments in fuel channels A, B, C, and D in element $6 \ldots \ldots \ldots$. . . . . . . . . . . . . . . . . . .

6.31 Problem 5.2.1 reference solution Shift power peaking factors for axial segments in fuel channels E, F, G, H, J, K, L, and M in element 1. . . . . . . . . . . . . . . 184

6.32 Problem 5.2.1 reference solution MPACT power peaking factors for axial segments in fuel channels E, F, G, H, J, K, L, and M in element 1. . . . . . . . . . . . . . 185

6.33 Problem 5.2.1 reference solution Shift power peaking factors for axial segments in fuel channels E, F, G, H, J, K, L, and M in element 2. . . . . . . . . . . . . . 186

6.34 Problem 5.2.1 reference solution MPACT power peaking factors for axial segments in fuel channels E, F, G, H, J, K, L, and M in element 2. . . . . . . . . . . . . . 187

6.35 Problem 5.2.1 reference solution Shift power peaking factors for axial segments in fuel channels E, F, G, H, J, K, L, and M in element 3. . . . . . . . . . . . . . 188

6.36 Problem 5.2.1 reference solution MPACT power peaking factors for axial segments in fuel channels E, F, G, H, J, K, L, and M in element 3. . . . . . . . . . . . . . . . . . . 189

6.37 Problem 5.2.1 reference solution Shift power peaking factors for axial segments in fuel channels E, F, G, H, J, K, L, and M in element 4. . . . . . . . . . . . . . . . . 190

6.38 Problem 5.2.1 reference solution MPACT power peaking factors for axial segments in fuel channels E, F, G, H, J, K, L, and M in element 4. . . . . . . . . . . . . . . . . . . 191

6.39 Problem 5.2.1 reference solution Shift power peaking factors for axial segments in fuel channels E, F, G, H, J, K, L, and M in element 5. . . . . . . . . . . . . . . . . 192

6.40 Problem 5.2.1 reference solution MPACT power peaking factors for axial segments in fuel channels E, F, G, H, J, K, L, and M in element 5. . . . . . . . . . . . . . . . . . . 193

6.41 Problem 5.2.1 reference solution Shift power peaking factors for axial segments in fuel channels E, F, G, H, J, K, L, and M in element 6. . . . . . . . . . . . . . . . . 194

6.42 Problem 5.2.1 reference solution MPACT power peaking factors for axial segments in fuel channels E, F, G, H, J, K, L, and M in element 6 . . . . . . . . . . . . . . . 195

6.43 Problem 5.2.1 reference solution Shift power peaking factors for axial segments in fuel channels $\mathrm{P}, \mathrm{Q}, \mathrm{R}$, and $\mathrm{S}$ in element $1 \ldots \ldots \ldots$. . . . . . . . . . . . . . . . . . . . . . . 
6.44 Problem 5.2.1 reference solution MPACT power peaking factors for axial segments in fuel channels $\mathrm{P}, \mathrm{Q}, \mathrm{R}$, and $\mathrm{S}$ in element $1 . \ldots \ldots$. . . . . . . . . . 197

6.45 Problem 5.2.1 reference solution Shift power peaking factors for axial segments in fuel channels $\mathrm{P}, \mathrm{Q}, \mathrm{R}$, and $\mathrm{S}$ in element 2. . . . . . . . . . . . . . . . . . 198

6.46 Problem 5.2.1 reference solution MPACT power peaking factors for axial segments in fuel channels $\mathrm{P}, \mathrm{Q}, \mathrm{R}$, and $\mathrm{S}$ in element $2 . \quad \ldots \ldots$. . . . . . . . . . . . 199

6.47 Problem 5.2.1 reference solution Shift power peaking factors for axial segments in fuel

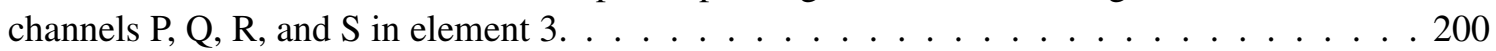

6.48 Problem 5.2.1 reference solution MPACT power peaking factors for axial segments in

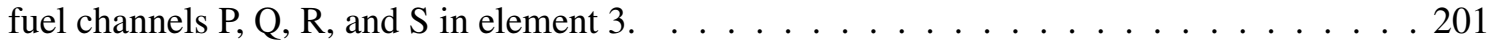

6.49 Problem 5.2.1 reference solution Shift power peaking factors for axial segments in fuel

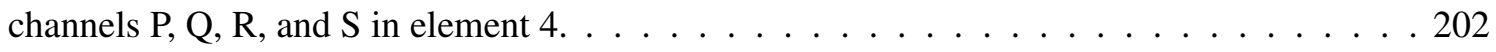

6.50 Problem 5.2.1 reference solution MPACT power peaking factors for axial segments in fuel channels $\mathrm{P}, \mathrm{Q}, \mathrm{R}$, and $\mathrm{S}$ in element $4 \ldots \ldots \ldots$. . . . . . . . . . . . . . . . . . .

6.51 Problem 5.2.1 reference solution Shift power peaking factors for axial segments in fuel channels $\mathrm{P}, \mathrm{Q}, \mathrm{R}$, and $\mathrm{S}$ in element $5 \ldots \ldots \ldots$. . . . . . . . . . . . 204

6.52 Problem 5.2.1 reference solution MPACT power peaking factors for axial segments in

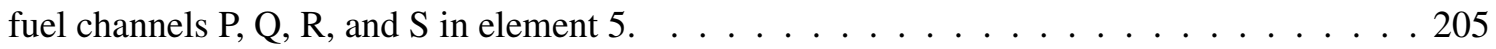

6.53 Problem 5.2.1 reference solution Shift power peaking factors for axial segments in fuel channels $\mathrm{P}, \mathrm{Q}, \mathrm{R}$, and $\mathrm{S}$ in element 6. . . . . . . . . . . . . . . 206

6.54 Problem 5.2.1 reference solution MPACT power peaking factors for axial segments in fuel channels $\mathrm{P}, \mathrm{Q}, \mathrm{R}$, and $\mathrm{S}$ in element $6 . \ldots \ldots$. . . . . . . . . . . 207

7.1 Neutron multiplication factor for the reference solution of Problem 6.1.1. . . . . . . 211

7.2 Timing variables for the reference solution of Problem 6.1.1 . . . . . . . . . 211

7.3 Problem 6.1.1 reference solution Shift power peaking factors for axial segments in element 1 for charge-pan I. . . . . . . . . . . . . . . . . . . 213

7.4 Problem 6.1.1 reference solution MPACT power peaking factors for axial segments in element 1 for charge-pan I. . . . . . . . . . . . . . . . . . . . . . . 214

7.5 Problem 6.1.1 reference solution Shift power peaking factors for axial segments in element 2 for charge-pan I. . . . . . . . . . . . . . . . . . . . 215

7.6 Problem 6.1.1 reference solution MPACT power peaking factors for axial segments in element 2 for charge-pan I. . . . . . . . . . . . . . . . . . . . 216

7.7 Problem 6.1.1 reference solution Shift power peaking factors for axial segments in element 3 for charge-pan I. . . . . . . . . . . . . . . . . . . . . . . 217

7.8 Problem 6.1.1 reference solution MPACT power peaking factors for axial segments in element 3 for charge-pan I. . . . . . . . . . . . . . . . . . . . . 218

7.9 Problem 6.1.1 reference solution Shift power peaking factors for axial segments in element 4 for charge-pan I. . . . . . . . . . . . . . . . . . . . . . . . 219

7.10 Problem 6.1.1 reference solution MPACT power peaking factors for axial segments in element 4 for charge-pan I. . . . . . . . . . . . . . . . . . . . . 220

7.11 Problem 6.1.1 reference solution Shift power peaking factors for axial segments in element 5 for charge-pan I. . . . . . . . . . . . . . . . . . . . . 221

7.12 Problem 6.1.1 reference solution MPACT power peaking factors for axial segments in element 5 for charge-pan I. . . . . . . . . . . . . . . . . . . . . 222 
7.13 Problem 6.1.1 reference solution Shift power peaking factors for axial segments in element 1 for charge-pan II. . . . . . . . . . . . . . . . . . . . . . 223

7.14 Problem 6.1.1 reference solution MPACT power peaking factors for axial segments in element 1 for charge-pan II. . . . . . . . . . . . . . . . . . . . . . . . 224

7.15 Problem 6.1.1 reference solution Shift power peaking factors for axial segments in element 2 for charge-pan II. . . . . . . . . . . . . . . . . . . . . 225

7.16 Problem 6.1.1 reference solution MPACT power peaking factors for axial segments in element 2 for charge-pan II. . . . . . . . . . . . . . . . . . . 226

7.17 Problem 6.1.1 reference solution Shift power peaking factors for axial segments in element 3 for charge-pan II. . . . . . . . . . . . . . . . . . . . . 227

7.18 Problem 6.1.1 reference solution MPACT power peaking factors for axial segments in element 3 for charge-pan II. . . . . . . . . . . . . . . . . . . . . . 228

7.19 Problem 6.1.1 reference solution Shift power peaking factors for axial segments in element 4 for charge-pan II. . . . . . . . . . . . . . . . . . . . . . . . . . . . . 229

7.20 Problem 6.1.1 reference solution MPACT power peaking factors for axial segments in element 4 for charge-pan II. . . . . . . . . . . . . . . . . . . . . . . 230

7.21 Problem 6.1.1 reference solution Shift power peaking factors for axial segments in element 5 for charge-pan II. . . . . . . . . . . . . . . . . . . . 231

7.22 Problem 6.1.1 reference solution MPACT power peaking factors for axial segments in element 5 for charge-pan II. . . . . . . . . . . . . . . . . . . . . 232

7.23 Problem 6.1.1 reference solution Shift power peaking factors for axial segments in element 6 for charge-pan II. . . . . . . . . . . . . . . . . . . . . . 233

7.24 Problem 6.1.1 reference solution MPACT power peaking factors for axial segments in element 6 for charge-pan II. . . . . . . . . . . . . . . . . . . . . . . 234

7.25 Problem 6.1.1 reference solution Shift power peaking factors for axial segments in element 1 for charge-pan III. . . . . . . . . . . . . . . . . . . . . . 235

7.26 Problem 6.1.1 reference solution MPACT power peaking factors for axial segments in element 1 for charge-pan III. . . . . . . . . . . . . . . . . . . . 236

7.27 Problem 6.1.1 reference solution Shift power peaking factors for axial segments in element 2 for charge-pan III. . . . . . . . . . . . . . . . . . . . . 237

7.28 Problem 6.1.1 reference solution MPACT power peaking factors for axial segments in element 2 for charge-pan III. . . . . . . . . . . . . . . . . . . . . 238

7.29 Problem 6.1.1 reference solution Shift power peaking factors for axial segments in element 3 for charge-pan III. . . . . . . . . . . . . . . . . . . . . . . . . 239

7.30 Problem 6.1.1 reference solution MPACT power peaking factors for axial segments in element 3 for charge-pan III. . . . . . . . . . . . . . . . . . . . . . . 240

7.31 Problem 6.1.1 reference solution Shift power peaking factors for axial segments in element 4 for charge-pan III. . . . . . . . . . . . . . . . . . . . . . . . . 241

7.32 Problem 6.1.1 reference solution MPACT power peaking factors for axial segments in element 4 for charge-pan III. . . . . . . . . . . . . . . . . . . . . . 242

7.33 Problem 6.1.1 reference solution Shift power peaking factors for axial segments in element 5 for charge-pan III. . . . . . . . . . . . . . . . . . . . . . . 243

7.34 Problem 6.1.1 reference solution MPACT power peaking factors for axial segments in element 5 for charge-pan III. . . . . . . . . . . . . . . . . . . . . . . 244 
7.35 Problem 6.1.1 reference solution Shift power peaking factors for axial segments in element 6 for charge-pan III. . . . . . . . . . . . . . . . . . . . . . . 245

7.36 Problem 6.1.1 reference solution MPACT power peaking factors for axial segments in element 6 for charge-pan III. . . . . . . . . . . . . . . . . . . . . . 246

7.37 Problem 6.1.1 reference solution Shift power peaking factors for axial segments in element 1 for charge-pan IV. . . . . . . . . . . . . . . . . . . . . . 247

7.38 Problem 6.1.1 reference solution MPACT power peaking factors for axial segments in element 1 for charge-pan IV. . . . . . . . . . . . . . . . . . . . . . . . 248

7.39 Problem 6.1.1 reference solution Shift power peaking factors for axial segments in element 2 for charge-pan IV. . . . . . . . . . . . . . . . . . . . . . . . 249

7.40 Problem 6.1.1 reference solution MPACT power peaking factors for axial segments in element 2 for charge-pan IV. . . . . . . . . . . . . . . . . . . . 250

7.41 Problem 6.1.1 reference solution Shift power peaking factors for axial segments in element 3 for charge-pan IV. . . . . . . . . . . . . . . . . . . . 251

7.42 Problem 6.1.1 reference solution MPACT power peaking factors for axial segments in element 3 for charge-pan IV. . . . . . . . . . . . . . . . . . . . . . 252

7.43 Problem 6.1.1 reference solution Shift power peaking factors for axial segments in element 4 for charge-pan IV. . . . . . . . . . . . . . . . . . . . 253

7.44 Problem 6.1.1 reference solution MPACT power peaking factors for axial segments in element 4 for charge-pan IV. . . . . . . . . . . . . . . . . . . . . 254

7.45 Problem 6.1.1 reference solution Shift power peaking factors for axial segments in element 5 for charge-pan IV. . . . . . . . . . . . . . . . . . . . 255

7.46 Problem 6.1.1 reference solution MPACT power peaking factors for axial segments in element 5 for charge-pan IV. . . . . . . . . . . . . . . . . . . 256

7.47 Problem 6.1.1 reference solution Shift power peaking factors for axial segments in element 6 for charge-pan IV. . . . . . . . . . . . . . . . . . . . 257

7.48 Problem 6.1.1 reference solution MPACT power peaking factors for axial segments in element 6 for charge-pan IV. . . . . . . . . . . . . . . . . . . . . 258

7.49 Neutron multiplication factor for the reference solution of Problem 6.2.1 . . . . . . . . 259

7.50 Timing variables for the reference solution of Problem 6.2.1 . . . . . . . . . . 260

7.51 Problem 6.2.1 reference solution power peaking factors in element 1 for charge-pan I (1/2). . 261

7.52 Problem 6.2.1 reference solution power peaking factors in element 1 for charge-pan I (2/2). . 262

7.53 Problem 6.2.1 reference solution power peaking factors in element 2 for charge-pan (1/2). . . 263

7.54 Problem 6.2.1 reference solution power peaking factors in element 2 for charge-pan I (2/2). . 264

7.55 Problem 6.2.1 reference solution power peaking factors in element 3 for charge-pan (1/2). . . 265

7.56 Problem 6.2.1 reference solution power peaking factors in element 3 for charge-pan I (2/2). . 266

7.57 Problem 6.2.1 reference solution power peaking factors in element 4 for charge-pan (1/2). . . 267

7.58 Problem 6.2.1 reference solution power peaking factors in element 4 for charge-pan I (2/2). . 268

7.59 Problem 6.2.1 reference solution power peaking factors in element 5 for charge-pan (1/2). . . 269

7.60 Problem 6.2.1 reference solution power peaking factors in element 5 for charge-pan I (2/2). . 270

7.61 Problem 6.2.1 reference solution power peaking factors in element 1 for charge-pan II (1/2). 271

7.62 Problem 6.2.1 reference solution power peaking factors in element 1 for charge-pan II (2/2). 272

7.63 Problem 6.2.1 reference solution power peaking factors in element 2 for charge-pan II (1/2). 273

7.64 Problem 6.2.1 reference solution power peaking factors in element 2 for charge-pan II (2/2). 274

7.65 Problem 6.2.1 reference solution power peaking factors in element 3 for charge-pan II (1/2). 275 
7.66 Problem 6.2.1 reference solution power peaking factors in element 3 for charge-pan II (2/2). 276

7.67 Problem 6.2.1 reference solution power peaking factors in element 4 for charge-pan II (1/2). 277

7.68 Problem 6.2.1 reference solution power peaking factors in element 4 for charge-pan II (2/2). 278

7.69 Problem 6.2.1 reference solution power peaking factors in element 5 for charge-pan II (1/2). 279

7.70 Problem 6.2.1 reference solution power peaking factors in element 5 for charge-pan II (2/2). 280

7.71 Problem 6.2.1 reference solution power peaking factors in element 6 for charge-pan II (1/2). 281

7.72 Problem 6.2.1 reference solution power peaking factors in element 6 for charge-pan II (2/2). 282

7.73 Problem 6.2.1 reference solution power peaking factors in element 1 for charge-pan III (1/2). 283

7.74 Problem 6.2.1 reference solution power peaking factors in element 1 for charge-pan III (2/2). 284

7.75 Problem 6.2.1 reference solution power peaking factors in element 2 for charge-pan III (1/2). 285

7.76 Problem 6.2.1 reference solution power peaking factors in element 2 for charge-pan III (2/2). 286

7.77 Problem 6.2.1 reference solution power peaking factors in element 3 for charge-pan III (1/2). 287

7.78 Problem 6.2.1 reference solution power peaking factors in element 3 for charge-pan III (2/2). 288

7.79 Problem 6.2.1 reference solution power peaking factors in element 4 for charge-pan III (1/2). 289

7.80 Problem 6.2.1 reference solution power peaking factors in element 4 for charge-pan III (2/2). 290

7.81 Problem 6.2.1 reference solution power peaking factors in element 5 for charge-pan III (1/2). 291

7.82 Problem 6.2.1 reference solution power peaking factors in element 5 for charge-pan III (2/2). 292

7.83 Problem 6.2.1 reference solution power peaking factors in element 6 for charge-pan III (1/2). 293

7.84 Problem 6.2.1 reference solution power peaking factors in element 6 for charge-pan III (2/2). 294

7.85 Problem 6.2.1 reference solution power peaking factors in element 1 for charge-pan IV (1/2). 295

7.86 Problem 6.2.1 reference solution power peaking factors in element 1 for charge-pan IV (2/2). 296

7.87 Problem 6.2.1 reference solution power peaking factors in element 2 for charge-pan IV (1/2). 297

7.88 Problem 6.2.1 reference solution power peaking factors in element 2 for charge-pan IV (2/2). 298

7.89 Problem 6.2.1 reference solution power peaking factors in element 3 for charge-pan IV (1/2). 299

7.90 Problem 6.2.1 reference solution power peaking factors in element 3 for charge-pan IV (2/2). 300

7.91 Problem 6.2.1 reference solution power peaking factors in element 4 for charge-pan IV (1/2). 301

7.92 Problem 6.2.1 reference solution power peaking factors in element 4 for charge-pan IV (2/2). 302

7.93 Problem 6.2.1 reference solution power peaking factors in element 5 for charge-pan IV (1/2). 303

7.94 Problem 6.2.1 reference solution power peaking factors in element 5 for charge-pan IV (2/2). 304

7.95 Problem 6.2.1 reference solution power peaking factors in element 6 for charge-pan IV (1/2). 305

7.96 Problem 6.2.1 reference solution power peaking factors in element 6 for charge-pan IV (2/2). 306

8.1 Summary of BOC cases of progression problems and eigenvalue comparisons. . . . . . . 308

8.2 Summary of depletion cases of progression problems and eigenvalue comparisons with root mean square $(\mathrm{RMS})$ deviations. . . . . . . . . . . . . . . . . . 308 


\section{ACRONYMS}

\begin{tabular}{|c|c|}
\hline ARI & all rods in \\
\hline ARO & all rods out \\
\hline BOC & beginning of cycle \\
\hline $\mathrm{CP}$ & charge-pan \\
\hline EOC & end of cycle \\
\hline GWd/MTU & gigawatt-day per metric ton of uranium \\
\hline MC & Monte Carlo \\
\hline MWd/MTU & megawatt-day per metric ton of uranium \\
\hline NNSA & National Nuclear Security Administration \\
\hline $\begin{array}{l}\text { ORNL } \\
\text { pcm }\end{array}$ & $\begin{array}{l}\text { Oak Ridge National Laboratory } \\
\text { per cent mille }\left(10^{-5}\right)\end{array}$ \\
\hline
\end{tabular}




\section{ACKNOWLEDGMENTS}

The authors would like to acknowledge support for this work provided by the Office of Defense Nuclear Nonproliferation Research and Development of the US Department of Energy's National Nuclear Security Administration. The authors acknowledge support from MPACT developers Aaron Graham, Tarek Ghaddar, and Shane Stimpson (formerly) and Shift developers Seth Johnson and Greg Davidson of Oak Ridge National Laboratory. Mark Baird, Jordan Lefebvre, and the Compute and Data Environment for Science (CADES) at Oak Ridge National Laboratory helped provide computational support. Reviews of the document by Austin Lo and Richard Reed are much appreciated. Emily Huckabay was instrumental in formatting the document for release.

This research used resources of the Compute and Data Environment for Science (CADES) at the Oak Ridge National Laboratory, which is supported by the Office of Science of the U.S. Department of Energy under Contract No. DE-AC05-00OR22725. 


\section{EXECUTIVE SUMMARY}

The goal of this document is to provide a set of comprehensive benchmarking neutronics problems for gas-cooled, graphite-moderated reactors. The problems are designed to be representative problems that increase in complexity to test performance of various codes. For the simulation of the benchmarking problems, the Shift Monte Carlo code and MPACT deterministic code are chosen because of their state-of-the-art capabilities, which are optimized for various reactor designs. Because these codes have been developed primarily for light water reactor applications, their application to graphite-moderated, gas-cooled reactors has not been extensively explored. This report attempts to evaluate benchmarks for a Magnox-style reactor, one that is graphite-moderated and gas-cooled. Parameters that are calculated and presented in this benchmark include the eigenvalue, peaking factors, and isotope concentrations. Results show good agreement between Shift and MPACT, and demonstrate the capability of MPACT to model graphite-moderated systems well. 


\section{INTRODUCTION}

High-fidelity tools have been developed to accommodate the next generation of modeling and simulation for advanced nuclear reactors. Several of these advanced reactor concepts use a graphite moderator, gas coolant, or a combination of both. Advanced modeling and simulation tools will require validation for these types of reactors, as well as others reactors with novel fuel designs, moderators, spectra, and geometry.

The goal of this document is threefold. The first goal is to create, model, and simulate a set of progression problems for graphite-moderated, gas-cooled reactors such that the results from any neutron transport code can be compared to these reference solutions. The second goal is to test the performance and capability of the MPACT code in comparison with a Monte Carlo code. As discussed later, MPACT was originally developed for light water reactors but can be leveraged for any reactor design. The third goal is to provide a set of benchmarks to which experimental measurements of spent fuel from Magnox reactors can be compared against in the future.

Previous series of progression problems have been developed for pressurized water reactor (PWR) and reference solutions provided [5]. An earlier iteration of 2D progression problems were compared with the Serpent Monte Carlo code [11, 12]. Development of full-core simulations has compared performance for larger-scale problems [1]. More information on the Magnox reactor design can also be found in Refs. $[7,1]$.

The set of progression problems contains six main geometries, each one progressively more complex than the previous problem. Progression problems include both two-dimensional (2D) and three-dimensional (3D) geometries. The set of geometries is intended to span from the simplest geometry, a fuel pin cell, to the most complex, a 3D (quarter) core. The set of six problem geometries is listed in Table 1.1.

The progression problems will come in the form of three numbers: X.X.X. The first number is the geometry indicator as listed in Table 1.1. The second number indicates whether the problem is a beginning of cycle (BOC) calculation (1) or a depletion calculation (2). The third number indicates the problem progression in terms of complexity. This number can indicate a variety of things, from a variation in temperatures to a change in the control rod insertion depth. Generally, the goal with each progression problem is to compare performance with each degree of complexity.

Table 1.1. Summary of naming convention for progression problems for geometry and solution method.

\begin{aligned} & \hline Problem Geometry \\ & \hline 1.X.X 2D Fuel Cell \\ & 2.X.X 2D Charge Pan \\ & 3.X.X 2D Quarter Core \\ & 4.X.X 3D Fuel Channel \\ & 5.X.X 3D Charge Pan \\ & 6.X.X Mini-Core \\ & \hline X.1.X BOC \\ & X.2.X Depletion \\ & \hline\end{aligned}




\subsection{MODELING AND SIMULATION TOOLS}

In the recent decades, advances in modeling and simulation tools have greatly increased the capability of neutron transport for reactor performance and safety analyses. Focus on the increased accuracy, flexibility, and solution convergence permits the study on an ever-increasing variety of designs for the next generation of reactors. The two codes were developed primarily for PWR cores through the Consortium for Advanced Light Water Reactor (CASL) energy simulation hub. Both the Shift Monte Carlo code and MPACT are capable of modeling complex geometries and are used to solve the benchmark problems in this report.

Throughout this report, the Shift and MPACT solutions were compared. Parameters to be compared include the neutron multiplication factor, i.e., eigenvalue $\left(k_{\mathrm{eff}}\right)$, isotopics, and power peaking factors. Owing to the nature of an MC (Shift) vs. deterministic (MPACT) code, listing of the uncertainty will be different. Shift will list the statistical uncertainty provided by the calculation, and MPACT will list the eigenvalue convergence criteria used in the input. For the 3D problems with complex geometries, not all values are provided for brevity.

This report documents the applicability of the capability of these codes to simulate Magnox reactors although they were originally developed to simulate LWRs. While Magnox and LWR are different designs, MPACT implementation in particular is alleviated by some similar design features. Key similarities include solid cylindrical fuel, square lattice, and thermal neutron spectra. Evident differences in a Magnox reactor include moderator type (graphite), coolant type $\left(\mathrm{CO}_{2}\right)$, lower operating temperature, metal fuel, and larger core size.

\subsubsection{SHIFT}

The Shift Monte Carlo (MC) code is a massively parallel transport package developed at Oak Ridge National Laboratory (ORNL) to enable high-performance neutronic analysis [15]. Shift is used as the MC solver in the Exnihilo code suite, which also contains the Denovo deterministic transport solver [3]. Shift leverages deterministic capabilities for variance reduction. Shift is a general purpose radiation transport solver that can solve eigenvalue and fixed source calculations with the options for neutron and photon transport. More recently, Shift couples to the ORIGEN module [4] for nuclide depletion calculations and has also been incorporated into the SCALE [17] code system. The Omnibus [8] front-end runner to the Exnihilo code suite is used to generate cell power tallies and isotope concentration tracking for depletion simulations.

These progression problems typically use 300 skipped cycles and 1200 active cycles. The number of particles per generation ranges from $2 \times 10^{4}$ to $5 \times 10^{5}$ to achieve at least a threshold of 10 and $20 \mathrm{pcm}$ errors for the neutron multiplication factor for BOC and depletion problems, respectively.

\subsubsection{MPACT}

MPACT is a 3D full-core neutron transport code that uses the " $2 \mathrm{D} / 1 \mathrm{D}$ " method $[2,10]$. This method uses the method of characteristics (MOC) to solve the radial (2D) solution. The coarse mesh finite difference acceleration is used in conjunction with the axial transport solver to various orders. MPACT was developed jointly by the University of Michigan and ORNL. MPACT requires group-specific cross section libraries, 
and a specific 69-group neutron flux library has been developed for Magnox reactors [9]. For depletion analyses, MPACT is also coupled to ORIGEN [4].

For all problems, a ray spacing of at least 0.03 is used. In several problems, a ray spacing of 0.01 is used to obtain better results. For the 3D progression problems, as stated later, the full height P3 equations are used to obtain better results. Additionally, MPACT uses a uniform axial

\subsection{GEOMETRIES}

Table 1.2 lists the relevant geometric parameters for the Magnox reactor core. The fuel used is close to natural uranium, and the cladding is Magnox (listed in Table 1.3). Unlike a traditional light water reactor (LWR), which has water as a coolant and a moderator, the Magnox fuel pin has a radial gas channel, and outside of the channel is the graphite reflector. The reactor design has three variable coolant channel radii, labeled A, B, and C. These problems only factor in neutronic calculations, thus the thermal hydraulic feedback is not calculated. However, the fuel channel radius greatly influences the moderating capability of the surrounding graphite.

The fuel channels in the Magnox design are organized into charge pans (analogous to LWR assemblies), which contain $16(4 \times 4)$ channels. At the center of a charge pan is a control rod channel for reactivity control, and it can be left empty for gas to flow. The charge pans are organized in a square pitch within the core. Each fuel element is just over one meter in length. All charge pans contain channels with either five or six fuel elements stacked axially with spacers (made of Magnox) in between the elements. Each relevant geometry will be discussed more in its own section.

Table 1.2. Geometrical parameters of gas-cooled, graphite-moderated reactor.

\begin{tabular}{llllll}
\hline \multicolumn{1}{c}{ Geometry } & Symbol & Value & Units & References \\
\hline Radius & & & & & \\
& Fuel & $r_{\text {fuel }}$ & 1.46 & $\mathrm{~cm}$ & [7] p. 12 \\
& Clad & $r_{\text {clad }}$ & 2.04 & $\mathrm{~cm}$ & [14] p. 4 \\
& Coolant Channel Zone A & $r_{\text {cool, A }}$ & 5.2832 & $\mathrm{~cm}$ & [7] p. 22 \\
& Coolant Channel Zone B & $r_{\text {cool, B }}$ & 5.0165 & $\mathrm{~cm}$ & [7] p. 22 \\
& Coolant Channel Zone C & $r_{\text {cool,C }}$ & 4.5847 & $\mathrm{~cm}$ & [7] p. 22 \\
& Control Rod Channel & $r_{\text {cr,chan }}$ & 4.1275 & $\mathrm{~cm}$ & From drawings \\
& Control Rod Channel & $r_{\text {cr,OD }}$ & 4.1275 & $\mathrm{~cm}$ & From drawings \\
Pitch & (Square) & & & & \\
& Pin & $p_{\text {pin }}$ & 20.32 & $\mathrm{~cm}$ & [7] p. 12 \\
& Charge-Pan & $p_{\text {pan }}$ & 81.28 & $\mathrm{~cm}$ & \\
& Fuel Element Active Length & $L_{\text {elem,act }}$ & $\mathrm{cm}$ & 101.6254 & \\
& Fuel Element Total Length & $L_{\text {elem,tot }}$ & $\mathrm{cm}$ & 109.3724 & \\
& Core Axial Upper Reflector & $L_{\text {refl,up }}$ & $\mathrm{cm}$ & 66.5 & \\
& Core Axial Lower Reflector & $L_{\text {refl,lo }}$ & $\mathrm{cm}$ & 77.5 & \\
& Channel Total Length & $L_{\text {chan }}$ & $\mathrm{cm}$ & 800.2344 & \\
\hline
\end{tabular}




\subsection{MATERIALS}

The progression problems contain five primary materials throughout the problems, which are described in Table 1.3. Unless otherwise noted, the same material properties are used in all problems. Although temperatures may vary in the problems, these progression problems do not consider geometric or density changes due to temperature changes. Although the graphite density will decrease with irradiation, the graphite composition is not depleted or altered with burnup for this work.

Table 1.3. Compositions of materials used in progression problems.

\begin{tabular}{|c|c|c|c|c|c|c|}
\hline Material & & Composition & & Value & Units & References \\
\hline \multirow{4}{*}{\multicolumn{2}{|c|}{ Fuel }} & \multirow[t]{4}{*}{ Uranium Metal } & & & & [7] p. 12 \\
\hline & & & ${ }^{234} \mathrm{U}$ & 0.0057 & wt $\%$ & [13] p. 331 \\
\hline & & & ${ }^{235} \mathrm{U}$ & 0.7204 & wt $\%$ & [13] p. 331 \\
\hline & & & ${ }^{238} \mathrm{U}$ & 99.2739 & wt $\%$ & [13] p. 331 \\
\hline \multirow{4}{*}{\multicolumn{2}{|c|}{ Clad }} & \multirow[t]{4}{*}{ Magnox-C Alloy } & & & & [13] p. 21 \\
\hline & & & ${ }^{\mathrm{Nat}} \mathrm{Al}$ & 0.8 & $\mathrm{wt} \%$ & [18] p. 711 \\
\hline & & & ${ }^{\mathrm{Nat}} \mathrm{Be}$ & 0.005 & $\mathrm{wt} \%$ & [18] p. 711 \\
\hline & & & ${ }^{\mathrm{Nat}} \mathrm{Mg}$ & 99.195 & $\mathrm{wt} \%$ & [18] p. 711 \\
\hline \multirow{3}{*}{\multicolumn{2}{|c|}{ Coolant }} & \multirow[t]{3}{*}{ Carbon Dioxide } & & & & [7] p. 12 \\
\hline & & & ${ }^{\mathrm{Nat}} \mathrm{C}$ & 27.2912 & $\mathrm{wt} \%$ & [13] p. 67 \\
\hline & & & ${ }^{\mathrm{Nat}} \mathrm{O}$ & 72.7088 & $\mathrm{wt} \%$ & [13] p. 67 \\
\hline \multirow{2}{*}{\multicolumn{2}{|c|}{ Moderator }} & \multirow[t]{2}{*}{ Graphite } & & & & [7] p. 12 \\
\hline & & & ${ }^{\mathrm{Nat}} \mathrm{C}$ & 100.0 & $\mathrm{wt} \%$ & [13] p. $70^{a}$ \\
\hline \multicolumn{7}{|l|}{ Control Rod } \\
\hline & Sheath & Stainless Steel & & & & {$[13]$} \\
\hline & Absorber & $3 \mathrm{wt} \%$ Boronated Steel & & & & [13] \\
\hline
\end{tabular}

Table 1.4. Densities.

\begin{tabular}{lccl}
\hline \multicolumn{1}{c}{ Densities } & Value & Units & \multicolumn{1}{c}{ References and Notes } \\
\hline Uranium Metal & 18.9 & $\mathrm{~g} / \mathrm{cm}^{3}$ & [6] p. $15[16] \mathrm{p} .657$ \\
Magnox-C Alloy & 1.74 & $\mathrm{~g} / \mathrm{cm}^{3}$ & [16] p. 657 \\
Carbon Dioxide & 0.0081 & $\mathrm{~g} / \mathrm{cm}^{3}$ & Estimated \\
Graphite & 1.65 & $\mathrm{~g} / \mathrm{cm}^{3}$ & [16] p. $173 \mathrm{p} .192 \mathrm{p} .657$ \\
\hline
\end{tabular}




\subsection{OPERATING CONDITIONS}

The progression problems use a consistent power based on the amount of the fuel in model, regardless of $2 \mathrm{D}$ or $3 \mathrm{D}$ configurations. The full core power of $182 \mathrm{MWt}$ is used to calculate the powers of all progression problems [7]. The power levels for each problem are listed in Table 1.6. Note, problems 1-3 are 2D problems; therefore, linear power is used. For problems 4-6, the 3D geometries use absolute power.

Both single state-point eigenvalue (i.e., BOC) and depletion calculations are performed. The naming convention for BOC and depletion is described in Table 1.1. Table 1.5 lists the burnup steps for the depletion cases. Note that the end-of-cycle (EOC) burnup is low at $1330 \mathrm{MWd} / \mathrm{MtU}$, which is orders of magnitudes lower than commercial light water reactor fuel at discharge. Table 1.6 shows the power values for each problem, all calculated from the total core power. Only fuel materials are depleted for all cases, and no decay time following irradiation is considered.

Table 1.5. Burnup points of comparison.

\begin{tabular}{rc}
\hline Step & $\begin{array}{c}\text { Burnup } \\
{[\mathrm{MWd} / \mathrm{MTU}]}\end{array}$ \\
\hline 0 & 0.0 \\
1 & 25.0 \\
2 & 50.0 \\
3 & 100.0 \\
4 & 200.0 \\
5 & 300.0 \\
6 & 400.0 \\
7 & 500.0 \\
8 & 600.0 \\
9 & 700.0 \\
10 & 800.0 \\
11 & 900.0 \\
12 & 1000.0 \\
13 & 1100.0 \\
14 & 1200.0 \\
15 & 1300.0 \\
\hline
\end{tabular}


Table 1.6. Power levels for various progression problems derived from total core power [7]

\begin{tabular}{lrlll}
\hline \multicolumn{1}{c}{ Parameter } & \multicolumn{1}{c}{ Value } & Units & Problem no. \\
\hline Power Density & 1.45 & $\left.\frac{\mathrm{MW}}{\mathrm{MtU}}\right]$ & - \\
Rod 2D & 183.840 & $\left.\frac{\mathrm{W}}{\mathrm{cm}}\right]$ & 1 \\
Charge Pan 2D & 2.941 & $\left.\frac{\mathrm{kW}}{\mathrm{cm}}\right]$ & 2 \\
Quarter Core 2D & 77.948 & $\left.\frac{\mathrm{kW}}{\mathrm{cm}}\right]$ & 3 \\
Six-Element Channel 3D & 112.069 & {$[\mathrm{~kW}]$} & 4 \\
Charge Pan 3D & 1.625 & {$[\mathrm{MW}]$} & 5 \\
Mini-Core 3D & 6.874 & {$[\mathrm{MW}]$} & 6 \\
Core Power & 182 & {$[\mathrm{MW}]$} & \\
& & & \\
\hline
\end{tabular}





\section{PROBLEM 1: PIN-CELL 2D}

Problem 1 demonstrates the capability to solve 2D Magnox pin-cell problems. Geometric dimensions are listed in Table 1.2, and the geometry is illustrated in Figure 1. Material densities are listed in Table 1.4. All external boundaries are fully reflected in this problem.

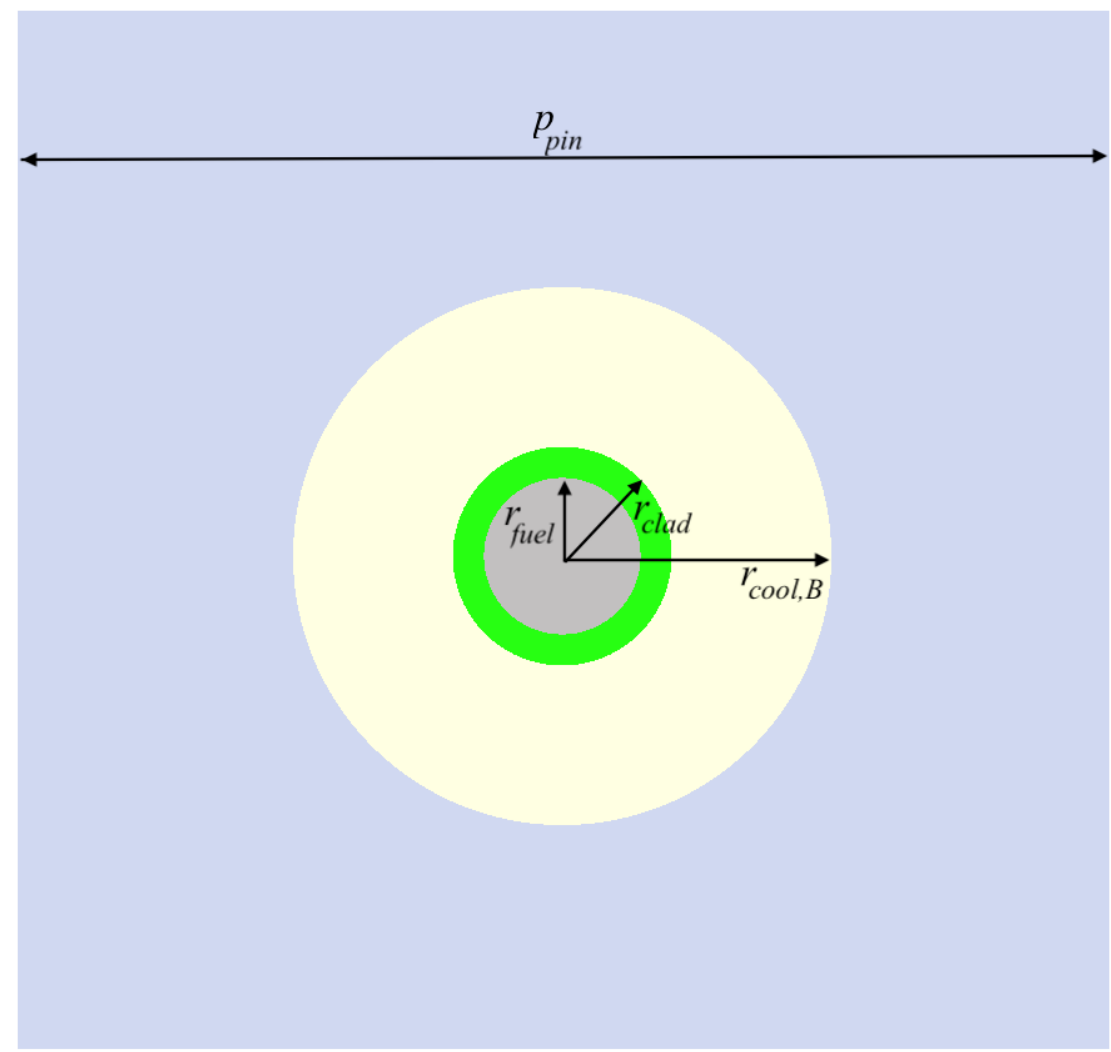

Figure 1. Magnox pin-cell geometry with Zone B coolant channel. 


\subsection{BEGINNING-OF-CYCLE PROBLEMS}

\subsubsection{Problem 1.1.1}

\subsubsection{Problem Description}

For all regions, Problem 1.1.1 uses temperatures shown in Table 2.1. These temperature values are selected because continuous-energy incident neutron libraries exist at these temperatures for the reference solution. Comparing results at these temperatures attempts to eliminate errors due to on-the-fly Doppler broadening techniques.

Table 2.1. Temperatures for Problem 1.1.1.

\begin{tabular}{lll}
\hline Temperature & Value & Units \\
\hline Fuel & 900.0 & $\mathrm{~K}$ \\
Clad & 600.0 & $\mathrm{~K}$ \\
Coolant & 600.0 & $\mathrm{~K}$ \\
Moderator & 600.0 & $\mathrm{~K}$ \\
\hline
\end{tabular}

\subsubsection{Reference Solution}

This first progression problem compares a Magnox pin cell at library temperatures. The neutron multiplication factors are shown in Table 2.2. They agree to $33 \mathrm{pcm}$. The uncertainties in parentheses refer to the absolute error of the highest precision digit(s) for each code or combined error in calculating the difference. The specific run parameters variables and wall time are shown in Table 2.3. MPACT converges to its solution with an order of magnitude less computational time.

Table 2.2. Neutron multiplication for the reference solution of Problem 1.1.1.

\begin{tabular}{rrrrr}
\hline Step & $\begin{array}{c}\text { Burnup } \\
{\left[\frac{\mathrm{MWd}}{\mathrm{MtU}}\right]}\end{array}$ & $\begin{array}{c}\text { Shift } \\
\mathrm{k}_{\mathrm{eff}}(1 \sigma)\end{array}$ & $\begin{array}{c}\text { MPACT } \\
\mathrm{k}_{\mathrm{eff}}(1 \sigma)\end{array}$ & $\begin{array}{c}\text { Difference } \\
{[\mathrm{pcm}]}\end{array}$ \\
\hline 0 & 0.0 & $1.06271(7)$ & 1.06304 & $33(7)$ \\
\hline
\end{tabular}


Table 2.3. Timing variables for the reference solution of Problem 1.1.1

\begin{tabular}{ccccccc}
\hline \hline \multicolumn{1}{c}{ Shift } & & & \\
\hline $\begin{array}{c}\text { Depletion } \\
\text { Solves }\end{array}$ & $\begin{array}{c}\text { Total } \\
\text { Cycles per } \\
\text { Transport } \\
\text { Solve }\end{array}$ & $\begin{array}{c}\text { Active } \\
\text { Cycles per } \\
\text { Transport } \\
\text { Solve }\end{array}$ & $\begin{array}{c}\text { Histories } \\
\text { per Cycle }\end{array}$ & $\begin{array}{c}\text { Compute } \\
\text { Nodes }\end{array}$ & $\begin{array}{c}\text { Cores } \\
\text { per Node }\end{array}$ & $\begin{array}{c}\text { Wall } \\
\text { Total Time }\end{array}$ \\
\hline 1 & 1500 & 1200 & 100000 & 1 & 48 & 2.23 \\
\hline $\begin{array}{c}k_{\text {eff }} \\
\text { Sepletion }\end{array}$ & $\begin{array}{c}\text { Source } \\
\text { Convergence } \\
\text { Criteria }\end{array}$ & $\begin{array}{c}\text { Convergence } \\
\text { Criteria }\end{array}$ & $\begin{array}{c}\text { Ray } \\
\text { Spacing } \\
{[\mathrm{cm}]}\end{array}$ & $\begin{array}{c}\text { Compute } \\
\text { Nodes }\end{array}$ & $\begin{array}{c}\text { Cores } \\
\text { per Node }\end{array}$ & $\begin{array}{c}\text { Total Time } \\
\text { [hours] }\end{array}$ \\
\hline 1 & $1.0 \times 10^{-06}$ & $1.0 \times 10^{-06}$ & 0.01 & 1 & 1 & 0.05 \\
\hline
\end{tabular}




\subsubsection{Problem 1.1.2}

\subsubsection{Problem Description}

The only difference between this problem and Problem 1.1.1 is the temperatures used. Temperatures used in this problem are typical of the operating conditions of a Magnox reactor. Both codes can use on-the-fly Doppler broadening to modify the cross-sections for the given temperatures. For all regions, Problem 1.1.2 uses average temperatures shown in Table 2.4.

Table 2.4. Temperatures for Problem 1.1.2.

\begin{tabular}{lcll}
\hline Temperature & Value & Units & References \& Notes \\
\hline Fuel & 698.15 & $\mathrm{~K}$ & [7] p. 22 \\
Clad & 533.59 & $\mathrm{~K}$ & Estimated \\
Coolant & 511.15 & $\mathrm{~K}$ & Estimated \\
Moderator & 523.15 & $\mathrm{~K}$ & [7] p. 22 \\
\hline
\end{tabular}

\subsubsection{Reference Solution}

The goal of this problem is to utilize operating temperatures in a Magnox reactor and to compare the codes at these temperatures. The neutron multiplication factors are shown in Table 2.5 The codes agree to approximately $13 \mathrm{pcm}$. The specific run parameters variables and wall time are shown in Table 2.6. MPACT converges to its solution with an order of magnitude less computational time.

Table 2.5. Neutron multiplication for the reference solution of Problem 1.1.2.

\begin{tabular}{rrrrr}
\hline Step & $\begin{array}{c}\text { Burnup } \\
{\left[\frac{\mathrm{MWd}}{\mathrm{MtU}}\right]}\end{array}$ & $\begin{array}{c}\text { Shift } \\
\mathrm{k}_{\mathrm{eff}}(1 \sigma)\end{array}$ & $\begin{array}{c}\text { MPACT } \\
\mathrm{k}_{\mathrm{eff}}(1 \sigma)\end{array}$ & $\begin{array}{c}\text { Difference } \\
{[\mathrm{pcm}]}\end{array}$ \\
\hline 0 & 0.0 & $1.06769(7)$ & 1.06782 & $13(7)$ \\
\hline
\end{tabular}


Table 2.6. Timing variables for the reference solution of Problem 1.1.2

\begin{tabular}{|c|c|c|c|c|c|c|}
\hline \multicolumn{7}{|c|}{ Shift } \\
\hline $\begin{array}{c}\text { Depletion } \\
\text { Solves }\end{array}$ & $\begin{array}{c}\text { Total } \\
\text { Cycles per } \\
\text { Transport } \\
\text { Solve } \\
\end{array}$ & $\begin{array}{c}\text { Active } \\
\text { Cycles per } \\
\text { Transport } \\
\text { Solve }\end{array}$ & $\begin{array}{l}\text { Histories } \\
\text { per Cycle }\end{array}$ & $\begin{array}{c}\text { Compute } \\
\text { Nodes }\end{array}$ & $\begin{array}{c}\text { Cores } \\
\text { per Node }\end{array}$ & $\begin{array}{c}\text { Wall } \\
\text { Total Time } \\
\text { [hours] }\end{array}$ \\
\hline 1 & 1500 & 1200 & 100000 & 1 & 48 & 2.17 \\
\hline \multicolumn{7}{|c|}{ MPACT } \\
\hline $\begin{array}{c}\text { Depletion } \\
\text { Solves }\end{array}$ & $\begin{array}{c}k_{\mathrm{eff}} \\
\text { Convergence } \\
\text { Criteria }\end{array}$ & $\begin{array}{c}\text { Source } \\
\text { Convergence } \\
\text { Criteria }\end{array}$ & $\begin{array}{c}\text { Ray } \\
\text { Spacing } \\
{[\mathrm{cm}]}\end{array}$ & $\begin{array}{c}\text { Compute } \\
\text { Nodes }\end{array}$ & $\begin{array}{c}\text { Cores } \\
\text { per Node }\end{array}$ & $\begin{array}{c}\text { Wall } \\
\text { Total Time } \\
\text { [hours] }\end{array}$ \\
\hline 1 & $1.0 \times 10^{-06}$ & $1.0 \times 10^{-06}$ & 0.01 & 1 & 1 & 0.05 \\
\hline
\end{tabular}




\subsection{DEPLETION}

Burnup states used for comparison are listed in Table 1.5 and the total system specific power is listed in Table 1.6.

\subsubsection{Problem 1.2.1}

\subsubsection{Problem Description}

Temperatures used for this problem are listed in Table 2.4.

\subsubsection{Reference Solution}

The neutron multiplication factor agrees well between Shift and MPACT. The BOC difference is $46 \mathrm{pcm}$, and the EOC difference increases to $150 \mathrm{pcm}$. The burnups for the pin match identically, which is used for normalization.

The isotope concentrations in the pin cell are also compared. The percent difference for major actinides and fission products are shown in Figure 2. The percent differences are also tabulated in Tables 2.19-2.22. Some isotope differences are omitted as some minor fission products $\left({ }^{84,86,87} \mathrm{Sr},{ }^{132,134,135} \mathrm{Ba},{ }^{85} \mathrm{Rb}\right)$ have not been benchmarked for MPACT/VERA due to being low priority. Other than these isotopes, all the major actinides and fission products are within 3-4\% for most of the burn cycle.

Table 2.7. Neutron multiplication factor for the reference solution of Problem 1.2.1.

\begin{tabular}{rrccr}
\hline Step & $\begin{array}{r}\text { Burnup } \\
{\left[\frac{\mathrm{MWd}}{\mathrm{MtU}}\right]}\end{array}$ & $\begin{array}{c}\text { Shift } \\
\mathrm{k}_{\mathrm{eff}}(1 \sigma)\end{array}$ & $\begin{array}{c}\text { MPACT } \\
\mathrm{k}_{\text {eff }}(1 \sigma)\end{array}$ & $\begin{array}{c}\text { Difference } \\
{[\mathrm{pcm}]}\end{array}$ \\
\hline 0 & 0.0 & $1.06741(16)$ & 1.06787 & $46(16)$ \\
1 & 25.0 & $1.05237(16)$ & 1.05282 & $45(16)$ \\
2 & 50.0 & $1.05198(16)$ & 1.05258 & $60(16)$ \\
3 & 100.0 & $1.05283(16)$ & 1.05373 & $91(16)$ \\
4 & 200.0 & $1.05683(16)$ & 1.05749 & $66(16)$ \\
5 & 300.0 & $1.05999(17)$ & 1.06096 & $97(16)$ \\
6 & 400.0 & $1.06242(16)$ & 1.06381 & $139(16)$ \\
7 & 500.0 & $1.0648(17)$ & 1.06609 & $129(17)$ \\
8 & 600.0 & $1.06662(17)$ & 1.06779 & $117(16)$ \\
9 & 700.0 & $1.06787(17)$ & 1.06920 & $133(17)$ \\
10 & 800.0 & $1.06887(17)$ & 1.07027 & $140(17)$ \\
11 & 900.0 & $1.06991(17)$ & 1.07105 & $114(17)$ \\
12 & 1000.0 & $1.07024(17)$ & 1.07159 & $135(16)$ \\
13 & 1100.0 & $1.07013(17)$ & 1.07191 & $178(16)$ \\
14 & 1200.0 & $1.07017(18)$ & 1.07205 & $188(17)$ \\
15 & 1300.0 & $1.07054(17)$ & 1.07203 & $150(17)$ \\
\hline
\end{tabular}



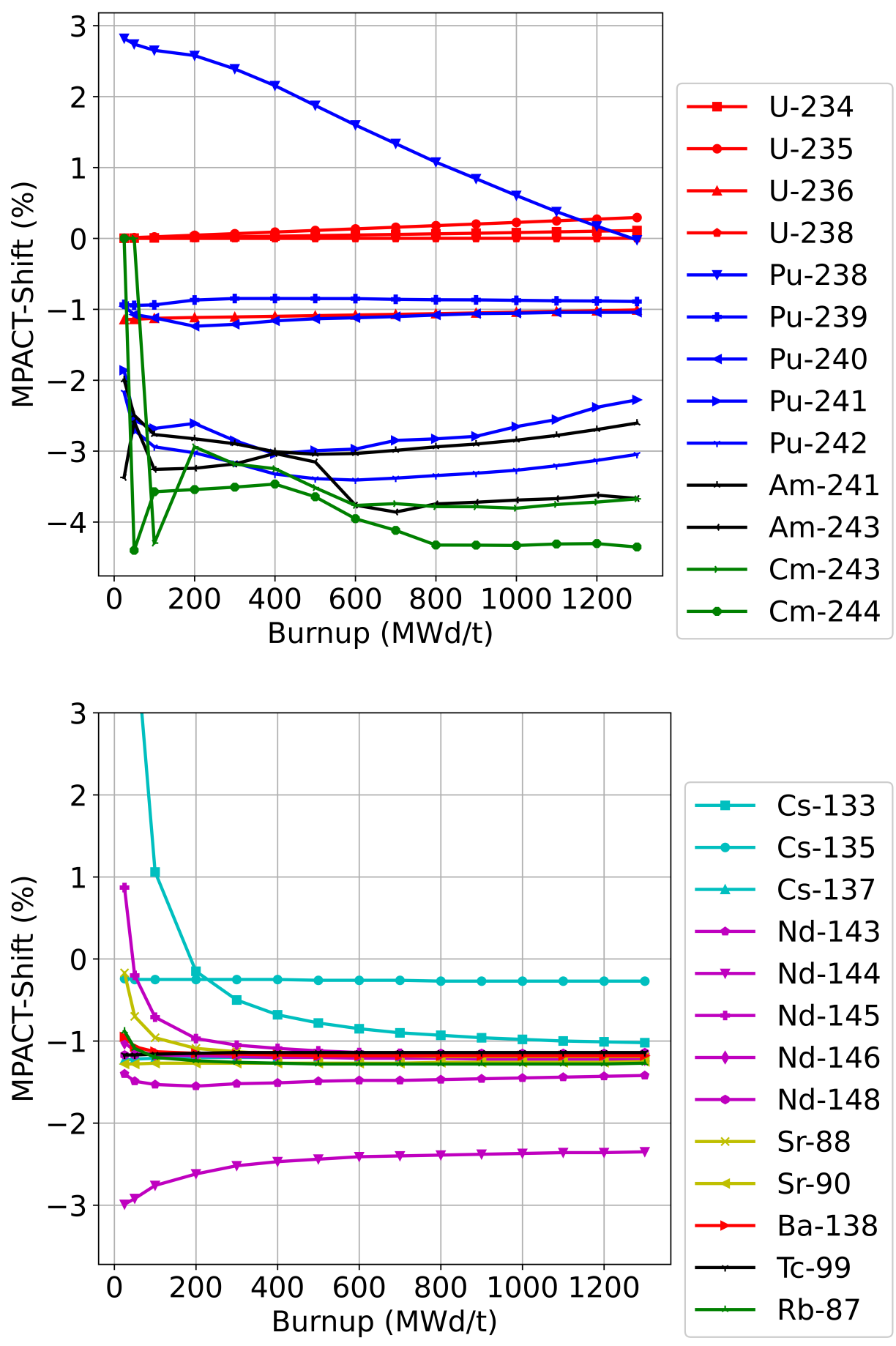

Figure 2. Percent difference in isotope concentrations between MPACT and Shift for actinides (top) and major fission products (bottom) 
Table 2.8. Timing variables for the reference solution of Problem 1.2.1

\begin{tabular}{|c|c|c|c|c|c|c|}
\hline \multicolumn{7}{|c|}{ 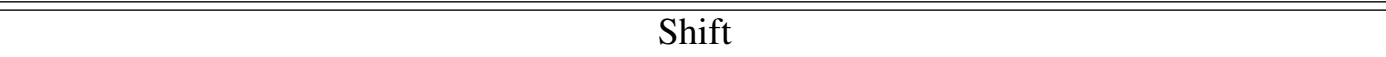 } \\
\hline $\begin{array}{c}\text { Depletion } \\
\text { Solves }\end{array}$ & $\begin{array}{c}\text { Total } \\
\text { Cycles per } \\
\text { Transport } \\
\text { Solve }\end{array}$ & $\begin{array}{l}\text { Active } \\
\text { Cycles per } \\
\text { Transport } \\
\text { Solve }\end{array}$ & $\begin{array}{l}\text { Histories } \\
\text { per Cycle }\end{array}$ & $\begin{array}{c}\text { Compute } \\
\text { Nodes }\end{array}$ & $\begin{array}{c}\text { Cores } \\
\text { per Node }\end{array}$ & $\begin{array}{c}\text { Wall } \\
\text { Total Time } \\
\text { [hours] }\end{array}$ \\
\hline 15 & 1500 & 1200 & 20000 & 2 & 48 & 25.90 \\
\hline \multicolumn{7}{|c|}{ MPACT } \\
\hline $\begin{array}{c}\text { Depletion } \\
\text { Solves }\end{array}$ & $\begin{array}{c}k_{\mathrm{eff}} \\
\text { Convergence } \\
\text { Criteria }\end{array}$ & $\begin{array}{c}\text { Source } \\
\text { Convergence } \\
\text { Criteria }\end{array}$ & $\begin{array}{c}\text { Ray } \\
\text { Spacing } \\
{[\mathrm{cm}]}\end{array}$ & $\begin{array}{c}\text { Compute } \\
\text { Nodes }\end{array}$ & $\begin{array}{c}\text { Cores } \\
\text { per Node }\end{array}$ & $\begin{array}{c}\text { Wall } \\
\text { Total Time } \\
\text { [hours] }\end{array}$ \\
\hline 15 & $1.0 \times 10^{-06}$ & $1.0 \times 10^{-06}$ & 0.03 & 1 & 1 & 0.44 \\
\hline
\end{tabular}


Table 2.9. Uranium isotope concentrations for the reference solution of Problem 1.2.1.

\begin{tabular}{|c|c|c|c|c|c|}
\hline \multirow{3}{*}{ Step } & \multirow{3}{*}{$\begin{array}{l}\text { Burnup } \\
{\left[\frac{\mathrm{MWd}}{\mathrm{MtU}}\right]}\end{array}$} & \multicolumn{4}{|c|}{ Shift } \\
\hline & & ${ }^{234} \mathrm{U}$ & ${ }^{235} \mathrm{U}$ & & ${ }^{238} \mathrm{U}$ \\
\hline & & {$\left[\frac{\text { atoms }}{b-\mathrm{cm}}\right]$} & {$\left[\frac{\text { atoms }}{b-\mathrm{cm}}\right]$} & {$\left[\frac{\text { atoms }}{b-\mathrm{cm}}\right]$} & {$\left[\frac{\text { atoms }}{\mathrm{b}-\mathrm{cm}}\right]$} \\
\hline 0 & 0.0 & $2.5775 \times 10^{-06}$ & $3.4449 \times 10^{-04}$ & $0.0000 \times 10^{-00}$ & $4.7470 \times 10^{-02}$ \\
\hline 1 & 25.0 & $2.5750 \times 10^{-06}$ & $3.4308 \times 10^{-04}$ & $2.2397 \times 10^{-07}$ & $4.7469 \times 10^{-02}$ \\
\hline 2 & 50.0 & $2.5724 \times 10^{-06}$ & $3.4169 \times 10^{-04}$ & $4.4612 \times 10^{-07}$ & $4.7467 \times 10^{-02}$ \\
\hline 3 & 100.0 & $2.5674 \times 10^{-06}$ & $3.3893 \times 10^{-04}$ & $8.8470 \times 10^{-07}$ & $4.7465 \times 10^{-02}$ \\
\hline 4 & 200.0 & $2.5575 \times 10^{-06}$ & $3.3355 \times 10^{-04}$ & $1.7406 \times 10^{-06}$ & $4.7459 \times 10^{-02}$ \\
\hline 5 & 300.0 & $2.5476 \times 10^{-06}$ & $3.2833 \times 10^{-04}$ & $2.5703 \times 10^{-06}$ & $4.7454 \times 10^{-02}$ \\
\hline 6 & 400.0 & $2.5380 \times 10^{-06}$ & $3.2327 \times 10^{-04}$ & $3.3758 \times 10^{-06}$ & $4.7449 \times 10^{-02}$ \\
\hline 7 & 500.0 & $2.5284 \times 10^{-06}$ & $3.1834 \times 10^{-04}$ & $4.1591 \times 10^{-06}$ & $4.7444 \times 10^{-02}$ \\
\hline 8 & 600.0 & $2.5189 \times 10^{-06}$ & $3.1354 \times 10^{-04}$ & $4.9218 \times 10^{-06}$ & $4.7439 \times 10^{-02}$ \\
\hline 9 & .0 & $2.5096 \times 10^{-06}$ & $3.0886 \times 10^{-04}$ & $5.6653 \times 10^{-06}$ & $4.7434 \times 10^{-02}$ \\
\hline 10 & 1.0 & $2.5003 \times 10^{-06}$ & $3.0430 \times 10^{-04}$ & $6.3907 \times 10^{-06}$ & $4.7428 \times 10^{-02}$ \\
\hline 11 & 900.0 & $2.4911 \times 10^{-06}$ & $2.9983 \times 10^{-04}$ & $7.0991 \times 10^{-06}$ & $4.7423 \times 10^{-02}$ \\
\hline 12 & 1000.0 & $2.4820 \times 10^{-06}$ & $2.9547 \times 10^{-04}$ & $7.7914 \times 10^{-06}$ & $4.7418 \times 10^{-02}$ \\
\hline 13 & & $2.4729 \times 10^{-06}$ & $2.9120 \times 10^{-04}$ & $8.4685 \times 10^{-06}$ & $4.7413 \times 10^{-02}$ \\
\hline 14 & 0 & $2.4639 \times 10^{-06}$ & $2.8702 \times 10^{-04}$ & $9.1314 \times 10^{-06}$ & $4.7408 \times 10^{-02}$ \\
\hline \multirow[t]{2}{*}{15} & .0 & $0 \times 10^{-06}$ & $292 \times 10^{-04}$ & $9.7803 \times 10^{-06}$ & $4.7403 \times 10^{-02}$ \\
\hline & & \multicolumn{4}{|c|}{ MPACT } \\
\hline \multirow[t]{2}{*}{ Step } & & ${ }^{234} \mathrm{U}$ & ${ }^{235} \mathrm{U}$ & ${ }^{236} \mathrm{U}$ & ${ }^{238} \mathrm{U}$ \\
\hline & & toms & aton & & \\
\hline 0 & 0. & $\frac{|\mathrm{b}-\mathrm{cm}|}{5775 \times 10^{-06}}$ & $49 \times 10^{-04}$ & $\frac{\mathrm{b}-\mathrm{cm}]}{0 \times 10^{-00}}$ & $70 \times 10^{-02}$ \\
\hline 1 & 25.0 & $0 \times 10^{-06}$ & $10 \times 10^{-04}$ & $2.2073 \times 10^{-07}$ & $4.7469 \times 10^{-02}$ \\
\hline 2 & 50.0 & $2.5726 \times 10^{-06}$ & $3.4172 \times 10^{-04}$ & $4.3968 \times 10^{-07}$ & $4.7467 \times 10^{-02}$ \\
\hline 3 & 100.0 & $2.5677 \times 10^{-06}$ & $3.3900 \times 10^{-04}$ & $8.7205 \times 10^{-07}$ & $4.7465 \times 10^{-02}$ \\
\hline 4 & 200.0 & $2.5581 \times 10^{-06}$ & $3.3369 \times 10^{-04}$ & $1.7159 \times 10^{-06}$ & $4.7460 \times 10^{-02}$ \\
\hline 5 & 300.0 & $2.5486 \times 10^{-06}$ & $3.2855 \times 10^{-04}$ & $2.5340 \times 10^{-06}$ & $4.7454 \times 10^{-02}$ \\
\hline 6 & 400.0 & $2.5392 \times 10^{-06}$ & $3.2355 \times 10^{-04}$ & $3.3283 \times 10^{-06}$ & $4.7449 \times 10^{-02}$ \\
\hline 7 & 500.0 & $2.5300 \times 10^{-06}$ & $3.1869 \times 10^{-04}$ & $4.1009 \times 10^{-06}$ & $4.7444 \times 10^{-02}$ \\
\hline 8 & 600.0 & $2.5208 \times 10^{-06}$ & $3.1395 \times 10^{-04}$ & $4.8533 \times 10^{-06}$ & $4.7439 \times 10^{-02}$ \\
\hline 9 & 700.0 & $2.5118 \times 10^{-06}$ & $3.0934 \times 10^{-04}$ & $5.5869 \times 10^{-06}$ & $4.7434 \times 10^{-02}$ \\
\hline 10 & 800.0 & $2.5028 \times 10^{-06}$ & $3.0483 \times 10^{-04}$ & $6.3029 \times 10^{-06}$ & $4.7429 \times 10^{-02}$ \\
\hline 11 & 900.0 & $2.4939 \times 10^{-06}$ & $3.0042 \times 10^{-04}$ & $7.0022 \times 10^{-06}$ & $4.7424 \times 10^{-02}$ \\
\hline 12 & 1000.0 & $2.4851 \times 10^{-06}$ & $2.9612 \times 10^{-04}$ & $7.6858 \times 10^{-06}$ & $4.7419 \times 10^{-02}$ \\
\hline 13 & 1100.0 & $2.4764 \times 10^{-06}$ & $2.9190 \times 10^{-04}$ & $8.3546 \times 10^{-06}$ & $4.7414 \times 10^{-02}$ \\
\hline 14 & 1200.0 & $2.4678 \times 10^{-06}$ & $2.8778 \times 10^{-04}$ & $9.0093 \times 10^{-06}$ & $4.7409 \times 10^{-02}$ \\
\hline 15 & 1300.0 & $2.4592 \times 10^{-06}$ & $2.8373 \times 10^{-04}$ & $9.6506 \times 10^{-06}$ & $4.7405 \times 10^{-02}$ \\
\hline
\end{tabular}


Table 2.10. Plutonium isotope concentrations for the reference solution of Problem 1.2.1.

\begin{tabular}{|c|c|c|c|c|c|c|}
\hline Step & $\begin{array}{l}\text { Burnup } \\
{\left[\frac{\mathrm{MWd}}{\mathrm{MtU}}\right]}\end{array}$ & $\begin{array}{l}{ }^{238} \mathrm{Pu} \\
{\left[\frac{\text { atoms }}{\mathrm{b}-\mathrm{cm}}\right]}\end{array}$ & $\begin{array}{l}{ }^{239} \mathrm{Pu} \\
{\left[\frac{\text { atoms }}{\mathrm{b}-\mathrm{cm}}\right]}\end{array}$ & $\begin{array}{l}\text { Shift } \\
{ }^{240} \mathrm{Pu} \\
{\left[\frac{\text { atoms }}{\mathrm{b}-\mathrm{cm}}\right]}\end{array}$ & $\begin{array}{l}{ }^{241} \mathrm{Pu} \\
\left\lceil\frac{\text { atoms }}{\mathrm{b}-\mathrm{cm}}\right]\end{array}$ & $\begin{array}{l}{ }^{242} \mathrm{Pu} \\
{\left[\frac{\text { atoms }}{\mathrm{b}-\mathrm{cm}}\right]}\end{array}$ \\
\hline 0 & 0.0 & $0.0000 \times 10^{-00}$ & $0.0000 \times 10^{-00}$ & $0.0000 \times 10^{-00}$ & $0.0000 \times 10^{-00}$ & $0.0000 \times 10^{-00}$ \\
\hline 1 & 25.0 & $8590 \times 10^{-13}$ & $1.0020 \times 10^{-06}$ & $2.0451 \times 10^{-09}$ & $5.2842 \times 10^{-12}$ & $3.8469 \times 10^{-15}$ \\
\hline 2 & 50.0 & $5.7709 \times 10^{-12}$ & $2.2366 \times 10^{-06}$ & $9.2493 \times 10^{-09}$ & $4.9838 \times 10^{-11}$ & $7.3959 \times 10^{-14}$ \\
\hline 3 & 100.0 & $3.3362 \times 10^{-11}$ & $4.6546 \times 10^{-06}$ & $3.8875 \times 10^{-08}$ & $4.2901 \times 10^{-10}$ & $1.3004 \times 10^{-12}$ \\
\hline 4 & 200.0 & $1.6274 \times 10^{-10}$ & $9.2764 \times 10^{-06}$ & $1.5431 \times 10^{-07}$ & $3.4184 \times 10^{-09}$ & $2.0980 \times 10^{-11}$ \\
\hline 5 & 300.0 & $3.9322 \times 10^{-10}$ & $1.3644 \times 10^{-05}$ & $3.3602 \times 10^{-07}$ & $1.1123 \times 10^{-08}$ & $1.0250 \times 10^{-10}$ \\
\hline 6 & 400.0 & $7.2754 \times 10^{-10}$ & $1.7784 \times 10^{-05}$ & $5.7548 \times 10^{-07}$ & $2.5151 \times 10^{-08}$ & $3.0932 \times 10^{-10}$ \\
\hline 7 & 500.0 & $1.1691 \times 10^{-09}$ & $2.1715 \times 10^{-05}$ & $8.6582 \times 10^{-07}$ & $4.6556 \times 10^{-08}$ & $7.1765 \times 10^{-10}$ \\
\hline 8 & 600.0 & $1.7215 \times 10^{-09}$ & $2.5454 \times 10^{-05}$ & $1.2013 \times 10^{-06}$ & $7.6116 \times 10^{-08}$ & $1.4114 \times 10^{-09}$ \\
\hline 9 & .0 & & $2.9020 \times 10^{-05}$ & $1.5767 \times 10^{-06}$ & $1.1413 \times 10^{-07}$ & $2.4777 \times 10^{-09}$ \\
\hline 10 & 0.0 & $3.1766 \times 1$ & $3.2422 \times 1$ & $1.9878 \times 10^{-06}$ & $1.6098 \times 1$ & $4.0052 \times 10^{-09}$ \\
\hline 11 & 900.0 & $4.0899 \times 10^{-09}$ & $3.5673 \times 10^{-05}$ & $2.4312 \times 10^{-06}$ & $2.1656 \times 10^{-07}$ & $6.0815 \times 10^{-09}$ \\
\hline 12 & 1000.0 & $1367 \times 1$ & $3.8786 \times 1$ & $2.9041 \times 10^{-06}$ & $2.8043 \times 1$ & $8.7907 \times 10^{-09}$ \\
\hline 13 & & $6.3244 \times 1$ & $4.1768 \times 10^{-05}$ & $3.4034 \times 10^{-06}$ & $3.5264 \times 10^{-07}$ & $1.2211 \times 10^{-08}$ \\
\hline 14 & 1200.0 & $7.6608 \times 10^{-09}$ & $4.4627 \times 10^{-05}$ & $3.9274 \times 10^{-06}$ & $4.3244 \times 10^{-07}$ & $1.6419 \times 10^{-08}$ \\
\hline 15 & .0 & $63 \times 10^{-09}$ & $373 \times 1$ & $4.4736 \times 10^{-06}$ & $5.2003 \times$ & $2.1485 \times 10^{-08}$ \\
\hline Step & $\begin{array}{l}\text { Burnup } \\
{\left[\frac{\mathrm{MWd}}{\mathrm{MtU}}\right]}\end{array}$ & $\begin{array}{l}{ }^{238} \mathrm{Pu} \\
{\left[\frac{\text { atoms }}{\mathrm{b}-\mathrm{cm}}\right]}\end{array}$ & $\begin{array}{l}{ }^{239} \mathrm{Pu} \\
{\left[\frac{\text { atoms }}{\mathrm{b}-\mathrm{cm}}\right]}\end{array}$ & $\begin{array}{c}\text { MPACT } \\
{ }^{240} \mathrm{Pu} \\
{\left[\frac{\text { atoms }}{\mathrm{b}-\mathrm{cm}}\right]}\end{array}$ & $\begin{array}{l}{ }^{241} \mathrm{Pu} \\
{\left[\frac{\text { atoms }}{\mathrm{b}-\mathrm{cm}}\right]}\end{array}$ & $\begin{array}{l}{ }^{242} \mathrm{Pu} \\
{\left[\frac{\text { atoms }}{\mathrm{b}-\mathrm{cm}}\right]}\end{array}$ \\
\hline 0 & 0.0 & $0.0000 \times 10^{-00}$ & $0.0000 \times 10^{-00}$ & $0.0000 \times 10^{-00}$ & $0.0000 \times 10^{-00}$ & $0.0000 \times 10^{-00}$ \\
\hline 1 & 25.0 & & $9.8194 \times 1$ & $2.0167 \times 10^{-09}$ & $5.1210 \times$ & $3.7974 \times 10^{-15}$ \\
\hline 2 & 50.0 & $5.7371 \times 10^{-12}$ & $2.1914 \times 10^{-06}$ & $9.1223 \times 10^{-09}$ & $4.8041 \times 10^{-11}$ & $7.2832 \times 10^{-14}$ \\
\hline 3 & 100.0 & $3.3140 \times 10^{-11}$ & $4.5601 \times 10^{-06}$ & $3.8351 \times 10^{-08}$ & $4.1348 \times 10^{-10}$ & $1.2798 \times 10^{-12}$ \\
\hline 4 & 200.0 & $5 \times 10^{-10}$ & $9.0920 \times 10^{-06}$ & $1.5213 \times 10^{-07}$ & $3.2994 \times 10^{-09}$ & $2.0658 \times 10^{-11}$ \\
\hline 5 & 300.0 & $3.8965 \times 10^{-10}$ & $1.3373 \times 10^{-05}$ & $3.3146 \times 10^{-07}$ & $1.0714 \times 10^{-08}$ & $1.0088 \times 10^{-10}$ \\
\hline 6 & 400.0 & $7.1934 \times 10^{-10}$ & $1.7425 \times 10^{-05}$ & $5.6808 \times 10^{-07}$ & $2.4191 \times 10^{-08}$ & $3.0423 \times 10^{-10}$ \\
\hline 7 & 500.0 & $1.1529 \times 10^{-09}$ & $2.1271 \times 10^{-05}$ & $8.5507 \times 10^{-07}$ & $4.4822 \times 10^{-08}$ & $7.0601 \times 10^{-10}$ \\
\hline 8 & 600.0 & $1.6934 \times 10^{-09}$ & $2.4927 \times 10^{-05}$ & $1.1866 \times 10^{-06}$ & $7.3335 \times 10^{-08}$ & $1.3895 \times 10^{-09}$ \\
\hline 9 & & $3 \times 1$ & $2.8409 \times 1$ & $1.5579 \times 10^{-06}$ & $016 \times 1$ & $2.4423 \times 10^{-09}$ \\
\hline 10 & 800.0 & $3.1101 \times 10^{-09}$ & $3.1731 \times 10^{-05}$ & $1.9647 \times 10^{-06}$ & $1.5549 \times 10^{-07}$ & $3.9531 \times 10^{-09}$ \\
\hline 11 & 900.0 & $3.9963 \times 10^{-09}$ & $3.4903 \times 10^{-05}$ & $2.4034 \times 10^{-06}$ & $2.0934 \times 10^{-07}$ & $6.0099 \times 10^{-09}$ \\
\hline 12 & 1000.0 & $5.0091 \times 10^{-09}$ & $3.7937 \times 10^{-05}$ & $2.8710 \times 10^{-06}$ & $2.7158 \times 10^{-07}$ & $8.6984 \times 10^{-09}$ \\
\hline 13 & 1100.0 & $6.1559 \times 10^{-09}$ & $4.0842 \times 10^{-05}$ & $3.3650 \times 10^{-06}$ & $3.4198 \times 10^{-07}$ & $1.2101 \times 10^{-08}$ \\
\hline 14 & 1200.0 & $7.4449 \times 10^{-09}$ & $4.3626 \times 10^{-05}$ & $3.8828 \times 10^{-06}$ & $4.2025 \times 10^{-07}$ & $1.6297 \times 10^{-08}$ \\
\hline 15 & 1300.0 & $8.8855 \times 10^{-09}$ & $4.6297 \times 10^{-05}$ & $4.4227 \times 10^{-06}$ & $5.0604 \times 10^{-07}$ & $2.1360 \times 10^{-08}$ \\
\hline
\end{tabular}


Table 2.11. Americium isotope concentrations for the reference solution of Problem 1.2.1.

\begin{tabular}{|c|c|c|c|}
\hline \multirow[b]{2}{*}{ Step } & \multirow[b]{2}{*}{$\begin{array}{l}\text { Burnup } \\
{\left[\frac{\mathrm{MWd}}{\mathrm{MtU}}\right]}\end{array}$} & \multicolumn{2}{|c|}{ Shift } \\
\hline & & $\begin{array}{l}{ }^{241} \mathrm{Am} \\
{\left[\frac{\text { atoms }}{\mathrm{b}-\mathrm{cm}}\right]}\end{array}$ & $\begin{array}{l}{ }^{243} \mathrm{Am} \\
{\left[\frac{\text { atoms }}{\mathrm{b}-\mathrm{cm}}\right]}\end{array}$ \\
\hline 0 & 0.0 & $0.0000 \times 10^{-00}$ & $0.0000 \times 10^{-00}$ \\
\hline 1 & 25.0 & $8011 \times 10^{-15}$ & $6.4345 \times 10^{-19}$ \\
\hline 2 & 50.0 & $5.3970 \times 10^{-14}$ & $2.6387 \times 10^{-17}$ \\
\hline 3 & 100.0 & $9.5392 \times 10^{-13}$ & $9.7729 \times 10^{-16}$ \\
\hline 4 & 200.0 & $1.5520 \times 10^{-11}$ & $3.2591 \times 10^{-14}$ \\
\hline 5 & 300.0 & $7.6518 \times 10^{-11}$ & $2.4314 \times 10^{-13}$ \\
\hline 6 & 0 & $2.3274 \times 10^{-10}$ & $9.8991 \times 10^{-13}$ \\
\hline 7 & 0 & $5.4359 \times$ & $2.9092 \times 10^{-12}$ \\
\hline 8 & 0 & $0751 \times$ & $6.9882 \times 10^{-12}$ \\
\hline 9 & 700.0 & $1.8966 \times 10^{-09}$ & $1.4486 \times 10^{-11}$ \\
\hline 10 & & $3.0785 \times$ & $2.7014 \times 10^{-11}$ \\
\hline 11 & 0 & $4.6908 \times$ & $4.6602 \times 10^{-11}$ \\
\hline 12 & 0 & $6.7995 \times$ & $7.5558 \times 10^{-11}$ \\
\hline 13 & & )$^{-09}$ & $1.1655 \times$ \\
\hline 14 & & $.2752 \times 10^{-08}$ & $1.7248 \times 10^{-10}$ \\
\hline \multirow[t]{2}{*}{15} & & $9 \times 10^{-08}$ & $2.4687>$ \\
\hline & & \multicolumn{2}{|c|}{ MPACT } \\
\hline Step & $\begin{array}{l}\text { Burnup } \\
{\left[\frac{\mathrm{MWd}}{\mathrm{MtU}}\right]}\end{array}$ & $\begin{array}{l}{ }^{241} \mathrm{Am} \\
{\left[\frac{\mathrm{atoms}}{\mathrm{b}-\mathrm{cm}}\right]}\end{array}$ & 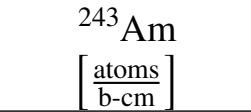 \\
\hline 0 & 0.0 & $0.0000 \times 10^{-00}$ & $0.0000 \times 10^{-00}$ \\
\hline 1 & 25.0 & $2.7078 \times 10^{-15}$ & $6.1871 \times 10^{-19}$ \\
\hline 2 & 0 & $5.2032 \times 10^{-14}$ & $2.5678 \times 10^{-17}$ \\
\hline 3 & 0 & $9.1823 \times 10^{-13}$ & $9.4651 \times 10^{-16}$ \\
\hline 4 & 200.0 & $1.4941 \times 10^{-11}$ & $3.1640 \times 10^{-14}$ \\
\hline 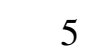 & 30 & $7.3634 \times 10^{-11}$ & $2.3637 \times 10^{-13}$ \\
\hline 6 & 40 & $2.2376 \times 10^{-10}$ & $9.6466 \times 10^{-13}$ \\
\hline 7 & & $5.2258 \times 10^{-10}$ & $2.8340 \times 10^{-12}$ \\
\hline 8 & & $1.0340 \times 10^{-09}$ & $6.7708 \times 10^{-12}$ \\
\hline 9 & 700.0 & $1.8253 \times 10^{-09}$ & $1.4034 \times 10^{-11}$ \\
\hline 10 & & $2.9651 \times 10^{-09}$ & $2.6226 \times 10^{-11}$ \\
\hline 11 & 900.0 & $4.5211 \times 10^{-09}$ & $4.5292 \times 10^{-11}$ \\
\hline 12 & 1000.0 & $6.5587 \times 10^{-09}$ & $7.3519 \times 10^{-11}$ \\
\hline 13 & 1100.0 & $9.1403 \times 10^{-09}$ & $1.1352 \times 10^{-10}$ \\
\hline 14 & 1200.0 & $1.2325 \times 10^{-08}$ & $1.6822 \times 10^{-10}$ \\
\hline 15 & 1300.0 & $1.6166 \times 10^{-08}$ & $2.4084 \times 10^{-10}$ \\
\hline
\end{tabular}


Table 2.12. Curium isotope concentrations for the reference solution of Problem 1.2.1.

\begin{tabular}{|c|c|c|c|}
\hline \multirow[b]{2}{*}{ Step } & \multirow[b]{2}{*}{$\begin{array}{l}\text { Burnup } \\
{\left[\frac{\mathrm{MWd}}{\mathrm{MtU}}\right]}\end{array}$} & \multicolumn{2}{|c|}{ Shift } \\
\hline & & $\begin{array}{l}{ }^{243} \mathrm{Cm} \\
{\left[\frac{\text { atoms }}{\mathrm{b}-\mathrm{cm}}\right]}\end{array}$ & $\begin{array}{l}{ }^{244} \mathrm{Cm} \\
{\left[\frac{\text { atoms }}{\mathrm{b}-\mathrm{cm}}\right]}\end{array}$ \\
\hline 0 & 0.0 & $0.0000 \times 10^{+00}$ & $0.0000 \times 10^{+00}$ \\
\hline 1 & 25.0 & $0000 \times 10^{+00}$ & $0.0000 \times 10^{+00}$ \\
\hline 2 & 50.0 & $0.0000 \times 10^{+00}$ & $1.9235 \times 10^{-20}$ \\
\hline 3 & 100.0 & $5.5931 \times 10^{-19}$ & $1.4704 \times 10^{-18}$ \\
\hline 4 & 200.0 & $3.7278 \times 10^{-17}$ & $9.8996 \times 10^{-17}$ \\
\hline 5 & 300.0 & $4.0205 \times 10^{-16}$ & $1.0983 \times 10^{-15}$ \\
\hline 6 & 0 & $0913 \times 10^{-15}$ & $5.9027 \times 10^{-15}$ \\
\hline 7 & 0 & $0^{-15}$ & $2.1417 \times 10^{-14}$ \\
\hline 8 & 0 & $83 \times 10^{-14}$ & $6.0738 \times 10^{-14}$ \\
\hline 9 & 700.0 & $4.6449 \times 10^{-14}$ & $1.4549 \times 10^{-13}$ \\
\hline 10 & & $23 \times 10^{-14}$ & $3.0821 \times 10^{-13}$ \\
\hline 11 & 0 & -13 & $5.9479 \times 10^{-13}$ \\
\hline 12 & 0 & $0691 \times 10^{-13}$ & $1.0669 \times 10^{-12}$ \\
\hline 13 & & )$^{-13}$ & $1.8041 \times 10^{-12}$ \\
\hline 14 & & $8122 \times 10^{-13}$ & $2.9067 \times 10^{-12}$ \\
\hline \multirow[t]{2}{*}{15} & & $\times 10^{-12}$ & $4.4974 \times 10^{-12}$ \\
\hline & & \multicolumn{2}{|c|}{ MPACT } \\
\hline Step & $\begin{array}{l}\text { Burnup } \\
{\left[\frac{\mathrm{MWd}}{\mathrm{MtU}}\right]}\end{array}$ & $\left.\begin{array}{l}{ }^{243} \mathrm{Cm} \\
{\left[\frac{\text { atoms }}{\mathrm{b}-\mathrm{cm}}\right.}\end{array}\right]$ & 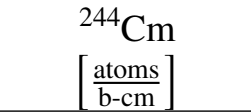 \\
\hline 0 & 0.0 & $000 \times 10^{-00}$ & $0.0000 \times 10^{-00}$ \\
\hline 1 & 25.0 & $0000 \times 10^{-00}$ & $0.0000 \times 10^{-00}$ \\
\hline 2 & & $0 \times$ & $1.4601 \times 10^{-20}$ \\
\hline 3 & .0 & $32 \times 10^{-19}$ & $1.2624 \times 10^{-18}$ \\
\hline 4 & 200.0 & $3.2610 \times 10^{-17}$ & $8.6225 \times 10^{-17}$ \\
\hline 5 & 300.0 & $23 \times 10^{-16}$ & $9.7499 \times 10^{-16}$ \\
\hline 6 & 400.0 & $1.8403 \times 10^{-15}$ & $5.3348 \times 10^{-15}$ \\
\hline 7 & & $19 \times 10^{-15}$ & $1.9675 \times 10^{-14}$ \\
\hline 8 & & $1.7732 \times 10^{-14}$ & $5.6618 \times 10^{-14}$ \\
\hline 9 & 700.0 & $4.1069 \times 10^{-14}$ & $1.3739 \times 10^{-13}$ \\
\hline 10 & & $8.4066 \times 10^{-14}$ & $2.9438 \times 10^{-13}$ \\
\hline 11 & 900.0 & $1.5666 \times 10^{-13}$ & $5.7377 \times 10^{-13}$ \\
\hline 12 & 1000.0 & $2.7122 \times 10^{-13}$ & $1.0380 \times 10^{-12}$ \\
\hline 13 & 1100.0 & $4.4254 \times 10^{-13}$ & $1.7684 \times 10^{-12}$ \\
\hline 14 & 1200.0 & $6.8778 \times 10^{-13}$ & $2.8673 \times 10^{-12}$ \\
\hline 15 & 1300.0 & $1.0264 \times 10^{-12}$ & $4.4602 \times 10^{-12}$ \\
\hline
\end{tabular}


Table 2.13. Cesium isotope concentrations for the reference solution of Problem 1.2.1.

\begin{tabular}{|c|c|c|c|c|}
\hline Step & $\begin{array}{l}\text { Burnup } \\
{\left[\frac{\mathrm{MWd}}{\mathrm{MtU}}\right]}\end{array}$ & $\begin{array}{l}{ }^{133} \mathrm{Cs} \\
{\left[\frac{\text { atoms }}{\mathrm{b}-\mathrm{cm}}\right]}\end{array}$ & $\begin{array}{l}\text { Shift } \\
{ }^{135} \mathrm{Cs} \\
{\left[\frac{\text { atoms }}{\mathrm{b}-\mathrm{cm}}\right]}\end{array}$ & $\begin{array}{l}{ }^{137} \mathrm{Cs} \\
{\left[\frac{\text { atoms }}{\mathrm{b}-\mathrm{cm}}\right]}\end{array}$ \\
\hline 0 & 0.0 & $0.0000 \times 10^{-00}$ & $0.0000 \times 10^{-00}$ & $0.0000 \times 10^{-00}$ \\
\hline 1 & 25.0 & $4.5614 \times 10^{-08}$ & $4.8829 \times 10^{-08}$ & $7.8444 \times 10^{-08}$ \\
\hline 2 & 50.0 & $1.2645 \times 10^{-07}$ & $9.9978 \times 10^{-08}$ & $1.5681 \times 10^{-07}$ \\
\hline 3 & 100.0 & $2.9598 \times 10^{-07}$ & $2.0268 \times 10^{-07}$ & $3.1333 \times 10^{-07}$ \\
\hline 4 & 200.0 & $6.3567 \times 10$ & $4.0961 \times 10^{-07}$ & $6.2553 \times 10^{-07}$ \\
\hline 5 & 300.0 & $9.7497 \times 10^{-07}$ & $6.1839 \times 10^{-07}$ & $9.3662 \times 10^{-07}$ \\
\hline 6 & 400.0 & $1.3139 \times 10^{-06}$ & $8.2881 \times 10^{-07}$ & $1.2466 \times 10^{-06}$ \\
\hline 7 & .0 & $1.6523 \times 10^{-06}$ & $1.0407 \times 10^{-06}$ & $1.5554 \times 10^{-06}$ \\
\hline 8 & 0.0 & $1.9904 \times 10^{-06}$ & $1.2540 \times 10^{-06}$ & $1.8630 \times 10^{-06}$ \\
\hline 9 & & $2.3280 \times 1$ & $1.4685 \times 10^{-06}$ & $2.1694 \times 10^{-06}$ \\
\hline 10 & .0 & $2.6651 \times 10^{-06}$ & $1.6841 \times 10^{-06}$ & $2.4747 \times 10^{-06}$ \\
\hline 11 & 900.0 & $3.0019 \times 10^{-06}$ & $1.9008 \times 10^{-06}$ & $2.7787 \times 10^{-06}$ \\
\hline 12 & .0 & $32 \times 10^{-06}$ & $2.1185 \times 10^{-06}$ & $3.0816 \times 10^{-06}$ \\
\hline 13 & & -06 & $2.3370 \times 10^{-06}$ & $3.3832 \times 10^{-06}$ \\
\hline 14 & & $4.0094 \times 10^{-06}$ & $2.5564 \times 10^{-06}$ & $3.6837 \times 10^{-06}$ \\
\hline 15 & & & $2.7765 \times 10^{-06}$ & $3.9829 \times 10^{-06}$ \\
\hline Step & & $\left\lceil\frac{\text { atoms }}{\mathrm{b}-\mathrm{cm}}\right]$ & $\begin{array}{c}\text { MPACT } \\
{ }^{135} \mathrm{Cs} \\
{\left[\frac{\text { atoms }}{\mathrm{b}-\mathrm{cm}}\right]}\end{array}$ & $\begin{array}{l}{ }^{137} \mathrm{Cs} \\
{\left[\frac{\text { atoms }}{\mathrm{b}-\mathrm{cm}}\right]}\end{array}$ \\
\hline 0 & 0.0 & $0000 \times 10^{-00}$ & $0.0000 \times 10^{-00}$ & $0.0000 \times 10^{-00}$ \\
\hline 1 & 25.0 & $.0919 \times 10^{-08}$ & $4.8535 \times 10^{-08}$ & $7.7474 \times 10^{-08}$ \\
\hline 2 & .0 & $4 \times 10^{-07}$ & $9.9359 \times 10^{-08}$ & $1.5489 \times 10^{-07}$ \\
\hline 3 & 100.0 & $2.9909 \times 10^{-07}$ & $2.0141 \times 10^{-07}$ & $3.0952 \times 10^{-07}$ \\
\hline 4 & & $8 \times$ & $4.0700 \times 10$ & $6.1800 \times 10^{-07}$ \\
\hline 5 & & $9.7001 \times 10^{-07}$ & $6.1436 \times 10^{-07}$ & $9.2536 \times 10^{-07}$ \\
\hline 6 & 0.0 & $1.3049 \times 10^{-06}$ & $8.2330 \times 10^{-07}$ & $1.2316 \times 10^{-06}$ \\
\hline 7 & & $94 \times 10^{-06}$ & $1.0337 \times 10^{-06}$ & $1.5367 \times 10^{-06}$ \\
\hline 8 & & $1.9734 \times 10^{-06}$ & $1.2454 \times 10^{-06}$ & $1.8406 \times 10^{-06}$ \\
\hline 9 & & $2.3071 \times 10^{-06}$ & $1.4583 \times 10^{-06}$ & $2.1434 \times 10^{-06}$ \\
\hline 10 & & $2.6403 \times 10^{-06}$ & $1.6722 \times 10^{-06}$ & $2.4450 \times 10^{-06}$ \\
\hline 11 & 0.0 & $2.9731 \times 10^{-06}$ & $1.8872 \times 10^{-06}$ & $2.7455 \times 10^{-06}$ \\
\hline 12 & 1000.0 & $3.3055 \times 10^{-06}$ & $2.1031 \times 10^{-06}$ & $3.0448 \times 10^{-06}$ \\
\hline 13 & 1100.0 & $3.6374 \times 10^{-06}$ & $2.3199 \times 10^{-06}$ & $3.3429 \times 10^{-06}$ \\
\hline 14 & 1200.0 & $3.9690 \times 10^{-06}$ & $2.5375 \times 10^{-06}$ & $3.6398 \times 10^{-06}$ \\
\hline 15 & 1300.0 & $4.3001 \times 10^{-06}$ & $2.7558 \times 10^{-06}$ & $3.9355 \times 10^{-06}$ \\
\hline
\end{tabular}


Table 2.14. Neodymium isotope concentrations for the reference solution of Problem 1.2.1.

\begin{tabular}{|c|c|c|c|c|c|c|}
\hline Step & $\begin{array}{l}\text { Burnup } \\
{\left[\frac{\mathrm{MWd}}{\mathrm{MtU}}\right]}\end{array}$ & $\begin{array}{l}{ }^{143} \mathrm{Nd} \\
{\left[\frac{\text { atoms }}{\mathrm{b}-\mathrm{cm}}\right]}\end{array}$ & $\begin{array}{l}{ }^{144} \mathrm{Nd} \\
{\left[\frac{\text { atoms }}{\mathrm{b}-\mathrm{cm}}\right]}\end{array}$ & $\begin{array}{l}\text { Shift } \\
{ }^{145} \mathrm{Nd} \\
{\left[\frac{\text { atoms }}{\mathrm{b}-\mathrm{cm}}\right]}\end{array}$ & $\begin{array}{l}{ }^{146} \mathrm{Nd} \\
{\left[\frac{\text { atoms }}{\mathrm{b}-\mathrm{cm}}\right]}\end{array}$ & $\begin{array}{l}{ }^{148} \mathrm{Nd} \\
{\left[\frac{\text { atoms }}{\mathrm{b}-\mathrm{cm}}\right]}\end{array}$ \\
\hline 0 & 0.0 & $0.0000 \times 10^{-00}$ & $0.0000 \times 10^{-00}$ & $0.0000 \times 10^{-00}$ & $0.0000 \times 10^{-00}$ & $0.0000 \times 10^{-00}$ \\
\hline 1 & 25.0 & $2.0333 \times 10^{-08}$ & $1.4520 \times 10^{-09}$ & $4.8749 \times 10^{-08}$ & $3.8290 \times 10^{-08}$ & $2.1624 \times 10^{-08}$ \\
\hline 2 & 50.0 & $7.1914 \times 10^{-08}$ & $5.7729 \times 10^{-09}$ & $9.8409 \times 10^{-08}$ & $7.6615 \times 10^{-08}$ & $4.3272 \times 10^{-08}$ \\
\hline 3 & 100.0 & $2.0669 \times 10^{-07}$ & $2.2636 \times 10^{-08}$ & $1.9734 \times 10^{-07}$ & $1.5313 \times 10^{-07}$ & $8.6558 \times 10^{-08}$ \\
\hline 4 & 200.0 & $4.9683 \times 10^{-07}$ & $8.6269 \times 10^{-08}$ & $3.9378 \times 10^{-07}$ & $3.0566 \times 10^{-07}$ & $1.7302 \times 10^{-07}$ \\
\hline 5 & 300.0 & $7.8494 \times 10^{-07}$ & $1.8452 \times 10^{-07}$ & $5.8846 \times 10^{-07}$ & $4.5763 \times 10^{-07}$ & $2.5935 \times 10^{-07}$ \\
\hline 6 & 400.0 & $1.0688 \times 10^{-06}$ & $3.1190 \times 10^{-07}$ & $7.8152 \times 10^{-07}$ & $6.0909 \times 10^{-07}$ & $3.4554 \times 10^{-07}$ \\
\hline 7 & 500.0 & $1.3485 \times 10^{-06}$ & $4.6382 \times 10^{-07}$ & $9.7307 \times 10^{-07}$ & $7.6011 \times 10^{-07}$ & $4.3162 \times 10^{-07}$ \\
\hline 8 & 600.0 & $1.6242 \times 10^{-06}$ & $6.3641 \times 10^{-07}$ & $1.1632 \times 10^{-06}$ & $9.1073 \times 10^{-07}$ & $5.1758 \times 10^{-07}$ \\
\hline 9 & 700.0 & $1.8963 \times 10^{-06}$ & $8.2641 \times 10^{-07}$ & $1.3520 \times 10^{-06}$ & $1.0610 \times 10^{-06}$ & $6.0345 \times 10^{-07}$ \\
\hline 10 & .0 & $2.1649 \times 10^{-06}$ & $1.0311 \times 10^{-06}$ & $1.5396 \times 10^{-06}$ & $1.2109 \times 10^{-06}$ & $6.8921 \times 10^{-07}$ \\
\hline 11 & 900.0 & $2.4300 \times 10^{-06}$ & $1.2482 \times 10^{-06}$ & $1.7259 \times 10^{-06}$ & $1.3606 \times 10^{-06}$ & $7.7488 \times 10^{-07}$ \\
\hline 12 & 1000.0 & $2.6919 \times 10^{-06}$ & $1.4757 \times 10^{-06}$ & $1.9111 \times 10^{-06}$ & $1.5100 \times 10^{-06}$ & $8.6047 \times 10^{-07}$ \\
\hline 13 & & $2.9507 \times 10^{-06}$ & $1.7121 \times 10^{-06}$ & $2.0953 \times 10^{-06}$ & $1.6592 \times 10^{-06}$ & $9.4598 \times 10^{-07}$ \\
\hline 14 & & & & $2.2784 \times 10^{-06}$ & $1.8081 \times$ & $1.0314 \times$ \\
\hline 15 & 0.0 & $592 \times 10^{-06}$ & $062 \times 10^{-06}$ & $2.4605 \times 10^{-06}$ & $1.9569 \times$ & $1.1168 \times$ \\
\hline Step & $\begin{array}{l}\text { Burnup } \\
{\left[\frac{\mathrm{MWd}}{\mathrm{MtU}}\right]}\end{array}$ & $\left.\begin{array}{l}{ }^{143} \mathrm{Nd} \\
{\left[\frac{\text { atoms }}{\mathrm{b}-\mathrm{cm}}\right.}\end{array}\right]$ & $\begin{array}{l}{ }^{144} \mathrm{Nd} \\
{\left[\frac{\text { atoms }}{\mathrm{b}-\mathrm{cm}}\right]}\end{array}$ & $\begin{array}{c}\text { MPACT } \\
{ }^{145} \mathrm{Nd} \\
{\left[\frac{\text { atoms }}{\mathrm{b}-\mathrm{cm}}\right]}\end{array}$ & $\begin{array}{l}{ }^{146} \mathrm{Nd} \\
{\left[\frac{\text { atoms }}{\mathrm{b}-\mathrm{cm}}\right]}\end{array}$ & $\begin{array}{l}{ }^{148} \mathrm{Nd} \\
{\left[\frac{\text { atoms }}{\mathrm{b}-\mathrm{cm}}\right]}\end{array}$ \\
\hline 0 & 0.0 & $00 \times 10^{-00}$ & $00 \times 10^{-00}$ & $00 \times 10^{-00}$ & $00 \times 10^{-00}$ & $00 \times 10^{-00}$ \\
\hline 1 & 25.0 & $1 \times 10^{-08}$ & $8 \times 1$ & $72 \times 10^{-08}$ & $3.7892 \times 10^{-08}$ & $2.1366 \times 10^{-08}$ \\
\hline 2 & 50.0 & $7.0852 \times 10^{-08}$ & $5.6062 \times 10^{-09}$ & $9.8214 \times 10^{-08}$ & $7.5741 \times 10^{-08}$ & $4.2755 \times 10^{-08}$ \\
\hline 3 & 100.0 & $2.0354 \times 10^{-07}$ & $2.2020 \times 10^{-08}$ & $1.9593 \times 10^{-07}$ & $1.5131 \times 10^{-07}$ & $8.5528 \times 10^{-08}$ \\
\hline 4 & 200.0 & $4.8914 \times 10^{-07}$ & $8.4059 \times 10^{-08}$ & $3.8998 \times 10^{-07}$ & $3.0198 \times 10^{-07}$ & $1.7098 \times 10^{-07}$ \\
\hline 5 & 300.0 & $7.7301 \times 10^{-07}$ & $1.7997 \times 10^{-07}$ & $5.8228 \times 10^{-07}$ & $4.5207 \times 10^{-07}$ & $2.5630 \times 10^{-07}$ \\
\hline 6 & & $6 \times 10^{-06}$ & $40 \times 10$ & $7.7298 \times 10^{-07}$ & $6.0164 \times 10^{-07}$ & $3.4148 \times 10^{-07}$ \\
\hline 7 & 500.0 & $1.3282 \times 10^{-06}$ & $4.5285 \times 10^{-07}$ & $9.6219 \times 10^{-07}$ & $7.5076 \times 10^{-07}$ & $4.2654 \times 10^{-07}$ \\
\hline 8 & 600.0 & $1.6000 \times 10^{-06}$ & $6.2152 \times 10^{-07}$ & $1.1500 \times 10^{-06}$ & $8.9948 \times 10^{-07}$ & $5.1149 \times 10^{-07}$ \\
\hline 9 & 700.0 & $1.8681 \times 10^{-06}$ & $8.0726 \times 10^{-07}$ & $1.3365 \times 10^{-06}$ & $1.0479 \times 10^{-06}$ & $5.9635 \times 10^{-07}$ \\
\hline 10 & 800.0 & $2.1327 \times 10^{-06}$ & $1.0074 \times 10^{-06}$ & $1.5218 \times 10^{-06}$ & $1.1959 \times 10^{-06}$ & $6.8111 \times 10^{-07}$ \\
\hline 11 & 900.0 & $2.3940 \times 10^{-06}$ & $1.2196 \times 10^{-06}$ & $1.7060 \times 10^{-06}$ & $1.3437 \times 10^{-06}$ & $7.6579 \times 10^{-07}$ \\
\hline 12 & 1000.0 & $2.6522 \times 10^{-06}$ & $1.4421 \times 10^{-06}$ & $1.8890 \times 10^{-06}$ & $1.4911 \times 10^{-06}$ & $8.5038 \times 10^{-07}$ \\
\hline 13 & 1100.0 & $2.9072 \times 10^{-06}$ & $1.6733 \times 10^{-06}$ & $2.0710 \times 10^{-06}$ & $1.6384 \times 10^{-06}$ & $9.3489 \times 10^{-07}$ \\
\hline 14 & 1200.0 & $3.1593 \times 10^{-06}$ & $1.9119 \times 10^{-06}$ & $2.2520 \times 10^{-06}$ & $1.7855 \times 10^{-06}$ & $1.0193 \times 10^{-06}$ \\
\hline 15 & 1300.0 & $3.4084 \times 10^{-06}$ & $2.1566 \times 10^{-06}$ & $2.4320 \times 10^{-06}$ & $1.9323 \times 10^{-06}$ & $1.1037 \times 10^{-06}$ \\
\hline
\end{tabular}


Table 2.15. Strontium isotope concentrations for the reference solution of Problem 1.2.1.

\begin{tabular}{|c|c|c|c|c|c|c|}
\hline Step & $\begin{array}{l}\text { Burnup } \\
{\left[\frac{\mathrm{MWd}}{\mathrm{MtU}}\right]}\end{array}$ & $\begin{array}{c}{ }^{84} \mathrm{Sr} \\
{\left[\frac{\text { atoms }}{\mathrm{b}-\mathrm{cm}}\right]}\end{array}$ & $\begin{array}{c}{ }^{86} \mathrm{Sr} \\
{\left[\frac{\text { atoms }}{\mathrm{b}-\mathrm{cm}}\right]}\end{array}$ & $\begin{array}{c}\text { Shift } \\
{ }^{87} \mathrm{Sr} \\
{\left[\frac{\text { atoms }}{\mathrm{b}-\mathrm{cm}}\right]}\end{array}$ & $\begin{array}{c}{ }^{88} \mathrm{Sr} \\
{\left[\frac{\text { atoms }}{\mathrm{b}-\mathrm{cm}}\right]}\end{array}$ & $\begin{array}{c}{ }^{90} \mathrm{Sr} \\
{\left[\frac{\text { atoms }}{\mathrm{b}-\mathrm{cm}}\right]}\end{array}$ \\
\hline 0 & 0.0 & $0.0000 \times 10^{-00}$ & $0.0000 \times 10^{-00}$ & $0.0000 \times 10^{-00}$ & $0.0000 \times 10^{-00}$ & $0.0000 \times 10^{-00}$ \\
\hline 1 & 25.0 & $2.9632 \times 10^{-19}$ & $3.2991 \times 10^{-14}$ & $1.6835 \times 10^{-14}$ & $4.3634 \times 10^{-08}$ & $7.1265 \times 10^{-08}$ \\
\hline 2 & 50.0 & $1.1645 \times 10^{-18}$ & $1.5560 \times 10^{-13}$ & $3.6459 \times 10^{-14}$ & $8.7491 \times 10^{-08}$ & $1.4204 \times 10^{-07}$ \\
\hline 3 & 100.0 & $4.5198 \times 10^{-18}$ & $7.5028 \times 10^{-13}$ & $8.4102 \times 10^{-14}$ & $1.7451 \times 10^{-07}$ & $2.8221 \times 10^{-07}$ \\
\hline 4 & 200.0 & $1.7166 \times 10^{-17}$ & $3.4961 \times 10^{-12}$ & $2.1111 \times 10^{-13}$ & $3.4596 \times 10^{-07}$ & $5.5738 \times 10^{-07}$ \\
\hline 5 & 300.0 & $3.7493 \times 10^{-17}$ & $8.3374 \times 10^{-12}$ & $3.7699 \times 10^{-13}$ & $5.1423 \times 10^{-07}$ & $8.2610 \times 10^{-07}$ \\
\hline 6 & 400.0 & $6.5289 \times 10^{-17}$ & $1.5236 \times 10^{-11}$ & $5.7832 \times 10^{-13}$ & $6.7959 \times 10^{-07}$ & $1.0888 \times 10^{-06}$ \\
\hline 7 & 500.0 & $1.0054 \times 10^{-16}$ & $2.4135 \times 10^{-11}$ & $8.1235 \times 10^{-13}$ & $8.4226 \times 10^{-07}$ & $1.3460 \times 10^{-06}$ \\
\hline 8 & 600.0 & $1.4302 \times 10^{-16}$ & $3.4987 \times 10^{-11}$ & $1.0766 \times 10^{-12}$ & $1.0025 \times 10^{-06}$ & $1.5980 \times 10^{-06}$ \\
\hline 9 & 700.0 & $1.9275 \times 10^{-16}$ & $4.7763 \times 10^{-11}$ & $1.3689 \times 10^{-12}$ & $1.1603 \times 10^{-06}$ & $1.8450 \times 10^{-06}$ \\
\hline 10 & 800.0 & $2.4960 \times 10^{-16}$ & $6.2413 \times 10^{-11}$ & $1.6874 \times 10^{-12}$ & $1.3160 \times 10^{-06}$ & $2.0874 \times 10^{-06}$ \\
\hline 11 & 900.0 & $3.1394 \times 10^{-16}$ & $7.8915 \times 10^{-11}$ & $2.0306 \times 10^{-12}$ & $1.4697 \times 10^{-06}$ & $2.3253 \times 10^{-06}$ \\
\hline 12 & 1000.0 & $3.8583 \times 10^{-16}$ & $9.7250 \times 10^{-11}$ & $2.3970 \times 10^{-12}$ & $1.6215 \times 10^{-06}$ & $2.5591 \times 10^{-06}$ \\
\hline 13 & 1100.0 & $4.6470 \times 10$ & $1.1743 \times 10^{-10}$ & $2.7852 \times 10^{-12}$ & $1.7714 \times 10^{-06}$ & $2.7888 \times 10^{-06}$ \\
\hline 14 & 1 & $5.5169 \times 10^{-16}$ & $1.3935 \times 10^{-10}$ & $3.1943 \times 10^{-12}$ & $1.9196 \times 10^{-06}$ & $3.0146 \times 10^{-06}$ \\
\hline 15 & 1300.0 & $30 \times$ & $1.6306 \times 10^{-10}$ & $3.6229 \times 10^{-12}$ & $2.0662 \times 10^{-06}$ & $3.2367 \times 10^{-06}$ \\
\hline Step & $\begin{array}{l}\text { Burnup } \\
{\left[\frac{\mathrm{MWd}}{\mathrm{MtU}}\right]}\end{array}$ & $\begin{array}{c}{ }^{84} \mathrm{Sr} \\
{\left[\frac{\text { atoms }}{\mathrm{b}-\mathrm{cm}}\right]}\end{array}$ & $\begin{array}{c}{ }^{86} \mathrm{Sr} \\
{\left[\frac{\text { atoms }}{\mathrm{b}-\mathrm{cm}}\right]}\end{array}$ & $\begin{array}{c}\text { MPACT } \\
{ }^{87} \mathrm{Sr} \\
{\left[\frac{\text { atoms }}{\mathrm{b}-\mathrm{cm}}\right]}\end{array}$ & $\begin{array}{c}{ }^{88} \mathrm{Sr} \\
{\left[\frac{\text { atoms }}{\mathrm{b}-\mathrm{cm}}\right]}\end{array}$ & $\begin{array}{c}{ }^{90} \mathrm{Sr} \\
{\left[\frac{\text { atoms }}{\mathrm{b}-\mathrm{cm}}\right]}\end{array}$ \\
\hline 0 & 0.0 & $0.0000 \times 10^{-00}$ & $0.0000 \times 10^{-00}$ & $0.0000 \times 10^{-00}$ & $0.0000 \times 10^{-00}$ & $0.0000 \times 10^{-00}$ \\
\hline 1 & 25.0 & $1.5878 \times 10^{-18}$ & $2.2275 \times 10^{-14}$ & $1.7027 \times 10^{-14}$ & $4.3574 \times 10^{-08}$ & $7.0375 \times 10^{-08}$ \\
\hline 2 & 50.0 & $3.3119 \times$ & $7.9740 \times$ & $3.7451 \times 1($ & $8.6905 \times 1$ & $1.4027 \times 10^{-07}$ \\
\hline 3 & 100.0 & $7.1491 \times 10^{-18}$ & $2.7124 \times 10^{-13}$ & $8.8555 \times 10^{-14}$ & $1.7289 \times 10^{-07}$ & $2.7872 \times 10^{-07}$ \\
\hline 4 & 200.0 & $1.6287 \times 10^{-17}$ & $8.8742 \times 10^{-13}$ & $2.2979 \times 10^{-13}$ & $3.4230 \times 10^{-07}$ & $5.5051 \times 10^{-07}$ \\
\hline 5 & 300.0 & $2.7220 \times 10^{-17}$ & $1.7705 \times 10^{-12}$ & $4.1961 \times 10^{-13}$ & $5.0856 \times 10^{-07}$ & $8.1591 \times 10^{-07}$ \\
\hline 6 & 400.0 & $3.9796 \times 10^{-17}$ & $2.8976 \times 10^{-12}$ & $6.5450 \times 10^{-13}$ & $6.7195 \times 10^{-07}$ & $1.0754 \times 10^{-06}$ \\
\hline 7 & 500.0 & $5.3883 \times 10^{-17}$ & $4.2533 \times 10^{-12}$ & $9.3146 \times 10^{-13}$ & $8.3268 \times 10^{-07}$ & $1.3294 \times 10^{-06}$ \\
\hline 8 & 600.0 & $6.9375 \times 10^{-17}$ & $5.8256 \times 10^{-12}$ & $1.2480 \times 10^{-12}$ & $9.9097 \times 10^{-07}$ & $1.5783 \times 10^{-06}$ \\
\hline 9 & 700.0 & $8.6174 \times 10^{-17}$ & $7.6044 \times 10^{-12}$ & $1.6018 \times 10^{-12}$ & $1.1470 \times 10^{-06}$ & $1.8223 \times 10^{-06}$ \\
\hline 10 & 800.0 & $1.0420 \times 10^{-16}$ & $9.5811 \times 10^{-12}$ & $1.9909 \times 10^{-12}$ & $1.3009 \times 10^{-06}$ & $2.0617 \times 10^{-06}$ \\
\hline 11 & 900.0 & $1.2338 \times 10^{-16}$ & $1.1749 \times 10^{-11}$ & $2.4137 \times 10^{-12}$ & $1.4527 \times 10^{-06}$ & $2.2968 \times 10^{-06}$ \\
\hline 12 & 1000.0 & $1.4364 \times 10^{-16}$ & $1.4101 \times 10^{-11}$ & $2.8686 \times 10^{-12}$ & $1.6027 \times 10^{-06}$ & $2.5277 \times 10^{-06}$ \\
\hline 13 & 1100.0 & $1.6494 \times 10^{-16}$ & $1.6634 \times 10^{-11}$ & $3.3541 \times 10^{-12}$ & $1.7509 \times 10^{-06}$ & $2.7546 \times 10^{-06}$ \\
\hline 14 & 1200.0 & $1.8721 \times 10^{-16}$ & $1.9342 \times 10^{-11}$ & $3.8691 \times 10^{-12}$ & $1.8974 \times 10^{-06}$ & $2.9778 \times 10^{-06}$ \\
\hline 15 & 1300.0 & $2.1042 \times 10^{-16}$ & $2.2223 \times 10^{-11}$ & $4.4125 \times 10^{-12}$ & $2.0423 \times 10^{-06}$ & $3.1973 \times 10^{-06}$ \\
\hline
\end{tabular}


Table 2.16. Barium isotope concentrations for the reference solution of Problem 1.2.1.

\begin{tabular}{|c|c|c|c|c|c|c|}
\hline Step & $\begin{array}{l}\text { Burnup } \\
{\left[\frac{\mathrm{MWd}}{\mathrm{MtU}}\right]}\end{array}$ & $\begin{array}{c}{ }^{132} \mathrm{Ba} \\
{\left[\frac{\text { atoms }}{\mathrm{b}-\mathrm{cm}}\right]}\end{array}$ & $\begin{array}{c}{ }^{134} \mathrm{Ba} \\
{\left[\frac{\text { atoms }}{\mathrm{b}-\mathrm{cm}}\right]}\end{array}$ & $\begin{array}{c}\text { Shift } \\
{ }^{135} \mathrm{Ba} \\
{\left[\frac{\text { atoms }}{\mathrm{b}-\mathrm{cm}}\right]}\end{array}$ & $\begin{array}{c}{ }^{136} \mathrm{Ba} \\
{\left[\frac{\text { atoms }}{\mathrm{b}-\mathrm{cm}}\right]}\end{array}$ & $\begin{array}{c}{ }^{138} \mathrm{Ba} \\
{\left[\frac{\text { atoms }}{\mathrm{b}-\mathrm{cm}}\right]}\end{array}$ \\
\hline 0 & 0.0 & $0.0000 \times 10^{-00}$ & $0.0000 \times 10^{-00}$ & $0.0000 \times 10^{-00}$ & $0.0000 \times 10^{-00}$ & $0.0000 \times 10^{-00}$ \\
\hline 1 & 25.0 & $1.0103 \times 10^{-17}$ & $2.5861 \times 10^{-14}$ & $1.7001 \times 10^{-15}$ & $3.6169 \times 10^{-11}$ & $8.4864 \times 10^{-08}$ \\
\hline 2 & 50.0 & $3.0395 \times 10^{-17}$ & $3.4304 \times 10^{-13}$ & $5.3205 \times 10^{-15}$ & $1.0079 \times 10^{-10}$ & $1.6983 \times 10^{-07}$ \\
\hline 3 & 100.0 & $9.0693 \times 10^{-17}$ & $3.7510 \times 10^{-12}$ & $1.8306 \times 10^{-14}$ & $2.8493 \times 10^{-10}$ & $3.3950 \times 10^{-07}$ \\
\hline 4 & 200.0 & $2.8506 \times 10^{-16}$ & $3.5152 \times 10^{-11}$ & $6.7438 \times 10^{-14}$ & $8.1997 \times 10^{-10}$ & $6.7784 \times 10^{-07}$ \\
\hline 5 & 300.0 & $5.8463 \times 10^{-16}$ & $1.2362 \times 10^{-10}$ & $1.4920 \times 10^{-13}$ & $1.5532 \times 10^{-09}$ & $1.0150 \times 10^{-06}$ \\
\hline 6 & 400.0 & $9.9027 \times 10^{-16}$ & $2.9622 \times 10^{-10}$ & $2.6799 \times 10^{-13}$ & $2.4715 \times 10^{-09}$ & $1.3510 \times 10^{-06}$ \\
\hline 7 & 500.0 & $1.5040 \times 10^{-15}$ & $5.7783 \times 10^{-10}$ & $4.3042 \times 10^{-13}$ & $3.5646 \times 10^{-09}$ & $1.6860 \times 10^{-06}$ \\
\hline 8 & 600.0 & $2.1225 \times 10^{-15}$ & $9.9142 \times 10^{-10}$ & $6.4504 \times 10^{-13}$ & $4.8247 \times 10^{-09}$ & $2.0201 \times 10^{-06}$ \\
\hline 9 & 700.0 & $2.8552 \times 10^{-15}$ & $1.5582 \times 10^{-09}$ & $9.2251 \times 10^{-13}$ & $6.2441 \times 10^{-09}$ & $2.3532 \times 10^{-06}$ \\
\hline 10 & 800.0 & $3.6968 \times 10^{-15}$ & $2.2977 \times 10^{-09}$ & $1.2747 \times 10^{-12}$ & $7.8168 \times 10^{-09}$ & $2.6855 \times 10^{-06}$ \\
\hline 11 & 900.0 & $4.6597 \times 10^{-15}$ & $3.2282 \times 10^{-09}$ & $1.7153 \times 10^{-12}$ & $9.5359 \times 10^{-09}$ & $3.0171 \times 10^{-06}$ \\
\hline 12 & 1000.0 & $5.7319 \times 10^{-15}$ & $4.3663 \times 10^{-09}$ & $2.2593 \times 10^{-12}$ & $1.1398 \times 10^{-08}$ & $3.3479 \times 10^{-06}$ \\
\hline 13 & 1100.0 & $6.9164 \times 10$ & $5.7280 \times 1$ & $2.9230 \times 10^{-12}$ & $1.3398 \times 10^{-08}$ & $3.6780 \times 10^{-06}$ \\
\hline 14 & 1200.0 & $8.2432 \times 10^{-15}$ & $7.3274 \times 10^{-09}$ & $3.7219 \times 10^{-12}$ & $1.5532 \times 10^{-08}$ & $4.0074 \times 10^{-06}$ \\
\hline 15 & 1300.0 & $79 x$ & $.1778 \times 10^{-09}$ & $4.6794 \times 10^{-12}$ & $1.7798 \times 10^{-08}$ & $4.3362 \times 10^{-06}$ \\
\hline Step & $\begin{array}{l}\text { Burnup } \\
{\left[\frac{\mathrm{MWd}}{\mathrm{MtU}}\right]}\end{array}$ & $\begin{array}{l}{ }^{132} \mathrm{Ba} \\
{\left[\frac{\text { atoms }}{\mathrm{b}-\mathrm{cm}}\right]}\end{array}$ & $\begin{array}{l}{ }^{134} \mathrm{Ba} \\
{\left[\frac{\text { atoms }}{\mathrm{b}-\mathrm{cm}}\right]}\end{array}$ & $\begin{array}{c}\text { MPACT } \\
{ }^{135} \mathrm{Ba} \\
{\left[\frac{\text { atoms }}{\mathrm{b}-\mathrm{cm}}\right]}\end{array}$ & $\begin{array}{l}{ }^{136} \mathrm{Ba} \\
{\left[\frac{\text { atoms }}{\mathrm{b}-\mathrm{cm}}\right]}\end{array}$ & $\begin{array}{l}{ }^{138} \mathrm{Ba} \\
{\left[\frac{\text { atoms }}{\mathrm{b}-\mathrm{cm}}\right]}\end{array}$ \\
\hline 0 & 0.0 & $0.0000 \times 10^{-00}$ & $0.0000 \times 10^{-00}$ & $0.0000 \times 10^{-00}$ & $0.0000 \times 10^{-00}$ & $0.0000 \times 10^{-00}$ \\
\hline 1 & 25 & $0.0000 \times 10^{-00}$ & $2.9481 \times 10^{-14}$ & $1.0788 \times 10^{-15}$ & $1.1071 \times 10^{-11}$ & $8.4065 \times 10^{-08}$ \\
\hline 2 & 0 & & 3.4 & $3.4532 \times 10^{-15}$ & -11 & $1.6803 \times 10^{-07}$ \\
\hline 3 & 100.0 & $1.0020 \times 10^{-21}$ & $3.5217 \times 10^{-12}$ & $1.2166 \times 10^{-14}$ & $1.2176 \times 10^{-10}$ & $3.3569 \times 10^{-07}$ \\
\hline 4 & 200.0 & $8.5373 \times 10^{-20}$ & $3.1935 \times 10^{-11}$ & $4.6062 \times 10^{-14}$ & $3.8195 \times 10^{-10}$ & $6.7007 \times 10^{-07}$ \\
\hline 5 & 300.0 & $4.2871 \times 10^{-19}$ & $1.1115 \times 10^{-10}$ & $1.0456 \times 10^{-13}$ & $7.5347 \times 10^{-10}$ & $1.0032 \times 10^{-06}$ \\
\hline 6 & 400.0 & $1.3394 \times 10^{-18}$ & $2.6497 \times 10^{-10}$ & $1.9291 \times 10^{-13}$ & $1.2300 \times 10^{-09}$ & $1.3353 \times 10^{-06}$ \\
\hline 7 & 500.0 & $3.2424 \times 10^{-18}$ & $5.1523 \times 10^{-10}$ & $3.1853 \times 10^{-13}$ & $1.8070 \times 10^{-09}$ & $1.6663 \times 10^{-06}$ \\
\hline 8 & 600.0 & $6.6726 \times 10^{-18}$ & $8.8205 \times 10^{-10}$ & $4.9083 \times 10^{-13}$ & $2.4804 \times 10^{-09}$ & $1.9964 \times 10^{-06}$ \\
\hline 9 & 700.0 & $1.2268 \times 10^{-17}$ & $1.3840 \times 10^{-09}$ & $7.2098 \times 10^{-13}$ & $3.2470 \times 10^{-09}$ & $2.3257 \times 10^{-06}$ \\
\hline 10 & 800.0 & $2.0765 \times 10^{-17}$ & $2.0381 \times 10^{-09}$ & $1.0218 \times 10^{-12}$ & $4.1038 \times 10^{-09}$ & $2.6541 \times 10^{-06}$ \\
\hline 11 & 900.0 & $3.2990 \times 10^{-17}$ & $2.8601 \times 10^{-09}$ & $1.4077 \times 10^{-12}$ & $5.0482 \times 10^{-09}$ & $2.9818 \times 10^{-06}$ \\
\hline 12 & 1000.0 & $4.9857 \times 10^{-17}$ & $3.8648 \times 10^{-09}$ & $1.8944 \times 10^{-12}$ & $6.0782 \times 10^{-09}$ & $3.3087 \times 10^{-06}$ \\
\hline 13 & 1100.0 & $7.2363 \times 10^{-17}$ & $5.0655 \times 10^{-09}$ & $2.4989 \times 10^{-12}$ & $7.1917 \times 10^{-09}$ & $3.6349 \times 10^{-06}$ \\
\hline 14 & 1200.0 & $1.0158 \times 10^{-16}$ & $6.4748 \times 10^{-09}$ & $3.2396 \times 10^{-12}$ & $8.3871 \times 10^{-09}$ & $3.9605 \times 10^{-06}$ \\
\hline 15 & 1300.0 & $1.3866 \times 10^{-16}$ & $8.1041 \times 10^{-09}$ & $4.1357 \times 10^{-12}$ & $9.6627 \times 10^{-09}$ & $4.2855 \times 10^{-06}$ \\
\hline
\end{tabular}


Table 2.17. Technetium isotope concentrations for the reference solution of Problem 1.2.1.

\begin{tabular}{rrc}
\hline & & MPACT \\
Step & $\begin{array}{c}\text { Burnup } \\
{\left[\frac{\mathrm{MWd}}{\mathrm{MtU}}\right]}\end{array}$ & {$\left[\frac{\mathrm{atoms}}{\mathrm{b}-\mathrm{cm}}\right]$} \\
\hline 0 & 0.0 & $0.0000 \times 10^{-00}$ \\
1 & 25.0 & $5.8550 \times 10^{-08}$ \\
2 & 50.0 & $1.3587 \times 10^{-07}$ \\
3 & 100.0 & $2.9087 \times 10^{-07}$ \\
4 & 200.0 & $6.0046 \times 10^{-07}$ \\
5 & 300.0 & $9.0954 \times 10^{-07}$ \\
6 & 400.0 & $1.2181 \times 10^{-06}$ \\
7 & 500.0 & $1.5262 \times 10^{-06}$ \\
8 & 600.0 & $1.8338 \times 10^{-06}$ \\
9 & 700.0 & $2.1409 \times 10^{-06}$ \\
10 & 800.0 & $2.4476 \times 10^{-06}$ \\
11 & 900.0 & $2.7538 \times 10^{-06}$ \\
12 & 1000.0 & $3.0595 \times 10^{-06}$ \\
13 & 1100.0 & $3.3648 \times 10^{-06}$ \\
14 & 1200.0 & $3.6697 \times 10^{-06}$ \\
15 & 1300.0 & $3.9741 \times 10^{-06}$ \\
\hline & & MPACT \\
Step & Burnup & $99 \mathrm{Tc}$ \\
& {$\left[\frac{\mathrm{MWd}}{\mathrm{MtU}}\right]$} & {$\left[\frac{\mathrm{atoms}}{\mathrm{b}-\mathrm{cm}}\right]$} \\
\hline 0 & 0.0 & $0.0000 \times 10^{-00}$ \\
1 & 25.0 & $5.7859 \times 10^{-08}$ \\
2 & 50.0 & $1.3427 \times 10^{-07}$ \\
3 & 100.0 & $2.8747 \times 10^{-07}$ \\
4 & 200.0 & $5.9351 \times 10^{-07}$ \\
5 & 300.0 & $8.9909 \times 10^{-07}$ \\
6 & 400.0 & $1.2042 \times 10^{-06}$ \\
7 & 500.0 & $1.5087 \times 10^{-06}$ \\
8 & 600.0 & $1.8128 \times 10^{-06}$ \\
9 & 700.0 & $2.1165 \times 10^{-06}$ \\
10 & 800.0 & $2.4196 \times 10^{-06}$ \\
11 & 900.0 & $2.7224 \times 10^{-06}$ \\
12 & 1000.0 & $3.0247 \times 10^{-06}$ \\
13 & 1100.0 & $3.3265 \times 10^{-06}$ \\
14 & 1200.0 & $3.6280 \times 10^{-06}$ \\
15 & 1300.0 & $3.9290 \times 10^{-06}$ \\
\hline & &
\end{tabular}


Table 2.18. Rubidium isotope concentrations for the reference solution of Problem 1.2.1.

\begin{tabular}{|c|c|c|c|}
\hline \multirow[b]{2}{*}{ Step } & \multirow[b]{2}{*}{$\begin{array}{l}\text { Burnup } \\
{\left[\frac{\mathrm{MWd}}{\mathrm{MtU}}\right]}\end{array}$} & \multicolumn{2}{|c|}{ Shift } \\
\hline & & $\begin{array}{l}{ }^{85} \mathrm{Rb} \\
{\left[\frac{\text { atoms }}{\mathrm{b}-\mathrm{cm}}\right]}\end{array}$ & $\begin{array}{l}{ }^{87} \mathrm{Rb} \\
{\left[\frac{\text { atoms }}{\mathrm{b}-\mathrm{cm}}\right]}\end{array}$ \\
\hline 0 & 0.0 & $0.0000 \times 10^{-00}$ & $0.0000 \times 10^{-}$ \\
\hline 1 & 25.0 & $2545 \times 10^{-08}$ & $3.1617 \times 10^{-08}$ \\
\hline 2 & 50.0 & $2.5233 \times 10^{-08}$ & $6.3199 \times 10^{-08}$ \\
\hline 3 & 100.0 & $5.0456 \times 10^{-08}$ & $1.2588 \times 10^{-07}$ \\
\hline 4 & 200.0 & $1.0034 \times 10^{-07}$ & $2.4944 \times 10^{-07}$ \\
\hline 5 & 300.0 & $1.4954 \times 10^{-07}$ & $3.7079 \times 10^{-07}$ \\
\hline 6 & 0 & $9813 \times 10^{-07}$ & $4.9011 \times 10^{-07}$ \\
\hline 7 & 50 & $2.4617 \times 10^{-07}$ & $6.0758 \times 10^{-07}$ \\
\hline 8 & 0 & $2.9369 \times 10^{-07}$ & $7.2331 \times 10^{-07}$ \\
\hline 9 & 700.0 & $3.4074 \times 10^{-07}$ & $8.3744 \times 10^{-07}$ \\
\hline 10 & & $3.8736 \times 10^{-07}$ & $9.5006 \times 10^{-07}$ \\
\hline 11 & 0 & $4.3358 \times$ & $1.0613 \times 10^{-06}$ \\
\hline 12 & 0 & $4.7942 \times$ & $1.1711 \times 10^{-06}$ \\
\hline 13 & & $5.2490 \times$ & $1.2797 \times 10^{-06}$ \\
\hline 14 & & $5.7006 \times 10^{-07}$ & $1.3872 \times 10^{-06}$ \\
\hline \multirow[t]{2}{*}{15} & & $90 \times 10^{-07}$ & $1.4934 \times 10^{-06}$ \\
\hline & & \multicolumn{2}{|c|}{ MPACT } \\
\hline Step & $\begin{array}{l}\text { Burnup } \\
{\left[\frac{\mathrm{MWd}}{\mathrm{MtU}}\right]}\end{array}$ & $\begin{array}{l}{ }^{85} \mathrm{Rb} \\
{\left[\frac{\text { atoms }}{\mathrm{b}-\mathrm{cm}}\right]}\end{array}$ & $\begin{array}{l}{ }^{87} \mathrm{Rb} \\
{\left[\frac{\text { atoms }}{\mathrm{b}-\mathrm{cm}}\right]}\end{array}$ \\
\hline 0 & 0.0 & $0.0000 \times 10^{-00}$ & $0.0000 \times 10^{-00}$ \\
\hline 1 & 25.0 & $3.2985 \times 10^{-11}$ & $3.1345 \times 10^{-08}$ \\
\hline 2 & 0 & $7.6130 \times 10^{-11}$ & $6.2523 \times 10^{-08}$ \\
\hline 3 & 0 & $1.9262 \times 10^{-10}$ & $1.2441 \times 10^{-07}$ \\
\hline 4 & 200.0 & $5.4431 \times 10^{-10}$ & $2.4640 \times 10^{-07}$ \\
\hline 5 & 30 & $1.0508 \times 10^{-09}$ & $3.6621 \times 10^{-07}$ \\
\hline 6 & 0 & $1.7077 \times 10^{-09}$ & $4.8401 \times 10^{-07}$ \\
\hline$r$ & & $2.5112 \times 10^{-09}$ & $5.9998 \times 10^{-07}$ \\
\hline 8 & & $3.4572 \times 10^{-09}$ & $7.1425 \times 10^{-07}$ \\
\hline 9 & 700.0 & $4.5423 \times 10^{-09}$ & $8.2694 \times 10^{-07}$ \\
\hline 10 & & $5.7629 \times 10^{-09}$ & $9.3814 \times 10^{-07}$ \\
\hline 11 & .0 & $7.1157 \times 10^{-09}$ & $1.0480 \times 10^{-06}$ \\
\hline 12 & 1000.0 & $8.5974 \times 10^{-09}$ & $1.1565 \times 10^{-06}$ \\
\hline 13 & 1100.0 & $1.0205 \times 10^{-08}$ & $1.2637 \times 10^{-06}$ \\
\hline 14 & 1200.0 & $1.1935 \times 10^{-08}$ & $1.3698 \times 10^{-06}$ \\
\hline 15 & 1300.0 & $1.3786 \times 10^{-08}$ & $1.4747 \times 10^{-06}$ \\
\hline
\end{tabular}


Table 2.19. Relative difference between MPACT and Shift concentrations for uranium and plutonium isotopes

\begin{tabular}{rrccccccccc}
\hline Step & $\begin{array}{c}\text { Burnup } \\
{\left[\frac{\mathrm{MWd}}{\mathrm{MtU}}\right]}\end{array}$ & $\begin{array}{r}{ }^{234} \mathrm{U} \\
{[\%]}\end{array}$ & $\begin{array}{c}{ }^{235} \mathrm{U} \\
{[\%]}\end{array}$ & $\begin{array}{c}{ }^{236} \mathrm{U} \\
{[\%]}\end{array}$ & $\begin{array}{c}{ }^{238} \mathrm{U} \\
{[\%]}\end{array}$ & $\begin{array}{c}{ }^{238} \mathrm{Pu} \\
{[\%]}\end{array}$ & $\begin{array}{c}{ }^{239} \mathrm{Pu} \\
{[\%]}\end{array}$ & $\begin{array}{c}{ }^{240} \mathrm{Pu} \\
{[\%]}\end{array}$ & $\begin{array}{c}{ }^{24} \mathrm{Pu} \\
{[\%]}\end{array}$ & $\begin{array}{c}{ }^{242} \mathrm{Pu} \\
{[\%]}\end{array}$ \\
\hline 0 & 0.0 & - & - & - & - & - & - & - & - & - \\
1 & 25.0 & 0.00 & 0.01 & -1.14 & 0.00 & 2.82 & -0.93 & -0.96 & -1.86 & -2.17 \\
2 & 50.0 & 0.00 & 0.01 & -1.14 & 0.00 & 2.74 & -0.94 & -1.07 & -2.57 & -2.70 \\
3 & 100.0 & 0.01 & 0.02 & -1.13 & 0.00 & 2.65 & -0.94 & -1.12 & -2.68 & -2.94 \\
4 & 200.0 & 0.01 & 0.04 & -1.12 & 0.00 & 2.58 & -0.87 & -1.24 & -2.61 & -3.02 \\
5 & 300.0 & 0.02 & 0.07 & -1.11 & 0.00 & 2.39 & -0.85 & -1.21 & -2.85 & -3.17 \\
6 & 400.0 & 0.03 & 0.09 & -1.10 & 0.00 & 2.15 & -0.85 & -1.16 & -3.04 & -3.32 \\
7 & 500.0 & 0.04 & 0.11 & -1.09 & 0.00 & 1.87 & -0.85 & -1.13 & -2.99 & -3.39 \\
8 & 600.0 & 0.05 & 0.13 & -1.08 & 0.00 & 1.60 & -0.85 & -1.12 & -2.97 & -3.41 \\
9 & 700.0 & 0.06 & 0.16 & -1.07 & 0.00 & 1.34 & -0.86 & -1.10 & -2.85 & -3.38 \\
10 & 800.0 & 0.06 & 0.18 & -1.06 & 0.00 & 1.08 & -0.87 & -1.08 & -2.83 & -3.34 \\
11 & 900.0 & 0.07 & 0.20 & -1.05 & 0.00 & 0.84 & -0.87 & -1.06 & -2.79 & -3.31 \\
12 & 1000.0 & 0.08 & 0.23 & -1.04 & 0.00 & 0.61 & -0.87 & -1.06 & -2.65 & -3.27 \\
13 & 1100.0 & 0.09 & 0.25 & -1.03 & 0.00 & 0.38 & -0.88 & -1.04 & -2.55 & -3.21 \\
14 & 1200.0 & 0.10 & 0.27 & -1.02 & 0.00 & 0.17 & -0.88 & -1.04 & -2.38 & -3.13 \\
15 & 1300.0 & 0.11 & 0.29 & -1.01 & 0.00 & -0.02 & -0.89 & -1.04 & -2.28 & -3.05 \\
\hline
\end{tabular}

Table 2.20. Relative difference between MPACT and Shift concentrations for americium and curium isotopes

\begin{tabular}{rrcccc}
\hline Step & $\begin{array}{c}\text { Burnup } \\
{\left[\frac{\mathrm{MWd}}{\mathrm{MtU}}\right]}\end{array}$ & $\begin{array}{c}{ }^{241} \mathrm{Am} \\
{[\%]}\end{array}$ & $\begin{array}{c}{ }^{243} \mathrm{Am} \\
{[\%]}\end{array}$ & $\begin{array}{c}{ }^{243} \mathrm{Cm} \\
{[\%]}\end{array}$ & $\begin{array}{c}{ }^{244} \mathrm{Cm} \\
{[\%]}\end{array}$ \\
\hline 0 & 0.0 & - & - & - & - \\
1 & 25.0 & -2.00 & -3.37 & - & - \\
2 & 50.0 & -2.49 & -2.58 & - & -4.40 \\
3 & 100.0 & -2.77 & -3.26 & -4.30 & -3.57 \\
4 & 200.0 & -2.83 & -3.24 & -2.94 & -3.54 \\
5 & 300.0 & -2.90 & -3.18 & -3.18 & -3.51 \\
6 & 400.0 & -3.01 & -3.03 & -3.25 & -3.47 \\
7 & 500.0 & -3.04 & -3.15 & -3.52 & -3.64 \\
8 & 600.0 & -3.03 & -3.76 & -3.77 & -3.95 \\
9 & 700.0 & -2.99 & -3.86 & -3.74 & -4.12 \\
10 & 800.0 & -2.94 & -3.74 & -3.78 & -4.32 \\
11 & 900.0 & -2.90 & -3.72 & -3.78 & -4.33 \\
12 & 1000.0 & -2.85 & -3.69 & -3.81 & -4.33 \\
13 & 1100.0 & -2.78 & -3.67 & -3.75 & -4.31 \\
14 & 1200.0 & -2.70 & -3.62 & -3.72 & -4.30 \\
15 & 1300.0 & -2.60 & -3.67 & -3.67 & -4.35 \\
\hline
\end{tabular}


Table 2.21. Relative difference between MPACT and Shift concentrations for cesium and neodymium isotopes

\begin{tabular}{rrrccccccc}
\hline Step & $\begin{array}{c}\text { Burnup } \\
{\left[\frac{\mathrm{MWd}}{\mathrm{MtU}}\right]}\end{array}$ & $\begin{array}{c}{ }^{33} \mathrm{Cs} \\
{[\%]}\end{array}$ & $\begin{array}{c}{ }^{135} \mathrm{Cs} \\
{[\%]}\end{array}$ & $\begin{array}{c}{ }^{137} \mathrm{Cs} \\
{[\%]}\end{array}$ & $\begin{array}{c}{ }^{143} \mathrm{Nd} \\
{[\%]}\end{array}$ & $\begin{array}{c}{ }^{144} \mathrm{Nd} \\
{[\%]}\end{array}$ & $\begin{array}{c}{ }^{145} \mathrm{Nd} \\
{[\%]}\end{array}$ & $\begin{array}{c}{ }^{146} \mathrm{Nd} \\
{[\%]}\end{array}$ & $\begin{array}{c}{ }^{148} \mathrm{Nd} \\
{[\%]}\end{array}$ \\
\hline 0 & 0.0 & - & - & - & - & - & - & - & - \\
1 & 25.0 & 11.64 & -0.24 & -1.23 & -1.40 & -2.99 & 0.87 & -1.02 & -1.17 \\
2 & 50.0 & 4.04 & -0.25 & -1.22 & -1.49 & -2.92 & -0.20 & -1.12 & -1.17 \\
3 & 100.0 & 1.06 & -0.25 & -1.21 & -1.53 & -2.76 & -0.71 & -1.17 & -1.16 \\
4 & 200.0 & -0.15 & -0.25 & -1.20 & -1.55 & -2.62 & -0.97 & -1.18 & -1.15 \\
5 & 300.0 & -0.50 & -0.25 & -1.20 & -1.52 & -2.52 & -1.05 & -1.19 & -1.15 \\
6 & 400.0 & -0.68 & -0.25 & -1.20 & -1.51 & -2.47 & -1.09 & -1.20 & -1.15 \\
7 & 500.0 & -0.78 & -0.26 & -1.20 & -1.49 & -2.44 & -1.12 & -1.20 & -1.15 \\
8 & 600.0 & -0.85 & -0.26 & -1.20 & -1.48 & -2.41 & -1.14 & -1.21 & -1.15 \\
9 & 700.0 & -0.90 & -0.26 & -1.20 & -1.48 & -2.40 & -1.15 & -1.21 & -1.15 \\
10 & 800.0 & -0.93 & -0.27 & -1.20 & -1.47 & -2.39 & -1.16 & -1.21 & -1.15 \\
11 & 900.0 & -0.96 & -0.27 & -1.20 & -1.46 & -2.38 & -1.16 & -1.22 & -1.15 \\
12 & 1000.0 & -0.98 & -0.27 & -1.20 & -1.45 & -2.37 & -1.17 & -1.22 & -1.15 \\
13 & 1100.0 & -1.00 & -0.27 & -1.20 & -1.44 & -2.36 & -1.17 & -1.22 & -1.15 \\
14 & 1200.0 & -1.01 & -0.27 & -1.20 & -1.43 & -2.36 & -1.17 & -1.22 & -1.15 \\
15 & 1300.0 & -1.02 & -0.27 & -1.19 & -1.42 & -2.35 & -1.17 & -1.22 & -1.14 \\
\hline
\end{tabular}

Table 2.22. Relative difference between MPACT and Shift concentrations for miscellaneous fission product isotopes

\begin{tabular}{rrccccc}
\hline Step & $\begin{array}{c}\text { Burnup } \\
{\left[\frac{\mathrm{MWd}}{\mathrm{MtU}}\right]}\end{array}$ & $\begin{array}{c}{ }^{88} \mathrm{Sr} \\
{[\%]}\end{array}$ & $\begin{array}{c}{ }^{90} \mathrm{Sr} \\
{[\%]}\end{array}$ & $\begin{array}{c}{ }^{138} \mathrm{Ba} \\
{[\%]}\end{array}$ & $\begin{array}{c}{ }^{99} \mathrm{Tc} \\
{[\%]}\end{array}$ & $\begin{array}{c}{ }^{87} \mathrm{Rb} \\
{[\%]}\end{array}$ \\
\hline 0 & 0.0 & - & - & - & - & - \\
1 & 25.0 & -0.17 & -1.28 & -0.95 & -1.17 & -0.88 \\
2 & 50.0 & -0.70 & -1.28 & -1.07 & -1.17 & -1.09 \\
3 & 100.0 & -0.96 & -1.27 & -1.13 & -1.16 & -1.20 \\
4 & 200.0 & -1.09 & -1.27 & -1.15 & -1.15 & -1.24 \\
5 & 300.0 & -1.13 & -1.27 & -1.16 & -1.14 & -1.26 \\
6 & 400.0 & -1.16 & -1.27 & -1.17 & -1.14 & -1.27 \\
7 & 500.0 & -1.17 & -1.27 & -1.18 & -1.14 & -1.28 \\
8 & 600.0 & -1.18 & -1.27 & -1.18 & -1.14 & -1.28 \\
9 & 700.0 & -1.18 & -1.27 & -1.18 & -1.14 & -1.28 \\
10 & 800.0 & -1.19 & -1.26 & -1.18 & -1.14 & -1.28 \\
11 & 900.0 & -1.19 & -1.26 & -1.18 & -1.14 & -1.28 \\
12 & 1000.0 & -1.19 & -1.26 & -1.18 & -1.14 & -1.28 \\
13 & 1100.0 & -1.19 & -1.26 & -1.18 & -1.14 & -1.28 \\
14 & 1200.0 & -1.19 & -1.26 & -1.18 & -1.14 & -1.28 \\
15 & 1300.0 & -1.19 & -1.25 & -1.18 & -1.14 & -1.27 \\
\hline
\end{tabular}




\section{PROBLEM 2: CHARGE-PAN 2D}

In the parlance of Magnox reactors, a charge-pan is roughly equivalent to a fuel assembly or bundle. The primary difference is that Magnox fuel elements in adjacent channels are not physically bound to each other like those in a fuel assembly. Despite this difference, Problem 2 is essentially a lattice physics problem. Geometric dimensions are listed in Table 1.2. All external boundaries are fully reflected in this problem. Problem 2 is more complex than Problem 1 because it adds multiple fuel channels and the central control rod X-hole.

\subsection{BEGINNING-OF-CYCLE}

\subsubsection{Problem 2.1.1}

\subsubsection{Description}

This problem uses coolant channel radius $\mathrm{C}$, which has the smallest radius. The geometry is shown in Figure 3. The layout of pins within the charge pan is shown in Figure 4. The $\mathrm{x}$-axis (horizontal) and y-axis (vertical) projections of channels $\mathrm{A}, \mathrm{B}, \mathrm{C}$, and $\mathrm{D}$ do not overlap with axis projections of the central $\mathrm{X}$-hole. Owing to the $1 / 8$ symmetry, fuel channels A, B, C and D will have the same power. Also because of symmetry, fuel channels E, F, G, H, J, K, L, and M will have the same power, and fuel channels P, Q, R, and $\mathrm{S}$ will have the same power. Material densities are listed in Table 1.4. Temperatures are listed in Table 2.4.

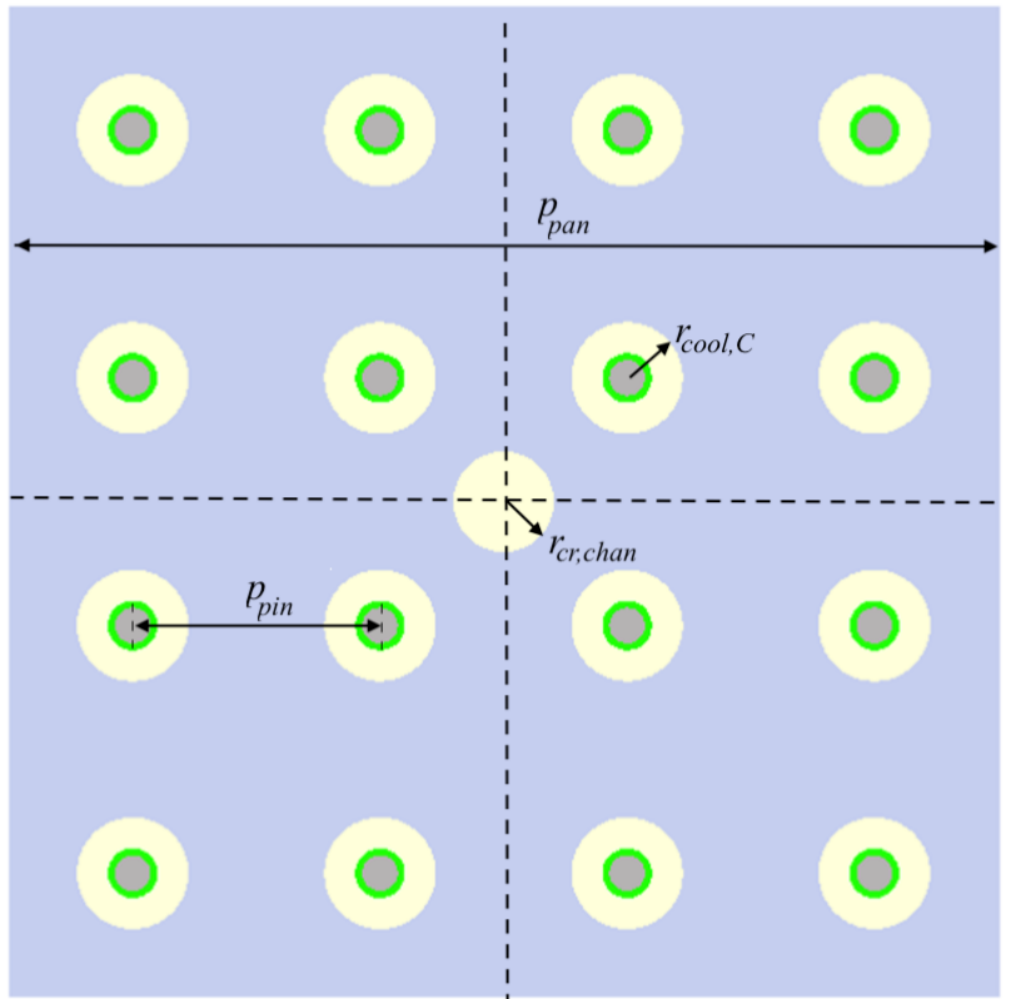

Figure 3. Magnox 2D charge-pan with Zone C coolant channel. 


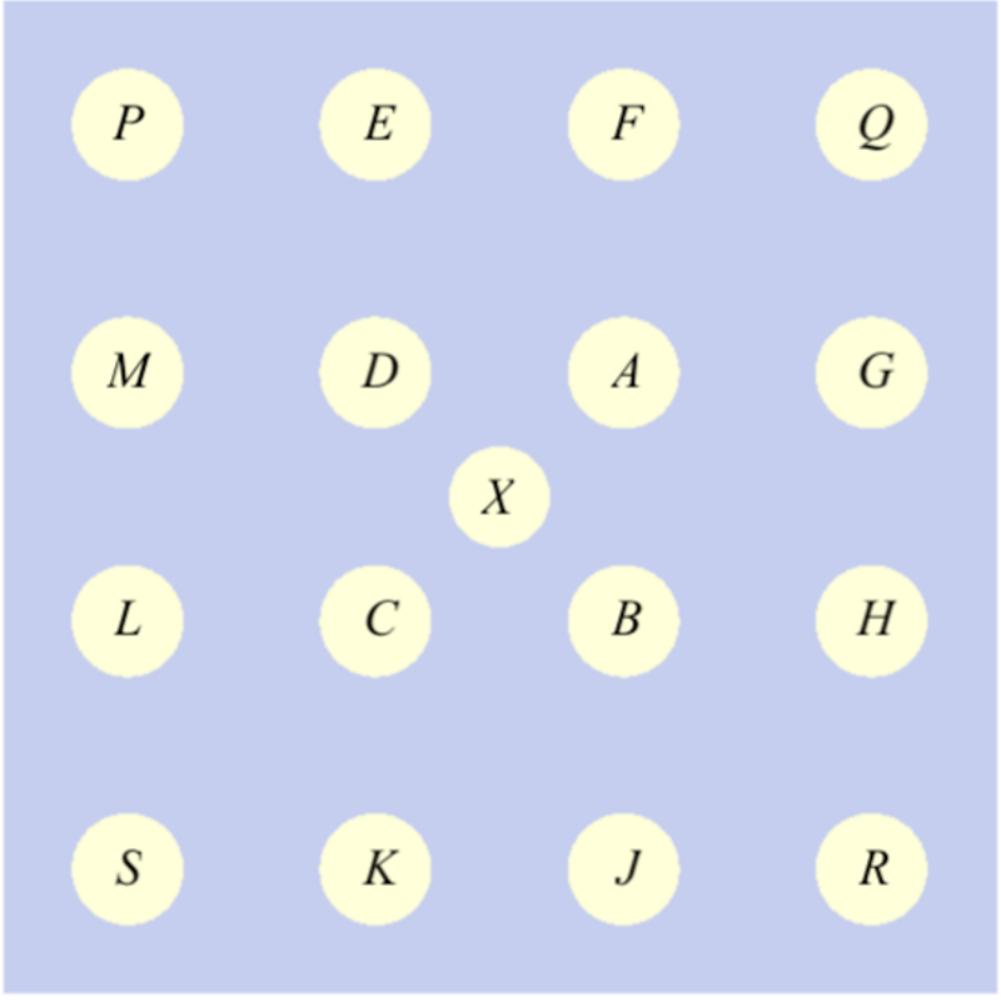

Figure 4. Holes within a charge-pan. 


\subsubsection{Reference Solution}

Table 3.1. Neutron multiplication for the reference solution of Problem 2.1.1.

\begin{tabular}{rrccc}
\hline Step & $\begin{array}{c}\text { Burnup } \\
{\left[\frac{\mathrm{MWd}}{\mathrm{MtU}}\right]}\end{array}$ & $\begin{array}{c}\text { Shift } \\
\mathrm{k}_{\mathrm{eff}}(1 \sigma)\end{array}$ & $\begin{array}{c}\text { MPACT } \\
\mathrm{k}_{\mathrm{eff}}(1 \sigma)\end{array}$ & $\begin{array}{c}\text { Difference } \\
{[\mathrm{pcm}]}\end{array}$ \\
\hline 0 & 0.0 & $1.06949(10)$ & 1.06976 & $28(10)$ \\
\hline
\end{tabular}

Table 3.2. Power peaking factors for the reference solution of Problem 2.1.1.

\begin{tabular}{lccc}
\hline \multicolumn{1}{c}{ Channels } & $\begin{array}{c}\text { Shift } \\
\text { Peaking Factor }\end{array}$ & $\begin{array}{c}\text { MPACT } \\
\text { Peaking Factor }\end{array}$ & $\begin{array}{c}\text { Difference } \\
\text { (MPACT-Shift) }\end{array}$ \\
\hline A,B,C,D & 0.992 & 0.992 & 0.000 \\
E,F,G,H,J,K,L,M & 1.002 & 1.002 & 0.000 \\
P,Q,R,S & 1.004 & 1.004 & 0.000 \\
\hline
\end{tabular}




\subsubsection{Problem 2.1.2}

\subsubsection{Description}

Problem 2.1.2 uses the same geometry as Problem 2.1.1, except it uses coolant channel radius A shown in Figure 5. In this geometry, the $\mathrm{x}$-axis (horizontal) and $\mathrm{y}$-axis (vertical) projections of channels $\mathrm{A}, \mathrm{B}, \mathrm{C}$, and D overlap with axis projections of the central X-hole. Material densities are listed in Table 1.4. Temperatures are listed in Table 2.4.

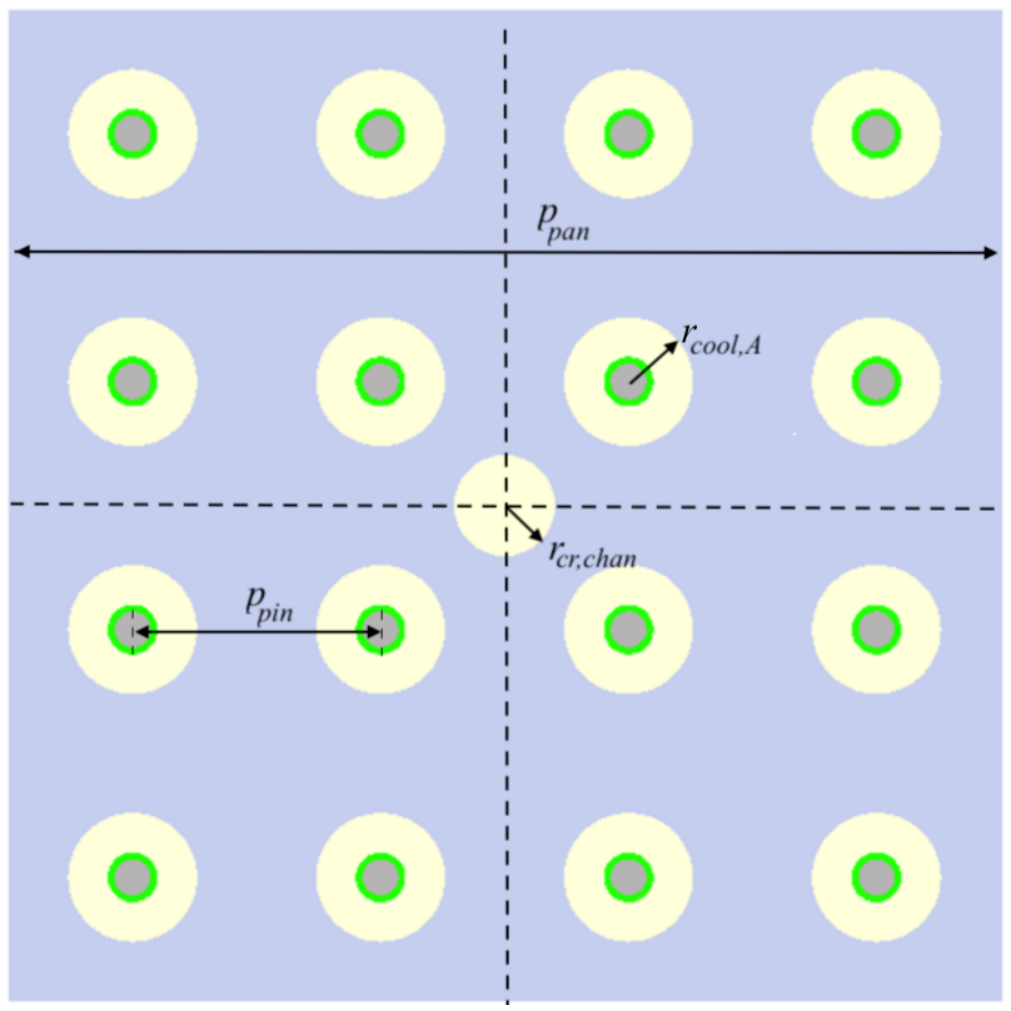

Figure 5. Magnox 2D charge-pan with Zone A coolant channel. 


\subsubsection{Reference Solution}

Table 3.3. Neutron multiplication for the reference solution of Problem 2.1.2.

\begin{tabular}{crccc}
\hline Step & $\begin{array}{c}\text { Burnup } \\
{\left[\frac{\mathrm{MWd}}{\mathrm{MtU}}\right]}\end{array}$ & $\begin{array}{c}\text { Shift } \\
\mathrm{k}_{\mathrm{eff}}(1 \sigma)\end{array}$ & $\begin{array}{c}\text { MPACT } \\
\mathrm{k}_{\mathrm{eff}}(1 \sigma)\end{array}$ & $\begin{array}{c}\text { Difference } \\
{[\mathrm{pcm}]}\end{array}$ \\
\hline 0 & 0.0 & $1.06460(10)$ & 1.06518 & $58(10)$ \\
\hline
\end{tabular}

Table 3.4. Power peaking factors for the reference solution of Problem 2.1.2

\begin{tabular}{lccc}
\hline \multicolumn{1}{c}{ Channels } & $\begin{array}{c}\text { Shift } \\
\text { Peaking Factor }\end{array}$ & $\begin{array}{c}\text { MPACT } \\
\text { Peaking Factor }\end{array}$ & $\begin{array}{c}\text { Difference } \\
\text { (MPACT-Shift) }\end{array}$ \\
\hline A, B, C, D & 0.992 & 0.992 & 0.000 \\
E, F, G, H, J, K, L, M & 1.002 & 1.002 & 0.0000 \\
P, Q, R, S & 1.004 & 1.004 & -0.001 \\
\hline
\end{tabular}

\subsubsection{Problem 2.1.3}

\subsubsection{Description}

Problem 2.1.3 uses the same geometry as Problem 2.1.2, except it has a control rod inserted in the central coolant channel. The control rod has an outer stainless steel sheath and an internal stainless steel portion doped with $3 \mathrm{wt} \%$ boron. Similar to the geometry of Problems 2.1.1 and 2.1.2, the pins will still be symmetric within the charge pan. However, channels A, B, C, and D will have greatly suppressed power due to being nearest to the control rod. The goal of this problem is to introduce a burnable absorber material in comparing Shift and MPACT. Material densities are listed in Table 1.4. Temperatures are listed in Table 2.4. 


\subsubsection{Reference Solution}

Table 3.5. Neutron multiplication for the reference solution of Problem 2.1.3.

\begin{tabular}{crccc}
\hline Step & $\begin{array}{c}\text { Burnup } \\
{\left[\frac{\mathrm{MWd}}{\mathrm{MtU}}\right]}\end{array}$ & $\begin{array}{c}\text { Shift } \\
\mathrm{k}_{\mathrm{eff}}(1 \sigma)\end{array}$ & $\begin{array}{c}\text { MPACT } \\
\mathrm{k}_{\mathrm{eff}}(1 \sigma)\end{array}$ & $\begin{array}{c}\text { Difference } \\
{[\mathrm{pcm}]}\end{array}$ \\
\hline 0 & 0.0 & $0.94269(10)$ & 0.94290 & $20(10)$ \\
\hline
\end{tabular}

Table 3.6. Power peaking factors for the reference solution of Problem 2.1.3.

\begin{tabular}{lccc}
\hline \multicolumn{1}{c}{ Channels } & $\begin{array}{c}\text { Shift } \\
\text { Peaking Factor }\end{array}$ & $\begin{array}{c}\text { MPACT } \\
\text { Peaking Factor }\end{array}$ & $\begin{array}{c}\text { Difference } \\
\text { (MPACT-Shift) }\end{array}$ \\
\hline A, B, C, D & 0.8812 & 0.8804 & -0.0008 \\
E, F, G, H, J, K, L, M & 1.0282 & 1.0284 & 0.0002 \\
P, Q, R, S & 1.0624 & 1.0628 & 0.0004 \\
\hline
\end{tabular}




\subsection{DEPLETION}

\subsubsection{Problem 2.2.1}

\subsubsection{Description}

This problem uses exactly the same geometry as Problem 2.1.2, using coolant channel radius A, shown in Figure 5. Material densities are listed in Table 1.4. Temperatures are listed in Table 2.4. Burnup states used for comparison are listed in Table 1.5, and the total system specific power is listed in Table 1.6.

\subsubsection{Reference Solution}

Table 3.7. Neutron multiplication factor for the reference solution of Problem 2.2.1.

\begin{tabular}{rrccr}
\hline Step & $\begin{array}{r}\text { Burnup } \\
{\left[\frac{\mathrm{MWd}}{\mathrm{MtU}}\right]}\end{array}$ & $\begin{array}{c}\text { Shift } \\
\mathrm{k}_{\mathrm{eff}}(1 \sigma)\end{array}$ & $\begin{array}{c}\text { MPACT } \\
\mathrm{k}_{\text {eff }}(1 \sigma)\end{array}$ & $\begin{array}{c}\text { Difference } \\
{[\mathrm{pcm}]}\end{array}$ \\
\hline 0 & 0.0 & $1.06462(10)$ & $1.06496(0)$ & $33(10)$ \\
1 & 25.0 & $1.04963(10)$ & $1.04999(0)$ & $36(10)$ \\
2 & 50.0 & $1.04954(10)$ & $1.04978(0)$ & $24(10)$ \\
3 & 100.0 & $1.05037(10)$ & $1.05099(0)$ & $63(10)$ \\
4 & 200.0 & $1.05396(10)$ & $1.05484(0)$ & $88(10)$ \\
5 & 300.0 & $1.05755(11)$ & $1.05838(0)$ & $83(11)$ \\
6 & 400.0 & $1.06013(10)$ & $1.06129(0)$ & $116(10)$ \\
7 & 500.0 & $1.06262(11)$ & $1.06362(0)$ & $100(11)$ \\
8 & 600.0 & $1.06417(11)$ & $1.06547(0)$ & $130(11)$ \\
9 & 700.0 & $1.06564(11)$ & $1.06691(0)$ & $127(11)$ \\
10 & 800.0 & $1.06675(11)$ & $1.06802(0)$ & $126(11)$ \\
11 & 900.0 & $1.06740(11)$ & $1.06883(0)$ & $142(11)$ \\
12 & 1000.0 & $1.06779(11)$ & $1.06939(0)$ & $161(11)$ \\
13 & 1100.0 & $1.06832(10)$ & $1.06974(0)$ & $142(10)$ \\
14 & 1200.0 & $1.06834(11)$ & $1.06991(0)$ & $157(11)$ \\
\hline
\end{tabular}




\section{Fuel Pins $A-D$}

Table 3.8. Power peaking factors for fuel channels A-D of the reference solution of Problem 2.2.1.

\begin{tabular}{rrrrr}
\hline Step & $\begin{array}{c}\text { Burnup } \\
{\left[\frac{\mathrm{MWd}}{\mathrm{MtU}}\right]}\end{array}$ & $\begin{array}{c}\text { Shift } \\
\text { Peaking Factor }\end{array}$ & $\begin{array}{c}\text { MPACT } \\
\text { Peaking Factor }\end{array}$ & $\begin{array}{c}\text { Difference } \\
\text { [MPACT-Shift] }\end{array}$ \\
\hline 1 & 0.0 & 0.9930 & 0.9921 & -0.0009 \\
2 & 25.0 & 0.9926 & 0.9921 & -0.0005 \\
3 & 50.0 & 0.9928 & 0.9921 & -0.0007 \\
4 & 100.0 & 0.9927 & 0.9920 & -0.0006 \\
5 & 200.0 & 0.9930 & 0.9920 & -0.0010 \\
6 & 300.0 & 0.9925 & 0.9919 & -0.0006 \\
7 & 400.0 & 0.9923 & 0.9919 & -0.0004 \\
8 & 500.0 & 0.9927 & 0.9919 & -0.0008 \\
9 & 600.0 & 0.9924 & 0.9919 & -0.0005 \\
10 & 700.0 & 0.9922 & 0.9919 & -0.0003 \\
11 & 800.0 & 0.9924 & 0.9919 & -0.0005 \\
12 & 900.0 & 0.9929 & 0.9919 & -0.0010 \\
13 & 1000.0 & 0.9923 & 0.9919 & -0.0004 \\
14 & 1100.0 & 0.9926 & 0.9919 & -0.0006 \\
15 & 1200.0 & 0.9924 & 0.9919 & -0.0004 \\
16 & 1300.0 & 0.9927 & 0.9919 & -0.0008 \\
\hline
\end{tabular}

Table 3.9. Uranium isotope concentrations for fuel channels A-D of the reference solution of Problem 2.2.1.

\begin{tabular}{rrcccc}
\hline Step & $\begin{array}{c}\text { Burnup } \\
{\left[\frac{\mathrm{MWd}}{\mathrm{MtU}}\right]}\end{array}$ & $\begin{array}{c}{ }^{234} \mathrm{U} \\
{\left[\frac{\mathrm{atoms}}{\mathrm{b} \text {-cm }}\right]}\end{array}$ & $\begin{array}{c}{ }^{235} \mathrm{U} \\
{\left[\frac{\mathrm{atoms}}{\mathrm{b}-\mathrm{cm}}\right]}\end{array}$ & $\begin{array}{c}{ }^{236} \mathrm{U} \\
{\left[\frac{\mathrm{atoms}}{\mathrm{b}-\mathrm{cm}}\right]}\end{array}$ & $\begin{array}{c}{ }^{238} \mathrm{U} \\
{\left[\frac{\mathrm{atoms}}{\mathrm{b}-\mathrm{cm}}\right]}\end{array}$ \\
\hline 0 & 0.0 & $2.5775 \times 10^{-06}$ & $3.4449 \times 10^{-04}$ & $0.0000 \times 10^{+00}$ & $4.7470 \times 10^{-02}$ \\
1 & 25.0 & $2.5750 \times 10^{-06}$ & $3.4309 \times 10^{-04}$ & $2.2440 \times 10^{-07}$ & $4.7469 \times 10^{-02}$ \\
2 & 50.0 & $2.5724 \times 10^{-06}$ & $3.4171 \times 10^{-04}$ & $4.4675 \times 10^{-07}$ & $4.7467 \times 10^{-02}$ \\
3 & 100.0 & $2.5675 \times 10^{-06}$ & $3.3897 \times 10^{-04}$ & $8.8514 \times 10^{-07}$ & $4.7465 \times 10^{-02}$ \\
4 & 200.0 & $2.5577 \times 10^{-06}$ & $3.3364 \times 10^{-04}$ & $1.7385 \times 10^{-06}$ & $4.7459 \times 10^{-02}$ \\
5 & 300.0 & $2.5481 \times 10^{-06}$ & $3.2849 \times 10^{-04}$ & $2.5633 \times 10^{-06}$ & $4.7454 \times 10^{-02}$ \\
6 & 400.0 & $2.5387 \times 10^{-06}$ & $3.2349 \times 10^{-04}$ & $3.3627 \times 10^{-06}$ & $4.7449 \times 10^{-02}$ \\
7 & 500.0 & $2.5294 \times 10^{-06}$ & $3.1864 \times 10^{-04}$ & $4.1384 \times 10^{-06}$ & $4.7444 \times 10^{-02}$ \\
8 & 600.0 & $2.5203 \times 10^{-06}$ & $3.1391 \times 10^{-04}$ & $4.8921 \times 10^{-06}$ & $4.7439 \times 10^{-02}$ \\
9 & 700.0 & $2.5114 \times 10^{-06}$ & $3.0931 \times 10^{-04}$ & $5.6259 \times 10^{-06}$ & $4.7434 \times 10^{-02}$ \\
10 & 800.0 & $2.5025 \times 10^{-06}$ & $3.0482 \times 10^{-04}$ & $6.3410 \times 10^{-06}$ & $4.7429 \times 10^{-02}$ \\
11 & 900.0 & $2.4938 \times 10^{-06}$ & $3.0044 \times 10^{-04}$ & $7.0385 \times 10^{-06}$ & $4.7425 \times 10^{-02}$ \\
12 & 1000.0 & $2.4852 \times 10^{-06}$ & $2.9615 \times 10^{-04}$ & $7.7198 \times 10^{-06}$ & $4.7420 \times 10^{-02}$ \\
13 & 1100.0 & $2.4766 \times 10^{-06}$ & $2.9196 \times 10^{-04}$ & $8.3858 \times 10^{-06}$ & $4.7415 \times 10^{-02}$ \\
14 & 1200.0 & $2.4682 \times 10^{-06}$ & $2.8786 \times 10^{-04}$ & $9.0368 \times 10^{-06}$ & $4.7410 \times 10^{-02}$ \\
15 & 1300.0 & $2.4598 \times 10^{-06}$ & $2.8383 \times 10^{-04}$ & $9.6742 \times 10^{-06}$ & $4.7406 \times 10^{-02}$ \\
\hline
\end{tabular}


Table 3.10. Plutonium isotope concentrations for fuel channels A through $D$ of the reference solution of Problem 2.2.1

\begin{tabular}{rrccccc}
\hline Step & $\begin{array}{c}\text { Burnup } \\
{\left[\frac{\mathrm{MWd}}{\mathrm{MtU}}\right]}\end{array}$ & $\begin{array}{c}{ }^{238} \mathrm{Pu} \\
{\left[\frac{\mathrm{atoms}}{\mathrm{b}-\mathrm{cm}}\right]}\end{array}$ & $\begin{array}{c}{ }^{239} \mathrm{Pu} \\
{\left[\frac{\mathrm{atoms}}{\mathrm{b}-\mathrm{cm}}\right]}\end{array}$ & $\begin{array}{c}{ }^{240} \mathrm{Pu} \\
{\left[\frac{\mathrm{atoms}}{\mathrm{b}-\mathrm{cm}}\right]}\end{array}$ & $\begin{array}{c}{ }^{241} \mathrm{Pu} \\
{\left[\frac{\mathrm{atoms}}{\mathrm{b}-\mathrm{cm}}\right]}\end{array}$ & $\begin{array}{c}{ }^{242} \mathrm{Pu} \\
{\left[\frac{\mathrm{atoms}}{\mathrm{b}-\mathrm{cm}}\right]}\end{array}$ \\
\hline 0 & 0.0 & $0.0000 \times 10^{+00}$ & $0.0000 \times 10^{+00}$ & $0.0000 \times 10^{+00}$ & $0.0000 \times 10^{+00}$ & $0.0000 \times 10^{+00}$ \\
1 & 25.0 & $7.5702 \times 10^{-13}$ & $9.9886 \times 10^{-07}$ & $2.2382 \times 10^{-09}$ & $5.8112 \times 10^{-12}$ & $4.4670 \times 10^{-15}$ \\
2 & 50.0 & $5.6451 \times 10^{-12}$ & $2.2372 \times 10^{-06}$ & $1.0355 \times 10^{-08}$ & $5.5697 \times 10^{-11}$ & $8.8064 \times 10^{-14}$ \\
3 & 100.0 & $3.2822 \times 10^{-11}$ & $4.6487 \times 10^{-06}$ & $4.3994 \times 10^{-08}$ & $4.8390 \times 10^{-10}$ & $1.5679 \times 10^{-12}$ \\
4 & 200.0 & $1.5930 \times 10^{-10}$ & $9.2130 \times 10^{-06}$ & $1.7512 \times 10^{-07}$ & $3.8596 \times 10^{-09}$ & $2.5379 \times 10^{-11}$ \\
5 & 300.0 & $3.8140 \times 10^{-10}$ & $1.3465 \times 10^{-05}$ & $3.8172 \times 10^{-07}$ & $1.2502 \times 10^{-08}$ & $1.2389 \times 10^{-10}$ \\
6 & 400.0 & $6.9884 \times 10^{-10}$ & $1.7439 \times 10^{-05}$ & $6.5398 \times 10^{-07}$ & $2.8224 \times 10^{-08}$ & $3.7374 \times 10^{-10}$ \\
7 & 500.0 & $1.1119 \times 10^{-09}$ & $2.1160 \times 10^{-05}$ & $9.8346 \times 10^{-07}$ & $5.2391 \times 10^{-08}$ & $8.6840 \times 10^{-10}$ \\
8 & 600.0 & $1.6218 \times 10^{-09}$ & $2.4651 \times 10^{-05}$ & $1.3628 \times 10^{-06}$ & $8.6011 \times 10^{-08}$ & $1.7127 \times 10^{-09}$ \\
9 & 700.0 & $2.2309 \times 10^{-09}$ & $2.7932 \times 10^{-05}$ & $1.7861 \times 10^{-06}$ & $1.2986 \times 10^{-07}$ & $3.0202 \times 10^{-09}$ \\
10 & 800.0 & $2.9429 \times 10^{-09}$ & $3.1021 \times 10^{-05}$ & $2.2478 \times 10^{-06}$ & $1.8445 \times 10^{-07}$ & $4.9092 \times 10^{-09}$ \\
11 & 900.0 & $3.7625 \times 10^{-09}$ & $3.3934 \times 10^{-05}$ & $2.7431 \times 10^{-06}$ & $2.5016 \times 10^{-07}$ & $7.5010 \times 10^{-09}$ \\
12 & 1000.0 & $4.6962 \times 10^{-09}$ & $3.6684 \times 10^{-05}$ & $3.2682 \times 10^{-06}$ & $3.2723 \times 10^{-07}$ & $1.0920 \times 10^{-08}$ \\
13 & 1100.0 & $5.7516 \times 10^{-09}$ & $3.9284 \times 10^{-05}$ & $3.8193 \times 10^{-06}$ & $4.1575 \times 10^{-07}$ & $1.5289 \times 10^{-08}$ \\
14 & 1200.0 & $6.9372 \times 10^{-09}$ & $4.1742 \times 10^{-05}$ & $4.3927 \times 10^{-06}$ & $5.1567 \times 10^{-07}$ & $2.0728 \times 10^{-08}$ \\
15 & 1300.0 & $8.2641 \times 10^{-09}$ & $4.4070 \times 10^{-05}$ & $4.9859 \times 10^{-06}$ & $6.2700 \times 10^{-07}$ & $2.7363 \times 10^{-08}$ \\
\hline
\end{tabular}

Table 3.11. Americium isotope concentrations for fuel channels A-D of the reference solution of Problem 2.2.1.

\begin{tabular}{rrcc}
\hline Step & $\begin{array}{r}\text { Burnup } \\
{\left[\frac{\mathrm{MWd}}{\mathrm{MtU}}\right]}\end{array}$ & $\begin{array}{c}{ }^{241} \mathrm{Am} \\
{\left[\frac{\mathrm{atoms}}{\mathrm{b}-\mathrm{cm}}\right]}\end{array}$ & $\begin{array}{c}{ }^{243} \mathrm{Am} \\
{\left[\frac{\mathrm{atoms}}{\mathrm{b}-\mathrm{cm}}\right]}\end{array}$ \\
\hline 0 & 0.0 & $0.0000 \times 10^{+00}$ & $0.0000 \times 10^{+00}$ \\
1 & 25.0 & $2.8778 \times 10^{-15}$ & $7.3895 \times 10^{-19}$ \\
2 & 50.0 & $5.6876 \times 10^{-14}$ & $3.1225 \times 10^{-17}$ \\
3 & 100.0 & $1.0177 \times 10^{-12}$ & $1.1626 \times 10^{-15}$ \\
4 & 200.0 & $1.6614 \times 10^{-11}$ & $3.8584 \times 10^{-14}$ \\
5 & 300.0 & $8.1672 \times 10^{-11}$ & $2.8475 \times 10^{-13}$ \\
6 & 400.0 & $2.4776 \times 10^{-10}$ & $1.1493 \times 10^{-12}$ \\
7 & 500.0 & $5.7839 \times 10^{-10}$ & $3.3440 \times 10^{-12}$ \\
8 & 600.0 & $1.1453 \times 10^{-09}$ & $7.9214 \times 10^{-12}$ \\
9 & 700.0 & $2.0258 \times 10^{-09}$ & $1.6307 \times 10^{-11}$ \\
10 & 800.0 & $3.3005 \times 10^{-09}$ & $3.0306 \times 10^{-11}$ \\
11 & 900.0 & $5.0516 \times 10^{-09}$ & $5.2115 \times 10^{-11}$ \\
12 & 1000.0 & $7.3615 \times 10^{-09}$ & $8.4333 \times 10^{-11}$ \\
13 & 1100.0 & $1.0312 \times 10^{-08}$ & $1.2994 \times 10^{-10}$ \\
14 & 1200.0 & $1.3983 \times 10^{-08}$ & $1.9227 \times 10^{-10}$ \\
15 & 1300.0 & $1.8452 \times 10^{-08}$ & $2.7510 \times 10^{-10}$ \\
\hline
\end{tabular}


Table 3.12. Curium isotope concentrations for fuel channels A-D of the reference solution of Problem 2.2.1.

\begin{tabular}{rrcc}
\hline Step & $\begin{array}{c}\text { Burnup } \\
{\left[\frac{\mathrm{MWd}}{\mathrm{MtU}}\right]}\end{array}$ & $\begin{array}{c}{ }^{243} \mathrm{Cm} \\
{\left[\frac{\mathrm{atoms}}{\mathrm{b}-\mathrm{cm}}\right]}\end{array}$ & $\begin{array}{c}{ }^{244} \mathrm{Cm} \\
{\left[\frac{\mathrm{atoms}}{\mathrm{b}-\mathrm{cm}}\right]}\end{array}$ \\
\hline 0 & 0.0 & $0.0000 \times 10^{+00}$ & $0.0000 \times 10^{+00}$ \\
1 & 25.0 & $0.0000 \times 10^{+00}$ & $0.0000 \times 10^{+00}$ \\
2 & 50.0 & $0.0000 \times 10^{+00}$ & $2.0426 \times 10^{-20}$ \\
3 & 100.0 & $5.7970 \times 10^{-19}$ & $1.5593 \times 10^{-18}$ \\
4 & 200.0 & $3.8570 \times 10^{-17}$ & $1.0474 \times 10^{-16}$ \\
5 & 300.0 & $4.1548 \times 10^{-16}$ & $1.1602 \times 10^{-15}$ \\
6 & 400.0 & $2.1600 \times 10^{-15}$ & $6.2305 \times 10^{-15}$ \\
7 & 500.0 & $7.5686 \times 10^{-15}$ & $2.2588 \times 10^{-14}$ \\
8 & 600.0 & $2.0709 \times 10^{-14}$ & $6.3982 \times 10^{-14}$ \\
9 & 700.0 & $4.7868 \times 10^{-14}$ & $1.5312 \times 10^{-13}$ \\
10 & 800.0 & $9.7866 \times 10^{-14}$ & $3.2412 \times 10^{-13}$ \\
11 & 900.0 & $1.8230 \times 10^{-13}$ & $6.2497 \times 10^{-13}$ \\
12 & 1000.0 & $3.1577 \times 10^{-13}$ & $1.1203 \times 10^{-12}$ \\
13 & 1100.0 & $5.1582 \times 10^{-13}$ & $1.8933 \times 10^{-12}$ \\
14 & 1200.0 & $8.0293 \times 10^{-13}$ & $3.0480 \times 10^{-12}$ \\
15 & 1300.0 & $1.2008 \times 10^{-12}$ & $4.7128 \times 10^{-12}$ \\
\hline
\end{tabular}

Table 3.13. Cesium isotope concentrations for fuel channels A-D of the reference solution of Problem 2.2.1.

\begin{tabular}{rrccc}
\hline Step & $\begin{array}{c}\text { Burnup } \\
{\left[\frac{\mathrm{MWd}}{\mathrm{MtU}}\right]}\end{array}$ & $\begin{array}{c}{ }^{133} \mathrm{Cs} \\
{\left[\frac{\mathrm{atoms}}{\mathrm{b}-\mathrm{cm}}\right]}\end{array}$ & $\begin{array}{c}{ }^{135} \mathrm{Cs} \\
{\left[\frac{\mathrm{atoms}}{\mathrm{b}-\mathrm{cm}}\right]}\end{array}$ & $\begin{array}{c}137 \mathrm{Cs} \\
{\left[\frac{\mathrm{atoms}}{\mathrm{b}-\mathrm{cm}}\right]}\end{array}$ \\
\hline 0 & 0.0 & $0.0000 \times 10^{+00}$ & $0.0000 \times 10^{+00}$ & $0.0000 \times 10^{+00}$ \\
1 & 25.0 & $4.3758 \times 10^{-08}$ & $4.7992 \times 10^{-08}$ & $7.7813 \times 10^{-08}$ \\
2 & 50.0 & $1.2326 \times 10^{-07}$ & $9.8338 \times 10^{-08}$ & $1.5559 \times 10^{-07}$ \\
3 & 100.0 & $2.9133 \times 10^{-07}$ & $1.9948 \times 10^{-07}$ & $3.1094 \times 10^{-07}$ \\
4 & 200.0 & $6.2839 \times 10^{-07}$ & $4.0344 \times 10^{-07}$ & $6.2087 \times 10^{-07}$ \\
5 & 300.0 & $9.6515 \times 10^{-07}$ & $6.0949 \times 10^{-07}$ & $9.2986 \times 10^{-07}$ \\
6 & 400.0 & $1.3017 \times 10^{-06}$ & $8.1746 \times 10^{-07}$ & $1.2380 \times 10^{-06}$ \\
7 & 500.0 & $1.6379 \times 10^{-06}$ & $1.0271 \times 10^{-06}$ & $1.5451 \times 10^{-06}$ \\
8 & 600.0 & $1.9738 \times 10^{-06}$ & $1.2383 \times 10^{-06}$ & $1.8511 \times 10^{-06}$ \\
9 & 700.0 & $2.3093 \times 10^{-06}$ & $1.4508 \times 10^{-06}$ & $2.1562 \times 10^{-06}$ \\
10 & 800.0 & $2.6445 \times 10^{-06}$ & $1.6646 \times 10^{-06}$ & $2.4602 \times 10^{-06}$ \\
11 & 900.0 & $2.9794 \times 10^{-06}$ & $1.8796 \times 10^{-06}$ & $2.7630 \times 10^{-06}$ \\
12 & 1000.0 & $3.3139 \times 10^{-06}$ & $2.0956 \times 10^{-06}$ & $3.0649 \times 10^{-06}$ \\
13 & 1100.0 & $3.6482 \times 10^{-06}$ & $2.3126 \times 10^{-06}$ & $3.3658 \times 10^{-06}$ \\
14 & 1200.0 & $3.9819 \times 10^{-06}$ & $2.5305 \times 10^{-06}$ & $3.6653 \times 10^{-06}$ \\
15 & 1300.0 & $4.3153 \times 10^{-06}$ & $2.7491 \times 10^{-06}$ & $3.9639 \times 10^{-06}$ \\
\hline
\end{tabular}


Table 3.14. Neodymium isotope concentrations for fuel channels A-D of the reference solution of Problem 2.2.1.

\begin{tabular}{rrccccc}
\hline Step & $\begin{array}{c}\text { Burnup } \\
{\left[\frac{\mathrm{MWd}}{\mathrm{MtU}}\right]}\end{array}$ & $\begin{array}{c}{ }^{143} \mathrm{Nd} \\
{\left[\frac{\mathrm{atoms}}{\mathrm{b}-\mathrm{cm}}\right]}\end{array}$ & $\begin{array}{c}{ }^{144} \mathrm{Nd} \\
{\left[\frac{\mathrm{atoms}}{\mathrm{b}-\mathrm{cm}}\right]}\end{array}$ & $\begin{array}{c}145 \mathrm{Nd} \\
{\left[\frac{\mathrm{atoms}}{\mathrm{b}-\mathrm{cm}}\right]}\end{array}$ & $\begin{array}{c}146 \mathrm{Nd} \\
{\left[\frac{\mathrm{atoms}}{\mathrm{b}-\mathrm{cm}}\right]}\end{array}$ & $\begin{array}{c}148 \mathrm{Nd} \\
{\left[\frac{\text { atoms }}{\mathrm{b}-\mathrm{cm}}\right]}\end{array}$ \\
\hline 0 & 0.0 & $0.0000 \times 10^{+00}$ & $0.0000 \times 10^{+00}$ & $0.0000 \times 10^{+00}$ & $0.0000 \times 10^{+00}$ & $0.0000 \times 10^{+00}$ \\
1 & 25.0 & $1.9157 \times 10^{-08}$ & $1.3685 \times 10^{-09}$ & $4.8296 \times 10^{-08}$ & $3.7979 \times 10^{-08}$ & $2.1454 \times 10^{-08}$ \\
2 & 50.0 & $6.8828 \times 10^{-08}$ & $5.4467 \times 10^{-09}$ & $9.7560 \times 10^{-08}$ & $7.5998 \times 10^{-08}$ & $4.2933 \times 10^{-08}$ \\
3 & 100.0 & $2.0081 \times 10^{-07}$ & $2.1393 \times 10^{-08}$ & $1.9567 \times 10^{-07}$ & $1.5187 \times 10^{-07}$ & $8.5874 \times 10^{-08}$ \\
4 & 200.0 & $4.8786 \times 10^{-07}$ & $8.1755 \times 10^{-08}$ & $3.9034 \times 10^{-07}$ & $3.0303 \times 10^{-07}$ & $1.7162 \times 10^{-07}$ \\
5 & 300.0 & $7.7343 \times 10^{-07}$ & $1.7527 \times 10^{-07}$ & $5.8318 \times 10^{-07}$ & $4.5353 \times 10^{-07}$ & $2.5720 \times 10^{-07}$ \\
6 & 400.0 & $1.0548 \times 10^{-06}$ & $2.9691 \times 10^{-07}$ & $7.7441 \times 10^{-07}$ & $6.0351 \times 10^{-07}$ & $3.4268 \times 10^{-07}$ \\
7 & 500.0 & $1.3320 \times 10^{-06}$ & $4.4241 \times 10^{-07}$ & $9.6409 \times 10^{-07}$ & $7.5298 \times 10^{-07}$ & $4.2802 \times 10^{-07}$ \\
8 & 600.0 & $1.6054 \times 10^{-06}$ & $6.0816 \times 10^{-07}$ & $1.1523 \times 10^{-06}$ & $9.0194 \times 10^{-07}$ & $5.1321 \times 10^{-07}$ \\
9 & 700.0 & $1.8751 \times 10^{-06}$ & $7.9111 \times 10^{-07}$ & $1.3392 \times 10^{-06}$ & $1.0505 \times 10^{-06}$ & $5.9830 \times 10^{-07}$ \\
10 & 800.0 & $2.1413 \times 10^{-06}$ & $9.8866 \times 10^{-07}$ & $1.5248 \times 10^{-06}$ & $1.1988 \times 10^{-06}$ & $6.8330 \times 10^{-07}$ \\
11 & 900.0 & $2.4041 \times 10^{-06}$ & $1.1986 \times 10^{-06}$ & $1.7093 \times 10^{-06}$ & $1.3467 \times 10^{-06}$ & $7.6819 \times 10^{-07}$ \\
12 & 1000.0 & $2.6639 \times 10^{-06}$ & $1.4192 \times 10^{-06}$ & $1.8927 \times 10^{-06}$ & $1.4943 \times 10^{-06}$ & $8.5301 \times 10^{-07}$ \\
13 & 1100.0 & $2.9206 \times 10^{-06}$ & $1.6488 \times 10^{-06}$ & $2.0750 \times 10^{-06}$ & $1.6417 \times 10^{-06}$ & $9.3775 \times 10^{-07}$ \\
14 & 1200.0 & $3.1742 \times 10^{-06}$ & $1.8860 \times 10^{-06}$ & $2.2562 \times 10^{-06}$ & $1.7888 \times 10^{-06}$ & $1.0224 \times 10^{-06}$ \\
15 & 1300.0 & $3.4250 \times 10^{-06}$ & $2.1299 \times 10^{-06}$ & $2.4366 \times 10^{-06}$ & $1.9356 \times 10^{-06}$ & $1.1069 \times 10^{-06}$ \\
\hline
\end{tabular}

Table 3.15. Strontium isotope concentrations for fuel channels A-D of the reference solution of Problem 2.2.1.

\begin{tabular}{rrccccc}
\hline Step & $\begin{array}{c}\text { Burnup } \\
{\left[\frac{\mathrm{MWd}}{\mathrm{MtU}}\right]}\end{array}$ & $\begin{array}{c}{ }^{84} \mathrm{Sr} \\
{\left[\frac{\mathrm{atoms}}{\mathrm{b}-\mathrm{cm}}\right]}\end{array}$ & $\begin{array}{c}{ }^{86} \mathrm{Sr} \\
{\left[\frac{\mathrm{atoms}}{\mathrm{b}-\mathrm{cm}}\right]}\end{array}$ & $\begin{array}{c}{ }^{87} \mathrm{Sr} \\
{\left[\frac{\mathrm{atoms}}{\mathrm{b}-\mathrm{cm}}\right]}\end{array}$ & $\begin{array}{c}{ }^{88} \mathrm{Sr} \\
{\left[\frac{\mathrm{atoms}}{\mathrm{b}-\mathrm{cm}}\right]}\end{array}$ & $\begin{array}{c}{ }^{90} \mathrm{Sr} \\
{\left[\frac{\mathrm{atoms}}{\mathrm{b}-\mathrm{cm}}\right]}\end{array}$ \\
\hline 0 & 0.0 & $0.0000 \times 10^{+00}$ & $0.0000 \times 10^{+00}$ & $0.0000 \times 10^{+00}$ & $0.0000 \times 10^{+00}$ & $0.0000 \times 10^{+00}$ \\
1 & 25.0 & $2.8266 \times 10^{-19}$ & $3.1548 \times 10^{-14}$ & $1.6788 \times 10^{-14}$ & $4.3235 \times 10^{-08}$ & $7.0656 \times 10^{-08}$ \\
2 & 50.0 & $1.1157 \times 10^{-18}$ & $1.5006 \times 10^{-13}$ & $3.6682 \times 10^{-14}$ & $8.6731 \times 10^{-08}$ & $1.4085 \times 10^{-07}$ \\
3 & 100.0 & $4.3497 \times 10^{-18}$ & $7.2961 \times 10^{-13}$ & $8.5744 \times 10^{-14}$ & $1.7297 \times 10^{-07}$ & $2.7979 \times 10^{-07}$ \\
4 & 200.0 & $1.6663 \times 10^{-17}$ & $3.4180 \times 10^{-12}$ & $2.1865 \times 10^{-13}$ & $3.4266 \times 10^{-07}$ & $5.5221 \times 10^{-07}$ \\
5 & 300.0 & $3.6202 \times 10^{-17}$ & $8.1433 \times 10^{-12}$ & $3.9405 \times 10^{-13}$ & $5.0899 \times 10^{-07}$ & $8.1798 \times 10^{-07}$ \\
6 & 400.0 & $6.2461 \times 10^{-17}$ & $1.4819 \times 10^{-11}$ & $6.0803 \times 10^{-13}$ & $6.7233 \times 10^{-07}$ & $1.0777 \times 10^{-06}$ \\
7 & 500.0 & $9.5027 \times 10^{-17}$ & $2.3357 \times 10^{-11}$ & $8.5711 \times 10^{-13}$ & $8.3290 \times 10^{-07}$ & $1.3318 \times 10^{-06}$ \\
8 & 600.0 & $1.3354 \times 10^{-16}$ & $3.3679 \times 10^{-11}$ & $1.1383 \times 10^{-12}$ & $9.9085 \times 10^{-07}$ & $1.5805 \times 10^{-06}$ \\
9 & 700.0 & $1.7772 \times 10^{-16}$ & $4.5723 \times 10^{-11}$ & $1.4491 \times 10^{-12}$ & $1.1465 \times 10^{-06}$ & $1.8244 \times 10^{-06}$ \\
10 & 800.0 & $2.2733 \times 10^{-16}$ & $5.9435 \times 10^{-11}$ & $1.7874 \times 10^{-12}$ & $1.2999 \times 10^{-06}$ & $2.0636 \times 10^{-06}$ \\
11 & 900.0 & $2.8216 \times 10^{-16}$ & $7.4767 \times 10^{-11}$ & $2.1511 \times 10^{-12}$ & $1.4512 \times 10^{-06}$ & $2.2984 \times 10^{-06}$ \\
12 & 1000.0 & $3.4206 \times 10^{-16}$ & $9.1679 \times 10^{-11}$ & $2.5387 \times 10^{-12}$ & $1.6007 \times 10^{-06}$ & $2.5291 \times 10^{-06}$ \\
13 & 1100.0 & $4.0686 \times 10^{-16}$ & $1.1014 \times 10^{-10}$ & $2.9485 \times 10^{-12}$ & $1.7483 \times 10^{-06}$ & $2.7558 \times 10^{-06}$ \\
14 & 1200.0 & $4.7639 \times 10^{-16}$ & $1.3010 \times 10^{-10}$ & $3.3790 \times 10^{-12}$ & $1.8942 \times 10^{-06}$ & $2.9787 \times 10^{-06}$ \\
15 & 1300.0 & $5.5054 \times 10^{-16}$ & $1.5155 \times 10^{-10}$ & $3.8292 \times 10^{-12}$ & $2.0385 \times 10^{-06}$ & $3.1980 \times 10^{-06}$ \\
\hline
\end{tabular}


Table 3.16. Barium isotope concentrations for fuel channels A-D of the reference solution of Problem 2.2.1.

\begin{tabular}{rrccccc}
\hline Step & $\begin{array}{c}\text { Burnup } \\
{\left[\frac{\mathrm{MWd}}{\mathrm{MtU}}\right]}\end{array}$ & $\begin{array}{c}{ }^{132} \mathrm{Ba} \\
{\left[\frac{\mathrm{atoms}}{\mathrm{b}-\mathrm{cm}}\right]}\end{array}$ & $\begin{array}{c}{ }^{134} \mathrm{Ba} \\
{\left[\frac{\mathrm{atoms}}{\mathrm{b}-\mathrm{cm}}\right]}\end{array}$ & $\begin{array}{c}{ }^{135} \mathrm{Ba} \\
{\left[\frac{\mathrm{atoms}}{\mathrm{b}-\mathrm{cm}}\right]}\end{array}$ & $\begin{array}{c}{ }^{136} \mathrm{Ba} \\
{\left[\frac{\mathrm{atoms}}{\mathrm{b}-\mathrm{cm}}\right]}\end{array}$ & $\begin{array}{c}{ }^{138} \mathrm{Ba} \\
{\left[\frac{\mathrm{atoms}}{\mathrm{b}-\mathrm{cm}}\right]}\end{array}$ \\
\hline 0 & 0.0 & $0.0000 \times 10^{+00}$ & $0.0000 \times 10^{+00}$ & $0.0000 \times 10^{+00}$ & $0.0000 \times 10^{+00}$ & $0.0000 \times 10^{+00}$ \\
1 & 25.0 & $0.0000 \times 10^{+00}$ & $2.3356 \times 10^{-14}$ & $1.6999 \times 10^{-15}$ & $3.5277 \times 10^{-11}$ & $8.4156 \times 10^{-08}$ \\
2 & 50.0 & $0.0000 \times 10^{+00}$ & $3.1273 \times 10^{-13}$ & $5.3931 \times 10^{-15}$ & $9.8943 \times 10^{-11}$ & $1.6847 \times 10^{-07}$ \\
3 & 100.0 & $0.0000 \times 10^{+00}$ & $3.4516 \times 10^{-12}$ & $1.8739 \times 10^{-14}$ & $2.8360 \times 10^{-10}$ & $3.3679 \times 10^{-07}$ \\
4 & 200.0 & $0.0000 \times 10^{+00}$ & $3.2480 \times 10^{-11}$ & $6.9167 \times 10^{-14}$ & $8.2899 \times 10^{-10}$ & $6.7238 \times 10^{-07}$ \\
5 & 300.0 & $0.0000 \times 10^{+00}$ & $1.1414 \times 10^{-10}$ & $1.5244 \times 10^{-13}$ & $1.5820 \times 10^{-09}$ & $1.0068 \times 10^{-06}$ \\
6 & 400.0 & $0.0000 \times 10^{+00}$ & $2.7297 \times 10^{-10}$ & $2.7190 \times 10^{-13}$ & $2.5269 \times 10^{-09}$ & $1.3402 \times 10^{-06}$ \\
7 & 500.0 & $0.0000 \times 10^{+00}$ & $5.3128 \times 10^{-10}$ & $4.3267 \times 10^{-13}$ & $3.6513 \times 10^{-09}$ & $1.6727 \times 10^{-06}$ \\
8 & 600.0 & $0.0000 \times 10^{+00}$ & $9.0934 \times 10^{-10}$ & $6.4143 \times 10^{-13}$ & $4.9442 \times 10^{-09}$ & $2.0041 \times 10^{-06}$ \\
9 & 700.0 & $0.0000 \times 10^{+00}$ & $1.4257 \times 10^{-09}$ & $9.0637 \times 10^{-13}$ & $6.3967 \times 10^{-09}$ & $2.3347 \times 10^{-06}$ \\
10 & 800.0 & $0.0000 \times 10^{+00}$ & $2.0972 \times 10^{-09}$ & $1.2368 \times 10^{-12}$ & $8.0011 \times 10^{-09}$ & $2.6646 \times 10^{-06}$ \\
11 & 900.0 & $0.0000 \times 10^{+00}$ & $2.9395 \times 10^{-09}$ & $1.6430 \times 10^{-12}$ & $9.7502 \times 10^{-09}$ & $2.9937 \times 10^{-06}$ \\
12 & 1000.0 & $0.0000 \times 10^{+00}$ & $3.9668 \times 10^{-09}$ & $2.1366 \times 10^{-12}$ & $1.1638 \times 10^{-08}$ & $3.3222 \times 10^{-06}$ \\
13 & 1100.0 & $0.0000 \times 10^{+00}$ & $5.1923 \times 10^{-09}$ & $2.7298 \times 10^{-12}$ & $1.3660 \times 10^{-08}$ & $3.6501 \times 10^{-06}$ \\
14 & 1200.0 & $0.0000 \times 10^{+00}$ & $6.6282 \times 10^{-09}$ & $3.4354 \times 10^{-12}$ & $1.5810 \times 10^{-08}$ & $3.9772 \times 10^{-06}$ \\
15 & 1300.0 & $0.0000 \times 10^{+00}$ & $8.2854 \times 10^{-09}$ & $4.2674 \times 10^{-12}$ & $1.8084 \times 10^{-08}$ & $4.3037 \times 10^{-06}$ \\
\hline
\end{tabular}

Table 3.17. Technetium isotope concentrations for fuel channels A-D of the reference solution of Problem 2.2.1.

\begin{tabular}{rrc}
\hline Step & $\begin{array}{c}\text { Burnup } \\
{\left[\frac{\mathrm{MWd}}{\mathrm{MtU}}\right]}\end{array}$ & $\begin{array}{c}99 \\
{\left[\frac{\mathrm{atoms}}{\mathrm{b}-\mathrm{cm}}\right]}\end{array}$ \\
\hline 0 & 0.0 & $0.0000 \times 10^{+00}$ \\
1 & 25.0 & $5.7134 \times 10^{-08}$ \\
2 & 50.0 & $1.3377 \times 10^{-07}$ \\
3 & 100.0 & $2.8757 \times 10^{-07}$ \\
4 & 200.0 & $5.9473 \times 10^{-07}$ \\
5 & 300.0 & $9.0142 \times 10^{-07}$ \\
6 & 400.0 & $1.2078 \times 10^{-06}$ \\
7 & 500.0 & $1.5137 \times 10^{-06}$ \\
8 & 600.0 & $1.8191 \times 10^{-06}$ \\
9 & 700.0 & $2.1242 \times 10^{-06}$ \\
10 & 800.0 & $2.4289 \times 10^{-06}$ \\
11 & 900.0 & $2.7332 \times 10^{-06}$ \\
12 & 1000.0 & $3.0371 \times 10^{-06}$ \\
13 & 1100.0 & $3.3407 \times 10^{-06}$ \\
14 & 1200.0 & $3.6437 \times 10^{-06}$ \\
15 & 1300.0 & $3.9464 \times 10^{-06}$ \\
\hline
\end{tabular}


Table 3.18. Rubidium isotope concentrations for fuel channels A-D of the reference solution of Problem 2.2.1

\begin{tabular}{rrcc}
\hline Step & $\begin{array}{c}\text { Burnup } \\
{\left[\frac{\mathrm{MWd}}{\mathrm{MtU}}\right]}\end{array}$ & $\begin{array}{c}{ }^{85} \mathrm{Rb} \\
{\left[\frac{\text { atoms }}{\mathrm{b}-\mathrm{cm}}\right]}\end{array}$ & $\begin{array}{c}87 \mathrm{Rb} \\
{\left[\frac{\mathrm{atoms}}{\mathrm{b}-\mathrm{cm}}\right]}\end{array}$ \\
\hline 0 & 0.0 & $0.0000 \times 10^{+00}$ & $0.0000 \times 10^{+00}$ \\
1 & 25.0 & $1.2427 \times 10^{-08}$ & $3.1341 \times 10^{-08}$ \\
2 & 50.0 & $2.5010 \times 10^{-08}$ & $6.2662 \times 10^{-08}$ \\
3 & 100.0 & $5.0007 \times 10^{-08}$ & $1.2478 \times 10^{-07}$ \\
4 & 200.0 & $9.9382 \times 10^{-08}$ & $2.4707 \times 10^{-07}$ \\
5 & 300.0 & $1.4802 \times 10^{-07}$ & $3.6702 \times 10^{-07}$ \\
6 & 400.0 & $1.9602 \times 10^{-07}$ & $4.8489 \times 10^{-07}$ \\
7 & 500.0 & $2.4343 \times 10^{-07}$ & $6.0082 \times 10^{-07}$ \\
8 & 600.0 & $2.9029 \times 10^{-07}$ & $7.1493 \times 10^{-07}$ \\
9 & 700.0 & $3.3667 \times 10^{-07}$ & $8.2741 \times 10^{-07}$ \\
10 & 800.0 & $3.8259 \times 10^{-07}$ & $9.3836 \times 10^{-07}$ \\
11 & 900.0 & $4.2811 \times 10^{-07}$ & $1.0479 \times 10^{-06}$ \\
12 & 1000.0 & $4.7324 \times 10^{-07}$ & $1.1561 \times 10^{-06}$ \\
13 & 1100.0 & $5.1803 \times 10^{-07}$ & $1.2630 \times 10^{-06}$ \\
14 & 1200.0 & $5.6246 \times 10^{-07}$ & $1.3687 \times 10^{-06}$ \\
15 & 1300.0 & $6.0658 \times 10^{-07}$ & $1.4733 \times 10^{-06}$ \\
\hline
\end{tabular}


Fuel Pins $E-M$

Table 3.19. Power peaking factors for fuel channels E-M of the reference solution of Problem 2.2.1.

\begin{tabular}{rrrrr}
\hline Step & $\begin{array}{r}\text { Burnup } \\
{\left[\frac{\mathrm{MWd}}{\mathrm{MtU}}\right]}\end{array}$ & $\begin{array}{r}\text { Shift } \\
\text { Peaking Factor }\end{array}$ & $\begin{array}{r}\text { MPACT } \\
\text { Peaking Factor }\end{array}$ & $\begin{array}{c}\text { Difference } \\
\text { [MPACT-Shift] }\end{array}$ \\
\hline 1 & 0.0 & 1.0027 & 1.0020 & -0.0007 \\
2 & 25.0 & 1.0027 & 1.0020 & -0.0007 \\
3 & 50.0 & 1.0027 & 1.0020 & -0.0007 \\
4 & 100.0 & 1.0028 & 1.0021 & -0.0007 \\
5 & 200.0 & 1.0026 & 1.0021 & -0.0005 \\
6 & 300.0 & 1.0028 & 1.0021 & -0.0008 \\
7 & 400.0 & 1.0028 & 1.0021 & -0.0008 \\
8 & 500.0 & 1.0030 & 1.0021 & -0.0009 \\
9 & 600.0 & 1.0026 & 1.0021 & -0.0005 \\
10 & 700.0 & 1.0030 & 1.0021 & -0.0009 \\
11 & 800.0 & 1.0028 & 1.0021 & -0.0006 \\
12 & 900.0 & 1.0028 & 1.0021 & -0.0007 \\
13 & 1000.0 & 1.0028 & 1.0021 & -0.0007 \\
14 & 1100.0 & 1.0025 & 1.0021 & -0.0004 \\
15 & 1200.0 & 1.0028 & 1.0021 & -0.0007 \\
16 & 1300.0 & 1.0030 & 1.0021 & -0.0009 \\
\hline
\end{tabular}

Table 3.20. Uranium isotope concentrations for fuel channels E-M of the reference solution of Problem 2.2.1.

\begin{tabular}{rrcccc}
\hline Step & $\begin{array}{c}\text { Burnup } \\
{\left[\frac{\mathrm{MWd}}{\mathrm{MtU}}\right]}\end{array}$ & $\begin{array}{c}{ }^{234} \mathrm{U} \\
{\left[\frac{\mathrm{atoms}}{\mathrm{b}-\mathrm{cm}}\right]}\end{array}$ & $\begin{array}{c}{ }^{235} \mathrm{U} \\
{\left[\frac{\mathrm{atoms}}{\mathrm{b}-\mathrm{cm}}\right]}\end{array}$ & $\begin{array}{c}{ }^{236} \mathrm{U} \\
{\left[\frac{\mathrm{atoms}}{\mathrm{b}-\mathrm{cm}}\right]}\end{array}$ & $\left.\begin{array}{c}{ }^{238} \mathrm{U} \\
\mathrm{atoms}\end{array}\right]$ \\
\hline 0 & 0.0 & $2.5775 \times 10^{-06}$ & $3.4449 \times 10^{-04}$ & $0.0000 \times 10^{+00}$ & $4.7470 \times 10^{-02}$ \\
1 & 25.0 & $2.5749 \times 10^{-06}$ & $3.4308 \times 10^{-04}$ & $2.2666 \times 10^{-07}$ & $4.7469 \times 10^{-02}$ \\
2 & 50.0 & $2.5724 \times 10^{-06}$ & $3.4167 \times 10^{-04}$ & $4.5123 \times 10^{-07}$ & $4.7467 \times 10^{-02}$ \\
3 & 100.0 & $2.5674 \times 10^{-06}$ & $3.3891 \times 10^{-04}$ & $8.9408 \times 10^{-07}$ & $4.7465 \times 10^{-02}$ \\
4 & 200.0 & $2.5575 \times 10^{-06}$ & $3.3352 \times 10^{-04}$ & $1.7562 \times 10^{-06}$ & $4.7459 \times 10^{-02}$ \\
5 & 300.0 & $2.5479 \times 10^{-06}$ & $3.2831 \times 10^{-04}$ & $2.5892 \times 10^{-06}$ & $4.7454 \times 10^{-02}$ \\
6 & 400.0 & $2.5384 \times 10^{-06}$ & $3.2326 \times 10^{-04}$ & $3.3963 \times 10^{-06}$ & $4.7449 \times 10^{-02}$ \\
7 & 500.0 & $2.5291 \times 10^{-06}$ & $3.1835 \times 10^{-04}$ & $4.1791 \times 10^{-06}$ & $4.7444 \times 10^{-02}$ \\
8 & 600.0 & $2.5199 \times 10^{-06}$ & $3.1358 \times 10^{-04}$ & $4.9400 \times 10^{-06}$ & $4.7439 \times 10^{-02}$ \\
9 & 700.0 & $2.5109 \times 10^{-06}$ & $3.0893 \times 10^{-04}$ & $5.6804 \times 10^{-06}$ & $4.7434 \times 10^{-02}$ \\
10 & 800.0 & $2.5020 \times 10^{-06}$ & $3.0440 \times 10^{-04}$ & $6.4016 \times 10^{-06}$ & $4.7429 \times 10^{-02}$ \\
11 & 900.0 & $2.4932 \times 10^{-06}$ & $2.9997 \times 10^{-04}$ & $7.1052 \times 10^{-06}$ & $4.7424 \times 10^{-02}$ \\
12 & 1000.0 & $2.4845 \times 10^{-06}$ & $2.9564 \times 10^{-04}$ & $7.7921 \times 10^{-06}$ & $4.7420 \times 10^{-02}$ \\
13 & 1100.0 & $2.4760 \times 10^{-06}$ & $2.9141 \times 10^{-04}$ & $8.4633 \times 10^{-06}$ & $4.7415 \times 10^{-02}$ \\
14 & 1200.0 & $2.4675 \times 10^{-06}$ & $2.8727 \times 10^{-04}$ & $9.1198 \times 10^{-06}$ & $4.7410 \times 10^{-02}$ \\
15 & 1300.0 & $2.4590 \times 10^{-06}$ & $2.8321 \times 10^{-04}$ & $9.7622 \times 10^{-06}$ & $4.7405 \times 10^{-02}$ \\
\hline
\end{tabular}


Table 3.21. Plutonium isotope concentrations for fuel channels E-M of the reference solution of Problem 2.2.1.

\begin{tabular}{rrccccc}
\hline Step & $\begin{array}{c}\text { Burnup } \\
{\left[\frac{\mathrm{MWd}}{\mathrm{MtU}}\right]}\end{array}$ & $\begin{array}{c}{ }^{238} \mathrm{Pu} \\
{\left[\frac{\mathrm{atoms}}{\mathrm{b}-\mathrm{cm}}\right]}\end{array}$ & $\begin{array}{c}{ }^{239} \mathrm{Pu} \\
{\left[\frac{\mathrm{atoms}}{\mathrm{b}-\mathrm{cm}}\right]}\end{array}$ & $\begin{array}{c}{ }^{240} \mathrm{Pu} \\
{\left[\frac{\mathrm{atoms}}{\mathrm{b}-\mathrm{cm}}\right]}\end{array}$ & $\begin{array}{c}{ }^{241} \mathrm{Pu} \\
{\left[\frac{\mathrm{atoms}}{\mathrm{b}-\mathrm{cm}}\right]}\end{array}$ & $\begin{array}{c}{ }^{242} \mathrm{Pu} \\
{\left[\frac{\mathrm{atoms}}{\mathrm{b}-\mathrm{cm}}\right]}\end{array}$ \\
\hline 0 & 0.0 & $0.0000 \times 10^{+00}$ & $0.0000 \times 10^{+00}$ & $0.0000 \times 10^{+00}$ & $0.0000 \times 10^{+00}$ & $0.0000 \times 10^{+00}$ \\
1 & 25.0 & $7.6665 \times 10^{-13}$ & $1.0040 \times 10^{-06}$ & $2.2708 \times 10^{-09}$ & $5.9041 \times 10^{-12}$ & $4.5877 \times 10^{-15}$ \\
2 & 50.0 & $5.7168 \times 10^{-12}$ & $2.2485 \times 10^{-06}$ & $1.0506 \times 10^{-08}$ & $5.6589 \times 10^{-11}$ & $9.0450 \times 10^{-14}$ \\
3 & 100.0 & $3.3248 \times 10^{-11}$ & $4.6721 \times 10^{-06}$ & $4.4645 \times 10^{-08}$ & $4.9180 \times 10^{-10}$ & $1.6112 \times 10^{-12}$ \\
4 & 200.0 & $1.6144 \times 10^{-10}$ & $9.2585 \times 10^{-06}$ & $1.7774 \times 10^{-07}$ & $3.9236 \times 10^{-09}$ & $2.6092 \times 10^{-11}$ \\
5 & 300.0 & $3.8657 \times 10^{-10}$ & $1.3529 \times 10^{-05}$ & $3.8737 \times 10^{-07}$ & $1.2707 \times 10^{-08}$ & $1.2735 \times 10^{-10}$ \\
6 & 400.0 & $7.0835 \times 10^{-10}$ & $1.7516 \times 10^{-05}$ & $6.6353 \times 10^{-07}$ & $2.8676 \times 10^{-08}$ & $3.8407 \times 10^{-10}$ \\
7 & 500.0 & $1.1270 \times 10^{-09}$ & $2.1248 \times 10^{-05}$ & $9.9752 \times 10^{-07}$ & $5.3207 \times 10^{-08}$ & $8.9201 \times 10^{-10}$ \\
8 & 600.0 & $1.6440 \times 10^{-09}$ & $2.4747 \times 10^{-05}$ & $1.3821 \times 10^{-06}$ & $8.7337 \times 10^{-08}$ & $1.7591 \times 10^{-09}$ \\
9 & 700.0 & $2.2617 \times 10^{-09}$ & $2.8035 \times 10^{-05}$ & $1.8110 \times 10^{-06}$ & $1.3181 \times 10^{-07}$ & $3.1011 \times 10^{-09}$ \\
10 & 800.0 & $2.9835 \times 10^{-09}$ & $3.1128 \times 10^{-05}$ & $2.2786 \times 10^{-06}$ & $1.8716 \times 10^{-07}$ & $5.0388 \times 10^{-09}$ \\
11 & 900.0 & $3.8147 \times 10^{-09}$ & $3.4043 \times 10^{-05}$ & $2.7803 \times 10^{-06}$ & $2.5377 \times 10^{-07}$ & $7.6973 \times 10^{-09}$ \\
12 & 1000.0 & $4.7615 \times 10^{-09}$ & $3.6793 \times 10^{-05}$ & $3.3117 \times 10^{-06}$ & $3.3183 \times 10^{-07}$ & $1.1202 \times 10^{-08}$ \\
13 & 1100.0 & $5.8318 \times 10^{-09}$ & $3.9392 \times 10^{-05}$ & $3.8692 \times 10^{-06}$ & $4.2145 \times 10^{-07}$ & $1.5678 \times 10^{-08}$ \\
14 & 1200.0 & $7.0350 \times 10^{-09}$ & $4.1849 \times 10^{-05}$ & $4.4495 \times 10^{-06}$ & $5.2266 \times 10^{-07}$ & $2.1254 \times 10^{-08}$ \\
15 & 1300.0 & $8.3816 \times 10^{-09}$ & $4.4174 \times 10^{-05}$ & $5.0495 \times 10^{-06}$ & $6.3533 \times 10^{-07}$ & $2.8050 \times 10^{-08}$ \\
\hline
\end{tabular}

Table 3.22. Americium isotope concentrations for fuel channels E-M of the reference solution of Problem 2.2.1.

\begin{tabular}{rrcc}
\hline Step & $\begin{array}{c}\text { Burnup } \\
{\left[\frac{\mathrm{MWd}}{\mathrm{MtU}}\right]}\end{array}$ & $\begin{array}{c}{ }^{241} \mathrm{Am} \\
{\left[\frac{\mathrm{atoms}}{\mathrm{b}-\mathrm{cm}}\right]}\end{array}$ & $\begin{array}{c}{ }^{43} \mathrm{Am} \\
{\left[\frac{\mathrm{atoms}}{\mathrm{b}-\mathrm{cm}}\right]}\end{array}$ \\
\hline 0 & 0.0 & $0.0000 \times 10^{+00}$ & $0.0000 \times 10^{+00}$ \\
1 & 25.0 & $2.9308 \times 10^{-15}$ & $7.5808 \times 10^{-19}$ \\
2 & 50.0 & $5.7905 \times 10^{-14}$ & $3.2007 \times 10^{-17}$ \\
3 & 100.0 & $1.0359 \times 10^{-12}$ & $1.1917 \times 10^{-15}$ \\
4 & 200.0 & $1.6911 \times 10^{-11}$ & $3.9568 \times 10^{-14}$ \\
5 & 300.0 & $8.3125 \times 10^{-11}$ & $2.9207 \times 10^{-13}$ \\
6 & 400.0 & $2.5208 \times 10^{-10}$ & $1.1779 \times 10^{-12}$ \\
7 & 500.0 & $5.8824 \times 10^{-10}$ & $3.4256 \times 10^{-12}$ \\
8 & 600.0 & $1.1645 \times 10^{-09}$ & $8.1152 \times 10^{-12}$ \\
9 & 700.0 & $2.0591 \times 10^{-09}$ & $1.6702 \times 10^{-11}$ \\
10 & 800.0 & $3.3539 \times 10^{-09}$ & $3.1037 \times 10^{-11}$ \\
11 & 900.0 & $5.1321 \times 10^{-09}$ & $5.3363 \times 10^{-11}$ \\
12 & 1000.0 & $7.4770 \times 10^{-09}$ & $8.6326 \times 10^{-11}$ \\
13 & 1100.0 & $1.0471 \times 10^{-08}$ & $1.3297 \times 10^{-10}$ \\
14 & 1200.0 & $1.4195 \times 10^{-08}$ & $1.9675 \times 10^{-10}$ \\
15 & 1300.0 & $1.8727 \times 10^{-08}$ & $2.8148 \times 10^{-10}$ \\
\hline
\end{tabular}


Table 3.23. Curium isotope concentrations for fuel channels E-M of the reference solution of Problem 2.2.1.

\begin{tabular}{rrcc}
\hline Step & $\begin{array}{c}\text { Burnup } \\
{\left[\frac{\mathrm{MWd}}{\mathrm{MtU}}\right]}\end{array}$ & $\begin{array}{c}{ }^{243} \mathrm{Cm} \\
{\left[\frac{\mathrm{atoms}}{\mathrm{b}-\mathrm{cm}}\right]}\end{array}$ & $\begin{array}{c}{ }^{244} \mathrm{Cm} \\
{\left[\frac{\mathrm{atoms}}{\mathrm{b}-\mathrm{cm}}\right]}\end{array}$ \\
\hline 0 & 0.0 & $0.0000 \times 10^{+00}$ & $0.0000 \times 10^{+00}$ \\
1 & 25.0 & $0.0000 \times 10^{+00}$ & $0.0000 \times 10^{+00}$ \\
2 & 50.0 & $0.0000 \times 10^{+00}$ & $2.1019 \times 10^{-20}$ \\
3 & 100.0 & $5.9654 \times 10^{-19}$ & $1.6045 \times 10^{-18}$ \\
4 & 200.0 & $3.9703 \times 10^{-17}$ & $1.0785 \times 10^{-16}$ \\
5 & 300.0 & $4.2775 \times 10^{-16}$ & $1.1950 \times 10^{-15}$ \\
6 & 400.0 & $2.2217 \times 10^{-15}$ & $6.4115 \times 10^{-15}$ \\
7 & 500.0 & $7.7814 \times 10^{-15}$ & $2.3232 \times 10^{-14}$ \\
8 & 600.0 & $2.1293 \times 10^{-14}$ & $6.5814 \times 10^{-14}$ \\
9 & 700.0 & $4.9204 \times 10^{-14}$ & $1.5748 \times 10^{-13}$ \\
10 & 800.0 & $1.0058 \times 10^{-13}$ & $3.3330 \times 10^{-13}$ \\
11 & 900.0 & $1.8731 \times 10^{-13}$ & $6.4260 \times 10^{-13}$ \\
12 & 1000.0 & $3.2432 \times 10^{-13}$ & $1.1515 \times 10^{-12}$ \\
13 & 1100.0 & $5.2959 \times 10^{-13}$ & $1.9455 \times 10^{-12}$ \\
14 & 1200.0 & $8.2429 \times 10^{-13}$ & $3.1319 \times 10^{-12}$ \\
15 & 1300.0 & $1.2326 \times 10^{-12}$ & $4.8422 \times 10^{-12}$ \\
\hline
\end{tabular}

Table 3.24. Cesium isotope concentrations for fuel channels E-M of the reference solution of Problem 2.2.1.

\begin{tabular}{rrccc}
\hline Step & $\begin{array}{c}\text { Burnup } \\
{\left[\frac{\mathrm{MWd}}{\mathrm{MtU}}\right]}\end{array}$ & $\begin{array}{c}{ }^{133} \mathrm{Cs} \\
{\left[\frac{\mathrm{atoms}}{\mathrm{b}-\mathrm{cm}}\right]}\end{array}$ & $\begin{array}{c}{ }^{135} \mathrm{Cs} \\
{\left[\frac{\mathrm{atoms}}{\mathrm{b}-\mathrm{cm}}\right]}\end{array}$ & $\begin{array}{c}137 \mathrm{Cs} \\
{\left[\frac{\mathrm{atoms}}{\mathrm{b}-\mathrm{cm}}\right]}\end{array}$ \\
\hline 0 & 0.0 & $0.0000 \times 10^{+00}$ & $0.0000 \times 10^{+00}$ & $0.0000 \times 10^{+00}$ \\
1 & 25.0 & $4.4188 \times 10^{-08}$ & $4.8254 \times 10^{-08}$ & $7.8578 \times 10^{-08}$ \\
2 & 50.0 & $1.2446 \times 10^{-07}$ & $9.8859 \times 10^{-08}$ & $1.5709 \times 10^{-07}$ \\
3 & 100.0 & $2.9413 \times 10^{-07}$ & $2.0053 \times 10^{-07}$ & $3.1393 \times 10^{-07}$ \\
4 & 200.0 & $6.3448 \times 10^{-07}$ & $4.0560 \times 10^{-07}$ & $6.2691 \times 10^{-07}$ \\
5 & 300.0 & $9.7456 \times 10^{-07}$ & $6.1279 \times 10^{-07}$ & $9.3896 \times 10^{-07}$ \\
6 & 400.0 & $1.3142 \times 10^{-06}$ & $8.2183 \times 10^{-07}$ & $1.2499 \times 10^{-06}$ \\
7 & 500.0 & $1.6535 \times 10^{-06}$ & $1.0326 \times 10^{-06}$ & $1.5599 \times 10^{-06}$ \\
8 & 600.0 & $1.9926 \times 10^{-06}$ & $1.2449 \times 10^{-06}$ & $1.8688 \times 10^{-06}$ \\
9 & 700.0 & $2.3312 \times 10^{-06}$ & $1.4585 \times 10^{-06}$ & $2.1767 \times 10^{-06}$ \\
10 & 800.0 & $2.6695 \times 10^{-06}$ & $1.6735 \times 10^{-06}$ & $2.4835 \times 10^{-06}$ \\
11 & 900.0 & $3.0074 \times 10^{-06}$ & $1.8895 \times 10^{-06}$ & $2.7892 \times 10^{-06}$ \\
12 & 1000.0 & $3.3449 \times 10^{-06}$ & $2.1066 \times 10^{-06}$ & $3.0937 \times 10^{-06}$ \\
13 & 1100.0 & $3.6820 \times 10^{-06}$ & $2.3247 \times 10^{-06}$ & $3.3971 \times 10^{-06}$ \\
14 & 1200.0 & $4.0187 \times 10^{-06}$ & $2.5436 \times 10^{-06}$ & $3.6995 \times 10^{-06}$ \\
15 & 1300.0 & $4.3551 \times 10^{-06}$ & $2.7634 \times 10^{-06}$ & $4.0006 \times 10^{-06}$ \\
\hline
\end{tabular}


Table 3.25. Neodymium isotope concentrations for fuel channels E-M of the reference solution of Problem 2.2.1.

\begin{tabular}{rrccccc}
\hline Step & $\begin{array}{c}\text { Burnup } \\
{\left[\frac{\mathrm{MWd}}{\mathrm{MtU}}\right]}\end{array}$ & $\begin{array}{c}{ }^{143} \mathrm{Nd} \\
{\left[\frac{\mathrm{atoms}}{\mathrm{b}-\mathrm{cm}}\right]}\end{array}$ & $\begin{array}{c}{ }^{144} \mathrm{Nd} \\
{\left[\frac{\mathrm{atoms}}{\mathrm{b}-\mathrm{cm}}\right]}\end{array}$ & $\begin{array}{c}145 \mathrm{Nd} \\
{\left[\frac{\mathrm{atoms}}{\mathrm{b}-\mathrm{cm}}\right]}\end{array}$ & $\begin{array}{c}146 \mathrm{Nd} \\
{\left[\frac{\mathrm{atoms}}{\mathrm{b}-\mathrm{cm}}\right]}\end{array}$ & $\begin{array}{c}148 \mathrm{Nd} \\
{\left[\frac{\text { atoms }}{\mathrm{b}-\mathrm{cm}}\right]}\end{array}$ \\
\hline 0 & 0.0 & $0.0000 \times 10^{+00}$ & $0.0000 \times 10^{+00}$ & $0.0000 \times 10^{+00}$ & $0.0000 \times 10^{+00}$ & $0.0000 \times 10^{+00}$ \\
1 & 25.0 & $1.9347 \times 10^{-08}$ & $1.3823 \times 10^{-09}$ & $4.8771 \times 10^{-08}$ & $3.8351 \times 10^{-08}$ & $2.1663 \times 10^{-08}$ \\
2 & 50.0 & $6.9500 \times 10^{-08}$ & $5.5014 \times 10^{-09}$ & $9.8500 \times 10^{-08}$ & $7.6726 \times 10^{-08}$ & $4.3344 \times 10^{-08}$ \\
3 & 100.0 & $2.0275 \times 10^{-07}$ & $2.1608 \times 10^{-08}$ & $1.9755 \times 10^{-07}$ & $1.5332 \times 10^{-07}$ & $8.6695 \times 10^{-08}$ \\
4 & 200.0 & $4.9257 \times 10^{-07}$ & $8.2586 \times 10^{-08}$ & $3.9412 \times 10^{-07}$ & $3.0595 \times 10^{-07}$ & $1.7328 \times 10^{-07}$ \\
5 & 300.0 & $7.8090 \times 10^{-07}$ & $1.7706 \times 10^{-07}$ & $5.8885 \times 10^{-07}$ & $4.5793 \times 10^{-07}$ & $2.5970 \times 10^{-07}$ \\
6 & 400.0 & $1.0648 \times 10^{-06}$ & $2.9995 \times 10^{-07}$ & $7.8181 \times 10^{-07}$ & $6.0927 \times 10^{-07}$ & $3.4595 \times 10^{-07}$ \\
7 & 500.0 & $1.3445 \times 10^{-06}$ & $4.4692 \times 10^{-07}$ & $9.7321 \times 10^{-07}$ & $7.6010 \times 10^{-07}$ & $4.3207 \times 10^{-07}$ \\
8 & 600.0 & $1.6203 \times 10^{-06}$ & $6.1435 \times 10^{-07}$ & $1.1632 \times 10^{-06}$ & $9.1049 \times 10^{-07}$ & $5.1807 \times 10^{-07}$ \\
9 & 700.0 & $1.8924 \times 10^{-06}$ & $7.9914 \times 10^{-07}$ & $1.3518 \times 10^{-06}$ & $1.0604 \times 10^{-06}$ & $6.0395 \times 10^{-07}$ \\
10 & 800.0 & $2.1609 \times 10^{-06}$ & $9.9869 \times 10^{-07}$ & $1.5391 \times 10^{-06}$ & $1.2100 \times 10^{-06}$ & $6.8973 \times 10^{-07}$ \\
11 & 900.0 & $2.4260 \times 10^{-06}$ & $1.2108 \times 10^{-06}$ & $1.7252 \times 10^{-06}$ & $1.3593 \times 10^{-06}$ & $7.7539 \times 10^{-07}$ \\
12 & 1000.0 & $2.6878 \times 10^{-06}$ & $1.4336 \times 10^{-06}$ & $1.9101 \times 10^{-06}$ & $1.5082 \times 10^{-06}$ & $8.6096 \times 10^{-07}$ \\
13 & 1100.0 & $2.9465 \times 10^{-06}$ & $1.6654 \times 10^{-06}$ & $2.0940 \times 10^{-06}$ & $1.6568 \times 10^{-06}$ & $9.4642 \times 10^{-07}$ \\
14 & 1200.0 & $3.2021 \times 10^{-06}$ & $1.9050 \times 10^{-06}$ & $2.2768 \times 10^{-06}$ & $1.8053 \times 10^{-06}$ & $1.0318 \times 10^{-06}$ \\
15 & 1300.0 & $3.4549 \times 10^{-06}$ & $2.1513 \times 10^{-06}$ & $2.4587 \times 10^{-06}$ & $1.9534 \times 10^{-06}$ & $1.1171 \times 10^{-06}$ \\
\hline
\end{tabular}

Table 3.26. Strontium isotope concentrations for fuel channels E-M of the reference solution of Problem 2.2.1.

\begin{tabular}{rrccccc}
\hline Step & $\begin{array}{c}\text { Burnup } \\
{\left[\frac{\mathrm{MWd}}{\mathrm{MtU}}\right]}\end{array}$ & $\begin{array}{c}{ }^{84} \mathrm{Sr} \\
{\left[\frac{\mathrm{atoms}}{\mathrm{b}-\mathrm{cm}}\right]}\end{array}$ & $\begin{array}{c}{ }^{86} \mathrm{Sr} \\
{\left[\frac{\mathrm{atoms}}{\mathrm{b}-\mathrm{cm}}\right]}\end{array}$ & $\begin{array}{c}{ }^{87} \mathrm{Sr} \\
{\left[\frac{\mathrm{atoms}}{\mathrm{b}-\mathrm{cm}}\right]}\end{array}$ & $\begin{array}{c}{ }^{88} \mathrm{Sr} \\
{\left[\frac{\mathrm{atoms}}{\mathrm{b}-\mathrm{cm}}\right]}\end{array}$ & $\begin{array}{c}{ }^{90} \mathrm{Sr} \\
{\left[\frac{\mathrm{atoms}}{\mathrm{b}-\mathrm{cm}}\right]}\end{array}$ \\
\hline 0 & 0.0 & $0.0000 \times 10^{+00}$ & $0.0000 \times 10^{+00}$ & $0.0000 \times 10^{+00}$ & $0.0000 \times 10^{+00}$ & $0.0000 \times 10^{+00}$ \\
1 & 25.0 & $2.8586 \times 10^{-19}$ & $3.1871 \times 10^{-14}$ & $1.6984 \times 10^{-14}$ & $4.3721 \times 10^{-08}$ & $7.1450 \times 10^{-08}$ \\
2 & 50.0 & $1.1307 \times 10^{-18}$ & $1.5153 \times 10^{-13}$ & $3.7120 \times 10^{-14}$ & $8.7702 \times 10^{-08}$ & $1.4243 \times 10^{-07}$ \\
3 & 100.0 & $4.4240 \times 10^{-18}$ & $7.3659 \times 10^{-13}$ & $8.6820 \times 10^{-14}$ & $1.7492 \times 10^{-07}$ & $2.8295 \times 10^{-07}$ \\
4 & 200.0 & $1.7029 \times 10^{-17}$ & $3.4507 \times 10^{-12}$ & $2.2157 \times 10^{-13}$ & $3.4656 \times 10^{-07}$ & $5.5850 \times 10^{-07}$ \\
5 & 300.0 & $3.7087 \times 10^{-17}$ & $8.2208 \times 10^{-12}$ & $3.9943 \times 10^{-13}$ & $5.1476 \times 10^{-07}$ & $8.2726 \times 10^{-07}$ \\
6 & 400.0 & $6.4074 \times 10^{-17}$ & $1.4958 \times 10^{-11}$ & $6.1639 \times 10^{-13}$ & $6.7990 \times 10^{-07}$ & $1.0899 \times 10^{-06}$ \\
7 & 500.0 & $9.7575 \times 10^{-17}$ & $2.3572 \times 10^{-11}$ & $8.6886 \times 10^{-13}$ & $8.4217 \times 10^{-07}$ & $1.3466 \times 10^{-06}$ \\
8 & 600.0 & $1.3724 \times 10^{-16}$ & $3.3987 \times 10^{-11}$ & $1.1539 \times 10^{-12}$ & $1.0018 \times 10^{-06}$ & $1.5981 \times 10^{-06}$ \\
9 & 700.0 & $1.8281 \times 10^{-16}$ & $4.6136 \times 10^{-11}$ & $1.4690 \times 10^{-12}$ & $1.1591 \times 10^{-06}$ & $1.8445 \times 10^{-06}$ \\
10 & 800.0 & $2.3399 \times 10^{-16}$ & $5.9964 \times 10^{-11}$ & $1.8117 \times 10^{-12}$ & $1.3141 \times 10^{-06}$ & $2.0861 \times 10^{-06}$ \\
11 & 900.0 & $2.9058 \times 10^{-16}$ & $7.5424 \times 10^{-11}$ & $2.1802 \times 10^{-12}$ & $1.4670 \times 10^{-06}$ & $2.3233 \times 10^{-06}$ \\
12 & 1000.0 & $3.5241 \times 10^{-16}$ & $9.2473 \times 10^{-11}$ & $2.5726 \times 10^{-12}$ & $1.6179 \times 10^{-06}$ & $2.5562 \times 10^{-06}$ \\
13 & 1100.0 & $4.1928 \times 10^{-16}$ & $1.1108 \times 10^{-10}$ & $2.9876 \times 10^{-12}$ & $1.7669 \times 10^{-06}$ & $2.7851 \times 10^{-06}$ \\
14 & 1200.0 & $4.9108 \times 10^{-16}$ & $1.3120 \times 10^{-10}$ & $3.4236 \times 10^{-12}$ & $1.9143 \times 10^{-06}$ & $3.0102 \times 10^{-06}$ \\
15 & 1300.0 & $5.6764 \times 10^{-16}$ & $1.5282 \times 10^{-10}$ & $3.8793 \times 10^{-12}$ & $2.0599 \times 10^{-06}$ & $3.2316 \times 10^{-06}$ \\
\hline
\end{tabular}


Table 3.27. Barium isotope concentrations for fuel channels E-M of the reference solution of Problem 2.2.1.

\begin{tabular}{rrccccc}
\hline Step & $\begin{array}{c}\text { Burnup } \\
{\left[\frac{\mathrm{MWd}}{\mathrm{MtU}}\right]}\end{array}$ & $\begin{array}{c}{ }^{132} \mathrm{Ba} \\
{\left[\frac{\mathrm{atoms}}{\mathrm{b}-\mathrm{cm}}\right]}\end{array}$ & $\begin{array}{c}{ }^{134} \mathrm{Ba} \\
{\left[\frac{\mathrm{atoms}}{\mathrm{b}-\mathrm{cm}}\right]}\end{array}$ & $\begin{array}{c}{ }^{135} \mathrm{Ba} \\
{\left[\frac{\mathrm{atoms}}{\mathrm{b}-\mathrm{cm}}\right]}\end{array}$ & $\begin{array}{c}{ }^{136} \mathrm{Ba} \\
{\left[\frac{\mathrm{atoms}}{\mathrm{b}-\mathrm{cm}}\right]}\end{array}$ & $\begin{array}{c}{ }^{138} \mathrm{Ba} \\
{\left[\frac{\mathrm{atoms}}{\mathrm{b}-\mathrm{cm}}\right]}\end{array}$ \\
\hline 0 & 0.0 & $0.0000 \times 10^{+00}$ & $0.0000 \times 10^{+00}$ & $0.0000 \times 10^{+00}$ & $0.0000 \times 10^{+00}$ & $0.0000 \times 10^{+00}$ \\
1 & 25.0 & $0.0000 \times 10^{+00}$ & $2.3695 \times 10^{-14}$ & $1.7168 \times 10^{-15}$ & $3.5635 \times 10^{-11}$ & $8.4987 \times 10^{-08}$ \\
2 & 50.0 & $0.0000 \times 10^{+00}$ & $3.1722 \times 10^{-13}$ & $5.4476 \times 10^{-15}$ & $9.9952 \times 10^{-11}$ & $1.7010 \times 10^{-07}$ \\
3 & 100.0 & $0.0000 \times 10^{+00}$ & $3.5008 \times 10^{-12}$ & $1.8933 \times 10^{-14}$ & $2.8656 \times 10^{-10}$ & $3.4004 \times 10^{-07}$ \\
4 & 200.0 & $0.0000 \times 10^{+00}$ & $3.2947 \times 10^{-11}$ & $6.9902 \times 10^{-14}$ & $8.3810 \times 10^{-10}$ & $6.7893 \times 10^{-07}$ \\
5 & 300.0 & $0.0000 \times 10^{+00}$ & $1.1579 \times 10^{-10}$ & $1.5409 \times 10^{-13}$ & $1.5998 \times 10^{-09}$ & $1.0166 \times 10^{-06}$ \\
6 & 400.0 & $0.0000 \times 10^{+00}$ & $2.7691 \times 10^{-10}$ & $2.7480 \times 10^{-13}$ & $2.5554 \times 10^{-09}$ & $1.3531 \times 10^{-06}$ \\
7 & 500.0 & $0.0000 \times 10^{+00}$ & $5.3889 \times 10^{-10}$ & $4.3730 \times 10^{-13}$ & $3.6921 \times 10^{-09}$ & $1.6886 \times 10^{-06}$ \\
8 & 600.0 & $0.0000 \times 10^{+00}$ & $9.2229 \times 10^{-10}$ & $6.4840 \times 10^{-13}$ & $4.9996 \times 10^{-09}$ & $2.0232 \times 10^{-06}$ \\
9 & 700.0 & $0.0000 \times 10^{+00}$ & $1.4459 \times 10^{-09}$ & $9.1633 \times 10^{-13}$ & $6.4683 \times 10^{-09}$ & $2.3569 \times 10^{-06}$ \\
10 & 800.0 & $0.0000 \times 10^{+00}$ & $2.1269 \times 10^{-09}$ & $1.2506 \times 10^{-12}$ & $8.0904 \times 10^{-09}$ & $2.6898 \times 10^{-06}$ \\
11 & 900.0 & $0.0000 \times 10^{+00}$ & $2.9811 \times 10^{-09}$ & $1.6616 \times 10^{-12}$ & $9.8586 \times 10^{-09}$ & $3.0220 \times 10^{-06}$ \\
12 & 1000.0 & $0.0000 \times 10^{+00}$ & $4.0228 \times 10^{-09}$ & $2.1611 \times 10^{-12}$ & $1.1767 \times 10^{-08}$ & $3.3533 \times 10^{-06}$ \\
13 & 1100.0 & $0.0000 \times 10^{+00}$ & $5.2654 \times 10^{-09}$ & $2.7614 \times 10^{-12}$ & $1.3810 \times 10^{-08}$ & $3.6840 \times 10^{-06}$ \\
14 & 1200.0 & $0.0000 \times 10^{+00}$ & $6.7211 \times 10^{-09}$ & $3.4758 \times 10^{-12}$ & $1.5982 \times 10^{-08}$ & $4.0141 \times 10^{-06}$ \\
15 & 1300.0 & $0.0000 \times 10^{+00}$ & $8.4013 \times 10^{-09}$ & $4.3184 \times 10^{-12}$ & $1.8280 \times 10^{-08}$ & $4.3435 \times 10^{-06}$ \\
\hline
\end{tabular}

Table 3.28. Technetium isotope concentrations for fuel channels E-M of the reference solution of Problem 2.2.1.

\begin{tabular}{rrc}
\hline Step & $\begin{array}{r}\text { Burnup } \\
{\left[\frac{\mathrm{MWd}}{\mathrm{MtU}}\right]}\end{array}$ & $\begin{array}{c}{ }^{99} \mathrm{Tc} \\
{\left[\frac{\mathrm{atoms}}{\mathrm{b}-\mathrm{cm}}\right]}\end{array}$ \\
\hline 0 & 0.0 & $0.0000 \times 10^{+00}$ \\
1 & 25.0 & $5.7696 \times 10^{-08}$ \\
2 & 50.0 & $1.3507 \times 10^{-07}$ \\
3 & 100.0 & $2.9033 \times 10^{-07}$ \\
4 & 200.0 & $6.0050 \times 10^{-07}$ \\
5 & 300.0 & $9.1022 \times 10^{-07}$ \\
6 & 400.0 & $1.2194 \times 10^{-06}$ \\
7 & 500.0 & $1.5281 \times 10^{-06}$ \\
8 & 600.0 & $1.8365 \times 10^{-06}$ \\
9 & 700.0 & $2.1444 \times 10^{-06}$ \\
10 & 800.0 & $2.4518 \times 10^{-06}$ \\
11 & 900.0 & $2.7589 \times 10^{-06}$ \\
12 & 1000.0 & $3.0655 \times 10^{-06}$ \\
13 & 1100.0 & $3.3716 \times 10^{-06}$ \\
14 & 1200.0 & $3.6774 \times 10^{-06}$ \\
15 & 1300.0 & $3.9828 \times 10^{-06}$ \\
\hline
\end{tabular}


Table 3.29. Rubidium isotope concentrations for fuel channels $E-M$ of the reference solution of Problem 2.2.1.

\begin{tabular}{rrcc}
\hline Step & $\begin{array}{c}\text { Burnup } \\
{\left[\frac{\mathrm{MWd}}{\mathrm{MtU}}\right]}\end{array}$ & $\begin{array}{c}{ }^{85} \mathrm{Rb} \\
{\left[\frac{\mathrm{atoms}}{\mathrm{b}-\mathrm{cm}}\right]}\end{array}$ & $\begin{array}{c}87 \mathrm{Rb} \\
{\left[\frac{\mathrm{atoms}}{\mathrm{b}-\mathrm{cm}}\right]}\end{array}$ \\
\hline 0 & 0.0 & $0.0000 \times 10^{+00}$ & $0.0000 \times 10^{+00}$ \\
1 & 25.0 & $1.2551 \times 10^{-08}$ & $3.1652 \times 10^{-08}$ \\
2 & 50.0 & $2.5254 \times 10^{-08}$ & $6.3272 \times 10^{-08}$ \\
3 & 100.0 & $5.0493 \times 10^{-08}$ & $1.2599 \times 10^{-07}$ \\
4 & 200.0 & $1.0035 \times 10^{-07}$ & $2.4948 \times 10^{-07}$ \\
5 & 300.0 & $1.4947 \times 10^{-07}$ & $3.7060 \times 10^{-07}$ \\
6 & 400.0 & $1.9791 \times 10^{-07}$ & $4.8953 \times 10^{-07}$ \\
7 & 500.0 & $2.4574 \times 10^{-07}$ & $6.0651 \times 10^{-07}$ \\
8 & 600.0 & $2.9304 \times 10^{-07}$ & $7.2168 \times 10^{-07}$ \\
9 & 700.0 & $3.3984 \times 10^{-07}$ & $8.3518 \times 10^{-07}$ \\
10 & 800.0 & $3.8618 \times 10^{-07}$ & $9.4713 \times 10^{-07}$ \\
11 & 900.0 & $4.3210 \times 10^{-07}$ & $1.0576 \times 10^{-06}$ \\
12 & 1000.0 & $4.7762 \times 10^{-07}$ & $1.1667 \times 10^{-06}$ \\
13 & 1100.0 & $5.2277 \times 10^{-07}$ & $1.2745 \times 10^{-06}$ \\
14 & 1200.0 & $5.6759 \times 10^{-07}$ & $1.3811 \times 10^{-06}$ \\
15 & 1300.0 & $6.1209 \times 10^{-07}$ & $1.4866 \times 10^{-06}$ \\
\hline
\end{tabular}




\section{Fuel Pins P-S}

Table 3.30. Power peaking factors for fuel channels P-S of the reference solution of Problem 2.2.1.

\begin{tabular}{rrrrr}
\hline Step & $\begin{array}{c}\text { Burnup } \\
{\left[\frac{\mathrm{MWd}}{\mathrm{MtU}}\right]}\end{array}$ & $\begin{array}{r}\text { Shift } \\
\text { Peaking Factor }\end{array}$ & $\begin{array}{r}\text { MPACT } \\
\text { Peaking Factor }\end{array}$ & $\begin{array}{c}\text { Difference } \\
\text { [MPACT-Shift }]\end{array}$ \\
\hline 1 & 0.0 & 1.0044 & 1.0039 & -0.0005 \\
2 & 25.0 & 1.0047 & 1.0039 & -0.0008 \\
3 & 50.0 & 1.0045 & 1.0039 & -0.0006 \\
4 & 100.0 & 1.0046 & 1.0039 & -0.0007 \\
5 & 200.0 & 1.0044 & 1.0039 & -0.0006 \\
6 & 300.0 & 1.0046 & 1.0039 & -0.0007 \\
7 & 400.0 & 1.0048 & 1.0039 & -0.0009 \\
8 & 500.0 & 1.0043 & 1.0039 & -0.0004 \\
9 & 600.0 & 1.0050 & 1.0039 & -0.0011 \\
10 & 700.0 & 1.0048 & 1.0039 & -0.0009 \\
11 & 800.0 & 1.0048 & 1.0039 & -0.0009 \\
12 & 900.0 & 1.0043 & 1.0039 & -0.0004 \\
13 & 1000.0 & 1.0048 & 1.0039 & -0.0009 \\
14 & 1100.0 & 1.0049 & 1.0039 & -0.0010 \\
15 & 1200.0 & 1.0048 & 1.0039 & -0.0009 \\
16 & 1300.0 & 1.0043 & 1.0039 & -0.0005 \\
\hline
\end{tabular}

Table 3.31. Uranium isotope concentrations for fuel channels $\mathbf{P}-\mathrm{S}$ of the reference solution of Problem 2.2.1.

\begin{tabular}{rrcccc}
\hline Step & $\begin{array}{c}\text { Burnup } \\
{\left[\frac{\mathrm{MWd}}{\mathrm{MtU}}\right]}\end{array}$ & $\begin{array}{c}{ }^{234} \mathrm{U} \\
{\left[\frac{\mathrm{atoms}}{\mathrm{b} \text {-cm }}\right]}\end{array}$ & $\begin{array}{c}{ }^{235} \mathrm{U} \\
{\left[\frac{\mathrm{atoms}}{\mathrm{b}-\mathrm{cm}}\right]}\end{array}$ & $\begin{array}{c}{ }^{236} \mathrm{U} \\
{\left[\frac{\mathrm{atoms}}{\mathrm{b}-\mathrm{cm}}\right]}\end{array}$ & $\begin{array}{c}{ }^{238} \mathrm{U} \\
{\left[\frac{\mathrm{atoms}}{\mathrm{b}-\mathrm{cm}}\right]}\end{array}$ \\
\hline 0 & 0.0 & $2.5775 \times 10^{-06}$ & $3.4449 \times 10^{-04}$ & $0.0000 \times 10^{+00}$ & $4.7470 \times 10^{-02}$ \\
1 & 25.0 & $2.5749 \times 10^{-06}$ & $3.4308 \times 10^{-04}$ & $2.2666 \times 10^{-07}$ & $4.7469 \times 10^{-02}$ \\
2 & 50.0 & $2.5724 \times 10^{-06}$ & $3.4167 \times 10^{-04}$ & $4.5123 \times 10^{-07}$ & $4.7467 \times 10^{-02}$ \\
3 & 100.0 & $2.5674 \times 10^{-06}$ & $3.3891 \times 10^{-04}$ & $8.9408 \times 10^{-07}$ & $4.7465 \times 10^{-02}$ \\
4 & 200.0 & $2.5575 \times 10^{-06}$ & $3.3352 \times 10^{-04}$ & $1.7562 \times 10^{-06}$ & $4.7459 \times 10^{-02}$ \\
5 & 300.0 & $2.5479 \times 10^{-06}$ & $3.2831 \times 10^{-04}$ & $2.5892 \times 10^{-06}$ & $4.7454 \times 10^{-02}$ \\
6 & 400.0 & $2.5384 \times 10^{-06}$ & $3.2326 \times 10^{-04}$ & $3.3963 \times 10^{-06}$ & $4.7449 \times 10^{-02}$ \\
7 & 500.0 & $2.5291 \times 10^{-06}$ & $3.1835 \times 10^{-04}$ & $4.1791 \times 10^{-06}$ & $4.7444 \times 10^{-02}$ \\
8 & 600.0 & $2.5199 \times 10^{-06}$ & $3.1358 \times 10^{-04}$ & $4.9400 \times 10^{-06}$ & $4.7439 \times 10^{-02}$ \\
9 & 700.0 & $2.5109 \times 10^{-06}$ & $3.0893 \times 10^{-04}$ & $5.6804 \times 10^{-06}$ & $4.7434 \times 10^{-02}$ \\
10 & 800.0 & $2.5020 \times 10^{-06}$ & $3.0440 \times 10^{-04}$ & $6.4016 \times 10^{-06}$ & $4.7429 \times 10^{-02}$ \\
11 & 900.0 & $2.4932 \times 10^{-06}$ & $2.9997 \times 10^{-04}$ & $7.1052 \times 10^{-06}$ & $4.7424 \times 10^{-02}$ \\
12 & 1000.0 & $2.4845 \times 10^{-06}$ & $2.9564 \times 10^{-04}$ & $7.7921 \times 10^{-06}$ & $4.7420 \times 10^{-02}$ \\
13 & 1100.0 & $2.4760 \times 10^{-06}$ & $2.9141 \times 10^{-04}$ & $8.4633 \times 10^{-06}$ & $4.7415 \times 10^{-02}$ \\
14 & 1200.0 & $2.4675 \times 10^{-06}$ & $2.8727 \times 10^{-04}$ & $9.1198 \times 10^{-06}$ & $4.7410 \times 10^{-02}$ \\
15 & 1300.0 & $2.4590 \times 10^{-06}$ & $2.8321 \times 10^{-04}$ & $9.7622 \times 10^{-06}$ & $4.7405 \times 10^{-02}$ \\
\hline
\end{tabular}


Table 3.32. Plutonium isotope concentrations for fuel channels P-S of the reference solution of Problem 2.2.1.

\begin{tabular}{rrccccc}
\hline Step & $\begin{array}{c}\text { Burnup } \\
{\left[\frac{\mathrm{MWd}}{\mathrm{MtU}}\right]}\end{array}$ & $\begin{array}{c}{ }^{238} \mathrm{Pu} \\
{\left[\frac{\mathrm{atoms}}{\mathrm{b}-\mathrm{cm}}\right]}\end{array}$ & $\begin{array}{c}{ }^{239} \mathrm{Pu} \\
{\left[\frac{\mathrm{atoms}}{\mathrm{b}-\mathrm{cm}}\right]}\end{array}$ & $\begin{array}{c}{ }^{240} \mathrm{Pu} \\
{\left[\frac{\mathrm{atoms}}{\mathrm{b}-\mathrm{cm}}\right]}\end{array}$ & $\begin{array}{c}{ }^{241} \mathrm{Pu} \\
{\left[\frac{\mathrm{atoms}}{\mathrm{b}-\mathrm{cm}}\right]}\end{array}$ & $\begin{array}{c}{ }^{242} \mathrm{Pu} \\
{\left[\frac{\text { atoms }}{\mathrm{b}-\mathrm{cm}}\right]}\end{array}$ \\
\hline 0 & 0.0 & $0.0000 \times 10^{+00}$ & $0.0000 \times 10^{+00}$ & $0.0000 \times 10^{+00}$ & $0.0000 \times 10^{+00}$ & $0.0000 \times 10^{+00}$ \\
1 & 25.0 & $7.6665 \times 10^{-13}$ & $1.0040 \times 10^{-06}$ & $2.2708 \times 10^{-09}$ & $5.9041 \times 10^{-12}$ & $4.5877 \times 10^{-15}$ \\
2 & 50.0 & $5.7168 \times 10^{-12}$ & $2.2485 \times 10^{-06}$ & $1.0506 \times 10^{-08}$ & $5.6589 \times 10^{-11}$ & $9.0450 \times 10^{-14}$ \\
3 & 100.0 & $3.3248 \times 10^{-11}$ & $4.6721 \times 10^{-06}$ & $4.4645 \times 10^{-08}$ & $4.9180 \times 10^{-10}$ & $1.6112 \times 10^{-12}$ \\
4 & 200.0 & $1.6144 \times 10^{-10}$ & $9.2585 \times 10^{-06}$ & $1.7774 \times 10^{-07}$ & $3.9236 \times 10^{-09}$ & $2.6092 \times 10^{-11}$ \\
5 & 300.0 & $3.8657 \times 10^{-10}$ & $1.3529 \times 10^{-05}$ & $3.8737 \times 10^{-07}$ & $1.2707 \times 10^{-08}$ & $1.2735 \times 10^{-10}$ \\
6 & 400.0 & $7.0835 \times 10^{-10}$ & $1.7516 \times 10^{-05}$ & $6.6353 \times 10^{-07}$ & $2.8676 \times 10^{-08}$ & $3.8407 \times 10^{-10}$ \\
7 & 500.0 & $1.1270 \times 10^{-09}$ & $2.1248 \times 10^{-05}$ & $9.9752 \times 10^{-07}$ & $5.3207 \times 10^{-08}$ & $8.9201 \times 10^{-10}$ \\
8 & 600.0 & $1.6440 \times 10^{-09}$ & $2.4747 \times 10^{-05}$ & $1.3821 \times 10^{-06}$ & $8.7337 \times 10^{-08}$ & $1.7591 \times 10^{-09}$ \\
9 & 700.0 & $2.2617 \times 10^{-09}$ & $2.8035 \times 10^{-05}$ & $1.8110 \times 10^{-06}$ & $1.3181 \times 10^{-07}$ & $3.1011 \times 10^{-09}$ \\
10 & 800.0 & $2.9835 \times 10^{-09}$ & $3.1128 \times 10^{-05}$ & $2.2786 \times 10^{-06}$ & $1.8716 \times 10^{-07}$ & $5.0388 \times 10^{-09}$ \\
11 & 900.0 & $3.8147 \times 10^{-09}$ & $3.4043 \times 10^{-05}$ & $2.7803 \times 10^{-06}$ & $2.5377 \times 10^{-07}$ & $7.6973 \times 10^{-09}$ \\
12 & 1000.0 & $4.7615 \times 10^{-09}$ & $3.6793 \times 10^{-05}$ & $3.3117 \times 10^{-06}$ & $3.3183 \times 10^{-07}$ & $1.1202 \times 10^{-08}$ \\
13 & 1100.0 & $5.8318 \times 10^{-09}$ & $3.9392 \times 10^{-05}$ & $3.8692 \times 10^{-06}$ & $4.2145 \times 10^{-07}$ & $1.5678 \times 10^{-08}$ \\
14 & 1200.0 & $7.0350 \times 10^{-09}$ & $4.1849 \times 10^{-05}$ & $4.4495 \times 10^{-06}$ & $5.2266 \times 10^{-07}$ & $2.1254 \times 10^{-08}$ \\
15 & 1300.0 & $8.3816 \times 10^{-09}$ & $4.4174 \times 10^{-05}$ & $5.0495 \times 10^{-06}$ & $6.3533 \times 10^{-07}$ & $2.8050 \times 10^{-08}$ \\
\hline
\end{tabular}

Table 3.33. Americium isotope concentrations for fuel channels $\mathrm{P}-\mathrm{S}$ of the reference solution of Problem 2.2.1.

\begin{tabular}{rrcc}
\hline Step & $\begin{array}{c}\text { Burnup } \\
{\left[\frac{\mathrm{MWd}}{\mathrm{MtU}}\right]}\end{array}$ & $\begin{array}{c}{ }^{241} \mathrm{Am} \\
{\left[\frac{\mathrm{atoms}}{\mathrm{b}-\mathrm{cm}}\right]}\end{array}$ & $\begin{array}{c}{ }^{43} \mathrm{Am} \\
{\left[\frac{\mathrm{atoms}}{\mathrm{b}-\mathrm{cm}}\right]}\end{array}$ \\
\hline 0 & 0.0 & $0.0000 \times 10^{+00}$ & $0.0000 \times 10^{+00}$ \\
1 & 25.0 & $2.9235 \times 10^{-15}$ & $7.6205 \times 10^{-19}$ \\
2 & 50.0 & $5.7785 \times 10^{-14}$ & $3.2204 \times 10^{-17}$ \\
3 & 100.0 & $1.0342 \times 10^{-12}$ & $1.1998 \times 10^{-15}$ \\
4 & 200.0 & $1.6886 \times 10^{-11}$ & $3.9849 \times 10^{-14}$ \\
5 & 300.0 & $8.3003 \times 10^{-11}$ & $2.9405 \times 10^{-13}$ \\
6 & 400.0 & $2.5173 \times 10^{-10}$ & $1.1865 \times 10^{-12}$ \\
7 & 500.0 & $5.8747 \times 10^{-10}$ & $3.4507 \times 10^{-12}$ \\
8 & 600.0 & $1.1630 \times 10^{-09}$ & $8.1742 \times 10^{-12}$ \\
9 & 700.0 & $2.0565 \times 10^{-09}$ & $1.6822 \times 10^{-11}$ \\
10 & 800.0 & $3.3494 \times 10^{-09}$ & $3.1251 \times 10^{-11}$ \\
11 & 900.0 & $5.1249 \times 10^{-09}$ & $5.3729 \times 10^{-11}$ \\
12 & 1000.0 & $7.4661 \times 10^{-09}$ & $8.6912 \times 10^{-11}$ \\
13 & 1100.0 & $1.0455 \times 10^{-08}$ & $1.3387 \times 10^{-10}$ \\
14 & 1200.0 & $1.4173 \times 10^{-08}$ & $1.9807 \times 10^{-10}$ \\
15 & 1300.0 & $1.8698 \times 10^{-08}$ & $2.8334 \times 10^{-10}$ \\
\hline
\end{tabular}


Table 3.34. Curium isotope concentrations for fuel channels P-S of the reference solution of Problem 2.2.1.

\begin{tabular}{rrcc}
\hline Step & $\begin{array}{c}\text { Burnup } \\
{\left[\frac{\mathrm{MWd}}{\mathrm{MtU}}\right]}\end{array}$ & $\begin{array}{c}{ }^{243} \mathrm{Cm} \\
{\left[\frac{\mathrm{atoms}}{\mathrm{b}-\mathrm{cm}}\right]}\end{array}$ & $\begin{array}{c}{ }^{244} \mathrm{Cm} \\
{\left[\frac{\mathrm{atoms}}{\mathrm{b}-\mathrm{cm}}\right]}\end{array}$ \\
\hline 0 & 0.0 & $0.0000 \times 10^{+00}$ & $0.0000 \times 10^{+00}$ \\
1 & 25.0 & $0.0000 \times 10^{+00}$ & $0.0000 \times 10^{+00}$ \\
2 & 50.0 & $0.0000 \times 10^{+00}$ & $2.1120 \times 10^{-20}$ \\
3 & 100.0 & $5.9659 \times 10^{-19}$ & $1.6135 \times 10^{-18}$ \\
4 & 200.0 & $3.9719 \times 10^{-17}$ & $1.0849 \times 10^{-16}$ \\
5 & 300.0 & $4.2780 \times 10^{-16}$ & $1.2017 \times 10^{-15}$ \\
6 & 400.0 & $2.2234 \times 10^{-15}$ & $6.4513 \times 10^{-15}$ \\
7 & 500.0 & $7.7867 \times 10^{-15}$ & $2.3377 \times 10^{-14}$ \\
8 & 600.0 & $2.1306 \times 10^{-14}$ & $6.6220 \times 10^{-14}$ \\
9 & 700.0 & $4.9230 \times 10^{-14}$ & $1.5843 \times 10^{-13}$ \\
10 & 800.0 & $1.0060 \times 10^{-13}$ & $3.3521 \times 10^{-13}$ \\
11 & 900.0 & $1.8736 \times 10^{-13}$ & $6.4626 \times 10^{-13}$ \\
12 & 1000.0 & $3.2438 \times 10^{-13}$ & $1.1580 \times 10^{-12}$ \\
13 & 1100.0 & $5.2969 \times 10^{-13}$ & $1.9563 \times 10^{-12}$ \\
14 & 1200.0 & $8.2445 \times 10^{-13}$ & $3.1493 \times 10^{-12}$ \\
15 & 1300.0 & $1.2327 \times 10^{-12}$ & $4.8685 \times 10^{-12}$ \\
\hline
\end{tabular}

Table 3.35. Cesium isotope concentrations for fuel channels $\mathrm{P}-\mathrm{S}$ of the reference solution of Problem 2.2.1.

\begin{tabular}{rrccc}
\hline Step & $\begin{array}{c}\text { Burnup } \\
{\left[\frac{\mathrm{MWd}}{\mathrm{MtU}}\right]}\end{array}$ & $\begin{array}{c}{ }^{133} \mathrm{Cs} \\
{\left[\frac{\mathrm{atoms}}{\mathrm{b}-\mathrm{cm}}\right]}\end{array}$ & $\begin{array}{c}{ }^{135} \mathrm{Cs} \\
{\left[\frac{\mathrm{atoms}}{\mathrm{b}-\mathrm{cm}}\right]}\end{array}$ & $\begin{array}{c}137 \mathrm{Cs} \\
{\left[\frac{\mathrm{atoms}}{\mathrm{b}-\mathrm{cm}}\right]}\end{array}$ \\
\hline 0 & 0.0 & $0.0000 \times 10^{+00}$ & $0.0000 \times 10^{+00}$ & $0.0000 \times 10^{+00}$ \\
1 & 25.0 & $4.4243 \times 10^{-08}$ & $4.8288 \times 10^{-08}$ & $7.8677 \times 10^{-08}$ \\
2 & 50.0 & $1.2463 \times 10^{-07}$ & $9.8939 \times 10^{-08}$ & $1.5731 \times 10^{-07}$ \\
3 & 100.0 & $2.9458 \times 10^{-07}$ & $2.0070 \times 10^{-07}$ & $3.1441 \times 10^{-07}$ \\
4 & 200.0 & $6.3549 \times 10^{-07}$ & $4.0597 \times 10^{-07}$ & $6.2791 \times 10^{-07}$ \\
5 & 300.0 & $9.7606 \times 10^{-07}$ & $6.1333 \times 10^{-07}$ & $9.4040 \times 10^{-07}$ \\
6 & 400.0 & $1.3164 \times 10^{-06}$ & $8.2260 \times 10^{-07}$ & $1.2520 \times 10^{-06}$ \\
7 & 500.0 & $1.6562 \times 10^{-06}$ & $1.0335 \times 10^{-06}$ & $1.5624 \times 10^{-06}$ \\
8 & 600.0 & $1.9958 \times 10^{-06}$ & $1.2460 \times 10^{-06}$ & $1.8719 \times 10^{-06}$ \\
9 & 700.0 & $2.3349 \times 10^{-06}$ & $1.4599 \times 10^{-06}$ & $2.1802 \times 10^{-06}$ \\
10 & 800.0 & $2.6736 \times 10^{-06}$ & $1.6749 \times 10^{-06}$ & $2.4873 \times 10^{-06}$ \\
11 & 900.0 & $3.0120 \times 10^{-06}$ & $1.8912 \times 10^{-06}$ & $2.7934 \times 10^{-06}$ \\
12 & 1000.0 & $3.3499 \times 10^{-06}$ & $2.1084 \times 10^{-06}$ & $3.0984 \times 10^{-06}$ \\
13 & 1100.0 & $3.6875 \times 10^{-06}$ & $2.3266 \times 10^{-06}$ & $3.4022 \times 10^{-06}$ \\
14 & 1200.0 & $4.0247 \times 10^{-06}$ & $2.5457 \times 10^{-06}$ & $3.7049 \times 10^{-06}$ \\
15 & 1300.0 & $4.3614 \times 10^{-06}$ & $2.7656 \times 10^{-06}$ & $4.0064 \times 10^{-06}$ \\
\hline
\end{tabular}


Table 3.36. Neodymium isotope concentrations for fuel channels $P-S$ of the reference solution of Problem 2.2.1.

\begin{tabular}{rrccccc}
\hline Step & $\begin{array}{c}\text { Burnup } \\
{\left[\frac{\mathrm{MWd}}{\mathrm{MtU}}\right]}\end{array}$ & $\begin{array}{c}{ }^{143} \mathrm{Nd} \\
{\left[\frac{\mathrm{atoms}}{\mathrm{b}-\mathrm{cm}}\right]}\end{array}$ & $\begin{array}{c}{ }^{144} \mathrm{Nd} \\
{\left[\frac{\mathrm{atoms}}{\mathrm{b}-\mathrm{cm}}\right]}\end{array}$ & $\begin{array}{c}145 \mathrm{Nd} \\
{\left[\frac{\mathrm{atoms}}{\mathrm{b}-\mathrm{cm}}\right]}\end{array}$ & $\begin{array}{c}146 \mathrm{Nd} \\
{\left[\frac{\mathrm{atoms}}{\mathrm{b}-\mathrm{cm}}\right]}\end{array}$ & $\begin{array}{c}{ }^{48} \mathrm{Nd} \\
{\left[\frac{\mathrm{atoms}}{\mathrm{b}-\mathrm{cm}}\right]}\end{array}$ \\
\hline 0 & 0.0 & $0.0000 \times 10^{+00}$ & $0.0000 \times 10^{+00}$ & $0.0000 \times 10^{+00}$ & $0.0000 \times 10^{+00}$ & $0.0000 \times 10^{+00}$ \\
1 & 25.0 & $1.9371 \times 10^{-08}$ & $1.3840 \times 10^{-09}$ & $4.8832 \times 10^{-08}$ & $3.8398 \times 10^{-08}$ & $2.1690 \times 10^{-08}$ \\
2 & 50.0 & $6.9594 \times 10^{-08}$ & $5.5091 \times 10^{-09}$ & $9.8639 \times 10^{-08}$ & $7.6834 \times 10^{-08}$ & $4.3405 \times 10^{-08}$ \\
3 & 100.0 & $2.0305 \times 10^{-07}$ & $2.1641 \times 10^{-08}$ & $1.9785 \times 10^{-07}$ & $1.5356 \times 10^{-07}$ & $8.6828 \times 10^{-08}$ \\
4 & 200.0 & $4.9335 \times 10^{-07}$ & $8.2721 \times 10^{-08}$ & $3.9475 \times 10^{-07}$ & $3.0644 \times 10^{-07}$ & $1.7355 \times 10^{-07}$ \\
5 & 300.0 & $7.8210 \times 10^{-07}$ & $1.7736 \times 10^{-07}$ & $5.8975 \times 10^{-07}$ & $4.5863 \times 10^{-07}$ & $2.6010 \times 10^{-07}$ \\
6 & 400.0 & $1.0665 \times 10^{-06}$ & $3.0045 \times 10^{-07}$ & $7.8308 \times 10^{-07}$ & $6.1027 \times 10^{-07}$ & $3.4652 \times 10^{-07}$ \\
7 & 500.0 & $1.3467 \times 10^{-06}$ & $4.4769 \times 10^{-07}$ & $9.7479 \times 10^{-07}$ & $7.6135 \times 10^{-07}$ & $4.3278 \times 10^{-07}$ \\
8 & 600.0 & $1.6229 \times 10^{-06}$ & $6.1541 \times 10^{-07}$ & $1.1650 \times 10^{-06}$ & $9.1197 \times 10^{-07}$ & $5.1891 \times 10^{-07}$ \\
9 & 700.0 & $1.8953 \times 10^{-06}$ & $8.0052 \times 10^{-07}$ & $1.3539 \times 10^{-06}$ & $1.0621 \times 10^{-06}$ & $6.0492 \times 10^{-07}$ \\
10 & 800.0 & $2.1641 \times 10^{-06}$ & $1.0004 \times 10^{-06}$ & $1.5414 \times 10^{-06}$ & $1.2119 \times 10^{-06}$ & $6.9079 \times 10^{-07}$ \\
11 & 900.0 & $2.4295 \times 10^{-06}$ & $1.2128 \times 10^{-06}$ & $1.7278 \times 10^{-06}$ & $1.3614 \times 10^{-06}$ & $7.7657 \times 10^{-07}$ \\
12 & 1000.0 & $2.6917 \times 10^{-06}$ & $1.4360 \times 10^{-06}$ & $1.9130 \times 10^{-06}$ & $1.5105 \times 10^{-06}$ & $8.6225 \times 10^{-07}$ \\
13 & 1100.0 & $2.9506 \times 10^{-06}$ & $1.6682 \times 10^{-06}$ & $2.0971 \times 10^{-06}$ & $1.6593 \times 10^{-06}$ & $9.4783 \times 10^{-07}$ \\
14 & 1200.0 & $3.2066 \times 10^{-06}$ & $1.9082 \times 10^{-06}$ & $2.2802 \times 10^{-06}$ & $1.8079 \times 10^{-06}$ & $1.0333 \times 10^{-06}$ \\
15 & 1300.0 & $3.4597 \times 10^{-06}$ & $2.1548 \times 10^{-06}$ & $2.4622 \times 10^{-06}$ & $1.9563 \times 10^{-06}$ & $1.1187 \times 10^{-06}$ \\
\hline
\end{tabular}

Table 3.37. Strontium isotope concentrations for fuel channels P-S of the reference solution of Problem 2.2.1.

\begin{tabular}{rrccccc}
\hline Step & $\begin{array}{c}\text { Burnup } \\
{\left[\frac{\mathrm{MWd}}{\mathrm{MtU}}\right]}\end{array}$ & $\begin{array}{c}{ }^{84} \mathrm{Sr} \\
{\left[\frac{\mathrm{atoms}}{\mathrm{b}-\mathrm{cm}}\right]}\end{array}$ & $\begin{array}{c}{ }^{86} \mathrm{Sr} \\
{\left[\frac{\mathrm{atoms}}{\mathrm{b}-\mathrm{cm}}\right]}\end{array}$ & $\begin{array}{c}{ }^{87} \mathrm{Sr} \\
{\left[\frac{\mathrm{atoms}}{\mathrm{b}-\mathrm{cm}}\right]}\end{array}$ & $\begin{array}{c}{ }^{88} \mathrm{Sr} \\
{\left[\frac{\mathrm{atoms}}{\mathrm{b}-\mathrm{cm}}\right]}\end{array}$ & $\begin{array}{c}{ }^{90} \mathrm{Sr} \\
{\left[\frac{\mathrm{atoms}}{\mathrm{b}-\mathrm{cm}}\right]}\end{array}$ \\
\hline 0 & 0.0 & $0.0000 \times 10^{+00}$ & $0.0000 \times 10^{+00}$ & $0.0000 \times 10^{+00}$ & $0.0000 \times 10^{+00}$ & $0.0000 \times 10^{+00}$ \\
1 & 25.0 & $2.8586 \times 10^{-19}$ & $3.1871 \times 10^{-14}$ & $1.6984 \times 10^{-14}$ & $4.3721 \times 10^{-08}$ & $7.1450 \times 10^{-08}$ \\
2 & 50.0 & $1.1307 \times 10^{-18}$ & $1.5153 \times 10^{-13}$ & $3.7120 \times 10^{-14}$ & $8.7702 \times 10^{-08}$ & $1.4243 \times 10^{-07}$ \\
3 & 100.0 & $4.4240 \times 10^{-18}$ & $7.3659 \times 10^{-13}$ & $8.6820 \times 10^{-14}$ & $1.7492 \times 10^{-07}$ & $2.8295 \times 10^{-07}$ \\
4 & 200.0 & $1.7029 \times 10^{-17}$ & $3.4507 \times 10^{-12}$ & $2.2157 \times 10^{-13}$ & $3.4656 \times 10^{-07}$ & $5.5850 \times 10^{-07}$ \\
5 & 300.0 & $3.7087 \times 10^{-17}$ & $8.2208 \times 10^{-12}$ & $3.9943 \times 10^{-13}$ & $5.1476 \times 10^{-07}$ & $8.2726 \times 10^{-07}$ \\
6 & 400.0 & $6.4074 \times 10^{-17}$ & $1.4958 \times 10^{-11}$ & $6.1639 \times 10^{-13}$ & $6.7990 \times 10^{-07}$ & $1.0899 \times 10^{-06}$ \\
7 & 500.0 & $9.7575 \times 10^{-17}$ & $2.3572 \times 10^{-11}$ & $8.6886 \times 10^{-13}$ & $8.4217 \times 10^{-07}$ & $1.3466 \times 10^{-06}$ \\
8 & 600.0 & $1.3724 \times 10^{-16}$ & $3.3987 \times 10^{-11}$ & $1.1539 \times 10^{-12}$ & $1.0018 \times 10^{-06}$ & $1.5981 \times 10^{-06}$ \\
9 & 700.0 & $1.8281 \times 10^{-16}$ & $4.6136 \times 10^{-11}$ & $1.4690 \times 10^{-12}$ & $1.1591 \times 10^{-06}$ & $1.8445 \times 10^{-06}$ \\
10 & 800.0 & $2.3399 \times 10^{-16}$ & $5.9964 \times 10^{-11}$ & $1.8117 \times 10^{-12}$ & $1.3141 \times 10^{-06}$ & $2.0861 \times 10^{-06}$ \\
11 & 900.0 & $2.9058 \times 10^{-16}$ & $7.5424 \times 10^{-11}$ & $2.1802 \times 10^{-12}$ & $1.4670 \times 10^{-06}$ & $2.3233 \times 10^{-06}$ \\
12 & 1000.0 & $3.5241 \times 10^{-16}$ & $9.2473 \times 10^{-11}$ & $2.5726 \times 10^{-12}$ & $1.6179 \times 10^{-06}$ & $2.5562 \times 10^{-06}$ \\
13 & 1100.0 & $4.1928 \times 10^{-16}$ & $1.1108 \times 10^{-10}$ & $2.9876 \times 10^{-12}$ & $1.7669 \times 10^{-06}$ & $2.7851 \times 10^{-06}$ \\
14 & 1200.0 & $4.9108 \times 10^{-16}$ & $1.3120 \times 10^{-10}$ & $3.4236 \times 10^{-12}$ & $1.9143 \times 10^{-06}$ & $3.0102 \times 10^{-06}$ \\
15 & 1300.0 & $5.6764 \times 10^{-16}$ & $1.5282 \times 10^{-10}$ & $3.8793 \times 10^{-12}$ & $2.0599 \times 10^{-06}$ & $3.2316 \times 10^{-06}$ \\
\hline
\end{tabular}


Table 3.38. Barium isotope concentrations for fuel channels $\mathrm{P}-\mathrm{S}$ of the reference solution of Problem 2.2.1.

\begin{tabular}{rrccccc}
\hline Step & $\begin{array}{c}\text { Burnup } \\
{\left[\frac{\mathrm{MWd}}{\mathrm{MtU}}\right]}\end{array}$ & $\begin{array}{c}{ }^{132} \mathrm{Ba} \\
{\left[\frac{\mathrm{atoms}}{\mathrm{b}-\mathrm{cm}}\right]}\end{array}$ & $\begin{array}{c}{ }^{134} \mathrm{Ba} \\
{\left[\frac{\mathrm{atoms}}{\mathrm{b}-\mathrm{cm}}\right]}\end{array}$ & $\begin{array}{c}{ }^{135} \mathrm{Ba} \\
{\left[\frac{\mathrm{atoms}}{\mathrm{b}-\mathrm{cm}}\right]}\end{array}$ & $\begin{array}{c}{ }^{136} \mathrm{Ba} \\
{\left[\frac{\mathrm{atoms}}{\mathrm{b}-\mathrm{cm}}\right]}\end{array}$ & $\begin{array}{c}{ }^{138} \mathrm{Ba} \\
{\left[\frac{\mathrm{atoms}}{\mathrm{b}-\mathrm{cm}}\right]}\end{array}$ \\
\hline 0 & 0.0 & $0.0000 \times 10^{+00}$ & $0.0000 \times 10^{+00}$ & $0.0000 \times 10^{+00}$ & $0.0000 \times 10^{+00}$ & $0.0000 \times 10^{+00}$ \\
1 & 25.0 & $0.0000 \times 10^{+00}$ & $2.3726 \times 10^{-14}$ & $1.7187 \times 10^{-15}$ & $3.5680 \times 10^{-11}$ & $8.5094 \times 10^{-08}$ \\
2 & 50.0 & $0.0000 \times 10^{+00}$ & $3.1771 \times 10^{-13}$ & $5.4544 \times 10^{-15}$ & $1.0009 \times 10^{-10}$ & $1.7034 \times 10^{-07}$ \\
3 & 100.0 & $0.0000 \times 10^{+00}$ & $3.5071 \times 10^{-12}$ & $1.8960 \times 10^{-14}$ & $2.8699 \times 10^{-10}$ & $3.4057 \times 10^{-07}$ \\
4 & 200.0 & $0.0000 \times 10^{+00}$ & $3.3011 \times 10^{-11}$ & $7.0013 \times 10^{-14}$ & $8.3942 \times 10^{-10}$ & $6.8002 \times 10^{-07}$ \\
5 & 300.0 & $0.0000 \times 10^{+00}$ & $1.1601 \times 10^{-10}$ & $1.5433 \times 10^{-13}$ & $1.6022 \times 10^{-09}$ & $1.0182 \times 10^{-06}$ \\
6 & 400.0 & $0.0000 \times 10^{+00}$ & $2.7746 \times 10^{-10}$ & $2.7531 \times 10^{-13}$ & $2.5594 \times 10^{-09}$ & $1.3553 \times 10^{-06}$ \\
7 & 500.0 & $0.0000 \times 10^{+00}$ & $5.3999 \times 10^{-10}$ & $4.3816 \times 10^{-13}$ & $3.6981 \times 10^{-09}$ & $1.6914 \times 10^{-06}$ \\
8 & 600.0 & $0.0000 \times 10^{+00}$ & $9.2421 \times 10^{-10}$ & $6.4978 \times 10^{-13}$ & $5.0075 \times 10^{-09}$ & $2.0265 \times 10^{-06}$ \\
9 & 700.0 & $0.0000 \times 10^{+00}$ & $1.4489 \times 10^{-09}$ & $9.1840 \times 10^{-13}$ & $6.4783 \times 10^{-09}$ & $2.3607 \times 10^{-06}$ \\
10 & 800.0 & $0.0000 \times 10^{+00}$ & $2.1313 \times 10^{-09}$ & $1.2535 \times 10^{-12}$ & $8.1021 \times 10^{-09}$ & $2.6940 \times 10^{-06}$ \\
11 & 900.0 & $0.0000 \times 10^{+00}$ & $2.9871 \times 10^{-09}$ & $1.6658 \times 10^{-12}$ & $9.8726 \times 10^{-09}$ & $3.0266 \times 10^{-06}$ \\
12 & 1000.0 & $0.0000 \times 10^{+00}$ & $4.0308 \times 10^{-09}$ & $2.1668 \times 10^{-12}$ & $1.1783 \times 10^{-08}$ & $3.3584 \times 10^{-06}$ \\
13 & 1100.0 & $0.0000 \times 10^{+00}$ & $5.2757 \times 10^{-09}$ & $2.7691 \times 10^{-12}$ & $1.3828 \times 10^{-08}$ & $3.6895 \times 10^{-06}$ \\
14 & 1200.0 & $0.0000 \times 10^{+00}$ & $6.7342 \times 10^{-09}$ & $3.4860 \times 10^{-12}$ & $1.6003 \times 10^{-08}$ & $4.0200 \times 10^{-06}$ \\
15 & 1300.0 & $0.0000 \times 10^{+00}$ & $8.4175 \times 10^{-09}$ & $4.3314 \times 10^{-12}$ & $1.8304 \times 10^{-08}$ & $4.3498 \times 10^{-06}$ \\
\hline
\end{tabular}

Table 3.39. Technetium isotope concentrations for fuel channels $\mathrm{P}-\mathrm{S}$ of the reference solution of Problem 2.2.1.

\begin{tabular}{rrc}
\hline Step & $\begin{array}{c}\text { Burnup } \\
{\left[\frac{\mathrm{MWd}}{\mathrm{MtU}}\right]}\end{array}$ & $\begin{array}{c}{ }^{99} \mathrm{Tc} \\
{\left[\frac{\mathrm{atoms}}{\mathrm{b}-\mathrm{cm}}\right]}\end{array}$ \\
\hline 0 & 0.0 & $0.0000 \times 10^{+00}$ \\
1 & 25.0 & $5.7768 \times 10^{-08}$ \\
2 & 50.0 & $1.3525 \times 10^{-07}$ \\
3 & 100.0 & $2.9078 \times 10^{-07}$ \\
4 & 200.0 & $6.0145 \times 10^{-07}$ \\
5 & 300.0 & $9.1161 \times 10^{-07}$ \\
6 & 400.0 & $1.2214 \times 10^{-06}$ \\
7 & 500.0 & $1.5306 \times 10^{-06}$ \\
8 & 600.0 & $1.8394 \times 10^{-06}$ \\
9 & 700.0 & $2.1478 \times 10^{-06}$ \\
10 & 800.0 & $2.4556 \times 10^{-06}$ \\
11 & 900.0 & $2.7631 \times 10^{-06}$ \\
12 & 1000.0 & $3.0701 \times 10^{-06}$ \\
13 & 1100.0 & $3.3766 \times 10^{-06}$ \\
14 & 1200.0 & $3.6828 \times 10^{-06}$ \\
15 & 1300.0 & $3.9886 \times 10^{-06}$ \\
\hline
\end{tabular}


Table 3.40. Rubidium isotope concentrations for fuel channels $\mathrm{P}-\mathrm{S}$ of the reference solution of Problem 2.2.1.

\begin{tabular}{rrcc}
\hline Step & $\begin{array}{c}\text { Burnup } \\
{\left[\frac{\mathrm{MWd}}{\mathrm{MtU}}\right]}\end{array}$ & $\begin{array}{c}{ }^{85} \mathrm{Rb} \\
{\left[\frac{\text { atoms }}{\mathrm{b}-\mathrm{cm}}\right]}\end{array}$ & $\begin{array}{c}87 \mathrm{Rb} \\
{\left[\frac{\mathrm{atoms}}{\mathrm{b}-\mathrm{cm}}\right]}\end{array}$ \\
\hline 0 & 0.0 & $0.0000 \times 10^{+00}$ & $0.0000 \times 10^{+00}$ \\
1 & 25.0 & $1.2567 \times 10^{-08}$ & $3.1692 \times 10^{-08}$ \\
2 & 50.0 & $2.5290 \times 10^{-08}$ & $6.3362 \times 10^{-08}$ \\
3 & 100.0 & $5.0571 \times 10^{-08}$ & $1.2618 \times 10^{-07}$ \\
4 & 200.0 & $1.0051 \times 10^{-07}$ & $2.4988 \times 10^{-07}$ \\
5 & 300.0 & $1.4970 \times 10^{-07}$ & $3.7117 \times 10^{-07}$ \\
6 & 400.0 & $1.9823 \times 10^{-07}$ & $4.9033 \times 10^{-07}$ \\
7 & 500.0 & $2.4615 \times 10^{-07}$ & $6.0750 \times 10^{-07}$ \\
8 & 600.0 & $2.9352 \times 10^{-07}$ & $7.2285 \times 10^{-07}$ \\
9 & 700.0 & $3.4038 \times 10^{-07}$ & $8.3651 \times 10^{-07}$ \\
10 & 800.0 & $3.8678 \times 10^{-07}$ & $9.4858 \times 10^{-07}$ \\
11 & 900.0 & $4.3276 \times 10^{-07}$ & $1.0592 \times 10^{-06}$ \\
12 & 1000.0 & $4.7834 \times 10^{-07}$ & $1.1685 \times 10^{-06}$ \\
13 & 1100.0 & $5.2355 \times 10^{-07}$ & $1.2764 \times 10^{-06}$ \\
14 & 1200.0 & $5.6843 \times 10^{-07}$ & $1.3832 \times 10^{-06}$ \\
15 & 1300.0 & $6.1298 \times 10^{-07}$ & $1.4887 \times 10^{-06}$ \\
\hline
\end{tabular}





\section{PROBLEM 3: 2D CORE}

Problem 3 is a 2D transverse slice of a Magnox core and radial reflector region. The core contains three regions, each with its own coolant channel radius. This problem uses fully reflected boundary conditions on the west and south (internal) faces, and vacuum (zero-return) boundary conditions on the north and east (external) faces.

Problem 3 is more complex than the previous problems because it adds multiple charge pans, multiple coolant channel zones, the peripheral region with the radial reflector, and strong absorbers in the control rods.

\subsection{BEGINNING-OF-CYCLE}

\subsubsection{Problem 3.1.1}

\subsubsection{Description}

Figure 6 shows the quarter core geometry for Problem 3.1.1, but the geometry is $1 / 8$ symmetric and may be modeled as such. The diagonal dashed line illustrates an axis of reflected symmetry. The core is composed of three zones (A, B, and C), where each zone represents the coolant channel radius of each rod in the charge-pan as noted in Table 1.2. Zone A comprises the following charge-pans: 0727, 0728, 0827, and 0828. Zone B comprises the following charge-pans: 0729, 0730, 0829, 0830, 0927, 0928, 0929, 1027, and 1028. Zone C comprises the following charge-pans: 0721, 0732, 0831, 0832, 0930, 0931, 0932, 1029, $1030,1031,1127,1128,1129,1130,1227$, and 1228. Coolant channel radii for each zone and other geometric dimensions are listed in Table 1.2. Problem 3.1.1 models all rods out (ARO), and the empty coolant channels are shown in blue in Figure 6. Material densities are listed in Table 1.4. Temperatures are listed in Table 2.4 .

\subsubsection{Reference Solution}

Table 4.1 shows the neutron multiplication factor comparison for Shift and MPACT. Timing variables are shown in Table 4.2. The power peaking factors and their difference are shown in Figures 7-8. The power peaking factors are tabulated in Tables 4.3-4.4. The power peaking factors agree well, within 0.005 absolute difference between MPACT and Shift.

Table 4.1. Neutron multiplication for the reference solution of Problem 3.1.1.

\begin{tabular}{rrccr}
\hline Step & $\begin{array}{c}\text { Burnup } \\
{\left[\frac{\mathrm{MWd}}{\mathrm{MtU}}\right]}\end{array}$ & $\begin{array}{c}\text { Shift } \\
\mathrm{k}_{\mathrm{eff}}\end{array}(1 \sigma)$ & $\begin{array}{c}\text { MPACT } \\
\mathrm{k}_{\mathrm{eff}}(1 \sigma)\end{array}$ & $\begin{array}{c}\text { Difference } \\
{[\mathrm{pcm}]}\end{array}$ \\
\hline 0 & 0.0 & $1.05021(3)$ & 1.05028 & $7(3)$ \\
\hline
\end{tabular}




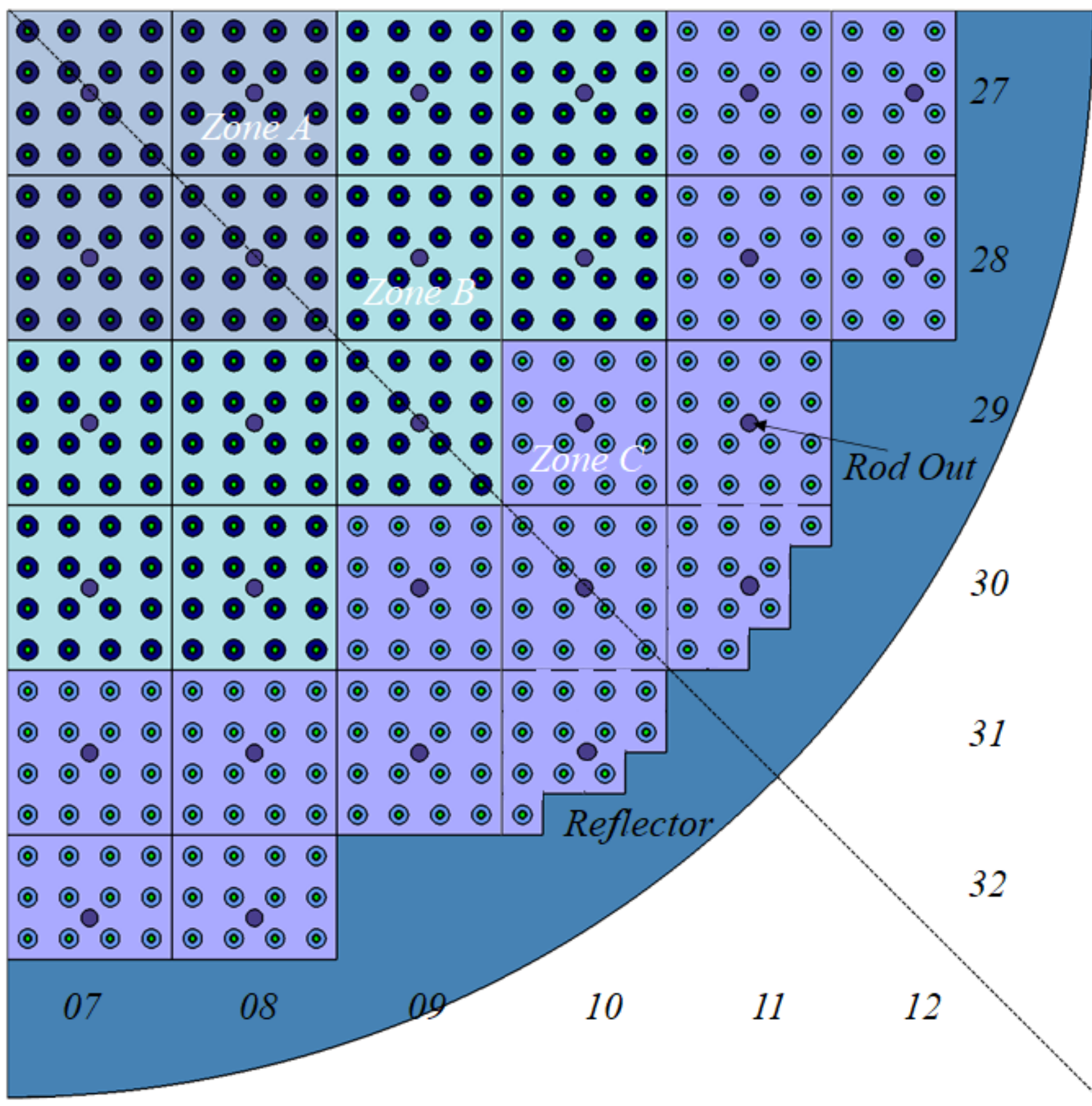

Figure 6. Quarter core 2D with all rods out (ARO). 
pin_powers: exposure 0 


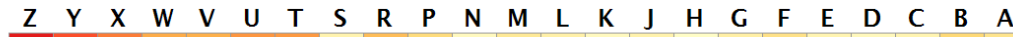

0.00613

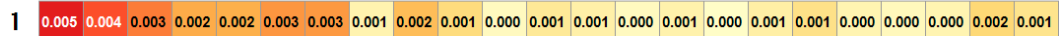

$2 \begin{array}{llllllllllllllllllllllllllll}2 & 0.004 & 0.003 & 0.003 & 0.003 & 0.004 & 0.000 & 0.001 & 0.002 & 0.003 & 0.000 & 0.000 & 0.001 & 0.001 & 0.001 & 0.001 & 0.000 & 0.001 & 0.000 & 0.002 & 0.000 & 0.000 & 0.001 & 0.001\end{array}$

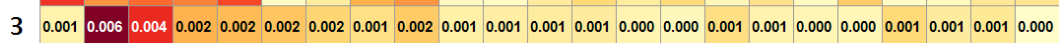

$\begin{array}{llllllllllllllllllllllll}4 & 0.004 & 0.005 & 0.003 & 0.004 & 0.003 & 0.000 & 0.000 & 0.001 & 0.001 & 0.002 & 0.000 & 0.002 & 0.002 & 0.002 & 0.001 & 0.000 & 0.001 & 0.000 & 0.000 & 0.000 & 0.002 & 0.001 & 0.000\end{array}$

0.006

0.005

0.004

$\begin{array}{llllllllllllllllllllllllllllll}5 & 0.001 & 0.003 & 0.003 & 0.003 & 0.003 & 0.001 & 0.002 & 0.001 & 0.000 & 0.000 & 0.001 & 0.001 & 0.000 & 0.000 & 0.001 & 0.001 & 0.000 & 0.000 & 0.000 & 0.000 & 0.001 & 0.001 & 0.001\end{array}$

0.003

$\begin{array}{lllllllllllllllllllllllllllllllll}6 & 0.002 & 0.005 & 0.003 & 0.002 & 0.001 & 0.001 & 0.000 & 0.001 & 0.001 & 0.001 & 0.003 & 0.002 & 0.002 & 0.001 & 0.001 & 0.001 & 0.000 & 0.001 & 0.001 & 0.001 & 0.000 & 0.001 & 0.000\end{array}$

0.002

$7 \begin{array}{lllllllllllllllllllllllllllllllll}7 & 0.004 & 0.001 & 0.003 & 0.003 & 0.002 & 0.001 & 0.000 & 0.001 & 0.002 & 0.000 & 0.001 & 0.004 & 0.003 & 0.001 & 0.001 & 0.000 & 0.001 & 0.000 & 0.000 & 0.001 & 0.000 & 0.000 & 0.001\end{array}$

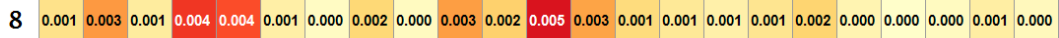

$\begin{array}{lllllllllllllllllllllllllllll}9 & 0.001 & 0.002 & 0.002 & 0.002 & 0.001 & 0.000 & 0.001 & 0.000 & 0.001 & 0.001 & 0.002 & 0.002 & 0.001 & 0.001 & 0.001 & 0.001 & 0.001 & 0.001 & 0.000 & 0.000\end{array}$

$\begin{array}{llllllllllllllllllllllllll}10 & 0.003 & 0.003 & 0.001 & 0.003 & 0.000 & 0.001 & 0.001 & 0.000 & 0.000 & 0.001 & 0.002 & 0.002 & 0.002 & 0.003 & 0.002 & 0.001 & 0.001 & 0.001 & 0.000 & 0.001\end{array}$

0.001

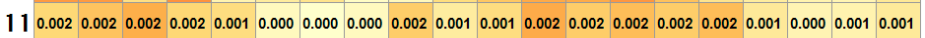

$\begin{array}{lllllllllllllllllllll}12 & 0.002 & 0.003 & 0.002 & 0.001 & 0.001 & 0.000 & 0.000 & 0.001 & 0.000 & 0.000 & 0.000 & 0.001 & 0.002 & 0.001 & 0.001 & 0.002 & 0.002 & 0.002 & 0.001 & 0.001\end{array}$

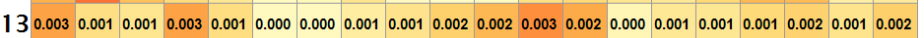

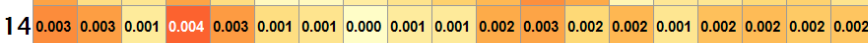

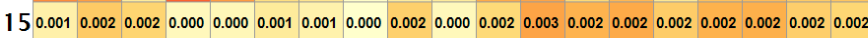

\begin{tabular}{ll|l|l|l|l|l|l|l|l|l|l|l|l|l|l|l|l|l|l|l}
16 & 0.002 & 0.002 & 0.001 & 0.000 & 0.000 & 0.001 & 0.000 & 0.002 & 0.001 & 0.000 & 0.002 & 0.002 & 0.003 & 0.002 & 0.001 & 0.001 & 0.000 & 0.001
\end{tabular}

$\begin{array}{llllllllllllllllll}170.001 & 0.000 & 0.001 & 0.001 & 0.001 & 0.000 & 0.000 & 0.000 & 0.001 & 0.001 & 0.000 & 0.002 & 0.002 & 0.002 & 0.002 & 0.002\end{array}$

$\begin{array}{lllllllllllllllll}18 & 0.001 & 0.000 & 0.000 & 0.001 & 0.002 & 0.001 & 0.001 & 0.001 & 0.001 & 0.001 & 0.001 & 0.001 & 0.002 & 0.002 & 0.001 & 0.002\end{array}$

$\begin{array}{llllllllllllllll}19 & 0.001 & 0.002 & 0.001 & 0.001 & 0.001 & 0.001 & 0.000 & 0.001 & 0.001 & 0.001 & 0.000 & 0.001 & 0.001 & 0.001 & 0.002\end{array}$

$200.0000 .0000 .0010 .0010 .003 \quad 0.0020 .0010 .0010 .0000 .0000 .0000 .0010 .001$

210.0000 .0010 .0010 .0010 .0010 .0010 .0010 .000

220.0000 .0000 .0010 .0000 .0000 .0010 .0010 .001

230.0010 .0000 .0000 .0010 .0010 .0000 .0010 .001

pin_powers: exposure 0

Figure 8. Absolute difference between Shift and MPACT power peaking factors for Problem 3.1.1 
Table 4.2. Timing variables for the reference solution of Problem 3.1.1

\begin{tabular}{|c|c|c|c|c|c|c|}
\hline \multicolumn{7}{|c|}{ Shift } \\
\hline $\begin{array}{c}\text { Depletion } \\
\text { Solves }\end{array}$ & $\begin{array}{c}\text { Total } \\
\text { Cycles per } \\
\text { Transport } \\
\text { Solve } \\
\end{array}$ & $\begin{array}{c}\text { Active } \\
\text { Cycles per } \\
\text { Transport } \\
\text { Solve }\end{array}$ & $\begin{array}{l}\text { Histories } \\
\text { per Cycle }\end{array}$ & $\begin{array}{c}\text { Compute } \\
\text { Nodes }\end{array}$ & $\begin{array}{c}\text { Cores } \\
\text { per Node }\end{array}$ & $\begin{array}{c}\text { Wall } \\
\text { Total Time } \\
\text { [hours] }\end{array}$ \\
\hline 1 & 1500 & 1200 & 500000 & 2 & 48 & 9.64 \\
\hline \multicolumn{7}{|c|}{ MPACT } \\
\hline $\begin{array}{c}\text { Depletion } \\
\text { Solves }\end{array}$ & $\begin{array}{c}k_{\mathrm{eff}} \\
\text { Convergence } \\
\text { Criteria }\end{array}$ & $\begin{array}{c}\text { Source } \\
\text { Convergence } \\
\text { Criteria }\end{array}$ & $\begin{array}{c}\text { Ray } \\
\text { Spacing } \\
{[\mathrm{cm}]}\end{array}$ & $\begin{array}{c}\text { Compute } \\
\text { Nodes }\end{array}$ & $\begin{array}{c}\text { Cores } \\
\text { per Node }\end{array}$ & $\begin{array}{c}\text { Wall } \\
\text { Total Time } \\
\text { [hours] }\end{array}$ \\
\hline 1 & $1.0 \times 10^{-04}$ & $1.0 \times 10^{-04}$ & 0.01 & 3 & 128 & 5.30 \\
\hline
\end{tabular}


Table 4.3. Power peaking factors A-H and P-S of the reference solution of Problem 3.1.1.

\begin{tabular}{|c|c|c|c|c|c|c|c|c|}
\hline \multirow[t]{2}{*}{ Charge Pans } & \multicolumn{8}{|c|}{ Shift Power Peaking Factors } \\
\hline & $\begin{array}{c}\text { A } \\
\text { (A) }\end{array}$ & $\begin{array}{c}\text { B } \\
\text { (D) }\end{array}$ & $\begin{array}{c}\mathrm{C} \\
\text { (C) }\end{array}$ & $\begin{array}{c}\mathrm{D} \\
\text { (B) }\end{array}$ & $\begin{array}{c}\mathrm{P} \\
(\mathrm{R})\end{array}$ & $\begin{array}{c}\mathrm{Q} \\
(\mathrm{Q})\end{array}$ & $\begin{array}{c}\mathrm{R} \\
\text { (P) }\end{array}$ & $\begin{array}{c}S \\
(S)\end{array}$ \\
\hline 0727 & 1632 & 1.621 & 1.629 & 1.643 & 1.672 & 1.642 & 1.608 & 1.640 \\
\hline 327 (0728) & & & 1.559 & 1.571 & 1.621 & & 502 & \\
\hline 729) & & 1.371 & 1.422 & 1.431 & 1.496 & 44 & 13 & 1.465 \\
\hline 730) & & & 1.178 & & 1.279 & & & \\
\hline 31) & & 0 & 0.878 & & 0.988 & .720 & 91 & 0.958 \\
\hline 32) & & 0.4 & 0.512 & 21 & 0.625 & - & - & 0.594 \\
\hline 0828 & & & 1.467 & & 1.567 & 80 & 92 & 477 \\
\hline 0829) & & 1.2 & 1.326 & 1.356 & 1.444 & 1.289 & .202 & 1.350 \\
\hline & & & & & & & & \\
\hline 31) & & & 0.788 & & 0.937 & 0.671 & 0.601 & 0.847 \\
\hline 32) & & & 35 & & 0.576 & . & - & 537 \\
\hline 0929 & & & 51 & & 0 & & & 59 \\
\hline 1029) & & & 0.933 & & 1. & & 42 & 0.952 \\
\hline 31) & & & 0 & & & & & 63 \\
\hline 1030 & & & 0.687 & & 3 & & 0.505 & 0.684 \\
\hline 1130) & & & 0.425 & 0.469 & 0.606 & 0.424 & - & 0.461 \\
\hline \multirow[t]{2}{*}{ Charge Pans } & \multicolumn{8}{|c|}{ MPACT Power Peaking Factors } \\
\hline & $\begin{array}{c}\text { A } \\
\text { (A) }\end{array}$ & B & $\begin{array}{c}\mathrm{C} \\
\text { (C) }\end{array}$ & $\begin{array}{l}\text { D } \\
\text { (B) }\end{array}$ & $\begin{array}{c}\mathrm{P} \\
\text { (R) }\end{array}$ & $\begin{array}{c}\mathrm{Q} \\
(\mathrm{Q})\end{array}$ & $\begin{array}{c}\mathrm{R} \\
(\mathrm{P})\end{array}$ & $\begin{array}{c}\mathrm{S} \\
(\mathrm{S})\end{array}$ \\
\hline 0727 & 1.635 & 40 & 1.635 & 1.646 & 1.676 & 1.644 & 1.612 & 1.644 \\
\hline 0728) & & & 1. & & 23 & & 3 & 1.591 \\
\hline 29) & & & 21 & & 9 & & & 1.466 \\
\hline & & & & & & & 36 & 1.246 \\
\hline 31) & 0 & 0 & 0.878 & & 39 & 21 & 91 & 958 \\
\hline 32) & & & 0.513 & & 0.624 & & - & 0.596 \\
\hline & & & 1.469 & & 1.569 & & & 1.481 \\
\hline $0928(0829)$ & & & 1.326 & & 1.444 & & 1.197 & 1.350 \\
\hline & & & 1.088 & & 1.225 & & 0.927 & 1.137 \\
\hline 11 & 0. & 0.70 & 0.787 & 0. & 0.937 & 0.671 & 0.601 & 0.846 \\
\hline 0832) & & & 0.436 & & 0.576 & - & - & 0.537 \\
\hline & & & & & 1.3 & & & 59 \\
\hline 1029) & & 0 & 0.931 & & 1.099 & 0.887 & 0.740 & 0.950 \\
\hline 11 & & & 0.623 & & 0.807 & 96 & 0.461 & 0.661 \\
\hline & & & & & 0.890 & 0.682 & 0.504 & 0.682 \\
\hline $1031(1130)$ & 0.422 & 0.389 & 0.424 & 0.467 & 0.604 & 0.423 & - & 0.461 \\
\hline
\end{tabular}


Table 4.4. Power peaking factors E-M of the reference solution of Problem 3.1.1.

\begin{tabular}{|c|c|c|c|c|c|c|c|c|}
\hline \multirow[t]{2}{*}{ Charge Pans } & \multicolumn{8}{|c|}{ Shift Power Peaking Factors } \\
\hline & $\begin{array}{c}E \\
(H)\end{array}$ & $\begin{array}{c}\mathrm{F} \\
(\mathrm{G})\end{array}$ & $\begin{array}{c}\mathrm{G} \\
(\mathrm{F})\end{array}$ & $\begin{array}{c}\mathrm{H} \\
(\mathrm{E})\end{array}$ & $\begin{array}{c}\mathrm{J} \\
(\mathrm{M})\end{array}$ & $\begin{array}{c}\mathrm{K} \\
(\mathrm{L})\end{array}$ & $\begin{array}{c}\mathrm{L} \\
(\mathrm{K})\end{array}$ & $\begin{array}{l}M \\
(J)\end{array}$ \\
\hline 0727 & 1.664 & 1.654 & 1.633 & 1.623 & 1.622 & 1.631 & 1.657 & .664 \\
\hline 327 (0728) & 91 & .561 & 1.525 & 15 & 1.531 & .561 & & .610 \\
\hline 29) & 51 & 1.400 & 1.336 & 1.326 & 1.368 & 18 & & 1.487 \\
\hline 730) & & & 1.061 & & 1.107 & & & 1.272 \\
\hline 31) & & & 0.715 & 04 & 0.784 & 73 & & 0.981 \\
\hline 32) & & 0.478 & & - & 0.450 & & & 0.618 \\
\hline 0828 & & & 1.452 & 22 & 1.421 & & & 539 \\
\hline 0829) & 98 & 1.347 & 1.262 & 1.234 & 1.253 & .306 & & 1.416 \\
\hline & & & 0.988 & & & & & 199 \\
\hline 31) & & & 0.646 & 18 & 0.676 & & & 0.910 \\
\hline 32) & & & - & 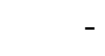 & 0.369 & & & 551 \\
\hline 0929 & & & 15 & & .062 & & & 261 \\
\hline 1029) & & & 0.844 & & 0.815 & & & 1.056 \\
\hline 31) & & & 0.562 & & & & & 762 \\
\hline 1030 & & & 0.622 & & 0.560 & & & 0.825 \\
\hline 1130) & & & & 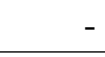 & - & 11 & & .547 \\
\hline \multirow[t]{2}{*}{ Charge Pans } & \multicolumn{8}{|c|}{ MPACT Power Peaking Factors } \\
\hline & $\begin{array}{c}\mathrm{E} \\
(\mathrm{H})\end{array}$ & $\mathrm{F}$ & $\begin{array}{l}\text { G } \\
(F)\end{array}$ & $\begin{array}{l}\mathrm{H} \\
\text { (E) }\end{array}$ & $\begin{array}{c}\mathrm{J} \\
(\mathrm{M})\end{array}$ & $\begin{array}{c}\mathrm{K} \\
(\mathrm{L})\end{array}$ & $\begin{array}{c}\mathrm{L} \\
(\mathrm{K})\end{array}$ & $\begin{array}{l}M \\
(J)\end{array}$ \\
\hline 0727 & 1.668 & 657 & 1.636 & 625 & 1.625 & 1.636 & 657 & 1.668 \\
\hline 0728) & & & 27 & & 1. & 51 & & 1.614 \\
\hline 29) & & & 36 & & 57 & & & 1.490 \\
\hline & & & 1.060 & & & & & 1.271 \\
\hline 31) & 4 & 4 & 0.715 & 05 & 0.783 & & 72 & 982 \\
\hline 32) & & & - & 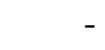 & 0.451 & & & 0.618 \\
\hline & & & 1.452 & & & & & 1.540 \\
\hline 0829) & & & 1.261 & & 52 & & & 1.415 \\
\hline & & & 0.987 & & 0.998 & & & 1.197 \\
\hline 11 & 0.852 & 0.763 & 0.646 & 0.619 & 0.677 & 61 & 79 & 0.909 \\
\hline 0832) & & 0.431 & & - & 0.369 & 0.450 & 0.529 & 0.551 \\
\hline & & & & & 1. & & & 1.261 \\
\hline 1029) & 1.032 & 0.961 & 0.843 & 0.793 & 0.814 & & 1.004 & 1.054 \\
\hline (093 & & & 0.563 & 0.513 & 0.503 & 79 & 0.712 & 0.761 \\
\hline & & & 0.620 & 0.559 & 0.559 & 0.620 & 0.754 & 0.823 \\
\hline $1031(1130)$ & 0.526 & 0.459 & 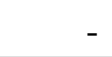 & - & 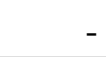 & 0.411 & 0.490 & 0.545 \\
\hline
\end{tabular}




\subsubsection{Problem 3.1.2}

\subsubsection{Description}

Figure 9 shows the quarter core geometry for Problem 3.1.2, which is the same as Problem 3.1.1 except Problem 3.1.2 models all rods in (ARI). The goal of this problem is to test the effect of control rods. As boron was not inserted in the graphite as an impurity material, this is the first problem with inserted neutron poisons aside from fission products. The geometry remains $1 / 8$ symmetric. The diagonal dashed line illustrates an axis of reflected symmetry. The following charge-pans are uncontrolled (no control rod present): 0731, 0732, 0830, 0832, 0931, 1028, 1031, 1127, 1129, 1130, 1227, and 1228. Material densities are listed in Table 1.4. Temperatures are listed in Table 10.

\subsubsection{Reference Solution}

Table 4.5 shows the neutron multiplication factor comparison for Shift and MPACT. Timing variables are shown in Table 4.6. The power peaking factors and their difference are shown in Figures 10-11. Note that they are flipped along the x-axis. The power peaking factors are tabulated in Tables 4.7-4.8. The power peaking factors agree well, within 0.007 absolute difference between MPACT and Shift. The largest differences can be seen near the control rod locations and radial edges of the core.

Table 4.5. Neutron multiplication for the reference solution of Problem 3.1.2.

\begin{tabular}{rrccc}
\hline Step & $\begin{array}{c}\text { Burnup } \\
{\left[\frac{\mathrm{MWd}}{\mathrm{MtU}}\right]}\end{array}$ & $\begin{array}{c}\text { Shift } \\
\mathrm{k}_{\mathrm{eff}}(1 \sigma)\end{array}$ & $\begin{array}{c}\text { MPACT } \\
\mathrm{k}_{\mathrm{eff}}(1 \sigma)\end{array}$ & $\begin{array}{c}\text { Difference } \\
{[\mathrm{pcm}]}\end{array}$ \\
\hline 0 & 0.0 & $0.98085(3)$ & 0.98024 & $-61(3)$ \\
\hline
\end{tabular}

Table 4.6. Timing variables for the reference solution of Problem 3.1.2

\begin{tabular}{ccccccc}
\hline \hline \multicolumn{2}{c}{ Shift } & & & \\
\hline $\begin{array}{c}\text { Depletion } \\
\text { Solves }\end{array}$ & $\begin{array}{c}\text { Total } \\
\text { Cycles per } \\
\text { Transport } \\
\text { Solve }\end{array}$ & $\begin{array}{c}\text { Active } \\
\text { Cycles per } \\
\text { Transport } \\
\text { Solve }\end{array}$ & $\begin{array}{c}\text { Histories } \\
\text { per Cycle }\end{array}$ & $\begin{array}{c}\text { Compute } \\
\text { Nodes }\end{array}$ & $\begin{array}{c}\text { Cores } \\
\text { per Node }\end{array}$ & $\begin{array}{c}\text { Wall } \\
\text { Total Time }\end{array}$ \\
\hline 1 & 1500 & 1200 & 500000 & 4 & 48 & 4.99 \\
\hline $\begin{array}{c}\text { Depletion } \\
\text { Solves }\end{array}$ & $\begin{array}{c}k_{\text {eff }} \\
\text { Convergence } \\
\text { Criteria }\end{array}$ & $\begin{array}{c}\text { Sonvergence } \\
\text { Criteria }\end{array}$ & $\begin{array}{c}\text { Spacing } \\
{[\mathrm{cm}]}\end{array}$ & Nodes & per Node & $\begin{array}{c}\text { Total Time } \\
\text { [hours] }\end{array}$ \\
\hline 1 & $1.0 \times 10^{-05}$ & $1.0 \times 10^{-05}$ & 0.03 & 6 & 32 & 0.87 \\
\hline
\end{tabular}




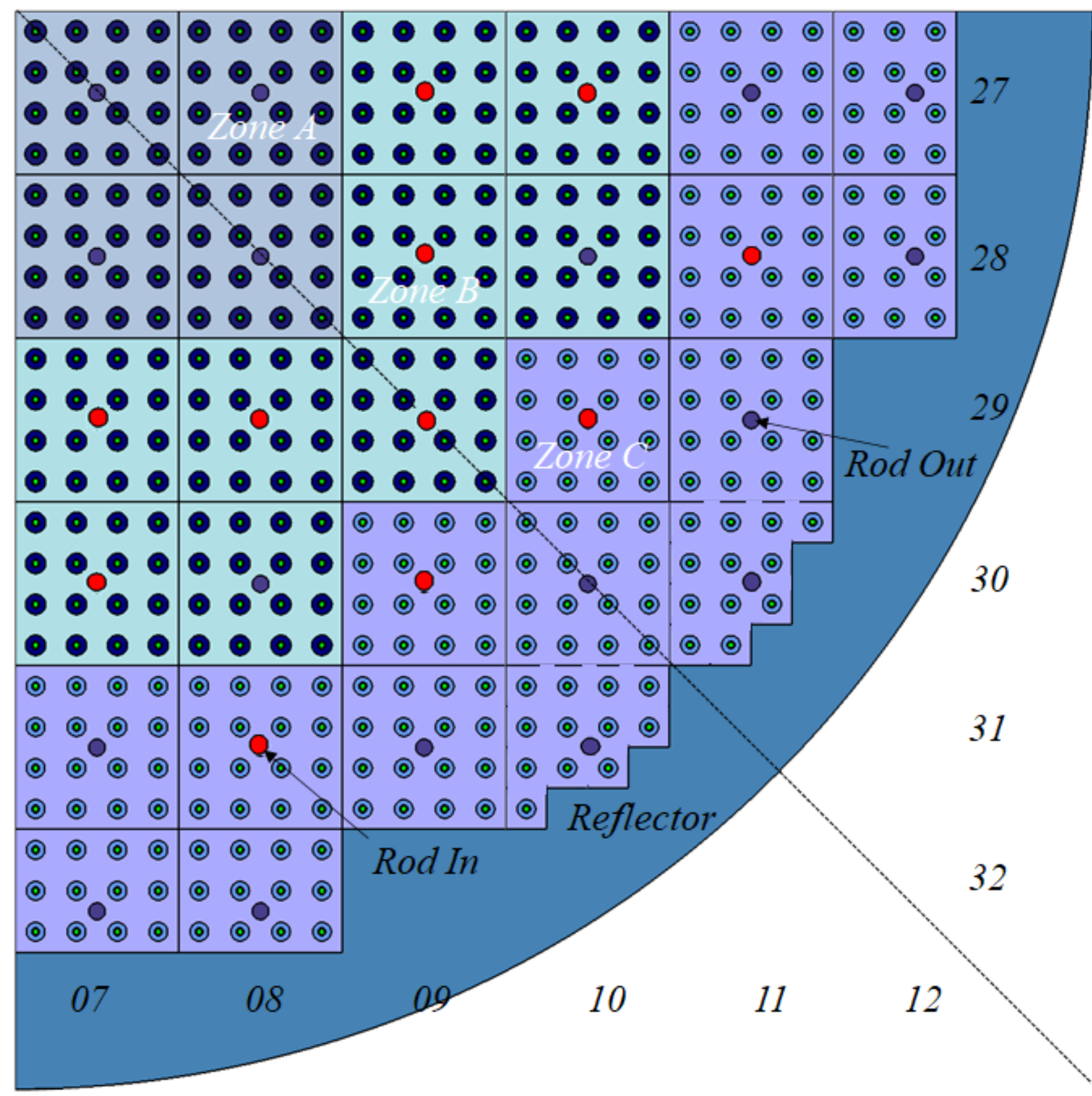

Figure 9. Quarter core 2D with all rods in (ARI), where red denotes the charge-pans with control rods inserted. 


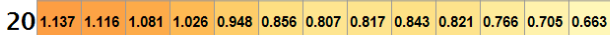

211.0451 .0270 .9940 .9480 .8860 .8190 .7710 .786

$220.9310 .9070 .8810 .8490 .795 \quad 0.7290 .6810 .695$

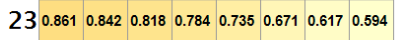

\section{pin_powers: exposure 0}

$\begin{array}{lllllllllllllllllllllll}Z & Y & X & W & V & U & T & S & R & P & N & M & L & K & J & H & G & F & E & D & C & B & A\end{array}$

11.8241 .7841 .7131 .6051 .4551 .2871 .1851 .1511 .0991 .0250 .9971 .0171 .0191 .0001 .0321 .1181 .1851 .2111 .1941 .1391 .0480 .9360 .867

$\begin{array}{llllllllllllllllllllllllll}1.784 & 1.730 & 1.667 & 1.576 & 1.409 & 1.105 & 1.011 & 1.116 & 1.068 & 0.879 & 0.852 & 0.989 & 0.991 & 0.856 & 0.884 & 1.086 & 1.169 & 1.182 & 1.164 & 1.120 & 1.029 & 0.911 & 0.848\end{array}$

$\begin{array}{lllllllllllllllllllllllll}1.713 & 1.667 & 1.614 & 1.538 & 1.390 & 1.102 & 1.018 & 1.125 & 1.077 & 0.886 & 0.860 & 1.000 & 1.004 & 0.869 & 0.892 & 1.082 & 1.151 & 1.155 & 1.131 & 1.084 & 0.997 & 0.884 & 0.823\end{array}$

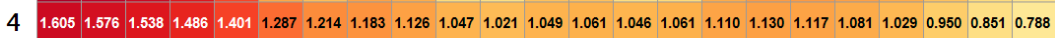

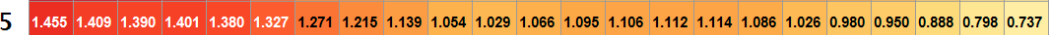

$\begin{array}{llllllllllllllllllllllll}6 & 1.287 & 1.105 & 1.102 & 1.287 & 1.327 & 1.297 & 1.258 & 1.209 & 1.109 & 0.901 & 0.877 & 1.039 & 1.098 & 1.106 & 1.105 & 1.094 & 1.022 & 0.828 & 0.775 & 0.858 & 0.821 & 0.731 & 0.674\end{array}$

$\begin{array}{lllllllllllllllllllllllll}7 & 1.185 & 1.011 & 1.018 & 1.214 & 1.271 & 1.258 & 1.230 & 1.187 & 1.091 & 0.887 & 0.865 & 1.027 & 1.085 & 1.092 & 1.087 & 1.069 & 0.989 & 0.790 & 0.730 & 0.809 & 0.773 & 0.685 & 0.619\end{array}$

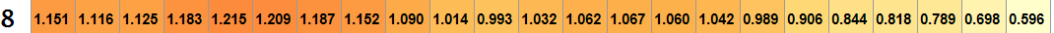

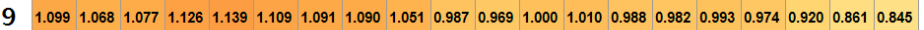

$\begin{array}{llllllllllllllllllllllll}10 & 1.025 & 0.879 & 0.886 & 1.047 & 1.054 & 0.901 & 0.887 & 1.014 & 0.987 & 0.821 & 0.804 & 0.939 & 0.941 & 0.803 & 0.797 & 0.930 & 0.940 & 0.889 & 0.833 & 0.823\end{array}$

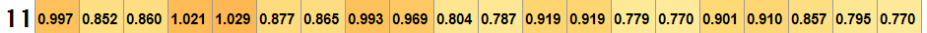

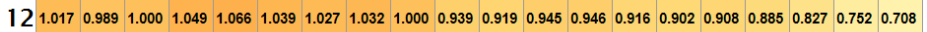

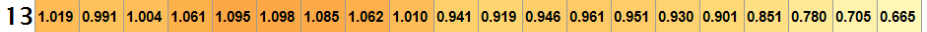

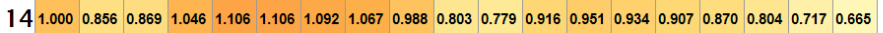

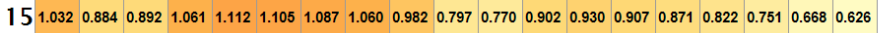

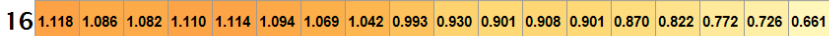

$\begin{array}{llllllllllllllll}171.185 & 1.169 & 1.151 & 1.130 & 1.086 & 1.022 & 0.989 & 0.989 & 0.974 & 0.940 & 0.910 & 0.885 & 0.851 & 0.804 & 0.751 & 0.726\end{array}$

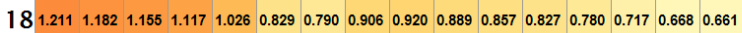

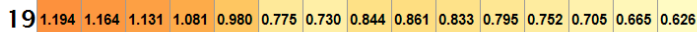

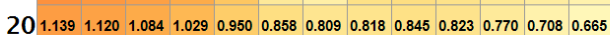

211.0481 .0300 .9970 .9500 .8880 .8210 .7730 .789

220.9360 .9110 .8840 .8510 .7980 .7310 .6850 .698

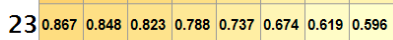

pin_powers: exposure 0

Figure 10. Shift (top) and MPACT (bottom) power peaking factors for Problem 3.1.2 


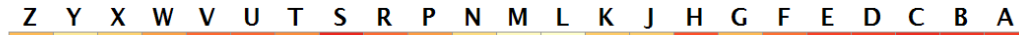

0.00749

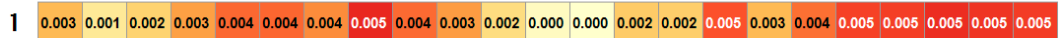

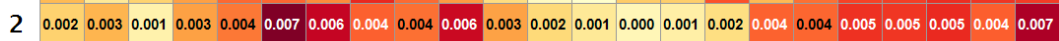

$\begin{array}{llllllllllllllllllllllllllllllllll}3 & 0.003 & 0.001 & 0.001 & 0.001 & 0.005 & 0.007 & 0.007 & 0.005 & 0.003 & 0.005 & 0.003 & 0.000 & 0.000 & 0.001 & 0.000 & 0.003 & 0.004 & 0.003 & 0.005 & 0.006 & 0.004 & 0.004 & 0.006\end{array}$

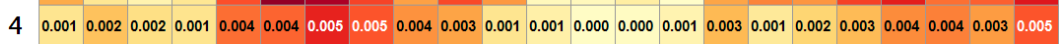

$\begin{array}{lllllllllllllllllllllllllllll}5 & 0.002 & 0.002 & 0.003 & 0.004 & 0.002 & 0.002 & 0.002 & 0.003 & 0.001 & 0.002 & 0.002 & 0.001 & 0.002 & 0.002 & 0.002 & 0.002 & 0.002 & 0.003 & 0.003 & 0.002 & 0.001 & 0.002 & 0.004\end{array}$

$\begin{array}{llllllllllllllllllllllllllll}6 & 0.004 & 0.007 & 0.006 & 0.003 & 0.002 & 0.002 & 0.001 & 0.002 & 0.003 & 0.004 & 0.003 & 0.000 & 0.002 & 0.002 & 0.001 & 0.001 & 0.000 & 0.000 & 0.003 & 0.001 & 0.002 & 0.001 & 0.003\end{array}$

$7 \begin{array}{lllllllllllllllllllllllllllll}7 & 0.002 & 0.006 & 0.006 & 0.004 & 0.002 & 0.001 & 0.003 & 0.001 & 0.002 & 0.005 & 0.002 & 0.002 & 0.001 & 0.003 & 0.002 & 0.001 & 0.000 & 0.001 & 0.001 & 0.001 & 0.002 & 0.003 & 0.003\end{array}$

$\begin{array}{llllllllllllllllllllllllllll}8 & 0.001 & 0.004 & 0.003 & 0.003 & 0.002 & 0.002 & 0.002 & 0.003 & 0.002 & 0.002 & 0.002 & 0.001 & 0.000 & 0.000 & 0.003 & 0.002 & 0.002 & 0.001 & 0.000 & 0.001 & 0.003 & 0.002 & 0.002\end{array}$

$\begin{array}{llllllllllllllllllllllllllllllllll}9 & 0.002 & 0.002 & 0.003 & 0.002 & 0.001 & 0.001 & 0.001 & 0.000 & 0.002 & 0.001 & 0.002 & 0.003 & 0.001 & 0.001 & 0.001 & 0.001 & 0.002 & 0.002 & 0.001 & 0.001\end{array}$

$\begin{array}{llllllllllllllllllllllllllll}10 & 0.003 & 0.004 & 0.005 & 0.003 & 0.001 & 0.005 & 0.004 & 0.001 & 0.002 & 0.003 & 0.003 & 0.001 & 0.001 & 0.003 & 0.001 & 0.002 & 0.000 & 0.001 & 0.001 & 0.001\end{array}$

0.007

0.006

0.005

0.004

0.003

0.002

0.001

2.23e-05

(Unitless)

$\begin{array}{llllllllllllllllllllllllll}11 & 0.002 & 0.004 & 0.004 & 0.003 & 0.001 & 0.004 & 0.003 & 0.001 & 0.001 & 0.005 & 0.004 & 0.000 & 0.000 & 0.003 & 0.001 & 0.000 & 0.002 & 0.001 & 0.002 & 0.002\end{array}$

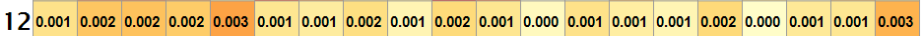
$\begin{array}{llllllllllllllllllllll}13 & 0.002 & 0.003 & 0.001 & 0.002 & 0.002 & 0.000 & 0.002 & 0.001 & 0.001 & 0.001 & 0.001 & 0.000 & 0.000 & 0.002 & 0.001 & 0.000 & 0.001 & 0.001 & 0.002 & 0.002\end{array}$ $\begin{array}{lllllllllllllllllllllllllllll}14 & 0.002 & 0.004 & 0.004 & 0.002 & 0.002 & 0.001 & 0.002 & 0.000 & 0.002 & 0.004 & 0.002 & 0.001 & 0.000 & 0.000 & 0.001 & 0.002 & 0.001 & 0.001 & 0.002\end{array}$

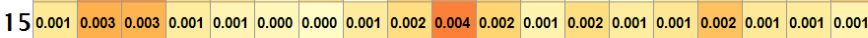
$160.0020 .0010 .0010 .0000 .0020 .0010 .0010 .0000 .001 \quad 0.001 \quad 0.000 \quad 0.000 \quad 0.002 \quad 0.000 \quad 0.001 \quad 0.002 \quad 0.0020 .002$ \begin{tabular}{ll|l|l|l|l|l|l|l|l|l|l|l|l|l|l}
170.004 & 0.005 & 0.003 & 0.003 & 0.001 & 0.002 & 0.001 & 0.000 & 0.002 & 0.000 & 0.001 & 0.000 & 0.001 & 0.001 & 0.002 & 0.000
\end{tabular}

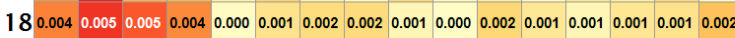

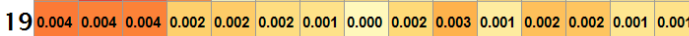

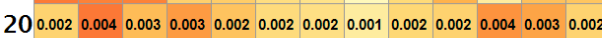

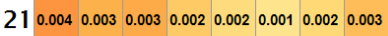
$220.0050 .0040 .003 \quad 0.0020 .003 \quad 0.0020 .0030 .003$ 230.0060 .0060 .0050 .0040 .0020 .0030 .0030 .002

pin_powers: exposure 0

Figure 11. Absolute difference between Shift and MPACT power peaking factors for Problem 3.1.2 
Table 4.7. Power peaking factors A-H and P-S of the reference solution of Problem 3.1.2.

\begin{tabular}{|c|c|c|c|c|c|c|c|c|}
\hline \multirow[t]{2}{*}{ Charge Pans } & \multicolumn{8}{|c|}{ Shift Power Peaking Factors } \\
\hline & $\begin{array}{c}\text { A } \\
\text { (A) }\end{array}$ & $\begin{array}{c}\text { B } \\
\text { (D) }\end{array}$ & $\begin{array}{c}\mathrm{C} \\
\text { (C) }\end{array}$ & $\begin{array}{c}\mathrm{D} \\
\text { (B) }\end{array}$ & $\begin{array}{c}\mathrm{P} \\
(\mathrm{R})\end{array}$ & $\begin{array}{c}\mathrm{Q} \\
(\mathrm{Q})\end{array}$ & $\begin{array}{c}\mathrm{R} \\
\text { (P) }\end{array}$ & $\begin{array}{c}S \\
(S)\end{array}$ \\
\hline 0727 & 1.667 & 1.615 & 1.668 & 1.733 & 1.827 & 1.608 & 1.488 & 1.606 \\
\hline 827 (0728) & & 025 & 1.109 & 1.113 & 1.459 & & & 1.405 \\
\hline 729) & 855 & 864 & 0.891 & 0.885 & 1.103 & 18 & 1.048 & .129 \\
\hline 730) & & & 870 & & 1.019 & & & .061 \\
\hline 731) & & 1. & 1.151 & 1.178 & 1.182 & 1.134 & 1.025 & 1.129 \\
\hline 32) & & 7 & 879 & 06 & 1.043 & - & - & 0.946 \\
\hline 0828 & & & 1.258 & 99 & 1.382 & & & 1.217 \\
\hline 0829) & 1 & 0.868 & 0.892 & 0.904 & 1.140 & 1.065 & 33 & .092 \\
\hline & & & & & & & & 062 \\
\hline 31) & & & 0.791 & 29 & 1.084 & 0.948 & & 0.987 \\
\hline 32) & & & 0.681 & 29 & 0.887 & . & - & .786 \\
\hline 0929 & & & 0.810 & & 053 & & & .001 \\
\hline 1029) & & & 0.781 & & 1.010 & 2 & & 0.947 \\
\hline 31) & & & 57 & & & & & 885 \\
\hline 1030 & & & 0.907 & & 0.961 & 0.901 & 0.769 & 0.899 \\
\hline 1130) & & & 0.667 & 0.716 & 0.850 & 0.662 & - & 0.724 \\
\hline \multirow[t]{2}{*}{ Charge Pans } & \multicolumn{8}{|c|}{ MPACT Power Peaking Factors } \\
\hline & $\begin{array}{l}\text { A } \\
\text { (A) }\end{array}$ & $\begin{array}{l}\text { B } \\
\text { (D) }\end{array}$ & $\begin{array}{c}\mathrm{C} \\
\text { (C) }\end{array}$ & $\begin{array}{l}\text { D } \\
\text { (B) }\end{array}$ & $\begin{array}{c}\mathrm{P} \\
\text { (R) }\end{array}$ & $\begin{array}{c}\mathrm{Q} \\
(\mathrm{Q})\end{array}$ & $\begin{array}{c}\mathrm{R} \\
\text { (P) }\end{array}$ & $\begin{array}{c}\mathrm{S} \\
(\mathrm{S})\end{array}$ \\
\hline 0727 & 1.667 & 5 & 1.667 & 1.730 & 1.824 & 1.605 & 6 & 1.605 \\
\hline 28) & 1 & 1.0 & 2 & 5 & 5 & 51 & & 401 \\
\hline & & & 36 & & 99 & & & 1.125 \\
\hline & & & 0.869 & & & & & 1.061 \\
\hline 31) & 1 & 1 & 1.155 & 82 & 1.185 & 39 & 29 & .130 \\
\hline 32) & & & 0.884 & & 1.048 & . & - & 0.950 \\
\hline 0828 & & & 1.258 & 97 & 79 & & & 1.215 \\
\hline & & & 0.887 & & & 1.066 & 32 & 1.090 \\
\hline & & & 1. & & 95 & 1.114 & & 1.062 \\
\hline 31) & 0 . & 0 & 0.790 & 28 & 1.086 & 50 & 18 & 0.989 \\
\hline$(0832)$ & & & 0.685 & & 0.888 & - & - & 0.789 \\
\hline & & & 0.804 & 1 & & & & 1.000 \\
\hline & & & 0.779 & & 1.010 & & 0.908 & 0.946 \\
\hline 11 & & & 0.857 & 89 & 0.974 & 0.845 & 0.708 & 0.885 \\
\hline & & & 0.908 & & 0.961 & 0.901 & 0.772 & 0.901 \\
\hline $1031(1130)$ & 0.665 & 0.626 & 0.668 & 0.717 & 0.852 & 0.665 & - & 0.726 \\
\hline
\end{tabular}


Table 4.8. Power peaking factors E-M of the reference solution of Problem 3.1.2.

\begin{tabular}{|c|c|c|c|c|c|c|c|c|}
\hline \multirow[t]{2}{*}{ Charge Pans } & \multicolumn{8}{|c|}{ Shift Power Peaking Factors } \\
\hline & $\begin{array}{c}E \\
(H)\end{array}$ & $\begin{array}{c}\mathrm{F} \\
(\mathrm{G})\end{array}$ & $\begin{array}{c}\mathrm{G} \\
(\mathrm{F})\end{array}$ & $\begin{array}{c}\mathrm{H} \\
(\mathrm{E})\end{array}$ & $\begin{array}{c}\mathrm{J} \\
(\mathrm{M})\end{array}$ & $\begin{array}{c}\mathrm{K} \\
(\mathrm{L})\end{array}$ & $\begin{array}{c}\mathrm{L} \\
(\mathrm{K})\end{array}$ & $\begin{array}{l}M \\
(J)\end{array}$ \\
\hline 0727 & 1.786 & 1.715 & 1.580 & 1.539 & 1.539 & 1.579 & 1.716 & .787 \\
\hline 327 (0728) & 291 & .189 & 1.120 & & 1.220 & .292 & & 1.413 \\
\hline 29) & 28 & 998 & 0.990 & 1.000 & 1.022 & 50 & & 1.072 \\
\hline 730) & & & 1.084 & & 1.061 & & & .989 \\
\hline 31) & & & 1.115 & 1.078 & 1.079 & 15 & & 164 \\
\hline 32) & 1 & 0.862 & - & - & 0.783 & & & .024 \\
\hline 0828 & & 2 & 1.211 & & 1.189 & 211 & & .329 \\
\hline 0829) & 056 & 1.031 & 1.040 & 1.029 & 0.996 & .016 & & 1.111 \\
\hline & & & 1.093 & & & & & 096 \\
\hline 31) & & 0.911 & 0.857 & 0.808 & 0.843 & & & 1.022 \\
\hline 32) & & & - & - & 0. & & & 819 \\
\hline 0929 & & & 0.940 & & 20 & & & 989 \\
\hline (1029) & & & 0.928 & 00 & 0.903 & 17 & & 0.942 \\
\hline 31) & & & 0.822 & & & & & 940 \\
\hline 1030 & & 0.930 & 0.868 & 820 & 0.821 & & & 0.950 \\
\hline 1130) & & & & 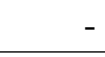 & - & 0.659 & & .803 \\
\hline \multirow[t]{2}{*}{ Charge Pans } & \multicolumn{8}{|c|}{ MPACT Power Peaking Factors } \\
\hline & $\begin{array}{c}\mathrm{E} \\
(\mathrm{H})\end{array}$ & $\mathrm{F}$ & $\begin{array}{l}\text { G } \\
(F)\end{array}$ & $\begin{array}{l}\mathrm{H} \\
\text { (E) }\end{array}$ & $\begin{array}{c}\mathrm{J} \\
(\mathrm{M})\end{array}$ & $\begin{array}{c}\mathrm{K} \\
(\mathrm{L})\end{array}$ & $\begin{array}{c}\mathrm{L} \\
(\mathrm{K})\end{array}$ & $\begin{array}{l}M \\
(J)\end{array}$ \\
\hline 0727 & 1.784 & 712 & 1.576 & 538 & 1.538 & 1.576 & 71 & 1.784 \\
\hline 0728) & 1 & & 6 & 25 & 1.214 & 37 & & 1.409 \\
\hline 29) & & & 89 & 00 & 21 & & & 1.068 \\
\hline & & & 1.086 & & & & & 0.990 \\
\hline 31) & 1 & 4 & 1.120 & 84 & 1 . & 17 & & .169 \\
\hline 732) & & & - & - & 0.788 & & & 1.030 \\
\hline 0828 & & 1 & 1.209 & & & & & 1.327 \\
\hline $0928(0829)$ & & & 1.039 & 27 & & 14 & & 1.109 \\
\hline & & & 1.094 & 1.069 & 1.060 & & & 1.097 \\
\hline 112 & 6 & 0. & 0.858 & 0.809 & 0.844 & 6 & & 1.022 \\
\hline$(0832)$ & & & & 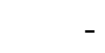 & 0.596 & 0.698 & & 0.821 \\
\hline & & & 0.939 & & & & & 0.987 \\
\hline 1029) & & 0.982 & 0.930 & 0.901 & 0.902 & 0.916 & 0.919 & 0.941 \\
\hline 093 & & & 0.823 & 0.770 & 0.752 & 0.827 & 10 & 0.940 \\
\hline & & & 0.870 & 0.822 & 0.822 & & & 0.951 \\
\hline $1031(1130)$ & 0.780 & 0.705 & - & 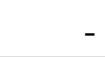 & 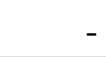 & 0.661 & 0.751 & 0.804 \\
\hline
\end{tabular}




\subsection{DEPLETION}

\subsubsection{Problem 3.2.1}

\subsubsection{Description}

Figure 12 shows the quarter core geometry for Problem 3.1.1, which models the depletion with no control rods inserted. The geometry remains $1 / 8$ symmetric. The diagonal dashed line illustrates an axis of reflected symmetry. The main control bank is inserted in the following charge-pans: 0729, 0730, 0927, 0929, and 1027. Material densities are listed in Table1.4. Temperatures are listed in Table 10. Burnup states used for comparison are listed in Table 1.5 and the total system specific power is listed in Table 1.6. 


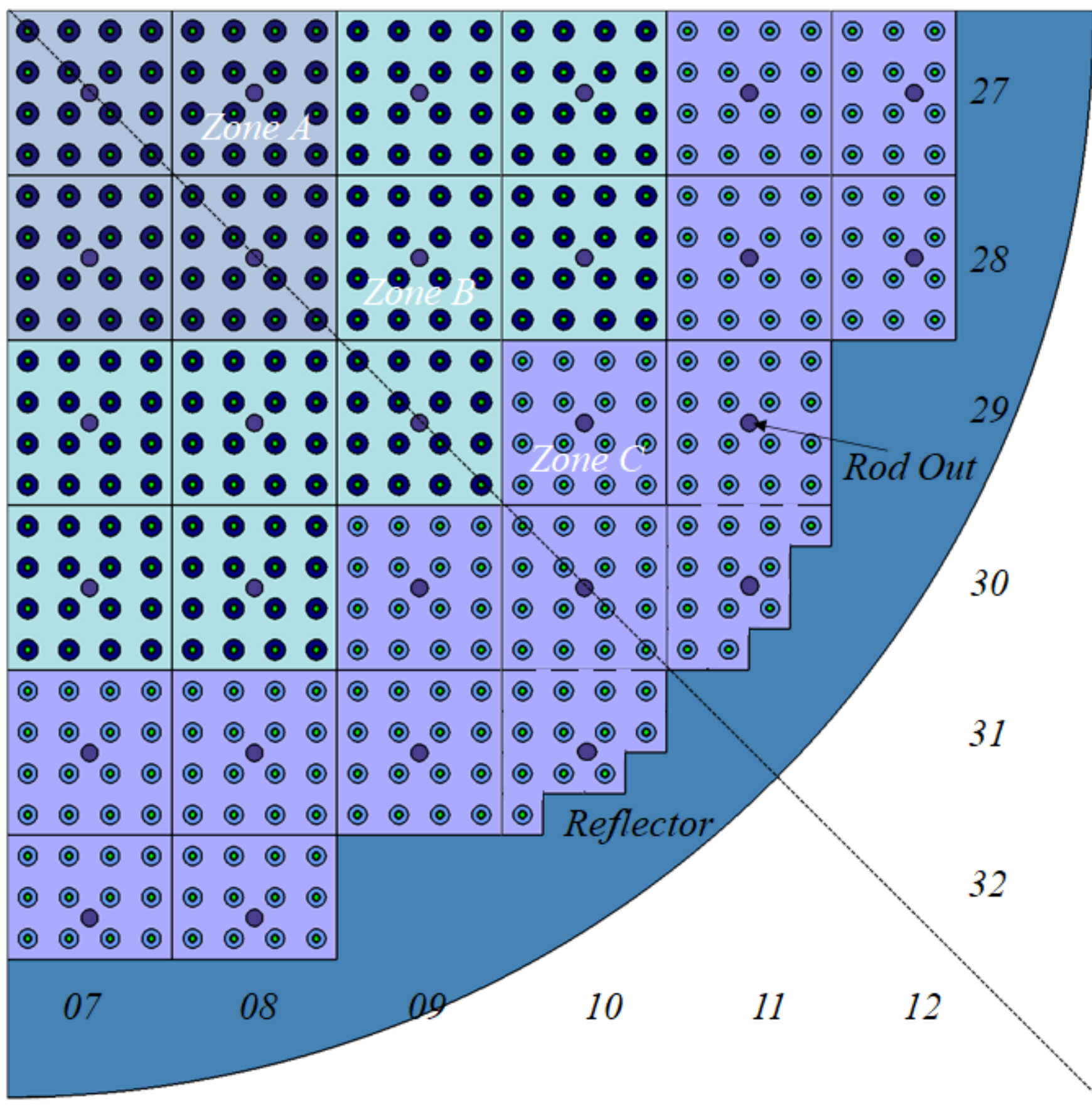

Figure 12. Quarter core 2D with all rods out (ARO). 


\subsubsection{Reference Solution}

The neutron multiplication factor results are shown in Table 4.9. The timing variables for Shift and MPACT are shown in Table 4.11. Power peaking factors are displayed for BOC and EOC in Figures 13-14 and fully tabulated for all time steps in Tables 4.23-4.39.

The neutron multiplication factor results agree decently well over the whole cycle. The peak difference is after the first time step and decreases throughout the cycle. The MPACT power peaking factors appear nearly perfectly symmetric. The Shift results are slightly asymmetric, despite high statistics, i.e., less than $10 \mathrm{pcm}$ statistical error and $0.3 \%$ or less relative error for tallies in each fuel region.

Table 4.9. Neutron multiplication factor for the reference solution of Problem 3.2.1.

\begin{tabular}{rrrrr}
\hline Step & $\begin{array}{r}\text { Burnup } \\
{\left[\frac{\mathrm{MWd}}{\mathrm{MtU}}\right]}\end{array}$ & $\begin{array}{c}\text { Shift } \\
\mathrm{k}_{\mathrm{eff}}(1 \sigma)\end{array}$ & \multicolumn{1}{c}{$\begin{array}{c}\text { MPACT } \\
\mathrm{k}_{\mathrm{eff}}(1 \sigma)\end{array}$} & $\begin{array}{r}\text { Difference } \\
{[\mathrm{pcm}]}\end{array}$ \\
\hline 0 & 0.0 & $1.05248(7)$ & $1.05031(1)$ & $-217(7)$ \\
1 & 25.0 & $1.03862(7)$ & $1.03401(1)$ & $-461(7)$ \\
2 & 50.0 & $1.03837(7)$ & $1.03414(1)$ & $-424(7)$ \\
3 & 100.0 & $1.03906(7)$ & $1.03599(1)$ & $-307(7)$ \\
4 & 200.0 & $1.04161(7)$ & $1.04054(1)$ & $-107(7)$ \\
5 & 300.0 & $1.04411(8)$ & $1.04432(1)$ & $22(8)$ \\
6 & 400.0 & $1.04639(7)$ & $1.04722(1)$ & $84(7)$ \\
7 & 500.0 & $1.04823(8)$ & $1.04939(1)$ & $117(8)$ \\
8 & 600.0 & $1.04983(7)$ & $1.05097(1)$ & $114(7)$ \\
9 & 700.0 & $1.05098(8)$ & $1.05207(1)$ & $109(8)$ \\
10 & 800.0 & $1.05194(7)$ & $1.0528(1)$ & $86(8)$ \\
11 & 900.0 & $1.0525(8)$ & $1.05321(1)$ & $71(8)$ \\
12 & 1000.0 & $1.05306(8)$ & $1.05337(1)$ & $30(8)$ \\
13 & 1100.0 & $1.05359(8)$ & $1.05331(1)$ & $-28(8)$ \\
14 & 1200.0 & $1.05373(8)$ & $1.05308(1)$ & $-65(8)$ \\
15 & 1300.0 & $1.05386(8)$ & $1.0527(1)$ & $-116(8)$ \\
\hline
\end{tabular}


pin_powers: exposure 1.3

\section{Figure 13. Shift power peaking factors for Problem 3.2.1 at BOC (top) and EOC (bottom)}


11

$\begin{array}{llllllllllllllllllllllllll}12 & 1.343 & 1.336 & 1.325 & 1.311 & 1.289 & 1.261 & 1.230 & 1.197 & 1.159 & 1.112 & 1.062 & 1.011 & 0.950 & 0.885 & 0.814 & 0.740 & 0.661 & 0.579 & 0.503 & 0.460\end{array}$

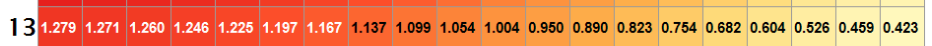

$\begin{array}{llllllllllllllllllllllllll}14 & 1.208 & 1.189 & 1.179 & 1.176 & 1.155 & 1.115 & 1.088 & 1.069 & 1.032 & 0.978 & 0.931 & 0.885 & 0.823 & 0.748 & 0.684 & 0.620 & 0.545 & 0.467 & 0.422\end{array}$

$\begin{array}{lllllllllllllllllllllll}15 & 1.137 & 1.122 & 1.112 & 1.105 & 1.084 & 1.049 & 1.021 & 0.998 & 0.961 & 0.911 & 0.864 & 0.814 & 0.754 & 0.684 & 0.623 & 0.559 & 0.490 & 0.424 & 0.389\end{array}$

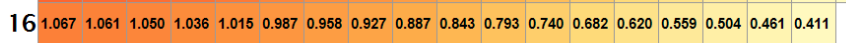

$\begin{array}{lllllllllllllllllll}17 & 0.989 & 0.982 & 0.972 & 0.958 & 0.937 & 0.909 & 0.879 & 0.846 & 0.807 & 0.761 & 0.712 & 0.661 & 0.604 & 0.545 & 0.490 & 0.461\end{array}$

$\begin{array}{lllllllllllllllll}18 & 0.904 & 0.888 & 0.878 & 0.873 & 0.852 & 0.816 & 0.787 & 0.761 & 0.722 & 0.668 & 0.623 & 0.579 & 0.526 & 0.467 & 0.423 & 0.411\end{array}$

$\begin{array}{llllllllllllllll}19 & 0.814 & 0.803 & 0.793 & 0.784 & 0.763 & 0.732 & 0.705 & 0.677 & 0.641 & 0.595 & 0.551 & 0.503 & 0.458 & 0.422 & 0.389\end{array}$

$\begin{array}{lllllllllllllllllll}20 & 0.721 & 0.715 & 0.705 & 0.691 & 0.671 & 0.646 & 0.619 & 0.601 & 0.596 & 0.563 & 0.513 & 0.460 & 0.423\end{array}$

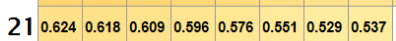

220.5330 .5220 .5130 .5050 .4860 .4570 .4360 .450

\begin{tabular}{lll|l|l|l|l|l|l|l|l|l|l}
23 & 0.479 & 0.472 & 0.463 & 0.451 & 0.431 & 0.403 & 0.377 & 0.369
\end{tabular}

pin_powers: exposure 0

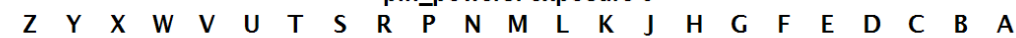

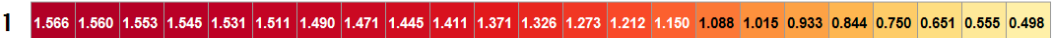

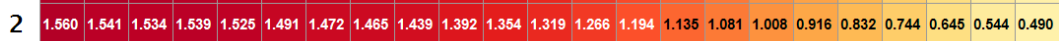

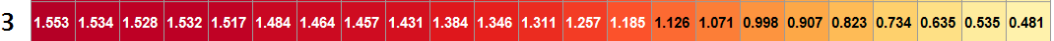

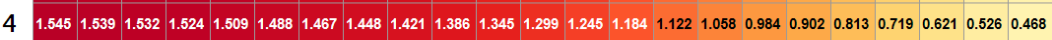

$\begin{array}{lllllllllllllllllllllllllll}5 & 1.531 & 1.525 & 1.517 & 1.509 & 1.494 & 1.473 & 1.451 & 1.432 & 1.405 & 1.369 & 1.328 & 1.282 & 1.227 & 1.166 & 1.102 & 1.038 & 0.964 & 0.882 & 0.793 & 0.699 & 0.601 & 0.506 & 0.447\end{array}$

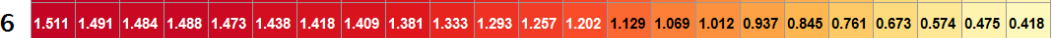

\begin{tabular}{ll|l|l|l|l|l|l|l|l|l|llllllllllll}
1.490 & 1.472 & 1.464 & 1.467 & 1.451 & 1.418 & 1.397 & 1.386 & 1.357 & 1.309 & 1.269 & 1.231 & 1.176 & 1.104 & 1.043 & 0.984 & 0.908 & 0.816 & 0.732 & 0.645 & 0.550 & 0.453 & 0.391
\end{tabular}

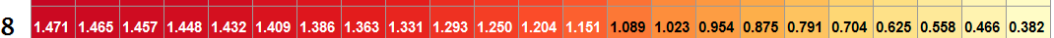

$9 \begin{array}{lllllllllllllllllllll}1.445 & 1.439 & 1.431 & 1.421 & 1.405 & 1.381 & 1.357 & 1.331 & 1.298 & 1.257 & 1.214 & 1.170 & 1.116 & 1.055 & 0.988 & 0.916 & 0.836 & 0.750 & 0.667 & 0.619\end{array}$

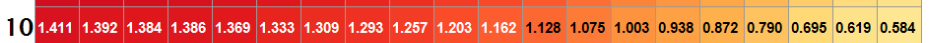

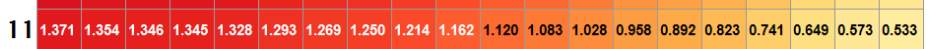

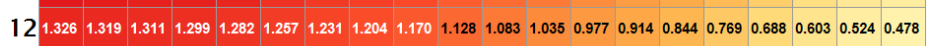

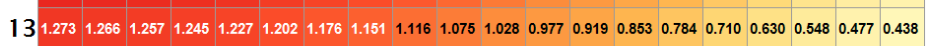

$\begin{array}{lllllllllllllllllllll}14 & 1.212 & 1.194 & 1.185 & 1.184 & 1.166 & 1.129 & 1.104 & 1.089 & 1.055 & 1.003 & 0.958 & 0.914 & 0.853 & 0.778 & 0.712 & 0.646 & 0.568 & 0.486 & 0.438\end{array}$

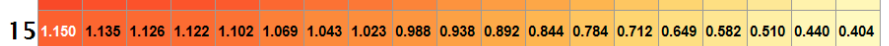

$\begin{array}{llllllllllllllllllllll}16 & 1.088 & 1.081 & 1.071 & 1.058 & 1.038 & 1.012 & 0.984 & 0.954 & 0.916 & 0.872 & 0.823 & 0.769 & 0.710 & 0.646 & 0.582 & 0.525 & 0.478 & 0.426\end{array}$

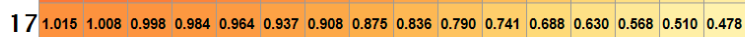

$\begin{array}{lllllllllllllllll}18 & 0.933 & 0.916 & 0.907 & 0.902 & 0.882 & 0.845 & 0.816 & 0.791 & 0.750 & 0.695 & 0.649 & 0.603 & 0.548 & 0.486 & 0.440 & 0.426\end{array}$

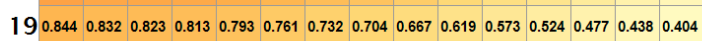

$\begin{array}{llllllllllllllll}20 & 0.750 & 0.744 & 0.734 & 0.719 & 0.699 & 0.673 & 0.645 & 0.625 & 0.619 & 0.684 & 0.633 & 0.478 & 0.438\end{array}$

$\begin{array}{lllllllll}21 & 0.651 & 0.645 & 0.635 & 0.621 & 0.601 & 0.574 & 0.550 & 0.558\end{array}$

$\begin{array}{lllllllll}22 & 0.555 & 0.544 & 0.535 & 0.526 & 0.506 & 0.475 & 0.453 & 0.466\end{array}$

$\begin{array}{lllllllll}23 & 0.498 & 0.490 & 0.481 & 0.468 & 0.447 & 0.418 & 0.391 & 0.382\end{array}$

pin_powers: exposure 1.3

Figure 14. MPACT power peaking factors for Problem 3.2.1 at BOC (top) and EOC (bottom) 
Table 4.10. Timing variables for the reference solution of Problem 3.2.1

\begin{tabular}{|c|c|c|c|c|c|c|}
\hline \multicolumn{7}{|c|}{ Shift } \\
\hline $\begin{array}{c}\text { Depletion } \\
\text { Solves }\end{array}$ & $\begin{array}{c}\text { Total } \\
\text { Cycles per } \\
\text { Transport } \\
\text { Solve }\end{array}$ & $\begin{array}{c}\text { Active } \\
\text { Cycles per } \\
\text { Transport } \\
\text { Solve }\end{array}$ & $\begin{array}{l}\text { Histories } \\
\text { per Cycle }\end{array}$ & $\begin{array}{c}\text { Compute } \\
\text { Nodes }\end{array}$ & $\begin{array}{c}\text { Cores } \\
\text { per Node }\end{array}$ & $\begin{array}{c}\text { Wall } \\
\text { Total Time } \\
\text { [hours] }\end{array}$ \\
\hline 1 & 1500 & 1200 & 500000 & 2 & 48 & 9.64 \\
\hline \multicolumn{7}{|c|}{ MPACT } \\
\hline $\begin{array}{c}\text { Depletion } \\
\text { Solves }\end{array}$ & $\begin{array}{c}k_{\mathrm{eff}} \\
\text { Convergence } \\
\text { Criteria }\end{array}$ & $\begin{array}{c}\text { Source } \\
\text { Convergence } \\
\text { Criteria }\end{array}$ & $\begin{array}{c}\text { Ray } \\
\text { Spacing } \\
{[\mathrm{cm}]}\end{array}$ & $\begin{array}{c}\text { Compute } \\
\text { Nodes }\end{array}$ & $\begin{array}{c}\text { Cores } \\
\text { per Node }\end{array}$ & $\begin{array}{c}\text { Wall } \\
\text { Total Time } \\
\text { [hours] }\end{array}$ \\
\hline 1 & $1.0 \times 10^{-04}$ & $1.0 \times 10^{-04}$ & 0.01 & 3 & 128 & 5.30 \\
\hline
\end{tabular}

Table 4.11. Timing variables for the reference solution of Problem 3.2.1

\begin{tabular}{|c|c|c|c|c|c|c|}
\hline \multicolumn{7}{|c|}{ Shift } \\
\hline $\begin{array}{c}\text { Depletion } \\
\text { Solves }\end{array}$ & $\begin{array}{c}\text { Total } \\
\text { Cycles per } \\
\text { Transport } \\
\text { Solve }\end{array}$ & $\begin{array}{c}\text { Active } \\
\text { Cycles per } \\
\text { Transport } \\
\text { Solve }\end{array}$ & $\begin{array}{l}\text { Histories } \\
\text { per Cycle }\end{array}$ & $\begin{array}{c}\text { Compute } \\
\text { Nodes }\end{array}$ & $\begin{array}{c}\text { Cores } \\
\text { per Node }\end{array}$ & $\begin{array}{c}\text { Wall } \\
\text { Total Time } \\
\text { [hours] }\end{array}$ \\
\hline 15 & 1500 & 1200 & 100000 & 8 & 48 & 13.91 \\
\hline \multicolumn{7}{|c|}{ MPACT } \\
\hline $\begin{array}{c}\text { Depletion } \\
\text { Solves }\end{array}$ & $\begin{array}{c}k_{\mathrm{eff}} \\
\text { Convergence } \\
\text { Criteria }\end{array}$ & $\begin{array}{c}\text { Source } \\
\text { Convergence } \\
\text { Criteria }\end{array}$ & $\begin{array}{c}\text { Ray } \\
\text { Spacing } \\
{[\mathrm{cm}]}\end{array}$ & $\begin{array}{c}\text { Compute } \\
\text { Nodes }\end{array}$ & $\begin{array}{c}\text { Cores } \\
\text { per Node }\end{array}$ & $\begin{array}{c}\text { Wall } \\
\text { Total Time } \\
\text { [hours] }\end{array}$ \\
\hline 15 & $1.0 \times 10^{-04}$ & $1.0 \times 10^{-04}$ & 0.03 & 3 & 128 & 21.09 \\
\hline
\end{tabular}




\section{Charge-Pan 0727}

Table 4.12. Power peaking factors for charge-pan 0727 of the reference solution of Problem 3.2.1.

\begin{tabular}{|c|c|c|c|c|c|c|c|c|c|c|c|}
\hline \multirow[t]{2}{*}{ Step } & Burnup & \multicolumn{10}{|c|}{ Shift Power Peaking Factors } \\
\hline & {$\left[\frac{\mathrm{MWd}}{\mathrm{MtU}}\right.$} & A & $\mathrm{B}, \mathrm{D}$ & $\mathrm{C}$ & $\mathrm{G}, \mathrm{F}$ & $\mathrm{H}, \mathrm{E}$ & $\mathrm{J}, \mathrm{M}$ & $\mathrm{K}, \mathrm{L}$ & Q & $\mathrm{R}, \mathrm{P}$ & S \\
\hline 0 & 0.0 & 1.620 & 1.627 & 1.637 & 1.619 & 1.628 & 1.653 & 1.662 & 1.607 & 1.637 & 1.673 \\
\hline 1 & 25.0 & 1.669 & 1.677 & 1.688 & 1.666 & 1.677 & 1.698 & 1.710 & 1.654 & 1.004 & 1.719 \\
\hline 2 & 50.0 & 1.668 & 1.675 & 1.689 & 1.665 & 1.676 & 1.698 & 1.711 & 1.648 & 1.686 & 1.720 \\
\hline 3 & 100.0 & 1.649 & 1.666 & 1.676 & 1.648 & 1.664 & 1.686 & 1.694 & 1.634 & 1.672 & 1.703 \\
\hline 4 & 200.0 & 1.616 & 1.642 & 1.654 & 1.621 & 1.643 & 1.660 & 1.671 & 1.606 & 1.649 & 1.680 \\
\hline 5 & 300.0 & 1.601 & 1.628 & 1.641 & 1.603 & 1.632 & 1.644 & 1.655 & 1.586 & 1.634 & 1.666 \\
\hline 6 & 400.0 & 1.585 & 1.626 & 1.632 & 1.588 & 1.625 & 1.636 & 1.648 & 75 & 1.624 & 1.654 \\
\hline 7 & 500.0 & & 1.629 & 1.638 & 1.592 & & 1.642 & & & 1.629 & 1.660 \\
\hline 8 & 600.0 & 1.589 & 1.636 & 1.648 & 1.597 & 1.638 & 1.649 & 1.660 & 1.576 & 1.640 & 1.669 \\
\hline 9 & 700.0 & 590 & 1.640 & 1.651 & 1.596 & 1.643 & 1.653 & 1.664 & 76 & 1.643 & 1.671 \\
\hline 10 & 8 & 1.600 & 1.661 & 1.670 & 1.612 & 1.663 & 1.674 & 35 & & 1.659 & 1.693 \\
\hline 11 & 900.0 & 1.603 & 1.664 & 1.672 & 1.614 & 1.665 & 1.675 & 1. & 88 & 1.667 & 1.695 \\
\hline 12 & 1000.0 & & & 1 & 1.623 & & & & & 78 & 1.706 \\
\hline 13 & 1 & 1.622 & 1.685 & 1.695 & 1.636 & 1.691 & 1.703 & 1.710 & & 1.692 & 1.719 \\
\hline 14 & 1200 & 1. & 1.698 & 1.706 & 1.645 & 1.700 & 1.715 & 1.7 & 14 & 1.704 & 1.729 \\
\hline 15 & 1300.0 & 0 & 1.708 & 1.715 & 1.654 & 1.714 & 1.724 & 1.737 & 19 & 1.713 & 1.743 \\
\hline \multirow[t]{2}{*}{ Step } & Burnup & \multicolumn{10}{|c|}{ MPACT Power Peaking Factors } \\
\hline & & A & $\mathrm{B}, \mathrm{D}$ & $\mathrm{C}$ & $\mathrm{G}, \mathrm{F}$ & $\mathrm{H}, \mathrm{E}$ & $\mathrm{J}, \mathrm{M}$ & $\mathrm{K}, \mathrm{L}$ & Q & $\mathrm{R}, \mathrm{P}$ & $\mathrm{S}$ \\
\hline 0 & 0.0 & 1.625 & 1.636 & 1.646 & 1.625 & 1.636 & 1.657 & 1.668 & 1.612 & 1.644 & 1.677 \\
\hline 1 & & 1.537 & 1.544 & 1.552 & 1.539 & & 1.564 & & 1.530 & 1.555 & 1.579 \\
\hline 2 & & 1.554 & 1.562 & 1.570 & 1.556 & & 1.583 & & 1.546 & 1.572 & 1.598 \\
\hline 3 & 100.0 & 1.585 & 1.594 & 1.603 & 1.586 & 1.596 & 1.615 & 1.624 & 1.574 & 1.603 & 1.632 \\
\hline 4 & 200.0 & 1.626 & 1.636 & 1.646 & 1.626 & 1.6 & 1.658 & 1.669 & 1.612 & 1.645 & 1.677 \\
\hline 5 & 300.0 & 1.644 & 1.655 & 1.666 & 1.644 & 1.655 & 1.678 & 1. & 30 & 1.664 & 1.698 \\
\hline 6 & 400.0 & 1.651 & 1.662 & 1.673 & 1.651 & 1.662 & 1.685 & 1.696 & 1.637 & 1.671 & 1.705 \\
\hline 7 & 500.0 & 1.650 & 1.660 & 1.671 & 1.650 & 1.6 & 1.683 & 1.694 & 1.636 & 1.669 & 1.703 \\
\hline 8 & 600.0 & 1.642 & 1.653 & 1.663 & 1.643 & 1.653 & 1.675 & 1.686 & 1.629 & 1.662 & 1.695 \\
\hline J & 700.0 & 1.631 & 1.641 & 1.650 & 1.631 & 1.642 & 1.663 & 1.673 & 1.618 & 1.650 & 1.681 \\
\hline 10 & 800.0 & 1.616 & 1.626 & 1.635 & 1.618 & 1.628 & 1.647 & 1.657 & 1.605 & 1.635 & 1.665 \\
\hline 11 & 900.0 & 1.600 & 1.609 & 1.618 & 1.602 & 1.611 & 1.630 & 1.639 & 1.591 & 1.619 & 1.647 \\
\hline 12 & 1000.0 & & 1.591 & 1.599 & 1.585 & 1.594 & 1.612 & 1.620 & 1.575 & 1.601 & 1.628 \\
\hline 13 & 1100.0 & 1.565 & 1.572 & 1.580 & 1.568 & 1.576 & 1.592 & 1.600 & 1.558 & 1.583 & 1.607 \\
\hline 14 & 1200.0 & 1.546 & 1.553 & 1.560 & 1.550 & 1.557 & 1.572 & 1.580 & 1.541 & 1.564 & 1.586 \\
\hline 15 & 1300.0 & 1.528 & 1.534 & 1.541 & 1.532 & 1.539 & 1.553 & 1.560 & 1.524 & 1.545 & 1.566 \\
\hline
\end{tabular}




\section{Charge-Pans 0827 and 0728}

Table 4.13. Problem 3.2.1 reference solution power peaking factors for charge-pans 0827 and 0728.

\begin{tabular}{|c|c|c|c|c|c|c|c|c|c|}
\hline \multirow[t]{3}{*}{ Step } & \multirow{3}{*}{$\begin{array}{l}\text { Burnup } \\
{\left[\frac{\mathrm{MWd}}{\mathrm{MtU}}\right]}\end{array}$} & \multicolumn{8}{|c|}{ Shift Power Peaking Factors } \\
\hline & & A & B & $\mathrm{C}$ & $\mathrm{D}$ & $\mathrm{P}$ & Q & $\mathrm{R}$ & $\mathrm{S}$ \\
\hline & & (A) & (D) & (C) & (B) & (R) & $(\mathrm{Q})$ & (P) & (S) \\
\hline 0 & 0.0 & 1.527 & 1.536 & 1.566 & 1.554 & 1.586 & 1.497 & 1.533 & 1.615 \\
\hline 1 & 25.0 & 1.566 & 1.579 & 1.607 & 1.598 & 1.624 & 1.531 & 1.572 & 1.662 \\
\hline 2 & 50.0 & 1.568 & 1.582 & 1.611 & 1.597 & 1.626 & 1.530 & 1.574 & 1.665 \\
\hline 3 & 100.0 & 1.554 & 1.566 & 1.598 & 1.586 & 1.613 & 1.521 & 1.560 & 1.649 \\
\hline 4 & 200.0 & 1.531 & 1.546 & 1.574 & 1.559 & 1.590 & 1.503 & 1.537 & 1.627 \\
\hline 5 & 300.0 & 1.521 & 1.534 & 1.560 & 1.548 & 1.583 & 1.491 & 1.526 & 1.607 \\
\hline 6 & 400.0 & 1.508 & 1.525 & 1.555 & 1.539 & 1.570 & 1.485 & 1.519 & 1.600 \\
\hline 7 & 500.0 & 1.518 & 1.536 & 1.563 & 1.545 & 1.577 & 1.492 & 1.528 & 1.601 \\
\hline 8 & 600.0 & 1.525 & 1.545 & 1.569 & 1.552 & 1.584 & 1.501 & 1.535 & 1.613 \\
\hline 9 & 700.0 & 1.529 & 1.550 & 1.572 & 1.555 & 1.587 & 1.504 & 1.542 & 1.614 \\
\hline 10 & 800.0 & 1.542 & 1.565 & 1.589 & 1.568 & 1.605 & 1.514 & 1.552 & 1.631 \\
\hline 11 & 900.0 & 1.546 & 1.567 & 1.594 & 1.572 & 1.606 & 1.517 & 1.563 & 1.637 \\
\hline 12 & 1000.0 & 1.557 & 1.584 & 1.607 & 1.584 & 1.618 & 1.531 & 1.577 & 1.649 \\
\hline 13 & 1100.0 & 1.572 & 1.596 & 1.621 & 1.598 & 1.627 & 1.543 & 1.589 & 1.668 \\
\hline 14 & 1200.0 & 1.580 & 1.603 & 1.632 & 1.606 & 1.636 & 1.548 & 1.599 & 1.677 \\
\hline 15 & 1300.0 & 1.592 & 1.616 & 1.643 & 1.614 & 1.644 & 1.557 & 1.608 & 1.688 \\
\hline \multirow[t]{3}{*}{ Step } & Burnup & \multicolumn{8}{|c|}{ MPACT Power Peaking Factors } \\
\hline & & A & B & $\mathrm{C}$ & D & $\mathrm{P}$ & Q & $\mathrm{R}$ & $S$ \\
\hline & & (A) & (D) & (C) & (B) & (R) & $(\mathrm{Q})$ & (P) & (S) \\
\hline 0 & 0.0 & 1.533 & 1.543 & 1.572 & 1.561 & 1.591 & 1.503 & 1.535 & 1.623 \\
\hline 1 & 25.0 & 1.466 & 1.474 & 1.495 & 1.487 & 1.513 & 1.446 & 1.472 & 1.538 \\
\hline 2 & 50.0 & 1.479 & 1.487 & 1.510 & 1.502 & 1.528 & 1.456 & 1.484 & 1.555 \\
\hline 3 & 100.0 & 1.502 & 1.511 & 1.536 & 1.527 & 1.555 & 1.476 & 1.505 & 1.584 \\
\hline 4 & 200.0 & 1.533 & 1.543 & 1.571 & 1.561 & 1.591 & 1.502 & 1.535 & 1.623 \\
\hline 5 & 300.0 & 1.547 & 1.558 & 1.588 & 1.577 & 1.607 & 1.515 & 1.549 & 1.641 \\
\hline 6 & 400.0 & 1.553 & 1.564 & 1.594 & 1.583 & 1.614 & 1.520 & 1.555 & 1.648 \\
\hline 7 & 500.0 & 1.553 & 1.564 & 1.593 & 1.583 & 1.613 & 1.521 & 1.555 & 1.647 \\
\hline 8 & 600.0 & 1.548 & 1.558 & 1.587 & 1.577 & 1.607 & 1.517 & 1.550 & 1.640 \\
\hline 9 & 700.0 & 1.540 & 1.550 & 1.578 & 1.568 & 1.597 & 1.510 & 1.543 & 1.629 \\
\hline 10 & 800.0 & 1.530 & 1.539 & 1.566 & 1.557 & 1.585 & 1.502 & 1.533 & 1.616 \\
\hline 11 & 900.0 & 1.518 & 1.527 & 1.552 & 1.544 & 1.572 & 1.493 & 1.522 & 1.600 \\
\hline 12 & 1000.0 & 1.505 & 1.514 & 1.538 & 1.529 & 1.557 & 1.482 & 1.510 & 1.584 \\
\hline 13 & 1100.0 & 1.492 & 1.500 & 1.523 & 1.515 & 1.541 & 1.471 & 1.498 & 1.566 \\
\hline 14 & 1200.0 & 1.478 & 1.486 & 1.507 & 1.499 & 1.525 & 1.459 & 1.484 & 1.548 \\
\hline 15 & 1300.0 & 1.464 & 1.472 & 1.491 & 1.484 & 1.509 & 1.448 & 1.471 & 1.531 \\
\hline
\end{tabular}


Table 4.14. Problem 3.2.1 reference solution power peaking factors for charge-pans 0827 and 0728 .

\begin{tabular}{|c|c|c|c|c|c|c|c|c|c|}
\hline \multirow[t]{3}{*}{ Step } & \multirow{3}{*}{$\begin{array}{l}\text { Burnup } \\
{\left[\frac{\mathrm{MWd}}{\mathrm{MtU}}\right]}\end{array}$} & \multicolumn{8}{|c|}{ Shift Power Peaking Factors } \\
\hline & & E & $\mathrm{F}$ & G & $\mathrm{H}$ & $\mathbf{J}$ & K & $\mathrm{L}$ & M \\
\hline & & $(\mathrm{H})$ & $(\mathrm{G})$ & $(\mathrm{F})$ & $(\mathrm{E})$ & $(\mathrm{M})$ & $(\mathrm{L})$ & $(\mathrm{K})$ & $(\mathrm{J})$ \\
\hline 0 & 0.0 & 1.557 & 1.526 & 1.510 & 1.521 & 1.559 & 1.590 & 1.605 & 1.597 \\
\hline 1 & 25.0 & 1.593 & 1.558 & 1.550 & 1.563 & 1.600 & 1.632 & 1.656 & 1.643 \\
\hline 2 & 50.0 & 1.592 & 1.562 & 1.549 & 1.567 & 1.602 & 1.635 & 1.654 & 1.641 \\
\hline 3 & 100.0 & 1.580 & 1.547 & 1.537 & 1.550 & 1.587 & 1.619 & 1.642 & 1.630 \\
\hline 4 & 200.0 & 1.562 & 1.529 & 1.516 & 1.533 & 1.564 & 1.594 & 1.618 & 1.603 \\
\hline 5 & 300.0 & 1.549 & 1.520 & 1.507 & 1.523 & 1.553 & 1.579 & 1.603 & 1.589 \\
\hline 6 & 400.0 & 1.543 & 1.509 & 1.495 & 1.512 & 1.541 & 1.572 & 1.595 & 1.580 \\
\hline 7 & 500.0 & 1.549 & 1.519 & 1.501 & 1.522 & 1.553 & 1.578 & 1.601 & 1.583 \\
\hline 8 & 600.0 & 1.552 & 1.526 & 1.509 & 1.532 & 1.562 & 1.590 & 1.609 & 1.593 \\
\hline 9 & 700.0 & 1.557 & 1.530 & 1.513 & 1.537 & 1.567 & 1.593 & 1.615 & 1.599 \\
\hline 10 & 800.0 & 1.570 & 1.543 & 1.526 & 1.549 & 1.581 & 1.608 & 1.633 & 1.614 \\
\hline 11 & 900.0 & 1.575 & 1.547 & 1.531 & 1.556 & 1.585 & 1.613 & 1.635 & 1.615 \\
\hline 12 & 1000.0 & 1.586 & 1.559 & 1.548 & 1.574 & 1.600 & 1.629 & 1.650 & 1.629 \\
\hline 13 & 1100.0 & 1.597 & 1.568 & 1.557 & 1.588 & 1.615 & 1.643 & 1.666 & 1.643 \\
\hline 14 & 1200.0 & 1.605 & 1.574 & 1.567 & 1.597 & 1.625 & 1.653 & 1.673 & 1.657 \\
\hline 15 & 1300.0 & 1.615 & 1.582 & 1.578 & 1.603 & 1.633 & 1.663 & 1.685 & 1.661 \\
\hline \multirow[t]{3}{*}{ Step } & Burnup & \multicolumn{8}{|c|}{ MPACT Power Peaking Factors } \\
\hline & {$\left[\frac{\mathrm{MWd}}{\mathrm{MtU}}\right]$} & E & F & $\mathrm{G}$ & $\mathrm{H}$ & $\mathrm{J}$ & K & $\mathrm{L}$ & M \\
\hline & & $(\mathrm{H})$ & $(\mathrm{G})$ & $(\mathrm{F})$ & $(\mathrm{E})$ & $(\mathrm{M})$ & (L) & $(\mathrm{K})$ & $(\mathrm{J})$ \\
\hline 0 & 0.0 & 1.561 & 1.532 & 1.516 & 1.527 & 1.564 & 1.594 & 1.614 & 1.604 \\
\hline 1 & 25.0 & 1.490 & 1.467 & 1.456 & 1.465 & 1.492 & 1.515 & 1.531 & 1.523 \\
\hline 2 & 50.0 & 1.504 & 1.479 & 1.468 & 1.477 & 1.506 & 1.531 & 1.548 & 1.539 \\
\hline 3 & 100.0 & 1.528 & 1.501 & 1.488 & 1.498 & 1.531 & 1.558 & 1.576 & 1.567 \\
\hline 4 & 200.0 & 1.561 & 1.531 & 1.516 & 1.527 & 1.564 & 1.594 & 1.615 & 1.604 \\
\hline 5 & 300.0 & 1.577 & 1.546 & 1.530 & 1.541 & 1.579 & 1.611 & 1.633 & 1.621 \\
\hline 6 & 400.0 & 1.583 & 1.551 & 1.535 & 1.546 & 1.585 & 1.617 & 1.639 & 1.628 \\
\hline 7 & 500.0 & 1.583 & 1.551 & 1.535 & 1.546 & 1.585 & 1.616 & 1.638 & 1.627 \\
\hline 8 & 600.0 & 1.577 & 1.547 & 1.531 & 1.542 & 1.580 & 1.610 & 1.631 & 1.621 \\
\hline 9 & 700.0 & 1.569 & 1.539 & 1.524 & 1.535 & 1.571 & 1.600 & 1.621 & 1.610 \\
\hline 10 & 800.0 & 1.558 & 1.529 & 1.515 & 1.525 & 1.560 & 1.588 & 1.608 & 1.598 \\
\hline 11 & 900.0 & 1.545 & 1.518 & 1.505 & 1.515 & 1.547 & 1.574 & 1.592 & 1.583 \\
\hline 12 & 1000.0 & 1.532 & 1.506 & 1.494 & 1.503 & 1.534 & 1.559 & 1.576 & 1.567 \\
\hline 13 & 1100.0 & 1.518 & 1.493 & 1.482 & 1.491 & 1.519 & 1.543 & 1.559 & 1.551 \\
\hline 14 & 1200.0 & 1.503 & 1.480 & 1.470 & 1.478 & 1.505 & 1.527 & 1.542 & 1.534 \\
\hline 15 & 1300.0 & 1.488 & 1.467 & 1.457 & 1.465 & 1.490 & 1.511 & 1.525 & 1.517 \\
\hline
\end{tabular}




\section{Charge-Pans 0927 and 0729}

Table 4.15. Problem 3.2.1 reference solution power peaking factors for charge-pans 0927 and 0729.

\begin{tabular}{|c|c|c|c|c|c|c|c|c|c|}
\hline \multirow[t]{3}{*}{ Step } & \multirow{3}{*}{$\begin{array}{l}\text { Burnup } \\
{\left[\frac{\mathrm{MWd}}{\mathrm{MtU}}\right]}\end{array}$} & \multicolumn{8}{|c|}{ Shift Power Peaking Factors } \\
\hline & & A & B & $\mathrm{C}$ & $\mathrm{D}$ & $\mathrm{P}$ & Q & $\mathrm{R}$ & $\mathrm{S}$ \\
\hline & & (A) & (D) & (C) & (B) & $(\mathrm{R})$ & $(\mathrm{Q})$ & $(\mathrm{P})$ & (S) \\
\hline 0 & 0.0 & 1.369 & 1.381 & 1.429 & 1.419 & 1.461 & 1.307 & 1.345 & 1.495 \\
\hline 1 & 25.0 & 1.406 & 1.420 & 1.470 & 1.455 & 1.494 & 1.339 & 1.386 & 1.539 \\
\hline 2 & 50.0 & 1.411 & 1.422 & 1.471 & 1.461 & 1.495 & 1.344 & 1.390 & 1.540 \\
\hline 3 & 100.0 & 1.396 & 1.410 & 1.456 & 1.443 & 1.481 & 1.330 & 1.377 & 1.524 \\
\hline 4 & 200.0 & 1.375 & 1.387 & 1.434 & 1.421 & 1.462 & 1.313 & 1.352 & 1.500 \\
\hline 5 & 300.0 & 1.362 & 1.375 & 1.421 & 1.410 & 1.452 & 1.304 & 1.334 & 1.484 \\
\hline 6 & 400.0 & 1.354 & 1.365 & 1.413 & 1.400 & 1.442 & 1.300 & 1.330 & 1.475 \\
\hline 7 & 500.0 & 1.359 & 1.372 & 1.422 & 1.407 & 1.452 & 1.303 & 1.334 & 1.480 \\
\hline 8 & 600.0 & 1.362 & 1.378 & 1.427 & 1.413 & 1.456 & 1.309 & 1.341 & 1.486 \\
\hline 9 & 700.0 & 1.370 & 1.384 & 1.434 & 1.414 & 1.461 & 1.311 & 1.345 & 1.490 \\
\hline 10 & 800.0 & 1.375 & 1.390 & 1.441 & 1.425 & 1.473 & 1.318 & 1.352 & 1.503 \\
\hline 11 & 900.0 & 1.384 & 1.400 & 1.453 & 1.432 & 1.477 & 1.329 & 1.365 & 1.511 \\
\hline 12 & 1000.0 & 1.400 & 1.419 & 1.467 & 1.447 & 1.489 & 1.339 & 1.379 & 1.527 \\
\hline 13 & 1100.0 & 1.410 & 1.431 & 1.479 & 1.456 & 1.499 & 1.351 & 1.393 & 1.537 \\
\hline 14 & 1200.0 & 1.419 & 1.442 & 1.492 & 1.463 & 1.508 & 1.360 & 1.407 & 1.551 \\
\hline 15 & 1300.0 & 1.430 & 1.447 & 1.498 & 1.476 & 1.515 & 1.366 & 1.414 & 1.559 \\
\hline \multirow[t]{3}{*}{ Step } & Burnup & \multicolumn{8}{|c|}{ MPACT Power Peaking Factors } \\
\hline & $\left.\frac{\mathrm{MWd}}{\mathrm{MtU}}\right]$ & A & B & $\mathrm{C}$ & $\mathrm{D}$ & $\mathrm{P}$ & Q & $\mathrm{R}$ & $\mathrm{S}$ \\
\hline & & (A) & (D) & (C) & (B) & $(\mathrm{R})$ & $(\mathrm{Q})$ & $(\mathrm{P})$ & (S) \\
\hline 0 & 0.0 & 1.372 & 1.382 & 1.431 & 1.421 & 1.466 & 1.311 & 1.343 & 1.499 \\
\hline 1 & 25.0 & 1.339 & 1.347 & 1.387 & 1.379 & 1.417 & 1.290 & 1.317 & 1.443 \\
\hline 2 & 50.0 & 1.344 & 1.353 & 1.395 & 1.386 & 1.426 & 1.293 & 1.322 & 1.454 \\
\hline 3 & 100.0 & 1.355 & 1.364 & 1.410 & 1.400 & 1.443 & 1.300 & 1.330 & 1.472 \\
\hline 4 & 200.0 & 1.370 & 1.381 & 1.430 & 1.420 & 1.465 & 1.310 & 1.342 & 1.498 \\
\hline 5 & 300.0 & 1.378 & 1.389 & 1.440 & 1.430 & 1.476 & 1.315 & 1.349 & 1.510 \\
\hline 6 & 400.0 & 1.382 & 1.392 & 1.445 & 1.434 & 1.481 & 1.318 & 1.352 & 1.516 \\
\hline 7 & 500.0 & 1.383 & 1.393 & 1.445 & 1.434 & 1.481 & 1.319 & 1.353 & 1.516 \\
\hline 8 & 600.0 & 1.381 & 1.392 & 1.443 & 1.432 & 1.479 & 1.319 & 1.352 & 1.512 \\
\hline 9 & 700.0 & 1.378 & 1.389 & 1.438 & 1.428 & 1.474 & 1.317 & 1.350 & 1.506 \\
\hline 10 & 800.0 & 1.374 & 1.384 & 1.432 & 1.422 & 1.467 & 1.316 & 1.347 & 1.498 \\
\hline 11 & 900.0 & 1.370 & 1.379 & 1.425 & 1.416 & 1.459 & 1.313 & 1.344 & 1.489 \\
\hline 12 & 1000.0 & 1.364 & 1.373 & 1.418 & 1.409 & 1.450 & 1.310 & 1.340 & 1.479 \\
\hline 13 & 1100.0 & 1.358 & 1.367 & 1.409 & 1.401 & 1.441 & 1.307 & 1.335 & 1.468 \\
\hline 14 & 1200.0 & 1.352 & 1.360 & 1.401 & 1.393 & 1.431 & 1.303 & 1.331 & 1.457 \\
\hline 15 & 1300.0 & 1.346 & 1.354 & 1.392 & 1.384 & 1.421 & 1.299 & 1.326 & 1.445 \\
\hline
\end{tabular}


Table 4.16. Problem 3.2.1 reference solution power peaking factors for charge-pans 0927 and 0729.

\begin{tabular}{|c|c|c|c|c|c|c|c|c|c|}
\hline \multirow[t]{2}{*}{ Step } & \multirow{2}{*}{$\begin{array}{l}\text { Burnup } \\
{\left[\frac{\mathrm{MWd}}{\mathrm{MtU}}\right]}\end{array}$} & \multicolumn{8}{|c|}{ Shift Power Peaking Factors } \\
\hline & & $\begin{array}{c}E \\
(H)\end{array}$ & $\begin{array}{c}F \\
(G)\end{array}$ & $\begin{array}{c}G \\
(F)\end{array}$ & $\begin{array}{l}\mathrm{H} \\
(\mathrm{E})\end{array}$ & $\begin{array}{c}\mathrm{J} \\
(\mathrm{M})\end{array}$ & $\begin{array}{c}\mathrm{K} \\
(\mathrm{L})\end{array}$ & $\begin{array}{c}\mathrm{L} \\
(\mathrm{K})\end{array}$ & $\begin{array}{l}\mathrm{M} \\
(\mathrm{J})\end{array}$ \\
\hline 0 & 0.0 & 1.414 & 1.366 & 1.325 & 1.336 & 1.399 & 1.451 & 1.488 & 1.476 \\
\hline 1 & 25.0 & 1.448 & 1.396 & 1.362 & 1.378 & 1.440 & 1.496 & 1.527 & 1.517 \\
\hline 2 & 50.0 & 1.450 & 1.400 & 1.367 & 1.381 & 1.441 & 1.494 & 1.529 & 1.515 \\
\hline 3 & 100.0 & 1.437 & 1.387 & 1.352 & 1.367 & 1.428 & 1.478 & 1.514 & 1.499 \\
\hline 4 & 200.0 & 1.417 & 1.371 & 1.328 & 1.341 & 1.405 & 1.456 & 1.491 & 1.477 \\
\hline 5 & 300.0 & 1.412 & 1.358 & 1.317 & 1.333 & 1.389 & 1.441 & 1.477 & 1.465 \\
\hline 6 & 400.0 & 1.401 & 1.352 & 1.310 & 1.325 & 1.384 & 1.432 & 1.468 & 1.455 \\
\hline 7 & 500.0 & 1.406 & 1.359 & 1.314 & 1.330 & 1.389 & 1.440 & 1.478 & 1.459 \\
\hline 8 & 600.0 & 1.416 & 1.363 & 1.322 & 1.338 & 1.394 & 1.444 & 1.484 & 1.467 \\
\hline 9 & 700.0 & 1.417 & 1.367 & 1.323 & 1.343 & 1.396 & 1.452 & 1.486 & 1.470 \\
\hline 10 & 800.0 & 1.426 & 1.375 & 1.333 & 1.349 & 1.407 & 1.458 & 1.498 & 1.481 \\
\hline 11 & 900.0 & 1.435 & 1.383 & 1.343 & 1.363 & 1.418 & 1.471 & 1.506 & 1.488 \\
\hline 12 & 1000.0 & 1.446 & 1.394 & 1.356 & 1.374 & 1.431 & 1.484 & 1.527 & 1.502 \\
\hline 13 & 1100.0 & 1.456 & 1.406 & 1.368 & 1.394 & 1.449 & 1.502 & 1.538 & 1.513 \\
\hline 14 & 1200.0 & 1.465 & 1.414 & 1.378 & 1.398 & 1.460 & 1.511 & 1.544 & 1.522 \\
\hline 15 & 1300.0 & 1.471 & 1.420 & 1.388 & 1.407 & 1.466 & 1.521 & 1.555 & 1.531 \\
\hline \multirow[t]{2}{*}{ Step } & Burnup & \multicolumn{8}{|c|}{ MPACT Power Peaking Factors } \\
\hline & {$\left[\frac{\mathrm{MWd}}{\mathrm{M}}\right]$} & $\begin{array}{c}E \\
(H)\end{array}$ & $\begin{array}{c}F \\
(G)\end{array}$ & $\begin{array}{c}\mathrm{G} \\
(\mathrm{F})\end{array}$ & $\begin{array}{c}\mathrm{H} \\
\text { (E) }\end{array}$ & $\begin{array}{c}\mathrm{J} \\
(\mathrm{M})\end{array}$ & $\begin{array}{c}\mathrm{K} \\
(\mathrm{L})\end{array}$ & $\begin{array}{c}\mathrm{L} \\
(\mathrm{K})\end{array}$ & $\begin{array}{l}M \\
(\mathrm{~J})\end{array}$ \\
\hline 0 & 0.0 & 1.420 & 1.367 & 1.325 & 1.336 & 1.400 & 1.452 & 1.490 & 1.479 \\
\hline 1 & 25.0 & 1.380 & 1.337 & 1.302 & 1.311 & 1.364 & 1.407 & 1.436 & 1.428 \\
\hline 2 & 50.0 & 1.387 & 1.342 & 1.305 & 1.315 & 1.370 & 1.415 & 1.446 & 1.437 \\
\hline 3 & 100.0 & 1.401 & 1.352 & 1.313 & 1.323 & 1.382 & 1.430 & 1.465 & 1.455 \\
\hline 4 & 200.0 & 1.419 & 1.367 & 1.324 & 1.335 & 1.399 & 1.452 & 1.490 & 1.479 \\
\hline 5 & 300.0 & 1.428 & 1.374 & 1.330 & 1.341 & 1.407 & 1.462 & 1.502 & 1.491 \\
\hline 6 & 400.0 & 1.432 & 1.377 & 1.333 & 1.344 & 1.411 & 1.467 & 1.507 & 1.496 \\
\hline 7 & 500.0 & 1.433 & 1.378 & 1.334 & 1.345 & 1.412 & 1.467 & 1.507 & 1.496 \\
\hline 8 & 600.0 & 1.431 & 1.377 & 1.333 & 1.344 & 1.411 & 1.465 & 1.504 & 1.493 \\
\hline 9 & 700.0 & 1.427 & 1.375 & 1.332 & 1.343 & 1.407 & 1.460 & 1.498 & 1.487 \\
\hline 10 & 800.0 & 1.422 & 1.371 & 1.329 & 1.340 & 1.403 & 1.454 & 1.490 & 1.480 \\
\hline 11 & 900.0 & 1.416 & 1.367 & 1.326 & 1.337 & 1.397 & 1.446 & 1.481 & 1.471 \\
\hline 12 & 1000.0 & 1.409 & 1.362 & 1.323 & 1.333 & 1.391 & 1.438 & 1.471 & 1.462 \\
\hline 13 & 1100.0 & 1.402 & 1.357 & 1.319 & 1.329 & 1.385 & 1.430 & 1.461 & 1.452 \\
\hline 14 & 1200.0 & 1.394 & 1.351 & 1.315 & 1.324 & 1.378 & 1.420 & 1.450 & 1.441 \\
\hline 15 & 1300.0 & 1.386 & 1.345 & 1.311 & 1.319 & 1.371 & 1.411 & 1.439 & 1.431 \\
\hline
\end{tabular}


Charge-Pans 1027 and (0730)

Table 4.17. Problem 3.2.1 reference solution power peaking factors for charge-pans 1027 and 0730 .

\begin{tabular}{|c|c|c|c|c|c|c|c|c|c|}
\hline \multirow[t]{3}{*}{ Step } & \multirow{3}{*}{$\begin{array}{l}\text { Burnup } \\
{\left[\frac{\mathrm{MWd}}{\mathrm{MtU}}\right]}\end{array}$} & \multicolumn{8}{|c|}{ Shift Power Peaking Factors } \\
\hline & & A & B & $\mathrm{C}$ & $\mathrm{D}$ & $\mathrm{P}$ & Q & $\mathrm{R}$ & $\mathrm{S}$ \\
\hline & & (A) & (D) & (C) & (B) & $(\mathrm{R})$ & $(\mathrm{Q})$ & $(\mathrm{P})$ & (S) \\
\hline 0 & 0.0 & 1.113 & 1.123 & 1.189 & 1.179 & 1.247 & 1.037 & 1.067 & 1.278 \\
\hline 1 & 25.0 & 1.157 & 1.171 & 1.239 & 1.222 & 1.283 & 1.074 & 1.118 & 1.331 \\
\hline 2 & 50.0 & 1.156 & 1.171 & 1.235 & 1.223 & 1.282 & 1.076 & 1.119 & 1.330 \\
\hline 3 & 100.0 & 1.142 & 1.158 & 1.222 & 1.207 & 1.268 & 1.062 & 1.105 & 1.312 \\
\hline 4 & 200.0 & 1.119 & 1.127 & 1.195 & 1.182 & 1.251 & 1.042 & 1.075 & 1.281 \\
\hline 5 & 300.0 & 1.099 & 1.112 & 1.179 & 1.165 & 1.235 & 1.027 & 1.059 & 1.262 \\
\hline 6 & 400.0 & 1.094 & 1.105 & 1.169 & 1.154 & 1.225 & 1.025 & 1.053 & 1.251 \\
\hline 7 & 500.0 & 1.097 & 1.111 & 1.171 & 1.163 & 1.229 & 1.026 & 1.052 & 1.250 \\
\hline 8 & 600.0 & 1.099 & 1.114 & 1.179 & 1.164 & 1.234 & 1.033 & 1.058 & 1.256 \\
\hline 9 & 700.0 & 1.101 & 1.118 & 1.184 & 1.167 & 1.234 & 1.036 & 1.063 & 1.262 \\
\hline 10 & 800.0 & 1.105 & 1.121 & 1.185 & 1.170 & 1.242 & 1.037 & 1.063 & 1.262 \\
\hline 11 & 900.0 & 1.117 & 1.136 & 1.200 & 1.184 & 1.251 & 1.054 & 1.083 & 1.272 \\
\hline 12 & 1000.0 & 1.1 & 1.144 & 1.210 & 1.191 & 1.260 & .058 & 1.087 & 1.285 \\
\hline 13 & 1100.0 & 1.139 & 1.158 & 1.225 & 1.204 & 1.272 & 1.071 & 1.103 & 1.298 \\
\hline 14 & 1200.0 & 1.149 & 1.169 & 1.232 & 1.213 & 1.281 & 1.080 & 1.114 & 1.310 \\
\hline 15 & 1300.0 & 1.153 & 1.179 & 1.243 & 1.220 & 1.287 & 1.087 & 1.122 & 1.316 \\
\hline \multirow[t]{3}{*}{ Step } & Burnup & \multicolumn{8}{|c|}{ MPACT Power Peaking Factors } \\
\hline & $\frac{\mathrm{MWd}}{\mathrm{MtU}}$ & A & B & $\mathrm{C}$ & $\mathrm{D}$ & $\mathrm{P}$ & Q & $\mathrm{R}$ & $\mathrm{S}$ \\
\hline & & (A) & (D) & (C) & (B) & (R) & $(\mathrm{Q})$ & $(\mathrm{P})$ & $(\mathrm{S})$ \\
\hline 0 & 0.0 & 1.112 & 1.122 & 1.189 & 1.179 & 1.246 & 1.036 & 1.067 & 1.279 \\
\hline 1 & 25.0 & 1.117 & 1.126 & 1.184 & 1.175 & 1.236 & 1.050 & 1.079 & 1.263 \\
\hline 2 & 50.0 & 1.115 & 1.124 & 1.184 & 1.175 & 1.237 & 1.047 & 1.076 & 1.265 \\
\hline 3 & 100.0 & 1.113 & 1.122 & 1.185 & 1.176 & 1.240 & 1.042 & 1.072 & 1.270 \\
\hline 4 & 200.0 & 1.111 & 1.121 & 1.188 & 1.178 & 1.245 & 1.035 & 1.067 & 1.278 \\
\hline 5 & 300.0 & 1.111 & 1.121 & 1.189 & 1.179 & 1.249 & 1.033 & 1.065 & 1.282 \\
\hline 6 & 400.0 & 1.111 & 1.121 & 1.191 & 1.180 & 1.251 & 1.032 & 1.065 & 1.284 \\
\hline 7 & 500.0 & 1.112 & 1.122 & 1.192 & 1.182 & 1.252 & 1.033 & 1.066 & 1.285 \\
\hline 8 & 600.0 & 1.114 & 1.124 & 1.193 & 1.183 & 1.252 & 1.035 & 1.067 & 1.285 \\
\hline 9 & 700.0 & 1.115 & 1.126 & 1.194 & 1.183 & 1.252 & 1.038 & 1.070 & 1.285 \\
\hline 10 & 800.0 & 1.117 & 1.127 & 1.194 & 1.184 & 1.252 & 1.041 & 1.073 & 1.284 \\
\hline 11 & 900.0 & 1.119 & 1.129 & 1.194 & 1.185 & 1.251 & 1.044 & 1.076 & 1.282 \\
\hline 12 & 1000.0 & 1.121 & 1.131 & 1.195 & 1.185 & 1.250 & 1.048 & 1.079 & 1.280 \\
\hline 13 & 1100.0 & 1.123 & 1.132 & 1.195 & 1.185 & 1.249 & 1.051 & 1.082 & 1.278 \\
\hline 14 & 1200.0 & 1.125 & 1.134 & 1.194 & 1.185 & 1.247 & 1.055 & 1.085 & 1.275 \\
\hline 15 & 1300.0 & 1.126 & 1.135 & 1.194 & 1.185 & 1.245 & 1.058 & 1.088 & 1.273 \\
\hline
\end{tabular}


Table 4.18. Problem 3.2.1 reference solution power peaking factors for charge-pans 1027 and 0730 .

\begin{tabular}{|c|c|c|c|c|c|c|c|c|c|}
\hline \multirow[t]{2}{*}{ Step } & \multirow{2}{*}{$\begin{array}{l}\text { Burnup } \\
{\left[\frac{\mathrm{MWd}}{\mathrm{MtU}}\right]}\end{array}$} & \multicolumn{8}{|c|}{ Shift Power Peaking Factors } \\
\hline & & $\begin{array}{c}E \\
(H)\end{array}$ & $\begin{array}{c}F \\
(\mathrm{G})\end{array}$ & $\begin{array}{c}\mathrm{G} \\
(\mathrm{F})\end{array}$ & $\begin{array}{c}\mathrm{H} \\
(\mathrm{E})\end{array}$ & $\begin{array}{c}\mathrm{J} \\
(\mathrm{M})\end{array}$ & $\begin{array}{c}\mathrm{K} \\
(\mathrm{L})\end{array}$ & $\begin{array}{c}\mathrm{L} \\
(\mathrm{K})\end{array}$ & $\begin{array}{l}\mathrm{M} \\
(\mathrm{J})\end{array}$ \\
\hline 0 & 0.0 & 1.176 & 1.108 & 1.049 & 1.060 & 1.137 & 1.211 & 1.271 & 1.261 \\
\hline 1 & 25.0 & 1.213 & 1.144 & 1.096 & 1.111 & 1.191 & 1.260 & 1.316 & 1.301 \\
\hline 2 & 50.0 & 1.212 & 1.145 & 1.097 & 1.108 & 1.188 & 1.260 & 1.318 & 1.303 \\
\hline 3 & 100.0 & 1.203 & 1.130 & 1.080 & 1.097 & 1.175 & 1.244 & 1.303 & 1.291 \\
\hline 4 & 200.0 & 1.181 & 1.115 & 1.058 & 1.071 & 1.144 & 1.214 & 1.278 & 1.262 \\
\hline 5 & 300.0 & 1.167 & 1.095 & 1.039 & 1.053 & 1.126 & 1.196 & 1.259 & 1.245 \\
\hline 6 & 400.0 & 1.162 & 1.090 & 1.035 & 1.046 & 1.117 & 1.187 & 1.250 & 1.237 \\
\hline 7 & 500.0 & 1.162 & 1.093 & 1.033 & 1.050 & 1.119 & 1.188 & 1.248 & 1.237 \\
\hline 8 & 600.0 & 1.167 & 1.098 & 1.042 & 1.056 & 1.124 & 1.192 & 1.250 & 1.241 \\
\hline 9 & 700.0 & 1.170 & 1.104 & 1.046 & 1.061 & 1.131 & 1.198 & 1.257 & 1.245 \\
\hline 10 & 800.0 & 1.176 & 1.102 & 1.045 & 1.062 & 1.128 & 1.198 & 1.260 & 1.247 \\
\hline 11 & 900.0 & 1.186 & 1.116 & 1.060 & 1.076 & 1.146 & 1.214 & 1.272 & 1.260 \\
\hline 12 & 1000.0 & 1.197 & 1.125 & 1.066 & 1.083 & 1.155 & 1.225 & 1.285 & 1.272 \\
\hline 13 & 1100.0 & 1.206 & 1.137 & 1.083 & 1.101 & 1.165 & 1.236 & 1.297 & 1.280 \\
\hline 14 & 1200.0 & 1.216 & 1.146 & 1.091 & 1.110 & 1.183 & 1.248 & 1.306 & 1.289 \\
\hline 15 & 1300.0 & 1.220 & 1.154 & 1.096 & 1.120 & 1.188 & 1.256 & 1.315 & 1.296 \\
\hline \multirow[t]{2}{*}{ Step } & Burnup & \multicolumn{8}{|c|}{ MPACT Power Peaking Factors } \\
\hline & $\frac{\mathrm{MWd}}{\mathrm{M}}$ & $\begin{array}{c}E \\
(H)\end{array}$ & $\begin{array}{c}F \\
(G)\end{array}$ & $\begin{array}{c}G \\
(F)\end{array}$ & $\begin{array}{l}\mathrm{H} \\
(\mathrm{E})\end{array}$ & $\begin{array}{c}\mathrm{J} \\
(\mathrm{M})\end{array}$ & $\begin{array}{c}\mathrm{K} \\
\text { (L) }\end{array}$ & $\begin{array}{c}\mathrm{L} \\
(\mathrm{K})\end{array}$ & $\begin{array}{l}\text { M } \\
(J)\end{array}$ \\
\hline 0 & 0.0 & 1.176 & 1.105 & 1.050 & 1.060 & 1.137 & 1.208 & 1.271 & 1.260 \\
\hline 1 & 25.0 & 1.174 & 1.112 & 1.063 & 1.073 & 1.140 & 1.202 & 1.256 & 1.247 \\
\hline 2 & 50.0 & 1.174 & 1.110 & 1.060 & 1.070 & 1.139 & 1.202 & 1.258 & 1.249 \\
\hline 3 & 100.0 & 1.174 & 1.108 & 1.055 & 1.065 & 1.137 & 1.204 & 1.263 & 1.253 \\
\hline 4 & 200.0 & 1.175 & 1.105 & 1.050 & 1.060 & 1.136 & 1.207 & 1.270 & 1.259 \\
\hline 5 & 300.0 & 1.176 & 1.104 & 1.047 & 1.058 & 1.136 & 1.209 & 1.274 & 1.263 \\
\hline 6 & 400.0 & 1.177 & 1.104 & 1.047 & 1.058 & 1.137 & 1.210 & 1.276 & 1.265 \\
\hline 7 & 500.0 & 1.179 & 1.105 & 1.048 & 1.059 & 1.138 & 1.212 & 1.277 & 1.266 \\
\hline 8 & 600.0 & 1.180 & 1.107 & 1.050 & 1.060 & 1.139 & 1.213 & 1.277 & 1.266 \\
\hline 9 & 700.0 & 1.181 & 1.109 & 1.052 & 1.063 & 1.141 & 1.213 & 1.277 & 1.266 \\
\hline 10 & 800.0 & 1.182 & 1.111 & 1.055 & 1.066 & 1.143 & 1.214 & 1.276 & 1.265 \\
\hline 11 & 900.0 & 1.183 & 1.113 & 1.058 & 1.069 & 1.144 & 1.214 & 1.275 & 1.264 \\
\hline 12 & 1000.0 & 1.183 & 1.115 & 1.062 & 1.072 & 1.146 & 1.214 & 1.273 & 1.263 \\
\hline 13 & 1100.0 & 1.184 & 1.118 & 1.065 & 1.075 & 1.147 & 1.213 & 1.271 & 1.261 \\
\hline 14 & 1200.0 & 1.184 & 1.120 & 1.068 & 1.078 & 1.149 & 1.213 & 1.268 & 1.259 \\
\hline 15 & 1300.0 & 1.185 & 1.122 & 1.071 & 1.081 & 1.150 & 1.212 & 1.266 & 1.257 \\
\hline
\end{tabular}


Charge-Pans 1127 and (0731)

Table 4.19. Problem 3.2.1 reference solution power peaking factors for charge-pans 1127 and 0731.

\begin{tabular}{|c|c|c|c|c|c|c|c|c|c|}
\hline \multirow[t]{3}{*}{ Step } & \multirow{3}{*}{$\begin{array}{l}\text { Burnup } \\
{\left[\frac{\mathrm{MWd}}{\mathrm{MtU}}\right]}\end{array}$} & \multicolumn{8}{|c|}{ Shift Power Peaking Factors } \\
\hline & & A & B & $\mathrm{C}$ & $\mathrm{D}$ & $\mathrm{P}$ & Q & $\mathrm{R}$ & $\mathrm{S}$ \\
\hline & & (A) & (D) & (C) & (B) & $(\mathrm{R})$ & $(\mathrm{Q})$ & $(\mathrm{P})$ & (S) \\
\hline 0 & 0.0 & 0.794 & 0.803 & 0.888 & 0.879 & 0.956 & 0.693 & 0.724 & 0.989 \\
\hline 1 & 25.0 & 0.835 & 0.850 & 0.935 & 0.923 & 0.999 & 0.727 & 0.766 & 1.044 \\
\hline 2 & 50.0 & 0.838 & 0.850 & 0.937 & 0.922 & 1.000 & 0.728 & 0.767 & 1.041 \\
\hline 3 & 100.0 & 0.823 & 0.835 & 0.921 & 0.911 & 0.986 & 0.716 & 0.753 & 1.029 \\
\hline 4 & 200.0 & 0.801 & 0.811 & 0.897 & 0.886 & 0.962 & 0.694 & 0.728 & 0.997 \\
\hline 5 & 300.0 & 0.789 & 0.796 & 0.880 & 0.871 & 0.942 & 0.683 & 0.713 & 0.978 \\
\hline 6 & 400.0 & 0.784 & 0.790 & 0.876 & 0.868 & 0.938 & 0.676 & 0.707 & 0.971 \\
\hline 7 & 500.0 & 0.784 & 0.792 & 0.877 & 0.868 & 0.936 & 0.675 & 0.709 & 0.970 \\
\hline 8 & 600.0 & 0.790 & 0.795 & 0.879 & 0.875 & 0.941 & 0.678 & 0.711 & 0.974 \\
\hline 9 & 700.0 & 0.791 & 0.797 & 0.882 & 0.878 & 0.941 & 0.681 & 0.712 & 0.978 \\
\hline 10 & 800.0 & 0.792 & 0.796 & 0.882 & 0.878 & 0.942 & 0.680 & 0.710 & 0.977 \\
\hline 11 & 900.0 & 0.807 & 0.813 & 0.899 & 0.892 & 0.958 & 0.691 & 0.727 & 0.992 \\
\hline 12 & 1000.0 & 0.813 & 0.820 & 0.906 & 0.899 & 0.961 & 0.697 & .731 & 0.997 \\
\hline 13 & 1100.0 & 0.827 & 0.835 & 0.921 & 0.915 & 0.974 & 0.707 & 0.744 & 1.015 \\
\hline 14 & 1200.0 & 0.835 & 0.842 & 0.927 & 0.924 & 0.980 & 0.712 & 0.752 & 1.026 \\
\hline 15 & 1300.0 & 0.843 & 0.850 & 0.936 & 0.929 & 0.987 & 0.719 & 0.759 & 1.036 \\
\hline \multirow[t]{3}{*}{ Step } & Burnup & \multicolumn{8}{|c|}{ MPACT Power Peaking Factors } \\
\hline & $\left.\frac{\mathrm{MWd}}{\mathrm{MtU}}\right]$ & A & B & $\mathrm{C}$ & $\mathrm{D}$ & $\mathrm{P}$ & Q & $\mathrm{R}$ & $\mathrm{S}$ \\
\hline & & (A) & (D) & (C) & (B) & $(\mathrm{R})$ & $(\mathrm{Q})$ & $(\mathrm{P})$ & (S) \\
\hline 0 & 0.0 & 0.793 & 0.803 & 0.888 & 0.878 & 0.958 & 0.691 & 0.721 & 0.989 \\
\hline 1 & 25.0 & 0.823 & 0.832 & 0.913 & 0.904 & 0.979 & 0.724 & 0.753 & 1.008 \\
\hline 2 & 50.0 & 0.818 & 0.827 & 0.908 & 0.899 & 0.975 & 0.718 & 0.747 & 1.004 \\
\hline 3 & 100.0 & 0.807 & 0.817 & 0.899 & 0.890 & 0.967 & 0.707 & 0.736 & 0.998 \\
\hline 4 & 200.0 & 0.794 & 0.803 & 0.888 & 0.878 & 0.958 & 0.692 & 0.721 & 0.989 \\
\hline 5 & 300.0 & 0.787 & 0.797 & 0.882 & 0.872 & 0.953 & 0.684 & 0.714 & 0.985 \\
\hline 6 & 400.0 & 0.784 & 0.794 & 0.880 & 0.870 & 0.952 & 0.681 & 0.711 & 0.984 \\
\hline 7 & 500.0 & 0.784 & 0.794 & 0.881 & 0.871 & 0.952 & 0.680 & 0.710 & 0.985 \\
\hline 8 & 600.0 & 0.786 & 0.796 & 0.883 & 0.873 & 0.954 & 0.682 & 0.712 & 0.987 \\
\hline 9 & 700.0 & 0.790 & 0.799 & 0.886 & 0.876 & 0.958 & 0.685 & 0.716 & 0.990 \\
\hline 10 & 800.0 & 0.794 & 0.804 & 0.890 & 0.880 & 0.961 & 0.690 & 0.720 & 0.993 \\
\hline 11 & 900.0 & 0.799 & 0.809 & 0.895 & 0.885 & 0.966 & 0.695 & 0.725 & 0.997 \\
\hline 12 & 1000.0 & 0.804 & 0.814 & 0.900 & 0.890 & 0.970 & 0.700 & 0.731 & 1.001 \\
\hline 13 & 1100.0 & 0.810 & 0.820 & 0.905 & 0.896 & 0.975 & 0.707 & 0.737 & 1.006 \\
\hline 14 & 1200.0 & 0.817 & 0.826 & 0.911 & 0.901 & 0.980 & 0.713 & 0.743 & 1.010 \\
\hline 15 & 1300.0 & 0.823 & 0.832 & 0.916 & 0.907 & 0.984 & 0.719 & 0.750 & 1.015 \\
\hline
\end{tabular}


Table 4.20. Problem 3.2.1 reference solution power peaking factors for charge-pans 1127 and 0731 .

\begin{tabular}{|c|c|c|c|c|c|c|c|c|c|}
\hline \multirow[t]{2}{*}{ Step } & \multirow{2}{*}{$\begin{array}{l}\text { Burnup } \\
{\left[\frac{\mathrm{MWd}}{\mathrm{MtU}}\right]}\end{array}$} & \multicolumn{8}{|c|}{ Shift Power Peaking Factors } \\
\hline & & $\begin{array}{c}E \\
(H)\end{array}$ & $\begin{array}{c}F \\
(G)\end{array}$ & $\begin{array}{l}G \\
(F)\end{array}$ & $\begin{array}{l}\mathrm{H} \\
(\mathrm{E})\end{array}$ & $\begin{array}{c}\mathrm{J} \\
(\mathrm{M})\end{array}$ & $\begin{array}{c}\mathrm{K} \\
(\mathrm{L})\end{array}$ & $\begin{array}{c}\mathrm{L} \\
(\mathrm{K})\end{array}$ & $\begin{array}{l}M \\
(J)\end{array}$ \\
\hline 0 & 0.0 & 0.875 & 0.786 & 0.706 & 0.719 & 0.816 & 0.905 & 0.983 & 0.973 \\
\hline 1 & 25.0 & 0.913 & 0.822 & 0.742 & 0.759 & 0.863 & 0.955 & 1.030 & 1.015 \\
\hline 2 & 50.0 & 0.915 & 0.823 & 0.746 & 0.757 & 0.862 & 0.954 & 1.030 & 1.018 \\
\hline 3 & 100.0 & 0.901 & 0.810 & 0.735 & 0.747 & 0.847 & 0.940 & 1.020 & 1.006 \\
\hline 4 & 200.0 & 0.881 & 0.788 & 0.714 & 0.724 & 0.824 & 0.914 & 0.990 & 0.982 \\
\hline 5 & 300.0 & 0.861 & 0.775 & 0.699 & 0.708 & 0.805 & 0.894 & 0.973 & 0.966 \\
\hline 6 & 400.0 & 0.857 & 0.769 & 0.698 & 0.704 & 0.799 & 0.887 & 0.968 & 0.959 \\
\hline 7 & 500.0 & 0.856 & 0.770 & 0.697 & 0.705 & 0.796 & 0.888 & 0.969 & 0.962 \\
\hline 8 & 600.0 & 0.859 & 0.774 & 0.701 & 0.707 & 0.802 & 0.892 & 0.973 & 0.967 \\
\hline 9 & 700.0 & 0.862 & 0.775 & 0.704 & 0.711 & 0.806 & 0.895 & 0.978 & 0.972 \\
\hline 10 & 800.0 & 0.862 & 0.780 & 0.705 & 0.711 & 0.803 & 0.891 & 0.976 & 0.974 \\
\hline 11 & 900.0 & 0.878 & 0.793 & 0.721 & 0.725 & 0.819 & 0.908 & 0.993 & 0.986 \\
\hline 12 & 1000.0 & 0.880 & 0.797 & 0.725 & 0.731 & 0.825 & 0.916 & 1.000 & 0.997 \\
\hline 13 & 1100.0 & 0.896 & 0.808 & 0.736 & 0.744 & 0.840 & 0.933 & 1.016 & 1.010 \\
\hline 14 & 1200.0 & 0.902 & 0.814 & 0.744 & 0.751 & 0.848 & 0.940 & 1.023 & 1.018 \\
\hline 15 & 1300.0 & 0.909 & 0.822 & 0.751 & 0.759 & 0.854 & 0.945 & 1.032 & 1.027 \\
\hline \multirow[t]{2}{*}{ Step } & Burnup & \multicolumn{8}{|c|}{ MPACT Power Peaking Factors } \\
\hline & $\underline{\mathrm{MWd}}$ & $\begin{array}{c}E \\
(H)\end{array}$ & $\begin{array}{c}\text { F } \\
(G)\end{array}$ & $\begin{array}{l}\mathrm{G} \\
(\mathrm{F})\end{array}$ & $\begin{array}{c}\mathrm{H} \\
(\mathrm{E})\end{array}$ & $\begin{array}{c}\mathrm{J} \\
(\mathrm{M})\end{array}$ & $\begin{array}{c}\mathrm{K} \\
(\mathrm{L})\end{array}$ & $\begin{array}{c}\mathrm{L} \\
(\mathrm{K})\end{array}$ & $\begin{array}{l}\text { M } \\
(\mathrm{J})\end{array}$ \\
\hline 0 & 0.0 & 0.873 & 0.783 & 0.705 & 0.715 & 0.814 & 0.904 & 0.982 & 0.971 \\
\hline 1 & 25.0 & 0.900 & 0.814 & 0.738 & 0.747 & 0.844 & 0.929 & 1.001 & 0.992 \\
\hline 2 & 50.0 & 0.895 & 0.809 & 0.732 & 0.742 & 0.838 & 0.924 & 0.997 & 0.988 \\
\hline 3 & 100.0 & 0.885 & 0.798 & 0.721 & 0.731 & 0.828 & 0.916 & 0.991 & 0.980 \\
\hline 4 & 200.0 & 0.873 & 0.784 & 0.706 & 0.716 & 0.814 & 0.904 & 0.982 & 0.971 \\
\hline 5 & 300.0 & 0.867 & 0.777 & 0.698 & 0.708 & 0.808 & 0.899 & 0.978 & 0.967 \\
\hline 6 & 400.0 & 0.865 & 0.774 & 0.695 & 0.705 & 0.805 & 0.897 & 0.977 & 0.966 \\
\hline 7 & 500.0 & 0.865 & 0.774 & 0.695 & 0.705 & 0.805 & 0.897 & 0.977 & 0.967 \\
\hline 8 & 600.0 & 0.867 & 0.776 & 0.696 & 0.706 & 0.807 & 0.899 & 0.979 & 0.969 \\
\hline 9 & 700.0 & 0.871 & 0.780 & 0.700 & 0.710 & 0.811 & 0.903 & 0.982 & 0.972 \\
\hline 10 & 800.0 & 0.875 & 0.784 & 0.704 & 0.714 & 0.815 & 0.907 & 0.986 & 0.975 \\
\hline 11 & 900.0 & 0.880 & 0.789 & 0.709 & 0.719 & 0.820 & 0.911 & 0.990 & 0.980 \\
\hline 12 & 1000.0 & 0.885 & 0.795 & 0.715 & 0.725 & 0.826 & 0.916 & 0.994 & 0.984 \\
\hline 13 & 1100.0 & 0.891 & 0.801 & 0.721 & 0.731 & 0.832 & 0.922 & 0.999 & 0.988 \\
\hline 14 & 1200.0 & 0.897 & 0.807 & 0.727 & 0.737 & 0.838 & 0.927 & 1.003 & 0.993 \\
\hline 15 & 1300.0 & 0.902 & 0.813 & 0.734 & 0.744 & 0.844 & 0.933 & 1.008 & 0.998 \\
\hline
\end{tabular}


Charge-Pans 1227 and (0732)

Table 4.21. Problem 3.2.1 reference solution power peaking factors for charge-pans 1227 and 0732 .

\begin{tabular}{|c|c|c|c|c|c|c|c|}
\hline \multirow[t]{3}{*}{ Step } & \multirow{3}{*}{$\begin{array}{l}\text { Burnup } \\
{\left[\frac{\mathrm{MWd}}{\mathrm{MtU}}\right]}\end{array}$} & \multicolumn{6}{|c|}{ Shift Power Peaking Factors } \\
\hline & & A & B & $\mathrm{C}$ & $\mathrm{D}$ & $\mathrm{P}$ & $\mathrm{S}$ \\
\hline & & (A) & (D) & (C) & (B) & $(\mathrm{R})$ & (Q) \\
\hline 0 & 0.0 & 0.466 & 0.474 & 0.525 & 0.516 & 0.598 & 0.626 \\
\hline 1 & 25.0 & 0.491 & 0.500 & 0.556 & 0.545 & 0.628 & 0.664 \\
\hline 2 & 50.0 & 91 & 0.502 & 0.555 & 0.545 & 0.628 & 0.665 \\
\hline 3 & 100.0 & 0.483 & 0.492 & 0.548 & 0.537 & 0.620 & 0.652 \\
\hline 4 & 200.0 & 1 & 0.477 & 0.530 & 0.519 & 0.602 & 631 \\
\hline 5 & 300.0 & 0.457 & 0.464 & 0.516 & 0.506 & 0.589 & 0.617 \\
\hline 6 & 400.0 & 0.456 & 0.461 & 0.513 & 0.502 & 0.587 & 0.612 \\
\hline 7 & 500.0 & 4 & 0.460 & 0.512 & 0.503 & 0.585 & .608 \\
\hline 8 & 600.0 & 0.456 & 0.462 & 0.516 & 0.505 & 0.590 & 0.612 \\
\hline 9 & .0 & & 0.462 & & 06 & 590 & .615 \\
\hline 10 & 800.0 & 0.461 & 0.464 & 0.518 & 0.507 & 0.593 & 0.614 \\
\hline 11 & 900.0 & 0.469 & 0.474 & 0.530 & 0.516 & 0.603 & 0.626 \\
\hline 12 & 1000.0 & 3 & 0.475 & 34 & 0.520 & 0.607 & 632 \\
\hline 13 & 1100.0 & 0.481 & 0.486 & 0.543 & 0.529 & 0.616 & 0.644 \\
\hline 14 & 1200.0 & 7 & 0.489 & 0.4 & 0.535 & 0.622 & 0.649 \\
\hline 15 & 1300.0 & 91 & 0.492 & 0.554 & 0.539 & 0.627 & 0.654 \\
\hline \multirow[t]{3}{*}{ Step } & Burnup & \multicolumn{6}{|c|}{ MPACT Power Peaking Factors } \\
\hline & & A & B & $\mathrm{C}$ & $\mathrm{D}$ & $\mathrm{P}$ & $S$ \\
\hline & & (A) & (D) & (C) & (B) & (R) & (Q) \\
\hline 0 & 0.0 & 0.463 & 0.472 & 0.522 & 0.513 & 0.595 & 0.624 \\
\hline 1 & 25.0 & 0.494 & 0.503 & 0.553 & 0.545 & 0.628 & 0.657 \\
\hline 2 & 50.0 & 0.489 & 0.498 & 0.548 & 0.539 & 0.623 & 0.651 \\
\hline 3 & 100.0 & 0.479 & 0.488 & 0.538 & 0.529 & 0.612 & 0.641 \\
\hline 4 & 200.0 & 0.465 & 0.473 & 0.523 & 0.514 & 0.597 & 0.625 \\
\hline 5 & 300.0 & 0.457 & 0.466 & 0.515 & 0.507 & 0.589 & 0.617 \\
\hline 6 & 400.0 & 0.453 & 0.462 & 0.512 & 0.503 & 0.585 & 0.614 \\
\hline 7 & 500.0 & 0.452 & 0.460 & 0.511 & 0.502 & 0.584 & 0.613 \\
\hline 8 & 600.0 & 0.452 & 0.461 & 0.512 & 0.503 & 0.586 & 0.615 \\
\hline 9 & 700.0 & 0.455 & 0.463 & 0.514 & 0.505 & 0.589 & 0.618 \\
\hline 10 & 800.0 & 0.458 & 0.466 & 0.518 & 0.509 & 0.593 & 0.622 \\
\hline 11 & 900.0 & 0.461 & 0.470 & 0.522 & 0.513 & 0.597 & 0.627 \\
\hline 12 & 1000.0 & 0.466 & 0.475 & 0.527 & 0.518 & 0.603 & 0.632 \\
\hline 13 & 1100.0 & 0.471 & 0.480 & 0.532 & 0.523 & 0.608 & 0.638 \\
\hline 14 & 1200.0 & 0.476 & 0.485 & 0.538 & 0.529 & 0.615 & 0.644 \\
\hline 15 & 1300.0 & 0.481 & 0.490 & 0.544 & 0.535 & 0.621 & 0.651 \\
\hline
\end{tabular}


Table 4.22. Problem 3.2.1 reference solution power peaking factors for charge-pans 1227 and 0732 .

\begin{tabular}{|c|c|c|c|c|c|c|c|}
\hline \multirow[t]{2}{*}{ Step } & \multirow{2}{*}{$\begin{array}{l}\text { Burnup } \\
{\left[\frac{\mathrm{MWd}}{\mathrm{MtU}}\right]}\end{array}$} & \multicolumn{6}{|c|}{ Shift Power Peaking Factors } \\
\hline & & $\begin{array}{c}E \\
(H)\end{array}$ & $\begin{array}{c}F \\
(G)\end{array}$ & $\begin{array}{c}\mathrm{J} \\
(\mathrm{F})\end{array}$ & $\begin{array}{l}K \\
\text { (E) }\end{array}$ & $\begin{array}{c}\mathrm{L} \\
(\mathrm{M})\end{array}$ & $\begin{array}{l}\text { M } \\
\text { (L) }\end{array}$ \\
\hline 0 & 0.0 & 0.509 & 0.454 & 0.483 & 0.536 & 0.620 & 0.611 \\
\hline 1 & 25.0 & & 0.478 & 10 & 0.567 & & \\
\hline 2 & 50.0 & 35 & 0.476 & 0.509 & 0.569 & 0.654 & דודט. \\
\hline 3 & 100.0 & 528 & 0.471 & 0.500 & 0.557 & 0.647 & 0.636 \\
\hline 4 & 200.0 & & 0.456 & 0.485 & 0.538 & & \\
\hline 5 & 300.0 & 1 & 0.446 & 0.473 & 0.527 & 2 & 0.605 \\
\hline 6 & 400.0 & & 0.445 & 0.469 & 0.521 & 0 & 602 \\
\hline 7 & 500.0 & & 0.444 & 0.468 & 0.520 & 9 & \\
\hline 8 & 600.0 & 8 & 0.446 & 0.472 & 0.524 & 88 & 0.603 \\
\hline 9 & 70 & & 0.450 & & 0.524 & & \\
\hline 10 & 800.0 & & 0.451 & 0.474 & 0.527 & & 608 \\
\hline 11 & 900.0 & & 0.461 & 0.483 & 0.537 & 5 & 20 \\
\hline 12 & 1000.0 & & 0.462 & 38 & 0.542 & & 25 \\
\hline 13 & 00.0 & & 0.471 & 0.495 & 0.549 & & 0.636 \\
\hline 14 & 00.0 & & 0.477 & 1 & 0.557 & & \\
\hline 15 & 00.0 & & 0.481 & 0.505 & 0.561 & 0.655 & 0.649 \\
\hline \multirow[t]{2}{*}{ Step } & Burnup & \multicolumn{6}{|c|}{ MPACT Power Peaking Factors } \\
\hline & MWd & $\begin{array}{c}E \\
(H)\end{array}$ & $\begin{array}{c}\mathrm{F} \\
(\mathrm{G})\end{array}$ & $\begin{array}{c}\text { J } \\
(\mathrm{F})\end{array}$ & $\begin{array}{c}\mathrm{K} \\
\text { (E) }\end{array}$ & $\begin{array}{c}\mathrm{L} \\
(\mathrm{M})\end{array}$ & $\begin{array}{l}\text { M } \\
\text { (L) }\end{array}$ \\
\hline 0 & 0.0 & 0.505 & 0.451 & 0.479 & 0.533 & 0.618 & 0.609 \\
\hline 1 & 25.0 & & 0.481 & 10 & 0.565 & & 42 \\
\hline 2 & 50.0 & & 0.476 & 0.505 & 0.559 & 0.6 & 0.636 \\
\hline 3 & 100.0 & 1 & 0.466 & 0.495 & 0.549 & 0.6 & 0.625 \\
\hline 4 & 200.0 & 6 & 0.452 & 80 & 0.534 & 9 & 0.610 \\
\hline 5 & 300.0 & & 0.445 & 0.472 & 0.526 & & 0.602 \\
\hline 6 & 400.0 & 0.4 & 0.441 & 0.469 & 0.522 & 88 & .598 \\
\hline 7 & 500.0 & & 0.440 & 0.467 & 0.521 & 0.607 & 0.598 \\
\hline 0 & 600.0 & & 0.440 & 0.468 & 0.522 & 0.609 & 0.599 \\
\hline 9 & 700.0 & 0.497 & 0.442 & 0.470 & 0.525 & 0.612 & 0.602 \\
\hline 10 & 800.0 & 0.501 & 0.445 & 0.473 & 0.528 & 0.616 & 0.606 \\
\hline 11 & 900.0 & 0.505 & 0.449 & 0.477 & 0.533 & 0.620 & 0.611 \\
\hline 12 & 1000.0 & 0.510 & 0.453 & 0.482 & 0.538 & 0.626 & 0.616 \\
\hline 13 & 1100.0 & & 0.458 & 0.487 & 0.543 & 0.632 & 0.622 \\
\hline 14 & 1200.0 & 0.521 & 0.463 & 0.492 & 0.549 & 0.638 & 0.628 \\
\hline 15 & 1300.0 & 0.526 & 0.468 & 0.498 & 0.555 & 0.645 & 0.635 \\
\hline
\end{tabular}




\section{Charge-Pan 0828}

Table 4.23. Problem 3.2.1 reference solution power peaking factors for charge-pan 0828 .

\begin{tabular}{|c|c|c|c|c|c|c|c|c|c|c|c|}
\hline \multirow[t]{2}{*}{ Step } & Burnup & \multicolumn{10}{|c|}{ Shift Power Peaking Factors } \\
\hline & $\mathrm{MWd}$ & A & $\mathrm{B}, \mathrm{D}$ & $\mathrm{C}$ & $\mathrm{G}, \mathrm{F}$ & $\mathrm{H}, \mathrm{E}$ & $\mathrm{J}, \mathrm{M}$ & $\mathrm{K}, \mathrm{L}$ & Q & $\mathrm{R}, \mathrm{P}$ & $S$ \\
\hline 0 & 0.0 & 1.436 & 1.465 & 1.496 & 1.415 & 1.446 & 1.502 & 1.536 & 1.387 & 1.474 & 1.566 \\
\hline 1 & 25.0 & 1.454 & 1.483 & 1.513 & 1.430 & 1.467 & 1.530 & 1.564 & 1.392 & .500 & 1.596 \\
\hline 2 & 50.0 & 1.450 & 1.484 & 1.517 & 1.429 & 1.466 & 1.533 & 1.562 & 1.393 & 1.502 & 1.594 \\
\hline 3 & 100.0 & 1.444 & 1.477 & 1.509 & 1.423 & 1.461 & 1.525 & 1.559 & 1.387 & 1.494 & 1.590 \\
\hline 4 & 200.0 & 1.432 & 1.474 & 1.503 & 1.416 & 1.457 & 1.513 & 1.543 & 1.384 & 1.484 & 1.575 \\
\hline 5 & 300.0 & 26 & 1.471 & 1. & 1.409 & 1.457 & 1.507 & 1.540 & 1.379 & 181 & .569 \\
\hline 6 & 400.0 & 1.418 & 1.468 & 1.496 & 1.403 & 1.452 & 1.503 & 1.532 & 1.377 & 1.475 & 1.563 \\
\hline 7 & 500.0 & 1.420 & 1.475 & 1.501 & 1.406 & 1.458 & 1.511 & 1.537 & 1.378 & 1.484 & 1.570 \\
\hline 8 & 600.0 & 23 & 1.479 & 1.511 & 1.408 & 1.464 & 1.519 & 1.547 & 1.381 & 90 & 1.576 \\
\hline 9 & 700.0 & 1.423 & 1.486 & 1.509 & 1.411 & 1.468 & 1.522 & 1.552 & 1.378 & 1.497 & 1.583 \\
\hline 10 & .0 & & 1.493 & 1.5 & 1.412 & 1.474 & 1.535 & 1.564 & 1.382 & 03 & 1.597 \\
\hline 11 & 900.0 & 1 & 1.494 & 1.518 & 1.412 & 1.477 & 1.537 & 1.564 & 1.380 & 1.509 & 1.597 \\
\hline 12 & 1000.0 & 1.4 & 1.500 & 1.531 & 1.420 & 1.483 & 1.544 & 1.574 & 1.386 & 1. & 1.606 \\
\hline 13 & 1100.0 & & 1.509 & & 1.423 & 38 & 1.551 & 1.583 & 1.385 & 28 & 1.616 \\
\hline 14 & 1200.0 & 1.442 & 1.513 & 1.540 & 1.425 & 1.491 & 1.556 & 1.588 & 1.385 & 1.528 & 1.623 \\
\hline 15 & 1300.0 & & 1. & & 1.429 & 1.496 & 1.563 & 1.597 & 1.388 & & 1.630 \\
\hline \multirow[t]{2}{*}{ Step } & Burnup & \multicolumn{10}{|c|}{ MPACT Power Peaking Factors } \\
\hline & $\frac{\mathrm{MWd}}{\mathrm{MtU}}$ & A & $\mathrm{B}, \mathrm{D}$ & $\mathrm{C}$ & $\mathrm{G}, \mathrm{F}$ & $\mathrm{H}, \mathrm{E}$ & $\mathrm{J}, \mathrm{M}$ & $\mathrm{K}, \mathrm{L}$ & Q & $\mathrm{R}, \mathrm{P}$ & $S$ \\
\hline 0 & 0.0 & 1.440 & 1.469 & 1.497 & 1.421 & 1.452 & 1.510 & 1.540 & 1.390 & 1.481 & 1.569 \\
\hline 1 & 25.0 & 1.392 & 1.415 & 1.437 & 1.380 & 1.405 & 1.450 & 1.473 & 1.355 & 1.428 & 1.497 \\
\hline 2 & 50.0 & 1.401 & 1.425 & 1.448 & 1.387 & 1.413 & 1.461 & 1.486 & 1.361 & 1.438 & 1.511 \\
\hline 3 & 100.0 & 1. & 1.443 & 1.469 & 1.401 & 1.429 & 1.482 & 1.509 & 1.373 & 1.456 & 1.536 \\
\hline 4 & 200.0 & 1.439 & 1.468 & 1.496 & 1.420 & 1.451 & 1.510 & 1.540 & 1.389 & 1.481 & 1.569 \\
\hline 5 & 300.0 & & 1.480 & 1.5 & 1.430 & 1.462 & 1.523 & 1.554 & 1.397 & 1.493 & 1.585 \\
\hline 6 & 400.0 & 1.455 & 1.485 & 1.515 & 1.434 & 1.467 & 1.528 & 1.560 & 1.401 & 1.498 & 1.591 \\
\hline 7 & 500.0 & 1.455 & 1.485 & 1.515 & 1.435 & 1.467 & 1.528 & 1.560 & 1.402 & 1.498 & 1.591 \\
\hline 8 & 600.0 & 1.453 & 1.482 & 1.511 & 1.433 & 1.465 & 1.524 & 1.555 & 1.401 & 1.495 & 1.585 \\
\hline 9 & 700.0 & 1.448 & 1.476 & 1.504 & 1.429 & 1.460 & 1.518 & 1.547 & 1.398 & 1.489 & 1.576 \\
\hline 10 & 800.0 & 1.441 & 1.468 & 1.496 & 1.424 & 1.453 & 1.509 & 1.537 & 1.393 & 1.481 & 1.565 \\
\hline 11 & 900.0 & 1.433 & 1.460 & 1.486 & 1.417 & 1.446 & 1.499 & 1.526 & 1.388 & 1.473 & 1.552 \\
\hline 12 & 1000.0 & 1.425 & 1.450 & 1.474 & 1.410 & 1.437 & 1.488 & 1.513 & 1.382 & 1.463 & 1.539 \\
\hline 13 & 1100.0 & 1.416 & 1.439 & 1.463 & 1.402 & 1.428 & 1.476 & 1.500 & 1.376 & 1.453 & 1.524 \\
\hline 14 & 1200.0 & 1.406 & 1.429 & 1.451 & 1.394 & 1.419 & 1.464 & 1.486 & 1.369 & 1.442 & 1.509 \\
\hline 15 & 1300.0 & 1.397 & 1.418 & 1.438 & 1.386 & 1.409 & 1.451 & 1.473 & 1.363 & 1.432 & 1.494 \\
\hline
\end{tabular}


Charge-Pans 0928 and (0829)

Table 4.24. Problem 3.2.1 reference solution power peaking factors for charge-pans 0928 and 0829 .

\begin{tabular}{|c|c|c|c|c|c|c|c|c|c|}
\hline \multirow[t]{3}{*}{ Step } & \multirow{3}{*}{$\begin{array}{l}\text { Burnup } \\
{\left[\frac{\mathrm{MWd}}{\mathrm{MtU}}\right]}\end{array}$} & \multicolumn{8}{|c|}{ Shift Power Peaking Factors } \\
\hline & & A & B & $\mathrm{C}$ & $\mathrm{D}$ & $\mathrm{P}$ & Q & $\mathrm{R}$ & S \\
\hline & & (A) & (D) & (C) & (B) & $(\mathrm{R})$ & $(\mathrm{Q})$ & (P) & (S) \\
\hline 0 & 0.0 & 1.274 & 1.301 & 1.349 & 1.322 & 1.345 & 1.194 & 1.289 & 1.436 \\
\hline 1 & 25.0 & 1.280 & 1.317 & 1.365 & 1.329 & 1.349 & 1.193 & 1.308 & 1.463 \\
\hline 2 & 50.0 & 1.284 & 1.320 & 1.368 & 1.329 & 1.349 & 1.196 & 1.315 & 1.462 \\
\hline 3 & 100.0 & 1.276 & 1.314 & 1.364 & 1.326 & 1.346 & 1.196 & 1.305 & 1.456 \\
\hline 4 & 200.0 & 1.276 & 1.310 & 1.356 & 1.324 & 1.349 & 1.199 & 1.299 & 1.448 \\
\hline 5 & 300.0 & 1.273 & 1.305 & 1.354 & 1.320 & 1.350 & 1.197 & 1.290 & 1.441 \\
\hline 6 & 400.0 & 1.270 & 1.305 & 1.349 & 1.319 & 1.349 & 1.199 & 1.289 & 1.440 \\
\hline 7 & 500.0 & 1.276 & 1.310 & 1.360 & 1.326 & 1.354 & 1.202 & 1.296 & 1.444 \\
\hline 8 & 600.0 & 1.280 & 1.316 & 1.366 & 1.329 & 1.355 & 1.205 & 1.303 & 1.449 \\
\hline 9 & 700.0 & 1.283 & 1.320 & 1.368 & 1.330 & 1.358 & 1.209 & 1.305 & 1.454 \\
\hline 10 & 800.0 & 1.286 & 1.323 & 1.372 & 1.335 & 1.359 & 1.209 & 1.312 & 1.462 \\
\hline 11 & 900.0 & 1.286 & 1.326 & 1.376 & 1.336 & 1.356 & 1.210 & 1.318 & 1.467 \\
\hline 12 & 1000.0 & 1.292 & 1.336 & 1.385 & 1.342 & 1.361 & 1.214 & 1.330 & 1.476 \\
\hline 13 & 1100.0 & 1.297 & 1.342 & 1.390 & 1.344 & 1.363 & 1.217 & 1.339 & 1.485 \\
\hline 14 & 1200.0 & 1.297 & 1.342 & 1.391 & 1.345 & 1.363 & 1.219 & 1.342 & 1.491 \\
\hline 15 & 1300.0 & 1.298 & 1.349 & 1.397 & 1.345 & 1.360 & 1.214 & 1.348 & 1.496 \\
\hline \multirow[t]{3}{*}{ Step } & Burnup & \multicolumn{8}{|c|}{ MPACT Power Peaking Factors } \\
\hline & $\frac{\mathrm{MWd}}{\mathrm{MtU}}$ & A & B & $\mathrm{C}$ & $\mathrm{D}$ & $\mathrm{P}$ & Q & $\mathrm{R}$ & S \\
\hline & & (A) & (D) & (C) & (B) & (R) & $(\mathrm{Q})$ & $(\mathrm{P})$ & (S) \\
\hline 0 & 0.0 & 1.277 & 1.307 & 1.356 & 1.326 & 1.350 & 1.197 & 1.289 & 1.444 \\
\hline 1 & 25.0 & 1.260 & 1.284 & 1.325 & 1.301 & 1.322 & 1.194 & 1.272 & 1.400 \\
\hline 2 & 50.0 & 1.262 & 1.288 & 1.330 & 1.305 & 1.327 & 1.194 & 1.275 & 1.408 \\
\hline 3 & 100.0 & 1.268 & 1.295 & 1.340 & 1.313 & 1.336 & 1.194 & 1.280 & 1.423 \\
\hline 4 & 200.0 & 1.276 & 1.305 & 1.355 & 1.325 & 1.349 & 1.197 & 1.288 & 1.443 \\
\hline 5 & 300.0 & 1.280 & 1.311 & 1.362 & 1.331 & 1.356 & 1.198 & 1.293 & 1.454 \\
\hline 6 & 400.0 & 1.283 & 1.314 & 1.365 & 1.334 & 1.359 & 1.199 & 1.295 & 1.458 \\
\hline 7 & 500.0 & 1.284 & 1.315 & 1.366 & 1.335 & 1.360 & 1.201 & 1.296 & 1.459 \\
\hline 8 & 600.0 & 1.284 & 1.314 & 1.365 & 1.334 & 1.359 & 1.202 & 1.296 & 1.456 \\
\hline 9 & 700.0 & 1.283 & 1.313 & 1.363 & 1.333 & 1.357 & 1.202 & 1.296 & 1.452 \\
\hline 10 & 800.0 & 1.282 & 1.311 & 1.359 & 1.330 & 1.354 & 1.203 & 1.294 & 1.446 \\
\hline 11 & 900.0 & 1.280 & 1.308 & 1.355 & 1.327 & 1.350 & 1.204 & 1.292 & 1.439 \\
\hline 12 & 1000.0 & 1.278 & 1.305 & 1.350 & 1.323 & 1.346 & 1.204 & 1.290 & 1.431 \\
\hline 13 & 1100.0 & 1.275 & 1.301 & 1.345 & 1.319 & 1.341 & 1.204 & 1.288 & 1.422 \\
\hline 14 & 1200.0 & 1.272 & 1.298 & 1.339 & 1.314 & 1.336 & 1.204 & 1.285 & 1.414 \\
\hline 15 & 1300.0 & 1.269 & 1.293 & 1.333 & 1.309 & 1.331 & 1.204 & 1.282 & 1.405 \\
\hline
\end{tabular}


Table 4.25. Problem 3.2.1 reference solution power peaking factors for charge-pans 0928 and 0829.

\begin{tabular}{|c|c|c|c|c|c|c|c|c|c|}
\hline \multirow[t]{2}{*}{ Step } & \multirow{2}{*}{$\begin{array}{l}\text { Burnup } \\
{\left[\frac{\mathrm{MWd}}{\mathrm{MtU}}\right]}\end{array}$} & \multicolumn{8}{|c|}{ Shift Power Peaking Factors } \\
\hline & & $\begin{array}{c}E \\
(H)\end{array}$ & $\begin{array}{c}F \\
(\mathrm{G})\end{array}$ & $\begin{array}{c}\mathrm{G} \\
(\mathrm{F})\end{array}$ & $\begin{array}{c}\mathrm{H} \\
(\mathrm{E})\end{array}$ & $\begin{array}{c}\mathrm{J} \\
(\mathrm{M})\end{array}$ & $\begin{array}{c}\mathrm{K} \\
(\mathrm{L})\end{array}$ & $\begin{array}{c}\mathrm{L} \\
(\mathrm{K})\end{array}$ & $\begin{array}{l}M \\
(J)\end{array}$ \\
\hline 0 & 0.0 & 1.298 & 1.248 & 1.229 & 1.261 & 1.343 & 1.394 & 1.409 & 1.379 \\
\hline 1 & 25.0 & 1.298 & 1.246 & 1.234 & 1.272 & 1.362 & 1.416 & 1.424 & 1.387 \\
\hline 2 & 50.0 & 1.301 & 1.250 & 1.238 & 1.277 & 1.368 & 1.420 & 1.431 & 1.389 \\
\hline 3 & 100.0 & 1.296 & 1.248 & 1.234 & 1.274 & 1.361 & 1.413 & 1.422 & 1.385 \\
\hline 4 & 200.0 & 1.305 & 1.252 & 1.231 & 1.265 & 1.350 & 1.403 & 1.418 & 1.386 \\
\hline 5 & 300.0 & 1.303 & 1.251 & 1.230 & 1.262 & 1.345 & 1.397 & 1.412 & 1.382 \\
\hline 6 & 400.0 & 1.302 & 1.252 & 1.227 & 1.259 & 1.342 & 1.392 & 1.409 & 1.378 \\
\hline 7 & 500.0 & 1.307 & 1.257 & 1.231 & 1.265 & 1.350 & 1.399 & 1.417 & 1.383 \\
\hline 8 & 600.0 & 1.309 & 1.258 & 1.236 & 1.271 & 1.356 & 1.406 & 1.422 & 1.387 \\
\hline 9 & 700.0 & 1.310 & 1.259 & 1.238 & 1.273 & 1.360 & 1.411 & 1.424 & 1.390 \\
\hline 10 & 800.0 & 1.311 & 1.259 & 1.242 & 1.282 & 1.366 & 1.418 & 1.432 & 1.391 \\
\hline 11 & 900.0 & 1.310 & 1.262 & 1.243 & 1.288 & 1.372 & 1.424 & 1.431 & 1.392 \\
\hline 12 & 1000.0 & 1.314 & 1.263 & 1.250 & 1.292 & 1.384 & 1.435 & 1.440 & 1.398 \\
\hline 13 & 1100.0 & 1.316 & 1.268 & 1.254 & 1.299 & 1.394 & 1.445 & 1.446 & 1.400 \\
\hline 14 & 1200.0 & 1.313 & 1.262 & 1.258 & 1.303 & 1.398 & 1.449 & 1.449 & 1.401 \\
\hline 15 & 1300.0 & 1.313 & 1.262 & 1.257 & 1.305 & 1.405 & 1.455 & 1.453 & 1.404 \\
\hline \multirow[t]{2}{*}{ Step } & Burnup & \multicolumn{8}{|c|}{ MPACT Power Peaking Factors } \\
\hline & $\frac{\mathrm{MWd}}{\mathrm{M}}$ & $\begin{array}{c}E \\
(H)\end{array}$ & $\begin{array}{c}F \\
(G)\end{array}$ & $\begin{array}{c}G \\
(F)\end{array}$ & $\begin{array}{l}\mathrm{H} \\
(\mathrm{E})\end{array}$ & $\begin{array}{c}\mathrm{J} \\
(\mathrm{M})\end{array}$ & $\begin{array}{c}\mathrm{K} \\
\text { (L) }\end{array}$ & $\begin{array}{c}\mathrm{L} \\
(\mathrm{K})\end{array}$ & $\begin{array}{l}\mathrm{M} \\
(\mathrm{J})\end{array}$ \\
\hline 0 & 0.0 & 1.303 & 1.252 & 1.230 & 1.261 & 1.346 & 1.398 & 1.415 & 1.383 \\
\hline 1 & 25.0 & 1.283 & 1.240 & 1.221 & 1.247 & 1.319 & 1.362 & 1.375 & 1.349 \\
\hline 2 & 50.0 & 1.286 & 1.241 & 1.222 & 1.249 & 1.324 & 1.368 & 1.382 & 1.355 \\
\hline 3 & 100.0 & 1.293 & 1.245 & 1.225 & 1.253 & 1.332 & 1.380 & 1.395 & 1.367 \\
\hline 4 & 200.0 & 1.302 & 1.251 & 1.229 & 1.260 & 1.345 & 1.397 & 1.414 & 1.382 \\
\hline 5 & 300.0 & 1.307 & 1.254 & 1.232 & 1.263 & 1.351 & 1.406 & 1.423 & 1.390 \\
\hline 6 & 400.0 & 1.310 & 1.256 & 1.234 & 1.266 & 1.355 & 1.409 & 1.427 & 1.394 \\
\hline 7 & 500.0 & 1.311 & 1.257 & 1.235 & 1.267 & 1.355 & 1.410 & 1.428 & 1.395 \\
\hline 8 & 600.0 & 1.311 & 1.258 & 1.236 & 1.267 & 1.355 & 1.409 & 1.426 & 1.394 \\
\hline 9 & 700.0 & 1.310 & 1.258 & 1.236 & 1.267 & 1.353 & 1.405 & 1.422 & 1.391 \\
\hline 10 & 800.0 & 1.308 & 1.257 & 1.236 & 1.266 & 1.350 & 1.401 & 1.417 & 1.387 \\
\hline 11 & 900.0 & 1.306 & 1.256 & 1.235 & 1.265 & 1.346 & 1.396 & 1.411 & 1.382 \\
\hline 12 & 1000.0 & 1.303 & 1.255 & 1.235 & 1.264 & 1.342 & 1.390 & 1.404 & 1.376 \\
\hline 13 & 1100.0 & 1.300 & 1.253 & 1.234 & 1.262 & 1.338 & 1.383 & 1.397 & 1.370 \\
\hline 14 & 1200.0 & 1.296 & 1.252 & 1.233 & 1.260 & 1.333 & 1.376 & 1.389 & 1.364 \\
\hline 15 & 1300.0 & 1.293 & 1.250 & 1.231 & 1.257 & 1.328 & 1.369 & 1.381 & 1.357 \\
\hline
\end{tabular}


Charge-Pans 1028 and (0830)

Table 4.26. Problem 3.2.1 reference solution power peaking factors for charge-pans 1028 and 0830 .

\begin{tabular}{|c|c|c|c|c|c|c|c|c|c|}
\hline \multirow[t]{3}{*}{ Step } & \multirow{3}{*}{$\begin{array}{l}\text { Burnup } \\
{\left[\frac{\mathrm{MWd}}{\mathrm{MtU}}\right]}\end{array}$} & \multicolumn{8}{|c|}{ Shift Power Peaking Factors } \\
\hline & & A & B & $\mathrm{C}$ & $\mathrm{D}$ & $\mathrm{P}$ & Q & $\mathrm{R}$ & S \\
\hline & & (A) & (D) & (C) & (B) & $(\mathrm{R})$ & $(\mathrm{Q})$ & $(\mathrm{P})$ & (S) \\
\hline 0 & 0.0 & 1.021 & 1.047 & 1.115 & 1.090 & 1.137 & 0.929 & 1.016 & 1.226 \\
\hline 1 & 25.0 & 1.031 & 1.067 & 1.131 & 1.099 & 1.137 & 0.932 & 1.042 & 1.249 \\
\hline 2 & 50.0 & 1.037 & 1.069 & 1.134 & 1.097 & 1.136 & 0.937 & 1.044 & 1.251 \\
\hline 3 & 100.0 & 1.031 & 1.065 & 1.130 & 1.095 & 1.137 & 0.937 & 1.037 & 1.241 \\
\hline 4 & 200.0 & 1.026 & 1.057 & 1.123 & 1.090 & 1.137 & 0.932 & 1.024 & 1.230 \\
\hline 5 & 300.0 & 1.020 & 1.048 & 1.110 & 1.083 & 1.134 & 0.927 & 1.016 & 1.220 \\
\hline 6 & 400.0 & 1.018 & 1.045 & 1.111 & 1.083 & 1.135 & 0.927 & 1.012 & 1.217 \\
\hline 7 & 500.0 & 1.021 & 1.050 & 1.114 & 1.086 & 1.141 & .931 & 1.016 & 1.222 \\
\hline 8 & 600.0 & 1.026 & 1.052 & 1.119 & 1.092 & 1.140 & 0.936 & 1.023 & 1.226 \\
\hline 9 & 700.0 & 1.031 & 1.059 & 1.124 & 1.095 & 1.146 & 0.938 & 1.028 & 1.231 \\
\hline 10 & 800.0 & 1.030 & 1.062 & 1.130 & 1.097 & 1.147 & 0.940 & 1.028 & 1.238 \\
\hline 11 & 900.0 & 1.039 & 1.071 & 1.136 & 1.103 & 1.152 & 0.947 & 1.042 & 1.246 \\
\hline 12 & 1000.0 & 1.045 & 1.077 & 1.141 & 1.109 & 1.157 & 52 & 46 & 1.255 \\
\hline 13 & 1100.0 & 1.051 & 1.085 & 1.150 & 1.114 & 1.156 & 0.958 & 1.058 & 1.266 \\
\hline 14 & 1200.0 & 1.054 & 1.092 & 1.154 & 1.120 & 1.160 & 0.960 & 1.066 & 1.272 \\
\hline 15 & 1300.0 & 1.061 & 1.096 & 1.160 & 1.124 & 1.161 & 0.964 & 1.074 & 1.277 \\
\hline \multirow[t]{3}{*}{ Step } & Burnup & \multicolumn{8}{|c|}{ MPACT Power Peaking Factors } \\
\hline & & A & B & $\mathrm{C}$ & $\mathrm{D}$ & $\mathrm{P}$ & Q & $\mathrm{R}$ & S \\
\hline & & (A) & (D) & (C) & (B) & $(\mathrm{R})$ & $(\mathrm{Q})$ & $(\mathrm{P})$ & (S) \\
\hline 0 & 0.0 & 1.021 & 1.049 & 1.115 & 1.088 & 1.137 & 0.927 & 1.015 & 1.225 \\
\hline 1 & 25.0 & 1.035 & 1.060 & 1.120 & 1.095 & 1.141 & 0.950 & 1.031 & 1.217 \\
\hline 2 & 50.0 & 1.032 & 1.058 & 1.118 & 1.093 & 1.140 & 0.945 & 1.028 & 1.218 \\
\hline 3 & 100.0 & 1.027 & 1.053 & 1.116 & 1.090 & 1.138 & 0.937 & 1.022 & 1.220 \\
\hline 4 & 200.0 & 1.021 & 1.048 & 1.114 & 1.087 & 1.137 & 0.926 & 1.014 & 1.224 \\
\hline 5 & 300.0 & 1.018 & 1.046 & 1.114 & 1.086 & 1.137 & 0.922 & 1.011 & 1.227 \\
\hline 6 & 400.0 & 1.017 & 1.046 & 1.115 & 1.086 & 1.137 & 0.920 & 1.010 & 1.228 \\
\hline 7 & 500.0 & 1.018 & 1.047 & 1.116 & 1.087 & 1.138 & 0.920 & 1.011 & 1.230 \\
\hline 8 & 600.0 & 1.020 & 1.049 & 1.117 & 1.089 & 1.140 & 0.923 & 1.013 & 1.230 \\
\hline 9 & 700.0 & 1.023 & 1.051 & 1.119 & 1.091 & 1.141 & 0.926 & 1.016 & 1.231 \\
\hline 10 & 800.0 & 1.026 & 1.054 & 1.121 & 1.093 & 1.143 & 0.930 & 1.019 & 1.231 \\
\hline 11 & 900.0 & 1.029 & 1.057 & 1.123 & 1.095 & 1.145 & 0.934 & 1.023 & 1.231 \\
\hline 12 & 1000.0 & 1.032 & 1.060 & 1.124 & 1.097 & 1.147 & 0.939 & 1.027 & 1.230 \\
\hline 13 & 1100.0 & 1.036 & 1.063 & 1.126 & 1.100 & 1.148 & 0.944 & 1.031 & 1.229 \\
\hline 14 & 1200.0 & 1.039 & 1.066 & 1.128 & 1.102 & 1.150 & 0.949 & 1.035 & 1.228 \\
\hline 15 & 1300.0 & 1.043 & 1.069 & 1.129 & 1.104 & 1.151 & 0.954 & 1.038 & 1.227 \\
\hline
\end{tabular}


Table 4.27. Problem 3.2.1 reference solution power peaking factors for charge-pans 1028 and 0830 .

\begin{tabular}{|c|c|c|c|c|c|c|c|c|c|}
\hline \multirow[t]{3}{*}{ Step } & \multirow{3}{*}{$\begin{array}{l}\text { Burnup } \\
{\left[\frac{\mathrm{MWd}}{\mathrm{MtU}}\right]}\end{array}$} & \multicolumn{8}{|c|}{ Shift Power Peaking Factors } \\
\hline & & E & $\mathrm{F}$ & $\mathrm{G}$ & $\mathrm{H}$ & $\mathbf{J}$ & $\mathrm{K}$ & $\mathrm{L}$ & M \\
\hline & & $(\mathrm{H})$ & $(\mathrm{G})$ & $(\mathrm{F})$ & $(\mathrm{E})$ & $(\mathrm{M})$ & $(\mathrm{L})$ & $(\mathrm{K})$ & $(\mathrm{J})$ \\
\hline 0 & 0.0 & 1.069 & 1.001 & 0.959 & 0.990 & 1.086 & 1.157 & 1.195 & 1.167 \\
\hline 1 & 25.0 & 1.073 & 1.003 & 0.971 & 1.008 & 1.114 & 1.179 & 1.213 & 1.174 \\
\hline 2 & 50.0 & 1.071 & 1.003 & 0.973 & 1.012 & 1.113 & 1.182 & 1.214 & 1.174 \\
\hline 3 & 100.0 & 1.071 & 1.004 & 0.969 & 1.003 & 1.106 & 1.172 & 1.208 & 1.169 \\
\hline 4 & 200.0 & 1.071 & 1.001 & 0.964 & 0.997 & 1.094 & 1.163 & 1.200 & 1.165 \\
\hline 5 & 300.0 & 1.071 & 1.000 & 0.957 & 0.986 & 1.083 & 1.151 & 1.191 & 1.162 \\
\hline 6 & 400.0 & 1.070 & 0.998 & 0.957 & 0.987 & 1.080 & 1.152 & 1.192 & 1.161 \\
\hline 7 & 500.0 & 1.073 & 1.001 & 0.958 & 0.988 & 1.083 & 1.152 & 1.193 & 1.166 \\
\hline 8 & 600.0 & 1.076 & 1.008 & 0.963 & 0.998 & 1.088 & 1.161 & 1.200 & 1.171 \\
\hline 9 & 700.0 & 1.082 & 1.011 & 0.970 & 1.001 & 1.094 & 1.163 & 1.205 & 1.173 \\
\hline 10 & 800.0 & 1.079 & 1.008 & 0.969 & 1.001 & 1.098 & 1.168 & 1.208 & 1.177 \\
\hline 11 & 900.0 & 1.088 & 1.016 & 0.978 & 1.010 & 1.110 & 1.180 & 1.215 & 1.180 \\
\hline 12 & 1000.0 & 1.093 & 1.022 & 0.982 & 1.018 & 1.116 & 1.186 & 1.226 & 1.186 \\
\hline 13 & 1100.0 & 1.094 & 1.026 & 0.991 & 1.027 & 1.128 & 1.197 & 1.226 & 1.191 \\
\hline 14 & 1200.0 & 1.097 & 1.030 & 0.997 & 1.032 & 1.134 & 1.201 & 1.233 & 1.196 \\
\hline 15 & 1300.0 & 1.098 & 1.035 & 1.002 & 1.040 & 1.140 & 1.212 & 1.238 & 1.198 \\
\hline \multirow[t]{3}{*}{ Step } & Burnup & \multicolumn{8}{|c|}{ MPACT Power Peaking Factors } \\
\hline & {$\left[\frac{\mathrm{MWd}}{\mathrm{MtU}}\right]$} & E & F & $\mathrm{G}$ & $\mathrm{H}$ & $\mathrm{J}$ & K & $\mathrm{L}$ & M \\
\hline & & $(\mathrm{H})$ & $(\mathrm{G})$ & $(\mathrm{F})$ & $(\mathrm{E})$ & $(\mathrm{M})$ & $(\mathrm{L})$ & $(\mathrm{K})$ & $(\mathrm{J})$ \\
\hline 0 & 0.0 & 1.069 & 0.998 & 0.958 & 0.987 & 1.084 & 1.155 & 1.197 & 1.167 \\
\hline 1 & 25.0 & 1.080 & 1.016 & 0.978 & 1.006 & 1.093 & 1.156 & 1.192 & 1.166 \\
\hline 2 & 50.0 & 1.078 & 1.012 & 0.974 & 1.002 & 1.091 & 1.155 & 1.192 & 1.166 \\
\hline 3 & 100.0 & 1.073 & 1.006 & 0.967 & 0.995 & 1.087 & 1.154 & 1.193 & 1.165 \\
\hline 4 & 200.0 & 1.069 & 0.998 & 0.957 & 0.987 & 1.084 & 1.154 & 1.196 & 1.166 \\
\hline 5 & 300.0 & 1.067 & 0.995 & 0.953 & 0.983 & 1.082 & 1.154 & 1.197 & 1.167 \\
\hline 6 & 400.0 & 1.066 & 0.994 & 0.952 & 0.982 & 1.082 & 1.155 & 1.199 & 1.168 \\
\hline 7 & 500.0 & 1.067 & 0.994 & 0.952 & 0.983 & 1.083 & 1.156 & 1.200 & 1.169 \\
\hline 8 & 600.0 & 1.069 & 0.996 & 0.955 & 0.985 & 1.085 & 1.158 & 1.201 & 1.170 \\
\hline 9 & 700.0 & 1.072 & 0.999 & 0.958 & 0.988 & 1.087 & 1.159 & 1.202 & 1.171 \\
\hline 10 & 800.0 & 1.074 & 1.003 & 0.961 & 0.992 & 1.090 & 1.161 & 1.202 & 1.172 \\
\hline 11 & 900.0 & 1.077 & 1.006 & 0.966 & 0.996 & 1.092 & 1.162 & 1.203 & 1.174 \\
\hline 12 & 1000.0 & 1.080 & 1.010 & 0.970 & 1.000 & 1.095 & 1.163 & 1.203 & 1.174 \\
\hline 13 & 1100.0 & 1.083 & 1.015 & 0.975 & 1.004 & 1.097 & 1.164 & 1.203 & 1.175 \\
\hline 14 & 1200.0 & 1.086 & 1.019 & 0.979 & 1.008 & 1.100 & 1.165 & 1.203 & 1.176 \\
\hline 15 & 1300.0 & 1.089 & 1.023 & 0.984 & 1.012 & 1.102 & 1.166 & 1.202 & 1.176 \\
\hline
\end{tabular}


Charge-Pans 1128 and (0831)

Table 4.28. Problem 3.2.1 reference solution power peaking factors for charge-pans 1128 and 0831 .

\begin{tabular}{|c|c|c|c|c|c|c|c|c|c|}
\hline \multirow[t]{3}{*}{ Step } & \multirow{3}{*}{$\begin{array}{l}\text { Burnup } \\
{\left[\frac{\mathrm{MWd}}{\mathrm{MtU}}\right]}\end{array}$} & \multicolumn{8}{|c|}{ Shift Power Peaking Factors } \\
\hline & & A & B & $\mathrm{C}$ & $\mathrm{D}$ & $\mathrm{P}$ & Q & $\mathrm{R}$ & $S$ \\
\hline & & (A) & (D) & (C) & (B) & $(\mathrm{R})$ & (Q) & $(\mathrm{P})$ & (S) \\
\hline 0 & 0.0 & 0.706 & 0.735 & 0.816 & 0.786 & 0.844 & 0.603 & 0.672 & 0.937 \\
\hline 1 & 25.0 & 0.724 & 0.758 & 0.840 & 0.807 & 0.857 & 0.613 & 0.701 & 0.967 \\
\hline 2 & 50.0 & 0.721 & 0.758 & 0.841 & 0.805 & 0.855 & 0.613 & 0.701 & 0.970 \\
\hline 3 & 100.0 & 0.720 & 0.753 & 0.835 & 0.800 & 0.853 & 0.613 & 0.694 & 0.960 \\
\hline 4 & 200.0 & 0.710 & 0.739 & 0.823 & 0.794 & 0.849 & 0.605 & 0.679 & 0.943 \\
\hline 5 & 300.0 & 0.700 & 0.729 & 0.811 & 0.784 & 0.844 & 0.600 & 0.667 & 0.930 \\
\hline 6 & 400.0 & 0.702 & 0.729 & 0.812 & 0.784 & 0.845 & 0.602 & 0.668 & 0.927 \\
\hline 7 & 500.0 & 0.702 & 0.728 & 0.811 & 0.784 & 0.843 & 0.601 & 0.667 & 0.928 \\
\hline 8 & 600.0 & 0.705 & 0.734 & 0.816 & 0.787 & 0.849 & 0.605 & 0.672 & 0.933 \\
\hline 9 & 700.0 & 0.709 & 0.737 & 0.818 & 0.791 & 0.853 & 0.610 & 0.675 & 0.938 \\
\hline 10 & 800.0 & 0.709 & 0.736 & 0.821 & 0.794 & 0.854 & 0.609 & 0.676 & 0.937 \\
\hline 11 & 900.0 & 0.722 & 0.748 & 0.833 & 0.803 & 0.860 & 0.618 & 0.689 & 0.951 \\
\hline 12 & 1000.0 & 0.726 & 0.753 & 0.841 & 0.807 & 0.865 & 0.621 & 0.694 & 0.957 \\
\hline 13 & 1100.0 & 0.733 & 0.764 & 0.847 & 0.818 & 0.871 & 0.627 & 0.705 & 0.968 \\
\hline 14 & 1200.0 & 0.737 & 0.771 & 0.853 & 0.821 & 0.873 & 0.631 & 0.711 & 0.976 \\
\hline 15 & 1300.0 & 0.741 & 0.774 & 0.859 & 0.824 & 0.878 & 0.636 & 0.717 & 0.983 \\
\hline \multirow[t]{3}{*}{ Step } & Burnup & \multicolumn{8}{|c|}{ MPACT Power Peaking Factors } \\
\hline & $\frac{\mathrm{MWd}}{\mathrm{MtU}}$ & A & B & $\mathrm{C}$ & $\mathrm{D}$ & $\mathrm{P}$ & Q & $\mathrm{R}$ & S \\
\hline & & (A) & (D) & (C) & (B) & (R) & $(\mathrm{Q})$ & $(\mathrm{P})$ & (S) \\
\hline 0 & 0.0 & 0.704 & 0.732 & 0.816 & 0.787 & 0.846 & 0.601 & 0.671 & 0.937 \\
\hline 1 & 25.0 & 0.736 & 0.763 & 0.844 & 0.817 & 0.874 & 0.634 & 0.704 & 0.960 \\
\hline 2 & 50.0 & 0.730 & 0.758 & 0.839 & 0.811 & 0.868 & 0.628 & 0.698 & 0.955 \\
\hline 3 & 100.0 & 0.720 & 0.747 & 0.829 & 0.801 & 0.859 & 0.617 & 0.687 & 0.947 \\
\hline 4 & 200.0 & 0.705 & 0.732 & 0.816 & 0.787 & 0.846 & 0.601 & 0.672 & 0.937 \\
\hline 5 & 300.0 & 0.698 & 0.725 & 0.810 & 0.781 & 0.840 & 0.593 & 0.664 & 0.932 \\
\hline 6 & 400.0 & 0.694 & 0.722 & 0.807 & 0.778 & 0.838 & 0.590 & 0.661 & 0.930 \\
\hline 7 & 500.0 & 0.694 & 0.722 & 0.807 & 0.778 & 0.838 & 0.589 & 0.660 & 0.931 \\
\hline 8 & 600.0 & 0.696 & 0.724 & 0.809 & 0.780 & 0.840 & 0.590 & 0.662 & 0.933 \\
\hline 9 & 700.0 & 0.699 & 0.727 & 0.812 & 0.783 & 0.843 & 0.593 & 0.665 & 0.936 \\
\hline 10 & 800.0 & 0.703 & 0.731 & 0.817 & 0.788 & 0.847 & 0.597 & 0.669 & 0.940 \\
\hline 11 & 900.0 & 0.708 & 0.736 & 0.822 & 0.793 & 0.852 & 0.602 & 0.674 & 0.944 \\
\hline 12 & 1000.0 & 0.714 & 0.742 & 0.827 & 0.798 & 0.858 & 0.607 & 0.680 & 0.949 \\
\hline 13 & 1100.0 & 0.720 & 0.748 & 0.833 & 0.804 & 0.863 & 0.613 & 0.686 & 0.954 \\
\hline 14 & 1200.0 & 0.726 & 0.754 & 0.839 & 0.810 & 0.869 & 0.619 & 0.692 & 0.959 \\
\hline 15 & 1300.0 & 0.732 & 0.761 & 0.845 & 0.816 & 0.875 & 0.625 & 0.699 & 0.964 \\
\hline
\end{tabular}


Table 4.29. Problem 3.2.1 reference solution power peaking factors for charge-pans 1128 and 0831 .

\begin{tabular}{|c|c|c|c|c|c|c|c|c|c|}
\hline \multirow[t]{2}{*}{ Step } & \multirow{2}{*}{$\begin{array}{l}\text { Burnup } \\
{\left[\frac{\mathrm{MWd}}{\mathrm{MtU}}\right]}\end{array}$} & \multicolumn{8}{|c|}{ Shift Power Peaking Factors } \\
\hline & & $\begin{array}{c}E \\
(H)\end{array}$ & $\begin{array}{c}F \\
(G)\end{array}$ & $\begin{array}{l}G \\
(F)\end{array}$ & $\begin{array}{l}\mathrm{H} \\
(\mathrm{E})\end{array}$ & $\begin{array}{c}\mathrm{J} \\
(\mathrm{M})\end{array}$ & $\begin{array}{c}\mathrm{K} \\
(\mathrm{L})\end{array}$ & $\begin{array}{c}\mathrm{L} \\
(\mathrm{K})\end{array}$ & $\begin{array}{l}\text { M } \\
(\mathrm{J})\end{array}$ \\
\hline 0 & 0.0 & 0.763 & 0.677 & 0.622 & 0.648 & 0.763 & 0.852 & 0.909 & 0.877 \\
\hline 1 & 25.0 & 0.773 & 0.691 & 0.636 & 0.669 & 0.795 & 0.885 & 0.933 & 0.895 \\
\hline 2 & 50.0 & 0.772 & 0.687 & 0.637 & 0.671 & 0.794 & 0.886 & 0.935 & 0.897 \\
\hline 3 & 100.0 & 0.772 & 0.689 & 0.635 & 0.666 & 0.789 & 0.876 & 0.926 & 0.890 \\
\hline 4 & 200.0 & 0.766 & 0.680 & 0.626 & 0.656 & 0.769 & 0.862 & 0.914 & 0.880 \\
\hline 5 & 300.0 & 0.759 & 0.674 & 0.616 & 0.644 & 0.760 & 0.847 & 0.904 & 0.874 \\
\hline 6 & 400.0 & 0.759 & 0.674 & 0.618 & 0.644 & 0.759 & 0.846 & 0.901 & 0.874 \\
\hline 7 & 500.0 & 0.760 & 0.677 & 0.617 & 0.643 & 0.756 & 0.847 & 0.900 & 0.874 \\
\hline 8 & 600.0 & 0.767 & 0.680 & 0.620 & 0.649 & 0.762 & 0.852 & 0.906 & 0.879 \\
\hline 9 & 700.0 & 0.770 & 0.684 & 0.625 & 0.651 & 0.765 & 0.853 & 0.910 & 0.883 \\
\hline 10 & 800.0 & 0.770 & 0.684 & 0.626 & 0.650 & 0.768 & 0.857 & 0.912 & 0.885 \\
\hline 11 & 900.0 & 0.780 & 0.695 & 0.637 & 0.663 & 0.781 & 0.871 & 0.921 & 0.892 \\
\hline 12 & 1000.0 & 0.784 & 0.697 & 0.639 & 0.666 & 0.788 & 0.876 & 0.926 & 0.897 \\
\hline 13 & 1100.0 & 0.789 & 0.704 & 0.647 & 0.676 & 0.798 & 0.886 & 0.938 & 0.906 \\
\hline 14 & 1200.0 & 0.795 & 0.709 & 0.652 & 0.681 & 0.803 & 0.894 & 0.944 & 0.911 \\
\hline 15 & 1300.0 & 0.797 & 0.710 & 0.655 & 0.687 & 0.812 & 0.900 & 0.951 & 0.917 \\
\hline \multirow[t]{2}{*}{ Step } & Burnup & \multicolumn{8}{|c|}{ MPACT Power Peaking Factors } \\
\hline & {$[\mathrm{MWd}]$} & $\begin{array}{c}E \\
(H)\end{array}$ & $\begin{array}{c}F \\
(G)\end{array}$ & $\begin{array}{l}\text { G } \\
(\mathrm{F})\end{array}$ & $\begin{array}{l}\mathrm{H} \\
\text { (E) }\end{array}$ & $\begin{array}{c}\mathrm{J} \\
(\mathrm{M})\end{array}$ & $\begin{array}{c}\mathrm{K} \\
(\mathrm{L})\end{array}$ & $\begin{array}{c}\mathrm{L} \\
(\mathrm{K})\end{array}$ & $\begin{array}{l}M \\
(\mathrm{~J})\end{array}$ \\
\hline 0 & 0.0 & 0.761 & 0.677 & 0.619 & 0.646 & 0.763 & 0.852 & 0.909 & 0.879 \\
\hline 1 & 25.0 & 0.792 & 0.709 & 0.652 & 0.678 & 0.794 & 0.880 & 0.933 & 0.905 \\
\hline 2 & 50.0 & 0.787 & 0.703 & 0.646 & 0.673 & 0.788 & 0.875 & 0.929 & 0.900 \\
\hline 3 & 100.0 & 0.776 & 0.692 & 0.635 & 0.662 & 0.778 & 0.865 & 0.920 & 0.891 \\
\hline 4 & 200.0 & 0.762 & 0.677 & 0.620 & 0.646 & 0.763 & 0.852 & 0.909 & 0.879 \\
\hline 5 & 300.0 & 0.755 & 0.670 & 0.612 & 0.639 & 0.756 & 0.846 & 0.904 & 0.873 \\
\hline 6 & 400.0 & 0.752 & 0.666 & 0.609 & 0.635 & 0.753 & 0.844 & 0.902 & 0.871 \\
\hline 7 & 500.0 & 0.752 & 0.666 & 0.608 & 0.635 & 0.753 & 0.844 & 0.902 & 0.872 \\
\hline 8 & 600.0 & 0.754 & 0.667 & 0.609 & 0.636 & 0.755 & 0.846 & 0.905 & 0.874 \\
\hline 9 & 700.0 & 0.757 & 0.670 & 0.612 & 0.639 & 0.759 & 0.849 & 0.908 & 0.877 \\
\hline 10 & 800.0 & 0.761 & 0.675 & 0.616 & 0.643 & 0.763 & 0.854 & 0.912 & 0.881 \\
\hline 11 & 900.0 & 0.766 & 0.680 & 0.621 & 0.648 & 0.768 & 0.859 & 0.917 & 0.886 \\
\hline 12 & 1000.0 & 0.772 & 0.685 & 0.626 & 0.654 & 0.774 & 0.864 & 0.921 & 0.891 \\
\hline 13 & 1100.0 & 0.778 & 0.691 & 0.632 & 0.660 & 0.780 & 0.870 & 0.927 & 0.896 \\
\hline 14 & 1200.0 & 0.784 & 0.697 & 0.638 & 0.666 & 0.786 & 0.876 & 0.932 & 0.902 \\
\hline 15 & 1300.0 & 0.791 & 0.704 & 0.645 & 0.673 & 0.793 & 0.882 & 0.937 & 0.908 \\
\hline
\end{tabular}


Charge-Pans 1228 and (0832)

Table 4.30. Problem 3.2.1 reference solution power peaking factors for charge-pans 1228 and 0832 .

\begin{tabular}{rrrccccc}
\hline Step & Burnup & \multicolumn{5}{c}{ Shift Power Peaking Factors } \\
& {$\left[\frac{\mathrm{MWd}}{\mathrm{MtU}}\right]$} & $\mathrm{A}$ & $\mathrm{B}$ & $\mathrm{C}$ & $\mathrm{D}$ & $\mathrm{P}$ & $\mathrm{S}$ \\
& & $(\mathrm{A})$ & $(\mathrm{D})$ & $(\mathrm{C})$ & $(\mathrm{B})$ & $(\mathrm{R})$ & $(\mathrm{Q})$ \\
\hline 0 & 0.0 & 0.380 & 0.405 & 0.460 & 0.438 & 0.540 & 0.579 \\
1 & 25.0 & 0.393 & 0.423 & 0.477 & 0.453 & 0.551 & 0.603 \\
2 & 50.0 & 0.392 & 0.420 & 0.478 & 0.452 & 0.550 & 0.604 \\
3 & 100.0 & 0.388 & 0.416 & 0.473 & 0.448 & 0.549 & 0.597 \\
4 & 200.0 & 0.382 & 0.407 & 0.462 & 0.439 & 0.541 & 0.579 \\
5 & 300.0 & 0.376 & 0.398 & 0.454 & 0.433 & 0.534 & 0.569 \\
6 & 400.0 & 0.375 & 0.397 & 0.453 & 0.431 & 0.535 & 0.568 \\
7 & 500.0 & 0.375 & 0.395 & 0.454 & 0.431 & 0.533 & 0.566 \\
8 & 600.0 & 0.375 & 0.395 & 0.455 & 0.433 & 0.534 & 0.569 \\
9 & 700.0 & 0.378 & 0.399 & 0.459 & 0.434 & 0.540 & 0.572 \\
10 & 800.0 & 0.379 & 0.398 & 0.460 & 0.434 & 0.539 & 0.572 \\
11 & 900.0 & 0.386 & 0.406 & 0.470 & 0.444 & 0.549 & 0.582 \\
12 & 1000.0 & 0.390 & 0.409 & 0.473 & 0.445 & 0.550 & 0.585 \\
13 & 1100.0 & 0.394 & 0.414 & 0.480 & 0.450 & 0.556 & 0.595 \\
14 & 1200.0 & 0.397 & 0.417 & 0.482 & 0.452 & 0.558 & 0.600 \\
15 & 1300.0 & 0.401 & 0.422 & 0.486 & 0.458 & 0.563 & 0.606 \\
\hline Step & Burnup & & MPACT & Power Peaking & Factors & \\
& {$\left[\frac{\mathrm{MWd}}{\mathrm{MtU}}\right]$} & $\mathrm{A}$ & $\mathrm{B}$ & $\mathrm{C}$ & $\mathrm{D}$ & $\mathrm{P}$ & $\mathrm{S}$ \\
& & $(\mathrm{A})$ & $(\mathrm{D})$ & $(\mathrm{C})$ & $(\mathrm{B})$ & $(\mathrm{R})$ & $(\mathrm{Q})$ \\
\hline 0 & 0.0 & 0.377 & 0.403 & 0.456 & 0.436 & 0.537 & 0.576 \\
1 & 25.0 & 0.404 & 0.431 & 0.486 & 0.464 & 0.569 & 0.609 \\
2 & 50.0 & 0.400 & 0.427 & 0.481 & 0.460 & 0.564 & 0.603 \\
3 & 100.0 & 0.391 & 0.418 & 0.472 & 0.450 & 0.553 & 0.592 \\
4 & 200.0 & 0.379 & 0.404 & 0.458 & 0.437 & 0.538 & 0.577 \\
5 & 300.0 & 0.372 & 0.397 & 0.450 & 0.430 & 0.530 & 0.569 \\
6 & 400.0 & 0.369 & 0.394 & 0.447 & 0.426 & 0.526 & 0.566 \\
7 & 500.0 & 0.367 & 0.393 & 0.446 & 0.425 & 0.525 & 0.565 \\
8 & 600.0 & 0.368 & 0.393 & 0.446 & 0.426 & 0.526 & 0.566 \\
9 & 700.0 & 0.369 & 0.395 & 0.448 & 0.427 & 0.528 & 0.569 \\
10 & 800.0 & 0.372 & 0.397 & 0.452 & 0.430 & 0.532 & 0.573 \\
11 & 900.0 & 0.375 & 0.401 & 0.455 & 0.434 & 0.536 & 0.577 \\
12 & 1000.0 & 0.378 & 0.405 & 0.460 & 0.438 & 0.541 & 0.583 \\
13 & 1100.0 & 0.382 & 0.409 & 0.464 & 0.443 & 0.546 & 0.588 \\
\hline 1200.0 & 0.387 & 0.413 & 0.470 & 0.448 & 0.552 & 0.594 \\
& 1300.0 & 0.391 & 0.418 & 0.475 & 0.453 & 0.558 & 0.601 \\
\hline & & & & & & & \\
10 & &
\end{tabular}


Table 4.31. Problem 3.2.1 reference solution power peaking factors for charge-pans 1228 and 0832 .

\begin{tabular}{|c|c|c|c|c|c|c|c|}
\hline \multirow[t]{3}{*}{ Step } & \multirow{3}{*}{$\begin{array}{l}\text { Burnup } \\
{\left[\frac{\mathrm{MWd}}{\mathrm{MtU}}\right]}\end{array}$} & \multicolumn{6}{|c|}{ Shift Power Peaking Factors } \\
\hline & & $E$ & $\mathrm{~F}$ & $\mathbf{J}$ & K & L & M \\
\hline & & $(\mathrm{H})$ & $(\mathrm{G})$ & $(\mathrm{F})$ & $(\mathrm{E})$ & $(\mathrm{M})$ & $(\mathrm{L})$ \\
\hline 0 & 0.0 & 0.451 & 0.372 & 0.433 & 0.488 & 0.555 & 0.531 \\
\hline 1 & 25.0 & & 0.382 & 0.452 & 0.510 & & \\
\hline 2 & 50.0 & 462 & 0.382 & 0.452 & 0.509 & 0.574 & 545 \\
\hline 3 & 100.0 & 461 & 0.380 & 0.448 & 0.506 & 0.569 & 544 \\
\hline 4 & 200.0 & & 0.374 & 0.436 & 0.492 & & 535 \\
\hline 5 & 300.0 & 7 & 0.371 & 0.428 & 0.481 & 17 & 525 \\
\hline 6 & 400.0 & & 0.370 & 0.427 & 0.480 & 6 & .527 \\
\hline 7 & 500.0 & & 0.371 & 0. & 0.481 & & \\
\hline 8 & 600.0 & 9 & 0.371 & 0.428 & 0.481 & 18 & 527 \\
\hline 9 & 70 & & 0.373 & & 0.482 & & \\
\hline 10 & 800.0 & & 0.374 & 0.433 & 0.485 & 53 & 33 \\
\hline 11 & 900.0 & & 0.382 & 0.441 & 0.495 & 2 & \\
\hline 12 & 1000.0 & & 0.387 & & 0.498 & 66 & 40 \\
\hline 13 & 00.0 & & 0.390 & 0. & 0.507 & 6 & 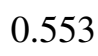 \\
\hline 14 & 00.0 & & 0.392 & & 11 & & \\
\hline 15 & 00.0 & & 0.396 & 0.460 & 0.515 & 0.583 & 0.560 \\
\hline \multirow[t]{3}{*}{ Step } & Burnup & \multicolumn{6}{|c|}{ MPACT Power Peaking Factors } \\
\hline & $\frac{\mathrm{MWd}}{\mathrm{MtU}}$ & F & $F$ & $\mathbf{J}$ & K & & M \\
\hline & & $(\mathrm{H})$ & $(\mathrm{G})$ & $(\mathrm{F})$ & (E) & $(\mathrm{M})$ & $(\mathrm{L})$ \\
\hline 0 & 0.0 & 0.450 & 0.369 & 0.431 & 0.486 & 0.551 & 0.529 \\
\hline 1 & 25.0 & 9 & 0.396 & 50 & 0.517 & 33 & 60 \\
\hline 2 & 50.0 & 0.475 & 0.392 & 0.455 & 0.512 & 0.578 & 0.555 \\
\hline 3 & 100.0 & 0.465 & 0.384 & 0.446 & 0.502 & 0.567 & 0.544 \\
\hline 4 & 200.0 & 1 & 0.371 & 32 & 0.487 & 2 & .529 \\
\hline 5 & 300.0 & & 0.365 & & 0.480 & 0.5 & 0.522 \\
\hline 6 & 400.0 & 0.440 & 0.361 & 0.421 & 0.476 & 41 & 0.518 \\
\hline 7 & 500.0 & & 0.360 & 0.420 & 0.475 & 0.540 & 0.517 \\
\hline 0 & 600.0 & 0.439 & 0.360 & 0.420 & 0.476 & 0.541 & 0.518 \\
\hline 9 & 700.0 & 0.441 & 0.362 & 0.422 & 0.478 & 0.544 & 0.521 \\
\hline 10 & 800.0 & 0.444 & 0.364 & 0.425 & 0.481 & 0.547 & 0.524 \\
\hline 11 & 900.0 & 0.447 & 0.367 & 0.428 & 0.485 & 0.552 & 0.528 \\
\hline 12 & 1000.0 & & 0.370 & 0.433 & 0.490 & 0.557 & 0.533 \\
\hline 13 & 1100.0 & 0.456 & 0.374 & 0.437 & 0.495 & 0.562 & 0.539 \\
\hline 14 & 1200.0 & 0.461 & 0.378 & 0.442 & 0.501 & 0.568 & 0.544 \\
\hline 15 & 1300.0 & 0.466 & 0.382 & 0.447 & 0.506 & 0.574 & 0.550 \\
\hline
\end{tabular}




\section{Charge-Pan 0929}

Table 4.32. Problem 3.2.1 reference solution power peaking factors for charge-pan 0929.

\begin{tabular}{|c|c|c|c|c|c|c|c|c|c|c|c|}
\hline \multirow[t]{2}{*}{ Step } & Burnup & \multicolumn{10}{|c|}{ Shift Power Peaking Factors } \\
\hline & $\mathrm{MWd}$ & A & $\mathrm{B}, \mathrm{D}$ & $\mathrm{C}$ & $\mathrm{G}, \mathrm{F}$ & $\mathrm{H}, \mathrm{E}$ & $\mathrm{J}, \mathrm{M}$ & $\mathrm{K}, \mathrm{L}$ & Q & $\mathrm{R}, \mathrm{P}$ & $S$ \\
\hline 0 & 0.0 & 1.104 & 1.156 & 1.197 & 1.062 & 1.113 & 1.208 & 1.258 & 1.011 & 1.159 & 1.307 \\
\hline 1 & 25.0 & 1.081 & 1.133 & 1.182 & 1.041 & 1.098 & 1.195 & 1.248 & 0.988 & 1.148 & 1.297 \\
\hline 2 & 50.0 & 1.084 & 1.135 & 1.183 & 1.042 & 1.098 & 1.199 & 1.250 & 0.987 & 1.149 & 1.301 \\
\hline 3 & 100.0 & 1.090 & 1.146 & 1.187 & 1.050 & 1.103 & 1.203 & 1.254 & 0.996 & 1.153 & 1.302 \\
\hline 4 & 200.0 & 1.104 & 1.160 & 1.199 & 1.063 & 1.115 & 1.212 & 1.262 & 1.008 & 1.163 & 1.312 \\
\hline 5 & 300.0 & 8 & 1. & 1.206 & 1.066 & 1.119 & 1.215 & 1.267 & 18 & 64 & 1.313 \\
\hline 6 & 400.0 & 1.110 & 1.173 & 1.208 & 1.071 & 1.119 & 1.218 & 1.265 & 1.023 & 1.166 & 1.313 \\
\hline 7 & 500.0 & 1.113 & 1.172 & 1.210 & 1.073 & 1.126 & 1.220 & 1.270 & 1.025 & 1.170 & 1.318 \\
\hline 8 & 600.0 & & 1.171 & 1.208 & 1.072 & 1.126 & 1.220 & 1.269 & 1.022 & 173 & 1.318 \\
\hline 9 & 700.0 & 1. & 1.168 & 1.209 & 1.070 & 1.126 & 1.222 & 1.272 & 1.021 & 1.174 & 1.317 \\
\hline 10 & 800.0 & & 1. & 1.204 & 1.066 & 1.122 & 1.225 & 1.271 & 15 & 74 & 1.318 \\
\hline 11 & 900.0 & 2 & 1.157 & 1.203 & 1.066 & 1.123 & 1.223 & 1.271 & 1.013 & 1.173 & 1.317 \\
\hline 12 & 1000.0 & 1. & 1.152 & 1.201 & 1.065 & 1.122 & 1.221 & 1.270 & 07 & 1.173 & 1.316 \\
\hline 13 & 1100.0 & & 1.146 & 1.198 & 1.058 & 21 & 1.221 & 1.269 & & 74 & 1.316 \\
\hline 14 & 1200.0 & 1.089 & 1.138 & 1.194 & 1.053 & 1.119 & 1.219 & 1.267 & 0.998 & 1.173 & 1.313 \\
\hline 15 & 1300.0 & & 2 & 1. & 1.050 & 1.114 & 1.216 & 1.264 & & 69 & 1.310 \\
\hline \multirow[t]{2}{*}{ Step } & Burnup & \multicolumn{10}{|c|}{ MPACT Power Peaking Factors } \\
\hline & $\frac{\mathrm{MWd}}{\mathrm{MtU}}$ & A & $\mathrm{B}, \mathrm{D}$ & $\mathrm{C}$ & $\mathrm{G}, \mathrm{F}$ & $\mathrm{H}, \mathrm{E}$ & $\mathrm{J}, \mathrm{M}$ & $\mathrm{K}, \mathrm{L}$ & Q & $\mathrm{R}, \mathrm{P}$ & S \\
\hline 0 & 0.0 & 1.105 & 1.159 & 1.200 & 1.062 & 1.112 & 1.210 & 1.261 & 1.011 & 1.159 & 1.309 \\
\hline 1 & 25.0 & 1.111 & 1.161 & 1.193 & 1.074 & 1.119 & 1.204 & 1.247 & 1.028 & 1.161 & 1.289 \\
\hline 2 & 50.0 & 1.109 & 1.160 & 1.194 & 1.071 & 1.117 & 1.204 & 1.249 & 1.025 & 1.160 & 1.292 \\
\hline 3 & 100.0 & 1.107 & 1.159 & 1.195 & 1.067 & 1.115 & 1.206 & 1.253 & 1.018 & 1.159 & 1.299 \\
\hline 4 & 200.0 & 04 & 1.158 & 1.199 & 1.062 & 1.112 & 1.209 & 1.260 & 1.010 & 1.158 & 1.309 \\
\hline 5 & 300.0 & & 1.159 & 1.201 & 1.060 & 1.111 & 1.211 & 1.263 & 1.007 & 1.159 & 1.314 \\
\hline 6 & 400.0 & 1.104 & 1.160 & 1.202 & 1.059 & 1.111 & 1.212 & 1.266 & 1.006 & 1.160 & 1.317 \\
\hline 7 & 500.0 & 1.105 & 1.161 & 1.203 & 1.060 & 1.112 & 1.214 & 1.267 & 1.007 & 1.161 & 1.318 \\
\hline 8 & 600.0 & 1.106 & 1.162 & 1.204 & 1.062 & 1.114 & 1.215 & 1.267 & 1.009 & 1.162 & 1.317 \\
\hline 9 & 700.0 & 1.108 & 1.164 & 1.205 & 1.064 & 1.116 & 1.215 & 1.267 & 1.012 & 1.164 & 1.316 \\
\hline 10 & 800.0 & 1.110 & 1.165 & 1.205 & 1.067 & 1.118 & 1.216 & 1.266 & 1.015 & 1.165 & 1.314 \\
\hline 11 & 900.0 & 1.112 & 1.166 & 1.205 & 1.070 & 1.120 & 1.216 & 1.265 & 1.019 & 1.166 & 1.312 \\
\hline 12 & 1000.0 & 1.114 & 1.167 & 1.205 & 1.073 & 1.122 & 1.216 & 1.263 & 1.023 & 1.167 & 1.309 \\
\hline 13 & 1100.0 & 1.116 & 1.169 & 1.205 & 1.076 & 1.124 & 1.215 & 1.262 & 1.027 & 1.169 & 1.306 \\
\hline 14 & 1200.0 & 1.118 & 1.170 & 1.204 & 1.080 & 1.126 & 1.215 & 1.260 & 1.031 & 1.170 & 1.302 \\
\hline 15 & 1300.0 & 1.120 & 1.170 & 1.203 & 1.083 & 1.128 & 1.214 & 1.257 & 1.035 & 1.170 & 1.298 \\
\hline
\end{tabular}


Charge-Pans 1029 and (0930)

Table 4.33. Problem 3.2.1 reference solution power peaking factors for charge-pans 1029 and 0930 .

\begin{tabular}{|c|c|c|c|c|c|c|c|c|c|}
\hline \multirow[t]{3}{*}{ Step } & \multirow{3}{*}{$\begin{array}{l}\text { Burnup } \\
{\left[\frac{\mathrm{MWd}}{\mathrm{MtU}}\right]}\end{array}$} & \multicolumn{8}{|c|}{ Shift Power Peaking Factors } \\
\hline & & A & B & $\mathrm{C}$ & $\mathrm{D}$ & $P$ & Q & $\mathrm{R}$ & S \\
\hline & & (A) & (D) & (C) & (B) & $(\mathrm{R})$ & $(\mathrm{Q})$ & (P) & (S) \\
\hline 0 & 0.0 & 0.865 & 0.913 & 0.980 & 0.932 & 0.952 & 0.742 & 0.889 & 1.097 \\
\hline 1 & 25.0 & 0.850 & 0.899 & 0.966 & 0.916 & 0.928 & 0.728 & 0.887 & 1.090 \\
\hline 2 & 50.0 & 0.851 & 0.902 & 0.966 & 0.917 & 0.928 & 0.724 & 0.887 & 1.092 \\
\hline 3 & 100.0 & 0.855 & 0.905 & 0.972 & 0.922 & 0.937 & 0.732 & 0.889 & 1.093 \\
\hline 4 & 200.0 & 0.863 & 0.911 & 0.978 & 0.930 & 0.949 & 739 & 0.891 & 1.102 \\
\hline 5 & 300.0 & 0.865 & 0.913 & 0.980 & 0.933 & 0.955 & 0.746 & 0.889 & 1.099 \\
\hline 6 & 400.0 & 0.867 & 0.915 & 0.982 & 0.935 & 0.960 & 0.748 & 0.892 & 1.103 \\
\hline 7 & 500.0 & 0.871 & 0.918 & 0.985 & 0.938 & 0.962 & 0.749 & 0.895 & 1.102 \\
\hline 8 & 600.0 & 0.870 & 0.919 & 0.988 & 0.940 & 0.961 & 0.754 & 0.900 & 1.105 \\
\hline 9 & 700.0 & 0.873 & 0.923 & 0.989 & 0.940 & 0.959 & 55 & .904 & 1.111 \\
\hline 10 & 800.0 & 0.871 & 0.919 & 0.987 & 0.937 & 0.955 & 0.749 & 0.903 & 1.108 \\
\hline 11 & 900.0 & 0.874 & 0.927 & 0.989 & 0.939 & 0.954 & 0.753 & 0.908 & 1.110 \\
\hline 12 & 1000.0 & 0.875 & 0.926 & 0.993 & 0.937 & 0.950 & 0.751 & 0.910 & 1.112 \\
\hline 13 & 1100.0 & 0.871 & 0.926 & 0.989 & 0.932 & 0.945 & 0.747 & 0.915 & 1.113 \\
\hline 14 & 1200.0 & 0.870 & 0.927 & 0.990 & 0.932 & 0.942 & 0.747 & 0.920 & 1.114 \\
\hline 15 & 1300.0 & 0.868 & 0.924 & 0.988 & 0.928 & 0.936 & 0.745 & 0.916 & 1.113 \\
\hline \multirow[t]{3}{*}{ Step } & Burnup & \multicolumn{8}{|c|}{ MPACT Power Peaking Factors } \\
\hline & & A & B & $\mathrm{C}$ & D & $\mathrm{P}$ & Q & $\mathrm{R}$ & S \\
\hline & & (A) & (D) & (C) & (B) & (R) & $(\mathrm{Q})$ & (P) & (S) \\
\hline 0 & 0.0 & 0.864 & 0.911 & 0.978 & 0.931 & 0.950 & 0.740 & 0.887 & 1.099 \\
\hline 1 & 25.0 & 0.891 & 0.935 & 0.997 & 0.953 & 0.972 & 0.772 & 0.913 & 1.107 \\
\hline 2 & 50.0 & 0.885 & 0.930 & 0.993 & 0.949 & 0.968 & 0.766 & 0.908 & 1.105 \\
\hline 3 & 100.0 & 0.876 & 0.922 & 0.986 & 0.941 & 0.960 & 0.755 & 0.899 & 1.102 \\
\hline 4 & 200.0 & 0.864 & 0.910 & 0.978 & 0.930 & 0.950 & 0.741 & 0.887 & 1.098 \\
\hline 5 & 300.0 & 0.858 & 0.905 & 0.974 & 0.926 & 0.945 & 0.733 & 0.881 & 1.097 \\
\hline 6 & 400.0 & 0.856 & 0.904 & 0.972 & 0.924 & 0.944 & 0.730 & 0.879 & 1.097 \\
\hline 7 & 500.0 & 0.856 & 0.904 & 0.973 & 0.924 & 0.944 & 0.730 & 0.880 & 1.098 \\
\hline 8 & 600.0 & 0.858 & 0.906 & 0.975 & 0.926 & 0.946 & 0.732 & 0.882 & 1.099 \\
\hline 9 & 700.0 & 0.861 & 0.909 & 0.978 & 0.930 & 0.949 & 0.735 & 0.885 & 1.102 \\
\hline 10 & 800.0 & 0.865 & 0.913 & 0.982 & 0.934 & 0.953 & 0.739 & 0.889 & 1.104 \\
\hline 11 & 900.0 & 0.870 & 0.918 & 0.986 & 0.938 & 0.958 & 0.744 & 0.894 & 1.106 \\
\hline 12 & 1000.0 & 0.875 & 0.923 & 0.990 & 0.943 & 0.962 & 0.750 & 0.899 & 1.109 \\
\hline 13 & 1100.0 & 0.881 & 0.928 & 0.994 & 0.948 & 0.967 & 0.756 & 0.904 & 1.111 \\
\hline 14 & 1200.0 & 0.887 & 0.933 & 0.999 & 0.953 & 0.972 & 0.763 & 0.910 & 1.114 \\
\hline 15 & 1300.0 & 0.892 & 0.938 & 1.003 & 0.958 & 0.977 & 0.769 & 0.916 & 1.116 \\
\hline
\end{tabular}


Table 4.34. Problem 3.2.1 reference solution power peaking factors for charge-pans 1029 and 0930 .

\begin{tabular}{|c|c|c|c|c|c|c|c|c|c|}
\hline \multirow[t]{3}{*}{ Step } & \multirow{3}{*}{$\begin{array}{l}\text { Burnup } \\
{\left[\frac{\mathrm{MWd}}{\mathrm{MtU}}\right]}\end{array}$} & \multicolumn{8}{|c|}{ Shift Power Peaking Factors } \\
\hline & & $\mathrm{E}$ & $\mathrm{F}$ & $\mathrm{G}$ & $\mathrm{H}$ & $\mathbf{J}$ & K & $\mathrm{L}$ & M \\
\hline & & $(\mathrm{H})$ & $(\mathrm{G})$ & $(\mathrm{F})$ & $(\mathrm{E})$ & $(\mathrm{M})$ & $(\mathrm{L})$ & $(\mathrm{K})$ & $(\mathrm{J})$ \\
\hline 0 & 0.0 & 0.883 & 0.816 & 0.796 & 0.843 & 0.961 & 1.035 & 1.056 & 1.005 \\
\hline 1 & 25.0 & 0.866 & 0.797 & 0.783 & 0.836 & 0.958 & 1.027 & 1.039 & 0.985 \\
\hline 2 & 50.0 & 0.866 & 0.798 & 0.784 & 0.837 & 0.956 & 1.025 & 1.042 & 0.988 \\
\hline 3 & 100.0 & 0.872 & 0.805 & 0.788 & 0.842 & 0.962 & 1.030 & 1.046 & 0.992 \\
\hline 4 & 200.0 & 0.884 & 0.815 & 0.795 & 0.845 & 0.965 & 1.033 & 1.055 & 1.001 \\
\hline 5 & 300.0 & 0.890 & 0.818 & 0.798 & 0.847 & 0.963 & 1.032 & 1.053 & 1.008 \\
\hline 6 & 400.0 & 0.894 & 0.823 & 0.799 & 0.850 & 0.964 & 1.034 & 1.060 & 1.009 \\
\hline 7 & 500.0 & 0.895 & 0.824 & 0.801 & 0.851 & 0.970 & 1.037 & 1.058 & 1.010 \\
\hline 8 & 600.0 & 0.897 & 0.827 & 0.805 & 0.855 & 0.972 & 1.043 & 1.063 & 1.012 \\
\hline 9 & 700.0 & 0.895 & 0.827 & 0.808 & 0.858 & 0.977 & 1.045 & 1.065 & 1.009 \\
\hline 10 & 800.0 & 0.894 & 0.823 & 0.803 & 0.855 & 0.975 & 1.044 & 1.061 & 1.006 \\
\hline 11 & 900.0 & 0.892 & 0.826 & 0.807 & 0.861 & 0.980 & 1.049 & 1.063 & 1.007 \\
\hline 12 & 1000.0 & 0.891 & 0.822 & 0.807 & 0.864 & 0.983 & 1.052 & 1.063 & 1.007 \\
\hline 13 & 1100.0 & 0.884 & 0.817 & 0.805 & 0.862 & 0.985 & 1.055 & 1.060 & 1.000 \\
\hline 14 & 1200.0 & 0.880 & 0.819 & 0.803 & 0.865 & 0.987 & 1.055 & 1.059 & 1.002 \\
\hline 15 & 1300.0 & 0.879 & 0.815 & 0.802 & 0.864 & 0.988 & 1.055 & 1.058 & 0.996 \\
\hline \multirow[t]{2}{*}{ Step } & Burnup & \multicolumn{8}{|c|}{ MPACT Power Peaking Factors } \\
\hline & {$\left[\frac{\mathrm{MWd}}{\underline{T}}\right]$} & $\begin{array}{c}E \\
(H)\end{array}$ & $\begin{array}{c}\mathrm{F} \\
(\mathrm{G})\end{array}$ & $\begin{array}{c}\mathrm{G} \\
(\mathrm{F})\end{array}$ & $\begin{array}{l}\mathrm{H} \\
\text { (E) }\end{array}$ & $\begin{array}{c}\mathrm{J} \\
(\mathrm{M})\end{array}$ & $\begin{array}{c}\mathrm{K} \\
(\mathrm{L})\end{array}$ & $\begin{array}{c}\mathrm{L} \\
(\mathrm{K})\end{array}$ & $\begin{array}{l}\text { M } \\
(\mathrm{J})\end{array}$ \\
\hline 0 & 0.0 & 0.885 & 0.814 & 0.793 & 0.843 & 0.961 & 1.032 & 1.054 & 1.004 \\
\hline 1 & 25.0 & 0.911 & 0.844 & 0.824 & 0.871 & 0.982 & 1.047 & 1.067 & 1.021 \\
\hline 2 & 50.0 & 0.906 & 0.839 & 0.818 & 0.866 & 0.978 & 1.044 & 1.064 & 1.018 \\
\hline 3 & 100.0 & 0.897 & 0.828 & 0.808 & 0.856 & 0.970 & 1.039 & 1.059 & 1.011 \\
\hline 4 & 200.0 & 0.885 & 0.815 & 0.794 & 0.843 & 0.961 & 1.032 & 1.053 & 1.003 \\
\hline 5 & 300.0 & 0.879 & 0.808 & 0.787 & 0.837 & 0.957 & 1.029 & 1.051 & 1.000 \\
\hline 6 & 400.0 & 0.877 & 0.805 & 0.784 & 0.834 & 0.955 & 1.028 & 1.050 & 0.999 \\
\hline 7 & 500.0 & 0.877 & 0.805 & 0.784 & 0.834 & 0.956 & 1.029 & 1.051 & 0.999 \\
\hline 8 & 600.0 & 0.879 & 0.807 & 0.786 & 0.836 & 0.958 & 1.031 & 1.053 & 1.001 \\
\hline 9 & 700.0 & 0.882 & 0.811 & 0.789 & 0.840 & 0.961 & 1.034 & 1.056 & 1.004 \\
\hline 10 & 800.0 & 0.887 & 0.815 & 0.794 & 0.844 & 0.965 & 1.037 & 1.059 & 1.008 \\
\hline 11 & 900.0 & 0.891 & 0.820 & 0.799 & 0.849 & 0.969 & 1.040 & 1.062 & 1.012 \\
\hline 12 & 1000.0 & 0.897 & 0.826 & 0.804 & 0.854 & 0.973 & 1.044 & 1.065 & 1.016 \\
\hline 13 & 1100.0 & 0.902 & 0.832 & 0.810 & 0.860 & 0.978 & 1.048 & 1.068 & 1.020 \\
\hline 14 & 1200.0 & 0.908 & 0.838 & 0.817 & 0.866 & 0.983 & 1.051 & 1.072 & 1.024 \\
\hline 15 & 1300.0 & 0.914 & 0.844 & 0.823 & 0.872 & 0.988 & 1.055 & 1.075 & 1.028 \\
\hline
\end{tabular}


Charge-Pans 1129 and (0931)

Table 4.35. Problem 3.2.1 reference solution power peaking factors for charge-pans 1129 and 0931.

\begin{tabular}{|c|c|c|c|c|c|c|c|c|c|}
\hline \multirow[t]{3}{*}{ Step } & \multirow{3}{*}{$\begin{array}{l}\text { Burnup } \\
{\left[\frac{\mathrm{MWd}}{\mathrm{MtU}}\right]}\end{array}$} & \multicolumn{8}{|c|}{ Shift Power Peaking Factors } \\
\hline & & A & B & $\mathrm{C}$ & $\mathrm{D}$ & $\mathrm{P}$ & Q & $\mathrm{R}$ & $S$ \\
\hline & & (A) & (D) & (C) & (B) & $(\mathrm{R})$ & (Q) & $(\mathrm{P})$ & (S) \\
\hline 0 & 0.0 & 0.552 & 0.598 & 0.671 & 0.624 & 0.663 & 0.463 & 0.598 & 0.811 \\
\hline 1 & 25.0 & 0.549 & 0.596 & 0.668 & 0.621 & 0.653 & 0.458 & 0.605 & 0.809 \\
\hline 2 & 50.0 & 0.548 & 0.597 & 0.668 & 0.618 & 0.651 & 0.458 & 0.601 & 0.810 \\
\hline 3 & 100.0 & 0.550 & 0.600 & 0.670 & 0.620 & 0.654 & 0.460 & 0.604 & 0.812 \\
\hline 4 & 200.0 & 0.551 & 0.594 & 0.667 & 0.624 & 0.661 & 0.463 & 0.600 & 0.808 \\
\hline 5 & 300.0 & 0.553 & 0.598 & 0.669 & 0.627 & 0.663 & 0.465 & 0.596 & 0.806 \\
\hline 6 & 400.0 & 0.553 & 0.597 & 0.670 & 0.627 & 0.667 & 0.467 & 0.598 & 0.804 \\
\hline 7 & 500.0 & 0.554 & 0.597 & 0.672 & 0.629 & 0.668 & 0.467 & 0.599 & 0.807 \\
\hline 8 & 600.0 & 0.555 & 0.600 & 0.673 & 0.629 & 0.667 & 0.469 & 0.601 & 0.810 \\
\hline 9 & 700.0 & 0.558 & 0.602 & 0.675 & 0.633 & 0.671 & 0.471 & 0.604 & 0.813 \\
\hline 10 & 800.0 & 0.558 & 0.604 & 0.676 & 0.631 & 0.667 & 0.472 & 0.606 & 0.813 \\
\hline 11 & 900.0 & 0.564 & 0.610 & 0.682 & 0.636 & 0.675 & 0.476 & 0.615 & 0.821 \\
\hline 12 & 1000.0 & 0.564 & 0.611 & 0.684 & 0.636 & 0.671 & 0.476 & 0.617 & 0.824 \\
\hline 13 & 1100.0 & 0.567 & 0.614 & 0.686 & 0.639 & 0.672 & 0.478 & 0.621 & 0.828 \\
\hline 14 & 1200.0 & 0.567 & 0.616 & 0.689 & 0.641 & 0.671 & 0.479 & 0.625 & 0.830 \\
\hline 15 & 1300.0 & 0.569 & 0.618 & 0.689 & 0.640 & 0.674 & 0.479 & 0.629 & 0.833 \\
\hline \multirow[t]{3}{*}{ Step } & Burnup & \multicolumn{8}{|c|}{ MPACT Power Peaking Factors } \\
\hline & $\frac{\mathrm{MWd}}{\mathrm{MtU}}$ & A & B & $\mathrm{C}$ & $\mathrm{D}$ & $\mathrm{P}$ & Q & $\mathrm{R}$ & S \\
\hline & & (A) & (D) & (C) & (B) & (R) & $(\mathrm{Q})$ & $(\mathrm{P})$ & (S) \\
\hline 0 & 0.0 & 0.551 & 0.595 & 0.668 & 0.623 & 0.661 & 0.460 & 0.596 & 0.807 \\
\hline 1 & 25.0 & 0.582 & 0.627 & 0.700 & 0.655 & 0.694 & 0.491 & 0.629 & 0.836 \\
\hline 2 & 50.0 & 0.576 & 0.621 & 0.694 & 0.650 & 0.688 & 0.486 & 0.623 & 0.831 \\
\hline 3 & 100.0 & 0.566 & 0.610 & 0.684 & 0.639 & 0.677 & 0.476 & 0.612 & 0.821 \\
\hline 4 & 200.0 & 0.551 & 0.595 & 0.669 & 0.624 & 0.661 & 0.462 & 0.596 & 0.807 \\
\hline 5 & 300.0 & 0.543 & 0.588 & 0.661 & 0.616 & 0.654 & 0.454 & 0.588 & 0.801 \\
\hline 6 & 400.0 & 0.540 & 0.584 & 0.658 & 0.613 & 0.650 & 0.450 & 0.584 & 0.798 \\
\hline 7 & 500.0 & 0.539 & 0.583 & 0.657 & 0.612 & 0.650 & 0.449 & 0.583 & 0.798 \\
\hline 8 & 600.0 & 0.540 & 0.584 & 0.659 & 0.613 & 0.651 & 0.450 & 0.584 & 0.800 \\
\hline 9 & 700.0 & 0.542 & 0.587 & 0.662 & 0.616 & 0.654 & 0.452 & 0.587 & 0.803 \\
\hline 10 & 800.0 & 0.546 & 0.591 & 0.666 & 0.620 & 0.659 & 0.455 & 0.591 & 0.808 \\
\hline 11 & 900.0 & 0.551 & 0.596 & 0.671 & 0.625 & 0.664 & 0.458 & 0.595 & 0.813 \\
\hline 12 & 1000.0 & 0.555 & 0.601 & 0.677 & 0.630 & 0.669 & 0.463 & 0.601 & 0.818 \\
\hline 13 & 1100.0 & 0.561 & 0.607 & 0.682 & 0.636 & 0.675 & 0.467 & 0.606 & 0.824 \\
\hline 14 & 1200.0 & 0.567 & 0.613 & 0.689 & 0.642 & 0.682 & 0.472 & 0.612 & 0.830 \\
\hline 15 & 1300.0 & 0.573 & 0.619 & 0.695 & 0.649 & 0.688 & 0.478 & 0.619 & 0.836 \\
\hline
\end{tabular}


Table 4.36. Problem 3.2.1 reference solution power peaking factors for charge-pans 1129 and 0931.

\begin{tabular}{|c|c|c|c|c|c|c|c|c|c|}
\hline \multirow[t]{2}{*}{ Step } & \multirow{2}{*}{$\begin{array}{l}\text { Burnup } \\
{\left[\frac{\mathrm{MWd}}{\mathrm{MtU}}\right]}\end{array}$} & \multicolumn{8}{|c|}{ Shift Power Peaking Factors } \\
\hline & & $\begin{array}{c}E \\
(H)\end{array}$ & $\begin{array}{c}F \\
(\mathrm{G})\end{array}$ & $\begin{array}{c}\mathrm{G} \\
(\mathrm{F})\end{array}$ & $\begin{array}{c}\mathrm{H} \\
(\mathrm{E})\end{array}$ & $\begin{array}{c}\mathrm{J} \\
(\mathrm{M})\end{array}$ & $\begin{array}{c}\mathrm{K} \\
(\mathrm{L})\end{array}$ & $\begin{array}{c}\mathrm{L} \\
(\mathrm{K})\end{array}$ & $\begin{array}{l}M \\
(J)\end{array}$ \\
\hline 0 & 0.0 & 0.581 & 0.505 & 0.515 & 0.566 & 0.643 & 0.725 & 0.766 & 0.713 \\
\hline 1 & 25.0 & 0.574 & 0.501 & 0.513 & 0.566 & 0.648 & 0.728 & 0.761 & 0.707 \\
\hline 2 & 50.0 & 0.572 & 0.499 & 0.513 & 0.567 & 0.648 & 0.728 & 0.759 & 0.704 \\
\hline 3 & 100.0 & 0.577 & 0.501 & 0.515 & 0.569 & 0.648 & 0.728 & 0.761 & 0.709 \\
\hline 4 & 200.0 & 0.580 & 0.503 & 0.514 & 0.565 & 0.645 & 0.723 & 0.760 & 0.712 \\
\hline 5 & 300.0 & 0.582 & 0.507 & 0.516 & 0.566 & 0.642 & 0.721 & 0.760 & 0.713 \\
\hline 6 & 400.0 & 0.585 & 0.508 & 0.518 & 0.568 & 0.641 & 0.720 & 0.759 & 0.715 \\
\hline 7 & 500.0 & 0.586 & 0.510 & 0.520 & 0.568 & 0.643 & 0.725 & 0.764 & 0.717 \\
\hline 8 & 600.0 & 0.587 & 0.512 & 0.519 & 0.569 & 0.646 & 0.726 & 0.763 & 0.718 \\
\hline 9 & 700.0 & 0.590 & 0.511 & 0.524 & 0.574 & 0.648 & 0.730 & 0.768 & 0.722 \\
\hline 10 & 800.0 & 0.589 & 0.515 & 0.524 & 0.572 & 0.649 & 0.731 & 0.768 & 0.721 \\
\hline 11 & 900.0 & 0.594 & 0.518 & 0.529 & 0.582 & 0.658 & 0.739 & 0.775 & 0.724 \\
\hline 12 & 1000.0 & 0.593 & 0.516 & 0.530 & 0.582 & 0.659 & 0.740 & 0.773 & 0.725 \\
\hline 13 & 1100.0 & 0.594 & 0.517 & 0.533 & 0.585 & 0.666 & 0.745 & 0.777 & 0.724 \\
\hline 14 & 1200.0 & 0.595 & 0.519 & 0.534 & 0.589 & 0.667 & 0.750 & 0.779 & 0.726 \\
\hline 15 & 1300.0 & 0.592 & 0.520 & 0.536 & 0.590 & 0.669 & 0.751 & 0.777 & 0.727 \\
\hline \multirow[t]{2}{*}{ Step } & Burnup & \multicolumn{8}{|c|}{ MPACT Power Peaking Factors } \\
\hline & $\frac{\mathrm{MWd}}{\mathrm{M}}$ & $\begin{array}{c}E \\
(H)\end{array}$ & $\begin{array}{c}F \\
(G)\end{array}$ & $\begin{array}{c}\mathrm{G} \\
(\mathrm{F})\end{array}$ & $\begin{array}{c}\mathrm{H} \\
(\mathrm{E})\end{array}$ & $\begin{array}{c}\mathrm{J} \\
(\mathrm{M})\end{array}$ & $\begin{array}{c}\mathrm{K} \\
\text { (L) }\end{array}$ & $\begin{array}{c}\mathrm{L} \\
(\mathrm{K})\end{array}$ & $\begin{array}{l}\mathrm{M} \\
(\mathrm{J})\end{array}$ \\
\hline 0 & 0.0 & 0.579 & 0.503 & 0.513 & 0.563 & 0.641 & 0.722 & 0.761 & 0.712 \\
\hline 1 & 25.0 & 0.611 & 0.534 & 0.545 & 0.596 & 0.674 & 0.753 & 0.792 & 0.744 \\
\hline 2 & 50.0 & 0.606 & 0.529 & 0.540 & 0.591 & 0.668 & 0.747 & 0.786 & 0.739 \\
\hline 3 & 100.0 & 0.595 & 0.519 & 0.529 & 0.580 & 0.657 & 0.737 & 0.776 & 0.728 \\
\hline 4 & 200.0 & 0.580 & 0.504 & 0.514 & 0.564 & 0.642 & 0.722 & 0.761 & 0.713 \\
\hline 5 & 300.0 & 0.572 & 0.497 & 0.506 & 0.555 & 0.634 & 0.715 & 0.754 & 0.705 \\
\hline 6 & 400.0 & 0.568 & 0.493 & 0.502 & 0.551 & 0.630 & 0.712 & 0.751 & 0.702 \\
\hline 7 & 500.0 & 0.568 & 0.492 & 0.501 & 0.550 & 0.630 & 0.711 & 0.751 & 0.702 \\
\hline 8 & 600.0 & 0.569 & 0.493 & 0.502 & 0.551 & 0.631 & 0.713 & 0.753 & 0.704 \\
\hline 9 & 700.0 & 0.572 & 0.495 & 0.504 & 0.554 & 0.634 & 0.716 & 0.756 & 0.707 \\
\hline 10 & 800.0 & 0.575 & 0.499 & 0.507 & 0.557 & 0.638 & 0.721 & 0.761 & 0.711 \\
\hline 11 & 900.0 & 0.580 & 0.503 & 0.512 & 0.562 & 0.643 & 0.726 & 0.766 & 0.716 \\
\hline 12 & 1000.0 & 0.585 & 0.507 & 0.516 & 0.567 & 0.649 & 0.731 & 0.771 & 0.722 \\
\hline 13 & 1100.0 & 0.591 & 0.513 & 0.521 & 0.573 & 0.655 & 0.737 & 0.777 & 0.728 \\
\hline 14 & 1200.0 & 0.597 & 0.518 & 0.527 & 0.578 & 0.661 & 0.744 & 0.784 & 0.734 \\
\hline 15 & 1300.0 & 0.603 & 0.524 & 0.533 & 0.584 & 0.667 & 0.750 & 0.790 & 0.741 \\
\hline
\end{tabular}




\section{Charge-Pan 1030}

Table 4.37. Problem 3.2.1 reference solution power peaking factors for charge-pan 1030 .

\begin{tabular}{|c|c|c|c|c|c|c|c|c|c|c|c|}
\hline \multirow[t]{2}{*}{ Step } & Burnup & \multicolumn{10}{|c|}{ Shift Power Peaking Factors } \\
\hline & $\left.\frac{\mathrm{MWd}}{\mathrm{MtU}}\right]$ & A & $\mathrm{B}, \mathrm{D}$ & $\mathrm{C}$ & $\mathrm{G}, \mathrm{F}$ & $\mathrm{H}, \mathrm{E}$ & $\mathrm{J}, \mathrm{M}$ & $\mathrm{K}, \mathrm{L}$ & Q & $\mathrm{R}, \mathrm{P}$ & $S$ \\
\hline 0 & 0.0 & 0.624 & 0.681 & 0.749 & 0.561 & 0.621 & 0.756 & 0.824 & 0.505 & 0.683 & 0.889 \\
\hline 1 & 25.0 & 0.603 & 0.657 & 0.727 & 0.541 & 0.602 & 0.737 & 0.801 & 0.487 & 0.668 & 0.866 \\
\hline 2 & 50.0 & 0.603 & 0.657 & 0.725 & 0.543 & 0.603 & 0.734 & 0.799 & 0.489 & 0.667 & 0.864 \\
\hline 3 & 100.0 & 0.612 & 0.665 & 0.736 & 0.549 & 0.611 & 0.742 & 0.810 & 0.493 & 0.673 & 0.874 \\
\hline 4 & 200.0 & 0.621 & 0.680 & 0.745 & 0.556 & 0.618 & 0.752 & 0.822 & 0.502 & 0.680 & 0.885 \\
\hline 5 & 300.0 & 0.626 & 0.688 & 0.753 & 0.564 & 0.626 & 0.758 & 0.827 & 0.510 & 0.687 & 0.892 \\
\hline 6 & 400.0 & 0.630 & 0.696 & 0.755 & 0.566 & 0.626 & 0.760 & 0.829 & 0.513 & 0.690 & 0.896 \\
\hline 7 & 500.0 & 0.633 & 0.693 & 0.757 & 0.570 & 0.630 & 0.761 & 0.830 & 0.516 & 0.690 & 0.897 \\
\hline 8 & 600.0 & 0.634 & 0.693 & 0.756 & 0.570 & 0.630 & 0.763 & 0.831 & 0.515 & 0.692 & 0.895 \\
\hline 9 & 700.0 & 0.630 & 0.688 & 0.754 & 0.568 & 0.629 & 0.761 & 0.829 & 0.515 & 0.693 & 0.893 \\
\hline 10 & 800.0 & 0.628 & 0.686 & 0.751 & 0.565 & 0.625 & 0.761 & 0.829 & 0.512 & 0.690 & 0.890 \\
\hline 11 & 900.0 & 0.627 & 0.684 & 0.750 & 0.567 & 0.627 & 0.761 & 0.826 & 0.512 & 0.693 & 0.888 \\
\hline 12 & 1000.0 & 0.625 & 0.676 & 0.746 & 0.561 & 0.624 & 0.758 & 0.825 & 0.506 & 0.690 & 0.886 \\
\hline 13 & 1100.0 & 0.617 & 0.669 & 0.742 & 0.557 & 0.621 & 0.754 & 0.818 & 0.504 & 0.687 & 0.878 \\
\hline 14 & 1200.0 & 0.615 & 0.668 & 0.737 & 0.557 & 0.618 & 0.752 & 0.816 & 0.503 & 0.687 & 0.876 \\
\hline 15 & 1300.0 & 0.612 & 0.661 & 0.736 & 0.553 & 0.618 & 0.749 & 0.813 & 0.501 & 0.684 & 0.872 \\
\hline \multirow[t]{2}{*}{ Step } & Burnup & \multicolumn{10}{|c|}{ MPACT Power Peaking Factors } \\
\hline & $\frac{\mathrm{MWd}}{\mathrm{MtU}}$ & A & $\mathrm{B}, \mathrm{D}$ & $\mathrm{C}$ & $\mathrm{G}, \mathrm{F}$ & $\mathrm{H}, \mathrm{E}$ & $\mathrm{J}, \mathrm{M}$ & $\mathrm{K}, \mathrm{L}$ & Q & $\mathrm{R}, \mathrm{P}$ & $S$ \\
\hline 0 & 0.0 & 0.623 & 0.682 & 0.748 & 0.559 & 0.620 & 0.754 & 0.823 & 0.504 & 0.682 & 0.890 \\
\hline 1 & 25.0 & 0.656 & 0.715 & 0.780 & 0.591 & 0.653 & 0.786 & 0.853 & 0.536 & 0.715 & 0.917 \\
\hline 2 & 50.0 & 0.650 & 0.709 & 0.774 & 0.586 & 0.647 & 0.780 & 0.847 & 0.531 & 0.709 & 0.911 \\
\hline 3 & 100.0 & 0.639 & 0.698 & 0.764 & 0.575 & 0.636 & 0.769 & 0.837 & 0.520 & 0.698 & 0.902 \\
\hline 4 & 200.0 & 0.624 & 0.682 & 0.749 & 0.559 & 0.621 & 0.755 & 0.824 & 0.505 & 0.682 & 0.890 \\
\hline 5 & 300.0 & 0.616 & 0.675 & 0.742 & 0.552 & 0.613 & 0.747 & 0.817 & 0.497 & 0.675 & 0.885 \\
\hline 6 & 400.0 & 0.612 & 0.671 & 0.739 & 0.548 & 0.609 & 0.744 & 0.814 & 0.493 & 0.671 & 0.882 \\
\hline 7 & 500.0 & 0.611 & 0.671 & 0.738 & 0.547 & 0.608 & 0.744 & 0.814 & 0.492 & 0.671 & 0.883 \\
\hline 8 & 600.0 & 0.613 & 0.672 & 0.740 & 0.548 & 0.610 & 0.746 & 0.816 & 0.493 & 0.672 & 0.885 \\
\hline 9 & 700.0 & 0.616 & 0.676 & 0.744 & 0.551 & 0.613 & 0.749 & 0.820 & 0.495 & 0.676 & 0.888 \\
\hline 10 & 800.0 & 0.620 & 0.680 & 0.748 & 0.554 & 0.617 & 0.754 & 0.824 & 0.499 & 0.680 & 0.892 \\
\hline 11 & 900.0 & 0.625 & 0.685 & 0.753 & 0.559 & 0.622 & 0.759 & 0.829 & 0.503 & 0.685 & 0.897 \\
\hline 12 & 1000.0 & 0.630 & 0.691 & 0.759 & 0.564 & 0.627 & 0.765 & 0.835 & 0.508 & 0.691 & 0.902 \\
\hline 13 & 1100.0 & 0.636 & 0.697 & 0.765 & 0.570 & 0.633 & 0.771 & 0.841 & 0.513 & 0.697 & 0.908 \\
\hline 14 & 1200.0 & 0.642 & 0.703 & 0.771 & 0.576 & 0.640 & 0.777 & 0.847 & 0.519 & 0.703 & 0.914 \\
\hline 15 & 1300.0 & 0.649 & 0.710 & 0.778 & 0.582 & 0.646 & 0.784 & 0.853 & 0.525 & 0.710 & 0.919 \\
\hline
\end{tabular}


Charge-Pans 1130 and (1031)

Table 4.38. Problem 3.2.1 reference solution power peaking factors for charge-pans 1130 and 1031.

\begin{tabular}{|c|c|c|c|c|c|c|c|c|}
\hline \multirow[t]{3}{*}{ Step } & \multirow{3}{*}{$\begin{array}{l}\text { Burnup } \\
{\left[\frac{\mathrm{MWd}}{\mathrm{MtU}}\right]}\end{array}$} & \multicolumn{7}{|c|}{ Shift Power Peaking Factors } \\
\hline & & A & B & $\mathrm{C}$ & $\mathrm{D}$ & $\mathrm{P}$ & $\mathrm{R}$ & S \\
\hline & & (A) & (D) & (C) & (B) & $(\mathrm{R})$ & (P) & (S) \\
\hline 0 & 0.0 & 0.392 & 0.423 & 0.468 & 0.425 & 0.461 & 0.426 & 0.606 \\
\hline 1 & 25.0 & & 414 & 0.458 & 0.415 & 0.448 & & 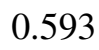 \\
\hline 2 & 50.0 & & 14 & 0.457 & 0.412 & 0.446 & 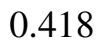 & .592 \\
\hline 3 & 100.0 & & 419 & 0.463 & 0.418 & 0.453 & 0.423 & .597 \\
\hline 4 & 200.0 & & 423 & 0.465 & 0.424 & 0. & & 00 \\
\hline 5 & 30 & & & 0.470 & 429 & 53 & 26 & 605 \\
\hline 6 & 400.0 & & 425 & 0.471 & 0.428 & 0.467 & 28 & 0.604 \\
\hline 7 & 500.0 & & & 0.473 & 0.431 & 0.467 & & 605 \\
\hline 8 & 600.0 & & 29 & 0.474 & 0.432 & 0.467 & 29 & 606 \\
\hline 9 & 700.0 & & 31 & 0.476 & 0.431 & 0.466 & 0.431 & 0.607 \\
\hline 10 & & & & & & & & 82 \\
\hline 11 & 900.0 & & & 0.474 & 0.433 & 0.466 & 4 & .606 \\
\hline 12 & 00.0 & & & 0.473 & 29 & 0 & & 603 \\
\hline 13 & 0 & & & 0.472 & 0.427 & & & 602 \\
\hline 14 & J & & & 0.472 & 0.428 & & & .604 \\
\hline 15 & 00.0 & & +30 & 0.472 & 0.426 & 0.458 & 0.436 & 600 \\
\hline \multirow[t]{3}{*}{ Step } & Burnup & \multicolumn{7}{|c|}{ MPACT Power Peaking Factors } \\
\hline & & A & B & $\mathrm{C}$ & $\mathrm{D}$ & $\mathrm{P}$ & $\mathrm{R}$ & $\mathrm{S}$ \\
\hline & & 1) & D) & (C) & (B) & $(\mathrm{R})$ & (1) & $(\mathrm{S})$ \\
\hline 0 & 0.0 & 389 & 422 & 0.467 & 0.424 & 0.461 & 4 & .604 \\
\hline 1 & & & & 0.497 & 0.452 & 0.4 & & .637 \\
\hline 2 & 0 & 3 & 6 & 92 & 48 & 86 & 17 & 632 \\
\hline 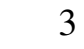 & 100.0 & & & 0.482 & 0.438 & 0.476 & 0. & 0.621 \\
\hline 4 & 200.0 & & 3 & 0.468 & 0.425 & 0.461 & 0. & .605 \\
\hline 5 & 300.0 & & & .460 & 0.417 & 0.454 & & .597 \\
\hline 6 & & & & & 0.414 & 0.450 & & .594 \\
\hline 7 & 500.0 & & 1 & 0.456 & 0.412 & 0.448 & 2 & .593 \\
\hline 8 & 600.0 & & & 0.456 & 0.413 & 0.449 & 0.412 & .594 \\
\hline (2) & 700.0 & & & 0.459 & 0.415 & 0.451 & 0.414 & 0.597 \\
\hline 10 & 800.0 & & & 0.462 & 0.418 & 0.454 & 0.417 & 0.601 \\
\hline 11 & 900.0 & & & 0.466 & 0.421 & 0.458 & 0.420 & 0.606 \\
\hline 12 & 1000.0 & 0.391 & 0.423 & 0.470 & 0.425 & 0.463 & 0.424 & 0.611 \\
\hline 13 & 1100.0 & & & 0.475 & 0.430 & 0.468 & 0.429 & 0.617 \\
\hline 14 & & & & & & & & 0.624 \\
\hline 15 & 1300.0 & 0.404 & 0.438 & 0.486 & 0.440 & 0.478 & 0.438 & 0.630 \\
\hline
\end{tabular}


Table 4.39. Problem 3.2.1 reference solution power peaking factors for charge-pans 1130 and 1031.

\begin{tabular}{|c|c|c|c|c|c|c|}
\hline \multirow[t]{3}{*}{ Step } & \multirow{3}{*}{$\begin{array}{l}\text { Burnup } \\
{\left[\frac{\mathrm{MWd}}{\mathrm{MtU}}\right]}\end{array}$} & \multicolumn{5}{|c|}{ Shift Power Peaking Factors } \\
\hline & & $\mathrm{E}$ & $\mathrm{J}$ & K & $\mathrm{L}$ & M \\
\hline & & (H) & (M) & (L) & $(\mathrm{K})$ & $(\mathrm{J})$ \\
\hline 0 & 0.0 & 0.412 & 0.463 & 0.529 & 0.548 & 0.491 \\
\hline 1 & 25.0 & 0.401 & 0.453 & 0.518 & 0.533 & 0.478 \\
\hline 2 & 50.0 & 0.400 & 0.451 & 0.517 & 0.530 & 0.475 \\
\hline 3 & 100.0 & 0.405 & 0.455 & 0.521 & 0.539 & 0.483 \\
\hline 4 & 200.0 & 0.409 & 0.459 & 0.523 & 0.544 & 0.489 \\
\hline 5 & 300.0 & 0.415 & 0.461 & 0.526 & 0.547 & 0.495 \\
\hline 6 & 400.0 & 0.418 & 0.462 & 0.527 & 0.549 & 0.496 \\
\hline 7 & 500.0 & 0.419 & 0.462 & 0.528 & 0.550 & 0.497 \\
\hline 8 & 600.0 & 0.421 & 0.462 & 0.530 & 0.551 & 0.497 \\
\hline 9 & 700.0 & 0.419 & 0.463 & 0.530 & 0.552 & 0.499 \\
\hline 10 & 800.0 & 0.418 & 0.463 & 0.527 & 0.550 & 0.497 \\
\hline 11 & 900.0 & 0.418 & 0.467 & 0.531 & 0.553 & 0.497 \\
\hline 12 & 1000.0 & 0.417 & 0.464 & 0.531 & 0.549 & 0.494 \\
\hline 13 & 1100.0 & 0.415 & 0.465 & 0.530 & 0.549 & 0.491 \\
\hline 14 & 1200.0 & 0.416 & 0.465 & 0.530 & 0.548 & 0.490 \\
\hline 15 & 1300.0 & 0.412 & 0.466 & 0.531 & 0.546 & 0.489 \\
\hline \multirow[t]{3}{*}{ Step } & Burnup & \multicolumn{5}{|c|}{ MPACT Power Peaking Factors } \\
\hline & & $\mathrm{E}$ & $\mathbf{J}$ & K & $\mathrm{L}$ & M \\
\hline & & (H) & $(\mathrm{M})$ & (L) & $(\mathrm{K})$ & $(\mathrm{J})$ \\
\hline 0 & 0.0 & 0.411 & 0.459 & 0.526 & 0.545 & 0.490 \\
\hline 1 & 25.0 & 0.440 & 0.488 & 0.557 & 0.577 & 0.521 \\
\hline 2 & 50.0 & 0.435 & 0.483 & 0.552 & 0.572 & 0.516 \\
\hline 3 & 100.0 & 0.426 & 0.474 & 0.542 & 0.561 & 0.506 \\
\hline 4 & 200.0 & 0.412 & 0.460 & 0.527 & 0.546 & 0.491 \\
\hline 5 & 300.0 & 0.405 & 0.452 & 0.519 & 0.538 & 0.483 \\
\hline 6 & 400.0 & 0.401 & 0.448 & 0.515 & 0.534 & 0.479 \\
\hline 7 & 500.0 & 0.399 & 0.447 & 0.514 & 0.533 & 0.478 \\
\hline 8 & 600.0 & 0.400 & 0.448 & 0.515 & 0.535 & 0.479 \\
\hline 9 & 700.0 & 0.402 & 0.450 & 0.518 & 0.537 & 0.482 \\
\hline 10 & 800.0 & 0.404 & 0.453 & 0.521 & 0.541 & 0.485 \\
\hline 11 & 900.0 & 0.408 & 0.457 & 0.526 & 0.545 & 0.489 \\
\hline 12 & 1000.0 & 0.412 & 0.461 & 0.531 & 0.550 & 0.494 \\
\hline 13 & 1100.0 & 0.416 & 0.466 & 0.536 & 0.556 & 0.499 \\
\hline 14 & 1200.0 & 0.421 & 0.471 & 0.542 & 0.562 & 0.504 \\
\hline 15 & 1300.0 & 0.426 & 0.477 & 0.548 & 0.568 & 0.510 \\
\hline
\end{tabular}

\subsubsection{Problem 3.2.2}

\subsubsection{Description}


Figure 9 shows the quarter core geometry for Problem 3.2.2, which is the same as Problem 3.1.2. The geometry remains $1 / 8$ symmetric. The diagonal dashed line illustrates an axis of reflected symmetry. The main control bank is inserted in the following charge-pans: 0729, 0730, 0927, 0929, and 1027. Material densities are listed in Table1.4. Temperatures are listed in Table 10. Burnup states used for comparison are listed in Table 1.5 and the total system specific power is listed in Table 1.6. 


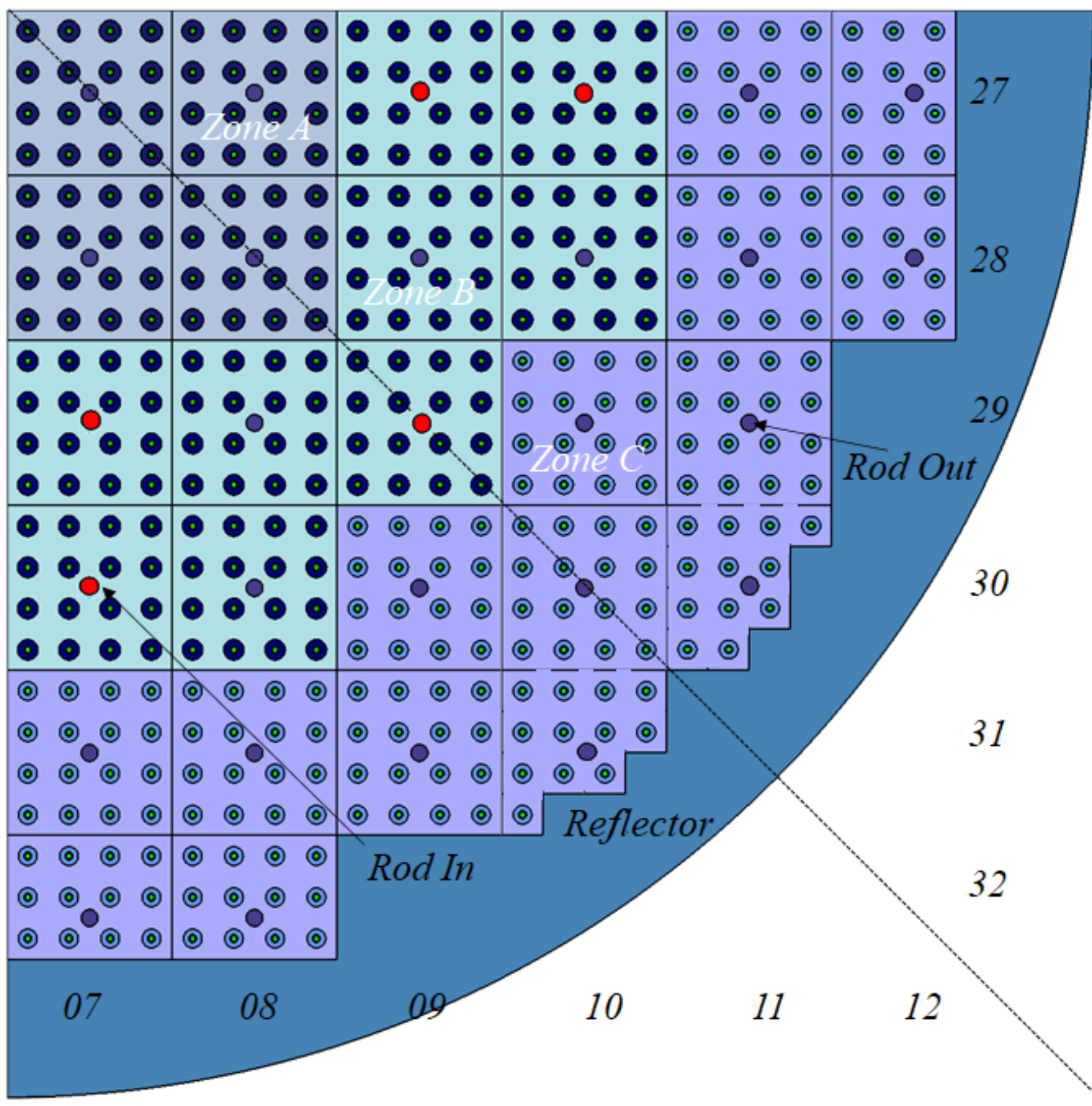

Figure 15. Quarter core 2D with main control bank inserted. 


\subsubsection{Reference Solution}

Table 4.40. Neutron multiplication factor for the reference solution of Problem 3.2.2.

\begin{tabular}{rrrrr}
\hline Step & $\begin{array}{r}\text { Burnup } \\
{\left[\frac{\mathrm{MWd}}{\mathrm{MtU}}\right]}\end{array}$ & $\begin{array}{c}\text { Shift } \\
\mathrm{k}_{\mathrm{eff}}(1 \sigma)\end{array}$ & \multicolumn{1}{c}{ MPACT } \\
$\mathrm{k}_{\text {eff }}(1 \sigma)$ & $\begin{array}{r}\text { Difference } \\
{[\mathrm{pcm}]}\end{array}$ \\
\hline 0 & 0.0 & $1.02371(5)$ & $1.02365(1)$ & $-6(5)$ \\
1 & 25.0 & $1.01060(5)$ & $1.008(1)$ & $-260(5)$ \\
2 & 50.0 & $1.01070(5)$ & $1.0083(1)$ & $-240(5)$ \\
3 & 100.0 & $1.01148(5)$ & $1.01032(1)$ & $-116(5)$ \\
4 & 200.0 & $1.01444(5)$ & $1.0151(1)$ & $66(5)$ \\
5 & 300.0 & $1.01740(5)$ & $1.01904(1)$ & $165(5)$ \\
6 & 400.0 & $1.01995(5)$ & $1.02205(1)$ & $211(6)$ \\
7 & 500.0 & $1.02200(5)$ & $1.0243(1)$ & $229(5)$ \\
8 & 600.0 & $1.02392(5)$ & $1.02594(1)$ & $202(5)$ \\
9 & 700.0 & $1.02530(6)$ & $1.0271(1)$ & $180(6)$ \\
10 & 800.0 & $1.0266(5)$ & $1.0279(1)$ & $130(5)$ \\
11 & 900.0 & $1.02736(5)$ & $1.0284(1)$ & $103(5)$ \\
12 & 1000.0 & $1.02834(5)$ & $1.02866(1)$ & $32(5)$ \\
13 & 1100.0 & $1.02890(5)$ & $1.02872(1)$ & $-18(5)$ \\
14 & 1200.0 & $1.02936(5)$ & $1.02862(1)$ & $-74(5)$ \\
15 & 1300.0 & $1.02950(5)$ & $1.02838(1)$ & $-112(5)$ \\
\hline
\end{tabular}

Table 4.41. Timing variables for the reference solution of Problem 3.2.2

\begin{tabular}{|c|c|c|c|c|c|c|}
\hline \multicolumn{7}{|c|}{$\overline{\text { Shift }}$} \\
\hline $\begin{array}{l}\text { Depletion } \\
\text { Solves }\end{array}$ & $\begin{array}{c}\text { Total } \\
\text { Cycles per } \\
\text { Transport } \\
\text { Solve }\end{array}$ & $\begin{array}{c}\text { Active } \\
\text { Cycles per } \\
\text { Transport } \\
\text { Solve }\end{array}$ & $\begin{array}{l}\text { Histories } \\
\text { per Cycle }\end{array}$ & $\begin{array}{c}\text { Compute } \\
\text { Nodes }\end{array}$ & $\begin{array}{c}\text { Cores } \\
\text { per Node }\end{array}$ & $\begin{array}{c}\text { Wall } \\
\text { Total Time } \\
\text { [hours] }\end{array}$ \\
\hline 15 & 1500 & 1200 & 200000 & 4 & 48 & 41.13 \\
\hline \multicolumn{7}{|c|}{ MPACT } \\
\hline $\begin{array}{c}\text { Depletion } \\
\text { Solves }\end{array}$ & $\begin{array}{c}k_{\mathrm{eff}} \\
\text { Convergence } \\
\text { Criteria }\end{array}$ & $\begin{array}{c}\text { Source } \\
\text { Convergence } \\
\text { Criteria }\end{array}$ & $\begin{array}{c}\text { Ray } \\
\text { Spacing } \\
{[\mathrm{cm}]}\end{array}$ & $\begin{array}{c}\text { Compute } \\
\text { Nodes }\end{array}$ & $\begin{array}{c}\text { Cores } \\
\text { per Node }\end{array}$ & $\begin{array}{c}\text { Wall } \\
\text { Total Time } \\
\text { [hours] }\end{array}$ \\
\hline 15 & $1.0 \times 10^{-04}$ & $1.0 \times 10^{-04}$ & 0.01 & 3 & 128 & 39.11 \\
\hline
\end{tabular}




\section{Charge-Pan 0727}

Table 4.42. Power peaking factors for charge-pan 0727 of the reference solution of Problem 3.2.2.

\begin{tabular}{|c|c|c|c|c|c|c|c|c|c|c|c|}
\hline \multirow[t]{2}{*}{ Step } & Burnup & \multicolumn{10}{|c|}{ Shift Power Peaking Factors } \\
\hline & {$\left[\frac{\mathrm{MWd}}{\mathrm{MtU}}\right]$} & A & $\mathrm{B}, \mathrm{D}$ & $\mathrm{C}$ & $\mathrm{G}, \mathrm{F}$ & $\mathrm{H}, \mathrm{E}$ & $\mathrm{J}, \mathrm{M}$ & $\mathrm{K}, \mathrm{L}$ & Q & $\mathrm{R}, \mathrm{P}$ & $S$ \\
\hline 0 & 0.0 & 1.965 & 1.996 & 2.031 & 1.929 & 1.960 & 2.030 & 2.071 & 1.884 & 1.978 & 2.099 \\
\hline 1 & 25.0 & 2.047 & 2.083 & 2.120 & 2.008 & 2.043 & 2.125 & 2.160 & 1.961 & 2.060 & 2.189 \\
\hline 2 & 50.0 & 2.033 & 2.069 & 2.106 & 1.998 & 2.029 & 2.104 & 2.148 & 1.950 & 2.048 & 2.173 \\
\hline 3 & 100.0 & 1.998 & 2.035 & 2.072 & 1.961 & 1.999 & 2.072 & 2.116 & 1.913 & 2.016 & 2.137 \\
\hline 4 & 200.0 & 1.961 & 2.002 & 2.038 & 1.930 & 1.965 & 2.038 & 2.078 & 1.880 & 1.983 & 2.097 \\
\hline 5 & 300.0 & 1.935 & 1.981 & 2.016 & 1.907 & 1.946 & 2.017 & 2.053 & 1.861 & 1.961 & 2.077 \\
\hline 6 & 400.0 & 1.906 & 1.963 & 2.001 & 1.883 & 1.929 & 1.996 & 2.034 & 1.837 & 1.945 & 2.059 \\
\hline 7 & 500.0 & 1.909 & 1.969 & 2.004 & 1.885 & 1.934 & 1.999 & 2.039 & 1.833 & 1.948 & 2.061 \\
\hline 8 & 600.0 & 1.913 & 1.979 & 2.014 & 1.892 & 1.944 & 2.011 & 2.051 & 1.838 & 1.957 & 2.071 \\
\hline 9 & 700.0 & 1.914 & 1.986 & 2.021 & 1.895 & 1.944 & 2.020 & 2.055 & 1.842 & 1.964 & 2.082 \\
\hline 10 & 800.0 & 1.923 & 1.997 & 2.029 & 1.902 & 1.962 & 2.027 & 2.066 & 1.847 & 1.976 & 2.087 \\
\hline 11 & 900.0 & 1.924 & 2.003 & 2.037 & 1.909 & 1.965 & 2.037 & 2.072 & 1.850 & 1.981 & 2.095 \\
\hline 12 & 1000.0 & 1.937 & 2.019 & 2.058 & 1.922 & 1.985 & 2.055 & 2.094 & 1.865 & 2.001 & 2.120 \\
\hline 13 & 1100.0 & 1.970 & 2.054 & 2.085 & 1.951 & 2.015 & 2.088 & 2.129 & 1.892 & 2.033 & 2.153 \\
\hline 14 & 1200.0 & 1.973 & 2.056 & 2.094 & 1.958 & 2.024 & 2.089 & 2.132 & 1.892 & 2.035 & 2.154 \\
\hline 15 & 1300.0 & 1.977 & 2.067 & 2.103 & 1.962 & 2.028 & 2.102 & 2.144 & 1.902 & 2.045 & 2.165 \\
\hline \multirow[t]{2}{*}{ Step } & Burnup & \multicolumn{10}{|c|}{ MPACT Power Peaking Factors } \\
\hline & $\frac{\mathrm{MWd}}{\mathrm{MtU}}$ & A & $\mathrm{B}, \mathrm{D}$ & $\mathrm{C}$ & $\mathrm{G}, \mathrm{F}$ & $\mathrm{H}, \mathrm{E}$ & $\mathrm{J}, \mathrm{M}$ & $\mathrm{K}, \mathrm{L}$ & Q & $\mathrm{R}, \mathrm{P}$ & S \\
\hline 0 & 0.0 & 1.978 & 2.011 & 2.046 & 1.943 & 1.973 & 2.048 & 2.087 & 1.899 & 1.992 & 2.111 \\
\hline 1 & 25.0 & 1.804 & 1.830 & 1.859 & 1.778 & 1.802 & 1.863 & 1.894 & 1.744 & 1.817 & 1.914 \\
\hline 2 & 50.0 & 1.845 & 1.873 & 1.903 & 1.817 & 1.843 & 1.907 & 1.940 & 1.780 & 1.859 & 1.961 \\
\hline 3 & 100.0 & 1.908 & 1.938 & 1.971 & 1.876 & 1.904 & 1.974 & 2.011 & 1.836 & 1.922 & 2.033 \\
\hline 4 & 200.0 & 1.989 & 2.022 & 2.059 & 1.954 & 1.985 & 2.061 & 2.101 & 1.909 & 2.004 & 2.125 \\
\hline 5 & 300.0 & 2.022 & 2.056 & 2.094 & 1.985 & 2.017 & 2.096 & 2.137 & 1.939 & 2.037 & 2.162 \\
\hline 6 & 400.0 & 2.030 & 2.065 & 2.102 & 1.994 & 2.026 & 2.105 & 2.146 & 1.947 & 2.045 & 2.170 \\
\hline 7 & 500.0 & 2.020 & 2.054 & 2.090 & 1.985 & 2.016 & 2.093 & 2.133 & 1.939 & 2.035 & 2.157 \\
\hline 8 & 600.0 & 1.998 & 2.031 & 2.066 & 1.964 & 1.994 & 2.069 & 2.108 & 1.921 & 2.013 & 2.131 \\
\hline 9 & 700.0 & 1.969 & 2.000 & 2.033 & 1.937 & 1.966 & 2.037 & 2.073 & 1.895 & 1.983 & 2.096 \\
\hline 10 & 800.0 & 1.935 & 1.964 & 1.996 & 1.905 & 1.932 & 1.999 & 2.034 & 1.865 & 1.949 & 2.055 \\
\hline 11 & 900.0 & 1.898 & 1.925 & 1.955 & 1.870 & 1.896 & 1.959 & 1.992 & 1.833 & 1.911 & 2.012 \\
\hline 12 & 1000.0 & 1.860 & 1.886 & 1.914 & 1.834 & 1.858 & 1.918 & 1.949 & 1.800 & 1.873 & 1.968 \\
\hline 13 & 1100.0 & 1.822 & 1.846 & 1.872 & 1.798 & 1.821 & 1.877 & 1.906 & 1.766 & 1.835 & 1.924 \\
\hline 14 & 1200.0 & 1.783 & 1.806 & 1.831 & 1.762 & 1.783 & 1.836 & 1.863 & 1.732 & 1.796 & 1.880 \\
\hline 15 & 1300.0 & 1.746 & 1.767 & 1.790 & 1.727 & 1.746 & 1.795 & 1.821 & 1.699 & 1.758 & 1.836 \\
\hline
\end{tabular}




\section{Charge-Pans 0827 and 0728}

Table 4.43. Problem 3.2.2 reference solution power peaking factors for charge-pans 0827 and 0728.

\begin{tabular}{|c|c|c|c|c|c|c|c|c|c|}
\hline \multirow[t]{3}{*}{ Step } & \multirow{3}{*}{$\begin{array}{l}\text { Burnup } \\
{\left[\frac{\mathrm{MWd}}{\mathrm{MtU}}\right]}\end{array}$} & \multicolumn{8}{|c|}{ Shift Power Peaking Factors } \\
\hline & & A & B & $\mathrm{C}$ & $\mathrm{D}$ & $\mathrm{P}$ & Q & $\mathrm{R}$ & S \\
\hline & & (A) & (D) & (C) & (B) & $(\mathrm{R})$ & (Q) & $(\mathrm{P})$ & (S) \\
\hline 0 & 0.0 & 1.637 & 1.645 & 1.759 & 1.744 & 1.823 & 1.520 & 1.517 & 1.894 \\
\hline 1 & 25.0 & 1.694 & 1.705 & 1.823 & 1.811 & 1.889 & 1.569 & 1.574 & 1.972 \\
\hline 2 & 50.0 & 1.687 & 1.696 & 1.818 & 1.798 & 1.879 & 1.563 & 1.566 & 1.959 \\
\hline 3 & 100.0 & 1.660 & 1.672 & 1.789 & 1.768 & 1.850 & 1.541 & 1.540 & 1.928 \\
\hline 4 & 200.0 & 1.638 & 1.647 & 1.766 & 1.744 & 1.827 & 1.524 & 1.520 & 1.901 \\
\hline 5 & 300.0 & 1.625 & 1.637 & 1.751 & 1.729 & 1.812 & 1.514 & 1.510 & 1.883 \\
\hline 6 & 400.0 & 1.614 & 1.625 & 1.740 & 1.713 & 1.798 & 1.505 & 1.496 & 1.865 \\
\hline 7 & 500.0 & 1.616 & 1.629 & 1.743 & 1.721 & 1.801 & 1.511 & 1.503 & 1.868 \\
\hline 8 & 600.0 & 1.620 & 1.639 & 1.750 & 1.727 & 1.809 & 1.517 & 1.512 & 1.878 \\
\hline 9 & 700.0 & 1.626 & 1.642 & 1.758 & 1.731 & 1.813 & 1.521 & 1.516 & 1.885 \\
\hline 10 & 800.0 & 1.639 & 1.657 & 1.770 & 1.742 & 1.825 & 1.534 & 1.528 & 1.899 \\
\hline 11 & 900.0 & 1.643 & 1.662 & 1.774 & 1.748 & 1.830 & 1.537 & 1.536 & 1.906 \\
\hline 12 & 1000.0 & 1.655 & 1.673 & 1.788 & 1.760 & 1.843 & 1.547 & 1.543 & 1.922 \\
\hline 13 & 1100.0 & 1.678 & 1.696 & 1.814 & 1.788 & 1.875 & 1.564 & 1.569 & 1.952 \\
\hline 14 & 1200.0 & 1.687 & 1.702 & 1.822 & 1.793 & 1.877 & 1.569 & 1.572 & 1.953 \\
\hline 15 & 1300.0 & 1.689 & 1.706 & 1.827 & 1.801 & 1.887 & 1.574 & 1.578 & 1.962 \\
\hline \multirow[t]{3}{*}{ Step } & Burnup & \multicolumn{8}{|c|}{ MPACT Power Peaking Factors } \\
\hline & $\frac{\mathrm{MWd}}{\mathrm{MtU}}$ & A & B & $\mathrm{C}$ & $\mathrm{D}$ & $\mathrm{P}$ & Q & $\mathrm{R}$ & S \\
\hline & & (A) & (D) & (C) & (B) & $(\mathrm{R})$ & $(\mathrm{Q})$ & $(\mathrm{P})$ & $(\mathrm{S})$ \\
\hline 0 & 0.0 & 1.637 & 1.645 & 1.759 & 1.744 & 1.823 & 1.520 & 1.517 & 1.894 \\
\hline 1 & 25.0 & 1.694 & 1.705 & 1.823 & 1.811 & 1.889 & 1.569 & 1.574 & 1.972 \\
\hline 2 & 50.0 & 1.687 & 1.696 & 1.818 & 1.798 & 1.879 & 1.563 & 1.566 & 1.959 \\
\hline 3 & 100.0 & 1.660 & 1.672 & 1.789 & 1.768 & 1.850 & 1.541 & 1.540 & 1.928 \\
\hline 4 & 200.0 & 1.638 & 1.647 & 1.766 & 1.744 & 1.827 & 1.524 & 1.520 & 1.901 \\
\hline 5 & 300.0 & 1.625 & 1.637 & 1.751 & 1.729 & 1.812 & 1.514 & 1.510 & 1.883 \\
\hline 6 & 400.0 & 1.614 & 1.625 & 1.740 & 1.713 & 1.798 & 1.505 & 1.496 & 1.865 \\
\hline 7 & 500.0 & 1.616 & 1.629 & 1.743 & 1.721 & 1.801 & 1.511 & 1.503 & 1.868 \\
\hline 8 & 600.0 & 1.620 & 1.639 & 1.750 & 1.727 & 1.809 & 1.517 & 1.512 & 1.878 \\
\hline 9 & 700.0 & 1.626 & 1.642 & 1.758 & 1.731 & 1.813 & 1.521 & 1.516 & 1.885 \\
\hline 10 & 800.0 & 1.639 & 1.657 & 1.770 & 1.742 & 1.825 & 1.534 & 1.528 & 1.899 \\
\hline 11 & 900.0 & 1.643 & 1.662 & 1.774 & 1.748 & 1.830 & 1.537 & 1.536 & 1.906 \\
\hline 12 & 1000.0 & 1.655 & 1.673 & 1.788 & 1.760 & 1.843 & 1.547 & 1.543 & 1.922 \\
\hline 13 & 1100.0 & 1.678 & 1.696 & 1.814 & 1.788 & 1.875 & 1.564 & 1.569 & 1.952 \\
\hline 14 & 1200.0 & 1.687 & 1.702 & 1.822 & 1.793 & 1.877 & 1.569 & 1.572 & 1.953 \\
\hline 15 & 1300.0 & 1.689 & 1.706 & 1.827 & 1.801 & 1.887 & 1.574 & 1.578 & 1.962 \\
\hline
\end{tabular}


Table 4.44. Problem 3.2.2 reference solution power peaking factors for charge-pans 0827 and 0728.

\begin{tabular}{|c|c|c|c|c|c|c|c|c|c|}
\hline \multirow[t]{2}{*}{ Step } & \multirow{2}{*}{$\begin{array}{l}\text { Burnup } \\
{\left[\frac{\mathrm{MWd}}{\mathrm{MtU}}\right]}\end{array}$} & \multicolumn{8}{|c|}{ Shift Power Peaking Factors } \\
\hline & & $\begin{array}{c}E \\
(H)\end{array}$ & $\begin{array}{c}F \\
(G)\end{array}$ & $\begin{array}{c}G \\
(F)\end{array}$ & $\begin{array}{l}\mathrm{H} \\
(\mathrm{E})\end{array}$ & $\begin{array}{c}\mathrm{J} \\
(\mathrm{M})\end{array}$ & $\begin{array}{c}\mathrm{K} \\
(\mathrm{L})\end{array}$ & $\begin{array}{c}\mathrm{L} \\
(\mathrm{K})\end{array}$ & $\begin{array}{l}M \\
(J)\end{array}$ \\
\hline 0 & 0.0 & 1.740 & 1.638 & 1.510 & 1.510 & 1.663 & 1.789 & 1.880 & 1.859 \\
\hline 1 & 25.0 & 1.797 & 1.689 & 1.564 & 1.567 & 1.726 & 1.858 & 1.952 & 1.930 \\
\hline 2 & 50.0 & 1.789 & 1.687 & 1.557 & 1.557 & 1.716 & 1.847 & 1.947 & 1.920 \\
\hline 3 & 100.0 & 1.763 & 1.659 & 1.533 & 1.532 & 1.691 & 1.816 & 1.913 & 1.886 \\
\hline 4 & 200.0 & 1.740 & 1.641 & 1.516 & 1.515 & 1.668 & 1.796 & 1.890 & 1.861 \\
\hline 5 & 300.0 & 1.728 & 1.627 & 1.505 & 1.507 & 1.655 & 1.776 & 1.872 & 1.842 \\
\hline 6 & 400.0 & 1.715 & 1.618 & 1.494 & 1.495 & 1.640 & 1.762 & 1.858 & 1.826 \\
\hline 7 & 500.0 & 1.715 & 1.625 & 1.496 & 1.501 & 1.642 & 1.765 & 1.858 & 1.831 \\
\hline 8 & 600.0 & 1.728 & 1.629 & 1.502 & 1.510 & 1.654 & 1.775 & 1.870 & 1.839 \\
\hline 9 & 700.0 & 1.729 & 1.634 & 1.504 & 1.511 & 1.658 & 1.782 & 1.875 & 1.842 \\
\hline 10 & 800.0 & 1.743 & 1.645 & 1.520 & 1.526 & 1.673 & 1.795 & 1.887 & 1.856 \\
\hline 11 & 900.0 & 1.751 & 1.653 & 1.526 & 1.536 & 1.678 & 1.800 & 1.893 & 1.863 \\
\hline 12 & 1000.0 & 1.758 & 1.660 & 1.536 & 1.541 & 1.689 & 1.814 & 1.911 & 1.878 \\
\hline 13 & 1100.0 & 1.787 & 1.681 & 1.553 & 1.560 & 1.714 & 1.842 & 1.945 & 1.907 \\
\hline 14 & 1200.0 & 1.789 & 1.687 & 1.558 & 1.565 & 1.718 & 1.843 & 1.944 & 1.912 \\
\hline 15 & 1300.0 & 1.797 & 1.694 & 1.568 & 1.575 & 1.725 & 1.856 & 1.951 & 1.914 \\
\hline \multirow[t]{2}{*}{ Step } & Burnup & \multicolumn{8}{|c|}{ MPACT Power Peaking Factors } \\
\hline & MWd & $\begin{array}{c}E \\
(H)\end{array}$ & $\begin{array}{c}\text { F } \\
(G)\end{array}$ & $\begin{array}{l}\mathrm{G} \\
(\mathrm{F})\end{array}$ & $\begin{array}{c}\mathrm{H} \\
(\mathrm{E})\end{array}$ & $\begin{array}{c}\mathrm{J} \\
(\mathrm{M})\end{array}$ & $\begin{array}{c}\mathrm{K} \\
(\mathrm{L})\end{array}$ & $\begin{array}{c}\mathrm{L} \\
(\mathrm{K})\end{array}$ & $\begin{array}{l}\text { M } \\
(\mathrm{J})\end{array}$ \\
\hline 0 & 0.0 & 1.740 & 1.638 & 1.510 & 1.510 & 1.663 & 1.789 & 1.880 & 1.859 \\
\hline 1 & 25.0 & 1.797 & 1.689 & 1.564 & 1.567 & 1.726 & 1.858 & 1.952 & 1.930 \\
\hline 2 & 50.0 & 1.789 & 1.687 & 1.557 & 1.557 & 1.716 & 1.847 & 1.947 & 1.920 \\
\hline 3 & 100.0 & 1.763 & 1.659 & 1.533 & 1.532 & 1.691 & 1.816 & 1.913 & 1.886 \\
\hline 4 & 200.0 & 1.740 & 1.641 & 1.516 & 1.515 & 1.668 & 1.796 & 1.890 & 1.861 \\
\hline 5 & 300.0 & 1.728 & 1.627 & 1.505 & 1.507 & 1.655 & 1.776 & 1.872 & 1.842 \\
\hline 6 & 400.0 & 1.715 & 1.618 & 1.494 & 1.495 & 1.640 & 1.762 & 1.858 & 1.826 \\
\hline 7 & 500.0 & 1.715 & 1.625 & 1.496 & 1.501 & 1.642 & 1.765 & 1.858 & 1.831 \\
\hline 8 & 600.0 & 1.728 & 1.629 & 1.502 & 1.510 & 1.654 & 1.775 & 1.870 & 1.839 \\
\hline 9 & 700.0 & 1.729 & 1.634 & 1.504 & 1.511 & 1.658 & 1.782 & 1.875 & 1.842 \\
\hline 10 & 800.0 & 1.743 & 1.645 & 1.520 & 1.526 & 1.673 & 1.795 & 1.887 & 1.856 \\
\hline 11 & 900.0 & 1.751 & 1.653 & 1.526 & 1.536 & 1.678 & 1.800 & 1.893 & 1.863 \\
\hline 12 & 1000.0 & 1.758 & 1.660 & 1.536 & 1.541 & 1.689 & 1.814 & 1.911 & 1.878 \\
\hline 13 & 1100.0 & 1.787 & 1.681 & 1.553 & 1.560 & 1.714 & 1.842 & 1.945 & 1.907 \\
\hline 14 & 1200.0 & 1.789 & 1.687 & 1.558 & 1.565 & 1.718 & 1.843 & 1.944 & 1.912 \\
\hline 15 & 1300.0 & 1.797 & 1.694 & 1.568 & 1.575 & 1.725 & 1.856 & 1.951 & 1.914 \\
\hline
\end{tabular}




\section{Charge-Pans 0927 and 0729}

Table 4.45. Problem 3.2.2 reference solution power peaking factors for charge-pans 0927 and 0729.

\begin{tabular}{|c|c|c|c|c|c|c|c|c|c|}
\hline \multirow[t]{3}{*}{ Step } & \multirow{3}{*}{$\begin{array}{l}\text { Burnup } \\
{\left[\frac{\mathrm{MWd}}{\mathrm{MtU}}\right]}\end{array}$} & \multicolumn{8}{|c|}{ Shift Power Peaking Factors } \\
\hline & & A & B & $\mathrm{C}$ & $\mathrm{D}$ & $\mathrm{P}$ & Q & $\mathrm{R}$ & $\mathrm{S}$ \\
\hline & & (A) & (D) & (C) & (B) & $(\mathrm{R})$ & $(\mathrm{Q})$ & (P) & $(\mathrm{S})$ \\
\hline 0 & 0.0 & 0.911 & 0.880 & 0.997 & 1.023 & 1.381 & 1.069 & 0.965 & 1.336 \\
\hline 1 & 25.0 & 0.936 & 0.906 & 1.032 & 1.052 & 1.422 & 1.089 & 0.993 & 1.387 \\
\hline 2 & 50.0 & 0.935 & 0.906 & 1.028 & 1.053 & 1.414 & 1.091 & 0.992 & 1.379 \\
\hline 3 & 100.0 & 0.924 & 0.892 & 1.013 & 1.035 & 1.395 & 1.083 & 0.983 & 1.361 \\
\hline 4 & 200.0 & 0.912 & 0.886 & 1.002 & 1.025 & 1.384 & 1.068 & 0.967 & 1.339 \\
\hline 5 & 300.0 & 0.907 & 0.880 & 0.998 & 1.018 & 1.373 & 1.063 & 0.962 & 1.331 \\
\hline 6 & 400.0 & 0.904 & 0.876 & 0.995 & 1.010 & 1.366 & 1.060 & 0.959 & 1.318 \\
\hline 7 & 500.0 & 0.906 & 0.879 & 0.995 & 1.015 & 1.372 & 1.065 & 0.960 & 1.321 \\
\hline 8 & 600.0 & 0.911 & 0.887 & 1.004 & 1.021 & 1.379 & 1.070 & 0.970 & 1.332 \\
\hline 9 & 700.0 & 0.911 & 0.885 & 1.004 & 1.019 & 1.376 & 1.068 & 0.970 & 1.333 \\
\hline 10 & 800.0 & 0.924 & 0.893 & 1.017 & 1.031 & 1.389 & 1.080 & 0.978 & 1.344 \\
\hline 11 & 900.0 & 0.926 & 0.901 & 1.018 & 1.035 & 1.399 & 1.086 & 0.986 & 1.351 \\
\hline 12 & 1000.0 & 0.931 & 0.906 & 1.024 & 1.041 & 1.403 & 1.092 & 0.992 & 1.358 \\
\hline 13 & 1100.0 & 0.938 & 0.913 & 1.037 & 1.054 & 1.420 & 1.102 & 1.001 & 1.380 \\
\hline 14 & 1200.0 & 0.945 & 0.919 & 1.039 & 1.057 & 1.419 & 1.105 & 1.009 & 1.382 \\
\hline 15 & 1300.0 & 0.951 & 0.928 & 1.049 & 1.062 & 1.431 & 1.114 & 1.016 & 1.391 \\
\hline \multirow[t]{3}{*}{ Step } & Burnup & \multicolumn{8}{|c|}{ MPACT Power Peaking Factors } \\
\hline & & A & B & $\mathrm{C}$ & $\mathrm{D}$ & $\mathrm{P}$ & Q & $\mathrm{R}$ & $\mathrm{S}$ \\
\hline & & (A) & (D) & (C) & (B) & (R) & (Q) & (P) & $(\mathrm{S})$ \\
\hline 0 & 0.0 & 0.909 & 0.879 & 0.998 & 1.022 & 1.386 & 1.068 & 0.965 & 1.343 \\
\hline 1 & 25.0 & 0.886 & 0.855 & 0.956 & 0.980 & 1.317 & 1.054 & 0.950 & 1.269 \\
\hline 2 & 50.0 & 0.892 & 0.861 & 0.966 & 0.990 & 1.333 & 1.058 & 0.954 & 1.286 \\
\hline 3 & 100.0 & 0.900 & 0.870 & 0.981 & 1.005 & 1.357 & 1.063 & 0.960 & 1.313 \\
\hline 4 & 200.0 & 0.912 & 0.883 & 1.002 & 1.025 & 1.390 & 1.070 & 0.969 & 1.349 \\
\hline 5 & 300.0 & 0.918 & 0.889 & 1.012 & 1.035 & 1.405 & 1.074 & 0.973 & 1.365 \\
\hline 6 & 400.0 & 0.922 & 0.892 & 1.017 & 1.039 & 1.411 & 1.077 & 0.976 & 1.371 \\
\hline 7 & 500.0 & 0.923 & 0.894 & 1.017 & 1.040 & 1.410 & 1.079 & 0.978 & 1.370 \\
\hline 8 & 600.0 & 0.923 & 0.893 & 1.015 & 1.038 & 1.405 & 1.080 & 0.978 & 1.363 \\
\hline 9 & 700.0 & 0.922 & 0.892 & 1.010 & 1.034 & 1.396 & 1.080 & 0.978 & 1.354 \\
\hline 10 & 800.0 & 0.920 & 0.890 & 1.005 & 1.028 & 1.386 & 1.080 & 0.977 & 1.342 \\
\hline 11 & 900.0 & 0.917 & 0.887 & 0.998 & 1.022 & 1.374 & 1.079 & 0.976 & 1.328 \\
\hline 12 & 1000.0 & 0.914 & 0.884 & 0.991 & 1.015 & 1.361 & 1.078 & 0.975 & 1.314 \\
\hline 13 & 1100.0 & 0.911 & 0.880 & 0.984 & 1.008 & 1.348 & 1.076 & 0.973 & 1.299 \\
\hline 14 & 1200.0 & 0.907 & 0.877 & 0.976 & 1.001 & 1.334 & 1.075 & 0.971 & 1.284 \\
\hline 15 & 1300.0 & 0.904 & 0.873 & 0.968 & 0.993 & 1.320 & 1.073 & 0.969 & 1.269 \\
\hline
\end{tabular}


Table 4.46. Problem 3.2.2 reference solution power peaking factors for charge-pans 0927 and 0729.

\begin{tabular}{|c|c|c|c|c|c|c|c|c|c|}
\hline \multirow[t]{2}{*}{ Step } & \multirow{2}{*}{$\begin{array}{l}\text { Burnup } \\
{\left[\frac{\mathrm{MWd}}{\mathrm{MtU}}\right]}\end{array}$} & \multicolumn{8}{|c|}{ Shift Power Peaking Factors } \\
\hline & & $\begin{array}{c}E \\
(H)\end{array}$ & $\begin{array}{c}F \\
(G)\end{array}$ & $\begin{array}{l}G \\
(F)\end{array}$ & $\begin{array}{l}\mathrm{H} \\
(\mathrm{E})\end{array}$ & $\begin{array}{c}\mathrm{J} \\
(\mathrm{M})\end{array}$ & $\begin{array}{c}\mathrm{K} \\
(\mathrm{L})\end{array}$ & $\begin{array}{c}\mathrm{L} \\
(\mathrm{K})\end{array}$ & $\begin{array}{l}M \\
(J)\end{array}$ \\
\hline 0 & 0.0 & 1.226 & 1.119 & 0.978 & 0.947 & 1.024 & 1.146 & 1.312 & 1.324 \\
\hline 1 & 25.0 & 1.257 & 1.147 & 1.005 & 0.975 & 1.057 & 1.187 & 1.358 & 1.370 \\
\hline 2 & 50.0 & 1.256 & 1.148 & 1.006 & 0.972 & 1.056 & 1.184 & 1.351 & 1.366 \\
\hline 3 & 100.0 & 1.240 & 1.135 & 0.994 & 0.962 & 1.040 & 1.168 & 1.333 & 1.344 \\
\hline 4 & 200.0 & 1.229 & 1.122 & 0.983 & 0.951 & 1.025 & 1.149 & 1.312 & 1.328 \\
\hline 5 & 300.0 & 1.221 & 1.117 & 0.976 & 0.947 & 1.021 & 1.145 & 1.309 & 1.318 \\
\hline 6 & 400.0 & 1.214 & 1.112 & 0.971 & 0.945 & 1.017 & 1.136 & 1.298 & 1.309 \\
\hline 7 & 500.0 & 1.221 & 1.116 & 0.976 & 0.947 & 1.019 & 1.139 & 1.303 & 1.315 \\
\hline 8 & 600.0 & 1.227 & 1.124 & 0.982 & 0.955 & 1.028 & 1.147 & 1.312 & 1.321 \\
\hline 9 & 700.0 & 1.227 & 1.121 & 0.979 & 0.954 & 1.026 & 1.147 & 1.313 & 1.320 \\
\hline 10 & 800.0 & 1.238 & 1.134 & 0.992 & 0.965 & 1.036 & 1.158 & 1.326 & 1.333 \\
\hline 11 & 900.0 & 1.243 & 1.140 & 0.999 & 0.970 & 1.041 & 1.166 & 1.330 & 1.335 \\
\hline 12 & 1000.0 & 1.251 & 1.143 & 1.004 & 0.980 & 1.047 & 1.173 & 1.339 & 1.345 \\
\hline 13 & 1100.0 & 1.263 & 1.154 & 1.013 & 0.986 & 1.060 & 1.189 & 1.357 & 1.363 \\
\hline 14 & 1200.0 & 1.267 & 1.162 & 1.016 & 0.991 & 1.065 & 1.191 & 1.362 & 1.366 \\
\hline 15 & 1300.0 & 1.275 & 1.165 & 1.024 & 0.998 & 1.073 & 1.202 & 1.368 & 1.375 \\
\hline \multirow[t]{2}{*}{ Step } & Burnup & \multicolumn{8}{|c|}{ MPACT Power Peaking Factors } \\
\hline & $\frac{\mathrm{MWd}}{\mathrm{M} I \mathrm{I}}$ & $\begin{array}{c}E \\
(H)\end{array}$ & $\begin{array}{c}\text { F } \\
(G)\end{array}$ & $\begin{array}{l}\mathrm{G} \\
(\mathrm{F})\end{array}$ & $\begin{array}{c}\mathrm{H} \\
(\mathrm{E})\end{array}$ & $\begin{array}{c}\mathrm{J} \\
(\mathrm{M})\end{array}$ & $\begin{array}{c}\mathrm{K} \\
(\mathrm{L})\end{array}$ & $\begin{array}{c}\mathrm{L} \\
(\mathrm{K})\end{array}$ & $\begin{array}{l}\text { M } \\
(\mathrm{J})\end{array}$ \\
\hline 0 & 0.0 & 1.228 & 1.122 & 0.980 & 0.946 & 1.024 & 1.151 & 1.318 & 1.331 \\
\hline 1 & 25.0 & 1.182 & 1.094 & 0.967 & 0.933 & 0.994 & 1.100 & 1.246 & 1.262 \\
\hline 2 & 50.0 & 1.193 & 1.101 & 0.971 & 0.937 & 1.002 & 1.113 & 1.263 & 1.278 \\
\hline 3 & 100.0 & 1.209 & 1.111 & 0.976 & 0.942 & 1.013 & 1.131 & 1.288 & 1.303 \\
\hline 4 & 200.0 & 1.232 & 1.125 & 0.983 & 0.950 & 1.029 & 1.156 & 1.323 & 1.336 \\
\hline 5 & 300.0 & 1.243 & 1.132 & 0.987 & 0.954 & 1.036 & 1.167 & 1.339 & 1.352 \\
\hline 6 & 400.0 & 1.248 & 1.136 & 0.990 & 0.957 & 1.040 & 1.172 & 1.345 & 1.358 \\
\hline 7 & 500.0 & 1.248 & 1.138 & 0.992 & 0.959 & 1.042 & 1.172 & 1.344 & 1.357 \\
\hline 8 & 600.0 & 1.246 & 1.137 & 0.993 & 0.960 & 1.041 & 1.169 & 1.338 & 1.351 \\
\hline 9 & 700.0 & 1.241 & 1.135 & 0.993 & 0.960 & 1.038 & 1.164 & 1.329 & 1.342 \\
\hline 10 & 800.0 & 1.235 & 1.132 & 0.993 & 0.959 & 1.035 & 1.157 & 1.317 & 1.332 \\
\hline 11 & 900.0 & 1.227 & 1.129 & 0.992 & 0.958 & 1.031 & 1.148 & 1.304 & 1.319 \\
\hline 12 & 1000.0 & 1.219 & 1.125 & 0.991 & 0.957 & 1.026 & 1.139 & 1.291 & 1.306 \\
\hline 13 & 1100.0 & 1.211 & 1.120 & 0.990 & 0.956 & 1.021 & 1.130 & 1.277 & 1.292 \\
\hline 14 & 1200.0 & 1.202 & 1.115 & 0.988 & 0.954 & 1.016 & 1.121 & 1.262 & 1.278 \\
\hline 15 & 1300.0 & 1.193 & 1.110 & 0.986 & 0.952 & 1.011 & 1.111 & 1.248 & 1.265 \\
\hline
\end{tabular}


Charge-Pans 1027 and (0730)

Table 4.47. Problem 3.2.2 reference solution power peaking factors for charge-pans 1027 and 0730.

\begin{tabular}{|c|c|c|c|c|c|c|c|c|c|}
\hline \multirow[t]{3}{*}{ Step } & \multirow{3}{*}{$\begin{array}{l}\text { Burnup } \\
{\left[\frac{\mathrm{MWd}}{\mathrm{MtU}}\right]}\end{array}$} & \multicolumn{8}{|c|}{ Shift Power Peaking Factors } \\
\hline & & A & B & $\mathrm{C}$ & $\mathrm{D}$ & $\mathrm{P}$ & Q & $\mathrm{R}$ & S \\
\hline & & (A) & (D) & (C) & (B) & $(\mathrm{R})$ & $(\mathrm{Q})$ & $(\mathrm{P})$ & (S) \\
\hline 0 & 0.0 & 0.719 & 0.693 & 0.722 & 0.755 & 1.002 & 0.892 & 0.820 & 0.902 \\
\hline 1 & 25.0 & 0.739 & 0.713 & 0.743 & 0.772 & 1.024 & 0.911 & 0.846 & 0.927 \\
\hline 2 & 50.0 & 0.739 & 0.713 & 0.741 & 0.771 & 1.024 & 0.912 & 0.849 & 0.927 \\
\hline 3 & 100.0 & 0.734 & 0.707 & 0.734 & 0.765 & 1.013 & 0.906 & 0.836 & 0.914 \\
\hline 4 & 200.0 & 0.722 & 0.695 & 0.721 & 0.751 & 1.003 & 0.891 & 0.822 & 0.897 \\
\hline 5 & 300.0 & 0.714 & 0.688 & 0.716 & 0.745 & 0.995 & 0.883 & 0.810 & 0.892 \\
\hline 6 & 400.0 & 0.713 & 0.685 & 0.715 & 0.742 & 0.993 & 0.882 & 0.810 & 0.885 \\
\hline 7 & 500.0 & 0.713 & 0.689 & 0.715 & 0.746 & 0.997 & 0.885 & 0.812 & 0.885 \\
\hline 8 & 600.0 & 0.716 & 0.693 & 0.722 & 0.749 & 1.002 & 0.887 & 0.816 & 0.890 \\
\hline 9 & 700.0 & 0.715 & 0.691 & 0.717 & 0.747 & 1.000 & 0.888 & 0.814 & 0.885 \\
\hline 10 & 800.0 & 0.726 & 0.700 & 0.727 & 0.756 & 1.007 & 0.902 & 0.829 & 0.896 \\
\hline 11 & 900.0 & 0.727 & 0.705 & 0.732 & 0.760 & 1.013 & 0.905 & 0.834 & 0.902 \\
\hline 12 & 1000.0 & 0.732 & 0.709 & 0.737 & 0.765 & 1.019 & 0.909 & .838 & 0.907 \\
\hline 13 & 1100.0 & 0.736 & 0.712 & 0.743 & 0.768 & 1.026 & 0.913 & 0.841 & 0.913 \\
\hline 14 & 1200.0 & 0.740 & 0.719 & 0.746 & 0.773 & 1.031 & 0.920 & 0.851 & 0.918 \\
\hline 15 & 1300.0 & 0.748 & 0.731 & 0.757 & 0.782 & 1.038 & 0.931 & 0.863 & 0.930 \\
\hline \multirow[t]{3}{*}{ Step } & Burnup & \multicolumn{8}{|c|}{ MPACT Power Peaking Factors } \\
\hline & $\left.\frac{\mathrm{MWd}}{\mathrm{MtU}}\right]$ & A & B & $\mathrm{C}$ & $\mathrm{D}$ & $\mathrm{P}$ & Q & $\mathrm{R}$ & $\mathrm{S}$ \\
\hline & & (A) & (D) & (C) & (B) & $(\mathrm{R})$ & $(\mathrm{Q})$ & $(\mathrm{P})$ & (S) \\
\hline 0 & 0.0 & 0.718 & 0.690 & 0.718 & 0.750 & 1.004 & 0.890 & 0.819 & 0.900 \\
\hline 1 & 25.0 & 0.738 & 0.709 & 0.726 & 0.759 & 1.004 & 0.922 & 0.850 & 0.898 \\
\hline 2 & 50.0 & 0.734 & 0.705 & 0.725 & 0.757 & 1.004 & 0.915 & 0.843 & 0.899 \\
\hline 3 & 100.0 & 0.727 & 0.699 & 0.722 & 0.755 & 1.004 & 0.904 & 0.833 & 0.900 \\
\hline 4 & 200.0 & 0.719 & 0.691 & 0.719 & 0.751 & 1.006 & 0.890 & 0.819 & 0.903 \\
\hline 5 & 300.0 & 0.716 & 0.688 & 0.719 & 0.750 & 1.007 & 0.884 & 0.814 & 0.905 \\
\hline 6 & 400.0 & 0.716 & 0.688 & 0.720 & 0.751 & 1.009 & 0.883 & 0.813 & 0.906 \\
\hline 7 & 500.0 & 0.717 & 0.689 & 0.721 & 0.753 & 1.011 & 0.885 & 0.815 & 0.908 \\
\hline 8 & 600.0 & 0.721 & 0.692 & 0.724 & 0.755 & 1.013 & 0.889 & 0.818 & 0.910 \\
\hline 9 & 700.0 & 0.724 & 0.696 & 0.726 & 0.758 & 1.015 & 0.894 & 0.824 & 0.911 \\
\hline 10 & 800.0 & 0.729 & 0.700 & 0.729 & 0.761 & 1.017 & 0.901 & 0.830 & 0.913 \\
\hline 11 & 900.0 & 0.734 & 0.705 & 0.732 & 0.764 & 1.018 & 0.908 & 0.836 & 0.914 \\
\hline 12 & 1000.0 & 0.739 & 0.710 & 0.735 & 0.767 & 1.020 & 0.915 & 0.843 & 0.915 \\
\hline 13 & 1100.0 & 0.744 & 0.715 & 0.738 & 0.770 & 1.021 & 0.922 & 0.850 & 0.916 \\
\hline 14 & 1200.0 & 0.749 & 0.720 & 0.741 & 0.773 & 1.022 & 0.929 & 0.857 & 0.917 \\
\hline 15 & 1300.0 & 0.754 & 0.725 & 0.744 & 0.776 & 1.022 & 0.936 & 0.864 & 0.917 \\
\hline
\end{tabular}


Table 4.48. Problem 3.2.2 reference solution power peaking factors for charge-pans 1027 and 0730 .

\begin{tabular}{|c|c|c|c|c|c|c|c|c|c|}
\hline \multirow[t]{2}{*}{ Step } & \multirow{2}{*}{$\begin{array}{l}\text { Burnup } \\
{\left[\frac{\mathrm{MWd}}{\mathrm{MtU}}\right]}\end{array}$} & \multicolumn{8}{|c|}{ Shift Power Peaking Factors } \\
\hline & & $\begin{array}{c}E \\
(H)\end{array}$ & $\begin{array}{c}F \\
(G)\end{array}$ & $\begin{array}{l}G \\
(F)\end{array}$ & $\begin{array}{l}\mathrm{H} \\
(\mathrm{E})\end{array}$ & $\begin{array}{c}\mathrm{J} \\
(\mathrm{M})\end{array}$ & $\begin{array}{c}\mathrm{K} \\
(\mathrm{L})\end{array}$ & $\begin{array}{c}\mathrm{L} \\
(\mathrm{K})\end{array}$ & $\begin{array}{l}M \\
(J)\end{array}$ \\
\hline 0 & 0.0 & 0.929 & 0.890 & 0.832 & 0.809 & 0.799 & 0.827 & 0.889 & 0.924 \\
\hline 1 & 25.0 & 0.949 & 0.908 & 0.853 & 0.833 & 0.825 & 0.853 & 0.915 & 0.948 \\
\hline 2 & 50.0 & 0.947 & 0.910 & 0.854 & 0.833 & 0.823 & 0.851 & 0.912 & 0.944 \\
\hline 3 & 100.0 & 0.942 & 0.903 & 0.845 & 0.824 & 0.812 & 0.840 & 0.902 & 0.936 \\
\hline 4 & 200.0 & 0.928 & 0.891 & 0.832 & 0.813 & 0.796 & 0.827 & 0.889 & 0.921 \\
\hline 5 & 300.0 & 0.922 & 0.883 & 0.820 & 0.797 & 0.788 & 0.819 & 0.880 & 0.912 \\
\hline 6 & 400.0 & 0.919 & 0.882 & 0.819 & 0.800 & 0.787 & 0.817 & 0.876 & 0.909 \\
\hline 7 & 500.0 & 0.922 & 0.885 & 0.823 & 0.803 & 0.789 & 0.817 & 0.876 & 0.911 \\
\hline 8 & 600.0 & 0.926 & 0.890 & 0.825 & 0.807 & 0.795 & 0.820 & 0.883 & 0.918 \\
\hline 9 & 700.0 & 0.924 & 0.886 & 0.823 & 0.806 & 0.791 & 0.818 & 0.881 & 0.913 \\
\hline 10 & 800.0 & 0.935 & 0.900 & 0.836 & 0.819 & 0.803 & 0.828 & 0.887 & 0.923 \\
\hline 11 & 900.0 & 0.941 & 0.903 & 0.840 & 0.825 & 0.810 & 0.834 & 0.893 & 0.927 \\
\hline 12 & 1000.0 & 0.947 & 0.908 & 0.845 & 0.827 & 0.816 & 0.839 & 0.901 & 0.931 \\
\hline 13 & 1100.0 & 0.952 & 0.909 & 0.848 & 0.831 & 0.819 & 0.845 & 0.909 & 0.937 \\
\hline 14 & 1200.0 & 0.956 & 0.918 & 0.856 & 0.841 & 0.827 & 0.852 & 0.914 & 0.944 \\
\hline 15 & 1300.0 & 0.966 & 0.928 & 0.866 & 0.852 & 0.839 & 0.860 & 0.920 & 0.950 \\
\hline \multirow[t]{2}{*}{ Step } & Burnup & \multicolumn{8}{|c|}{ MPACT Power Peaking Factors } \\
\hline & $\underline{\mathrm{MWd}}$ & $\begin{array}{c}E \\
(H)\end{array}$ & $\begin{array}{c}\text { F } \\
(G)\end{array}$ & $\begin{array}{l}\mathrm{G} \\
(\mathrm{F})\end{array}$ & $\begin{array}{c}\mathrm{H} \\
(\mathrm{E})\end{array}$ & $\begin{array}{c}\mathrm{J} \\
(\mathrm{M})\end{array}$ & $\begin{array}{c}\mathrm{K} \\
(\mathrm{L})\end{array}$ & $\begin{array}{c}\mathrm{L} \\
(\mathrm{K})\end{array}$ & $\begin{array}{l}\text { M } \\
(\mathrm{J})\end{array}$ \\
\hline 0 & 0.0 & 0.928 & 0.889 & 0.830 & 0.807 & 0.796 & 0.824 & 0.887 & 0.922 \\
\hline 1 & 25.0 & 0.939 & 0.911 & 0.861 & 0.838 & 0.817 & 0.834 & 0.886 & 0.920 \\
\hline 2 & 50.0 & 0.937 & 0.906 & 0.855 & 0.831 & 0.813 & 0.833 & 0.887 & 0.921 \\
\hline 3 & 100.0 & 0.933 & 0.899 & 0.844 & 0.821 & 0.807 & 0.830 & 0.888 & 0.922 \\
\hline 4 & 200.0 & 0.929 & 0.890 & 0.830 & 0.807 & 0.798 & 0.827 & 0.890 & 0.924 \\
\hline 5 & 300.0 & 0.928 & 0.886 & 0.825 & 0.802 & 0.795 & 0.826 & 0.892 & 0.926 \\
\hline 6 & 400.0 & 0.929 & 0.886 & 0.824 & 0.801 & 0.795 & 0.827 & 0.894 & 0.927 \\
\hline 7 & 500.0 & 0.931 & 0.888 & 0.826 & 0.802 & 0.797 & 0.829 & 0.896 & 0.929 \\
\hline 8 & 600.0 & 0.934 & 0.891 & 0.829 & 0.806 & 0.800 & 0.831 & 0.897 & 0.931 \\
\hline 9 & 700.0 & 0.937 & 0.896 & 0.835 & 0.812 & 0.804 & 0.834 & 0.899 & 0.933 \\
\hline 10 & 800.0 & 0.941 & 0.901 & 0.841 & 0.818 & 0.809 & 0.838 & 0.900 & 0.935 \\
\hline 11 & 900.0 & 0.944 & 0.906 & 0.848 & 0.824 & 0.814 & 0.841 & 0.902 & 0.936 \\
\hline 12 & 1000.0 & 0.948 & 0.912 & 0.855 & 0.831 & 0.819 & 0.844 & 0.903 & 0.937 \\
\hline 13 & 1100.0 & 0.951 & 0.917 & 0.862 & 0.838 & 0.824 & 0.847 & 0.904 & 0.938 \\
\hline 14 & 1200.0 & 0.955 & 0.923 & 0.869 & 0.845 & 0.829 & 0.850 & 0.905 & 0.939 \\
\hline 15 & 1300.0 & 0.958 & 0.928 & 0.876 & 0.852 & 0.835 & 0.853 & 0.905 & 0.940 \\
\hline
\end{tabular}


Charge-Pans 1127 and (0731)

Table 4.49. Problem 3.2.2 reference solution power peaking factors for charge-pans 1127 and 0731.

\begin{tabular}{|c|c|c|c|c|c|c|c|c|c|}
\hline \multirow[t]{3}{*}{ Step } & \multirow{3}{*}{$\begin{array}{l}\text { Burnup } \\
{\left[\frac{\mathrm{MWd}}{\mathrm{MtU}}\right]}\end{array}$} & \multicolumn{8}{|c|}{ Shift Power Peaking Factors } \\
\hline & & A & B & $\mathrm{C}$ & $\mathrm{D}$ & $\mathrm{P}$ & Q & $\mathrm{R}$ & $\mathrm{S}$ \\
\hline & & (A) & (D) & (C) & (B) & $(\mathrm{R})$ & $(\mathrm{Q})$ & $(\mathrm{P})$ & (S) \\
\hline 0 & 0.0 & 0.785 & 0.783 & 0.816 & 0.825 & 0.879 & 0.730 & 0.732 & 0.833 \\
\hline 1 & 25.0 & 0.810 & 0.814 & 0.847 & 0.853 & 0.902 & 0.751 & 0.761 & 0.863 \\
\hline 2 & 50.0 & 0.815 & 0.814 & 0.849 & 0.855 & 0.905 & 0.754 & 0.762 & 0.860 \\
\hline 3 & 100.0 & 0.805 & 0.805 & 0.838 & 0.845 & 0.895 & 0.748 & 0.753 & 0.851 \\
\hline 4 & 200.0 & 0.788 & 0.785 & 0.817 & 0.827 & 0.876 & 0.730 & 0.735 & 0.833 \\
\hline 5 & 300.0 & 0.772 & 0.769 & 0.802 & 0.814 & 0.864 & 0.715 & 0.716 & 0.819 \\
\hline 6 & 400.0 & 0.776 & 0.771 & 0.807 & 0.815 & 0.861 & 0.713 & 0.719 & 0.819 \\
\hline 7 & 500.0 & 0.778 & 0.772 & 0.805 & 0.819 & 0.862 & 0.716 & 0.719 & 0.819 \\
\hline 8 & 600.0 & 0.778 & 0.773 & 0.809 & 0.821 & 0.864 & 0.717 & 0.719 & 0.820 \\
\hline 9 & 700.0 & 0.779 & 0.772 & 0.808 & 0.823 & 0.863 & 0.712 & 0.718 & 0.816 \\
\hline 10 & 800.0 & 0.796 & 0.786 & 0.825 & 0.836 & 0.876 & 0.727 & 0.731 & 0.833 \\
\hline 11 & 900.0 & 0.798 & 0.792 & 0.828 & 0.840 & 0.878 & 0.731 & 0.737 & 0.838 \\
\hline 12 & 1000.0 & 0.803 & 0.795 & 0.831 & 0.843 & 0.882 & 0.730 & 0.738 & 0.842 \\
\hline 13 & 1100.0 & 0.805 & 0.799 & 0.833 & 0.845 & 0.882 & 0.733 & 0.742 & 0.843 \\
\hline 14 & 1200.0 & 0.812 & 0.808 & 0.843 & 0.857 & 0.890 & 0.740 & 0.752 & 0.854 \\
\hline 15 & 1300.0 & 0.827 & 0.824 & 0.860 & 0.872 & 0.905 & 0.751 & 0.764 & 0.865 \\
\hline \multirow[t]{3}{*}{ Step } & Burnup & \multicolumn{8}{|c|}{ MPACT Power Peaking Factors } \\
\hline & $\left.\frac{\mathrm{MWd}}{\mathrm{MtU}}\right]$ & A & B & $\mathrm{C}$ & $\mathrm{D}$ & $\mathrm{P}$ & Q & $\mathrm{R}$ & $\mathrm{S}$ \\
\hline & & (A) & (D) & (C) & (B) & (R) & (Q) & (P) & (S) \\
\hline 0 & 0.0 & 0.787 & 0.783 & 0.818 & 0.827 & 0.880 & 0.731 & 0.733 & 0.833 \\
\hline 1 & 25.0 & 0.836 & 0.833 & 0.863 & 0.872 & 0.920 & 0.782 & 0.785 & 0.872 \\
\hline 2 & 50.0 & 0.825 & 0.822 & 0.853 & 0.862 & 0.911 & 0.770 & 0.773 & 0.864 \\
\hline 3 & 100.0 & 0.808 & 0.805 & 0.837 & 0.846 & 0.897 & 0.753 & 0.756 & 0.850 \\
\hline 4 & 200.0 & 0.785 & 0.781 & 0.816 & 0.825 & 0.879 & 0.728 & 0.731 & 0.832 \\
\hline 5 & 300.0 & 0.774 & 0.771 & 0.807 & 0.816 & 0.871 & 0.717 & 0.720 & 0.825 \\
\hline 6 & 400.0 & 0.771 & 0.767 & 0.804 & 0.813 & 0.869 & 0.713 & 0.716 & 0.823 \\
\hline 7 & 500.0 & 0.771 & 0.768 & 0.805 & 0.814 & 0.870 & 0.714 & 0.716 & 0.824 \\
\hline 8 & 600.0 & 0.776 & 0.773 & 0.810 & 0.818 & 0.875 & 0.718 & 0.720 & 0.828 \\
\hline 9 & 700.0 & 0.782 & 0.779 & 0.816 & 0.825 & 0.881 & 0.724 & 0.726 & 0.834 \\
\hline 10 & 800.0 & 0.790 & 0.787 & 0.824 & 0.833 & 0.888 & 0.731 & 0.734 & 0.842 \\
\hline 11 & 900.0 & 0.799 & 0.796 & 0.833 & 0.841 & 0.896 & 0.740 & 0.743 & 0.849 \\
\hline 12 & 1000.0 & 0.809 & 0.805 & 0.842 & 0.850 & 0.905 & 0.750 & 0.753 & 0.858 \\
\hline 13 & 1100.0 & 0.818 & 0.815 & 0.851 & 0.860 & 0.913 & 0.759 & 0.763 & 0.866 \\
\hline 14 & 1200.0 & 0.828 & 0.825 & 0.861 & 0.869 & 0.922 & 0.770 & 0.773 & 0.875 \\
\hline 15 & 1300.0 & 0.839 & 0.836 & 0.870 & 0.879 & 0.930 & 0.780 & 0.784 & 0.883 \\
\hline
\end{tabular}


Table 4.50. Problem 3.2.2 reference solution power peaking factors for charge-pans 1127 and 0731 .

\begin{tabular}{|c|c|c|c|c|c|c|c|c|c|}
\hline \multirow[t]{2}{*}{ Step } & \multirow{2}{*}{$\begin{array}{l}\text { Burnup } \\
{\left[\frac{\mathrm{MWd}}{\mathrm{MtU}}\right]}\end{array}$} & \multicolumn{8}{|c|}{ Shift Power Peaking Factors } \\
\hline & & $\begin{array}{c}E \\
(H)\end{array}$ & $\begin{array}{c}F \\
(G)\end{array}$ & $\begin{array}{l}G \\
(F)\end{array}$ & $\begin{array}{l}\mathrm{H} \\
(\mathrm{E})\end{array}$ & $\begin{array}{c}\mathrm{J} \\
(\mathrm{M})\end{array}$ & $\begin{array}{c}\mathrm{K} \\
(\mathrm{L})\end{array}$ & $\begin{array}{c}\mathrm{L} \\
(\mathrm{K})\end{array}$ & $\begin{array}{l}M \\
(J)\end{array}$ \\
\hline 0 & 0.0 & 0.849 & 0.800 & 0.732 & 0.733 & 0.787 & 0.821 & 0.835 & 0.851 \\
\hline 1 & 25.0 & 0.869 & 0.819 & 0.756 & 0.759 & 0.817 & 0.855 & 0.863 & 0.875 \\
\hline 2 & 50.0 & 0.875 & 0.821 & 0.757 & 0.761 & 0.818 & 0.854 & 0.864 & 0.879 \\
\hline 3 & 100.0 & 0.865 & 0.813 & 0.752 & 0.751 & 0.809 & 0.844 & 0.855 & 0.871 \\
\hline 4 & 200.0 & 0.846 & 0.797 & 0.734 & 0.734 & 0.789 & 0.823 & 0.836 & 0.851 \\
\hline 5 & 300.0 & 0.834 & 0.785 & 0.719 & 0.717 & 0.771 & 0.805 & 0.821 & 0.841 \\
\hline 6 & 400.0 & 0.834 & 0.785 & 0.721 & 0.719 & 0.772 & 0.808 & 0.824 & 0.841 \\
\hline 7 & 500.0 & 0.833 & 0.785 & 0.723 & 0.721 & 0.772 & 0.809 & 0.827 & 0.842 \\
\hline 8 & 600.0 & 0.835 & 0.789 & 0.724 & 0.720 & 0.772 & 0.810 & 0.826 & 0.848 \\
\hline 9 & 700.0 & 0.833 & 0.788 & 0.726 & 0.722 & 0.773 & 0.809 & 0.826 & 0.848 \\
\hline 10 & 800.0 & 0.848 & 0.798 & 0.737 & 0.734 & 0.788 & 0.823 & 0.841 & 0.862 \\
\hline 11 & 900.0 & 0.854 & 0.804 & 0.744 & 0.738 & 0.794 & 0.829 & 0.846 & 0.866 \\
\hline 12 & 1000.0 & 0.857 & 0.805 & 0.745 & 0.741 & 0.794 & 0.830 & 0.851 & 0.869 \\
\hline 13 & 1100.0 & 0.857 & 0.806 & 0.750 & 0.747 & 0.797 & 0.834 & 0.853 & 0.873 \\
\hline 14 & 1200.0 & 0.864 & 0.816 & 0.759 & 0.755 & 0.808 & 0.844 & 0.862 & 0.883 \\
\hline 15 & 1300.0 & 0.879 & 0.829 & 0.773 & 0.771 & 0.824 & 0.859 & 0.875 & 0.896 \\
\hline \multirow[t]{2}{*}{ Step } & Burnup & \multicolumn{8}{|c|}{ MPACT Power Peaking Factors } \\
\hline & $\underline{\mathrm{MWd}}$ & $\begin{array}{c}E \\
(H)\end{array}$ & $\begin{array}{c}\text { F } \\
(G)\end{array}$ & $\begin{array}{l}\mathrm{G} \\
(\mathrm{F})\end{array}$ & $\begin{array}{c}\mathrm{H} \\
(\mathrm{E})\end{array}$ & $\begin{array}{c}\mathrm{J} \\
(\mathrm{M})\end{array}$ & $\begin{array}{c}\mathrm{K} \\
(\mathrm{L})\end{array}$ & $\begin{array}{c}\mathrm{L} \\
(\mathrm{K})\end{array}$ & $\begin{array}{l}\text { M } \\
(\mathrm{J})\end{array}$ \\
\hline 0 & 0.0 & 0.849 & 0.798 & 0.732 & 0.732 & 0.788 & 0.823 & 0.835 & 0.851 \\
\hline 1 & 25.0 & 0.895 & 0.847 & 0.783 & 0.784 & 0.838 & 0.869 & 0.875 & 0.891 \\
\hline 2 & 50.0 & 0.884 & 0.836 & 0.771 & 0.772 & 0.827 & 0.859 & 0.866 & 0.882 \\
\hline 3 & 100.0 & 0.868 & 0.819 & 0.754 & 0.754 & 0.810 & 0.843 & 0.852 & 0.868 \\
\hline 4 & 200.0 & 0.847 & 0.796 & 0.729 & 0.730 & 0.786 & 0.822 & 0.835 & 0.850 \\
\hline 5 & 300.0 & 0.838 & 0.785 & 0.718 & 0.719 & 0.776 & 0.812 & 0.827 & 0.843 \\
\hline 6 & 400.0 & 0.835 & 0.781 & 0.714 & 0.715 & 0.772 & 0.809 & 0.825 & 0.841 \\
\hline 7 & 500.0 & 0.836 & 0.782 & 0.715 & 0.715 & 0.773 & 0.811 & 0.826 & 0.842 \\
\hline 8 & 600.0 & 0.840 & 0.787 & 0.719 & 0.719 & 0.777 & 0.815 & 0.831 & 0.847 \\
\hline 9 & 700.0 & 0.847 & 0.793 & 0.725 & 0.725 & 0.784 & 0.821 & 0.837 & 0.853 \\
\hline 10 & 800.0 & 0.855 & 0.801 & 0.733 & 0.733 & 0.792 & 0.829 & 0.844 & 0.860 \\
\hline 11 & 900.0 & 0.863 & 0.810 & 0.741 & 0.742 & 0.801 & 0.838 & 0.852 & 0.868 \\
\hline 12 & 1000.0 & 0.873 & 0.820 & 0.751 & 0.752 & 0.811 & 0.847 & 0.860 & 0.876 \\
\hline 13 & 1100.0 & 0.882 & 0.829 & 0.761 & 0.762 & 0.821 & 0.857 & 0.868 & 0.885 \\
\hline 14 & 1200.0 & 0.892 & 0.840 & 0.771 & 0.772 & 0.831 & 0.866 & 0.877 & 0.893 \\
\hline 15 & 1300.0 & 0.901 & 0.850 & 0.781 & 0.782 & 0.841 & 0.876 & 0.886 & 0.902 \\
\hline
\end{tabular}


Charge-Pans 1227 and (0732)

Table 4.51. Problem 3.2.2 reference solution power peaking factors for charge-pans 1227 and 0732 .

\begin{tabular}{|c|c|c|c|c|c|c|c|}
\hline \multirow[t]{3}{*}{ Step } & \multirow{3}{*}{$\begin{array}{l}\text { Burnup } \\
{\left[\frac{\mathrm{MWd}}{\mathrm{MtU}}\right]}\end{array}$} & \multicolumn{6}{|c|}{ Shift Power Peaking Factors } \\
\hline & & A & B & $\mathrm{C}$ & $\mathrm{D}$ & $\mathrm{P}$ & S \\
\hline & & (A) & (D) & (C) & (B) & $(\mathrm{R})$ & (Q) \\
\hline 0 & 0.0 & 0.517 & 0.522 & 0.570 & 0.565 & 0.650 & 0.660 \\
\hline 1 & 25.0 & 0.538 & 0.545 & 0.592 & 0.585 & 0.669 & 0.687 \\
\hline 2 & 50.0 & 0.538 & 0.547 & 0.592 & 0.587 & 0.670 & 0.688 \\
\hline 3 & 100.0 & 0.534 & 0.540 & 0.588 & 0.579 & 0.663 & 0.681 \\
\hline 4 & 200.0 & 0.520 & 0.526 & 73 & 0.568 & 0.650 & 0.662 \\
\hline 5 & 300.0 & 0.508 & 0.509 & 0.557 & 0.552 & 0.636 & 0.645 \\
\hline 6 & 400.0 & 0.510 & 0.514 & 0.560 & 0.555 & 0.639 & 0.646 \\
\hline 7 & 500.0 & 9 & 0.511 & 0.561 & 0.553 & 0.637 & 0.643 \\
\hline 8 & 600.0 & 0.509 & 0.511 & 0.561 & 0.553 & 0.638 & 0.644 \\
\hline 9 & 700.0 & & 0.508 & & 0.550 & 37 & 0.642 \\
\hline 10 & 800.0 & 0.518 & 0.519 & 0.571 & 0.563 & 0.651 & 0.655 \\
\hline 11 & 900.0 & 0.524 & 0.524 & 0.578 & 0.567 & 0.656 & 0.661 \\
\hline 12 & 1000.0 & 0.523 & 0.521 & 78 & 0.568 & 0.656 & 0.661 \\
\hline 13 & 1100.0 & 0.526 & 0.525 & 0.581 & 0.570 & 0.658 & 0.662 \\
\hline 14 & 1200.0 & 4 & 0.533 & 88 & 0.577 & 0.666 & 0.674 \\
\hline 15 & 1300.0 & 4 & 0.542 & 0.599 & 0.586 & 0.679 & 0.685 \\
\hline \multirow[t]{3}{*}{ Step } & Burnup & \multicolumn{6}{|c|}{ MPACT Power Peaking Factors } \\
\hline & & A & B & $\mathrm{C}$ & D & $\mathrm{P}$ & $S$ \\
\hline & & (A) & (D) & (C) & (B) & (R) & (Q) \\
\hline 0 & 0.0 & 0.519 & 0.526 & 0.571 & 0.566 & 0.650 & 0.660 \\
\hline 1 & 25.0 & 0.564 & 0.571 & 0.618 & 0.613 & 0.699 & 0.711 \\
\hline 2 & 50.0 & 0.555 & 0.562 & 0.608 & 0.603 & 0.688 & 0.700 \\
\hline 3 & 100.0 & 0.539 & 0.546 & 0.591 & 0.586 & 0.671 & 0.682 \\
\hline 4 & 200.0 & 0.517 & 0.523 & 0.568 & 0.564 & 0.647 & 0.658 \\
\hline 5 & 300.0 & 0.506 & 0.513 & 0.558 & 0.553 & 0.636 & 0.646 \\
\hline 6 & 400.0 & 0.502 & 0.508 & 0.553 & 0.548 & 0.631 & 0.642 \\
\hline 7 & 500.0 & 0.501 & 0.507 & 0.553 & 0.548 & 0.631 & 0.642 \\
\hline 8 & 600.0 & 0.503 & 0.509 & & 0.551 & 0.635 & 0.645 \\
\hline 9 & 700.0 & 0.507 & 0.514 & 0.560 & 0.556 & 0.640 & 0.651 \\
\hline 10 & 800.0 & 0.513 & 0.520 & 0.567 & 0.562 & 0.647 & 0.659 \\
\hline 11 & 900.0 & 0.520 & 0.526 & 0.574 & 0.569 & 0.656 & 0.667 \\
\hline 12 & 1000.0 & 0.527 & 0.534 & 0.582 & 0.577 & 0.664 & 0.676 \\
\hline 13 & 1100.0 & 0.535 & 0.542 & 0.591 & 0.586 & 0.674 & 0.686 \\
\hline 14 & 1200.0 & 0.543 & 0.550 & 0.600 & 0.594 & 0.683 & 0.696 \\
\hline 15 & 1300.0 & 0.551 & 0.558 & 0.609 & 0.603 & 0.693 & 0.706 \\
\hline
\end{tabular}


Table 4.52. Problem 3.2.2 reference solution power peaking factors for charge-pans 1227 and 0732 .

\begin{tabular}{|c|c|c|c|c|c|c|c|}
\hline \multirow[t]{3}{*}{ Step } & \multirow{3}{*}{$\begin{array}{l}\text { Burnup } \\
{\left[\frac{\mathrm{MWd}}{\mathrm{MtU}}\right]}\end{array}$} & \multicolumn{6}{|c|}{ Shift Power Peaking Factors } \\
\hline & & & $\mathrm{F}$ & $\mathbf{J}$ & K & L & M \\
\hline & & $(\mathrm{H})$ & $(\mathrm{G})$ & $(\mathrm{F})$ & (E) & $(\mathrm{M})$ & $(\mathrm{L})$ \\
\hline 0 & 0.0 & 0.564 & 0.511 & 0.529 & 0.580 & 0.657 & 0.654 \\
\hline 1 & 25.0 & 81 & 0.527 & 0.551 & 0.601 & & 77 \\
\hline 2 & 50.0 & 582 & 0.529 & 0.555 & 0.604 & 0.683 & .677 \\
\hline 3 & 100.0 & .578 & 0.522 & 0.547 & 0.598 & 0.677 & 0.672 \\
\hline 4 & 200.0 & & 0.512 & & 0.581 & & \\
\hline 5 & 300.0 & 0 & 0.501 & 0.518 & 0.565 & & 0.642 \\
\hline 6 & 400.0 & & 0.504 & & 0.569 & 6 & 45 \\
\hline 7 & 500.0 & & 0.502 & 9 & 0.566 & & \\
\hline 8 & 600.0 & 6 & 0.504 & 0.519 & 0.566 & 5 & 0.648 \\
\hline 9 & 0 & & 0.502 & & 0.564 & & \\
\hline 10 & 800.0 & & 0.514 & 0 . & 0.575 & 55 & 657 \\
\hline 11 & 900.0 & & 0.519 & 4 & 0.581 & 61 & \\
\hline 12 & 1000.0 & & 0.518 & 32 & 0.579 & & 65 \\
\hline 13 & 1100.0 & & 0.519 & 0.537 & 0.584 & 7 & 70 \\
\hline 14 & 00.0 & & 0.528 & & 0.593 & & 78 \\
\hline 15 & 00.0 & & 0.539 & 0.555 & 0.605 & 0.690 & 0.688 \\
\hline \multirow[t]{2}{*}{ Step } & Burnup & \multicolumn{6}{|c|}{ MPACT Power Peaking Factors } \\
\hline & [MWd] & $\begin{array}{c}E \\
(H)\end{array}$ & $\begin{array}{c}\mathrm{F} \\
(\mathrm{G})\end{array}$ & $\begin{array}{c}\mathbf{J} \\
(\mathrm{F})\end{array}$ & $\begin{array}{l}\mathrm{K} \\
(\mathrm{E})\end{array}$ & $\begin{array}{c}\mathrm{L} \\
(\mathrm{M})\end{array}$ & $\begin{array}{l}\text { M } \\
\text { (L) }\end{array}$ \\
\hline 0 & 0.0 & 0.564 & 0.511 & 0.532 & 0.580 & 0.657 & 0.654 \\
\hline 1 & 25.0 & 0 & 0.555 & 78 & 0.628 & 8 & 704 \\
\hline 2 & 50.0 & 0.600 & 0.546 & 58 & 0.617 & 0.697 & 0.693 \\
\hline 3 & 100.0 & 4 & 0.530 & 0.552 & 0.601 & 0.679 & 0.676 \\
\hline 4 & 200.0 & 1 & 0.509 & 29 & 0.577 & 5 & 0.652 \\
\hline 5 & 300.0 & & 0.498 & 0.519 & 0.567 & & 0.641 \\
\hline 6 & 400.0 & 6 & 0.493 & 14 & 0.562 & 39 & 0.636 \\
\hline 7 & 500.0 & & 0.493 & 0.513 & 0.561 & 0.639 & 0.636 \\
\hline 0 & 600.0 & & 0.495 & 0.515 & 0.564 & 0.643 & 0.639 \\
\hline 9 & 700.0 & 0.5 & 0.499 & 0.520 & 0.569 & 0.648 & 0.645 \\
\hline 10 & 800.0 & 0.559 & 0.505 & 0.526 & 0.576 & 0.656 & 0.652 \\
\hline 11 & 900.0 & 0.567 & 0.511 & 0.533 & 0.583 & 0.664 & 0.661 \\
\hline 12 & 1000.0 & & 0.518 & 0.540 & 0.592 & 0.673 & 0.670 \\
\hline 13 & 1100.0 & & 0.526 & & 0.600 & 0.683 & 0.679 \\
\hline 14 & 1200.0 & 0.592 & 0.534 & 0.556 & 0.609 & 0.693 & 0.689 \\
\hline 15 & 1300.0 & 0.600 & 0.542 & 0.565 & 0.619 & 0.702 & 0.699 \\
\hline
\end{tabular}




\section{Charge-Pan 0828}

Table 4.53. Problem 3.2.2 reference solution power peaking factors for charge-pan 0828 .

\begin{tabular}{|c|c|c|c|c|c|c|c|c|c|c|c|}
\hline \multirow[t]{2}{*}{ Step } & Burnup & \multicolumn{10}{|c|}{ Shift Power Peaking Factors } \\
\hline & $\mathrm{MWd}$ & A & $\mathrm{B}, \mathrm{D}$ & $\mathrm{C}$ & $\mathrm{G}, \mathrm{F}$ & $\mathrm{H}, \mathrm{E}$ & $\mathrm{J}, \mathrm{M}$ & $\mathrm{K}, \mathrm{L}$ & Q & $\mathrm{R}, \mathrm{P}$ & $S$ \\
\hline 0 & 0.0 & 1.507 & 1.557 & 1.624 & 1.455 & 1.498 & 1.613 & 1.697 & 1.402 & 1.517 & 1.767 \\
\hline 1 & 25.0 & 1.539 & 1.596 & 1.665 & 1.483 & 1.529 & 1.661 & 1.753 & 1.421 & 1.561 & 1.827 \\
\hline 2 & 50.0 & 1.536 & 1.596 & 1.662 & 1.482 & 1.527 & 1.654 & 1.741 & 1.421 & 1.556 & 1.824 \\
\hline 3 & 100.0 & 1.523 & 1.577 & 1.642 & 1.468 & 1.512 & 1.634 & 1.722 & 1.411 & 1.537 & 1.794 \\
\hline 4 & 200.0 & 1.510 & 1.561 & 1.628 & 1.456 & 1.501 & 1.618 & 1.697 & 1.403 & 1.523 & 1.777 \\
\hline 5 & 300.0 & 1 & 1. & 1. & 1.454 & 196 & 1.608 & 1.689 & & 515 & 1.766 \\
\hline 6 & 400.0 & 1.490 & 1.549 & 1.615 & 1.445 & 1.487 & 1.597 & 1.682 & 1.393 & 1.511 & 1.754 \\
\hline 7 & 500.0 & 1.493 & 1.554 & 1.618 & 1.450 & 1.490 & 1.601 & 1.682 & 1.398 & 1.511 & 1.759 \\
\hline 8 & 600.0 & 1.498 & 1.558 & 1.623 & 1.453 & 1.496 & 1.607 & 1.691 & 1.402 & 1.517 & 1.767 \\
\hline 9 & 700.0 & 1.501 & 1.563 & 1.628 & 1.454 & 1.502 & 1.611 & 1.698 & 1.402 & 1.522 & 1.768 \\
\hline 10 & 800.0 & & 1.573 & 1.6 & 1.460 & 1.509 & 1.626 & 1.710 & 04 & 33 & 1.783 \\
\hline 11 & 900.0 & 1 & 1.575 & 1.637 & 1.461 & 1.514 & 1.629 & 1.714 & 1.403 & 1.538 & 1.784 \\
\hline 12 & 1000.0 & 1.514 & 1.585 & 1.649 & 1.468 & 1.523 & 1.638 & 1.722 & 1.411 & 1.544 & 1.799 \\
\hline 13 & 1100.0 & & 1.599 & 1.667 & 1.481 & 1.535 & 1.658 & 1.747 & 22 & 63 & 1.823 \\
\hline 14 & 1200.0 & 1.528 & 1.605 & 1.671 & 1.484 & 1.542 & 1.665 & 1.753 & 1.418 & 1.568 & 1.829 \\
\hline 15 & 1300.0 & & 1.611 & 1.6 & 1.485 & 1.548 & 1.672 & 1.758 & 22 & 72 & 1.834 \\
\hline \multirow[t]{2}{*}{ Step } & Burnup & \multicolumn{10}{|c|}{ MPACT Power Peaking Factors } \\
\hline & $\frac{\mathrm{MWd}}{\mathrm{MtU}}$ & A & $\mathrm{B}, \mathrm{D}$ & $\mathrm{C}$ & $\mathrm{G}, \mathrm{F}$ & $\mathrm{H}, \mathrm{E}$ & $\mathrm{J}, \mathrm{M}$ & $\mathrm{K}, \mathrm{L}$ & Q & $\mathrm{R}, \mathrm{P}$ & S \\
\hline 0 & 0.0 & 1.515 & 1.566 & 1.631 & 1.462 & 1.503 & 1.621 & 1.706 & 1.406 & 1.527 & 1.779 \\
\hline 1 & 25.0 & 1.438 & 1.477 & 1.529 & 1.398 & 1.429 & 1.521 & 1.590 & 1.354 & 1.444 & 1.649 \\
\hline 2 & 50.0 & 1.455 & 1.497 & 1.5 & 1.413 & 1.446 & 1.544 & 1.617 & 1.366 & 1.463 & 1.680 \\
\hline 3 & 100.0 & 1.482 & 1.528 & 1.5 & 1.435 & 1.472 & 1.579 & 1.658 & 1.384 & 1.492 & 1.726 \\
\hline 4 & 200.0 & 1.519 & 1.570 & 1.637 & 1.466 & 1.507 & 1.627 & 1.713 & 1.409 & 1.532 & 1.788 \\
\hline 5 & 300.0 & 1. & 1.589 & 1.6 & 1.480 & 1.523 & 1.647 & 1.736 & 1.421 & 1.549 & 1.814 \\
\hline 6 & 400.0 & 1.542 & 1.596 & 1.665 & 1.486 & 1.530 & 1.655 & 1.744 & 1.426 & 1.556 & 1.822 \\
\hline 7 & 500.0 & 1.541 & 1.594 & 1.662 & 1.485 & 1.529 & 1.652 & 1.740 & 1.427 & 1.554 & 1.816 \\
\hline 8 & 600.0 & 1.534 & 1.586 & 1.652 & 1.481 & 1.523 & 1.642 & 1.728 & 1.424 & 1.547 & 1.802 \\
\hline 9 & 700.0 & 1.524 & 1.574 & 1.637 & 1.473 & 1.513 & 1.628 & 1.710 & 1.418 & 1.536 & 1.781 \\
\hline 10 & 800.0 & 1.512 & 1.559 & 1.620 & 1.463 & 1.501 & 1.610 & 1.690 & 1.411 & 1.522 & 1.757 \\
\hline 11 & 900.0 & 1.498 & 1.542 & 1.600 & 1.452 & 1.488 & 1.591 & 1.667 & 1.402 & 1.507 & 1.731 \\
\hline 12 & 1000.0 & 1.483 & 1.525 & 1.579 & 1.440 & 1.474 & 1.571 & 1.643 & 1.393 & 1.491 & 1.703 \\
\hline 13 & 1100.0 & 1.467 & 1.506 & 1.558 & 1.428 & 1.459 & 1.550 & 1.618 & 1.383 & 1.474 & 1.676 \\
\hline 14 & 1200.0 & 1.451 & 1.488 & 1.537 & 1.415 & 1.444 & 1.529 & 1.594 & 1.372 & 1.457 & 1.648 \\
\hline 15 & 1300.0 & 1.435 & 1.470 & 1.515 & 1.402 & 1.428 & 1.508 & 1.569 & 1.362 & 1.440 & 1.620 \\
\hline
\end{tabular}


Charge-Pans 0928 and (0829)

Table 4.54. Problem 3.2.2 reference solution power peaking factors for charge-pans 0928 and 0829 .

\begin{tabular}{|c|c|c|c|c|c|c|c|c|c|}
\hline \multirow[t]{2}{*}{ Step } & \multirow{2}{*}{$\begin{array}{l}\text { Burnup } \\
{\left[\frac{\mathrm{MWd}}{\mathrm{MtU}}\right]}\end{array}$} & \multicolumn{8}{|c|}{ Shift Power Peaking Factors } \\
\hline & & $\begin{array}{c}\text { A } \\
\text { (A) }\end{array}$ & $\begin{array}{c}\text { B } \\
\text { (D) }\end{array}$ & $\begin{array}{c}C \\
(C)\end{array}$ & $\begin{array}{l}\mathrm{D} \\
\text { (B) }\end{array}$ & $\begin{array}{c}P \\
(\mathrm{R})\end{array}$ & $\begin{array}{c}\mathrm{Q} \\
(\mathrm{Q})\end{array}$ & $\begin{array}{l}\mathrm{R} \\
(\mathrm{P})\end{array}$ & $\begin{array}{c}S \\
(S)\end{array}$ \\
\hline 0 & 0.0 & 1.228 & 1.230 & 1.309 & 1.292 & 1.333 & 1.154 & 1.137 & 1.411 \\
\hline 1 & 25.0 & 1.238 & 1.249 & 1.327 & 1.310 & 1.344 & 1.158 & 1.158 & 1.442 \\
\hline 2 & 50.0 & 1.239 & 1.248 & 1.328 & 1.309 & 1.343 & 1.159 & 1.156 & 1.442 \\
\hline 3 & 100.0 & 1.231 & 1.240 & 1.318 & 1.301 & 1.336 & 1.154 & 1.149 & 1.428 \\
\hline 4 & 200.0 & 1.228 & 1.234 & 1.315 & 1.292 & 1.331 & 1.152 & 1.142 & 1.422 \\
\hline 5 & 300.0 & 1.223 & 1.234 & 1.314 & 1.290 & 1.327 & 1.152 & 1.140 & 1.416 \\
\hline 6 & 400.0 & 1.221 & 1.232 & 1.306 & 1.284 & 1.328 & 1.149 & 1.136 & 1.408 \\
\hline 7 & 500.0 & 1.227 & 1.239 & 1.312 & 1.289 & 1.332 & 1.151 & 1.142 & 1.413 \\
\hline 8 & 600.0 & 1.230 & 1.243 & 1.318 & 1.295 & 1.332 & 1.157 & 1.149 & 1.422 \\
\hline 9 & 700.0 & 1.228 & 1.244 & 1.320 & 1.294 & 1.335 & 1.157 & 1.147 & 1.422 \\
\hline 10 & 800.0 & 1.237 & 1.252 & 1.331 & 1.300 & 1.337 & 1.160 & 1.158 & 1.434 \\
\hline 11 & 900.0 & 1.237 & 1.257 & 1.333 & 1.303 & 1.340 & 1.162 & 1.162 & 1.439 \\
\hline 12 & 1000.0 & 1.247 & 1.264 & 1.340 & 1.307 & 1.343 & 1.167 & 1.168 & 1.445 \\
\hline 13 & 1100.0 & 1.249 & 1.271 & 1.346 & 1.314 & 1.353 & 1.170 & 1.176 & 1.464 \\
\hline 14 & 1200.0 & 1.251 & 1.269 & 1.349 & 1.315 & 1.350 & 1.171 & 1.180 & 1.463 \\
\hline 15 & 1300.0 & 1.256 & 1.280 & 1.358 & 1.321 & 1.354 & 1.176 & 1.190 & 1.473 \\
\hline \multirow[t]{2}{*}{ Step } & Burnup & \multicolumn{8}{|c|}{ MPACT Power Peaking Factors } \\
\hline & MWd & $\begin{array}{c}\text { A } \\
\text { (A) }\end{array}$ & $\begin{array}{c}\mathrm{B} \\
\text { (D) }\end{array}$ & $\begin{array}{c}\mathrm{C} \\
\text { (C) }\end{array}$ & $\begin{array}{c}\mathrm{D} \\
\text { (B) }\end{array}$ & $\begin{array}{c}\mathrm{P} \\
(\mathrm{R})\end{array}$ & $\begin{array}{c}\mathrm{Q} \\
(\mathrm{Q})\end{array}$ & $\begin{array}{c}\mathrm{R} \\
(\mathrm{P})\end{array}$ & $\begin{array}{c}S \\
(S)\end{array}$ \\
\hline 0 & 0.0 & 1.229 & 1.234 & 1.311 & 1.295 & 1.332 & 1.154 & 1.139 & 1.417 \\
\hline 1 & 25.0 & 1.209 & 1.209 & 1.271 & 1.261 & 1.293 & 1.154 & 1.126 & 1.353 \\
\hline 2 & 50.0 & 1.213 & 1.215 & 1.280 & 1.269 & 1.302 & 1.153 & 1.129 & 1.368 \\
\hline 3 & 100.0 & 1.220 & 1.223 & 1.294 & 1.280 & 1.315 & 1.153 & 1.134 & 1.391 \\
\hline 4 & 200.0 & 1.230 & 1.235 & 1.314 & 1.296 & 1.334 & 1.154 & 1.141 & 1.421 \\
\hline 5 & 300.0 & 1.235 & 1.242 & 1.323 & 1.305 & 1.344 & 1.155 & 1.145 & 1.435 \\
\hline 6 & 400.0 & 1.238 & 1.245 & 1.327 & 1.309 & 1.348 & 1.157 & 1.148 & 1.441 \\
\hline 7 & 500.0 & 1.240 & 1.247 & 1.328 & 1.310 & 1.349 & 1.159 & 1.149 & 1.441 \\
\hline 8 & 600.0 & 1.240 & 1.247 & 1.327 & 1.309 & 1.348 & 1.162 & 1.150 & 1.436 \\
\hline 9 & 700.0 & 1.240 & 1.245 & 1.323 & 1.306 & 1.344 & 1.164 & 1.151 & 1.428 \\
\hline 10 & 800.0 & 1.238 & 1.243 & 1.318 & 1.303 & 1.339 & 1.166 & 1.151 & 1.419 \\
\hline 11 & 900.0 & 1.236 & 1.240 & 1.312 & 1.298 & 1.334 & 1.167 & 1.150 & 1.407 \\
\hline 12 & 1000.0 & 1.234 & 1.237 & 1.305 & 1.292 & 1.327 & 1.169 & 1.149 & 1.395 \\
\hline 13 & 1100.0 & 1.231 & 1.233 & 1.298 & 1.287 & 1.320 & 1.170 & 1.147 & 1.383 \\
\hline 14 & 1200.0 & 1.228 & 1.228 & 1.290 & 1.281 & 1.313 & 1.171 & 1.146 & 1.370 \\
\hline 15 & 1300.0 & 1.224 & 1.224 & 1.283 & 1.274 & 1.305 & 1.171 & 1.144 & 1.357 \\
\hline
\end{tabular}


Table 4.55. Problem 3.2.2 reference solution power peaking factors for charge-pans 0928 and 0829 .

\begin{tabular}{|c|c|c|c|c|c|c|c|c|c|}
\hline \multirow[t]{2}{*}{ Step } & \multirow{2}{*}{$\begin{array}{l}\text { Burnup } \\
{\left[\frac{\mathrm{MWd}}{\mathrm{MtU}}\right]}\end{array}$} & \multicolumn{8}{|c|}{ Shift Power Peaking Factors } \\
\hline & & $\begin{array}{c}E \\
(H)\end{array}$ & $\begin{array}{c}F \\
(G)\end{array}$ & $\begin{array}{c}\mathrm{G} \\
(\mathrm{F})\end{array}$ & $\begin{array}{c}\mathrm{H} \\
(\mathrm{E})\end{array}$ & $\begin{array}{c}\mathbf{J} \\
(\mathrm{M})\end{array}$ & $\begin{array}{c}\mathrm{K} \\
(\mathrm{L})\end{array}$ & $\begin{array}{c}\mathrm{L} \\
(\mathrm{K})\end{array}$ & $\begin{array}{l}\mathrm{M} \\
(\mathrm{J})\end{array}$ \\
\hline 0 & 0.0 & 1.252 & 1.195 & 1.178 & 1.173 & 1.209 & 1.303 & 1.409 & 1.383 \\
\hline 1 & 25.0 & 1.264 & 1.200 & 1.189 & 1.188 & 1.230 & 1.329 & 1.435 & 1.404 \\
\hline 2 & 50.0 & 1.264 & 1.199 & 1.188 & 1.190 & 1.230 & 1.327 & 1.437 & 1.403 \\
\hline 3 & 100.0 & 1.256 & 1.195 & 1.183 & 1.180 & 1.220 & 1.314 & 1.425 & 1.392 \\
\hline 4 & 200.0 & 1.252 & 1.190 & 1.175 & 1.173 & 1.215 & 1.307 & 1.418 & 1.386 \\
\hline 5 & 300.0 & 1.250 & 1.190 & 1.174 & 1.173 & 1.212 & 1.303 & 1.414 & 1.379 \\
\hline 6 & 400.0 & 1.249 & 1.190 & 1.172 & 1.174 & 1.207 & 1.299 & 1.408 & 1.374 \\
\hline 7 & 500.0 & 1.253 & 1.193 & 1.176 & 1.178 & 1.213 & 1.305 & 1.414 & 1.379 \\
\hline 8 & 600.0 & 1.257 & 1.196 & 1.182 & 1.185 & 1.221 & 1.311 & 1.422 & 1.384 \\
\hline 9 & 700.0 & 1.255 & 1.198 & 1.178 & 1.183 & 1.221 & 1.312 & 1.424 & 1.387 \\
\hline 10 & 800.0 & 1.261 & 1.201 & 1.186 & 1.194 & 1.233 & 1.326 & 1.431 & 1.392 \\
\hline 11 & 900.0 & 1.260 & 1.201 & 1.190 & 1.202 & 1.236 & 1.330 & 1.436 & 1.396 \\
\hline 12 & 1000.0 & 1.269 & 1.208 & 1.194 & 1.203 & 1.242 & 1.337 & 1.445 & 1.402 \\
\hline 13 & 1100.0 & 1.274 & 1.213 & 1.203 & 1.208 & 1.251 & 1.346 & 1.454 & 1.410 \\
\hline 14 & 1200.0 & 1.272 & 1.214 & 1.201 & 1.212 & 1.253 & 1.351 & 1.455 & 1.410 \\
\hline 15 & 1300.0 & 1.279 & 1.218 & 1.206 & 1.220 & 1.264 & 1.358 & 1.463 & 1.416 \\
\hline \multirow[t]{2}{*}{ Step } & Burnup & \multicolumn{8}{|c|}{ MPACT Power Peaking Factors } \\
\hline & $\frac{\mathrm{MWd}}{\mathrm{M}}$ & $\begin{array}{c}E \\
(H)\end{array}$ & $\begin{array}{c}\text { F } \\
(\mathrm{G})\end{array}$ & $\begin{array}{l}G \\
(F)\end{array}$ & $\begin{array}{c}\mathrm{H} \\
(\mathrm{E})\end{array}$ & $\begin{array}{c}\mathrm{J} \\
(\mathrm{M})\end{array}$ & $\begin{array}{c}\mathrm{K} \\
(\mathrm{L})\end{array}$ & $\begin{array}{c}\mathrm{L} \\
(\mathrm{K})\end{array}$ & $\begin{array}{l}\text { M } \\
(J)\end{array}$ \\
\hline 0 & 0.0 & 1.255 & 1.194 & 1.179 & 1.175 & 1.211 & 1.305 & 1.415 & 1.386 \\
\hline 1 & 25.0 & 1.229 & 1.182 & 1.173 & 1.165 & 1.183 & 1.260 & 1.358 & 1.337 \\
\hline 2 & 50.0 & 1.235 & 1.184 & 1.174 & 1.167 & 1.189 & 1.270 & 1.371 & 1.348 \\
\hline 3 & 100.0 & 1.244 & 1.188 & 1.176 & 1.170 & 1.199 & 1.286 & 1.391 & 1.365 \\
\hline 4 & 200.0 & 1.256 & 1.195 & 1.180 & 1.176 & 1.213 & 1.308 & 1.419 & 1.389 \\
\hline 5 & 300.0 & 1.263 & 1.199 & 1.182 & 1.179 & 1.221 & 1.319 & 1.431 & 1.400 \\
\hline 6 & 400.0 & 1.267 & 1.202 & 1.185 & 1.182 & 1.224 & 1.323 & 1.437 & 1.405 \\
\hline 7 & 500.0 & 1.268 & 1.204 & 1.187 & 1.184 & 1.226 & 1.324 & 1.437 & 1.406 \\
\hline 8 & 600.0 & 1.268 & 1.205 & 1.188 & 1.185 & 1.225 & 1.322 & 1.433 & 1.403 \\
\hline 9 & 700.0 & 1.267 & 1.206 & 1.190 & 1.186 & 1.223 & 1.317 & 1.427 & 1.398 \\
\hline 10 & 800.0 & 1.265 & 1.206 & 1.191 & 1.186 & 1.221 & 1.311 & 1.418 & 1.391 \\
\hline 11 & 900.0 & 1.262 & 1.205 & 1.191 & 1.186 & 1.217 & 1.304 & 1.408 & 1.383 \\
\hline 12 & 1000.0 & 1.258 & 1.204 & 1.192 & 1.186 & 1.213 & 1.296 & 1.398 & 1.374 \\
\hline 13 & 1100.0 & 1.254 & 1.203 & 1.192 & 1.185 & 1.208 & 1.288 & 1.387 & 1.365 \\
\hline 14 & 1200.0 & 1.249 & 1.201 & 1.191 & 1.184 & 1.203 & 1.279 & 1.375 & 1.356 \\
\hline 15 & 1300.0 & 1.245 & 1.199 & 1.191 & 1.182 & 1.198 & 1.270 & 1.363 & 1.346 \\
\hline
\end{tabular}


Charge-Pans 1028 and (0830)

Table 4.56. Problem 3.2.2 reference solution power peaking factors for charge-pans 1028 and 0830 .

\begin{tabular}{|c|c|c|c|c|c|c|c|c|c|}
\hline \multirow[t]{3}{*}{ Step } & \multirow{3}{*}{$\begin{array}{l}\text { Burnup } \\
{\left[\frac{\mathrm{MWd}}{\mathrm{MtU}}\right]}\end{array}$} & \multicolumn{8}{|c|}{ Shift Power Peaking Factors } \\
\hline & & A & B & $\mathrm{C}$ & $\mathrm{D}$ & $\mathrm{P}$ & Q & $\mathrm{R}$ & S \\
\hline & & (A) & (D) & (C) & (B) & $(\mathrm{R})$ & $(\mathrm{Q})$ & (P) & (S) \\
\hline 0 & 0.0 & 1.017 & 1.003 & 1.048 & 1.064 & 1.120 & 0.983 & 0.940 & 1.071 \\
\hline 1 & 25.0 & 1.025 & 1.010 & 1.056 & 1.070 & 1.119 & 0.982 & 0.953 & 1.088 \\
\hline 2 & 50.0 & 1.023 & 1.013 & 1.056 & 1.073 & 1.121 & 0.984 & 0.959 & 1.091 \\
\hline 3 & 100.0 & 1.023 & 1.009 & 1.055 & 1.066 & 1.122 & 0.982 & 0.950 & 1.086 \\
\hline 4 & 200.0 & 1.015 & 1.002 & 1.050 & 1.063 & 1.119 & 0.981 & 0.942 & 1.075 \\
\hline 5 & 300.0 & 1.015 & 0.998 & 1.043 & 1.064 & 1.121 & 0.975 & 0.938 & 1.071 \\
\hline 6 & 400.0 & 1.018 & 1.002 & 1.048 & 1.066 & 1.123 & 0.983 & 0.939 & 1.071 \\
\hline 7 & 500.0 & 1.021 & 1.004 & 1.048 & 1.064 & 1.123 & 0.981 & 0.944 & 1.075 \\
\hline 8 & 600.0 & 1.023 & 1.008 & 1.054 & 1.072 & 1.129 & 0.990 & 0.944 & 1.081 \\
\hline 9 & 700.0 & 1.022 & 1.006 & 1.052 & 1.071 & 1.129 & 0.987 & 0.947 & 1.078 \\
\hline 10 & 800.0 & 1.033 & 1.016 & 1.063 & 1.079 & 1.135 & 0.995 & 0.958 & 1.092 \\
\hline 11 & 900.0 & 1.035 & 1.023 & 1.066 & 1.082 & 1.139 & 0.999 & 0.962 & 1.095 \\
\hline 12 & 1000.0 & 1. & 1.028 & 1.072 & 90 & 1.142 & 01 & 68 & 102 \\
\hline 13 & 1100.0 & 1.040 & 1.028 & 1.075 & 1.088 & 1.146 & 0.999 & 0.970 & 1.107 \\
\hline 14 & 1200.0 & 1.045 & 1.031 & 1.079 & 1.094 & 1.146 & 1.009 & 0.980 & 1.111 \\
\hline 15 & 1300.0 & 1.051 & 1.040 & 1.084 & 1.097 & 1.151 & 1.011 & 0.986 & 1.117 \\
\hline \multirow[t]{3}{*}{ Step } & Burnup & \multicolumn{8}{|c|}{ MPACT Power Peaking Factors } \\
\hline & & A & B & $\mathrm{C}$ & $\mathrm{D}$ & $\mathrm{P}$ & Q & $\mathrm{R}$ & $\mathrm{S}$ \\
\hline & & (A) & (D) & (C) & (B) & $(\mathrm{R})$ & $(\mathrm{Q})$ & $(\mathrm{P})$ & $(\mathrm{S})$ \\
\hline 0 & 0.0 & 1.018 & 1.002 & 1.047 & 1.064 & 1.118 & 0.981 & 0.940 & 1.074 \\
\hline 1 & 25.0 & 1.045 & 1.026 & 1.062 & 1.082 & 1.129 & 1.017 & 0.972 & 1.075 \\
\hline 2 & 50.0 & 1.038 & 1.020 & 1.058 & 1.077 & 1.126 & 1.008 & 0.965 & 1.074 \\
\hline 3 & 100.0 & 1.028 & 1.011 & 1.053 & 1.070 & 1.122 & 0.995 & 0.953 & 1.074 \\
\hline 4 & 200.0 & 1.016 & 1.000 & 1.046 & 1.063 & 1.117 & 0.978 & 0.938 & 1.075 \\
\hline 5 & 300.0 & 1.012 & 0.996 & 1.044 & 1.060 & 1.116 & 0.971 & 0.932 & 1.076 \\
\hline 6 & 400.0 & 1.011 & 0.996 & 1.045 & 1.060 & 1.117 & 0.969 & 0.931 & 1.078 \\
\hline 7 & 500.0 & 1.013 & 0.998 & 1.047 & 1.063 & 1.120 & 0.971 & 0.933 & 1.080 \\
\hline 8 & 600.0 & 1.017 & 1.002 & 1.050 & 1.066 & 1.123 & 0.975 & 0.937 & 1.082 \\
\hline 9 & 700.0 & 1.022 & 1.007 & 1.054 & 1.071 & 1.126 & 0.981 & 0.943 & 1.084 \\
\hline 10 & 800.0 & 1.028 & 1.012 & 1.059 & 1.075 & 1.130 & 0.989 & 0.949 & 1.086 \\
\hline 11 & 900.0 & 1.034 & 1.018 & 1.063 & 1.080 & 1.134 & 0.996 & 0.957 & 1.088 \\
\hline 12 & 1000.0 & 1.041 & 1.024 & 1.067 & 1.085 & 1.137 & 1.004 & 0.964 & 1.090 \\
\hline 13 & 1100.0 & 1.047 & 1.030 & 1.071 & 1.089 & 1.141 & 1.012 & 0.971 & 1.091 \\
\hline 14 & 1200.0 & 1.053 & 1.036 & 1.075 & 1.094 & 1.144 & 1.020 & 0.978 & 1.092 \\
\hline 15 & 1300.0 & 1.059 & 1.041 & 1.079 & 1.098 & 1.147 & 1.028 & 0.986 & 1.093 \\
\hline
\end{tabular}


Table 4.57. Problem 3.2.2 reference solution power peaking factors for charge-pans 1028 and 0830 .

\begin{tabular}{|c|c|c|c|c|c|c|c|c|c|}
\hline \multirow[t]{2}{*}{ Step } & \multirow{2}{*}{$\begin{array}{l}\text { Burnup } \\
{\left[\frac{\mathrm{MWd}}{\mathrm{MtU}}\right]}\end{array}$} & \multicolumn{8}{|c|}{ Shift Power Peaking Factors } \\
\hline & & $\begin{array}{c}E \\
(H)\end{array}$ & $\begin{array}{c}F \\
(G)\end{array}$ & $\begin{array}{l}G \\
(F)\end{array}$ & $\begin{array}{l}\mathrm{H} \\
(\mathrm{E})\end{array}$ & $\begin{array}{c}\mathrm{J} \\
(\mathrm{M})\end{array}$ & $\begin{array}{c}\mathrm{K} \\
(\mathrm{L})\end{array}$ & $\begin{array}{c}\mathrm{L} \\
(\mathrm{K})\end{array}$ & $\begin{array}{l}M \\
(J)\end{array}$ \\
\hline 0 & 0.0 & 1.077 & 1.030 & 0.982 & 0.967 & 0.969 & 1.013 & 1.113 & 1.125 \\
\hline 1 & 25.0 & 1.074 & 1.029 & 0.987 & 0.977 & 0.985 & 1.030 & 1.125 & 1.133 \\
\hline 2 & 50.0 & 1.078 & 1.031 & 0.988 & 0.979 & 0.987 & 1.030 & 1.126 & 1.136 \\
\hline 3 & 100.0 & 1.078 & 1.031 & 0.984 & 0.975 & 0.977 & 1.023 & 1.120 & 1.131 \\
\hline 4 & 200.0 & 1.077 & 1.031 & 0.980 & 0.970 & 0.970 & 1.016 & 1.114 & 1.126 \\
\hline 5 & 300.0 & 1.075 & 1.031 & 0.975 & 0.965 & 0.966 & 1.013 & 1.111 & 1.124 \\
\hline 6 & 400.0 & 1.078 & 1.034 & 0.979 & 0.970 & 0.968 & 1.012 & 1.113 & 1.126 \\
\hline 7 & 500.0 & 1.084 & 1.036 & 0.981 & 0.973 & 0.970 & 1.016 & 1.114 & 1.127 \\
\hline 8 & 600.0 & 1.086 & 1.039 & 0.986 & 0.976 & 0.977 & 1.021 & 1.120 & 1.137 \\
\hline 9 & 700.0 & 1.090 & 1.040 & 0.983 & 0.975 & 0.975 & 1.020 & 1.117 & 1.132 \\
\hline 10 & 800.0 & 1.094 & 1.050 & 0.995 & 0.987 & 0.986 & 1.030 & 1.128 & 1.139 \\
\hline 11 & 900.0 & 1.094 & 1.049 & 0.999 & 0.991 & 0.992 & 1.038 & 1.133 & 1.147 \\
\hline 12 & 1000.0 & 1.101 & 1.052 & 1.001 & 0.994 & 0.998 & 1.041 & 1.139 & 1.147 \\
\hline 13 & 1100.0 & 1.104 & 1.055 & 1.002 & 0.996 & 0.999 & 1.043 & 1.144 & 1.156 \\
\hline 14 & 1200.0 & 1.107 & 1.059 & 1.007 & 1.004 & 1.005 & 1.049 & 1.144 & 1.158 \\
\hline 15 & 1300.0 & 1.109 & 1.065 & 1.017 & 1.011 & 1.015 & 1.057 & 1.152 & 1.160 \\
\hline \multirow[t]{2}{*}{ Step } & Burnup & \multicolumn{8}{|c|}{ MPACT Power Peaking Factors } \\
\hline & MWd & $\begin{array}{c}E \\
(H)\end{array}$ & $\begin{array}{c}\text { F } \\
(G)\end{array}$ & $\begin{array}{l}\mathrm{G} \\
(\mathrm{F})\end{array}$ & $\begin{array}{c}\mathrm{H} \\
(\mathrm{E})\end{array}$ & $\begin{array}{c}\mathrm{J} \\
(\mathrm{M})\end{array}$ & $\begin{array}{c}\mathrm{K} \\
(\mathrm{L})\end{array}$ & $\begin{array}{c}\mathrm{L} \\
(\mathrm{K})\end{array}$ & $\begin{array}{l}\text { M } \\
(\mathrm{J})\end{array}$ \\
\hline 0 & 0.0 & 1.076 & 1.030 & 0.980 & 0.968 & 0.969 & 1.013 & 1.113 & 1.126 \\
\hline 1 & 25.0 & 1.097 & 1.060 & 1.015 & 1.001 & 0.992 & 1.026 & 1.116 & 1.133 \\
\hline 2 & 50.0 & 1.092 & 1.052 & 1.006 & 0.993 & 0.987 & 1.023 & 1.115 & 1.130 \\
\hline 3 & 100.0 & 1.084 & 1.041 & 0.994 & 0.981 & 0.978 & 1.019 & 1.114 & 1.128 \\
\hline 4 & 200.0 & 1.075 & 1.028 & 0.978 & 0.966 & 0.968 & 1.013 & 1.113 & 1.126 \\
\hline 5 & 300.0 & 1.071 & 1.023 & 0.971 & 0.960 & 0.964 & 1.012 & 1.114 & 1.126 \\
\hline 6 & 400.0 & 1.071 & 1.022 & 0.969 & 0.958 & 0.964 & 1.013 & 1.116 & 1.127 \\
\hline 7 & 500.0 & 1.074 & 1.024 & 0.971 & 0.960 & 0.966 & 1.015 & 1.118 & 1.129 \\
\hline 8 & 600.0 & 1.077 & 1.028 & 0.976 & 0.965 & 0.970 & 1.018 & 1.120 & 1.132 \\
\hline 9 & 700.0 & 1.082 & 1.034 & 0.982 & 0.971 & 0.975 & 1.022 & 1.123 & 1.135 \\
\hline 10 & 800.0 & 1.087 & 1.040 & 0.989 & 0.977 & 0.980 & 1.026 & 1.125 & 1.138 \\
\hline 11 & 900.0 & 1.093 & 1.047 & 0.996 & 0.984 & 0.986 & 1.029 & 1.128 & 1.141 \\
\hline 12 & 1000.0 & 1.098 & 1.053 & 1.004 & 0.992 & 0.991 & 1.033 & 1.130 & 1.144 \\
\hline 13 & 1100.0 & 1.103 & 1.060 & 1.012 & 0.999 & 0.997 & 1.037 & 1.131 & 1.146 \\
\hline 14 & 1200.0 & 1.108 & 1.067 & 1.019 & 1.007 & 1.003 & 1.041 & 1.133 & 1.148 \\
\hline 15 & 1300.0 & 1.113 & 1.073 & 1.027 & 1.014 & 1.008 & 1.044 & 1.134 & 1.150 \\
\hline
\end{tabular}


Charge-Pans 1128 and (0831)

Table 4.58. Problem 3.2.2 reference solution power peaking factors for charge-pans 1128 and 0831.

\begin{tabular}{|c|c|c|c|c|c|c|c|c|c|}
\hline \multirow[t]{3}{*}{ Step } & \multirow{3}{*}{$\begin{array}{l}\text { Burnup } \\
{\left[\frac{\mathrm{MWd}}{\mathrm{MtU}}\right]}\end{array}$} & \multicolumn{8}{|c|}{ Shift Power Peaking Factors } \\
\hline & & A & B & $\mathrm{C}$ & $\mathrm{D}$ & $\mathrm{P}$ & Q & $\mathrm{R}$ & $\mathrm{S}$ \\
\hline & & (A) & (D) & (C) & (B) & $(\mathrm{R})$ & $(\mathrm{Q})$ & $(\mathrm{P})$ & (S) \\
\hline 0 & 0.0 & 0.780 & 0.789 & 0.854 & 0.850 & 0.919 & 0.692 & 0.727 & 0.905 \\
\hline 1 & 25.0 & 0.787 & 0.802 & 0.867 & 0.856 & 0.919 & 0.698 & 0.741 & 0.924 \\
\hline 2 & 50.0 & 0.788 & 0.803 & 0.869 & 0.859 & 0.922 & 0.698 & 0.745 & 0.927 \\
\hline 3 & 100.0 & 0.783 & 0.801 & 0.863 & 0.856 & 0.920 & 0.695 & 0.737 & 0.918 \\
\hline 4 & 200.0 & 0.777 & 0.787 & 0.851 & 0.847 & 0.918 & 0.693 & 0.727 & 0.906 \\
\hline 5 & 300.0 & 0.772 & 0.781 & 0.846 & 0.845 & 0.913 & 0.689 & 0.718 & 0.898 \\
\hline 6 & 400.0 & 0.774 & 0.785 & 0.849 & 0.847 & 0.917 & 0.693 & 0.723 & 0.897 \\
\hline 7 & 500.0 & 0.775 & 0.788 & 0.853 & 0.850 & 0.919 & 0.694 & 0.724 & 0.899 \\
\hline 8 & 600.0 & 0.779 & 0.789 & 0.854 & 0.853 & 0.923 & 0.697 & 0.726 & 0.903 \\
\hline 9 & 700.0 & 0.780 & 0.788 & 0.853 & 0.851 & 0.922 & 0.697 & 0.724 & 0.903 \\
\hline 10 & 800.0 & 0.788 & 0.799 & 0.865 & 0.863 & 0.933 & 0.706 & 0.737 & 0.916 \\
\hline 11 & 900.0 & 0.794 & 0.805 & 0.870 & 0.866 & 0.934 & 0.711 & 0.743 & 0.919 \\
\hline 12 & 1000.0 & 0.793 & 0.806 & 0.873 & 0.868 & 0.936 & 0.709 & 0.744 & 0.923 \\
\hline 13 & 1100.0 & 0.794 & 0.807 & 0.871 & 0.867 & 0.936 & 0.711 & 0.742 & 0.925 \\
\hline 14 & 1200.0 & 0.803 & 0.815 & 0.882 & 0.877 & 0.946 & 0.718 & 0.754 & 0.933 \\
\hline 15 & 1300.0 & 0.812 & 0.824 & 0.893 & 0.883 & 0.949 & 0.724 & 0.766 & 0.946 \\
\hline \multirow[t]{3}{*}{ Step } & Burnup & \multicolumn{8}{|c|}{ MPACT Power Peaking Factors } \\
\hline & $\left.\frac{\mathrm{MWd}}{\mathrm{MtU}}\right]$ & A & B & $\mathrm{C}$ & $\mathrm{D}$ & $\mathrm{P}$ & Q & $\mathrm{R}$ & $\mathrm{S}$ \\
\hline & & (A) & (D) & (C) & (B) & $(\mathrm{R})$ & $(\mathrm{Q})$ & $(\mathrm{P})$ & (S) \\
\hline 0 & 0.0 & 0.779 & 0.789 & 0.854 & 0.851 & 0.919 & 0.693 & 0.726 & 0.906 \\
\hline 1 & 25.0 & 0.826 & 0.837 & 0.900 & 0.896 & 0.960 & 0.740 & 0.776 & 0.946 \\
\hline 2 & 50.0 & 0.815 & 0.826 & 0.889 & 0.885 & 0.950 & 0.729 & 0.764 & 0.937 \\
\hline 3 & 100.0 & 0.798 & 0.809 & 0.873 & 0.869 & 0.935 & 0.712 & 0.747 & 0.923 \\
\hline 4 & 200.0 & 0.775 & 0.786 & 0.851 & 0.847 & 0.915 & 0.689 & 0.723 & 0.904 \\
\hline 5 & 300.0 & 0.765 & 0.776 & 0.842 & 0.838 & 0.907 & 0.679 & 0.712 & 0.896 \\
\hline 6 & 400.0 & 0.761 & 0.772 & 0.839 & 0.834 & 0.904 & 0.674 & 0.708 & 0.894 \\
\hline 7 & 500.0 & 0.762 & 0.773 & 0.840 & 0.836 & 0.905 & 0.674 & 0.708 & 0.896 \\
\hline 8 & 600.0 & 0.766 & 0.777 & 0.845 & 0.840 & 0.910 & 0.678 & 0.712 & 0.900 \\
\hline 9 & 700.0 & 0.772 & 0.783 & 0.851 & 0.846 & 0.916 & 0.683 & 0.718 & 0.906 \\
\hline 10 & 800.0 & 0.780 & 0.791 & 0.859 & 0.854 & 0.924 & 0.690 & 0.726 & 0.914 \\
\hline 11 & 900.0 & 0.788 & 0.800 & 0.867 & 0.863 & 0.932 & 0.698 & 0.734 & 0.922 \\
\hline 12 & 1000.0 & 0.797 & 0.809 & 0.876 & 0.872 & 0.941 & 0.707 & 0.744 & 0.930 \\
\hline 13 & 1100.0 & 0.807 & 0.818 & 0.886 & 0.881 & 0.950 & 0.716 & 0.753 & 0.939 \\
\hline 14 & 1200.0 & 0.816 & 0.828 & 0.895 & 0.890 & 0.959 & 0.725 & 0.763 & 0.947 \\
\hline 15 & 1300.0 & 0.826 & 0.838 & 0.905 & 0.900 & 0.968 & 0.734 & 0.773 & 0.956 \\
\hline
\end{tabular}


Table 4.59. Problem 3.2.2 reference solution power peaking factors for charge-pans 1128 and 0831 .

\begin{tabular}{|c|c|c|c|c|c|c|c|c|c|}
\hline \multirow[t]{2}{*}{ Step } & \multirow{2}{*}{$\begin{array}{l}\text { Burnup } \\
{\left[\frac{\mathrm{MWd}}{\mathrm{MtU}}\right]}\end{array}$} & \multicolumn{8}{|c|}{ Shift Power Peaking Factors } \\
\hline & & $\begin{array}{c}E \\
(H)\end{array}$ & $\begin{array}{c}F \\
(G)\end{array}$ & $\begin{array}{c}G \\
(F)\end{array}$ & $\begin{array}{c}\mathrm{H} \\
(\mathrm{E})\end{array}$ & $\begin{array}{c}\mathrm{J} \\
(\mathrm{M})\end{array}$ & $\begin{array}{c}\mathrm{K} \\
(\mathrm{L})\end{array}$ & $\begin{array}{c}\mathrm{L} \\
(\mathrm{K})\end{array}$ & $\begin{array}{l}\mathrm{M} \\
(\mathrm{J})\end{array}$ \\
\hline 0 & 0.0 & 0.845 & 0.769 & 0.700 & 0.716 & 0.800 & 0.860 & 0.920 & 0.926 \\
\hline 1 & 25.0 & 0.848 & 0.771 & 0.708 & 0.726 & 0.815 & 0.879 & 0.931 & 0.933 \\
\hline 2 & 50.0 & 0.849 & 0.771 & 0.710 & 0.728 & 0.820 & 0.882 & 0.932 & 0.934 \\
\hline 3 & 100.0 & 0.848 & 0.771 & 0.706 & 0.725 & 0.811 & 0.871 & 0.930 & 0.931 \\
\hline 4 & 200.0 & 0.845 & 0.767 & 0.699 & 0.715 & 0.799 & 0.860 & 0.916 & 0.923 \\
\hline 5 & 300.0 & 0.843 & 0.763 & 0.695 & 0.708 & 0.792 & 0.851 & 0.912 & 0.918 \\
\hline 6 & 400.0 & 0.848 & 0.766 & 0.696 & 0.710 & 0.793 & 0.857 & 0.916 & 0.920 \\
\hline 7 & 500.0 & 0.845 & 0.769 & 0.698 & 0.712 & 0.795 & 0.855 & 0.916 & 0.922 \\
\hline 8 & 600.0 & 0.852 & 0.771 & 0.702 & 0.714 & 0.801 & 0.858 & 0.919 & 0.926 \\
\hline 9 & 700.0 & 0.850 & 0.771 & 0.701 & 0.715 & 0.796 & 0.858 & 0.917 & 0.927 \\
\hline 10 & 800.0 & 0.861 & 0.781 & 0.712 & 0.724 & 0.810 & 0.871 & 0.928 & 0.935 \\
\hline 11 & 900.0 & 0.862 & 0.787 & 0.716 & 0.732 & 0.817 & 0.877 & 0.934 & 0.938 \\
\hline 12 & 1000.0 & 0.864 & 0.785 & 0.715 & 0.731 & 0.817 & 0.878 & 0.938 & 0.946 \\
\hline 13 & 1100.0 & 0.864 & 0.784 & 0.717 & 0.731 & 0.820 & 0.880 & 0.937 & 0.942 \\
\hline 14 & 1200.0 & 0.873 & 0.790 & 0.724 & 0.737 & 0.830 & 0.890 & 0.947 & 0.953 \\
\hline 15 & 1300.0 & 0.877 & 0.799 & 0.732 & 0.750 & 0.841 & 0.902 & 0.958 & 0.958 \\
\hline \multirow[t]{2}{*}{ Step } & Burnup & \multicolumn{8}{|c|}{ MPACT Power Peaking Factors } \\
\hline & $\frac{\mathrm{MWd}}{\mathrm{M} I I}$ & $\begin{array}{c}E \\
(H)\end{array}$ & $\begin{array}{c}F \\
(G)\end{array}$ & $\begin{array}{c}\mathrm{G} \\
(\mathrm{F})\end{array}$ & $\begin{array}{c}\mathrm{H} \\
(\mathrm{E})\end{array}$ & $\begin{array}{c}\mathrm{J} \\
(\mathrm{M})\end{array}$ & $\begin{array}{c}\mathrm{K} \\
\text { (L) }\end{array}$ & $\begin{array}{c}\mathrm{L} \\
(\mathrm{K})\end{array}$ & $\begin{array}{l}\mathrm{M} \\
(\mathrm{J})\end{array}$ \\
\hline 0 & 0.0 & 0.846 & 0.768 & 0.700 & 0.714 & 0.799 & 0.860 & 0.921 & 0.925 \\
\hline 1 & 25.0 & 0.892 & 0.814 & 0.747 & 0.763 & 0.848 & 0.906 & 0.961 & 0.966 \\
\hline 2 & 50.0 & 0.881 & 0.803 & 0.736 & 0.752 & 0.837 & 0.895 & 0.951 & 0.956 \\
\hline 3 & 100.0 & 0.865 & 0.786 & 0.720 & 0.735 & 0.820 & 0.879 & 0.937 & 0.941 \\
\hline 4 & 200.0 & 0.843 & 0.764 & 0.696 & 0.711 & 0.797 & 0.858 & 0.918 & 0.922 \\
\hline 5 & 300.0 & 0.833 & 0.753 & 0.686 & 0.700 & 0.786 & 0.848 & 0.910 & 0.913 \\
\hline 6 & 400.0 & 0.830 & 0.749 & 0.682 & 0.696 & 0.783 & 0.845 & 0.908 & 0.911 \\
\hline 7 & 500.0 & 0.831 & 0.750 & 0.682 & 0.697 & 0.784 & 0.846 & 0.909 & 0.913 \\
\hline 8 & 600.0 & 0.835 & 0.754 & 0.685 & 0.700 & 0.788 & 0.851 & 0.914 & 0.917 \\
\hline 9 & 700.0 & 0.841 & 0.760 & 0.691 & 0.706 & 0.794 & 0.857 & 0.920 & 0.923 \\
\hline 10 & 800.0 & 0.849 & 0.767 & 0.698 & 0.714 & 0.802 & 0.865 & 0.928 & 0.931 \\
\hline 11 & 900.0 & 0.858 & 0.776 & 0.707 & 0.722 & 0.811 & 0.874 & 0.936 & 0.939 \\
\hline 12 & 1000.0 & 0.867 & 0.785 & 0.715 & 0.731 & 0.820 & 0.883 & 0.944 & 0.948 \\
\hline 13 & 1100.0 & 0.876 & 0.794 & 0.724 & 0.740 & 0.830 & 0.893 & 0.953 & 0.957 \\
\hline 14 & 1200.0 & 0.886 & 0.803 & 0.734 & 0.750 & 0.840 & 0.902 & 0.962 & 0.965 \\
\hline 15 & 1300.0 & 0.895 & 0.813 & 0.743 & 0.760 & 0.850 & 0.912 & 0.970 & 0.974 \\
\hline
\end{tabular}


Charge-Pans 1228 and (0832)

Table 4.60. Problem 3.2.2 reference solution power peaking factors for charge-pans 1228 and 0832 .

\begin{tabular}{|c|c|c|c|c|c|c|c|}
\hline \multirow[t]{3}{*}{ Step } & \multirow{3}{*}{$\begin{array}{l}\text { Burnup } \\
{\left[\frac{\mathrm{MWd}}{\mathrm{MtU}}\right]}\end{array}$} & \multicolumn{6}{|c|}{ Shift Power Peaking Factors } \\
\hline & & A & B & $\mathrm{C}$ & $\mathrm{D}$ & $\mathrm{P}$ & S \\
\hline & & (A) & (D) & (C) & (B) & $(\mathrm{R})$ & (Q) \\
\hline 0 & 0.0 & 0.443 & 0.469 & 0.524 & 0.508 & 0.627 & 0.641 \\
\hline 1 & 25.0 & 0.452 & 0.479 & 0.536 & 0.514 & 0.634 & 0.656 \\
\hline 2 & 50.0 & 0.452 & 0.478 & 0.536 & 0.517 & 0.635 & 0.656 \\
\hline 3 & 100.0 & 0.449 & 0.476 & 0.534 & 0.514 & 0.632 & 0.651 \\
\hline 4 & 200.0 & & 0.468 & 0.526 & 0.507 & 0.628 & 0.638 \\
\hline 5 & 300.0 & 0.437 & 0.458 & 0.518 & 0.498 & 0.619 & 0.626 \\
\hline 6 & 400.0 & 0.439 & 0.460 & 0.520 & 0.503 & 0.623 & 0.628 \\
\hline 7 & 500.0 & 0 & 0.457 & 0.520 & 0.499 & 0.622 & 0.628 \\
\hline 8 & 600.0 & 0.440 & 0.458 & 0.523 & 0.503 & 0.626 & 0.632 \\
\hline 9 & 700.0 & & 0.458 & & 0.502 & 27 & 29 \\
\hline 10 & 800.0 & 0 . & 0.464 & 0.529 & 0.508 & 0.633 & 0.641 \\
\hline 11 & 900.0 & 0.450 & 0.469 & 0.537 & 0.514 & 0.639 & 0.645 \\
\hline 12 & 1000.0 & 0 & 0.467 & 0 . & 0.513 & 0.637 & .645 \\
\hline 13 & 1100.0 & 0.452 & 0.469 & 0.537 & 0.513 & 0.638 & 0.645 \\
\hline 14 & 1200.0 & 0 & 0.474 & 0. & 0.519 & 0.645 & 0.652 \\
\hline 15 & 1300.0 & 66 & 0.483 & 0.552 & 0.527 & 0.653 & 0.664 \\
\hline \multirow[t]{3}{*}{ Step } & Burnup & \multicolumn{6}{|c|}{ MPACT Power Peaking Factors } \\
\hline & & A & B & $\mathrm{C}$ & D & $\mathrm{P}$ & $S$ \\
\hline & & (A) & (D) & (C) & (B) & (R) & (Q) \\
\hline$\overline{0}$ & 0.0 & 0.443 & 0.468 & 0.524 & 0.507 & 0.628 & 0.639 \\
\hline 1 & 25.0 & 0.480 & 0.508 & 0.567 & 0.548 & 0.674 & 0.688 \\
\hline 2 & 50.0 & 0.472 & 0.499 & 57 & 0.539 & 0.663 & 0.677 \\
\hline 3 & 100.0 & 0.459 & 0.485 & 0.543 & 0.524 & 0.647 & 0.660 \\
\hline 4 & 200.0 & 0.440 & 0.465 & 0.522 & 0.504 & 0.624 & 0.637 \\
\hline 5 & 300.0 & 0.431 & 0.456 & 0.512 & 0.495 & 0.613 & 0.626 \\
\hline 6 & 400.0 & 0.427 & 0.451 & 0.507 & 0.490 & 0.608 & 0.621 \\
\hline 7 & 500.0 & 0.426 & 0.451 & 0.507 & 0.490 & 0.608 & 0.621 \\
\hline 8 & 600.0 & 0.428 & 0.452 & 0.509 & 0.492 & 0.611 & 0.624 \\
\hline 9 & 700.0 & 0.431 & 0.456 & 0.514 & 0.496 & 0.616 & 0.630 \\
\hline 10 & 800.0 & 0.436 & 0.461 & 0.519 & 0.501 & 0.622 & 0.637 \\
\hline 11 & 900.0 & 0.441 & 0.467 & 0.526 & 0.508 & 0.630 & 0.645 \\
\hline 12 & 1000.0 & 0.447 & 0.473 & 0.533 & 0.514 & 0.638 & 0.653 \\
\hline 13 & 1100.0 & 0.453 & 0.480 & 0.540 & 0.522 & 0.646 & 0.662 \\
\hline 14 & 1200.0 & 0.460 & 0.487 & 0.548 & 0.529 & 0.655 & 0.672 \\
\hline 15 & 1300.0 & 0.467 & 0.494 & 0.556 & 0.537 & 0.664 & 0.681 \\
\hline
\end{tabular}


Table 4.61. Problem 3.2.2 reference solution power peaking factors for charge-pans 1228 and 0832 .

\begin{tabular}{|c|c|c|c|c|c|c|c|}
\hline \multirow[t]{3}{*}{ Step } & \multirow{3}{*}{$\begin{array}{l}\text { Burnup } \\
{\left[\frac{\mathrm{MWd}}{\mathrm{MtU}}\right]}\end{array}$} & \multicolumn{6}{|c|}{ Shift Power Peaking Factors } \\
\hline & & $\mathrm{E}$ & $\mathrm{F}$ & $\mathrm{J}$ & K & L & M \\
\hline & & $(\mathrm{H})$ & $(\mathrm{G})$ & $(\mathrm{F})$ & $(\mathrm{E})$ & $(\mathrm{M})$ & $(\mathrm{L})$ \\
\hline 0 & 0.0 & 0.530 & 0.439 & 0.493 & 0.552 & 0.624 & 0.609 \\
\hline 1 & 25.0 & & 0.446 & 0.508 & 0.566 & & \\
\hline 2 & 50.0 & 38 & 0.445 & 0.509 & 0.566 & 0.636 & .617 \\
\hline 3 & 100.0 & 534 & 0.443 & 0.505 & 0.560 & 0.632 & 0.614 \\
\hline 4 & 200.0 & & 0.439 & 0.495 & 0.552 & & \\
\hline 5 & 300.0 & 4 & 0.436 & 0.485 & 0.541 & & 0.603 \\
\hline 6 & 400.0 & & 0.437 & 0.489 & 0.543 & 16 & .605 \\
\hline 7 & 500.0 & & 0.436 & 0.486 & 0.541 & & 03 \\
\hline 8 & 600.0 & 3 & 0.440 & 0.489 & 0.546 & 21 & 0.609 \\
\hline 9 & 70 & & 0.441 & & 0.542 & & \\
\hline 10 & 800.0 & & 0.446 & 0.497 & 0.554 & 27 & 615 \\
\hline 11 & 900.0 & & 0.451 & 0 . & 0.558 & & 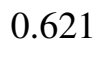 \\
\hline 12 & 1000.0 & & 0.449 & 00 & 0.556 & & 20 \\
\hline 13 & 00.0 & & 0.452 & 0.506 & 0.560 & & 0.621 \\
\hline 14 & 00.0 & & 0.457 & & 0.565 & & \\
\hline 15 & 00.0 & & 0.464 & 0.521 & 0.576 & 0.654 & 0.635 \\
\hline \multirow[t]{3}{*}{ Step } & Burnup & \multicolumn{6}{|c|}{ MPACT Power Peaking Factors } \\
\hline & $\frac{\mathrm{MWd}}{\mathrm{MtU}}$ & $\mathrm{E}$ & $\mathrm{F}$ & $\mathrm{J}$ & K & $(M)$ & M \\
\hline & & $(\mathrm{H})$ & $(\mathrm{G})$ & $(\mathrm{F})$ & (E) & $(\mathrm{M})$ & $(\mathrm{L})$ \\
\hline 0 & 0.0 & 0.530 & 0.438 & 0.494 & 0.550 & 0.623 & 0.608 \\
\hline 1 & 25.0 & 2 & 0.475 & 36 & 0.595 & 9 & 53 \\
\hline 2 & 50.0 & 3 & 0.467 & 0.527 & 0.585 & 0.6 & 0.643 \\
\hline 3 & 100.0 & 8 & 0.454 & 0.512 & 0.569 & 0.6 & 0.627 \\
\hline 4 & 200.0 & 7 & 0.436 & 91 & 0.548 & & 0.604 \\
\hline 5 & 300.0 & & 0.427 & 0.481 & 0.537 & 0.6 & 0.594 \\
\hline 6 & 400.0 & 2 & 0.422 & 0.477 & 0.533 & 5 & .590 \\
\hline 7 & 500.0 & & 0.422 & 0.476 & 0.532 & 0.605 & 0.589 \\
\hline 0 & 600.0 & & 0.423 & 0.478 & 0.535 & 0.608 & 0.592 \\
\hline 0 & 700.0 & & 0.426 & 0.482 & 0.539 & 0.613 & 0.597 \\
\hline 10 & 800.0 & 0.523 & 0.431 & 0.487 & 0.545 & 0.620 & 0.604 \\
\hline 11 & 900.0 & 0.530 & 0.436 & 0.493 & 0.552 & 0.627 & 0.611 \\
\hline 12 & 1000.0 & & 0.442 & 0.500 & 0.560 & 0.636 & 0.619 \\
\hline 13 & 1100.0 & & 0.448 & 0.508 & 0.568 & 0.644 & 0.627 \\
\hline 14 & 1200.0 & 0.551 & 0.454 & 0.515 & 0.577 & 0.653 & 0.636 \\
\hline 15 & 1300.0 & 0.559 & 0.460 & 0.523 & 0.585 & 0.662 & 0.645 \\
\hline
\end{tabular}




\section{Charge-Pan 0929}

Table 4.62. Problem 3.2.2 reference solution power peaking factors for charge-pan 0929 .

\begin{tabular}{|c|c|c|c|c|c|c|c|c|c|c|c|}
\hline \multirow[t]{2}{*}{ Step } & Burnup & \multicolumn{10}{|c|}{ Shift Power Peaking Factors } \\
\hline & $\left.\frac{\mathrm{MWd}}{\mathrm{MtU}}\right]$ & A & $\mathrm{B}, \mathrm{D}$ & $\mathrm{C}$ & $\mathrm{G}, \mathrm{F}$ & $\mathrm{H}, \mathrm{E}$ & $\mathrm{J}, \mathrm{M}$ & $\mathrm{K}, \mathrm{L}$ & Q & $\mathrm{R}, \mathrm{P}$ & $S$ \\
\hline 0 & 0.0 & 0.851 & 1.098 & 0.931 & 0.986 & 1.022 & 1.098 & 1.151 & 0.997 & 1.097 & 1.248 \\
\hline 1 & 25.0 & 0.837 & 1.075 & 0.926 & 0.969 & 1.010 & 1.099 & 1.151 & 0.973 & 1.093 & 1.252 \\
\hline 2 & 50.0 & 0.837 & 1.081 & 0.927 & 0.969 & 1.010 & 1.097 & 1.151 & 0.972 & 1.095 & 1.252 \\
\hline 3 & 100.0 & 0.845 & 1.090 & 0.935 & 0.979 & 1.020 & 1.100 & 1.152 & 0.980 & 1.099 & 1.251 \\
\hline 4 & 200.0 & 0.849 & 1.097 & 0.937 & 0.982 & 1.025 & 1.104 & 1.157 & 0.994 & 1.102 & 1.252 \\
\hline 5 & 300.0 & 0.855 & 1.108 & 0.942 & 0.988 & 1.032 & 1.107 & 1.161 & 0.997 & 1.104 & 1.258 \\
\hline 6 & 400.0 & 0.857 & 1.110 & 0.945 & 0.989 & 1.036 & 1.110 & 1.160 & 1.003 & 1.107 & 1.256 \\
\hline 7 & 500.0 & 0.858 & 1.113 & 0.944 & 0.992 & 1.034 & 1.111 & 1.163 & 1.004 & 1.112 & 1.259 \\
\hline 8 & 600.0 & 0.859 & 1.113 & 0.948 & 0.994 & 1.042 & 1.117 & 1.168 & 1.006 & 1.117 & 1.262 \\
\hline 9 & 700.0 & 0.859 & 1.112 & 0.947 & 0.995 & 1.043 & 1.119 & 1.166 & 1.006 & 1.118 & 1.265 \\
\hline 10 & 800.0 & 0.858 & 1.103 & 0.947 & 0.991 & 1.041 & 1.119 & 1.168 & 1.000 & 1.118 & 1.261 \\
\hline 11 & 900.0 & 0.855 & 1.099 & 0.945 & 0.989 & 1.038 & 1.120 & 1.169 & 0.998 & 1.116 & 1.263 \\
\hline 12 & 1000.0 & 0.858 & 1.100 & 0.947 & 0.992 & 1.043 & 1.122 & 1.171 & 0.997 & 1.123 & 1.269 \\
\hline 13 & 1100.0 & 0.852 & 1.092 & 0.949 & 0.985 & 1.040 & 1.125 & 1.176 & 0.989 & 1.124 & 1.268 \\
\hline 14 & 1200.0 & 0.850 & 1.086 & 0.943 & 0.988 & 1.038 & 1.123 & 1.174 & 0.989 & 1.121 & 1.264 \\
\hline 15 & 1300.0 & 0.847 & 1.084 & 0.943 & 0.984 & 1.036 & 1.126 & 1.176 & 0.983 & 1.125 & 1.270 \\
\hline \multirow[t]{2}{*}{ Step } & Burnup & \multicolumn{10}{|c|}{ MPACT Power Peaking Factors } \\
\hline & $\frac{\mathrm{MWd}}{\mathrm{MtU}}$ & A & $\mathrm{B}, \mathrm{D}$ & $\mathrm{C}$ & $\mathrm{G}, \mathrm{F}$ & $\mathrm{H}, \mathrm{E}$ & $\mathrm{J}, \mathrm{M}$ & $\mathrm{K}, \mathrm{L}$ & Q & $\mathrm{R}, \mathrm{P}$ & $S$ \\
\hline 0 & 0.0 & 0.846 & 1.097 & 0.929 & 0.983 & 1.019 & 1.098 & 1.150 & 0.992 & 1.097 & 1.249 \\
\hline 1 & 25.0 & 0.859 & 1.103 & 0.924 & 1.003 & 1.033 & 1.094 & 1.135 & 1.019 & 1.103 & 1.222 \\
\hline 2 & 50.0 & 0.855 & 1.101 & 0.925 & 0.998 & 1.029 & 1.094 & 1.138 & 1.012 & 1.101 & 1.228 \\
\hline 3 & 100.0 & 0.851 & 1.098 & 0.926 & 0.990 & 1.024 & 1.095 & 1.143 & 1.002 & 1.098 & 1.237 \\
\hline 4 & 200.0 & 0.846 & 1.096 & 0.929 & 0.981 & 1.018 & 1.098 & 1.151 & 0.990 & 1.096 & 1.251 \\
\hline 5 & 300.0 & 0.844 & 1.096 & 0.931 & 0.978 & 1.017 & 1.101 & 1.156 & 0.985 & 1.096 & 1.258 \\
\hline 6 & 400.0 & 0.845 & 1.098 & 0.934 & 0.979 & 1.018 & 1.103 & 1.159 & 0.985 & 1.098 & 1.262 \\
\hline 7 & 500.0 & 0.847 & 1.100 & 0.936 & 0.981 & 1.020 & 1.105 & 1.161 & 0.987 & 1.100 & 1.263 \\
\hline 8 & 600.0 & 0.851 & 1.103 & 0.938 & 0.985 & 1.024 & 1.107 & 1.162 & 0.991 & 1.103 & 1.263 \\
\hline 9 & 700.0 & 0.854 & 1.106 & 0.939 & 0.990 & 1.028 & 1.109 & 1.162 & 0.997 & 1.106 & 1.261 \\
\hline 10 & 800.0 & 0.858 & 1.109 & 0.940 & 0.995 & 1.032 & 1.110 & 1.162 & 1.003 & 1.109 & 1.259 \\
\hline 11 & 900.0 & 0.863 & 1.112 & 0.942 & 1.000 & 1.036 & 1.111 & 1.161 & 1.009 & 1.112 & 1.255 \\
\hline 12 & 1000.0 & 0.867 & 1.115 & 0.942 & 1.006 & 1.040 & 1.112 & 1.159 & 1.016 & 1.115 & 1.251 \\
\hline 13 & 1100.0 & 0.871 & 1.118 & 0.943 & 1.011 & 1.044 & 1.113 & 1.157 & 1.022 & 1.118 & 1.247 \\
\hline 14 & 1200.0 & 0.875 & 1.120 & 0.943 & 1.016 & 1.048 & 1.113 & 1.155 & 1.029 & 1.120 & 1.242 \\
\hline 15 & 1300.0 & 0.878 & 1.122 & 0.944 & 1.021 & 1.052 & 1.113 & 1.153 & 1.035 & 1.122 & 1.237 \\
\hline
\end{tabular}


Charge-Pans 1029 and (0930)

Table 4.63. Problem 3.2.2 reference solution power peaking factors for charge-pans 1029 and 0930 .

\begin{tabular}{|c|c|c|c|c|c|c|c|c|c|}
\hline \multirow[t]{3}{*}{ Step } & \multirow{3}{*}{$\begin{array}{l}\text { Burnup } \\
{\left[\frac{\mathrm{MWd}}{\mathrm{MtU}}\right]}\end{array}$} & \multicolumn{8}{|c|}{ Shift Power Peaking Factors } \\
\hline & & A & B & $\mathrm{C}$ & $\mathrm{D}$ & $\mathrm{P}$ & Q & $\mathrm{R}$ & $S$ \\
\hline & & (A) & (D) & (C) & (B) & $(\mathrm{R})$ & $(\mathrm{Q})$ & (P) & $(\mathrm{S})$ \\
\hline 0 & 0.0 & 0.954 & 0.985 & 1.021 & 0.992 & 0.993 & 0.862 & 0.966 & 1.086 \\
\hline 1 & 25.0 & 0.934 & 0.971 & 1.009 & 0.973 & 0.967 & 0.838 & 0.959 & 1.081 \\
\hline 2 & 50.0 & 0.936 & 0.975 & 1.007 & 0.973 & 0.968 & 0.842 & 0.962 & 1.083 \\
\hline 3 & 100.0 & 0.945 & 0.982 & 1.018 & 0.981 & 0.979 & 0.850 & 0.965 & 1.085 \\
\hline 4 & 200.0 & 0.949 & 0.983 & 1.020 & 0.989 & 0.988 & 0.858 & 0.966 & 1.086 \\
\hline 5 & 300.0 & 0.954 & 0.988 & 1.024 & 0.991 & 0.995 & 0.861 & 0.966 & 1.089 \\
\hline 6 & 400.0 & 0.958 & 0.993 & 1.026 & 0.996 & 0.999 & 0.868 & 0.971 & 1.091 \\
\hline 7 & 500.0 & 0.962 & 0.993 & 1.028 & 0.998 & 1.001 & 0.866 & 0.973 & 1.092 \\
\hline 8 & 600.0 & 0.963 & 0.997 & 1.033 & 1.002 & 1.005 & 0.870 & 0.979 & 1.099 \\
\hline 9 & 700.0 & 0.965 & 0.998 & 1.035 & 1.003 & 1.005 & 0.872 & 978 & 1.099 \\
\hline 10 & 800.0 & 0.964 & 1.002 & 1.036 & 1.001 & 0.999 & 0.872 & 0.986 & 1.098 \\
\hline 11 & 900.0 & 0.963 & 1.000 & 1.033 & 1.000 & 0.996 & 0.868 & 0.987 & 1.101 \\
\hline 12 & 1000.0 & 0.964 & 1.002 & 1.038 & 1.001 & 0.999 & 0.869 & 0.989 & 1.107 \\
\hline 13 & 1100.0 & 0.955 & 0.998 & 1.034 & 0.994 & 0.989 & 0.863 & 0.986 & 1.107 \\
\hline 14 & 1200.0 & 0.962 & 1.002 & 1.038 & 0.995 & 0.987 & 0.865 & .990 & 1.109 \\
\hline 15 & 1300.0 & 0.956 & 1.002 & 1.036 & 0.991 & 0.983 & 0.862 & 0.995 & 1.109 \\
\hline \multirow[t]{3}{*}{ Step } & Burnup & \multicolumn{8}{|c|}{ MPACT Power Peaking Factors } \\
\hline & {$\left[\frac{\mathrm{MWd}}{\mathrm{MtU}}\right]$} & A & $\mathrm{B}$ & $\mathrm{C}$ & $\mathrm{D}$ & $P$ & $\mathrm{Q}$ & $R$ & $\mathrm{~S}$ \\
\hline & & (A) & (D) & (C) & (B) & $(\mathrm{R})$ & $(\mathrm{Q})$ & $(\mathrm{P})$ & (S) \\
\hline 0 & 0.0 & 0.951 & 0.983 & 1.020 & 0.988 & 0.987 & 0.857 & 0.965 & 1.086 \\
\hline 1 & 25.0 & 0.989 & 1.019 & 1.049 & 1.021 & 1.020 & 0.901 & 1.003 & 1.102 \\
\hline 2 & 50.0 & 0.979 & 1.010 & 1.041 & 1.012 & 1.011 & 0.890 & 0.994 & 1.098 \\
\hline 3 & 100.0 & 0.965 & 0.997 & 1.031 & 1.000 & 0.999 & 0.874 & 0.979 & 1.092 \\
\hline 4 & 200.0 & 0.947 & 0.980 & 1.017 & 0.984 & 0.984 & 0.853 & 0.961 & 1.085 \\
\hline 5 & 300.0 & 0.939 & 0.973 & 1.012 & 0.978 & 0.978 & 0.844 & 0.954 & 1.083 \\
\hline 6 & 400.0 & 0.937 & 0.971 & 1.011 & 0.977 & 0.977 & 0.840 & 0.951 & 1.083 \\
\hline 7 & 500.0 & 0.939 & 0.973 & 1.013 & 0.979 & 0.979 & 0.842 & 0.953 & 1.085 \\
\hline 8 & 600.0 & 0.943 & 0.978 & 1.017 & 0.983 & 0.984 & 0.846 & 0.958 & 1.089 \\
\hline 9 & 700.0 & 0.949 & 0.984 & 1.023 & 0.989 & 0.989 & 0.852 & 0.964 & 1.093 \\
\hline 10 & 800.0 & 0.957 & 0.991 & 1.029 & 0.996 & 0.996 & 0.860 & 0.971 & 1.098 \\
\hline 11 & 900.0 & 0.965 & 0.998 & 1.036 & 1.003 & 1.004 & 0.868 & 0.979 & 1.102 \\
\hline 12 & 1000.0 & 0.973 & 1.006 & 1.043 & 1.011 & 1.011 & 0.877 & 0.988 & 1.107 \\
\hline 13 & 1100.0 & 0.981 & 1.014 & 1.050 & 1.018 & 1.018 & 0.886 & 0.996 & 1.111 \\
\hline 14 & 1200.0 & 0.989 & 1.022 & 1.056 & 1.026 & 1.026 & 0.895 & 1.004 & 1.116 \\
\hline 15 & 1300.0 & 0.998 & 1.030 & 1.063 & 1.033 & 1.033 & 0.905 & 1.013 & 1.119 \\
\hline
\end{tabular}


Table 4.64. Problem 3.2.2 reference solution power peaking factors for charge-pans 1029 and 0930.

\begin{tabular}{|c|c|c|c|c|c|c|c|c|c|}
\hline \multirow[t]{3}{*}{ Step } & \multirow{3}{*}{$\begin{array}{l}\text { Burnup } \\
{\left[\frac{\mathrm{MWd}}{\mathrm{MtU}}\right]}\end{array}$} & \multicolumn{8}{|c|}{ Shift Power Peaking Factors } \\
\hline & & $\mathrm{E}$ & $\mathrm{F}$ & $\mathrm{G}$ & $\mathrm{H}$ & $\mathbf{J}$ & K & $\mathrm{L}$ & M \\
\hline & & $(\mathrm{H})$ & $(\mathrm{G})$ & $(\mathrm{F})$ & $(\mathrm{E})$ & $(\mathrm{M})$ & $(\mathrm{L})$ & $(\mathrm{K})$ & $(\mathrm{J})$ \\
\hline 0 & 0.0 & 0.966 & 0.922 & 0.905 & 0.940 & 1.018 & 1.063 & 1.046 & 1.014 \\
\hline 1 & 25.0 & 0.943 & 0.900 & 0.887 & 0.928 & 1.014 & 1.053 & 1.034 & 0.992 \\
\hline 2 & 50.0 & 0.944 & 0.898 & 0.888 & 0.929 & 1.012 & 1.058 & 1.034 & 0.997 \\
\hline 3 & 100.0 & 0.956 & 0.912 & 0.898 & 0.937 & 1.016 & 1.058 & 1.042 & 1.005 \\
\hline 4 & 200.0 & 0.962 & 0.919 & 0.902 & 0.938 & 1.018 & 1.056 & 1.044 & 1.012 \\
\hline 5 & 300.0 & 0.969 & 0.923 & 0.902 & 0.940 & 1.016 & 1.058 & 1.048 & 1.017 \\
\hline 6 & 400.0 & 0.974 & 0.929 & 0.909 & 0.947 & 1.023 & 1.065 & 1.053 & 1.020 \\
\hline 7 & 500.0 & 0.975 & 0.930 & 0.909 & 0.950 & 1.024 & 1.066 & 1.053 & 1.023 \\
\hline 8 & 600.0 & 0.978 & 0.933 & 0.913 & 0.952 & 1.027 & 1.069 & 1.059 & 1.024 \\
\hline 9 & 700.0 & 0.979 & 0.934 & 0.910 & 0.954 & 1.032 & 1.072 & 1.061 & 1.028 \\
\hline 10 & 800.0 & 0.976 & 0.931 & 0.915 & 0.959 & 1.038 & 1.077 & 1.061 & 1.024 \\
\hline 11 & 900.0 & 0.972 & 0.926 & 0.913 & 0.957 & 1.037 & 1.079 & 1.057 & 1.020 \\
\hline 12 & 1000.0 & 0.973 & 0.928 & 0.913 & 0.958 & 1.040 & 1.084 & 1.060 & 1.024 \\
\hline 13 & 1100.0 & 0.967 & 0.921 & 0.908 & 0.952 & 1.038 & 1.082 & 1.058 & 1.015 \\
\hline 14 & 1200.0 & 0.964 & 0.922 & 0.913 & 0.958 & 1.042 & 1.086 & 1.060 & 1.014 \\
\hline 15 & 1300.0 & 0.960 & 0.918 & 0.911 & 0.961 & 1.050 & 1.087 & 1.057 & 1.014 \\
\hline \multirow[t]{3}{*}{ Step } & Burnup & \multicolumn{8}{|c|}{ MPACT Power Peaking Factors } \\
\hline & {$\left[\frac{\mathrm{MWd}}{\mathrm{MtU}}\right]$} & E & $\mathrm{F}$ & G & $\mathrm{H}$ & J & K & $\mathrm{L}$ & M \\
\hline & & $(\mathrm{H})$ & $(\mathrm{G})$ & $(\mathrm{F})$ & $(\mathrm{E})$ & $(\mathrm{M})$ & $(\mathrm{L})$ & $(\mathrm{K})$ & $(\mathrm{J})$ \\
\hline 0 & 0.0 & 0.962 & 0.917 & 0.901 & 0.938 & 1.017 & 1.059 & 1.045 & 1.010 \\
\hline 1 & 25.0 & 0.999 & 0.958 & 0.943 & 0.978 & 1.050 & 1.084 & 1.067 & 1.037 \\
\hline 2 & 50.0 & 0.990 & 0.948 & 0.933 & 0.968 & 1.041 & 1.077 & 1.061 & 1.030 \\
\hline 3 & 100.0 & 0.976 & 0.933 & 0.917 & 0.953 & 1.029 & 1.068 & 1.053 & 1.020 \\
\hline 4 & 200.0 & 0.958 & 0.913 & 0.897 & 0.934 & 1.014 & 1.057 & 1.043 & 1.008 \\
\hline 5 & 300.0 & 0.951 & 0.905 & 0.888 & 0.925 & 1.008 & 1.053 & 1.039 & 1.003 \\
\hline 6 & 400.0 & 0.949 & 0.902 & 0.885 & 0.923 & 1.007 & 1.052 & 1.039 & 1.002 \\
\hline 7 & 500.0 & 0.951 & 0.903 & 0.886 & 0.925 & 1.009 & 1.054 & 1.042 & 1.005 \\
\hline 8 & 600.0 & 0.955 & 0.908 & 0.891 & 0.929 & 1.013 & 1.058 & 1.046 & 1.009 \\
\hline 9 & 700.0 & 0.961 & 0.914 & 0.897 & 0.935 & 1.019 & 1.064 & 1.051 & 1.014 \\
\hline 10 & 800.0 & 0.969 & 0.922 & 0.905 & 0.943 & 1.026 & 1.069 & 1.056 & 1.021 \\
\hline 11 & 900.0 & 0.977 & 0.930 & 0.913 & 0.951 & 1.033 & 1.076 & 1.062 & 1.027 \\
\hline 12 & 1000.0 & 0.985 & 0.939 & 0.922 & 0.960 & 1.040 & 1.082 & 1.067 & 1.034 \\
\hline 13 & 1100.0 & 0.993 & 0.948 & 0.931 & 0.968 & 1.048 & 1.088 & 1.073 & 1.040 \\
\hline 14 & 1200.0 & 1.001 & 0.957 & 0.940 & 0.977 & 1.055 & 1.094 & 1.078 & 1.047 \\
\hline 15 & 1300.0 & 1.009 & 0.965 & 0.949 & 0.986 & 1.062 & 1.099 & 1.083 & 1.053 \\
\hline
\end{tabular}


Charge-Pans 1129 and (0931)

Table 4.65. Problem 3.2.2 reference solution power peaking factors for charge-pans 1129 and 0931.

\begin{tabular}{|c|c|c|c|c|c|c|c|c|c|}
\hline \multirow[t]{3}{*}{ Step } & \multirow{3}{*}{$\begin{array}{l}\text { Burnup } \\
{\left[\frac{\mathrm{MWd}}{\mathrm{MtU}}\right]}\end{array}$} & \multicolumn{8}{|c|}{ Shift Power Peaking Factors } \\
\hline & & A & B & $\mathrm{C}$ & $\mathrm{D}$ & $\mathrm{P}$ & Q & $\mathrm{R}$ & $\mathrm{S}$ \\
\hline & & (A) & (D) & (C) & (B) & $(\mathrm{R})$ & $(\mathrm{Q})$ & $(\mathrm{P})$ & (S) \\
\hline 0 & 0.0 & 0.664 & 0.704 & 0.782 & 0.742 & 0.788 & 0.567 & 0.700 & 0.903 \\
\hline 1 & 25.0 & 0.655 & 0.699 & 0.772 & 0.730 & 0.767 & 0.557 & 0.700 & 0.896 \\
\hline 2 & 50.0 & 0.654 & 0.697 & 0.773 & 0.732 & 0.770 & 0.560 & 0.702 & 0.899 \\
\hline 3 & 100.0 & 0.659 & 0.700 & 0.776 & 0.735 & 0.777 & 0.562 & 0.700 & 0.900 \\
\hline 4 & 200.0 & 0.658 & 0.701 & 0.776 & 0.738 & 0.781 & 0.565 & 0.699 & 0.897 \\
\hline 5 & 300.0 & 0.658 & 0.698 & 0.776 & 0.736 & 0.782 & 0.567 & 0.699 & 0.895 \\
\hline 6 & 400.0 & 0.665 & 0.703 & 0.778 & 0.743 & 0.788 & 0.572 & 0.701 & 0.900 \\
\hline 7 & 500.0 & 0.664 & 0.706 & 0.783 & 0.743 & 0.787 & 0.573 & 0.702 & 0.903 \\
\hline 8 & 600.0 & 0.667 & 0.709 & 0.783 & 0.746 & 0.792 & 0.576 & 0.705 & 0.906 \\
\hline 9 & 700.0 & 0.669 & 0.711 & 0.785 & 0.746 & 0.796 & 0.576 & 0.708 & 0.903 \\
\hline 10 & 800.0 & 0.670 & 0.714 & 0.792 & 0.750 & 0.795 & 0.580 & 0.713 & 0.911 \\
\hline 11 & 900.0 & 0.672 & 0.715 & 0.794 & 0.751 & 0.794 & 0.579 & 0.720 & 0.915 \\
\hline 12 & 1000.0 & 0.671 & 0.713 & 0.792 & 0.751 & 0.795 & 0.576 & 717 & 0.915 \\
\hline 13 & 1100.0 & 0.669 & 0.713 & 0.791 & 0.747 & 0.790 & 0.576 & 0.716 & 0.914 \\
\hline 14 & 1200.0 & 0.674 & 0.720 & 0.795 & 0.753 & 0.792 & 0.582 & 0.722 & 0.920 \\
\hline 15 & 1300.0 & 0.677 & 0.723 & 0.798 & 0.756 & 0.791 & 0.583 & 0.728 & 0.925 \\
\hline \multirow[t]{3}{*}{ Step } & Burnup & \multicolumn{8}{|c|}{ MPACT Power Peaking Factors } \\
\hline & $\left.\frac{\mathrm{MWd}}{\mathrm{MtU}}\right]$ & A & B & $\mathrm{C}$ & $\mathrm{D}$ & $\mathrm{P}$ & Q & $\mathrm{R}$ & $\mathrm{S}$ \\
\hline & & (A) & (D) & (C) & (B) & $(\mathrm{R})$ & $(\mathrm{Q})$ & $(\mathrm{P})$ & (S) \\
\hline 0 & 0.0 & 0.661 & 0.703 & 0.778 & 0.738 & 0.783 & 0.565 & 0.700 & 0.900 \\
\hline 1 & 25.0 & 0.703 & 0.747 & 0.822 & 0.782 & 0.827 & 0.606 & 0.747 & 0.943 \\
\hline 2 & 50.0 & 0.693 & 0.736 & 0.811 & 0.772 & 0.817 & 0.596 & 0.736 & 0.932 \\
\hline 3 & 100.0 & 0.677 & 0.720 & 0.795 & 0.756 & 0.800 & 0.582 & 0.719 & 0.916 \\
\hline 4 & 200.0 & 0.656 & 0.698 & 0.773 & 0.734 & 0.778 & 0.561 & 0.695 & 0.896 \\
\hline 5 & 300.0 & 0.646 & 0.688 & 0.763 & 0.724 & 0.769 & 0.551 & 0.684 & 0.887 \\
\hline 6 & 400.0 & 0.642 & 0.684 & 0.760 & 0.720 & 0.765 & 0.547 & 0.680 & 0.884 \\
\hline 7 & 500.0 & 0.642 & 0.684 & 0.761 & 0.721 & 0.766 & 0.546 & 0.680 & 0.885 \\
\hline 8 & 600.0 & 0.645 & 0.688 & 0.764 & 0.724 & 0.770 & 0.549 & 0.683 & 0.890 \\
\hline 9 & 700.0 & 0.650 & 0.693 & 0.770 & 0.730 & 0.776 & 0.553 & 0.688 & 0.896 \\
\hline 10 & 800.0 & 0.657 & 0.700 & 0.778 & 0.737 & 0.783 & 0.559 & 0.695 & 0.904 \\
\hline 11 & 900.0 & 0.664 & 0.708 & 0.786 & 0.745 & 0.791 & 0.565 & 0.703 & 0.912 \\
\hline 12 & 1000.0 & 0.672 & 0.716 & 0.795 & 0.754 & 0.800 & 0.572 & 0.712 & 0.921 \\
\hline 13 & 1100.0 & 0.680 & 0.725 & 0.804 & 0.762 & 0.809 & 0.579 & 0.721 & 0.930 \\
\hline 14 & 1200.0 & 0.689 & 0.734 & 0.813 & 0.771 & 0.818 & 0.586 & 0.730 & 0.939 \\
\hline 15 & 1300.0 & 0.697 & 0.742 & 0.822 & 0.780 & 0.827 & 0.594 & 0.739 & 0.948 \\
\hline
\end{tabular}


Table 4.66. Problem 3.2.2 reference solution power peaking factors for charge-pans 1129 and 0931.

\begin{tabular}{|c|c|c|c|c|c|c|c|c|c|}
\hline \multirow[t]{2}{*}{ Step } & \multirow{2}{*}{$\begin{array}{l}\text { Burnup } \\
{\left[\frac{\mathrm{MWd}}{\mathrm{MtU}}\right]}\end{array}$} & \multicolumn{8}{|c|}{ Shift Power Peaking Factors } \\
\hline & & $\begin{array}{c}E \\
(H)\end{array}$ & $\begin{array}{c}F \\
(G)\end{array}$ & $\begin{array}{c}G \\
(F)\end{array}$ & $\begin{array}{c}\mathrm{H} \\
(\mathrm{E})\end{array}$ & $\begin{array}{c}\mathrm{J} \\
(\mathrm{M})\end{array}$ & $\begin{array}{c}\mathrm{K} \\
(\mathrm{L})\end{array}$ & $\begin{array}{c}\mathrm{L} \\
(\mathrm{K})\end{array}$ & $\begin{array}{l}\mathrm{M} \\
(\mathrm{J})\end{array}$ \\
\hline 0 & 0.0 & 0.702 & 0.617 & 0.622 & 0.673 & 0.743 & 0.825 & 0.870 & 0.833 \\
\hline 1 & 25.0 & 0.688 & 0.605 & 0.617 & 0.669 & 0.743 & 0.820 & 0.861 & 0.818 \\
\hline 2 & 50.0 & 0.688 & 0.607 & 0.616 & 0.670 & 0.744 & 0.821 & 0.861 & 0.818 \\
\hline 3 & 100.0 & 0.695 & 0.612 & 0.619 & 0.672 & 0.745 & 0.822 & 0.866 & 0.826 \\
\hline 4 & 200.0 & 0.696 & 0.612 & 0.618 & 0.671 & 0.743 & 0.822 & 0.865 & 0.825 \\
\hline 5 & 300.0 & 0.697 & 0.613 & 0.620 & 0.670 & 0.740 & 0.819 & 0.867 & 0.829 \\
\hline 6 & 400.0 & 0.702 & 0.620 & 0.627 & 0.677 & 0.743 & 0.824 & 0.870 & 0.833 \\
\hline 7 & 500.0 & 0.702 & 0.620 & 0.628 & 0.678 & 0.746 & 0.825 & 0.872 & 0.832 \\
\hline 8 & 600.0 & 0.706 & 0.623 & 0.633 & 0.680 & 0.749 & 0.830 & 0.876 & 0.837 \\
\hline 9 & 700.0 & 0.709 & 0.623 & 0.632 & 0.679 & 0.749 & 0.828 & 0.874 & 0.838 \\
\hline 10 & 800.0 & 0.712 & 0.627 & 0.634 & 0.687 & 0.757 & 0.837 & 0.880 & 0.842 \\
\hline 11 & 900.0 & 0.711 & 0.626 & 0.639 & 0.690 & 0.757 & 0.839 & 0.880 & 0.839 \\
\hline 12 & 1000.0 & 0.708 & 0.625 & 0.636 & 0.688 & 0.758 & 0.839 & 0.880 & 0.839 \\
\hline 13 & 1100.0 & 0.708 & 0.623 & 0.634 & 0.686 & 0.758 & 0.835 & 0.878 & 0.836 \\
\hline 14 & 1200.0 & 0.713 & 0.626 & 0.641 & 0.694 & 0.765 & 0.845 & 0.883 & 0.841 \\
\hline 15 & 1300.0 & 0.712 & 0.630 & 0.644 & 0.697 & 0.769 & 0.848 & 0.886 & 0.843 \\
\hline \multirow[t]{2}{*}{ Step } & Burnup & \multicolumn{8}{|c|}{ MPACT Power Peaking Factors } \\
\hline & {$\left[\frac{\mathrm{MWd}}{\mathrm{M} t \mathrm{tI}}\right]$} & $\begin{array}{c}E \\
(H)\end{array}$ & $\begin{array}{c}F \\
(G)\end{array}$ & $\begin{array}{c}\mathrm{G} \\
\text { (F) }\end{array}$ & $\begin{array}{c}\mathrm{H} \\
\text { (E) }\end{array}$ & $\begin{array}{c}\mathrm{J} \\
(\mathrm{M})\end{array}$ & $\begin{array}{c}\mathrm{K} \\
(\mathrm{L})\end{array}$ & $\begin{array}{c}\mathrm{L} \\
(\mathrm{K})\end{array}$ & $\begin{array}{l}\mathrm{M} \\
(\mathrm{J})\end{array}$ \\
\hline 0 & 0.0 & 0.698 & 0.614 & 0.622 & 0.672 & 0.744 & 0.822 & 0.868 & 0.829 \\
\hline 1 & 25.0 & 0.741 & 0.655 & 0.665 & 0.718 & 0.789 & 0.867 & 0.911 & 0.873 \\
\hline 2 & 50.0 & 0.731 & 0.645 & 0.655 & 0.707 & 0.778 & 0.856 & 0.901 & 0.862 \\
\hline 3 & 100.0 & 0.715 & 0.630 & 0.639 & 0.690 & 0.762 & 0.840 & 0.885 & 0.846 \\
\hline 4 & 200.0 & 0.693 & 0.609 & 0.617 & 0.667 & 0.739 & 0.818 & 0.864 & 0.824 \\
\hline 5 & 300.0 & 0.683 & 0.600 & 0.607 & 0.657 & 0.729 & 0.808 & 0.854 & 0.815 \\
\hline 6 & 400.0 & 0.679 & 0.596 & 0.602 & 0.652 & 0.725 & 0.805 & 0.851 & 0.811 \\
\hline 7 & 500.0 & 0.680 & 0.596 & 0.602 & 0.652 & 0.725 & 0.806 & 0.852 & 0.812 \\
\hline 8 & 600.0 & 0.683 & 0.598 & 0.604 & 0.655 & 0.729 & 0.810 & 0.857 & 0.816 \\
\hline 9 & 700.0 & 0.689 & 0.603 & 0.609 & 0.660 & 0.735 & 0.816 & 0.863 & 0.823 \\
\hline 10 & 800.0 & 0.695 & 0.609 & 0.615 & 0.666 & 0.742 & 0.824 & 0.871 & 0.830 \\
\hline 11 & 900.0 & 0.703 & 0.616 & 0.622 & 0.674 & 0.750 & 0.832 & 0.879 & 0.839 \\
\hline 12 & 1000.0 & 0.711 & 0.624 & 0.630 & 0.682 & 0.759 & 0.841 & 0.888 & 0.847 \\
\hline 13 & 1100.0 & 0.720 & 0.632 & 0.638 & 0.691 & 0.768 & 0.850 & 0.897 & 0.857 \\
\hline 14 & 1200.0 & 0.729 & 0.640 & 0.646 & 0.699 & 0.777 & 0.860 & 0.906 & 0.866 \\
\hline 15 & 1300.0 & 0.737 & 0.648 & 0.654 & 0.708 & 0.786 & 0.869 & 0.915 & 0.875 \\
\hline
\end{tabular}




\section{Charge-Pan 1030}

Table 4.67. Problem 3.2.2 reference solution power peaking factors for charge-pan 1030 .

\begin{tabular}{|c|c|c|c|c|c|c|c|c|c|c|c|}
\hline \multirow[t]{2}{*}{ Step } & Burnup & \multicolumn{10}{|c|}{ Shift Power Peaking Factors } \\
\hline & {$\left[\frac{\mathrm{MWd}}{\mathrm{MtU}}\right.$} & A & $\mathrm{B}, \mathrm{D}$ & $\mathrm{C}$ & $\mathrm{G}, \mathrm{F}$ & $\mathrm{H}, \mathrm{E}$ & $\mathrm{J}, \mathrm{M}$ & $\mathrm{K}, \mathrm{L}$ & Q & $\mathrm{R}, \mathrm{P}$ & $\mathrm{S}$ \\
\hline 0 & 0.0 & 0.752 & 0.810 & 0.865 & 0.687 & 0.751 & 0.872 & 0.924 & 0.628 & 0.809 & 0.964 \\
\hline 1 & 25.0 & 0.725 & 0.779 & 0.839 & 0.665 & 0.729 & 0.849 & & 0.606 & 789 & 0.934 \\
\hline 2 & 50.0 & 0.726 & 0.778 & 0.839 & 0.665 & 0.728 & 0.848 & 00 & 607 & 788 & 0.940 \\
\hline 3 & 100.0 & 0.739 & 0.792 & 0.847 & 0.674 & 0.737 & 0.862 & 0.913 & 0.615 & 0.800 & 0.948 \\
\hline 4 & 200.0 & 0.746 & 0.802 & 0.858 & 0.680 & 0.744 & 0.866 & 0.921 & 0.627 & 0.803 & 0.959 \\
\hline 5 & 300.0 & 0.752 & 0.814 & 0.863 & 0.690 & 0.751 & 0.875 & 0.927 & 0.632 & 0.811 & 0.965 \\
\hline 6 & 400.0 & 0.761 & 0.820 & 0.872 & 0.696 & 0.756 & 0.878 & 0.933 & 38 & 0.817 & 0.971 \\
\hline 7 & 500.0 & 58 & 0.822 & 0.872 & 0.694 & & 0.876 & & & 814 & .974 \\
\hline 8 & 600.0 & 0.760 & 0.816 & 0.870 & 0.697 & 0.756 & 0.882 & 0.934 & 0.638 & 0.817 & 0.973 \\
\hline 9 & 700.0 & 759 & 0.816 & 0.870 & 0.697 & 0.757 & 0.881 & 0.932 & 38 & 0.819 & .970 \\
\hline 10 & 8 & 55 & 0.807 & 0.862 & 0.690 & 54 & 0.880 & & & 0.816 & .964 \\
\hline 11 & 900.0 & 0.752 & 0.808 & 0.860 & 0.688 & 0.750 & 0.875 & 0.925 & 0. & 0.815 & 0.961 \\
\hline 12 & 1000.0 & & & & 0.688 & & 75 & & & 12 & 960 \\
\hline 13 & 0 & 44 & 0.790 & 0.852 & 0.684 & 0. & 0.868 & & & 0.811 & 0.954 \\
\hline 14 & 1200 & 0 & 0.787 & 0.851 & 0.680 & 0.7 & 0.866 & 0. & 0 . & 0.810 & 0.948 \\
\hline 15 & 1300.0 & 35 & 0.778 & 44 & 0.677 & 0.740 & 0.866 & 0.912 & 18 & 0.807 & 0.946 \\
\hline \multirow[t]{2}{*}{ Step } & Burnup & \multicolumn{10}{|c|}{ MPACT Power Peaking Factors } \\
\hline & & A & $\mathrm{B}, \mathrm{D}$ & $\mathrm{C}$ & $\mathrm{G}, \mathrm{F}$ & $\mathrm{H}, \mathrm{E}$ & $\mathrm{J}, \mathrm{M}$ & $\mathrm{K}, \mathrm{L}$ & Q & $\mathrm{R}, \mathrm{P}$ & $S$ \\
\hline 0 & 0.0 & 0.748 & 0.805 & 0.861 & 0.683 & 0.746 & 0.869 & 0.921 & .625 & 0.805 & 0.961 \\
\hline 1 & & 0.791 & 0.850 & 0.904 & 0.726 & & 0.912 & 0. & 0.667 & 0.850 & 0.998 \\
\hline 2 & & 0.781 & 0.839 & 0.8 & 0.716 & & 0.9 & & & 0.839 & 0.988 \\
\hline 3 & 100.0 & 0.765 & 0.823 & 0.877 & 0.700 & 0.763 & 0.886 & 36 & 0.641 & 0.823 & 0.975 \\
\hline 4 & 200.0 & 0.743 & 0.801 & 0.857 & 0.678 & 41 & 0.865 & & 0.620 & 0.801 & 0.957 \\
\hline 5 & 300.0 & 0.733 & 0.791 & 0.848 & 0.668 & 0.731 & 0.856 & 8 & 10 & 0.791 & .950 \\
\hline 6 & 400.0 & 0.729 & 0.788 & 0.844 & 0.664 & & 0.852 & & 0.606 & 0.788 & 0.948 \\
\hline 7 & 500.0 & 0.730 & 0.788 & 0.846 & 0.665 & 0.728 & 0.854 & & 0.606 & 0.788 & 0.950 \\
\hline 8 & 600.0 & 0.734 & 0.793 & 0.850 & 0.668 & 0.732 & 0.858 & 0.912 & 0.609 & 0.793 & 0.955 \\
\hline ( & 700.0 & 0.739 & 0.799 & 0.856 & 0.673 & 0.738 & 0.865 & 0.918 & 0.614 & 0.799 & 0.961 \\
\hline 10 & 800.0 & 0.747 & 0.806 & 0.864 & 0.680 & 0.745 & 0.872 & 0.926 & 0.620 & 0.806 & 0.968 \\
\hline 11 & 900.0 & 0.755 & 0.815 & 0.872 & 0.688 & 0.753 & 0.881 & 0.934 & 0.627 & 0.815 & 0.976 \\
\hline 12 & 1000.0 & 0.763 & 0.823 & 0.881 & 0.696 & 0.761 & 0.890 & & 0.635 & 0.823 & 0.984 \\
\hline 13 & 1100.0 & 0.772 & 0.832 & 0.890 & 0.704 & 0.770 & 0.899 & 0.952 & 0.643 & 0.832 & 0.992 \\
\hline 14 & 1200.0 & 0.781 & 0.842 & 0.899 & 0.713 & 0.779 & 0.908 & 0.960 & 0.651 & 0.842 & 1.000 \\
\hline 15 & 1300.0 & 0.790 & 0.851 & 0.908 & 0.722 & 0.788 & 0.917 & 0.969 & 0.659 & 0.851 & 1.008 \\
\hline
\end{tabular}


Charge-Pans 1130 and (1031)

Table 4.68. Problem 3.2.2 reference solution power peaking factors for charge-pans 1130 and 1031.

\begin{tabular}{|c|c|c|c|c|c|c|c|c|}
\hline \multirow[t]{3}{*}{ Step } & \multirow{3}{*}{$\begin{array}{l}\text { Burnup } \\
{\left[\frac{\mathrm{MWd}}{\mathrm{MtU}}\right]}\end{array}$} & \multicolumn{7}{|c|}{ Shift Power Peaking Factors } \\
\hline & & A & B & $\mathrm{C}$ & D & $\mathrm{P}$ & $\mathrm{R}$ & S \\
\hline & & (A) & (D) & (C) & (B) & (R) & (P) & (S) \\
\hline 0 & 0.0 & 0.494 & 0.528 & 0.582 & 0.533 & 0.579 & 0.529 & 0.731 \\
\hline 1 & 25.0 & 0.479 & 0.517 & 0.565 & 0.515 & 0.559 & 0.518 & 0.714 \\
\hline 2 & 50.0 & 0.481 & 0.519 & 0.568 & 0.518 & 0.560 & 0.518 & 0.715 \\
\hline 3 & 100.0 & 0.485 & 0.523 & 0.573 & 0.524 & 0.569 & 0.521 & 0.720 \\
\hline 4 & 200.0 & 0.491 & 0.526 & 0.579 & 0.528 & 0.575 & 0.523 & 0.725 \\
\hline 5 & 300.0 & 0.494 & 0.530 & 0.581 & 0.532 & 0.580 & 0.526 & 0.727 \\
\hline 6 & 400.0 & 0.497 & 0.533 & 0.587 & 0.539 & 0.585 & 0.530 & 0.731 \\
\hline 7 & 500.0 & 0.495 & 0.535 & 0.585 & 0.536 & 0.582 & 0.531 & 0.728 \\
\hline 8 & 600.0 & 0.499 & 0.538 & 0.588 & 0.539 & 0.585 & 0.536 & 0.731 \\
\hline 9 & 700.0 & 0.498 & 0.537 & 0.588 & 0.539 & 0.586 & 0.534 & 0.731 \\
\hline 10 & 800.0 & 0.499 & 0.539 & 0.588 & 0.538 & 0.582 & 0.536 & 0.731 \\
\hline 11 & 900.0 & 0.497 & 0.536 & 0.588 & 0.536 & 0.582 & 0.537 & 0.729 \\
\hline 12 & 1000.0 & 0.496 & 0.536 & 0.587 & 0.536 & 0.578 & 0.534 & 0.728 \\
\hline 13 & 1100.0 & 0.496 & 0.534 & 0.584 & 0.533 & 0.576 & 0.533 & 0.727 \\
\hline 14 & 1200.0 & 0.495 & 0.537 & 0.583 & 0.532 & 0.575 & 0.538 & 0.726 \\
\hline 15 & 1300.0 & 0.495 & 0.534 & 0.583 & 0.533 & 0.572 & 0.538 & 0.726 \\
\hline \multirow[t]{3}{*}{ Step } & Burnup & \multicolumn{7}{|c|}{ MPACT Power Peaking Factors } \\
\hline & $\frac{\mathrm{MWd}}{\mathrm{MtU}}$ & A & B & $\mathrm{C}$ & $\mathrm{D}$ & $\mathrm{P}$ & $\mathrm{R}$ & S \\
\hline & & (A) & (D) & (C) & (B) & (R) & (P) & (S) \\
\hline 0 & 0.0 & 0.490 & 0.527 & 0.579 & 0.530 & 0.577 & 0.525 & 0.728 \\
\hline 1 & 25.0 & 0.527 & 0.565 & 0.619 & 0.568 & 0.618 & 0.564 & 0.772 \\
\hline 2 & 50.0 & 0.519 & 0.556 & 0.609 & 0.559 & 0.608 & 0.555 & 0.762 \\
\hline 3 & 100.0 & 0.505 & 0.542 & 0.595 & 0.545 & 0.593 & 0.541 & 0.746 \\
\hline 4 & 200.0 & 0.487 & 0.523 & 0.574 & 0.526 & 0.572 & 0.521 & 0.724 \\
\hline 5 & 300.0 & 0.478 & 0.513 & 0.565 & 0.517 & 0.562 & 0.512 & 0.714 \\
\hline 6 & 400.0 & 0.473 & 0.509 & 0.561 & 0.513 & 0.558 & 0.508 & 0.710 \\
\hline 7 & 500.0 & 0.473 & 0.509 & 0.561 & 0.512 & 0.558 & 0.507 & 0.710 \\
\hline 8 & 600.0 & 0.475 & 0.511 & 0.563 & 0.515 & 0.560 & 0.509 & 0.714 \\
\hline 9 & 700.0 & 0.478 & 0.515 & 0.568 & 0.519 & 0.565 & 0.513 & 0.720 \\
\hline 10 & 800.0 & 0.483 & 0.520 & 0.574 & 0.524 & 0.570 & 0.518 & 0.727 \\
\hline 11 & 900.0 & 0.489 & 0.526 & 0.580 & 0.530 & 0.577 & 0.524 & 0.735 \\
\hline 12 & 1000.0 & 0.495 & 0.532 & 0.587 & 0.536 & 0.584 & 0.530 & 0.743 \\
\hline 13 & 1100.0 & 0.501 & 0.539 & 0.595 & 0.543 & 0.591 & 0.537 & 0.752 \\
\hline 14 & 1200.0 & 0.507 & 0.546 & 0.602 & 0.550 & 0.599 & 0.544 & 0.761 \\
\hline 15 & 1300.0 & 0.514 & 0.554 & 0.610 & 0.558 & 0.607 & 0.551 & 0.770 \\
\hline
\end{tabular}


Table 4.69. Problem 3.2.2 reference solution power peaking factors for charge-pans 1130 and 1031.

\begin{tabular}{rrrcccc}
\hline Step & Burnup & \multicolumn{5}{c}{ Power Peaking Factors } \\
& {$\left[\frac{\text { MWd }}{\text { MtU }}\right]$} & E & J & K & L & M \\
& & $(\mathrm{H})$ & $(\mathrm{M})$ & $(\mathrm{L})$ & $(\mathrm{K})$ & $(\mathrm{J})$ \\
\hline 0 & 0.0 & 0.521 & 0.570 & 0.647 & 0.670 & 0.610 \\
1 & 25.0 & 0.504 & 0.557 & 0.633 & 0.651 & 0.591 \\
2 & 50.0 & 0.505 & 0.557 & 0.632 & 0.651 & 0.592 \\
3 & 100.0 & 0.512 & 0.561 & 0.639 & 0.660 & 0.601 \\
4 & 200.0 & 0.515 & 0.565 & 0.640 & 0.664 & 0.606 \\
5 & 300.0 & 0.523 & 0.565 & 0.643 & 0.668 & 0.611 \\
6 & 400.0 & 0.528 & 0.570 & 0.646 & 0.677 & 0.616 \\
7 & 500.0 & 0.525 & 0.568 & 0.645 & 0.674 & 0.612 \\
8 & 600.0 & 0.530 & 0.573 & 0.648 & 0.676 & 0.617 \\
9 & 700.0 & 0.529 & 0.572 & 0.650 & 0.677 & 0.616 \\
10 & 800.0 & 0.528 & 0.574 & 0.648 & 0.676 & 0.615 \\
11 & 900.0 & 0.526 & 0.574 & 0.648 & 0.674 & 0.612 \\
12 & 1000.0 & 0.524 & 0.572 & 0.646 & 0.671 & 0.610 \\
13 & 1100.0 & 0.524 & 0.571 & 0.645 & 0.672 & 0.609 \\
14 & 1200.0 & 0.520 & 0.571 & 0.647 & 0.668 & 0.606 \\
15 & 1300.0 & 0.518 & 0.573 & 0.646 & 0.668 & 0.604 \\
\hline Step & Burnup & \multicolumn{2}{c}{ MPACT Power Peaking Factors } \\
& {$\left[\frac{\text { Mwd }}{\text { MtU }}\right]$} & E & J & K & L & M \\
& & $(\mathrm{H})$ & $(\mathrm{M})$ & $(\mathrm{L})$ & $(\mathrm{K})$ & $(\mathrm{J})$ \\
\hline 0 & 0.0 & 0.518 & 0.567 & 0.643 & 0.667 & 0.608 \\
1 & 25.0 & 0.556 & 0.606 & 0.685 & 0.710 & 0.649 \\
2 & 50.0 & 0.547 & 0.597 & 0.675 & 0.700 & 0.639 \\
3 & 100.0 & 0.533 & 0.582 & 0.660 & 0.684 & 0.624 \\
4 & 200.0 & 0.514 & 0.562 & 0.639 & 0.662 & 0.603 \\
5 & 300.0 & 0.504 & 0.553 & 0.629 & 0.653 & 0.593 \\
6 & 400.0 & 0.500 & 0.549 & 0.625 & 0.648 & 0.589 \\
7 & 500.0 & 0.500 & 0.548 & 0.625 & 0.649 & 0.589 \\
8 & 600.0 & 0.502 & 0.551 & 0.628 & 0.652 & 0.592 \\
9 & 700.0 & 0.505 & 0.555 & 0.633 & 0.657 & 0.597 \\
10 & 800.0 & 0.510 & 0.561 & 0.640 & 0.664 & 0.603 \\
11 & 900.0 & 0.516 & 0.567 & 0.647 & 0.671 & 0.610 \\
12 & 1000.0 & 0.523 & 0.574 & 0.655 & 0.679 & 0.617 \\
13 & 1100.0 & 0.529 & 0.582 & 0.663 & 0.688 & 0.625 \\
14 & 1200.0 & 0.536 & 0.589 & 0.671 & 0.696 & 0.633 \\
15 & 1300.0 & 0.543 & 0.597 & 0.679 & 0.705 & 0.641 \\
\hline & & & & & &
\end{tabular}




\section{PROBLEM 4: FUEL CHANNEL 3D}

Problem 4 is a single 3D fuel channel with upper and lower axial reflector regions. The top and bottom have vacuum (zero-return) boundaries, and the north, south, east, and west boundaries are fully reflected. Problem 4 is more complex than previous problems because it is $3 \mathrm{D}$ and includes axial reflectors.

\subsection{BEGINNING-OF-CYCLE}

\subsubsection{Problem 4.1.1}

\subsubsection{Description}

Figure 16 shows the axial fuel channel geometry for Problem 4.1.1. Six fuel rods are stacked on one another within the fuel channel. Within each fuel element, the active (uranium-bearing) length is centered in the total length such that two end cap regions are formed at the top and bottom of each element. These regions are each

$$
L_{\text {cap }}=\frac{L_{\text {elem,tot }}-L_{\text {elem,act }}}{2}
$$

in length and composed entirely of cladding material. In the reference solution for Problem 4.1.1, each fuel element is divided into 20 segments, each representing $5 \%$ of the active fuel length. The values reported are the average values over each segment. The transverse geometry is illustrated by Figure 16. Zone B coolant channel radius is used for this problem. Geometric dimensions are listed in Table 1.2. Material densities are listed in Table 1.4. Temperatures are listed in Table 2.4. 


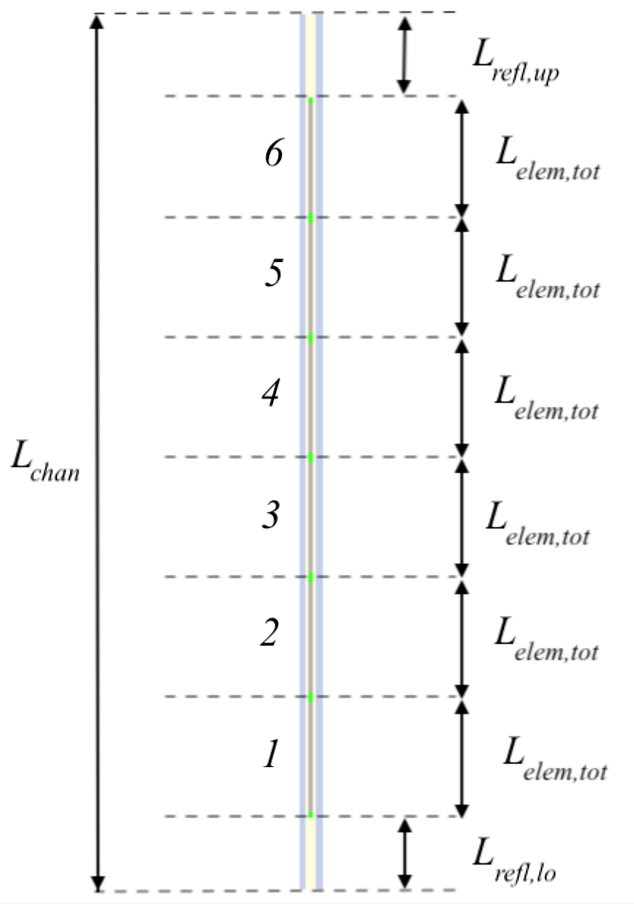

(a) Fuel channel 3D, six fuel element stack

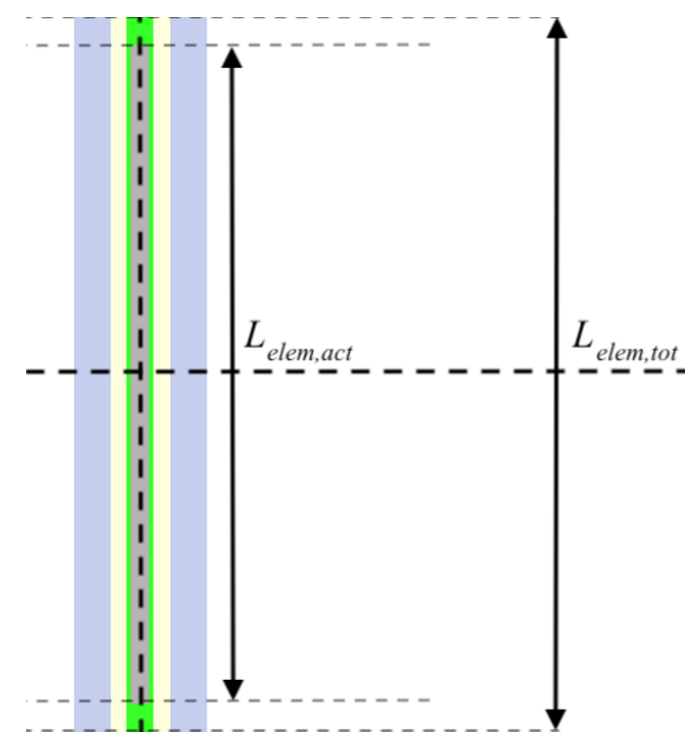

(b) Single fuel element

Figure 16. 3D fuel channel. 


\subsubsection{Reference Solution}

The neutron multiplication factor results are shown in Table 5.1. The timing variables for Shift and MPACT are shown in Table 5.2. Power peaking factors are displayed for BOC and EOC in Figure 17 and fully tabulated for all time steps in Tables 5.3-5.8. MPACT and Shift agree well for this case.

Table 5.1. Neutron multiplication factor for the reference solution of Problem 4.1.1.

\begin{tabular}{ccccc}
\hline Step & $\begin{array}{c}\text { Burnup } \\
{\left[\frac{\mathrm{MWd}}{\mathrm{MtU}}\right]}\end{array}$ & $\begin{array}{c}\text { Shift } \\
\mathrm{k}_{\mathrm{eff}}(1 \sigma)\end{array}$ & $\begin{array}{c}\text { MPACT } \\
\mathrm{k}_{\mathrm{eff}}\end{array}(1 \sigma)$ & $\begin{array}{c}\text { Difference } \\
{[\mathrm{pcm}]}\end{array}$ \\
\hline 0 & 0.0 & $1.05183(5)$ & $1.05091(10)$ & $-98(11)$ \\
\hline
\end{tabular}

Table 5.2. Timing variables for the reference solution of Problem 4.1.1

\begin{tabular}{|c|c|c|c|c|c|c|}
\hline \multicolumn{7}{|c|}{ Shift } \\
\hline $\begin{array}{c}\text { Depletion } \\
\text { Solves }\end{array}$ & $\begin{array}{c}\text { Total } \\
\text { Cycles per } \\
\text { Transport } \\
\text { Solve }\end{array}$ & $\begin{array}{c}\text { Active } \\
\text { Cycles per } \\
\text { Transport } \\
\text { Solve }\end{array}$ & $\begin{array}{l}\text { Histories } \\
\text { per Cycle }\end{array}$ & $\begin{array}{c}\text { Compute } \\
\text { Nodes }\end{array}$ & $\begin{array}{c}\text { Cores } \\
\text { per Node }\end{array}$ & $\begin{array}{c}\text { Wall } \\
\text { Total Time } \\
\text { [hours] }\end{array}$ \\
\hline 1 & 1500 & 1200 & 250000 & 2 & 48 & 1.95 \\
\hline \multicolumn{7}{|c|}{ MPACT } \\
\hline $\begin{array}{c}\text { Depletion } \\
\text { Solves }\end{array}$ & $\begin{array}{c}k_{\mathrm{eff}} \\
\text { Convergence } \\
\text { Criteria }\end{array}$ & $\begin{array}{c}\text { Source } \\
\text { Convergence } \\
\text { Criteria } \\
\end{array}$ & $\begin{array}{c}\text { Ray } \\
\text { Spacing } \\
{[\mathrm{cm}]}\end{array}$ & $\begin{array}{c}\text { Compute } \\
\text { Nodes }\end{array}$ & $\begin{array}{c}\text { Cores } \\
\text { per Node }\end{array}$ & $\begin{array}{c}\text { Wall } \\
\text { Total Time } \\
\text { [hours] }\end{array}$ \\
\hline 1 & $1.0 \times 10^{-04}$ & $1.0 \times 10^{-03}$ & 0.01 & 4 & 32 & 0.72 \\
\hline
\end{tabular}

Table 5.3. Problem 4.1.1 reference solution power peaking factors for axial segments in element 1.

\begin{tabular}{lcccccccccc}
\hline & & \multicolumn{8}{c}{ Power Peaking Factors } \\
& 1 & 2 & 3 & 4 & 5 & 6 & 7 & 8 & 9 & 10 \\
\hline Shift & 0.467 & 0.455 & 0.461 & 0.473 & 0.489 & 0.508 & 0.528 & 0.551 & 0.574 & 0.597 \\
MPACT & 0.453 & 0.448 & 0.453 & 0.465 & 0.481 & 0.500 & 0.521 & 0.544 & 0.567 & 0.591 \\
\hline \hline & 11 & 12 & 13 & 14 & 15 & 16 & 17 & 18 & 19 & 20 \\
\hline Shift & 0.621 & 0.646 & 0.672 & 0.698 & 0.725 & 0.755 & 0.787 & 0.821 & 0.857 & 0.925 \\
MPACT & 0.616 & 0.641 & 0.667 & 0.694 & 0.722 & 0.752 & 0.784 & 0.819 & 0.859 & 0.911 \\
\hline
\end{tabular}



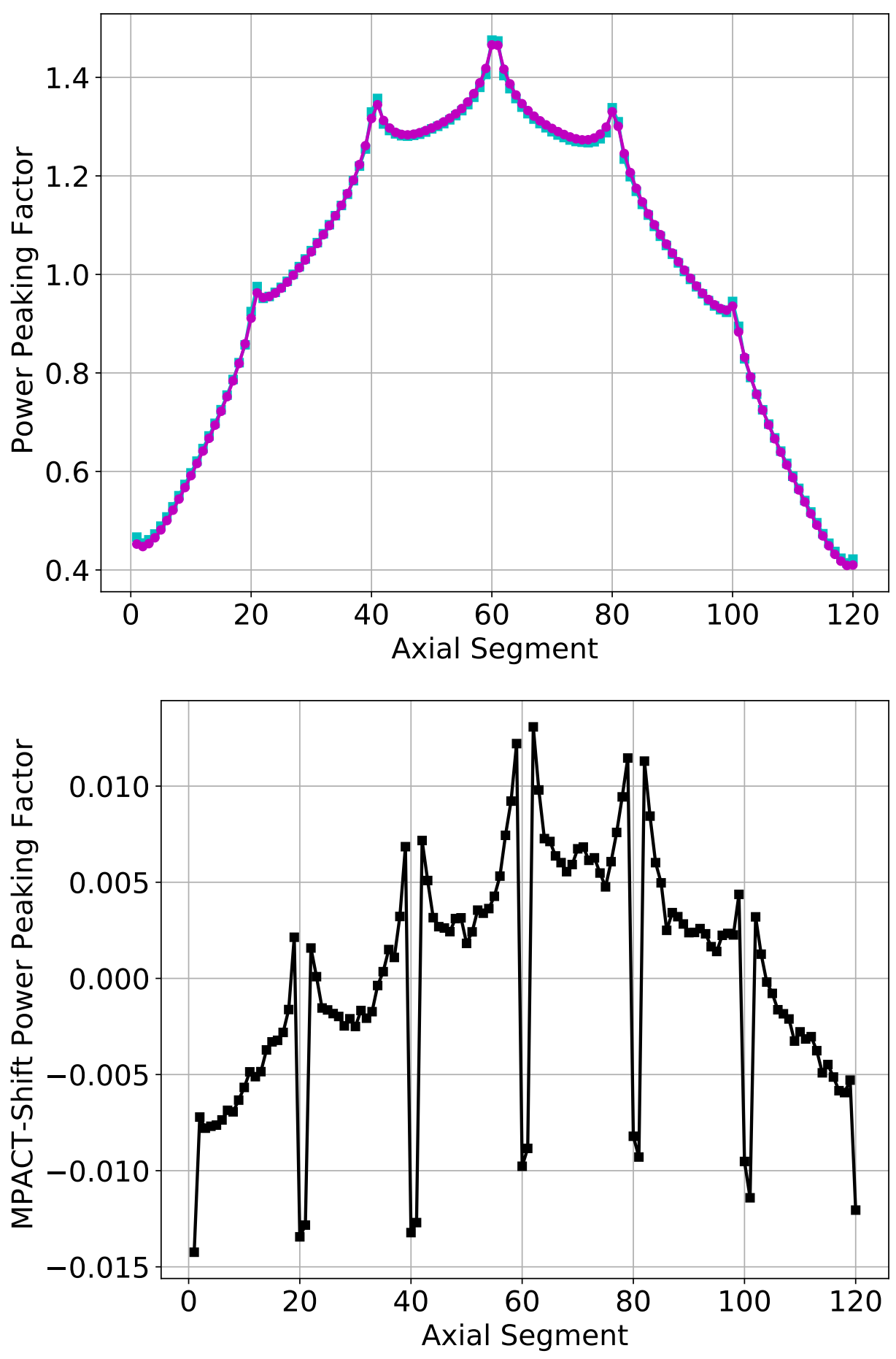

Figure 17. Power peaking factors for Problem 4.1.1 for Shift and MPACT (top) and their absolute difference (bottom). 
Table 5.4. Problem 4.1.1 reference solution power peaking factors for axial segments in element 2 .

\begin{tabular}{lcccccccccc}
\hline & & \multicolumn{10}{c}{ Power Peaking Factors } \\
& 1 & 2 & 3 & 4 & 5 & 6 & 7 & 8 & 9 & 10 \\
\hline Shift & 0.975 & 0.951 & 0.955 & 0.964 & 0.974 & 0.986 & 1.000 & 1.016 & 1.031 & 1.048 \\
MPACT & 0.962 & 0.953 & 0.955 & 0.962 & 0.972 & 0.984 & 0.998 & 1.013 & 1.029 & 1.046 \\
\hline \hline & 11 & 12 & 13 & 14 & 15 & 16 & 17 & 18 & 19 & 20 \\
\hline Shift & 1.065 & 1.083 & 1.101 & 1.119 & 1.140 & 1.163 & 1.190 & 1.220 & 1.254 & 1.330 \\
MPACT & 1.063 & 1.081 & 1.099 & 1.119 & 1.140 & 1.164 & 1.191 & 1.223 & 1.261 & 1.316 \\
\hline
\end{tabular}

Table 5.5. Problem 4.1.1 reference solution power peaking factors for axial segments in element 3 .

\begin{tabular}{lcccccccccc}
\hline & \multicolumn{10}{c}{ Power Peaking Factors } \\
& 1 & 2 & 3 & 4 & 5 & 6 & 7 & 8 & 9 & 10 \\
\hline Shift & 1.357 & 1.305 & 1.292 & 1.285 & 1.282 & 1.281 & 1.282 & 1.285 & 1.289 & 1.295 \\
MPACT & 1.344 & 1.312 & 1.297 & 1.288 & 1.284 & 1.283 & 1.285 & 1.288 & 1.292 & 1.297 \\
\hline \hline & 11 & 12 & 13 & 14 & 15 & 16 & 17 & 18 & 19 & 20 \\
\hline Shift & 1.301 & 1.306 & 1.314 & 1.322 & 1.332 & 1.345 & 1.360 & 1.380 & 1.406 & 1.476 \\
MPACT & 1.303 & 1.310 & 1.317 & 1.326 & 1.337 & 1.350 & 1.367 & 1.389 & 1.418 & 1.466 \\
\hline
\end{tabular}

Table 5.6. Problem 4.1.1 reference solution power peaking factors for axial segments in element 4 .

\begin{tabular}{lcccccccccc}
\hline & \multicolumn{10}{c}{ Power Peaking Factors } \\
& 1 & 2 & 3 & 4 & 5 & 6 & 7 & 8 & 9 & 10 \\
\hline Shift & 1.474 & 1.404 & 1.377 & 1.357 & 1.340 & 1.326 & 1.315 & 1.306 & 1.298 & 1.290 \\
MPACT & 1.465 & 1.417 & 1.387 & 1.364 & 1.347 & 1.333 & 1.321 & 1.312 & 1.304 & 1.296 \\
\hline \hline & 11 & 12 & 13 & 14 & 15 & 16 & 17 & 18 & 19 & 20 \\
\hline Shift & 1.283 & 1.278 & 1.273 & 1.270 & 1.268 & 1.267 & 1.269 & 1.275 & 1.288 & 1.338 \\
MPACT & 1.290 & 1.284 & 1.279 & 1.275 & 1.273 & 1.273 & 1.277 & 1.285 & 1.299 & 1.330 \\
\hline
\end{tabular}

Table 5.7. Problem 4.1.1 reference solution power peaking factors for axial segments in element 5.

\begin{tabular}{lcccccccccc}
\hline & \multicolumn{10}{c}{ Power Peaking Factors } \\
& 1 & 2 & 3 & 4 & 5 & 6 & 7 & 8 & 9 & 10 \\
\hline Shift & 1.310 & 1.234 & 1.198 & 1.169 & 1.142 & 1.120 & 1.097 & 1.077 & 1.059 & 1.041 \\
MPACT & 1.301 & 1.245 & 1.207 & 1.175 & 1.147 & 1.123 & 1.101 & 1.081 & 1.062 & 1.043 \\
\hline \hline & 11 & 12 & 13 & 14 & 15 & 16 & 17 & 18 & 19 & 20 \\
\hline Shift & 1.023 & 1.006 & 0.990 & 0.975 & 0.961 & 0.947 & 0.936 & 0.928 & 0.923 & 0.945 \\
MPACT & 1.026 & 1.009 & 0.992 & 0.976 & 0.962 & 0.949 & 0.938 & 0.931 & 0.927 & 0.936 \\
\hline
\end{tabular}


Table 5.8. Problem 4.1.1 reference solution power peaking factors for axial segments in element 6 .

\begin{tabular}{lcccccccccc}
\hline & \multicolumn{10}{c}{ Power Peaking Factors } \\
& 1 & 2 & 3 & 4 & 5 & 6 & 7 & 8 & 9 & 10 \\
\hline Shift & 0.895 & 0.829 & 0.791 & 0.757 & 0.725 & 0.696 & 0.668 & 0.641 & 0.616 & 0.590 \\
MPACT & 0.883 & 0.832 & 0.792 & 0.757 & 0.724 & 0.695 & 0.666 & 0.639 & 0.613 & 0.588 \\
\hline \hline & 11 & 12 & 13 & 14 & 15 & 16 & 17 & 18 & 19 & 20 \\
\hline Shift & 0.566 & 0.541 & 0.518 & 0.496 & 0.474 & 0.454 & 0.438 & 0.424 & 0.414 & 0.422 \\
MPACT & 0.562 & 0.538 & 0.514 & 0.491 & 0.469 & 0.449 & 0.432 & 0.418 & 0.409 & 0.410 \\
\hline
\end{tabular}




\subsection{DEPLETION}

\subsubsection{Problem 4.2.1}

\subsubsection{Description}

This problem uses exactly the same geometry as Problem 4.1.1 shown in Figure 16. Material densities are listed in Table 1.4. Temperatures are listed in Table 2.4. The total system specific power is listed in Table 1.6.

\subsubsection{Reference Solution}

The neutron multiplication factor results are shown in Table 5.9. The timing variables for Shift and MPACT are shown in Table 5.10. For this problem, the MPACT solution converges much quicker than the Shift solution. This is because MPACT uses the converged solution from the previous step as the initial source distribution for the next step. While MC codes do this as well, as with Shift, its convergence is solely proportional to the number of neutron histories run. Additionally, loose convergence criteria relative to the $2 \mathrm{D}$ problems was required for the problem to finish. It is hypothesized that the non-fueled end cap regions cause the MPACT solution to remain unconverged if the convergence criteria is too precise.

Power peaking factors are displayed for BOC and EOC in Figures 18-19 and fully tabulated for all time steps in Tables 5.11-5.22. The peaking factors agree to about $2 \%$.

Table 5.9. Neutron multiplication factor for the reference solution of Problem 4.2.1.

\begin{tabular}{rrccr}
\hline Step & $\begin{array}{r}\text { Burnup } \\
{\left[\frac{\mathrm{MWd}}{\mathrm{MtU}}\right]}\end{array}$ & $\begin{array}{c}\text { Shift } \\
\mathrm{k}_{\mathrm{eff}}(1 \sigma)\end{array}$ & $\begin{array}{c}\text { MPACT } \\
\mathrm{k}_{\mathrm{eff}}(1 \sigma)\end{array}$ & $\begin{array}{c}\text { Difference } \\
{[\mathrm{pcm}]}\end{array}$ \\
\hline 0 & 0.0 & $1.05187(10)$ & $1.05093(10)$ & $-94(14)$ \\
1 & 25.0 & $1.03587(11)$ & $1.03527(10)$ & $-60(15)$ \\
2 & 50.0 & $1.03563(11)$ & $1.03543(10)$ & $-19(15)$ \\
3 & 100.0 & $1.03720(10)$ & $1.03688(10)$ & $-32(14)$ \\
4 & 200.0 & $1.04119(11)$ & $1.04137(10)$ & $18(15)$ \\
5 & 300.0 & $1.04465(10)$ & $1.04530(10)$ & $65(14)$ \\
6 & 400.0 & $1.04736(10)$ & $1.04794(10)$ & $59(14)$ \\
7 & 500.0 & $1.04937(10)$ & $1.05029(10)$ & $93(14)$ \\
8 & 600.0 & $1.05112(11)$ & $1.05218(10)$ & $105(15)$ \\
9 & 700.0 & $1.05196(11)$ & $1.05315(10)$ & $119(15)$ \\
10 & 800.0 & $1.05280(10)$ & $1.05418(10)$ & $138(14)$ \\
11 & 900.0 & $1.05334(11)$ & $1.05465(10)$ & $131(15)$ \\
12 & 1000.0 & $1.05336(11)$ & $1.05510(10)$ & $174(15)$ \\
13 & 1100.0 & $1.05349(10)$ & $1.05536(10)$ & $186(14)$ \\
14 & 1200.0 & $1.05325(11)$ & $1.05512(10)$ & $187(15)$ \\
15 & 1300.0 & $1.05310(11)$ & $1.05488(10)$ & $178(15)$ \\
\hline
\end{tabular}



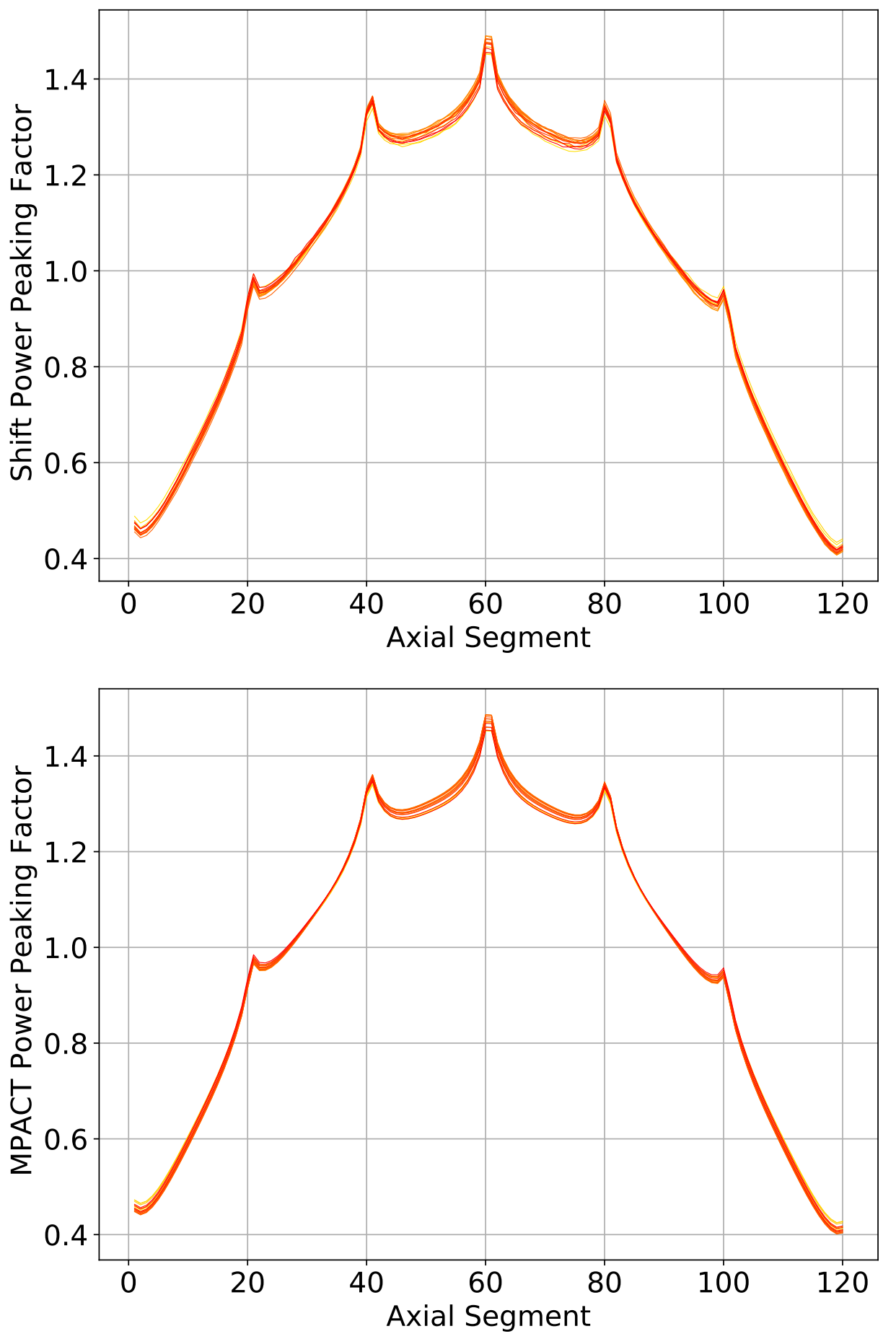

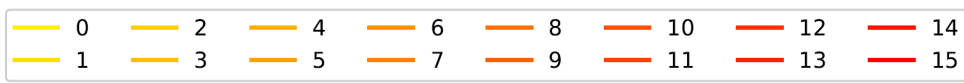

Figure 18. Power peaking factors for Problem 4.2.1 for Shift (top) and MPACT (bottom). Yellow represents time step 0 and red represents time step 15, with a gradient of colors in between. 


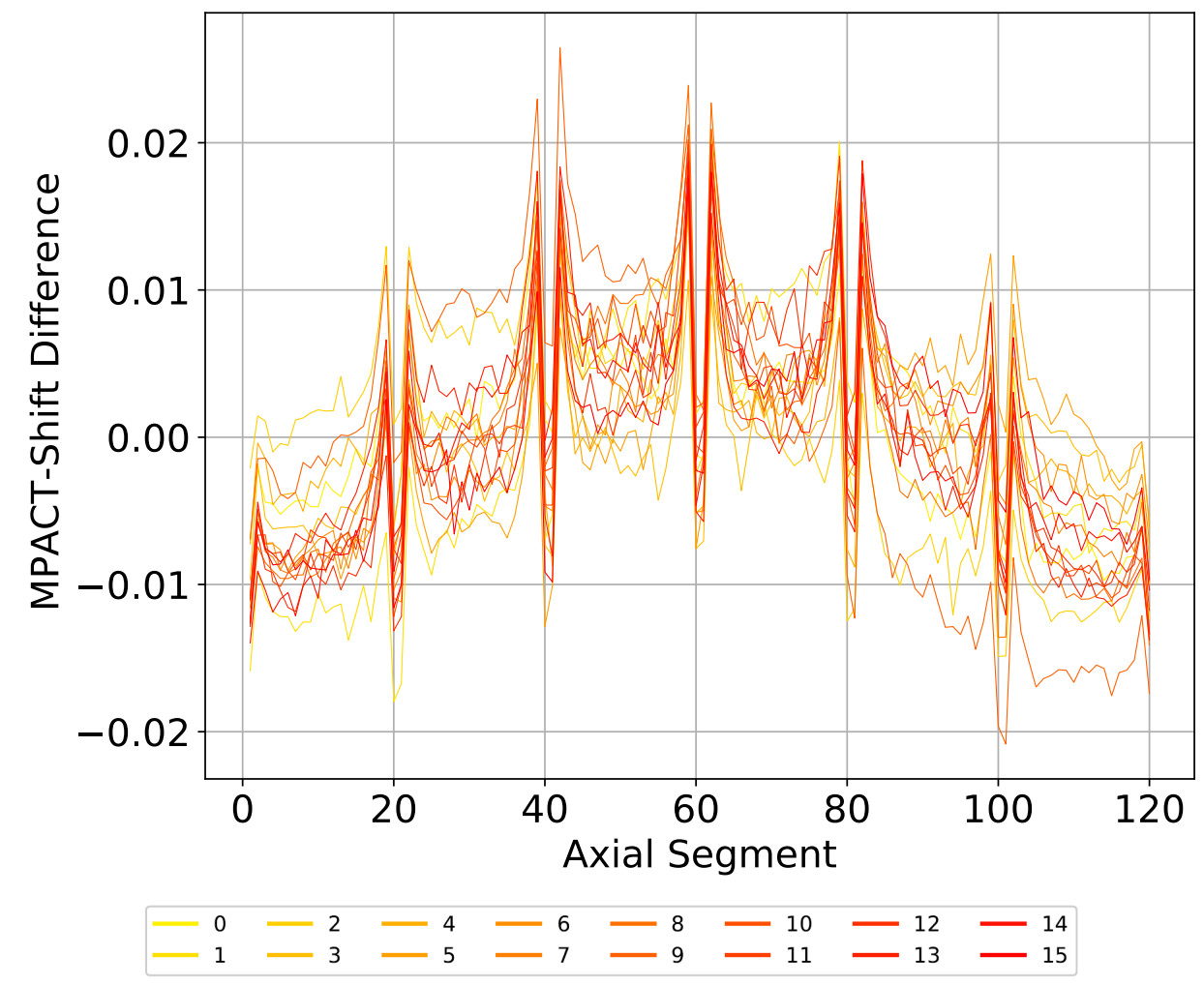

Figure 19. Power peaking factors absolute difference MPACT-Shift. Yellow represents time step 0 and red represents time step 15, with a gradient of colors in between. 
Table 5.10. Timing variables for the reference solution of Problem 4.2.1

\begin{tabular}{|c|c|c|c|c|c|c|}
\hline \multicolumn{7}{|c|}{$\overline{\text { Shift }}$} \\
\hline $\begin{array}{c}\text { Depletion } \\
\text { Solves }\end{array}$ & $\begin{array}{c}\text { Total } \\
\text { Cycles per } \\
\text { Transport } \\
\text { Solve }\end{array}$ & $\begin{array}{c}\text { Active } \\
\text { Cycles per } \\
\text { Transport } \\
\text { Solve }\end{array}$ & $\begin{array}{l}\text { Histories } \\
\text { per Cycle }\end{array}$ & $\begin{array}{c}\text { Compute } \\
\text { Nodes }\end{array}$ & $\begin{array}{c}\text { Cores } \\
\text { per Node }\end{array}$ & $\begin{array}{c}\text { Wall } \\
\text { Total Time } \\
\text { [hours] }\end{array}$ \\
\hline 15 & 1500 & 1200 & 50000 & 2 & 48 & 15.93 \\
\hline \multicolumn{7}{|c|}{ MPACT } \\
\hline $\begin{array}{c}\text { Depletion } \\
\text { Solves }\end{array}$ & $\begin{array}{c}k_{\mathrm{eff}} \\
\text { Convergence } \\
\text { Criteria }\end{array}$ & $\begin{array}{c}\text { Source } \\
\text { Convergence } \\
\text { Criteria }\end{array}$ & $\begin{array}{c}\text { Ray } \\
\text { Spacing } \\
{[\mathrm{cm}]}\end{array}$ & $\begin{array}{c}\text { Compute } \\
\text { Nodes }\end{array}$ & $\begin{array}{c}\text { Cores } \\
\text { per Node }\end{array}$ & $\begin{array}{c}\text { Wall } \\
\text { Total Time } \\
\text { [hours] }\end{array}$ \\
\hline 15 & $1.0 \times 10^{-04}$ & $1.0 \times 10^{-03}$ & 0.01 & 1 & 128 & 1.39 \\
\hline
\end{tabular}


Table 5.11. Problem 4.2.1 reference solution power peaking factors for axial segments 1-10 in element 1.

\begin{tabular}{|c|c|c|c|c|c|c|c|c|c|c|c|}
\hline \multirow[t]{2}{*}{ Step } & \multirow{2}{*}{$\begin{array}{l}\text { Burnup } \\
{\left[\frac{\mathrm{MWd}}{\mathrm{MtU}}\right]}\end{array}$} & \multicolumn{10}{|c|}{ Shift Power Peaking Factors } \\
\hline & & 1 & 2 & 3 & 4 & 5 & 6 & 7 & 8 & 9 & 10 \\
\hline 0 & 0.0 & 0.465 & 0.454 & 0.461 & 0.472 & 0.488 & 0.506 & 0.527 & 0.549 & 0.572 & 0.596 \\
\hline 1 & 25.0 & 488 & .474 & 0.481 & 0.493 & 0.508 & 0.527 & 0.549 & .571 & 0.594 & 0.616 \\
\hline 2 & 50.0 & 475 & 0.464 & 0.469 & 0.482 & 0.497 & 0.516 & 0.535 & .557 & .580 & 0.604 \\
\hline 3 & 100.0 & 479 & 0.464 & 0.472 & 0.484 & 0.499 & 0.518 & 0.539 & .561 & 0.585 & 0.608 \\
\hline 4 & 200.0 & 469 & 0.455 & 0.460 & 0.474 & 0.489 & 0.508 & 0.529 & 0.552 & 0.574 & 0.598 \\
\hline 5 & 300.0 & 6 & & 60 & 0.472 & 88 & 0.508 & 28 & & 72 & 597 \\
\hline 6 & 400.0 & 463 & 450 & 0.457 & 0.468 & 0.485 & 0.503 & 0.524 & .546 & 0.569 & 0.592 \\
\hline 7 & 500.0 & 460 & 0.448 & 0.455 & 0.467 & 0.483 & 0.502 & 0.523 & 0.544 & 0.569 & 0.593 \\
\hline 8 & 600.0 & & & & 0.466 & 0.483 & 0.501 & & & 567 & .591 \\
\hline 9 & 700.0 & 56 & 0.443 & 0.448 & 0.460 & 0.478 & 0.497 & 17 & 38 & 0.562 & 0.585 \\
\hline 10 & 800.0 & & 9 & & 0.469 & 0.485 & 0.503 & 23 & & 70 & .593 \\
\hline 11 & 900.0 & 163 & 0 & 58 & 0.469 & 0.485 & 0.505 & 27 & & .570 & 0.595 \\
\hline 12 & 1000.0 & 466 & 0.4 & 0.461 & 0.472 & 0.489 & 0.510 & 0. & 52 & 0.574 & 0.600 \\
\hline 13 & 00.0 & & & 51 & 0.473 & 89 & 0.508 & & & 75 & 599 \\
\hline 14 & 1200.0 & 474 & 0.462 & 0.468 & 0.481 & 0.496 & 0.515 & 0. & 59 & 0.582 & 0.608 \\
\hline 15 & 1300.0 & & 2 & 59 & 0.482 & 0.498 & 0.516 & 39 & 62 & 586 & 0.611 \\
\hline \multirow[t]{2}{*}{ Step } & Burnup & \multicolumn{10}{|c|}{ MPACT Power Peaking Factors } \\
\hline & {$\left[\frac{\mathrm{MWd}}{\mathrm{MtU}}\right]$} & 1 & 2 & 3 & 4 & 5 & 6 & 7 & 8 & 9 & 10 \\
\hline 0 & 0.0 & 458 & 52 & 456 & 0.467 & 0.483 & 0.501 & 0.522 & 544 & 0.568 & 0.592 \\
\hline 1 & 25.0 & 0 & & 0.470 & 0.481 & 0.496 & 0.515 & & & 0.581 & 0.605 \\
\hline 2 & 50.0 & 72 & 55 & 0.470 & 0.481 & 0.497 & 0.515 & 36 & 59 & 582 & .606 \\
\hline 3 & 100.0 & 0.469 & 0.462 & 0.467 & 0.478 & 0.493 & 0.512 & 0 . & 55 & 0.578 & 0.602 \\
\hline 4 & 200.0 & 0.461 & 0. & 0.459 & 0.470 & 0.485 & 0.504 & 0.525 & 0.547 & 0.571 & 0.595 \\
\hline 5 & 300.0 & 55 & 0. & 0.453 & 0.464 & 0.480 & 0.499 & 0.519 & 42 & 0.565 & 0.589 \\
\hline 6 & 400.0 & & & & 0.461 & 0.476 & 0.495 & & & 0.561 & 0.585 \\
\hline 7 & 500.0 & 0.449 & 0.442 & 0.447 & 0.458 & 0.474 & 0.493 & 0.514 & 36 & 560 & .584 \\
\hline 8 & 600.0 & 0.448 & 0.441 & 0.446 & 0.457 & 0.473 & 0.492 & 0.513 & 35 & 0.559 & 0.583 \\
\hline 9 & 700.0 & 0.449 & 0.442 & 0.447 & 0.458 & 0.474 & 0.493 & 0.514 & 36 & 0.560 & 0.584 \\
\hline 10 & 800.0 & 0.450 & 0.443 & 0.448 & 0.459 & 0.475 & 0.494 & 0.515 & 0.537 & 0.561 & 0.585 \\
\hline 11 & 900.0 & 0.452 & 0.445 & 0.450 & 0.461 & 0.477 & 0.496 & 0.518 & 0.540 & 0.564 & 0.588 \\
\hline 12 & 1000.0 & 0.453 & 0.447 & 0.452 & 0.463 & 0.479 & 0.498 & 0.519 & 0.542 & 0.566 & 0.590 \\
\hline 13 & 1100.0 & 0.455 & 0.448 & 0.453 & 0.465 & 0.481 & 0.500 & 0.521 & 0.544 & 0.568 & 0.593 \\
\hline 14 & 1200.0 & & & & 0.469 & & 0.505 & & & 0.573 & 0.597 \\
\hline 15 & 1300.0 & 0.463 & 0.456 & 0.461 & 0.473 & 0.489 & 0.509 & 0.530 & 0.553 & 0.577 & 0.602 \\
\hline
\end{tabular}


Table 5.12. Problem 4.2.1 reference solution power peaking factors for axial segments 11-20 in element 1.

\begin{tabular}{|c|c|c|c|c|c|c|c|c|c|c|c|}
\hline \multirow[t]{2}{*}{ Step } & \multirow[t]{2}{*}{ Burnup } & \multicolumn{10}{|c|}{ Shift Power Peaking Factors } \\
\hline & & 11 & 12 & 13 & 14 & 15 & 16 & 17 & 18 & 19 & 20 \\
\hline 0 & 0.0 & 0.619 & 0.645 & 0.671 & 0.696 & 0.722 & 0.752 & 0.785 & 0.818 & 0.856 & 0.923 \\
\hline 1 & 25.0 & 0.642 & 0.666 & 0.691 & 0.720 & 0.746 & 0.773 & 0.808 & 0.840 & 0.879 & 0.946 \\
\hline 2 & 50.0 & 0.629 & 0.654 & 0.677 & 0.706 & 0.733 & 0.762 & 0.792 & 0.825 & 0.861 & 0.929 \\
\hline 3 & 100.0 & 0.633 & 0.658 & 0.682 & 0.708 & 0.736 & 0.764 & 0.796 & 0.829 & 0.868 & 0.934 \\
\hline 4 & 200.0 & 0.621 & 0.646 & 0.672 & 0.696 & 0.725 & 0.756 & 0.784 & 0.820 & 0.858 & 0.927 \\
\hline 5 & 300.0 & 0.622 & 0.647 & 0.675 & 0.700 & 0.727 & 0.757 & 0.789 & 0.823 & 0.860 & 0.929 \\
\hline 6 & 400.0 & 0.617 & 0.641 & 0.670 & 0.694 & 0.724 & 0.752 & 0.785 & 0.818 & 0.855 & 0.925 \\
\hline 7 & 500.0 & 0.617 & 0.641 & 0.667 & 0.695 & 0.721 & 0.752 & 0.779 & 0.817 & 0.855 & 0.923 \\
\hline 8 & 600.0 & 0.617 & 0.641 & 0.666 & 0.693 & 0.719 & 0.749 & 0.781 & 0.815 & 0.852 & 0.922 \\
\hline 9 & 700.0 & 0 & 0 . & 0.660 & 0.687 & 0.715 & 0.744 & 75 & 08 & 46 & .917 \\
\hline 10 & 800.0 & 0.618 & 0.644 & 0.668 & 0.696 & 0.723 & 0.752 & 0.782 & 0.818 & 0.856 & 0.929 \\
\hline 11 & 900.0 & 0.620 & 0.646 & 0.671 & 0.698 & 0.727 & 0.755 & 0.786 & 0.820 & 0.857 & 0.928 \\
\hline 12 & 1000.0 & 0.625 & 0.649 & 0.677 & 0.703 & 0.731 & 0.760 & 0.793 & 0.825 & 0.866 & .936 \\
\hline 13 & 1100.0 & 0.623 & 0.649 & 0.676 & 0.701 & 0.729 & 0.757 & 0.789 & 0.823 & 0.861 & 0.933 \\
\hline 14 & 1200.0 & 0 & 0.6 & 0.682 & 0.708 & 0.736 & 0.765 & 0.797 & 31 & 67 & 0.939 \\
\hline 15 & 1300.0 & 6 & 0.660 & 0.686 & 0.713 & 0.740 & 0.772 & 0.802 & 0.837 & 0.873 & 0.945 \\
\hline \multirow[t]{2}{*}{ Step } & Burnup & \multicolumn{10}{|c|}{ MPACT Power Peaking Factors } \\
\hline & & 11 & 12 & 13 & 14 & 15 & 16 & 17 & 18 & 19 & 20 \\
\hline 0 & $\overline{0.0}$ & 0.616 & 0.641 & 0.667 & 0.693 & 0.721 & 0.751 & 0.783 & 0.819 & 0.861 & 0.916 \\
\hline 1 & 25.0 & 0.630 & 0.655 & 0.680 & 0.706 & 0.734 & 0.763 & 0.795 & 0.831 & 0.872 & 0.928 \\
\hline 2 & 50.0 & 0.631 & 0.656 & 0.681 & 0.708 & 0.735 & 0.765 & 0.797 & 0.833 & 0.874 & 0.930 \\
\hline 3 & 100.0 & 0.627 & 0.652 & 0.677 & 0.704 & 0.731 & 0.761 & 0.793 & 0.829 & 0.870 & 0.926 \\
\hline 4 & 200.0 & 0.619 & 0.644 & 0.670 & 0.696 & 0.724 & 0.754 & 0.786 & 0.822 & 0.864 & 0.921 \\
\hline 5 & 300.0 & 0.614 & 0.639 & 0.665 & 0.692 & 0.720 & 0.750 & 0.782 & 0.819 & 0.861 & 0.918 \\
\hline 6 & 400.0 & 0.610 & 0.635 & 0.661 & 0.688 & 0.716 & 0.746 & 0.778 & 0.815 & 0.857 & 0.914 \\
\hline 7 & 500.0 & 0.608 & 0.634 & 0.659 & 0.686 & 0.714 & 0.744 & 0.777 & 0.814 & 0.856 & 0.914 \\
\hline 8 & 600.0 & 0.608 & 0.633 & 0.659 & 0.686 & 0.715 & 0.745 & 0.778 & 0.815 & 0.857 & 0.915 \\
\hline 9 & 700.0 & 0.609 & 0.634 & 0.660 & 0.687 & 0.715 & 0.745 & 0.778 & 0.815 & 0.857 & 0.915 \\
\hline 10 & 800.0 & 0.610 & 0.635 & 0.661 & 0.688 & 0.716 & 0.746 & 0.779 & 0.816 & 0.859 & 0.917 \\
\hline 11 & 900.0 & 0.613 & 0.639 & 0.664 & 0.691 & 0.720 & 0.750 & 0.782 & 0.819 & 0.862 & 0.920 \\
\hline 12 & 1000.0 & 0.615 & 0.641 & 0.667 & 0.694 & 0.722 & 0.752 & 0.785 & 0.821 & 0.864 & 0.922 \\
\hline 13 & 1100.0 & 0.618 & 0.643 & 0.669 & 0.696 & 0.725 & 0.755 & 0.788 & 0.825 & 0.868 & 0.926 \\
\hline 14 & 1200.0 & 0.623 & 0.648 & 0.674 & 0.701 & 0.729 & 0.759 & 0.792 & 0.829 & 0.871 & 0.930 \\
\hline 15 & 1300.0 & 0.627 & 0.652 & 0.678 & 0.705 & 0.733 & 0.763 & 0.796 & 0.833 & 0.875 & 0.934 \\
\hline
\end{tabular}


Table 5.13. Problem 4.2.1 reference solution power peaking factors for axial segments 1-10 in element 2.

\begin{tabular}{|c|c|c|c|c|c|c|c|c|c|c|c|}
\hline \multirow[t]{2}{*}{ Step } & \multirow{2}{*}{$\begin{array}{l}\text { Burnup } \\
{\left[\frac{\mathrm{MWd}}{\mathrm{MtU}}\right]}\end{array}$} & \multicolumn{10}{|c|}{ Shift Power Peaking Factors } \\
\hline & & 1 & 2 & 3 & 4 & 5 & 6 & 7 & 8 & 9 & 10 \\
\hline 0 & 0.0 & 0.970 & 0.947 & .952 & 0.960 & 0.969 & 0.982 & 0.995 & 1.012 & 1.027 & $\overline{1.042}$ \\
\hline 1 & 25.0 & 994 & 0.965 & 0.968 & 0.975 & 0.986 & 0.995 & 1.007 & 1.023 & 1.036 & 1.052 \\
\hline 2 & 50.0 & .977 & 0.952 & 0.955 & 0.962 & 0.971 & 0.981 & 95 & 09 & 1.024 & 1.040 \\
\hline 3 & 100.0 & 982 & 0.957 & 0.960 & 0.967 & 0.976 & 0.987 & 1.003 & & 031 & 1.047 \\
\hline 4 & 200.0 & 0.974 & 0.948 & 0.953 & 0.963 & 0.970 & 0.983 & 0.998 & 1.011 & 1.028 & 1.045 \\
\hline 5 & 300.0 & 981 & 0.955 & 0.959 & 0.967 & 0.979 & 0.990 & 1.004 & 1.018 & 1.035 & 1.051 \\
\hline 6 & 400.0 & 975 & 0.950 & 955 & 0.960 & 0.973 & 0.985 & 1.000 & 1.014 & 1.031 & 1.047 \\
\hline 7 & 500.0 & 0.973 & 0.948 & 0.953 & 0.961 & 0.971 & 0.982 & 0 . & 13 & 1.029 & 1.045 \\
\hline 8 & 600.0 & & & & 0.960 & 70 & 0.982 & & & & 1.044 \\
\hline 9 & 700.0 & 968 & 0.941 & 0.943 & 0.951 & 0.962 & 0.974 & 0.988 & 1.003 & 1.019 & 1.036 \\
\hline 10 & 800.0 & 79 & 53 & 0.956 & 0.965 & 0.975 & 0.988 & 1.003 & 18 & 34 & 1.048 \\
\hline 11 & 900.0 & 0 & 54 & 56 & 0.963 & 0.976 & 0.987 & 1.001 & & 31 & 1.048 \\
\hline 12 & 1000.0 & 986 & 0.958 & 0.960 & 0.968 & 0.976 & 0.991 & 1.005 & 18 & 1.036 & 1.050 \\
\hline 13 & 0.0 & & & & 0.965 & 0.972 & 0.985 & 1.000 & & 32 & 1.046 \\
\hline 14 & 1200.0 & 87 & 59 & 0.963 & 0.970 & 0.979 & 0.990 & 1.006 & 19 & 1.033 & 1.050 \\
\hline 15 & 1300.0 & & & & 0.974 & 0. & 0.995 & 1. & & & 1.056 \\
\hline \multirow[t]{2}{*}{ Step } & Dumup & \multicolumn{10}{|c|}{ MPACT Power Peaking Factors } \\
\hline & $\frac{\mathrm{MWd}}{\mathrm{MtU}}$ & 1 & 2 & 3 & 4 & 5 & 6 & 7 & 8 & 9 & 10 \\
\hline 0 & 0.0 & 0.967 & 0.954 & 0.955 & 0.961 & 0.971 & 0.983 & 0.997 & 1.012 & 1.028 & 1.045 \\
\hline 1 & 25.0 & 0.977 & 0.963 & 0.962 & 0.968 & 76 & 0.988 & 1.001 & & 1.031 & 1.046 \\
\hline 2 & 50.0 & 0.979 & 65 & 0.964 & 0.969 & 0.978 & 0.989 & 1.002 & 16 & 1.031 & 1.047 \\
\hline 3 & 100.0 & 76 & 0 & 0.961 & 0.966 & 0.975 & 0.987 & 1.000 & & 1.030 & 1.046 \\
\hline 4 & 200.0 & .971 & 0.957 & 0.957 & 0.963 & 0.972 & 0.984 & 0.998 & 13 & 1.029 & 1.045 \\
\hline 5 & 300.0 & 0.969 & 55 & 0.956 & 0.961 & 0.971 & 0.983 & 0.997 & 1.013 & 1.029 & 1.045 \\
\hline 6 & 400.0 & 0.966 & 0.952 & 0.952 & 0.959 & 0.969 & 0.981 & 0.996 & 1.011 & 1.028 & 1.045 \\
\hline 7 & 500.0 & 0.966 & 0.952 & 0.952 & 0.958 & 0.968 & 0.981 & 0.996 & 1.012 & 1.028 & 1.045 \\
\hline 8 & 600.0 & 0.967 & 0.953 & 0.953 & 0.959 & 0.969 & 0.982 & 0.996 & 1.012 & 1.028 & 1.045 \\
\hline 9 & 700.0 & & & 0.953 & 0.959 & 0.969 & 0.982 & & & 1.029 & 1.046 \\
\hline 10 & 800.0 & 0.969 & 0.954 & 0.954 & 0.961 & 0.971 & 0.983 & 0.998 & 1.013 & 1.030 & 1.047 \\
\hline 11 & 900.0 & 0.972 & 0.957 & 0.957 & 0.963 & 0.973 & 0.985 & 0.999 & 1.015 & 1.031 & 1.048 \\
\hline 12 & 1000.0 & 0.974 & 0.959 & 0.959 & 0.964 & 0.974 & 0.986 & 1.001 & 1.016 & 1.032 & 1.048 \\
\hline 13 & 1100.0 & 0.977 & 0.962 & 0.961 & 0.967 & 0.976 & 0.988 & 1.002 & 1.017 & 1.033 & 1.049 \\
\hline 14 & 1200.0 & 0.980 & 0.964 & 0.964 & 0.969 & 0.978 & 0.990 & 1.004 & 1.019 & 1.034 & 1.050 \\
\hline 15 & 1300.0 & 0.984 & 0.968 & 0.967 & 0.972 & 0.981 & 0.993 & 1.006 & 1.021 & 1.036 & 1.052 \\
\hline
\end{tabular}


Table 5.14. Problem 4.2.1 reference solution power peaking factors for axial segments 11-20 in element 2.

\begin{tabular}{|c|c|c|c|c|c|c|c|c|c|c|c|}
\hline \multirow[t]{2}{*}{ Step } & \multirow[t]{2}{*}{ Burnup } & \multicolumn{10}{|c|}{ Shift Power Peaking Factors } \\
\hline & & 11 & 12 & 13 & 14 & 15 & 16 & 17 & 18 & 19 & 20 \\
\hline 0 & 0.0 & 1.062 & 1.076 & 1.094 & 1.116 & 1.136 & 1.159 & 1.182 & 1.212 & 1.246 & 1.322 \\
\hline 1 & 25.0 & 1.067 & 1.084 & 1.102 & 1.119 & 1.139 & 1.162 & 1.187 & 1.216 & 1.247 & 1.322 \\
\hline 2 & 50.0 & 1.054 & 1.071 & 1.088 & 1.108 & 1.127 & 1.152 & 1.175 & 1.203 & 1.240 & 1.312 \\
\hline 3 & 100.0 & 1.065 & 1.083 & 1.100 & 1.118 & 1.137 & 1.161 & 1.186 & 1.217 & 1.249 & 1.326 \\
\hline 4 & 200.0 & 1.061 & 1.080 & 1.098 & 1.117 & 1.137 & 1.161 & 1.186 & 1.217 & 1.252 & 1.327 \\
\hline 5 & 300.0 & 1.067 & 1.085 & 1.104 & 1.123 & 1.146 & 1.168 & 1.193 & 1.221 & 1.260 & 1.340 \\
\hline 6 & 400.0 & 1.065 & 1.083 & 1.101 & 1.121 & 1.144 & 1.165 & 1.191 & 1.222 & 1.257 & 1.336 \\
\hline 7 & 500.0 & 1.063 & 1.080 & 1.099 & 1.118 & 1.139 & 1.159 & 1.189 & 1.221 & 1.254 & 1.334 \\
\hline 8 & 600.0 & 1.061 & 1.081 & 1.098 & 1.117 & 1.137 & 1.160 & 1.188 & 1.217 & 1.256 & 1.334 \\
\hline 9 & .0 & 1.055 & 1.0 & $1 .($ & 1.109 & & 1.154 & 1.181 & 1.210 & 46 & 1.327 \\
\hline 10 & 800.0 & 1.064 & 1.083 & 1.104 & 1.121 & 1.142 & 1.166 & 1.189 & 1.221 & 1.257 & 1.336 \\
\hline 11 & 900.0 & 1.065 & 1.082 & 1.100 & 1.120 & 1.140 & 1.164 & 1.192 & 1.216 & 1.253 & 1.333 \\
\hline 12 & 1000.0 & 1.069 & 1.084 & 1.100 & 1.121 & 1.139 & 1.162 & 1.188 & 1.217 & 1.253 & 1.334 \\
\hline 13 & 1100.0 & 1.063 & 1.078 & 1.096 & 1.117 & 1.137 & 1.160 & 1.182 & 1.211 & 1.246 & 1.327 \\
\hline 14 & 1200.0 & 1.064 & 1.080 & 1.099 & 1.116 & 1.135 & 1.158 & 1.182 & 1.214 & 1.246 & 1.325 \\
\hline 15 & 1300.0 & 1 & 1.088 & 1.104 & 1.122 & 1.143 & 1.164 & 1.188 & 1.215 & 1.250 & 1.330 \\
\hline \multirow[t]{2}{*}{ Step } & Burnup & \multicolumn{10}{|c|}{ MPACT Power Peaking Factors } \\
\hline & & 11 & 12 & 13 & 14 & 15 & 16 & 17 & 18 & 19 & 20 \\
\hline 0 & $\overline{0.0}$ & 1.062 & 1.080 & 1.098 & 1.117 & 1.139 & 1.162 & 1.190 & 1.223 & 1.263 & 1.324 \\
\hline 1 & 25.0 & 1.063 & 1.079 & 1.097 & 1.115 & 1.136 & 1.158 & 1.185 & 1.217 & 1.256 & 1.315 \\
\hline 2 & 50.0 & 1.063 & 1.079 & 1.096 & 1.115 & 1.135 & 1.158 & 1.184 & 1.216 & 1.255 & 1.314 \\
\hline 3 & 100.0 & 1.062 & 1.079 & 1.097 & 1.115 & 1.136 & 1.159 & 1.186 & 1.218 & 1.258 & 1.319 \\
\hline 4 & 200.0 & 1.062 & 1.080 & 1.098 & 1.117 & 1.138 & 1.161 & 1.189 & 1.222 & 1.262 & 1.323 \\
\hline 5 & 300.0 & 1.062 & 1.080 & 1.098 & 1.118 & 1.139 & 1.163 & 1.190 & 1.224 & 1.265 & 1.327 \\
\hline 6 & 400.0 & 1.062 & 1.080 & 1.099 & 1.118 & 1.140 & 1.164 & 1.192 & 1.226 & 1.268 & 1.331 \\
\hline 7 & 500.0 & 1.063 & 1.081 & 1.099 & 1.119 & 1.141 & 1.165 & 1.193 & 1.227 & 1.269 & 1.332 \\
\hline 8 & 600.0 & 1.063 & 1.081 & 1.099 & 1.119 & 1.141 & 1.165 & 1.193 & 1.227 & 1.269 & 1.332 \\
\hline 9 & 700.0 & 1.063 & 1.081 & 1.100 & 1.120 & 1.141 & 1.165 & 1.193 & 1.227 & 1.269 & 1.333 \\
\hline 10 & 800.0 & 1.064 & 1.082 & 1.100 & 1.120 & 1.141 & 1.165 & 1.193 & 1.227 & 1.268 & 1.331 \\
\hline 11 & 900.0 & 1.065 & 1.082 & 1.100 & 1.120 & 1.141 & 1.165 & 1.192 & 1.226 & 1.267 & 1.331 \\
\hline 12 & 1000.0 & 1.065 & 1.083 & 1.101 & 1.120 & 1.141 & 1.165 & 1.192 & 1.225 & 1.266 & 1.329 \\
\hline 13 & 1100.0 & 1.066 & 1.083 & 1.101 & 1.120 & 1.140 & 1.164 & 1.191 & 1.223 & 1.264 & 1.327 \\
\hline 14 & 1200.0 & 1.067 & 1.084 & 1.101 & 1.120 & 1.140 & 1.163 & 1.190 & 1.222 & 1.262 & 1.324 \\
\hline 15 & 1300.0 & 1.068 & 1.084 & 1.101 & 1.120 & 1.140 & 1.162 & 1.188 & 1.220 & 1.260 & 1.321 \\
\hline
\end{tabular}


Table 5.15. Problem 4.2.1 reference solution power peaking factors for axial segments 1-10 in element 3.

\begin{tabular}{|c|c|c|c|c|c|c|c|c|c|c|c|}
\hline \multirow[t]{2}{*}{ Step } & Burnup & \multicolumn{10}{|c|}{ Shift Power Peaking Factors } \\
\hline & {$\left[\frac{\mathrm{MWd}}{\mathrm{MtU}}\right]$} & 1 & 2 & 3 & 4 & 5 & 6 & 7 & 8 & 9 & 10 \\
\hline 0 & 0.0 & 1.351 & 1.300 & 1.290 & 1.280 & 1.278 & 1.275 & 1.277 & 1.279 & 1.285 & 1.291 \\
\hline 1 & 25.0 & 1.346 & 1.291 & 1.277 & 1.270 & 1.263 & 1.264 & 1.265 & 1.266 & 1.268 & 1.274 \\
\hline 2 & 50.0 & 1.339 & 1.287 & 1.273 & 1.265 & 1.264 & 1.259 & 1.262 & 1.266 & 1.270 & 1.273 \\
\hline 3 & 100.0 & 1.353 & 1.296 & 1.285 & 1.277 & 1.271 & 1.271 & 1.273 & 1.274 & 1.280 & 1.286 \\
\hline 4 & 200.0 & 1.359 & 1.303 & 1.290 & 1.285 & 1.279 & 1.279 & 1.279 & 1.282 & 1.287 & 1.288 \\
\hline 5 & 300.0 & 1.365 & 1.309 & 1.294 & 1.286 & 1.285 & 1.284 & 1.284 & 1.289 & 1.292 & 1.298 \\
\hline 6 & 400.0 & 1.365 & 1.307 & 1.295 & 1.288 & 1.285 & 1.287 & 1.287 & 1.291 & 1.292 & 1.298 \\
\hline 7 & 500.0 & 1.364 & 1.306 & 1.295 & 1.287 & 1.284 & 1.282 & 1.284 & 1.288 & 1.294 & 1.298 \\
\hline 8 & 600.0 & 1.361 & 1.304 & 1.290 & 1.283 & 1.282 & 1.280 & 1.280 & 1.285 & 1.288 & 1.294 \\
\hline 9 & 700.0 & 1.355 & 1.295 & 1.285 & 1.277 & 1.275 & 1.274 & 1.275 & 1.281 & 1.286 & 1.291 \\
\hline 10 & 800.0 & 1.364 & 1.303 & 1.292 & 1.283 & 1.280 & 1.278 & 1.279 & 1.284 & 1.285 & 1.293 \\
\hline 11 & 900.0 & 1.360 & 1.300 & 1.289 & 1.281 & 1.278 & 1.274 & 1.279 & 1.282 & 1.287 & 1.289 \\
\hline 12 & 1000.0 & 1.363 & 1.301 & 1.289 & 1.283 & 1.280 & 1.276 & 1.280 & 1.283 & 1.289 & 1.292 \\
\hline 13 & 1100.0 & 1.351 & 1.294 & 1.279 & 1.274 & 1.270 & 1.268 & 1.273 & 1.275 & 1.279 & 1.284 \\
\hline 14 & 1200.0 & 1.350 & 1.293 & 1.280 & 1.270 & 1.268 & 1.266 & 1.269 & 1.273 & 1.275 & 1.280 \\
\hline 15 & 1300.0 & 1.356 & 1.294 & 1.282 & 1.273 & 1.268 & 1.266 & 1.269 & 1.271 & 1.274 & 1.279 \\
\hline \multirow[t]{2}{*}{ Step } & Burnup & \multicolumn{10}{|c|}{ MPACT Power Peaking Factors } \\
\hline & $\frac{\mathrm{MWd}}{\mathrm{MtU}}$ & 1 & 2 & 3 & 4 & 5 & 6 & 7 & 8 & 9 & 10 \\
\hline 0 & 0.0 & 1.351 & 1.314 & 1.296 & 1.287 & 1.282 & 1.281 & 1.283 & 1.286 & 1.290 & 1.295 \\
\hline 1 & 25.0 & 1.341 & 1.303 & 1.285 & 1.275 & 1.270 & 1.269 & 1.270 & 1.272 & 1.276 & 1.281 \\
\hline 2 & 50.0 & 1.340 & 1.303 & 1.284 & 1.274 & 1.269 & 1.267 & 1.268 & 1.271 & 1.275 & 1.280 \\
\hline 3 & 100.0 & 1.345 & 1.306 & 1.288 & 1.277 & 1.272 & 1.271 & 1.272 & 1.275 & 1.279 & 1.284 \\
\hline 4 & 200.0 & 1.350 & 1.312 & 1.294 & 1.284 & 1.279 & 1.278 & 1.279 & 1.282 & 1.287 & 1.292 \\
\hline 5 & 300.0 & 1.355 & 1.316 & 1.298 & 1.288 & 1.283 & 1.282 & 1.284 & 1.287 & 1.291 & 1.297 \\
\hline 6 & 400.0 & 1.360 & 1.320 & 1.301 & 1.291 & 1.287 & 1.286 & 1.288 & 1.291 & 1.296 & 1.301 \\
\hline 7 & 500.0 & 1.360 & 1.321 & 1.303 & 1.293 & 1.288 & 1.287 & 1.289 & 1.293 & 1.297 & 1.303 \\
\hline 8 & 600.0 & 1.360 & 1.321 & 1.303 & 1.293 & 1.288 & 1.287 & 1.289 & 1.293 & 1.297 & 1.303 \\
\hline 9 & 700.0 & 1.361 & 1.321 & 1.302 & 1.292 & 1.287 & 1.286 & 1.288 & 1.292 & 1.296 & 1.302 \\
\hline 10 & 800.0 & 1.359 & 1.320 & 1.301 & 1.291 & 1.286 & 1.285 & 1.287 & 1.290 & 1.295 & 1.300 \\
\hline 11 & 900.0 & 1.358 & 1.318 & 1.298 & 1.288 & 1.283 & 1.282 & 1.283 & 1.287 & 1.291 & 1.296 \\
\hline 12 & 1000.0 & 1.355 & 1.315 & 1.297 & 1.286 & 1.281 & 1.280 & 1.281 & 1.285 & 1.289 & 1.294 \\
\hline 13 & 1100.0 & 1.353 & 1.313 & 1.294 & 1.283 & 1.278 & 1.277 & 1.279 & 1.282 & 1.286 & 1.291 \\
\hline 14 & 1200.0 & 1.350 & 1.309 & 1.290 & 1.279 & 1.274 & 1.273 & 1.274 & 1.277 & 1.281 & 1.285 \\
\hline 15 & 1300.0 & 1.346 & 1.306 & 1.286 & 1.275 & 1.270 & 1.268 & 1.269 & 1.272 & 1.276 & 1.280 \\
\hline
\end{tabular}


Table 5.16. Problem 4.2.1 reference solution power peaking factors for axial segments 11-20 in element 3.

\begin{tabular}{|c|c|c|c|c|c|c|c|c|c|c|c|}
\hline \multirow[t]{2}{*}{ Step } & \multirow[t]{2}{*}{ Burnup } & \multicolumn{10}{|c|}{ Shift Power Peaking Factors } \\
\hline & & 11 & 12 & 13 & 14 & 15 & 16 & 17 & 18 & 19 & 20 \\
\hline 0 & 0.0 & 1.295 & 1.303 & 1.311 & 1.321 & 1.331 & 1.341 & 1.360 & 1.377 & 1.404 & 1.474 \\
\hline 1 & 25.0 & 1.278 & 1.283 & 1.293 & 1.297 & 1.307 & 1.321 & 1.336 & 1.357 & 1.381 & 1.452 \\
\hline 2 & 50.0 & 1.279 & 1.285 & 1.293 & 1.300 & 1.308 & 1.323 & 1.337 & 1.357 & 1.383 & 1.455 \\
\hline 3 & 100.0 & 1.291 & 1.298 & 1.304 & 1.311 & 1.325 & 1.336 & 1.350 & 1.370 & 1.395 & 1.467 \\
\hline 4 & 200.0 & 1.296 & 1.302 & 1.310 & 1.315 & 1.327 & 1.340 & 1.359 & 1.378 & 1.400 & 1.475 \\
\hline 5 & 300.0 & 1.302 & 1.309 & 1.319 & 1.325 & 1.333 & 1.347 & 1.362 & 1.383 & 1.406 & 1.485 \\
\hline 6 & 400.0 & 1.304 & 1.312 & 1.319 & 1.328 & 1.338 & 1.351 & 1.368 & 1.388 & 1.412 & 1.490 \\
\hline 7 & 500.0 & 1.305 & 1.314 & 1.317 & 1.327 & 1.336 & 1.350 & 1.366 & 1.387 & 1.414 & 1.490 \\
\hline 8 & 600.0 & 1.300 & 1.306 & 1.313 & 1.321 & 1.331 & 1.345 & 1.361 & 1.380 & 1.406 & 1.483 \\
\hline 9 & 700.0 & 1.296 & 1.303 & 1.2 & 1.320 & 1.331 & 1.343 & 1.359 & 1.379 & 1.407 & 1.484 \\
\hline 10 & 800.0 & 1.300 & 1.303 & 1.313 & 1.320 & 1.331 & 1.344 & 1.357 & 1.379 & 1.405 & 1.482 \\
\hline 11 & 900.0 & 1.298 & 1.301 & 1.312 & 1.318 & 1.330 & 1.338 & 1.356 & 1.374 & 1.401 & 1.477 \\
\hline 12 & 1000.0 & 1.297 & 1.301 & 1.310 & 1.318 & 1.323 & 1.340 & 1.354 & 1.374 & 1.398 & 1.474 \\
\hline 13 & 1100.0 & 1.290 & 1.297 & 1.302 & 1.311 & 1.318 & 1.335 & 1.352 & 1.373 & 1.395 & 1.473 \\
\hline 14 & 1200.0 & 1.286 & 1.290 & 1.298 & 1.306 & 1.3 & 1.329 & 1.343 & 1.365 & 1.386 & 1.465 \\
\hline 15 & 1300.0 & 3 & 1.290 & 1.295 & 1.304 & 1.314 & 1.324 & 1.339 & 1.358 & 1.381 & 1.455 \\
\hline \multirow[t]{2}{*}{ Step } & Burnup & \multicolumn{10}{|c|}{ MPACT Power Peaking Factors } \\
\hline & & 11 & 12 & 13 & 14 & 15 & 16 & 17 & 18 & 19 & 20 \\
\hline 0 & $\overline{0.0}$ & 1.301 & 1.308 & 1.315 & 1.323 & 1.334 & 1.347 & 1.365 & 1.388 & 1.420 & 1.473 \\
\hline 1 & 25.0 & 1.286 & 1.292 & 1.299 & 1.308 & 1.318 & 1.331 & 1.347 & 1.370 & 1.401 & 1.454 \\
\hline 2 & 50.0 & 1.285 & 1.291 & 1.298 & 1.306 & 1.316 & 1.329 & 1.346 & 1.369 & 1.400 & 1.452 \\
\hline 3 & 100.0 & 1.289 & 1.295 & 1.302 & 1.310 & 1.321 & 1.334 & 1.351 & 1.374 & 1.406 & 1.460 \\
\hline 4 & 200.0 & 1.297 & 1.304 & 1.311 & 1.319 & 1.330 & 1.343 & 1.360 & 1.384 & 1.416 & 1.470 \\
\hline 5 & 300.0 & 1.303 & 1.309 & 1.316 & 1.325 & 1.335 & 1.349 & 1.366 & 1.390 & 1.422 & 1.477 \\
\hline 6 & 400.0 & 1.307 & 1.314 & 1.321 & 1.330 & 1.340 & 1.354 & 1.371 & 1.395 & 1.428 & 1.485 \\
\hline 7 & 500.0 & 1.309 & 1.315 & 1.323 & 1.331 & 1.342 & 1.355 & 1.373 & 1.397 & 1.430 & 1.486 \\
\hline 8 & 600.0 & 1.309 & 1.315 & 1.323 & 1.331 & 1.342 & 1.355 & 1.373 & 1.397 & 1.430 & 1.486 \\
\hline 9 & 700.0 & 1.308 & 1.314 & 1.321 & 1.330 & 1.340 & 1.354 & 1.371 & 1.395 & 1.429 & 1.486 \\
\hline 10 & 800.0 & 1.306 & 1.312 & 1.320 & 1.328 & 1.338 & 1.352 & 1.369 & 1.393 & 1.426 & 1.482 \\
\hline 11 & 900.0 & 1.302 & 1.308 & 1.315 & 1.324 & 1.334 & 1.347 & 1.364 & 1.388 & 1.421 & 1.478 \\
\hline 12 & 1000.0 & 1.300 & 1.306 & 1.313 & 1.321 & 1.331 & 1.344 & 1.361 & 1.384 & 1.417 & 1.473 \\
\hline 13 & 1100.0 & 1.296 & 1.302 & 1.309 & 1.317 & 1.327 & 1.340 & 1.357 & 1.380 & 1.413 & 1.468 \\
\hline 14 & 1200.0 & 1.291 & 1.296 & 1.303 & 1.311 & 1.321 & 1.333 & 1.350 & 1.373 & 1.405 & 1.460 \\
\hline 15 & 1300.0 & 1.285 & 1.291 & 1.297 & 1.305 & 1.315 & 1.327 & 1.344 & 1.366 & 1.398 & 1.453 \\
\hline
\end{tabular}


Table 5.17. Problem 4.2.1 reference solution power peaking factors for axial segments 1-10 in element 4.

\begin{tabular}{|c|c|c|c|c|c|c|c|c|c|c|c|}
\hline \multirow[t]{2}{*}{ Step } & \multirow{2}{*}{$\begin{array}{l}\text { Burnup } \\
{\left[\frac{\mathrm{MWd}}{\mathrm{MtU}}\right]}\end{array}$} & \multicolumn{10}{|c|}{ Shift Power Peaking Factors } \\
\hline & & 1 & 2 & 3 & 4 & 5 & 6 & 7 & 8 & 9 & 10 \\
\hline 0 & 0.0 & 1.474 & 1.403 & 1.378 & 1.355 & 1.340 & 1.328 & 1.315 & 1.307 & 1.299 & 1.290 \\
\hline 1 & 25.0 & 1.450 & 1.379 & 1.355 & 1.334 & 1.317 & 1.303 & 1.295 & 1.285 & 1.279 & 1.270 \\
\hline 2 & 50.0 & 1.453 & 1.385 & 1.358 & 1.338 & 1.323 & 1.309 & 1.298 & 1.289 & 1.283 & 1.279 \\
\hline 3 & 100.0 & 1.466 & 1.395 & 1.368 & 1.347 & 1.330 & 1.320 & 1.306 & 1.294 & 1.288 & 1.280 \\
\hline 4 & 200.0 & 1.474 & 1.402 & 1.379 & 1.356 & 1.338 & 1.324 & 1.312 & 1.305 & 1.298 & 1.291 \\
\hline 5 & 300.0 & 1.481 & 1.410 & 1.381 & 1.359 & 1.344 & 1.331 & 1.319 & 1.307 & 1.301 & 1.291 \\
\hline 6 & 400.0 & 1.489 & 1.412 & 1.385 & 1.363 & 1.348 & 1.333 & 1.324 & 1.312 & 1.305 & 1.297 \\
\hline 7 & 500.0 & 1.486 & 1.411 & 1.384 & 1.363 & 1.348 & 1.334 & 1. & 1.313 & 1. & 1.300 \\
\hline 8 & 600.0 & & 5 & 1.379 & 1.358 & 1.341 & 1.329 & & & & 1.297 \\
\hline 9 & 700.0 & 1.483 & 1.406 & 1.377 & 1.361 & 1.345 & 1.331 & 1.322 & 1.312 & 1.306 & 1.297 \\
\hline 10 & 800.0 & 1.482 & 1.404 & 1.380 & 1.357 & 1.339 & 1.327 & 14 & 1.307 & 1.299 & 1.290 \\
\hline 11 & 900.0 & 1.475 & 1.401 & 1.376 & 1.352 & 1.336 & 1.324 & 15 & 1.307 & 1.297 & 1.290 \\
\hline 12 & 1000.0 & 1.471 & 1.396 & 1.369 & 1.348 & 1.330 & 1.322 & 8 & 1.298 & 1.290 & 1.285 \\
\hline 13 & 0.0 & 3 & 1 & 1.369 & 1.350 & 1.335 & 1.322 & & 03 & 95 & 1.290 \\
\hline 14 & 1200.0 & 1.461 & 1. & 1.359 & 1.339 & 1.323 & 1.311 & 02 & 1.294 & 1.288 & 1.280 \\
\hline 15 & 1300.0 & & & & 1.335 & 1.3 & 1.305 & & & & 1.275 \\
\hline \multirow[t]{2}{*}{ Step } & Dumup & \multicolumn{10}{|c|}{ MPACT Power Peaking Factors } \\
\hline & $\frac{\mathrm{MWd}}{\mathrm{MtU}}$ & 1 & 2 & 3 & 4 & 5 & 6 & 7 & 8 & 9 & 10 \\
\hline 0 & 0.0 & 1.472 & 1.418 & 1.386 & 1.362 & 1.344 & 1.330 & 1.319 & 1.310 & 1.302 & 1.295 \\
\hline 1 & 25.0 & 1.453 & 1.400 & 1.368 & 1.345 & 1.327 & 1.314 & & 1.294 & 1.287 & 1.280 \\
\hline 2 & 50.0 & 1.452 & 1.398 & 1.367 & 1.343 & 1.326 & 1.312 & 02 & 1.293 & 1.285 & 1.279 \\
\hline 3 & 100.0 & 1.459 & 1.404 & 1.372 & 1.348 & 1.330 & 1.317 & 1. & 1.297 & 1.289 & 1.283 \\
\hline 4 & 200.0 & 1.469 & 1.414 & 1.381 & 1.358 & 1.340 & 1.326 & 1.315 & 1.306 & 1.298 & 1.291 \\
\hline 5 & 300.0 & 1.477 & 1.421 & 1.388 & 1.363 & 1.345 & 1.331 & 1.320 & 1.311 & 1.303 & 1.296 \\
\hline 6 & 400.0 & 1.484 & 1.427 & 1.393 & 1.368 & 1.350 & 1.336 & 1.325 & 1.315 & 1.307 & 1.300 \\
\hline 7 & 500.0 & 1.485 & 1.428 & 1.395 & 1.370 & 1.352 & 1.338 & 1.326 & 1.317 & 1.309 & 1.302 \\
\hline 8 & 600.0 & 1.485 & 1.428 & 1.394 & 1.370 & 1.352 & 1.338 & 1.326 & 1.317 & 1.309 & 1.302 \\
\hline 9 & 700.0 & 1.485 & 1.427 & 1.393 & 1.368 & 1.350 & 1.336 & 1.325 & 1.316 & 1.308 & 1.301 \\
\hline 10 & 800.0 & 1.481 & 1.424 & 1.391 & 1.366 & 1.348 & 1.334 & 1.323 & 1.314 & 1.306 & 1.299 \\
\hline 11 & 900.0 & 1.477 & 1.419 & 1.386 & 1.361 & 1.343 & 1.330 & 1.319 & 1.310 & 1.302 & 1.295 \\
\hline 12 & 1000.0 & 1.472 & 1.415 & 1.382 & 1.358 & 1.340 & 1.327 & 1.316 & 1.307 & 1.300 & 1.293 \\
\hline 13 & 1100.0 & 1.467 & 1.411 & 1.378 & 1.354 & 1.337 & 1.323 & 1.313 & 1.304 & 1.296 & 1.290 \\
\hline 14 & 1200.0 & 1.459 & 1.404 & 1.371 & 1.347 & 1.330 & 1.317 & & 1.298 & 1.291 & 1.284 \\
\hline 15 & 1300.0 & 1.452 & 1.397 & 1.365 & 1.341 & 1.324 & 1.311 & 1.301 & 1.293 & 1.286 & 1.279 \\
\hline
\end{tabular}


Table 5.18. Problem 4.2.1 reference solution power peaking factors for axial segments 11-20 in element 4.

\begin{tabular}{|c|c|c|c|c|c|c|c|c|c|c|c|}
\hline \multirow[t]{2}{*}{ Step } & \multirow[t]{2}{*}{ Burnup } & \multicolumn{10}{|c|}{ Shift Power Peaking Factors } \\
\hline & & 11 & 12 & 13 & 14 & 15 & 16 & 17 & 18 & 19 & 20 \\
\hline 0 & 0.0 & 1.283 & 1.278 & 1.273 & 1.270 & 1.267 & 1.267 & 1.271 & 1.278 & 1.290 & 1.339 \\
\hline 1 & 25.0 & 1.265 & 1.259 & 1.254 & 1.249 & 1.249 & 1.250 & 1.252 & 1.261 & 1.271 & 1.323 \\
\hline 2 & 50.0 & 1.273 & 1.269 & 1.265 & 1.261 & 1.258 & 1.261 & 1.266 & 1.274 & 1.286 & 1.339 \\
\hline 3 & 100.0 & 1.275 & 1.269 & 1.265 & 1.262 & 1.259 & 1.257 & 1.263 & 1.267 & 1.278 & 1.333 \\
\hline 4 & 200.0 & 1.282 & 1.276 & 1.272 & 1.270 & 1.268 & 1.267 & 1.271 & 1.277 & 1.291 & 1.344 \\
\hline 5 & 300.0 & 1.286 & 1.280 & 1.273 & 1.268 & 1.266 & 1.267 & 1.268 & 1.275 & 1.288 & 1.341 \\
\hline 6 & 400.0 & 1.294 & 1.284 & 1.278 & 1.275 & 1.270 & 1.272 & 1.274 & 1.279 & 1.290 & 1.347 \\
\hline 7 & 500.0 & 1.292 & 1.283 & 1.278 & 1.277 & 1.273 & 1.272 & 1.277 & 1.282 & 1.293 & 1.348 \\
\hline 8 & 600.0 & 1.291 & 1.287 & 1.281 & 1.277 & 1.273 & 1.272 & 1.274 & 1.281 & 1.294 & 1.349 \\
\hline 9 & 0.0 & 1.294 & 1.288 & 1.283 & 1.278 & 1.277 & 1.277 & 1.279 & 1.287 & 1.299 & 1.355 \\
\hline 10 & 800.0 & 1.286 & 1.280 & 1.275 & 1.271 & 1.268 & 1.271 & 1.270 & 1.275 & 1.289 & 1.343 \\
\hline 11 & 900.0 & 1.286 & 1.280 & 1.275 & 1.272 & 1.266 & 1.267 & 1.267 & 1.277 & 1.286 & 1.343 \\
\hline 12 & 0.0 & 1.280 & 1.272 & 1.266 & 1.266 & 1.258 & 1.259 & 1.262 & 1.271 & 1.283 & 1.339 \\
\hline 13 & 1100.0 & 1.285 & 1.278 & 1.271 & 1.269 & 1.267 & 1.265 & 1.268 & 1.274 & 1.285 & 1.344 \\
\hline 14 & 1200.0 & 1.274 & 1.272 & 1.266 & 1.259 & 1.2 & 1.260 & 1.261 & 1.270 & 1.282 & 1.339 \\
\hline 15 & 1300.0 & 1.269 & 1.265 & 1.258 & 1.258 & 1.255 & 1.254 & 1.258 & 1.264 & 1.277 & 1.333 \\
\hline \multirow[t]{2}{*}{ Step } & Burnup & \multicolumn{10}{|c|}{ MPACT Power Peaking Factors } \\
\hline & & 11 & 12 & 13 & 14 & 15 & 16 & 17 & 18 & 19 & 20 \\
\hline 0 & 0.0 & 1.288 & 1.282 & 1.277 & 1.273 & 1.271 & 1.271 & 1.275 & 1.284 & 1.301 & 1.337 \\
\hline 1 & 25.0 & 1.274 & 1.269 & 1.264 & 1.261 & 1.259 & 1.260 & 1.264 & 1.274 & 1.291 & 1.327 \\
\hline 2 & 50.0 & 1.273 & 1.268 & 1.263 & 1.260 & 1.258 & 1.259 & 1.263 & 1.273 & 1.290 & 1.327 \\
\hline 3 & 100.0 & 1.277 & 1.271 & 1.267 & 1.263 & 1.261 & 1.262 & 1.266 & 1.276 & 1.294 & 1.331 \\
\hline 4 & 200.0 & 1.284 & 1.279 & 1.274 & 1.270 & 1.268 & 1.268 & 1.272 & 1.282 & 1.299 & 1.336 \\
\hline 5 & 0.0 & 1.289 & 1.283 & 1.278 & 1.274 & 1.272 & 1.272 & 1.276 & 1.285 & 1.302 & 1.340 \\
\hline 6 & 400.0 & 1.293 & 1.287 & 1.282 & 1.278 & 1.275 & 1.275 & 1.279 & 1.289 & 1.306 & 1.344 \\
\hline 7 & 500.0 & 1.295 & 1.289 & 1.284 & 1.279 & 1.277 & 1.277 & 1.280 & 1.290 & 1.307 & 1.345 \\
\hline 8 & 600.0 & 1.295 & 1.289 & 1.284 & 1.279 & 1.277 & 1.277 & 1.280 & 1.289 & 1.307 & 1.345 \\
\hline 9 & 700.0 & 1.294 & 1.288 & 1.283 & 1.278 & 1.276 & 1.276 & 1.280 & 1.289 & 1.307 & 1.346 \\
\hline 10 & 800.0 & 1.293 & 1.287 & 1.281 & 1.277 & 1.275 & 1.275 & 1.279 & 1.288 & 1.306 & 1.344 \\
\hline 11 & 900.0 & 1.289 & 1.283 & 1.278 & 1.274 & 1.272 & 1.272 & 1.276 & 1.286 & 1.304 & 1.343 \\
\hline 12 & 1000.0 & 1.287 & 1.281 & 1.276 & 1.272 & 1.270 & 1.270 & 1.274 & 1.284 & 1.302 & 1.341 \\
\hline 13 & 1100.0 & 1.283 & 1.278 & 1.273 & 1.269 & 1.267 & 1.268 & 1.272 & 1.282 & 1.300 & 1.339 \\
\hline 14 & 1200.0 & 1.278 & 1.273 & 1.268 & 1.265 & 1.263 & 1.264 & 1.268 & 1.278 & 1.296 & 1.336 \\
\hline 15 & 1300.0 & 1.274 & 1.268 & 1.264 & 1.261 & 1.259 & 1.260 & 1.265 & 1.275 & 1.293 & 1.333 \\
\hline
\end{tabular}


Table 5.19. Problem 4.2.1 reference solution power peaking factors for axial segments 1-10 in element 5.

\begin{tabular}{|c|c|c|c|c|c|c|c|c|c|c|c|}
\hline \multirow[t]{2}{*}{ Step } & Burnup & \multicolumn{10}{|c|}{ Shift Power Peaking Factors } \\
\hline & {$\left[\frac{\mathrm{MWd}}{\mathrm{MtU}}\right]$} & 1 & 2 & 3 & 4 & 5 & 6 & 7 & 8 & 9 & 10 \\
\hline 0 & 0.0 & 1.313 & 1.236 & 1.201 & 1.173 & 1.145 & 1.123 & 1.102 & 1.083 & 1.064 & 1.046 \\
\hline 1 & 25.0 & 1.298 & 1.225 & 1.192 & 1.163 & 1.136 & 1.114 & 1.093 & 1.075 & 1.058 & 1.042 \\
\hline 2 & 50.0 & 1.311 & 1.237 & 1.202 & 1.174 & 1.150 & 1.127 & 1.108 & 1.087 & 1.069 & 1.052 \\
\hline 3 & 100.0 & 1.306 & 1.227 & 1.193 & 1.163 & 1.138 & 1.116 & 1.096 & 1.076 & 1.057 & 1.041 \\
\hline 4 & 200.0 & 1.316 & 1.238 & 1.200 & 1.170 & 1.143 & 1.120 & 1.099 & 1.079 & 1.060 & 1.043 \\
\hline 5 & 300.0 & 1.311 & 1.233 & 1.199 & 1.167 & 1.140 & 1.118 & 1.094 & 1.075 & 1.055 & 1.037 \\
\hline 6 & 400.0 & 1.316 & 1.235 & 1.201 & 1.170 & 1.140 & 1.119 & 1.097 & 1.076 & 1.056 & 1.039 \\
\hline 7 & 500.0 & 1.319 & 1.241 & 1.202 & 1.172 & 1.145 & 1.120 & 1.097 & 1.076 & 1.058 & 1.042 \\
\hline 8 & 600.0 & 1.321 & 1.239 & 1.204 & 1.172 & 1.146 & 1.123 & 1.104 & 1.084 & 1.064 & 1.047 \\
\hline 9 & 700.0 & 1.329 & 1.246 & 1.212 & 1.181 & 1.154 & 1.132 & 1.108 & 1.089 & 1.071 & 1.054 \\
\hline 10 & 800.0 & 1.316 & 1.237 & 1.200 & 1.170 & 1.144 & 1.122 & 1.101 & 1.081 & 1.062 & 1.043 \\
\hline 11 & 900.0 & 1.316 & 1.236 & 1.200 & 1.171 & 1.144 & 1.119 & 1.103 & 1.079 & 1.063 & 1.045 \\
\hline 12 & 1000.0 & 1.309 & 1.231 & 1.196 & 1.167 & 1.140 & 1.118 & 1.100 & 1.080 & 1.060 & 1.043 \\
\hline 13 & 1100.0 & 1.317 & 1.237 & 1.201 & 1.171 & 1.144 & 1.122 & 1.102 & 1.083 & 1.064 & 1.046 \\
\hline 14 & 1200.0 & 1.313 & 1.232 & 1.199 & 1.171 & 1.143 & 1.123 & 1.104 & 1.081 & 1.066 & 1.050 \\
\hline 15 & 1300.0 & 1.308 & 1.227 & 1.194 & 1.164 & 1.138 & 1.118 & 1.099 & 1.080 & 1.061 & 1.043 \\
\hline \multirow[t]{2}{*}{ Step } & Burnup & \multicolumn{10}{|c|}{ MPACT Power Peaking Factors } \\
\hline & $\frac{\mathrm{MWd}}{\mathrm{MtU}}$ & 1 & 2 & 3 & 4 & 5 & 6 & 7 & 8 & 9 & 10 \\
\hline 0 & 0.0 & 1.308 & 1.248 & 1.207 & 1.174 & 1.146 & 1.121 & 1.100 & 1.080 & 1.061 & 1.043 \\
\hline 1 & 25.0 & 1.300 & 1.241 & 1.201 & 1.169 & 1.142 & 1.119 & 1.098 & 1.079 & 1.061 & 1.044 \\
\hline 2 & 50.0 & 1.299 & 1.240 & 1.201 & 1.169 & 1.142 & 1.119 & 1.098 & 1.079 & 1.061 & 1.044 \\
\hline 3 & 100.0 & 1.303 & 1.243 & 1.202 & 1.170 & 1.143 & 1.119 & 1.098 & 1.079 & 1.061 & 1.043 \\
\hline 4 & 200.0 & 1.307 & 1.246 & 1.205 & 1.172 & 1.145 & 1.120 & 1.099 & 1.079 & 1.061 & 1.043 \\
\hline 5 & 300.0 & 1.310 & 1.248 & 1.207 & 1.173 & 1.145 & 1.121 & 1.099 & 1.079 & 1.060 & 1.042 \\
\hline 6 & 400.0 & 1.315 & 1.251 & 1.209 & 1.175 & 1.146 & 1.122 & 1.100 & 1.079 & 1.060 & 1.042 \\
\hline 7 & 500.0 & 1.315 & 1.252 & 1.210 & 1.176 & 1.147 & 1.122 & 1.100 & 1.080 & 1.061 & 1.042 \\
\hline 8 & 600.0 & 1.315 & 1.252 & 1.209 & 1.175 & 1.147 & 1.122 & 1.100 & 1.080 & 1.061 & 1.042 \\
\hline 9 & 700.0 & 1.316 & 1.252 & 1.210 & 1.176 & 1.147 & 1.123 & 1.101 & 1.080 & 1.061 & 1.043 \\
\hline 10 & 800.0 & 1.315 & 1.252 & 1.210 & 1.176 & 1.147 & 1.123 & 1.101 & 1.081 & 1.062 & 1.043 \\
\hline 11 & 900.0 & 1.314 & 1.251 & 1.209 & 1.175 & 1.147 & 1.123 & 1.101 & 1.081 & 1.062 & 1.044 \\
\hline 12 & 1000.0 & 1.312 & 1.250 & 1.208 & 1.175 & 1.147 & 1.123 & 1.102 & 1.082 & 1.063 & 1.045 \\
\hline 13 & 1100.0 & 1.311 & 1.248 & 1.207 & 1.174 & 1.146 & 1.123 & 1.102 & 1.082 & 1.064 & 1.046 \\
\hline 14 & 1200.0 & 1.309 & 1.247 & 1.206 & 1.173 & 1.146 & 1.123 & 1.102 & 1.083 & 1.065 & 1.047 \\
\hline 15 & 1300.0 & 1.306 & 1.245 & 1.205 & 1.172 & 1.146 & 1.123 & 1.102 & 1.084 & 1.066 & 1.049 \\
\hline
\end{tabular}


Table 5.20. Problem 4.2.1 reference solution power peaking factors for axial segments 11-20 in element 5.

\begin{tabular}{|c|c|c|c|c|c|c|c|c|c|c|c|}
\hline \multirow[t]{2}{*}{ Step } & \multirow[t]{2}{*}{ Burnup } & \multicolumn{10}{|c|}{ Shift Power Peaking Factors } \\
\hline & & 11 & 12 & 13 & 14 & 15 & 16 & 17 & 18 & 19 & 20 \\
\hline 0 & 0.0 & 1.031 & 1.012 & 0.996 & 0.981 & 0.968 & 0.953 & 0.944 & 0.934 & 0.930 & 0.951 \\
\hline 1 & 25.0 & 1.025 & 1.010 & 0.996 & 0.978 & 0.966 & 0.954 & 0.946 & 0.938 & .933 & 0.958 \\
\hline 2 & 50.0 & 1.034 & 1.020 & 1.003 & 0.993 & 0.976 & 0.963 & 0.955 & 0.948 & 0.943 & 0.968 \\
\hline 3 & 100.0 & 1.023 & 1.007 & 0.991 & 0.976 & 0.961 & 0.950 & 0.940 & 0.932 & 0.928 & 0.952 \\
\hline 4 & 200.0 & 1.027 & 1.010 & 0.992 & 0.975 & 0.967 & 0.952 & 0.940 & 0.933 & .926 & .953 \\
\hline 5 & 300.0 & 1.018 & 1.003 & 0.986 & 0.972 & 0.953 & 0.943 & 0.931 & 0.921 & 0.917 & 0.939 \\
\hline 6 & 400.0 & 1.023 & 1.004 & 0.987 & 0.971 & 0.954 & 0.942 & 0.930 & 0.922 & 0.916 & 0.942 \\
\hline 7 & 500.0 & 1.024 & 1.008 & 0.988 & 0.973 & 0.957 & 0.943 & 0.935 & 0.925 & 0.920 & 0.943 \\
\hline 8 & 600.0 & 1.028 & 1.011 & 0.995 & 0.979 & 0.963 & 0.950 & 0.942 & 0.931 & 0.926 & 0.952 \\
\hline 9 & .0 & 1.0 & 1.018 & 3 & 0.987 & 72 & 0.957 & & 40 & 36 & .958 \\
\hline 10 & 800.0 & 1.026 & 1.011 & 0.993 & 0.978 & 0.964 & 0.950 & 0.938 & 0.927 & 0.925 & 0.948 \\
\hline 11 & 900.0 & 1.028 & 1.010 & 0.993 & 0.979 & 0.964 & 0.952 & 0.938 & 0.930 & 0.928 & 0.952 \\
\hline 12 & 1000.0 & 1.026 & 1.009 & 0.992 & 0.978 & 0.962 & 0.949 & 0.940 & 0.932 & 0.928 & .953 \\
\hline 13 & 1100.0 & 1.030 & 1.015 & 1.001 & 0.983 & 0.970 & 0.957 & 0.946 & 0.937 & 0.932 & 0.959 \\
\hline 14 & 1200.0 & 1.033 & 1.016 & 1.001 & 0.985 & 0.972 & 0.960 & 0.948 & 38 & 36 & 0.961 \\
\hline 15 & 1300.0 & 1.029 & 1.0 & 98 & 0.981 & 0.967 & 0.956 & 0.946 & 0.939 & 0.933 & 0.961 \\
\hline \multirow[t]{2}{*}{ Step } & Burnup & \multicolumn{10}{|c|}{ MPACT Power Peaking Factors } \\
\hline & & 11 & 12 & 13 & 14 & 15 & 16 & 17 & 18 & 19 & 20 \\
\hline 0 & $\overline{0.0}$ & 1.025 & 1.008 & 0.991 & 0.975 & 0.961 & 0.948 & 0.937 & 0.930 & 0.929 & 0.940 \\
\hline 1 & 25.0 & 1.027 & 1.011 & 0.995 & 0.980 & 0.966 & 0.954 & 0.945 & 0.939 & 0.938 & 0.951 \\
\hline 2 & 50.0 & 1.028 & 1.012 & 0.996 & 0.981 & 0.968 & 0.956 & 0.946 & 0.940 & 0.940 & 0.953 \\
\hline 3 & 100.0 & 1.027 & 1.010 & 0.994 & 0.979 & 0.965 & 0.953 & 0.943 & 0.937 & 0.936 & 0.949 \\
\hline 4 & 200.0 & 1.025 & 1.008 & 0.992 & 0.976 & 0.962 & 0.949 & 0.939 & 0.932 & 0.931 & 0.944 \\
\hline 5 & 300.0 & 1.025 & 1.008 & 0.991 & 0.975 & 0.960 & 0.948 & 0.937 & 0.930 & 0.929 & 0.942 \\
\hline 6 & 400.0 & 1.024 & 1.006 & 0.989 & 0.973 & 0.958 & 0.945 & 0.934 & 0.927 & 0.925 & 0.938 \\
\hline 7 & 500.0 & 1.024 & 1.006 & 0.989 & 0.973 & 0.958 & 0.944 & 0.933 & 0.926 & 0.925 & 0.937 \\
\hline 8 & 600.0 & 1.024 & 1.007 & 0.990 & 0.974 & 0.959 & 0.945 & 0.934 & 0.927 & 0.926 & 0.938 \\
\hline 9 & 700.0 & 1.025 & 1.007 & 0.990 & 0.974 & 0.959 & 0.945 & 0.934 & 0.927 & 0.926 & 0.939 \\
\hline 10 & 800.0 & 1.026 & 1.008 & 0.991 & 0.975 & 0.960 & 0.946 & 0.936 & 0.928 & 0.927 & 0.940 \\
\hline 11 & 900.0 & 1.027 & 1.009 & 0.993 & 0.977 & 0.962 & 0.949 & 0.938 & 0.931 & 0.930 & 0.943 \\
\hline 12 & 1000.0 & 1.028 & 1.011 & 0.994 & 0.978 & 0.963 & 0.950 & 0.940 & 0.933 & 0.932 & 0.945 \\
\hline 13 & 1100.0 & 1.029 & 1.012 & 0.996 & 0.980 & 0.965 & 0.953 & 0.942 & 0.936 & 0.935 & 0.949 \\
\hline 14 & 1200.0 & 1.030 & 1.014 & 0.998 & 0.982 & 0.968 & 0.955 & 0.945 & 0.939 & 0.938 & 0.952 \\
\hline 15 & 1300.0 & 1.032 & 1.016 & 1.000 & 0.984 & 0.970 & 0.958 & 0.948 & 0.942 & 0.942 & 0.956 \\
\hline
\end{tabular}


Table 5.21. Problem 4.2.1 reference solution power peaking factors for axial segments 1-10 in element 6.

\begin{tabular}{|c|c|c|c|c|c|c|c|c|c|c|c|}
\hline \multirow[t]{2}{*}{ Step } & \multirow{2}{*}{$\begin{array}{l}\text { Burnup } \\
{\left[\frac{\mathrm{MWd}}{\mathrm{MtU}}\right]}\end{array}$} & \multicolumn{10}{|c|}{ Shift Power Peaking Factors } \\
\hline & & 1 & 2 & 3 & 4 & 5 & 6 & 7 & 8 & 9 & 10 \\
\hline 0 & 0.0 & 0.900 & 0.835 & 0.796 & 0.764 & 0.730 & 0.702 & 0.674 & 0.647 & 0.620 & 0.595 \\
\hline 1 & 25.0 & 909 & .841 & .804 & 0.770 & 0.739 & 0.712 & 0.684 & .657 & .631 & 0.606 \\
\hline 2 & 50.0 & 917 & 0.852 & 0.814 & 0.779 & 0.748 & 0.719 & 0.692 & .665 & 0.639 & 0.614 \\
\hline 3 & 100.0 & 900 & 0.835 & 0.798 & 0.764 & 0.732 & 0.703 & 0.675 & .649 & 0.623 & .599 \\
\hline 4 & 200.0 & 898 & 0.831 & 0.793 & 0.762 & 0.729 & 0.698 & 0.670 & 0.644 & 0.618 & 0.593 \\
\hline 5 & 300.0 & & 1 & 84 & 0.751 & 18 & 0.689 & 63 & & 507 & 583 \\
\hline 6 & 400.0 & 890 & .820 & 0.783 & 0.749 & 0.718 & 0.688 & 0.663 & 0.634 & 0.608 & 0.584 \\
\hline 7 & 500.0 & 891 & 0.826 & 0.786 & 0.752 & 0.719 & 0.690 & 0.662 & 0.637 & 0.610 & 0.585 \\
\hline 8 & 600.0 & & 9 & 792 & 0.758 & 0.726 & 0.696 & 0.667 & & 15 & .589 \\
\hline 9 & 700.0 & 906 & 0.837 & 0.799 & 0.764 & 0.733 & 0.702 & 0.674 & 0.646 & 0.620 & 0.595 \\
\hline 10 & 800.0 & & 9 & 791 & 0.755 & 0.724 & 0.695 & 0.667 & & 15 & .589 \\
\hline 11 & 900.0 & 01 & 3 & 794 & 0.759 & 0.727 & 0.698 & 0.671 & & 0.618 & 0.592 \\
\hline 12 & 1000.0 & 02 & 0.833 & 0.795 & 0.760 & 0.729 & 0.699 & 0.672 & 45 & 0.619 & 0.594 \\
\hline 13 & 00.0 & & & 97 & 0.764 & 34 & 0.703 & & & 22 & 598 \\
\hline 14 & 1200.0 & 910 & 0.840 & 0.803 & 0.768 & 0.735 & 0.706 & 0.679 & 50 & 0.624 & 0.598 \\
\hline 15 & 1300.0 & & 40 & 03 & 0.766 & 0.736 & 0.709 & 0.680 & 53 & 27 & 0.600 \\
\hline \multirow[t]{2}{*}{ Step } & Burnup & \multicolumn{10}{|c|}{ MPACT Power Peaking Factors } \\
\hline & {$\left[\frac{\mathrm{MWd}}{\mathrm{MtU}}\right]$} & 1 & 2 & 3 & 4 & 5 & 6 & 7 & 8 & 9 & 10 \\
\hline 0 & 0.0 & 888 & 833 & .792 & 0.756 & 723 & 0.693 & 0.665 & 638 & 0.612 & 0.587 \\
\hline 1 & 25.0 & 0.900 & 0 . & 0.804 & 0.768 & 0.736 & 0.706 & & 52 & 0.626 & 0.601 \\
\hline 2 & 50.0 & 0.902 & 47 & 0.806 & 0.770 & 38 & 0.708 & 80 & 53 & 0.627 & 0.602 \\
\hline 3 & 100.0 & 0.898 & 0.843 & 0.801 & 0.765 & 0.733 & 0.704 & 0.676 & 0. & 0.623 & 0.598 \\
\hline 4 & 200.0 & 92 & 0.836 & 0.795 & 0.759 & 0.726 & 0.696 & 0.668 & 0.641 & 0.616 & 0.590 \\
\hline 5 & 300.0 & 39 & 0.833 & 0.791 & 0.755 & 0.722 & 0.692 & 0.663 & 0.636 & 0.610 & 0.585 \\
\hline 6 & 400.0 & & & 0.786 & 0.750 & 0.717 & 0.687 & & & 0.606 & 0.581 \\
\hline 7 & 500.0 & 0.884 & 0.827 & 0.785 & 0.748 & 0.716 & 0.686 & 0.657 & 0.630 & 0.604 & .579 \\
\hline 8 & 600.0 & 0.885 & 0.828 & 0.786 & 0.749 & 0.716 & 0.686 & 0.657 & 0.630 & 0.604 & 0.578 \\
\hline 9 & 700.0 & 0.885 & 0.828 & 0.786 & 0.749 & 0.716 & 0.686 & 0.658 & 0.631 & 0.604 & 0.579 \\
\hline 10 & 800.0 & & 0.830 & 0.787 & 0.751 & 0.718 & 0.687 & 0.659 & 0.632 & 0.606 & 0.580 \\
\hline 11 & 900.0 & 0.890 & 0.833 & 0.790 & 0.754 & 0.721 & 0.690 & 0.662 & 0.635 & 0.609 & 0.583 \\
\hline 12 & 1000.0 & 0.893 & 0.835 & 0.793 & 0.756 & 0.723 & 0.693 & 0.664 & 0.637 & 0.611 & 0.585 \\
\hline 13 & 1100.0 & & 0.839 & 0.796 & 0.759 & 0.726 & 0.696 & 0.667 & 0.640 & 0.613 & 0.588 \\
\hline 14 & 1200.0 & & & & 0.763 & & 0.700 & & & 0.618 & 0.592 \\
\hline 15 & 1300.0 & 0.905 & 0.847 & 0.805 & 0.768 & 0.735 & 0.705 & 0.676 & 0.649 & 0.623 & 0.597 \\
\hline
\end{tabular}


Table 5.22. Problem 4.2.1 reference solution power peaking factors for axial segments 11-20 in element 6.

\begin{tabular}{|c|c|c|c|c|c|c|c|c|c|c|c|}
\hline \multirow[t]{2}{*}{ Step } & \multirow[t]{2}{*}{ Burnup } & \multicolumn{10}{|c|}{ Shift Power Peaking Factors } \\
\hline & & 11 & 12 & 13 & 14 & 15 & 16 & 17 & 18 & 19 & 20 \\
\hline 0 & 0.0 & 0.570 & 0.545 & 0.522 & 0.501 & 0.479 & 0.459 & 0.441 & 0.427 & 0.418 & 0.426 \\
\hline 1 & 25.0 & 0.581 & 0.559 & 0.535 & 0.511 & 0.489 & 0.469 & 0.451 & 0.439 & 0.429 & 0.437 \\
\hline 2 & 50.0 & 0.589 & 0.564 & 0.540 & 0.516 & 0.494 & 0.476 & 0.458 & 0.443 & 0.434 & 0.441 \\
\hline 3 & 100.0 & 0.574 & 0.550 & 0.526 & 0.505 & 0.482 & 0.463 & 0.445 & 0.432 & 0.422 & 0.430 \\
\hline 4 & 200.0 & 0.569 & 0.544 & 0.521 & 0.498 & 0.476 & 0.457 & 0.440 & 0.426 & .418 & .425 \\
\hline 5 & 300.0 & 0.559 & 0.535 & 0.512 & 0.490 & 0.469 & 0.450 & 0.433 & 0.417 & 0.409 & 0.417 \\
\hline 6 & 400.0 & 0.557 & 0.535 & 0.512 & 0.488 & 0.468 & 0.449 & 0.430 & 0.417 & 0.407 & 0.414 \\
\hline 7 & 500.0 & 0.560 & 0.536 & 0.513 & 0.490 & 0.468 & 0.449 & 0.430 & 0.418 & 0.407 & 0.415 \\
\hline 8 & 600.0 & 0.564 & 0.539 & 0.515 & 0.492 & 0.471 & 0.449 & 0.433 & 0.418 & 0.410 & 0.418 \\
\hline 9 & 700.0 & 0 & & & 0.498 & 0.478 & 0.456 & & & 14 & .422 \\
\hline 10 & 800.0 & 0.564 & 0.540 & 0.515 & 0.493 & 0.472 & 0.452 & 0.433 & 0.419 & 0.411 & 0.418 \\
\hline 11 & 900.0 & 0.566 & 0.543 & 0.519 & 0.496 & 0.473 & 0.453 & 0.436 & 0.421 & 0.411 & 0.419 \\
\hline 12 & 1000.0 & 0.569 & 0.544 & 0.520 & 0.498 & 0.475 & 0.454 & 0.437 & 23 & 0.412 & .422 \\
\hline 13 & 1100.0 & 0.573 & 0.547 & 0.524 & 0.500 & 0.479 & 0.458 & 0.440 & 0.425 & 0.416 & 0.424 \\
\hline 14 & 1200.0 & 0 & 0.5 & 0.524 & 0.501 & 0.479 & 0.458 & 0.442 & 27 & 0.418 & 0.425 \\
\hline 15 & 1300.0 & 5 & 2 & 26 & 0.503 & 0.482 & 0.461 & 0.445 & 0.429 & 0.419 & 0.428 \\
\hline \multirow[t]{2}{*}{ Step } & Burnup & \multicolumn{10}{|c|}{ MPACT Power Peaking Factors } \\
\hline & & 11 & 12 & 13 & 14 & 15 & 16 & 17 & 18 & 19 & 20 \\
\hline 0 & $\overline{0.0}$ & 0.562 & 0.538 & 0.514 & 0.491 & 0.469 & 0.450 & 0.433 & 0.419 & 0.411 & 0.414 \\
\hline 1 & 25.0 & 0.576 & 0.552 & 0.528 & 0.505 & 0.483 & 0.463 & 0.446 & 0.432 & 0.425 & 0.427 \\
\hline 2 & 50.0 & 0.577 & 0.552 & 0.528 & 0.505 & 0.483 & 0.463 & 0.446 & 0.433 & 0.425 & 0.428 \\
\hline 3 & 100.0 & 0.573 & 0.549 & 0.525 & 0.502 & 0.480 & 0.460 & 0.443 & 0.430 & 0.422 & 0.425 \\
\hline 4 & 200.0 & 0.565 & 0.541 & 0.517 & 0.494 & 0.472 & 0.452 & 0.435 & 0.422 & 0.414 & 0.417 \\
\hline 5 & 300.0 & 0.560 & 0.535 & 0.511 & 0.488 & 0.467 & 0.447 & 0.430 & 0.416 & 0.409 & 0.411 \\
\hline 6 & 400.0 & 0.556 & 0.531 & 0.507 & 0.484 & 0.463 & 0.443 & 0.426 & 0.413 & 0.405 & 0.407 \\
\hline 7 & 500.0 & 0.554 & 0.529 & 0.505 & 0.482 & 0.461 & 0.441 & 0.424 & 0.410 & 0.403 & 0.405 \\
\hline 8 & 600.0 & 0.553 & 0.528 & 0.504 & 0.481 & 0.459 & 0.440 & 0.422 & 0.409 & 0.401 & 0.404 \\
\hline 9 & 700.0 & 0.554 & 0.529 & 0.505 & 0.482 & 0.460 & 0.440 & 0.423 & 0.410 & 0.402 & 0.404 \\
\hline 10 & 800.0 & 0.555 & 0.530 & 0.506 & 0.483 & 0.461 & 0.441 & 0.424 & 0.411 & 0.403 & 0.405 \\
\hline 11 & 900.0 & 0.558 & 0.533 & 0.509 & 0.486 & 0.464 & 0.444 & 0.426 & 0.413 & 0.405 & 0.407 \\
\hline 12 & 1000.0 & 0.560 & 0.535 & 0.511 & 0.487 & 0.465 & 0.445 & 0.428 & 0.414 & 0.406 & 0.408 \\
\hline 13 & 1100.0 & 0.562 & 0.537 & 0.513 & 0.489 & 0.467 & 0.447 & 0.429 & 0.416 & 0.408 & 0.410 \\
\hline 14 & 1200.0 & 0.567 & 0.542 & 0.518 & 0.494 & 0.472 & 0.451 & 0.434 & 0.420 & 0.412 & 0.414 \\
\hline 15 & 1300.0 & 0.571 & 0.546 & 0.522 & 0.498 & 0.476 & 0.455 & 0.437 & 0.424 & 0.415 & 0.418 \\
\hline
\end{tabular}




\section{PROBLEM 5: CHARGE-PAN 3D}

Problem 5 is a single 3D charge-pan with upper and lower axial reflector regions. The top and bottom problem edges have vacuum boundaries, and the north, south, east, and west boundaries are fully reflected. Problem 5 is more complex than previous problems because it adds multiple fuel channels and control rods in $3 \mathrm{D}$.

\subsection{BEGINNING-OF-CYCLE}

\subsubsection{Problem 5.1.1}

\subsubsection{Description}

Figure 20 shows the axial fuel channel geometry for Problem 5.1.1. Six fuel rods are stacked on one another within each fuel channel. While problem 4 describes the single 3D fuel channel geometry, this problem considers the entire charge-pan in 3D. The transverse geometry is illustrated by Figure 3. Zone C coolant channel radius is used for this problem. Geometric dimensions are listed in Table 1.2. Recall that owing to the $1 / 8$ symmetry, fuel channels A, B, C, and D will have the same power. Also because of symmetry, fuel channels E, F, G, H, J, K, L, and M will have the same power, and fuel channels P, Q, R, and $\mathrm{S}$ will have the same power. Material densities are listed in Table 1.4. Temperatures are listed in Table 2.4.

\subsubsection{Reference Solution}

The neutron multiplication factor results are shown in Table 6.1. The timing variables for Shift and MPACT are shown in Table 6.2. Power peaking factors for the three types of charge pan pins are displayed for BOC and EOC in Figure 21 and fully tabulated in Tables 6.3-6.8. The neutron multiplication factor agrees to under $100 \mathrm{pcm}$ and peaking factors agree to about $1.5 \%$.

Table 6.1. Neutron multiplication factor for the reference solution of Problem 5.1.1.

\begin{tabular}{rrrrr}
\hline Step & $\begin{array}{c}\text { Burnup } \\
{\left[\frac{\mathrm{MWd}}{\mathrm{MtU}}\right]}\end{array}$ & $\begin{array}{c}\text { Shift } \\
\mathrm{k}_{\mathrm{eff}}(1 \sigma)\end{array}$ & \multicolumn{2}{c}{ MPACT } \\
$\mathrm{k}_{\mathrm{eff}}$ & $(1 \sigma)$ & $\begin{array}{c}\text { Difference } \\
{[\mathrm{pcm}]}\end{array}$ \\
\hline 0 & 0.0 & $1.05470(7)$ & $1.05488(10)$ & $18(12)$ \\
\hline
\end{tabular}




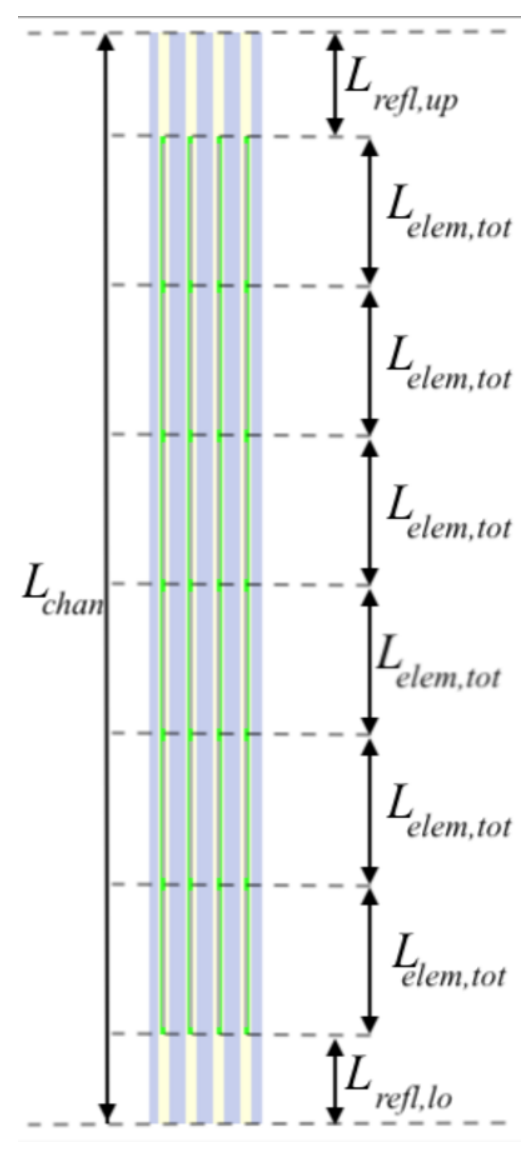

Figure 20. 3D charge-pan with six fuel element stacks. 

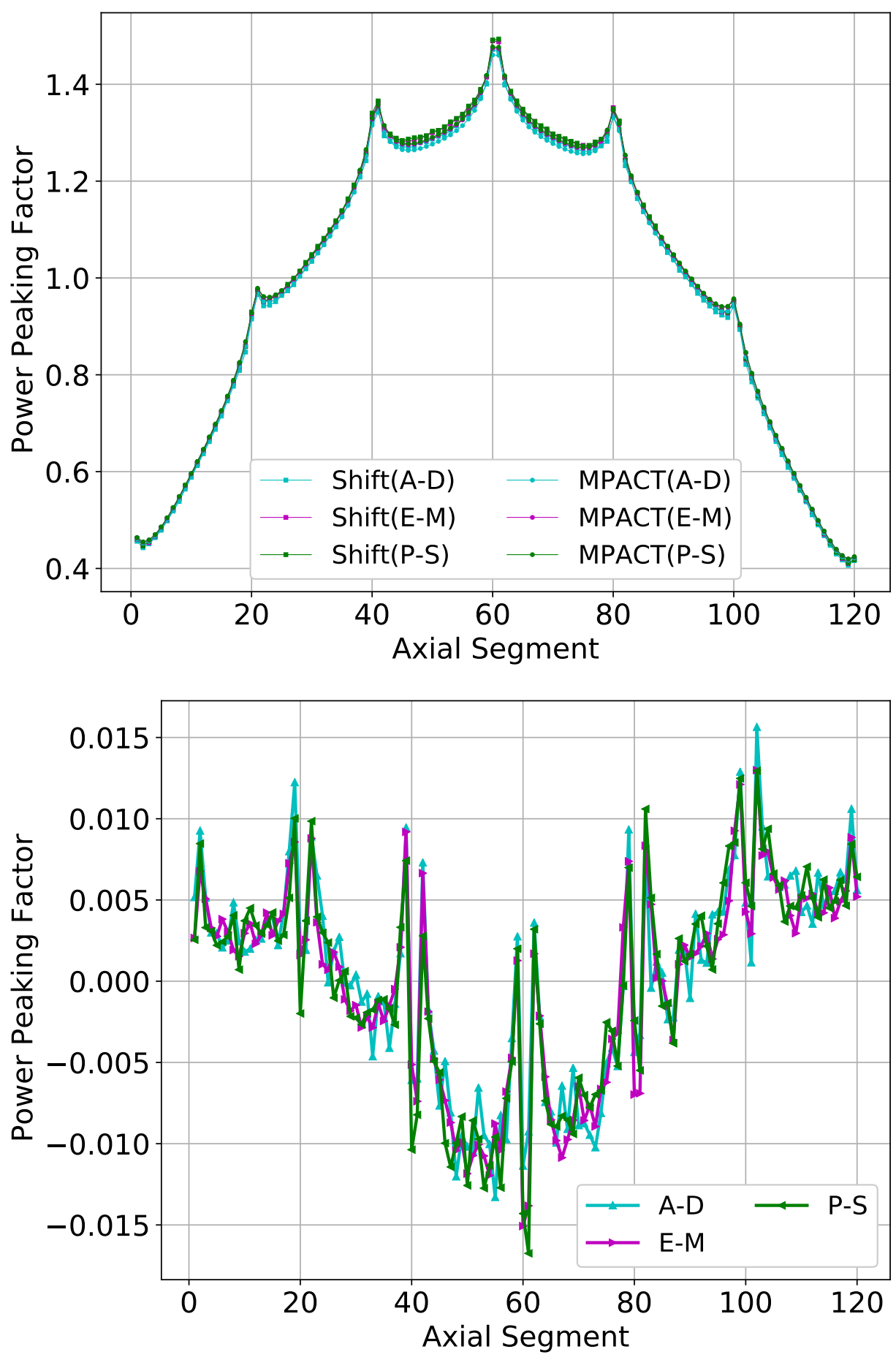

Figure 21. Power peaking factors for Problem 5.1.1 for Shift and MPACT (top) and their absolute difference (bottom). The three series correspond to pins ABCD (central), EFGHJKLM (edge), and PQRS (corner). 
Table 6.2. Timing variables for the reference solution of Problem 5.1.1

\begin{tabular}{|c|c|c|c|c|c|c|}
\hline \multicolumn{7}{|c|}{ Shift } \\
\hline $\begin{array}{c}\text { Depletion } \\
\text { Solves }\end{array}$ & $\begin{array}{c}\text { Total } \\
\text { Cycles per } \\
\text { Transport } \\
\text { Solve }\end{array}$ & $\begin{array}{c}\text { Active } \\
\text { Cycles per } \\
\text { Transport } \\
\text { Solve }\end{array}$ & $\begin{array}{l}\text { Histories } \\
\text { per Cycle }\end{array}$ & $\begin{array}{c}\text { Compute } \\
\text { Nodes }\end{array}$ & $\begin{array}{c}\text { Cores } \\
\text { per Node }\end{array}$ & $\begin{array}{c}\text { Wall } \\
\text { Total Time } \\
\text { [hours] }\end{array}$ \\
\hline 1 & 1500 & 1200 & 100000 & 4 & 48 & 0.54 \\
\hline \multicolumn{7}{|c|}{ MPACT } \\
\hline $\begin{array}{c}\text { Depletion } \\
\text { Solves }\end{array}$ & $\begin{array}{c}k_{\mathrm{eff}} \\
\text { Convergence } \\
\text { Criteria }\end{array}$ & $\begin{array}{c}\text { Source } \\
\text { Convergence } \\
\text { Criteria } \\
\end{array}$ & $\begin{array}{c}\text { Ray } \\
\text { Spacing } \\
{[\mathrm{cm}]}\end{array}$ & $\begin{array}{c}\text { Compute } \\
\text { Nodes }\end{array}$ & $\begin{array}{c}\text { Cores } \\
\text { per Node }\end{array}$ & $\begin{array}{c}\text { Wall } \\
\text { Total Time } \\
\text { [hours] }\end{array}$ \\
\hline 1 & $1.0 \times 10^{-04}$ & $1.0 \times 10^{-03}$ & 0.01 & 5 & 128 & 1.73 \\
\hline
\end{tabular}

Table 6.3. Problem 5.1.1 reference solution power peaking factors for axial segments in element 1.

\begin{tabular}{rcccccccccc}
\hline Shift & & \multicolumn{8}{c}{ Power Peaking Factors } \\
Channels & 1 & 2 & 3 & 4 & 5 & 6 & 7 & 8 & 9 & 10 \\
\hline A, B, C, D & 0.456 & 0.443 & 0.451 & 0.464 & 0.479 & 0.498 & 0.519 & 0.538 & 0.564 & 0.588 \\
E, F, G, H, J, K, L, M & 0.461 & 0.448 & 0.453 & 0.466 & 0.482 & 0.500 & 0.522 & 0.546 & 0.570 & 0.593 \\
P, Q, R, S & 0.461 & 0.446 & 0.456 & 0.467 & 0.484 & 0.502 & 0.523 & 0.545 & 0.572 & 0.593 \\
\hline \hline Channels & 11 & 12 & 13 & 14 & 15 & 16 & 17 & 18 & 19 & 20 \\
\hline A, B, C, D & 0.613 & 0.637 & 0.662 & 0.687 & 0.715 & 0.746 & 0.776 & 0.809 & 0.847 & 0.915 \\
E, F, G, H, J, K, L, M & 0.617 & 0.643 & 0.668 & 0.693 & 0.722 & 0.751 & 0.783 & 0.817 & 0.859 & 0.925 \\
P, Q, R, S & 0.617 & 0.643 & 0.669 & 0.695 & 0.722 & 0.754 & 0.786 & 0.820 & 0.859 & 0.930 \\
\hline MPACT & & & & & Power Peaking Factors & & & \\
Channels & 1 & 2 & 3 & 4 & 5 & 6 & 7 & 8 & 9 & 10 \\
\hline A, B, C, D & 0.461 & 0.452 & 0.456 & 0.467 & 0.482 & 0.500 & 0.521 & 0.543 & 0.566 & 0.590 \\
E, F, G, H, J, K, L, M & 0.463 & 0.454 & 0.459 & 0.470 & 0.485 & 0.504 & 0.525 & 0.548 & 0.571 & 0.595 \\
P, Q, R, S & 0.464 & 0.455 & 0.459 & 0.470 & 0.486 & 0.505 & 0.526 & 0.549 & 0.572 & 0.596 \\
\hline \hline Channels & 11 & 12 & 13 & 14 & 15 & 16 & 17 & 18 & 19 & 20 \\
\hline A, B, C, D & 0.615 & 0.638 & 0.663 & 0.690 & 0.717 & 0.747 & 0.779 & 0.816 & 0.858 & 0.917 \\
E, F, G, H, J, K, L, M & 0.619 & 0.644 & 0.669 & 0.696 & 0.724 & 0.754 & 0.786 & 0.823 & 0.866 & 0.925 \\
P, Q, R, S & 0.620 & 0.645 & 0.670 & 0.697 & 0.725 & 0.755 & 0.787 & 0.824 & 0.868 & 0.927 \\
\hline
\end{tabular}


Table 6.4. Problem 5.1.1 reference solution power peaking factors for axial segments in element 2.

\begin{tabular}{|c|c|c|c|c|c|c|c|c|c|c|}
\hline Shift & \multicolumn{10}{|c|}{ Power Peaking Factors } \\
\hline Channels & 1 & 2 & 3 & 4 & 5 & 6 & 7 & 8 & 9 & 10 \\
\hline $\mathrm{A}, \mathrm{B}, \mathrm{C}, \mathrm{D}$ & 0.966 & 0.942 & 0.944 & 0.951 & 0.964 & 0.974 & 0.986 & 1.003 & 1.019 & 1.034 \\
\hline $\mathrm{E}, \mathrm{F}, \mathrm{G}, \mathrm{H}, \mathrm{J}, \mathrm{K}, \mathrm{L}, \mathrm{M}$ & 0.975 & 0.951 & 0.955 & 0.963 & 0.972 & 0.982 & 0.997 & 1.014 & 1.030 & 1.046 \\
\hline $\mathrm{P}, \mathrm{Q}, \mathrm{R}, \mathrm{S}$ & 0.975 & 0.952 & 0.956 & 0.962 & 0.972 & 0.987 & 0.999 & 1.014 & 1.032 & 1.048 \\
\hline Channels & 11 & 12 & 13 & 14 & 15 & 16 & 17 & 18 & 19 & 20 \\
\hline $\mathrm{A}, \mathrm{B}, \mathrm{C}, \mathrm{D}$ & 1.052 & 1.069 & 1.091 & 1.106 & 1.127 & 1.154 & 1.178 & 1.208 & 1.242 & 1.321 \\
\hline $\mathrm{E}, \mathrm{F}, \mathrm{G}, \mathrm{H}, \mathrm{J}, \mathrm{K}, \mathrm{L}, \mathrm{M}$ & 1.064 & 1.080 & 1.099 & 1.117 & 1.139 & 1.161 & 1.188 & 1.219 & 1.254 & 1.333 \\
\hline $\mathrm{P}, \mathrm{Q}, \mathrm{R}, \mathrm{S}$ & 1.066 & 1.082 & 1.100 & 1.118 & 1.139 & 1.163 & 1.192 & 1.220 & 1.258 & 1.341 \\
\hline MPACT & \multicolumn{10}{|c|}{ Power Peaking Factors } \\
\hline Channels & 1 & 2 & 3 & 4 & 5 & 6 & 7 & 8 & 9 & 10 \\
\hline $\mathrm{A}, \mathrm{B}, \mathrm{C}, \mathrm{D}$ & 0.968 & 0.951 & 0.950 & 0.955 & 0.964 & 0.975 & 0.989 & 1.003 & 1.019 & 1.035 \\
\hline $\mathrm{E}, \mathrm{F}, \mathrm{G}, \mathrm{H}, \mathrm{J}, \mathrm{K}, \mathrm{L}, \mathrm{M}$ & 0.977 & 0.960 & 0.959 & 0.964 & 0.973 & 0.984 & 0.998 & 1.013 & 1.028 & 1.044 \\
\hline $\mathrm{P}, \mathrm{Q}, \mathrm{R}, \mathrm{S}$ & 0.979 & 0.962 & 0.960 & 0.965 & 0.974 & 0.986 & 0.999 & 1.014 & 1.030 & 1.046 \\
\hline Channels & 11 & 12 & 13 & 14 & 15 & 16 & 17 & 18 & 19 & 20 \\
\hline $\mathrm{A}, \mathrm{B}, \mathrm{C}, \mathrm{D}$ & 1.051 & 1.068 & 1.086 & 1.105 & 1.126 & 1.150 & 1.177 & 1.210 & 1.252 & 1.315 \\
\hline $\mathrm{E}, \mathrm{F}, \mathrm{G}, \mathrm{H}, \mathrm{J}, \mathrm{K}, \mathrm{L}, \mathrm{M}$ & 1.061 & 1.078 & 1.096 & 1.115 & 1.136 & 1.160 & 1.187 & 1.221 & 1.263 & 1.328 \\
\hline $\mathrm{P}, \mathrm{Q}, \mathrm{R}, \mathrm{S}$ & 1.063 & 1.080 & 1.098 & 1.117 & 1.138 & 1.162 & 1.189 & 1.223 & 1.265 & 1.330 \\
\hline
\end{tabular}

Table 6.5. Problem 5.1.1 reference solution power peaking factors for axial segments in element 3 .

\begin{tabular}{|c|c|c|c|c|c|c|c|c|c|c|}
\hline Shift & \multicolumn{10}{|c|}{ Power Peaking Factors } \\
\hline Channels & 1 & 2 & 3 & 4 & 5 & 6 & 7 & 8 & 9 & 10 \\
\hline $\mathrm{A}, \mathrm{B}, \mathrm{C}, \mathrm{D}$ & 1.348 & 1.293 & 1.283 & 1.275 & 1.273 & 1.268 & 1.273 & 1.279 & 1.281 & 1.287 \\
\hline $\mathrm{E}, \mathrm{F}, \mathrm{G}, \mathrm{H}, \mathrm{J}, \mathrm{K}, \mathrm{L}, \mathrm{M}$ & 1.363 & 1.306 & 1.295 & 1.286 & 1.282 & 1.282 & 1.285 & 1.289 & 1.293 & 1.300 \\
\hline $\mathrm{P}, \mathrm{Q}, \mathrm{R}, \mathrm{S}$ & 1.366 & 1.312 & 1.297 & 1.289 & 1.284 & 1.287 & 1.290 & 1.291 & 1.294 & 1.303 \\
\hline Channels & 11 & 12 & 13 & 14 & 15 & 16 & 17 & 18 & 19 & 20 \\
\hline $\mathrm{A}, \mathrm{B}, \mathrm{C}, \mathrm{D}$ & 1.293 & 1.295 & 1.305 & 1.314 & 1.328 & 1.336 & 1.356 & 1.373 & 1.400 & 1.472 \\
\hline $\mathrm{E}, \mathrm{F}, \mathrm{G}, \mathrm{H}, \mathrm{J}, \mathrm{K}, \mathrm{L}, \mathrm{M}$ & 1.305 & 1.310 & 1.318 & 1.328 & 1.335 & 1.350 & 1.365 & 1.387 & 1.415 & 1.490 \\
\hline $\mathrm{P}, \mathrm{Q}, \mathrm{R}, \mathrm{S}$ & 1.305 & 1.312 & 1.322 & 1.330 & 1.338 & 1.355 & 1.367 & 1.390 & 1.417 & 1.491 \\
\hline MPACT & \multicolumn{10}{|c|}{ Power Peaking Factors } \\
\hline Channels & 1 & 2 & 3 & 4 & 5 & 6 & 7 & 8 & 9 & 10 \\
\hline $\mathrm{A}, \mathrm{B}, \mathrm{C}, \mathrm{D}$ & 1.342 & 1.301 & 1.281 & 1.270 & 1.265 & 1.263 & 1.265 & 1.267 & 1.271 & 1.276 \\
\hline $\mathrm{E}, \mathrm{F}, \mathrm{G}, \mathrm{H}, \mathrm{J}, \mathrm{K}, \mathrm{L}, \mathrm{M}$ & 1.355 & 1.313 & 1.293 & 1.282 & 1.276 & 1.275 & 1.276 & 1.279 & 1.283 & 1.288 \\
\hline $\mathrm{P}, \mathrm{Q}, \mathrm{R}, \mathrm{S}$ & 1.357 & 1.315 & 1.295 & 1.284 & 1.278 & 1.277 & 1.278 & 1.281 & 1.285 & 1.291 \\
\hline Channels & 11 & 12 & 13 & 14 & 15 & 16 & 17 & 18 & 19 & 20 \\
\hline $\mathrm{A}, \mathrm{B}, \mathrm{C}, \mathrm{D}$ & 1.282 & 1.288 & 1.296 & 1.304 & 1.315 & 1.328 & 1.346 & 1.370 & 1.403 & 1.461 \\
\hline $\mathrm{E}, \mathrm{F}, \mathrm{G}, \mathrm{H}, \mathrm{J}, \mathrm{K}, \mathrm{L}, \mathrm{M}$ & 1.294 & 1.300 & 1.307 & 1.316 & 1.327 & 1.340 & 1.358 & 1.382 & 1.416 & 1.475 \\
\hline $\mathrm{P}, \mathrm{Q}, \mathrm{R}, \mathrm{S}$ & 1.296 & 1.303 & 1.310 & 1.318 & 1.329 & 1.342 & 1.360 & 1.385 & 1.419 & 1.477 \\
\hline
\end{tabular}


Table 6.6. Problem 5.1.1 reference solution power peaking factors for axial segments in element 4.

\begin{tabular}{|c|c|c|c|c|c|c|c|c|c|c|}
\hline Shift & \multicolumn{10}{|c|}{ Power Peaking Factors } \\
\hline Channels & 1 & 2 & 3 & 4 & 5 & 6 & 7 & 8 & 9 & 10 \\
\hline $\mathrm{A}, \mathrm{B}, \mathrm{C}, \mathrm{D}$ & 1.469 & .399 & 1.371 & .352 & 1.334 & 1.322 & 1.307 & 1.301 & 1.290 & 1.286 \\
\hline , H, J, K, L, M & 1.488 & 1.413 & 383 & 362 & 1.346 & 1.334 & 1.324 & 314 & 305 & 1.296 \\
\hline $\mathrm{P}, \mathrm{Q}, \mathrm{R}, \mathrm{S}$ & 1.493 & 1.414 & 1.386 & 1.366 & 1.349 & 1.335 & 1.323 & 1.315 & 1.308 & 1.298 \\
\hline Channels & 11 & 12 & 13 & $\overline{14}$ & 15 & 16 & $\overline{17}$ & 18 & 19 & 20 \\
\hline $\mathrm{A}, \mathrm{B}$, & 280 & 1.275 & .272 & .266 & 1.262 & 1.262 & 1.268 & 1.273 & .282 & 1.336 \\
\hline $\mathrm{H}, \mathrm{J}, \mathrm{K}, \mathrm{L}, \mathrm{M}$ & 1.292 & 1.285 & 1.282 & 1.276 & 1.274 & 1.272 & 1.277 & 1.281 & 1.296 & 1.352 \\
\hline $\mathrm{P}, \mathrm{Q}, \mathrm{R}, \mathrm{S}$ & 2 & 1.288 & 1.282 & .278 & 1.272 & 1.274 & 1.281 & 1.287 & 1.299 & 1.350 \\
\hline MPACT & \multicolumn{10}{|c|}{ Power Peaking Factors } \\
\hline Channels & 1 & 2 & 3 & 4 & 5 & 6 & 7 & 8 & 9 & 10 \\
\hline $\mathrm{A}, \mathrm{B}, \mathrm{C}, \mathrm{D}$ & 1.460 & 1.402 & 1.369 & 1.344 & 1.326 & 1.312 & 1.301 & 1.292 & 1.284 & 1.277 \\
\hline $\mathrm{J}, \mathrm{H}, \mathrm{J}, \mathrm{K}, \mathrm{L}, \mathrm{M}$ & 1.474 & 1.415 & 1.381 & 1.356 & 1.338 & 1.324 & 1.313 & 1.304 & 1.296 & 1.289 \\
\hline $\mathrm{P}, \mathrm{Q}, \mathrm{R}, \mathrm{S}$ & 1.477 & 1.418 & 1.383 & 1.358 & 1.340 & 1.326 & 1.315 & 1.306 & 1.298 & 1.292 \\
\hline Channels & 11 & 12 & 13 & 14 & 15 & 16 & 17 & 18 & 19 & 20 \\
\hline $\mathrm{A}, \mathrm{B}, \mathrm{C}, \mathrm{D}$ & 1.271 & 1.266 & 1.261 & 1.258 & 1.256 & 1.258 & 1.262 & 1.273 & 1.291 & 1.332 \\
\hline $\mathrm{E}, \mathrm{F}, \mathrm{G}, \mathrm{H}, \mathrm{J}, \mathrm{K}, \mathrm{L}, \mathrm{M}$ & 1.283 & 1.278 & 1.273 & 1.270 & 1.268 & 1.269 & 1.274 & 1.284 & 1.303 & 1.345 \\
\hline $\mathrm{P}, \mathrm{Q}, \mathrm{R}, \mathrm{S}$ & 1.285 & 1.280 & 1.275 & 1.272 & 1.270 & 1.271 & 1.276 & 1.286 & 1.306 & 1.347 \\
\hline
\end{tabular}

Table 6.7. Problem 5.1.1 reference solution power peaking factors for axial segments in element 5.

\begin{tabular}{|c|c|c|c|c|c|c|c|c|c|c|}
\hline Shift & \multicolumn{10}{|c|}{ Power Peaking Factors } \\
\hline Channels & 1 & 2 & 3 & 4 & 5 & 6 & 7 & 8 & 9 & 10 \\
\hline $\mathrm{A}, \mathrm{B}, \mathrm{C}, \mathrm{D}$ & 1.307 & 1.232 & 1.199 & 1.164 & 1.137 & 1.116 & 1.094 & 1.071 & 1.052 & 1.038 \\
\hline $\mathrm{E}, \mathrm{F}, \mathrm{G}, \mathrm{H}, \mathrm{J}, \mathrm{K}, \mathrm{L}, \mathrm{M}$ & 1.323 & 1.243 & 1.205 & 1.175 & 1.147 & 1.125 & 1.106 & 1.081 & 1.062 & 1.045 \\
\hline $\mathrm{P}, \mathrm{Q}, \mathrm{R}, \mathrm{S}$ & 1.324 & 1.243 & 1.206 & 1.176 & 1.151 & 1.127 & 1.108 & 1.082 & 1.065 & 1.047 \\
\hline Channels & 11 & 12 & 13 & 14 & 15 & 16 & 17 & 18 & 19 & 20 \\
\hline $\mathrm{A}, \mathrm{B}, \mathrm{C}, \mathrm{D}$ & 1.016 & 1.002 & 0.986 & 0.968 & 0.954 & 0.942 & 0.930 & 0.923 & 0.918 & 0.942 \\
\hline $\mathrm{E}, \mathrm{F}, \mathrm{G}, \mathrm{H}, \mathrm{J}, \mathrm{K}, \mathrm{L}, \mathrm{M}$ & 1.028 & 1.011 & 0.994 & 0.980 & 0.965 & 0.952 & 0.940 & 0.930 & 0.928 & 0.951 \\
\hline $\mathrm{P}, \mathrm{Q}, \mathrm{R}, \mathrm{S}$ & 1.028 & 1.010 & 0.996 & 0.982 & 0.965 & 0.950 & 0.938 & 0.933 & 0.929 & 0.951 \\
\hline MPACT & \multicolumn{10}{|c|}{ Power Peaking Factors } \\
\hline Channels & 1 & 2 & 3 & 4 & 5 & 6 & 7 & 8 & 9 & 10 \\
\hline $\mathrm{A}, \mathrm{B}, \mathrm{C}, \mathrm{D}$ & 1.304 & 1.240 & 1.198 & 1.165 & 1.137 & 1.113 & 1.092 & 1.073 & 1.054 & 1.037 \\
\hline $\mathrm{E}, \mathrm{F}, \mathrm{G}, \mathrm{H}, \mathrm{J}, \mathrm{K}, \mathrm{L}, \mathrm{M}$ & 1.316 & 1.252 & 1.209 & 1.175 & 1.147 & 1.123 & 1.102 & 1.082 & 1.064 & 1.046 \\
\hline $\mathrm{P}, \mathrm{Q}, \mathrm{R}, \mathrm{S}$ & 1.319 & 1.254 & 1.211 & 1.177 & 1.149 & 1.125 & 1.104 & 1.084 & 1.066 & 1.048 \\
\hline Channels & 11 & 12 & 13 & 14 & 15 & 16 & 17 & 18 & 19 & 20 \\
\hline $\mathrm{A}, \mathrm{B}, \mathrm{C}, \mathrm{D}$ & 1.020 & 1.003 & 0.987 & 0.972 & 0.958 & 0.946 & 0.937 & 0.931 & 0.931 & 0.946 \\
\hline $\mathrm{E}, \mathrm{F}, \mathrm{G}, \mathrm{H}, \mathrm{J}, \mathrm{K}, \mathrm{L}, \mathrm{M}$ & 1.029 & 1.013 & 0.997 & 0.981 & 0.967 & 0.955 & 0.945 & 0.940 & 0.940 & 0.956 \\
\hline $\mathrm{P}, \mathrm{Q}, \mathrm{R}, \mathrm{S}$ & 1.031 & 1.014 & 0.998 & 0.983 & 0.969 & 0.956 & 0.947 & 0.941 & 0.941 & 0.957 \\
\hline
\end{tabular}


Table 6.8. Problem 5.1.1 reference solution power peaking factors for axial segments in element 6.

\begin{tabular}{ccccccccccc}
\hline Shift & \multicolumn{10}{c}{ Power Peaking Factors } \\
Channels & 1 & 2 & 3 & 4 & 5 & 6 & 7 & 8 & 9 & 10 \\
\hline A, B, C, D & 0.894 & 0.821 & 0.785 & 0.752 & 0.719 & 0.690 & 0.662 & 0.635 & 0.609 & 0.586 \\
E, F, G, H, J, K, L, M & 0.901 & 0.832 & 0.794 & 0.757 & 0.726 & 0.697 & 0.668 & 0.643 & 0.618 & 0.591 \\
P, Q, R, S & 0.900 & 0.833 & 0.795 & 0.757 & 0.727 & 0.697 & 0.671 & 0.644 & 0.618 & 0.591 \\
\hline \hline Channels & 11 & 12 & 13 & 14 & 15 & 16 & 17 & 18 & 19 & 20 \\
\hline A, B, C, D & 0.561 & 0.538 & 0.511 & 0.490 & 0.468 & 0.448 & 0.430 & 0.418 & 0.406 & 0.416 \\
E, F, G, H, J, K, L, M & 0.566 & 0.541 & 0.518 & 0.494 & 0.471 & 0.453 & 0.434 & 0.421 & 0.410 & 0.419 \\
P, Q, R, S & 0.565 & 0.542 & 0.519 & 0.493 & 0.473 & 0.452 & 0.434 & 0.422 & 0.411 & 0.418 \\
MPACT & & & & \multicolumn{2}{c}{ Power Peaking Factors } & & & \\
Channels & 1 & 2 & 3 & 4 & 5 & 6 & 7 & 8 & 9 & 10 \\
\hline A, B, C, D & 0.895 & 0.837 & 0.795 & 0.758 & 0.726 & 0.696 & 0.668 & 0.641 & 0.616 & 0.590 \\
E, F, G, H, J, K, L, M & 0.903 & 0.845 & 0.802 & 0.765 & 0.732 & 0.702 & 0.674 & 0.647 & 0.621 & 0.596 \\
P, Q, R, S & 0.905 & 0.846 & 0.803 & 0.766 & 0.733 & 0.703 & 0.675 & 0.648 & 0.622 & 0.597 \\
\hline \hline Channels & 11 & 12 & 13 & 14 & 15 & 16 & 17 & 18 & 19 & 20 \\
\hline A, B, C, D & 0.566 & 0.541 & 0.517 & 0.495 & 0.473 & 0.453 & 0.437 & 0.424 & 0.417 & 0.422 \\
E, F, G, H, J, K, L, M & 0.571 & 0.546 & 0.522 & 0.499 & 0.477 & 0.457 & 0.439 & 0.426 & 0.419 & 0.424 \\
P, Q, R, S & 0.572 & 0.547 & 0.523 & 0.499 & 0.477 & 0.457 & 0.440 & 0.427 & 0.420 & 0.424 \\
\hline
\end{tabular}




\subsubsection{Problem 5.1.2}

\subsubsection{Description}

Problem 5.1.2 uses the same geometry, temperatures and materials as Problem 5.1.1, except Problem 5.1.2 has the control rod inserted half way into the the fuel from the top. The bottom of the control rod coincides with the boundary between elements 3 and 4, at the boundary between both end caps. The boundary conditions are vacuum at the axial bottom and top of the reflectors and reflective at the four sides of the charge-pan. As with Problem 4, each fuel element is divided into 20 segments, each representing 5\% of the active fuel length.

\subsubsection{Reference Solution}

The neutron multiplication factor results are shown in Table 6.9. The timing variables for Shift and MPACT are shown in Table 6.10. Power peaking factors for the three types of charge pan pins are displayed for BOC and EOC in Figure 22 and fully tabulated in Tables 6.11-6.16. The neutron multiplication factor agrees to under $50 \mathrm{pcm}$.

The power peaking factors in Figure 22 show noticeable differences between the MPACT and Shift peaking factors. The overall shape agrees well. The MPACT peaking factors reveal a larger flux depression with the control rod inserted compared to it fully withdrawn in Problem 5.1.1. This issue is still under investigation. Geometry and material composition issues were confirmed to be non-issues. It is suggested that the axial solver in MPACT has trouble with the axial heterogeneity with the added control rod.

Table 6.9. Neutron multiplication factor for the reference solution of Problem 5.1.2

\begin{tabular}{ccccc}
\hline Step & $\begin{array}{c}\text { Burnup } \\
{\left[\frac{\mathrm{MWd}}{\mathrm{MtU}}\right]}\end{array}$ & $\begin{array}{c}\text { Shift } \\
\mathrm{k}_{\mathrm{eff}}(1 \sigma)\end{array}$ & $\begin{array}{c}\text { MPACT } \\
\mathrm{k}_{\mathrm{eff}}\end{array}(1 \sigma)$ & $\begin{array}{c}\text { Difference } \\
{[\mathrm{pcm}]}\end{array}$ \\
\hline 0 & 0.0 & $1.02824(7)$ & $1.02867(10)$ & $44(12)$ \\
\hline
\end{tabular}

Table 6.10. Timing variables for the reference solution of Problem 5.1.2

\begin{tabular}{|c|c|c|c|c|c|c|}
\hline \multicolumn{7}{|c|}{$\overline{\text { Shift }}$} \\
\hline $\begin{array}{c}\text { Depletion } \\
\text { Solves }\end{array}$ & $\begin{array}{c}\text { Total } \\
\text { Cycles per } \\
\text { Transport } \\
\text { Solve }\end{array}$ & $\begin{array}{l}\text { Active } \\
\text { Cycles per } \\
\text { Transport } \\
\text { Solve }\end{array}$ & $\begin{array}{l}\text { Histories } \\
\text { per Cycle }\end{array}$ & $\begin{array}{l}\text { Compute } \\
\text { Nodes }\end{array}$ & $\begin{array}{c}\text { Cores } \\
\text { per Node }\end{array}$ & $\begin{array}{c}\text { Wall } \\
\text { Total Time } \\
\text { [hours] }\end{array}$ \\
\hline 1 & 1500 & 1200 & 100000 & 2 & 48 & 0.93 \\
\hline \multicolumn{7}{|c|}{ MPACT } \\
\hline $\begin{array}{c}\text { Depletion } \\
\text { Solves }\end{array}$ & $\begin{array}{c}k_{\mathrm{eff}} \\
\text { Convergence } \\
\text { Criteria }\end{array}$ & $\begin{array}{c}\text { Source } \\
\text { Convergence } \\
\text { Criteria }\end{array}$ & $\begin{array}{c}\text { Ray } \\
\text { Spacing } \\
{[\mathrm{cm}]}\end{array}$ & $\begin{array}{c}\text { Compute } \\
\text { Nodes }\end{array}$ & $\begin{array}{c}\text { Cores } \\
\text { per Node }\end{array}$ & $\begin{array}{c}\text { Wall } \\
\text { Total Time } \\
\text { [hours] }\end{array}$ \\
\hline 1 & $1.0 \times 10^{-04}$ & $1.0 \times 10^{-03}$ & 0.01 & 2 & 128 & 0.74 \\
\hline
\end{tabular}



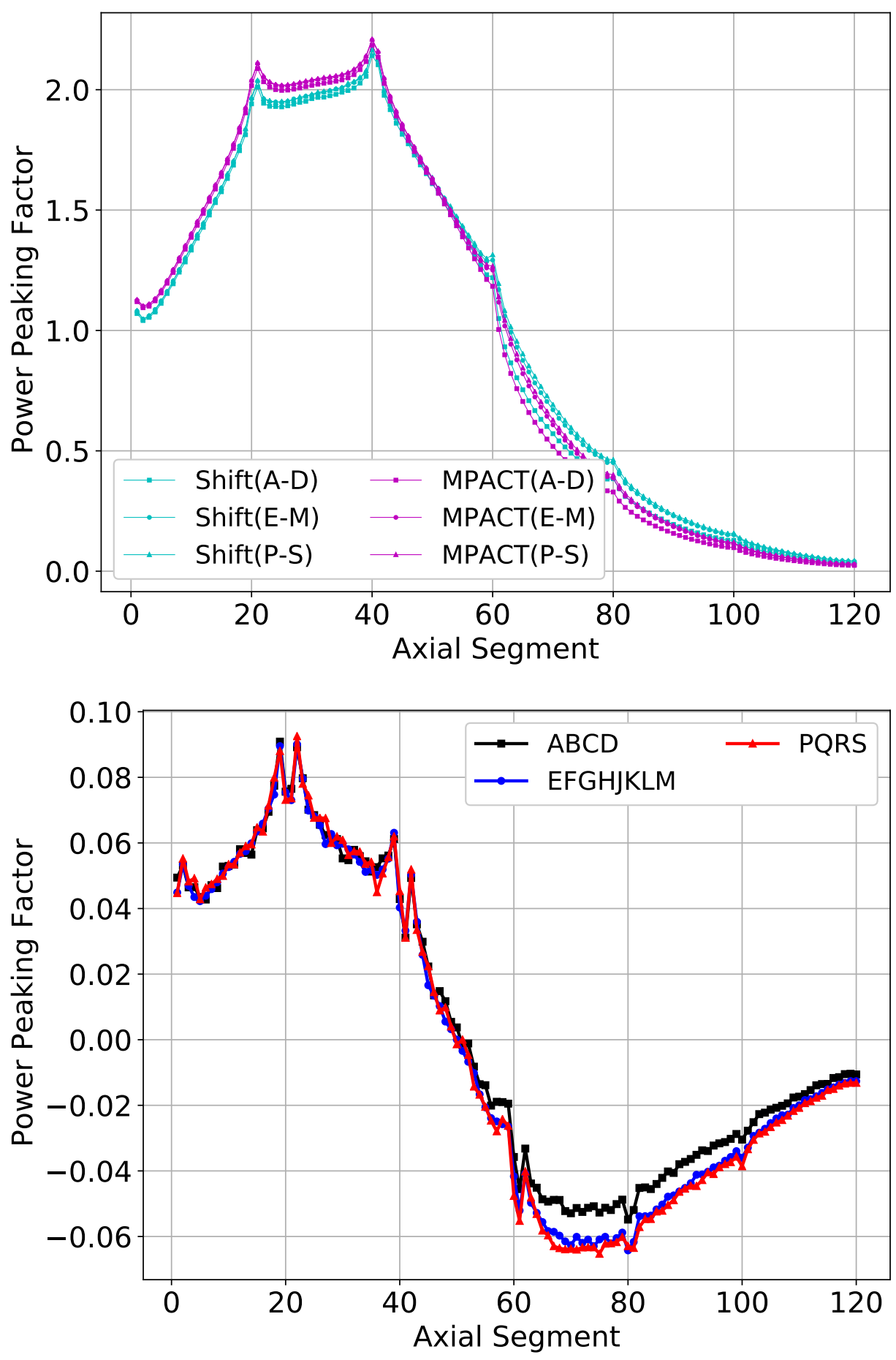

Figure 22. Power peaking factors for Problem 5.1.2 for Shift and MPACT (top) and their absolute difference (bottom). The three series correspond to pins ABCD (central), EFGHJKLM (edge), and PQRS (corner). 
Table 6.11. Problem 5.1.2 reference solution power peaking factors for axial segments in element 1.

\begin{tabular}{|c|c|c|c|c|c|c|c|c|c|c|}
\hline Shift & \multicolumn{10}{|c|}{ Power Peaking Factors } \\
\hline Channels & 1 & 2 & 3 & 4 & 5 & 6 & 7 & 8 & 9 & 10 \\
\hline $\mathrm{A}, \mathrm{B}, \mathrm{C}, \mathrm{D}$ & 1.070 & 1.041 & 1.055 & 1.078 & 1.113 & 1.151 & 1.194 & 1.241 & 1.290 & 1.336 \\
\hline $\mathrm{E}, \mathrm{F}, \mathrm{G}, \mathrm{H}, \mathrm{J}, \mathrm{K}, \mathrm{L}, \mathrm{M}$ & 1.081 & 1.048 & 1.061 & 1.086 & 1.121 & 1.160 & 1.206 & 1.253 & 1.301 & 1.350 \\
\hline $\mathrm{P}, \mathrm{Q}, \mathrm{R}, \mathrm{S}$ & 1.082 & 1.048 & 1.061 & 1.090 & 1.123 & 1.161 & 1.209 & 1.255 & 1.302 & 1.351 \\
\hline Channels & 11 & 12 & 13 & 14 & 15 & 16 & 17 & 18 & 19 & 20 \\
\hline $\mathrm{A}, \mathrm{B}, \mathrm{C}, \mathrm{D}$ & 1.382 & 1.433 & 1.480 & 1.527 & 1.577 & 1.635 & 1.689 & 1.751 & 1.821 & 1.949 \\
\hline $\mathrm{E}, \mathrm{F}, \mathrm{G}, \mathrm{H}, \mathrm{J}, \mathrm{K}, \mathrm{L}, \mathrm{M}$ & 1.399 & 1.449 & 1.497 & 1.546 & 1.598 & 1.651 & 1.706 & 1.769 & 1.840 & 1.971 \\
\hline $\mathrm{P}, \mathrm{Q}, \mathrm{R}, \mathrm{S}$ & 1.397 & 1.452 & 1.500 & 1.546 & 1.605 & 1.654 & 1.710 & 1.768 & 1.842 & 1.973 \\
\hline MPACT & \multicolumn{10}{|c|}{ Power Peaking Factors } \\
\hline Channels & 1 & 2 & 3 & 4 & 5 & 6 & 7 & 8 & 9 & 10 \\
\hline $\mathrm{A}, \mathrm{B}, \mathrm{C}, \mathrm{D}$ & 1.117 & 1.092 & 1.099 & 1.122 & 1.155 & 1.195 & 1.240 & 1.287 & 1.336 & 1.385 \\
\hline $\mathrm{E}, \mathrm{F}, \mathrm{G}, \mathrm{H}, \mathrm{J}, \mathrm{K}, \mathrm{L}, \mathrm{M}$ & 1.123 & 1.099 & 1.106 & 1.129 & 1.163 & 1.205 & 1.250 & 1.299 & 1.348 & 1.399 \\
\hline $\mathrm{P}, \mathrm{Q}, \mathrm{R}, \mathrm{S}$ & 1.124 & 1.100 & 1.107 & 1.131 & 1.165 & 1.207 & 1.253 & 1.301 & 1.351 & 1.401 \\
\hline Channels & 11 & 12 & 13 & 14 & 15 & 16 & 17 & 18 & 19 & 20 \\
\hline $\mathrm{A}, \mathrm{B}, \mathrm{C}, \mathrm{D}$ & 1.435 & 1.485 & 1.535 & 1.585 & 1.638 & 1.693 & 1.754 & 1.821 & 1.901 & 2.013 \\
\hline $\mathrm{E}, \mathrm{F}, \mathrm{G}, \mathrm{H}, \mathrm{J}, \mathrm{K}, \mathrm{L}, \mathrm{M}$ & 1.449 & 1.499 & 1.549 & 1.600 & 1.653 & 1.709 & 1.770 & 1.838 & 1.919 & 2.033 \\
\hline $\mathrm{P}, \mathrm{Q}, \mathrm{R}, \mathrm{S}$ & 1.451 & 1.502 & 1.552 & 1.603 & 1.656 & 1.712 & 1.773 & 1.842 & 1.923 & 2.037 \\
\hline
\end{tabular}

Table 6.12. Problem 5.1.2 reference solution power peaking factors for axial segments in element 2.

\begin{tabular}{|c|c|c|c|c|c|c|c|c|c|c|}
\hline Shift & \multicolumn{10}{|c|}{ Power Peaking Factors } \\
\hline Channels & 1 & 2 & 3 & 4 & 5 & 6 & 7 & 8 & 9 & 10 \\
\hline $\mathrm{A}, \mathrm{B}, \mathrm{C}, \mathrm{D}$ & 2.018 & 1.951 & 1.936 & 1.936 & 1.934 & 1.937 & 1.938 & 1.945 & 1.955 & 1.961 \\
\hline $\mathrm{E}, \mathrm{F}, \mathrm{G}, \mathrm{H}, \mathrm{J}, \mathrm{K}, \mathrm{L}, \mathrm{M}$ & 2.038 & 1.968 & 1.957 & 1.954 & 1.955 & 1.956 & 1.961 & 1.969 & 1.977 & 1.980 \\
\hline $\mathrm{P}, \mathrm{Q}, \mathrm{R}, \mathrm{S}$ & 2.046 & 1.971 & 1.957 & 1.956 & 1.962 & 1.957 & 1.965 & 1.972 & 1.981 & 1.986 \\
\hline Channels & 11 & 12 & 13 & 14 & 15 & 16 & 17 & 18 & 19 & 20 \\
\hline $\mathrm{A}, \mathrm{B}, \mathrm{C}, \mathrm{D}$ & 1.966 & 1.974 & 1.978 & 1.983 & 1.991 & 1.995 & 2.013 & 2.027 & 2.056 & 2.140 \\
\hline $\mathrm{E}, \mathrm{F}, \mathrm{G}, \mathrm{H}, \mathrm{J}, \mathrm{K}, \mathrm{L}, \mathrm{M}$ & 1.989 & 1.994 & 1.998 & 2.003 & 2.011 & 2.018 & 2.033 & 2.049 & 2.074 & 2.167 \\
\hline $\mathrm{P}, \mathrm{Q}, \mathrm{R}, \mathrm{S}$ & 1.992 & 1.998 & 2.003 & 2.006 & 2.014 & 2.021 & 2.037 & 2.051 & 2.081 & 2.169 \\
\hline MPACT & \multicolumn{10}{|c|}{ Power Peaking Factors } \\
\hline Channels & 1 & 2 & 3 & 4 & 5 & 6 & 7 & 8 & 9 & 10 \\
\hline $\mathrm{A}, \mathrm{B}, \mathrm{C}, \mathrm{D}$ & 2.084 & 2.030 & 2.008 & 1.998 & 1.995 & 1.996 & 2.000 & 2.005 & 2.011 & 2.016 \\
\hline $\mathrm{F}, \mathrm{G}, \mathrm{H}, \mathrm{J}, \mathrm{K}, \mathrm{L}, \mathrm{M}$ & 2.105 & 2.049 & 2.027 & 2.016 & 2.013 & 2.015 & 2.019 & 2.025 & 2.030 & 2.036 \\
\hline $\mathrm{P}, \mathrm{Q}, \mathrm{R}, \mathrm{S}$ & 2.109 & 2.053 & 2.031 & 2.020 & 2.017 & 2.019 & 2.023 & 2.028 & 2.034 & 2.039 \\
\hline Channels & 11 & 12 & 13 & 14 & 15 & 16 & 17 & 18 & 19 & 20 \\
\hline $\mathrm{A}, \mathrm{B}, \mathrm{C}, \mathrm{D}$ & 2.021 & 2.025 & 2.029 & 2.033 & 2.038 & 2.047 & 2.060 & 2.081 & 2.114 & 2.181 \\
\hline $\mathrm{E}, \mathrm{F}, \mathrm{G}, \mathrm{H}, \mathrm{J}, \mathrm{K}, \mathrm{L}, \mathrm{M}$ & 2.040 & 2.045 & 2.048 & 2.052 & 2.058 & 2.066 & 2.079 & 2.101 & 2.134 & 2.203 \\
\hline $\mathrm{P}, \mathrm{Q}, \mathrm{R}, \mathrm{S}$ & 2.044 & 2.048 & 2.052 & 2.056 & 2.061 & 2.070 & 2.083 & 2.104 & 2.138 & 2.207 \\
\hline
\end{tabular}


Table 6.13. Problem 5.1.2 reference solution power peaking factors for axial segments in element 3.

\begin{tabular}{|c|c|c|c|c|c|c|c|c|c|c|}
\hline Shift & \multicolumn{10}{|c|}{ Power Peaking Factors } \\
\hline Channels & 1 & 2 & 3 & 4 & 5 & 6 & 7 & 8 & 9 & 10 \\
\hline $\mathrm{A}, \mathrm{B}, \mathrm{C}, \mathrm{D}$ & 2.098 & 1.974 & 1.914 & 1.861 & 1.813 & 1.775 & 1.729 & 1.689 & 1.651 & 1.613 \\
\hline $\mathrm{E}, \mathrm{F}, \mathrm{G}, \mathrm{H}, \mathrm{J}, \mathrm{K}, \mathrm{L}, \mathrm{M}$ & 2.122 & 1.993 & 1.936 & 1.879 & 1.832 & 1.791 & 1.748 & 1.707 & 1.666 & 1.626 \\
\hline $\mathrm{P}, \mathrm{Q}, \mathrm{R}, \mathrm{S}$ & 2.125 & 1.998 & 1.943 & 1.887 & 1.834 & 1.794 & 1.749 & 1.711 & 1.670 & 1.632 \\
\hline Channels & 11 & 12 & 13 & 14 & 15 & 16 & 17 & 18 & 19 & 20 \\
\hline $\mathrm{A}, \mathrm{B}, \mathrm{C}, \mathrm{D}$ & 1.569 & 1.532 & 1.488 & 1.445 & 1.403 & 1.358 & 1.313 & 1.269 & 1.231 & 1.216 \\
\hline $\mathrm{E}, \mathrm{F}, \mathrm{G}, \mathrm{H}, \mathrm{J}, \mathrm{K}, \mathrm{L}, \mathrm{M}$ & 1.587 & 1.549 & 1.508 & 1.468 & 1.426 & 1.385 & 1.348 & 1.314 & 1.281 & 1.292 \\
\hline $\mathrm{P}, \mathrm{Q}, \mathrm{R}, \mathrm{S}$ & 1.595 & 1.554 & 1.513 & 1.474 & 1.433 & 1.393 & 1.357 & 1.329 & 1.295 & 1.308 \\
\hline MPACT & \multicolumn{10}{|c|}{ Power Peaking Factors } \\
\hline Channels & 1 & 2 & 3 & 4 & 5 & 6 & 7 & 8 & 9 & 10 \\
\hline $\mathrm{A}, \mathrm{B}, \mathrm{C}, \mathrm{D}$ & 2.131 & 2.023 & 1.950 & 1.889 & 1.836 & 1.787 & 1.742 & 1.698 & 1.655 & 1.612 \\
\hline $\mathrm{E}, \mathrm{F}, \mathrm{G}, \mathrm{H}, \mathrm{J}, \mathrm{K}, \mathrm{L}, \mathrm{M}$ & 2.153 & 2.043 & 1.968 & 1.907 & 1.853 & 1.804 & 1.759 & 1.715 & 1.672 & 1.629 \\
\hline $\mathrm{P}, \mathrm{Q}, \mathrm{R}, \mathrm{S}$ & 2.157 & 2.047 & 1.972 & 1.910 & 1.856 & 1.808 & 1.762 & 1.719 & 1.676 & 1.633 \\
\hline Channels & 11 & 12 & 13 & 14 & 15 & 16 & 17 & 18 & 19 & 20 \\
\hline $\mathrm{A}, \mathrm{B}, \mathrm{C}, \mathrm{D}$ & 1.569 & 1.525 & 1.480 & 1.434 & 1.388 & 1.342 & 1.296 & 1.252 & 1.210 & 1.181 \\
\hline $\mathrm{E}, \mathrm{F}, \mathrm{G}, \mathrm{H}, \mathrm{J}, \mathrm{K}, \mathrm{L}, \mathrm{M}$ & 1.586 & 1.542 & 1.497 & 1.453 & 1.409 & 1.366 & 1.326 & 1.290 & 1.261 & 1.252 \\
\hline $\mathrm{P}, \mathrm{Q}, \mathrm{R}, \mathrm{S}$ & 1.589 & 1.546 & 1.502 & 1.458 & 1.414 & 1.373 & 1.334 & 1.300 & 1.274 & 1.269 \\
\hline
\end{tabular}

Table 6.14. Problem 5.1.2 reference solution power peaking factors for axial segments in element 4.

\begin{tabular}{ccccccccccc}
\hline Shift & & \multicolumn{8}{c}{ Power Peaking Factors } \\
Channels & 1 & 2 & 3 & 4 & 5 & 6 & 7 & 8 & 9 & 10 \\
\hline A, B, C, D & 1.048 & 0.930 & 0.863 & 0.801 & 0.751 & 0.705 & 0.666 & 0.631 & 0.599 & 0.566 \\
E, F, G, H, J, K, L, M & 1.168 & 1.057 & 0.988 & 0.928 & 0.874 & 0.826 & 0.781 & 0.739 & 0.702 & 0.666 \\
P, Q, R, S & 1.193 & 1.081 & 1.015 & 0.957 & 0.899 & 0.851 & 0.801 & 0.764 & 0.724 & 0.688 \\
\hline \hline Channels & 11 & 12 & 13 & 14 & 15 & 16 & 17 & 18 & 19 & 20 \\
\hline A, B, C, D & 0.539 & 0.512 & 0.490 & 0.467 & 0.445 & 0.427 & 0.411 & 0.394 & 0.384 & 0.382 \\
E, F, G, H, J, K, L, M & 0.633 & 0.604 & 0.576 & 0.548 & 0.524 & 0.502 & 0.483 & 0.465 & 0.449 & 0.449 \\
P, Q, R, S & 0.654 & 0.623 & 0.595 & 0.568 & 0.543 & 0.519 & 0.499 & 0.480 & 0.465 & 0.463 \\
\hline MPACT & & & & & Power Peaking Factors & & & \\
Channels & 1 & 2 & 3 & 4 & 5 & 6 & 7 & 8 & 9 & 10 \\
\hline A, B, C, D & 1.002 & 0.898 & 0.821 & 0.758 & 0.705 & 0.659 & 0.619 & 0.582 & 0.549 & 0.519 \\
E, F, G, H, J, K, L, M & 1.119 & 1.021 & 0.945 & 0.880 & 0.823 & 0.772 & 0.727 & 0.685 & 0.647 & 0.611 \\
P, Q, R, S & 1.142 & 1.045 & 0.970 & 0.905 & 0.848 & 0.797 & 0.750 & 0.708 & 0.669 & 0.632 \\
\hline \hline Channels & 11 & 12 & 13 & 14 & 15 & 16 & 17 & 18 & 19 & 20 \\
\hline A, B, C, D & 0.491 & 0.465 & 0.440 & 0.417 & 0.397 & 0.378 & 0.361 & 0.346 & 0.335 & 0.330 \\
E, F, G, H, J, K, L, M & 0.578 & 0.548 & 0.519 & 0.492 & 0.468 & 0.445 & 0.425 & 0.409 & 0.395 & 0.390 \\
P, Q, R, S & 0.598 & 0.567 & 0.537 & 0.509 & 0.484 & 0.461 & 0.441 & 0.423 & 0.410 & 0.403 \\
\hline
\end{tabular}


Table 6.15. Problem 5.1.2 reference solution power peaking factors for axial segments in element 5.

\begin{tabular}{rcccccccccc}
\hline Shift & \multicolumn{1}{c}{ Power Peaking Factors } \\
Channels & 1 & 2 & 3 & 4 & 5 & 6 & 7 & 8 & 9 & 10 \\
\hline A, B, C, D & 0.343 & 0.310 & 0.290 & 0.272 & 0.255 & 0.240 & 0.226 & 0.216 & 0.204 & 0.194 \\
E, F, G, H, J, K, L, M & 0.404 & 0.366 & 0.341 & 0.320 & 0.301 & 0.283 & 0.269 & 0.254 & 0.239 & 0.228 \\
P, Q, R, S & 0.417 & 0.376 & 0.351 & 0.330 & 0.311 & 0.293 & 0.277 & 0.261 & 0.250 & 0.237 \\
\hline \hline Channels & 11 & 12 & 13 & 14 & 15 & 16 & 17 & 18 & 19 & 20 \\
\hline A, B, C, D & 0.185 & 0.176 & 0.167 & 0.159 & 0.150 & 0.144 & 0.137 & 0.132 & 0.128 & 0.128 \\
E, F, G, H, J, K, L, M & 0.217 & 0.206 & 0.196 & 0.187 & 0.177 & 0.170 & 0.162 & 0.156 & 0.149 & 0.150 \\
P, Q, R, S & 0.225 & 0.213 & 0.203 & 0.193 & 0.185 & 0.175 & 0.168 & 0.162 & 0.156 & 0.155 \\
\hline MPACT & & & & & Power Peaking Factors & & & \\
Channels & 1 & 2 & 3 & 4 & 5 & 6 & 7 & 8 & 9 & 10 \\
\hline A, B, C, D & 0.292 & 0.266 & 0.246 & 0.229 & 0.214 & 0.201 & 0.189 & 0.178 & 0.168 & 0.158 \\
E, F, G, H, J, K, L, M & 0.345 & 0.314 & 0.291 & 0.270 & 0.253 & 0.237 & 0.223 & 0.210 & 0.198 & 0.187 \\
P, Q, R, S & 0.357 & 0.325 & 0.301 & 0.280 & 0.262 & 0.245 & 0.231 & 0.217 & 0.205 & 0.193 \\
\hline \hline Channels & 11 & 12 & 13 & 14 & 15 & 16 & 17 & 18 & 19 & 20 \\
\hline A, B, C, D & 0.149 & 0.141 & 0.134 & 0.127 & 0.120 & 0.114 & 0.109 & 0.104 & 0.100 & 0.098 \\
E, F, G, H, J, K, L, M & 0.176 & 0.167 & 0.158 & 0.149 & 0.142 & 0.135 & 0.128 & 0.123 & 0.119 & 0.116 \\
P, Q, R, S & 0.183 & 0.173 & 0.163 & 0.155 & 0.147 & 0.139 & 0.133 & 0.127 & 0.123 & 0.121 \\
\hline
\end{tabular}

Table 6.16. Problem 5.1.2 reference solution power peaking factors for axial segments in element 6.

\begin{tabular}{ccccccccccc}
\hline Shift & & \multicolumn{1}{c}{ Power Peaking Factors } \\
Channels & 1 & 2 & 3 & 4 & 5 & 6 & 7 & 8 & 9 & 10 \\
\hline A, B, C, D & 0.114 & 0.102 & 0.095 & 0.088 & 0.082 & 0.076 & 0.072 & 0.068 & 0.063 & 0.059 \\
E, F, G, H, J, K, L, M & 0.134 & 0.119 & 0.111 & 0.104 & 0.097 & 0.091 & 0.085 & 0.080 & 0.075 & 0.070 \\
P, Q, R, S & 0.138 & 0.123 & 0.115 & 0.108 & 0.100 & 0.093 & 0.087 & 0.082 & 0.078 & 0.072 \\
\hline \hline Channels & 11 & 12 & 13 & 14 & 15 & 16 & 17 & 18 & 19 & 20 \\
\hline A, B, C, D & 0.055 & 0.052 & 0.049 & 0.045 & 0.043 & 0.040 & 0.038 & 0.036 & 0.034 & 0.034 \\
E, F, G, H, J, K, L, M & 0.066 & 0.062 & 0.058 & 0.054 & 0.050 & 0.047 & 0.045 & 0.042 & 0.040 & 0.040 \\
P, Q, R, S & 0.068 & 0.064 & 0.059 & 0.056 & 0.052 & 0.049 & 0.046 & 0.044 & 0.042 & 0.042 \\
\hline MPACT & & & & & Power Peaking Factors & & & \\
Channels & 1 & 2 & 3 & 4 & 5 & 6 & 7 & 8 & 9 & 10 \\
\hline A, B, C, D & 0.087 & 0.079 & 0.072 & 0.067 & 0.062 & 0.058 & 0.054 & 0.050 & 0.047 & 0.044 \\
E, F, G, H, J, K, L, M & 0.102 & 0.093 & 0.085 & 0.079 & 0.073 & 0.068 & 0.064 & 0.060 & 0.056 & 0.052 \\
P, Q, R, S & 0.106 & 0.096 & 0.089 & 0.082 & 0.076 & 0.071 & 0.066 & 0.062 & 0.058 & 0.054 \\
\hline \hline Channels & 11 & 12 & 13 & 14 & 15 & 16 & 17 & 18 & 19 & 20 \\
\hline A, B, C, D & 0.041 & 0.038 & 0.036 & 0.033 & 0.031 & 0.029 & 0.027 & 0.026 & 0.025 & 0.025 \\
E, F, G, H, J, K, L, M & 0.049 & 0.045 & 0.042 & 0.039 & 0.037 & 0.034 & 0.032 & 0.031 & 0.029 & 0.029 \\
P, Q, R, S & 0.050 & 0.047 & 0.044 & 0.041 & 0.038 & 0.036 & 0.033 & 0.032 & 0.030 & 0.030 \\
\hline
\end{tabular}




\subsection{DEPLETION}

\subsubsection{Problem 5.2.1}

\subsubsection{Description}

Problem 5.2.1 uses the same geometry, temperatures, and materials as Problem 5.1.2. The control rod does not move during the depletion. As with Problems 5.1.1 and 5.1.2, each fuel element is divided into 20 segments, each representing $5 \%$ of the active fuel length.

\subsubsection{Reference Solution}

The neutron multiplication factor results are shown in Table 6.17. The timing variables for Shift and MPACT are shown in Table 6.18. Power peaking factors for the three types of charge pan pins are displayed for all time steps in Figures 23-25. The peaking factors are fully tabulated in Tables 6.19-6.54. The neutron multiplication factor agrees to approximately $200 \mathrm{pcm}$ throughout the entire burnup.

As with Problem 5.1.2, the power peaking factors show noticeable differences between the MPACT and Shift peaking factors. The overall shape agrees well. The MPACT peaking factors reveal a larger flux depression with the control rod inserted compared to it fully withdrawn in Problem 5.1.1. The addition that Problem 5.2.1 confirms is that the discrepancy is not due to differences in burnup. This behavior is noted for all three charge pan pins, revealing that the discrepancy had no radial dependence.

Table 6.17. Neutron multiplication factor for the reference solution of Problem 5.2.1.

\begin{tabular}{rrrrr}
\hline Step & $\begin{array}{r}\text { Burnup } \\
{\left[\frac{\mathrm{MWd}}{\mathrm{MtU}}\right]}\end{array}$ & $\begin{array}{c}\text { Shift } \\
\mathrm{k}_{\mathrm{eff}}(1 \sigma)\end{array}$ & \multicolumn{1}{c}{$\begin{array}{c}\text { MPACT } \\
\mathrm{k}_{\mathrm{eff}}(1 \sigma)\end{array}$} & $\begin{array}{c}\text { Difference } \\
{[\mathrm{pcm}]}\end{array}$ \\
\hline 0 & 0.0 & $1.02840(7)$ & $1.02710(20)$ & $-129(21)$ \\
1 & 25.0 & $1.00952(7)$ & $1.00872(20)$ & $-80(21)$ \\
2 & 50.0 & $1.0102(7)$ & $1.01006(20)$ & $-14(21)$ \\
3 & 100.0 & $1.01305(7)$ & $1.01366(20)$ & $60(21)$ \\
4 & 200.0 & $1.01834(7)$ & $1.01966(20)$ & $132(21)$ \\
5 & 300.0 & $1.02207(7)$ & $1.02389(20)$ & $183(21)$ \\
6 & 400.0 & $1.02462(7)$ & $1.02668(20)$ & $206(21)$ \\
7 & 500.0 & $1.02621(7)$ & $1.02840(20)$ & $219(21)$ \\
8 & 600.0 & $1.02732(7)$ & $1.02933(20)$ & $200(21)$ \\
9 & 700.0 & $1.02762(7)$ & $1.02981(20)$ & $220(21)$ \\
10 & 800.0 & $1.02763(8)$ & $1.02947(20)$ & $184(21)$ \\
11 & 900.0 & $1.0273(7)$ & $1.02906(20)$ & $176(21)$ \\
12 & 1000.0 & $1.02665(8)$ & $1.02808(20)$ & $143(21)$ \\
13 & 1100.0 & $1.02575(8)$ & $1.02699(20)$ & $124(21)$ \\
14 & 1200.0 & $1.0246(8)$ & $1.0257(20)$ & $109(21)$ \\
15 & 1300.0 & $1.02323(8)$ & $1.02423(20)$ & $99(21)$ \\
\hline
\end{tabular}



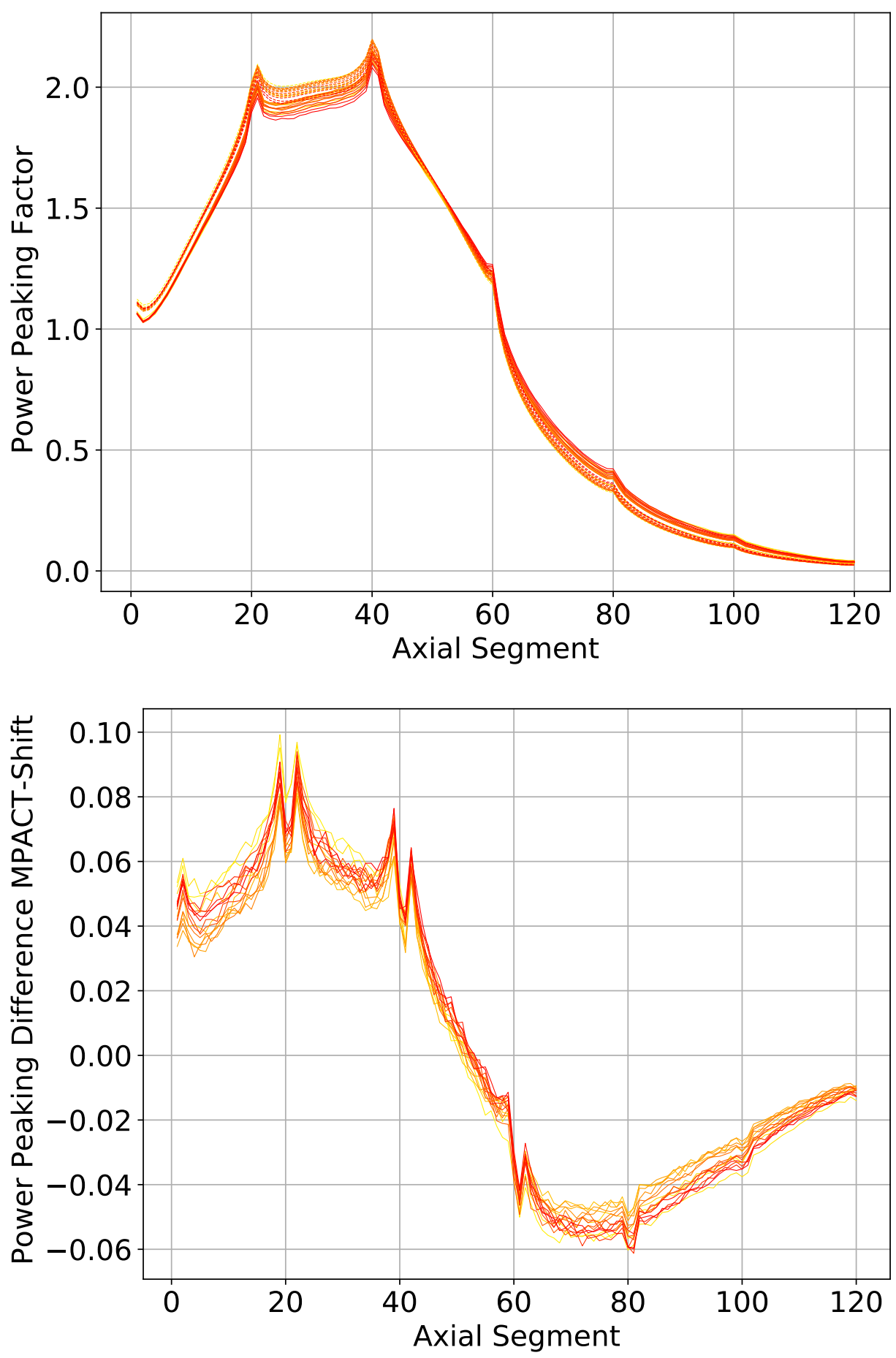

Figure 23. Power peaking factors for rods A-D in Problem 5.2.1 for Shift and MPACT with the latter having dashed lines (top). The absolute difference in the peaking factors MPACT-Shift (bottom). Yellow represents time step 0 and red represents time step 15, with a gradient of colors in between. 

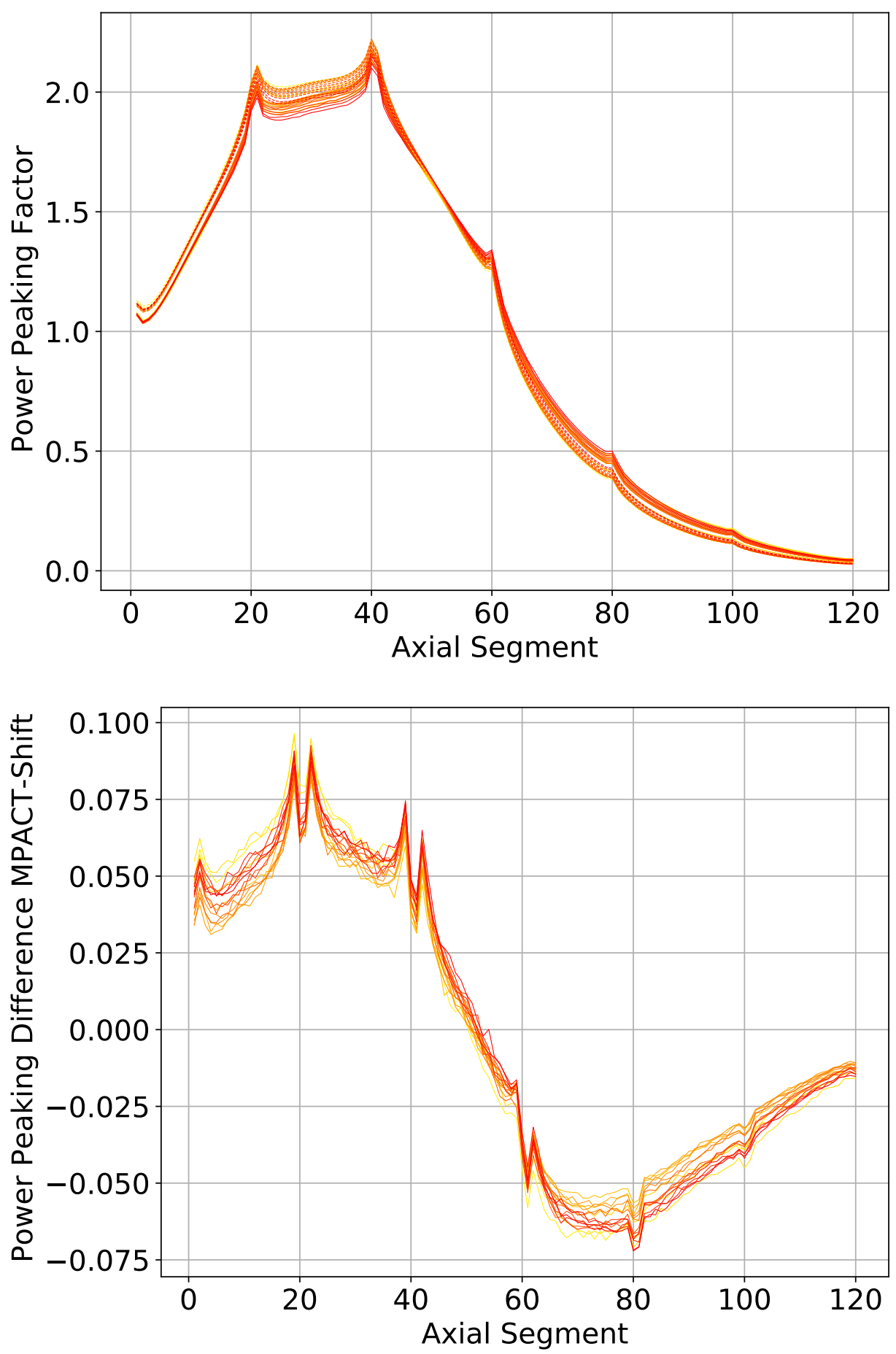

Figure 24. Power peaking factors for rods E-M in Problem 5.2.1 for Shift and MPACT with the latter having dashed lines (top). The absolute difference in the peaking factors MPACT-Shift (bottom). Yellow represents time step 0 and red represents time step 15, with a gradient of colors in between. 

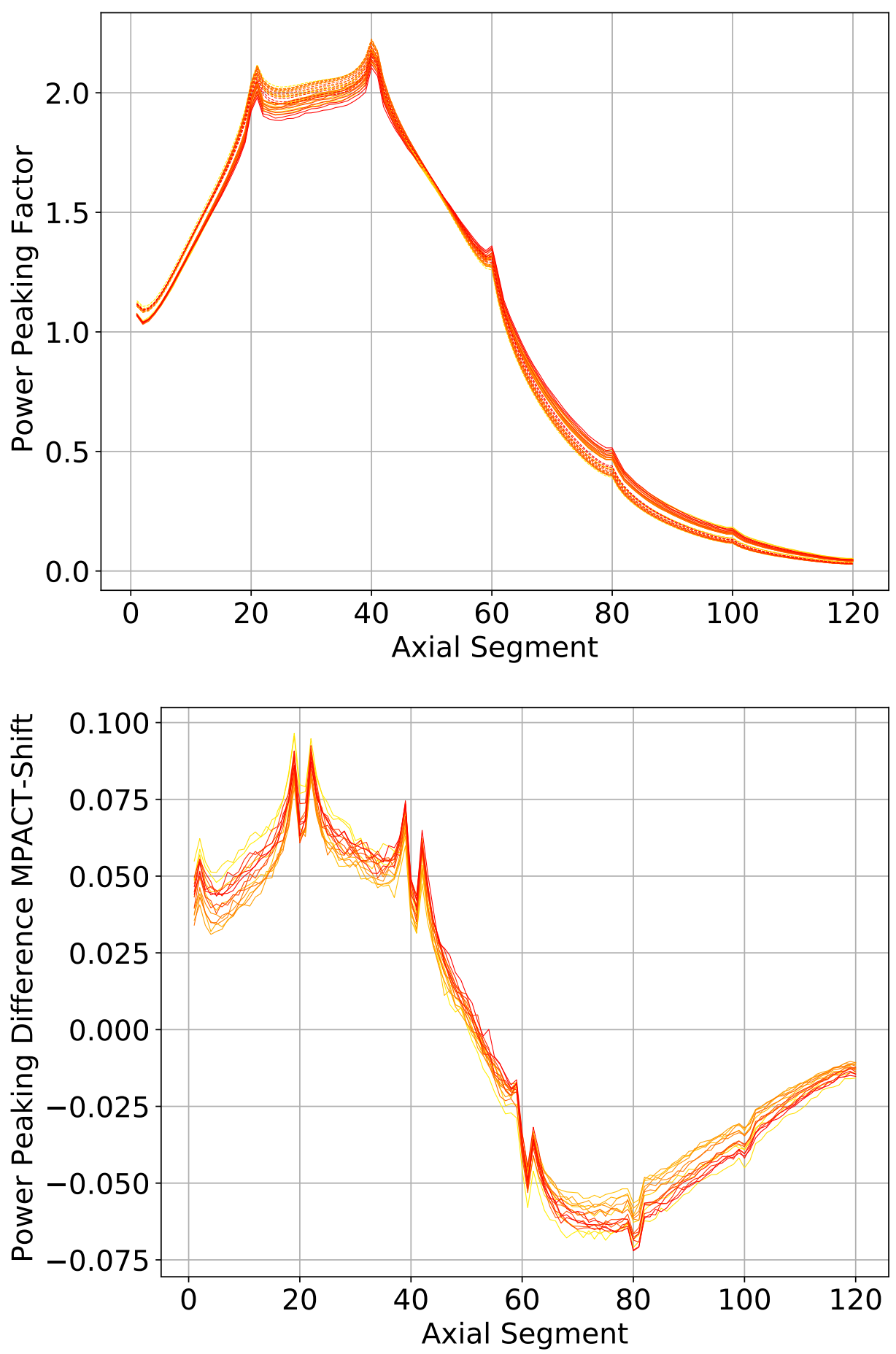

Figure 25. Power peaking factors for rods P-S in Problem 5.2.1 for Shift and MPACT with the latter having dashed lines (top). The absolute difference in the peaking factors MPACT-Shift (bottom). Yellow represents time step 0 and red represents time step 15, with a gradient of colors in between. 
Table 6.18. Timing variables for the reference solution of Problem 5.2.1

\begin{tabular}{|c|c|c|c|c|c|c|}
\hline \multicolumn{7}{|c|}{$\overline{\text { Shift }}$} \\
\hline $\begin{array}{c}\text { Depletion } \\
\text { Solves }\end{array}$ & $\begin{array}{c}\text { Total } \\
\text { Cycles per } \\
\text { Transport } \\
\text { Solve }\end{array}$ & $\begin{array}{l}\text { Active } \\
\text { Cycles per } \\
\text { Transport } \\
\text { Solve }\end{array}$ & $\begin{array}{l}\text { Histories } \\
\text { per Cycle }\end{array}$ & $\begin{array}{c}\text { Compute } \\
\text { Nodes }\end{array}$ & $\begin{array}{c}\text { Cores } \\
\text { per Node }\end{array}$ & $\begin{array}{c}\text { Wall } \\
\text { Total Time } \\
\text { [hours] }\end{array}$ \\
\hline 15 & 1500 & 1200 & 100000 & 8 & 48 & 11.26 \\
\hline \multicolumn{7}{|c|}{ MPACT } \\
\hline $\begin{array}{c}\text { Depletion } \\
\text { Solves }\end{array}$ & $\begin{array}{c}k_{\mathrm{eff}} \\
\text { Convergence } \\
\text { Criteria }\end{array}$ & $\begin{array}{c}\text { Source } \\
\text { Convergence } \\
\text { Criteria }\end{array}$ & $\begin{array}{c}\text { Ray } \\
\text { Spacing } \\
{[\mathrm{cm}]}\end{array}$ & $\begin{array}{c}\text { Compute } \\
\text { Nodes }\end{array}$ & $\begin{array}{c}\text { Cores } \\
\text { per Node }\end{array}$ & $\begin{array}{c}\text { Wall } \\
\text { Total Time } \\
\text { [hours] }\end{array}$ \\
\hline 15 & $1.0 \times 10^{-04}$ & $1.0 \times 10^{-03}$ & 0.01 & 2 & 128 & 3.81 \\
\hline
\end{tabular}


Fuel Channels A, B, C, and D

Table 6.19. Problem 5.2.1 reference solution Shift power peaking factors for axial segments in fuel channels $A, B, C$, and $D$ in element 1 .

\begin{tabular}{|c|c|c|c|c|c|c|c|c|c|c|c|}
\hline \multirow[t]{2}{*}{ Step } & \multirow{2}{*}{$\begin{array}{l}\text { Burnup } \\
{\left[\frac{\mathrm{MWd}}{\mathrm{M} \mathrm{dU}}\right]}\end{array}$} & \multicolumn{10}{|c|}{ Power Peaking Factors } \\
\hline & & 1 & 2 & 3 & 4 & 5 & 6 & 7 & 8 & 9 & 10 \\
\hline 0 & 0.0 & 1.069 & 1.036 & 1.051 & 1.076 & 1.112 & 1.149 & 1.196 & 1.240 & 1.284 & 1.333 \\
\hline 1 & 25.0 & 1.064 & 1.030 & 1.044 & 1.068 & 1.101 & 1.138 & 1.179 & 1.224 & 1.270 & 1.315 \\
\hline 2 & 50.0 & 1.065 & 1.029 & 1.042 & 1.069 & 1.099 & 1.138 & 1.179 & 1.226 & 1.271 & 1.317 \\
\hline 3 & 100.0 & 1.057 & 1.025 & 1.036 & 1.063 & 1.097 & 1.136 & 1.177 & 1.221 & 1.270 & 1.315 \\
\hline 4 & 200.0 & 1.065 & 1.031 & 1.045 & 1.068 & 1.105 & 1.143 & 1.185 & 1.230 & 1.277 & 1.322 \\
\hline 5 & 300.0 & 1.062 & 1.026 & 1.038 & 1.065 & 1.100 & 1.140 & 1.182 & 1.230 & 1.276 & 1.326 \\
\hline 6 & 400.0 & 1.065 & 1.033 & 1.045 & 1.071 & 1.108 & 1.147 & 1.192 & 1.235 & 1.286 & 1.336 \\
\hline 7 & 500.0 & 1.061 & 1.026 & 1.039 & 1.065 & 1.099 & 1.138 & 1.181 & 1.231 & 1.276 & 1.330 \\
\hline 8 & 600.0 & 1.061 & 1.029 & 1.040 & 1.069 & 1.103 & 1.144 & 1.186 & 1.235 & 1.279 & 1.326 \\
\hline 9 & 700.0 & 1.064 & 1.031 & 1.046 & 1.072 & 1.105 & 1.147 & 1.190 & 1.239 & 1.287 & 1.334 \\
\hline 10 & 800.0 & 1.061 & 1.025 & 1.039 & 1.067 & 1.103 & 1.139 & 1.184 & 1.231 & 1.279 & 1.329 \\
\hline 11 & 900.0 & 1.060 & 1.028 & 1.043 & 1.072 & 1.105 & 1.142 & 1.188 & 1.232 & 1.282 & 1.327 \\
\hline 12 & 1000.0 & 1.065 & 1.030 & 1.045 & 1.070 & 1.102 & 1.146 & 1.190 & 1.235 & 1.283 & 1.330 \\
\hline 13 & 1100.0 & 1.059 & 1.022 & 1.039 & 1.063 & 1.100 & 1.141 & 1.185 & 1.228 & 1.275 & 1.324 \\
\hline 14 & 1200.0 & 1.061 & 1.026 & 1.043 & 1.070 & 1.100 & 1.143 & 1.188 & 1.233 & 1.278 & 1.326 \\
\hline 15 & 1300.0 & 1.060 & 1.024 & 1.043 & 1.067 & 1.102 & 1.141 & 1.187 & 1.229 & 1.279 & 1.325 \\
\hline & & 11 & 12 & 13 & 14 & 15 & 16 & 17 & 18 & 19 & 20 \\
\hline 0 & 0.0 & 1.381 & 1.430 & 1.477 & 1.526 & 1.576 & 1.630 & 1.686 & 1.749 & 1.813 & 1.941 \\
\hline 1 & 25.0 & 1.360 & 1.412 & 1.455 & 1.503 & 1.554 & 1.606 & 1.659 & 1.718 & 1.781 & 1.913 \\
\hline 2 & 50.0 & 1.363 & 1.410 & 1.454 & 1.503 & 1.551 & 1.604 & 1.658 & 1.717 & 1.778 & 1.912 \\
\hline 3 & 100.0 & 1.363 & 1.410 & 1.460 & 1.508 & 1.554 & 1.611 & 1.665 & 1.723 & 1.793 & 1.922 \\
\hline 4 & 200.0 & 1.373 & 1.419 & 1.468 & 1.520 & 1.569 & 1.622 & 1.675 & 1.739 & 1.808 & 1.943 \\
\hline 5 & 300.0 & 1.373 & 1.421 & 1.472 & 1.519 & 1.572 & 1.622 & 1.679 & 1.739 & 1.813 & 1.946 \\
\hline 6 & 400.0 & 1.384 & 1.433 & 1.478 & 1.529 & 1.576 & 1.633 & 1.689 & 1.750 & 1.819 & 1.956 \\
\hline 7 & 500.0 & 1.377 & 1.424 & 1.476 & 1.526 & 1.577 & 1.629 & 1.685 & 1.743 & 1.813 & 1.951 \\
\hline 8 & 600.0 & 1.375 & 1.425 & 1.477 & 1.524 & 1.574 & 1.630 & 1.679 & 1.745 & 1.815 & 1.949 \\
\hline 9 & 700.0 & 1.380 & 1.430 & 1.478 & 1.524 & 1.576 & 1.629 & 1.688 & 1.746 & 1.813 & 1.949 \\
\hline 10 & 800.0 & 1.377 & 1.426 & 1.474 & 1.525 & 1.570 & 1.625 & 1.679 & 1.742 & 1.808 & 1.946 \\
\hline 11 & 900.0 & 1.375 & 1.421 & 1.469 & 1.519 & 1.567 & 1.619 & 1.675 & 1.731 & 1.797 & 1.938 \\
\hline 12 & 1000.0 & 1.377 & 1.421 & 1.469 & 1.516 & 1.564 & 1.616 & 1.667 & 1.729 & 1.794 & 1.929 \\
\hline 13 & 1100.0 & 1.369 & 1.412 & 1.462 & 1.512 & 1.562 & 1.614 & 1.663 & 1.718 & 1.790 & 1.921 \\
\hline 14 & 1200.0 & 1.370 & 1.422 & 1.470 & 1.509 & 1.562 & 1.607 & 1.661 & 1.722 & 1.788 & 1.916 \\
\hline 15 & 1300.0 & 1.372 & 1.417 & 1.462 & 1.509 & 1.550 & 1.601 & 1.653 & 1.710 & 1.774 & 1.908 \\
\hline
\end{tabular}


Table 6.20. Problem 5.2.1 reference solution MPACT power peaking factors for axial segments in fuel channels $\mathrm{A}, \mathrm{B}, \mathrm{C}$, and $\mathrm{D}$ in element 1.

\begin{tabular}{|c|c|c|c|c|c|c|c|c|c|c|c|}
\hline \multirow[t]{2}{*}{ Step } & \multirow{2}{*}{$\begin{array}{l}\text { Burnup } \\
{\left[\frac{\mathrm{MWd}}{\mathrm{MtU}}\right]}\end{array}$} & \multicolumn{10}{|c|}{ Power Peaking Factors } \\
\hline & & 1 & 2 & 3 & 4 & 5 & 6 & 7 & 8 & 9 & 10 \\
\hline 0 & 0.0 & 1.124 & 1.099 & 1.105 & 1.128 & 1.161 & 1.201 & 1.246 & 1.294 & 1.343 & 1.392 \\
\hline 1 & 25.0 & 1.116 & 1.090 & 1.096 & 1.117 & 1.149 & 1.188 & 1.232 & 1.278 & 1.326 & 1.374 \\
\hline 2 & 50.0 & 1.112 & 1.086 & 1.092 & 1.113 & 1.145 & 1.184 & 1.228 & 1.274 & 1.322 & 1.370 \\
\hline 3 & 100.0 & 1.105 & 1.079 & 1.084 & 1.106 & 1.139 & 1.178 & 1.222 & 1.269 & 1.317 & 1.366 \\
\hline 4 & 200.0 & 1.101 & 1.075 & 1.081 & 1.103 & 1.136 & 1.177 & 1.221 & 1.269 & 1.318 & 1.368 \\
\hline 5 & 300.0 & 1.098 & 1.073 & 1.080 & 1.103 & 1.136 & 1.177 & 1.222 & 1.270 & 1.320 & 1.370 \\
\hline 6 & 400.0 & 1.099 & 1.074 & 1.081 & 1.104 & 1.138 & 1.179 & 1.224 & 1.272 & 1.322 & 1.372 \\
\hline 7 & 500.0 & 1.101 & 1.075 & 1.082 & 1.105 & 1.139 & 1.181 & 1.226 & 1.275 & 1.324 & 1.375 \\
\hline 8 & 600.0 & 1.103 & 1.078 & 1.084 & 1.108 & 1.141 & 1.183 & 1.228 & 1.277 & 1.326 & 1.376 \\
\hline 9 & 700.0 & 1.105 & 1.080 & 1.087 & 1.110 & 1.144 & 1.185 & 1.231 & 1.279 & 1.328 & 1.378 \\
\hline 10 & 800.0 & 1.107 & 1.081 & 1.088 & 1.111 & 1.145 & 1.186 & 1.231 & 1.279 & 1.329 & 1.378 \\
\hline 11 & 900.0 & 1.108 & 1.083 & 1.090 & 1.113 & 1.147 & 1.188 & 1.233 & 1.281 & 1.330 & 1.379 \\
\hline 12 & 1000.0 & 1.110 & 1.084 & 1.091 & 1.114 & 1.147 & 1.188 & 1.233 & 1.281 & 1.329 & 1.378 \\
\hline 13 & 1100.0 & 1.110 & 1.085 & 1.092 & 1.115 & 1.149 & 1.189 & 1.234 & 1.282 & 1.330 & 1.378 \\
\hline 14 & 1200.0 & 1.110 & 1.084 & 1.091 & 1.113 & 1.147 & 1.187 & 1.232 & 1.279 & 1.327 & 1.375 \\
\hline 15 & 1300.0 & 1.110 & 1.085 & 1.092 & 1.115 & 1.148 & 1.188 & 1.233 & 1.279 & 1.327 & 1.374 \\
\hline & & 11 & 12 & 13 & 14 & 15 & 16 & 17 & 18 & 19 & 20 \\
\hline 0 & 0.0 & 1.442 & 1.492 & 1.542 & 1.594 & 1.646 & 1.702 & 1.763 & 1.831 & 1.911 & 2.024 \\
\hline 1 & 25.0 & 1.422 & 1.471 & 1.519 & 1.568 & 1.619 & 1.673 & 1.731 & 1.798 & 1.876 & 1.987 \\
\hline 2 & 50.0 & 1.418 & 1.467 & 1.515 & 1.564 & 1.615 & 1.669 & 1.728 & 1.794 & 1.871 & 1.982 \\
\hline 3 & 100.0 & 1.415 & 1.464 & 1.514 & 1.564 & 1.615 & 1.670 & 1.730 & 1.798 & 1.877 & 1.991 \\
\hline 4 & 200.0 & 1.417 & 1.467 & 1.517 & 1.568 & 1.621 & 1.677 & 1.738 & 1.807 & 1.888 & 2.005 \\
\hline 5 & 300.0 & 1.420 & 1.470 & 1.521 & 1.572 & 1.626 & 1.682 & 1.743 & 1.813 & 1.895 & 2.013 \\
\hline 6 & 400.0 & 1.423 & 1.473 & 1.524 & 1.575 & 1.629 & 1.685 & 1.746 & 1.816 & 1.898 & 2.017 \\
\hline 7 & 500.0 & 1.425 & 1.475 & 1.526 & 1.577 & 1.630 & 1.687 & 1.748 & 1.817 & 1.899 & 2.018 \\
\hline 8 & 600.0 & 1.427 & 1.477 & 1.527 & 1.578 & 1.631 & 1.687 & 1.748 & 1.816 & 1.898 & 2.017 \\
\hline 9 & 700.0 & 1.428 & 1.478 & 1.528 & 1.578 & 1.630 & 1.686 & 1.746 & 1.813 & 1.894 & 2.010 \\
\hline 10 & 800.0 & 1.428 & 1.477 & 1.527 & 1.577 & 1.629 & 1.684 & 1.744 & 1.812 & 1.892 & 2.009 \\
\hline 11 & 900.0 & 1.428 & 1.477 & 1.526 & 1.576 & 1.627 & 1.681 & 1.740 & 1.806 & 1.885 & 2.000 \\
\hline 12 & 1000.0 & 1.427 & 1.476 & 1.524 & 1.574 & 1.624 & 1.678 & 1.736 & 1.802 & 1.881 & 1.996 \\
\hline 13 & 1100.0 & 1.427 & 1.475 & 1.523 & 1.571 & 1.621 & 1.673 & 1.731 & 1.795 & 1.872 & 1.984 \\
\hline 14 & 1200.0 & 1.422 & 1.470 & 1.517 & 1.565 & 1.614 & 1.666 & 1.723 & 1.787 & 1.864 & 1.976 \\
\hline 15 & 1300.0 & 1.422 & 1.469 & 1.515 & 1.562 & 1.611 & 1.662 & 1.717 & 1.780 & 1.855 & 1.964 \\
\hline
\end{tabular}


Table 6.21. Problem 5.2.1 reference solution Shift power peaking factors for axial segments in fuel channels A, B, C, and D in element 2.

\begin{tabular}{|c|c|c|c|c|c|c|c|c|c|c|c|}
\hline \multirow[t]{2}{*}{ Step } & Burnup & \multicolumn{10}{|c|}{ Power Peaking Factors } \\
\hline & {$\left[\frac{\mathrm{MWd}}{\mathrm{MtU}}\right]$} & 1 & 2 & 3 & 4 & 5 & 6 & 7 & 8 & 9 & 10 \\
\hline 0 & 0.0 & 2.012 & 1.945 & 1.934 & 1.930 & 1.928 & 1.934 & 1.939 & 1.943 & 1.954 & 1.961 \\
\hline 1 & 25.0 & 1.974 & 1.904 & 1.895 & 1.891 & 1.890 & 1.893 & 1.900 & 1.906 & 1.906 & 1.919 \\
\hline 2 & 50.0 & 1.979 & 1.905 & 1.893 & 1.890 & 1.890 & 1.898 & 1.903 & 1.908 & 1.914 & 1.921 \\
\hline 3 & 100.0 & 1.990 & 1.918 & 1.902 & 1.901 & 1.906 & 1.910 & 1.917 & 1.922 & 1.930 & 1.940 \\
\hline 4 & 200.0 & 2.011 & 1.937 & 1.924 & 1.924 & 1.925 & 1.927 & 1.928 & 1.940 & 1.945 & 1.948 \\
\hline 5 & 300.0 & 2.013 & 1.939 & 1.930 & 1.924 & 1.929 & 1.931 & 1.936 & 1.942 & 1.946 & 1.958 \\
\hline 6 & 400.0 & 2.028 & 1.952 & 1.942 & 1.937 & 1.934 & 1.942 & 1.947 & 1.952 & 1.961 & 1.966 \\
\hline 7 & 500.0 & 2.022 & 1.944 & 1.938 & 1.926 & 1.932 & 1.935 & 1.943 & 1.948 & 1.958 & 1.961 \\
\hline 8 & 600.0 & 2.013 & 1.941 & 1.928 & 1.925 & 1.930 & 1.931 & 1.938 & 1.944 & 1.959 & 1.960 \\
\hline 9 & 700.0 & 2.020 & 1.938 & 1.926 & 1.926 & 1.929 & 1.932 & 1.933 & 1.943 & 1.950 & 1.955 \\
\hline 10 & 800.0 & 2.014 & 1.936 & 1.922 & 1.916 & 1.919 & 1.924 & 1.931 & 1.938 & 1.943 & 1.954 \\
\hline 11 & 900.0 & 2.004 & 1.927 & 1.912 & 1.909 & 1.911 & 1.915 & 1.919 & 1.924 & 1.935 & 1.941 \\
\hline 12 & 1000.0 & 1.996 & 1.915 & 1.906 & 1.902 & 1.899 & 1.909 & 1.915 & 1.918 & 1.929 & 1.936 \\
\hline 13 & 1100.0 & 1.986 & 1.910 & 1.897 & 1.893 & 1.892 & 1.896 & 1.906 & 1.914 & 1.919 & 1.930 \\
\hline 14 & 1200.0 & 1.984 & 1.903 & 1.891 & 1.891 & 1.892 & 1.895 & 1.901 & 1.906 & 1.921 & 1.921 \\
\hline 15 & 1300.0 & 1.969 & 1.894 & 1.885 & 1.880 & 1.877 & 1.886 & 1.887 & 1.896 & 1.901 & 1.914 \\
\hline & & 11 & 12 & 13 & 14 & 15 & 16 & 17 & 18 & 19 & 20 \\
\hline 0 & 0.0 & 1.963 & 1.968 & 1.976 & 1.979 & 1.992 & 1.999 & 2.006 & 2.026 & 2.047 & 2.147 \\
\hline 1 & 25.0 & 1.927 & 1.931 & 1.935 & 1.940 & 1.948 & 1.957 & 1.974 & 1.993 & 2.015 & 2.103 \\
\hline 2 & 50.0 & 1.927 & 1.934 & 1.939 & 1.946 & 1.952 & 1.961 & 1.978 & 1.995 & 2.018 & 2.111 \\
\hline 3 & 100.0 & 1.943 & 1.947 & 1.957 & 1.962 & 1.973 & 1.981 & 1.992 & 2.009 & 2.034 & 2.131 \\
\hline 4 & 200.0 & 1.962 & 1.964 & 1.970 & 1.978 & 1.985 & 1.988 & 2.007 & 2.018 & 2.047 & 2.142 \\
\hline 5 & 300.0 & 1.963 & 1.970 & 1.973 & 1.984 & 1.990 & 1.998 & 2.011 & 2.031 & 2.055 & 2.152 \\
\hline 6 & 400.0 & 1.971 & 1.977 & 1.982 & 1.988 & 1.991 & 2.000 & 2.013 & 2.034 & 2.059 & 2.149 \\
\hline 7 & 500.0 & 1.970 & 1.976 & 1.980 & 1.992 & 1.996 & 2.001 & 2.018 & 2.035 & 2.058 & 2.160 \\
\hline 8 & 600.0 & 1.971 & 1.972 & 1.981 & 1.984 & 1.990 & 1.993 & 2.006 & 2.027 & 2.052 & 2.153 \\
\hline 9 & 700.0 & 1.966 & 1.973 & 1.974 & 1.981 & 1.984 & 1.993 & 2.006 & 2.025 & 2.048 & 2.152 \\
\hline 10 & 800.0 & 1.956 & 1.965 & 1.965 & 1.979 & 1.980 & 1.989 & 2.002 & 2.025 & 2.042 & 2.150 \\
\hline 11 & 900.0 & 1.948 & 1.952 & 1.957 & 1.964 & 1.972 & 1.981 & 1.991 & 2.005 & 2.038 & 2.132 \\
\hline 12 & 1000.0 & 1.940 & 1.947 & 1.956 & 1.959 & 1.960 & 1.970 & 1.985 & 2.000 & 2.024 & 2.127 \\
\hline 13 & 1100.0 & 1.932 & 1.939 & 1.944 & 1.952 & 1.959 & 1.966 & 1.978 & 1.996 & 2.024 & 2.121 \\
\hline 14 & 1200.0 & 1.927 & 1.932 & 1.939 & 1.944 & 1.949 & 1.959 & 1.971 & 1.989 & 2.016 & 2.114 \\
\hline 15 & 1300.0 & 1.913 & 1.918 & 1.928 & 1.931 & 1.941 & 1.945 & 1.958 & 1.974 & 2.004 & 2.097 \\
\hline
\end{tabular}


Table 6.22. Problem 5.2.1 reference solution MPACT power peaking factors for axial segments in fuel channels A, B, C, and D in element 2.

\begin{tabular}{|c|c|c|c|c|c|c|c|c|c|c|c|}
\hline \multirow[t]{2}{*}{ Step } & Burnup & \multicolumn{10}{|c|}{ Power Peaking Factors } \\
\hline & {$\left[\frac{\mathrm{MWd}}{\mathrm{MtU}}\right]$} & 1 & 2 & 3 & 4 & 5 & 6 & 7 & 8 & 9 & 10 \\
\hline 0 & 0.0 & 2.095 & 2.040 & 2.018 & 2.007 & 2.003 & 2.005 & 2.008 & 2.013 & 2.019 & 2.024 \\
\hline 1 & 25.0 & 2.055 & 1.999 & 1.976 & 1.965 & 1.961 & 1.962 & 1.966 & 1.971 & 1.976 & .981 \\
\hline 2 & 50.0 & 2.051 & 1.996 & 1.975 & 1.964 & 1.961 & 1.963 & 1.967 & 1.972 & 1.978 & 1.983 \\
\hline 3 & 100.0 & 2.061 & 2.005 & 1.982 & 1.972 & 1.969 & 1.970 & 1.975 & 1.980 & 1.986 & 1.991 \\
\hline 4 & 200.0 & 2.077 & 2.019 & 1.997 & 1.986 & 1.984 & 1.986 & 1.990 & 1.996 & 2.002 & 2.008 \\
\hline 5 & 300.0 & 2.085 & 2.027 & 2.004 & 1.994 & 1.991 & 1.993 & 1.998 & 2.004 & 2.010 & 2.016 \\
\hline 6 & 400.0 & 2.090 & 2.031 & 2.008 & 1.997 & 1.994 & 1.996 & 2.001 & 2.007 & 2.013 & 2.019 \\
\hline 7 & 500.0 & 2.090 & 2.031 & 2.007 & 1.997 & 1.994 & 1.996 & 2. & 2.006 & 3 & 2.018 \\
\hline 8 & 600.0 & 8 & 2.028 & 2.0 & & 1.991 & 1.993 & & & & 2.015 \\
\hline 9 & 700.0 & 2.081 & 2.022 & 2.000 & 1.989 & 1.987 & 1.989 & 1. & 1.999 & 2.005 & 2.011 \\
\hline 10 & 800.0 & .078 & 2.0 & 1.9 & 1.983 & 1.980 & 1.982 & & 1.9 & 8 & .003 \\
\hline 11 & 900.0 & 2.068 & 2.009 & 1.9 & 1.976 & 1.973 & 1.975 & & 1.985 & 1.9 & 1.996 \\
\hline 12 & 1000.0 & 2.063 & 2.003 & 1.9 & 1.968 & 1.964 & 1.966 & 1. & 1. & 1 & 1.986 \\
\hline 13 & 1100.0 & 0 & 1.9 & & & 55 & 1.957 & & & & 978 \\
\hline 14 & 1200.0 & 2.040 & 1.981 & 1.957 & 1.946 & 1.942 & 1.943 & 1.947 & 1.952 & 1.958 & 1.963 \\
\hline 15 & 1300.0 & 2.027 & 1.970 & 1.947 & 1.937 & 1.933 & 1.935 & 1.939 & 1.944 & 1.950 & 1.955 \\
\hline & & 11 & 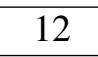 & & 14 & 15 & 16 & & 18 & 17 & 20 \\
\hline 0 & 0.0 & 2.028 & 0 & 2.0 & 040 & 2.045 & 2.054 & 2.0 & 2.088 & 2.121 & 2.188 \\
\hline 1 & 25.0 & 1.986 & 1.990 & & 1.999 & 2.005 & 2.014 & & 2.050 & 2.084 & 2.154 \\
\hline 2 & 50.0 & 1.988 & 1.993 & 1.9 & 2.001 & 2.008 & 2.016 & 2.1 & 2.052 & 2.085 & 2.153 \\
\hline 3 & 100.0 & 1.997 & 2.001 & 2.006 & 2.010 & 2.017 & 2.026 & 2.0 & 2.062 & 2.097 & 2.168 \\
\hline 4 & 200.0 & 2.013 & 2.018 & 2.022 & 2.027 & 2.034 & 2.043 & 2.0 & 2.079 & 2.114 & 2.187 \\
\hline 5 & & 2.021 & 2.026 & 2.030 & 2.035 & 2.041 & 2.050 & & 2.087 & 2.122 & 2.196 \\
\hline 6 & 400.0 & 2.024 & 2.029 & 2.033 & 2.038 & 2.044 & 2.053 & & 2.089 & 2.125 & 2.199 \\
\hline 7 & 500.0 & 2.024 & 2.028 & 2.032 & 2.037 & 2.043 & 2.052 & 2.066 & 2.088 & 2.124 & 2.199 \\
\hline 8 & 600.0 & 2.020 & 2.024 & 2.029 & 2.033 & 2.039 & 2.048 & 2.062 & 2.085 & 2.121 & 2.195 \\
\hline 9 & 700.0 & 2.016 & 2.021 & 2.025 & 2.029 & 2.035 & 2.043 & 2.057 & 2.079 & 2.114 & 2.187 \\
\hline 10 & 800.0 & 2.008 & 2.013 & 2.017 & 2.021 & 2.027 & 2.036 & 2.050 & 2.073 & 2.109 & 2.183 \\
\hline 11 & 900.0 & 2.001 & 2.006 & 2.010 & 2.014 & 2.020 & 2.029 & 2.042 & 2.064 & 2.099 & 2.172 \\
\hline 12 & 1000.0 & 1.991 & 1.995 & 2.000 & 2.004 & 2.010 & 2.019 & 2.034 & 2.056 & 2.092 & 2.166 \\
\hline 13 & 1100.0 & 1.983 & 1.987 & 1.991 & 1.995 & 2.001 & 2.010 & 2.024 & 2.046 & 2.081 & 2.152 \\
\hline 14 & 1200.0 & 1.968 & 1.972 & & 1.982 & 1.988 & 1.997 & & 2.034 & 2.070 & 2.143 \\
\hline 15 & 1300.0 & 1.960 & 1.964 & 1.968 & 1.973 & 1.979 & 1.988 & 2.002 & 2.024 & 2.059 & 2.129 \\
\hline
\end{tabular}


Table 6.23. Problem 5.2.1 reference solution Shift power peaking factors for axial segments in fuel channels A, B, C, and D in element 3.

\begin{tabular}{|c|c|c|c|c|c|c|c|c|c|c|c|}
\hline \multirow[t]{2}{*}{ Step } & \multirow{2}{*}{$\begin{array}{l}\text { Burnup } \\
{\left[\frac{\mathrm{MWd}}{\mathrm{MtU}}\right]}\end{array}$} & \multicolumn{10}{|c|}{ Power Peaking Factors } \\
\hline & & 1 & 2 & 3 & 4 & 5 & 6 & 7 & 8 & 9 & 10 \\
\hline 0 & 0.0 & 2.096 & 1.969 & 1.913 & 1.861 & 1.812 & 1.769 & 1.730 & 1.693 & 1.649 & 1.610 \\
\hline 1 & 25.0 & 2.067 & 1.939 & 1.886 & 1.838 & 1.792 & 1.753 & 1.711 & 1.676 & 1.637 & 1.600 \\
\hline 2 & 50.0 & 2.072 & 1.954 & 1.888 & 1.845 & 1.794 & 1.751 & 1.715 & 1.677 & 1.637 & 1.603 \\
\hline 3 & 100.0 & 2.085 & 1.958 & 1.903 & 1.851 & 1.805 & 1.760 & 1.719 & 1.682 & 1.643 & 1.605 \\
\hline 4 & 200.0 & 2.104 & 1.973 & 1.913 & 1.863 & 1.812 & 1.767 & 1.726 & 1.684 & 1.650 & 1.610 \\
\hline 5 & 300.0 & 2.108 & 1.975 & 1.917 & 1.868 & 1.821 & 1.772 & 1.735 & 1.692 & 1.655 & 1.614 \\
\hline 6 & 400.0 & 2.111 & 1.977 & 1.917 & 1.864 & 1.819 & 1.769 & 1.731 & 1.690 & 1.651 & 1.614 \\
\hline 7 & 500.0 & 2.114 & 1.980 & 1.921 & 1.871 & 1.824 & 1.775 & 1.738 & 1.700 & 1.657 & 1.617 \\
\hline 8 & 600.0 & 2.114 & 1.980 & 1.915 & 1.866 & 1.814 & 1.775 & 1.736 & 1.696 & 1.661 & 1.620 \\
\hline 9 & 700.0 & 2.110 & 1.972 & 1.912 & 1.862 & 1.820 & 1.778 & 1.735 & 1.696 & 1.657 & 1.618 \\
\hline 10 & 800.0 & 2.106 & 1.973 & 1.912 & 1.863 & 1.819 & 1.772 & 1.734 & 1.693 & 1.661 & 1.615 \\
\hline 11 & 900.0 & 2.091 & 1.966 & 1.907 & 1.856 & 1.812 & 1.772 & 1.732 & 1.693 & 1.655 & 1.618 \\
\hline 12 & 1000.0 & 2.083 & 1.960 & 1.900 & 1.848 & 1.808 & 1.766 & 1.725 & 1.690 & 1.653 & 1.619 \\
\hline 13 & 1100.0 & 2.081 & 1.956 & 1.897 & 1.849 & 1.808 & 1.766 & 1.725 & 1.688 & 1.652 & 1.618 \\
\hline 14 & 1200.0 & 2.079 & 1.942 & 1.891 & 1.842 & 1.799 & 1.760 & 1.720 & 1.689 & 1.649 & 1.615 \\
\hline 15 & 1300.0 & 2.065 & 1.931 & 1.884 & 1.835 & 1.794 & 1.756 & 1.718 & 1.684 & 1.652 & 1.613 \\
\hline & & 11 & 12 & 13 & 14 & 15 & 16 & 17 & 18 & 19 & 20 \\
\hline 0 & 0.0 & 1.573 & 1.532 & 1.487 & 1.444 & 1.404 & 1.365 & 1.320 & 1.279 & 1.235 & 1.222 \\
\hline 1 & 25.0 & 1.558 & 1.523 & 1.487 & 1.446 & 1.408 & 1.368 & 1.326 & 1.282 & 1.243 & 1.232 \\
\hline 2 & 50.0 & 1.565 & 1.525 & 1.491 & 1.448 & 1.403 & 1.365 & 1.323 & 1.279 & 1.242 & 1.232 \\
\hline 3 & 100.0 & 1.565 & 1.521 & 1.486 & 1.443 & 1.405 & 1.358 & 1.317 & 1.281 & 1.240 & 1.225 \\
\hline 4 & 200.0 & 1.567 & 1.528 & 1.488 & 1.444 & 1.401 & 1.360 & 1.318 & 1.274 & 1.230 & 1.219 \\
\hline 5 & 300.0 & 1.573 & 1.534 & 1.492 & 1.449 & 1.408 & 1.364 & 1.318 & 1.275 & 1.233 & 1.222 \\
\hline 6 & 400.0 & 1.572 & 1.530 & 1.485 & 1.446 & 1.404 & 1.359 & 1.313 & 1.276 & 1.233 & 1.222 \\
\hline 7 & 500.0 & 1.575 & 1.533 & 1.494 & 1.452 & 1.411 & 1.364 & 1.323 & 1.276 & 1.233 & 1.228 \\
\hline 8 & 600.0 & 1.576 & 1.535 & 1.496 & 1.454 & 1.412 & 1.366 & 1.322 & 1.280 & 1.235 & 1.231 \\
\hline 9 & 700.0 & 1.577 & 1.535 & 1.495 & 1.451 & 1.409 & 1.367 & 1.321 & 1.276 & 1.236 & 1.229 \\
\hline 10 & 800.0 & 1.577 & 1.540 & 1.497 & 1.453 & 1.414 & 1.371 & 1.327 & 1.286 & 1.244 & 1.236 \\
\hline 11 & 900.0 & 1.579 & 1.541 & 1.502 & 1.460 & 1.420 & 1.373 & 1.329 & 1.289 & 1.250 & 1.241 \\
\hline 12 & 1000.0 & 1.578 & 1.541 & 1.500 & 1.458 & 1.413 & 1.374 & 1.333 & 1.290 & 1.250 & 1.247 \\
\hline 13 & 1100.0 & 1.580 & 1.542 & 1.502 & 1.462 & 1.419 & 1.381 & 1.341 & 1.297 & 1.260 & 1.254 \\
\hline 14 & 1200.0 & 1.577 & 1.540 & 1.504 & 1.461 & 1.421 & 1.378 & 1.340 & 1.297 & 1.257 & 1.253 \\
\hline 15 & 1300.0 & 1.581 & 1.541 & 1.506 & 1.464 & 1.425 & 1.387 & 1.344 & 1.303 & 1.267 & 1.261 \\
\hline
\end{tabular}


Table 6.24. Problem 5.2.1 reference solution MPACT power peaking factors for axial segments in fuel channels A, B, C, and D in element 3.

\begin{tabular}{|c|c|c|c|c|c|c|c|c|c|c|c|}
\hline \multirow[t]{2}{*}{ Step } & \multirow{2}{*}{$\begin{array}{l}\text { Burnup } \\
{\left[\frac{\mathrm{MWd}}{\mathrm{MtU}}\right]}\end{array}$} & \multicolumn{10}{|c|}{ Power Peaking Factors } \\
\hline & & 1 & 2 & 3 & 4 & 5 & 6 & 7 & 8 & 9 & 10 \\
\hline 0 & 0.0 & 2.138 & 2.029 & 1.954 & 1.893 & 1.839 & 1.790 & 1.744 & 1.700 & 1.657 & 1.613 \\
\hline 1 & 25.0 & 2.108 & 2.001 & 1.929 & 1.870 & 1.818 & 1.772 & 1.729 & 1.688 & 1.647 & 1.606 \\
\hline 2 & 50.0 & 2.107 & 2.001 & 1.930 & 1.871 & 1.820 & 1.774 & 1.731 & 1.689 & 1.648 & 1.607 \\
\hline 3 & 100.0 & 2.121 & 2.011 & 1.938 & 1.878 & 1.825 & 1.778 & 1.734 & 1.692 & 1.650 & 1.609 \\
\hline 4 & 200.0 & 2.138 & 2.026 & 1.951 & 1.889 & 1.836 & 1.788 & 1.743 & 1.699 & 1.657 & 1.614 \\
\hline 5 & 300.0 & 2.147 & 2.033 & 1.957 & 1.895 & 1.841 & 1.792 & 1.747 & 1.703 & 1.660 & 1.617 \\
\hline 6 & 400.0 & 2.150 & 2.036 & 1.960 & 1.897 & 1.844 & 1.795 & 1.749 & 1.706 & 1.663 & 1.619 \\
\hline 7 & 500.0 & 2.150 & 2.035 & 1.960 & 1.898 & 1.844 & 1.796 & 1.751 & 1.707 & 1.664 & 1.621 \\
\hline 8 & 600.0 & 2.147 & 2.033 & 1.958 & 1.896 & 1.843 & 1.795 & 1.750 & 1.707 & 1.665 & 1.622 \\
\hline 9 & 700.0 & 2.140 & 2.028 & 1.954 & 1.894 & 1.842 & 1.794 & 1.750 & 1.708 & 1.666 & 1.624 \\
\hline 10 & 800.0 & 2.137 & 2.025 & 1.951 & 1.891 & 1.839 & 1.792 & 1.749 & 1.707 & 1.665 & 1.623 \\
\hline 11 & 900.0 & 2.127 & 2.017 & 1.945 & 1.886 & 1.836 & 1.790 & 1.747 & 1.706 & 1.666 & 1.625 \\
\hline 12 & 1000.0 & 2.122 & 2.012 & 1.940 & 1.882 & 1.832 & 1.787 & 1.745 & 1.704 & 1.664 & 1.624 \\
\hline 13 & 1100.0 & 2.110 & 2.003 & 1.934 & 1.877 & 1.828 & 1.784 & 1.743 & 1.703 & 1.664 & 1.625 \\
\hline 14 & 1200.0 & 2.102 & 1.996 & 1.926 & 1.870 & 1.822 & 1.779 & 1.739 & 1.700 & 1.662 & 1.623 \\
\hline 15 & 1300.0 & 2.090 & 1.987 & 1.919 & 1.865 & 1.818 & 1.776 & 1.737 & 1.699 & 1.662 & 1.624 \\
\hline & & 11 & 12 & 13 & 14 & 15 & 16 & 17 & 18 & 19 & 20 \\
\hline 0 & 0.0 & 1.569 & 1.524 & 1.479 & 1.433 & 1.387 & 1.341 & 1.295 & 1.250 & 1.208 & 1.179 \\
\hline 1 & 25.0 & 1.565 & 1.523 & 1.480 & 1.436 & 1.393 & 1.349 & 1.305 & 1.263 & 1.224 & 1.198 \\
\hline 2 & 50.0 & 1.566 & 1.524 & 1.481 & 1.437 & 1.393 & 1.349 & 1.305 & 1.262 & 1.222 & 1.196 \\
\hline 3 & 100.0 & 1.566 & 1.524 & 1.480 & 1.436 & 1.391 & 1.346 & 1.302 & 1.260 & 1.220 & 1.194 \\
\hline 4 & 200.0 & 1.571 & 1.527 & 1.482 & 1.437 & 1.391 & 1.345 & 1.300 & 1.257 & 1.216 & 1.189 \\
\hline 5 & 300.0 & 1.573 & 1.529 & 1.484 & 1.438 & 1.392 & 1.346 & 1.300 & 1.256 & 1.215 & 1.188 \\
\hline 6 & 400.0 & 1.575 & 1.531 & 1.486 & 1.440 & 1.393 & 1.347 & 1.301 & 1.257 & 1.216 & 1.189 \\
\hline 7 & 500.0 & 1.577 & 1.533 & 1.488 & 1.442 & 1.395 & 1.349 & 1.303 & 1.259 & 1.219 & 1.192 \\
\hline 8 & 600.0 & 1.579 & 1.535 & 1.490 & 1.444 & 1.398 & 1.352 & 1.306 & 1.263 & 1.222 & 1.196 \\
\hline 9 & 700.0 & 1.581 & 1.537 & 1.492 & 1.447 & 1.401 & 1.355 & 1.310 & 1.266 & 1.225 & 1.199 \\
\hline 10 & 800.0 & 1.581 & 1.538 & 1.494 & 1.449 & 1.403 & 1.358 & 1.313 & 1.270 & 1.230 & 1.205 \\
\hline 11 & 900.0 & 1.583 & 1.540 & 1.497 & 1.452 & 1.407 & 1.362 & 1.318 & 1.275 & 1.235 & 1.209 \\
\hline 12 & 1000.0 & 1.583 & 1.541 & 1.498 & 1.454 & 1.410 & 1.365 & 1.322 & 1.279 & 1.240 & 1.217 \\
\hline 13 & 1100.0 & 1.584 & 1.543 & 1.501 & 1.457 & 1.414 & 1.370 & 1.326 & 1.284 & 1.245 & 1.221 \\
\hline 14 & 1200.0 & 1.584 & 1.543 & 1.502 & 1.460 & 1.417 & 1.374 & 1.332 & 1.291 & 1.253 & 1.231 \\
\hline 15 & 1300.0 & 1.585 & 1.546 & 1.505 & 1.463 & 1.421 & 1.378 & 1.336 & 1.295 & 1.258 & 1.235 \\
\hline
\end{tabular}


Table 6.25. Problem 5.2.1 reference solution Shift power peaking factors for axial segments in fuel channels A, B, C, and D in element 4.

\begin{tabular}{|c|c|c|c|c|c|c|c|c|c|c|c|}
\hline \multirow[t]{2}{*}{ Step } & \multirow{2}{*}{$\begin{array}{l}\text { Burnup } \\
{\left[\frac{\mathrm{MWd}}{\mathrm{MtU}}\right]}\end{array}$} & \multicolumn{10}{|c|}{ Power Peaking Factors } \\
\hline & & 1 & 2 & 3 & 4 & 5 & 6 & 7 & 8 & 9 & 10 \\
\hline 0 & 0.0 & 1.051 & 0.935 & 0.866 & 0.806 & 0.754 & 0.708 & 0.669 & 0.633 & 0.599 & 0.570 \\
\hline 1 & 25.0 & 1.069 & 0.952 & 0.884 & 0.824 & 0.773 & 0.730 & 0.689 & 0.654 & 0.624 & 0.594 \\
\hline 2 & 50.0 & 1.065 & 0.949 & 0.879 & 0.821 & 0.772 & 0.728 & 0.688 & 0.653 & 0.623 & 0.590 \\
\hline 3 & 100.0 & 1.060 & 0.942 & 0.872 & 0.814 & 0.765 & 0.722 & 0.681 & 0.646 & 0.615 & 0.586 \\
\hline 4 & 200.0 & 1.052 & 0.935 & 0.863 & 0.806 & 0.756 & 0.713 & 0.673 & 0.638 & 0.605 & 0.572 \\
\hline 5 & 300.0 & 1.054 & 0.937 & 0.864 & 0.802 & 0.754 & 0.710 & 0.669 & 0.633 & 0.600 & 0.572 \\
\hline 6 & 400.0 & 1.050 & 0.932 & 0.859 & 0.802 & 0.749 & 0.707 & 0.665 & 0.632 & 0.598 & 0.565 \\
\hline 7 & 500.0 & 1.057 & 0.934 & 0.864 & 0.804 & 0.753 & 0.710 & 0.669 & 0.633 & 0.598 & 0.567 \\
\hline 8 & 600.0 & 1.059 & 0.939 & 0.870 & 0.808 & 0.757 & 0.711 & 0.670 & 0.633 & 0.603 & 0.573 \\
\hline 9 & 700.0 & 1.058 & 0.941 & 0.866 & 0.808 & 0.756 & 0.713 & 0.673 & 0.636 & 0.605 & 0.575 \\
\hline 10 & 800.0 & 1.067 & 0.946 & 0.875 & 0.815 & 0.761 & 0.715 & 0.677 & 0.641 & 0.608 & 0.577 \\
\hline 11 & 900.0 & 1.073 & 0.951 & 0.880 & 0.819 & 0.769 & 0.722 & 0.681 & 0.646 & 0.614 & 0.584 \\
\hline 12 & 1000.0 & 1.077 & 0.954 & 0.882 & 0.823 & 0.771 & 0.727 & 0.688 & 0.654 & 0.618 & 0.589 \\
\hline 13 & 1100.0 & 1.089 & 0.965 & 0.894 & 0.833 & 0.781 & 0.735 & 0.695 & 0.658 & 0.627 & 0.596 \\
\hline 14 & 1200.0 & 1.092 & 0.965 & 0.894 & 0.831 & 0.783 & 0.739 & 0.698 & 0.662 & 0.629 & 0.598 \\
\hline 15 & 1300.0 & 1.096 & 0.972 & 0.903 & 0.841 & 0.792 & 0.747 & 0.706 & 0.670 & 0.640 & 0.609 \\
\hline & & 11 & 12 & 13 & 14 & 15 & 16 & 17 & 18 & 19 & 20 \\
\hline 0 & 0.0 & 0.543 & 0.516 & 0.492 & 0.468 & 0.447 & 0.428 & 0.412 & 0.397 & 0.385 & 0.383 \\
\hline 1 & 25.0 & 0.566 & 0.540 & 0.518 & 0.495 & 0.473 & 0.455 & 0.439 & 0.423 & 0.411 & 0.410 \\
\hline 2 & 50.0 & 0.563 & 0.538 & 0.514 & 0.491 & 0.472 & 0.451 & 0.434 & 0.421 & 0.411 & 0.412 \\
\hline 3 & 100.0 & 0.556 & 0.530 & 0.509 & 0.484 & 0.465 & 0.447 & 0.429 & 0.414 & 0.402 & 0.403 \\
\hline 4 & 200.0 & 0.545 & 0.519 & 0.493 & 0.475 & 0.454 & 0.431 & 0.417 & 0.401 & 0.388 & 0.388 \\
\hline 5 & 300.0 & 0.543 & 0.519 & 0.492 & 0.469 & 0.449 & 0.430 & 0.413 & 0.397 & 0.384 & 0.382 \\
\hline 6 & 400.0 & 0.538 & 0.514 & 0.490 & 0.465 & 0.443 & 0.425 & 0.407 & 0.391 & 0.379 & 0.381 \\
\hline 7 & 500.0 & 0.540 & 0.515 & 0.487 & 0.466 & 0.444 & 0.424 & 0.407 & 0.392 & 0.379 & 0.380 \\
\hline 8 & 600.0 & 0.543 & 0.518 & 0.493 & 0.469 & 0.447 & 0.427 & 0.409 & 0.397 & 0.382 & 0.382 \\
\hline 9 & 700.0 & 0.545 & 0.517 & 0.494 & 0.471 & 0.450 & 0.431 & 0.411 & 0.397 & 0.384 & 0.382 \\
\hline 10 & 800.0 & 0.548 & 0.521 & 0.498 & 0.473 & 0.451 & 0.431 & 0.416 & 0.400 & 0.386 & 0.387 \\
\hline 11 & 900.0 & 0.553 & 0.527 & 0.500 & 0.480 & 0.458 & 0.438 & 0.418 & 0.405 & 0.393 & 0.391 \\
\hline 12 & 1000.0 & 0.560 & 0.534 & 0.506 & 0.484 & 0.464 & 0.444 & 0.425 & 0.410 & 0.399 & 0.397 \\
\hline 13 & 1100.0 & 0.566 & 0.542 & 0.514 & 0.490 & 0.467 & 0.449 & 0.431 & 0.414 & 0.404 & 0.403 \\
\hline 14 & 1200.0 & 0.570 & 0.544 & 0.518 & 0.495 & 0.475 & 0.455 & 0.436 & 0.419 & 0.408 & 0.407 \\
\hline 15 & 1300.0 & 0.579 & 0.549 & 0.526 & 0.501 & 0.479 & 0.459 & 0.441 & 0.427 & 0.414 & 0.416 \\
\hline
\end{tabular}


Table 6.26. Problem 5.2.1 reference solution MPACT power peaking factors for axial segments in fuel channels A, B, C, and D in element 4.

\begin{tabular}{|c|c|c|c|c|c|c|c|c|c|c|c|}
\hline \multirow[t]{2}{*}{ Step } & Burnup & \multicolumn{10}{|c|}{ Power Peaking Factors } \\
\hline & {$\left[\frac{\mathrm{MWd}}{\mathrm{MtU}}\right]$} & 1 & 2 & 3 & 4 & 5 & 6 & 7 & 8 & 9 & 10 \\
\hline 0 & 0.0 & 0.999 & 0.894 & 0.816 & 0.753 & 0.700 & 0.654 & 0.613 & 0.577 & 0.544 & 0.513 \\
\hline 1 & 25.0 & 1.021 & 0.916 & 0.839 & 0.776 & 0.724 & 0.679 & 0.639 & 0.603 & 0.570 & 0.540 \\
\hline 2 & 50.0 & 1.019 & 0.914 & 0.838 & 0.775 & 0.723 & 0.678 & 0.638 & 0.603 & 0.570 & 0.540 \\
\hline 3 & 100.0 & 1.016 & 0.911 & 0.834 & 0.771 & 0.718 & 0.673 & 0.633 & 0.597 & 0.565 & 0.535 \\
\hline 4 & 200.0 & 1.010 & 0.904 & 0.826 & 0.763 & 0.710 & 0.664 & 0.624 & 0.588 & 0.555 & 0.525 \\
\hline 5 & 300.0 & 1.008 & 0.901 & 0.823 & 0.760 & 0.707 & 0.661 & 0.620 & 0.584 & 0.551 & 0.520 \\
\hline 6 & 400.0 & 1.009 & 0.901 & 0.823 & 0.759 & 0.706 & 0.659 & 0.619 & 0.582 & 0.549 & 0.519 \\
\hline 7 & 500.0 & 1.011 & 0.903 & 0.824 & 0.761 & 0.707 & 0.661 & 0.620 & 0.583 & 0.550 & 0.519 \\
\hline 8 & 600.0 & 1.015 & 0.906 & 0.828 & 0.764 & 0.710 & 0.663 & 0.622 & 0.585 & 0.552 & 0.521 \\
\hline 9 & 700.0 & 1.017 & 0.909 & 0.831 & 0.767 & 0.713 & 0.667 & 0.626 & 0.589 & 0.555 & 0.524 \\
\hline 10 & 800.0 & 1.025 & 0.916 & 0.836 & 0.772 & 0.718 & 0.671 & 0.630 & 0.593 & 0.559 & 0.528 \\
\hline 11 & 900.0 & 1.029 & 0.920 & 0.841 & 0.777 & 0.723 & 0.677 & 0.635 & 0.598 & 0.565 & 0.534 \\
\hline 12 & 1000.0 & 1.037 & 0.928 & 0.848 & 0.784 & 0.730 & 0.683 & 0.641 & 0.604 & 0.570 & 0.539 \\
\hline 13 & 1100.0 & 1.042 & 0.933 & 0.854 & 0.790 & 0.736 & 0.689 & 0.648 & 0.611 & 0.577 & 0.546 \\
\hline 14 & 1200.0 & 1.053 & 0.943 & 0.864 & 0.799 & 0.745 & 0.698 & 0.657 & 0.620 & 0.586 & 0.554 \\
\hline 15 & 1300.0 & 1.058 & 0.948 & 0.869 & 0.805 & 0.751 & 0.704 & 0.663 & 0.626 & 0.592 & 0.561 \\
\hline & & 11 & 12 & 13 & 14 & 15 & 16 & 17 & 18 & 19 & 20 \\
\hline 0 & 0.0 & 0.485 & 0.459 & 0.434 & 0.411 & 0.391 & 0.372 & 0.355 & 0.340 & 0.329 & 0.324 \\
\hline 1 & 25.0 & 0.512 & 0.486 & 0.462 & 0.440 & 0.419 & 0.400 & 0.383 & 0.369 & 0.359 & 0.354 \\
\hline 2 & 50.0 & 0.512 & 0.487 & 0.462 & 0.440 & 0.419 & 0.400 & 0.384 & 0.370 & 0.359 & 0.355 \\
\hline 3 & 100.0 & 0.507 & 0.481 & 0.457 & 0.435 & 0.414 & 0.395 & 0.379 & 0.365 & 0.354 & 0.350 \\
\hline 4 & 200.0 & 0.497 & 0.471 & 0.447 & 0.424 & 0.404 & 0.385 & 0.368 & 0.354 & 0.343 & 0.339 \\
\hline 5 & 300.0 & 0.492 & 0.466 & 0.442 & 0.419 & 0.398 & 0.379 & 0.363 & 0.348 & 0.338 & 0.333 \\
\hline 6 & 400.0 & 0.490 & 0.464 & 0.439 & 0.417 & 0.396 & 0.377 & 0.360 & 0.345 & 0.334 & 0.330 \\
\hline 7 & 500.0 & 0.490 & 0.464 & 0.439 & 0.416 & 0.395 & 0.376 & 0.359 & 0.345 & 0.334 & 0.329 \\
\hline 8 & 600.0 & 0.492 & 0.466 & 0.441 & 0.418 & 0.397 & 0.377 & 0.360 & 0.346 & 0.335 & 0.330 \\
\hline 9 & 700.0 & 0.495 & 0.469 & 0.444 & 0.421 & 0.399 & 0.380 & 0.362 & 0.348 & 0.336 & 0.331 \\
\hline 10 & 800.0 & 0.499 & 0.472 & 0.447 & 0.424 & 0.403 & 0.383 & 0.366 & 0.351 & 0.340 & 0.335 \\
\hline 11 & 900.0 & 0.505 & 0.478 & 0.452 & 0.429 & 0.407 & 0.388 & 0.370 & 0.355 & 0.344 & 0.338 \\
\hline 12 & 1000.0 & 0.510 & 0.483 & 0.458 & 0.434 & 0.412 & 0.393 & 0.375 & 0.360 & 0.349 & 0.344 \\
\hline 13 & 1100.0 & 0.517 & 0.489 & 0.464 & 0.440 & 0.418 & 0.398 & 0.381 & 0.366 & 0.354 & 0.349 \\
\hline 14 & 1200.0 & 0.525 & 0.498 & 0.472 & 0.449 & 0.427 & 0.407 & 0.389 & 0.374 & 0.363 & 0.358 \\
\hline 15 & 1300.0 & 0.532 & 0.504 & 0.479 & 0.455 & 0.433 & 0.413 & 0.395 & 0.380 & 0.368 & 0.363 \\
\hline
\end{tabular}


Table 6.27. Problem 5.2.1 reference solution Shift power peaking factors for axial segments in fuel channels A, B, C, and D in element 5.

\begin{tabular}{|c|c|c|c|c|c|c|c|c|c|c|c|}
\hline \multirow[t]{2}{*}{ Step } & \multirow{2}{*}{$\begin{array}{l}\text { Burnup } \\
{\left[\frac{\mathrm{MWd}}{\mathrm{M} \mathrm{dI}}\right]}\end{array}$} & \multicolumn{10}{|c|}{ Power Peaking Factors } \\
\hline & & 1 & 2 & 3 & 4 & 5 & 6 & 7 & 8 & 9 & 10 \\
\hline 0 & 0.0 & 0.344 & 0.312 & 0.291 & 0.273 & 0.259 & 0.244 & 0.230 & 0.217 & 0.206 & 0.195 \\
\hline 1 & 25.0 & 0.373 & 0.338 & 0.318 & 0.300 & 0.283 & 0.269 & 0.254 & 0.241 & 0.231 & 0.218 \\
\hline 2 & 50.0 & 0.371 & 0.339 & 0.317 & 0.300 & 0.282 & 0.266 & 0.253 & 0.242 & 0.229 & 0.219 \\
\hline 3 & 100.0 & 0.363 & 0.329 & 0.309 & 0.290 & 0.275 & 0.260 & 0.246 & 0.233 & 0.222 & 0.211 \\
\hline 4 & 200.0 & 0.350 & 0.317 & 0.296 & 0.279 & 0.262 & 0.247 & 0.235 & 0.223 & 0.212 & 0.202 \\
\hline 5 & 300.0 & 0.345 & 0.313 & 0.293 & 0.274 & 0.257 & 0.243 & 0.230 & 0.217 & 0.209 & 0.198 \\
\hline 6 & 400.0 & 0.340 & 0.308 & 0.287 & 0.270 & 0.253 & 0.238 & 0.227 & 0.214 & 0.204 & 0.193 \\
\hline 7 & 500.0 & 0.342 & 0.308 & 0.287 & 0.269 & 0.254 & 0.238 & 0.225 & 0.213 & 0.203 & 0.191 \\
\hline 8 & 600.0 & 0.343 & 0.308 & 0.288 & 0.270 & 0.254 & 0.241 & 0.227 & 0.214 & 0.204 & 0.192 \\
\hline 9 & 700.0 & 0.345 & 0.310 & 0.289 & 0.271 & 0.255 & 0.239 & 0.227 & 0.216 & 0.205 & 0.193 \\
\hline 10 & 800.0 & 0.348 & 0.312 & 0.291 & 0.274 & 0.258 & 0.244 & 0.230 & 0.217 & 0.205 & 0.194 \\
\hline 11 & 900.0 & 0.354 & 0.317 & 0.296 & 0.279 & 0.263 & 0.247 & 0.233 & 0.221 & 0.210 & 0.199 \\
\hline 12 & 1000.0 & 0.358 & 0.322 & 0.302 & 0.282 & 0.267 & 0.250 & 0.238 & 0.225 & 0.212 & 0.202 \\
\hline 13 & 1100.0 & 0.364 & 0.328 & 0.305 & 0.285 & 0.269 & 0.253 & 0.241 & 0.228 & 0.216 & 0.205 \\
\hline 14 & 1200.0 & 0.366 & 0.328 & 0.308 & 0.288 & 0.274 & 0.257 & 0.242 & 0.231 & 0.217 & 0.207 \\
\hline 15 & 1300.0 & 0.374 & 0.338 & 0.315 & 0.295 & 0.281 & 0.264 & 0.250 & 0.236 & 0.225 & 0.213 \\
\hline & & 11 & 12 & 13 & 14 & 15 & 16 & 17 & 18 & 19 & 20 \\
\hline 0 & 0.0 & 0.186 & 0.176 & 0.167 & 0.161 & 0.152 & 0.145 & 0.140 & 0.134 & 0.129 & 0.129 \\
\hline 1 & 25.0 & 0.208 & 0.200 & 0.191 & 0.182 & 0.174 & 0.167 & 0.161 & 0.154 & 0.151 & 0.150 \\
\hline 2 & 50.0 & 0.210 & 0.199 & 0.189 & 0.181 & 0.173 & 0.165 & 0.160 & 0.153 & 0.149 & 0.149 \\
\hline 3 & 100.0 & 0.200 & 0.193 & 0.185 & 0.175 & 0.168 & 0.162 & 0.154 & 0.149 & 0.144 & 0.144 \\
\hline 4 & 200.0 & 0.192 & 0.182 & 0.173 & 0.166 & 0.158 & 0.151 & 0.143 & 0.140 & 0.135 & 0.134 \\
\hline 5 & 300.0 & 0.186 & 0.177 & 0.170 & 0.161 & 0.154 & 0.148 & 0.141 & 0.137 & 0.131 & 0.130 \\
\hline 6 & 400.0 & 0.183 & 0.173 & 0.166 & 0.157 & 0.149 & 0.145 & 0.137 & 0.132 & 0.127 & 0.128 \\
\hline 7 & 500.0 & 0.183 & 0.173 & 0.165 & 0.157 & 0.150 & 0.143 & 0.137 & 0.131 & 0.127 & 0.126 \\
\hline 8 & 600.0 & 0.182 & 0.173 & 0.165 & 0.157 & 0.149 & 0.144 & 0.138 & 0.131 & 0.127 & 0.126 \\
\hline 9 & 700.0 & 0.184 & 0.176 & 0.167 & 0.159 & 0.150 & 0.144 & 0.138 & 0.132 & 0.127 & 0.127 \\
\hline 10 & 800.0 & 0.186 & 0.175 & 0.169 & 0.160 & 0.152 & 0.143 & 0.138 & 0.132 & 0.127 & 0.128 \\
\hline 11 & 900.0 & 0.188 & 0.179 & 0.169 & 0.162 & 0.154 & 0.147 & 0.140 & 0.135 & 0.130 & 0.130 \\
\hline 12 & 1000.0 & 0.191 & 0.181 & 0.173 & 0.164 & 0.158 & 0.149 & 0.143 & 0.138 & 0.133 & 0.132 \\
\hline 13 & 1100.0 & 0.195 & 0.184 & 0.175 & 0.167 & 0.159 & 0.152 & 0.146 & 0.140 & 0.135 & 0.134 \\
\hline 14 & 1200.0 & 0.199 & 0.187 & 0.178 & 0.170 & 0.162 & 0.155 & 0.148 & 0.142 & 0.139 & 0.138 \\
\hline 15 & 1300.0 & 0.202 & 0.193 & 0.183 & 0.175 & 0.167 & 0.158 & 0.151 & 0.147 & 0.141 & 0.141 \\
\hline
\end{tabular}


Table 6.28. Problem 5.2.1 reference solution MPACT power peaking factors for axial segments in fuel channels A, B, C, and D in element 5.

\begin{tabular}{|c|c|c|c|c|c|c|c|c|c|c|c|}
\hline \multirow[t]{2}{*}{ Step } & Burnup & \multicolumn{10}{|c|}{ Power Peaking Factors } \\
\hline & {$\left[\frac{\mathrm{MWd}}{\mathrm{MtU}}\right]$} & 1 & 2 & 3 & 4 & 5 & 6 & 7 & 8 & 9 & 10 \\
\hline 0 & 0.0 & 0.287 & 0.261 & 0.241 & 0.224 & 0.209 & 0.196 & 0.184 & 0.173 & 0.163 & 0.154 \\
\hline 1 & 25.0 & 0.316 & 0.289 & 0.268 & 0.250 & 0.234 & 0.220 & 0.208 & 0.196 & 0.186 & 0.176 \\
\hline 2 & 50.0 & 0.317 & 0.289 & 0.269 & 0.251 & 0.235 & 0.222 & 0.209 & 0.198 & 0.187 & 0.178 \\
\hline 3 & 100.0 & 0.313 & 0.285 & 0.265 & 0.247 & 0.232 & 0.218 & 0.206 & 0.195 & 0.184 & 0.175 \\
\hline 4 & 200.0 & 0.302 & 0.275 & 0.255 & 0.238 & 0.222 & 0.209 & 0.197 & 0.186 & 0.176 & 0.166 \\
\hline 5 & 300.0 & 0.295 & 0.269 & 0.249 & 0.232 & 0.217 & 0.204 & 0.192 & 0.181 & 0.171 & 0.161 \\
\hline 6 & 400.0 & 0.292 & 0.266 & 0.246 & 0.228 & 0.214 & 0.200 & 0.188 & 0.177 & 0.167 & 0.158 \\
\hline 7 & 500.0 & 0.291 & 0.264 & 0.244 & 0.227 & 0.212 & 0.199 & 0.187 & 0.176 & 0.166 & 0.156 \\
\hline 8 & 600.0 & 0.291 & 0.265 & 0.245 & 0.227 & 0.212 & 0.199 & 0.187 & 0.176 & 0.166 & 0.156 \\
\hline 9 & 700.0 & 0.293 & 0.266 & 0.246 & 0.228 & 0.213 & 0.200 & 0.188 & 0.177 & 0.166 & 0.157 \\
\hline 10 & 800.0 & 0.296 & 0.269 & 0.248 & 0.231 & 0.215 & 0.202 & 0.189 & 0.178 & 0.168 & 0.158 \\
\hline 11 & 900.0 & 0.299 & 0.272 & 0.251 & 0.234 & 0.218 & 0.204 & 0.192 & 0.181 & 0.170 & 0.160 \\
\hline 12 & 1000.0 & 0.304 & 0.277 & 0.255 & 0.237 & 0.222 & 0.208 & 0.195 & 0.183 & 0.173 & 0.163 \\
\hline 13 & 1100.0 & 0.309 & 0.281 & 0.260 & 0.242 & 0.226 & 0.212 & 0.199 & 0.187 & 0.176 & 0.166 \\
\hline 14 & 1200.0 & 0.318 & 0.289 & 0.267 & 0.248 & 0.232 & 0.218 & 0.205 & 0.193 & 0.182 & 0.172 \\
\hline 15 & 1300.0 & 0.322 & 0.294 & 0.272 & 0.253 & 0.236 & 0.222 & 0.209 & 0.197 & 0.185 & 0.175 \\
\hline & & 11 & 12 & 13 & 14 & 15 & 16 & 17 & 18 & 19 & 20 \\
\hline 0 & 0.0 & 0.145 & 0.137 & 0.130 & 0.123 & 0.116 & 0.110 & 0.105 & 0.101 & 0.097 & 0.095 \\
\hline 1 & 25.0 & 0.167 & 0.158 & 0.150 & 0.143 & 0.136 & 0.129 & 0.124 & 0.119 & 0.115 & 0.113 \\
\hline 2 & 50.0 & 0.168 & 0.160 & 0.152 & 0.144 & 0.137 & 0.131 & 0.125 & 0.121 & 0.117 & 0.115 \\
\hline 3 & 100.0 & 0.166 & 0.157 & 0.149 & 0.142 & 0.135 & 0.129 & 0.123 & 0.118 & 0.115 & 0.113 \\
\hline 4 & 200.0 & 0.158 & 0.149 & 0.142 & 0.134 & 0.128 & 0.122 & 0.116 & 0.112 & 0.108 & 0.106 \\
\hline 5 & 300.0 & 0.153 & 0.144 & 0.137 & 0.130 & 0.123 & 0.117 & 0.112 & 0.107 & 0.104 & 0.102 \\
\hline 6 & 400.0 & 0.149 & 0.141 & 0.134 & 0.127 & 0.120 & 0.114 & 0.109 & 0.104 & 0.101 & 0.099 \\
\hline 7 & 500.0 & 0.148 & 0.140 & 0.132 & 0.125 & 0.119 & 0.113 & 0.107 & 0.103 & 0.099 & 0.097 \\
\hline 8 & 600.0 & 0.148 & 0.139 & 0.132 & 0.125 & 0.118 & 0.112 & 0.107 & 0.102 & 0.099 & 0.097 \\
\hline 9 & 700.0 & 0.148 & 0.140 & 0.132 & 0.125 & 0.119 & 0.113 & 0.107 & 0.103 & 0.099 & 0.097 \\
\hline 10 & 800.0 & 0.149 & 0.141 & 0.133 & 0.126 & 0.119 & 0.113 & 0.108 & 0.103 & 0.100 & 0.098 \\
\hline 11 & 900.0 & 0.151 & 0.143 & 0.135 & 0.128 & 0.121 & 0.115 & 0.110 & 0.105 & 0.101 & 0.099 \\
\hline 12 & 1000.0 & 0.154 & 0.145 & 0.137 & 0.130 & 0.123 & 0.117 & 0.111 & 0.106 & 0.103 & 0.101 \\
\hline 13 & 1100.0 & 0.157 & 0.148 & 0.140 & 0.133 & 0.126 & 0.120 & 0.114 & 0.109 & 0.105 & 0.103 \\
\hline 14 & 1200.0 & 0.162 & 0.153 & 0.145 & 0.137 & 0.130 & 0.124 & 0.118 & 0.113 & 0.109 & 0.107 \\
\hline 15 & 1300.0 & 0.166 & 0.157 & 0.148 & 0.140 & 0.133 & 0.126 & 0.121 & 0.115 & 0.111 & 0.110 \\
\hline
\end{tabular}


Table 6.29. Problem 5.2.1 reference solution Shift power peaking factors for axial segments in fuel channels A, B, C, and D in element 6.

\begin{tabular}{|c|c|c|c|c|c|c|c|c|c|c|c|}
\hline \multirow[t]{2}{*}{ Step } & Burnup & \multicolumn{10}{|c|}{ Power Peaking Factors } \\
\hline & $\frac{\mathrm{MWd}}{\mathrm{MtU}}$ & 1 & 2 & 3 & 4 & 5 & 6 & 7 & 8 & 9 & 10 \\
\hline 0 & 0.0 & 0.114 & 0.103 & 0.096 & 0.090 & 0.084 & 0.077 & 0.073 & 0.068 & 0.065 & .060 \\
\hline 1 & 25.0 & 0.135 & 0.122 & 0.113 & 0.105 & 0.101 & 0.093 & 0.088 & 0.083 & 0.078 & 0.072 \\
\hline 2 & 50.0 & 0.134 & 0.122 & 0.113 & 0.105 & 0.098 & 0.092 & 0.088 & 0.082 & 0.077 & 0.073 \\
\hline 3 & 100.0 & 0.129 & 0.116 & 0.108 & 0.102 & 0.095 & 0.089 & 0.084 & 0.079 & 0.074 & 0.069 \\
\hline 4 & 200.0 & 0.120 & 0.108 & 0.101 & 0.095 & 0.087 & 0.082 & 0.077 & 0.074 & 0.069 & 0.066 \\
\hline 5 & 300.0 & 0.117 & 0.104 & 0.097 & 0.091 & 0.086 & 0.080 & 0.074 & 0.071 & 0.068 & 0.062 \\
\hline 6 & 400.0 & 0.113 & 0.102 & 0.095 & 0.088 & 0.082 & 0.077 & 0.071 & 0.067 & 0.064 & 0.060 \\
\hline 7 & 500.0 & 0.112 & 0.101 & 0.094 & 0.088 & 0.082 & 0.076 & 0. & 88 & 0.063 & 0.059 \\
\hline 8 & 600.0 & & & & 36 & 0.081 & 0.076 & & & 63 & .059 \\
\hline 9 & 700.0 & 0.113 & 0.101 & 0.093 & 0.088 & 0.081 & 0.076 & 0.071 & 0.067 & 0.063 & 0.059 \\
\hline 10 & 800.0 & 14 & 0 & 0.095 & 0.088 & 0.082 & 0.078 & & 9 & 54 & 0.060 \\
\hline 11 & 900.0 & 0 & 0 . & 0.096 & 0.090 & 0.084 & 0.079 & & 0. & 0.065 & 0.061 \\
\hline 12 & 1000.0 & 0.117 & 0.106 & 0.098 & 0.091 & 0.086 & 0.080 & 0.075 & 0.070 & 0.065 & 0.062 \\
\hline 13 & 1100.0 & & & & & 0.087 & 81 & & & 67 & .063 \\
\hline 14 & 1200.0 & 0.122 & 0.110 & 0.102 & 0.096 & 0.088 & 0.084 & 0.077 & 0.072 & 0.069 & 0.064 \\
\hline 15 & 1300.0 & 0.125 & 0.112 & 0.104 & 0.097 & 0.092 & 0.086 & 0.080 & 0.075 & 0.071 & 0.066 \\
\hline & & 1 & & & 14 & 15 & 16 & 7 & 18 & 19 & 20 \\
\hline 0 & 0.0 & .057 & & 50 & 47 & .044 & 0.042 & 0.038 & 0.036 & 0.035 & 0.035 \\
\hline 1 & 25.0 & 0.068 & 0.0 & 0.061 & 0.057 & 0.054 & 0.050 & $0 .($ & 0.0 & 0.043 & 0.042 \\
\hline 2 & 50.0 & 0.069 & 0.064 & 0.060 & 0.057 & 0.053 & 0.050 & 0. & 0.044 & 0.043 & 0.043 \\
\hline 3 & 100.0 & 0.066 & & 0.0 & 0.053 & 0.051 & 0.048 & 0.0 & 0.042 & 0.041 & 0.041 \\
\hline 4 & 200.0 & 0.061 & 0.0 & 0.053 & 0.050 & 0.047 & 0.044 & 0.042 & 0.039 & 0.038 & 0.038 \\
\hline 5 & & & & & 0.049 & 0.045 & 0.042 & & 0.037 & 0.036 & 0.036 \\
\hline 6 & 400.0 & & & 0.050 & 0.046 & 0.043 & 0.041 & & 0.0 & 0.035 & 0.034 \\
\hline 7 & 500.0 & 0.0 & 0.0 & 0.048 & 0.046 & 0.043 & 0.040 & 0. & 0.035 & 034 & 0.034 \\
\hline 8 & 600.0 & 0.055 & 0.052 & 0.049 & 0.045 & 0.042 & 0.040 & 0.037 & 0.036 & 0.034 & 0.034 \\
\hline 9 & 700.0 & 0.056 & & 0.049 & 0.046 & 0.043 & 0.040 & 0.0 & 0.035 & 0.034 & 0.034 \\
\hline 10 & 800.0 & 0.057 & 0.053 & 0.049 & 0.046 & 0.043 & 0.041 & 0.038 & 0.036 & 0.034 & 0.034 \\
\hline 11 & & & & & 0.047 & 0.043 & 0.041 & & 0.036 & 0.035 & 0.035 \\
\hline 12 & 1000.0 & 0.058 & 0.055 & 0.050 & 0.048 & 0.044 & 0.042 & 0.039 & 0.037 & 0.036 & 0.035 \\
\hline 13 & 1100.0 & & & 0.052 & 0.049 & 0.045 & 0.043 & 0.040 & 0.038 & 0.036 & 0.036 \\
\hline 14 & 1200.0 & & & & 0.050 & 0.046 & 0.043 & 0.041 & & 0.037 & 0.037 \\
\hline 15 & 1300.0 & 0.062 & 0.058 & 0.054 & 0.051 & 0.047 & 0.044 & 0.042 & 0.040 & 0.038 & 0.037 \\
\hline
\end{tabular}


Table 6.30. Problem 5.2.1 reference solution MPACT power peaking factors for axial segments in fuel channels A, B, C, and D in element 6.

\begin{tabular}{|c|c|c|c|c|c|c|c|c|c|c|c|}
\hline \multirow[t]{2}{*}{ Step } & Burnup & \multicolumn{10}{|c|}{ Power Peaking Factors } \\
\hline & $\frac{\mathrm{MWd}}{\mathrm{MtU}}$ & 1 & 2 & 3 & 4 & 5 & 6 & 7 & 8 & 9 & 10 \\
\hline 0 & 0.0 & 0.084 & 0.076 & 0.070 & 0.065 & 0.060 & 0.056 & 0.052 & 0.049 & 0.045 & 0.042 \\
\hline 1 & 25.0 & 0.100 & 0.091 & 0.084 & 0.078 & 0.073 & 0.068 & 0.063 & 0.059 & 0.056 & 0.052 \\
\hline 2 & 50.0 & 0.102 & 0.093 & 0.086 & 0.080 & 0.074 & 0.069 & 0.065 & .061 & 0.057 & 0.053 \\
\hline 3 & 100.0 & 0.100 & 0.091 & 0.084 & 0.078 & 0.073 & 0.068 & 0.064 & 0.060 & 0.056 & 0.053 \\
\hline 4 & 200.0 & 0.094 & 0.086 & 0.079 & 0.073 & 0.068 & 0.064 & 0.060 & 56 & 0.052 & 0.049 \\
\hline 5 & 300.0 & 0 & 0 . & 0 . & 0 & 0.065 & 0.061 & 0 & & & .047 \\
\hline 6 & 400.0 & 0.087 & 0.079 & 0.073 & 0.068 & 0.063 & 0.059 & 0.055 & 0.051 & 0.048 & 0.045 \\
\hline 7 & 500.0 & 0.086 & 0.078 & 0.072 & 0.066 & 0.062 & 0.057 & 0.054 & 0.050 & 0.047 & 0.044 \\
\hline 8 & 600.0 & 0 . & 0.077 & 0.071 & 0.066 & 0.061 & 0.057 & & & & .043 \\
\hline 9 & 700.0 & 0.085 & 0.077 & 0.071 & 0.066 & 0.061 & 0.057 & 0.053 & 0.049 & 0.046 & 0.043 \\
\hline 10 & & & & & 0.066 & 61 & & & 50 & 46 & 0.043 \\
\hline 11 & 900.0 & 37 & 0.079 & 0.072 & 0.067 & 0.062 & 0.058 & 0. & 0.050 & 0.047 & 0.044 \\
\hline 12 & 1000.0 & 0.088 & 0.080 & 0.074 & 0.068 & 0.063 & 0.059 & 0.055 & 0.051 & 0.048 & 0.045 \\
\hline 13 & 1100.0 & 0.091 & 0.082 & 0. & 0.070 & 0.065 & 0.060 & & & & 0.046 \\
\hline 14 & 1200.0 & 0.094 & 0.085 & 0.078 & 0.072 & 0.067 & 0.063 & 0.058 & 0.054 & 0.051 & 0.048 \\
\hline 15 & 1300.0 & 0.096 & 0.087 & 0.080 & 0.074 & 0.069 & 0.064 & 0.060 & 0.056 & 0.052 & 0.049 \\
\hline & & 11 & 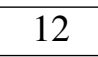 & 13 & 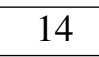 & 15 & 16 & 11 & 10 & 17 & 20 \\
\hline 0 & 0.0 & 0.040 & 0.037 & 0.034 & 0.032 & 0.030 & 0.028 & 0.026 & 0.025 & 0.024 & 0.024 \\
\hline 1 & 25.0 & 0.049 & 0.0 & 0.0 & 0.040 & 0.037 & 0.035 & 0. & 31 & 0.030 & 0.029 \\
\hline 2 & 50.0 & 0.050 & 0.047 & 0.044 & 0.041 & 0.038 & 0.036 & 0.034 & 0.032 & 0.031 & 0.030 \\
\hline 3 & 100.0 & 0.049 & 0.0 & 0.0 & 0.040 & 0.038 & 0.035 & 0. & 0.031 & 0.030 & 0.030 \\
\hline 4 & 200.0 & 0.046 & 0.043 & 0.0 & 0.037 & 0.035 & 0.033 & 0.031 & 0.029 & 0.028 & 0.028 \\
\hline 5 & 300.0 & 0.044 & 0.041 & 0.0 & 0.036 & 0.033 & 0.031 & 0.029 & 0.028 & 0.027 & 0.026 \\
\hline 6 & 400.0 & 0.042 & 0.039 & 0.0 & 0.034 & 0.032 & 0.030 & 0.028 & 0.026 & 0.025 & 0.025 \\
\hline 7 & 500.0 & 0.041 & 0.038 & 0.036 & 0.033 & 0.031 & 0.029 & 0.027 & 0.026 & 0.025 & 0.024 \\
\hline 8 & 600.0 & 0.040 & 0.038 & 0.035 & 0.033 & 0.031 & 0.029 & 0.027 & 0.025 & 0.024 & 0.024 \\
\hline 9 & 700.0 & 0.040 & 0.038 & 0.0 & 0.033 & 0.030 & 0.028 & 0.027 & 0.025 & 0.024 & 0.024 \\
\hline 10 & 800.0 & 0.040 & 0.038 & 0.035 & 0.033 & 0.031 & 0.029 & 0.027 & 0.025 & 0.024 & 0.024 \\
\hline 11 & 900.0 & & & & 0.033 & 0.031 & 0.029 & 0.027 & 0.026 & 0.025 & 0.024 \\
\hline 12 & 1000.0 & 0.042 & 0.039 & 0.036 & 0.034 & 0.031 & 0.029 & 0.028 & 0.026 & 0.025 & 0.025 \\
\hline 13 & 1100.0 & 0.043 & 0.040 & 0.037 & 0.035 & 0.032 & 0.030 & 0.028 & 0.027 & 0.026 & 0.025 \\
\hline 14 & 1200.0 & & & & 0.036 & 0.034 & 0.031 & & & 0.027 & 0.026 \\
\hline 15 & 1300.0 & 0.045 & 0.042 & 0.040 & 0.037 & 0.034 & 0.032 & 0.030 & 0.029 & 0.027 & 0.027 \\
\hline
\end{tabular}


Fuel Channels E, F, G, H, J, K, L, and M

Table 6.31. Problem 5.2.1 reference solution Shift power peaking factors for axial segments in fuel channels $E, F, G, H, J, K, L$, and $M$ in element 1.

\begin{tabular}{|c|c|c|c|c|c|c|c|c|c|c|c|}
\hline \multirow[t]{2}{*}{ Step } & \multirow{2}{*}{$\begin{array}{l}\text { Burnup } \\
{\left[\frac{\mathrm{MWd}}{\mathrm{MtU}}\right]}\end{array}$} & \multicolumn{10}{|c|}{ Power Peaking Factors } \\
\hline & & 1 & 2 & 3 & 4 & 5 & 6 & 7 & 8 & 9 & 10 \\
\hline 0 & 0.0 & 1.073 & 1.044 & 1.056 & 1.084 & 1.118 & 1.158 & 1.203 & 1.250 & 1.299 & 1.346 \\
\hline 1 & 25.0 & 1.069 & 1.038 & 1.049 & 1.075 & 1.109 & 1.146 & 1.191 & 1.235 & 1.282 & 1.327 \\
\hline 2 & 50.0 & 1.069 & 1.034 & 1.048 & 1.075 & 1.108 & 1.144 & 1.190 & 1.234 & 1.280 & 1.329 \\
\hline 3 & 100.0 & 1.066 & 1.032 & 1.045 & 1.072 & 1.104 & 1.145 & 1.188 & 1.235 & 1.283 & 1.328 \\
\hline 4 & 200.0 & 1.070 & 1.039 & 1.053 & 1.077 & 1.111 & 1.153 & 1.197 & 1.244 & 1.291 & 1.339 \\
\hline 5 & 300.0 & 1.067 & 1.034 & 1.047 & 1.073 & 1.110 & 1.150 & 1.194 & 1.241 & 1.290 & 1.338 \\
\hline 6 & 400.0 & 1.073 & 1.038 & 1.053 & 1.078 & 1.113 & 1.157 & 1.200 & 1.247 & 1.297 & 1.343 \\
\hline 7 & 500.0 & 1.066 & 1.034 & 1.048 & 1.075 & 1.110 & 1.150 & 1.196 & 1.243 & 1.292 & 1.341 \\
\hline 8 & 600.0 & 1.068 & 1.035 & 1.048 & 1.074 & 1.111 & 1.152 & 1.195 & 1.246 & 1.294 & 1.344 \\
\hline 9 & 700.0 & 1.070 & 1.038 & 1.054 & 1.079 & 1.114 & 1.153 & 1.201 & 1.248 & 1.297 & 1.345 \\
\hline 10 & 800.0 & 1.068 & 1.034 & 1.047 & 1.074 & 1.109 & 1.151 & 1.196 & 1.244 & 1.292 & 1.340 \\
\hline 11 & 900.0 & 1.069 & 1.035 & 1.050 & 1.076 & 1.112 & 1.155 & 1.199 & 1.245 & 1.294 & 1.343 \\
\hline 12 & 1000.0 & 1.071 & 1.038 & 1.054 & 1.079 & 1.116 & 1.155 & 1.201 & 1.248 & 1.296 & 1.344 \\
\hline 13 & 1100.0 & 1.066 & 1.030 & 1.045 & 1.072 & 1.107 & 1.149 & 1.195 & 1.240 & 1.287 & 1.336 \\
\hline 14 & 1200.0 & 1.069 & 1.038 & 1.050 & 1.077 & 1.114 & 1.153 & 1.197 & 1.245 & 1.290 & 1.336 \\
\hline 15 & 1300.0 & 1.065 & 1.035 & 1.049 & 1.072 & 1.111 & 1.151 & 1.199 & 1.244 & 1.289 & 1.338 \\
\hline & & 11 & 12 & 13 & 14 & 15 & 16 & 17 & 18 & 19 & 20 \\
\hline 0 & 0.0 & 1.395 & 1.442 & 1.492 & 1.543 & 1.594 & 1.646 & 1.702 & 1.763 & 1.834 & 1.963 \\
\hline 1 & 25.0 & 1.377 & 1.423 & 1.470 & 1.517 & 1.568 & 1.619 & 1.673 & 1.732 & 1.797 & 1.929 \\
\hline 2 & 50.0 & 1.377 & 1.424 & 1.471 & 1.519 & 1.566 & 1.621 & 1.673 & 1.736 & 1.801 & 1.934 \\
\hline 3 & 100.0 & 1.375 & 1.424 & 1.472 & 1.521 & 1.571 & 1.622 & 1.677 & 1.742 & 1.810 & 1.942 \\
\hline 4 & 200.0 & 1.386 & 1.435 & 1.483 & 1.536 & 1.585 & 1.639 & 1.693 & 1.757 & 1.824 & 1.963 \\
\hline 5 & 300.0 & 1.389 & 1.435 & 1.486 & 1.536 & 1.585 & 1.640 & 1.696 & 1.759 & 1.827 & 1.967 \\
\hline 6 & 400.0 & 1.393 & 1.445 & 1.492 & 1.541 & 1.592 & 1.646 & 1.704 & 1.772 & 1.839 & 1.980 \\
\hline 7 & 500.0 & 1.390 & 1.438 & 1.488 & 1.536 & 1.591 & 1.644 & 1.700 & 1.765 & 1.834 & 1.973 \\
\hline 8 & 600.0 & 1.391 & 1.440 & 1.488 & 1.538 & 1.589 & 1.642 & 1.698 & 1.761 & 1.830 & 1.972 \\
\hline 9 & 700.0 & 1.395 & 1.442 & 1.489 & 1.539 & 1.593 & 1.648 & 1.701 & 1.762 & 1.829 & 1.969 \\
\hline 10 & 800.0 & 1.390 & 1.438 & 1.487 & 1.537 & 1.588 & 1.638 & 1.694 & 1.759 & 1.826 & 1.966 \\
\hline 11 & 900.0 & 1.390 & 1.440 & 1.488 & 1.537 & 1.588 & 1.637 & 1.692 & 1.752 & 1.822 & 1.959 \\
\hline 12 & 1000.0 & 1.389 & 1.438 & 1.485 & 1.533 & 1.580 & 1.633 & 1.687 & 1.747 & 1.815 & 1.953 \\
\hline 13 & 1100.0 & 1.385 & 1.433 & 1.479 & 1.526 & 1.572 & 1.623 & 1.680 & 1.739 & 1.804 & 1.944 \\
\hline 14 & 1200.0 & 1.388 & 1.433 & 1.480 & 1.527 & 1.574 & 1.626 & 1.677 & 1.736 & 1.802 & 1.935 \\
\hline 15 & 1300.0 & 1.382 & 1.429 & 1.475 & 1.522 & 1.567 & 1.619 & 1.671 & 1.727 & 1.793 & 1.929 \\
\hline
\end{tabular}


Table 6.32. Problem 5.2.1 reference solution MPACT power peaking factors for axial segments in fuel channels $\mathrm{E}, \mathrm{F}, \mathrm{G}, \mathrm{H}, \mathrm{J}, \mathrm{K}, \mathrm{L}$, and $\mathrm{M}$ in element 1.

\begin{tabular}{|c|c|c|c|c|c|c|c|c|c|c|c|}
\hline \multirow[t]{2}{*}{ Step } & \multirow{2}{*}{$\begin{array}{l}\text { Burnup } \\
{\left[\frac{\mathrm{MWd}}{\mathrm{MtU}}\right]}\end{array}$} & \multicolumn{10}{|c|}{ Power Peaking Factors } \\
\hline & & 1 & 2 & 3 & 4 & 5 & 6 & 7 & 8 & 9 & 10 \\
\hline 0 & 0.0 & 1.130 & 1.105 & 1.112 & 1.135 & 1.169 & 1.210 & 1.256 & 1.305 & 1.355 & 1.405 \\
\hline 1 & 25.0 & 1.124 & 1.097 & 1.103 & 1.125 & 1.158 & 1.198 & 1.243 & 1.290 & 1.339 & 1.387 \\
\hline 2 & 50.0 & 1.118 & 1.092 & 1.098 & 1.120 & 1.154 & 1.194 & 1.238 & 1.286 & 1.334 & 1.383 \\
\hline 3 & 100.0 & 1.110 & 1.085 & 1.091 & 1.114 & 1.147 & 1.188 & 1.233 & 1.281 & 1.330 & 1.379 \\
\hline 4 & 200.0 & 1.107 & 1.081 & 1.088 & 1.111 & 1.145 & 1.186 & 1.232 & 1.281 & 1.330 & 1.381 \\
\hline 5 & 300.0 & 1.105 & 1.080 & 1.087 & 1.110 & 1.145 & 1.187 & 1.233 & 1.282 & 1.332 & 1.383 \\
\hline 6 & 400.0 & 1.107 & 1.082 & 1.089 & 1.112 & 1.147 & 1.189 & 1.235 & 1.285 & 1.335 & 1.386 \\
\hline 7 & 500.0 & 1.109 & 1.083 & 1.090 & 1.114 & 1.149 & 1.191 & 1.238 & 1.287 & 1.337 & 1.388 \\
\hline 8 & 600.0 & 1.109 & 1.084 & 1.091 & 1.115 & 1.150 & 1.192 & 1.239 & 1.288 & 1.339 & 1.390 \\
\hline 9 & 700.0 & 1.113 & 1.087 & 1.095 & 1.118 & 1.153 & 1.196 & 1.242 & 1.291 & 1.341 & 1.392 \\
\hline 10 & 800.0 & 1.114 & 1.088 & 1.095 & 1.119 & 1.154 & 1.196 & 1.242 & 1.291 & 1.341 & 1.392 \\
\hline 11 & 900.0 & 1.115 & 1.089 & 1.097 & 1.121 & 1.156 & 1.198 & 1.244 & 1.293 & 1.343 & 1.393 \\
\hline 12 & 1000.0 & 1.116 & 1.091 & 1.098 & 1.122 & 1.156 & 1.198 & 1.244 & 1.293 & 1.342 & 1.392 \\
\hline 13 & 1100.0 & 1.118 & 1.092 & 1.100 & 1.124 & 1.158 & 1.200 & 1.246 & 1.294 & 1.343 & 1.392 \\
\hline 14 & 1200.0 & 1.116 & 1.091 & 1.098 & 1.121 & 1.156 & 1.197 & 1.243 & 1.291 & 1.339 & 1.388 \\
\hline 15 & 1300.0 & 1.117 & 1.092 & 1.099 & 1.123 & 1.157 & 1.199 & 1.244 & 1.291 & 1.340 & 1.388 \\
\hline & & 11 & 12 & 13 & 14 & 15 & 16 & 17 & 18 & 19 & 20 \\
\hline 0 & 0.0 & 1.456 & 1.506 & 1.557 & 1.608 & 1.661 & 1.717 & 1.779 & 1.848 & 1.929 & 2.044 \\
\hline 1 & 25.0 & 1.436 & 1.485 & 1.533 & 1.583 & 1.634 & 1.688 & 1.747 & 1.814 & 1.894 & 2.007 \\
\hline 2 & 50.0 & 1.432 & 1.481 & 1.530 & 1.579 & 1.630 & 1.684 & 1.744 & 1.811 & 1.890 & 2.002 \\
\hline 3 & 100.0 & 1.429 & 1.478 & 1.528 & 1.578 & 1.631 & 1.686 & 1.746 & 1.815 & 1.896 & 2.012 \\
\hline 4 & 200.0 & 1.431 & 1.482 & 1.532 & 1.583 & 1.637 & 1.693 & 1.754 & 1.824 & 1.907 & 2.025 \\
\hline 5 & 300.0 & 1.434 & 1.485 & 1.536 & 1.588 & 1.641 & 1.698 & 1.760 & 1.830 & 1.913 & 2.033 \\
\hline 6 & 400.0 & 1.437 & 1.488 & 1.539 & 1.591 & 1.644 & 1.701 & 1.763 & 1.833 & 1.917 & 2.038 \\
\hline 7 & 500.0 & 1.439 & 1.490 & 1.541 & 1.593 & 1.646 & 1.703 & 1.764 & 1.835 & 1.918 & 2.039 \\
\hline 8 & 600.0 & 1.441 & 1.491 & 1.542 & 1.593 & 1.646 & 1.703 & 1.764 & 1.834 & 1.917 & 2.037 \\
\hline 9 & 700.0 & 1.442 & 1.493 & 1.543 & 1.594 & 1.646 & 1.702 & 1.762 & 1.831 & 1.913 & 2.031 \\
\hline 10 & 800.0 & 1.442 & 1.492 & 1.542 & 1.592 & 1.645 & 1.700 & 1.760 & 1.829 & 1.910 & 2.029 \\
\hline 11 & 900.0 & 1.443 & 1.492 & 1.541 & 1.591 & 1.643 & 1.697 & 1.756 & 1.823 & 1.904 & 2.020 \\
\hline 12 & 1000.0 & 1.441 & 1.490 & 1.539 & 1.589 & 1.640 & 1.693 & 1.752 & 1.819 & 1.899 & 2.016 \\
\hline 13 & 1100.0 & 1.441 & 1.489 & 1.537 & 1.586 & 1.636 & 1.689 & 1.747 & 1.812 & 1.890 & 2.004 \\
\hline 14 & 1200.0 & 1.436 & 1.484 & 1.532 & 1.580 & 1.629 & 1.682 & 1.739 & 1.804 & 1.882 & 1.995 \\
\hline 15 & 1300.0 & 1.436 & 1.483 & 1.530 & 1.577 & 1.626 & 1.677 & 1.733 & 1.797 & 1.873 & 1.983 \\
\hline
\end{tabular}


Table 6.33. Problem 5.2.1 reference solution Shift power peaking factors for axial segments in fuel channels E, F, G, H, J, K, L, and $M$ in element 2.

\begin{tabular}{|c|c|c|c|c|c|c|c|c|c|c|c|}
\hline \multirow[t]{2}{*}{ Step } & \multirow{2}{*}{$\begin{array}{l}\text { Burnup } \\
{\left[\frac{\mathrm{MWd}}{\mathrm{MtU}}\right]}\end{array}$} & \multicolumn{10}{|c|}{ Power Peaking Factors } \\
\hline & & 1 & 2 & 3 & 4 & 5 & 6 & 7 & 8 & 9 & 10 \\
\hline 0 & 0.0 & 2.031 & 1.961 & 1.953 & 1.948 & 1.950 & 1.950 & 1.957 & 1.966 & 1.972 & 1.976 \\
\hline 1 & 25.0 & 1.998 & 1.924 & 1.914 & 1.907 & 1.907 & 1.913 & 1.916 & 1.923 & 1.933 & 1.939 \\
\hline 2 & 50.0 & 2.001 & 1.927 & 1.915 & 1.911 & 1.911 & 1.915 & 1.922 & 1.928 & 1.934 & 1.943 \\
\hline 3 & 100.0 & 2.014 & 1.939 & 1.927 & 1.923 & 1.926 & 1.927 & 1.936 & 1.942 & 1.947 & 1.955 \\
\hline 4 & 200.0 & 2.033 & 1.957 & 1.944 & 1.937 & 1.940 & 1.945 & 1.953 & 1.958 & 1.966 & 1.972 \\
\hline 5 & 300.0 & 2.037 & 1.958 & 1.949 & 1.948 & 1.947 & 1.950 & 1.955 & 1.966 & 1.972 & 1.976 \\
\hline 6 & 400.0 & 2.052 & 1.971 & 1.956 & 1.952 & 1.955 & 1.961 & 1.965 & 1.974 & 1.978 & 1.987 \\
\hline 7 & 500.0 & 2.044 & 1.963 & 1.954 & 1.949 & 1.952 & 1.956 & 1.959 & 1.971 & 1.979 & 1.986 \\
\hline 8 & 600.0 & 2.043 & 1.961 & 1.950 & 1.947 & 1.946 & 1.952 & 1.956 & 1.965 & 1.972 & 1.980 \\
\hline 9 & 700.0 & 2.041 & 1.961 & 1.950 & 1.946 & 1.942 & 1.952 & 1.955 & 1.964 & 1.971 & 1.978 \\
\hline 10 & 800.0 & 2.033 & 1.954 & 1.938 & 1.938 & 1.942 & 1.942 & 1.949 & 1.958 & 1.965 & 1.973 \\
\hline 11 & 900.0 & 2.025 & 1.945 & 1.937 & 1.930 & 1.929 & 1.932 & 1.941 & 1.945 & 1.957 & 1.964 \\
\hline 12 & 1000.0 & 2.020 & 1.939 & 1.925 & 1.922 & 1.921 & 1.928 & 1.935 & 1.943 & 1.950 & 1.955 \\
\hline 13 & 1100.0 & 2.007 & 1.928 & 1.918 & 1.911 & 1.912 & 1.918 & 1.927 & 1.935 & 1.938 & 1.947 \\
\hline 14 & 1200.0 & 2.000 & 1.923 & 1.913 & 1.908 & 1.907 & 1.910 & 1.916 & 1.926 & 1.934 & 1.940 \\
\hline 15 & 1300.0 & 1.992 & 1.911 & 1.902 & 1.895 & 1.899 & 1.901 & 1.908 & 1.916 & 1.922 & 1.927 \\
\hline & & 11 & 12 & 13 & 14 & 15 & 16 & 17 & 18 & 19 & 20 \\
\hline 0 & 0.0 & 1.985 & 1.990 & 1.998 & 2.005 & 2.011 & 2.017 & 2.030 & 2.049 & 2.072 & 2.163 \\
\hline 1 & 25.0 & 1.947 & 1.948 & 1.952 & 1.960 & 1.970 & 1.980 & 1.993 & 2.011 & 2.038 & 2.129 \\
\hline 2 & 50.0 & 1.946 & 1.958 & 1.961 & 1.968 & 1.974 & 1.982 & 1.992 & 2.019 & 2.041 & 2.135 \\
\hline 3 & 100.0 & 1.962 & 1.969 & 1.974 & 1.983 & 1.990 & 1.998 & 2.011 & 2.032 & 2.054 & 2.149 \\
\hline 4 & 200.0 & 1.979 & 1.985 & 1.989 & 1.997 & 2.002 & 2.013 & 2.023 & 2.045 & 2.069 & 2.166 \\
\hline 5 & 300.0 & 1.983 & 1.990 & 1.994 & 2.003 & 2.010 & 2.017 & 2.033 & 2.051 & 2.077 & 2.176 \\
\hline 6 & 400.0 & 1.993 & 1.999 & 2.004 & 2.008 & 2.014 & 2.025 & 2.035 & 2.052 & 2.078 & 2.175 \\
\hline 7 & 500.0 & 1.992 & 1.999 & 2.005 & 2.012 & 2.015 & 2.023 & 2.037 & 2.054 & 2.080 & 2.184 \\
\hline 8 & 600.0 & 1.986 & 1.991 & 1.995 & 2.003 & 2.011 & 2.021 & 2.031 & 2.050 & 2.076 & 2.176 \\
\hline 9 & 700.0 & 1.985 & 1.988 & 1.993 & 2.002 & 2.006 & 2.016 & 2.025 & 2.046 & 2.073 & 2.175 \\
\hline 10 & 800.0 & 1.981 & 1.984 & 1.991 & 1.993 & 2.000 & 2.011 & 2.021 & 2.037 & 2.066 & 2.168 \\
\hline 11 & 900.0 & 1.972 & 1.975 & 1.981 & 1.985 & 1.990 & 2.000 & 2.016 & 2.030 & 2.057 & 2.156 \\
\hline 12 & 1000.0 & 1.962 & 1.963 & 1.968 & 1.977 & 1.984 & 1.991 & 2.003 & 2.021 & 2.047 & 2.145 \\
\hline 13 & 1100.0 & 1.950 & 1.957 & 1.962 & 1.969 & 1.975 & 1.984 & 1.999 & 2.016 & 2.041 & 2.140 \\
\hline 14 & 1200.0 & 1.948 & 1.951 & 1.957 & 1.963 & 1.969 & 1.976 & 1.989 & 2.006 & 2.031 & 2.129 \\
\hline 15 & 1300.0 & 1.933 & 1.939 & 1.944 & 1.949 & 1.959 & 1.964 & 1.980 & 1.993 & 2.021 & 2.121 \\
\hline
\end{tabular}


Table 6.34. Problem 5.2.1 reference solution MPACT power peaking factors for axial segments in fuel channels E, F, G, H, J, K, L, and $M$ in element 2.

\begin{tabular}{|c|c|c|c|c|c|c|c|c|c|c|c|}
\hline \multirow[t]{2}{*}{ Step } & \multirow{2}{*}{$\begin{array}{l}\text { Burnup } \\
{\left[\frac{\mathrm{MWd}}{\mathrm{MtU}}\right]}\end{array}$} & \multicolumn{10}{|c|}{ Power Peaking Factors } \\
\hline & & 1 & 2 & 3 & 4 & 5 & 6 & 7 & 8 & 9 & 10 \\
\hline 0 & 0.0 & 2.116 & 2.059 & 2.036 & 2.025 & 2.022 & 2.023 & 2.027 & 2.032 & 2.038 & 2.043 \\
\hline 1 & 25.0 & 2.075 & 2.018 & 1.995 & 1.983 & 1.980 & 1.981 & 1.984 & 1.990 & 1.995 & 2.000 \\
\hline 2 & 50.0 & 2.071 & 2.016 & 1.993 & 1.983 & 1.980 & 1.981 & 1.986 & 1.991 & 1.997 & 2.003 \\
\hline 3 & 100.0 & 2.082 & 2.025 & 2.001 & 1.991 & 1.987 & 1.989 & 1.994 & 1.999 & 2.005 & 2.011 \\
\hline 4 & 200.0 & 2.098 & 2.039 & 2.016 & 2.005 & 2.003 & 2.005 & 2.010 & 2.016 & 2.022 & 2.028 \\
\hline 5 & 300.0 & 2.107 & 2.047 & 2.024 & 2.013 & 2.010 & 2.013 & 2.018 & 2.024 & 2.030 & 2.036 \\
\hline 6 & 400.0 & 2.111 & 2.051 & 2.027 & 2.016 & 2.014 & 2.016 & 2.021 & 2.027 & 2.034 & 2.040 \\
\hline 7 & 500.0 & 2.112 & 2.051 & 2.027 & 2.016 & 2.013 & 2.015 & 2.020 & 2.026 & 2.033 & 2.039 \\
\hline 8 & 600.0 & 2.109 & 2.048 & 2.024 & 2.013 & 2.010 & 2.012 & 2.017 & 2.023 & 2.029 & 2.035 \\
\hline 9 & 700.0 & 2.102 & 2.042 & 2.019 & 2.008 & 2.006 & 2.008 & 2.013 & 2.019 & 2.025 & 2.031 \\
\hline 10 & 800.0 & 2.099 & 2.038 & 2.013 & 2.002 & 1.999 & 2.001 & 2.005 & 2.011 & 2.017 & 2.023 \\
\hline 11 & 900.0 & 2.088 & 2.029 & 2.005 & 1.994 & 1.992 & 1.994 & 1.998 & 2.004 & 2.010 & 2.016 \\
\hline 12 & 1000.0 & 2.083 & 2.022 & 1.998 & 1.986 & 1.983 & 1.984 & 1.989 & 1.994 & 2.000 & 2.006 \\
\hline 13 & 1100.0 & 2.070 & 2.011 & 1.988 & 1.977 & 1.974 & 1.975 & 1.980 & 1.985 & 1.991 & 1.997 \\
\hline 14 & 1200.0 & 2.059 & 1.999 & 1.975 & 1.963 & 1.960 & 1.961 & 1.965 & 1.971 & 1.977 & 1.982 \\
\hline 15 & 1300.0 & 2.046 & 1.988 & 1.965 & 1.954 & 1.951 & 1.953 & 1.957 & 1.962 & 1.968 & 1.974 \\
\hline & & 11 & 12 & 13 & 14 & 15 & 16 & 17 & 18 & 19 & 20 \\
\hline 0 & 0.0 & 2.048 & 2.051 & 2.055 & 2.059 & 2.064 & 2.072 & 2.086 & 2.107 & 2.141 & 2.210 \\
\hline 1 & 25.0 & 2.005 & 2.009 & 2.013 & 2.018 & 2.024 & 2.033 & 2.047 & 2.069 & 2.104 & 2.175 \\
\hline 2 & 50.0 & 2.008 & 2.012 & 2.016 & 2.021 & 2.027 & 2.035 & 2.049 & 2.071 & 2.106 & 2.175 \\
\hline 3 & 100.0 & 2.016 & 2.021 & 2.025 & 2.030 & 2.036 & 2.045 & 2.059 & 2.082 & 2.118 & 2.190 \\
\hline 4 & 200.0 & 2.034 & 2.038 & 2.043 & 2.047 & 2.053 & 2.062 & 2.076 & 2.099 & 2.135 & 2.209 \\
\hline 5 & 300.0 & 2.042 & 2.046 & 2.051 & 2.055 & 2.061 & 2.070 & 2.084 & 2.107 & 2.143 & 2.218 \\
\hline 6 & 400.0 & 2.045 & 2.049 & 2.054 & 2.058 & 2.064 & 2.073 & 2.087 & 2.110 & 2.146 & 2.222 \\
\hline 7 & 500.0 & 2.044 & 2.048 & 2.053 & 2.057 & 2.063 & 2.072 & 2.086 & 2.109 & 2.145 & 2.221 \\
\hline 8 & 600.0 & 2.040 & 2.045 & 2.049 & 2.053 & 2.059 & 2.068 & 2.082 & 2.105 & 2.142 & 2.218 \\
\hline 9 & 700.0 & 2.036 & 2.041 & 2.045 & 2.049 & 2.054 & 2.063 & 2.077 & 2.099 & 2.135 & 2.209 \\
\hline 10 & 800.0 & 2.028 & 2.032 & 2.036 & 2.041 & 2.047 & 2.056 & 2.070 & 2.093 & 2.129 & 2.205 \\
\hline 11 & 900.0 & 2.021 & 2.025 & 2.029 & 2.033 & 2.039 & 2.048 & 2.061 & 2.084 & 2.119 & 2.193 \\
\hline 12 & 1000.0 & 2.011 & 2.015 & 2.019 & 2.023 & 2.029 & 2.038 & 2.052 & 2.075 & 2.112 & 2.187 \\
\hline 13 & 1100.0 & 2.002 & 2.006 & 2.010 & 2.014 & 2.020 & 2.029 & 2.042 & 2.065 & 2.100 & 2.173 \\
\hline 14 & 1200.0 & 1.987 & 1.991 & 1.995 & 2.000 & 2.006 & 2.015 & 2.030 & 2.052 & 2.089 & 2.163 \\
\hline 15 & 1300.0 & 1.978 & 1.983 & 1.987 & 1.991 & 1.997 & 2.006 & 2.020 & 2.042 & 2.078 & 2.150 \\
\hline
\end{tabular}


Table 6.35. Problem 5.2.1 reference solution Shift power peaking factors for axial segments in fuel channels E, F, G, H, J, K, L, and $M$ in element 3.

\begin{tabular}{|c|c|c|c|c|c|c|c|c|c|c|c|}
\hline \multirow[t]{2}{*}{ Step } & \multirow{2}{*}{$\begin{array}{l}\text { Burnup } \\
{\left[\frac{\mathrm{MWd}}{\mathrm{MtU}}\right]}\end{array}$} & \multicolumn{10}{|c|}{ Power Peaking Factors } \\
\hline & & 1 & 2 & 3 & 4 & 5 & 6 & 7 & 8 & 9 & 10 \\
\hline 0 & 0.0 & 2.118 & 1.991 & 1.933 & 1.881 & 1.832 & 1.789 & 1.748 & 1.710 & 1.668 & 1.628 \\
\hline 1 & 25.0 & 2.091 & 1.968 & 1.908 & 1.854 & 1.809 & 1.769 & 1.731 & 1.695 & 1.658 & 1.615 \\
\hline 2 & 50.0 & 2.096 & 1.970 & 1.909 & 1.859 & 1.810 & 1.773 & 1.732 & 1.694 & 1.656 & 1.617 \\
\hline 3 & 100.0 & 2.107 & 1.979 & 1.919 & 1.870 & 1.822 & 1.780 & 1.739 & 1.699 & 1.662 & 1.624 \\
\hline 4 & 200.0 & 2.124 & 1.993 & 1.929 & 1.877 & 1.829 & 1.786 & 1.745 & 1.707 & 1.669 & 1.627 \\
\hline 5 & 300.0 & 2.134 & 1.999 & 1.938 & 1.884 & 1.835 & 1.791 & 1.753 & 1.713 & 1.672 & 1.633 \\
\hline 6 & 400.0 & 2.135 & 1.999 & 1.934 & 1.884 & 1.834 & 1.793 & 1.749 & 1.710 & 1.668 & 1.631 \\
\hline 7 & 500.0 & 2.143 & 2.004 & 1.941 & 1.886 & 1.841 & 1.797 & 1.755 & 1.716 & 1.677 & 1.637 \\
\hline 8 & 600.0 & 2.133 & 1.999 & 1.940 & 1.885 & 1.841 & 1.795 & 1.756 & 1.716 & 1.673 & 1.636 \\
\hline 9 & 700.0 & 2.129 & 1.993 & 1.933 & 1.880 & 1.837 & 1.793 & 1.750 & 1.713 & 1.673 & 1.634 \\
\hline 10 & 800.0 & 2.130 & 1.992 & 1.933 & 1.881 & 1.830 & 1.790 & 1.753 & 1.716 & 1.674 & 1.636 \\
\hline 11 & 900.0 & 2.117 & 1.984 & 1.926 & 1.873 & 1.831 & 1.791 & 1.751 & 1.711 & 1.675 & 1.635 \\
\hline 12 & 1000.0 & 2.109 & 1.974 & 1.919 & 1.870 & 1.824 & 1.785 & 1.745 & 1.710 & 1.673 & 1.635 \\
\hline 13 & 1100.0 & 2.104 & 1.973 & 1.913 & 1.866 & 1.824 & 1.787 & 1.745 & 1.709 & 1.673 & 1.639 \\
\hline 14 & 1200.0 & 2.095 & 1.967 & 1.910 & 1.858 & 1.819 & 1.779 & 1.741 & 1.706 & 1.670 & 1.635 \\
\hline 15 & 1300.0 & 2.082 & 1.958 & 1.901 & 1.851 & 1.810 & 1.773 & 1.735 & 1.702 & 1.666 & 1.633 \\
\hline & & 11 & 12 & 13 & 14 & 15 & 16 & 17 & 18 & 19 & 20 \\
\hline 0 & 0.0 & 1.590 & 1.548 & 1.510 & 1.471 & 1.428 & 1.389 & 1.348 & 1.316 & 1.286 & 1.293 \\
\hline 1 & 25.0 & 1.582 & 1.544 & 1.505 & 1.466 & 1.428 & 1.392 & 1.357 & 1.324 & 1.296 & 1.306 \\
\hline 2 & 50.0 & 1.580 & 1.542 & 1.504 & 1.469 & 1.429 & 1.389 & 1.354 & 1.321 & 1.293 & 1.306 \\
\hline 3 & 100.0 & 1.584 & 1.544 & 1.505 & 1.465 & 1.427 & 1.389 & 1.352 & 1.321 & 1.292 & 1.300 \\
\hline 4 & 200.0 & 1.587 & 1.547 & 1.508 & 1.466 & 1.426 & 1.385 & 1.350 & 1.317 & 1.287 & 1.297 \\
\hline 5 & 300.0 & 1.591 & 1.551 & 1.512 & 1.471 & 1.430 & 1.389 & 1.350 & 1.315 & 1.285 & 1.297 \\
\hline 6 & 400.0 & 1.591 & 1.547 & 1.506 & 1.464 & 1.424 & 1.384 & 1.347 & 1.314 & 1.284 & 1.295 \\
\hline 7 & 500.0 & 1.597 & 1.556 & 1.516 & 1.471 & 1.432 & 1.391 & 1.353 & 1.319 & 1.290 & 1.299 \\
\hline 8 & 600.0 & 1.599 & 1.557 & 1.516 & 1.477 & 1.433 & 1.394 & 1.355 & 1.319 & 1.292 & 1.303 \\
\hline 9 & 700.0 & 1.594 & 1.553 & 1.514 & 1.473 & 1.434 & 1.392 & 1.352 & 1.319 & 1.292 & 1.304 \\
\hline 10 & 800.0 & 1.601 & 1.560 & 1.516 & 1.476 & 1.436 & 1.397 & 1.362 & 1.326 & 1.296 & 1.312 \\
\hline 11 & 900.0 & 1.597 & 1.559 & 1.520 & 1.478 & 1.439 & 1.402 & 1.365 & 1.329 & 1.301 & 1.318 \\
\hline 12 & 1000.0 & 1.598 & 1.560 & 1.522 & 1.481 & 1.440 & 1.399 & 1.365 & 1.334 & 1.306 & 1.318 \\
\hline 13 & 1100.0 & 1.600 & 1.561 & 1.525 & 1.486 & 1.445 & 1.409 & 1.373 & 1.341 & 1.315 & 1.328 \\
\hline 14 & 1200.0 & 1.596 & 1.560 & 1.522 & 1.482 & 1.442 & 1.406 & 1.371 & 1.337 & 1.312 & 1.329 \\
\hline 15 & 1300.0 & 1.597 & 1.560 & 1.523 & 1.487 & 1.448 & 1.410 & 1.375 & 1.342 & 1.316 & 1.335 \\
\hline
\end{tabular}


Table 6.36. Problem 5.2.1 reference solution MPACT power peaking factors for axial segments in fuel channels E, F, G, H, J, K, L, and $M$ in element 3.

\begin{tabular}{|c|c|c|c|c|c|c|c|c|c|c|c|}
\hline \multirow[t]{2}{*}{ Step } & Burnup & \multicolumn{10}{|c|}{ Power Peaking Factors } \\
\hline & {$\left[\frac{\mathrm{MWd}}{\mathrm{MtU}}\right]$} & 1 & 2 & 3 & 4 & 5 & 6 & 7 & 8 & 9 & 10 \\
\hline 0 & 0.0 & 2.159 & 2.048 & 1.972 & 1.910 & 1.855 & 1.806 & 1.760 & 1.716 & 1.673 & 1.629 \\
\hline 1 & 25.0 & 2.129 & 2.020 & 1.947 & 1.887 & 1.835 & 1.789 & 1.746 & 1.704 & 1.663 & 1.623 \\
\hline 2 & 50.0 & 2.129 & 2.021 & 1.948 & 1.888 & 1.837 & 1.790 & 1.747 & 1.706 & 1.665 & 1.624 \\
\hline 3 & 100.0 & 2.143 & 2.031 & 1.957 & 1.895 & 1.843 & 1.795 & 1.751 & 1.709 & 1.667 & 1.625 \\
\hline 4 & 200.0 & 2.160 & 2.046 & 1.970 & 1.907 & 1.853 & 1.805 & 1.760 & 1.716 & 1.674 & 1.631 \\
\hline 5 & 300.0 & 2.169 & 2.053 & 1.976 & 1.913 & 1.859 & 1.810 & 1.764 & 1.720 & 1.677 & 1.634 \\
\hline 6 & 400.0 & 2.172 & 2.056 & 1.979 & 1.915 & 1.861 & 1.812 & 1.767 & 1.723 & 1.680 & 1.636 \\
\hline 7 & 500.0 & 2.172 & 2.056 & 1.979 & 1.916 & 1.862 & 1.813 & 1.768 & 1.724 & 1.681 & 1.638 \\
\hline 8 & 600.0 & 2.169 & 2.053 & 1.977 & 1.914 & 1.861 & 1.813 & 1.768 & 1.725 & 1.682 & 1.639 \\
\hline 9 & 700.0 & 2.161 & 2.048 & 1.973 & 1.912 & 1.859 & 1.812 & 1.768 & 1.725 & 1.683 & 1.641 \\
\hline 10 & 800.0 & 2.159 & 2.045 & 1.970 & 1.908 & 1.856 & 1.809 & 1.766 & 1.724 & 1.682 & 1.640 \\
\hline 11 & 900.0 & 2.148 & 2.037 & 1.964 & 1.904 & 1.853 & 1.807 & 1.765 & 1.723 & 1.683 & 1.642 \\
\hline 12 & 1000.0 & 2.143 & 2.032 & 1.959 & 1.899 & 1.849 & 1.804 & 1.762 & 1.721 & 1.681 & 1.641 \\
\hline 13 & 1100.0 & 2.131 & 2.022 & 1.951 & 1.894 & 1.845 & 1.801 & 1.760 & 1.720 & 1.681 & 1.642 \\
\hline 14 & 1200.0 & 2.123 & 2.014 & 1.944 & 1.887 & 1.839 & 1.796 & 1.756 & 1.717 & 1.679 & 1.640 \\
\hline 15 & 1300.0 & 2.110 & 2.005 & 1.937 & 1.882 & 1.835 & 1.793 & 1.754 & 1.716 & 1.678 & 1.641 \\
\hline & & 11 & 12 & 13 & 14 & 15 & 16 & 17 & 18 & 19 & 20 \\
\hline 0 & 0.0 & 1.585 & 1.541 & 1.496 & 1.451 & 1.406 & 1.363 & 1.322 & 1.286 & 1.256 & 1.247 \\
\hline 1 & 25.0 & 1.581 & 1.539 & 1.497 & 1.455 & 1.412 & 1.372 & 1.333 & 1.300 & 1.273 & 1.267 \\
\hline 2 & 50.0 & 1.583 & 1.540 & 1.498 & 1.455 & 1.413 & 1.372 & 1.333 & 1.299 & 1.272 & 1.264 \\
\hline 3 & 100.0 & 1.583 & 1.541 & 1.497 & 1.454 & 1.411 & 1.370 & 1.331 & 1.296 & 1.269 & 1.263 \\
\hline 4 & 200.0 & 1.588 & 1.544 & 1.499 & 1.455 & 1.411 & 1.369 & 1.329 & 1.293 & 1.265 & 1.258 \\
\hline 5 & 300.0 & 1.590 & 1.546 & 1.501 & 1.456 & 1.412 & 1.369 & 1.328 & 1.293 & 1.265 & 1.257 \\
\hline 6 & 400.0 & 1.593 & 1.548 & 1.503 & 1.458 & 1.414 & 1.370 & 1.330 & 1.294 & 1.266 & 1.258 \\
\hline 7 & 500.0 & 1.594 & 1.550 & 1.505 & 1.460 & 1.416 & 1.373 & 1.332 & 1.296 & 1.268 & 1.261 \\
\hline 8 & 600.0 & 1.596 & 1.552 & 1.507 & 1.463 & 1.418 & 1.375 & 1.335 & 1.299 & 1.271 & 1.265 \\
\hline 9 & 700.0 & 1.598 & 1.555 & 1.510 & 1.466 & 1.422 & 1.379 & 1.338 & 1.303 & 1.275 & 1.268 \\
\hline 10 & 800.0 & 1.598 & 1.555 & 1.511 & 1.467 & 1.424 & 1.382 & 1.342 & 1.307 & 1.280 & 1.275 \\
\hline 11 & 900.0 & 1.600 & 1.558 & 1.514 & 1.471 & 1.428 & 1.386 & 1.346 & 1.311 & 1.284 & 1.278 \\
\hline 12 & 1000.0 & 1.600 & 1.558 & 1.516 & 1.473 & 1.430 & 1.389 & 1.350 & 1.316 & 1.290 & 1.286 \\
\hline 13 & 1100.0 & 1.601 & 1.560 & 1.518 & 1.476 & 1.434 & 1.393 & 1.355 & 1.321 & 1.295 & 1.291 \\
\hline 14 & 1200.0 & 1.601 & 1.561 & 1.520 & 1.479 & 1.438 & 1.398 & 1.360 & 1.328 & 1.303 & 1.301 \\
\hline 15 & 1300.0 & 1.602 & 1.563 & 1.522 & 1.482 & 1.441 & 1.402 & 1.365 & 1.332 & 1.308 & 1.305 \\
\hline
\end{tabular}


Table 6.37. Problem 5.2.1 reference solution Shift power peaking factors for axial segments in fuel channels $E, F, G, H, J, K, L$, and $M$ in element 4.

\begin{tabular}{|c|c|c|c|c|c|c|c|c|c|c|c|}
\hline \multirow[t]{2}{*}{ Step } & \multirow{2}{*}{$\begin{array}{l}\text { Burnup } \\
{\left[\frac{\mathrm{MWd}}{\mathrm{MtU}}\right]}\end{array}$} & \multicolumn{10}{|c|}{ Power Peaking Factors } \\
\hline & & 1 & 2 & 3 & 4 & 5 & 6 & 7 & 8 & 9 & 10 \\
\hline 0 & 0.0 & 1.170 & 1.062 & 0.993 & 0.932 & 0.877 & 0.830 & 0.784 & 0.742 & 0.707 & 0.672 \\
\hline 1 & 25.0 & 1.188 & 1.079 & 1.013 & 0.953 & 0.899 & 0.851 & 0.808 & 0.768 & 0.731 & 0.696 \\
\hline 2 & 50.0 & 1.186 & 1.075 & 1.009 & 0.949 & 0.895 & 0.849 & 0.804 & 0.765 & 0.730 & 0.693 \\
\hline 3 & 100.0 & 1.180 & 1.068 & 1.001 & 0.942 & 0.889 & 0.840 & 0.797 & 0.755 & 0.718 & 0.686 \\
\hline 4 & 200.0 & 1.173 & 1.060 & 0.993 & 0.932 & 0.878 & 0.831 & 0.787 & 0.743 & 0.708 & 0.673 \\
\hline 5 & 300.0 & 1.173 & 1.060 & 0.993 & 0.931 & 0.878 & 0.827 & 0.784 & 0.742 & 0.705 & 0.669 \\
\hline 6 & 400.0 & 1.169 & 1.057 & 0.989 & 0.927 & 0.871 & 0.823 & 0.777 & 0.739 & 0.700 & 0.665 \\
\hline 7 & 500.0 & 1.175 & 1.061 & 0.993 & 0.929 & 0.878 & 0.828 & 0.783 & 0.741 & 0.703 & 0.667 \\
\hline 8 & 600.0 & 1.182 & 1.066 & 0.997 & 0.933 & 0.881 & 0.832 & 0.786 & 0.746 & 0.708 & 0.672 \\
\hline 9 & 700.0 & 1.180 & 1.064 & 0.996 & 0.935 & 0.879 & 0.831 & 0.786 & 0.747 & 0.708 & 0.672 \\
\hline 10 & 800.0 & 1.187 & 1.072 & 1.002 & 0.940 & 0.886 & 0.837 & 0.792 & 0.752 & 0.714 & 0.677 \\
\hline 11 & 900.0 & 1.194 & 1.075 & 1.008 & 0.949 & 0.894 & 0.844 & 0.799 & 0.758 & 0.719 & 0.684 \\
\hline 12 & 1000.0 & 1.196 & 1.081 & 1.015 & 0.953 & 0.899 & 0.849 & 0.806 & 0.764 & 0.727 & 0.692 \\
\hline 13 & 1100.0 & 1.206 & 1.091 & 1.022 & 0.962 & 0.905 & 0.857 & 0.813 & 0.772 & 0.733 & 0.698 \\
\hline 14 & 1200.0 & 1.209 & 1.092 & 1.026 & 0.966 & 0.909 & 0.862 & 0.817 & 0.774 & 0.739 & 0.702 \\
\hline 15 & 1300.0 & 1.217 & 1.101 & 1.031 & 0.970 & 0.917 & 0.868 & 0.824 & 0.785 & 0.746 & 0.712 \\
\hline & & 11 & 12 & 13 & 14 & 15 & 16 & 17 & 18 & 19 & 20 \\
\hline 0 & 0.0 & 0.638 & 0.607 & 0.578 & 0.551 & 0.527 & 0.504 & 0.485 & 0.466 & 0.453 & 0.451 \\
\hline 1 & 25.0 & 0.666 & 0.635 & 0.607 & 0.581 & 0.557 & 0.535 & 0.513 & 0.498 & 0.484 & 0.485 \\
\hline 2 & 50.0 & 0.664 & 0.632 & 0.603 & 0.575 & 0.553 & 0.532 & 0.512 & 0.496 & 0.484 & 0.483 \\
\hline 3 & 100.0 & 0.653 & 0.623 & 0.596 & 0.571 & 0.545 & 0.524 & 0.504 & 0.487 & 0.472 & 0.474 \\
\hline 4 & 200.0 & 0.641 & 0.611 & 0.582 & 0.556 & 0.531 & 0.508 & 0.489 & 0.471 & 0.457 & 0.458 \\
\hline 5 & 300.0 & 0.638 & 0.604 & 0.578 & 0.551 & 0.528 & 0.504 & 0.483 & 0.466 & 0.452 & 0.453 \\
\hline 6 & 400.0 & 0.632 & 0.603 & 0.573 & 0.546 & 0.521 & 0.499 & 0.479 & 0.461 & 0.448 & 0.448 \\
\hline 7 & 500.0 & 0.634 & 0.603 & 0.575 & 0.547 & 0.523 & 0.501 & 0.481 & 0.463 & 0.449 & 0.448 \\
\hline 8 & 600.0 & 0.639 & 0.609 & 0.580 & 0.552 & 0.527 & 0.505 & 0.482 & 0.465 & 0.452 & 0.450 \\
\hline 9 & 700.0 & 0.639 & 0.609 & 0.580 & 0.555 & 0.529 & 0.506 & 0.483 & 0.467 & 0.453 & 0.452 \\
\hline 10 & 800.0 & 0.643 & 0.613 & 0.585 & 0.557 & 0.531 & 0.508 & 0.489 & 0.473 & 0.456 & 0.455 \\
\hline 11 & 900.0 & 0.653 & 0.622 & 0.590 & 0.564 & 0.538 & 0.517 & 0.496 & 0.477 & 0.462 & 0.463 \\
\hline 12 & 1000.0 & 0.658 & 0.626 & 0.597 & 0.571 & 0.545 & 0.522 & 0.501 & 0.484 & 0.467 & 0.469 \\
\hline 13 & 1100.0 & 0.664 & 0.634 & 0.603 & 0.577 & 0.551 & 0.527 & 0.507 & 0.489 & 0.476 & 0.475 \\
\hline 14 & 1200.0 & 0.669 & 0.638 & 0.608 & 0.581 & 0.556 & 0.534 & 0.512 & 0.493 & 0.480 & 0.479 \\
\hline 15 & 1300.0 & 0.679 & 0.648 & 0.616 & 0.591 & 0.564 & 0.540 & 0.519 & 0.504 & 0.487 & 0.488 \\
\hline
\end{tabular}


Table 6.38. Problem 5.2.1 reference solution MPACT power peaking factors for axial segments in fuel channels $E, F, G, H, J, K, L$, and $M$ in element 4.

\begin{tabular}{|c|c|c|c|c|c|c|c|c|c|c|c|}
\hline \multirow[t]{2}{*}{ Step } & Burnup & \multicolumn{10}{|c|}{ Power Peaking Factors } \\
\hline & $\frac{\mathrm{MWd}}{\mathrm{MtU}}$ & 1 & 2 & 3 & 4 & 5 & 6 & 7 & 8 & 9 & 10 \\
\hline 0 & 0.0 & 1.113 & 1.014 & 0.938 & 0.872 & 0.815 & 0.764 & 0.718 & .676 & 0.638 & 0.603 \\
\hline 1 & 25.0 & 1.137 & 1.039 & 0.964 & 0.899 & 0.843 & 0.793 & 0.748 & 0.707 & 0.669 & 0.634 \\
\hline 2 & 50.0 & 1.135 & 1.037 & 0.962 & 0.898 & 0.842 & 0.792 & 0.747 & 0.706 & 0.669 & 0.634 \\
\hline 3 & 100.0 & 1.132 & 1.033 & 0.958 & 0.893 & 0.837 & 0.786 & 0.741 & 0.700 & 0.663 & 0.628 \\
\hline 4 & 200.0 & 1.126 & 1.026 & 0.950 & 0.884 & 0.827 & 0.777 & 0.731 & 0.690 & 0.652 & 0.617 \\
\hline 5 & 300.0 & 1.124 & 1.023 & 0.946 & 0.881 & 0.823 & 0.772 & 0.727 & 0.685 & 0.647 & 0.611 \\
\hline 6 & 400.0 & 1.124 & 1.023 & 0.946 & 0.880 & 0.822 & 0.771 & 0.725 & 0.683 & 0.645 & 0.609 \\
\hline 7 & 500.0 & 1.127 & 1.025 & 0.948 & 0.882 & 0.824 & 0.772 & 0 . & 0.684 & 0. & 0.610 \\
\hline 8 & 600.0 & & & & 35 & 0.827 & 0.775 & & & & 0.612 \\
\hline 9 & 700.0 & 1.134 & 1.032 & 0.955 & 0.889 & 0.831 & 0.779 & 0.733 & 0.691 & 0.652 & 0.616 \\
\hline 10 & 800.0 & 1.1 & 1.0 & 1 & 0.894 & 0.836 & 0.784 & & 96 & & 0.621 \\
\hline 11 & 900.0 & 1. & 1.044 & 0.966 & 0.900 & 0.842 & 0.791 & & 0.702 & & 0.627 \\
\hline 12 & 1000.0 & 1.1 & 1.0 & 0.974 & 0.907 & 0.849 & 0.797 & 0.7 & 0.708 & 0.669 & 0.633 \\
\hline 13 & 1100.0 & & & & & 56 & 05 & & 16 & & 641 \\
\hline 14 & 1200.0 & 1.171 & 1.068 & 0.991 & 0.924 & 0.866 & 0.815 & 0.768 & 0.726 & 0.687 & 0.651 \\
\hline 15 & 1300.0 & 1.176 & 1.074 & 0.997 & 0.931 & 0.873 & 0.822 & 0.776 & 0.734 & 0.695 & 0.658 \\
\hline & & 11 & 12 & 13 & 14 & 15 & 16 & 17 & 18 & 19 & 20 \\
\hline 0 & 0.0 & 0.570 & 0 & 10 & 84 & 459 & 0.437 & 0.417 & 0.400 & 0.387 & 0.381 \\
\hline 1 & 25.0 & 0.601 & & & 0.516 & 0.492 & 0.470 & & 0.434 & 0.422 & 0.417 \\
\hline 2 & 50.0 & 0.602 & 1 & 43 & 0.517 & 0.493 & 0.471 & & 0.435 & 0.422 & 0.417 \\
\hline 3 & 100.0 & & & & 0.511 & 0.487 & 0.465 & 0.446 & 0.429 & 0.417 & 0.412 \\
\hline 4 & 200.0 & 0 . & 0.5 & 0.525 & 0.499 & 0.475 & 0.453 & 0.433 & 0.417 & 0.404 & 0.399 \\
\hline 5 & 300.0 & & & & 0.493 & 0.468 & 0.446 & & 0.410 & 0.397 & 0.392 \\
\hline 6 & 400.0 & & & & 0.490 & 0.465 & 0.443 & & 0.406 & 0.393 & 0.388 \\
\hline 7 & 500.0 & 0 . & 0.5 & 17 & 0.490 & 0.465 & 0.443 & 0. & 0.406 & 93 & 0.387 \\
\hline 8 & 600.0 & 0.579 & 0.5 & 0.519 & 0.492 & 0.467 & 0.444 & 0.424 & 0.407 & 0.394 & 0.388 \\
\hline 9 & 700.0 & & & 0.522 & 0.495 & 0.470 & 0.447 & 0.427 & 0.409 & 0.396 & 0.390 \\
\hline 10 & 800.0 & & 0.555 & 0.526 & 0.499 & 0.474 & 0.451 & 0.430 & 0.413 & 0.400 & 0.394 \\
\hline 11 & & & & & 0.504 & 0.479 & 0.456 & & 0.418 & 0.404 & 0.399 \\
\hline 12 & 1000.0 & 0.599 & 0.568 & 0.538 & 0.510 & 0.485 & 0.462 & 0.441 & 0.424 & 0.410 & 0.405 \\
\hline 13 & 1100.0 & 0.607 & 0.5 & 0.545 & 0.518 & 0.492 & 0.469 & 0.448 & 0.430 & 0.417 & 0.411 \\
\hline 14 & 1200.0 & & & & 0.527 & 0.502 & 0.478 & & & 0.427 & 0.422 \\
\hline 15 & 1300.0 & 0.624 & 0.592 & 0.562 & 0.535 & 0.509 & 0.485 & 0.464 & 0.447 & 0.433 & 0.428 \\
\hline
\end{tabular}


Table 6.39. Problem 5.2.1 reference solution Shift power peaking factors for axial segments in fuel channels E, F, G, H, J, K, L, and $M$ in element 5.

\begin{tabular}{|c|c|c|c|c|c|c|c|c|c|c|c|}
\hline \multirow[t]{2}{*}{ Step } & Burnup & \multicolumn{10}{|c|}{ Power Peaking Factors } \\
\hline & {$\left[\frac{\mathrm{MWd}}{\mathrm{MtU}}\right]$} & 1 & 2 & 3 & 4 & 5 & 6 & 7 & 8 & 9 & 10 \\
\hline 0 & 0.0 & 0.405 & 0.366 & 0.344 & 0.322 & 0.303 & 0.286 & 0.271 & 0.256 & 0.243 & 0.231 \\
\hline 1 & 25.0 & 0.439 & 0.398 & 0.373 & 0.352 & 0.333 & 0.316 & 0.298 & 0.284 & 0.271 & 0.259 \\
\hline 2 & 50.0 & 0.436 & 0.397 & 0.372 & 0.352 & 0.331 & 0.314 & 0.297 & 0.283 & 0.270 & 0.256 \\
\hline 3 & 100.0 & 0.428 & 0.388 & 0.363 & 0.343 & 0.323 & 0.306 & 0.290 & 0.275 & 0.262 & 0.248 \\
\hline 4 & 200.0 & 0.414 & 0.374 & 0.350 & 0.328 & 0.310 & 0.292 & 0.277 & 0.262 & 0.249 & 0.237 \\
\hline 5 & 300.0 & 0.407 & 0.367 & 0.344 & 0.323 & 0.303 & 0.287 & 0.272 & 0.257 & 0.244 & 0.232 \\
\hline 6 & 400.0 & 0.400 & 0.361 & 0.339 & 0.318 & 0.299 & 0.283 & 0.267 & 0.252 & 0.239 & 0.228 \\
\hline 7 & 500.0 & 0.401 & 0.362 & 0.339 & 0.317 & 0.298 & 0.281 & 0.267 & 0.251 & 0.238 & 0.227 \\
\hline 8 & 600.0 & 0.403 & 0.363 & 0.340 & 0.319 & 0.299 & 0.282 & 0.267 & 0.253 & 0.239 & 0.227 \\
\hline 9 & 700.0 & 0.406 & 0.365 & 0.341 & 0.321 & 0.301 & 0.283 & 0.267 & 0.254 & 0.241 & 0.228 \\
\hline 10 & 800.0 & 0.409 & 0.369 & 0.345 & 0.324 & 0.303 & 0.287 & 0.270 & 0.256 & 0.242 & 0.230 \\
\hline 11 & 900.0 & 0.415 & 0.374 & 0.349 & 0.326 & 0.308 & 0.291 & 0.275 & 0.260 & 0.246 & 0.233 \\
\hline 12 & 1000.0 & 0.421 & 0.379 & 0.355 & 0.333 & 0.312 & 0.295 & 0.279 & 0.265 & 0.251 & 0.238 \\
\hline 13 & 1100.0 & 0.427 & 0.385 & 0.360 & 0.337 & 0.317 & 0.300 & 0.284 & 0.269 & 0.254 & 0.241 \\
\hline 14 & 1200.0 & 0.433 & 0.388 & 0.364 & 0.342 & 0.321 & 0.303 & 0.288 & 0.271 & 0.258 & 0.244 \\
\hline 15 & 1300.0 & 0.442 & 0.398 & 0.372 & 0.349 & 0.328 & 0.312 & 0.293 & 0.278 & 0.264 & 0.252 \\
\hline & & 11 & 12 & 13 & 14 & 15 & 16 & 17 & 18 & 19 & 20 \\
\hline 0 & 0.0 & 0.218 & 0.208 & 0.197 & 0.188 & 0.179 & 0.171 & 0.164 & 0.157 & 0.152 & 0.151 \\
\hline 1 & 25.0 & 0.247 & 0.235 & 0.225 & 0.215 & 0.206 & 0.196 & 0.190 & 0.182 & 0.177 & 0.176 \\
\hline 2 & 50.0 & 0.245 & 0.234 & 0.223 & 0.214 & 0.204 & 0.196 & 0.187 & 0.182 & 0.175 & 0.176 \\
\hline 3 & 100.0 & 0.238 & 0.226 & 0.215 & 0.206 & 0.197 & 0.188 & 0.182 & 0.176 & 0.169 & 0.169 \\
\hline 4 & 200.0 & 0.225 & 0.214 & 0.204 & 0.194 & 0.185 & 0.179 & 0.171 & 0.164 & 0.159 & 0.158 \\
\hline 5 & 300.0 & 0.221 & 0.209 & 0.200 & 0.190 & 0.182 & 0.173 & 0.166 & 0.160 & 0.156 & 0.153 \\
\hline 6 & 400.0 & 0.215 & 0.205 & 0.194 & 0.186 & 0.178 & 0.170 & 0.162 & 0.156 & 0.151 & 0.149 \\
\hline 7 & 500.0 & 0.215 & 0.204 & 0.194 & 0.185 & 0.176 & 0.168 & 0.161 & 0.155 & 0.148 & 0.149 \\
\hline 8 & 600.0 & 0.216 & 0.205 & 0.194 & 0.186 & 0.177 & 0.167 & 0.161 & 0.154 & 0.149 & 0.148 \\
\hline 9 & 700.0 & 0.217 & 0.205 & 0.195 & 0.185 & 0.177 & 0.169 & 0.162 & 0.156 & 0.150 & 0.150 \\
\hline 10 & 800.0 & 0.218 & 0.207 & 0.197 & 0.188 & 0.179 & 0.171 & 0.163 & 0.157 & 0.152 & 0.151 \\
\hline 11 & 900.0 & 0.222 & 0.211 & 0.200 & 0.190 & 0.182 & 0.173 & 0.166 & 0.160 & 0.154 & 0.154 \\
\hline 12 & 1000.0 & 0.227 & 0.214 & 0.204 & 0.193 & 0.185 & 0.177 & 0.168 & 0.162 & 0.156 & 0.155 \\
\hline 13 & 1100.0 & 0.227 & 0.218 & 0.207 & 0.197 & 0.188 & 0.178 & 0.171 & 0.165 & 0.159 & 0.158 \\
\hline 14 & 1200.0 & 0.233 & 0.222 & 0.210 & 0.200 & 0.190 & 0.183 & 0.174 & 0.168 & 0.163 & 0.161 \\
\hline 15 & 1300.0 & 0.238 & 0.227 & 0.215 & 0.205 & 0.196 & 0.186 & 0.179 & 0.171 & 0.168 & 0.165 \\
\hline
\end{tabular}


Table 6.40. Problem 5.2.1 reference solution MPACT power peaking factors for axial segments in fuel channels $\mathrm{E}, \mathrm{F}, \mathrm{G}, \mathrm{H}, \mathrm{J}, \mathrm{K}, \mathrm{L}$, and $\mathrm{M}$ in element 5.

\begin{tabular}{|c|c|c|c|c|c|c|c|c|c|c|c|}
\hline \multirow[t]{2}{*}{ Step } & \multirow{2}{*}{$\begin{array}{l}\text { Burnup } \\
{\left[\frac{\mathrm{MWd}}{\mathrm{M} \mathrm{dI}}\right]}\end{array}$} & \multicolumn{10}{|c|}{ Power Peaking Factors } \\
\hline & & 1 & 2 & 3 & 4 & 5 & 6 & 7 & 8 & 9 & 10 \\
\hline 0 & 0.0 & 0.337 & 0.307 & 0.284 & 0.264 & 0.246 & 0.230 & 0.216 & 0.204 & 0.192 & 0.181 \\
\hline 1 & 25.0 & 0.372 & 0.339 & 0.315 & 0.294 & 0.276 & 0.259 & 0.244 & 0.231 & 0.219 & 0.207 \\
\hline 2 & 50.0 & 0.372 & 0.340 & 0.316 & 0.295 & 0.277 & 0.261 & 0.246 & 0.233 & 0.220 & 0.209 \\
\hline 3 & 100.0 & 0.368 & 0.336 & 0.312 & 0.291 & 0.273 & 0.257 & 0.242 & 0.229 & 0.217 & 0.205 \\
\hline 4 & 200.0 & 0.355 & 0.324 & 0.300 & 0.279 & 0.262 & 0.246 & 0.232 & 0.219 & 0.207 & 0.196 \\
\hline 5 & 300.0 & 0.348 & 0.317 & 0.293 & 0.273 & 0.255 & 0.240 & 0.225 & 0.213 & 0.201 & 0.190 \\
\hline 6 & 400.0 & 0.344 & 0.313 & 0.289 & 0.269 & 0.251 & 0.236 & 0.221 & 0.209 & 0.197 & 0.186 \\
\hline 7 & 500.0 & 0.342 & 0.311 & 0.288 & 0.267 & 0.250 & 0.234 & 0.220 & 0.207 & 0.195 & 0.184 \\
\hline 8 & 600.0 & 0.343 & 0.312 & 0.288 & 0.268 & 0.250 & 0.234 & 0.220 & 0.207 & 0.195 & 0.184 \\
\hline 9 & 700.0 & 0.345 & 0.313 & 0.289 & 0.269 & 0.251 & 0.235 & 0.221 & 0.208 & 0.196 & 0.185 \\
\hline 10 & 800.0 & 0.349 & 0.317 & 0.292 & 0.272 & 0.253 & 0.237 & 0.223 & 0.210 & 0.197 & 0.186 \\
\hline 11 & 900.0 & 0.352 & 0.320 & 0.296 & 0.275 & 0.257 & 0.240 & 0.226 & 0.212 & 0.200 & 0.189 \\
\hline 12 & 1000.0 & 0.358 & 0.326 & 0.301 & 0.280 & 0.261 & 0.244 & 0.230 & 0.216 & 0.203 & 0.192 \\
\hline 13 & 1100.0 & 0.364 & 0.331 & 0.306 & 0.285 & 0.266 & 0.249 & 0.234 & 0.220 & 0.208 & 0.196 \\
\hline 14 & 1200.0 & 0.374 & 0.340 & 0.315 & 0.293 & 0.273 & 0.256 & 0.241 & 0.227 & 0.214 & 0.202 \\
\hline 15 & 1300.0 & 0.380 & 0.346 & 0.320 & 0.298 & 0.278 & 0.261 & 0.246 & 0.231 & 0.218 & 0.206 \\
\hline & & 11 & 12 & 13 & 14 & 15 & 16 & 17 & 18 & 19 & 20 \\
\hline 0 & 0.0 & 0.171 & 0.161 & 0.153 & 0.144 & 0.137 & 0.130 & 0.124 & 0.119 & 0.114 & 0.112 \\
\hline 1 & 25.0 & 0.196 & 0.186 & 0.177 & 0.168 & 0.160 & 0.152 & 0.145 & 0.140 & 0.135 & 0.133 \\
\hline 2 & 50.0 & 0.198 & 0.188 & 0.178 & 0.170 & 0.161 & 0.154 & 0.147 & 0.142 & 0.137 & 0.135 \\
\hline 3 & 100.0 & 0.195 & 0.185 & 0.176 & 0.167 & 0.159 & 0.152 & 0.145 & 0.140 & 0.135 & 0.133 \\
\hline 4 & 200.0 & 0.185 & 0.176 & 0.167 & 0.158 & 0.150 & 0.143 & 0.137 & 0.132 & 0.127 & 0.126 \\
\hline 5 & 300.0 & 0.179 & 0.170 & 0.161 & 0.153 & 0.145 & 0.138 & 0.132 & 0.127 & 0.122 & 0.120 \\
\hline 6 & 400.0 & 0.176 & 0.166 & 0.157 & 0.149 & 0.141 & 0.135 & 0.128 & 0.123 & 0.119 & 0.117 \\
\hline 7 & 500.0 & 0.174 & 0.164 & 0.155 & 0.147 & 0.140 & 0.133 & 0.126 & 0.121 & 0.117 & 0.115 \\
\hline 8 & 600.0 & 0.174 & 0.164 & 0.155 & 0.147 & 0.139 & 0.132 & 0.126 & 0.121 & 0.116 & 0.114 \\
\hline 9 & 700.0 & 0.174 & 0.165 & 0.156 & 0.147 & 0.139 & 0.132 & 0.126 & 0.121 & 0.116 & 0.114 \\
\hline 10 & 800.0 & 0.176 & 0.166 & 0.157 & 0.148 & 0.141 & 0.133 & 0.127 & 0.122 & 0.117 & 0.115 \\
\hline 11 & 900.0 & 0.178 & 0.168 & 0.159 & 0.150 & 0.143 & 0.135 & 0.129 & 0.123 & 0.119 & 0.117 \\
\hline 12 & 1000.0 & 0.181 & 0.171 & 0.162 & 0.153 & 0.145 & 0.138 & 0.131 & 0.125 & 0.121 & 0.119 \\
\hline 13 & 1100.0 & 0.185 & 0.175 & 0.165 & 0.156 & 0.148 & 0.141 & 0.134 & 0.128 & 0.124 & 0.121 \\
\hline 14 & 1200.0 & 0.191 & 0.181 & 0.171 & 0.162 & 0.153 & 0.146 & 0.139 & 0.133 & 0.128 & 0.126 \\
\hline 15 & 1300.0 & 0.195 & 0.184 & 0.174 & 0.165 & 0.157 & 0.149 & 0.142 & 0.136 & 0.131 & 0.129 \\
\hline
\end{tabular}


Table 6.41. Problem 5.2.1 reference solution Shift power peaking factors for axial segments in fuel channels E, F, G, H, J, K, L, and $M$ in element 6.

\begin{tabular}{|c|c|c|c|c|c|c|c|c|c|c|c|}
\hline \multirow[t]{2}{*}{ Step } & Burnup & \multicolumn{10}{|c|}{ Power Peaking Factors } \\
\hline & {$\left[\frac{\mathrm{MWd}}{\mathrm{MtU}}\right]$} & 1 & 2 & 3 & 4 & 5 & 6 & 7 & 8 & 9 & 10 \\
\hline 0 & 0.0 & 0.135 & 0.121 & 0.113 & 0.105 & 0.099 & 0.092 & 0.086 & 0.081 & 0.076 & 0.071 \\
\hline 1 & 25.0 & 0.159 & 0.143 & 0.134 & 0.125 & 0.118 & 0.110 & 0.104 & 0.098 & 0.091 & 0.086 \\
\hline 2 & 50.0 & 0.158 & 0.142 & 0.133 & 0.125 & 0.116 & 0.110 & 0.103 & 0.097 & 0.092 & 0.086 \\
\hline 3 & 100.0 & 0.152 & 0.137 & 0.127 & 0.120 & 0.112 & 0.105 & 0.099 & 0.093 & 0.088 & 0.082 \\
\hline 4 & 200.0 & 0.141 & 0.128 & 0.120 & 0.111 & 0.105 & 0.098 & 0.092 & 0.086 & 0.081 & 0.076 \\
\hline 5 & 300.0 & 0.138 & 0.124 & 0.115 & 0.107 & 0.100 & 0.095 & 0.089 & 0.083 & 0.078 & 0.073 \\
\hline 6 & 400.0 & 0.133 & 0.120 & 0.112 & 0.104 & 0.097 & 0.090 & 0.085 & 0.080 & 0.075 & 0.070 \\
\hline 7 & 500.0 & 0.132 & 0.119 & 0.111 & 0.103 & 0.096 & 0.090 & 0.084 & 0.079 & 0.075 & 0.070 \\
\hline 8 & 600.0 & 0.132 & 0.118 & 0.110 & 0.103 & 0.096 & 0.090 & 0.084 & 0.079 & 0.074 & 0.069 \\
\hline 9 & 700.0 & 0.132 & 0.119 & 0.110 & 0.103 & 0.097 & 0.091 & 0.085 & 0.079 & 0.074 & 0.070 \\
\hline 10 & 800.0 & 0.134 & 0.120 & 0.113 & 0.104 & 0.097 & 0.091 & 0.085 & 0.080 & 0.075 & 0.071 \\
\hline 11 & 900.0 & 0.136 & 0.122 & 0.113 & 0.106 & 0.098 & 0.093 & 0.086 & 0.081 & 0.076 & 0.071 \\
\hline 12 & 1000.0 & 0.139 & 0.124 & 0.115 & 0.107 & 0.101 & 0.094 & 0.088 & 0.083 & 0.077 & 0.073 \\
\hline 13 & 1100.0 & 0.140 & 0.126 & 0.117 & 0.109 & 0.103 & 0.096 & 0.090 & 0.084 & 0.079 & 0.074 \\
\hline 14 & 1200.0 & 0.144 & 0.129 & 0.120 & 0.112 & 0.105 & 0.098 & 0.092 & 0.086 & 0.081 & 0.075 \\
\hline 15 & 1300.0 & 0.148 & 0.132 & 0.123 & 0.115 & 0.107 & 0.101 & 0.094 & 0.089 & 0.084 & 0.078 \\
\hline & & 11 & 12 & 13 & 14 & 15 & 16 & 17 & 18 & 19 & 20 \\
\hline 0 & 0.0 & 0.067 & 0.063 & 0.059 & 0.055 & 0.051 & 0.049 & 0.046 & 0.043 & 0.041 & 0.041 \\
\hline 1 & 25.0 & 0.081 & 0.076 & 0.071 & 0.067 & 0.062 & 0.059 & 0.056 & 0.053 & 0.050 & 0.051 \\
\hline 2 & 50.0 & 0.081 & 0.076 & 0.071 & 0.067 & 0.063 & 0.059 & 0.055 & 0.053 & 0.051 & 0.050 \\
\hline 3 & 100.0 & 0.077 & 0.072 & 0.067 & 0.064 & 0.060 & 0.056 & 0.053 & 0.050 & 0.048 & 0.048 \\
\hline 4 & 200.0 & 0.071 & 0.067 & 0.063 & 0.059 & 0.055 & 0.052 & 0.049 & 0.046 & 0.044 & 0.044 \\
\hline 5 & 300.0 & 0.068 & 0.065 & 0.060 & 0.057 & 0.053 & 0.050 & 0.047 & 0.044 & 0.043 & 0.043 \\
\hline 6 & 400.0 & 0.067 & 0.062 & 0.058 & 0.054 & 0.051 & 0.048 & 0.045 & 0.042 & 0.041 & 0.041 \\
\hline 7 & 500.0 & 0.066 & 0.061 & 0.057 & 0.053 & 0.050 & 0.047 & 0.044 & 0.042 & 0.040 & 0.040 \\
\hline 8 & 600.0 & 0.065 & 0.061 & 0.057 & 0.053 & 0.050 & 0.047 & 0.044 & 0.042 & 0.041 & 0.040 \\
\hline 9 & 700.0 & 0.066 & 0.061 & 0.058 & 0.054 & 0.050 & 0.047 & 0.044 & 0.042 & 0.040 & 0.040 \\
\hline 10 & 800.0 & 0.066 & 0.062 & 0.058 & 0.054 & 0.051 & 0.048 & 0.045 & 0.042 & 0.040 & 0.040 \\
\hline 11 & 900.0 & 0.067 & 0.062 & 0.058 & 0.054 & 0.051 & 0.048 & 0.045 & 0.043 & 0.041 & 0.041 \\
\hline 12 & 1000.0 & 0.068 & 0.064 & 0.059 & 0.056 & 0.052 & 0.049 & 0.046 & 0.044 & 0.042 & 0.042 \\
\hline 13 & 1100.0 & 0.069 & 0.065 & 0.061 & 0.057 & 0.053 & 0.050 & 0.047 & 0.044 & 0.043 & 0.042 \\
\hline 14 & 1200.0 & 0.071 & 0.066 & 0.063 & 0.058 & 0.055 & 0.051 & 0.048 & 0.046 & 0.043 & 0.043 \\
\hline 15 & 1300.0 & 0.073 & 0.068 & 0.064 & 0.060 & 0.056 & 0.052 & 0.050 & 0.047 & 0.045 & 0.045 \\
\hline
\end{tabular}


Table 6.42. Problem 5.2.1 reference solution MPACT power peaking factors for axial segments in fuel channels E, F, G, H, J, K, L, and $M$ in element 6 .

\begin{tabular}{|c|c|c|c|c|c|c|c|c|c|c|c|}
\hline \multirow[t]{2}{*}{ Step } & \multirow{2}{*}{$\begin{array}{l}\text { Burnup } \\
{\left[\frac{\mathrm{MWd}}{\mathrm{MtU}}\right]}\end{array}$} & \multicolumn{10}{|c|}{ Power Peaking Factors } \\
\hline & & 1 & 2 & 3 & 4 & 5 & 6 & 7 & 8 & 9 & 10 \\
\hline 0 & 0.0 & 0.099 & 0.089 & 0.082 & 0.076 & 0.071 & 0.066 & 0.061 & 0.057 & 0.053 & 0.050 \\
\hline 1 & 25.0 & 0.118 & 0.107 & 0.099 & 0.092 & 0.085 & 0.080 & 0.075 & 0.070 & 0.065 & 0.061 \\
\hline 2 & 50.0 & 0.120 & 0.109 & 0.101 & 0.093 & 0.087 & 0.081 & 0.076 & 0.071 & 0.067 & 0.063 \\
\hline 3 & 100.0 & 0.118 & 0.107 & 0.099 & 0.092 & 0.086 & 0.080 & 0.075 & 0.070 & 0.066 & 0.062 \\
\hline 4 & 200.0 & 0.111 & 0.101 & 0.093 & 0.086 & 0.080 & 0.075 & 0.070 & 0.066 & 0.062 & 0.058 \\
\hline 5 & 300.0 & 0.106 & 0.097 & 0.089 & 0.083 & 0.077 & 0.072 & 0.067 & 0.063 & 0.059 & 0.055 \\
\hline 6 & 400.0 & 0.103 & 0.093 & 0.086 & 0.080 & 0.074 & 0.069 & 0.064 & 0.060 & 0.056 & 0.053 \\
\hline 7 & 500.0 & 0.101 & 0.092 & 0.084 & 0.078 & 0.073 & 0.068 & 0.063 & 0.059 & 0.055 & 0.051 \\
\hline 8 & 600.0 & 0.100 & 0.091 & 0.084 & 0.077 & 0.072 & 0.067 & 0.062 & 0.058 & 0.054 & 0.051 \\
\hline 9 & 700.0 & 0.100 & 0.091 & 0.084 & 0.077 & 0.072 & 0.067 & 0.062 & 0.058 & 0.054 & 0.051 \\
\hline 10 & 800.0 & 0.101 & 0.092 & 0.084 & 0.078 & 0.072 & 0.067 & 0.063 & 0.058 & 0.055 & 0.051 \\
\hline 11 & 900.0 & 0.102 & 0.093 & 0.085 & 0.079 & 0.073 & 0.068 & 0.063 & 0.059 & 0.055 & 0.052 \\
\hline 12 & 1000.0 & 0.104 & 0.094 & 0.087 & 0.080 & 0.074 & 0.069 & 0.065 & 0.060 & 0.056 & 0.052 \\
\hline 13 & 1100.0 & 0.106 & 0.096 & 0.089 & 0.082 & 0.076 & 0.071 & 0.066 & 0.062 & 0.057 & 0.054 \\
\hline 14 & 1200.0 & 0.111 & 0.100 & 0.092 & 0.085 & 0.079 & 0.074 & 0.069 & 0.064 & 0.060 & 0.056 \\
\hline \multirow[t]{2}{*}{15} & 1300.0 & 0.113 & 0.102 & 0.094 & 0.087 & 0.081 & 0.075 & 0.070 & 0.066 & 0.061 & 0.057 \\
\hline & & 11 & 12 & 13 & 14 & 15 & 16 & 17 & 18 & 19 & 20 \\
\hline 0 & 0.0 & 0.046 & 0.043 & 0.040 & 0.038 & 0.035 & 0.033 & 0.031 & 0.029 & 0.028 & 0.028 \\
\hline 1 & 25.0 & 0.057 & 0.054 & 0.050 & 0.047 & 0.044 & 0.041 & 0.038 & 0.037 & 0.035 & 0.035 \\
\hline 2 & 50.0 & 0.059 & 0.055 & 0.051 & 0.048 & 0.045 & 0.042 & 0.040 & 0.038 & 0.036 & 0.036 \\
\hline 3 & 100.0 & 0.058 & 0.054 & 0.051 & 0.047 & 0.044 & 0.042 & 0.039 & 0.037 & 0.036 & 0.035 \\
\hline 4 & 200.0 & 0.054 & 0.050 & 0.047 & 0.044 & 0.041 & 0.039 & 0.036 & 0.034 & 0.033 & 0.033 \\
\hline 5 & 300.0 & 0.051 & 0.048 & 0.045 & 0.042 & 0.039 & 0.037 & 0.034 & 0.033 & 0.031 & 0.031 \\
\hline 6 & 400.0 & 0.049 & 0.046 & 0.043 & 0.040 & 0.037 & 0.035 & 0.033 & 0.031 & 0.030 & 0.030 \\
\hline 7 & 500.0 & 0.048 & 0.045 & 0.042 & 0.039 & 0.036 & 0.034 & 0.032 & 0.030 & 0.029 & 0.029 \\
\hline 8 & 600.0 & 0.047 & 0.044 & 0.041 & 0.039 & 0.036 & 0.034 & 0.032 & 0.030 & 0.029 & 0.028 \\
\hline 9 & 700.0 & 0.047 & 0.044 & 0.041 & 0.038 & 0.036 & 0.033 & 0.031 & 0.030 & 0.029 & 0.028 \\
\hline 10 & 800.0 & 0.048 & 0.044 & 0.041 & 0.039 & 0.036 & 0.034 & 0.032 & 0.030 & 0.029 & 0.028 \\
\hline 11 & 900.0 & 0.048 & 0.045 & 0.042 & 0.039 & 0.036 & 0.034 & 0.032 & 0.030 & 0.029 & 0.029 \\
\hline 12 & 1000.0 & 0.049 & 0.046 & 0.043 & 0.040 & 0.037 & 0.035 & 0.032 & 0.031 & 0.030 & 0.029 \\
\hline 13 & 1100.0 & 0.050 & 0.047 & 0.044 & 0.041 & 0.038 & 0.035 & 0.033 & 0.031 & 0.030 & 0.030 \\
\hline 14 & 1200.0 & 0.052 & 0.049 & 0.045 & 0.042 & 0.040 & 0.037 & 0.035 & 0.033 & 0.032 & 0.031 \\
\hline 15 & 1300.0 & 0.053 & 0.050 & 0.047 & 0.043 & 0.040 & 0.038 & 0.036 & 0.034 & 0.032 & 0.032 \\
\hline
\end{tabular}


Fuel Channels $P, Q, R$, and $S$

Table 6.43. Problem 5.2.1 reference solution Shift power peaking factors for axial segments in fuel channels $P, Q, R$, and $S$ in element 1 .

\begin{tabular}{|c|c|c|c|c|c|c|c|c|c|c|c|}
\hline \multirow[t]{2}{*}{ Step } & \multirow{2}{*}{$\begin{array}{l}\text { Burnup } \\
{\left[\frac{\mathrm{MWd}}{\mathrm{MtU}}\right]}\end{array}$} & \multicolumn{10}{|c|}{ Power Peaking Factors } \\
\hline & & 1 & 2 & 3 & 4 & 5 & 6 & 7 & 8 & 9 & 10 \\
\hline 0 & 0.0 & 1.077 & 1.046 & 1.063 & 1.085 & 1.120 & 1.160 & 1.202 & 1.252 & 1.299 & 1.344 \\
\hline 1 & 25.0 & 1.070 & 1.038 & 1.052 & 1.077 & 1.110 & 1.146 & 1.188 & 1.236 & 1.279 & 1.330 \\
\hline 2 & 50.0 & 1.072 & 1.035 & 1.049 & 1.075 & 1.109 & 1.149 & 1.191 & 1.236 & 1.284 & 1.332 \\
\hline 3 & 100.0 & 1.064 & 1.034 & 1.046 & 1.073 & 1.106 & 1.147 & 1.190 & 1.234 & 1.283 & 1.330 \\
\hline 4 & 200.0 & 1.076 & 1.038 & 1.052 & 1.077 & 1.113 & 1.150 & 1.200 & 1.244 & 1.296 & 1.340 \\
\hline 5 & 300.0 & 1.067 & 1.036 & 1.047 & 1.077 & 1.110 & 1.151 & 1.198 & 1.246 & 1.289 & 1.341 \\
\hline 6 & 400.0 & 1.072 & 1.041 & 1.055 & 1.082 & 1.116 & 1.161 & 1.204 & 1.250 & 1.299 & 1.347 \\
\hline 7 & 500.0 & 1.067 & 1.031 & 1.044 & 1.069 & 1.107 & 1.152 & 1.199 & 1.243 & 1.292 & 1.339 \\
\hline 8 & 600.0 & 1.069 & 1.035 & 1.050 & 1.079 & 1.113 & 1.154 & 1.201 & 1.245 & 1.298 & 1.341 \\
\hline 9 & 700.0 & 1.073 & 1.040 & 1.055 & 1.079 & 1.118 & 1.159 & 1.204 & 1.250 & 1.300 & 1.348 \\
\hline 10 & 800.0 & 1.066 & 1.033 & 1.049 & 1.073 & 1.111 & 1.153 & 1.200 & 1.247 & 1.294 & 1.346 \\
\hline 11 & 900.0 & 1.071 & 1.039 & 1.052 & 1.078 & 1.115 & 1.157 & 1.202 & 1.247 & 1.296 & 1.347 \\
\hline 12 & 1000.0 & 1.073 & 1.039 & 1.053 & 1.082 & 1.114 & 1.157 & 1.200 & 1.252 & 1.293 & 1.343 \\
\hline 13 & 1100.0 & 1.067 & 1.031 & 1.045 & 1.074 & 1.109 & 1.151 & 1.196 & 1.247 & 1.291 & 1.337 \\
\hline 14 & 1200.0 & 1.067 & 1.035 & 1.051 & 1.077 & 1.117 & 1.154 & 1.200 & 1.246 & 1.292 & 1.341 \\
\hline 15 & 1300.0 & 1.066 & 1.033 & 1.048 & 1.073 & 1.110 & 1.155 & 1.195 & 1.243 & 1.290 & 1.338 \\
\hline & & 11 & 12 & 13 & 14 & 15 & 16 & 17 & 18 & 19 & 20 \\
\hline 0 & 0.0 & 1.393 & 1.442 & 1.494 & 1.547 & 1.594 & 1.647 & 1.704 & 1.771 & 1.835 & 1.970 \\
\hline 1 & 25.0 & 1.377 & 1.429 & 1.470 & 1.520 & 1.567 & 1.619 & 1.673 & 1.734 & 1.807 & 1.934 \\
\hline 2 & 50.0 & 1.379 & 1.425 & 1.472 & 1.522 & 1.572 & 1.623 & 1.677 & 1.737 & 1.803 & 1.938 \\
\hline 3 & 100.0 & 1.381 & 1.429 & 1.474 & 1.524 & 1.575 & 1.627 & 1.682 & 1.744 & 1.815 & 1.947 \\
\hline 4 & 200.0 & 1.391 & 1.440 & 1.490 & 1.536 & 1.588 & 1.644 & 1.697 & 1.760 & 1.830 & 1.966 \\
\hline 5 & 300.0 & 1.393 & 1.442 & 1.487 & 1.540 & 1.592 & 1.642 & 1.701 & 1.764 & 1.833 & 1.966 \\
\hline 6 & 400.0 & 1.396 & 1.449 & 1.498 & 1.546 & 1.597 & 1.652 & 1.708 & 1.772 & 1.841 & 1.984 \\
\hline 7 & 500.0 & 1.392 & 1.444 & 1.492 & 1.544 & 1.597 & 1.648 & 1.704 & 1.765 & 1.840 & 1.978 \\
\hline 8 & 600.0 & 1.396 & 1.442 & 1.488 & 1.539 & 1.590 & 1.646 & 1.700 & 1.764 & 1.835 & 1.975 \\
\hline 9 & 700.0 & 1.397 & 1.449 & 1.495 & 1.549 & 1.593 & 1.647 & 1.701 & 1.765 & 1.837 & 1.972 \\
\hline 10 & 800.0 & 1.393 & 1.442 & 1.490 & 1.538 & 1.586 & 1.638 & 1.698 & 1.758 & 1.831 & 1.972 \\
\hline 11 & 900.0 & 1.390 & 1.438 & 1.490 & 1.533 & 1.583 & 1.639 & 1.696 & 1.751 & 1.826 & 1.968 \\
\hline 12 & 1000.0 & 1.395 & 1.441 & 1.486 & 1.535 & 1.586 & 1.633 & 1.692 & 1.749 & 1.814 & 1.956 \\
\hline 13 & 1100.0 & 1.384 & 1.432 & 1.485 & 1.527 & 1.575 & 1.626 & 1.684 & 1.740 & 1.810 & 1.944 \\
\hline 14 & 1200.0 & 1.392 & 1.437 & 1.482 & 1.526 & 1.578 & 1.623 & 1.677 & 1.738 & 1.803 & 1.937 \\
\hline 15 & 1300.0 & 1.384 & 1.432 & 1.479 & 1.524 & 1.573 & 1.620 & 1.671 & 1.733 & 1.794 & 1.928 \\
\hline
\end{tabular}


Table 6.44. Problem 5.2.1 reference solution MPACT power peaking factors for axial segments in fuel channels $P, Q, R$, and $S$ in element 1.

\begin{tabular}{|c|c|c|c|c|c|c|c|c|c|c|c|}
\hline \multirow[t]{2}{*}{ Step } & \multirow{2}{*}{$\begin{array}{l}\text { Burnup } \\
{\left[\frac{\mathrm{MWd}}{\mathrm{MtU}}\right]}\end{array}$} & \multicolumn{10}{|c|}{ Power Peaking Factors } \\
\hline & & 1 & 2 & 3 & 4 & 5 & 6 & 7 & 8 & 9 & 10 \\
\hline 0 & 0.0 & 1.131 & 1.106 & 1.113 & 1.136 & 1.171 & 1.212 & 1.258 & 1.307 & 1.357 & 1.407 \\
\hline 1 & 25.0 & 1.125 & 1.098 & 1.104 & 1.126 & 1.160 & 1.200 & 1.245 & 1.292 & 1.340 & 1.389 \\
\hline 2 & 50.0 & 1.120 & 1.093 & 1.099 & 1.122 & 1.155 & 1.195 & 1.240 & 1.287 & 1.336 & 1.385 \\
\hline 3 & 100.0 & 1.112 & 1.086 & 1.092 & 1.115 & 1.149 & 1.189 & 1.235 & 1.282 & 1.332 & 1.381 \\
\hline 4 & 200.0 & 1.108 & 1.082 & 1.089 & 1.112 & 1.146 & 1.188 & 1.234 & 1.282 & 1.332 & 1.382 \\
\hline 5 & 300.0 & 1.107 & 1.081 & 1.088 & 1.112 & 1.146 & 1.188 & 1.235 & 1.284 & 1.334 & 1.385 \\
\hline 6 & 400.0 & 1.108 & 1.082 & 1.090 & 1.113 & 1.148 & 1.190 & 1.237 & 1.286 & 1.337 & 1.388 \\
\hline 7 & 500.0 & 1.109 & 1.083 & 1.091 & 1.115 & 1.150 & 1.192 & 1.239 & 1.288 & 1.339 & 1.390 \\
\hline 8 & 600.0 & 1.111 & 1.085 & 1.092 & 1.116 & 1.152 & 1.194 & 1.241 & 1.290 & 1.341 & 1.392 \\
\hline 9 & 700.0 & 1.113 & 1.088 & 1.095 & 1.119 & 1.154 & 1.197 & 1.243 & 1.293 & 1.343 & 1.394 \\
\hline 10 & 800.0 & 1.115 & 1.089 & 1.096 & 1.120 & 1.155 & 1.197 & 1.244 & 1.293 & 1.343 & 1.394 \\
\hline 11 & 900.0 & 1.117 & 1.091 & 1.098 & 1.122 & 1.157 & 1.200 & 1.246 & 1.295 & 1.345 & 1.395 \\
\hline 12 & 1000.0 & 1.116 & 1.091 & 1.098 & 1.122 & 1.157 & 1.199 & 1.246 & 1.294 & 1.344 & 1.393 \\
\hline 13 & 1100.0 & 1.118 & 1.093 & 1.100 & 1.124 & 1.159 & 1.201 & 1.247 & 1.295 & 1.344 & 1.394 \\
\hline 14 & 1200.0 & 1.117 & 1.091 & 1.099 & 1.123 & 1.157 & 1.199 & 1.244 & 1.292 & 1.341 & 1.390 \\
\hline 15 & 1300.0 & 1.118 & 1.093 & 1.100 & 1.124 & 1.159 & 1.200 & 1.245 & 1.293 & 1.341 & 1.390 \\
\hline & & 11 & 12 & 13 & 14 & 15 & 16 & 17 & 18 & 19 & 20 \\
\hline 0 & 0.0 & 1.458 & 1.508 & 1.559 & 1.610 & 1.663 & 1.719 & 1.781 & 1.850 & 1.931 & 2.047 \\
\hline 1 & 25.0 & 1.438 & 1.487 & 1.535 & 1.585 & 1.636 & 1.690 & 1.750 & 1.817 & 1.896 & 2.010 \\
\hline 2 & 50.0 & 1.434 & 1.483 & 1.531 & 1.581 & 1.632 & 1.687 & 1.746 & 1.813 & 1.892 & 2.005 \\
\hline 3 & 100.0 & 1.431 & 1.480 & 1.530 & 1.580 & 1.633 & 1.688 & 1.748 & 1.817 & 1.898 & 2.014 \\
\hline 4 & 200.0 & 1.433 & 1.483 & 1.534 & 1.585 & 1.639 & 1.695 & 1.756 & 1.826 & 1.909 & 2.028 \\
\hline 5 & 300.0 & 1.436 & 1.487 & 1.538 & 1.590 & 1.643 & 1.700 & 1.762 & 1.832 & 1.916 & 2.036 \\
\hline 6 & 400.0 & 1.439 & 1.490 & 1.541 & 1.593 & 1.646 & 1.703 & 1.765 & 1.836 & 1.919 & 2.040 \\
\hline 7 & 500.0 & 1.441 & 1.492 & 1.543 & 1.594 & 1.648 & 1.705 & 1.766 & 1.837 & 1.920 & 2.041 \\
\hline 8 & 600.0 & 1.442 & 1.493 & 1.544 & 1.595 & 1.648 & 1.705 & 1.766 & 1.836 & 1.919 & 2.040 \\
\hline 9 & 700.0 & 1.444 & 1.494 & 1.545 & 1.595 & 1.648 & 1.703 & 1.764 & 1.833 & 1.915 & 2.033 \\
\hline 10 & 800.0 & 1.444 & 1.494 & 1.544 & 1.594 & 1.647 & 1.702 & 1.762 & 1.831 & 1.913 & 2.032 \\
\hline 11 & 900.0 & 1.444 & 1.494 & 1.543 & 1.593 & 1.644 & 1.699 & 1.758 & 1.826 & 1.906 & 2.022 \\
\hline 12 & 1000.0 & 1.443 & 1.492 & 1.541 & 1.590 & 1.641 & 1.695 & 1.754 & 1.821 & 1.901 & 2.018 \\
\hline 13 & 1100.0 & 1.443 & 1.491 & 1.539 & 1.588 & 1.638 & 1.691 & 1.749 & 1.814 & 1.893 & 2.006 \\
\hline 14 & 1200.0 & 1.438 & 1.486 & 1.534 & 1.582 & 1.631 & 1.684 & 1.741 & 1.806 & 1.884 & 1.997 \\
\hline 15 & 1300.0 & 1.437 & 1.485 & 1.532 & 1.579 & 1.628 & 1.679 & 1.735 & 1.799 & 1.875 & 1.986 \\
\hline
\end{tabular}


Table 6.45. Problem 5.2.1 reference solution Shift power peaking factors for axial segments in fuel channels $\mathbf{P}, \mathbf{Q}, \mathbf{R}$, and $\mathrm{S}$ in element 2.

\begin{tabular}{|c|c|c|c|c|c|c|c|c|c|c|c|}
\hline \multirow[t]{2}{*}{ Step } & Burnup & \multicolumn{10}{|c|}{ Power Peaking Factors } \\
\hline & {$\left[\frac{\mathrm{MWd}}{\mathrm{MtU}}\right]$} & 1 & 2 & 3 & 4 & 5 & 6 & 7 & 8 & 9 & 10 \\
\hline 0 & 0.0 & 2.041 & 1.966 & 1.958 & 1.952 & 1.951 & 1.958 & 1.962 & 1.967 & 1.977 & 1.982 \\
\hline 1 & 25.0 & 2.000 & 1.927 & 1.915 & 1.910 & 1.911 & 1.917 & 1.917 & 1.928 & 1.933 & 1.948 \\
\hline 2 & 50.0 & 2.005 & 1.931 & 1.917 & 1.912 & 1.916 & 1.919 & 1.925 & 1.929 & 1.938 & 1.944 \\
\hline 3 & 100.0 & 2.015 & 1.941 & 1.929 & 1.927 & 1.928 & 1.931 & 1.939 & 1.944 & 1.955 & 1.957 \\
\hline 4 & 200.0 & 2.033 & 1.958 & 1.952 & 1.945 & 1.945 & 1.949 & 1.953 & 1.962 & 1.971 & 1.975 \\
\hline 5 & 300.0 & 2.042 & 1.964 & 1.953 & 1.948 & 1.950 & 1.954 & 1.960 & 1.970 & 1.976 & 1.981 \\
\hline 6 & 400.0 & 2.053 & 1.976 & 1.962 & 1.960 & 1.956 & 1.967 & 1.966 & 1.974 & 1.983 & 1.989 \\
\hline 7 & 500.0 & 2.045 & 1.968 & 1.958 & 1.955 & 1.954 & 1.958 & 1.965 & 1.974 & 1.982 & 1.987 \\
\hline 8 & 600.0 & 2.042 & 1.963 & 1.954 & 1.953 & 1.950 & 1.956 & 1.960 & 1.965 & 1.972 & 1.985 \\
\hline 9 & 700.0 & 2.045 & 1.967 & 1.954 & 1.950 & 1.946 & 1.951 & 1.961 & 1.968 & 1.976 & 1.984 \\
\hline 10 & 800.0 & 2.040 & 1.951 & 1.946 & 1.937 & 1.944 & 1.946 & 1.952 & 1.964 & 1.966 & 1.980 \\
\hline 11 & 900.0 & 2.024 & 1.948 & 1.935 & 1.933 & 1.933 & 1.938 & 1.938 & 1.951 & 1.958 & 1.965 \\
\hline 12 & 1000.0 & 2.024 & 1.945 & 1.925 & 1.924 & 1.925 & 1.932 & 1.936 & 1.940 & 1.953 & 1.958 \\
\hline 13 & 1100.0 & 2.013 & 1.932 & 1.917 & 1.914 & 1.918 & 1.922 & 1.927 & 1.933 & 1.939 & 1.952 \\
\hline 14 & 1200.0 & 2.006 & 1.926 & 1.918 & 1.909 & 1.907 & 1.917 & 1.923 & 1.930 & 1.938 & 1.942 \\
\hline 15 & 1300.0 & 1.990 & 1.911 & 1.901 & 1.898 & 1.900 & 1.904 & 1.911 & 1.920 & 1.931 & 1.933 \\
\hline & & 11 & 12 & 13 & 14 & 15 & 16 & 17 & 18 & 19 & 20 \\
\hline 0 & 0.0 & 1.987 & 1.994 & 2.000 & 2.002 & 2.011 & 2.022 & 2.034 & 2.050 & 2.078 & 2.167 \\
\hline 1 & 25.0 & 1.950 & 1.954 & 1.962 & 1.965 & 1.974 & 1.982 & 1.996 & 2.015 & 2.039 & 2.133 \\
\hline 2 & 50.0 & 1.950 & 1.961 & 1.965 & 1.967 & 1.976 & 1.985 & 1.999 & 2.026 & 2.047 & 2.139 \\
\hline 3 & 100.0 & 1.965 & 1.969 & 1.981 & 1.983 & 1.995 & 2.000 & 2.014 & 2.032 & 2.060 & 2.154 \\
\hline 4 & 200.0 & 1.984 & 1.984 & 1.998 & 2.000 & 2.007 & 2.014 & 2.028 & 2.047 & 2.073 & 2.171 \\
\hline 5 & 300.0 & 1.987 & 1.994 & 2.001 & 2.006 & 2.014 & 2.027 & 2.037 & 2.052 & 2.081 & 2.181 \\
\hline 6 & 400.0 & 1.994 & 2.002 & 2.002 & 2.010 & 2.014 & 2.024 & 2.037 & 2.059 & 2.086 & 2.183 \\
\hline 7 & 500.0 & 1.994 & 2.002 & 2.006 & 2.014 & 2.019 & 2.025 & 2.039 & 2.061 & 2.086 & 2.184 \\
\hline 8 & 600.0 & 1.993 & 1.995 & 2.003 & 2.007 & 2.014 & 2.016 & 2.034 & 2.049 & 2.075 & 2.176 \\
\hline 9 & 700.0 & 1.984 & 1.990 & 2.000 & 2.005 & 2.007 & 2.016 & 2.031 & 2.048 & 2.074 & 2.174 \\
\hline 10 & 800.0 & 1.986 & 1.988 & 1.992 & 2.003 & 2.008 & 2.016 & 2.030 & 2.043 & 2.071 & 2.174 \\
\hline 11 & 900.0 & 1.972 & 1.976 & 1.984 & 1.988 & 1.996 & 2.005 & 2.014 & 2.033 & 2.061 & 2.163 \\
\hline 12 & 1000.0 & 1.964 & 1.971 & 1.977 & 1.980 & 1.983 & 1.992 & 2.006 & 2.024 & 2.051 & 2.150 \\
\hline 13 & 1100.0 & 1.955 & 1.962 & 1.963 & 1.973 & 1.980 & 1.990 & 1.998 & 2.015 & 2.043 & 2.146 \\
\hline 14 & 1200.0 & 1.950 & 1.952 & 1.953 & 1.965 & 1.972 & 1.982 & 1.996 & 2.010 & 2.036 & 2.131 \\
\hline 15 & 1300.0 & 1.940 & 1.943 & 1.947 & 1.954 & 1.963 & 1.969 & 1.983 & 2.001 & 2.022 & 2.126 \\
\hline
\end{tabular}


Table 6.46. Problem 5.2.1 reference solution MPACT power peaking factors for axial segments in fuel channels $\mathbf{P}, \mathbf{Q}, \mathbf{R}$, and $\mathrm{S}$ in element 2.

\begin{tabular}{|c|c|c|c|c|c|c|c|c|c|c|c|}
\hline \multirow[t]{2}{*}{ Step } & \multirow{2}{*}{$\begin{array}{l}\text { Burnup } \\
{\left[\frac{\mathrm{MWd}}{\mathrm{MtU}}\right]}\end{array}$} & \multicolumn{10}{|c|}{ Power Peaking Factors } \\
\hline & & 1 & 2 & 3 & 4 & 5 & 6 & 7 & 8 & 9 & 10 \\
\hline 0 & 0.0 & 2.118 & 2.062 & 2.039 & 2.028 & 2.024 & 2.026 & 2.030 & 2.035 & 2.041 & 2.046 \\
\hline 1 & 25.0 & 2.078 & 2.021 & 1.997 & 1.986 & 1.982 & 1.983 & 1.987 & 1.992 & 1.998 & 2.003 \\
\hline 2 & 50.0 & 2.074 & 2.018 & 1.996 & 1.985 & 1.982 & 1.984 & 1.988 & 1.994 & 2.000 & 2.005 \\
\hline 3 & 100.0 & 2.085 & 2.027 & 2.004 & 1.993 & 1.990 & 1.992 & 1.996 & 2.002 & 2.008 & 2.014 \\
\hline 4 & 200.0 & 2.101 & 2.042 & 2.019 & 2.008 & 2.005 & 2.007 & 2.012 & 2.018 & 2.025 & 2.031 \\
\hline 5 & 300.0 & 2.110 & 2.050 & 2.026 & 2.016 & 2.013 & 2.015 & 2.020 & 2.026 & 2.033 & 2.039 \\
\hline 6 & 400.0 & 2.114 & 2.053 & 2.029 & 2.019 & 2.016 & 2.018 & 2.023 & 2.030 & 2.036 & 2.042 \\
\hline 7 & 500.0 & 2.114 & 2.053 & 2.029 & 2.018 & 2.016 & 2.018 & 2.023 & 2.029 & 2.035 & 2.041 \\
\hline 8 & 600.0 & 2.112 & 2.051 & 2.026 & 2.015 & 2.012 & 2.014 & 2.019 & 2.025 & 2.032 & 2.038 \\
\hline 9 & 700.0 & 2.104 & 2.045 & 2.021 & 2.011 & 2.008 & 2.010 & 2.015 & 2.021 & 2.028 & 2.034 \\
\hline 10 & 800.0 & 2.102 & 2.040 & 2.016 & 2.004 & 2.001 & 2.003 & 2.008 & 2.014 & 2.020 & 2.025 \\
\hline 11 & 900.0 & 2.091 & 2.031 & 2.008 & 1.997 & 1.994 & 1.996 & 2.001 & 2.007 & 2.013 & 2.018 \\
\hline 12 & 1000.0 & 2.085 & 2.024 & 2.000 & 1.988 & 1.985 & 1.987 & 1.991 & 1.996 & 2.002 & 2.008 \\
\hline 13 & 1100.0 & 2.072 & 2.013 & 1.990 & 1.979 & 1.976 & 1.977 & 1.982 & 1.988 & 1.994 & 1.999 \\
\hline 14 & 1200.0 & 2.062 & 2.002 & 1.977 & 1.966 & 1.962 & 1.963 & 1.968 & 1.973 & 1.979 & 1.984 \\
\hline 15 & 1300.0 & 2.049 & 1.991 & 1.967 & 1.956 & 1.953 & 1.955 & 1.959 & 1.965 & 1.970 & 1.976 \\
\hline & & 11 & 12 & 13 & 14 & 15 & 16 & 17 & 18 & 19 & 20 \\
\hline 0 & 0.0 & 2.050 & 2.054 & 2.058 & 2.061 & 2.067 & 2.075 & 2.088 & 2.110 & 2.144 & 2.213 \\
\hline 1 & 25.0 & 2.008 & 2.012 & 2.016 & 2.020 & 2.026 & 2.035 & 2.050 & 2.072 & 2.107 & 2.178 \\
\hline 2 & 50.0 & 2.010 & 2.015 & 2.019 & 2.023 & 2.029 & 2.038 & 2.052 & 2.074 & 2.108 & 2.178 \\
\hline 3 & 100.0 & 2.019 & 2.023 & 2.028 & 2.032 & 2.038 & 2.048 & 2.062 & 2.085 & 2.120 & 2.193 \\
\hline 4 & 200.0 & 2.036 & 2.041 & 2.045 & 2.050 & 2.056 & 2.065 & 2.079 & 2.102 & 2.138 & 2.212 \\
\hline 5 & 300.0 & 2.044 & 2.049 & 2.053 & 2.058 & 2.064 & 2.073 & 2.087 & 2.110 & 2.146 & 2.221 \\
\hline 6 & 400.0 & 2.047 & 2.052 & 2.056 & 2.060 & 2.066 & 2.075 & 2.089 & 2.112 & 2.149 & 2.225 \\
\hline 7 & 500.0 & 2.047 & 2.051 & 2.055 & 2.059 & 2.065 & 2.074 & 2.088 & 2.111 & 2.148 & 2.224 \\
\hline 8 & 600.0 & 2.043 & 2.047 & 2.051 & 2.056 & 2.061 & 2.070 & 2.084 & 2.107 & 2.144 & 2.220 \\
\hline 9 & 700.0 & 2.039 & 2.043 & 2.047 & 2.051 & 2.057 & 2.065 & 2.079 & 2.101 & 2.137 & 2.212 \\
\hline 10 & 800.0 & 2.030 & 2.035 & 2.039 & 2.043 & 2.049 & 2.058 & 2.072 & 2.095 & 2.132 & 2.208 \\
\hline 11 & 900.0 & 2.023 & 2.028 & 2.032 & 2.036 & 2.041 & 2.050 & 2.064 & 2.086 & 2.122 & 2.196 \\
\hline 12 & 1000.0 & 2.013 & 2.017 & 2.021 & 2.026 & 2.032 & 2.040 & 2.055 & 2.078 & 2.114 & 2.189 \\
\hline 13 & 1100.0 & 2.004 & 2.008 & 2.012 & 2.017 & 2.022 & 2.031 & 2.045 & 2.067 & 2.103 & 2.176 \\
\hline 14 & 1200.0 & 1.989 & 1.994 & 1.998 & 2.002 & 2.008 & 2.017 & 2.032 & 2.055 & 2.091 & 2.166 \\
\hline 15 & 1300.0 & 1.981 & 1.985 & 1.989 & 1.993 & 1.999 & 2.008 & 2.022 & 2.044 & 2.080 & 2.152 \\
\hline
\end{tabular}


Table 6.47. Problem 5.2.1 reference solution Shift power peaking factors for axial segments in fuel channels $P, Q, R$, and $S$ in element 3 .

\begin{tabular}{|c|c|c|c|c|c|c|c|c|c|c|c|}
\hline \multirow[t]{2}{*}{ Step } & Burnup & \multicolumn{10}{|c|}{ Power Peaking Factors } \\
\hline & {$\left[\frac{\mathrm{MWd}}{\mathrm{MtU}}\right.$} & 1 & 2 & 3 & 4 & 5 & 6 & 7 & 8 & 9 & 10 \\
\hline 0 & 0.0 & 2.129 & 1.999 & 1.936 & 1.883 & 1.834 & 1.792 & 1.747 & 1.712 & 1.672 & 1.633 \\
\hline 1 & 25.0 & 2.097 & 1.967 & 1.910 & 1.861 & 1.817 & 1.774 & 1.732 & 1.695 & 1.661 & 1.624 \\
\hline 2 & 50.0 & 2.101 & 1.972 & 1.910 & 1.860 & 1.814 & 1.776 & 1.739 & 1.696 & 1.662 & 1.623 \\
\hline 3 & 100.0 & 2.116 & 1.985 & 1.928 & 1.876 & 1.828 & 1.781 & 1.740 & 1.700 & 1.665 & 1.626 \\
\hline 4 & 200.0 & 2.126 & 1.996 & 1.932 & 1.880 & 1.834 & 1.787 & 1.746 & 1.708 & 1.666 & 1.631 \\
\hline 5 & 300.0 & 2.141 & 1.998 & 1.942 & 1.886 & 1.844 & 1.794 & 1.751 & 1.712 & 1.676 & 1.639 \\
\hline 6 & 400.0 & 2.138 & 2.001 & 1.940 & 1.889 & 1.839 & 1.798 & 1.753 & 1.714 & 1.671 & 1.634 \\
\hline 7 & 500.0 & 2.144 & 2.004 & 1.945 & 1.892 & 1.839 & 1.797 & 1.756 & 1.718 & 1.676 & 1.639 \\
\hline 8 & 600.0 & 2.137 & 2.002 & 1.940 & 1.891 & 1.843 & 1.795 & 1.761 & 1.721 & 1.679 & 1.642 \\
\hline 9 & 700.0 & 2.127 & 1.994 & 1.934 & 1.881 & 1.835 & 1.796 & 1.757 & 1.713 & 1.675 & 1.636 \\
\hline 10 & 800.0 & 2.126 & 1.989 & 1.934 & 1.881 & 1.833 & 1.794 & 1.755 & 1.718 & 1.681 & 1.641 \\
\hline 11 & 900.0 & 2.123 & 1.983 & 1.928 & 1.881 & 1.832 & 1.795 & 1.755 & 1.716 & 1.673 & 1.639 \\
\hline 12 & 1000.0 & 2.113 & 1.983 & 1.922 & 1.873 & 1.831 & 1.784 & 1.753 & 1.717 & 1.677 & 1.637 \\
\hline 13 & 1100.0 & 2.108 & 1.975 & 1.924 & 1.874 & 1.827 & 1.790 & 1.751 & 1.713 & 1.679 & 1.640 \\
\hline 14 & 1200.0 & 2.099 & 1.969 & 1.915 & 1.865 & 1.821 & 1.785 & 1.742 & 1.707 & 1.672 & 1.638 \\
\hline 15 & 1300.0 & 2.086 & 1.958 & 1.904 & 1.858 & 1.811 & 1.775 & 1.742 & 1.701 & 1.671 & 1.632 \\
\hline & & 11 & 12 & 13 & 14 & 15 & 16 & 17 & 18 & 19 & 20 \\
\hline 0 & 0.0 & 1.595 & 1.554 & 1.512 & 1.476 & 1.436 & 1.394 & 1.359 & 1.326 & 1.303 & 1.310 \\
\hline 1 & 25.0 & 1.585 & 1.546 & 1.509 & 1.474 & 1.434 & 1.397 & 1.364 & 1.333 & 1.310 & 1.326 \\
\hline 2 & 50.0 & 1.586 & 1.551 & 1.507 & 1.469 & 1.431 & 1.393 & 1.361 & 1.331 & 1.307 & 1.321 \\
\hline 3 & 100.0 & 1.593 & 1.551 & 1.511 & 1.471 & 1.433 & 1.397 & 1.363 & 1.332 & 1.304 & 1.318 \\
\hline 4 & 200.0 & 1.590 & 1.551 & 1.511 & 1.472 & 1.431 & 1.391 & 1.361 & 1.327 & 1.297 & 1.312 \\
\hline 5 & 300.0 & 1.601 & 1.554 & 1.516 & 1.475 & 1.438 & 1.398 & 1.363 & 1.325 & 1.303 & 1.315 \\
\hline 6 & 400.0 & 1.594 & 1.553 & 1.513 & 1.471 & 1.429 & 1.391 & 1.356 & 1.322 & 1.298 & 1.308 \\
\hline 7 & 500.0 & 1.599 & 1.560 & 1.519 & 1.480 & 1.437 & 1.401 & 1.365 & 1.330 & 1.302 & 1.319 \\
\hline 8 & 600.0 & 1.596 & 1.560 & 1.521 & 1.482 & 1.442 & 1.397 & 1.364 & 1.334 & 1.305 & 1.321 \\
\hline 9 & 700.0 & 1.593 & 1.560 & 1.517 & 1.480 & 1.442 & 1.396 & 1.363 & 1.329 & 1.306 & 1.325 \\
\hline 10 & 800.0 & 1.603 & 1.564 & 1.523 & 1.478 & 1.447 & 1.405 & 1.370 & 1.339 & 1.312 & 1.326 \\
\hline 11 & 900.0 & 1.599 & 1.564 & 1.522 & 1.483 & 1.445 & 1.407 & 1.372 & 1.343 & 1.316 & 1.335 \\
\hline 12 & 1000.0 & 1.600 & 1.564 & 1.523 & 1.485 & 1.446 & 1.408 & 1.374 & 1.344 & 1.317 & 1.338 \\
\hline 13 & 1100.0 & 1.605 & 1.569 & 1.528 & 1.486 & 1.452 & 1.413 & 1.387 & 1.354 & 1.327 & 1.341 \\
\hline 14 & 1200.0 & 1.599 & 1.564 & 1.525 & 1.486 & 1.449 & 1.414 & 1.377 & 1.349 & 1.327 & 1.345 \\
\hline 15 & 1300.0 & 1.602 & 1.562 & 1.526 & 1.490 & 1.453 & 1.419 & 1.387 & 1.358 & 1.334 & 1.353 \\
\hline
\end{tabular}


Table 6.48. Problem 5.2.1 reference solution MPACT power peaking factors for axial segments in fuel channels $P, Q, R$, and $S$ in element 3 .

\begin{tabular}{|c|c|c|c|c|c|c|c|c|c|c|c|}
\hline \multirow[t]{2}{*}{ Step } & Burnup & \multicolumn{10}{|c|}{ Power Peaking Factors } \\
\hline & {$\left[\frac{\mathrm{MWd}}{\mathrm{MtU}}\right.$} & 1 & 2 & 3 & 4 & 5 & 6 & 7 & 8 & 9 & 10 \\
\hline 0 & 0.0 & 2.162 & 2.051 & 1.975 & 1.912 & 1.858 & 1.809 & 1.763 & 1.719 & 1.675 & 1.632 \\
\hline 1 & 25.0 & 2.132 & 2.023 & 1.949 & 1.889 & 1.838 & 1.791 & 1.748 & 1.707 & 1.666 & 1.625 \\
\hline 2 & 50.0 & 2.131 & 2.023 & 1.951 & 1.891 & 1.839 & 1.793 & 1.750 & 1.708 & 1.667 & 1.626 \\
\hline 3 & 100.0 & 2.145 & 2.034 & 1.959 & 1.898 & 1.845 & 1.798 & 1.753 & 1.711 & 1.670 & 1.628 \\
\hline 4 & 200.0 & 2.163 & 2.048 & 1.972 & 1.910 & 1.856 & 1.807 & 1.762 & 1.719 & 1.676 & 1.633 \\
\hline 5 & 300.0 & 2.171 & 2.056 & 1.978 & 1.915 & 1.861 & 1.812 & 1.767 & 1.723 & 1.680 & 1.637 \\
\hline 6 & 400.0 & 2.175 & 2.058 & 1.981 & 1.918 & 1.863 & 1.815 & 1.769 & 1.725 & 1.682 & 1.639 \\
\hline 7 & 500.0 & 2.175 & 2.058 & 1.981 & 1.918 & 1.864 & 1.815 & 1.770 & 1.726 & 1.684 & 1.641 \\
\hline 8 & 600.0 & 2.172 & 2.056 & 1.979 & 1.917 & 1.863 & 1.815 & 1.770 & 1.727 & 1.684 & 1.642 \\
\hline 9 & 700.0 & 2.164 & 2.050 & 1.975 & 1.914 & 1.861 & 1.814 & 1.770 & 1.727 & 1.685 & 1.643 \\
\hline 10 & 800.0 & 2.161 & 2.047 & 1.972 & 1.911 & 1.858 & 1.812 & 1.768 & 1.726 & 1.684 & 1.643 \\
\hline 11 & 900.0 & 2.150 & 2.039 & 1.966 & 1.906 & 1.855 & 1.809 & 1.767 & 1.726 & 1.685 & 1.644 \\
\hline 12 & 1000.0 & 2.146 & 2.034 & 1.961 & 1.902 & 1.851 & 1.806 & 1.764 & 1.723 & 1.683 & 1.643 \\
\hline 13 & 1100.0 & 2.133 & 2.025 & 1.954 & 1.896 & 1.847 & 1.803 & 1.762 & 1.722 & 1.683 & 1.644 \\
\hline 14 & 1200.0 & 2.125 & 2.017 & 1.946 & 1.889 & 1.841 & 1.798 & 1.758 & 1.719 & 1.681 & 1.642 \\
\hline \multirow[t]{2}{*}{15} & 1300.0 & 2.112 & 2.007 & 1.939 & 1.884 & 1.837 & 1.795 & 1.756 & 1.718 & 1.681 & 1.643 \\
\hline & & 11 & 12 & 13 & 14 & 15 & 16 & 17 & 18 & 19 & 20 \\
\hline 0 & 0.0 & 1.588 & 1.544 & 1.499 & 1.454 & 1.410 & 1.368 & 1.328 & 1.293 & 1.266 & 1.259 \\
\hline 1 & 25.0 & 1.584 & 1.542 & 1.500 & 1.458 & 1.416 & 1.377 & 1.340 & 1.307 & 1.283 & 1.279 \\
\hline 2 & 50.0 & 1.585 & 1.543 & 1.501 & 1.459 & 1.417 & 1.377 & 1.339 & 1.307 & 1.282 & 1.277 \\
\hline 3 & 100.0 & 1.586 & 1.543 & 1.500 & 1.457 & 1.415 & 1.375 & 1.337 & 1.304 & 1.279 & 1.275 \\
\hline 4 & 200.0 & 1.590 & 1.547 & 1.502 & 1.458 & 1.415 & 1.373 & 1.335 & 1.301 & 1.275 & 1.271 \\
\hline 5 & 300.0 & 1.593 & 1.549 & 1.504 & 1.460 & 1.416 & 1.374 & 1.335 & 1.300 & 1.274 & 1.270 \\
\hline 6 & 400.0 & 1.595 & 1.551 & 1.506 & 1.462 & 1.418 & 1.375 & 1.336 & 1.301 & 1.275 & 1.271 \\
\hline 7 & 500.0 & 1.597 & 1.553 & 1.508 & 1.464 & 1.420 & 1.377 & 1.338 & 1.304 & 1.278 & 1.273 \\
\hline 8 & 600.0 & 1.598 & 1.555 & 1.510 & 1.466 & 1.422 & 1.380 & 1.341 & 1.307 & 1.281 & 1.277 \\
\hline 9 & 700.0 & 1.601 & 1.557 & 1.513 & 1.469 & 1.426 & 1.383 & 1.344 & 1.310 & 1.284 & 1.280 \\
\hline 10 & 800.0 & 1.601 & 1.558 & 1.514 & 1.471 & 1.428 & 1.386 & 1.348 & 1.315 & 1.289 & 1.287 \\
\hline 11 & 900.0 & 1.603 & 1.560 & 1.517 & 1.474 & 1.432 & 1.390 & 1.352 & 1.319 & 1.294 & 1.291 \\
\hline 12 & 1000.0 & 1.602 & 1.561 & 1.518 & 1.476 & 1.434 & 1.394 & 1.356 & 1.324 & 1.300 & 1.299 \\
\hline 13 & 1100.0 & 1.604 & 1.563 & 1.521 & 1.479 & 1.438 & 1.398 & 1.361 & 1.329 & 1.305 & 1.303 \\
\hline 14 & 1200.0 & 1.603 & 1.563 & 1.523 & 1.482 & 1.442 & 1.403 & 1.367 & 1.336 & 1.313 & 1.313 \\
\hline 15 & 1300.0 & 1.605 & 1.565 & 1.525 & 1.485 & 1.445 & 1.407 & 1.371 & 1.340 & 1.317 & 1.317 \\
\hline
\end{tabular}


Table 6.49. Problem 5.2.1 reference solution Shift power peaking factors for axial segments in fuel channels $P, Q, R$, and $S$ in element 4.

\begin{tabular}{|c|c|c|c|c|c|c|c|c|c|c|c|}
\hline \multirow[t]{2}{*}{ Step } & Burnup & \multicolumn{10}{|c|}{ Power Peaking Factors } \\
\hline & {$\left[\frac{\mathrm{MWd}}{\mathrm{MtU}}\right]$} & 1 & 2 & 3 & 4 & 5 & 6 & 7 & 8 & 9 & 10 \\
\hline 0 & 0.0 & 1.193 & 1.085 & 1.020 & 0.958 & 0.905 & 0.855 & 0.810 & 0.769 & 0.728 & 0.692 \\
\hline 1 & 25.0 & 1.212 & 1.101 & 1.038 & 0.976 & 0.925 & 0.878 & 0.831 & 0.794 & 0.754 & 0.721 \\
\hline 2 & 50.0 & 1.208 & 1.097 & 1.033 & 0.974 & 0.922 & 0.873 & 0.830 & 0.790 & 0.750 & 0.717 \\
\hline 3 & 100.0 & 1.206 & 1.098 & 1.031 & 0.969 & 0.913 & 0.866 & 0.824 & 0.782 & 0.743 & 0.706 \\
\hline 4 & 200.0 & 1.195 & 1.085 & 1.019 & 0.959 & 0.906 & 0.856 & 0.810 & 0.770 & 0.730 & 0.693 \\
\hline 5 & 300.0 & 1.199 & 1.086 & 1.018 & 0.958 & 0.901 & 0.852 & 0.809 & 0.769 & 0.730 & 0.692 \\
\hline 6 & 400.0 & 1.196 & 1.082 & 1.011 & 0.953 & 0.897 & 0.850 & 0.803 & 0.762 & 0.724 & 0.686 \\
\hline 7 & 500.0 & 1.196 & 1.083 & 1.014 & 0.956 & 0.902 & 0.852 & 0.807 & 0.764 & 0.727 & 0.688 \\
\hline 8 & 600.0 & 1.202 & 1.088 & 1.023 & 0.963 & 0.909 & 0.857 & 0.811 & 0.768 & 0.730 & 0.695 \\
\hline 9 & 700.0 & 1.205 & 1.089 & 1.022 & 0.959 & 0.904 & 0.857 & 0.812 & 0.770 & 0.731 & 0.696 \\
\hline 10 & 800.0 & 1.210 & 1.096 & 1.029 & 0.967 & 0.914 & 0.861 & 0.820 & 0.776 & 0.736 & 0.701 \\
\hline 11 & 900.0 & 1.220 & 1.099 & 1.033 & 0.973 & 0.919 & 0.870 & 0.823 & 0.783 & 0.745 & 0.707 \\
\hline 12 & 1000.0 & 1.223 & 1.107 & 1.039 & 0.978 & 0.923 & 0.876 & 0.832 & 0.788 & 0.750 & 0.713 \\
\hline 13 & 1100.0 & 1.229 & 1.114 & 1.046 & 0.987 & 0.936 & 0.885 & 0.838 & 0.797 & 0.758 & 0.721 \\
\hline 14 & 1200.0 & 1.235 & 1.119 & 1.048 & 0.988 & 0.934 & 0.885 & 0.841 & 0.802 & 0.761 & 0.723 \\
\hline 15 & 1300.0 & 1.239 & 1.126 & 1.057 & 0.998 & 0.940 & 0.897 & 0.852 & 0.809 & 0.771 & 0.735 \\
\hline & & 11 & 12 & 13 & 14 & 15 & 16 & 17 & 18 & 19 & 20 \\
\hline 0 & 0.0 & 0.661 & 0.630 & 0.599 & 0.569 & 0.545 & 0.522 & 0.500 & 0.483 & 0.469 & 0.468 \\
\hline 1 & 25.0 & 0.687 & 0.655 & 0.627 & 0.602 & 0.575 & 0.553 & 0.533 & 0.515 & 0.500 & 0.503 \\
\hline 2 & 50.0 & 0.682 & 0.652 & 0.625 & 0.595 & 0.571 & 0.550 & 0.530 & 0.514 & 0.499 & 0.502 \\
\hline 3 & 100.0 & 0.675 & 0.643 & 0.613 & 0.589 & 0.562 & 0.541 & 0.521 & 0.502 & 0.489 & 0.489 \\
\hline 4 & 200.0 & 0.663 & 0.631 & 0.601 & 0.572 & 0.550 & 0.528 & 0.507 & 0.487 & 0.475 & 0.473 \\
\hline 5 & 300.0 & 0.658 & 0.628 & 0.597 & 0.569 & 0.545 & 0.523 & 0.503 & 0.485 & 0.468 & 0.469 \\
\hline 6 & 400.0 & 0.654 & 0.623 & 0.591 & 0.563 & 0.540 & 0.516 & 0.495 & 0.476 & 0.463 & 0.463 \\
\hline 7 & 500.0 & 0.658 & 0.623 & 0.594 & 0.569 & 0.540 & 0.517 & 0.496 & 0.478 & 0.463 & 0.463 \\
\hline 8 & 600.0 & 0.661 & 0.628 & 0.598 & 0.571 & 0.544 & 0.521 & 0.498 & 0.481 & 0.467 & 0.466 \\
\hline 9 & 700.0 & 0.663 & 0.630 & 0.599 & 0.573 & 0.547 & 0.521 & 0.502 & 0.483 & 0.468 & 0.466 \\
\hline 10 & 800.0 & 0.666 & 0.633 & 0.605 & 0.579 & 0.548 & 0.527 & 0.504 & 0.487 & 0.472 & 0.471 \\
\hline 11 & 900.0 & 0.673 & 0.640 & 0.611 & 0.587 & 0.559 & 0.532 & 0.512 & 0.494 & 0.478 & 0.476 \\
\hline 12 & 1000.0 & 0.678 & 0.648 & 0.618 & 0.586 & 0.564 & 0.538 & 0.519 & 0.500 & 0.483 & 0.486 \\
\hline 13 & 1100.0 & 0.686 & 0.656 & 0.625 & 0.595 & 0.570 & 0.549 & 0.524 & 0.507 & 0.491 & 0.493 \\
\hline 14 & 1200.0 & 0.690 & 0.659 & 0.629 & 0.600 & 0.576 & 0.549 & 0.532 & 0.511 & 0.496 & 0.498 \\
\hline 15 & 1300.0 & 0.700 & 0.671 & 0.639 & 0.609 & 0.584 & 0.562 & 0.539 & 0.520 & 0.504 & 0.506 \\
\hline
\end{tabular}


Table 6.50. Problem 5.2.1 reference solution MPACT power peaking factors for axial segments in fuel channels $P, Q, R$, and $S$ in element 4.

\begin{tabular}{|c|c|c|c|c|c|c|c|c|c|c|c|}
\hline \multirow[t]{2}{*}{ Step } & \multirow{2}{*}{$\begin{array}{l}\text { Burnup } \\
{\left[\frac{\mathrm{MWd}}{\mathrm{MtU}}\right]}\end{array}$} & \multicolumn{10}{|c|}{ Power Peaking Factors } \\
\hline & & 1 & 2 & 3 & 4 & 5 & 6 & 7 & 8 & 9 & 10 \\
\hline 0 & 0.0 & 1.130 & 1.032 & 0.956 & 0.891 & 0.834 & 0.782 & 0.736 & 0.693 & 0.654 & 0.618 \\
\hline 1 & 25.0 & 1.154 & 1.057 & 0.982 & 0.918 & 0.862 & 0.811 & 0.766 & 0.724 & 0.686 & 0.650 \\
\hline 2 & 50.0 & 1.152 & 1.055 & 0.981 & 0.917 & 0.861 & 0.811 & 0.765 & 0.724 & 0.686 & 0.650 \\
\hline 3 & 100.0 & 1.149 & 1.051 & 0.976 & 0.912 & 0.855 & 0.805 & 0.759 & 0.718 & 0.679 & 0.644 \\
\hline 4 & 200.0 & 1.143 & 1.043 & 0.968 & 0.903 & 0.846 & 0.795 & 0.749 & 0.707 & 0.668 & 0.632 \\
\hline 5 & 300.0 & 1.141 & 1.041 & 0.965 & 0.899 & 0.842 & 0.790 & 0.744 & 0.702 & 0.663 & 0.627 \\
\hline 6 & 400.0 & 1.141 & 1.040 & 0.964 & 0.898 & 0.841 & 0.789 & 0.742 & 0.700 & 0.661 & 0.624 \\
\hline 7 & 500.0 & 1.144 & 1.043 & 0.966 & 0.900 & 0.842 & 0.790 & 0.743 & 0.701 & 0.661 & 0.625 \\
\hline 8 & 600.0 & 1.148 & 1.046 & 0.969 & 0.903 & 0.845 & 0.793 & 0.746 & 0.704 & 0.664 & 0.628 \\
\hline 9 & 700.0 & 1.150 & 1.050 & 0.973 & 0.907 & 0.849 & 0.797 & 0.750 & 0.708 & 0.668 & 0.631 \\
\hline 10 & 800.0 & 1.158 & 1.056 & 0.979 & 0.913 & 0.854 & 0.802 & 0.755 & 0.712 & 0.673 & 0.636 \\
\hline 11 & 900.0 & 1.163 & 1.061 & 0.985 & 0.919 & 0.861 & 0.809 & 0.762 & 0.719 & 0.679 & 0.642 \\
\hline 12 & 1000.0 & 1.171 & 1.069 & 0.992 & 0.926 & 0.868 & 0.816 & 0.769 & 0.726 & 0.686 & 0.649 \\
\hline 13 & 1100.0 & 1.177 & 1.075 & 0.999 & 0.933 & 0.875 & 0.823 & 0.776 & 0.733 & 0.694 & 0.657 \\
\hline 14 & 1200.0 & 1.188 & 1.086 & 1.009 & 0.943 & 0.885 & 0.833 & 0.786 & 0.743 & 0.704 & 0.667 \\
\hline 15 & 1300.0 & 1.193 & 1.092 & 1.016 & 0.950 & 0.892 & 0.841 & 0.794 & 0.751 & 0.711 & 0.674 \\
\hline & & 11 & 12 & 13 & 14 & 15 & 16 & 17 & 18 & 19 & 20 \\
\hline 0 & 0.0 & 0.584 & 0.553 & 0.524 & 0.496 & 0.471 & 0.448 & 0.428 & 0.411 & 0.397 & 0.391 \\
\hline 1 & 25.0 & 0.617 & 0.586 & 0.557 & 0.530 & 0.505 & 0.482 & 0.462 & 0.445 & 0.433 & 0.428 \\
\hline 2 & 50.0 & 0.617 & 0.586 & 0.557 & 0.530 & 0.505 & 0.483 & 0.463 & 0.446 & 0.433 & 0.428 \\
\hline 3 & 100.0 & 0.611 & 0.580 & 0.551 & 0.524 & 0.499 & 0.477 & 0.457 & 0.440 & 0.428 & 0.423 \\
\hline 4 & 200.0 & 0.599 & 0.568 & 0.539 & 0.512 & 0.487 & 0.464 & 0.444 & 0.428 & 0.415 & 0.409 \\
\hline 5 & 300.0 & 0.593 & 0.562 & 0.533 & 0.505 & 0.480 & 0.458 & 0.438 & 0.421 & 0.408 & 0.402 \\
\hline 6 & 400.0 & 0.591 & 0.559 & 0.530 & 0.502 & 0.477 & 0.454 & 0.434 & 0.417 & 0.404 & 0.398 \\
\hline 7 & 500.0 & 0.591 & 0.559 & 0.530 & 0.502 & 0.477 & 0.454 & 0.434 & 0.416 & 0.403 & 0.397 \\
\hline 8 & 600.0 & 0.593 & 0.562 & 0.532 & 0.504 & 0.479 & 0.456 & 0.435 & 0.418 & 0.404 & 0.398 \\
\hline 9 & 700.0 & 0.597 & 0.565 & 0.535 & 0.507 & 0.482 & 0.458 & 0.438 & 0.420 & 0.406 & 0.400 \\
\hline 10 & 800.0 & 0.602 & 0.569 & 0.539 & 0.512 & 0.486 & 0.462 & 0.442 & 0.424 & 0.410 & 0.404 \\
\hline 11 & 900.0 & 0.608 & 0.576 & 0.545 & 0.517 & 0.491 & 0.468 & 0.447 & 0.429 & 0.415 & 0.409 \\
\hline 12 & 1000.0 & 0.614 & 0.582 & 0.552 & 0.523 & 0.497 & 0.474 & 0.453 & 0.435 & 0.421 & 0.415 \\
\hline 13 & 1100.0 & 0.622 & 0.590 & 0.559 & 0.531 & 0.505 & 0.481 & 0.460 & 0.442 & 0.428 & 0.422 \\
\hline 14 & 1200.0 & 0.632 & 0.600 & 0.569 & 0.541 & 0.515 & 0.491 & 0.470 & 0.452 & 0.438 & 0.433 \\
\hline 15 & 1300.0 & 0.640 & 0.607 & 0.577 & 0.548 & 0.522 & 0.498 & 0.476 & 0.458 & 0.444 & 0.439 \\
\hline
\end{tabular}


Table 6.51. Problem 5.2.1 reference solution Shift power peaking factors for axial segments in fuel channels $P, Q, R$, and $S$ in element 5.

\begin{tabular}{|c|c|c|c|c|c|c|c|c|c|c|c|}
\hline \multirow[t]{2}{*}{ Step } & Burnup & \multicolumn{10}{|c|}{ Power Peaking Factors } \\
\hline & {$\left[\frac{\mathrm{MWd}}{\mathrm{MtU}}\right]$} & 1 & 2 & 3 & 4 & 5 & 6 & 7 & 8 & 9 & 10 \\
\hline 0 & 0.0 & 0.420 & 0.379 & 0.354 & 0.331 & 0.311 & 0.295 & 0.279 & 0.264 & 0.251 & 0.239 \\
\hline 1 & 25.0 & 0.455 & 0.411 & 0.386 & 0.364 & 0.345 & 0.325 & 0.309 & 0.294 & 0.280 & 0.267 \\
\hline 2 & 50.0 & 0.452 & 0.408 & 0.384 & 0.362 & 0.344 & 0.324 & 0.309 & 0.292 & 0.277 & 0.265 \\
\hline 3 & 100.0 & 0.443 & 0.400 & 0.377 & 0.354 & 0.333 & 0.317 & 0.299 & 0.285 & 0.271 & 0.258 \\
\hline 4 & 200.0 & 0.427 & 0.384 & 0.362 & 0.338 & 0.319 & 0.301 & 0.285 & 0.272 & 0.258 & 0.244 \\
\hline 5 & 300.0 & 0.421 & 0.381 & 0.357 & 0.333 & 0.315 & 0.295 & 0.281 & 0.266 & 0.251 & 0.240 \\
\hline 6 & 400.0 & 0.415 & 0.375 & 0.352 & 0.330 & 0.309 & 0.290 & 0.275 & 0.263 & 0.248 & 0.235 \\
\hline 7 & 500.0 & 0.415 & 0.374 & 0.351 & 0.328 & 0.307 & 0.290 & 0.276 & 0.260 & 0.248 & 0.233 \\
\hline 8 & 600.0 & 0.418 & 0.379 & 0.352 & 0.331 & 0.309 & 0.292 & 0.276 & 0.262 & 0.247 & 0.234 \\
\hline 9 & 700.0 & 0.419 & 0.377 & 0.353 & 0.329 & 0.312 & 0.293 & 0.276 & 0.263 & 0.248 & 0.235 \\
\hline 10 & 800.0 & 0.423 & 0.383 & 0.356 & 0.335 & 0.316 & 0.297 & 0.280 & 0.267 & 0.251 & 0.239 \\
\hline 11 & 900.0 & 0.431 & 0.388 & 0.363 & 0.339 & 0.319 & 0.301 & 0.285 & 0.270 & 0.257 & 0.241 \\
\hline 12 & 1000.0 & 0.436 & 0.390 & 0.368 & 0.344 & 0.323 & 0.304 & 0.288 & 0.272 & 0.259 & 0.247 \\
\hline 13 & 1100.0 & 0.442 & 0.398 & 0.371 & 0.348 & 0.329 & 0.311 & 0.292 & 0.277 & 0.263 & 0.248 \\
\hline 14 & 1200.0 & 0.448 & 0.401 & 0.375 & 0.353 & 0.333 & 0.315 & 0.297 & 0.281 & 0.268 & 0.253 \\
\hline 15 & 1300.0 & 0.458 & 0.413 & 0.384 & 0.360 & 0.340 & 0.321 & 0.304 & 0.287 & 0.272 & 0.260 \\
\hline & & 11 & 12 & 13 & 14 & 15 & 16 & 17 & 18 & 19 & 20 \\
\hline 0 & 0.0 & 0.225 & 0.214 & 0.204 & 0.193 & 0.185 & 0.177 & 0.170 & 0.163 & 0.157 & 0.156 \\
\hline 1 & 25.0 & 0.255 & 0.241 & 0.232 & 0.223 & 0.213 & 0.203 & 0.197 & 0.188 & 0.183 & 0.183 \\
\hline 2 & 50.0 & 0.252 & 0.241 & 0.232 & 0.221 & 0.210 & 0.203 & 0.194 & 0.187 & 0.181 & 0.182 \\
\hline 3 & 100.0 & 0.244 & 0.235 & 0.225 & 0.214 & 0.203 & 0.195 & 0.188 & 0.182 & 0.176 & 0.175 \\
\hline 4 & 200.0 & 0.234 & 0.221 & 0.211 & 0.202 & 0.192 & 0.183 & 0.174 & 0.169 & 0.165 & 0.164 \\
\hline 5 & 300.0 & 0.229 & 0.216 & 0.206 & 0.197 & 0.189 & 0.180 & 0.171 & 0.165 & 0.161 & 0.161 \\
\hline 6 & 400.0 & 0.224 & 0.213 & 0.201 & 0.193 & 0.183 & 0.176 & 0.168 & 0.162 & 0.155 & 0.155 \\
\hline 7 & 500.0 & 0.222 & 0.209 & 0.200 & 0.190 & 0.182 & 0.175 & 0.166 & 0.158 & 0.154 & 0.153 \\
\hline 8 & 600.0 & 0.224 & 0.212 & 0.202 & 0.190 & 0.183 & 0.174 & 0.167 & 0.160 & 0.154 & 0.154 \\
\hline 9 & 700.0 & 0.223 & 0.212 & 0.201 & 0.193 & 0.184 & 0.175 & 0.166 & 0.159 & 0.155 & 0.155 \\
\hline 10 & 800.0 & 0.226 & 0.214 & 0.204 & 0.195 & 0.185 & 0.177 & 0.170 & 0.162 & 0.157 & 0.156 \\
\hline 11 & 900.0 & 0.230 & 0.217 & 0.207 & 0.197 & 0.188 & 0.178 & 0.172 & 0.164 & 0.160 & 0.158 \\
\hline 12 & 1000.0 & 0.233 & 0.220 & 0.210 & 0.200 & 0.189 & 0.183 & 0.175 & 0.167 & 0.161 & 0.160 \\
\hline 13 & 1100.0 & 0.236 & 0.225 & 0.213 & 0.204 & 0.194 & 0.184 & 0.176 & 0.171 & 0.164 & 0.163 \\
\hline 14 & 1200.0 & 0.241 & 0.229 & 0.218 & 0.208 & 0.198 & 0.189 & 0.180 & 0.173 & 0.167 & 0.165 \\
\hline 15 & 1300.0 & 0.246 & 0.233 & 0.224 & 0.212 & 0.202 & 0.194 & 0.185 & 0.178 & 0.172 & 0.171 \\
\hline
\end{tabular}


Table 6.52. Problem 5.2.1 reference solution MPACT power peaking factors for axial segments in fuel channels $P, Q, R$, and $S$ in element 5.

\begin{tabular}{|c|c|c|c|c|c|c|c|c|c|c|c|}
\hline \multirow[t]{2}{*}{ Step } & Burnup & \multicolumn{10}{|c|}{ Power Peaking Factors } \\
\hline & {$\left[\frac{\mathrm{MWd}}{\mathrm{MtU}}\right.$} & 1 & 2 & 3 & 4 & 5 & 6 & 7 & 8 & 9 & 10 \\
\hline 0 & 0.0 & 0.346 & 0.315 & 0.291 & 0.271 & 0.253 & 0.237 & 0.222 & 0.209 & 0.197 & 0.186 \\
\hline 1 & 25.0 & 0.382 & 0.348 & 0.323 & 0.302 & 0.283 & 0.266 & 0.251 & 0.237 & 0.224 & 0.212 \\
\hline 2 & 50.0 & 0.382 & 0.349 & 0.324 & 0.303 & 0.284 & 0.267 & 0.252 & 0.239 & 0.226 & 0.214 \\
\hline 3 & 100.0 & 0.377 & 0.345 & 0.320 & 0.299 & 0.280 & 0.263 & 0.248 & 0.235 & 0.222 & 0.211 \\
\hline 4 & 200.0 & 0.364 & 0.332 & 0.308 & 0.287 & 0.268 & 0.252 & 0.238 & 0.224 & 0.212 & 0.201 \\
\hline 5 & 300.0 & 0.357 & 0.325 & 0.301 & 0.280 & 0.262 & 0.246 & 0.231 & 0.218 & 0.206 & 0.195 \\
\hline 6 & 400.0 & 0.353 & 0.321 & 0.297 & 0.276 & 0.258 & 0.242 & 0.227 & 0.214 & 0.202 & 0.191 \\
\hline 7 & 500.0 & 0.351 & 0.319 & 0.295 & 0.274 & 0.256 & 0.240 & 0.226 & 0.212 & 0.200 & 0.189 \\
\hline 8 & 600.0 & 0.352 & 0.320 & 0.296 & 0.275 & 0.256 & 0.240 & 0.226 & 0.212 & 0.200 & 0.189 \\
\hline 9 & 700.0 & 0.354 & 0.321 & 0.297 & 0.276 & 0.258 & 0.241 & 0.227 & 0.213 & 0.201 & 0.189 \\
\hline 10 & 800.0 & 0.358 & 0.325 & 0.300 & 0.279 & 0.260 & 0.244 & 0.229 & 0.215 & 0.203 & 0.191 \\
\hline 11 & 900.0 & 0.362 & 0.329 & 0.304 & 0.282 & 0.263 & 0.247 & 0.232 & 0.218 & 0.205 & 0.194 \\
\hline 12 & 1000.0 & 0.368 & 0.334 & 0.309 & 0.287 & 0.268 & 0.251 & 0.235 & 0.222 & 0.209 & 0.197 \\
\hline 13 & 1100.0 & 0.374 & 0.340 & 0.314 & 0.292 & 0.273 & 0.255 & 0.240 & 0.226 & 0.213 & 0.201 \\
\hline 14 & 1200.0 & 0.384 & 0.349 & 0.323 & 0.300 & 0.281 & 0.263 & 0.247 & 0.233 & 0.220 & 0.207 \\
\hline 15 & 1300.0 & 0.390 & 0.355 & 0.328 & 0.305 & 0.286 & 0.268 & 0.252 & 0.237 & 0.224 & 0.211 \\
\hline & & 11 & 12 & 13 & 14 & 15 & 16 & 17 & 18 & 19 & 20 \\
\hline 0 & 0.0 & 0.175 & 0.166 & 0.157 & 0.148 & 0.141 & 0.133 & 0.127 & 0.122 & 0.117 & 0.115 \\
\hline 1 & 25.0 & 0.201 & 0.191 & 0.181 & 0.172 & 0.164 & 0.156 & 0.149 & 0.143 & 0.139 & 0.137 \\
\hline 2 & 50.0 & 0.203 & 0.193 & 0.183 & 0.174 & 0.166 & 0.158 & 0.151 & 0.145 & 0.141 & 0.139 \\
\hline 3 & 100.0 & 0.200 & 0.190 & 0.180 & 0.171 & 0.163 & 0.156 & 0.149 & 0.143 & 0.139 & 0.137 \\
\hline 4 & 200.0 & 0.190 & 0.180 & 0.171 & 0.162 & 0.154 & 0.147 & 0.141 & 0.135 & 0.131 & 0.129 \\
\hline 5 & 300.0 & 0.184 & 0.174 & 0.165 & 0.157 & 0.149 & 0.142 & 0.135 & 0.130 & 0.126 & 0.124 \\
\hline 6 & 400.0 & 0.180 & 0.171 & 0.161 & 0.153 & 0.145 & 0.138 & 0.132 & 0.126 & 0.122 & 0.120 \\
\hline 7 & 500.0 & 0.178 & 0.169 & 0.159 & 0.151 & 0.143 & 0.136 & 0.130 & 0.124 & 0.120 & 0.118 \\
\hline 8 & 600.0 & 0.178 & 0.168 & 0.159 & 0.151 & 0.143 & 0.136 & 0.129 & 0.124 & 0.119 & 0.117 \\
\hline 9 & 700.0 & 0.179 & 0.169 & 0.160 & 0.151 & 0.143 & 0.136 & 0.129 & 0.124 & 0.119 & 0.117 \\
\hline 10 & 800.0 & 0.180 & 0.170 & 0.161 & 0.152 & 0.144 & 0.137 & 0.130 & 0.125 & 0.120 & 0.118 \\
\hline 11 & 900.0 & 0.183 & 0.173 & 0.163 & 0.154 & 0.146 & 0.139 & 0.132 & 0.126 & 0.122 & 0.120 \\
\hline 12 & 1000.0 & 0.186 & 0.176 & 0.166 & 0.157 & 0.149 & 0.141 & 0.134 & 0.129 & 0.124 & 0.122 \\
\hline 13 & 1100.0 & 0.190 & 0.179 & 0.169 & 0.160 & 0.152 & 0.144 & 0.137 & 0.131 & 0.127 & 0.124 \\
\hline 14 & 1200.0 & 0.196 & 0.185 & 0.175 & 0.166 & 0.157 & 0.149 & 0.142 & 0.136 & 0.132 & 0.129 \\
\hline 15 & 1300.0 & 0.200 & 0.189 & 0.179 & 0.169 & 0.161 & 0.153 & 0.145 & 0.139 & 0.134 & 0.132 \\
\hline
\end{tabular}


Table 6.53. Problem 5.2.1 reference solution Shift power peaking factors for axial segments in fuel channels $P, Q, R$, and $S$ in element 6.

\begin{tabular}{|c|c|c|c|c|c|c|c|c|c|c|c|}
\hline \multirow[t]{2}{*}{ Step } & Burnup & \multicolumn{10}{|c|}{ Power Peaking Factors } \\
\hline & {$\left[\frac{\mathrm{MWd}}{\mathrm{MtU}}\right]$} & 1 & 2 & 3 & 4 & 5 & 6 & 7 & 8 & 9 & 10 \\
\hline 0 & 0.0 & 0.140 & 0.126 & 0.118 & 0.108 & 0.102 & 0.095 & 0.089 & 0.083 & 0.079 & 0.074 \\
\hline 1 & 25.0 & 0.163 & 0.148 & 0.138 & 0.128 & 0.120 & 0.114 & 0.107 & 0.101 & 0.095 & 0.089 \\
\hline 2 & 50.0 & 0.163 & 0.147 & 0.138 & 0.128 & 0.121 & 0.114 & 0.106 & 0.101 & 0.094 & 0.089 \\
\hline 3 & 100.0 & 0.157 & 0.142 & 0.131 & 0.123 & 0.115 & 0.108 & 0.103 & 0.096 & 0.090 & 0.085 \\
\hline 4 & 200.0 & 0.145 & 0.132 & 0.122 & 0.115 & 0.107 & 0.100 & 0.095 & 0.090 & 0.084 & 0.079 \\
\hline 5 & 300.0 & 0.142 & 0.128 & 0.119 & 0.111 & 0.103 & 0.098 & 0.092 & 0.086 & 0.080 & 0.076 \\
\hline 6 & 400.0 & 0.138 & 0.123 & 0.115 & 0.108 & 0.100 & 0.095 & 0.088 & 0.083 & 0.079 & 0.073 \\
\hline 7 & 500.0 & 0.137 & 0.122 & 0.115 & 0.106 & 0.099 & 0.093 & 0.087 & 0.081 & 0.077 & 0.072 \\
\hline 8 & 600.0 & 0.136 & 0.122 & 0.112 & 0.106 & 0.099 & 0.092 & 0.087 & 0.082 & 0.077 & 0.072 \\
\hline 9 & 700.0 & 0.137 & 0.122 & 0.115 & 0.107 & 0.100 & 0.093 & 0.086 & 0.082 & 0.077 & 0.073 \\
\hline 10 & 800.0 & 0.139 & 0.124 & 0.115 & 0.108 & 0.100 & 0.095 & 0.088 & 0.083 & 0.078 & 0.072 \\
\hline 11 & 900.0 & 0.141 & 0.126 & 0.117 & 0.109 & 0.102 & 0.095 & 0.089 & 0.084 & 0.079 & 0.074 \\
\hline 12 & 1000.0 & 0.143 & 0.128 & 0.119 & 0.112 & 0.104 & 0.097 & 0.092 & 0.086 & 0.080 & 0.076 \\
\hline 13 & 1100.0 & 0.145 & 0.130 & 0.121 & 0.113 & 0.105 & 0.099 & 0.092 & 0.086 & 0.082 & 0.076 \\
\hline 14 & 1200.0 & 0.148 & 0.134 & 0.125 & 0.115 & 0.109 & 0.102 & 0.096 & 0.088 & 0.084 & 0.079 \\
\hline 15 & 1300.0 & 0.153 & 0.137 & 0.128 & 0.119 & 0.110 & 0.104 & 0.098 & 0.091 & 0.085 & 0.081 \\
\hline & & 11 & 12 & 13 & 14 & 15 & 16 & 17 & 18 & 19 & 20 \\
\hline 0 & 0.0 & 0.069 & 0.065 & 0.060 & 0.057 & 0.053 & 0.051 & 0.048 & 0.045 & 0.043 & 0.043 \\
\hline 1 & 25.0 & 0.084 & 0.078 & 0.074 & 0.070 & 0.066 & 0.061 & 0.058 & 0.054 & 0.052 & 0.052 \\
\hline 2 & 50.0 & 0.084 & 0.078 & 0.074 & 0.069 & 0.065 & 0.061 & 0.057 & 0.055 & 0.052 & 0.052 \\
\hline 3 & 100.0 & 0.079 & 0.074 & 0.070 & 0.066 & 0.062 & 0.058 & 0.054 & 0.051 & 0.050 & 0.049 \\
\hline 4 & 200.0 & 0.074 & 0.070 & 0.066 & 0.061 & 0.058 & 0.054 & 0.050 & 0.048 & 0.046 & 0.045 \\
\hline 5 & 300.0 & 0.071 & 0.066 & 0.063 & 0.058 & 0.054 & 0.051 & 0.048 & 0.046 & 0.045 & 0.044 \\
\hline 6 & 400.0 & 0.068 & 0.065 & 0.059 & 0.057 & 0.053 & 0.049 & 0.047 & 0.044 & 0.042 & 0.042 \\
\hline 7 & 500.0 & 0.067 & 0.063 & 0.059 & 0.055 & 0.052 & 0.049 & 0.045 & 0.044 & 0.042 & 0.042 \\
\hline 8 & 600.0 & 0.068 & 0.064 & 0.059 & 0.055 & 0.052 & 0.049 & 0.045 & 0.043 & 0.042 & 0.042 \\
\hline 9 & 700.0 & 0.068 & 0.064 & 0.059 & 0.056 & 0.052 & 0.049 & 0.046 & 0.044 & 0.042 & 0.042 \\
\hline 10 & 800.0 & 0.069 & 0.064 & 0.060 & 0.056 & 0.053 & 0.049 & 0.046 & 0.043 & 0.042 & 0.042 \\
\hline 11 & 900.0 & 0.069 & 0.064 & 0.060 & 0.056 & 0.053 & 0.050 & 0.046 & 0.044 & 0.043 & 0.042 \\
\hline 12 & 1000.0 & 0.071 & 0.066 & 0.063 & 0.058 & 0.054 & 0.051 & 0.048 & 0.045 & 0.044 & 0.044 \\
\hline 13 & 1100.0 & 0.072 & 0.067 & 0.063 & 0.059 & 0.055 & 0.052 & 0.049 & 0.046 & 0.044 & 0.043 \\
\hline 14 & 1200.0 & 0.073 & 0.068 & 0.065 & 0.060 & 0.057 & 0.053 & 0.050 & 0.047 & 0.045 & 0.045 \\
\hline 15 & 1300.0 & 0.075 & 0.070 & 0.066 & 0.063 & 0.060 & 0.055 & 0.052 & 0.049 & 0.046 & 0.047 \\
\hline
\end{tabular}


Table 6.54. Problem 5.2.1 reference solution MPACT power peaking factors for axial segments in fuel channels $P, Q, R$, and $S$ in element 6.

\begin{tabular}{|c|c|c|c|c|c|c|c|c|c|c|c|}
\hline \multirow[t]{2}{*}{ Step } & \multirow{2}{*}{$\begin{array}{l}\text { Burnup } \\
{\left[\frac{\mathrm{MWd}}{\mathrm{M} \mathrm{dI}}\right]}\end{array}$} & \multicolumn{10}{|c|}{ Power Peaking Factors } \\
\hline & & 1 & 2 & 3 & 4 & 5 & 6 & 7 & 8 & 9 & 10 \\
\hline 0 & 0.0 & 0.101 & 0.092 & 0.084 & 0.078 & 0.072 & 0.067 & 0.063 & 0.059 & 0.055 & 0.051 \\
\hline 1 & 25.0 & 0.121 & 0.110 & 0.102 & 0.094 & 0.088 & 0.082 & 0.077 & 0.072 & 0.067 & 0.063 \\
\hline 2 & 50.0 & 0.123 & 0.112 & 0.103 & 0.096 & 0.089 & 0.083 & 0.078 & 0.073 & 0.069 & 0.064 \\
\hline 3 & 100.0 & 0.121 & 0.110 & 0.102 & 0.095 & 0.088 & 0.082 & 0.077 & 0.072 & 0.068 & 0.063 \\
\hline 4 & 200.0 & 0.114 & 0.104 & 0.096 & 0.089 & 0.083 & 0.077 & 0.072 & 0.067 & 0.063 & 0.059 \\
\hline 5 & 300.0 & 0.109 & 0.099 & 0.091 & 0.085 & 0.079 & 0.073 & 0.069 & 0.064 & 0.060 & 0.056 \\
\hline 6 & 400.0 & 0.106 & 0.096 & 0.088 & 0.082 & 0.076 & 0.071 & 0.066 & 0.062 & 0.058 & 0.054 \\
\hline 7 & 500.0 & 0.104 & 0.094 & 0.087 & 0.080 & 0.074 & 0.069 & 0.065 & 0.060 & 0.056 & 0.053 \\
\hline 8 & 600.0 & 0.103 & 0.093 & 0.086 & 0.079 & 0.074 & 0.069 & 0.064 & 0.060 & 0.056 & 0.052 \\
\hline 9 & 700.0 & 0.103 & 0.093 & 0.086 & 0.079 & 0.074 & 0.069 & 0.064 & 0.060 & 0.056 & 0.052 \\
\hline 10 & 800.0 & 0.104 & 0.094 & 0.086 & 0.080 & 0.074 & 0.069 & 0.064 & 0.060 & 0.056 & 0.052 \\
\hline 11 & 900.0 & 0.105 & 0.095 & 0.087 & 0.081 & 0.075 & 0.070 & 0.065 & 0.061 & 0.057 & 0.053 \\
\hline 12 & 1000.0 & 0.107 & 0.097 & 0.089 & 0.082 & 0.076 & 0.071 & 0.066 & 0.062 & 0.058 & 0.054 \\
\hline 13 & 1100.0 & 0.109 & 0.099 & 0.091 & 0.084 & 0.078 & 0.073 & 0.068 & 0.063 & 0.059 & 0.055 \\
\hline 14 & 1200.0 & 0.114 & 0.103 & 0.095 & 0.088 & 0.081 & 0.076 & 0.071 & 0.066 & 0.061 & 0.057 \\
\hline 15 & 1300.0 & 0.116 & 0.105 & 0.097 & 0.089 & 0.083 & 0.077 & 0.072 & 0.067 & 0.063 & 0.059 \\
\hline & & 11 & 12 & 13 & 14 & 15 & 16 & 17 & 18 & 19 & 20 \\
\hline 0 & 0.0 & 0.048 & 0.045 & 0.042 & 0.039 & 0.036 & 0.034 & 0.032 & 0.030 & 0.029 & 0.029 \\
\hline 1 & 25.0 & 0.059 & 0.055 & 0.051 & 0.048 & 0.045 & 0.042 & 0.040 & 0.037 & 0.036 & 0.036 \\
\hline 2 & 50.0 & 0.060 & 0.056 & 0.053 & 0.049 & 0.046 & 0.043 & 0.041 & 0.039 & 0.037 & 0.037 \\
\hline 3 & 100.0 & 0.059 & 0.056 & 0.052 & 0.049 & 0.045 & 0.043 & 0.040 & 0.038 & 0.037 & 0.036 \\
\hline 4 & 200.0 & 0.055 & 0.052 & 0.048 & 0.045 & 0.042 & 0.040 & 0.037 & 0.035 & 0.034 & 0.034 \\
\hline 5 & 300.0 & 0.053 & 0.049 & 0.046 & 0.043 & 0.040 & 0.038 & 0.035 & 0.033 & 0.032 & 0.032 \\
\hline 6 & 400.0 & 0.051 & 0.047 & 0.044 & 0.041 & 0.038 & 0.036 & 0.034 & 0.032 & 0.031 & 0.030 \\
\hline 7 & 500.0 & 0.049 & 0.046 & 0.043 & 0.040 & 0.037 & 0.035 & 0.033 & 0.031 & 0.030 & 0.030 \\
\hline 8 & 600.0 & 0.049 & 0.045 & 0.042 & 0.040 & 0.037 & 0.034 & 0.032 & 0.031 & 0.029 & 0.029 \\
\hline 9 & 700.0 & 0.049 & 0.045 & 0.042 & 0.039 & 0.037 & 0.034 & 0.032 & 0.031 & 0.029 & 0.029 \\
\hline 10 & 800.0 & 0.049 & 0.045 & 0.042 & 0.040 & 0.037 & 0.034 & 0.032 & 0.031 & 0.029 & 0.029 \\
\hline 11 & 900.0 & 0.049 & 0.046 & 0.043 & 0.040 & 0.037 & 0.035 & 0.033 & 0.031 & 0.030 & 0.029 \\
\hline 12 & 1000.0 & 0.050 & 0.047 & 0.044 & 0.041 & 0.038 & 0.035 & 0.033 & 0.032 & 0.030 & 0.030 \\
\hline 13 & 1100.0 & 0.051 & 0.048 & 0.045 & 0.042 & 0.039 & 0.036 & 0.034 & 0.032 & 0.031 & 0.031 \\
\hline 14 & 1200.0 & 0.054 & 0.050 & 0.047 & 0.043 & 0.041 & 0.038 & 0.036 & 0.034 & 0.032 & 0.032 \\
\hline 15 & 1300.0 & 0.055 & 0.051 & 0.048 & 0.044 & 0.042 & 0.039 & 0.036 & 0.035 & 0.033 & 0.033 \\
\hline
\end{tabular}





\section{PROBLEM 6: $2 \times 2$ MINI-CORE 3D}

Problem 6 is a mini-core with four charge-pans. Two are controlled, and one contains fuel stacks that are only five elements high. The top and bottom problem edges have vacuum (zero-return) boundary conditions, and the north, south, east, and west boundaries are fully reflected. Problem 6 is more complex than previous problems because it has both five-element and six-element fuel stacks, and it has control rods inserted in two of the four charge pans.

\subsection{BEGINNING-OF-CYCLE}

\subsubsection{Problem 6.1.1}

\subsubsection{Description}

Figure 26 shows the transverse mini-core geometry for Problem 6.1.1 at two different axial locations. Each charge-pan has been labeled with Roman numerals corresponding to its quadrant. The charge-pan in the upper right of Figure 26 (quadrant I, shown in dark grey) has fuel channels with five-element-high stacks, whereas all other charge-pans (quadrants II, III, and IV, shown in light grey) have six-element-high stacks. The axial geometry of the six-element stacks are shown in Figure 20, and the five-element high stacks are shown in Figure 27. Charge-pans in quadrants II and IV have control rods inserted halfway into the the fuel from the top (shown in red in Figure 26). The bottom of the control rods coincide with the bottom of fuel element four and the top of fuel element three.

Zone A coolant channel radius is used for all charge-pans in this problem. Geometric dimensions are listed in Table 1.2. Owing to the five-element and six-element stacks and the presence of control rods, the problem is asymmetric in the radial and axial dimensions. Material densities are listed in Table 1.4. Temperatures are listed in Table 2.4.

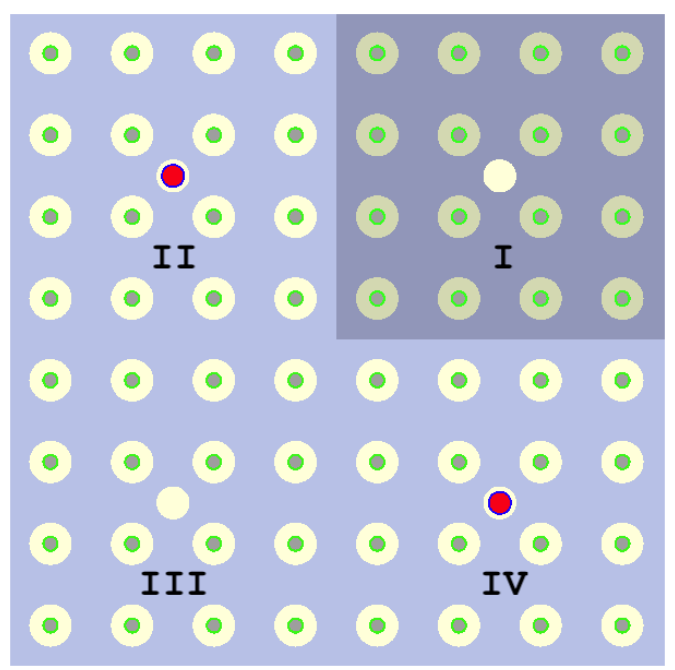

(a) At 5 th fuel element height and below.

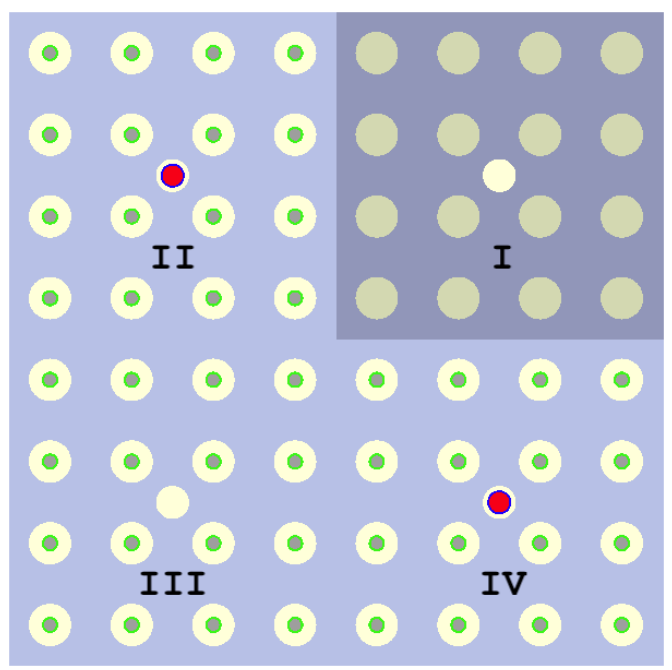

(b) Above 5th element height

Figure 26. $2 \times 2$ mini-core 3D. 


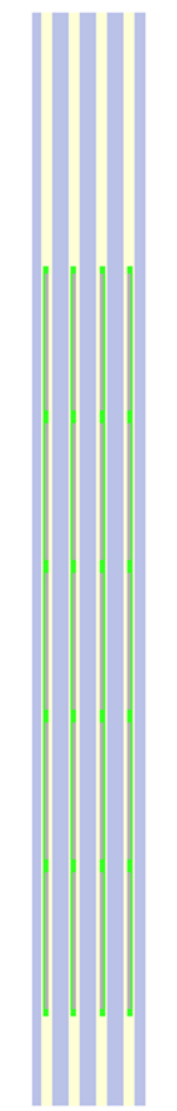

Figure 27. 3D charge-pan with five fuel element stacks. 


\subsubsection{Reference Solution}

The neutron multiplication factor results are shown in Table 7.1. The timing variables for Shift and MPACT are shown in Table 7.2. It can be seen that the MPACT problem took significantly longer than Shift. This can be attributed to the ray spacing of 0.01 and a high convergence criteria, both of which exacerbate the issue with the end caps, i.e. non-fueled regions, in between the elements.

In the reference solution for Problem 6.1.1, each fuel element is divided into axial segments as the two previous problems. Figure 28 shows the assembly-average power peaking factors for each axial segment in all four assemblies (I-IV) and their absolute difference between MPACT and Shift. It can be seen that the power peaking factors look to match up Tables 7.3-7.48 show the power peaking factors for each of the pins for all axial locations in mini-core.

The peaking factors agree decently between the two codes.

Table 7.1. Neutron multiplication factor for the reference solution of Problem 6.1.1.

\begin{tabular}{rrccc}
\hline Step & $\begin{array}{c}\text { Burnup } \\
{\left[\frac{\mathrm{MWd}}{\mathrm{MtU}}\right]}\end{array}$ & $\begin{array}{c}\text { Shift } \\
\mathrm{k}_{\mathrm{eff}}(1 \sigma)\end{array}$ & $\begin{array}{c}\text { MPACT } \\
\mathrm{k}_{\mathrm{eff}}\end{array}(1 \sigma)$ & $\begin{array}{c}\text { Difference } \\
{[\mathrm{pcm}]}\end{array}$ \\
\hline 0 & 0.0 & $1.02722(5)$ & $1.02518(10)$ & $-205(11)$ \\
\hline
\end{tabular}

Table 7.2. Timing variables for the reference solution of Problem 6.1.1

\begin{tabular}{|c|c|c|c|c|c|c|}
\hline \multicolumn{7}{|c|}{$\overline{\text { Shift }}$} \\
\hline $\begin{array}{c}\text { Depletion } \\
\text { Solves }\end{array}$ & $\begin{array}{c}\text { Total } \\
\text { Cycles per } \\
\text { Transport } \\
\text { Solve }\end{array}$ & $\begin{array}{c}\text { Active } \\
\text { Cycles per } \\
\text { Transport } \\
\text { Solve }\end{array}$ & $\begin{array}{l}\text { Histories } \\
\text { per Cycle }\end{array}$ & $\begin{array}{c}\text { Compute } \\
\text { Nodes }\end{array}$ & $\begin{array}{c}\text { Cores } \\
\text { per Node }\end{array}$ & $\begin{array}{c}\text { Wall } \\
\text { Total Time } \\
\text { [hours] }\end{array}$ \\
\hline 1 & 1500 & 1200 & 200000 & 2 & 48 & 1.74 \\
\hline \multicolumn{7}{|c|}{ MPACT } \\
\hline $\begin{array}{c}\text { Depletion } \\
\text { Solves }\end{array}$ & $\begin{array}{c}k_{\mathrm{eff}} \\
\text { Convergence } \\
\text { Criteria }\end{array}$ & $\begin{array}{c}\text { Source } \\
\text { Convergence } \\
\text { Criteria }\end{array}$ & $\begin{array}{c}\text { Ray } \\
\text { Spacing } \\
{[\mathrm{cm}]}\end{array}$ & $\begin{array}{c}\text { Compute } \\
\text { Nodes }\end{array}$ & $\begin{array}{c}\text { Cores } \\
\text { per Node }\end{array}$ & $\begin{array}{c}\text { Wall } \\
\text { Total Time } \\
\text { [hours] }\end{array}$ \\
\hline 1 & $1.0 \times 10^{-04}$ & $3.0 \times 10^{-04}$ & 0.01 & 12 & 128 & 4.12 \\
\hline
\end{tabular}



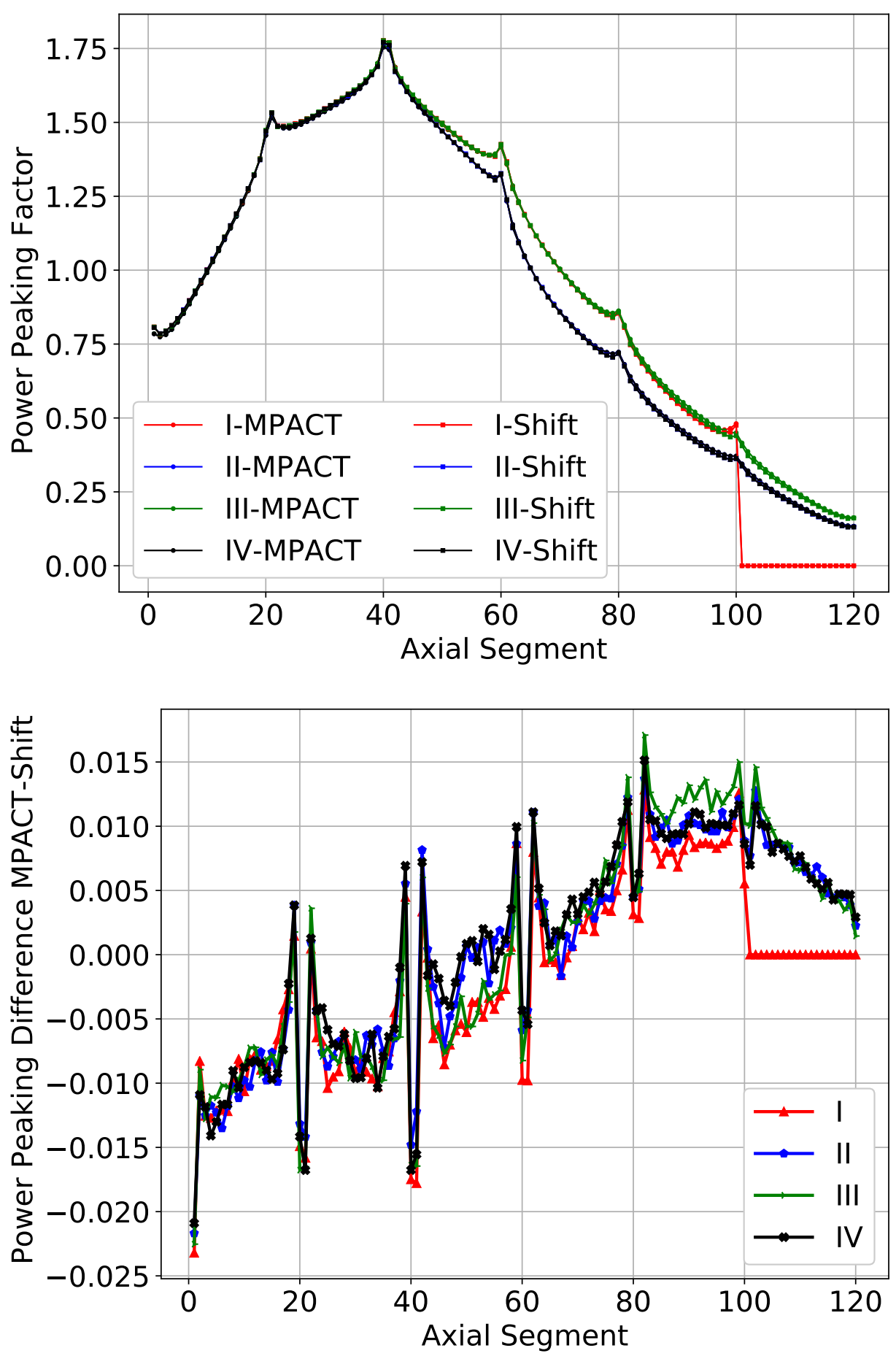

Figure 28. Power peaking factors for Problem 6.1.1 for Shift and MPACT (top) and their absolute difference (bottom). The three series correspond to charge-pans I-IV. Charge-pan I is the 5-element high stack, and charge-pans II and IV have the control rod inserted halfway. 


\section{Charge-Pan I}

Table 7.3. Problem 6.1.1 reference solution Shift power peaking factors for axial segments in element 1 for charge-pan I.

\begin{tabular}{|c|c|c|c|c|c|c|c|c|c|c|}
\hline \multirow[t]{2}{*}{ Channels } & \multicolumn{10}{|c|}{ Power Peaking Factors } \\
\hline & 1 & 2 & 3 & 4 & 5 & 6 & 7 & 8 & 9 & 10 \\
\hline A & 0.805 & 0.781 & 0.794 & 0.806 & 0.837 & 0.859 & 0.895 & 0.926 & 0.959 & 0.993 \\
\hline B & 0.802 & 0.778 & 0.790 & 0.805 & 0.834 & 0.867 & 0.890 & 0.923 & 0.950 & 0.991 \\
\hline $\mathrm{C}$ & 0.805 & 0.774 & 0.787 & 0.810 & 0.839 & 0.867 & 0.892 & 0.922 & 0.953 & 1.000 \\
\hline $\mathrm{D}$ & 0.796 & 0.787 & 0.787 & 0.805 & 0.830 & 0.856 & 0.891 & 0.923 & 0.953 & 0.993 \\
\hline $\mathrm{E}$ & 0.816 & 0.784 & 0.798 & 0.813 & 0.836 & 0.864 & 0.896 & 0.932 & 0.968 & 1.008 \\
\hline $\mathrm{F}$ & 0.810 & 0.776 & 0.798 & 0.809 & 0.833 & 0.862 & 0.896 & 0.928 & 0.965 & 0.998 \\
\hline G & 0.807 & 0.781 & 0.790 & 0.818 & 0.838 & 0.863 & 0.895 & 0.935 & 0.959 & 1.007 \\
\hline $\mathrm{H}$ & 0.805 & 0.787 & 0.797 & 0.817 & 0.831 & 0.861 & 0.899 & 0.928 & 0.966 & 1.006 \\
\hline $\mathrm{J}$ & 0.811 & 0.788 & 0.800 & 0.816 & 0.843 & 0.869 & 0.900 & 0.938 & 0.965 & 1.008 \\
\hline K & 0.807 & 0.788 & 0.797 & 0.814 & 0.846 & 0.874 & 0.902 & 0.939 & 0.970 & 1.000 \\
\hline $\mathrm{L}$ & 0.816 & 0.782 & 0.794 & 0.817 & 0.834 & 0.863 & 0.902 & 0.927 & 0.957 & 0.997 \\
\hline M & 0.808 & 0.779 & 0.796 & 0.811 & 0.831 & 0.869 & 0.905 & 0.925 & 0.968 & 1.005 \\
\hline $\mathrm{P}$ & 0.812 & 0.790 & 0.798 & 0.817 & 0.834 & 0.864 & 0.898 & 0.931 & 0.966 & 1.004 \\
\hline Q & 0.814 & 0.776 & 0.794 & 0.812 & 0.837 & 0.870 & 0.892 & 0.928 & 0.970 & 1.002 \\
\hline $\mathrm{R}$ & 0.812 & 0.786 & 0.795 & 0.812 & 0.837 & 0.865 & 0.904 & 0.933 & 0.972 & 1.013 \\
\hline S & 0.806 & 0.786 & 0.801 & 0.813 & 0.836 & 0.867 & 0.902 & 0.929 & 0.970 & 1.011 \\
\hline Channels & 11 & 12 & 13 & 14 & 15 & 16 & 17 & 18 & 19 & 20 \\
\hline A & 1.017 & 1.055 & 1.099 & 1.139 & 1.179 & 1.215 & 1.263 & 1.306 & 1.362 & 1.448 \\
\hline B & 1.033 & 1.074 & 1.099 & 1.151 & 1.181 & 1.216 & 1.265 & 1.311 & 1.366 & 1.465 \\
\hline $\mathrm{C}$ & 1.032 & 1.056 & 1.090 & 1.139 & 1.182 & 1.218 & 1.267 & 1.312 & 1.371 & 1.467 \\
\hline D & 1.029 & 1.061 & 1.102 & 1.138 & 1.175 & 1.227 & 1.267 & 1.316 & 1.363 & 1.453 \\
\hline $\mathrm{E}$ & 1.039 & 1.074 & 1.111 & 1.157 & 1.191 & 1.227 & 1.280 & 1.318 & 1.367 & 1.473 \\
\hline $\mathrm{F}$ & 1.041 & 1.074 & 1.116 & 1.158 & 1.200 & 1.230 & 1.275 & 1.328 & 1.384 & 1.472 \\
\hline G & 1.040 & 1.084 & 1.118 & 1.150 & 1.192 & 1.235 & 1.278 & 1.321 & 1.380 & 1.474 \\
\hline $\mathrm{H}$ & 1.039 & 1.075 & 1.116 & 1.159 & 1.194 & 1.234 & 1.268 & 1.330 & 1.378 & 1.471 \\
\hline $\mathrm{J}$ & 1.037 & 1.077 & 1.119 & 1.151 & 1.193 & 1.242 & 1.266 & 1.327 & 1.373 & 1.474 \\
\hline K & 1.037 & 1.075 & 1.127 & 1.152 & 1.191 & 1.235 & 1.280 & 1.330 & 1.378 & 1.479 \\
\hline $\mathrm{L}$ & 1.037 & 1.075 & 1.121 & 1.151 & 1.192 & 1.239 & 1.277 & 1.321 & 1.383 & 1.465 \\
\hline $\mathrm{M}$ & 1.035 & 1.084 & 1.112 & 1.149 & 1.189 & 1.233 & 1.277 & 1.333 & 1.370 & 1.479 \\
\hline $\mathrm{P}$ & 1.035 & 1.072 & 1.120 & 1.152 & 1.198 & 1.235 & 1.278 & 1.323 & 1.381 & 1.481 \\
\hline Q & 1.040 & 1.073 & 1.116 & 1.150 & 1.189 & 1.238 & 1.277 & 1.328 & 1.389 & 1.475 \\
\hline $\mathrm{R}$ & 1.045 & 1.078 & 1.109 & 1.160 & 1.196 & 1.233 & 1.279 & 1.328 & 1.388 & 1.484 \\
\hline $\mathrm{S}$ & 1.047 & 1.084 & 1.121 & 1.151 & 1.200 & 1.229 & 1.279 & 1.328 & 1.392 & 1.484 \\
\hline
\end{tabular}


Table 7.4. Problem 6.1.1 reference solution MPACT power peaking factors for axial segments in element 1 for charge-pan I.

\begin{tabular}{|c|c|c|c|c|c|c|c|c|c|c|}
\hline \multirow[t]{2}{*}{ Channels } & \multicolumn{10}{|c|}{ Power Peaking Factors } \\
\hline & 1 & 2 & 3 & 4 & 5 & 6 & 7 & 8 & 9 & 10 \\
\hline A & 0.782 & 0.771 & 0.778 & 0.795 & 0.819 & 0.847 & 0.879 & 0.913 & 0.948 & 0.984 \\
\hline B & 0.781 & 0.771 & 0.778 & 0.795 & 0.819 & 0.847 & 0.879 & 0.913 & 0.948 & 0.984 \\
\hline $\mathrm{C}$ & 0.781 & 0.771 & 0.778 & 0.795 & 0.819 & 0.847 & 0.879 & 0.913 & 0.948 & 0.984 \\
\hline $\mathrm{D}$ & 0.781 & 0.770 & 0.778 & 0.795 & 0.819 & 0.847 & 0.879 & 0.913 & 0.948 & 0.984 \\
\hline $\mathrm{E}$ & 0.786 & 0.776 & 0.783 & 0.801 & 0.825 & 0.854 & 0.887 & 0.921 & 0.957 & 0.993 \\
\hline $\mathrm{F}$ & 0.786 & 0.775 & 0.783 & 0.801 & 0.825 & 0.855 & 0.887 & 0.922 & 0.957 & 0.994 \\
\hline G & 0.786 & 0.776 & 0.783 & 0.801 & 0.825 & 0.855 & 0.887 & 0.922 & 0.957 & 0.994 \\
\hline $\mathrm{H}$ & 0.786 & 0.775 & 0.783 & 0.801 & 0.825 & 0.854 & 0.887 & 0.921 & 0.957 & 0.993 \\
\hline $\mathrm{J}$ & 0.786 & 0.775 & 0.783 & 0.801 & 0.825 & 0.854 & 0.887 & 0.921 & 0.957 & 0.994 \\
\hline K & 0.786 & 0.776 & 0.783 & 0.801 & 0.825 & 0.854 & 0.887 & 0.921 & 0.957 & 0.993 \\
\hline $\mathrm{L}$ & 0.785 & 0.775 & 0.783 & 0.801 & 0.825 & 0.854 & 0.887 & 0.921 & 0.957 & 0.993 \\
\hline M & 0.786 & 0.776 & 0.783 & 0.801 & 0.825 & 0.855 & 0.887 & 0.921 & 0.957 & 0.993 \\
\hline $\mathrm{P}$ & 0.786 & 0.776 & 0.784 & 0.802 & 0.826 & 0.856 & 0.888 & 0.923 & 0.958 & 0.995 \\
\hline Q & 0.787 & 0.777 & 0.785 & 0.802 & 0.827 & 0.856 & 0.888 & 0.923 & 0.959 & 0.995 \\
\hline $\mathrm{R}$ & 0.787 & 0.776 & 0.784 & 0.802 & 0.826 & 0.856 & 0.888 & 0.923 & 0.959 & 0.995 \\
\hline S & 0.787 & 0.776 & 0.784 & 0.802 & 0.826 & 0.856 & 0.888 & 0.923 & 0.958 & 0.995 \\
\hline Channels & 11 & 12 & 13 & 14 & $\overline{15}$ & $\overline{16}$ & 17 & $\overline{18}$ & 19 & 20 \\
\hline A & 1.020 & 1.057 & 1.095 & 1.133 & 1.173 & 1.215 & 1.260 & 1.310 & 1.367 & 1.445 \\
\hline B & 1.020 & 1.057 & 1.095 & 1.133 & 1.173 & 1.214 & 1.259 & 1.309 & 1.367 & 1.445 \\
\hline $\mathrm{C}$ & 1.020 & 1.057 & 1.095 & 1.133 & 1.173 & 1.214 & 1.259 & 1.309 & 1.367 & 1.445 \\
\hline D & 1.020 & 1.057 & 1.094 & 1.133 & 1.172 & 1.214 & 1.259 & 1.309 & 1.367 & 1.445 \\
\hline $\mathrm{E}$ & 1.030 & 1.068 & 1.105 & 1.144 & 1.184 & 1.226 & 1.272 & 1.323 & 1.381 & 1.460 \\
\hline $\mathrm{F}$ & 1.031 & 1.068 & 1.106 & 1.145 & 1.185 & 1.227 & 1.273 & 1.323 & 1.382 & 1.461 \\
\hline G & 1.031 & 1.068 & 1.106 & 1.145 & 1.185 & 1.227 & 1.273 & 1.323 & 1.382 & 1.461 \\
\hline $\mathrm{H}$ & 1.030 & 1.068 & 1.106 & 1.144 & 1.184 & 1.226 & 1.272 & 1.323 & 1.381 & 1.460 \\
\hline $\mathrm{J}$ & 1.031 & 1.068 & 1.106 & 1.144 & 1.184 & 1.227 & 1.272 & 1.323 & 1.381 & 1.461 \\
\hline K & 1.030 & 1.068 & 1.105 & 1.144 & 1.184 & 1.226 & 1.272 & 1.322 & 1.381 & 1.460 \\
\hline $\mathrm{L}$ & 1.030 & 1.068 & 1.105 & 1.144 & 1.184 & 1.226 & 1.272 & 1.322 & 1.381 & 1.460 \\
\hline $\mathrm{M}$ & 1.030 & 1.068 & 1.105 & 1.144 & 1.184 & 1.226 & 1.272 & 1.322 & 1.381 & 1.460 \\
\hline $\mathrm{P}$ & 1.032 & 1.069 & 1.107 & 1.146 & 1.186 & 1.228 & 1.273 & 1.324 & 1.382 & 1.460 \\
\hline Q & 1.032 & 1.070 & 1.108 & 1.146 & 1.186 & 1.229 & 1.274 & 1.325 & 1.383 & 1.461 \\
\hline $\mathrm{R}$ & 1.032 & 1.069 & 1.107 & 1.146 & 1.186 & 1.228 & 1.274 & 1.324 & 1.382 & 1.461 \\
\hline $\mathrm{S}$ & 1.032 & 1.069 & 1.107 & 1.146 & 1.186 & 1.228 & 1.273 & 1.324 & 1.382 & 1.461 \\
\hline
\end{tabular}


Table 7.5. Problem 6.1.1 reference solution Shift power peaking factors for axial segments in element 2 for charge-pan $I$.

\begin{tabular}{|c|c|c|c|c|c|c|c|c|c|c|}
\hline \multirow[t]{2}{*}{ Channels } & \multicolumn{10}{|c|}{ Power Peaking Factors } \\
\hline & 1 & 2 & 3 & 4 & 5 & 6 & 7 & 8 & 9 & 10 \\
\hline A & 1.518 & 1.475 & 1.474 & 1.474 & 1.481 & 1.487 & 1.497 & 1.508 & 1.522 & 1.534 \\
\hline B & 1.513 & 1.478 & 1.473 & 1.474 & 1.477 & 1.492 & 1.497 & 1.517 & 1.524 & 1.537 \\
\hline $\mathrm{C}$ & 1.514 & 1.469 & 1.479 & 1.484 & 1.482 & 1.492 & 1.502 & 1.509 & 1.506 & 1.535 \\
\hline $\mathrm{D}$ & 1.525 & 1.474 & 1.476 & 1.474 & 1.479 & 1.486 & 1.499 & 1.497 & 1.513 & 1.542 \\
\hline $\mathrm{E}$ & 1.538 & 1.494 & 1.485 & 1.492 & 1.498 & 1.505 & 1.513 & 1.524 & 1.535 & 1.550 \\
\hline $\mathrm{F}$ & 1.538 & 1.489 & 1.483 & 1.490 & 1.496 & 1.507 & 1.512 & 1.516 & 1.535 & 1.546 \\
\hline G & 1.529 & 1.485 & 1.497 & 1.492 & 1.503 & 1.512 & 1.521 & 1.531 & 1.537 & 1.545 \\
\hline $\mathrm{H}$ & 1.540 & 1.494 & 1.493 & 1.495 & 1.502 & 1.500 & 1.508 & 1.520 & 1.547 & 1.551 \\
\hline J & 1.539 & 1.506 & 1.493 & 1.484 & 1.499 & 1.516 & 1.518 & 1.527 & 1.535 & 1.551 \\
\hline $\mathrm{K}$ & 1.528 & 1.490 & 1.484 & 1.492 & 1.497 & 1.506 & 1.520 & 1.525 & 1.537 & 1.550 \\
\hline $\mathrm{L}$ & 1.540 & 1.485 & 1.496 & 1.495 & 1.504 & 1.505 & 1.517 & 1.518 & 1.541 & 1.549 \\
\hline M & 1.529 & 1.493 & 1.488 & 1.485 & 1.505 & 1.511 & 1.513 & 1.530 & 1.538 & 1.555 \\
\hline$P$ & 1.543 & 1.496 & 1.493 & 1.491 & 1.503 & 1.503 & 1.519 & 1.523 & 1.545 & 1.549 \\
\hline Q & 1.548 & 1.500 & 1.498 & 1.491 & 1.507 & 1.511 & 1.523 & 1.523 & 1.540 & 1.556 \\
\hline $\mathrm{R}$ & 1.537 & 1.497 & 1.493 & 1.496 & 1.509 & 1.516 & 1.517 & 1.531 & 1.538 & 1.551 \\
\hline $\mathrm{S}$ & 1.539 & 1.492 & 1.499 & 1.498 & 1.500 & 1.503 & 1.525 & 1.528 & 1.539 & 1.547 \\
\hline Channels & 11 & 12 & 13 & $\overline{14}$ & 15 & 16 & 17 & $\overline{18}$ & 19 & 20 \\
\hline A & 1.545 & 1.546 & 1.565 & 1.590 & 1.602 & 1.613 & 1.618 & 1.646 & 1.679 & 1.754 \\
\hline B & 1.549 & 1.558 & 1.570 & 1.588 & 1.605 & 1.613 & 1.633 & 1.653 & 1.682 & 1.760 \\
\hline $\mathrm{C}$ & 1.547 & 1.561 & 1.567 & 1.583 & 1.584 & 1.610 & 1.628 & 1.645 & 1.687 & 1.770 \\
\hline D & 1.545 & 1.556 & 1.572 & 1.584 & 1.593 & 1.613 & 1.635 & 1.666 & 1.681 & 1.764 \\
\hline $\mathrm{E}$ & 1.561 & 1.577 & 1.589 & 1.597 & 1.609 & 1.634 & 1.640 & 1.662 & 1.701 & 1.772 \\
\hline $\mathrm{F}$ & 1.559 & 1.573 & 1.585 & 1.605 & 1.617 & 1.620 & 1.651 & 1.673 & 1.690 & 1.764 \\
\hline G & 1.558 & 1.579 & 1.587 & 1.597 & 1.613 & 1.619 & 1.646 & 1.663 & 1.701 & 1.784 \\
\hline $\mathrm{H}$ & 1.561 & 1.568 & 1.589 & 1.595 & 1.607 & 1.637 & 1.639 & 1.673 & 1.705 & 1.782 \\
\hline J & 1.556 & 1.570 & 1.590 & 1.604 & 1.605 & 1.628 & 1.651 & 1.676 & 1.701 & 1.783 \\
\hline K & 1.564 & 1.574 & 1.587 & 1.588 & 1.612 & 1.624 & 1.645 & 1.678 & 1.708 & 1.785 \\
\hline $\mathrm{L}$ & 1.565 & 1.583 & 1.593 & 1.600 & 1.606 & 1.632 & 1.648 & 1.662 & 1.703 & 1.770 \\
\hline M & 1.560 & 1.575 & 1.584 & 1.600 & 1.616 & 1.627 & 1.648 & 1.680 & 1.696 & 1.787 \\
\hline $\mathrm{P}$ & 1.569 & 1.575 & 1.583 & 1.602 & 1.615 & 1.633 & 1.646 & 1.669 & 1.691 & 1.793 \\
\hline Q & 1.563 & 1.578 & 1.586 & 1.602 & 1.612 & 1.627 & 1.650 & 1.669 & 1.693 & 1.785 \\
\hline $\mathrm{R}$ & 1.563 & 1.573 & 1.593 & 1.605 & 1.623 & 1.625 & 1.644 & 1.675 & 1.707 & 1.793 \\
\hline $\mathrm{S}$ & 1.554 & 1.576 & 1.582 & 1.600 & 1.611 & 1.636 & 1.651 & 1.682 & 1.702 & 1.788 \\
\hline
\end{tabular}


Table 7.6. Problem 6.1.1 reference solution MPACT power peaking factors for axial segments in element 2 for charge-pan I.

\begin{tabular}{|c|c|c|c|c|c|c|c|c|c|c|}
\hline \multirow[t]{2}{*}{ Channels } & \multicolumn{10}{|c|}{ Power Peaking Factors } \\
\hline & 1 & 2 & 3 & 4 & 5 & 6 & 7 & 8 & 9 & 10 \\
\hline A & 1.505 & 1.478 & 1.470 & 1.470 & 1.475 & 1.482 & 1.492 & 1.503 & 1.514 & 1.526 \\
\hline B & 1.504 & 1.477 & 1.470 & 1.470 & 1.474 & 1.482 & 1.491 & 1.502 & 1.513 & 1.525 \\
\hline $\mathrm{C}$ & 1.504 & 1.477 & 1.470 & 1.469 & 1.474 & 1.482 & 1.491 & 1.502 & 1.514 & 1.525 \\
\hline D & 1.504 & 1.477 & 1.469 & 1.469 & 1.474 & 1.482 & 1.491 & 1.502 & 1.513 & 1.525 \\
\hline $\mathrm{E}$ & 1.520 & 1.492 & 1.484 & 1.484 & 1.489 & 1.497 & 1.507 & 1.518 & 1.529 & 1.541 \\
\hline $\mathrm{F}$ & 1.521 & 1.493 & 1.485 & 1.485 & 1.490 & 1.498 & 1.508 & 1.519 & 1.530 & 1.542 \\
\hline G & 1.521 & 1.493 & 1.485 & 1.485 & 1.490 & 1.498 & 1.508 & 1.519 & 1.530 & 1.542 \\
\hline $\mathrm{H}$ & 1.520 & 1.492 & 1.485 & 1.484 & 1.489 & 1.497 & 1.507 & 1.518 & 1.529 & 1.541 \\
\hline $\mathrm{J}$ & 1.521 & 1.493 & 1.485 & 1.485 & 1.489 & 1.497 & 1.507 & 1.518 & 1.529 & 1.541 \\
\hline K & 1.520 & 1.492 & 1.484 & 1.484 & 1.489 & 1.497 & 1.506 & 1.517 & 1.529 & 1.540 \\
\hline $\mathrm{L}$ & 1.520 & 1.492 & 1.484 & 1.484 & 1.489 & 1.496 & 1.506 & 1.517 & 1.529 & 1.540 \\
\hline M & 1.520 & 1.492 & 1.484 & 1.484 & 1.489 & 1.497 & 1.506 & 1.517 & 1.529 & 1.540 \\
\hline $\mathrm{P}$ & 1.521 & 1.493 & 1.486 & 1.486 & 1.491 & 1.499 & 1.509 & 1.520 & 1.531 & 1.543 \\
\hline Q & 1.522 & 1.494 & 1.487 & 1.487 & 1.492 & 1.500 & 1.510 & 1.521 & 1.533 & 1.544 \\
\hline $\mathrm{R}$ & 1.521 & 1.494 & 1.486 & 1.486 & 1.491 & 1.499 & 1.509 & 1.520 & 1.532 & 1.543 \\
\hline $\mathrm{S}$ & 1.521 & 1.493 & 1.486 & 1.486 & 1.491 & 1.499 & 1.509 & 1.520 & 1.531 & 1.543 \\
\hline Channels & 11 & 12 & 13 & 14 & 15 & 16 & 17 & 18 & 19 & 20 \\
\hline A & 1.537 & 1.549 & 1.561 & 1.574 & 1.588 & 1.605 & 1.626 & 1.652 & 1.688 & 1.747 \\
\hline B & 1.537 & 1.548 & 1.560 & 1.573 & 1.587 & 1.604 & 1.625 & 1.651 & 1.686 & 1.745 \\
\hline $\mathrm{C}$ & 1.537 & 1.548 & 1.560 & 1.573 & 1.587 & 1.604 & 1.624 & 1.650 & 1.685 & 1.743 \\
\hline D & 1.537 & 1.548 & 1.560 & 1.573 & 1.587 & 1.604 & 1.625 & 1.651 & 1.686 & 1.745 \\
\hline $\mathrm{E}$ & 1.553 & 1.565 & 1.577 & 1.590 & 1.604 & 1.621 & 1.642 & 1.669 & 1.705 & 1.765 \\
\hline $\mathrm{F}$ & 1.554 & 1.565 & 1.578 & 1.591 & 1.605 & 1.622 & 1.643 & 1.670 & 1.706 & 1.767 \\
\hline G & 1.554 & 1.566 & 1.578 & 1.591 & 1.605 & 1.622 & 1.643 & 1.670 & 1.706 & 1.766 \\
\hline $\mathrm{H}$ & 1.553 & 1.565 & 1.577 & 1.590 & 1.604 & 1.621 & 1.642 & 1.668 & 1.704 & 1.765 \\
\hline $\mathrm{J}$ & 1.552 & 1.564 & 1.576 & 1.589 & 1.603 & 1.620 & 1.640 & 1.667 & 1.702 & 1.762 \\
\hline K & 1.552 & 1.564 & 1.576 & 1.588 & 1.603 & 1.619 & 1.640 & 1.667 & 1.702 & 1.762 \\
\hline $\mathrm{L}$ & 1.552 & 1.564 & 1.576 & 1.589 & 1.603 & 1.619 & 1.640 & 1.667 & 1.702 & 1.762 \\
\hline M & 1.552 & 1.564 & 1.576 & 1.589 & 1.603 & 1.620 & 1.640 & 1.667 & 1.703 & 1.763 \\
\hline $\mathrm{P}$ & 1.554 & 1.566 & 1.578 & 1.591 & 1.605 & 1.622 & 1.642 & 1.668 & 1.704 & 1.763 \\
\hline Q & 1.556 & 1.568 & 1.580 & 1.593 & 1.608 & 1.625 & 1.646 & 1.673 & 1.709 & 1.770 \\
\hline $\mathrm{R}$ & 1.555 & 1.567 & 1.579 & 1.591 & 1.606 & 1.622 & 1.643 & 1.670 & 1.706 & 1.766 \\
\hline $\mathrm{S}$ & 1.554 & 1.566 & 1.578 & 1.591 & 1.605 & 1.621 & 1.642 & 1.668 & 1.704 & 1.762 \\
\hline
\end{tabular}


Table 7.7. Problem 6.1.1 reference solution Shift power peaking factors for axial segments in element 3 for charge-pan I.

\begin{tabular}{|c|c|c|c|c|c|c|c|c|c|c|}
\hline \multirow[t]{2}{*}{ Channels } & \multicolumn{10}{|c|}{ Power Peaking Factors } \\
\hline & 1 & 2 & 3 & 4 & 5 & 6 & 7 & 8 & 9 & 10 \\
\hline A & 1.750 & 1.667 & 1.627 & 1.614 & 1.584 & 1.556 & 1.544 & 1.521 & 1.505 & 1.493 \\
\hline B & 1.764 & 1.678 & 1.634 & 1.608 & 1.573 & 1.564 & 1.542 & 1.527 & 1.505 & 1.492 \\
\hline $\mathrm{C}$ & 1.760 & 1.671 & 1.643 & 1.601 & 1.577 & 1.561 & 1.545 & 1.521 & 1.509 & 1.499 \\
\hline D & 1.761 & 1.670 & 1.630 & 1.608 & 1.577 & 1.556 & 1.534 & 1.522 & 1.498 & 1.480 \\
\hline $\mathrm{E}$ & 1.771 & 1.678 & 1.656 & 1.619 & 1.588 & 1.576 & 1.550 & 1.533 & 1.515 & 1.493 \\
\hline $\mathrm{F}$ & 1.764 & 1.683 & 1.639 & 1.625 & 1.583 & 1.565 & 1.555 & 1.526 & 1.505 & 1.486 \\
\hline G & 1.778 & 1.685 & 1.642 & 1.623 & 1.606 & 1.577 & 1.548 & 1.534 & 1.519 & 1.492 \\
\hline $\mathrm{H}$ & 1.775 & 1.691 & 1.658 & 1.622 & 1.599 & 1.581 & 1.567 & 1.541 & 1.524 & 1.507 \\
\hline $\mathrm{J}$ & 1.779 & 1.695 & 1.655 & 1.637 & 1.601 & 1.583 & 1.568 & 1.540 & 1.528 & 1.512 \\
\hline K & 1.772 & 1.692 & 1.644 & 1.631 & 1.609 & 1.583 & 1.558 & 1.533 & 1.520 & 1.508 \\
\hline $\mathrm{L}$ & 1.780 & 1.688 & 1.650 & 1.623 & 1.589 & 1.574 & 1.547 & 1.525 & 1.510 & 1.492 \\
\hline M & 1.768 & 1.674 & 1.645 & 1.611 & 1.591 & 1.565 & 1.548 & 1.528 & 1.511 & 1.493 \\
\hline $\mathrm{P}$ & 1.772 & 1.689 & 1.650 & 1.622 & 1.590 & 1.569 & 1.551 & 1.535 & 1.512 & 1.495 \\
\hline Q & 1.768 & 1.692 & 1.652 & 1.624 & 1.599 & 1.584 & 1.546 & 1.534 & 1.520 & 1.494 \\
\hline $\mathrm{R}$ & 1.789 & 1.701 & 1.654 & 1.627 & 1.602 & 1.587 & 1.565 & 1.554 & 1.525 & 1.520 \\
\hline S & 1.773 & 1.682 & 1.648 & 1.616 & 1.602 & 1.578 & 1.549 & 1.532 & 1.516 & 1.504 \\
\hline Channels & 11 & 12 & 13 & 14 & 15 & 16 & 17 & 18 & 19 & 20 \\
\hline A & 1.463 & 1.450 & 1.437 & 1.421 & 1.420 & 1.399 & 1.385 & 1.390 & 1.376 & 1.426 \\
\hline B & 1.471 & 1.459 & 1.447 & 1.435 & 1.417 & 1.416 & 1.402 & 1.402 & 1.393 & 1.430 \\
\hline $\mathrm{C}$ & 1.471 & 1.456 & 1.442 & 1.420 & 1.409 & 1.406 & 1.385 & 1.384 & 1.379 & 1.420 \\
\hline D & 1.462 & 1.447 & 1.443 & 1.416 & 1.404 & 1.391 & 1.381 & 1.373 & 1.363 & 1.407 \\
\hline $\mathrm{E}$ & 1.479 & 1.455 & 1.431 & 1.420 & 1.404 & 1.389 & 1.378 & 1.369 & 1.361 & 1.404 \\
\hline $\mathrm{F}$ & 1.475 & 1.457 & 1.438 & 1.418 & 1.405 & 1.395 & 1.382 & 1.365 & 1.366 & 1.400 \\
\hline G & 1.488 & 1.475 & 1.462 & 1.443 & 1.425 & 1.415 & 1.403 & 1.409 & 1.401 & 1.448 \\
\hline $\mathrm{H}$ & 1.489 & 1.479 & 1.457 & 1.450 & 1.433 & 1.426 & 1.423 & 1.411 & 1.410 & 1.460 \\
\hline $\mathrm{J}$ & 1.489 & 1.484 & 1.466 & 1.452 & 1.440 & 1.422 & 1.420 & 1.416 & 1.412 & 1.452 \\
\hline K & 1.489 & 1.478 & 1.459 & 1.444 & 1.434 & 1.414 & 1.412 & 1.403 & 1.396 & 1.447 \\
\hline $\mathrm{L}$ & 1.477 & 1.454 & 1.436 & 1.417 & 1.410 & 1.385 & 1.379 & 1.377 & 1.374 & 1.413 \\
\hline $\mathrm{M}$ & 1.476 & 1.456 & 1.439 & 1.413 & 1.403 & 1.388 & 1.388 & 1.372 & 1.371 & 1.400 \\
\hline $\mathrm{P}$ & 1.477 & 1.453 & 1.436 & 1.425 & 1.399 & 1.390 & 1.384 & 1.377 & 1.365 & 1.407 \\
\hline Q & 1.473 & 1.454 & 1.438 & 1.429 & 1.413 & 1.396 & 1.393 & 1.369 & 1.380 & 1.422 \\
\hline $\mathrm{R}$ & 1.496 & 1.478 & 1.469 & 1.455 & 1.442 & 1.438 & 1.426 & 1.420 & 1.426 & 1.469 \\
\hline $\mathrm{S}$ & 1.482 & 1.460 & 1.454 & 1.428 & 1.416 & 1.399 & 1.382 & 1.377 & 1.374 & 1.409 \\
\hline
\end{tabular}


Table 7.8. Problem 6.1.1 reference solution MPACT power peaking factors for axial segments in element 3 for charge-pan I.

\begin{tabular}{|c|c|c|c|c|c|c|c|c|c|c|}
\hline \multirow[t]{2}{*}{ Channels } & \multicolumn{10}{|c|}{ Power Peaking Factors } \\
\hline & 1 & 2 & 3 & 4 & 5 & 6 & 7 & 8 & 9 & 10 \\
\hline A & 1.741 & 1.676 & 1.635 & 1.603 & 1.578 & 1.556 & 1.536 & 1.519 & 1.502 & 1.486 \\
\hline B & 1.738 & 1.673 & 1.632 & 1.601 & 1.574 & 1.552 & 1.533 & 1.515 & 1.497 & 1.481 \\
\hline $\mathrm{C}$ & 1.736 & 1.672 & 1.631 & 1.599 & 1.573 & 1.551 & 1.531 & 1.513 & 1.495 & 1.478 \\
\hline D & 1.738 & 1.673 & 1.633 & 1.601 & 1.575 & 1.552 & 1.533 & 1.515 & 1.497 & 1.481 \\
\hline $\mathrm{E}$ & 1.758 & 1.692 & 1.650 & 1.618 & 1.591 & 1.569 & 1.549 & 1.531 & 1.514 & 1.498 \\
\hline $\mathrm{F}$ & 1.760 & 1.694 & 1.653 & 1.621 & 1.595 & 1.573 & 1.553 & 1.536 & 1.519 & 1.503 \\
\hline G & 1.760 & 1.694 & 1.653 & 1.621 & 1.595 & 1.573 & 1.554 & 1.536 & 1.520 & 1.504 \\
\hline $\mathrm{H}$ & 1.758 & 1.692 & 1.650 & 1.618 & 1.591 & 1.569 & 1.549 & 1.531 & 1.514 & 1.498 \\
\hline $\mathrm{J}$ & 1.755 & 1.688 & 1.646 & 1.613 & 1.587 & 1.564 & 1.543 & 1.525 & 1.507 & 1.489 \\
\hline K & 1.755 & 1.688 & 1.646 & 1.613 & 1.586 & 1.563 & 1.543 & 1.524 & 1.506 & 1.488 \\
\hline $\mathrm{L}$ & 1.755 & 1.688 & 1.646 & 1.613 & 1.586 & 1.563 & 1.543 & 1.524 & 1.506 & 1.488 \\
\hline M & 1.755 & 1.689 & 1.646 & 1.614 & 1.587 & 1.564 & 1.543 & 1.525 & 1.507 & 1.489 \\
\hline $\mathrm{P}$ & 1.755 & 1.690 & 1.648 & 1.616 & 1.589 & 1.566 & 1.546 & 1.527 & 1.509 & 1.492 \\
\hline Q & 1.764 & 1.698 & 1.656 & 1.625 & 1.599 & 1.577 & 1.558 & 1.541 & 1.525 & 1.509 \\
\hline $\mathrm{R}$ & 1.758 & 1.692 & 1.649 & 1.617 & 1.590 & 1.567 & 1.547 & 1.528 & 1.510 & 1.492 \\
\hline $\mathrm{S}$ & 1.755 & 1.689 & 1.647 & 1.614 & 1.587 & 1.564 & 1.544 & 1.525 & 1.507 & 1.489 \\
\hline Channels & 11 & 12 & 13 & 14 & 15 & 16 & 17 & 18 & 19 & 20 \\
\hline A & 1.470 & 1.455 & 1.440 & 1.426 & 1.414 & 1.403 & 1.396 & 1.394 & 1.400 & 1.425 \\
\hline B & 1.465 & 1.449 & 1.433 & 1.419 & 1.405 & 1.394 & 1.386 & 1.383 & 1.388 & 1.412 \\
\hline $\mathrm{C}$ & 1.462 & 1.445 & 1.429 & 1.414 & 1.400 & 1.388 & 1.380 & 1.376 & 1.380 & 1.402 \\
\hline D & 1.465 & 1.449 & 1.433 & 1.418 & 1.405 & 1.394 & 1.386 & 1.383 & 1.388 & 1.411 \\
\hline $\mathrm{E}$ & 1.481 & 1.465 & 1.450 & 1.435 & 1.422 & 1.411 & 1.403 & 1.400 & 1.405 & 1.430 \\
\hline $\mathrm{F}$ & 1.488 & 1.472 & 1.458 & 1.444 & 1.431 & 1.421 & 1.415 & 1.413 & 1.420 & 1.446 \\
\hline G & 1.488 & 1.473 & 1.458 & 1.444 & 1.432 & 1.422 & 1.415 & 1.414 & 1.420 & 1.447 \\
\hline $\mathrm{H}$ & 1.481 & 1.465 & 1.450 & 1.435 & 1.422 & 1.411 & 1.403 & 1.400 & 1.406 & 1.430 \\
\hline $\mathrm{J}$ & 1.472 & 1.454 & 1.437 & 1.421 & 1.406 & 1.392 & 1.382 & 1.377 & 1.379 & 1.401 \\
\hline K & 1.470 & 1.453 & 1.436 & 1.419 & 1.403 & 1.390 & 1.380 & 1.374 & 1.376 & 1.397 \\
\hline $\mathrm{L}$ & 1.470 & 1.453 & 1.435 & 1.419 & 1.403 & 1.389 & 1.379 & 1.373 & 1.375 & 1.395 \\
\hline $\mathrm{M}$ & 1.472 & 1.454 & 1.437 & 1.421 & 1.405 & 1.392 & 1.382 & 1.377 & 1.379 & 1.400 \\
\hline $\mathrm{P}$ & 1.475 & 1.458 & 1.441 & 1.424 & 1.409 & 1.396 & 1.387 & 1.382 & 1.384 & 1.405 \\
\hline Q & 1.494 & 1.479 & 1.464 & 1.451 & 1.439 & 1.429 & 1.423 & 1.422 & 1.429 & 1.455 \\
\hline $\mathrm{R}$ & 1.475 & 1.458 & 1.441 & 1.425 & 1.409 & 1.396 & 1.387 & 1.382 & 1.384 & 1.405 \\
\hline $\mathrm{S}$ & 1.471 & 1.453 & 1.436 & 1.419 & 1.403 & 1.389 & 1.378 & 1.372 & 1.373 & 1.393 \\
\hline
\end{tabular}


Table 7.9. Problem 6.1.1 reference solution Shift power peaking factors for axial segments in element 4 for charge-pan $I$.

\begin{tabular}{|c|c|c|c|c|c|c|c|c|c|c|}
\hline \multirow[t]{2}{*}{ Channels } & \multicolumn{10}{|c|}{ Power Peaking Factors } \\
\hline & 1 & 2 & 3 & 4 & 5 & 6 & 7 & 8 & 9 & 10 \\
\hline A & 1.361 & 1.268 & 1.225 & 1.184 & 1.151 & 1.105 & 1.083 & 1.056 & 1.034 & 1.007 \\
\hline B & 1.386 & 1.285 & 1.247 & 1.196 & 1.172 & 1.133 & 1.103 & 1.070 & 1.050 & 1.023 \\
\hline $\mathrm{C}$ & 1.374 & 1.277 & 1.231 & 1.195 & 1.151 & 1.118 & 1.090 & 1.052 & 1.017 & 1.001 \\
\hline $\mathrm{D}$ & 1.345 & 1.263 & 1.212 & 1.179 & 1.133 & 1.107 & 1.078 & 1.040 & 1.014 & 0.991 \\
\hline $\mathrm{E}$ & 1.343 & 1.244 & 1.208 & 1.159 & 1.122 & 1.082 & 1.057 & 1.029 & 1.004 & 0.972 \\
\hline $\mathrm{F}$ & 1.352 & 1.255 & 1.197 & 1.165 & 1.127 & 1.089 & 1.062 & 1.034 & 1.008 & 0.969 \\
\hline G & 1.378 & 1.297 & 1.235 & 1.206 & 1.175 & 1.134 & 1.099 & 1.077 & 1.048 & 1.018 \\
\hline $\mathrm{H}$ & 1.413 & 1.315 & 1.263 & 1.227 & 1.184 & 1.156 & 1.125 & 1.090 & 1.057 & 1.035 \\
\hline $\mathrm{J}$ & 1.394 & 1.307 & 1.266 & 1.222 & 1.181 & 1.159 & 1.124 & 1.096 & 1.064 & 1.038 \\
\hline K & 1.381 & 1.299 & 1.250 & 1.209 & 1.173 & 1.133 & 1.104 & 1.076 & 1.046 & 1.020 \\
\hline $\mathrm{L}$ & 1.347 & 1.257 & 1.210 & 1.176 & 1.117 & 1.093 & 1.057 & 1.031 & 0.999 & 0.977 \\
\hline M & 1.338 & 1.256 & 1.200 & 1.161 & 1.122 & 1.085 & 1.053 & 1.025 & 0.996 & 0.965 \\
\hline $\mathrm{P}$ & 1.342 & 1.250 & 1.197 & 1.156 & 1.119 & 1.084 & 1.056 & 1.018 & 0.996 & 0.967 \\
\hline $\mathrm{Q}$ & 1.354 & 1.268 & 1.211 & 1.166 & 1.138 & 1.098 & 1.072 & 1.039 & 1.013 & 0.985 \\
\hline $\mathrm{R}$ & 1.410 & 1.317 & 1.283 & 1.236 & 1.196 & 1.170 & 1.140 & 1.112 & 1.083 & 1.049 \\
\hline S & 1.359 & 1.271 & 1.211 & 1.182 & 1.136 & 1.107 & 1.068 & 1.044 & 1.016 & 0.987 \\
\hline Channels & 11 & 12 & 13 & 14 & 15 & 16 & 17 & 18 & 19 & 20 \\
\hline A & 0.979 & 0.948 & 0.934 & 0.913 & 0.894 & 0.875 & 0.861 & 0.844 & 0.839 & 0.857 \\
\hline B & 1.000 & 0.976 & 0.959 & 0.936 & 0.908 & 0.900 & 0.887 & 0.867 & 0.855 & 0.876 \\
\hline $\mathrm{C}$ & 0.981 & 0.952 & 0.938 & 0.913 & 0.896 & 0.883 & 0.860 & 0.848 & 0.836 & 0.859 \\
\hline D & 0.959 & 0.945 & 0.926 & 0.900 & 0.878 & 0.865 & 0.848 & 0.834 & 0.824 & 0.847 \\
\hline $\mathrm{E}$ & 0.944 & 0.921 & 0.900 & 0.875 & 0.861 & 0.844 & 0.833 & 0.821 & 0.809 & 0.830 \\
\hline $\mathrm{F}$ & 0.946 & 0.931 & 0.905 & 0.879 & 0.866 & 0.848 & 0.834 & 0.828 & 0.823 & 0.835 \\
\hline G & 1.000 & 0.965 & 0.951 & 0.923 & 0.908 & 0.890 & 0.875 & 0.867 & 0.850 & 0.874 \\
\hline $\mathrm{H}$ & 1.023 & 0.989 & 0.965 & 0.950 & 0.928 & 0.916 & 0.900 & 0.892 & 0.882 & 0.890 \\
\hline $\mathrm{J}$ & 1.024 & 0.999 & 0.969 & 0.951 & 0.932 & 0.913 & 0.902 & 0.891 & 0.882 & 0.888 \\
\hline K & 0.993 & 0.971 & 0.954 & 0.926 & 0.914 & 0.891 & 0.881 & 0.867 & 0.856 & 0.870 \\
\hline $\mathrm{L}$ & 0.949 & 0.924 & 0.909 & 0.888 & 0.866 & 0.852 & 0.837 & 0.824 & 0.810 & 0.830 \\
\hline $\mathrm{M}$ & 0.938 & 0.925 & 0.897 & 0.877 & 0.863 & 0.852 & 0.826 & 0.820 & 0.813 & 0.822 \\
\hline $\mathrm{P}$ & 0.950 & 0.915 & 0.891 & 0.871 & 0.860 & 0.835 & 0.831 & 0.814 & 0.807 & 0.814 \\
\hline Q & 0.962 & 0.942 & 0.924 & 0.897 & 0.867 & 0.856 & 0.840 & 0.835 & 0.829 & 0.839 \\
\hline $\mathrm{R}$ & 1.024 & 1.008 & 0.990 & 0.964 & 0.945 & 0.926 & 0.909 & 0.895 & 0.890 & 0.906 \\
\hline $\mathrm{S}$ & 0.962 & 0.936 & 0.909 & 0.884 & 0.885 & 0.864 & 0.842 & 0.831 & 0.818 & 0.830 \\
\hline
\end{tabular}


Table 7.10. Problem 6.1.1 reference solution MPACT power peaking factors for axial segments in element 4 for charge-pan I.

\begin{tabular}{|c|c|c|c|c|c|c|c|c|c|c|}
\hline \multirow[t]{2}{*}{ Channels } & \multicolumn{10}{|c|}{ Power Peaking Factors } \\
\hline & 1 & 2 & 3 & 4 & 5 & 6 & 7 & 8 & 9 & 10 \\
\hline A & 1.371 & 1.299 & 1.248 & 1.205 & 1.167 & 1.133 & 1.103 & 1.074 & 1.048 & 1.023 \\
\hline B & 1.355 & 1.283 & 1.232 & 1.188 & 1.150 & 1.116 & 1.085 & 1.056 & 1.030 & 1.005 \\
\hline $\mathrm{C}$ & 1.344 & 1.272 & 1.220 & 1.176 & 1.138 & 1.104 & 1.073 & 1.044 & 1.017 & 0.992 \\
\hline D & 1.355 & 1.283 & 1.231 & 1.188 & 1.150 & 1.116 & 1.085 & 1.056 & 1.030 & 1.004 \\
\hline $\mathrm{E}$ & 1.374 & 1.301 & 1.249 & 1.205 & 1.166 & 1.132 & 1.101 & 1.073 & 1.046 & 1.021 \\
\hline $\mathrm{F}$ & 1.392 & 1.319 & 1.267 & 1.224 & 1.186 & 1.152 & 1.122 & 1.094 & 1.067 & 1.042 \\
\hline G & 1.392 & 1.320 & 1.268 & 1.224 & 1.187 & 1.153 & 1.122 & 1.094 & 1.068 & 1.042 \\
\hline $\mathrm{H}$ & 1.374 & 1.301 & 1.249 & 1.205 & 1.166 & 1.132 & 1.101 & 1.073 & 1.046 & 1.021 \\
\hline $\mathrm{J}$ & 1.339 & 1.265 & 1.212 & 1.166 & 1.127 & 1.092 & 1.060 & 1.031 & 1.004 & 0.979 \\
\hline K & 1.334 & 1.261 & 1.207 & 1.162 & 1.122 & 1.087 & 1.056 & 1.026 & 0.999 & 0.974 \\
\hline $\mathrm{L}$ & 1.333 & 1.260 & 1.206 & 1.161 & 1.122 & 1.087 & 1.055 & 1.026 & 0.999 & 0.974 \\
\hline M & 1.339 & 1.265 & 1.211 & 1.166 & 1.127 & 1.092 & 1.060 & 1.031 & 1.004 & 0.978 \\
\hline $\mathrm{P}$ & 1.344 & 1.272 & 1.219 & 1.174 & 1.135 & 1.100 & 1.068 & 1.039 & 1.012 & 0.986 \\
\hline Q & 1.402 & 1.330 & 1.278 & 1.235 & 1.197 & 1.164 & 1.134 & 1.106 & 1.079 & 1.054 \\
\hline $\mathrm{R}$ & 1.344 & 1.272 & 1.218 & 1.174 & 1.134 & 1.100 & 1.068 & 1.039 & 1.012 & 0.986 \\
\hline $\mathrm{S}$ & 1.330 & 1.257 & 1.203 & 1.158 & 1.119 & 1.083 & 1.052 & 1.022 & 0.995 & 0.969 \\
\hline Channels & 11 & 12 & 13 & 14 & 15 & 16 & 17 & 18 & 19 & 20 \\
\hline A & 0.999 & 0.976 & 0.955 & 0.935 & 0.916 & 0.900 & 0.886 & 0.876 & 0.871 & 0.878 \\
\hline B & 0.981 & 0.958 & 0.936 & 0.916 & 0.898 & 0.881 & 0.867 & 0.857 & 0.852 & 0.860 \\
\hline $\mathrm{C}$ & 0.968 & 0.945 & 0.923 & 0.903 & 0.885 & 0.869 & 0.855 & 0.845 & 0.840 & 0.847 \\
\hline D & 0.980 & 0.958 & 0.936 & 0.916 & 0.897 & 0.881 & 0.867 & 0.857 & 0.852 & 0.859 \\
\hline $\mathrm{E}$ & 0.997 & 0.974 & 0.952 & 0.932 & 0.913 & 0.896 & 0.882 & 0.872 & 0.868 & 0.875 \\
\hline $\mathrm{F}$ & 1.018 & 0.995 & 0.973 & 0.953 & 0.934 & 0.917 & 0.903 & 0.893 & 0.889 & 0.897 \\
\hline G & 1.019 & 0.996 & 0.974 & 0.953 & 0.935 & 0.918 & 0.904 & 0.894 & 0.889 & 0.897 \\
\hline $\mathrm{H}$ & 0.997 & 0.974 & 0.952 & 0.932 & 0.913 & 0.896 & 0.882 & 0.872 & 0.868 & 0.875 \\
\hline $\mathrm{J}$ & 0.955 & 0.932 & 0.910 & 0.890 & 0.871 & 0.855 & 0.841 & 0.831 & 0.826 & 0.833 \\
\hline K & 0.950 & 0.927 & 0.905 & 0.885 & 0.866 & 0.850 & 0.836 & 0.826 & 0.821 & 0.827 \\
\hline $\mathrm{L}$ & 0.950 & 0.927 & 0.905 & 0.885 & 0.866 & 0.850 & 0.836 & 0.826 & 0.822 & 0.829 \\
\hline M & 0.954 & 0.931 & 0.910 & 0.889 & 0.871 & 0.854 & 0.841 & 0.830 & 0.825 & 0.832 \\
\hline $\mathrm{P}$ & 0.962 & 0.939 & 0.917 & 0.897 & 0.878 & 0.862 & 0.848 & 0.837 & 0.832 & 0.839 \\
\hline Q & 1.030 & 1.007 & 0.985 & 0.965 & 0.946 & 0.929 & 0.915 & 0.905 & 0.900 & 0.908 \\
\hline $\mathrm{R}$ & 0.962 & 0.939 & 0.917 & 0.897 & 0.878 & 0.862 & 0.848 & 0.838 & 0.833 & 0.840 \\
\hline$S$ & 0.945 & 0.922 & 0.900 & 0.880 & 0.862 & 0.845 & 0.831 & 0.821 & 0.816 & 0.823 \\
\hline
\end{tabular}


Table 7.11. Problem 6.1.1 reference solution Shift power peaking factors for axial segments in element 5 for charge-pan I.

\begin{tabular}{|c|c|c|c|c|c|c|c|c|c|c|}
\hline \multirow[t]{2}{*}{ Channels } & \multicolumn{10}{|c|}{ Power Peaking Factors } \\
\hline & 1 & 2 & 3 & 4 & 5 & 6 & 7 & 8 & 9 & 10 \\
\hline A & 0.807 & 0.749 & 0.715 & 0.688 & 0.661 & 0.631 & 0.612 & 0.594 & 0.567 & 0.548 \\
\hline B & 0.822 & 0.771 & 0.740 & 0.700 & 0.669 & 0.643 & 0.620 & 0.601 & 0.581 & 0.564 \\
\hline $\mathrm{C}$ & 0.811 & 0.749 & 0.726 & 0.689 & 0.660 & 0.632 & 0.612 & 0.598 & 0.575 & 0.554 \\
\hline D & 0.792 & 0.744 & 0.706 & 0.674 & 0.652 & 0.627 & 0.608 & 0.583 & 0.562 & 0.544 \\
\hline $\mathrm{E}$ & 0.771 & 0.722 & 0.687 & 0.658 & 0.637 & 0.614 & 0.588 & 0.565 & 0.553 & 0.531 \\
\hline $\mathrm{F}$ & 0.781 & 0.726 & 0.692 & 0.667 & 0.641 & 0.618 & 0.590 & 0.571 & 0.551 & 0.533 \\
\hline G & 0.820 & 0.761 & 0.724 & 0.695 & 0.675 & 0.646 & 0.625 & 0.604 & 0.580 & 0.565 \\
\hline $\mathrm{H}$ & 0.844 & 0.776 & 0.749 & 0.718 & 0.697 & 0.666 & 0.642 & 0.618 & 0.593 & 0.573 \\
\hline $\mathrm{J}$ & 0.849 & 0.789 & 0.747 & 0.716 & 0.686 & 0.664 & 0.643 & 0.617 & 0.597 & 0.573 \\
\hline K & 0.820 & 0.757 & 0.725 & 0.700 & 0.669 & 0.645 & 0.623 & 0.603 & 0.584 & 0.555 \\
\hline $\mathrm{L}$ & 0.783 & 0.727 & 0.694 & 0.670 & 0.640 & 0.614 & 0.588 & 0.571 & 0.556 & 0.533 \\
\hline M & 0.779 & 0.716 & 0.685 & 0.664 & 0.635 & 0.609 & 0.589 & 0.565 & 0.547 & 0.531 \\
\hline $\mathrm{P}$ & 0.771 & 0.717 & 0.691 & 0.653 & 0.628 & 0.611 & 0.584 & 0.569 & 0.542 & 0.521 \\
\hline Q & 0.794 & 0.731 & 0.699 & 0.667 & 0.645 & 0.621 & 0.594 & 0.575 & 0.555 & 0.538 \\
\hline $\mathrm{R}$ & 0.861 & 0.793 & 0.762 & 0.725 & 0.696 & 0.671 & 0.651 & 0.628 & 0.602 & 0.582 \\
\hline $\mathrm{S}$ & 0.789 & 0.730 & 0.695 & 0.669 & 0.645 & 0.612 & 0.590 & 0.577 & 0.555 & 0.534 \\
\hline Channels & 11 & 12 & 13 & 14 & 15 & 16 & 17 & 18 & 19 & 20 \\
\hline A & 0.532 & 0.515 & 0.509 & 0.485 & 0.474 & 0.463 & 0.457 & 0.454 & 0.459 & 0.483 \\
\hline B & 0.545 & 0.527 & 0.510 & 0.493 & 0.481 & 0.472 & 0.471 & 0.468 & 0.470 & 0.493 \\
\hline $\mathrm{C}$ & 0.533 & 0.520 & 0.500 & 0.490 & 0.473 & 0.465 & 0.457 & 0.456 & 0.459 & 0.486 \\
\hline D & 0.528 & 0.509 & 0.495 & 0.474 & 0.465 & 0.457 & 0.448 & 0.449 & 0.451 & 0.480 \\
\hline $\mathrm{E}$ & 0.516 & 0.498 & 0.484 & 0.465 & 0.459 & 0.444 & 0.442 & 0.431 & 0.433 & 0.449 \\
\hline $\mathrm{F}$ & 0.515 & 0.500 & 0.477 & 0.470 & 0.455 & 0.448 & 0.439 & 0.437 & 0.436 & 0.455 \\
\hline G & 0.541 & 0.523 & 0.504 & 0.495 & 0.484 & 0.471 & 0.464 & 0.464 & 0.464 & 0.486 \\
\hline $\mathrm{H}$ & 0.554 & 0.535 & 0.519 & 0.509 & 0.493 & 0.487 & 0.476 & 0.472 & 0.476 & 0.504 \\
\hline $\mathbf{J}$ & 0.555 & 0.531 & 0.518 & 0.505 & 0.489 & 0.482 & 0.476 & 0.474 & 0.479 & 0.502 \\
\hline K & 0.544 & 0.531 & 0.507 & 0.491 & 0.481 & 0.469 & 0.462 & 0.462 & 0.466 & 0.486 \\
\hline $\mathrm{L}$ & 0.514 & 0.500 & 0.485 & 0.471 & 0.457 & 0.446 & 0.439 & 0.434 & 0.434 & 0.459 \\
\hline $\mathrm{M}$ & 0.516 & 0.503 & 0.482 & 0.466 & 0.456 & 0.441 & 0.437 & 0.432 & 0.430 & 0.450 \\
\hline $\mathrm{P}$ & 0.514 & 0.495 & 0.481 & 0.467 & 0.453 & 0.435 & 0.432 & 0.424 & 0.427 & 0.441 \\
\hline Q & 0.516 & 0.500 & 0.489 & 0.471 & 0.460 & 0.450 & 0.443 & 0.439 & 0.439 & 0.463 \\
\hline $\mathrm{R}$ & 0.562 & 0.538 & 0.525 & 0.509 & 0.501 & 0.489 & 0.477 & 0.476 & 0.471 & 0.509 \\
\hline $\mathrm{S}$ & 0.519 & 0.500 & 0.486 & 0.480 & 0.462 & 0.453 & 0.441 & 0.434 & 0.440 & 0.463 \\
\hline
\end{tabular}


Table 7.12. Problem 6.1.1 reference solution MPACT power peaking factors for axial segments in element 5 for charge-pan I.

\begin{tabular}{|c|c|c|c|c|c|c|c|c|c|c|}
\hline \multirow[t]{2}{*}{ Channels } & \multicolumn{10}{|c|}{ Power Peaking Factors } \\
\hline & 1 & 2 & 3 & 4 & 5 & 6 & 7 & 8 & 9 & 10 \\
\hline A & 0.829 & 0.779 & 0.742 & 0.710 & 0.682 & 0.656 & 0.633 & 0.611 & 0.591 & 0.571 \\
\hline B & 0.811 & 0.762 & 0.726 & 0.695 & 0.667 & 0.642 & 0.620 & 0.598 & 0.578 & 0.559 \\
\hline $\mathrm{C}$ & 0.799 & 0.751 & 0.715 & 0.685 & 0.657 & 0.633 & 0.611 & 0.590 & 0.570 & 0.551 \\
\hline $\mathrm{D}$ & 0.811 & 0.762 & 0.726 & 0.695 & 0.667 & 0.642 & 0.619 & 0.598 & 0.578 & 0.559 \\
\hline $\mathrm{E}$ & 0.826 & 0.776 & 0.739 & 0.707 & 0.679 & 0.654 & 0.630 & 0.609 & 0.588 & 0.569 \\
\hline $\mathrm{F}$ & 0.846 & 0.795 & 0.757 & 0.725 & 0.696 & 0.670 & 0.646 & 0.624 & 0.603 & 0.583 \\
\hline G & 0.847 & 0.796 & 0.758 & 0.725 & 0.696 & 0.670 & 0.646 & 0.624 & 0.603 & 0.583 \\
\hline $\mathrm{H}$ & 0.826 & 0.776 & 0.739 & 0.707 & 0.679 & 0.654 & 0.630 & 0.609 & 0.588 & 0.569 \\
\hline $\mathrm{J}$ & 0.786 & 0.738 & 0.703 & 0.673 & 0.646 & 0.622 & 0.600 & 0.580 & 0.560 & 0.542 \\
\hline K & 0.780 & 0.733 & 0.699 & 0.669 & 0.642 & 0.619 & 0.597 & 0.577 & 0.558 & 0.540 \\
\hline $\mathrm{L}$ & 0.781 & 0.734 & 0.699 & 0.669 & 0.643 & 0.619 & 0.597 & 0.577 & 0.558 & 0.540 \\
\hline $\mathrm{M}$ & 0.784 & 0.737 & 0.702 & 0.672 & 0.646 & 0.622 & 0.600 & 0.579 & 0.560 & 0.542 \\
\hline $\mathrm{P}$ & 0.791 & 0.744 & 0.708 & 0.678 & 0.651 & 0.627 & 0.605 & 0.584 & 0.564 & 0.546 \\
\hline Q & 0.857 & 0.806 & 0.767 & 0.734 & 0.705 & 0.679 & 0.655 & 0.632 & 0.611 & 0.590 \\
\hline $\mathrm{R}$ & 0.792 & 0.744 & 0.708 & 0.678 & 0.651 & 0.627 & 0.605 & 0.584 & 0.565 & 0.546 \\
\hline S & 0.776 & 0.730 & 0.695 & 0.665 & 0.639 & 0.616 & 0.594 & 0.574 & 0.556 & 0.538 \\
\hline Channels & 11 & 12 & 13 & 14 & 15 & 16 & 17 & 18 & 19 & 20 \\
\hline A & 0.553 & 0.535 & 0.519 & 0.504 & 0.492 & 0.482 & 0.476 & 0.475 & 0.482 & 0.501 \\
\hline B & 0.541 & 0.524 & 0.508 & 0.494 & 0.482 & 0.472 & 0.466 & 0.465 & 0.471 & 0.489 \\
\hline $\mathrm{C}$ & 0.534 & 0.517 & 0.502 & 0.488 & 0.476 & 0.466 & 0.460 & 0.459 & 0.464 & 0.481 \\
\hline D & 0.541 & 0.524 & 0.508 & 0.494 & 0.482 & 0.472 & 0.466 & 0.465 & 0.471 & 0.489 \\
\hline $\mathrm{E}$ & 0.550 & 0.533 & 0.517 & 0.502 & 0.489 & 0.479 & 0.472 & 0.471 & 0.477 & 0.495 \\
\hline $\mathrm{F}$ & 0.563 & 0.545 & 0.529 & 0.513 & 0.500 & 0.490 & 0.483 & 0.482 & 0.488 & 0.508 \\
\hline G & 0.564 & 0.546 & 0.529 & 0.513 & 0.500 & 0.490 & 0.483 & 0.482 & 0.488 & 0.508 \\
\hline $\mathrm{H}$ & 0.550 & 0.533 & 0.516 & 0.502 & 0.489 & 0.479 & 0.472 & 0.471 & 0.477 & 0.495 \\
\hline $\mathrm{J}$ & 0.525 & 0.508 & 0.492 & 0.478 & 0.466 & 0.455 & 0.448 & 0.445 & 0.448 & 0.461 \\
\hline K & 0.522 & 0.506 & 0.491 & 0.477 & 0.464 & 0.454 & 0.446 & 0.443 & 0.446 & 0.459 \\
\hline $\mathrm{L}$ & 0.523 & 0.506 & 0.491 & 0.477 & 0.464 & 0.454 & 0.447 & 0.443 & 0.446 & 0.459 \\
\hline M & 0.524 & 0.508 & 0.492 & 0.478 & 0.465 & 0.455 & 0.448 & 0.445 & 0.447 & 0.461 \\
\hline $\mathrm{P}$ & 0.528 & 0.511 & 0.496 & 0.481 & 0.468 & 0.458 & 0.450 & 0.447 & 0.450 & 0.464 \\
\hline Q & 0.571 & 0.552 & 0.535 & 0.520 & 0.506 & 0.495 & 0.489 & 0.488 & 0.494 & 0.514 \\
\hline $\mathrm{R}$ & 0.528 & 0.512 & 0.496 & 0.481 & 0.469 & 0.458 & 0.451 & 0.447 & 0.450 & 0.464 \\
\hline $\mathrm{S}$ & 0.521 & 0.505 & 0.489 & 0.475 & 0.462 & 0.451 & 0.443 & 0.438 & 0.439 & 0.449 \\
\hline
\end{tabular}




\section{Charge-Pan II}

Table 7.13. Problem 6.1.1 reference solution Shift power peaking factors for axial segments in element 1 for charge-pan II.

\begin{tabular}{|c|c|c|c|c|c|c|c|c|c|c|}
\hline \multirow[t]{2}{*}{ Channels } & \multicolumn{10}{|c|}{ Power Peaking Factors } \\
\hline & 1 & 2 & 3 & 4 & 5 & 6 & 7 & 8 & 9 & 10 \\
\hline A & 0.801 & 0.781 & 0.794 & 0.807 & 0.830 & 0.871 & 0.894 & 0.920 & 0.959 & 0.994 \\
\hline B & 0.800 & 0.784 & 0.791 & 0.810 & 0.827 & 0.856 & 0.887 & 0.931 & 0.968 & 0.998 \\
\hline $\mathrm{C}$ & 0.803 & 0.787 & 0.788 & 0.805 & 0.823 & 0.858 & 0.898 & 0.924 & 0.956 & 0.996 \\
\hline D & 0.804 & 0.778 & 0.796 & 0.809 & 0.831 & 0.861 & 0.890 & 0.923 & 0.961 & 1.000 \\
\hline $\mathrm{E}$ & 0.805 & 0.784 & 0.798 & 0.808 & 0.845 & 0.873 & 0.898 & 0.935 & 0.968 & 1.005 \\
\hline $\mathrm{F}$ & 0.808 & 0.789 & 0.793 & 0.814 & 0.835 & 0.861 & 0.898 & 0.927 & 0.971 & 1.005 \\
\hline G & 0.805 & 0.789 & 0.797 & 0.810 & 0.844 & 0.869 & 0.901 & 0.933 & 0.962 & 0.999 \\
\hline $\mathrm{H}$ & 0.810 & 0.786 & 0.794 & 0.815 & 0.839 & 0.869 & 0.898 & 0.934 & 0.966 & 1.005 \\
\hline $\mathrm{J}$ & 0.807 & 0.783 & 0.795 & 0.816 & 0.848 & 0.871 & 0.894 & 0.924 & 0.970 & 1.004 \\
\hline K & 0.812 & 0.784 & 0.798 & 0.812 & 0.833 & 0.870 & 0.898 & 0.936 & 0.969 & 1.007 \\
\hline $\mathrm{L}$ & 0.810 & 0.790 & 0.789 & 0.820 & 0.829 & 0.862 & 0.896 & 0.928 & 0.968 & 1.000 \\
\hline M & 0.806 & 0.785 & 0.795 & 0.802 & 0.833 & 0.865 & 0.899 & 0.931 & 0.969 & 0.998 \\
\hline $\mathrm{P}$ & 0.807 & 0.787 & 0.798 & 0.813 & 0.835 & 0.867 & 0.900 & 0.932 & 0.964 & 1.005 \\
\hline Q & 0.806 & 0.792 & 0.792 & 0.811 & 0.843 & 0.875 & 0.903 & 0.935 & 0.969 & 1.002 \\
\hline $\mathrm{R}$ & 0.806 & 0.781 & 0.795 & 0.809 & 0.840 & 0.866 & 0.896 & 0.934 & 0.972 & 0.997 \\
\hline S & 0.815 & 0.786 & 0.801 & 0.819 & 0.839 & 0.867 & 0.898 & 0.926 & 0.965 & 1.000 \\
\hline Channels & 11 & 12 & 13 & 14 & 15 & 16 & 17 & 18 & 19 & 20 \\
\hline A & 1.026 & 1.065 & 1.104 & 1.138 & 1.185 & 1.229 & 1.267 & 1.310 & 1.364 & 1.446 \\
\hline B & 1.027 & 1.061 & 1.096 & 1.147 & 1.175 & 1.231 & 1.275 & 1.319 & 1.369 & 1.450 \\
\hline $\mathrm{C}$ & 1.027 & 1.067 & 1.106 & 1.137 & 1.183 & 1.226 & 1.267 & 1.308 & 1.360 & 1.462 \\
\hline D & 1.032 & 1.061 & 1.099 & 1.143 & 1.186 & 1.215 & 1.267 & 1.317 & 1.367 & 1.459 \\
\hline $\mathrm{E}$ & 1.045 & 1.076 & 1.121 & 1.159 & 1.191 & 1.231 & 1.275 & 1.336 & 1.374 & 1.466 \\
\hline $\mathrm{F}$ & 1.042 & 1.075 & 1.118 & 1.150 & 1.185 & 1.237 & 1.281 & 1.329 & 1.382 & 1.476 \\
\hline G & 1.030 & 1.078 & 1.108 & 1.152 & 1.198 & 1.236 & 1.277 & 1.327 & 1.381 & 1.472 \\
\hline $\mathrm{H}$ & 1.037 & 1.073 & 1.109 & 1.155 & 1.195 & 1.235 & 1.277 & 1.323 & 1.374 & 1.470 \\
\hline $\mathrm{J}$ & 1.045 & 1.073 & 1.110 & 1.151 & 1.187 & 1.237 & 1.280 & 1.320 & 1.372 & 1.473 \\
\hline K & 1.045 & 1.077 & 1.115 & 1.151 & 1.184 & 1.234 & 1.275 & 1.327 & 1.376 & 1.476 \\
\hline $\mathrm{L}$ & 1.046 & 1.076 & 1.112 & 1.145 & 1.188 & 1.234 & 1.284 & 1.329 & 1.387 & 1.469 \\
\hline $\mathrm{M}$ & 1.045 & 1.076 & 1.110 & 1.155 & 1.199 & 1.234 & 1.275 & 1.316 & 1.372 & 1.480 \\
\hline $\mathrm{P}$ & 1.039 & 1.080 & 1.110 & 1.159 & 1.186 & 1.239 & 1.284 & 1.325 & 1.373 & 1.477 \\
\hline Q & 1.042 & 1.081 & 1.122 & 1.154 & 1.197 & 1.242 & 1.283 & 1.332 & 1.365 & 1.473 \\
\hline $\mathrm{R}$ & 1.040 & 1.076 & 1.115 & 1.161 & 1.183 & 1.233 & 1.271 & 1.329 & 1.381 & 1.476 \\
\hline $\mathrm{S}$ & 1.043 & 1.078 & 1.112 & 1.160 & 1.200 & 1.238 & 1.278 & 1.325 & 1.375 & 1.477 \\
\hline
\end{tabular}


Table 7.14. Problem 6.1.1 reference solution MPACT power peaking factors for axial segments in element 1 for charge-pan II.

\begin{tabular}{|c|c|c|c|c|c|c|c|c|c|c|}
\hline \multirow[t]{2}{*}{ Channels } & \multicolumn{10}{|c|}{ Power Peaking Factors } \\
\hline & 1 & 2 & 3 & 4 & 5 & 6 & 7 & 8 & 9 & 10 \\
\hline A & 0.781 & 0.771 & 0.778 & 0.795 & 0.819 & 0.847 & 0.879 & 0.913 & 0.948 & 0.984 \\
\hline B & 0.781 & 0.770 & 0.778 & 0.795 & 0.818 & 0.847 & 0.879 & 0.912 & 0.948 & 0.983 \\
\hline $\mathrm{C}$ & 0.781 & 0.770 & 0.778 & 0.795 & 0.818 & 0.847 & 0.879 & 0.912 & 0.948 & 0.983 \\
\hline D & 0.781 & 0.770 & 0.778 & 0.795 & 0.818 & 0.847 & 0.879 & 0.912 & 0.948 & 0.984 \\
\hline $\mathrm{E}$ & 0.786 & 0.775 & 0.783 & 0.800 & 0.825 & 0.854 & 0.886 & 0.921 & 0.956 & 0.993 \\
\hline $\mathrm{F}$ & 0.786 & 0.775 & 0.783 & 0.801 & 0.825 & 0.854 & 0.887 & 0.921 & 0.957 & 0.993 \\
\hline G & 0.786 & 0.776 & 0.783 & 0.801 & 0.825 & 0.855 & 0.887 & 0.921 & 0.957 & 0.994 \\
\hline $\mathrm{H}$ & 0.786 & 0.775 & 0.783 & 0.801 & 0.825 & 0.854 & 0.887 & 0.921 & 0.957 & 0.993 \\
\hline $\mathrm{J}$ & 0.786 & 0.775 & 0.783 & 0.801 & 0.825 & 0.854 & 0.887 & 0.921 & 0.957 & 0.994 \\
\hline K & 0.786 & 0.775 & 0.783 & 0.801 & 0.825 & 0.854 & 0.887 & 0.921 & 0.957 & 0.993 \\
\hline $\mathrm{L}$ & 0.786 & 0.775 & 0.783 & 0.801 & 0.825 & 0.854 & 0.887 & 0.921 & 0.957 & 0.993 \\
\hline M & 0.786 & 0.775 & 0.783 & 0.800 & 0.825 & 0.854 & 0.886 & 0.921 & 0.956 & 0.993 \\
\hline $\mathrm{P}$ & 0.787 & 0.776 & 0.784 & 0.802 & 0.826 & 0.855 & 0.888 & 0.922 & 0.958 & 0.995 \\
\hline Q & 0.787 & 0.777 & 0.784 & 0.802 & 0.826 & 0.856 & 0.888 & 0.923 & 0.958 & 0.995 \\
\hline $\mathrm{R}$ & 0.786 & 0.776 & 0.784 & 0.802 & 0.826 & 0.855 & 0.888 & 0.922 & 0.958 & 0.995 \\
\hline $\mathrm{S}$ & 0.787 & 0.776 & 0.784 & 0.802 & 0.826 & 0.856 & 0.888 & 0.923 & 0.958 & 0.995 \\
\hline Channels & 11 & 12 & 13 & 14 & 15 & 16 & 17 & 18 & 19 & 20 \\
\hline A & 1.020 & 1.057 & 1.094 & 1.133 & 1.172 & 1.214 & 1.259 & 1.309 & 1.366 & 1.444 \\
\hline B & 1.020 & 1.057 & 1.094 & 1.132 & 1.172 & 1.214 & 1.259 & 1.309 & 1.366 & 1.444 \\
\hline $\mathrm{C}$ & 1.020 & 1.057 & 1.094 & 1.132 & 1.172 & 1.214 & 1.259 & 1.309 & 1.366 & 1.444 \\
\hline D & 1.020 & 1.057 & 1.094 & 1.133 & 1.172 & 1.214 & 1.259 & 1.309 & 1.366 & 1.444 \\
\hline $\mathrm{E}$ & 1.029 & 1.067 & 1.104 & 1.143 & 1.183 & 1.225 & 1.270 & 1.320 & 1.378 & 1.456 \\
\hline $\mathrm{F}$ & 1.030 & 1.067 & 1.105 & 1.144 & 1.184 & 1.226 & 1.271 & 1.322 & 1.380 & 1.460 \\
\hline G & 1.031 & 1.068 & 1.106 & 1.144 & 1.184 & 1.226 & 1.272 & 1.323 & 1.381 & 1.460 \\
\hline $\mathrm{H}$ & 1.030 & 1.068 & 1.105 & 1.144 & 1.184 & 1.226 & 1.272 & 1.322 & 1.381 & 1.460 \\
\hline $\mathrm{J}$ & 1.030 & 1.068 & 1.106 & 1.144 & 1.184 & 1.226 & 1.272 & 1.323 & 1.381 & 1.460 \\
\hline K & 1.030 & 1.067 & 1.105 & 1.144 & 1.184 & 1.226 & 1.271 & 1.322 & 1.380 & 1.459 \\
\hline $\mathrm{L}$ & 1.030 & 1.067 & 1.105 & 1.144 & 1.184 & 1.226 & 1.271 & 1.322 & 1.380 & 1.459 \\
\hline $\mathrm{M}$ & 1.030 & 1.067 & 1.105 & 1.143 & 1.183 & 1.225 & 1.271 & 1.321 & 1.380 & 1.459 \\
\hline $\mathrm{P}$ & 1.032 & 1.069 & 1.107 & 1.145 & 1.185 & 1.227 & 1.273 & 1.323 & 1.381 & 1.459 \\
\hline Q & 1.032 & 1.069 & 1.107 & 1.146 & 1.186 & 1.228 & 1.273 & 1.324 & 1.382 & 1.460 \\
\hline $\mathrm{R}$ & 1.032 & 1.069 & 1.107 & 1.146 & 1.186 & 1.228 & 1.273 & 1.324 & 1.383 & 1.462 \\
\hline $\mathrm{S}$ & 1.032 & 1.069 & 1.107 & 1.146 & 1.186 & 1.228 & 1.273 & 1.324 & 1.382 & 1.460 \\
\hline
\end{tabular}


Table 7.15. Problem 6.1.1 reference solution Shift power peaking factors for axial segments in element 2 for charge-pan II.

\begin{tabular}{|c|c|c|c|c|c|c|c|c|c|c|}
\hline \multirow[t]{2}{*}{ Channels } & \multicolumn{10}{|c|}{ Power Peaking Factors } \\
\hline & 1 & 2 & 3 & 4 & 5 & 6 & 7 & 8 & 9 & 10 \\
\hline A & 1.517 & 1.474 & 1.479 & 1.476 & 1.480 & 1.487 & 1.492 & 1.511 & 1.521 & 1.532 \\
\hline B & 1.509 & 1.474 & 1.471 & 1.473 & 1.482 & 1.485 & 1.491 & 1.510 & 1.519 & 1.523 \\
\hline $\mathrm{C}$ & 1.518 & 1.482 & 1.472 & 1.481 & 1.486 & 1.490 & 1.498 & 1.507 & 1.517 & 1.529 \\
\hline $\mathrm{D}$ & 1.521 & 1.473 & 1.473 & 1.487 & 1.485 & 1.491 & 1.485 & 1.512 & 1.526 & 1.527 \\
\hline $\mathrm{E}$ & 1.523 & 1.491 & 1.487 & 1.491 & 1.494 & 1.499 & 1.508 & 1.527 & 1.537 & 1.552 \\
\hline $\mathrm{F}$ & 1.537 & 1.484 & 1.484 & 1.486 & 1.495 & 1.501 & 1.525 & 1.530 & 1.534 & 1.546 \\
\hline G & 1.532 & 1.490 & 1.486 & 1.487 & 1.499 & 1.510 & 1.520 & 1.517 & 1.545 & 1.549 \\
\hline $\mathrm{H}$ & 1.533 & 1.496 & 1.498 & 1.496 & 1.491 & 1.503 & 1.514 & 1.514 & 1.528 & 1.553 \\
\hline J & 1.528 & 1.489 & 1.482 & 1.483 & 1.492 & 1.496 & 1.512 & 1.518 & 1.533 & 1.542 \\
\hline K & 1.534 & 1.489 & 1.487 & 1.492 & 1.497 & 1.507 & 1.509 & 1.519 & 1.530 & 1.549 \\
\hline $\mathrm{L}$ & 1.531 & 1.488 & 1.490 & 1.484 & 1.488 & 1.507 & 1.520 & 1.525 & 1.537 & 1.538 \\
\hline M & 1.527 & 1.480 & 1.496 & 1.492 & 1.498 & 1.501 & 1.516 & 1.523 & 1.542 & 1.553 \\
\hline$P$ & 1.535 & 1.492 & 1.488 & 1.494 & 1.497 & 1.509 & 1.513 & 1.523 & 1.542 & 1.556 \\
\hline Q & 1.537 & 1.495 & 1.488 & 1.490 & 1.504 & 1.508 & 1.507 & 1.526 & 1.536 & 1.550 \\
\hline $\mathrm{R}$ & 1.546 & 1.506 & 1.486 & 1.496 & 1.503 & 1.509 & 1.522 & 1.533 & 1.542 & 1.548 \\
\hline $\mathrm{S}$ & 1.544 & 1.489 & 1.486 & 1.496 & 1.505 & 1.507 & 1.515 & 1.532 & 1.537 & 1.561 \\
\hline Channels & 11 & 12 & 13 & $\overline{14}$ & $\overline{15}$ & 16 & 17 & 18 & 19 & 20 \\
\hline A & 1.539 & 1.554 & 1.565 & 1.583 & 1.583 & 1.614 & 1.626 & 1.643 & 1.667 & 1.751 \\
\hline B & 1.548 & 1.554 & 1.567 & 1.583 & 1.595 & 1.619 & 1.616 & 1.644 & 1.672 & 1.751 \\
\hline $\mathrm{C}$ & 1.535 & 1.547 & 1.555 & 1.573 & 1.600 & 1.622 & 1.627 & 1.661 & 1.683 & 1.753 \\
\hline D & 1.549 & 1.550 & 1.568 & 1.576 & 1.586 & 1.608 & 1.630 & 1.635 & 1.672 & 1.754 \\
\hline $\mathrm{E}$ & 1.558 & 1.573 & 1.581 & 1.603 & 1.611 & 1.626 & 1.649 & 1.666 & 1.697 & 1.777 \\
\hline $\mathrm{F}$ & 1.558 & 1.571 & 1.573 & 1.589 & 1.609 & 1.627 & 1.646 & 1.684 & 1.704 & 1.782 \\
\hline G & 1.555 & 1.564 & 1.591 & 1.595 & 1.615 & 1.620 & 1.643 & 1.667 & 1.695 & 1.766 \\
\hline $\mathrm{H}$ & 1.568 & 1.566 & 1.584 & 1.591 & 1.608 & 1.627 & 1.645 & 1.672 & 1.697 & 1.780 \\
\hline J & 1.556 & 1.562 & 1.572 & 1.590 & 1.608 & 1.630 & 1.651 & 1.660 & 1.690 & 1.782 \\
\hline K & 1.566 & 1.564 & 1.590 & 1.588 & 1.610 & 1.626 & 1.641 & 1.659 & 1.699 & 1.769 \\
\hline $\mathrm{L}$ & 1.556 & 1.573 & 1.582 & 1.591 & 1.604 & 1.627 & 1.645 & 1.655 & 1.682 & 1.765 \\
\hline M & 1.566 & 1.569 & 1.575 & 1.594 & 1.618 & 1.619 & 1.641 & 1.664 & 1.686 & 1.770 \\
\hline $\mathrm{P}$ & 1.561 & 1.571 & 1.588 & 1.590 & 1.606 & 1.624 & 1.654 & 1.678 & 1.701 & 1.776 \\
\hline Q & 1.558 & 1.581 & 1.590 & 1.592 & 1.613 & 1.627 & 1.644 & 1.675 & 1.706 & 1.785 \\
\hline $\mathrm{R}$ & 1.565 & 1.583 & 1.584 & 1.599 & 1.604 & 1.623 & 1.650 & 1.678 & 1.697 & 1.786 \\
\hline $\mathrm{S}$ & 1.566 & 1.564 & 1.585 & 1.594 & 1.613 & 1.628 & 1.648 & 1.660 & 1.697 & 1.770 \\
\hline
\end{tabular}


Table 7.16. Problem 6.1.1 reference solution MPACT power peaking factors for axial segments in element 2 for charge-pan II.

\begin{tabular}{|c|c|c|c|c|c|c|c|c|c|c|}
\hline \multirow[t]{2}{*}{ Channels } & \multicolumn{10}{|c|}{ Power Peaking Factors } \\
\hline & 1 & 2 & 3 & 4 & 5 & 6 & 7 & 8 & 9 & 10 \\
\hline A & 1.503 & 1.476 & 1.469 & 1.469 & 1.473 & 1.481 & 1.490 & 1.501 & 1.512 & 1.523 \\
\hline B & 1.503 & 1.476 & 1.469 & 1.469 & 1.473 & 1.481 & 1.490 & 1.501 & 1.512 & 1.524 \\
\hline $\mathrm{C}$ & 1.503 & 1.476 & 1.469 & 1.468 & 1.473 & 1.481 & 1.490 & 1.501 & 1.512 & 1.524 \\
\hline $\mathrm{D}$ & 1.503 & 1.476 & 1.468 & 1.468 & 1.473 & 1.481 & 1.490 & 1.501 & 1.512 & 1.523 \\
\hline $\mathrm{E}$ & 1.516 & 1.489 & 1.482 & 1.482 & 1.487 & 1.495 & 1.504 & 1.515 & 1.527 & 1.538 \\
\hline $\mathrm{F}$ & 1.520 & 1.492 & 1.484 & 1.483 & 1.488 & 1.496 & 1.506 & 1.517 & 1.528 & 1.539 \\
\hline G & 1.520 & 1.492 & 1.484 & 1.484 & 1.489 & 1.497 & 1.506 & 1.517 & 1.529 & 1.540 \\
\hline $\mathrm{H}$ & 1.520 & 1.492 & 1.484 & 1.484 & 1.489 & 1.496 & 1.506 & 1.517 & 1.529 & 1.540 \\
\hline J & 1.520 & 1.492 & 1.484 & 1.484 & 1.489 & 1.497 & 1.506 & 1.517 & 1.529 & 1.540 \\
\hline K & 1.519 & 1.491 & 1.483 & 1.483 & 1.488 & 1.496 & 1.506 & 1.517 & 1.528 & 1.540 \\
\hline $\mathrm{L}$ & 1.518 & 1.491 & 1.483 & 1.483 & 1.488 & 1.496 & 1.505 & 1.516 & 1.528 & 1.539 \\
\hline M & 1.519 & 1.491 & 1.483 & 1.483 & 1.487 & 1.495 & 1.505 & 1.516 & 1.527 & 1.538 \\
\hline$P$ & 1.519 & 1.492 & 1.485 & 1.485 & 1.489 & 1.497 & 1.507 & 1.518 & 1.529 & 1.541 \\
\hline Q & 1.520 & 1.493 & 1.486 & 1.486 & 1.491 & 1.498 & 1.508 & 1.519 & 1.531 & 1.543 \\
\hline $\mathrm{R}$ & 1.522 & 1.494 & 1.486 & 1.486 & 1.491 & 1.499 & 1.508 & 1.519 & 1.531 & 1.543 \\
\hline $\mathrm{S}$ & 1.521 & 1.493 & 1.486 & 1.486 & 1.491 & 1.499 & 1.508 & 1.519 & 1.531 & 1.542 \\
\hline Channels & 11 & 12 & 13 & $\overline{14}$ & $\overline{15}$ & 16 & 17 & 18 & 19 & 20 \\
\hline A & 1.535 & 1.546 & 1.558 & 1.571 & 1.585 & 1.601 & 1.621 & 1.647 & 1.681 & 1.739 \\
\hline B & 1.535 & 1.547 & 1.558 & 1.571 & 1.585 & 1.601 & 1.622 & 1.647 & 1.682 & 1.740 \\
\hline $\mathrm{C}$ & 1.535 & 1.546 & 1.558 & 1.571 & 1.585 & 1.601 & 1.621 & 1.647 & 1.682 & 1.740 \\
\hline D & 1.535 & 1.546 & 1.558 & 1.570 & 1.584 & 1.601 & 1.621 & 1.646 & 1.681 & 1.739 \\
\hline $\mathrm{E}$ & 1.550 & 1.561 & 1.573 & 1.586 & 1.600 & 1.616 & 1.636 & 1.662 & 1.697 & 1.756 \\
\hline $\mathrm{F}$ & 1.551 & 1.563 & 1.574 & 1.587 & 1.601 & 1.617 & 1.638 & 1.664 & 1.699 & 1.758 \\
\hline G & 1.552 & 1.563 & 1.575 & 1.588 & 1.602 & 1.619 & 1.639 & 1.666 & 1.701 & 1.761 \\
\hline $\mathrm{H}$ & 1.552 & 1.563 & 1.575 & 1.588 & 1.602 & 1.619 & 1.639 & 1.666 & 1.701 & 1.761 \\
\hline J & 1.552 & 1.564 & 1.575 & 1.588 & 1.602 & 1.619 & 1.639 & 1.666 & 1.701 & 1.761 \\
\hline K & 1.551 & 1.563 & 1.575 & 1.588 & 1.602 & 1.619 & 1.639 & 1.666 & 1.701 & 1.761 \\
\hline $\mathrm{L}$ & 1.551 & 1.562 & 1.574 & 1.587 & 1.601 & 1.617 & 1.638 & 1.664 & 1.699 & 1.759 \\
\hline M & 1.550 & 1.561 & 1.573 & 1.586 & 1.599 & 1.616 & 1.636 & 1.662 & 1.697 & 1.756 \\
\hline $\mathrm{P}$ & 1.552 & 1.564 & 1.576 & 1.588 & 1.602 & 1.618 & 1.638 & 1.664 & 1.698 & 1.756 \\
\hline Q & 1.554 & 1.566 & 1.578 & 1.590 & 1.605 & 1.621 & 1.642 & 1.668 & 1.704 & 1.764 \\
\hline $\mathrm{R}$ & 1.554 & 1.566 & 1.578 & 1.590 & 1.605 & 1.621 & 1.642 & 1.668 & 1.704 & 1.764 \\
\hline $\mathrm{S}$ & 1.554 & 1.566 & 1.578 & 1.590 & 1.604 & 1.621 & 1.641 & 1.668 & 1.703 & 1.763 \\
\hline
\end{tabular}


Table 7.17. Problem 6.1.1 reference solution Shift power peaking factors for axial segments in element 3 for charge-pan II.

\begin{tabular}{|c|c|c|c|c|c|c|c|c|c|c|}
\hline \multirow[t]{2}{*}{ Channels } & \multicolumn{10}{|c|}{ Power Peaking Factors } \\
\hline & 1 & 2 & 3 & 4 & 5 & 6 & 7 & 8 & 9 & 10 \\
\hline A & 1.739 & 1.663 & 1.628 & 1.589 & 1.568 & 1.551 & 1.523 & 1.506 & 1.482 & 1.452 \\
\hline B & 1.738 & 1.657 & 1.627 & 1.584 & 1.551 & 1.535 & 1.528 & 1.496 & 1.478 & 1.452 \\
\hline $\mathrm{C}$ & 1.746 & 1.663 & 1.623 & 1.591 & 1.567 & 1.543 & 1.521 & 1.492 & 1.473 & 1.446 \\
\hline D & 1.755 & 1.654 & 1.623 & 1.603 & 1.571 & 1.547 & 1.522 & 1.501 & 1.481 & 1.460 \\
\hline $\mathrm{E}$ & 1.767 & 1.675 & 1.638 & 1.605 & 1.592 & 1.574 & 1.541 & 1.524 & 1.505 & 1.485 \\
\hline $\mathrm{F}$ & 1.767 & 1.675 & 1.643 & 1.609 & 1.587 & 1.574 & 1.543 & 1.526 & 1.501 & 1.483 \\
\hline G & 1.766 & 1.693 & 1.641 & 1.609 & 1.588 & 1.558 & 1.532 & 1.527 & 1.505 & 1.477 \\
\hline $\mathrm{H}$ & 1.766 & 1.680 & 1.654 & 1.608 & 1.586 & 1.570 & 1.534 & 1.514 & 1.505 & 1.480 \\
\hline $\mathrm{J}$ & 1.758 & 1.669 & 1.634 & 1.609 & 1.585 & 1.559 & 1.539 & 1.511 & 1.498 & 1.463 \\
\hline K & 1.754 & 1.670 & 1.631 & 1.614 & 1.578 & 1.553 & 1.542 & 1.505 & 1.483 & 1.464 \\
\hline $\mathrm{L}$ & 1.755 & 1.666 & 1.637 & 1.603 & 1.586 & 1.562 & 1.532 & 1.511 & 1.487 & 1.460 \\
\hline M & 1.759 & 1.680 & 1.638 & 1.608 & 1.581 & 1.561 & 1.532 & 1.512 & 1.496 & 1.481 \\
\hline $\mathrm{P}$ & 1.766 & 1.669 & 1.636 & 1.623 & 1.593 & 1.562 & 1.551 & 1.535 & 1.499 & 1.485 \\
\hline Q & 1.761 & 1.688 & 1.651 & 1.619 & 1.590 & 1.565 & 1.550 & 1.527 & 1.508 & 1.489 \\
\hline $\mathrm{R}$ & 1.774 & 1.668 & 1.648 & 1.619 & 1.579 & 1.575 & 1.550 & 1.520 & 1.494 & 1.485 \\
\hline $\mathrm{S}$ & 1.762 & 1.675 & 1.637 & 1.607 & 1.575 & 1.561 & 1.525 & 1.520 & 1.487 & 1.470 \\
\hline Channels & 11 & 12 & 13 & 14 & 15 & 16 & 17 & 18 & 19 & 20 \\
\hline A & 1.447 & 1.421 & 1.388 & 1.386 & 1.350 & 1.323 & 1.315 & 1.294 & 1.261 & 1.272 \\
\hline B & 1.440 & 1.410 & 1.388 & 1.374 & 1.352 & 1.323 & 1.305 & 1.282 & 1.258 & 1.254 \\
\hline $\mathrm{C}$ & 1.432 & 1.408 & 1.379 & 1.358 & 1.341 & 1.312 & 1.293 & 1.274 & 1.244 & 1.238 \\
\hline D & 1.436 & 1.423 & 1.392 & 1.370 & 1.354 & 1.331 & 1.307 & 1.282 & 1.247 & 1.260 \\
\hline $\mathrm{E}$ & 1.465 & 1.447 & 1.422 & 1.398 & 1.389 & 1.378 & 1.357 & 1.345 & 1.333 & 1.357 \\
\hline $\mathrm{F}$ & 1.457 & 1.448 & 1.433 & 1.416 & 1.382 & 1.381 & 1.356 & 1.343 & 1.328 & 1.366 \\
\hline G & 1.460 & 1.453 & 1.425 & 1.409 & 1.384 & 1.368 & 1.353 & 1.350 & 1.343 & 1.366 \\
\hline $\mathrm{H}$ & 1.462 & 1.437 & 1.424 & 1.403 & 1.381 & 1.364 & 1.359 & 1.338 & 1.327 & 1.351 \\
\hline $\mathrm{J}$ & 1.452 & 1.423 & 1.413 & 1.388 & 1.373 & 1.350 & 1.335 & 1.320 & 1.311 & 1.338 \\
\hline $\mathrm{K}$ & 1.438 & 1.422 & 1.407 & 1.381 & 1.360 & 1.337 & 1.325 & 1.304 & 1.288 & 1.316 \\
\hline $\mathrm{L}$ & 1.446 & 1.418 & 1.405 & 1.383 & 1.357 & 1.334 & 1.328 & 1.302 & 1.301 & 1.312 \\
\hline M & 1.453 & 1.423 & 1.403 & 1.393 & 1.370 & 1.344 & 1.326 & 1.317 & 1.303 & 1.333 \\
\hline $\mathrm{P}$ & 1.467 & 1.443 & 1.424 & 1.408 & 1.393 & 1.373 & 1.357 & 1.347 & 1.339 & 1.379 \\
\hline Q & 1.465 & 1.452 & 1.439 & 1.422 & 1.393 & 1.383 & 1.366 & 1.358 & 1.361 & 1.382 \\
\hline $\mathrm{R}$ & 1.471 & 1.445 & 1.432 & 1.414 & 1.394 & 1.374 & 1.358 & 1.358 & 1.343 & 1.382 \\
\hline $\mathrm{S}$ & 1.441 & 1.425 & 1.396 & 1.393 & 1.355 & 1.340 & 1.324 & 1.309 & 1.298 & 1.328 \\
\hline
\end{tabular}


Table 7.18. Problem 6.1.1 reference solution MPACT power peaking factors for axial segments in element 3 for charge-pan II.

\begin{tabular}{|c|c|c|c|c|c|c|c|c|c|c|}
\hline \multirow[t]{2}{*}{ Channels } & \multicolumn{10}{|c|}{ Power Peaking Factors } \\
\hline & 1 & 2 & 3 & 4 & 5 & 6 & 7 & 8 & 9 & 10 \\
\hline A & 1.730 & 1.665 & 1.623 & 1.590 & 1.562 & 1.538 & 1.517 & 1.496 & 1.476 & 1.457 \\
\hline B & 1.731 & 1.666 & 1.624 & 1.591 & 1.564 & 1.540 & 1.519 & 1.499 & 1.479 & 1.460 \\
\hline $\mathrm{C}$ & 1.731 & 1.666 & 1.624 & 1.590 & 1.563 & 1.539 & 1.517 & 1.497 & 1.477 & 1.457 \\
\hline D & 1.730 & 1.664 & 1.622 & 1.588 & 1.560 & 1.536 & 1.514 & 1.493 & 1.472 & 1.452 \\
\hline $\mathrm{E}$ & 1.747 & 1.680 & 1.637 & 1.603 & 1.574 & 1.550 & 1.528 & 1.507 & 1.486 & 1.466 \\
\hline $\mathrm{F}$ & 1.749 & 1.682 & 1.639 & 1.606 & 1.578 & 1.554 & 1.532 & 1.511 & 1.491 & 1.471 \\
\hline G & 1.753 & 1.686 & 1.643 & 1.610 & 1.583 & 1.559 & 1.538 & 1.518 & 1.499 & 1.480 \\
\hline $\mathrm{H}$ & 1.753 & 1.686 & 1.644 & 1.610 & 1.583 & 1.560 & 1.538 & 1.519 & 1.500 & 1.481 \\
\hline $\mathrm{J}$ & 1.753 & 1.686 & 1.644 & 1.611 & 1.583 & 1.560 & 1.539 & 1.519 & 1.500 & 1.482 \\
\hline K & 1.753 & 1.686 & 1.643 & 1.610 & 1.583 & 1.559 & 1.538 & 1.518 & 1.499 & 1.481 \\
\hline $\mathrm{L}$ & 1.750 & 1.683 & 1.640 & 1.606 & 1.578 & 1.554 & 1.532 & 1.511 & 1.491 & 1.471 \\
\hline M & 1.747 & 1.680 & 1.636 & 1.602 & 1.574 & 1.550 & 1.527 & 1.506 & 1.486 & 1.466 \\
\hline $\mathrm{P}$ & 1.747 & 1.680 & 1.638 & 1.604 & 1.575 & 1.551 & 1.528 & 1.507 & 1.487 & 1.466 \\
\hline Q & 1.755 & 1.688 & 1.646 & 1.612 & 1.585 & 1.561 & 1.540 & 1.521 & 1.502 & 1.483 \\
\hline $\mathrm{R}$ & 1.756 & 1.689 & 1.647 & 1.614 & 1.586 & 1.563 & 1.542 & 1.523 & 1.505 & 1.486 \\
\hline $\mathrm{S}$ & 1.755 & 1.688 & 1.645 & 1.612 & 1.585 & 1.561 & 1.540 & 1.520 & 1.502 & 1.483 \\
\hline Channels & 11 & 12 & 13 & 14 & 15 & 16 & 17 & 18 & 19 & 20 \\
\hline A & 1.436 & 1.416 & 1.394 & 1.373 & 1.350 & 1.328 & 1.305 & 1.284 & 1.264 & 1.254 \\
\hline B & 1.440 & 1.420 & 1.399 & 1.378 & 1.356 & 1.334 & 1.313 & 1.292 & 1.272 & 1.264 \\
\hline $\mathrm{C}$ & 1.437 & 1.416 & 1.395 & 1.373 & 1.351 & 1.328 & 1.306 & 1.284 & 1.264 & 1.255 \\
\hline D & 1.431 & 1.410 & 1.388 & 1.365 & 1.342 & 1.319 & 1.296 & 1.273 & 1.252 & 1.241 \\
\hline $\mathrm{E}$ & 1.445 & 1.424 & 1.402 & 1.381 & 1.360 & 1.340 & 1.322 & 1.308 & 1.300 & 1.308 \\
\hline $\mathrm{F}$ & 1.451 & 1.431 & 1.410 & 1.390 & 1.369 & 1.350 & 1.334 & 1.320 & 1.313 & 1.323 \\
\hline G & 1.462 & 1.443 & 1.423 & 1.405 & 1.386 & 1.370 & 1.355 & 1.345 & 1.341 & 1.355 \\
\hline $\mathrm{H}$ & 1.463 & 1.444 & 1.425 & 1.406 & 1.388 & 1.372 & 1.358 & 1.348 & 1.344 & 1.359 \\
\hline $\mathrm{J}$ & 1.463 & 1.444 & 1.425 & 1.406 & 1.388 & 1.372 & 1.358 & 1.348 & 1.344 & 1.358 \\
\hline $\mathrm{K}$ & 1.462 & 1.443 & 1.424 & 1.405 & 1.386 & 1.370 & 1.355 & 1.345 & 1.341 & 1.354 \\
\hline $\mathrm{L}$ & 1.451 & 1.431 & 1.410 & 1.390 & 1.370 & 1.351 & 1.334 & 1.321 & 1.314 & 1.325 \\
\hline $\mathrm{M}$ & 1.445 & 1.424 & 1.402 & 1.381 & 1.360 & 1.340 & 1.322 & 1.308 & 1.300 & 1.309 \\
\hline $\mathrm{P}$ & 1.445 & 1.424 & 1.403 & 1.382 & 1.361 & 1.342 & 1.325 & 1.313 & 1.307 & 1.318 \\
\hline Q & 1.464 & 1.445 & 1.427 & 1.408 & 1.390 & 1.375 & 1.362 & 1.353 & 1.351 & 1.368 \\
\hline $\mathrm{R}$ & 1.468 & 1.450 & 1.432 & 1.414 & 1.397 & 1.382 & 1.370 & 1.363 & 1.362 & 1.380 \\
\hline $\mathrm{S}$ & 1.464 & 1.445 & 1.427 & 1.408 & 1.391 & 1.375 & 1.362 & 1.353 & 1.352 & 1.368 \\
\hline
\end{tabular}


Table 7.19. Problem 6.1.1 reference solution Shift power peaking factors for axial segments in element 4 for charge-pan II.

\begin{tabular}{|c|c|c|c|c|c|c|c|c|c|c|}
\hline \multirow[t]{2}{*}{ Channels } & \multicolumn{10}{|c|}{ Power Peaking Factors } \\
\hline & 1 & 2 & 3 & 4 & 5 & 6 & 7 & 8 & 9 & 10 \\
\hline A & 1.137 & 1.045 & 0.989 & 0.940 & 0.899 & 0.862 & 0.834 & 0.811 & 0.792 & 0.764 \\
\hline B & 1.133 & 1.029 & 0.977 & 0.930 & 0.890 & 0.850 & 0.833 & 0.796 & 0.771 & 0.751 \\
\hline $\mathrm{C}$ & 1.113 & 1.021 & 0.958 & 0.901 & 0.866 & 0.833 & 0.803 & 0.778 & 0.753 & 0.730 \\
\hline $\mathrm{D}$ & 1.129 & 1.031 & 0.974 & 0.924 & 0.890 & 0.851 & 0.829 & 0.796 & 0.772 & 0.745 \\
\hline $\mathrm{E}$ & 1.282 & 1.183 & 1.142 & 1.097 & 1.057 & 1.019 & 0.989 & 0.960 & 0.939 & 0.904 \\
\hline $\mathrm{F}$ & 1.288 & 1.193 & 1.157 & 1.106 & 1.071 & 1.031 & 1.004 & 0.972 & 0.944 & 0.912 \\
\hline G & 1.294 & 1.204 & 1.147 & 1.112 & 1.066 & 1.029 & 1.003 & 0.963 & 0.939 & 0.921 \\
\hline $\mathrm{H}$ & 1.287 & 1.190 & 1.151 & 1.105 & 1.057 & 1.022 & 0.999 & 0.959 & 0.935 & 0.907 \\
\hline $\mathrm{J}$ & 1.255 & 1.155 & 1.107 & 1.059 & 1.019 & 0.986 & 0.947 & 0.920 & 0.892 & 0.866 \\
\hline $\mathrm{K}$ & 1.231 & 1.142 & 1.085 & 1.030 & 0.996 & 0.960 & 0.926 & 0.900 & 0.870 & 0.840 \\
\hline $\mathrm{L}$ & 1.232 & 1.131 & 1.074 & 1.029 & 1.000 & 0.962 & 0.931 & 0.902 & 0.875 & 0.843 \\
\hline $\mathrm{M}$ & 1.245 & 1.157 & 1.104 & 1.058 & 1.022 & 0.979 & 0.953 & 0.914 & 0.888 & 0.862 \\
\hline $\mathrm{P}$ & 1.305 & 1.211 & 1.163 & 1.113 & 1.082 & 1.045 & 1.015 & 0.982 & 0.954 & 0.935 \\
\hline Q & 1.320 & 1.235 & 1.191 & 1.137 & 1.100 & 1.067 & 1.035 & 1.001 & 0.977 & 0.950 \\
\hline $\mathrm{R}$ & 1.296 & 1.211 & 1.163 & 1.127 & 1.088 & 1.056 & 1.018 & 0.991 & 0.960 & 0.937 \\
\hline S & 1.249 & 1.157 & 1.107 & 1.053 & 1.017 & 0.986 & 0.956 & 0.917 & 0.893 & 0.860 \\
\hline "Channels & 11 & 12 & 13 & 14 & $\overline{15}$ & 16 & 17 & 18 & 19 & 20 \\
\hline A & 0.746 & 0.721 & 0.707 & 0.694 & 0.679 & 0.666 & 0.647 & 0.638 & 0.633 & 0.645 \\
\hline B & 0.728 & 0.709 & 0.684 & 0.679 & 0.656 & 0.644 & 0.629 & 0.620 & 0.610 & 0.623 \\
\hline $\mathrm{C}$ & 0.705 & 0.687 & 0.678 & 0.653 & 0.637 & 0.625 & 0.612 & 0.603 & 0.598 & 0.608 \\
\hline D & 0.731 & 0.710 & 0.693 & 0.675 & 0.658 & 0.646 & 0.629 & 0.619 & 0.620 & 0.633 \\
\hline $\mathrm{E}$ & 0.880 & 0.863 & 0.840 & 0.821 & 0.803 & 0.792 & 0.770 & 0.763 & 0.748 & 0.765 \\
\hline $\mathrm{F}$ & 0.890 & 0.871 & 0.851 & 0.822 & 0.808 & 0.793 & 0.780 & 0.768 & 0.761 & 0.773 \\
\hline G & 0.886 & 0.865 & 0.844 & 0.821 & 0.810 & 0.797 & 0.776 & 0.766 & 0.751 & 0.767 \\
\hline $\mathrm{H}$ & 0.882 & 0.863 & 0.844 & 0.824 & 0.804 & 0.787 & 0.771 & 0.766 & 0.751 & 0.769 \\
\hline $\mathrm{J}$ & 0.845 & 0.820 & 0.799 & 0.778 & 0.758 & 0.742 & 0.734 & 0.719 & 0.704 & 0.726 \\
\hline K & 0.819 & 0.796 & 0.780 & 0.756 & 0.737 & 0.725 & 0.702 & 0.695 & 0.689 & 0.690 \\
\hline $\mathrm{L}$ & 0.816 & 0.792 & 0.775 & 0.749 & 0.735 & 0.721 & 0.708 & 0.700 & 0.684 & 0.696 \\
\hline M & 0.836 & 0.817 & 0.791 & 0.777 & 0.757 & 0.742 & 0.728 & 0.715 & 0.710 & 0.725 \\
\hline $\mathrm{P}$ & 0.897 & 0.878 & 0.865 & 0.839 & 0.825 & 0.804 & 0.794 & 0.776 & 0.766 & 0.782 \\
\hline Q & 0.929 & 0.904 & 0.877 & 0.860 & 0.830 & 0.817 & 0.805 & 0.795 & 0.785 & 0.798 \\
\hline $\mathrm{R}$ & 0.904 & 0.880 & 0.866 & 0.844 & 0.825 & 0.807 & 0.785 & 0.769 & 0.770 & 0.788 \\
\hline $\mathrm{S}$ & 0.838 & 0.811 & 0.789 & 0.767 & 0.757 & 0.731 & 0.723 & 0.709 & 0.706 & 0.708 \\
\hline
\end{tabular}


Table 7.20. Problem 6.1.1 reference solution MPACT power peaking factors for axial segments in element 4 for charge-pan II.

\begin{tabular}{|c|c|c|c|c|c|c|c|c|c|c|}
\hline \multirow[t]{2}{*}{ Channels } & \multicolumn{10}{|c|}{ Power Peaking Factors } \\
\hline & 1 & 2 & 3 & 4 & 5 & 6 & 7 & 8 & 9 & 10 \\
\hline A & 1.126 & 1.040 & 0.979 & 0.930 & 0.890 & 0.856 & 0.826 & 0.799 & 0.775 & 0.753 \\
\hline B & 1.137 & 1.052 & 0.991 & 0.943 & 0.903 & 0.869 & 0.839 & 0.813 & 0.789 & 0.767 \\
\hline $\mathrm{C}$ & 1.127 & 1.041 & 0.980 & 0.931 & 0.890 & 0.856 & 0.826 & 0.799 & 0.775 & 0.753 \\
\hline $\mathrm{D}$ & 1.111 & 1.025 & 0.963 & 0.913 & 0.872 & 0.838 & 0.807 & 0.780 & 0.756 & 0.734 \\
\hline $\mathrm{E}$ & 1.226 & 1.149 & 1.091 & 1.042 & 1.000 & 0.963 & 0.929 & 0.899 & 0.872 & 0.846 \\
\hline $\mathrm{F}$ & 1.243 & 1.167 & 1.110 & 1.061 & 1.020 & 0.983 & 0.950 & 0.920 & 0.893 & 0.868 \\
\hline G & 1.281 & 1.204 & 1.148 & 1.101 & 1.060 & 1.024 & 0.992 & 0.963 & 0.936 & 0.911 \\
\hline $\mathrm{H}$ & 1.285 & 1.209 & 1.152 & 1.105 & 1.065 & 1.029 & 0.997 & 0.968 & 0.941 & 0.916 \\
\hline $\mathrm{J}$ & 1.284 & 1.208 & 1.152 & 1.106 & 1.065 & 1.029 & 0.997 & 0.968 & 0.942 & 0.917 \\
\hline $\mathrm{K}$ & 1.280 & 1.204 & 1.148 & 1.101 & 1.061 & 1.025 & 0.993 & 0.964 & 0.937 & 0.912 \\
\hline $\mathrm{L}$ & 1.245 & 1.168 & 1.110 & 1.062 & 1.020 & 0.984 & 0.951 & 0.921 & 0.894 & 0.868 \\
\hline $\mathrm{M}$ & 1.227 & 1.149 & 1.091 & 1.043 & 1.000 & 0.963 & 0.930 & 0.900 & 0.872 & 0.847 \\
\hline $\mathrm{P}$ & 1.241 & 1.166 & 1.110 & 1.061 & 1.020 & 0.982 & 0.949 & 0.918 & 0.890 & 0.864 \\
\hline Q & 1.299 & 1.224 & 1.169 & 1.123 & 1.083 & 1.047 & 1.014 & 0.985 & 0.957 & 0.932 \\
\hline $\mathrm{R}$ & 1.314 & 1.240 & 1.185 & 1.139 & 1.100 & 1.064 & 1.032 & 1.003 & 0.976 & 0.950 \\
\hline S & 1.299 & 1.225 & 1.170 & 1.124 & 1.083 & 1.048 & 1.015 & 0.986 & 0.959 & 0.933 \\
\hline Channels & 11 & 12 & 13 & 14 & 15 & 16 & 17 & 18 & 19 & 20 \\
\hline A & 0.732 & 0.713 & 0.695 & 0.678 & 0.663 & 0.649 & 0.638 & 0.630 & 0.625 & 0.630 \\
\hline B & 0.746 & 0.727 & 0.709 & 0.692 & 0.677 & 0.663 & 0.652 & 0.644 & 0.640 & 0.645 \\
\hline $\mathrm{C}$ & 0.733 & 0.713 & 0.695 & 0.679 & 0.663 & 0.650 & 0.639 & 0.631 & 0.626 & 0.631 \\
\hline D & 0.713 & 0.694 & 0.676 & 0.659 & 0.644 & 0.630 & 0.619 & 0.611 & 0.606 & 0.610 \\
\hline $\mathrm{E}$ & 0.822 & 0.800 & 0.779 & 0.760 & 0.742 & 0.726 & 0.713 & 0.704 & 0.699 & 0.704 \\
\hline $\mathrm{F}$ & 0.844 & 0.822 & 0.801 & 0.782 & 0.764 & 0.748 & 0.735 & 0.726 & 0.720 & 0.726 \\
\hline G & 0.887 & 0.865 & 0.844 & 0.824 & 0.806 & 0.791 & 0.777 & 0.768 & 0.763 & 0.769 \\
\hline $\mathrm{H}$ & 0.892 & 0.870 & 0.849 & 0.830 & 0.812 & 0.796 & 0.783 & 0.773 & 0.768 & 0.774 \\
\hline $\mathrm{J}$ & 0.893 & 0.871 & 0.850 & 0.830 & 0.813 & 0.797 & 0.784 & 0.775 & 0.770 & 0.776 \\
\hline K & 0.888 & 0.866 & 0.845 & 0.826 & 0.808 & 0.792 & 0.779 & 0.770 & 0.765 & 0.771 \\
\hline $\mathrm{L}$ & 0.845 & 0.822 & 0.802 & 0.782 & 0.765 & 0.749 & 0.736 & 0.727 & 0.722 & 0.727 \\
\hline M & 0.823 & 0.800 & 0.780 & 0.760 & 0.743 & 0.727 & 0.714 & 0.705 & 0.700 & 0.705 \\
\hline $\mathrm{P}$ & 0.839 & 0.817 & 0.795 & 0.775 & 0.757 & 0.741 & 0.728 & 0.718 & 0.713 & 0.718 \\
\hline Q & 0.908 & 0.885 & 0.863 & 0.843 & 0.825 & 0.809 & 0.795 & 0.785 & 0.780 & 0.786 \\
\hline $\mathrm{R}$ & 0.926 & 0.903 & 0.882 & 0.862 & 0.844 & 0.828 & 0.814 & 0.805 & 0.800 & 0.807 \\
\hline $\mathrm{S}$ & 0.909 & 0.886 & 0.865 & 0.845 & 0.827 & 0.811 & 0.797 & 0.788 & 0.783 & 0.789 \\
\hline
\end{tabular}


Table 7.21. Problem 6.1.1 reference solution Shift power peaking factors for axial segments in element 5 for charge-pan II.

\begin{tabular}{|c|c|c|c|c|c|c|c|c|c|c|}
\hline \multirow[t]{2}{*}{ Channels } & \multicolumn{10}{|c|}{ Power Peaking Factors } \\
\hline & 1 & 2 & 3 & 4 & 5 & 6 & 7 & 8 & 9 & 10 \\
\hline A & 0.607 & 0.560 & 0.534 & 0.511 & 0.489 & 0.473 & 0.457 & 0.439 & 0.429 & 0.416 \\
\hline B & 0.591 & 0.545 & 0.519 & 0.498 & 0.480 & 0.459 & 0.444 & 0.431 & 0.414 & 0.405 \\
\hline $\mathrm{C}$ & 0.567 & 0.529 & 0.502 & 0.485 & 0.462 & 0.449 & 0.429 & 0.418 & 0.404 & 0.388 \\
\hline $\mathrm{D}$ & 0.589 & 0.549 & 0.520 & 0.498 & 0.480 & 0.459 & 0.443 & 0.433 & 0.417 & 0.401 \\
\hline $\mathrm{E}$ & 0.723 & 0.671 & 0.641 & 0.617 & 0.590 & 0.566 & 0.546 & 0.531 & 0.509 & 0.494 \\
\hline $\mathrm{F}$ & 0.722 & 0.676 & 0.642 & 0.620 & 0.590 & 0.572 & 0.560 & 0.533 & 0.513 & 0.496 \\
\hline G & 0.729 & 0.672 & 0.642 & 0.615 & 0.593 & 0.569 & 0.551 & 0.533 & 0.517 & 0.498 \\
\hline $\mathrm{H}$ & 0.723 & 0.670 & 0.639 & 0.614 & 0.590 & 0.568 & 0.545 & 0.528 & 0.506 & 0.491 \\
\hline $\mathrm{J}$ & 0.679 & 0.621 & 0.607 & 0.585 & 0.554 & 0.536 & 0.511 & 0.495 & 0.479 & 0.463 \\
\hline K & 0.647 & 0.606 & 0.587 & 0.557 & 0.535 & 0.508 & 0.496 & 0.480 & 0.464 & 0.451 \\
\hline $\mathrm{L}$ & 0.659 & 0.610 & 0.577 & 0.560 & 0.536 & 0.515 & 0.500 & 0.482 & 0.464 & 0.452 \\
\hline $\mathrm{M}$ & 0.676 & 0.627 & 0.603 & 0.573 & 0.549 & 0.531 & 0.522 & 0.494 & 0.482 & 0.462 \\
\hline $\mathrm{P}$ & 0.740 & 0.684 & 0.657 & 0.629 & 0.600 & 0.581 & 0.560 & 0.544 & 0.527 & 0.504 \\
\hline Q & 0.756 & 0.701 & 0.670 & 0.636 & 0.616 & 0.597 & 0.572 & 0.549 & 0.531 & 0.510 \\
\hline $\mathrm{R}$ & 0.741 & 0.685 & 0.651 & 0.625 & 0.605 & 0.573 & 0.562 & 0.545 & 0.516 & 0.499 \\
\hline S & 0.671 & 0.619 & 0.592 & 0.572 & 0.547 & 0.524 & 0.511 & 0.490 & 0.474 & 0.455 \\
\hline Channels & 11 & 12 & 13 & 14 & 15 & 16 & 17 & 18 & 19 & 20 \\
\hline A & 0.397 & 0.387 & 0.372 & 0.360 & 0.353 & 0.340 & 0.331 & 0.326 & 0.321 & 0.321 \\
\hline B & 0.390 & 0.378 & 0.367 & 0.353 & 0.340 & 0.326 & 0.328 & 0.315 & 0.310 & 0.311 \\
\hline $\mathrm{C}$ & 0.376 & 0.361 & 0.352 & 0.343 & 0.331 & 0.318 & 0.312 & 0.306 & 0.299 & 0.301 \\
\hline D & 0.395 & 0.380 & 0.367 & 0.355 & 0.342 & 0.332 & 0.325 & 0.316 & 0.313 & 0.318 \\
\hline $\mathrm{E}$ & 0.480 & 0.463 & 0.450 & 0.441 & 0.428 & 0.415 & 0.407 & 0.391 & 0.387 & 0.387 \\
\hline $\mathrm{F}$ & 0.487 & 0.474 & 0.456 & 0.437 & 0.427 & 0.411 & 0.402 & 0.394 & 0.388 & 0.391 \\
\hline G & 0.479 & 0.465 & 0.451 & 0.435 & 0.426 & 0.414 & 0.401 & 0.396 & 0.391 & 0.394 \\
\hline $\mathrm{H}$ & 0.472 & 0.457 & 0.448 & 0.431 & 0.418 & 0.414 & 0.401 & 0.393 & 0.387 & 0.393 \\
\hline $\mathrm{J}$ & 0.447 & 0.432 & 0.423 & 0.405 & 0.396 & 0.384 & 0.374 & 0.367 & 0.354 & 0.360 \\
\hline K & 0.437 & 0.421 & 0.408 & 0.396 & 0.379 & 0.366 & 0.362 & 0.353 & 0.342 & 0.345 \\
\hline $\mathrm{L}$ & 0.435 & 0.419 & 0.403 & 0.395 & 0.383 & 0.370 & 0.363 & 0.354 & 0.347 & 0.344 \\
\hline M & 0.448 & 0.435 & 0.420 & 0.406 & 0.401 & 0.388 & 0.374 & 0.368 & 0.358 & 0.363 \\
\hline $\mathrm{P}$ & 0.489 & 0.476 & 0.460 & 0.451 & 0.433 & 0.425 & 0.413 & 0.402 & 0.390 & 0.398 \\
\hline Q & 0.497 & 0.484 & 0.468 & 0.457 & 0.440 & 0.431 & 0.424 & 0.413 & 0.408 & 0.413 \\
\hline $\mathrm{R}$ & 0.486 & 0.475 & 0.457 & 0.447 & 0.436 & 0.411 & 0.404 & 0.397 & 0.399 & 0.403 \\
\hline $\mathrm{S}$ & 0.441 & 0.425 & 0.415 & 0.402 & 0.389 & 0.379 & 0.365 & 0.356 & 0.349 & 0.354 \\
\hline
\end{tabular}


Table 7.22. Problem 6.1.1 reference solution MPACT power peaking factors for axial segments in element 5 for charge-pan II.

\begin{tabular}{|c|c|c|c|c|c|c|c|c|c|c|}
\hline \multirow[t]{2}{*}{ Channels } & \multicolumn{10}{|c|}{ Power Peaking Factors } \\
\hline & 1 & 2 & 3 & 4 & 5 & 6 & 7 & 8 & 9 & 10 \\
\hline A & 0.593 & 0.558 & 0.531 & 0.508 & 0.488 & 0.470 & 0.454 & 0.439 & 0.424 & 0.411 \\
\hline B & 0.608 & 0.571 & 0.544 & 0.521 & 0.501 & 0.482 & 0.466 & 0.450 & 0.436 & 0.422 \\
\hline $\mathrm{C}$ & 0.595 & 0.559 & 0.532 & 0.510 & 0.490 & 0.472 & 0.456 & 0.441 & 0.427 & 0.413 \\
\hline $\mathrm{D}$ & 0.575 & 0.540 & 0.514 & 0.492 & 0.473 & 0.456 & 0.440 & 0.425 & 0.411 & 0.398 \\
\hline $\mathrm{E}$ & 0.662 & 0.622 & 0.592 & 0.567 & 0.544 & 0.524 & 0.506 & 0.489 & 0.473 & 0.458 \\
\hline $\mathrm{F}$ & 0.683 & 0.642 & 0.611 & 0.585 & 0.562 & 0.541 & 0.522 & 0.505 & 0.488 & 0.472 \\
\hline G & 0.724 & 0.681 & 0.648 & 0.621 & 0.596 & 0.574 & 0.554 & 0.535 & 0.518 & 0.501 \\
\hline $\mathrm{H}$ & 0.730 & 0.686 & 0.654 & 0.626 & 0.601 & 0.579 & 0.559 & 0.540 & 0.523 & 0.506 \\
\hline $\mathrm{J}$ & 0.732 & 0.689 & 0.656 & 0.628 & 0.604 & 0.582 & 0.562 & 0.543 & 0.526 & 0.509 \\
\hline K & 0.728 & 0.684 & 0.652 & 0.624 & 0.600 & 0.578 & 0.559 & 0.540 & 0.523 & 0.507 \\
\hline $\mathrm{L}$ & 0.685 & 0.644 & 0.614 & 0.588 & 0.565 & 0.544 & 0.525 & 0.508 & 0.492 & 0.476 \\
\hline $\mathrm{M}$ & 0.664 & 0.623 & 0.593 & 0.568 & 0.546 & 0.526 & 0.507 & 0.490 & 0.475 & 0.459 \\
\hline $\mathrm{P}$ & 0.675 & 0.634 & 0.604 & 0.578 & 0.555 & 0.535 & 0.516 & 0.499 & 0.482 & 0.467 \\
\hline Q & 0.740 & 0.696 & 0.663 & 0.634 & 0.609 & 0.587 & 0.566 & 0.547 & 0.529 & 0.512 \\
\hline $\mathrm{R}$ & 0.761 & 0.715 & 0.681 & 0.652 & 0.627 & 0.604 & 0.583 & 0.563 & 0.545 & 0.528 \\
\hline S & 0.744 & 0.700 & 0.667 & 0.639 & 0.614 & 0.592 & 0.572 & 0.553 & 0.536 & 0.519 \\
\hline Channels & 11 & 12 & 13 & 14 & 15 & 16 & 17 & 18 & 19 & 20 \\
\hline A & 0.398 & 0.385 & 0.373 & 0.362 & 0.351 & 0.341 & 0.333 & 0.325 & 0.320 & 0.320 \\
\hline B & 0.408 & 0.396 & 0.383 & 0.372 & 0.361 & 0.351 & 0.342 & 0.335 & 0.330 & 0.330 \\
\hline $\mathrm{C}$ & 0.400 & 0.388 & 0.376 & 0.365 & 0.354 & 0.345 & 0.336 & 0.329 & 0.323 & 0.323 \\
\hline D & 0.386 & 0.374 & 0.362 & 0.351 & 0.341 & 0.331 & 0.323 & 0.316 & 0.310 & 0.309 \\
\hline $\mathrm{E}$ & 0.443 & 0.430 & 0.416 & 0.404 & 0.392 & 0.381 & 0.371 & 0.363 & 0.357 & 0.356 \\
\hline $\mathrm{F}$ & 0.457 & 0.443 & 0.429 & 0.416 & 0.403 & 0.392 & 0.382 & 0.374 & 0.368 & 0.367 \\
\hline G & 0.485 & 0.469 & 0.455 & 0.441 & 0.429 & 0.417 & 0.408 & 0.401 & 0.398 & 0.400 \\
\hline $\mathrm{H}$ & 0.490 & 0.475 & 0.460 & 0.446 & 0.434 & 0.422 & 0.413 & 0.406 & 0.402 & 0.405 \\
\hline $\mathrm{J}$ & 0.493 & 0.478 & 0.464 & 0.450 & 0.437 & 0.425 & 0.415 & 0.406 & 0.400 & 0.400 \\
\hline K & 0.491 & 0.476 & 0.462 & 0.448 & 0.435 & 0.423 & 0.413 & 0.404 & 0.398 & 0.397 \\
\hline $\mathrm{L}$ & 0.462 & 0.447 & 0.434 & 0.421 & 0.409 & 0.398 & 0.388 & 0.379 & 0.373 & 0.372 \\
\hline M & 0.445 & 0.431 & 0.418 & 0.405 & 0.394 & 0.383 & 0.373 & 0.365 & 0.359 & 0.358 \\
\hline $\mathrm{P}$ & 0.452 & 0.438 & 0.425 & 0.412 & 0.400 & 0.389 & 0.379 & 0.371 & 0.365 & 0.364 \\
\hline Q & 0.495 & 0.479 & 0.465 & 0.450 & 0.438 & 0.426 & 0.416 & 0.409 & 0.405 & 0.408 \\
\hline $\mathrm{R}$ & 0.511 & 0.495 & 0.480 & 0.466 & 0.453 & 0.441 & 0.431 & 0.423 & 0.419 & 0.421 \\
\hline $\mathrm{S}$ & 0.503 & 0.488 & 0.473 & 0.459 & 0.446 & 0.434 & 0.424 & 0.415 & 0.409 & 0.408 \\
\hline
\end{tabular}


Table 7.23. Problem 6.1.1 reference solution Shift power peaking factors for axial segments in element 6 for charge-pan II.

\begin{tabular}{|c|c|c|c|c|c|c|c|c|c|c|}
\hline \multirow[t]{2}{*}{ Channels } & \multicolumn{10}{|c|}{ Power Peaking Factors } \\
\hline & 1 & 2 & 3 & 4 & 5 & 6 & 7 & 8 & 9 & 10 \\
\hline A & 0.300 & 0.272 & 0.258 & 0.248 & 0.233 & 0.222 & 0.206 & 0.200 & 0.188 & 0.179 \\
\hline B & 0.288 & 0.262 & 0.250 & 0.240 & 0.226 & 0.215 & 0.202 & 0.192 & 0.184 & 0.175 \\
\hline $\mathrm{C}$ & 0.278 & 0.253 & 0.242 & 0.229 & 0.215 & 0.206 & 0.193 & 0.182 & 0.173 & 0.165 \\
\hline $\mathrm{D}$ & 0.287 & 0.266 & 0.249 & 0.235 & 0.222 & 0.211 & 0.205 & 0.193 & 0.183 & 0.176 \\
\hline $\mathrm{E}$ & 0.356 & 0.329 & 0.313 & 0.295 & 0.278 & 0.263 & 0.257 & 0.240 & 0.226 & 0.217 \\
\hline $\mathrm{F}$ & 0.361 & 0.327 & 0.312 & 0.296 & 0.277 & 0.267 & 0.253 & 0.240 & 0.234 & 0.216 \\
\hline G & 0.373 & 0.347 & 0.333 & 0.320 & 0.305 & 0.288 & 0.276 & 0.261 & 0.255 & 0.238 \\
\hline $\mathrm{H}$ & 0.374 & 0.341 & 0.329 & 0.318 & 0.303 & 0.287 & 0.274 & 0.262 & 0.248 & 0.239 \\
\hline $\mathrm{J}$ & 0.332 & 0.304 & 0.290 & 0.272 & 0.258 & 0.241 & 0.232 & 0.220 & 0.210 & 0.199 \\
\hline K & 0.319 & 0.295 & 0.273 & 0.262 & 0.249 & 0.234 & 0.222 & 0.207 & 0.201 & 0.188 \\
\hline $\mathrm{L}$ & 0.325 & 0.290 & 0.277 & 0.265 & 0.250 & 0.238 & 0.223 & 0.211 & 0.204 & 0.187 \\
\hline M & 0.336 & 0.308 & 0.287 & 0.273 & 0.258 & 0.246 & 0.232 & 0.222 & 0.208 & 0.198 \\
\hline $\mathrm{P}$ & 0.367 & 0.337 & 0.322 & 0.300 & 0.286 & 0.269 & 0.255 & 0.245 & 0.230 & 0.220 \\
\hline Q & 0.388 & 0.357 & 0.339 & 0.324 & 0.315 & 0.295 & 0.278 & 0.268 & 0.260 & 0.245 \\
\hline $\mathrm{R}$ & 0.381 & 0.351 & 0.338 & 0.319 & 0.303 & 0.293 & 0.282 & 0.267 & 0.253 & 0.245 \\
\hline S & 0.323 & 0.295 & 0.277 & 0.265 & 0.251 & 0.239 & 0.226 & 0.215 & 0.202 & 0.192 \\
\hline Channels & 11 & 12 & 13 & 14 & $\overline{15}$ & 16 & 17 & 18 & 19 & 20 \\
\hline A & 0.172 & 0.161 & 0.156 & 0.147 & 0.138 & 0.130 & 0.122 & 0.116 & 0.116 & 0.116 \\
\hline B & 0.164 & 0.156 & 0.146 & 0.141 & 0.132 & 0.126 & 0.119 & 0.114 & 0.111 & 0.112 \\
\hline $\mathrm{C}$ & 0.156 & 0.150 & 0.140 & 0.133 & 0.128 & 0.119 & 0.115 & 0.112 & 0.108 & 0.106 \\
\hline D & 0.163 & 0.155 & 0.148 & 0.140 & 0.136 & 0.126 & 0.123 & 0.119 & 0.114 & 0.114 \\
\hline $\mathrm{E}$ & 0.203 & 0.194 & 0.181 & 0.173 & 0.166 & 0.157 & 0.148 & 0.143 & 0.142 & 0.141 \\
\hline $\mathrm{F}$ & 0.207 & 0.196 & 0.184 & 0.175 & 0.167 & 0.162 & 0.150 & 0.144 & 0.141 & 0.142 \\
\hline G & 0.229 & 0.214 & 0.203 & 0.193 & 0.184 & 0.176 & 0.165 & 0.160 & 0.153 & 0.151 \\
\hline $\mathrm{H}$ & 0.226 & 0.213 & 0.202 & 0.191 & 0.184 & 0.175 & 0.164 & 0.151 & 0.147 & 0.148 \\
\hline $\mathrm{J}$ & 0.189 & 0.181 & 0.167 & 0.157 & 0.149 & 0.143 & 0.138 & 0.132 & 0.129 & 0.130 \\
\hline K & 0.183 & 0.172 & 0.160 & 0.152 & 0.146 & 0.138 & 0.131 & 0.125 & 0.123 & 0.125 \\
\hline $\mathrm{L}$ & 0.182 & 0.174 & 0.163 & 0.154 & 0.146 & 0.142 & 0.133 & 0.127 & 0.121 & 0.122 \\
\hline $\mathrm{M}$ & 0.192 & 0.180 & 0.173 & 0.165 & 0.154 & 0.146 & 0.138 & 0.132 & 0.129 & 0.127 \\
\hline $\mathrm{P}$ & 0.210 & 0.197 & 0.188 & 0.178 & 0.170 & 0.158 & 0.158 & 0.146 & 0.144 & 0.143 \\
\hline Q & 0.228 & 0.220 & 0.207 & 0.199 & 0.188 & 0.178 & 0.167 & 0.161 & 0.153 & 0.151 \\
\hline $\mathrm{R}$ & 0.231 & 0.221 & 0.204 & 0.193 & 0.183 & 0.174 & 0.165 & 0.159 & 0.151 & 0.151 \\
\hline $\mathrm{S}$ & 0.185 & 0.173 & 0.165 & 0.151 & 0.148 & 0.140 & 0.131 & 0.127 & 0.123 & 0.126 \\
\hline
\end{tabular}


Table 7.24. Problem 6.1.1 reference solution MPACT power peaking factors for axial segments in element 6 for charge-pan II.

\begin{tabular}{|c|c|c|c|c|c|c|c|c|c|c|}
\hline \multirow[t]{2}{*}{ Channels } & \multicolumn{10}{|c|}{ Power Peaking Factors } \\
\hline & 1 & 2 & 3 & 4 & 5 & 6 & 7 & 8 & 9 & 10 \\
\hline A & 0.296 & 0.275 & 0.259 & 0.245 & 0.232 & 0.221 & 0.210 & 0.199 & 0.189 & 0.180 \\
\hline B & 0.305 & 0.284 & 0.267 & 0.253 & 0.240 & 0.228 & 0.217 & 0.206 & 0.196 & 0.186 \\
\hline $\mathrm{C}$ & 0.297 & 0.276 & 0.260 & 0.245 & 0.232 & 0.221 & 0.210 & 0.199 & 0.189 & 0.180 \\
\hline $\mathrm{D}$ & 0.285 & 0.265 & 0.249 & 0.235 & 0.223 & 0.211 & 0.200 & 0.190 & 0.181 & 0.172 \\
\hline $\mathrm{E}$ & 0.327 & 0.304 & 0.286 & 0.270 & 0.255 & 0.242 & 0.230 & 0.218 & 0.207 & 0.197 \\
\hline $\mathrm{F}$ & 0.339 & 0.315 & 0.297 & 0.281 & 0.266 & 0.252 & 0.240 & 0.228 & 0.216 & 0.206 \\
\hline G & 0.379 & 0.357 & 0.340 & 0.325 & 0.311 & 0.297 & 0.284 & 0.271 & 0.258 & 0.246 \\
\hline $\mathrm{H}$ & 0.384 & 0.361 & 0.344 & 0.328 & 0.313 & 0.300 & 0.286 & 0.273 & 0.260 & 0.248 \\
\hline $\mathrm{J}$ & 0.369 & 0.343 & 0.323 & 0.306 & 0.290 & 0.275 & 0.262 & 0.249 & 0.236 & 0.225 \\
\hline K & 0.366 & 0.340 & 0.320 & 0.303 & 0.287 & 0.272 & 0.259 & 0.246 & 0.233 & 0.222 \\
\hline $\mathrm{L}$ & 0.343 & 0.319 & 0.300 & 0.284 & 0.269 & 0.255 & 0.242 & 0.230 & 0.219 & 0.207 \\
\hline M & 0.329 & 0.306 & 0.288 & 0.272 & 0.258 & 0.244 & 0.232 & 0.220 & 0.209 & 0.199 \\
\hline$P$ & 0.335 & 0.311 & 0.292 & 0.276 & 0.261 & 0.248 & 0.235 & 0.223 & 0.212 & 0.201 \\
\hline Q & 0.386 & 0.364 & 0.346 & 0.330 & 0.316 & 0.302 & 0.288 & 0.275 & 0.262 & 0.250 \\
\hline $\mathrm{R}$ & 0.396 & 0.371 & 0.352 & 0.335 & 0.320 & 0.305 & 0.291 & 0.277 & 0.264 & 0.251 \\
\hline $\mathrm{S}$ & 0.376 & 0.350 & 0.329 & 0.311 & 0.295 & 0.280 & 0.266 & 0.253 & 0.240 & 0.228 \\
\hline Channels & 11 & 12 & 13 & 14 & 15 & 16 & 17 & 18 & 19 & 20 \\
\hline A & 0.171 & 0.162 & 0.153 & 0.145 & 0.137 & 0.130 & 0.124 & 0.119 & 0.115 & 0.114 \\
\hline B & 0.176 & 0.167 & 0.158 & 0.150 & 0.142 & 0.135 & 0.128 & 0.123 & 0.119 & 0.118 \\
\hline $\mathrm{C}$ & 0.170 & 0.161 & 0.153 & 0.145 & 0.137 & 0.130 & 0.124 & 0.119 & 0.115 & 0.114 \\
\hline D & 0.163 & 0.154 & 0.146 & 0.138 & 0.131 & 0.124 & 0.118 & 0.113 & 0.110 & 0.109 \\
\hline $\mathrm{E}$ & 0.186 & 0.177 & 0.167 & 0.158 & 0.150 & 0.142 & 0.135 & 0.130 & 0.126 & 0.124 \\
\hline $\mathrm{F}$ & 0.195 & 0.185 & 0.175 & 0.166 & 0.157 & 0.149 & 0.142 & 0.136 & 0.131 & 0.130 \\
\hline G & 0.233 & 0.222 & 0.210 & 0.199 & 0.188 & 0.178 & 0.169 & 0.161 & 0.155 & 0.152 \\
\hline $\mathrm{H}$ & 0.235 & 0.223 & 0.212 & 0.201 & 0.190 & 0.180 & 0.171 & 0.163 & 0.156 & 0.153 \\
\hline J & 0.213 & 0.202 & 0.192 & 0.181 & 0.172 & 0.163 & 0.155 & 0.149 & 0.145 & 0.143 \\
\hline K & 0.210 & 0.200 & 0.189 & 0.179 & 0.170 & 0.161 & 0.153 & 0.147 & 0.143 & 0.142 \\
\hline $\mathrm{L}$ & 0.197 & 0.187 & 0.177 & 0.167 & 0.158 & 0.150 & 0.143 & 0.137 & 0.133 & 0.132 \\
\hline M & 0.189 & 0.179 & 0.169 & 0.160 & 0.152 & 0.144 & 0.137 & 0.131 & 0.127 & 0.126 \\
\hline $\mathrm{P}$ & 0.191 & 0.181 & 0.171 & 0.162 & 0.153 & 0.146 & 0.139 & 0.133 & 0.129 & 0.128 \\
\hline Q & 0.237 & 0.225 & 0.213 & 0.202 & 0.191 & 0.181 & 0.172 & 0.164 & 0.157 & 0.154 \\
\hline $\mathrm{R}$ & 0.239 & 0.227 & 0.215 & 0.203 & 0.193 & 0.183 & 0.173 & 0.166 & 0.160 & 0.157 \\
\hline $\mathrm{S}$ & 0.216 & 0.205 & 0.194 & 0.184 & 0.174 & 0.165 & 0.158 & 0.151 & 0.147 & 0.145 \\
\hline
\end{tabular}




\section{Charge-Pan III}

Table 7.25. Problem 6.1.1 reference solution Shift power peaking factors for axial segments in element 1 for charge-pan III.

\begin{tabular}{|c|c|c|c|c|c|c|c|c|c|c|}
\hline \multirow[t]{2}{*}{ Channels } & \multicolumn{10}{|c|}{ Power Peaking Factors } \\
\hline & 1 & 2 & 3 & 4 & 5 & 6 & 7 & 8 & 9 & 10 \\
\hline A & 0.805 & 0.783 & 0.790 & 0.811 & 0.828 & 0.860 & 0.887 & 0.929 & 0.957 & 0.996 \\
\hline B & 0.801 & 0.778 & 0.791 & 0.809 & 0.829 & 0.858 & 0.894 & 0.928 & 0.958 & 0.994 \\
\hline $\mathrm{C}$ & 0.808 & 0.781 & 0.786 & 0.802 & 0.825 & 0.862 & 0.887 & 0.923 & 0.957 & 0.988 \\
\hline $\mathrm{D}$ & 0.809 & 0.773 & 0.792 & 0.807 & 0.827 & 0.852 & 0.885 & 0.920 & 0.960 & 0.997 \\
\hline $\mathrm{E}$ & 0.804 & 0.783 & 0.795 & 0.812 & 0.837 & 0.861 & 0.898 & 0.928 & 0.973 & 0.997 \\
\hline $\mathrm{F}$ & 0.809 & 0.784 & 0.793 & 0.810 & 0.840 & 0.867 & 0.891 & 0.928 & 0.966 & 1.004 \\
\hline G & 0.803 & 0.787 & 0.800 & 0.812 & 0.836 & 0.857 & 0.896 & 0.934 & 0.965 & 1.005 \\
\hline $\mathrm{H}$ & 0.812 & 0.786 & 0.798 & 0.810 & 0.839 & 0.869 & 0.894 & 0.932 & 0.965 & 0.998 \\
\hline $\mathrm{J}$ & 0.809 & 0.786 & 0.797 & 0.809 & 0.834 & 0.865 & 0.905 & 0.933 & 0.964 & 1.002 \\
\hline K & 0.811 & 0.782 & 0.795 & 0.817 & 0.833 & 0.864 & 0.894 & 0.937 & 0.967 & 1.007 \\
\hline $\mathrm{L}$ & 0.811 & 0.784 & 0.794 & 0.814 & 0.842 & 0.860 & 0.897 & 0.932 & 0.965 & 1.002 \\
\hline M & 0.814 & 0.788 & 0.799 & 0.810 & 0.843 & 0.867 & 0.900 & 0.924 & 0.965 & 1.001 \\
\hline $\mathrm{P}$ & 0.808 & 0.789 & 0.799 & 0.812 & 0.845 & 0.873 & 0.896 & 0.938 & 0.971 & 1.001 \\
\hline Q & 0.802 & 0.786 & 0.797 & 0.811 & 0.832 & 0.860 & 0.894 & 0.929 & 0.966 & 1.005 \\
\hline $\mathrm{R}$ & 0.811 & 0.784 & 0.796 & 0.811 & 0.837 & 0.871 & 0.905 & 0.933 & 0.970 & 1.006 \\
\hline $\mathrm{S}$ & 0.805 & 0.781 & 0.795 & 0.815 & 0.833 & 0.863 & 0.905 & 0.935 & 0.969 & 0.999 \\
\hline Channels & 11 & 12 & 13 & 14 & 15 & 16 & 17 & 18 & 19 & 20 \\
\hline A & 1.037 & 1.069 & 1.105 & 1.142 & 1.176 & 1.220 & 1.268 & 1.302 & 1.366 & 1.465 \\
\hline B & 1.023 & 1.061 & 1.099 & 1.142 & 1.180 & 1.224 & 1.268 & 1.310 & 1.364 & 1.456 \\
\hline $\mathrm{C}$ & 1.024 & 1.059 & 1.104 & 1.151 & 1.183 & 1.213 & 1.263 & 1.316 & 1.364 & 1.463 \\
\hline D & 1.037 & 1.069 & 1.106 & 1.143 & 1.182 & 1.227 & 1.266 & 1.323 & 1.368 & 1.451 \\
\hline $\mathrm{E}$ & 1.042 & 1.078 & 1.111 & 1.144 & 1.195 & 1.239 & 1.279 & 1.336 & 1.379 & 1.474 \\
\hline $\mathrm{F}$ & 1.031 & 1.080 & 1.118 & 1.143 & 1.201 & 1.233 & 1.272 & 1.325 & 1.374 & 1.472 \\
\hline G & 1.034 & 1.074 & 1.108 & 1.154 & 1.194 & 1.242 & 1.277 & 1.327 & 1.373 & 1.485 \\
\hline $\mathrm{H}$ & 1.044 & 1.074 & 1.118 & 1.153 & 1.190 & 1.231 & 1.286 & 1.328 & 1.378 & 1.482 \\
\hline $\mathrm{J}$ & 1.038 & 1.073 & 1.110 & 1.156 & 1.188 & 1.233 & 1.282 & 1.329 & 1.384 & 1.474 \\
\hline K & 1.033 & 1.074 & 1.124 & 1.155 & 1.190 & 1.228 & 1.279 & 1.322 & 1.378 & 1.473 \\
\hline $\mathrm{L}$ & 1.041 & 1.075 & 1.116 & 1.153 & 1.197 & 1.242 & 1.274 & 1.325 & 1.390 & 1.478 \\
\hline $\mathrm{M}$ & 1.032 & 1.065 & 1.108 & 1.146 & 1.184 & 1.233 & 1.276 & 1.323 & 1.376 & 1.475 \\
\hline $\mathrm{P}$ & 1.034 & 1.077 & 1.125 & 1.157 & 1.198 & 1.238 & 1.277 & 1.323 & 1.386 & 1.484 \\
\hline Q & 1.038 & 1.081 & 1.114 & 1.150 & 1.188 & 1.234 & 1.282 & 1.322 & 1.383 & 1.480 \\
\hline $\mathrm{R}$ & 1.037 & 1.079 & 1.117 & 1.156 & 1.194 & 1.246 & 1.277 & 1.336 & 1.375 & 1.479 \\
\hline $\mathrm{S}$ & 1.043 & 1.076 & 1.116 & 1.154 & 1.193 & 1.236 & 1.271 & 1.327 & 1.380 & 1.479 \\
\hline
\end{tabular}


Table 7.26. Problem 6.1.1 reference solution MPACT power peaking factors for axial segments in element 1 for charge-pan III.

\begin{tabular}{|c|c|c|c|c|c|c|c|c|c|c|}
\hline \multirow[t]{2}{*}{ Channels } & \multicolumn{10}{|c|}{ Power Peaking Factors } \\
\hline & 1 & 2 & 3 & 4 & 5 & 6 & 7 & 8 & 9 & 10 \\
\hline A & 0.781 & 0.770 & 0.778 & 0.795 & 0.818 & 0.847 & 0.879 & 0.913 & 0.948 & 0.984 \\
\hline B & 0.781 & 0.771 & 0.778 & 0.795 & 0.819 & 0.847 & 0.879 & 0.913 & 0.948 & 0.984 \\
\hline $\mathrm{C}$ & 0.781 & 0.771 & 0.778 & 0.795 & 0.819 & 0.847 & 0.879 & 0.913 & 0.948 & 0.984 \\
\hline D & 0.781 & 0.771 & 0.778 & 0.795 & 0.819 & 0.847 & 0.879 & 0.913 & 0.948 & 0.984 \\
\hline $\mathrm{E}$ & 0.786 & 0.776 & 0.783 & 0.801 & 0.825 & 0.855 & 0.887 & 0.921 & 0.957 & 0.994 \\
\hline $\mathrm{F}$ & 0.786 & 0.776 & 0.783 & 0.801 & 0.825 & 0.854 & 0.887 & 0.921 & 0.957 & 0.993 \\
\hline G & 0.786 & 0.776 & 0.783 & 0.801 & 0.825 & 0.855 & 0.887 & 0.921 & 0.957 & 0.994 \\
\hline $\mathrm{H}$ & 0.786 & 0.776 & 0.783 & 0.801 & 0.825 & 0.854 & 0.887 & 0.921 & 0.957 & 0.993 \\
\hline $\mathrm{J}$ & 0.786 & 0.776 & 0.783 & 0.801 & 0.825 & 0.855 & 0.887 & 0.922 & 0.957 & 0.994 \\
\hline K & 0.787 & 0.776 & 0.783 & 0.801 & 0.825 & 0.854 & 0.887 & 0.921 & 0.957 & 0.993 \\
\hline $\mathrm{L}$ & 0.786 & 0.775 & 0.783 & 0.801 & 0.825 & 0.854 & 0.887 & 0.921 & 0.957 & 0.993 \\
\hline M & 0.786 & 0.775 & 0.783 & 0.801 & 0.825 & 0.854 & 0.887 & 0.921 & 0.957 & 0.993 \\
\hline $\mathrm{P}$ & 0.787 & 0.776 & 0.784 & 0.802 & 0.826 & 0.856 & 0.888 & 0.923 & 0.958 & 0.995 \\
\hline Q & 0.786 & 0.776 & 0.784 & 0.802 & 0.826 & 0.855 & 0.888 & 0.922 & 0.958 & 0.995 \\
\hline $\mathrm{R}$ & 0.787 & 0.776 & 0.784 & 0.802 & 0.826 & 0.855 & 0.888 & 0.923 & 0.958 & 0.995 \\
\hline S & 0.787 & 0.777 & 0.785 & 0.802 & 0.827 & 0.856 & 0.889 & 0.923 & 0.959 & 0.995 \\
\hline Channels & 11 & 12 & 13 & 14 & $\overline{15}$ & $\overline{16}$ & 17 & $\overline{18}$ & 19 & 20 \\
\hline A & 1.020 & 1.057 & 1.094 & 1.133 & 1.172 & 1.214 & 1.259 & 1.309 & 1.367 & 1.445 \\
\hline B & 1.020 & 1.057 & 1.095 & 1.133 & 1.173 & 1.215 & 1.260 & 1.310 & 1.367 & 1.445 \\
\hline $\mathrm{C}$ & 1.020 & 1.057 & 1.095 & 1.133 & 1.173 & 1.215 & 1.260 & 1.310 & 1.367 & 1.445 \\
\hline D & 1.020 & 1.057 & 1.095 & 1.133 & 1.173 & 1.214 & 1.259 & 1.309 & 1.367 & 1.445 \\
\hline $\mathrm{E}$ & 1.031 & 1.068 & 1.106 & 1.144 & 1.184 & 1.227 & 1.272 & 1.323 & 1.381 & 1.460 \\
\hline $\mathrm{F}$ & 1.030 & 1.068 & 1.106 & 1.144 & 1.184 & 1.226 & 1.272 & 1.323 & 1.381 & 1.460 \\
\hline G & 1.030 & 1.068 & 1.106 & 1.144 & 1.184 & 1.226 & 1.272 & 1.323 & 1.381 & 1.460 \\
\hline $\mathrm{H}$ & 1.030 & 1.068 & 1.105 & 1.144 & 1.184 & 1.226 & 1.272 & 1.322 & 1.381 & 1.460 \\
\hline $\mathrm{J}$ & 1.031 & 1.068 & 1.106 & 1.145 & 1.185 & 1.227 & 1.272 & 1.323 & 1.382 & 1.461 \\
\hline K & 1.030 & 1.068 & 1.105 & 1.144 & 1.184 & 1.226 & 1.272 & 1.322 & 1.380 & 1.458 \\
\hline $\mathrm{L}$ & 1.030 & 1.068 & 1.105 & 1.144 & 1.184 & 1.226 & 1.272 & 1.322 & 1.381 & 1.460 \\
\hline $\mathrm{M}$ & 1.030 & 1.067 & 1.105 & 1.144 & 1.184 & 1.226 & 1.272 & 1.322 & 1.381 & 1.460 \\
\hline $\mathrm{P}$ & 1.032 & 1.069 & 1.107 & 1.146 & 1.186 & 1.228 & 1.273 & 1.324 & 1.382 & 1.460 \\
\hline Q & 1.032 & 1.069 & 1.107 & 1.146 & 1.186 & 1.228 & 1.274 & 1.324 & 1.383 & 1.462 \\
\hline $\mathrm{R}$ & 1.032 & 1.069 & 1.107 & 1.146 & 1.186 & 1.228 & 1.274 & 1.324 & 1.382 & 1.461 \\
\hline $\mathrm{S}$ & 1.032 & 1.070 & 1.108 & 1.146 & 1.186 & 1.228 & 1.274 & 1.325 & 1.383 & 1.461 \\
\hline
\end{tabular}


Table 7.27. Problem 6.1.1 reference solution Shift power peaking factors for axial segments in element 2 for charge-pan III.

\begin{tabular}{|c|c|c|c|c|c|c|c|c|c|c|}
\hline \multirow[t]{2}{*}{ Channels } & \multicolumn{10}{|c|}{ Power Peaking Factors } \\
\hline & 1 & 2 & 3 & 4 & 5 & 6 & 7 & 8 & 9 & 10 \\
\hline A & 1.522 & 1.475 & 1.474 & 1.481 & 1.483 & 1.490 & 1.495 & 1.498 & 1.525 & 1.529 \\
\hline B & 1.519 & 1.466 & 1.475 & 1.479 & 1.487 & 1.495 & 1.502 & 1.512 & 1.525 & 1.535 \\
\hline $\mathrm{C}$ & 1.530 & 1.481 & 1.483 & 1.477 & 1.482 & 1.499 & 1.503 & 1.514 & 1.520 & 1.533 \\
\hline D & 1.525 & 1.464 & 1.466 & 1.486 & 1.473 & 1.486 & 1.501 & 1.522 & 1.527 & 1.532 \\
\hline $\mathrm{E}$ & 1.533 & 1.483 & 1.490 & 1.486 & 1.487 & 1.515 & 1.514 & 1.526 & 1.545 & 1.549 \\
\hline $\mathrm{F}$ & 1.532 & 1.491 & 1.489 & 1.495 & 1.497 & 1.509 & 1.513 & 1.523 & 1.539 & 1.555 \\
\hline G & 1.531 & 1.498 & 1.489 & 1.492 & 1.496 & 1.499 & 1.515 & 1.529 & 1.537 & 1.542 \\
\hline $\mathrm{H}$ & 1.533 & 1.487 & 1.493 & 1.490 & 1.501 & 1.506 & 1.522 & 1.521 & 1.539 & 1.544 \\
\hline $\mathrm{J}$ & 1.534 & 1.486 & 1.490 & 1.487 & 1.501 & 1.502 & 1.523 & 1.530 & 1.537 & 1.539 \\
\hline K & 1.542 & 1.499 & 1.486 & 1.489 & 1.490 & 1.499 & 1.518 & 1.514 & 1.529 & 1.544 \\
\hline $\mathrm{L}$ & 1.531 & 1.479 & 1.490 & 1.490 & 1.497 & 1.505 & 1.508 & 1.519 & 1.549 & 1.541 \\
\hline M & 1.537 & 1.485 & 1.483 & 1.495 & 1.496 & 1.507 & 1.507 & 1.529 & 1.538 & 1.540 \\
\hline $\mathrm{P}$ & 1.531 & 1.494 & 1.485 & 1.498 & 1.504 & 1.513 & 1.534 & 1.528 & 1.542 & 1.551 \\
\hline Q & 1.540 & 1.497 & 1.492 & 1.490 & 1.494 & 1.497 & 1.506 & 1.536 & 1.535 & 1.550 \\
\hline $\mathrm{R}$ & 1.545 & 1.490 & 1.488 & 1.494 & 1.495 & 1.501 & 1.521 & 1.522 & 1.535 & 1.558 \\
\hline$S$ & 1.541 & 1.490 & 1.491 & 1.495 & 1.508 & 1.504 & 1.511 & 1.523 & 1.549 & 1.556 \\
\hline Channels & 11 & 12 & 13 & 14 & 15 & 16 & 17 & 18 & 19 & 20 \\
\hline A & 1.542 & 1.556 & 1.568 & 1.576 & 1.590 & 1.610 & 1.636 & 1.656 & 1.678 & 1.753 \\
\hline B & 1.551 & 1.562 & 1.562 & 1.582 & 1.597 & 1.602 & 1.634 & 1.658 & 1.686 & 1.769 \\
\hline $\mathrm{C}$ & 1.546 & 1.544 & 1.566 & 1.592 & 1.596 & 1.617 & 1.629 & 1.648 & 1.686 & 1.768 \\
\hline D & 1.554 & 1.565 & 1.576 & 1.578 & 1.602 & 1.607 & 1.632 & 1.654 & 1.683 & 1.764 \\
\hline $\mathrm{E}$ & 1.563 & 1.573 & 1.591 & 1.607 & 1.614 & 1.626 & 1.650 & 1.687 & 1.701 & 1.783 \\
\hline $\mathrm{F}$ & 1.559 & 1.569 & 1.582 & 1.596 & 1.612 & 1.629 & 1.640 & 1.681 & 1.702 & 1.772 \\
\hline G & 1.564 & 1.562 & 1.591 & 1.604 & 1.608 & 1.625 & 1.649 & 1.672 & 1.700 & 1.783 \\
\hline $\mathrm{H}$ & 1.557 & 1.571 & 1.583 & 1.606 & 1.607 & 1.629 & 1.650 & 1.671 & 1.699 & 1.768 \\
\hline $\mathrm{J}$ & 1.560 & 1.568 & 1.586 & 1.590 & 1.626 & 1.631 & 1.646 & 1.679 & 1.697 & 1.777 \\
\hline K & 1.557 & 1.578 & 1.583 & 1.601 & 1.613 & 1.632 & 1.650 & 1.671 & 1.697 & 1.781 \\
\hline $\mathrm{L}$ & 1.548 & 1.585 & 1.589 & 1.605 & 1.615 & 1.625 & 1.642 & 1.675 & 1.698 & 1.780 \\
\hline M & 1.562 & 1.581 & 1.578 & 1.591 & 1.611 & 1.626 & 1.655 & 1.681 & 1.704 & 1.789 \\
\hline $\mathrm{P}$ & 1.570 & 1.572 & 1.598 & 1.606 & 1.622 & 1.636 & 1.656 & 1.686 & 1.705 & 1.786 \\
\hline Q & 1.556 & 1.571 & 1.588 & 1.604 & 1.621 & 1.638 & 1.650 & 1.680 & 1.702 & 1.785 \\
\hline $\mathrm{R}$ & 1.558 & 1.577 & 1.591 & 1.610 & 1.613 & 1.622 & 1.647 & 1.669 & 1.701 & 1.783 \\
\hline$S$ & 1.570 & 1.570 & 1.580 & 1.587 & 1.618 & 1.627 & 1.647 & 1.667 & 1.706 & 1.784 \\
\hline
\end{tabular}


Table 7.28. Problem 6.1.1 reference solution MPACT power peaking factors for axial segments in element 2 for charge-pan III.

\begin{tabular}{|c|c|c|c|c|c|c|c|c|c|c|}
\hline \multirow[t]{2}{*}{ Channels } & \multicolumn{10}{|c|}{ Power Peaking Factors } \\
\hline & 1 & 2 & 3 & 4 & 5 & 6 & 7 & 8 & 9 & 10 \\
\hline A & 1.504 & 1.477 & 1.469 & 1.469 & 1.474 & 1.482 & 1.491 & 1.502 & 1.513 & 1.525 \\
\hline B & 1.504 & 1.477 & 1.470 & 1.470 & 1.474 & 1.482 & 1.492 & 1.502 & 1.514 & 1.525 \\
\hline $\mathrm{C}$ & 1.505 & 1.478 & 1.470 & 1.470 & 1.475 & 1.482 & 1.492 & 1.503 & 1.514 & 1.526 \\
\hline D & 1.504 & 1.477 & 1.469 & 1.469 & 1.474 & 1.482 & 1.492 & 1.502 & 1.514 & 1.525 \\
\hline $\mathrm{E}$ & 1.520 & 1.493 & 1.485 & 1.484 & 1.489 & 1.497 & 1.507 & 1.518 & 1.529 & 1.541 \\
\hline $\mathrm{F}$ & 1.520 & 1.492 & 1.484 & 1.484 & 1.489 & 1.496 & 1.506 & 1.517 & 1.529 & 1.540 \\
\hline G & 1.520 & 1.492 & 1.484 & 1.484 & 1.489 & 1.497 & 1.507 & 1.517 & 1.529 & 1.541 \\
\hline $\mathrm{H}$ & 1.520 & 1.492 & 1.484 & 1.484 & 1.489 & 1.497 & 1.506 & 1.517 & 1.529 & 1.540 \\
\hline $\mathrm{J}$ & 1.521 & 1.493 & 1.485 & 1.485 & 1.490 & 1.498 & 1.507 & 1.518 & 1.530 & 1.542 \\
\hline K & 1.519 & 1.492 & 1.484 & 1.484 & 1.489 & 1.497 & 1.507 & 1.518 & 1.530 & 1.542 \\
\hline $\mathrm{L}$ & 1.520 & 1.492 & 1.484 & 1.484 & 1.489 & 1.497 & 1.507 & 1.518 & 1.530 & 1.542 \\
\hline M & 1.520 & 1.492 & 1.484 & 1.484 & 1.489 & 1.497 & 1.507 & 1.518 & 1.529 & 1.541 \\
\hline $\mathrm{P}$ & 1.521 & 1.493 & 1.486 & 1.486 & 1.491 & 1.499 & 1.509 & 1.520 & 1.531 & 1.543 \\
\hline Q & 1.522 & 1.494 & 1.486 & 1.486 & 1.491 & 1.499 & 1.509 & 1.520 & 1.531 & 1.543 \\
\hline $\mathrm{R}$ & 1.521 & 1.494 & 1.486 & 1.486 & 1.491 & 1.499 & 1.509 & 1.520 & 1.531 & 1.543 \\
\hline $\mathrm{S}$ & 1.521 & 1.494 & 1.487 & 1.487 & 1.492 & 1.500 & 1.510 & 1.521 & 1.533 & 1.545 \\
\hline Channels & 11 & 12 & 13 & 14 & 15 & 16 & 17 & 18 & 19 & 20 \\
\hline A & 1.537 & 1.548 & 1.560 & 1.573 & 1.588 & 1.604 & 1.625 & 1.651 & 1.686 & 1.745 \\
\hline B & 1.537 & 1.549 & 1.561 & 1.574 & 1.588 & 1.605 & 1.625 & 1.651 & 1.686 & 1.744 \\
\hline $\mathrm{C}$ & 1.537 & 1.549 & 1.561 & 1.574 & 1.589 & 1.606 & 1.626 & 1.653 & 1.688 & 1.748 \\
\hline D & 1.537 & 1.549 & 1.561 & 1.574 & 1.588 & 1.605 & 1.625 & 1.652 & 1.687 & 1.746 \\
\hline $\mathrm{E}$ & 1.553 & 1.564 & 1.576 & 1.589 & 1.603 & 1.620 & 1.641 & 1.667 & 1.703 & 1.763 \\
\hline $\mathrm{F}$ & 1.552 & 1.563 & 1.575 & 1.588 & 1.602 & 1.619 & 1.639 & 1.666 & 1.701 & 1.761 \\
\hline G & 1.552 & 1.564 & 1.576 & 1.589 & 1.603 & 1.620 & 1.640 & 1.667 & 1.703 & 1.763 \\
\hline $\mathrm{H}$ & 1.552 & 1.564 & 1.576 & 1.589 & 1.603 & 1.620 & 1.640 & 1.667 & 1.703 & 1.763 \\
\hline $\mathrm{J}$ & 1.553 & 1.565 & 1.577 & 1.590 & 1.605 & 1.622 & 1.642 & 1.669 & 1.705 & 1.766 \\
\hline K & 1.554 & 1.565 & 1.578 & 1.591 & 1.605 & 1.622 & 1.643 & 1.670 & 1.707 & 1.767 \\
\hline $\mathrm{L}$ & 1.554 & 1.565 & 1.578 & 1.591 & 1.605 & 1.622 & 1.643 & 1.670 & 1.707 & 1.767 \\
\hline $\mathrm{M}$ & 1.553 & 1.565 & 1.577 & 1.590 & 1.604 & 1.621 & 1.642 & 1.669 & 1.705 & 1.765 \\
\hline $\mathrm{P}$ & 1.555 & 1.567 & 1.579 & 1.591 & 1.606 & 1.622 & 1.643 & 1.670 & 1.705 & 1.766 \\
\hline Q & 1.555 & 1.566 & 1.578 & 1.591 & 1.605 & 1.622 & 1.643 & 1.669 & 1.705 & 1.765 \\
\hline $\mathrm{R}$ & 1.555 & 1.567 & 1.579 & 1.591 & 1.606 & 1.622 & 1.643 & 1.670 & 1.706 & 1.766 \\
\hline $\mathrm{S}$ & 1.556 & 1.568 & 1.581 & 1.594 & 1.608 & 1.625 & 1.646 & 1.673 & 1.710 & 1.770 \\
\hline
\end{tabular}


Table 7.29. Problem 6.1.1 reference solution Shift power peaking factors for axial segments in element 3 for charge-pan III.

\begin{tabular}{|c|c|c|c|c|c|c|c|c|c|c|}
\hline \multirow[t]{2}{*}{ Channels } & \multicolumn{10}{|c|}{ Power Peaking Factors } \\
\hline & 1 & 2 & 3 & 4 & 5 & 6 & 7 & 8 & 9 & 10 \\
\hline A & 1.752 & 1.666 & 1.631 & 1.609 & 1.583 & 1.560 & 1.546 & 1.517 & 1.506 & 1.485 \\
\hline B & 1.747 & 1.669 & 1.632 & 1.611 & 1.578 & 1.564 & 1.538 & 1.520 & 1.495 & 1.488 \\
\hline $\mathrm{C}$ & 1.752 & 1.672 & 1.633 & 1.612 & 1.585 & 1.562 & 1.540 & 1.525 & 1.505 & 1.485 \\
\hline $\mathrm{D}$ & 1.752 & 1.671 & 1.637 & 1.606 & 1.583 & 1.563 & 1.541 & 1.533 & 1.510 & 1.495 \\
\hline $\mathrm{E}$ & 1.774 & 1.690 & 1.655 & 1.630 & 1.605 & 1.580 & 1.563 & 1.545 & 1.516 & 1.511 \\
\hline $\mathrm{F}$ & 1.778 & 1.692 & 1.654 & 1.619 & 1.590 & 1.573 & 1.561 & 1.534 & 1.515 & 1.502 \\
\hline G & 1.774 & 1.682 & 1.646 & 1.621 & 1.604 & 1.569 & 1.543 & 1.522 & 1.507 & 1.494 \\
\hline $\mathrm{H}$ & 1.764 & 1.676 & 1.653 & 1.622 & 1.592 & 1.570 & 1.542 & 1.531 & 1.514 & 1.502 \\
\hline $\mathrm{J}$ & 1.775 & 1.684 & 1.647 & 1.606 & 1.585 & 1.570 & 1.544 & 1.519 & 1.502 & 1.489 \\
\hline $\mathrm{K}$ & 1.769 & 1.679 & 1.651 & 1.620 & 1.587 & 1.572 & 1.544 & 1.533 & 1.510 & 1.492 \\
\hline $\mathrm{L}$ & 1.779 & 1.692 & 1.651 & 1.628 & 1.605 & 1.581 & 1.556 & 1.535 & 1.516 & 1.498 \\
\hline $\mathrm{M}$ & 1.772 & 1.682 & 1.666 & 1.634 & 1.603 & 1.590 & 1.571 & 1.545 & 1.524 & 1.508 \\
\hline $\mathrm{P}$ & 1.797 & 1.693 & 1.665 & 1.623 & 1.606 & 1.589 & 1.572 & 1.546 & 1.532 & 1.525 \\
\hline Q & 1.780 & 1.682 & 1.648 & 1.624 & 1.595 & 1.572 & 1.553 & 1.535 & 1.513 & 1.495 \\
\hline $\mathrm{R}$ & 1.778 & 1.690 & 1.642 & 1.619 & 1.595 & 1.567 & 1.558 & 1.533 & 1.510 & 1.491 \\
\hline $\mathrm{S}$ & 1.772 & 1.682 & 1.662 & 1.618 & 1.595 & 1.570 & 1.551 & 1.535 & 1.517 & 1.500 \\
\hline "Channels & $\overline{11}$ & 12 & 13 & 14 & $\overline{15}$ & $\overline{16}$ & 17 & $\overline{18}$ & 19 & 20 \\
\hline A & 1.470 & 1.451 & 1.434 & 1.425 & 1.410 & 1.397 & 1.387 & 1.390 & 1.387 & 1.430 \\
\hline B & 1.458 & 1.457 & 1.427 & 1.413 & 1.400 & 1.390 & 1.382 & 1.376 & 1.378 & 1.407 \\
\hline $\mathrm{C}$ & 1.473 & 1.455 & 1.429 & 1.416 & 1.410 & 1.400 & 1.384 & 1.381 & 1.380 & 1.428 \\
\hline D & 1.481 & 1.461 & 1.454 & 1.429 & 1.419 & 1.408 & 1.394 & 1.394 & 1.386 & 1.428 \\
\hline $\mathrm{E}$ & 1.496 & 1.481 & 1.467 & 1.450 & 1.439 & 1.429 & 1.421 & 1.415 & 1.420 & 1.463 \\
\hline $\mathrm{F}$ & 1.488 & 1.474 & 1.460 & 1.448 & 1.430 & 1.423 & 1.411 & 1.403 & 1.396 & 1.436 \\
\hline G & 1.478 & 1.455 & 1.445 & 1.422 & 1.413 & 1.390 & 1.381 & 1.373 & 1.369 & 1.411 \\
\hline $\mathrm{H}$ & 1.484 & 1.456 & 1.435 & 1.417 & 1.406 & 1.395 & 1.375 & 1.364 & 1.374 & 1.398 \\
\hline $\mathrm{J}$ & 1.468 & 1.449 & 1.430 & 1.429 & 1.410 & 1.388 & 1.381 & 1.375 & 1.365 & 1.398 \\
\hline K & 1.476 & 1.465 & 1.438 & 1.430 & 1.410 & 1.394 & 1.375 & 1.380 & 1.375 & 1.415 \\
\hline $\mathrm{L}$ & 1.478 & 1.467 & 1.451 & 1.437 & 1.426 & 1.406 & 1.407 & 1.413 & 1.406 & 1.445 \\
\hline M & 1.495 & 1.475 & 1.469 & 1.450 & 1.432 & 1.424 & 1.417 & 1.415 & 1.414 & 1.457 \\
\hline $\mathrm{P}$ & 1.506 & 1.483 & 1.456 & 1.456 & 1.440 & 1.434 & 1.417 & 1.424 & 1.432 & 1.459 \\
\hline Q & 1.480 & 1.465 & 1.445 & 1.429 & 1.406 & 1.394 & 1.379 & 1.376 & 1.374 & 1.412 \\
\hline $\mathrm{R}$ & 1.473 & 1.464 & 1.436 & 1.414 & 1.400 & 1.393 & 1.387 & 1.369 & 1.364 & 1.404 \\
\hline $\mathrm{S}$ & 1.491 & 1.456 & 1.440 & 1.433 & 1.414 & 1.406 & 1.393 & 1.384 & 1.382 & 1.414 \\
\hline
\end{tabular}


Table 7.30. Problem 6.1.1 reference solution MPACT power peaking factors for axial segments in element 3 for charge-pan III.

\begin{tabular}{|c|c|c|c|c|c|c|c|c|c|c|}
\hline \multirow[t]{2}{*}{ Channels } & \multicolumn{10}{|c|}{ Power Peaking Factors } \\
\hline & 1 & 2 & 3 & 4 & 5 & 6 & 7 & 8 & 9 & 10 \\
\hline A & 1.738 & 1.673 & 1.632 & 1.600 & 1.574 & 1.551 & 1.531 & 1.513 & 1.495 & 1.478 \\
\hline B & 1.737 & 1.673 & 1.633 & 1.601 & 1.575 & 1.553 & 1.533 & 1.515 & 1.498 & 1.482 \\
\hline $\mathrm{C}$ & 1.741 & 1.676 & 1.636 & 1.604 & 1.578 & 1.556 & 1.537 & 1.519 & 1.502 & 1.486 \\
\hline D & 1.739 & 1.674 & 1.633 & 1.601 & 1.575 & 1.553 & 1.533 & 1.515 & 1.498 & 1.482 \\
\hline $\mathrm{E}$ & 1.755 & 1.689 & 1.647 & 1.614 & 1.587 & 1.564 & 1.544 & 1.525 & 1.507 & 1.489 \\
\hline $\mathrm{F}$ & 1.753 & 1.687 & 1.645 & 1.612 & 1.585 & 1.562 & 1.542 & 1.523 & 1.505 & 1.487 \\
\hline G & 1.755 & 1.689 & 1.646 & 1.613 & 1.586 & 1.563 & 1.543 & 1.524 & 1.506 & 1.488 \\
\hline $\mathrm{H}$ & 1.755 & 1.689 & 1.647 & 1.614 & 1.587 & 1.564 & 1.543 & 1.525 & 1.507 & 1.489 \\
\hline $\mathrm{J}$ & 1.759 & 1.693 & 1.651 & 1.618 & 1.592 & 1.570 & 1.550 & 1.532 & 1.515 & 1.498 \\
\hline K & 1.761 & 1.695 & 1.653 & 1.621 & 1.595 & 1.573 & 1.554 & 1.536 & 1.520 & 1.504 \\
\hline $\mathrm{L}$ & 1.761 & 1.695 & 1.653 & 1.621 & 1.595 & 1.573 & 1.554 & 1.536 & 1.520 & 1.504 \\
\hline M & 1.758 & 1.692 & 1.651 & 1.618 & 1.592 & 1.570 & 1.550 & 1.532 & 1.515 & 1.498 \\
\hline $\mathrm{P}$ & 1.758 & 1.692 & 1.649 & 1.617 & 1.590 & 1.567 & 1.547 & 1.528 & 1.510 & 1.492 \\
\hline Q & 1.758 & 1.691 & 1.649 & 1.616 & 1.588 & 1.565 & 1.545 & 1.526 & 1.507 & 1.489 \\
\hline $\mathrm{R}$ & 1.758 & 1.692 & 1.650 & 1.617 & 1.590 & 1.567 & 1.547 & 1.528 & 1.510 & 1.493 \\
\hline $\mathrm{S}$ & 1.764 & 1.698 & 1.657 & 1.625 & 1.599 & 1.578 & 1.559 & 1.541 & 1.525 & 1.510 \\
\hline Channels & 11 & 12 & 13 & 14 & 15 & 16 & 17 & 18 & 19 & 20 \\
\hline A & 1.462 & 1.445 & 1.429 & 1.414 & 1.400 & 1.389 & 1.380 & 1.376 & 1.380 & 1.403 \\
\hline B & 1.465 & 1.449 & 1.434 & 1.419 & 1.406 & 1.395 & 1.387 & 1.384 & 1.389 & 1.413 \\
\hline $\mathrm{C}$ & 1.471 & 1.455 & 1.441 & 1.427 & 1.414 & 1.404 & 1.397 & 1.395 & 1.401 & 1.427 \\
\hline D & 1.465 & 1.449 & 1.434 & 1.419 & 1.406 & 1.395 & 1.387 & 1.384 & 1.389 & 1.413 \\
\hline $\mathrm{E}$ & 1.472 & 1.454 & 1.437 & 1.421 & 1.406 & 1.392 & 1.382 & 1.377 & 1.379 & 1.401 \\
\hline $\mathrm{F}$ & 1.470 & 1.452 & 1.435 & 1.418 & 1.403 & 1.390 & 1.379 & 1.374 & 1.376 & 1.397 \\
\hline G & 1.470 & 1.453 & 1.436 & 1.419 & 1.403 & 1.390 & 1.380 & 1.374 & 1.376 & 1.397 \\
\hline $\mathrm{H}$ & 1.472 & 1.454 & 1.437 & 1.421 & 1.406 & 1.392 & 1.382 & 1.377 & 1.379 & 1.401 \\
\hline $\mathrm{J}$ & 1.482 & 1.466 & 1.450 & 1.436 & 1.422 & 1.411 & 1.404 & 1.401 & 1.406 & 1.431 \\
\hline K & 1.488 & 1.473 & 1.458 & 1.444 & 1.432 & 1.422 & 1.415 & 1.414 & 1.421 & 1.447 \\
\hline $\mathrm{L}$ & 1.488 & 1.473 & 1.458 & 1.444 & 1.432 & 1.422 & 1.416 & 1.414 & 1.421 & 1.447 \\
\hline M & 1.482 & 1.466 & 1.450 & 1.436 & 1.422 & 1.411 & 1.404 & 1.401 & 1.406 & 1.431 \\
\hline $\mathrm{P}$ & 1.475 & 1.458 & 1.441 & 1.425 & 1.410 & 1.397 & 1.387 & 1.382 & 1.385 & 1.406 \\
\hline Q & 1.472 & 1.454 & 1.436 & 1.419 & 1.404 & 1.390 & 1.379 & 1.373 & 1.375 & 1.396 \\
\hline $\mathrm{R}$ & 1.475 & 1.458 & 1.441 & 1.425 & 1.410 & 1.397 & 1.387 & 1.382 & 1.385 & 1.406 \\
\hline $\mathrm{S}$ & 1.494 & 1.479 & 1.465 & 1.451 & 1.439 & 1.430 & 1.424 & 1.423 & 1.430 & 1.456 \\
\hline
\end{tabular}


Table 7.31. Problem 6.1.1 reference solution Shift power peaking factors for axial segments in element 4 for charge-pan III.

\begin{tabular}{|c|c|c|c|c|c|c|c|c|c|c|}
\hline \multirow[t]{2}{*}{ Channels } & \multicolumn{10}{|c|}{ Power Peaking Factors } \\
\hline & 1 & 2 & 3 & 4 & 5 & 6 & 7 & 8 & 9 & 10 \\
\hline A & 1.362 & 1.283 & 1.224 & 1.179 & 1.145 & 1.119 & 1.081 & 1.052 & 1.023 & 1.006 \\
\hline B & 1.351 & 1.262 & 1.217 & 1.174 & 1.140 & 1.107 & 1.069 & 1.042 & 1.016 & 0.992 \\
\hline $\mathrm{C}$ & 1.364 & 1.272 & 1.226 & 1.187 & 1.154 & 1.115 & 1.089 & 1.055 & 1.027 & 1.006 \\
\hline $\mathrm{D}$ & 1.376 & 1.291 & 1.236 & 1.204 & 1.161 & 1.139 & 1.099 & 1.069 & 1.053 & 1.023 \\
\hline $\mathrm{E}$ & 1.395 & 1.314 & 1.263 & 1.221 & 1.186 & 1.160 & 1.120 & 1.091 & 1.063 & 1.038 \\
\hline $\mathrm{F}$ & 1.385 & 1.288 & 1.245 & 1.189 & 1.163 & 1.125 & 1.106 & 1.079 & 1.050 & 1.020 \\
\hline G & 1.340 & 1.260 & 1.214 & 1.161 & 1.125 & 1.098 & 1.060 & 1.027 & 1.004 & 0.975 \\
\hline $\mathrm{H}$ & 1.333 & 1.244 & 1.199 & 1.159 & 1.132 & 1.085 & 1.055 & 1.025 & 0.999 & 0.968 \\
\hline $\mathrm{J}$ & 1.342 & 1.242 & 1.202 & 1.166 & 1.126 & 1.087 & 1.058 & 1.024 & 0.997 & 0.973 \\
\hline $\mathrm{K}$ & 1.338 & 1.253 & 1.215 & 1.165 & 1.126 & 1.100 & 1.051 & 1.029 & 1.008 & 0.974 \\
\hline $\mathrm{L}$ & 1.376 & 1.295 & 1.248 & 1.211 & 1.173 & 1.133 & 1.101 & 1.071 & 1.047 & 1.019 \\
\hline $\mathrm{M}$ & 1.405 & 1.320 & 1.258 & 1.218 & 1.190 & 1.153 & 1.121 & 1.099 & 1.066 & 1.036 \\
\hline $\mathrm{P}$ & 1.413 & 1.318 & 1.276 & 1.238 & 1.200 & 1.165 & 1.133 & 1.103 & 1.079 & 1.060 \\
\hline Q & 1.352 & 1.260 & 1.214 & 1.173 & 1.132 & 1.098 & 1.066 & 1.029 & 1.009 & 0.992 \\
\hline $\mathrm{R}$ & 1.342 & 1.244 & 1.207 & 1.156 & 1.126 & 1.092 & 1.056 & 1.026 & 0.993 & 0.973 \\
\hline $\mathrm{S}$ & 1.343 & 1.266 & 1.216 & 1.168 & 1.141 & 1.090 & 1.073 & 1.041 & 1.011 & 0.985 \\
\hline "Channels & $\overline{11}$ & 12 & 13 & 14 & 15 & 16 & 17 & 18 & $\overline{19}$ & 20 \\
\hline A & 0.982 & 0.957 & 0.937 & 0.916 & 0.893 & 0.880 & 0.860 & 0.855 & 0.841 & 0.854 \\
\hline B & 0.962 & 0.949 & 0.924 & 0.899 & 0.887 & 0.866 & 0.857 & 0.839 & 0.832 & 0.844 \\
\hline $\mathrm{C}$ & 0.978 & 0.946 & 0.933 & 0.914 & 0.889 & 0.885 & 0.871 & 0.859 & 0.845 & 0.863 \\
\hline D & 1.002 & 0.976 & 0.954 & 0.930 & 0.909 & 0.892 & 0.877 & 0.874 & 0.860 & 0.879 \\
\hline $\mathrm{E}$ & 1.015 & 0.994 & 0.972 & 0.952 & 0.931 & 0.916 & 0.908 & 0.888 & 0.884 & 0.896 \\
\hline $\mathrm{F}$ & 0.992 & 0.972 & 0.952 & 0.930 & 0.908 & 0.894 & 0.876 & 0.869 & 0.859 & 0.879 \\
\hline G & 0.952 & 0.926 & 0.908 & 0.882 & 0.865 & 0.844 & 0.839 & 0.824 & 0.812 & 0.836 \\
\hline $\mathrm{H}$ & 0.946 & 0.926 & 0.900 & 0.874 & 0.862 & 0.850 & 0.834 & 0.819 & 0.811 & 0.824 \\
\hline $\mathrm{J}$ & 0.948 & 0.930 & 0.904 & 0.884 & 0.858 & 0.845 & 0.828 & 0.821 & 0.803 & 0.824 \\
\hline K & 0.951 & 0.931 & 0.913 & 0.896 & 0.873 & 0.855 & 0.830 & 0.830 & 0.818 & 0.839 \\
\hline $\mathrm{L}$ & 1.001 & 0.972 & 0.951 & 0.933 & 0.913 & 0.894 & 0.878 & 0.866 & 0.861 & 0.874 \\
\hline M & 1.016 & 0.996 & 0.971 & 0.956 & 0.929 & 0.920 & 0.909 & 0.891 & 0.880 & 0.899 \\
\hline $\mathrm{P}$ & 1.027 & 1.009 & 0.985 & 0.962 & 0.943 & 0.927 & 0.914 & 0.902 & 0.901 & 0.917 \\
\hline $\mathrm{Q}$ & 0.962 & 0.937 & 0.913 & 0.892 & 0.869 & 0.862 & 0.840 & 0.836 & 0.825 & 0.838 \\
\hline $\mathrm{R}$ & 0.940 & 0.919 & 0.898 & 0.872 & 0.856 & 0.843 & 0.823 & 0.812 & 0.801 & 0.816 \\
\hline $\mathrm{S}$ & 0.963 & 0.946 & 0.915 & 0.899 & 0.875 & 0.855 & 0.850 & 0.826 & 0.826 & 0.841 \\
\hline
\end{tabular}


Table 7.32. Problem 6.1.1 reference solution MPACT power peaking factors for axial segments in element 4 for charge-pan III.

\begin{tabular}{|c|c|c|c|c|c|c|c|c|c|c|}
\hline \multirow[t]{2}{*}{ Channels } & \multicolumn{10}{|c|}{ Power Peaking Factors } \\
\hline & 1 & 2 & 3 & 4 & 5 & 6 & 7 & 8 & 9 & 10 \\
\hline A & 1.345 & 1.273 & 1.221 & 1.177 & 1.139 & 1.105 & 1.074 & 1.045 & 1.019 & 0.993 \\
\hline B & 1.356 & 1.285 & 1.233 & 1.189 & 1.151 & 1.117 & 1.087 & 1.058 & 1.032 & 1.007 \\
\hline $\mathrm{C}$ & 1.372 & 1.301 & 1.249 & 1.206 & 1.169 & 1.135 & 1.105 & 1.077 & 1.050 & 1.026 \\
\hline $\mathrm{D}$ & 1.356 & 1.284 & 1.233 & 1.189 & 1.151 & 1.117 & 1.086 & 1.058 & 1.032 & 1.007 \\
\hline $\mathrm{E}$ & 1.339 & 1.265 & 1.212 & 1.167 & 1.128 & 1.093 & 1.061 & 1.032 & 1.005 & 0.980 \\
\hline $\mathrm{F}$ & 1.334 & 1.261 & 1.207 & 1.162 & 1.123 & 1.088 & 1.056 & 1.027 & 1.000 & 0.975 \\
\hline G & 1.335 & 1.261 & 1.207 & 1.162 & 1.123 & 1.088 & 1.056 & 1.027 & 1.000 & 0.975 \\
\hline $\mathrm{H}$ & 1.339 & 1.265 & 1.212 & 1.167 & 1.128 & 1.093 & 1.061 & 1.032 & 1.005 & 0.980 \\
\hline $\mathrm{J}$ & 1.375 & 1.302 & 1.250 & 1.206 & 1.168 & 1.134 & 1.103 & 1.075 & 1.048 & 1.023 \\
\hline K & 1.393 & 1.321 & 1.269 & 1.225 & 1.188 & 1.154 & 1.124 & 1.096 & 1.070 & 1.045 \\
\hline $\mathrm{L}$ & 1.393 & 1.321 & 1.269 & 1.226 & 1.188 & 1.155 & 1.124 & 1.096 & 1.070 & 1.045 \\
\hline M & 1.375 & 1.302 & 1.250 & 1.206 & 1.168 & 1.134 & 1.103 & 1.075 & 1.048 & 1.023 \\
\hline $\mathrm{P}$ & 1.345 & 1.272 & 1.219 & 1.175 & 1.136 & 1.101 & 1.069 & 1.040 & 1.013 & 0.988 \\
\hline $\mathrm{Q}$ & 1.333 & 1.259 & 1.205 & 1.159 & 1.120 & 1.084 & 1.053 & 1.023 & 0.996 & 0.970 \\
\hline $\mathrm{R}$ & 1.345 & 1.272 & 1.219 & 1.175 & 1.136 & 1.101 & 1.069 & 1.040 & 1.013 & 0.988 \\
\hline S & 1.403 & 1.331 & 1.280 & 1.237 & 1.199 & 1.166 & 1.136 & 1.108 & 1.082 & 1.057 \\
\hline Channels & 11 & 12 & 13 & 14 & 15 & 16 & 17 & 18 & 19 & 20 \\
\hline A & 0.970 & 0.947 & 0.926 & 0.906 & 0.888 & 0.871 & 0.858 & 0.848 & 0.844 & 0.851 \\
\hline B & 0.983 & 0.960 & 0.939 & 0.919 & 0.901 & 0.885 & 0.872 & 0.862 & 0.858 & 0.865 \\
\hline $\mathrm{C}$ & 1.002 & 0.980 & 0.958 & 0.938 & 0.920 & 0.904 & 0.891 & 0.881 & 0.877 & 0.885 \\
\hline D & 0.983 & 0.960 & 0.939 & 0.919 & 0.901 & 0.885 & 0.871 & 0.862 & 0.857 & 0.865 \\
\hline $\mathrm{E}$ & 0.956 & 0.933 & 0.912 & 0.892 & 0.873 & 0.857 & 0.844 & 0.834 & 0.829 & 0.836 \\
\hline $\mathrm{F}$ & 0.951 & 0.928 & 0.906 & 0.886 & 0.868 & 0.852 & 0.839 & 0.829 & 0.824 & 0.832 \\
\hline G & 0.951 & 0.928 & 0.906 & 0.886 & 0.868 & 0.852 & 0.838 & 0.829 & 0.824 & 0.832 \\
\hline $\mathrm{H}$ & 0.956 & 0.933 & 0.912 & 0.892 & 0.873 & 0.857 & 0.844 & 0.834 & 0.830 & 0.837 \\
\hline $\mathrm{J}$ & 0.999 & 0.976 & 0.955 & 0.935 & 0.916 & 0.900 & 0.886 & 0.877 & 0.873 & 0.881 \\
\hline K & 1.021 & 0.998 & 0.977 & 0.957 & 0.938 & 0.922 & 0.908 & 0.899 & 0.895 & 0.903 \\
\hline $\mathrm{L}$ & 1.021 & 0.999 & 0.977 & 0.957 & 0.939 & 0.922 & 0.909 & 0.900 & 0.896 & 0.905 \\
\hline M & 0.999 & 0.976 & 0.955 & 0.935 & 0.916 & 0.900 & 0.887 & 0.877 & 0.873 & 0.881 \\
\hline $\mathrm{P}$ & 0.964 & 0.941 & 0.919 & 0.899 & 0.881 & 0.865 & 0.851 & 0.841 & 0.837 & 0.844 \\
\hline Q & 0.946 & 0.923 & 0.902 & 0.882 & 0.863 & 0.847 & 0.833 & 0.823 & 0.819 & 0.826 \\
\hline $\mathrm{R}$ & 0.964 & 0.941 & 0.919 & 0.899 & 0.881 & 0.864 & 0.851 & 0.841 & 0.836 & 0.843 \\
\hline $\mathrm{S}$ & 1.033 & 1.011 & 0.989 & 0.969 & 0.951 & 0.934 & 0.921 & 0.911 & 0.907 & 0.916 \\
\hline
\end{tabular}


Table 7.33. Problem 6.1.1 reference solution Shift power peaking factors for axial segments in element 5 for charge-pan III.

\begin{tabular}{|c|c|c|c|c|c|c|c|c|c|c|}
\hline \multirow[t]{2}{*}{ Channels } & \multicolumn{10}{|c|}{ Power Peaking Factors } \\
\hline & 1 & 2 & 3 & 4 & 5 & 6 & 7 & 8 & 9 & 10 \\
\hline A & 0.814 & 0.754 & 0.715 & 0.695 & 0.667 & 0.644 & 0.623 & 0.601 & 0.575 & 0.564 \\
\hline B & 0.796 & 0.744 & 0.708 & 0.677 & 0.656 & 0.633 & 0.606 & 0.587 & 0.567 & 0.547 \\
\hline $\mathrm{C}$ & 0.819 & 0.748 & 0.720 & 0.693 & 0.664 & 0.641 & 0.620 & 0.599 & 0.582 & 0.565 \\
\hline $\mathrm{D}$ & 0.833 & 0.767 & 0.739 & 0.712 & 0.685 & 0.653 & 0.635 & 0.607 & 0.590 & 0.568 \\
\hline $\mathrm{E}$ & 0.848 & 0.782 & 0.754 & 0.724 & 0.700 & 0.673 & 0.647 & 0.628 & 0.609 & 0.590 \\
\hline $\mathrm{F}$ & 0.835 & 0.768 & 0.733 & 0.704 & 0.681 & 0.652 & 0.629 & 0.611 & 0.593 & 0.571 \\
\hline G & 0.784 & 0.726 & 0.696 & 0.664 & 0.642 & 0.622 & 0.594 & 0.573 & 0.555 & 0.536 \\
\hline $\mathrm{H}$ & 0.784 & 0.726 & 0.690 & 0.669 & 0.641 & 0.618 & 0.591 & 0.569 & 0.552 & 0.532 \\
\hline $\mathrm{J}$ & 0.786 & 0.723 & 0.692 & 0.662 & 0.636 & 0.619 & 0.592 & 0.568 & 0.555 & 0.537 \\
\hline K & 0.783 & 0.724 & 0.696 & 0.667 & 0.639 & 0.616 & 0.599 & 0.577 & 0.560 & 0.539 \\
\hline $\mathrm{L}$ & 0.822 & 0.758 & 0.734 & 0.705 & 0.671 & 0.655 & 0.627 & 0.609 & 0.591 & 0.568 \\
\hline $\mathrm{M}$ & 0.844 & 0.784 & 0.755 & 0.723 & 0.696 & 0.673 & 0.650 & 0.620 & 0.606 & 0.585 \\
\hline $\mathrm{P}$ & 0.859 & 0.800 & 0.774 & 0.733 & 0.705 & 0.677 & 0.658 & 0.637 & 0.616 & 0.596 \\
\hline Q & 0.789 & 0.741 & 0.702 & 0.671 & 0.644 & 0.629 & 0.603 & 0.589 & 0.561 & 0.546 \\
\hline $\mathrm{R}$ & 0.777 & 0.718 & 0.684 & 0.657 & 0.631 & 0.608 & 0.592 & 0.567 & 0.552 & 0.527 \\
\hline S & 0.790 & 0.734 & 0.704 & 0.670 & 0.647 & 0.621 & 0.602 & 0.580 & 0.559 & 0.540 \\
\hline Channels & 11 & 12 & 13 & 14 & 15 & 16 & 17 & 18 & 19 & 20 \\
\hline A & 0.542 & 0.524 & 0.511 & 0.500 & 0.481 & 0.470 & 0.452 & 0.447 & 0.438 & 0.444 \\
\hline B & 0.532 & 0.516 & 0.500 & 0.488 & 0.478 & 0.462 & 0.448 & 0.436 & 0.430 & 0.432 \\
\hline $\mathrm{C}$ & 0.543 & 0.527 & 0.509 & 0.494 & 0.480 & 0.471 & 0.456 & 0.450 & 0.436 & 0.441 \\
\hline D & 0.554 & 0.542 & 0.526 & 0.511 & 0.495 & 0.476 & 0.466 & 0.456 & 0.452 & 0.457 \\
\hline $\mathrm{E}$ & 0.577 & 0.551 & 0.535 & 0.522 & 0.510 & 0.493 & 0.482 & 0.467 & 0.452 & 0.465 \\
\hline $\mathrm{F}$ & 0.548 & 0.533 & 0.514 & 0.509 & 0.490 & 0.481 & 0.470 & 0.451 & 0.449 & 0.450 \\
\hline G & 0.528 & 0.511 & 0.493 & 0.478 & 0.460 & 0.451 & 0.435 & 0.430 & 0.424 & 0.426 \\
\hline $\mathrm{H}$ & 0.511 & 0.499 & 0.482 & 0.470 & 0.452 & 0.440 & 0.438 & 0.423 & 0.412 & 0.421 \\
\hline $\mathrm{J}$ & 0.521 & 0.499 & 0.480 & 0.468 & 0.453 & 0.446 & 0.434 & 0.426 & 0.415 & 0.424 \\
\hline K & 0.526 & 0.501 & 0.498 & 0.479 & 0.460 & 0.451 & 0.438 & 0.430 & 0.424 & 0.423 \\
\hline $\mathrm{L}$ & 0.555 & 0.534 & 0.517 & 0.508 & 0.487 & 0.479 & 0.467 & 0.460 & 0.449 & 0.449 \\
\hline M & 0.571 & 0.555 & 0.538 & 0.516 & 0.506 & 0.492 & 0.484 & 0.469 & 0.458 & 0.461 \\
\hline $\mathrm{P}$ & 0.582 & 0.560 & 0.540 & 0.529 & 0.509 & 0.499 & 0.483 & 0.473 & 0.468 & 0.470 \\
\hline Q & 0.530 & 0.514 & 0.495 & 0.482 & 0.471 & 0.455 & 0.442 & 0.433 & 0.422 & 0.429 \\
\hline $\mathrm{R}$ & 0.512 & 0.494 & 0.479 & 0.467 & 0.453 & 0.439 & 0.428 & 0.420 & 0.411 & 0.421 \\
\hline $\mathrm{S}$ & 0.520 & 0.509 & 0.491 & 0.485 & 0.468 & 0.457 & 0.445 & 0.436 & 0.430 & 0.427 \\
\hline
\end{tabular}


Table 7.34. Problem 6.1.1 reference solution MPACT power peaking factors for axial segments in element 5 for charge-pan III.

\begin{tabular}{|c|c|c|c|c|c|c|c|c|c|c|}
\hline \multirow[t]{2}{*}{ Channels } & \multicolumn{10}{|c|}{ Power Peaking Factors } \\
\hline & 1 & 2 & 3 & 4 & 5 & 6 & 7 & 8 & 9 & 10 \\
\hline A & 0.804 & 0.757 & 0.721 & 0.691 & 0.665 & 0.641 & 0.619 & 0.599 & 0.580 & 0.562 \\
\hline B & 0.818 & 0.770 & 0.734 & 0.703 & 0.676 & 0.652 & 0.630 & 0.610 & 0.591 & 0.572 \\
\hline $\mathrm{C}$ & 0.837 & 0.788 & 0.751 & 0.720 & 0.693 & 0.668 & 0.646 & 0.625 & 0.605 & 0.587 \\
\hline $\mathrm{D}$ & 0.818 & 0.769 & 0.734 & 0.703 & 0.676 & 0.652 & 0.630 & 0.610 & 0.590 & 0.572 \\
\hline $\mathrm{E}$ & 0.789 & 0.743 & 0.708 & 0.678 & 0.652 & 0.629 & 0.608 & 0.588 & 0.569 & 0.552 \\
\hline $\mathrm{F}$ & 0.785 & 0.738 & 0.703 & 0.674 & 0.648 & 0.624 & 0.603 & 0.583 & 0.565 & 0.547 \\
\hline G & 0.785 & 0.738 & 0.703 & 0.674 & 0.648 & 0.624 & 0.603 & 0.583 & 0.565 & 0.547 \\
\hline $\mathrm{H}$ & 0.791 & 0.744 & 0.709 & 0.679 & 0.653 & 0.629 & 0.608 & 0.588 & 0.569 & 0.552 \\
\hline $\mathrm{J}$ & 0.833 & 0.783 & 0.747 & 0.716 & 0.689 & 0.664 & 0.642 & 0.621 & 0.602 & 0.583 \\
\hline K & 0.854 & 0.804 & 0.767 & 0.735 & 0.707 & 0.683 & 0.660 & 0.639 & 0.619 & 0.600 \\
\hline $\mathrm{L}$ & 0.856 & 0.805 & 0.768 & 0.736 & 0.708 & 0.683 & 0.660 & 0.639 & 0.619 & 0.600 \\
\hline $\mathrm{M}$ & 0.833 & 0.784 & 0.747 & 0.716 & 0.689 & 0.664 & 0.642 & 0.621 & 0.602 & 0.583 \\
\hline $\mathrm{P}$ & 0.797 & 0.750 & 0.715 & 0.685 & 0.659 & 0.635 & 0.614 & 0.594 & 0.575 & 0.557 \\
\hline Q & 0.779 & 0.733 & 0.698 & 0.668 & 0.642 & 0.619 & 0.598 & 0.578 & 0.559 & 0.542 \\
\hline $\mathrm{R}$ & 0.796 & 0.749 & 0.714 & 0.685 & 0.658 & 0.635 & 0.613 & 0.594 & 0.575 & 0.557 \\
\hline S & 0.866 & 0.816 & 0.778 & 0.746 & 0.718 & 0.693 & 0.670 & 0.648 & 0.628 & 0.609 \\
\hline Channels & 11 & 12 & 13 & 14 & 15 & 16 & 17 & 18 & 19 & 20 \\
\hline A & 0.545 & 0.528 & 0.512 & 0.498 & 0.484 & 0.471 & 0.459 & 0.450 & 0.443 & 0.443 \\
\hline B & 0.555 & 0.538 & 0.522 & 0.507 & 0.493 & 0.480 & 0.469 & 0.459 & 0.452 & 0.452 \\
\hline $\mathrm{C}$ & 0.569 & 0.552 & 0.536 & 0.520 & 0.506 & 0.493 & 0.481 & 0.472 & 0.465 & 0.464 \\
\hline D & 0.555 & 0.538 & 0.522 & 0.507 & 0.493 & 0.480 & 0.469 & 0.459 & 0.453 & 0.452 \\
\hline $\mathrm{E}$ & 0.535 & 0.519 & 0.503 & 0.488 & 0.475 & 0.462 & 0.451 & 0.442 & 0.435 & 0.435 \\
\hline $\mathrm{F}$ & 0.530 & 0.514 & 0.499 & 0.484 & 0.470 & 0.458 & 0.446 & 0.437 & 0.431 & 0.430 \\
\hline G & 0.530 & 0.514 & 0.498 & 0.484 & 0.470 & 0.457 & 0.446 & 0.437 & 0.430 & 0.430 \\
\hline $\mathrm{H}$ & 0.535 & 0.519 & 0.503 & 0.489 & 0.475 & 0.462 & 0.451 & 0.442 & 0.435 & 0.435 \\
\hline $\mathrm{J}$ & 0.566 & 0.549 & 0.533 & 0.517 & 0.503 & 0.490 & 0.478 & 0.469 & 0.462 & 0.462 \\
\hline K & 0.582 & 0.565 & 0.548 & 0.532 & 0.518 & 0.504 & 0.492 & 0.483 & 0.476 & 0.475 \\
\hline $\mathrm{L}$ & 0.582 & 0.565 & 0.548 & 0.533 & 0.518 & 0.505 & 0.493 & 0.483 & 0.476 & 0.476 \\
\hline M & 0.566 & 0.549 & 0.533 & 0.517 & 0.503 & 0.490 & 0.478 & 0.468 & 0.462 & 0.461 \\
\hline $\mathrm{P}$ & 0.540 & 0.524 & 0.508 & 0.494 & 0.480 & 0.467 & 0.456 & 0.446 & 0.440 & 0.439 \\
\hline Q & 0.525 & 0.509 & 0.493 & 0.479 & 0.465 & 0.452 & 0.442 & 0.433 & 0.427 & 0.427 \\
\hline $\mathrm{R}$ & 0.540 & 0.524 & 0.508 & 0.494 & 0.480 & 0.467 & 0.456 & 0.446 & 0.440 & 0.439 \\
\hline $\mathrm{S}$ & 0.591 & 0.573 & 0.557 & 0.541 & 0.526 & 0.512 & 0.500 & 0.490 & 0.484 & 0.483 \\
\hline
\end{tabular}


Table 7.35. Problem 6.1.1 reference solution Shift power peaking factors for axial segments in element 6 for charge-pan III.

\begin{tabular}{|c|c|c|c|c|c|c|c|c|c|c|}
\hline \multirow[t]{2}{*}{ Channels } & \multicolumn{10}{|c|}{ Power Peaking Factors } \\
\hline & 1 & 2 & 3 & 4 & 5 & 6 & 7 & 8 & 9 & 10 \\
\hline A & 0.406 & 0.375 & 0.353 & 0.336 & 0.316 & 0.304 & 0.287 & 0.273 & 0.264 & 0.249 \\
\hline B & 0.402 & 0.363 & 0.346 & 0.329 & 0.313 & 0.300 & 0.286 & 0.272 & 0.256 & 0.241 \\
\hline $\mathrm{C}$ & 0.406 & 0.368 & 0.356 & 0.332 & 0.316 & 0.299 & 0.286 & 0.273 & 0.257 & 0.247 \\
\hline D & 0.420 & 0.387 & 0.364 & 0.343 & 0.326 & 0.309 & 0.294 & 0.281 & 0.270 & 0.255 \\
\hline $\mathrm{E}$ & 0.429 & 0.395 & 0.372 & 0.350 & 0.336 & 0.319 & 0.303 & 0.284 & 0.275 & 0.259 \\
\hline $\mathrm{F}$ & 0.416 & 0.381 & 0.361 & 0.343 & 0.323 & 0.304 & 0.297 & 0.275 & 0.265 & 0.251 \\
\hline G & 0.388 & 0.360 & 0.341 & 0.324 & 0.306 & 0.291 & 0.273 & 0.262 & 0.250 & 0.238 \\
\hline $\mathrm{H}$ & 0.387 & 0.354 & 0.338 & 0.313 & 0.302 & 0.289 & 0.272 & 0.260 & 0.248 & 0.236 \\
\hline $\mathrm{J}$ & 0.390 & 0.357 & 0.337 & 0.320 & 0.301 & 0.288 & 0.270 & 0.259 & 0.251 & 0.237 \\
\hline $\mathrm{K}$ & 0.393 & 0.356 & 0.338 & 0.322 & 0.305 & 0.290 & 0.275 & 0.258 & 0.251 & 0.237 \\
\hline $\mathrm{L}$ & 0.416 & 0.381 & 0.360 & 0.341 & 0.329 & 0.309 & 0.295 & 0.275 & 0.262 & 0.248 \\
\hline M & 0.423 & 0.395 & 0.373 & 0.352 & 0.337 & 0.319 & 0.298 & 0.289 & 0.274 & 0.260 \\
\hline $\mathrm{P}$ & 0.435 & 0.397 & 0.377 & 0.363 & 0.341 & 0.323 & 0.306 & 0.292 & 0.281 & 0.265 \\
\hline Q & 0.395 & 0.364 & 0.345 & 0.322 & 0.307 & 0.291 & 0.277 & 0.264 & 0.253 & 0.242 \\
\hline $\mathrm{R}$ & 0.387 & 0.356 & 0.336 & 0.318 & 0.303 & 0.293 & 0.280 & 0.261 & 0.247 & 0.238 \\
\hline S & 0.396 & 0.362 & 0.345 & 0.328 & 0.306 & 0.291 & 0.276 & 0.263 & 0.250 & 0.239 \\
\hline Channels & 11 & 12 & 13 & 14 & 15 & 16 & 17 & 18 & 19 & 20 \\
\hline A & 0.233 & 0.222 & 0.212 & 0.201 & 0.187 & 0.179 & 0.173 & 0.166 & 0.163 & 0.163 \\
\hline B & 0.226 & 0.220 & 0.207 & 0.194 & 0.184 & 0.177 & 0.168 & 0.159 & 0.161 & 0.157 \\
\hline $\mathrm{C}$ & 0.235 & 0.224 & 0.211 & 0.202 & 0.189 & 0.183 & 0.173 & 0.167 & 0.162 & 0.163 \\
\hline D & 0.240 & 0.230 & 0.215 & 0.206 & 0.194 & 0.185 & 0.177 & 0.173 & 0.166 & 0.169 \\
\hline $\mathrm{E}$ & 0.246 & 0.234 & 0.222 & 0.212 & 0.204 & 0.190 & 0.183 & 0.176 & 0.171 & 0.170 \\
\hline $\mathrm{F}$ & 0.239 & 0.228 & 0.213 & 0.205 & 0.191 & 0.183 & 0.174 & 0.168 & 0.163 & 0.164 \\
\hline G & 0.224 & 0.214 & 0.204 & 0.194 & 0.182 & 0.172 & 0.166 & 0.161 & 0.154 & 0.157 \\
\hline $\mathrm{H}$ & 0.224 & 0.207 & 0.201 & 0.189 & 0.177 & 0.170 & 0.161 & 0.157 & 0.150 & 0.151 \\
\hline J & 0.224 & 0.210 & 0.199 & 0.194 & 0.183 & 0.169 & 0.162 & 0.161 & 0.150 & 0.153 \\
\hline K & 0.225 & 0.215 & 0.202 & 0.192 & 0.180 & 0.175 & 0.165 & 0.159 & 0.151 & 0.153 \\
\hline $\mathrm{L}$ & 0.238 & 0.228 & 0.219 & 0.203 & 0.193 & 0.187 & 0.174 & 0.170 & 0.164 & 0.166 \\
\hline $\mathrm{M}$ & 0.244 & 0.236 & 0.226 & 0.213 & 0.201 & 0.195 & 0.183 & 0.178 & 0.169 & 0.172 \\
\hline $\mathrm{P}$ & 0.252 & 0.237 & 0.224 & 0.217 & 0.205 & 0.195 & 0.185 & 0.176 & 0.175 & 0.179 \\
\hline Q & 0.225 & 0.215 & 0.204 & 0.195 & 0.183 & 0.175 & 0.170 & 0.157 & 0.154 & 0.158 \\
\hline $\mathrm{R}$ & 0.223 & 0.213 & 0.202 & 0.193 & 0.182 & 0.171 & 0.163 & 0.157 & 0.151 & 0.152 \\
\hline $\mathrm{S}$ & 0.228 & 0.216 & 0.204 & 0.195 & 0.182 & 0.173 & 0.168 & 0.164 & 0.156 & 0.155 \\
\hline
\end{tabular}


Table 7.36. Problem 6.1.1 reference solution MPACT power peaking factors for axial segments in element 6 for charge-pan III.

\begin{tabular}{|c|c|c|c|c|c|c|c|c|c|c|}
\hline \multirow[t]{2}{*}{ Channels } & \multicolumn{10}{|c|}{ Power Peaking Factors } \\
\hline & 1 & 2 & 3 & 4 & 5 & 6 & 7 & 8 & 9 & 10 \\
\hline $\mathrm{A}$ & 0.408 & 0.380 & 0.358 & 0.338 & 0.320 & 0.304 & 0.289 & 0.275 & 0.261 & 0.248 \\
\hline B & 0.417 & 0.388 & 0.365 & 0.345 & 0.327 & 0.311 & 0.295 & 0.281 & 0.267 & 0.254 \\
\hline $\mathrm{C}$ & 0.429 & 0.399 & 0.376 & 0.355 & 0.337 & 0.320 & 0.304 & 0.289 & 0.275 & 0.261 \\
\hline D & 0.417 & 0.388 & 0.365 & 0.346 & 0.328 & 0.311 & 0.296 & 0.281 & 0.267 & 0.254 \\
\hline $\mathrm{E}$ & 0.401 & 0.373 & 0.351 & 0.331 & 0.314 & 0.298 & 0.283 & 0.269 & 0.256 & 0.243 \\
\hline $\mathrm{F}$ & 0.397 & 0.369 & 0.347 & 0.328 & 0.311 & 0.295 & 0.281 & 0.267 & 0.254 & 0.241 \\
\hline G & 0.397 & 0.369 & 0.347 & 0.328 & 0.311 & 0.295 & 0.281 & 0.267 & 0.253 & 0.241 \\
\hline $\mathrm{H}$ & 0.401 & 0.373 & 0.351 & 0.332 & 0.314 & 0.298 & 0.283 & 0.269 & 0.256 & 0.243 \\
\hline $\mathrm{J}$ & 0.426 & 0.396 & 0.373 & 0.353 & 0.334 & 0.318 & 0.302 & 0.287 & 0.273 & 0.259 \\
\hline K & 0.439 & 0.408 & 0.385 & 0.364 & 0.345 & 0.328 & 0.312 & 0.296 & 0.282 & 0.268 \\
\hline $\mathrm{L}$ & 0.440 & 0.409 & 0.385 & 0.364 & 0.345 & 0.328 & 0.312 & 0.296 & 0.282 & 0.268 \\
\hline M & 0.426 & 0.396 & 0.373 & 0.353 & 0.334 & 0.317 & 0.302 & 0.287 & 0.273 & 0.259 \\
\hline $\mathrm{P}$ & 0.405 & 0.377 & 0.355 & 0.335 & 0.318 & 0.302 & 0.287 & 0.273 & 0.259 & 0.246 \\
\hline$Q$ & 0.396 & 0.369 & 0.348 & 0.330 & 0.313 & 0.298 & 0.283 & 0.269 & 0.256 & 0.244 \\
\hline $\mathrm{R}$ & 0.405 & 0.377 & 0.355 & 0.335 & 0.318 & 0.302 & 0.287 & 0.273 & 0.259 & 0.246 \\
\hline$S$ & 0.447 & 0.415 & 0.391 & 0.370 & 0.351 & 0.333 & 0.317 & 0.301 & 0.287 & 0.272 \\
\hline Channels & 11 & 12 & 13 & 14 & 15 & 16 & 17 & 18 & 19 & 20 \\
\hline A & 0.235 & 0.223 & 0.212 & 0.201 & 0.190 & 0.181 & 0.173 & 0.166 & 0.161 & 0.160 \\
\hline B & 0.241 & 0.228 & 0.217 & 0.205 & 0.195 & 0.185 & 0.177 & 0.170 & 0.165 & 0.164 \\
\hline $\mathrm{C}$ & 0.248 & 0.235 & 0.223 & 0.212 & 0.201 & 0.191 & 0.182 & 0.175 & 0.170 & 0.169 \\
\hline D & 0.241 & 0.229 & 0.217 & 0.206 & 0.195 & 0.185 & 0.177 & 0.170 & 0.165 & 0.164 \\
\hline E & 0.231 & 0.219 & 0.207 & 0.197 & 0.186 & 0.177 & 0.169 & 0.162 & 0.157 & 0.156 \\
\hline $\mathrm{F}$ & 0.229 & 0.217 & 0.205 & 0.195 & 0.185 & 0.175 & 0.167 & 0.160 & 0.156 & 0.154 \\
\hline G & 0.229 & 0.217 & 0.205 & 0.195 & 0.184 & 0.175 & 0.167 & 0.160 & 0.156 & 0.154 \\
\hline $\mathrm{H}$ & 0.231 & 0.219 & 0.207 & 0.197 & 0.186 & 0.177 & 0.169 & 0.162 & 0.157 & 0.156 \\
\hline $\mathrm{J}$ & 0.246 & 0.234 & 0.221 & 0.210 & 0.199 & 0.189 & 0.180 & 0.173 & 0.168 & 0.167 \\
\hline $\mathrm{K}$ & 0.254 & 0.241 & 0.229 & 0.217 & 0.206 & 0.196 & 0.187 & 0.179 & 0.174 & 0.173 \\
\hline $\mathrm{L}$ & 0.254 & 0.241 & 0.229 & 0.217 & 0.206 & 0.196 & 0.187 & 0.179 & 0.174 & 0.173 \\
\hline M & 0.246 & 0.234 & 0.221 & 0.210 & 0.199 & 0.189 & 0.180 & 0.173 & 0.168 & 0.167 \\
\hline $\mathrm{P}$ & 0.234 & 0.222 & 0.210 & 0.199 & 0.189 & 0.179 & 0.171 & 0.164 & 0.159 & 0.158 \\
\hline Q & 0.231 & 0.219 & 0.208 & 0.197 & 0.187 & 0.177 & 0.169 & 0.162 & 0.157 & 0.155 \\
\hline $\mathrm{R}$ & 0.234 & 0.222 & 0.210 & 0.199 & 0.189 & 0.179 & 0.171 & 0.164 & 0.159 & 0.158 \\
\hline $\mathrm{S}$ & 0.259 & 0.246 & 0.233 & 0.221 & 0.209 & 0.199 & 0.190 & 0.182 & 0.177 & 0.176 \\
\hline
\end{tabular}




\section{Charge-Pan IV}

Table 7.37. Problem 6.1.1 reference solution Shift power peaking factors for axial segments in element 1 for charge-pan IV.

\begin{tabular}{|c|c|c|c|c|c|c|c|c|c|c|}
\hline \multirow[t]{2}{*}{ Channels } & \multicolumn{10}{|c|}{ Power Peaking Factors } \\
\hline & 1 & 2 & 3 & 4 & 5 & 6 & 7 & 8 & 9 & 10 \\
\hline A & 0.802 & 0.782 & 0.788 & 0.817 & 0.829 & 0.856 & 0.885 & 0.926 & 0.960 & 1.000 \\
\hline B & 0.804 & 0.776 & 0.786 & 0.811 & 0.835 & 0.861 & 0.887 & 0.925 & 0.960 & 0.991 \\
\hline $\mathrm{C}$ & 0.812 & 0.785 & 0.792 & 0.813 & 0.840 & 0.857 & 0.889 & 0.919 & 0.957 & 0.989 \\
\hline $\mathrm{D}$ & 0.803 & 0.782 & 0.790 & 0.816 & 0.833 & 0.858 & 0.893 & 0.924 & 0.964 & 0.991 \\
\hline $\mathrm{E}$ & 0.808 & 0.789 & 0.796 & 0.814 & 0.832 & 0.869 & 0.902 & 0.931 & 0.969 & 1.003 \\
\hline $\mathrm{F}$ & 0.805 & 0.786 & 0.799 & 0.812 & 0.844 & 0.867 & 0.906 & 0.928 & 0.966 & 0.996 \\
\hline G & 0.803 & 0.792 & 0.801 & 0.813 & 0.834 & 0.869 & 0.895 & 0.931 & 0.966 & 1.000 \\
\hline $\mathrm{H}$ & 0.811 & 0.784 & 0.790 & 0.813 & 0.835 & 0.867 & 0.898 & 0.931 & 0.967 & 1.004 \\
\hline $\mathrm{J}$ & 0.800 & 0.788 & 0.799 & 0.814 & 0.841 & 0.864 & 0.897 & 0.925 & 0.970 & 1.000 \\
\hline K & 0.809 & 0.787 & 0.801 & 0.810 & 0.832 & 0.865 & 0.899 & 0.926 & 0.961 & 1.001 \\
\hline $\mathrm{L}$ & 0.806 & 0.786 & 0.797 & 0.811 & 0.840 & 0.866 & 0.895 & 0.929 & 0.966 & 1.004 \\
\hline M & 0.803 & 0.784 & 0.790 & 0.813 & 0.838 & 0.865 & 0.897 & 0.939 & 0.971 & 1.004 \\
\hline $\mathrm{P}$ & 0.806 & 0.784 & 0.791 & 0.812 & 0.837 & 0.869 & 0.900 & 0.928 & 0.965 & 1.002 \\
\hline Q & 0.804 & 0.785 & 0.794 & 0.816 & 0.841 & 0.869 & 0.909 & 0.932 & 0.961 & 1.005 \\
\hline $\mathrm{R}$ & 0.810 & 0.790 & 0.792 & 0.815 & 0.839 & 0.865 & 0.899 & 0.933 & 0.973 & 1.010 \\
\hline S & 0.804 & 0.785 & 0.797 & 0.816 & 0.838 & 0.864 & 0.894 & 0.927 & 0.969 & 1.001 \\
\hline Channels & 11 & 12 & 13 & 14 & 15 & 16 & 17 & 18 & 19 & 20 \\
\hline A & 1.025 & 1.067 & 1.098 & 1.133 & 1.176 & 1.220 & 1.267 & 1.308 & 1.365 & 1.467 \\
\hline B & 1.025 & 1.072 & 1.103 & 1.140 & 1.180 & 1.228 & 1.267 & 1.302 & 1.365 & 1.454 \\
\hline $\mathrm{C}$ & 1.025 & 1.064 & 1.103 & 1.138 & 1.193 & 1.222 & 1.272 & 1.310 & 1.356 & 1.462 \\
\hline D & 1.021 & 1.067 & 1.100 & 1.143 & 1.182 & 1.221 & 1.262 & 1.310 & 1.357 & 1.455 \\
\hline $\mathrm{E}$ & 1.042 & 1.073 & 1.124 & 1.156 & 1.197 & 1.241 & 1.275 & 1.330 & 1.372 & 1.468 \\
\hline $\mathrm{F}$ & 1.044 & 1.081 & 1.114 & 1.153 & 1.197 & 1.242 & 1.284 & 1.327 & 1.376 & 1.469 \\
\hline G & 1.042 & 1.069 & 1.112 & 1.145 & 1.194 & 1.237 & 1.275 & 1.323 & 1.381 & 1.464 \\
\hline $\mathrm{H}$ & 1.038 & 1.076 & 1.109 & 1.154 & 1.193 & 1.243 & 1.290 & 1.328 & 1.385 & 1.485 \\
\hline $\mathrm{J}$ & 1.044 & 1.072 & 1.108 & 1.153 & 1.196 & 1.233 & 1.283 & 1.322 & 1.370 & 1.477 \\
\hline K & 1.041 & 1.078 & 1.118 & 1.150 & 1.198 & 1.236 & 1.275 & 1.323 & 1.378 & 1.470 \\
\hline $\mathrm{L}$ & 1.041 & 1.078 & 1.117 & 1.151 & 1.185 & 1.230 & 1.279 & 1.329 & 1.382 & 1.481 \\
\hline $\mathrm{M}$ & 1.030 & 1.080 & 1.116 & 1.153 & 1.189 & 1.235 & 1.277 & 1.322 & 1.382 & 1.467 \\
\hline $\mathrm{P}$ & 1.041 & 1.075 & 1.115 & 1.160 & 1.196 & 1.235 & 1.278 & 1.326 & 1.366 & 1.471 \\
\hline Q & 1.042 & 1.076 & 1.119 & 1.164 & 1.185 & 1.228 & 1.271 & 1.326 & 1.386 & 1.473 \\
\hline $\mathrm{R}$ & 1.044 & 1.080 & 1.116 & 1.159 & 1.203 & 1.242 & 1.290 & 1.328 & 1.380 & 1.483 \\
\hline $\mathrm{S}$ & 1.039 & 1.069 & 1.109 & 1.155 & 1.191 & 1.231 & 1.275 & 1.329 & 1.376 & 1.472 \\
\hline
\end{tabular}


Table 7.38. Problem 6.1.1 reference solution MPACT power peaking factors for axial segments in element 1 for charge-pan IV.

\begin{tabular}{|c|c|c|c|c|c|c|c|c|c|c|}
\hline \multirow[t]{2}{*}{ Channels } & \multicolumn{10}{|c|}{ Power Peaking Factors } \\
\hline & 1 & 2 & 3 & 4 & 5 & 6 & 7 & 8 & 9 & 10 \\
\hline A & 0.781 & 0.770 & 0.778 & 0.795 & 0.818 & 0.847 & 0.879 & 0.912 & 0.948 & 0.984 \\
\hline B & 0.781 & 0.770 & 0.778 & 0.795 & 0.818 & 0.847 & 0.879 & 0.912 & 0.948 & 0.984 \\
\hline $\mathrm{C}$ & 0.780 & 0.770 & 0.777 & 0.795 & 0.818 & 0.847 & 0.879 & 0.912 & 0.948 & 0.983 \\
\hline $\mathrm{D}$ & 0.780 & 0.770 & 0.777 & 0.795 & 0.818 & 0.847 & 0.879 & 0.912 & 0.947 & 0.983 \\
\hline $\mathrm{E}$ & 0.786 & 0.776 & 0.783 & 0.801 & 0.825 & 0.854 & 0.887 & 0.921 & 0.957 & 0.993 \\
\hline $\mathrm{F}$ & 0.786 & 0.775 & 0.783 & 0.801 & 0.825 & 0.854 & 0.887 & 0.921 & 0.957 & 0.993 \\
\hline G & 0.786 & 0.775 & 0.783 & 0.801 & 0.825 & 0.854 & 0.886 & 0.921 & 0.957 & 0.993 \\
\hline $\mathrm{H}$ & 0.786 & 0.775 & 0.783 & 0.801 & 0.825 & 0.854 & 0.887 & 0.921 & 0.957 & 0.993 \\
\hline $\mathrm{J}$ & 0.786 & 0.775 & 0.783 & 0.801 & 0.825 & 0.854 & 0.886 & 0.921 & 0.957 & 0.993 \\
\hline K & 0.785 & 0.775 & 0.783 & 0.800 & 0.825 & 0.854 & 0.886 & 0.921 & 0.957 & 0.993 \\
\hline $\mathrm{L}$ & 0.786 & 0.775 & 0.783 & 0.801 & 0.825 & 0.854 & 0.887 & 0.921 & 0.957 & 0.994 \\
\hline M & 0.786 & 0.775 & 0.783 & 0.801 & 0.825 & 0.854 & 0.887 & 0.921 & 0.957 & 0.993 \\
\hline $\mathrm{P}$ & 0.786 & 0.776 & 0.784 & 0.802 & 0.826 & 0.856 & 0.888 & 0.923 & 0.958 & 0.995 \\
\hline Q & 0.787 & 0.777 & 0.784 & 0.802 & 0.826 & 0.856 & 0.888 & 0.923 & 0.958 & 0.995 \\
\hline $\mathrm{R}$ & 0.787 & 0.776 & 0.784 & 0.802 & 0.826 & 0.855 & 0.888 & 0.922 & 0.958 & 0.995 \\
\hline S & 0.787 & 0.776 & 0.784 & 0.802 & 0.826 & 0.855 & 0.888 & 0.923 & 0.958 & 0.995 \\
\hline Channels & 11 & 12 & 13 & 14 & $\overline{15}$ & $\overline{16}$ & 17 & $\overline{18}$ & 19 & 20 \\
\hline A & 1.020 & 1.057 & 1.094 & 1.133 & 1.172 & 1.214 & 1.259 & 1.309 & 1.366 & 1.444 \\
\hline B & 1.020 & 1.057 & 1.094 & 1.133 & 1.172 & 1.214 & 1.259 & 1.309 & 1.367 & 1.444 \\
\hline $\mathrm{C}$ & 1.020 & 1.057 & 1.094 & 1.133 & 1.172 & 1.214 & 1.259 & 1.309 & 1.367 & 1.444 \\
\hline D & 1.020 & 1.057 & 1.094 & 1.133 & 1.172 & 1.214 & 1.259 & 1.309 & 1.367 & 1.444 \\
\hline $\mathrm{E}$ & 1.030 & 1.068 & 1.105 & 1.144 & 1.184 & 1.226 & 1.272 & 1.322 & 1.381 & 1.460 \\
\hline $\mathrm{F}$ & 1.030 & 1.068 & 1.105 & 1.144 & 1.184 & 1.226 & 1.272 & 1.322 & 1.381 & 1.460 \\
\hline G & 1.030 & 1.067 & 1.105 & 1.144 & 1.184 & 1.226 & 1.271 & 1.322 & 1.380 & 1.459 \\
\hline $\mathrm{H}$ & 1.030 & 1.067 & 1.105 & 1.144 & 1.184 & 1.226 & 1.271 & 1.322 & 1.380 & 1.459 \\
\hline $\mathrm{J}$ & 1.030 & 1.067 & 1.105 & 1.143 & 1.183 & 1.225 & 1.271 & 1.321 & 1.379 & 1.457 \\
\hline K & 1.030 & 1.067 & 1.105 & 1.143 & 1.183 & 1.226 & 1.271 & 1.322 & 1.380 & 1.459 \\
\hline $\mathrm{L}$ & 1.030 & 1.068 & 1.106 & 1.144 & 1.184 & 1.227 & 1.272 & 1.323 & 1.381 & 1.460 \\
\hline $\mathrm{M}$ & 1.030 & 1.068 & 1.105 & 1.144 & 1.184 & 1.226 & 1.272 & 1.322 & 1.381 & 1.460 \\
\hline $\mathrm{P}$ & 1.032 & 1.069 & 1.107 & 1.146 & 1.186 & 1.228 & 1.273 & 1.324 & 1.382 & 1.461 \\
\hline Q & 1.032 & 1.069 & 1.107 & 1.146 & 1.186 & 1.228 & 1.273 & 1.324 & 1.382 & 1.460 \\
\hline $\mathrm{R}$ & 1.032 & 1.069 & 1.107 & 1.145 & 1.185 & 1.228 & 1.273 & 1.324 & 1.382 & 1.460 \\
\hline $\mathrm{S}$ & 1.032 & 1.069 & 1.107 & 1.146 & 1.186 & 1.228 & 1.273 & 1.324 & 1.382 & 1.461 \\
\hline
\end{tabular}


Table 7.39. Problem 6.1.1 reference solution Shift power peaking factors for axial segments in element 2 for charge-pan IV.

\begin{tabular}{|c|c|c|c|c|c|c|c|c|c|c|}
\hline \multirow[t]{2}{*}{ Channels } & \multicolumn{10}{|c|}{ Power Peaking Factors } \\
\hline & 1 & 2 & 3 & 4 & 5 & 6 & 7 & 8 & 9 & 10 \\
\hline A & 1.517 & 1.469 & 1.471 & 1.476 & 1.478 & 1.480 & 1.497 & 1.505 & 1.515 & 1.535 \\
\hline B & 1.515 & 1.479 & 1.476 & 1.465 & 1.487 & 1.493 & 1.498 & 1.502 & 1.515 & 1.538 \\
\hline $\mathrm{C}$ & 1.517 & 1.473 & 1.473 & 1.474 & 1.486 & 1.490 & 1.500 & 1.499 & 1.509 & 1.531 \\
\hline $\mathrm{D}$ & 1.527 & 1.479 & 1.474 & 1.467 & 1.470 & 1.487 & 1.496 & 1.500 & 1.523 & 1.540 \\
\hline $\mathrm{E}$ & 1.536 & 1.484 & 1.491 & 1.485 & 1.502 & 1.499 & 1.512 & 1.524 & 1.537 & 1.545 \\
\hline $\mathrm{F}$ & 1.541 & 1.491 & 1.493 & 1.491 & 1.502 & 1.497 & 1.503 & 1.523 & 1.538 & 1.540 \\
\hline G & 1.538 & 1.488 & 1.484 & 1.489 & 1.492 & 1.504 & 1.503 & 1.522 & 1.536 & 1.549 \\
\hline $\mathrm{H}$ & 1.534 & 1.492 & 1.489 & 1.489 & 1.491 & 1.506 & 1.513 & 1.515 & 1.528 & 1.547 \\
\hline $\mathrm{J}$ & 1.535 & 1.498 & 1.487 & 1.494 & 1.494 & 1.510 & 1.507 & 1.532 & 1.550 & 1.551 \\
\hline $\mathrm{K}$ & 1.531 & 1.493 & 1.491 & 1.489 & 1.497 & 1.510 & 1.513 & 1.526 & 1.536 & 1.546 \\
\hline $\mathrm{L}$ & 1.537 & 1.490 & 1.487 & 1.486 & 1.485 & 1.501 & 1.516 & 1.523 & 1.541 & 1.550 \\
\hline $\mathrm{M}$ & 1.538 & 1.476 & 1.493 & 1.486 & 1.486 & 1.507 & 1.512 & 1.532 & 1.536 & 1.549 \\
\hline $\mathrm{P}$ & 1.537 & 1.498 & 1.488 & 1.492 & 1.494 & 1.501 & 1.524 & 1.529 & 1.539 & 1.556 \\
\hline Q & 1.530 & 1.495 & 1.488 & 1.493 & 1.495 & 1.501 & 1.525 & 1.531 & 1.544 & 1.554 \\
\hline $\mathrm{R}$ & 1.543 & 1.496 & 1.494 & 1.492 & 1.500 & 1.503 & 1.513 & 1.521 & 1.532 & 1.541 \\
\hline $\mathrm{S}$ & 1.541 & 1.490 & 1.480 & 1.482 & 1.494 & 1.505 & 1.520 & 1.526 & 1.547 & 1.558 \\
\hline "Channels & 11 & 12 & 13 & 14 & $\overline{15}$ & $\overline{16}$ & 17 & 18 & 19 & 20 \\
\hline A & 1.540 & 1.553 & 1.561 & 1.582 & 1.594 & 1.609 & 1.630 & 1.653 & 1.673 & 1.758 \\
\hline B & 1.543 & 1.558 & 1.558 & 1.577 & 1.591 & 1.607 & 1.630 & 1.648 & 1.666 & 1.744 \\
\hline $\mathrm{C}$ & 1.547 & 1.559 & 1.567 & 1.578 & 1.597 & 1.611 & 1.623 & 1.648 & 1.664 & 1.754 \\
\hline D & 1.548 & 1.553 & 1.566 & 1.587 & 1.600 & 1.606 & 1.627 & 1.643 & 1.670 & 1.752 \\
\hline $\mathrm{E}$ & 1.563 & 1.568 & 1.583 & 1.589 & 1.613 & 1.616 & 1.636 & 1.671 & 1.696 & 1.772 \\
\hline $\mathrm{F}$ & 1.563 & 1.564 & 1.579 & 1.589 & 1.602 & 1.618 & 1.638 & 1.658 & 1.692 & 1.779 \\
\hline G & 1.558 & 1.558 & 1.577 & 1.590 & 1.598 & 1.621 & 1.644 & 1.668 & 1.691 & 1.769 \\
\hline $\mathrm{H}$ & 1.555 & 1.568 & 1.577 & 1.604 & 1.613 & 1.627 & 1.645 & 1.665 & 1.693 & 1.787 \\
\hline $\mathrm{J}$ & 1.563 & 1.575 & 1.581 & 1.598 & 1.613 & 1.625 & 1.642 & 1.663 & 1.697 & 1.777 \\
\hline K & 1.561 & 1.570 & 1.590 & 1.608 & 1.608 & 1.629 & 1.655 & 1.664 & 1.703 & 1.776 \\
\hline $\mathrm{L}$ & 1.560 & 1.572 & 1.575 & 1.594 & 1.607 & 1.630 & 1.642 & 1.670 & 1.693 & 1.780 \\
\hline M & 1.566 & 1.573 & 1.580 & 1.608 & 1.607 & 1.620 & 1.645 & 1.665 & 1.692 & 1.780 \\
\hline $\mathrm{P}$ & 1.559 & 1.572 & 1.579 & 1.600 & 1.603 & 1.624 & 1.645 & 1.665 & 1.696 & 1.778 \\
\hline $\mathrm{Q}$ & 1.567 & 1.582 & 1.588 & 1.592 & 1.611 & 1.628 & 1.639 & 1.662 & 1.691 & 1.780 \\
\hline $\mathrm{R}$ & 1.553 & 1.571 & 1.585 & 1.602 & 1.622 & 1.626 & 1.646 & 1.666 & 1.698 & 1.771 \\
\hline $\mathrm{S}$ & 1.568 & 1.577 & 1.588 & 1.603 & 1.606 & 1.628 & 1.651 & 1.669 & 1.697 & 1.779 \\
\hline
\end{tabular}


Table 7.40. Problem 6.1.1 reference solution MPACT power peaking factors for axial segments in element 2 for charge-pan IV.

\begin{tabular}{|c|c|c|c|c|c|c|c|c|c|c|}
\hline \multirow[t]{2}{*}{ Channels } & \multicolumn{10}{|c|}{ Power Peaking Factors } \\
\hline & 1 & 2 & 3 & 4 & 5 & 6 & 7 & 8 & 9 & 10 \\
\hline A & 1.504 & 1.476 & 1.469 & 1.469 & 1.473 & 1.481 & 1.490 & 1.501 & 1.512 & 1.524 \\
\hline B & 1.504 & 1.476 & 1.469 & 1.469 & 1.473 & 1.481 & 1.490 & 1.501 & 1.512 & 1.523 \\
\hline $\mathrm{C}$ & 1.504 & 1.476 & 1.469 & 1.469 & 1.473 & 1.481 & 1.490 & 1.501 & 1.512 & 1.524 \\
\hline D & 1.504 & 1.476 & 1.469 & 1.469 & 1.473 & 1.481 & 1.491 & 1.501 & 1.512 & 1.524 \\
\hline $\mathrm{E}$ & 1.520 & 1.492 & 1.484 & 1.484 & 1.488 & 1.496 & 1.506 & 1.517 & 1.528 & 1.540 \\
\hline $\mathrm{F}$ & 1.520 & 1.492 & 1.484 & 1.484 & 1.488 & 1.496 & 1.506 & 1.517 & 1.528 & 1.540 \\
\hline G & 1.519 & 1.491 & 1.483 & 1.483 & 1.488 & 1.495 & 1.505 & 1.516 & 1.527 & 1.539 \\
\hline $\mathrm{H}$ & 1.518 & 1.491 & 1.483 & 1.483 & 1.488 & 1.495 & 1.505 & 1.516 & 1.527 & 1.539 \\
\hline $\mathrm{J}$ & 1.517 & 1.490 & 1.483 & 1.482 & 1.487 & 1.495 & 1.505 & 1.515 & 1.527 & 1.538 \\
\hline K & 1.519 & 1.491 & 1.483 & 1.483 & 1.488 & 1.496 & 1.505 & 1.516 & 1.528 & 1.539 \\
\hline $\mathrm{L}$ & 1.520 & 1.492 & 1.484 & 1.484 & 1.489 & 1.496 & 1.506 & 1.517 & 1.528 & 1.540 \\
\hline M & 1.520 & 1.492 & 1.484 & 1.484 & 1.488 & 1.496 & 1.506 & 1.517 & 1.528 & 1.540 \\
\hline $\mathrm{P}$ & 1.521 & 1.493 & 1.486 & 1.486 & 1.491 & 1.498 & 1.508 & 1.519 & 1.531 & 1.542 \\
\hline Q & 1.521 & 1.493 & 1.486 & 1.486 & 1.491 & 1.499 & 1.508 & 1.519 & 1.531 & 1.542 \\
\hline $\mathrm{R}$ & 1.520 & 1.493 & 1.485 & 1.485 & 1.490 & 1.498 & 1.507 & 1.518 & 1.530 & 1.541 \\
\hline S & 1.521 & 1.493 & 1.486 & 1.486 & 1.491 & 1.499 & 1.508 & 1.519 & 1.531 & 1.542 \\
\hline Channels & 11 & 12 & 13 & 14 & 15 & 16 & 17 & 18 & 19 & 20 \\
\hline A & 1.535 & 1.546 & 1.558 & 1.571 & 1.585 & 1.601 & 1.621 & 1.647 & 1.682 & 1.740 \\
\hline B & 1.535 & 1.546 & 1.558 & 1.570 & 1.584 & 1.600 & 1.620 & 1.645 & 1.679 & 1.736 \\
\hline $\mathrm{C}$ & 1.535 & 1.546 & 1.558 & 1.571 & 1.585 & 1.601 & 1.621 & 1.647 & 1.681 & 1.739 \\
\hline D & 1.535 & 1.547 & 1.558 & 1.571 & 1.585 & 1.602 & 1.622 & 1.647 & 1.682 & 1.740 \\
\hline $\mathrm{E}$ & 1.551 & 1.563 & 1.575 & 1.588 & 1.602 & 1.618 & 1.639 & 1.665 & 1.701 & 1.760 \\
\hline $\mathrm{F}$ & 1.552 & 1.563 & 1.575 & 1.588 & 1.602 & 1.618 & 1.639 & 1.665 & 1.701 & 1.760 \\
\hline G & 1.550 & 1.562 & 1.573 & 1.586 & 1.600 & 1.616 & 1.636 & 1.662 & 1.697 & 1.756 \\
\hline $\mathrm{H}$ & 1.550 & 1.562 & 1.573 & 1.586 & 1.600 & 1.616 & 1.636 & 1.662 & 1.697 & 1.756 \\
\hline $\mathbf{J}$ & 1.550 & 1.561 & 1.573 & 1.585 & 1.599 & 1.615 & 1.635 & 1.661 & 1.696 & 1.755 \\
\hline K & 1.551 & 1.562 & 1.574 & 1.587 & 1.601 & 1.617 & 1.637 & 1.664 & 1.699 & 1.758 \\
\hline $\mathrm{L}$ & 1.551 & 1.563 & 1.575 & 1.587 & 1.601 & 1.618 & 1.638 & 1.665 & 1.700 & 1.760 \\
\hline $\mathrm{M}$ & 1.552 & 1.563 & 1.575 & 1.588 & 1.602 & 1.619 & 1.639 & 1.665 & 1.701 & 1.761 \\
\hline $\mathrm{P}$ & 1.554 & 1.566 & 1.578 & 1.590 & 1.604 & 1.621 & 1.642 & 1.668 & 1.704 & 1.764 \\
\hline Q & 1.554 & 1.565 & 1.577 & 1.590 & 1.604 & 1.620 & 1.641 & 1.667 & 1.702 & 1.761 \\
\hline $\mathrm{R}$ & 1.552 & 1.564 & 1.575 & 1.588 & 1.602 & 1.618 & 1.638 & 1.663 & 1.698 & 1.756 \\
\hline $\mathrm{S}$ & 1.554 & 1.566 & 1.578 & 1.590 & 1.604 & 1.621 & 1.641 & 1.668 & 1.703 & 1.763 \\
\hline
\end{tabular}


Table 7.41. Problem 6.1.1 reference solution Shift power peaking factors for axial segments in element 3 for charge-pan IV.

\begin{tabular}{|c|c|c|c|c|c|c|c|c|c|c|}
\hline \multirow[t]{2}{*}{ Channels } & \multicolumn{10}{|c|}{ Power Peaking Factors } \\
\hline & 1 & 2 & 3 & 4 & 5 & 6 & 7 & 8 & 9 & 10 \\
\hline A & 1.742 & 1.650 & 1.618 & 1.586 & 1.563 & 1.546 & 1.516 & 1.491 & 1.466 & 1.447 \\
\hline B & 1.746 & 1.652 & 1.619 & 1.588 & 1.567 & 1.548 & 1.520 & 1.492 & 1.477 & 1.458 \\
\hline $\mathrm{C}$ & 1.739 & 1.663 & 1.626 & 1.583 & 1.565 & 1.549 & 1.517 & 1.498 & 1.476 & 1.458 \\
\hline D & 1.744 & 1.653 & 1.633 & 1.589 & 1.563 & 1.538 & 1.513 & 1.510 & 1.476 & 1.459 \\
\hline $\mathrm{E}$ & 1.754 & 1.677 & 1.645 & 1.620 & 1.585 & 1.554 & 1.536 & 1.517 & 1.496 & 1.467 \\
\hline $\mathrm{F}$ & 1.761 & 1.669 & 1.633 & 1.602 & 1.570 & 1.550 & 1.523 & 1.510 & 1.495 & 1.467 \\
\hline G & 1.760 & 1.680 & 1.648 & 1.606 & 1.574 & 1.544 & 1.534 & 1.511 & 1.485 & 1.472 \\
\hline $\mathrm{H}$ & 1.769 & 1.675 & 1.634 & 1.607 & 1.584 & 1.550 & 1.535 & 1.512 & 1.489 & 1.465 \\
\hline $\mathrm{J}$ & 1.772 & 1.675 & 1.639 & 1.611 & 1.582 & 1.557 & 1.543 & 1.518 & 1.494 & 1.479 \\
\hline K & 1.767 & 1.676 & 1.635 & 1.612 & 1.588 & 1.562 & 1.548 & 1.527 & 1.499 & 1.482 \\
\hline $\mathrm{L}$ & 1.772 & 1.670 & 1.654 & 1.608 & 1.579 & 1.562 & 1.545 & 1.516 & 1.504 & 1.476 \\
\hline M & 1.781 & 1.675 & 1.647 & 1.611 & 1.582 & 1.566 & 1.546 & 1.519 & 1.501 & 1.480 \\
\hline $\mathrm{P}$ & 1.756 & 1.691 & 1.642 & 1.616 & 1.585 & 1.570 & 1.543 & 1.517 & 1.502 & 1.482 \\
\hline Q & 1.764 & 1.679 & 1.644 & 1.600 & 1.574 & 1.547 & 1.531 & 1.509 & 1.481 & 1.463 \\
\hline $\mathrm{R}$ & 1.770 & 1.684 & 1.646 & 1.609 & 1.584 & 1.563 & 1.547 & 1.519 & 1.500 & 1.481 \\
\hline $\mathrm{S}$ & 1.780 & 1.682 & 1.650 & 1.616 & 1.597 & 1.580 & 1.554 & 1.529 & 1.511 & 1.489 \\
\hline Channels & 11 & 12 & 13 & 14 & 15 & 16 & 17 & 18 & 19 & 20 \\
\hline A & 1.425 & 1.402 & 1.382 & 1.366 & 1.346 & 1.316 & 1.299 & 1.272 & 1.238 & 1.245 \\
\hline B & 1.432 & 1.410 & 1.404 & 1.376 & 1.352 & 1.327 & 1.302 & 1.279 & 1.255 & 1.258 \\
\hline $\mathrm{C}$ & 1.447 & 1.411 & 1.392 & 1.373 & 1.356 & 1.331 & 1.315 & 1.284 & 1.258 & 1.266 \\
\hline D & 1.430 & 1.417 & 1.393 & 1.370 & 1.348 & 1.323 & 1.308 & 1.278 & 1.256 & 1.257 \\
\hline $\mathrm{E}$ & 1.454 & 1.433 & 1.403 & 1.387 & 1.367 & 1.352 & 1.330 & 1.314 & 1.301 & 1.327 \\
\hline $\mathrm{F}$ & 1.442 & 1.418 & 1.398 & 1.385 & 1.365 & 1.340 & 1.326 & 1.297 & 1.286 & 1.305 \\
\hline G & 1.439 & 1.426 & 1.395 & 1.380 & 1.357 & 1.337 & 1.322 & 1.296 & 1.288 & 1.314 \\
\hline $\mathrm{H}$ & 1.441 & 1.425 & 1.406 & 1.398 & 1.371 & 1.353 & 1.333 & 1.327 & 1.306 & 1.322 \\
\hline $\mathrm{J}$ & 1.467 & 1.444 & 1.419 & 1.409 & 1.387 & 1.370 & 1.349 & 1.348 & 1.337 & 1.363 \\
\hline K & 1.460 & 1.440 & 1.428 & 1.394 & 1.395 & 1.373 & 1.353 & 1.352 & 1.329 & 1.364 \\
\hline $\mathrm{L}$ & 1.459 & 1.452 & 1.426 & 1.396 & 1.390 & 1.368 & 1.355 & 1.342 & 1.333 & 1.365 \\
\hline $\mathrm{M}$ & 1.466 & 1.451 & 1.417 & 1.406 & 1.386 & 1.372 & 1.348 & 1.337 & 1.328 & 1.355 \\
\hline $\mathrm{P}$ & 1.469 & 1.448 & 1.429 & 1.403 & 1.385 & 1.374 & 1.361 & 1.355 & 1.347 & 1.374 \\
\hline Q & 1.443 & 1.424 & 1.407 & 1.374 & 1.368 & 1.341 & 1.330 & 1.313 & 1.304 & 1.331 \\
\hline $\mathrm{R}$ & 1.456 & 1.451 & 1.426 & 1.403 & 1.386 & 1.377 & 1.363 & 1.348 & 1.345 & 1.376 \\
\hline $\mathrm{S}$ & 1.477 & 1.463 & 1.427 & 1.420 & 1.408 & 1.389 & 1.366 & 1.359 & 1.355 & 1.393 \\
\hline
\end{tabular}


Table 7.42. Problem 6.1.1 reference solution MPACT power peaking factors for axial segments in element 3 for charge-pan IV.

\begin{tabular}{|c|c|c|c|c|c|c|c|c|c|c|}
\hline \multirow[t]{2}{*}{ Channels } & \multicolumn{10}{|c|}{ Power Peaking Factors } \\
\hline & 1 & 2 & 3 & 4 & 5 & 6 & 7 & 8 & 9 & 10 \\
\hline A & 1.731 & 1.665 & 1.623 & 1.590 & 1.563 & 1.539 & 1.517 & 1.496 & 1.477 & 1.457 \\
\hline B & 1.727 & 1.662 & 1.621 & 1.587 & 1.560 & 1.536 & 1.514 & 1.493 & 1.472 & 1.452 \\
\hline $\mathrm{C}$ & 1.731 & 1.665 & 1.623 & 1.590 & 1.563 & 1.539 & 1.517 & 1.496 & 1.477 & 1.457 \\
\hline D & 1.732 & 1.666 & 1.625 & 1.592 & 1.564 & 1.540 & 1.519 & 1.499 & 1.479 & 1.460 \\
\hline $\mathrm{E}$ & 1.752 & 1.686 & 1.643 & 1.610 & 1.583 & 1.559 & 1.538 & 1.519 & 1.500 & 1.481 \\
\hline $\mathrm{F}$ & 1.752 & 1.685 & 1.643 & 1.610 & 1.582 & 1.559 & 1.538 & 1.518 & 1.499 & 1.480 \\
\hline G & 1.747 & 1.681 & 1.638 & 1.604 & 1.576 & 1.552 & 1.531 & 1.510 & 1.490 & 1.471 \\
\hline $\mathrm{H}$ & 1.747 & 1.680 & 1.637 & 1.602 & 1.574 & 1.550 & 1.527 & 1.506 & 1.486 & 1.466 \\
\hline $\mathrm{J}$ & 1.745 & 1.678 & 1.635 & 1.602 & 1.573 & 1.549 & 1.527 & 1.506 & 1.486 & 1.465 \\
\hline K & 1.749 & 1.682 & 1.639 & 1.606 & 1.578 & 1.554 & 1.532 & 1.511 & 1.491 & 1.471 \\
\hline $\mathrm{L}$ & 1.751 & 1.685 & 1.642 & 1.609 & 1.582 & 1.558 & 1.537 & 1.518 & 1.499 & 1.480 \\
\hline M & 1.753 & 1.686 & 1.644 & 1.610 & 1.583 & 1.560 & 1.539 & 1.519 & 1.500 & 1.482 \\
\hline $\mathrm{P}$ & 1.756 & 1.689 & 1.647 & 1.613 & 1.586 & 1.563 & 1.542 & 1.523 & 1.504 & 1.486 \\
\hline Q & 1.752 & 1.687 & 1.644 & 1.611 & 1.584 & 1.561 & 1.540 & 1.520 & 1.501 & 1.483 \\
\hline $\mathrm{R}$ & 1.746 & 1.680 & 1.637 & 1.603 & 1.575 & 1.550 & 1.528 & 1.507 & 1.486 & 1.466 \\
\hline $\mathrm{S}$ & 1.755 & 1.688 & 1.646 & 1.612 & 1.585 & 1.561 & 1.540 & 1.521 & 1.502 & 1.483 \\
\hline Channels & 11 & 12 & 13 & 14 & 15 & 16 & 17 & 18 & 19 & 20 \\
\hline A & 1.437 & 1.416 & 1.394 & 1.373 & 1.350 & 1.328 & 1.306 & 1.284 & 1.264 & 1.254 \\
\hline B & 1.431 & 1.410 & 1.388 & 1.366 & 1.343 & 1.319 & 1.296 & 1.273 & 1.253 & 1.242 \\
\hline $\mathrm{C}$ & 1.437 & 1.416 & 1.395 & 1.373 & 1.350 & 1.328 & 1.306 & 1.284 & 1.264 & 1.254 \\
\hline D & 1.440 & 1.420 & 1.399 & 1.378 & 1.356 & 1.334 & 1.313 & 1.292 & 1.273 & 1.265 \\
\hline $\mathrm{E}$ & 1.463 & 1.444 & 1.425 & 1.407 & 1.389 & 1.372 & 1.358 & 1.348 & 1.345 & 1.359 \\
\hline $\mathrm{F}$ & 1.462 & 1.443 & 1.423 & 1.405 & 1.386 & 1.370 & 1.355 & 1.345 & 1.341 & 1.356 \\
\hline G & 1.451 & 1.430 & 1.410 & 1.389 & 1.369 & 1.350 & 1.334 & 1.321 & 1.314 & 1.325 \\
\hline $\mathrm{H}$ & 1.445 & 1.424 & 1.402 & 1.381 & 1.360 & 1.340 & 1.322 & 1.308 & 1.300 & 1.309 \\
\hline $\mathrm{J}$ & 1.445 & 1.424 & 1.402 & 1.381 & 1.360 & 1.340 & 1.322 & 1.308 & 1.300 & 1.309 \\
\hline $\mathrm{K}$ & 1.451 & 1.431 & 1.410 & 1.390 & 1.370 & 1.351 & 1.334 & 1.321 & 1.314 & 1.325 \\
\hline $\mathrm{L}$ & 1.461 & 1.442 & 1.423 & 1.404 & 1.386 & 1.370 & 1.355 & 1.345 & 1.342 & 1.356 \\
\hline $\mathrm{M}$ & 1.463 & 1.444 & 1.425 & 1.407 & 1.389 & 1.372 & 1.358 & 1.348 & 1.345 & 1.359 \\
\hline $\mathrm{P}$ & 1.468 & 1.450 & 1.431 & 1.414 & 1.397 & 1.382 & 1.370 & 1.363 & 1.362 & 1.380 \\
\hline Q & 1.464 & 1.445 & 1.426 & 1.408 & 1.390 & 1.375 & 1.362 & 1.353 & 1.351 & 1.368 \\
\hline $\mathrm{R}$ & 1.445 & 1.424 & 1.403 & 1.382 & 1.361 & 1.342 & 1.325 & 1.313 & 1.307 & 1.318 \\
\hline $\mathrm{S}$ & 1.464 & 1.445 & 1.427 & 1.408 & 1.391 & 1.375 & 1.362 & 1.353 & 1.352 & 1.368 \\
\hline
\end{tabular}


Table 7.43. Problem 6.1.1 reference solution Shift power peaking factors for axial segments in element 4 for charge-pan IV.

\begin{tabular}{|c|c|c|c|c|c|c|c|c|c|c|}
\hline \multirow[t]{2}{*}{ Channels } & \multicolumn{10}{|c|}{ Power Peaking Factors } \\
\hline & 1 & 2 & 3 & 4 & 5 & 6 & 7 & 8 & 9 & 10 \\
\hline A & 1.122 & 1.019 & 0.958 & 0.912 & 0.874 & 0.837 & 0.811 & 0.784 & 0.749 & 0.733 \\
\hline B & 1.121 & 1.023 & 0.978 & 0.927 & 0.889 & 0.858 & 0.820 & 0.795 & 0.768 & 0.755 \\
\hline $\mathrm{C}$ & 1.139 & 1.043 & 0.985 & 0.939 & 0.902 & 0.867 & 0.837 & 0.811 & 0.792 & 0.768 \\
\hline $\mathrm{D}$ & 1.128 & 1.026 & 0.967 & 0.928 & 0.892 & 0.852 & 0.829 & 0.794 & 0.768 & 0.750 \\
\hline $\mathrm{E}$ & 1.245 & 1.155 & 1.103 & 1.058 & 1.019 & 0.985 & 0.940 & 0.910 & 0.885 & 0.861 \\
\hline $\mathrm{F}$ & 1.241 & 1.143 & 1.086 & 1.040 & 0.998 & 0.961 & 0.927 & 0.891 & 0.864 & 0.844 \\
\hline G & 1.231 & 1.140 & 1.081 & 1.034 & 0.998 & 0.959 & 0.925 & 0.906 & 0.869 & 0.844 \\
\hline $\mathrm{H}$ & 1.250 & 1.158 & 1.105 & 1.060 & 1.016 & 0.980 & 0.958 & 0.923 & 0.887 & 0.859 \\
\hline $\mathrm{J}$ & 1.291 & 1.187 & 1.146 & 1.100 & 1.059 & 1.021 & 0.985 & 0.954 & 0.932 & 0.904 \\
\hline $\mathrm{K}$ & 1.294 & 1.198 & 1.156 & 1.102 & 1.062 & 1.022 & 1.001 & 0.963 & 0.940 & 0.908 \\
\hline $\mathrm{L}$ & 1.297 & 1.196 & 1.154 & 1.107 & 1.067 & 1.024 & 1.000 & 0.966 & 0.943 & 0.916 \\
\hline $\mathrm{M}$ & 1.282 & 1.194 & 1.142 & 1.098 & 1.059 & 1.023 & 0.991 & 0.958 & 0.933 & 0.907 \\
\hline $\mathrm{P}$ & 1.307 & 1.206 & 1.164 & 1.122 & 1.076 & 1.044 & 1.008 & 0.982 & 0.954 & 0.932 \\
\hline Q & 1.238 & 1.166 & 1.103 & 1.061 & 1.018 & 0.979 & 0.948 & 0.913 & 0.890 & 0.864 \\
\hline $\mathrm{R}$ & 1.314 & 1.214 & 1.163 & 1.122 & 1.092 & 1.052 & 1.012 & 0.980 & 0.952 & 0.927 \\
\hline S & 1.318 & 1.230 & 1.181 & 1.139 & 1.102 & 1.066 & 1.036 & 1.007 & 0.970 & 0.947 \\
\hline "Channels & $\overline{11}$ & 12 & 13 & 14 & 15 & 16 & 17 & 18 & $\overline{19}$ & 20 \\
\hline A & 0.707 & 0.688 & 0.669 & 0.656 & 0.636 & 0.619 & 0.606 & 0.598 & 0.594 & 0.605 \\
\hline B & 0.727 & 0.710 & 0.687 & 0.675 & 0.651 & 0.642 & 0.625 & 0.618 & 0.620 & 0.625 \\
\hline $\mathrm{C}$ & 0.745 & 0.720 & 0.702 & 0.687 & 0.674 & 0.662 & 0.643 & 0.631 & 0.628 & 0.642 \\
\hline D & 0.729 & 0.707 & 0.689 & 0.673 & 0.657 & 0.638 & 0.635 & 0.619 & 0.621 & 0.628 \\
\hline $\mathrm{E}$ & 0.843 & 0.816 & 0.794 & 0.779 & 0.761 & 0.743 & 0.727 & 0.713 & 0.706 & 0.718 \\
\hline $\mathrm{F}$ & 0.821 & 0.790 & 0.777 & 0.755 & 0.739 & 0.723 & 0.704 & 0.691 & 0.688 & 0.695 \\
\hline G & 0.818 & 0.797 & 0.773 & 0.756 & 0.738 & 0.723 & 0.708 & 0.695 & 0.679 & 0.701 \\
\hline $\mathrm{H}$ & 0.840 & 0.820 & 0.798 & 0.773 & 0.758 & 0.742 & 0.734 & 0.716 & 0.706 & 0.724 \\
\hline $\mathrm{J}$ & 0.879 & 0.870 & 0.835 & 0.817 & 0.801 & 0.784 & 0.771 & 0.760 & 0.757 & 0.765 \\
\hline K & 0.892 & 0.863 & 0.837 & 0.825 & 0.806 & 0.788 & 0.768 & 0.761 & 0.760 & 0.769 \\
\hline $\mathrm{L}$ & 0.887 & 0.866 & 0.849 & 0.829 & 0.812 & 0.786 & 0.773 & 0.771 & 0.758 & 0.772 \\
\hline M & 0.883 & 0.861 & 0.840 & 0.825 & 0.804 & 0.792 & 0.773 & 0.756 & 0.754 & 0.767 \\
\hline $\mathrm{P}$ & 0.903 & 0.881 & 0.859 & 0.837 & 0.820 & 0.805 & 0.787 & 0.780 & 0.768 & 0.790 \\
\hline Q & 0.831 & 0.809 & 0.795 & 0.768 & 0.747 & 0.730 & 0.720 & 0.712 & 0.694 & 0.710 \\
\hline $\mathrm{R}$ & 0.899 & 0.880 & 0.859 & 0.841 & 0.817 & 0.802 & 0.793 & 0.778 & 0.771 & 0.779 \\
\hline $\mathrm{S}$ & 0.923 & 0.900 & 0.876 & 0.852 & 0.840 & 0.819 & 0.802 & 0.790 & 0.784 & 0.803 \\
\hline
\end{tabular}


Table 7.44. Problem 6.1.1 reference solution MPACT power peaking factors for axial segments in element 4 for charge-pan IV.

\begin{tabular}{|c|c|c|c|c|c|c|c|c|c|c|}
\hline \multirow[t]{2}{*}{ Channels } & \multicolumn{10}{|c|}{ Power Peaking Factors } \\
\hline & 1 & 2 & 3 & 4 & 5 & 6 & 7 & 8 & 9 & 10 \\
\hline A & 1.126 & 1.040 & 0.979 & 0.930 & 0.890 & 0.856 & 0.826 & 0.799 & 0.775 & 0.753 \\
\hline B & 1.112 & 1.025 & 0.963 & 0.914 & 0.873 & 0.838 & 0.808 & 0.781 & 0.756 & 0.734 \\
\hline $\mathrm{C}$ & 1.126 & 1.041 & 0.980 & 0.931 & 0.890 & 0.856 & 0.826 & 0.799 & 0.775 & 0.753 \\
\hline $\mathrm{D}$ & 1.139 & 1.053 & 0.992 & 0.943 & 0.903 & 0.869 & 0.839 & 0.813 & 0.789 & 0.767 \\
\hline $\mathrm{E}$ & 1.285 & 1.209 & 1.153 & 1.106 & 1.065 & 1.030 & 0.998 & 0.968 & 0.941 & 0.916 \\
\hline $\mathrm{F}$ & 1.281 & 1.204 & 1.148 & 1.101 & 1.060 & 1.024 & 0.992 & 0.963 & 0.936 & 0.911 \\
\hline G & 1.245 & 1.167 & 1.110 & 1.062 & 1.020 & 0.983 & 0.950 & 0.920 & 0.893 & 0.868 \\
\hline $\mathrm{H}$ & 1.227 & 1.149 & 1.091 & 1.042 & 1.000 & 0.963 & 0.929 & 0.899 & 0.872 & 0.846 \\
\hline $\mathrm{J}$ & 1.227 & 1.149 & 1.092 & 1.043 & 1.000 & 0.963 & 0.930 & 0.900 & 0.872 & 0.847 \\
\hline K & 1.245 & 1.168 & 1.110 & 1.062 & 1.020 & 0.983 & 0.951 & 0.921 & 0.894 & 0.868 \\
\hline $\mathrm{L}$ & 1.281 & 1.205 & 1.149 & 1.102 & 1.061 & 1.025 & 0.993 & 0.964 & 0.937 & 0.912 \\
\hline $\mathrm{M}$ & 1.285 & 1.209 & 1.153 & 1.106 & 1.066 & 1.030 & 0.998 & 0.969 & 0.942 & 0.917 \\
\hline $\mathrm{P}$ & 1.313 & 1.239 & 1.185 & 1.139 & 1.099 & 1.064 & 1.032 & 1.003 & 0.976 & 0.950 \\
\hline Q & 1.299 & 1.224 & 1.170 & 1.123 & 1.083 & 1.047 & 1.014 & 0.985 & 0.957 & 0.932 \\
\hline $\mathrm{R}$ & 1.241 & 1.166 & 1.110 & 1.061 & 1.020 & 0.982 & 0.949 & 0.918 & 0.890 & 0.864 \\
\hline S & 1.299 & 1.225 & 1.170 & 1.124 & 1.083 & 1.048 & 1.015 & 0.986 & 0.959 & 0.933 \\
\hline "Channels & 11 & 12 & 13 & 14 & $\overline{15}$ & 16 & 17 & 18 & 19 & 20 \\
\hline A & 0.732 & 0.713 & 0.695 & 0.678 & 0.663 & 0.649 & 0.638 & 0.630 & 0.625 & 0.630 \\
\hline B & 0.713 & 0.694 & 0.676 & 0.659 & 0.644 & 0.630 & 0.619 & 0.611 & 0.606 & 0.610 \\
\hline $\mathrm{C}$ & 0.733 & 0.713 & 0.695 & 0.679 & 0.663 & 0.650 & 0.639 & 0.630 & 0.626 & 0.631 \\
\hline D & 0.746 & 0.727 & 0.709 & 0.692 & 0.677 & 0.663 & 0.652 & 0.644 & 0.639 & 0.644 \\
\hline $\mathrm{E}$ & 0.893 & 0.870 & 0.849 & 0.830 & 0.812 & 0.796 & 0.783 & 0.774 & 0.769 & 0.775 \\
\hline $\mathrm{F}$ & 0.887 & 0.865 & 0.844 & 0.824 & 0.806 & 0.791 & 0.777 & 0.768 & 0.763 & 0.769 \\
\hline G & 0.844 & 0.822 & 0.801 & 0.782 & 0.764 & 0.748 & 0.735 & 0.726 & 0.721 & 0.726 \\
\hline $\mathrm{H}$ & 0.822 & 0.800 & 0.779 & 0.760 & 0.742 & 0.726 & 0.713 & 0.704 & 0.698 & 0.703 \\
\hline $\mathrm{J}$ & 0.823 & 0.800 & 0.779 & 0.760 & 0.742 & 0.727 & 0.714 & 0.704 & 0.699 & 0.704 \\
\hline K & 0.845 & 0.822 & 0.802 & 0.782 & 0.765 & 0.749 & 0.736 & 0.727 & 0.722 & 0.727 \\
\hline $\mathrm{L}$ & 0.888 & 0.866 & 0.845 & 0.826 & 0.808 & 0.793 & 0.780 & 0.770 & 0.766 & 0.772 \\
\hline M & 0.893 & 0.871 & 0.850 & 0.830 & 0.813 & 0.797 & 0.784 & 0.774 & 0.770 & 0.776 \\
\hline $\mathrm{P}$ & 0.926 & 0.903 & 0.882 & 0.862 & 0.844 & 0.828 & 0.814 & 0.804 & 0.800 & 0.806 \\
\hline Q & 0.908 & 0.885 & 0.863 & 0.843 & 0.825 & 0.809 & 0.795 & 0.785 & 0.780 & 0.786 \\
\hline $\mathrm{R}$ & 0.839 & 0.816 & 0.795 & 0.775 & 0.757 & 0.741 & 0.727 & 0.717 & 0.712 & 0.716 \\
\hline $\mathrm{S}$ & 0.909 & 0.886 & 0.865 & 0.845 & 0.827 & 0.811 & 0.797 & 0.787 & 0.783 & 0.789 \\
\hline
\end{tabular}


Table 7.45. Problem 6.1.1 reference solution Shift power peaking factors for axial segments in element 5 for charge-pan IV.

\begin{tabular}{|c|c|c|c|c|c|c|c|c|c|c|}
\hline \multirow[t]{2}{*}{ Channels } & \multicolumn{10}{|c|}{ Power Peaking Factors } \\
\hline & 1 & 2 & 3 & 4 & 5 & 6 & 7 & 8 & 9 & 10 \\
\hline A & 0.567 & 0.528 & 0.507 & 0.483 & 0.463 & 0.450 & 0.431 & 0.411 & 0.403 & 0.389 \\
\hline B & 0.584 & 0.540 & 0.521 & 0.504 & 0.476 & 0.457 & 0.441 & 0.426 & 0.415 & 0.403 \\
\hline $\mathrm{C}$ & 0.595 & 0.555 & 0.535 & 0.511 & 0.492 & 0.475 & 0.458 & 0.444 & 0.427 & 0.413 \\
\hline $\mathrm{D}$ & 0.591 & 0.545 & 0.519 & 0.495 & 0.481 & 0.459 & 0.448 & 0.431 & 0.416 & 0.405 \\
\hline $\mathrm{E}$ & 0.677 & 0.631 & 0.600 & 0.574 & 0.554 & 0.533 & 0.516 & 0.503 & 0.481 & 0.467 \\
\hline $\mathrm{F}$ & 0.658 & 0.606 & 0.584 & 0.562 & 0.533 & 0.519 & 0.500 & 0.484 & 0.462 & 0.447 \\
\hline G & 0.648 & 0.608 & 0.583 & 0.553 & 0.531 & 0.518 & 0.494 & 0.473 & 0.467 & 0.450 \\
\hline $\mathrm{H}$ & 0.679 & 0.625 & 0.598 & 0.579 & 0.550 & 0.532 & 0.518 & 0.496 & 0.477 & 0.460 \\
\hline $\mathrm{J}$ & 0.717 & 0.661 & 0.640 & 0.614 & 0.588 & 0.566 & 0.541 & 0.524 & 0.511 & 0.491 \\
\hline K & 0.725 & 0.676 & 0.645 & 0.616 & 0.591 & 0.570 & 0.549 & 0.540 & 0.513 & 0.497 \\
\hline $\mathrm{L}$ & 0.727 & 0.675 & 0.645 & 0.619 & 0.595 & 0.578 & 0.554 & 0.535 & 0.515 & 0.494 \\
\hline $\mathrm{M}$ & 0.717 & 0.667 & 0.640 & 0.616 & 0.593 & 0.567 & 0.551 & 0.528 & 0.512 & 0.493 \\
\hline $\mathrm{P}$ & 0.737 & 0.683 & 0.654 & 0.630 & 0.607 & 0.583 & 0.563 & 0.538 & 0.529 & 0.505 \\
\hline Q & 0.673 & 0.624 & 0.595 & 0.563 & 0.545 & 0.522 & 0.509 & 0.487 & 0.466 & 0.456 \\
\hline $\mathrm{R}$ & 0.745 & 0.683 & 0.655 & 0.620 & 0.604 & 0.578 & 0.553 & 0.542 & 0.521 & 0.504 \\
\hline S & 0.757 & 0.694 & 0.665 & 0.635 & 0.620 & 0.595 & 0.571 & 0.554 & 0.541 & 0.520 \\
\hline Channels & 11 & 12 & 13 & 14 & 15 & 16 & 17 & 18 & 19 & 20 \\
\hline A & 0.380 & 0.363 & 0.354 & 0.342 & 0.334 & 0.322 & 0.311 & 0.305 & 0.299 & 0.303 \\
\hline B & 0.392 & 0.373 & 0.367 & 0.355 & 0.340 & 0.333 & 0.320 & 0.311 & 0.310 & 0.312 \\
\hline $\mathrm{C}$ & 0.398 & 0.383 & 0.374 & 0.360 & 0.349 & 0.343 & 0.334 & 0.325 & 0.319 & 0.317 \\
\hline D & 0.391 & 0.379 & 0.368 & 0.356 & 0.347 & 0.331 & 0.326 & 0.317 & 0.309 & 0.312 \\
\hline $\mathrm{E}$ & 0.446 & 0.434 & 0.427 & 0.411 & 0.399 & 0.384 & 0.375 & 0.369 & 0.361 & 0.363 \\
\hline $\mathrm{F}$ & 0.433 & 0.419 & 0.409 & 0.395 & 0.381 & 0.372 & 0.360 & 0.351 & 0.355 & 0.348 \\
\hline G & 0.430 & 0.422 & 0.405 & 0.387 & 0.382 & 0.374 & 0.359 & 0.352 & 0.347 & 0.352 \\
\hline $\mathrm{H}$ & 0.442 & 0.433 & 0.415 & 0.409 & 0.386 & 0.381 & 0.372 & 0.367 & 0.356 & 0.362 \\
\hline $\mathrm{J}$ & 0.472 & 0.464 & 0.449 & 0.431 & 0.420 & 0.405 & 0.401 & 0.397 & 0.385 & 0.393 \\
\hline K & 0.482 & 0.466 & 0.448 & 0.434 & 0.426 & 0.417 & 0.408 & 0.399 & 0.390 & 0.399 \\
\hline $\mathrm{L}$ & 0.481 & 0.466 & 0.448 & 0.445 & 0.426 & 0.414 & 0.408 & 0.393 & 0.382 & 0.387 \\
\hline M & 0.479 & 0.461 & 0.453 & 0.437 & 0.427 & 0.413 & 0.404 & 0.389 & 0.389 & 0.391 \\
\hline $\mathrm{P}$ & 0.489 & 0.475 & 0.464 & 0.446 & 0.440 & 0.426 & 0.413 & 0.402 & 0.396 & 0.398 \\
\hline Q & 0.442 & 0.427 & 0.412 & 0.399 & 0.387 & 0.375 & 0.366 & 0.357 & 0.354 & 0.355 \\
\hline $\mathrm{R}$ & 0.484 & 0.468 & 0.453 & 0.443 & 0.426 & 0.416 & 0.406 & 0.402 & 0.396 & 0.401 \\
\hline $\mathrm{S}$ & 0.501 & 0.483 & 0.470 & 0.454 & 0.442 & 0.433 & 0.422 & 0.409 & 0.402 & 0.411 \\
\hline
\end{tabular}


Table 7.46. Problem 6.1.1 reference solution MPACT power peaking factors for axial segments in element 5 for charge-pan IV.

\begin{tabular}{|c|c|c|c|c|c|c|c|c|c|c|}
\hline \multirow[t]{2}{*}{ Channels } & \multicolumn{10}{|c|}{ Power Peaking Factors } \\
\hline & 1 & 2 & 3 & 4 & 5 & 6 & 7 & 8 & 9 & 10 \\
\hline A & 0.593 & 0.557 & 0.531 & 0.508 & 0.488 & 0.470 & 0.454 & 0.439 & 0.424 & 0.411 \\
\hline B & 0.575 & 0.540 & 0.514 & 0.492 & 0.473 & 0.456 & 0.440 & 0.425 & 0.411 & 0.398 \\
\hline $\mathrm{C}$ & 0.594 & 0.559 & 0.532 & 0.510 & 0.490 & 0.472 & 0.456 & 0.441 & 0.427 & 0.413 \\
\hline D & 0.607 & 0.571 & 0.544 & 0.521 & 0.500 & 0.482 & 0.466 & 0.450 & 0.435 & 0.422 \\
\hline $\mathrm{E}$ & 0.731 & 0.687 & 0.654 & 0.626 & 0.602 & 0.579 & 0.559 & 0.540 & 0.523 & 0.506 \\
\hline $\mathrm{F}$ & 0.725 & 0.681 & 0.648 & 0.621 & 0.596 & 0.574 & 0.554 & 0.535 & 0.518 & 0.501 \\
\hline G & 0.684 & 0.642 & 0.612 & 0.585 & 0.562 & 0.541 & 0.522 & 0.505 & 0.488 & 0.472 \\
\hline $\mathrm{H}$ & 0.662 & 0.622 & 0.592 & 0.567 & 0.544 & 0.524 & 0.506 & 0.489 & 0.473 & 0.458 \\
\hline $\mathrm{J}$ & 0.663 & 0.623 & 0.593 & 0.568 & 0.545 & 0.525 & 0.507 & 0.490 & 0.474 & 0.459 \\
\hline K & 0.685 & 0.644 & 0.614 & 0.587 & 0.565 & 0.544 & 0.525 & 0.508 & 0.492 & 0.476 \\
\hline $\mathrm{L}$ & 0.728 & 0.685 & 0.652 & 0.625 & 0.601 & 0.579 & 0.559 & 0.541 & 0.523 & 0.507 \\
\hline M & 0.732 & 0.688 & 0.656 & 0.628 & 0.604 & 0.582 & 0.562 & 0.543 & 0.526 & 0.509 \\
\hline $\mathrm{P}$ & 0.761 & 0.715 & 0.681 & 0.652 & 0.627 & 0.604 & 0.583 & 0.563 & 0.545 & 0.528 \\
\hline Q & 0.740 & 0.696 & 0.663 & 0.634 & 0.609 & 0.587 & 0.566 & 0.547 & 0.529 & 0.512 \\
\hline $\mathrm{R}$ & 0.674 & 0.633 & 0.603 & 0.577 & 0.554 & 0.534 & 0.515 & 0.498 & 0.482 & 0.467 \\
\hline $\mathrm{S}$ & 0.744 & 0.700 & 0.667 & 0.639 & 0.614 & 0.592 & 0.572 & 0.553 & 0.536 & 0.519 \\
\hline Channels & 11 & 12 & 13 & 14 & 15 & 16 & 17 & 18 & 19 & 20 \\
\hline A & 0.398 & 0.385 & 0.373 & 0.362 & 0.351 & 0.341 & 0.332 & 0.325 & 0.320 & 0.320 \\
\hline B & 0.386 & 0.374 & 0.362 & 0.351 & 0.341 & 0.331 & 0.323 & 0.316 & 0.311 & 0.310 \\
\hline $\mathrm{C}$ & 0.400 & 0.388 & 0.376 & 0.365 & 0.354 & 0.345 & 0.336 & 0.329 & 0.323 & 0.323 \\
\hline D & 0.408 & 0.396 & 0.383 & 0.372 & 0.361 & 0.351 & 0.342 & 0.335 & 0.330 & 0.330 \\
\hline $\mathrm{E}$ & 0.490 & 0.474 & 0.460 & 0.446 & 0.434 & 0.422 & 0.413 & 0.406 & 0.402 & 0.405 \\
\hline $\mathrm{F}$ & 0.485 & 0.470 & 0.455 & 0.441 & 0.429 & 0.418 & 0.408 & 0.401 & 0.398 & 0.401 \\
\hline G & 0.457 & 0.443 & 0.429 & 0.416 & 0.403 & 0.392 & 0.382 & 0.374 & 0.368 & 0.367 \\
\hline $\mathrm{H}$ & 0.443 & 0.429 & 0.416 & 0.403 & 0.392 & 0.381 & 0.371 & 0.363 & 0.357 & 0.355 \\
\hline $\mathrm{J}$ & 0.445 & 0.431 & 0.418 & 0.405 & 0.394 & 0.383 & 0.373 & 0.365 & 0.359 & 0.358 \\
\hline K & 0.462 & 0.447 & 0.434 & 0.421 & 0.409 & 0.398 & 0.388 & 0.380 & 0.374 & 0.373 \\
\hline $\mathrm{L}$ & 0.491 & 0.476 & 0.462 & 0.448 & 0.435 & 0.424 & 0.413 & 0.405 & 0.398 & 0.398 \\
\hline M & 0.493 & 0.478 & 0.463 & 0.450 & 0.437 & 0.425 & 0.414 & 0.406 & 0.400 & 0.399 \\
\hline $\mathrm{P}$ & 0.511 & 0.495 & 0.480 & 0.466 & 0.453 & 0.441 & 0.431 & 0.423 & 0.419 & 0.421 \\
\hline Q & 0.495 & 0.479 & 0.465 & 0.450 & 0.438 & 0.426 & 0.416 & 0.409 & 0.406 & 0.408 \\
\hline $\mathrm{R}$ & 0.452 & 0.438 & 0.424 & 0.412 & 0.400 & 0.388 & 0.379 & 0.370 & 0.365 & 0.364 \\
\hline $\mathrm{S}$ & 0.503 & 0.488 & 0.473 & 0.459 & 0.446 & 0.434 & 0.424 & 0.415 & 0.409 & 0.408 \\
\hline
\end{tabular}


Table 7.47. Problem 6.1.1 reference solution Shift power peaking factors for axial segments in element 6 for charge-pan IV.

\begin{tabular}{|c|c|c|c|c|c|c|c|c|c|c|}
\hline \multirow[t]{2}{*}{ Channels } & \multicolumn{10}{|c|}{ Power Peaking Factors } \\
\hline & 1 & 2 & 3 & 4 & 5 & 6 & 7 & 8 & 9 & 10 \\
\hline A & 0.274 & 0.258 & 0.239 & 0.227 & 0.217 & 0.203 & 0.196 & 0.182 & 0.174 & 0.166 \\
\hline B & 0.289 & 0.265 & 0.250 & 0.235 & 0.226 & 0.213 & 0.203 & 0.193 & 0.182 & 0.174 \\
\hline $\mathrm{C}$ & 0.295 & 0.271 & 0.255 & 0.241 & 0.231 & 0.220 & 0.207 & 0.201 & 0.189 & 0.179 \\
\hline $\mathrm{D}$ & 0.289 & 0.265 & 0.253 & 0.236 & 0.228 & 0.215 & 0.199 & 0.193 & 0.180 & 0.170 \\
\hline $\mathrm{E}$ & 0.340 & 0.308 & 0.289 & 0.274 & 0.263 & 0.250 & 0.234 & 0.223 & 0.214 & 0.199 \\
\hline $\mathrm{F}$ & 0.322 & 0.296 & 0.277 & 0.265 & 0.253 & 0.238 & 0.223 & 0.211 & 0.205 & 0.190 \\
\hline G & 0.319 & 0.290 & 0.281 & 0.264 & 0.248 & 0.233 & 0.219 & 0.209 & 0.198 & 0.189 \\
\hline $\mathrm{H}$ & 0.333 & 0.303 & 0.285 & 0.268 & 0.255 & 0.246 & 0.233 & 0.221 & 0.210 & 0.197 \\
\hline $\mathrm{J}$ & 0.376 & 0.342 & 0.330 & 0.313 & 0.298 & 0.290 & 0.280 & 0.261 & 0.252 & 0.240 \\
\hline K & 0.376 & 0.349 & 0.336 & 0.318 & 0.303 & 0.287 & 0.275 & 0.265 & 0.254 & 0.239 \\
\hline $\mathrm{L}$ & 0.361 & 0.329 & 0.311 & 0.294 & 0.279 & 0.267 & 0.255 & 0.243 & 0.225 & 0.217 \\
\hline M & 0.361 & 0.328 & 0.310 & 0.290 & 0.281 & 0.260 & 0.253 & 0.235 & 0.224 & 0.213 \\
\hline $\mathrm{P}$ & 0.365 & 0.337 & 0.318 & 0.301 & 0.284 & 0.268 & 0.257 & 0.246 & 0.236 & 0.219 \\
\hline Q & 0.328 & 0.299 & 0.281 & 0.265 & 0.251 & 0.239 & 0.226 & 0.215 & 0.204 & 0.196 \\
\hline $\mathrm{R}$ & 0.387 & 0.354 & 0.335 & 0.322 & 0.309 & 0.291 & 0.280 & 0.266 & 0.254 & 0.241 \\
\hline S & 0.386 & 0.359 & 0.339 & 0.325 & 0.315 & 0.296 & 0.280 & 0.271 & 0.257 & 0.243 \\
\hline Channels & 11 & 12 & 13 & 14 & 15 & 16 & 17 & 18 & 19 & 20 \\
\hline A & 0.158 & 0.148 & 0.146 & 0.133 & 0.128 & 0.122 & 0.114 & 0.112 & 0.106 & 0.106 \\
\hline B & 0.165 & 0.156 & 0.151 & 0.143 & 0.134 & 0.128 & 0.118 & 0.115 & 0.112 & 0.111 \\
\hline $\mathrm{C}$ & 0.169 & 0.161 & 0.152 & 0.146 & 0.137 & 0.131 & 0.125 & 0.118 & 0.115 & 0.114 \\
\hline D & 0.163 & 0.159 & 0.149 & 0.140 & 0.133 & 0.129 & 0.121 & 0.114 & 0.110 & 0.110 \\
\hline $\mathrm{E}$ & 0.190 & 0.180 & 0.169 & 0.162 & 0.149 & 0.146 & 0.139 & 0.131 & 0.130 & 0.128 \\
\hline $\mathrm{F}$ & 0.179 & 0.174 & 0.165 & 0.155 & 0.149 & 0.141 & 0.132 & 0.130 & 0.124 & 0.123 \\
\hline G & 0.183 & 0.171 & 0.158 & 0.155 & 0.144 & 0.138 & 0.134 & 0.127 & 0.123 & 0.122 \\
\hline $\mathrm{H}$ & 0.192 & 0.180 & 0.167 & 0.161 & 0.149 & 0.144 & 0.137 & 0.131 & 0.129 & 0.129 \\
\hline $\mathrm{J}$ & 0.227 & 0.213 & 0.204 & 0.190 & 0.182 & 0.173 & 0.162 & 0.154 & 0.152 & 0.150 \\
\hline K & 0.225 & 0.218 & 0.206 & 0.194 & 0.185 & 0.175 & 0.164 & 0.155 & 0.151 & 0.148 \\
\hline $\mathrm{L}$ & 0.206 & 0.197 & 0.186 & 0.175 & 0.163 & 0.159 & 0.149 & 0.144 & 0.141 & 0.142 \\
\hline M & 0.203 & 0.191 & 0.182 & 0.175 & 0.165 & 0.156 & 0.149 & 0.142 & 0.138 & 0.141 \\
\hline $\mathrm{P}$ & 0.210 & 0.201 & 0.189 & 0.181 & 0.167 & 0.159 & 0.153 & 0.147 & 0.140 & 0.143 \\
\hline Q & 0.186 & 0.175 & 0.166 & 0.154 & 0.148 & 0.140 & 0.134 & 0.129 & 0.122 & 0.126 \\
\hline $\mathrm{R}$ & 0.228 & 0.219 & 0.207 & 0.196 & 0.183 & 0.176 & 0.167 & 0.156 & 0.151 & 0.150 \\
\hline $\mathrm{S}$ & 0.230 & 0.219 & 0.209 & 0.198 & 0.187 & 0.176 & 0.166 & 0.160 & 0.150 & 0.152 \\
\hline
\end{tabular}


Table 7.48. Problem 6.1.1 reference solution MPACT power peaking factors for axial segments in element 6 for charge-pan IV.

\begin{tabular}{|c|c|c|c|c|c|c|c|c|c|c|}
\hline \multirow[t]{2}{*}{ Channels } & \multicolumn{10}{|c|}{ Power Peaking Factors } \\
\hline & 1 & 2 & 3 & 4 & 5 & 6 & 7 & 8 & 9 & 10 \\
\hline A & 0.295 & 0.275 & 0.259 & 0.245 & 0.232 & 0.221 & 0.210 & 0.199 & 0.189 & 0.180 \\
\hline B & 0.285 & 0.265 & 0.249 & 0.235 & 0.223 & 0.211 & 0.201 & 0.190 & 0.181 & 0.172 \\
\hline $\mathrm{C}$ & 0.297 & 0.276 & 0.260 & 0.245 & 0.232 & 0.221 & 0.210 & 0.199 & 0.189 & 0.180 \\
\hline $\mathrm{D}$ & 0.305 & 0.284 & 0.267 & 0.253 & 0.240 & 0.228 & 0.217 & 0.206 & 0.196 & 0.186 \\
\hline $\mathrm{E}$ & 0.383 & 0.361 & 0.343 & 0.328 & 0.313 & 0.299 & 0.286 & 0.273 & 0.260 & 0.248 \\
\hline $\mathrm{F}$ & 0.380 & 0.357 & 0.340 & 0.325 & 0.311 & 0.297 & 0.284 & 0.271 & 0.258 & 0.246 \\
\hline G & 0.339 & 0.315 & 0.297 & 0.280 & 0.266 & 0.252 & 0.240 & 0.228 & 0.216 & 0.205 \\
\hline $\mathrm{H}$ & 0.327 & 0.304 & 0.285 & 0.270 & 0.255 & 0.242 & 0.230 & 0.218 & 0.207 & 0.197 \\
\hline $\mathrm{J}$ & 0.330 & 0.306 & 0.288 & 0.272 & 0.258 & 0.245 & 0.232 & 0.221 & 0.209 & 0.199 \\
\hline K & 0.343 & 0.319 & 0.300 & 0.284 & 0.269 & 0.255 & 0.242 & 0.230 & 0.219 & 0.208 \\
\hline $\mathrm{L}$ & 0.367 & 0.341 & 0.321 & 0.303 & 0.287 & 0.272 & 0.259 & 0.246 & 0.234 & 0.222 \\
\hline M & 0.369 & 0.343 & 0.323 & 0.305 & 0.290 & 0.275 & 0.261 & 0.249 & 0.236 & 0.224 \\
\hline$P$ & 0.396 & 0.371 & 0.352 & 0.335 & 0.320 & 0.305 & 0.291 & 0.277 & 0.264 & 0.251 \\
\hline Q & 0.387 & 0.364 & 0.346 & 0.331 & 0.316 & 0.302 & 0.288 & 0.275 & 0.262 & 0.250 \\
\hline $\mathrm{R}$ & 0.335 & 0.311 & 0.292 & 0.276 & 0.261 & 0.248 & 0.235 & 0.223 & 0.212 & 0.201 \\
\hline $\mathrm{S}$ & 0.376 & 0.350 & 0.329 & 0.311 & 0.295 & 0.280 & 0.266 & 0.253 & 0.240 & 0.228 \\
\hline Channels & 11 & 12 & 13 & 14 & 15 & 16 & 17 & 18 & 19 & 20 \\
\hline A & 0.171 & 0.162 & 0.153 & 0.145 & 0.137 & 0.130 & 0.124 & 0.119 & 0.115 & 0.114 \\
\hline B & 0.163 & 0.154 & 0.146 & 0.138 & 0.131 & 0.124 & 0.118 & 0.113 & 0.110 & 0.109 \\
\hline $\mathrm{C}$ & 0.170 & 0.161 & 0.153 & 0.145 & 0.137 & 0.130 & 0.124 & 0.119 & 0.115 & 0.114 \\
\hline D & 0.176 & 0.167 & 0.158 & 0.150 & 0.142 & 0.135 & 0.128 & 0.123 & 0.119 & 0.118 \\
\hline $\mathrm{E}$ & 0.235 & 0.223 & 0.212 & 0.201 & 0.190 & 0.180 & 0.171 & 0.163 & 0.157 & 0.154 \\
\hline $\mathrm{F}$ & 0.234 & 0.222 & 0.210 & 0.199 & 0.188 & 0.178 & 0.169 & 0.161 & 0.155 & 0.152 \\
\hline G & 0.195 & 0.185 & 0.175 & 0.166 & 0.157 & 0.149 & 0.141 & 0.136 & 0.131 & 0.130 \\
\hline $\mathrm{H}$ & 0.186 & 0.177 & 0.167 & 0.158 & 0.150 & 0.142 & 0.135 & 0.130 & 0.126 & 0.124 \\
\hline J & 0.189 & 0.179 & 0.169 & 0.160 & 0.152 & 0.144 & 0.137 & 0.131 & 0.127 & 0.126 \\
\hline K & 0.197 & 0.187 & 0.177 & 0.167 & 0.158 & 0.150 & 0.143 & 0.137 & 0.133 & 0.132 \\
\hline $\mathrm{L}$ & 0.211 & 0.200 & 0.189 & 0.179 & 0.170 & 0.161 & 0.153 & 0.147 & 0.143 & 0.142 \\
\hline M & 0.213 & 0.202 & 0.191 & 0.181 & 0.172 & 0.163 & 0.155 & 0.149 & 0.144 & 0.143 \\
\hline $\mathrm{P}$ & 0.239 & 0.227 & 0.215 & 0.204 & 0.193 & 0.183 & 0.174 & 0.166 & 0.160 & 0.157 \\
\hline Q & 0.237 & 0.225 & 0.213 & 0.202 & 0.191 & 0.181 & 0.172 & 0.164 & 0.157 & 0.154 \\
\hline $\mathrm{R}$ & 0.191 & 0.181 & 0.171 & 0.162 & 0.153 & 0.145 & 0.138 & 0.133 & 0.129 & 0.127 \\
\hline $\mathrm{S}$ & 0.216 & 0.205 & 0.194 & 0.184 & 0.174 & 0.165 & 0.158 & 0.151 & 0.147 & 0.146 \\
\hline
\end{tabular}




\subsection{DEPLETION}

\subsubsection{Problem 6.2.1}

\subsubsection{Description}

Problem 6.2.1 uses the same geometry as Problem 6.1.1, but the fuel material is depleted throughout the simulation. The control rod material is not depleted and the control rods do not move with depletion.

\subsubsection{Reference Solution}

The neutron multiplication factor for Problem 6.2.1 is shown in Table 7.49. The timing variables for both problems are shown in Table 7.50. Note that the number of particles per generation in Shift was increased and number of generations compared to Problem 6.1.1. This sped up the Shift calculation significantly.

In the reference solution for Problem 6.2.1, power peaking factors are reported by element rather than by axial segment. his is done to reduce the volume of data reported. The geometry model includes the 20 axial subdivisions of each fuel elements, as with the previous problems.

Table 7.49. Neutron multiplication factor for the reference solution of Problem 6.2.1.

\begin{tabular}{rrcrr}
\hline Step & $\begin{array}{r}\text { Burnup } \\
{\left[\frac{\mathrm{MWd}}{\mathrm{MtU}}\right]}\end{array}$ & $\begin{array}{c}\text { Shift } \\
\mathrm{k}_{\mathrm{eff}}(1 \sigma)\end{array}$ & $\begin{array}{c}\text { MPACT } \\
\mathrm{k}_{\mathrm{eff}}(1 \sigma)\end{array}$ & $\begin{array}{c}\text { Difference } \\
{[\mathrm{pcm}]}\end{array}$ \\
\hline 0 & 0.0 & $1.02749(11)$ & $1.02528(1)$ & $-221(11)$ \\
1 & 25.0 & $1.01553(10)$ & $1.00876(1)$ & $-676(10)$ \\
2 & 50.0 & $1.01539(10)$ & $1.00911(1)$ & $-629(11)$ \\
3 & 100.0 & $1.01642(11)$ & $1.01143(1)$ & $-500(11)$ \\
4 & 200.0 & $1.01912(10)$ & $1.01662(1)$ & $-250(10)$ \\
5 & 300.0 & $1.02168(11)$ & $1.02077(1)$ & $-91(11)$ \\
6 & 400.0 & $1.02367(10)$ & $1.02387(1)$ & $20(11)$ \\
7 & 500.0 & $1.02512(10)$ & $1.02628(1)$ & $115(10)$ \\
8 & 600.0 & $1.02642(10)$ & $1.02795(1)$ & $153(10)$ \\
9 & 700.0 & $1.02763(11)$ & $1.02912(1)$ & $149(11)$ \\
10 & 800.0 & $1.02832(11)$ & $1.0299(1)$ & $158(11)$ \\
11 & 900.0 & $1.02893(10)$ & $1.03038(1)$ & $145(10)$ \\
12 & 1000.0 & $1.02937(11)$ & $1.03053(1)$ & $116(11)$ \\
13 & 1100.0 & $1.02973(12)$ & $1.03047(1)$ & $74(12)$ \\
14 & 1200.0 & $1.02973(10)$ & $1.0302(1)$ & $47(10)$ \\
15 & 1300.0 & $1.02989(11)$ & $1.02978(1)$ & $-11(11)$ \\
\hline
\end{tabular}


Table 7.50. Timing variables for the reference solution of Problem 6.2.1

\begin{tabular}{|c|c|c|c|c|c|c|}
\hline \multicolumn{7}{|c|}{ Shift } \\
\hline $\begin{array}{c}\text { Depletion } \\
\text { Solves }\end{array}$ & $\begin{array}{c}\text { Total } \\
\text { Cycles per } \\
\text { Transport } \\
\text { Solve } \\
\end{array}$ & $\begin{array}{c}\text { Active } \\
\text { Cycles per } \\
\text { Transport } \\
\text { Solve }\end{array}$ & $\begin{array}{l}\text { Histories } \\
\text { per Cycle }\end{array}$ & $\begin{array}{c}\text { Compute } \\
\text { Nodes }\end{array}$ & $\begin{array}{c}\text { Cores } \\
\text { per Node }\end{array}$ & $\begin{array}{c}\text { Wall } \\
\text { Total Time } \\
\text { [hours] }\end{array}$ \\
\hline 15 & 1500 & 1200 & 500000 & 8 & 48 & 6.04 \\
\hline \multicolumn{7}{|c|}{ MPACT } \\
\hline $\begin{array}{c}\text { Depletion } \\
\text { Solves }\end{array}$ & $\begin{array}{c}k_{\mathrm{eff}} \\
\text { Convergence } \\
\text { Criteria }\end{array}$ & $\begin{array}{c}\text { Source } \\
\text { Convergence } \\
\text { Criteria }\end{array}$ & $\begin{array}{c}\text { Ray } \\
\text { Spacing } \\
{[\mathrm{cm}]}\end{array}$ & $\begin{array}{c}\text { Compute } \\
\text { Nodes }\end{array}$ & $\begin{array}{c}\text { Cores } \\
\text { per Node }\end{array}$ & $\begin{array}{c}\text { Wall } \\
\text { Total Time } \\
\text { [hours] }\end{array}$ \\
\hline 15 & $1.0 \times 10^{-04}$ & $3.0 \times 10^{-04}$ & 0.01 & 12 & 128 & 24.94 \\
\hline
\end{tabular}


Table 7.51. Problem 6.2.1 reference solution power peaking factors in element 1 for charge-pan I (1/2).

\begin{tabular}{|c|c|c|c|c|c|c|c|c|c|}
\hline \multirow[t]{2}{*}{ Step } & \multirow{2}{*}{$\begin{array}{l}\text { Burnup } \\
{\left[\frac{\mathrm{MWd}}{\mathrm{MtU}}\right]}\end{array}$} & \multicolumn{8}{|c|}{ Shift Power Peaking Factors } \\
\hline & & A & B & $\mathrm{C}$ & $\mathrm{D}$ & $\mathrm{P}$ & Q & $\mathrm{R}$ & $\mathrm{S}$ \\
\hline 0 & 0.0 & 1.034 & 1.034 & 1.032 & 1.033 & 1.044 & 1.046 & 1.047 & 1.048 \\
\hline 1 & 25.0 & 1.064 & 1.063 & 1.062 & 1.068 & 1.081 & 1.076 & 1.071 & 1.079 \\
\hline 2 & 50.0 & 1.066 & 1.062 & 1.065 & 1.068 & 1.082 & 1.078 & 1.074 & 1.079 \\
\hline 3 & 100.0 & 1.057 & 1.057 & 1.062 & 1.057 & 1.073 & 1.074 & 1.070 & 1.070 \\
\hline 4 & 200.0 & 1.041 & 1.046 & 1.052 & 1.035 & 1.056 & 1.066 & 1.063 & 1.053 \\
\hline 5 & 300.0 & 1.028 & 1.037 & 1.047 & 1.022 & 1.043 & 1.057 & 1.056 & 1.038 \\
\hline 6 & 400.0 & 1.019 & 1.031 & 1.041 & 1.011 & 1.034 & 1.054 & 1.052 & 1.036 \\
\hline 7 & 500.0 & 1.014 & 1.028 & 1.039 & 1.005 & 1.031 & 1.051 & 1.047 & 1.026 \\
\hline 8 & 600.0 & 1.013 & 1.028 & 1.041 & 1.002 & 1.031 & 1.050 & 1.046 & 1.025 \\
\hline 9 & 700.0 & 1.012 & 1.030 & 1.040 & 0.998 & 1.027 & 1.052 & 1.049 & 1.023 \\
\hline 10 & 800.0 & 1.014 & 1.029 & 1.039 & 0.997 & 1.031 & 1.052 & 1.045 & 1.025 \\
\hline 11 & 900.0 & 1.015 & 1.033 & 1.043 & 0.997 & 1.034 & 1.057 & 1.051 & 1.030 \\
\hline 12 & 1000.0 & 1.023 & 1.036 & 1.047 & 1.000 & 1.038 & 1.059 & 1.051 & 1.033 \\
\hline 13 & 1100.0 & 1.026 & 1.041 & 1.052 & 1.003 & 1.043 & 1.063 & 1.058 & 1.039 \\
\hline 14 & 1200.0 & 1.029 & 1.044 & 1.053 & 1.005 & 1.047 & 1.062 & 1.058 & 1.041 \\
\hline 15 & 1300.0 & 1.035 & 1.047 & 1.054 & 1.009 & 1.054 & 1.068 & 1.057 & 1.048 \\
\hline \multirow[t]{2}{*}{ Step } & Burnup & \multicolumn{8}{|c|}{ MPACT Power Peaking Factors } \\
\hline & $\frac{\mathrm{MWd}}{\mathrm{MtU}}$ & A & B & $\mathrm{C}$ & $\mathrm{D}$ & $\mathrm{P}$ & Q & $\mathrm{R}$ & S \\
\hline 0 & 0.0 & 1.046 & 1.046 & 1.046 & 1.046 & 1.058 & 1.058 & 1.058 & 1.058 \\
\hline 1 & 25.0 & 1.030 & 1.029 & 1.029 & 1.029 & 1.040 & 1.041 & 1.040 & 1.040 \\
\hline 2 & 50.0 & 1.030 & 1.031 & 1.030 & 1.031 & 1.041 & 1.042 & 1.041 & 1.041 \\
\hline 3 & 100.0 & 1.033 & 1.033 & 1.033 & 1.033 & 1.044 & 1.044 & 1.044 & 1.044 \\
\hline 4 & 200.0 & 1.036 & 1.036 & 1.036 & 1.035 & 1.047 & 1.047 & 1.047 & 1.047 \\
\hline 5 & 300.0 & 1.039 & 1.039 & 1.039 & 1.039 & 1.050 & 1.051 & 1.051 & 1.050 \\
\hline 6 & 400.0 & 1.040 & 1.040 & 1.040 & 1.040 & 1.052 & 1.052 & 1.052 & 1.052 \\
\hline 7 & 500.0 & 1.040 & 1.040 & 1.040 & 1.040 & 1.052 & 1.052 & 1.052 & 1.051 \\
\hline 8 & 600.0 & 1.040 & 1.040 & 1.040 & 1.040 & 1.052 & 1.052 & 1.051 & 1.051 \\
\hline 9 & 700.0 & 1.039 & 1.039 & 1.039 & 1.039 & 1.050 & 1.051 & 1.050 & 1.050 \\
\hline 10 & 800.0 & 1.038 & 1.038 & 1.038 & 1.038 & 1.050 & 1.050 & 1.049 & 1.049 \\
\hline 11 & 900.0 & 1.036 & 1.036 & 1.036 & 1.036 & 1.048 & 1.048 & 1.047 & 1.047 \\
\hline 12 & 1000.0 & 1.035 & 1.034 & 1.034 & 1.034 & 1.046 & 1.047 & 1.046 & 1.046 \\
\hline 13 & 1100.0 & 1.033 & 1.032 & 1.032 & 1.032 & 1.044 & 1.045 & 1.044 & 1.044 \\
\hline 14 & 1200.0 & 1.031 & 1.030 & 1.030 & 1.030 & 1.042 & 1.043 & 1.041 & 1.042 \\
\hline 15 & 1300.0 & 1.028 & 1.028 & 1.028 & 1.027 & 1.039 & 1.040 & 1.039 & 1.039 \\
\hline
\end{tabular}


Table 7.52. Problem 6.2.1 reference solution power peaking factors in element 1 for charge-pan I (2/2).

\begin{tabular}{|c|c|c|c|c|c|c|c|c|c|}
\hline \multirow[t]{2}{*}{ Step } & \multirow{2}{*}{$\begin{array}{l}\text { Burnup } \\
{\left[\frac{\mathrm{MWd}}{\mathrm{MtU}}\right]}\end{array}$} & \multicolumn{8}{|c|}{ Shift Power Peaking Factors } \\
\hline & & $\mathrm{E}$ & $\mathrm{F}$ & G & $\mathrm{H}$ & $\mathrm{J}$ & K & $\mathrm{L}$ & M \\
\hline 0 & 0.0 & 1.042 & 1.044 & 1.043 & 1.044 & 1.043 & 1.044 & 1.044 & 1.044 \\
\hline 1 & 25.0 & 1.073 & 1.073 & 1.073 & 1.072 & 1.069 & 1.074 & 1.075 & 1.075 \\
\hline 2 & 50.0 & 1.077 & 1.076 & 1.076 & 1.075 & 1.072 & 1.074 & 1.076 & 1.075 \\
\hline 3 & 100.0 & 1.070 & 1.073 & 1.069 & 1.066 & 1.064 & 1.066 & 1.070 & 1.070 \\
\hline 4 & 200.0 & 1.057 & 1.062 & 1.056 & 1.047 & 1.056 & 1.051 & 1.061 & 1.058 \\
\hline 5 & 300.0 & 1.050 & 1.056 & 1.045 & 1.033 & 1.051 & 1.043 & 1.051 & 1.049 \\
\hline 6 & 400.0 & 1.042 & 1.050 & 1.039 & 1.023 & 1.046 & 1.039 & 1.052 & 1.045 \\
\hline 7 & 500.0 & 1.040 & 1.049 & 1.035 & 1.013 & 1.044 & 1.036 & 1.050 & 1.040 \\
\hline 8 & 600.0 & 1.040 & 1.049 & 1.033 & 1.010 & 1.043 & 1.034 & 1.052 & 1.040 \\
\hline 9 & 700.0 & 1.040 & 1.049 & 1.036 & 1.009 & 1.046 & 1.035 & 1.052 & 1.039 \\
\hline 10 & 800.0 & 1.046 & 1.054 & 1.039 & 1.009 & 1.044 & 1.035 & 1.054 & 1.043 \\
\hline 11 & 900.0 & 1.046 & 1.058 & 1.044 & 1.009 & 1.053 & 1.041 & 1.057 & 1.046 \\
\hline 12 & 1000.0 & 1.055 & 1.061 & 1.050 & 1.009 & 1.054 & 1.045 & 1.063 & 1.052 \\
\hline 13 & 1100.0 & 1.059 & 1.068 & 1.056 & 1.017 & 1.059 & 1.051 & 1.069 & 1.060 \\
\hline 14 & 1200.0 & 1.063 & 1.066 & 1.054 & 1.017 & 1.061 & 1.054 & 1.069 & 1.061 \\
\hline 15 & 1300.0 & 1.069 & 1.074 & 1.063 & 1.017 & 1.060 & 1.056 & 1.072 & 1.064 \\
\hline \multirow[t]{2}{*}{ Step } & Burnup & \multicolumn{8}{|c|}{ MPACT Power Peaking Factors } \\
\hline & $\frac{\mathrm{MWd}}{\mathrm{MtU}}$ & $\mathrm{E}$ & $\mathrm{F}$ & G & $\mathrm{H}$ & $\mathrm{J}$ & K & $\mathrm{L}$ & M \\
\hline 0 & 0.0 & 1.056 & 1.056 & 1.056 & 1.056 & 1.055 & 1.055 & 1.055 & 1.055 \\
\hline 1 & 25.0 & 1.039 & 1.039 & 1.039 & 1.039 & 1.039 & 1.039 & 1.039 & 1.039 \\
\hline 2 & 50.0 & 1.040 & 1.040 & 1.040 & 1.039 & 1.039 & 1.039 & 1.039 & 1.040 \\
\hline 3 & 100.0 & 1.043 & 1.043 & 1.043 & 1.043 & 1.043 & 1.042 & 1.042 & 1.042 \\
\hline 4 & 200.0 & 1.046 & 1.046 & 1.046 & 1.045 & 1.045 & 1.045 & 1.046 & 1.045 \\
\hline 5 & 300.0 & 1.049 & 1.049 & 1.049 & 1.049 & 1.049 & 1.049 & 1.049 & 1.049 \\
\hline 6 & 400.0 & 1.050 & 1.050 & 1.050 & 1.050 & 1.050 & 1.050 & 1.050 & 1.049 \\
\hline 7 & 500.0 & 1.050 & 1.051 & 1.050 & 1.050 & 1.050 & 1.050 & 1.050 & 1.050 \\
\hline 8 & 600.0 & 1.050 & 1.050 & 1.050 & 1.050 & 1.049 & 1.049 & 1.050 & 1.050 \\
\hline 9 & 700.0 & 1.049 & 1.049 & 1.049 & 1.049 & 1.049 & 1.048 & 1.048 & 1.048 \\
\hline 10 & 800.0 & 1.048 & 1.048 & 1.048 & 1.048 & 1.047 & 1.047 & 1.048 & 1.047 \\
\hline 11 & 900.0 & 1.046 & 1.046 & 1.046 & 1.046 & 1.046 & 1.045 & 1.045 & 1.045 \\
\hline 12 & 1000.0 & 1.045 & 1.045 & 1.045 & 1.045 & 1.044 & 1.044 & 1.044 & 1.044 \\
\hline 13 & 1100.0 & 1.043 & 1.043 & 1.043 & 1.043 & 1.042 & 1.042 & 1.042 & 1.042 \\
\hline 14 & 1200.0 & 1.041 & 1.041 & 1.040 & 1.041 & 1.040 & 1.040 & 1.040 & 1.040 \\
\hline 15 & 1300.0 & 1.038 & 1.038 & 1.038 & 1.038 & 1.037 & 1.037 & 1.037 & 1.037 \\
\hline
\end{tabular}


Table 7.53. Problem 6.2.1 reference solution power peaking factors in element 2 for charge-pan (1/2).

\begin{tabular}{|c|c|c|c|c|c|c|c|c|c|}
\hline \multirow[t]{2}{*}{ Step } & \multirow{2}{*}{$\begin{array}{l}\text { Burnup } \\
{\left[\frac{\mathrm{MWd}}{\mathrm{MtU}}\right]}\end{array}$} & \multicolumn{8}{|c|}{ Shift Power Peaking Factors } \\
\hline & & A & B & $\mathrm{C}$ & $\mathrm{D}$ & $\mathrm{P}$ & Q & $\mathrm{R}$ & S \\
\hline 0 & 0.0 & 1.558 & 1.555 & 1.556 & 1.554 & 1.574 & 1.570 & 1.576 & 1.575 \\
\hline 1 & 25.0 & 1.592 & 1.580 & 1.585 & 1.586 & 1.615 & 1.608 & 1.597 & 1.614 \\
\hline 2 & 50.0 & 1.589 & 1.585 & 1.587 & 1.589 & 1.616 & 1.610 & 1.602 & 1.613 \\
\hline 3 & 100.0 & 1.578 & 1.582 & 1.581 & 1.580 & 1.602 & 1.606 & 1.596 & 1.603 \\
\hline 4 & 200.0 & 1.557 & 1.581 & 1.577 & 1.572 & 1.581 & 1.601 & 1.596 & 1.579 \\
\hline 5 & 300.0 & 1.536 & 1.571 & 1.571 & 1.557 & 1.560 & 1.589 & 1.584 & 1.561 \\
\hline 6 & 400.0 & 1.526 & 1.571 & 1.570 & 1.555 & 1.546 & 1.591 & 1.581 & 1.552 \\
\hline 7 & 500.0 & 1.516 & 1.568 & 1.563 & 1.548 & 1.539 & 1.588 & 1.579 & 1.538 \\
\hline 8 & 600.0 & 1.510 & 1.568 & 1.567 & 1.551 & 1.536 & 1.592 & 1.581 & 1.535 \\
\hline 9 & 700.0 & 1.502 & 1.566 & 1.568 & 1.552 & 1.531 & 1.588 & 1.583 & 1.532 \\
\hline 10 & 800.0 & 1.501 & 1.564 & 1.570 & 1.553 & 1.529 & 1.595 & 1.583 & 1.531 \\
\hline 11 & 900.0 & 1.503 & 1.570 & 1.573 & 1.555 & 1.528 & 1.599 & 1.583 & 1.533 \\
\hline 12 & 1000.0 & 1.502 & 1.569 & 1.575 & 1.554 & 1.530 & 1.599 & 1.583 & 1.531 \\
\hline 13 & 1100.0 & 1.505 & 1.571 & 1.579 & 1.562 & 1.536 & 1.608 & 1.587 & 1.538 \\
\hline 14 & 1200.0 & 1.507 & 1.574 & 1.584 & 1.566 & 1.539 & 1.611 & 1.592 & 1.539 \\
\hline 15 & 1300.0 & 1.510 & 1.573 & 1.588 & 1.570 & 1.541 & 1.613 & 1.591 & 1.545 \\
\hline \multirow[t]{2}{*}{ Step } & Burnup & \multicolumn{8}{|c|}{ MPACT Power Peaking Factors } \\
\hline & $\frac{\mathrm{MWd}}{\mathrm{MtU}}$ & A & B & $\mathrm{C}$ & $\mathrm{D}$ & $\mathrm{P}$ & Q & $\mathrm{R}$ & S \\
\hline 0 & 0.0 & 1.592 & 1.591 & 1.591 & 1.591 & 1.609 & 1.611 & 1.609 & 1.609 \\
\hline 1 & 25.0 & 1.552 & 1.551 & 1.551 & 1.551 & 1.568 & 1.571 & 1.569 & 1.568 \\
\hline 2 & 50.0 & 1.557 & 1.558 & 1.557 & 1.557 & 1.574 & 1.577 & 1.575 & 1.574 \\
\hline 3 & 100.0 & 1.570 & 1.569 & 1.569 & 1.570 & 1.586 & 1.589 & 1.588 & 1.586 \\
\hline 4 & 200.0 & 1.586 & 1.585 & 1.585 & 1.585 & 1.604 & 1.606 & 1.604 & 1.604 \\
\hline 5 & 300.0 & 1.596 & 1.595 & 1.595 & 1.595 & 1.613 & 1.616 & 1.614 & 1.613 \\
\hline 6 & 400.0 & 1.599 & 1.598 & 1.598 & 1.598 & 1.618 & 1.620 & 1.617 & 1.617 \\
\hline 7 & 500.0 & 1.600 & 1.599 & 1.599 & 1.599 & 1.618 & 1.620 & 1.618 & 1.617 \\
\hline 8 & 600.0 & 1.598 & 1.598 & 1.597 & 1.598 & 1.617 & 1.619 & 1.616 & 1.616 \\
\hline 9 & 700.0 & 1.595 & 1.594 & 1.593 & 1.594 & 1.613 & 1.615 & 1.612 & 1.612 \\
\hline 10 & 800.0 & 1.591 & 1.589 & 1.589 & 1.589 & 1.608 & 1.611 & 1.608 & 1.608 \\
\hline 11 & 900.0 & 1.585 & 1.583 & 1.583 & 1.583 & 1.602 & 1.605 & 1.602 & 1.601 \\
\hline 12 & 1000.0 & 1.579 & 1.577 & 1.577 & 1.577 & 1.596 & 1.598 & 1.595 & 1.595 \\
\hline 13 & 1100.0 & 1.572 & 1.570 & 1.569 & 1.570 & 1.588 & 1.591 & 1.588 & 1.588 \\
\hline 14 & 1200.0 & 1.564 & 1.562 & 1.562 & 1.562 & 1.581 & 1.584 & 1.580 & 1.580 \\
\hline 15 & 1300.0 & 1.556 & 1.554 & 1.554 & 1.554 & 1.572 & 1.575 & 1.572 & 1.572 \\
\hline
\end{tabular}


Table 7.54. Problem 6.2.1 reference solution power peaking factors in element 2 for charge-pan I (2/2).

\begin{tabular}{|c|c|c|c|c|c|c|c|c|c|}
\hline \multirow[t]{2}{*}{ Step } & \multirow{2}{*}{$\begin{array}{l}\text { Burnup } \\
{\left[\frac{\mathrm{MWd}}{\mathrm{MtU}}\right]}\end{array}$} & \multicolumn{8}{|c|}{ Shift Power Peaking Factors } \\
\hline & & $\mathrm{E}$ & $\mathrm{F}$ & G & $\mathrm{H}$ & $\mathrm{J}$ & K & $\mathrm{L}$ & M \\
\hline 0 & 0.0 & 1.570 & 1.570 & 1.573 & 1.571 & 1.575 & 1.571 & 1.572 & 1.570 \\
\hline 1 & 25.0 & 1.610 & 1.607 & 1.605 & 1.598 & 1.600 & 1.608 & 1.607 & 1.604 \\
\hline 2 & 50.0 & 1.611 & 1.607 & 1.608 & 1.596 & 1.604 & 1.606 & 1.606 & 1.608 \\
\hline 3 & 100.0 & 1.597 & 1.600 & 1.593 & 1.593 & 1.590 & 1.594 & 1.596 & 1.602 \\
\hline 4 & 200.0 & 1.582 & 1.588 & 1.575 & 1.585 & 1.585 & 1.575 & 1.585 & 1.599 \\
\hline 5 & 300.0 & 1.566 & 1.576 & 1.557 & 1.570 & 1.568 & 1.561 & 1.571 & 1.590 \\
\hline 6 & 400.0 & 1.554 & 1.574 & 1.551 & 1.567 & 1.568 & 1.554 & 1.566 & 1.592 \\
\hline 7 & 500.0 & 1.554 & 1.569 & 1.544 & 1.562 & 1.559 & 1.547 & 1.564 & 1.588 \\
\hline 8 & 600.0 & 1.553 & 1.572 & 1.543 & 1.567 & 1.562 & 1.546 & 1.566 & 1.590 \\
\hline 9 & 700.0 & 1.550 & 1.568 & 1.543 & 1.566 & 1.564 & 1.545 & 1.567 & 1.589 \\
\hline 10 & 800.0 & 1.549 & 1.574 & 1.539 & 1.563 & 1.561 & 1.545 & 1.571 & 1.591 \\
\hline 11 & 900.0 & 1.554 & 1.577 & 1.544 & 1.566 & 1.565 & 1.544 & 1.574 & 1.590 \\
\hline 12 & 1000.0 & 1.560 & 1.582 & 1.543 & 1.567 & 1.568 & 1.548 & 1.578 & 1.594 \\
\hline 13 & 1100.0 & 1.563 & 1.584 & 1.551 & 1.573 & 1.573 & 1.553 & 1.588 & 1.600 \\
\hline 14 & 1200.0 & 1.568 & 1.593 & 1.555 & 1.576 & 1.578 & 1.559 & 1.589 & 1.601 \\
\hline 15 & 1300.0 & 1.574 & 1.597 & 1.558 & 1.581 & 1.583 & 1.563 & 1.598 & 1.603 \\
\hline \multirow[t]{2}{*}{ Step } & Burnup & \multicolumn{8}{|c|}{ MPACT Power Peaking Factors } \\
\hline & $\frac{\mathrm{MWd}}{\mathrm{MtU}}$ & $\mathrm{E}$ & $\mathrm{F}$ & G & $\mathrm{H}$ & $\mathrm{J}$ & K & $\mathrm{L}$ & M \\
\hline 0 & 0.0 & 1.607 & 1.607 & 1.608 & 1.607 & 1.606 & 1.606 & 1.606 & 1.606 \\
\hline 1 & 25.0 & 1.568 & 1.569 & 1.569 & 1.568 & 1.567 & 1.567 & 1.567 & 1.567 \\
\hline 2 & 50.0 & 1.573 & 1.574 & 1.573 & 1.573 & 1.572 & 1.572 & 1.572 & 1.572 \\
\hline 3 & 100.0 & 1.586 & 1.587 & 1.586 & 1.586 & 1.585 & 1.584 & 1.584 & 1.584 \\
\hline 4 & 200.0 & 1.603 & 1.603 & 1.602 & 1.602 & 1.601 & 1.600 & 1.602 & 1.601 \\
\hline 5 & 300.0 & 1.613 & 1.613 & 1.613 & 1.612 & 1.611 & 1.610 & 1.610 & 1.611 \\
\hline 6 & 400.0 & 1.615 & 1.616 & 1.616 & 1.616 & 1.614 & 1.615 & 1.615 & 1.614 \\
\hline 7 & 500.0 & 1.616 & 1.617 & 1.617 & 1.616 & 1.615 & 1.615 & 1.615 & 1.615 \\
\hline 8 & 600.0 & 1.615 & 1.616 & 1.616 & 1.615 & 1.614 & 1.613 & 1.614 & 1.614 \\
\hline 9 & 700.0 & 1.611 & 1.612 & 1.612 & 1.611 & 1.610 & 1.609 & 1.610 & 1.610 \\
\hline 10 & 800.0 & 1.606 & 1.607 & 1.607 & 1.606 & 1.605 & 1.605 & 1.605 & 1.605 \\
\hline 11 & 900.0 & 1.600 & 1.601 & 1.601 & 1.600 & 1.599 & 1.599 & 1.599 & 1.599 \\
\hline 12 & 1000.0 & 1.594 & 1.595 & 1.595 & 1.594 & 1.592 & 1.592 & 1.592 & 1.593 \\
\hline 13 & 1100.0 & 1.587 & 1.588 & 1.588 & 1.587 & 1.585 & 1.585 & 1.585 & 1.586 \\
\hline 14 & 1200.0 & 1.579 & 1.580 & 1.580 & 1.579 & 1.577 & 1.577 & 1.577 & 1.578 \\
\hline 15 & 1300.0 & 1.571 & 1.572 & 1.572 & 1.571 & 1.569 & 1.569 & 1.569 & 1.570 \\
\hline
\end{tabular}


Table 7.55. Problem 6.2.1 reference solution power peaking factors in element 3 for charge-pan (1/2).

\begin{tabular}{|c|c|c|c|c|c|c|c|c|c|}
\hline \multirow[t]{2}{*}{ Step } & \multirow{2}{*}{$\begin{array}{l}\text { Burnup } \\
{\left[\frac{\mathrm{MWd}}{\mathrm{MtU}}\right]}\end{array}$} & \multicolumn{8}{|c|}{ Shift Power Peaking Factors } \\
\hline & & A & B & $\mathrm{C}$ & $\mathrm{D}$ & $\mathrm{P}$ & Q & $\mathrm{R}$ & $\mathrm{S}$ \\
\hline 0 & 0.0 & 1.502 & 1.507 & 1.501 & 1.499 & 1.507 & 1.511 & 1.533 & 1.511 \\
\hline 1 & 25.0 & 1.499 & 1.506 & 1.502 & 1.493 & 1.504 & 1.511 & 1.532 & 1.507 \\
\hline 2 & 50.0 & 1.499 & 1.504 & 1.501 & 1.495 & 1.497 & 1.511 & 1.533 & 1.506 \\
\hline 3 & 100.0 & 1.497 & 1.508 & 1.498 & 1.498 & 1.501 & 1.510 & 1.531 & 1.510 \\
\hline 4 & 200.0 & 1.503 & 1.517 & 1.500 & 1.506 & 1.506 & 1.514 & 1.534 & 1.514 \\
\hline 5 & 300.0 & 1.505 & 1.524 & 1.503 & 1.514 & 1.504 & 1.512 & 1.535 & 1.516 \\
\hline 6 & 400.0 & 1.501 & 1.524 & 1.499 & 1.514 & 1.507 & 1.513 & 1.531 & 1.514 \\
\hline 7 & 500.0 & 1.507 & 1.525 & 1.500 & 1.517 & 1.509 & 1.512 & 1.532 & 1.518 \\
\hline 8 & 600.0 & 1.507 & 1.529 & 1.502 & 1.518 & 1.507 & 1.511 & 1.536 & 1.521 \\
\hline 9 & 700.0 & 1.506 & 1.525 & 1.503 & 1.517 & 1.507 & 1.517 & 1.534 & 1.519 \\
\hline 10 & 800.0 & 1.506 & 1.530 & 1.505 & 1.519 & 1.512 & 1.517 & 1.536 & 1.523 \\
\hline 11 & 900.0 & 1.506 & 1.528 & 1.507 & 1.516 & 1.508 & 1.518 & 1.538 & 1.522 \\
\hline 12 & 1000.0 & 1.505 & 1.529 & 1.505 & 1.512 & 1.509 & 1.519 & 1.536 & 1.523 \\
\hline 13 & 1100.0 & 1.509 & 1.532 & 1.511 & 1.515 & 1.511 & 1.525 & 1.540 & 1.523 \\
\hline 14 & 1200.0 & 1.511 & 1.529 & 1.511 & 1.511 & 1.506 & 1.524 & 1.540 & 1.524 \\
\hline 15 & 1300.0 & 1.507 & 1.527 & 1.512 & 1.509 & 1.509 & 1.526 & 1.542 & 1.522 \\
\hline \multirow[t]{2}{*}{ Step } & Burnup & \multicolumn{8}{|c|}{ MPACT Power Peaking Factors } \\
\hline & $\frac{\mathrm{MWd}}{\mathrm{MtU}}$ & A & B & $\mathrm{C}$ & $\mathrm{D}$ & $\mathrm{P}$ & Q & $\mathrm{R}$ & $S$ \\
\hline 0 & 0.0 & 1.526 & 1.519 & 1.515 & 1.519 & 1.529 & 1.550 & 1.529 & 1.524 \\
\hline 1 & 25.0 & 1.503 & 1.497 & 1.493 & 1.496 & 1.505 & 1.527 & 1.506 & 1.500 \\
\hline 2 & 50.0 & 1.506 & 1.501 & 1.496 & 1.500 & 1.508 & 1.530 & 1.509 & 1.504 \\
\hline 3 & 100.0 & 1.514 & 1.508 & 1.504 & 1.508 & 1.516 & 1.539 & 1.517 & 1.512 \\
\hline 4 & 200.0 & 1.525 & 1.520 & 1.516 & 1.520 & 1.529 & 1.551 & 1.530 & 1.525 \\
\hline 5 & 300.0 & 1.532 & 1.526 & 1.522 & 1.526 & 1.535 & 1.557 & 1.535 & 1.530 \\
\hline 6 & 400.0 & 1.535 & 1.528 & 1.525 & 1.529 & 1.539 & 1.561 & 1.538 & 1.533 \\
\hline 7 & 500.0 & 1.536 & 1.530 & 1.526 & 1.530 & 1.540 & 1.562 & 1.539 & 1.534 \\
\hline 8 & 600.0 & 1.535 & 1.529 & 1.525 & 1.529 & 1.540 & 1.562 & 1.539 & 1.534 \\
\hline 9 & 700.0 & 1.535 & 1.528 & 1.524 & 1.529 & 1.538 & 1.560 & 1.538 & 1.533 \\
\hline 10 & 800.0 & 1.532 & 1.526 & 1.521 & 1.526 & 1.536 & 1.558 & 1.536 & 1.531 \\
\hline 11 & 900.0 & 1.529 & 1.523 & 1.518 & 1.523 & 1.533 & 1.554 & 1.533 & 1.528 \\
\hline 12 & 1000.0 & 1.526 & 1.519 & 1.515 & 1.520 & 1.529 & 1.551 & 1.529 & 1.524 \\
\hline 13 & 1100.0 & 1.522 & 1.515 & 1.511 & 1.516 & 1.525 & 1.547 & 1.526 & 1.520 \\
\hline 14 & 1200.0 & 1.518 & 1.511 & 1.507 & 1.512 & 1.521 & 1.542 & 1.521 & 1.516 \\
\hline 15 & 1300.0 & 1.514 & 1.507 & 1.502 & 1.508 & 1.516 & 1.537 & 1.517 & 1.511 \\
\hline
\end{tabular}


Table 7.56. Problem 6.2.1 reference solution power peaking factors in element 3 for charge-pan I (2/2).

\begin{tabular}{|c|c|c|c|c|c|c|c|c|c|}
\hline \multirow[t]{2}{*}{ Step } & \multirow{2}{*}{$\begin{array}{l}\text { Burnup } \\
{\left[\frac{\mathrm{MWd}}{\mathrm{MtU}}\right]}\end{array}$} & \multicolumn{8}{|c|}{ Shift Power Peaking Factors } \\
\hline & & $\mathrm{E}$ & $\mathrm{F}$ & G & $\mathrm{H}$ & $\mathrm{J}$ & K & $\mathrm{L}$ & M \\
\hline 0 & 0.0 & 1.504 & 1.507 & 1.519 & 1.525 & 1.528 & 1.518 & 1.507 & 1.504 \\
\hline 1 & 25.0 & 1.505 & 1.509 & 1.524 & 1.523 & 1.530 & 1.523 & 1.508 & 1.499 \\
\hline 2 & 50.0 & 1.502 & 1.503 & 1.521 & 1.524 & 1.526 & 1.523 & 1.504 & 1.501 \\
\hline 3 & 100.0 & 1.500 & 1.503 & 1.517 & 1.527 & 1.523 & 1.518 & 1.501 & 1.502 \\
\hline 4 & 200.0 & 1.490 & 1.497 & 1.510 & 1.536 & 1.522 & 1.511 & 1.497 & 1.508 \\
\hline 5 & 300.0 & 1.483 & 1.496 & 1.501 & 1.538 & 1.517 & 1.504 & 1.493 & 1.516 \\
\hline 6 & 400.0 & 1.475 & 1.490 & 1.491 & 1.545 & 1.512 & 1.496 & 1.487 & 1.519 \\
\hline 7 & 500.0 & 1.472 & 1.490 & 1.488 & 1.546 & 1.512 & 1.492 & 1.488 & 1.524 \\
\hline 8 & 600.0 & 1.467 & 1.486 & 1.481 & 1.546 & 1.509 & 1.486 & 1.484 & 1.522 \\
\hline 9 & 700.0 & 1.464 & 1.487 & 1.479 & 1.543 & 1.508 & 1.484 & 1.480 & 1.524 \\
\hline 10 & 800.0 & 1.463 & 1.490 & 1.478 & 1.545 & 1.508 & 1.480 & 1.484 & 1.525 \\
\hline 11 & 900.0 & 1.457 & 1.487 & 1.474 & 1.545 & 1.507 & 1.477 & 1.482 & 1.525 \\
\hline 12 & 1000.0 & 1.457 & 1.487 & 1.474 & 1.544 & 1.509 & 1.480 & 1.482 & 1.527 \\
\hline 13 & 1100.0 & 1.455 & 1.491 & 1.472 & 1.542 & 1.509 & 1.475 & 1.486 & 1.530 \\
\hline 14 & 1200.0 & 1.453 & 1.490 & 1.468 & 1.539 & 1.510 & 1.477 & 1.482 & 1.527 \\
\hline 15 & 1300.0 & 1.449 & 1.489 & 1.469 & 1.537 & 1.511 & 1.476 & 1.483 & 1.525 \\
\hline \multirow[t]{2}{*}{ Step } & Burnup & \multicolumn{8}{|c|}{ MPACT Power Peaking Factors } \\
\hline & $\frac{\mathrm{MWd}}{\mathrm{MtU}}$ & $\mathrm{E}$ & $\mathrm{F}$ & G & $\mathrm{H}$ & $\mathrm{J}$ & K & $\mathrm{L}$ & M \\
\hline 0 & 0.0 & 1.536 & 1.543 & 1.544 & 1.536 & 1.524 & 1.523 & 1.523 & 1.524 \\
\hline 1 & 25.0 & 1.514 & 1.521 & 1.521 & 1.514 & 1.502 & 1.500 & 1.500 & 1.502 \\
\hline 2 & 50.0 & 1.517 & 1.525 & 1.524 & 1.518 & 1.505 & 1.504 & 1.504 & 1.505 \\
\hline 3 & 100.0 & 1.525 & 1.533 & 1.533 & 1.526 & 1.514 & 1.512 & 1.512 & 1.513 \\
\hline 4 & 200.0 & 1.538 & 1.545 & 1.545 & 1.537 & 1.525 & 1.524 & 1.524 & 1.525 \\
\hline 5 & 300.0 & 1.544 & 1.551 & 1.551 & 1.543 & 1.531 & 1.530 & 1.530 & 1.531 \\
\hline 6 & 400.0 & 1.546 & 1.554 & 1.553 & 1.547 & 1.534 & 1.533 & 1.533 & 1.534 \\
\hline 7 & 500.0 & 1.548 & 1.555 & 1.555 & 1.549 & 1.536 & 1.534 & 1.535 & 1.536 \\
\hline 8 & 600.0 & 1.547 & 1.555 & 1.554 & 1.548 & 1.536 & 1.534 & 1.534 & 1.535 \\
\hline 9 & 700.0 & 1.546 & 1.554 & 1.554 & 1.546 & 1.534 & 1.533 & 1.533 & 1.534 \\
\hline 10 & 800.0 & 1.543 & 1.551 & 1.551 & 1.543 & 1.532 & 1.530 & 1.530 & 1.532 \\
\hline 11 & 900.0 & 1.540 & 1.548 & 1.548 & 1.540 & 1.528 & 1.527 & 1.527 & 1.529 \\
\hline 12 & 1000.0 & 1.537 & 1.545 & 1.545 & 1.537 & 1.525 & 1.524 & 1.524 & 1.526 \\
\hline 13 & 1100.0 & 1.532 & 1.541 & 1.541 & 1.533 & 1.521 & 1.520 & 1.520 & 1.522 \\
\hline 14 & 1200.0 & 1.528 & 1.537 & 1.537 & 1.528 & 1.517 & 1.516 & 1.516 & 1.518 \\
\hline 15 & 1300.0 & 1.524 & 1.532 & 1.532 & 1.524 & 1.512 & 1.511 & 1.511 & 1.513 \\
\hline
\end{tabular}


Table 7.57. Problem 6.2.1 reference solution power peaking factors in element 4 for charge-pan (1/2).

\begin{tabular}{|c|c|c|c|c|c|c|c|c|c|}
\hline \multirow[t]{2}{*}{ Step } & \multirow{2}{*}{$\begin{array}{l}\text { Burnup } \\
{\left[\frac{\mathrm{MWd}}{\mathrm{MtU}}\right]}\end{array}$} & \multicolumn{8}{|c|}{ Shift Power Peaking Factors } \\
\hline & & A & $\mathrm{B}$ & $\mathrm{C}$ & $\mathrm{D}$ & $\mathrm{P}$ & Q & $\mathrm{R}$ & $\mathrm{S}$ \\
\hline 0 & 0.0 & 1.026 & 1.043 & 1.025 & 1.012 & 0.993 & 1.008 & 1.074 & 1.009 \\
\hline 1 & 25.0 & 0.997 & 1.017 & 1.002 & 0.984 & 0.959 & 0.986 & 1.051 & 0.981 \\
\hline 2 & 50.0 & 0.999 & 1.018 & 1.002 & 0.987 & 0.959 & 0.983 & 1.050 & 0.979 \\
\hline 3 & 100.0 & 1.008 & 1.025 & 1.004 & 0.995 & 0.972 & 0.989 & 1.056 & 0.989 \\
\hline 4 & 200.0 & 1.024 & 1.035 & 1.011 & 1.009 & 0.987 & 0.994 & 1.065 & 1.003 \\
\hline 5 & 300.0 & 1.036 & 1.045 & 1.017 & 1.022 & 0.998 & 1.000 & 1.067 & 1.019 \\
\hline 6 & 400.0 & 1.039 & 1.048 & 1.018 & 1.027 & 1.005 & 0.998 & 1.075 & 1.022 \\
\hline 7 & 500.0 & 1.047 & 1.050 & 1.021 & 1.032 & 1.012 & 1.005 & 1.074 & 1.030 \\
\hline 8 & 600.0 & 1.046 & 1.052 & 1.018 & 1.034 & 1.011 & 1.001 & 1.073 & 1.030 \\
\hline 9 & 700.0 & 1.050 & 1.057 & 1.020 & 1.035 & 1.013 & 1.004 & 1.074 & 1.032 \\
\hline 10 & 800.0 & 1.047 & 1.055 & 1.019 & 1.036 & 1.010 & 1.001 & 1.074 & 1.030 \\
\hline 11 & 900.0 & 1.047 & 1.056 & 1.017 & 1.032 & 1.007 & 1.001 & 1.074 & 1.026 \\
\hline 12 & 1000.0 & 1.041 & 1.053 & 1.016 & 1.028 & 1.001 & 0.999 & 1.073 & 1.023 \\
\hline 13 & 1100.0 & 1.034 & 1.046 & 1.010 & 1.026 & 0.995 & 0.992 & 1.065 & 1.015 \\
\hline 14 & 1200.0 & 1.032 & 1.046 & 1.008 & 1.022 & 0.993 & 0.994 & 1.068 & 1.014 \\
\hline 15 & 1300.0 & 1.030 & 1.047 & 1.006 & 1.019 & 0.987 & 0.989 & 1.068 & 1.010 \\
\hline \multirow[t]{2}{*}{ Step } & Burnup & \multicolumn{8}{|c|}{ MPACT Power Peaking Factors } \\
\hline & {$\left[\frac{\mathrm{MWd}}{\mathrm{MtU}}\right.$} & A & B & $\mathrm{C}$ & $\mathrm{D}$ & $\mathrm{P}$ & Q & $\mathrm{R}$ & S \\
\hline 0 & 0.0 & 1.022 & 1.005 & 0.992 & 1.005 & 0.988 & 1.053 & 0.988 & 0.972 \\
\hline 1 & 25.0 & 1.043 & 1.025 & 1.013 & 1.025 & 1.008 & 1.073 & 1.008 & 0.992 \\
\hline 2 & 50.0 & 1.040 & 1.022 & 1.009 & 1.022 & 1.004 & 1.070 & 1.005 & 0.988 \\
\hline 3 & 100.0 & 1.034 & 1.016 & 1.003 & 1.016 & 0.999 & 1.064 & 0.999 & 0.983 \\
\hline 4 & 200.0 & 1.026 & 1.009 & 0.996 & 1.008 & 0.992 & 1.057 & 0.992 & 0.976 \\
\hline 5 & 300.0 & 1.022 & 1.004 & 0.992 & 1.004 & 0.988 & 1.052 & 0.988 & 0.971 \\
\hline 6 & 400.0 & 1.021 & 1.003 & 0.991 & 1.003 & 0.987 & 1.052 & 0.987 & 0.971 \\
\hline 7 & 500.0 & 1.021 & 1.004 & 0.992 & 1.004 & 0.988 & 1.053 & 0.988 & 0.972 \\
\hline 8 & 600.0 & 1.023 & 1.006 & 0.993 & 1.006 & 0.989 & 1.054 & 0.989 & 0.973 \\
\hline 9 & 700.0 & 1.027 & 1.009 & 0.997 & 1.009 & 0.993 & 1.058 & 0.993 & 0.977 \\
\hline 10 & 800.0 & 1.030 & 1.012 & 1.000 & 1.013 & 0.996 & 1.062 & 0.997 & 0.980 \\
\hline 11 & 900.0 & 1.034 & 1.017 & 1.004 & 1.017 & 1.001 & 1.066 & 1.001 & 0.985 \\
\hline 12 & 1000.0 & 1.038 & 1.021 & 1.008 & 1.021 & 1.005 & 1.069 & 1.005 & 0.989 \\
\hline 13 & 1100.0 & 1.043 & 1.025 & 1.013 & 1.026 & 1.009 & 1.074 & 1.009 & 0.993 \\
\hline 14 & 1200.0 & 1.048 & 1.030 & 1.017 & 1.031 & 1.014 & 1.079 & 1.014 & 0.998 \\
\hline 15 & 1300.0 & 1.053 & 1.035 & 1.022 & 1.036 & 1.019 & 1.084 & 1.019 & 1.003 \\
\hline
\end{tabular}


Table 7.58. Problem 6.2.1 reference solution power peaking factors in element 4 for charge-pan I (2/2).

\begin{tabular}{|c|c|c|c|c|c|c|c|c|c|}
\hline \multirow[t]{2}{*}{ Step } & \multirow{2}{*}{$\begin{array}{l}\text { Burnup } \\
{\left[\frac{\mathrm{MWd}}{\mathrm{MtU}}\right]}\end{array}$} & \multicolumn{8}{|c|}{ Shift Power Peaking Factors } \\
\hline & & $\mathrm{E}$ & $\mathrm{F}$ & G & $\mathrm{H}$ & $\mathrm{J}$ & K & $\mathrm{L}$ & M \\
\hline 0 & 0.0 & 0.995 & 1.000 & 1.041 & 1.063 & 1.063 & 1.040 & 1.002 & 0.996 \\
\hline 1 & 25.0 & 0.967 & 0.979 & 1.016 & 1.034 & 1.041 & 1.015 & 0.976 & 0.971 \\
\hline 2 & 50.0 & 0.968 & 0.976 & 1.017 & 1.036 & 1.040 & 1.016 & 0.977 & 0.969 \\
\hline 3 & 100.0 & 0.974 & 0.979 & 1.021 & 1.045 & 1.044 & 1.024 & 0.979 & 0.976 \\
\hline 4 & 200.0 & 0.986 & 0.982 & 1.032 & 1.061 & 1.049 & 1.035 & 0.984 & 0.984 \\
\hline 5 & 300.0 & 0.995 & 0.987 & 1.044 & 1.071 & 1.049 & 1.044 & 0.986 & 0.991 \\
\hline 6 & 400.0 & 1.000 & 0.986 & 1.047 & 1.078 & 1.051 & 1.049 & 0.983 & 1.000 \\
\hline 7 & 500.0 & 1.002 & 0.984 & 1.052 & 1.084 & 1.051 & 1.054 & 0.985 & 1.001 \\
\hline 8 & 600.0 & 1.006 & 0.982 & 1.051 & 1.084 & 1.049 & 1.054 & 0.983 & 1.002 \\
\hline 9 & 700.0 & 1.008 & 0.982 & 1.056 & 1.086 & 1.049 & 1.057 & 0.981 & 1.004 \\
\hline 10 & 800.0 & 1.006 & 0.977 & 1.052 & 1.089 & 1.044 & 1.057 & 0.979 & 1.005 \\
\hline 11 & 900.0 & 1.003 & 0.976 & 1.053 & 1.086 & 1.041 & 1.056 & 0.973 & 1.005 \\
\hline 12 & 1000.0 & 0.998 & 0.971 & 1.048 & 1.085 & 1.041 & 1.054 & 0.970 & 1.002 \\
\hline 13 & 1100.0 & 0.996 & 0.965 & 1.043 & 1.077 & 1.032 & 1.046 & 0.962 & 0.998 \\
\hline 14 & 1200.0 & 0.994 & 0.964 & 1.044 & 1.073 & 1.030 & 1.048 & 0.963 & 0.996 \\
\hline 15 & 1300.0 & 0.987 & 0.958 & 1.038 & 1.072 & 1.027 & 1.044 & 0.958 & 0.993 \\
\hline \multirow[t]{2}{*}{ Step } & Burnup & \multicolumn{8}{|c|}{ MPACT Power Peaking Factors } \\
\hline & $\frac{\mathrm{MWd}}{\mathrm{MtU}}$ & $\mathrm{E}$ & $\mathrm{F}$ & G & $\mathrm{H}$ & $\mathrm{J}$ & K & $\mathrm{L}$ & M \\
\hline 0 & 0.0 & 1.021 & 1.041 & 1.042 & 1.021 & 0.980 & 0.976 & 0.976 & 0.980 \\
\hline 1 & 25.0 & 1.041 & 1.062 & 1.062 & 1.041 & 1.001 & 0.996 & 0.996 & 1.001 \\
\hline 2 & 50.0 & 1.038 & 1.059 & 1.059 & 1.038 & 0.998 & 0.993 & 0.993 & 0.997 \\
\hline 3 & 100.0 & 1.032 & 1.052 & 1.053 & 1.032 & 0.991 & 0.987 & 0.987 & 0.991 \\
\hline 4 & 200.0 & 1.024 & 1.045 & 1.045 & 1.024 & 0.984 & 0.980 & 0.980 & 0.984 \\
\hline 5 & 300.0 & 1.021 & 1.041 & 1.041 & 1.020 & 0.980 & 0.976 & 0.976 & 0.980 \\
\hline 6 & 400.0 & 1.020 & 1.040 & 1.040 & 1.020 & 0.979 & 0.975 & 0.975 & 0.979 \\
\hline 7 & 500.0 & 1.020 & 1.041 & 1.040 & 1.021 & 0.980 & 0.975 & 0.975 & 0.980 \\
\hline 8 & 600.0 & 1.022 & 1.043 & 1.043 & 1.022 & 0.982 & 0.977 & 0.977 & 0.982 \\
\hline 9 & 700.0 & 1.025 & 1.046 & 1.047 & 1.025 & 0.985 & 0.981 & 0.981 & 0.985 \\
\hline 10 & 800.0 & 1.029 & 1.050 & 1.050 & 1.029 & 0.989 & 0.984 & 0.984 & 0.989 \\
\hline 11 & 900.0 & 1.033 & 1.055 & 1.054 & 1.033 & 0.993 & 0.989 & 0.988 & 0.993 \\
\hline 12 & 1000.0 & 1.037 & 1.058 & 1.058 & 1.037 & 0.997 & 0.992 & 0.992 & 0.997 \\
\hline 13 & 1100.0 & 1.042 & 1.063 & 1.063 & 1.042 & 1.002 & 0.997 & 0.997 & 1.002 \\
\hline 14 & 1200.0 & 1.047 & 1.068 & 1.068 & 1.047 & 1.006 & 1.002 & 1.002 & 1.007 \\
\hline 15 & 1300.0 & 1.052 & 1.073 & 1.073 & 1.052 & 1.011 & 1.007 & 1.007 & 1.011 \\
\hline
\end{tabular}


Table 7.59. Problem 6.2.1 reference solution power peaking factors in element 5 for charge-pan (1/2).

\begin{tabular}{|c|c|c|c|c|c|c|c|c|c|}
\hline \multirow[t]{2}{*}{ Step } & \multirow{2}{*}{$\begin{array}{l}\text { Burnup } \\
{\left[\frac{\mathrm{MWd}}{\mathrm{MtU}}\right]}\end{array}$} & \multicolumn{8}{|c|}{ Shift Power Peaking Factors } \\
\hline & & A & B & $\mathrm{C}$ & $\mathrm{D}$ & $\mathrm{P}$ & Q & $\mathrm{R}$ & $\mathrm{S}$ \\
\hline 0 & 0.0 & 0.571 & 0.584 & 0.571 & 0.563 & 0.545 & 0.557 & 0.605 & 0.556 \\
\hline 1 & 25.0 & 0.545 & 0.558 & 0.548 & 0.536 & 0.519 & 0.532 & 0.578 & 0.528 \\
\hline 2 & 50.0 & 0.548 & 0.561 & 0.549 & 0.540 & 0.518 & 0.532 & 0.582 & 0.530 \\
\hline 3 & 100.0 & 0.553 & 0.564 & 0.552 & 0.543 & 0.525 & 0.534 & 0.585 & 0.537 \\
\hline 4 & 200.0 & 0.566 & 0.572 & 0.560 & 0.554 & 0.539 & 0.544 & 0.594 & 0.548 \\
\hline 5 & 300.0 & 0.575 & 0.579 & 0.564 & 0.563 & 0.548 & 0.548 & 0.598 & 0.559 \\
\hline 6 & 400.0 & 0.584 & 0.585 & 0.568 & 0.568 & 0.555 & 0.552 & 0.602 & 0.565 \\
\hline 7 & 500.0 & 0.587 & 0.587 & 0.569 & 0.574 & 0.558 & 0.552 & 0.602 & 0.570 \\
\hline 8 & 600.0 & 0.589 & 0.589 & 0.568 & 0.575 & 0.562 & 0.552 & 0.603 & 0.574 \\
\hline 9 & 700.0 & 0.593 & 0.591 & 0.569 & 0.579 & 0.564 & 0.553 & 0.605 & 0.575 \\
\hline 10 & 800.0 & 0.590 & 0.586 & 0.563 & 0.573 & 0.559 & 0.548 & 0.599 & 0.570 \\
\hline 11 & 900.0 & 0.590 & 0.588 & 0.563 & 0.576 & 0.559 & 0.548 & 0.600 & 0.571 \\
\hline 12 & 1000.0 & 0.588 & 0.585 & 0.560 & 0.572 & 0.556 & 0.543 & 0.599 & 0.568 \\
\hline 13 & 1100.0 & 0.580 & 0.579 & 0.555 & 0.567 & 0.552 & 0.537 & 0.592 & 0.563 \\
\hline 14 & 1200.0 & 0.579 & 0.578 & 0.551 & 0.566 & 0.550 & 0.537 & 0.589 & 0.561 \\
\hline 15 & 1300.0 & 0.576 & 0.576 & 0.549 & 0.565 & 0.546 & 0.533 & 0.587 & 0.557 \\
\hline \multirow[t]{2}{*}{ Step } & Burnup & \multicolumn{8}{|c|}{ MPACT Power Peaking Factors } \\
\hline & $\frac{\mathrm{MWd}}{\mathrm{MtU}}$ & A & B & $\mathrm{C}$ & $\mathrm{D}$ & $\mathrm{P}$ & Q & $\mathrm{R}$ & $S$ \\
\hline 0 & 0.0 & 0.550 & 0.538 & 0.529 & 0.538 & 0.523 & 0.568 & 0.523 & 0.513 \\
\hline 1 & 25.0 & 0.591 & 0.579 & 0.570 & 0.578 & 0.563 & 0.610 & 0.563 & 0.553 \\
\hline 2 & 50.0 & 0.586 & 0.573 & 0.565 & 0.573 & 0.557 & 0.604 & 0.558 & 0.548 \\
\hline 3 & 100.0 & 0.573 & 0.561 & 0.553 & 0.561 & 0.545 & 0.592 & 0.546 & 0.536 \\
\hline 4 & 200.0 & 0.556 & 0.544 & 0.536 & 0.544 & 0.529 & 0.574 & 0.528 & 0.520 \\
\hline 5 & 300.0 & 0.545 & 0.533 & 0.525 & 0.533 & 0.519 & 0.563 & 0.519 & 0.509 \\
\hline 6 & 400.0 & 0.541 & 0.529 & 0.521 & 0.529 & 0.514 & 0.559 & 0.514 & 0.505 \\
\hline 7 & 500.0 & 0.539 & 0.527 & 0.519 & 0.528 & 0.513 & 0.558 & 0.513 & 0.503 \\
\hline 8 & 600.0 & 0.540 & 0.529 & 0.520 & 0.528 & 0.513 & 0.558 & 0.514 & 0.504 \\
\hline 9 & 700.0 & 0.543 & 0.531 & 0.523 & 0.531 & 0.516 & 0.561 & 0.517 & 0.507 \\
\hline 10 & 800.0 & 0.547 & 0.535 & 0.527 & 0.535 & 0.520 & 0.565 & 0.520 & 0.510 \\
\hline 11 & 900.0 & 0.552 & 0.540 & 0.532 & 0.540 & 0.525 & 0.571 & 0.525 & 0.516 \\
\hline 12 & 1000.0 & 0.558 & 0.546 & 0.537 & 0.545 & 0.530 & 0.576 & 0.530 & 0.521 \\
\hline 13 & 1100.0 & 0.564 & 0.552 & 0.544 & 0.552 & 0.537 & 0.583 & 0.537 & 0.527 \\
\hline 14 & 1200.0 & 0.571 & 0.559 & 0.551 & 0.559 & 0.544 & 0.591 & 0.543 & 0.534 \\
\hline 15 & 1300.0 & 0.579 & 0.567 & 0.558 & 0.567 & 0.551 & 0.598 & 0.552 & 0.542 \\
\hline
\end{tabular}


Table 7.60. Problem 6.2.1 reference solution power peaking factors in element 5 for charge-pan I (2/2).

\begin{tabular}{|c|c|c|c|c|c|c|c|c|c|}
\hline \multirow[t]{2}{*}{ Step } & \multirow{2}{*}{$\begin{array}{l}\text { Burnup } \\
{\left[\frac{\mathrm{MWd}}{\mathrm{MtU}}\right]}\end{array}$} & \multicolumn{8}{|c|}{ Shift Power Peaking Factors } \\
\hline & & $\mathrm{E}$ & $\mathrm{F}$ & G & $\mathrm{H}$ & $\mathrm{J}$ & K & $\mathrm{L}$ & M \\
\hline 0 & 0.0 & 0.549 & 0.553 & 0.581 & 0.596 & 0.595 & 0.580 & 0.551 & 0.548 \\
\hline 1 & 25.0 & 0.522 & 0.524 & 0.553 & 0.570 & 0.568 & 0.554 & 0.525 & 0.523 \\
\hline 2 & 50.0 & 0.523 & 0.526 & 0.556 & 0.575 & 0.572 & 0.555 & 0.525 & 0.522 \\
\hline 3 & 100.0 & 0.530 & 0.532 & 0.562 & 0.575 & 0.576 & 0.562 & 0.532 & 0.528 \\
\hline 4 & 200.0 & 0.543 & 0.543 & 0.577 & 0.587 & 0.587 & 0.576 & 0.544 & 0.536 \\
\hline 5 & 300.0 & 0.551 & 0.551 & 0.586 & 0.597 & 0.598 & 0.585 & 0.550 & 0.543 \\
\hline 6 & 400.0 & 0.559 & 0.558 & 0.594 & 0.603 & 0.603 & 0.594 & 0.558 & 0.549 \\
\hline 7 & 500.0 & 0.562 & 0.559 & 0.597 & 0.604 & 0.607 & 0.597 & 0.561 & 0.551 \\
\hline 8 & 600.0 & 0.566 & 0.564 & 0.601 & 0.606 & 0.609 & 0.600 & 0.562 & 0.551 \\
\hline 9 & 700.0 & 0.567 & 0.566 & 0.602 & 0.609 & 0.611 & 0.602 & 0.566 & 0.553 \\
\hline 10 & 800.0 & 0.565 & 0.561 & 0.597 & 0.606 & 0.608 & 0.596 & 0.562 & 0.548 \\
\hline 11 & 900.0 & 0.563 & 0.562 & 0.600 & 0.609 & 0.611 & 0.598 & 0.562 & 0.548 \\
\hline 12 & 1000.0 & 0.560 & 0.561 & 0.595 & 0.606 & 0.608 & 0.594 & 0.560 & 0.547 \\
\hline 13 & 1100.0 & 0.553 & 0.554 & 0.590 & 0.602 & 0.602 & 0.590 & 0.555 & 0.543 \\
\hline 14 & 1200.0 & 0.550 & 0.553 & 0.587 & 0.600 & 0.601 & 0.587 & 0.552 & 0.537 \\
\hline 15 & 1300.0 & 0.548 & 0.550 & 0.585 & 0.599 & 0.599 & 0.583 & 0.551 & 0.537 \\
\hline \multirow[t]{2}{*}{ Step } & Burnup & \multicolumn{8}{|c|}{ MPACT Power Peaking Factors } \\
\hline & $\frac{\mathrm{MWd}}{\mathrm{MtU}}$ & $\mathrm{E}$ & $\mathrm{F}$ & G & $\mathrm{H}$ & $\mathrm{J}$ & K & $\mathrm{L}$ & M \\
\hline 0 & 0.0 & 0.547 & 0.561 & 0.561 & 0.547 & 0.519 & 0.516 & 0.516 & 0.519 \\
\hline 1 & 25.0 & 0.588 & 0.602 & 0.603 & 0.588 & 0.559 & 0.556 & 0.556 & 0.558 \\
\hline 2 & 50.0 & 0.582 & 0.597 & 0.597 & 0.582 & 0.553 & 0.551 & 0.551 & 0.553 \\
\hline 3 & 100.0 & 0.570 & 0.584 & 0.584 & 0.570 & 0.541 & 0.539 & 0.539 & 0.541 \\
\hline 4 & 200.0 & 0.553 & 0.567 & 0.566 & 0.552 & 0.525 & 0.522 & 0.522 & 0.525 \\
\hline 5 & 300.0 & 0.542 & 0.556 & 0.556 & 0.542 & 0.515 & 0.512 & 0.512 & 0.515 \\
\hline 6 & 400.0 & 0.538 & 0.551 & 0.552 & 0.538 & 0.510 & 0.508 & 0.508 & 0.510 \\
\hline 7 & 500.0 & 0.536 & 0.550 & 0.550 & 0.536 & 0.509 & 0.506 & 0.506 & 0.509 \\
\hline 8 & 600.0 & 0.537 & 0.551 & 0.551 & 0.537 & 0.510 & 0.507 & 0.507 & 0.510 \\
\hline 9 & 700.0 & 0.540 & 0.554 & 0.554 & 0.540 & 0.512 & 0.510 & 0.510 & 0.512 \\
\hline 10 & 800.0 & 0.544 & 0.558 & 0.558 & 0.543 & 0.516 & 0.514 & 0.513 & 0.516 \\
\hline 11 & 900.0 & 0.549 & 0.564 & 0.563 & 0.549 & 0.521 & 0.519 & 0.519 & 0.521 \\
\hline 12 & 1000.0 & 0.554 & 0.569 & 0.569 & 0.555 & 0.527 & 0.524 & 0.524 & 0.526 \\
\hline 13 & 1100.0 & 0.561 & 0.576 & 0.575 & 0.561 & 0.533 & 0.530 & 0.531 & 0.533 \\
\hline 14 & 1200.0 & 0.568 & 0.583 & 0.583 & 0.568 & 0.540 & 0.537 & 0.537 & 0.540 \\
\hline 15 & 1300.0 & 0.576 & 0.591 & 0.591 & 0.576 & 0.547 & 0.545 & 0.545 & 0.547 \\
\hline
\end{tabular}




\section{Charge-Pan II}

Table 7.61. Problem 6.2.1 reference solution power peaking factors in element 1 for charge-pan II (1/2).

\begin{tabular}{|c|c|c|c|c|c|c|c|c|c|}
\hline \multirow[t]{2}{*}{ Step } & \multirow{2}{*}{$\begin{array}{l}\text { Burnup } \\
{\left[\frac{\mathrm{MWd}}{\mathrm{MtU}}\right]}\end{array}$} & \multicolumn{8}{|c|}{ Shift Power Peaking Factors } \\
\hline & & A & B & $\mathrm{C}$ & $\mathrm{D}$ & $\mathrm{P}$ & Q & $\mathrm{R}$ & $S$ \\
\hline 0 & 0.0 & 1.033 & 1.033 & 1.035 & 1.033 & 1.045 & 1.044 & 1.043 & 1.048 \\
\hline 1 & 25.0 & 1.070 & 1.070 & 1.072 & 1.070 & 1.078 & 1.080 & 1.082 & 1.083 \\
\hline 2 & 50.0 & 1.069 & 1.072 & 1.070 & 1.071 & 1.079 & 1.081 & 1.081 & 1.082 \\
\hline 3 & 100.0 & 1.060 & 1.061 & 1.061 & 1.061 & 1.070 & 1.071 & 1.075 & 1.073 \\
\hline 4 & 200.0 & 1.045 & 1.045 & 1.045 & 1.044 & 1.055 & 1.056 & 1.053 & 1.056 \\
\hline 5 & 300.0 & 1.032 & 1.033 & 1.031 & 1.032 & 1.041 & 1.044 & 1.041 & 1.047 \\
\hline 6 & 400.0 & 1.028 & 1.026 & 1.027 & 1.026 & 1.034 & 1.039 & 1.037 & 1.037 \\
\hline 7 & 500.0 & 1.020 & 1.021 & 1.020 & 1.022 & 1.029 & 1.032 & 1.029 & 1.033 \\
\hline 8 & 600.0 & 1.020 & 1.018 & 1.019 & 1.020 & 1.026 & 1.035 & 1.028 & 1.033 \\
\hline 9 & 700.0 & 1.018 & 1.019 & 1.021 & 1.018 & 1.027 & 1.031 & 1.028 & 1.031 \\
\hline 10 & 800.0 & 1.023 & 1.024 & 1.024 & 1.026 & 1.032 & 1.034 & 1.029 & 1.040 \\
\hline 11 & 900.0 & 1.027 & 1.025 & 1.026 & 1.028 & 1.034 & 1.037 & 1.037 & 1.041 \\
\hline 12 & 1000.0 & 1.034 & 1.032 & 1.033 & 1.034 & 1.041 & 1.044 & 1.038 & 1.048 \\
\hline 13 & 1100.0 & 1.038 & 1.036 & 1.040 & 1.040 & 1.046 & 1.047 & 1.048 & 1.052 \\
\hline 14 & 1200.0 & 1.044 & 1.045 & 1.047 & 1.044 & 1.050 & 1.053 & 1.050 & 1.058 \\
\hline 15 & 1300.0 & 1.045 & 1.048 & 1.049 & 1.049 & 1.053 & 1.060 & 1.052 & 1.061 \\
\hline \multirow[t]{2}{*}{ Step } & Burnup & \multicolumn{8}{|c|}{ MPACT Power Peaking Factors } \\
\hline & $\frac{\mathrm{MWd}}{\mathrm{MtU}}$ & A & B & $\mathrm{C}$ & D & $\mathrm{P}$ & Q & $\mathrm{R}$ & $\mathrm{S}$ \\
\hline 0 & 0.0 & 1.046 & 1.046 & 1.046 & 1.045 & 1.057 & 1.057 & 1.057 & 1.057 \\
\hline 1 & 25.0 & 1.029 & 1.029 & 1.029 & 1.029 & 1.040 & 1.040 & 1.040 & 1.040 \\
\hline 2 & 50.0 & 1.030 & 1.030 & 1.030 & 1.030 & 1.040 & 1.042 & 1.041 & 1.041 \\
\hline 3 & 100.0 & 1.033 & 1.033 & 1.033 & 1.033 & 1.043 & 1.044 & 1.044 & 1.044 \\
\hline 4 & 200.0 & 1.036 & 1.035 & 1.036 & 1.036 & 1.047 & 1.047 & 1.047 & 1.047 \\
\hline 5 & 300.0 & 1.039 & 1.039 & 1.039 & 1.039 & 1.050 & 1.050 & 1.050 & 1.050 \\
\hline 6 & 400.0 & 1.040 & 1.040 & 1.040 & 1.040 & 1.052 & 1.051 & 1.051 & 1.051 \\
\hline 7 & 500.0 & 1.040 & 1.040 & 1.040 & 1.040 & 1.051 & 1.052 & 1.052 & 1.052 \\
\hline 8 & 600.0 & 1.039 & 1.039 & 1.040 & 1.039 & 1.051 & 1.051 & 1.051 & 1.052 \\
\hline 9 & 700.0 & 1.038 & 1.038 & 1.038 & 1.038 & 1.050 & 1.050 & 1.050 & 1.050 \\
\hline 10 & 800.0 & 1.037 & 1.038 & 1.037 & 1.037 & 1.049 & 1.049 & 1.049 & 1.049 \\
\hline 11 & 900.0 & 1.036 & 1.036 & 1.035 & 1.035 & 1.047 & 1.047 & 1.047 & 1.047 \\
\hline 12 & 1000.0 & 1.034 & 1.034 & 1.034 & 1.034 & 1.046 & 1.046 & 1.046 & 1.046 \\
\hline 13 & 1100.0 & 1.032 & 1.032 & 1.032 & 1.032 & 1.044 & 1.044 & 1.044 & 1.044 \\
\hline 14 & 1200.0 & 1.030 & 1.030 & 1.030 & 1.030 & 1.041 & 1.041 & 1.042 & 1.042 \\
\hline 15 & 1300.0 & 1.027 & 1.028 & 1.027 & 1.027 & 1.039 & 1.039 & 1.039 & 1.039 \\
\hline
\end{tabular}


Table 7.62. Problem 6.2.1 reference solution power peaking factors in element 1 for charge-pan II (2/2).

\begin{tabular}{|c|c|c|c|c|c|c|c|c|c|}
\hline \multirow[t]{2}{*}{ Step } & \multirow{2}{*}{$\begin{array}{l}\text { Burnup } \\
{\left[\frac{\mathrm{MWd}}{\mathrm{MtU}}\right]}\end{array}$} & \multicolumn{8}{|c|}{ Shift Power Peaking Factors } \\
\hline & & $\mathrm{E}$ & $\mathrm{F}$ & G & $\mathrm{H}$ & $\mathrm{J}$ & K & $\mathrm{L}$ & M \\
\hline 0 & 0.0 & 1.044 & 1.044 & 1.042 & 1.045 & 1.044 & 1.045 & 1.043 & 1.041 \\
\hline 1 & 25.0 & 1.078 & 1.078 & 1.080 & 1.079 & 1.082 & 1.081 & 1.082 & 1.081 \\
\hline 2 & 50.0 & 1.078 & 1.080 & 1.079 & 1.078 & 1.082 & 1.082 & 1.083 & 1.082 \\
\hline 3 & 100.0 & 1.069 & 1.068 & 1.068 & 1.070 & 1.072 & 1.070 & 1.073 & 1.071 \\
\hline 4 & 200.0 & 1.053 & 1.054 & 1.054 & 1.052 & 1.053 & 1.055 & 1.054 & 1.055 \\
\hline 5 & 300.0 & 1.042 & 1.042 & 1.040 & 1.043 & 1.041 & 1.044 & 1.044 & 1.040 \\
\hline 6 & 400.0 & 1.032 & 1.034 & 1.035 & 1.035 & 1.038 & 1.037 & 1.036 & 1.036 \\
\hline 7 & 500.0 & 1.030 & 1.030 & 1.029 & 1.029 & 1.029 & 1.032 & 1.030 & 1.030 \\
\hline 8 & 600.0 & 1.028 & 1.030 & 1.030 & 1.027 & 1.026 & 1.031 & 1.031 & 1.031 \\
\hline 9 & 700.0 & 1.027 & 1.029 & 1.028 & 1.030 & 1.027 & 1.031 & 1.030 & 1.028 \\
\hline 10 & 800.0 & 1.030 & 1.032 & 1.034 & 1.031 & 1.031 & 1.038 & 1.033 & 1.033 \\
\hline 11 & 900.0 & 1.035 & 1.033 & 1.034 & 1.036 & 1.034 & 1.040 & 1.037 & 1.038 \\
\hline 12 & 1000.0 & 1.040 & 1.040 & 1.041 & 1.041 & 1.040 & 1.044 & 1.045 & 1.043 \\
\hline 13 & 1100.0 & 1.043 & 1.043 & 1.046 & 1.045 & 1.042 & 1.052 & 1.050 & 1.049 \\
\hline 14 & 1200.0 & 1.050 & 1.053 & 1.051 & 1.051 & 1.050 & 1.056 & 1.056 & 1.056 \\
\hline 15 & 1300.0 & 1.053 & 1.054 & 1.053 & 1.053 & 1.054 & 1.060 & 1.059 & 1.060 \\
\hline \multirow[t]{2}{*}{ Step } & Burnup & \multicolumn{8}{|c|}{ MPACT Power Peaking Factors } \\
\hline & $\frac{\mathrm{MWd}}{\mathrm{MtU}}$ & $\mathrm{E}$ & $\mathrm{F}$ & G & $\mathrm{H}$ & $\mathrm{J}$ & K & $\mathrm{L}$ & M \\
\hline 0 & 0.0 & 1.056 & 1.055 & 1.055 & 1.055 & 1.055 & 1.055 & 1.055 & 1.055 \\
\hline 1 & 25.0 & 1.038 & 1.039 & 1.039 & 1.039 & 1.039 & 1.038 & 1.038 & 1.038 \\
\hline 2 & 50.0 & 1.039 & 1.040 & 1.039 & 1.039 & 1.040 & 1.039 & 1.039 & 1.039 \\
\hline 3 & 100.0 & 1.042 & 1.042 & 1.042 & 1.042 & 1.043 & 1.042 & 1.042 & 1.042 \\
\hline 4 & 200.0 & 1.045 & 1.045 & 1.046 & 1.045 & 1.045 & 1.045 & 1.045 & 1.045 \\
\hline 5 & 300.0 & 1.048 & 1.049 & 1.049 & 1.048 & 1.049 & 1.048 & 1.049 & 1.048 \\
\hline 6 & 400.0 & 1.049 & 1.050 & 1.050 & 1.049 & 1.049 & 1.050 & 1.049 & 1.050 \\
\hline 7 & 500.0 & 1.049 & 1.050 & 1.049 & 1.050 & 1.050 & 1.050 & 1.049 & 1.050 \\
\hline 8 & 600.0 & 1.049 & 1.049 & 1.049 & 1.049 & 1.049 & 1.049 & 1.049 & 1.050 \\
\hline 9 & 700.0 & 1.048 & 1.048 & 1.048 & 1.049 & 1.048 & 1.048 & 1.048 & 1.049 \\
\hline 10 & 800.0 & 1.047 & 1.048 & 1.047 & 1.047 & 1.047 & 1.047 & 1.047 & 1.048 \\
\hline 11 & 900.0 & 1.045 & 1.045 & 1.045 & 1.046 & 1.045 & 1.045 & 1.045 & 1.045 \\
\hline 12 & 1000.0 & 1.044 & 1.044 & 1.044 & 1.044 & 1.044 & 1.044 & 1.044 & 1.044 \\
\hline 13 & 1100.0 & 1.042 & 1.042 & 1.042 & 1.042 & 1.042 & 1.042 & 1.042 & 1.042 \\
\hline 14 & 1200.0 & 1.039 & 1.040 & 1.039 & 1.040 & 1.039 & 1.039 & 1.039 & 1.040 \\
\hline 15 & 1300.0 & 1.037 & 1.037 & 1.037 & 1.038 & 1.037 & 1.037 & 1.037 & 1.037 \\
\hline
\end{tabular}


Table 7.63. Problem 6.2.1 reference solution power peaking factors in element 2 for charge-pan II (1/2).

\begin{tabular}{|c|c|c|c|c|c|c|c|c|c|}
\hline \multirow[t]{2}{*}{ Step } & \multirow{2}{*}{$\begin{array}{l}\text { Burnup } \\
{\left[\frac{\mathrm{MWd}}{\mathrm{MtU}}\right]}\end{array}$} & \multicolumn{8}{|c|}{ Shift Power Peaking Factors } \\
\hline & & A & B & $\mathrm{C}$ & $\mathrm{D}$ & $\mathrm{P}$ & Q & $\mathrm{R}$ & $\mathrm{S}$ \\
\hline 0 & 0.0 & 1.553 & 1.551 & 1.550 & 1.553 & 1.570 & 1.574 & 1.571 & 1.569 \\
\hline 1 & 25.0 & 1.596 & 1.597 & 1.598 & 1.599 & 1.613 & 1.616 & 1.619 & 1.618 \\
\hline 2 & 50.0 & 1.600 & 1.601 & 1.598 & 1.599 & 1.611 & 1.614 & 1.615 & 1.620 \\
\hline 3 & 100.0 & 1.587 & 1.588 & 1.584 & 1.586 & 1.602 & 1.603 & 1.603 & 1.606 \\
\hline 4 & 200.0 & 1.565 & 1.565 & 1.566 & 1.566 & 1.580 & 1.585 & 1.583 & 1.584 \\
\hline 5 & 300.0 & 1.547 & 1.543 & 1.548 & 1.546 & 1.562 & 1.565 & 1.565 & 1.565 \\
\hline 6 & 400.0 & 1.537 & 1.536 & 1.539 & 1.537 & 1.550 & 1.553 & 1.554 & 1.552 \\
\hline 7 & 500.0 & 1.526 & 1.526 & 1.525 & 1.525 & 1.543 & 1.545 & 1.543 & 1.546 \\
\hline 8 & 600.0 & 1.521 & 1.523 & 1.523 & 1.521 & 1.538 & 1.541 & 1.539 & 1.542 \\
\hline 9 & 700.0 & 1.521 & 1.523 & 1.518 & 1.521 & 1.533 & 1.537 & 1.534 & 1.538 \\
\hline 10 & 800.0 & 1.519 & 1.520 & 1.522 & 1.521 & 1.540 & 1.538 & 1.534 & 1.542 \\
\hline 11 & 900.0 & 1.521 & 1.523 & 1.524 & 1.523 & 1.537 & 1.539 & 1.534 & 1.543 \\
\hline 12 & 1000.0 & 1.523 & 1.524 & 1.525 & 1.524 & 1.540 & 1.544 & 1.540 & 1.544 \\
\hline 13 & 1100.0 & 1.533 & 1.531 & 1.535 & 1.535 & 1.543 & 1.547 & 1.543 & 1.555 \\
\hline 14 & 1200.0 & 1.536 & 1.533 & 1.538 & 1.537 & 1.546 & 1.550 & 1.545 & 1.557 \\
\hline 15 & 1300.0 & 1.538 & 1.540 & 1.542 & 1.540 & 1.553 & 1.556 & 1.547 & 1.563 \\
\hline \multirow[t]{2}{*}{ Step } & Burnup & \multicolumn{8}{|c|}{ MPACT Power Peaking Factors } \\
\hline & $\frac{\mathrm{MWd}}{\mathrm{MtU}}$ & A & B & $\mathrm{C}$ & $\mathrm{D}$ & $\mathrm{P}$ & Q & $\mathrm{R}$ & S \\
\hline 0 & 0.0 & 1.588 & 1.589 & 1.589 & 1.588 & 1.607 & 1.608 & 1.608 & 1.608 \\
\hline 1 & 25.0 & 1.549 & 1.549 & 1.549 & 1.549 & 1.566 & 1.568 & 1.568 & 1.568 \\
\hline 2 & 50.0 & 1.555 & 1.555 & 1.555 & 1.554 & 1.571 & 1.574 & 1.575 & 1.574 \\
\hline 3 & 100.0 & 1.568 & 1.568 & 1.568 & 1.567 & 1.584 & 1.587 & 1.587 & 1.587 \\
\hline 4 & 200.0 & 1.583 & 1.583 & 1.583 & 1.582 & 1.602 & 1.603 & 1.603 & 1.603 \\
\hline 5 & 300.0 & 1.593 & 1.593 & 1.593 & 1.592 & 1.611 & 1.613 & 1.613 & 1.613 \\
\hline 6 & 400.0 & 1.596 & 1.596 & 1.596 & 1.595 & 1.614 & 1.615 & 1.617 & 1.617 \\
\hline 7 & 500.0 & 1.597 & 1.597 & 1.597 & 1.596 & 1.615 & 1.618 & 1.618 & 1.618 \\
\hline 8 & 600.0 & 1.595 & 1.595 & 1.595 & 1.594 & 1.613 & 1.615 & 1.616 & 1.616 \\
\hline 9 & 700.0 & 1.591 & 1.592 & 1.591 & 1.591 & 1.610 & 1.612 & 1.613 & 1.612 \\
\hline 10 & 800.0 & 1.587 & 1.588 & 1.587 & 1.587 & 1.605 & 1.607 & 1.608 & 1.607 \\
\hline 11 & 900.0 & 1.581 & 1.582 & 1.581 & 1.581 & 1.599 & 1.601 & 1.602 & 1.601 \\
\hline 12 & 1000.0 & 1.575 & 1.576 & 1.575 & 1.575 & 1.593 & 1.595 & 1.596 & 1.595 \\
\hline 13 & 1100.0 & 1.567 & 1.569 & 1.568 & 1.568 & 1.585 & 1.587 & 1.588 & 1.587 \\
\hline 14 & 1200.0 & 1.560 & 1.561 & 1.560 & 1.561 & 1.578 & 1.579 & 1.581 & 1.579 \\
\hline 15 & 1300.0 & 1.552 & 1.553 & 1.552 & 1.552 & 1.569 & 1.571 & 1.572 & 1.571 \\
\hline
\end{tabular}


Table 7.64. Problem 6.2.1 reference solution power peaking factors in element 2 for charge-pan II (2/2).

\begin{tabular}{|c|c|c|c|c|c|c|c|c|c|}
\hline \multirow[t]{2}{*}{ Step } & \multirow{2}{*}{$\begin{array}{l}\text { Burnup } \\
{\left[\frac{\mathrm{MWd}}{\mathrm{MtU}}\right]}\end{array}$} & \multicolumn{8}{|c|}{ Shift Power Peaking Factors } \\
\hline & & $\mathrm{E}$ & $\mathrm{F}$ & G & $\mathrm{H}$ & $\mathrm{J}$ & K & $\mathrm{L}$ & M \\
\hline 0 & 0.0 & 1.568 & 1.571 & 1.568 & 1.568 & 1.567 & 1.566 & 1.565 & 1.567 \\
\hline 1 & 25.0 & 1.612 & 1.614 & 1.611 & 1.612 & 1.617 & 1.616 & 1.616 & 1.613 \\
\hline 2 & 50.0 & 1.611 & 1.614 & 1.611 & 1.614 & 1.617 & 1.616 & 1.619 & 1.613 \\
\hline 3 & 100.0 & 1.600 & 1.601 & 1.598 & 1.599 & 1.600 & 1.604 & 1.603 & 1.605 \\
\hline 4 & 200.0 & 1.578 & 1.582 & 1.578 & 1.582 & 1.580 & 1.580 & 1.583 & 1.579 \\
\hline 5 & 300.0 & 1.559 & 1.562 & 1.560 & 1.560 & 1.560 & 1.564 & 1.561 & 1.563 \\
\hline 6 & 400.0 & 1.550 & 1.549 & 1.548 & 1.551 & 1.552 & 1.552 & 1.550 & 1.551 \\
\hline 7 & 500.0 & 1.541 & 1.539 & 1.540 & 1.540 & 1.542 & 1.541 & 1.541 & 1.543 \\
\hline 8 & 600.0 & 1.538 & 1.541 & 1.537 & 1.538 & 1.534 & 1.538 & 1.537 & 1.536 \\
\hline 9 & 700.0 & 1.534 & 1.536 & 1.532 & 1.534 & 1.529 & 1.539 & 1.536 & 1.535 \\
\hline 10 & 800.0 & 1.537 & 1.535 & 1.531 & 1.533 & 1.530 & 1.537 & 1.537 & 1.537 \\
\hline 11 & 900.0 & 1.534 & 1.538 & 1.534 & 1.536 & 1.534 & 1.542 & 1.538 & 1.537 \\
\hline 12 & 1000.0 & 1.540 & 1.538 & 1.536 & 1.540 & 1.532 & 1.540 & 1.541 & 1.543 \\
\hline 13 & 1100.0 & 1.546 & 1.544 & 1.542 & 1.548 & 1.543 & 1.550 & 1.553 & 1.548 \\
\hline 14 & 1200.0 & 1.547 & 1.547 & 1.544 & 1.550 & 1.542 & 1.554 & 1.555 & 1.551 \\
\hline 15 & 1300.0 & 1.552 & 1.550 & 1.548 & 1.550 & 1.546 & 1.558 & 1.560 & 1.556 \\
\hline \multirow[t]{2}{*}{ Step } & Burnup & \multicolumn{8}{|c|}{ MPACT Power Peaking Factors } \\
\hline & $\frac{\mathrm{MWd}}{\mathrm{MtU}}$ & $\mathrm{E}$ & $\mathrm{F}$ & G & $\mathrm{H}$ & $\mathrm{J}$ & K & $\mathrm{L}$ & M \\
\hline 0 & 0.0 & 1.604 & 1.604 & 1.605 & 1.605 & 1.605 & 1.605 & 1.604 & 1.603 \\
\hline 1 & 25.0 & 1.564 & 1.565 & 1.566 & 1.566 & 1.566 & 1.566 & 1.565 & 1.564 \\
\hline 2 & 50.0 & 1.569 & 1.570 & 1.571 & 1.571 & 1.571 & 1.571 & 1.570 & 1.570 \\
\hline 3 & 100.0 & 1.582 & 1.583 & 1.584 & 1.584 & 1.584 & 1.584 & 1.583 & 1.581 \\
\hline 4 & 200.0 & 1.598 & 1.599 & 1.600 & 1.600 & 1.601 & 1.600 & 1.600 & 1.598 \\
\hline 5 & 300.0 & 1.608 & 1.609 & 1.610 & 1.610 & 1.610 & 1.610 & 1.609 & 1.608 \\
\hline 6 & 400.0 & 1.611 & 1.613 & 1.614 & 1.613 & 1.613 & 1.614 & 1.613 & 1.612 \\
\hline 7 & 500.0 & 1.612 & 1.614 & 1.614 & 1.615 & 1.614 & 1.614 & 1.614 & 1.613 \\
\hline 8 & 600.0 & 1.611 & 1.612 & 1.613 & 1.613 & 1.613 & 1.613 & 1.612 & 1.611 \\
\hline 9 & 700.0 & 1.607 & 1.608 & 1.609 & 1.610 & 1.609 & 1.609 & 1.608 & 1.607 \\
\hline 10 & 800.0 & 1.603 & 1.603 & 1.605 & 1.605 & 1.605 & 1.605 & 1.603 & 1.603 \\
\hline 11 & 900.0 & 1.597 & 1.597 & 1.598 & 1.599 & 1.599 & 1.599 & 1.597 & 1.597 \\
\hline 12 & 1000.0 & 1.590 & 1.591 & 1.592 & 1.593 & 1.592 & 1.592 & 1.591 & 1.590 \\
\hline 13 & 1100.0 & 1.583 & 1.584 & 1.585 & 1.586 & 1.585 & 1.585 & 1.583 & 1.583 \\
\hline 14 & 1200.0 & 1.576 & 1.576 & 1.577 & 1.578 & 1.578 & 1.578 & 1.576 & 1.575 \\
\hline 15 & 1300.0 & 1.567 & 1.567 & 1.569 & 1.570 & 1.569 & 1.569 & 1.567 & 1.567 \\
\hline
\end{tabular}


Table 7.65. Problem 6.2.1 reference solution power peaking factors in element 3 for charge-pan II (1/2).

\begin{tabular}{|c|c|c|c|c|c|c|c|c|c|}
\hline \multirow[t]{2}{*}{ Step } & \multirow{2}{*}{$\begin{array}{l}\text { Burnup } \\
{\left[\frac{\mathrm{MWd}}{\mathrm{M} t \mathrm{II}}\right]}\end{array}$} & \multicolumn{8}{|c|}{ Shift Power Peaking Factors } \\
\hline & & A & B & $\mathrm{C}$ & $\mathrm{D}$ & $\mathrm{P}$ & Q & $\mathrm{R}$ & S \\
\hline 0 & 0.0 & 1.459 & 1.457 & 1.448 & 1.455 & 1.493 & 1.501 & 1.498 & 1.471 \\
\hline 1 & 25.0 & 1.454 & 1.450 & 1.443 & 1.449 & 1.488 & 1.495 & 1.491 & 1.467 \\
\hline 2 & 50.0 & 1.454 & 1.451 & 1.443 & 1.450 & 1.486 & 1.495 & 1.492 & 1.468 \\
\hline 3 & 100.0 & 1.453 & 1.452 & 1.446 & 1.450 & 1.490 & 1.494 & 1.494 & 1.467 \\
\hline 4 & 200.0 & 1.455 & 1.451 & 1.446 & 1.452 & 1.493 & 1.498 & 1.495 & 1.472 \\
\hline 5 & 300.0 & 1.458 & 1.454 & 1.448 & 1.456 & 1.498 & 1.500 & 1.496 & 1.474 \\
\hline 6 & 400.0 & 1.461 & 1.459 & 1.451 & 1.457 & 1.494 & 1.501 & 1.499 & 1.475 \\
\hline 7 & 500.0 & 1.463 & 1.459 & 1.451 & 1.459 & 1.500 & 1.504 & 1.500 & 1.478 \\
\hline 8 & 600.0 & 1.463 & 1.457 & 1.451 & 1.457 & 1.501 & 1.502 & 1.498 & 1.473 \\
\hline 9 & 700.0 & 1.461 & 1.457 & 1.451 & 1.458 & 1.502 & 1.505 & 1.498 & 1.472 \\
\hline 10 & 800.0 & 1.465 & 1.461 & 1.452 & 1.458 & 1.506 & 1.506 & 1.503 & 1.479 \\
\hline 11 & 900.0 & 1.463 & 1.459 & 1.450 & 1.460 & 1.504 & 1.503 & 1.499 & 1.477 \\
\hline 12 & 1000.0 & 1.465 & 1.458 & 1.450 & 1.460 & 1.500 & 1.504 & 1.499 & 1.477 \\
\hline 13 & 1100.0 & 1.469 & 1.464 & 1.455 & 1.461 & 1.506 & 1.508 & 1.498 & 1.479 \\
\hline 14 & 1200.0 & 1.466 & 1.463 & 1.453 & 1.462 & 1.504 & 1.504 & 1.500 & 1.481 \\
\hline 15 & 1300.0 & 1.468 & 1.465 & 1.454 & 1.464 & 1.507 & 1.505 & 1.501 & 1.478 \\
\hline \multirow[t]{2}{*}{ Step } & Burnup & \multicolumn{8}{|c|}{ MPACT Power Peaking Factors } \\
\hline & $\frac{\mathrm{MWd}}{\mathrm{MtU}}$ & A & B & $\mathrm{C}$ & $\mathrm{D}$ & $\mathrm{P}$ & Q & $\mathrm{R}$ & $S$ \\
\hline 0 & 0.0 & 1.476 & 1.480 & 1.476 & 1.470 & 1.493 & 1.514 & 1.519 & 1.515 \\
\hline 1 & 25.0 & 1.453 & 1.457 & 1.453 & 1.447 & 1.470 & 1.492 & 1.496 & 1.492 \\
\hline 2 & 50.0 & 1.457 & 1.461 & 1.457 & 1.451 & 1.474 & 1.495 & 1.500 & 1.496 \\
\hline 3 & 100.0 & 1.464 & 1.469 & 1.465 & 1.459 & 1.481 & 1.504 & 1.509 & 1.504 \\
\hline 4 & 200.0 & 1.476 & 1.480 & 1.477 & 1.470 & 1.494 & 1.516 & 1.521 & 1.515 \\
\hline 5 & 300.0 & 1.482 & 1.486 & 1.483 & 1.476 & 1.500 & 1.522 & 1.527 & 1.522 \\
\hline 6 & 400.0 & 1.484 & 1.489 & 1.485 & 1.479 & 1.502 & 1.524 & 1.530 & 1.525 \\
\hline 7 & 500.0 & 1.487 & 1.491 & 1.486 & 1.480 & 1.504 & 1.527 & 1.531 & 1.527 \\
\hline 8 & 600.0 & 1.486 & 1.489 & 1.486 & 1.479 & 1.503 & 1.525 & 1.531 & 1.526 \\
\hline 9 & 700.0 & 1.484 & 1.489 & 1.485 & 1.479 & 1.502 & 1.524 & 1.530 & 1.525 \\
\hline 10 & 800.0 & 1.482 & 1.487 & 1.483 & 1.478 & 1.500 & 1.522 & 1.527 & 1.522 \\
\hline 11 & 900.0 & 1.479 & 1.484 & 1.480 & 1.475 & 1.497 & 1.519 & 1.525 & 1.519 \\
\hline 12 & 1000.0 & 1.476 & 1.481 & 1.476 & 1.472 & 1.494 & 1.516 & 1.521 & 1.516 \\
\hline 13 & 1100.0 & 1.472 & 1.478 & 1.473 & 1.468 & 1.490 & 1.511 & 1.517 & 1.512 \\
\hline 14 & 1200.0 & 1.469 & 1.474 & 1.469 & 1.464 & 1.486 & 1.507 & 1.513 & 1.508 \\
\hline 15 & 1300.0 & 1.464 & 1.469 & 1.464 & 1.460 & 1.482 & 1.503 & 1.509 & 1.503 \\
\hline
\end{tabular}


Table 7.66. Problem 6.2.1 reference solution power peaking factors in element 3 for charge-pan II (2/2).

\begin{tabular}{|c|c|c|c|c|c|c|c|c|c|}
\hline \multirow[t]{2}{*}{ Step } & \multirow{2}{*}{$\begin{array}{l}\text { Burnup } \\
{\left[\frac{\mathrm{MWd}}{\mathrm{MtU}}\right]}\end{array}$} & \multicolumn{8}{|c|}{ Shift Power Peaking Factors } \\
\hline & & $\mathrm{E}$ & $\mathrm{F}$ & G & $\mathrm{H}$ & $\mathrm{J}$ & K & $\mathrm{L}$ & M \\
\hline 0 & 0.0 & 1.491 & 1.492 & 1.491 & 1.492 & 1.482 & 1.469 & 1.470 & 1.479 \\
\hline 1 & 25.0 & 1.484 & 1.487 & 1.488 & 1.486 & 1.474 & 1.465 & 1.466 & 1.469 \\
\hline 2 & 50.0 & 1.483 & 1.484 & 1.489 & 1.489 & 1.472 & 1.466 & 1.465 & 1.474 \\
\hline 3 & 100.0 & 1.484 & 1.487 & 1.489 & 1.486 & 1.473 & 1.464 & 1.465 & 1.473 \\
\hline 4 & 200.0 & 1.487 & 1.487 & 1.492 & 1.490 & 1.476 & 1.469 & 1.468 & 1.475 \\
\hline 5 & 300.0 & 1.490 & 1.493 & 1.494 & 1.494 & 1.479 & 1.468 & 1.471 & 1.479 \\
\hline 6 & 400.0 & 1.490 & 1.492 & 1.493 & 1.490 & 1.482 & 1.474 & 1.472 & 1.480 \\
\hline 7 & 500.0 & 1.492 & 1.498 & 1.496 & 1.497 & 1.478 & 1.473 & 1.474 & 1.478 \\
\hline 8 & 600.0 & 1.493 & 1.493 & 1.494 & 1.494 & 1.483 & 1.472 & 1.472 & 1.483 \\
\hline 9 & 700.0 & 1.494 & 1.495 & 1.493 & 1.494 & 1.480 & 1.474 & 1.471 & 1.480 \\
\hline 10 & 800.0 & 1.498 & 1.497 & 1.498 & 1.499 & 1.483 & 1.475 & 1.474 & 1.483 \\
\hline 11 & 900.0 & 1.494 & 1.498 & 1.499 & 1.499 & 1.482 & 1.476 & 1.473 & 1.482 \\
\hline 12 & 1000.0 & 1.494 & 1.496 & 1.495 & 1.498 & 1.480 & 1.473 & 1.474 & 1.483 \\
\hline 13 & 1100.0 & 1.496 & 1.500 & 1.501 & 1.503 & 1.486 & 1.477 & 1.479 & 1.489 \\
\hline 14 & 1200.0 & 1.499 & 1.498 & 1.499 & 1.500 & 1.485 & 1.476 & 1.476 & 1.486 \\
\hline 15 & 1300.0 & 1.499 & 1.500 & 1.499 & 1.503 & 1.481 & 1.474 & 1.479 & 1.487 \\
\hline \multirow[t]{2}{*}{ Step } & Burnup & \multicolumn{8}{|c|}{ MPACT Power Peaking Factors } \\
\hline & $\frac{\mathrm{MWd}}{\mathrm{MtU}}$ & $\mathrm{E}$ & $\mathrm{F}$ & G & $\mathrm{H}$ & $\mathrm{J}$ & K & $\mathrm{L}$ & M \\
\hline 0 & 0.0 & 1.490 & 1.498 & 1.510 & 1.511 & 1.511 & 1.510 & 1.497 & 1.490 \\
\hline 1 & 25.0 & 1.468 & 1.475 & 1.488 & 1.489 & 1.489 & 1.488 & 1.476 & 1.468 \\
\hline 2 & 50.0 & 1.472 & 1.479 & 1.491 & 1.492 & 1.492 & 1.491 & 1.479 & 1.471 \\
\hline 3 & 100.0 & 1.480 & 1.487 & 1.499 & 1.501 & 1.501 & 1.499 & 1.487 & 1.479 \\
\hline 4 & 200.0 & 1.491 & 1.498 & 1.511 & 1.512 & 1.513 & 1.511 & 1.499 & 1.491 \\
\hline 5 & 300.0 & 1.498 & 1.504 & 1.517 & 1.518 & 1.518 & 1.518 & 1.505 & 1.497 \\
\hline 6 & 400.0 & 1.500 & 1.508 & 1.520 & 1.522 & 1.522 & 1.521 & 1.508 & 1.501 \\
\hline 7 & 500.0 & 1.502 & 1.509 & 1.521 & 1.523 & 1.523 & 1.522 & 1.509 & 1.502 \\
\hline 8 & 600.0 & 1.501 & 1.509 & 1.521 & 1.522 & 1.522 & 1.521 & 1.509 & 1.501 \\
\hline 9 & 700.0 & 1.500 & 1.507 & 1.520 & 1.522 & 1.522 & 1.520 & 1.508 & 1.500 \\
\hline 10 & 800.0 & 1.499 & 1.505 & 1.518 & 1.520 & 1.520 & 1.518 & 1.506 & 1.498 \\
\hline 11 & 900.0 & 1.496 & 1.502 & 1.515 & 1.517 & 1.517 & 1.515 & 1.503 & 1.496 \\
\hline 12 & 1000.0 & 1.492 & 1.499 & 1.512 & 1.514 & 1.514 & 1.512 & 1.500 & 1.492 \\
\hline 13 & 1100.0 & 1.488 & 1.495 & 1.508 & 1.510 & 1.510 & 1.508 & 1.496 & 1.488 \\
\hline 14 & 1200.0 & 1.485 & 1.491 & 1.504 & 1.506 & 1.506 & 1.504 & 1.492 & 1.484 \\
\hline 15 & 1300.0 & 1.480 & 1.486 & 1.500 & 1.501 & 1.502 & 1.500 & 1.487 & 1.480 \\
\hline
\end{tabular}


Table 7.67. Problem 6.2.1 reference solution power peaking factors in element 4 for charge-pan II (1/2).

\begin{tabular}{|c|c|c|c|c|c|c|c|c|c|}
\hline \multirow[t]{2}{*}{ Step } & \multirow{2}{*}{$\begin{array}{l}\text { Burnup } \\
{\left[\frac{\mathrm{MWd}}{\mathrm{M} t \mathrm{II}}\right]}\end{array}$} & \multicolumn{8}{|c|}{ Shift Power Peaking Factors } \\
\hline & & A & B & $\mathrm{C}$ & $\mathrm{D}$ & $\mathrm{P}$ & Q & $\mathrm{R}$ & S \\
\hline 0 & 0.0 & 0.794 & 0.780 & 0.763 & 0.782 & 0.959 & 0.974 & 0.958 & 0.889 \\
\hline 1 & 25.0 & 0.768 & 0.754 & 0.735 & 0.756 & 0.925 & 0.943 & 0.926 & 0.857 \\
\hline 2 & 50.0 & 0.769 & 0.755 & 0.738 & 0.754 & 0.921 & 0.942 & 0.928 & 0.860 \\
\hline 3 & 100.0 & 0.774 & 0.765 & 0.744 & 0.762 & 0.932 & 0.952 & 0.937 & 0.870 \\
\hline 4 & 200.0 & 0.788 & 0.775 & 0.755 & 0.773 & 0.949 & 0.966 & 0.951 & 0.882 \\
\hline 5 & 300.0 & 0.799 & 0.785 & 0.767 & 0.785 & 0.962 & 0.978 & 0.962 & 0.895 \\
\hline 6 & 400.0 & 0.804 & 0.790 & 0.772 & 0.790 & 0.968 & 0.984 & 0.967 & 0.898 \\
\hline 7 & 500.0 & 0.811 & 0.795 & 0.774 & 0.795 & 0.973 & 0.990 & 0.974 & 0.904 \\
\hline 8 & 600.0 & 0.810 & 0.795 & 0.776 & 0.798 & 0.975 & 0.991 & 0.971 & 0.906 \\
\hline 9 & 700.0 & 0.811 & 0.796 & 0.778 & 0.795 & 0.975 & 0.991 & 0.974 & 0.909 \\
\hline 10 & 800.0 & 0.809 & 0.796 & 0.777 & 0.798 & 0.976 & 0.994 & 0.974 & 0.911 \\
\hline 11 & 900.0 & 0.809 & 0.794 & 0.774 & 0.794 & 0.971 & 0.990 & 0.969 & 0.905 \\
\hline 12 & 1000.0 & 0.807 & 0.794 & 0.772 & 0.791 & 0.967 & 0.986 & 0.965 & 0.902 \\
\hline 13 & 1100.0 & 0.801 & 0.786 & 0.766 & 0.787 & 0.961 & 0.980 & 0.957 & 0.895 \\
\hline 14 & 1200.0 & 0.801 & 0.786 & 0.764 & 0.783 & 0.958 & 0.977 & 0.957 & 0.894 \\
\hline 15 & 1300.0 & 0.797 & 0.782 & 0.760 & 0.782 & 0.954 & 0.973 & 0.952 & 0.890 \\
\hline \multirow[t]{2}{*}{ Step } & Burnup & \multicolumn{8}{|c|}{ MPACT Power Peaking Factors } \\
\hline & $\frac{\mathrm{MWd}}{\mathrm{MtU}}$ & A & B & $\mathrm{C}$ & $\mathrm{D}$ & $\mathrm{P}$ & Q & $\mathrm{R}$ & S \\
\hline 0 & 0.0 & 0.765 & 0.778 & 0.765 & 0.746 & 0.871 & 0.936 & 0.954 & 0.938 \\
\hline 1 & 25.0 & 0.781 & 0.795 & 0.782 & 0.763 & 0.890 & 0.956 & 0.974 & 0.957 \\
\hline 2 & 50.0 & 0.779 & 0.793 & 0.780 & 0.761 & 0.887 & 0.952 & 0.970 & 0.954 \\
\hline 3 & 100.0 & 0.774 & 0.787 & 0.775 & 0.756 & 0.882 & 0.947 & 0.965 & 0.948 \\
\hline 4 & 200.0 & 0.768 & 0.781 & 0.769 & 0.750 & 0.875 & 0.941 & 0.958 & 0.942 \\
\hline 5 & 300.0 & 0.765 & 0.778 & 0.766 & 0.747 & 0.872 & 0.936 & 0.954 & 0.938 \\
\hline 6 & 400.0 & 0.764 & 0.777 & 0.765 & 0.746 & 0.871 & 0.936 & 0.953 & 0.937 \\
\hline 7 & 500.0 & 0.765 & 0.778 & 0.765 & 0.747 & 0.872 & 0.936 & 0.954 & 0.938 \\
\hline 8 & 600.0 & 0.766 & 0.779 & 0.767 & 0.749 & 0.874 & 0.938 & 0.955 & 0.939 \\
\hline 9 & 700.0 & 0.769 & 0.783 & 0.770 & 0.752 & 0.876 & 0.942 & 0.959 & 0.943 \\
\hline 10 & 800.0 & 0.773 & 0.786 & 0.773 & 0.755 & 0.880 & 0.945 & 0.962 & 0.946 \\
\hline 11 & 900.0 & 0.776 & 0.789 & 0.777 & 0.758 & 0.884 & 0.949 & 0.966 & 0.951 \\
\hline 12 & 1000.0 & 0.780 & 0.793 & 0.780 & 0.762 & 0.888 & 0.953 & 0.971 & 0.955 \\
\hline 13 & 1100.0 & 0.784 & 0.797 & 0.784 & 0.766 & 0.892 & 0.957 & 0.975 & 0.959 \\
\hline 14 & 1200.0 & 0.788 & 0.801 & 0.789 & 0.771 & 0.897 & 0.962 & 0.980 & 0.964 \\
\hline 15 & 1300.0 & 0.793 & 0.806 & 0.793 & 0.775 & 0.902 & 0.967 & 0.985 & 0.969 \\
\hline
\end{tabular}


Table 7.68. Problem 6.2.1 reference solution power peaking factors in element 4 for charge-pan II (2/2).

\begin{tabular}{|c|c|c|c|c|c|c|c|c|c|}
\hline \multirow[t]{2}{*}{ Step } & \multirow{2}{*}{$\begin{array}{l}\text { Burnup } \\
{\left[\frac{\mathrm{MWd}}{\mathrm{MtU}}\right]}\end{array}$} & \multicolumn{8}{|c|}{ Shift Power Peaking Factors } \\
\hline & & $\mathrm{E}$ & $\mathrm{F}$ & G & $\mathrm{H}$ & $\mathrm{J}$ & K & $\mathrm{L}$ & M \\
\hline 0 & 0.0 & 0.936 & 0.941 & 0.942 & 0.937 & 0.895 & 0.874 & 0.871 & 0.895 \\
\hline 1 & 25.0 & 0.905 & 0.910 & 0.913 & 0.908 & 0.864 & 0.842 & 0.843 & 0.863 \\
\hline 2 & 50.0 & 0.905 & 0.910 & 0.911 & 0.910 & 0.866 & 0.845 & 0.846 & 0.864 \\
\hline 3 & 100.0 & 0.912 & 0.918 & 0.922 & 0.918 & 0.876 & 0.853 & 0.851 & 0.872 \\
\hline 4 & 200.0 & 0.929 & 0.934 & 0.935 & 0.931 & 0.889 & 0.867 & 0.863 & 0.884 \\
\hline 5 & 300.0 & 0.941 & 0.945 & 0.947 & 0.943 & 0.898 & 0.877 & 0.879 & 0.897 \\
\hline 6 & 400.0 & 0.946 & 0.951 & 0.954 & 0.948 & 0.903 & 0.883 & 0.884 & 0.905 \\
\hline 7 & 500.0 & 0.953 & 0.960 & 0.959 & 0.957 & 0.909 & 0.888 & 0.889 & 0.909 \\
\hline 8 & 600.0 & 0.956 & 0.958 & 0.961 & 0.956 & 0.911 & 0.886 & 0.889 & 0.911 \\
\hline 9 & 700.0 & 0.954 & 0.962 & 0.961 & 0.957 & 0.911 & 0.890 & 0.889 & 0.912 \\
\hline 10 & 800.0 & 0.953 & 0.959 & 0.959 & 0.956 & 0.909 & 0.888 & 0.893 & 0.912 \\
\hline 11 & 900.0 & 0.952 & 0.957 & 0.960 & 0.953 & 0.908 & 0.884 & 0.887 & 0.909 \\
\hline 12 & 1000.0 & 0.948 & 0.954 & 0.956 & 0.952 & 0.904 & 0.882 & 0.886 & 0.905 \\
\hline 13 & 1100.0 & 0.943 & 0.950 & 0.953 & 0.945 & 0.896 & 0.874 & 0.877 & 0.900 \\
\hline 14 & 1200.0 & 0.938 & 0.944 & 0.948 & 0.945 & 0.894 & 0.871 & 0.877 & 0.896 \\
\hline 15 & 1300.0 & 0.936 & 0.943 & 0.945 & 0.942 & 0.891 & 0.869 & 0.872 & 0.894 \\
\hline \multirow[t]{2}{*}{ Step } & Burnup & \multicolumn{8}{|c|}{ MPACT Power Peaking Factors } \\
\hline & $\frac{\mathrm{MWd}}{\mathrm{MtU}}$ & $\mathrm{E}$ & $\mathrm{F}$ & G & $\mathrm{H}$ & $\mathrm{J}$ & K & $\mathrm{L}$ & M \\
\hline 0 & 0.0 & 0.854 & 0.875 & 0.916 & 0.921 & 0.922 & 0.917 & 0.876 & 0.854 \\
\hline 1 & 25.0 & 0.873 & 0.894 & 0.936 & 0.941 & 0.941 & 0.937 & 0.895 & 0.874 \\
\hline 2 & 50.0 & 0.871 & 0.892 & 0.932 & 0.938 & 0.939 & 0.934 & 0.892 & 0.871 \\
\hline 3 & 100.0 & 0.865 & 0.886 & 0.927 & 0.932 & 0.933 & 0.928 & 0.887 & 0.865 \\
\hline 4 & 200.0 & 0.859 & 0.880 & 0.921 & 0.926 & 0.926 & 0.921 & 0.880 & 0.859 \\
\hline 5 & 300.0 & 0.855 & 0.876 & 0.916 & 0.921 & 0.922 & 0.918 & 0.877 & 0.855 \\
\hline 6 & 400.0 & 0.855 & 0.875 & 0.916 & 0.921 & 0.922 & 0.917 & 0.876 & 0.855 \\
\hline 7 & 500.0 & 0.855 & 0.876 & 0.917 & 0.922 & 0.922 & 0.918 & 0.877 & 0.856 \\
\hline 8 & 600.0 & 0.857 & 0.878 & 0.919 & 0.923 & 0.924 & 0.920 & 0.879 & 0.858 \\
\hline 9 & 700.0 & 0.861 & 0.881 & 0.922 & 0.927 & 0.928 & 0.923 & 0.882 & 0.861 \\
\hline 10 & 800.0 & 0.864 & 0.884 & 0.925 & 0.930 & 0.931 & 0.926 & 0.886 & 0.864 \\
\hline 11 & 900.0 & 0.868 & 0.888 & 0.929 & 0.935 & 0.935 & 0.930 & 0.890 & 0.869 \\
\hline 12 & 1000.0 & 0.872 & 0.892 & 0.933 & 0.939 & 0.939 & 0.934 & 0.894 & 0.872 \\
\hline 13 & 1100.0 & 0.876 & 0.896 & 0.938 & 0.943 & 0.944 & 0.939 & 0.898 & 0.876 \\
\hline 14 & 1200.0 & 0.881 & 0.901 & 0.943 & 0.948 & 0.949 & 0.944 & 0.903 & 0.881 \\
\hline 15 & 1300.0 & 0.886 & 0.906 & 0.948 & 0.953 & 0.954 & 0.948 & 0.908 & 0.886 \\
\hline
\end{tabular}


Table 7.69. Problem 6.2.1 reference solution power peaking factors in element 5 for charge-pan II (1/2).

\begin{tabular}{|c|c|c|c|c|c|c|c|c|c|}
\hline \multirow[t]{2}{*}{ Step } & \multirow{2}{*}{$\begin{array}{l}\text { Burnup } \\
{\left[\frac{\mathrm{MWd}}{\mathrm{MtU}}\right]}\end{array}$} & \multicolumn{8}{|c|}{ Shift Power Peaking Factors } \\
\hline & & A & B & $\mathrm{C}$ & $\mathrm{D}$ & $\mathrm{P}$ & Q & $\mathrm{R}$ & $\mathrm{S}$ \\
\hline 0 & 0.0 & 0.423 & 0.411 & 0.400 & 0.414 & 0.521 & 0.532 & 0.518 & 0.469 \\
\hline 1 & 25.0 & 0.401 & 0.391 & 0.378 & 0.391 & 0.493 & 0.504 & 0.489 & 0.442 \\
\hline 2 & 50.0 & 0.401 & 0.390 & 0.378 & 0.393 & 0.492 & 0.503 & 0.490 & 0.443 \\
\hline 3 & 100.0 & 0.408 & 0.397 & 0.384 & 0.397 & 0.500 & 0.512 & 0.498 & 0.450 \\
\hline 4 & 200.0 & 0.417 & 0.406 & 0.394 & 0.408 & 0.514 & 0.525 & 0.509 & 0.462 \\
\hline 5 & 300.0 & 0.426 & 0.414 & 0.404 & 0.418 & 0.523 & 0.534 & 0.518 & 0.470 \\
\hline 6 & 400.0 & 0.430 & 0.419 & 0.405 & 0.423 & 0.531 & 0.540 & 0.524 & 0.476 \\
\hline 7 & 500.0 & 0.435 & 0.423 & 0.409 & 0.425 & 0.533 & 0.544 & 0.528 & 0.478 \\
\hline 8 & 600.0 & 0.437 & 0.425 & 0.413 & 0.427 & 0.536 & 0.548 & 0.531 & 0.483 \\
\hline 9 & 700.0 & 0.438 & 0.426 & 0.413 & 0.428 & 0.537 & 0.550 & 0.532 & 0.484 \\
\hline 10 & 800.0 & 0.437 & 0.424 & 0.413 & 0.427 & 0.534 & 0.546 & 0.529 & 0.483 \\
\hline 11 & 900.0 & 0.434 & 0.423 & 0.410 & 0.426 & 0.532 & 0.545 & 0.529 & 0.482 \\
\hline 12 & 1000.0 & 0.434 & 0.420 & 0.408 & 0.423 & 0.529 & 0.544 & 0.524 & 0.479 \\
\hline 13 & 1100.0 & 0.428 & 0.416 & 0.403 & 0.419 & 0.524 & 0.538 & 0.522 & 0.474 \\
\hline 14 & 1200.0 & 0.426 & 0.415 & 0.400 & 0.415 & 0.521 & 0.536 & 0.518 & 0.471 \\
\hline 15 & 1300.0 & 0.424 & 0.414 & 0.399 & 0.415 & 0.520 & 0.532 & 0.517 & 0.469 \\
\hline \multirow[t]{2}{*}{ Step } & Burnup & \multicolumn{8}{|c|}{ MPACT Power Peaking Factors } \\
\hline & $\frac{\mathrm{MWd}}{\mathrm{MtU}}$ & A & B & $\mathrm{C}$ & $\mathrm{D}$ & $\mathrm{P}$ & Q & $\mathrm{R}$ & S \\
\hline 0 & 0.0 & 0.388 & 0.398 & 0.390 & 0.376 & 0.441 & 0.486 & 0.500 & 0.491 \\
\hline 1 & 25.0 & 0.419 & 0.430 & 0.422 & 0.406 & 0.477 & 0.524 & 0.540 & 0.530 \\
\hline 2 & 50.0 & 0.415 & 0.427 & 0.418 & 0.403 & 0.472 & 0.519 & 0.535 & 0.525 \\
\hline 3 & 100.0 & 0.406 & 0.417 & 0.408 & 0.394 & 0.462 & 0.508 & 0.523 & 0.513 \\
\hline 4 & 200.0 & 0.393 & 0.404 & 0.396 & 0.381 & 0.447 & 0.492 & 0.507 & 0.497 \\
\hline 5 & 300.0 & 0.385 & 0.396 & 0.388 & 0.373 & 0.438 & 0.483 & 0.497 & 0.488 \\
\hline 6 & 400.0 & 0.382 & 0.392 & 0.384 & 0.370 & 0.434 & 0.478 & 0.492 & 0.483 \\
\hline 7 & 500.0 & 0.381 & 0.391 & 0.382 & 0.369 & 0.433 & 0.477 & 0.491 & 0.481 \\
\hline 8 & 600.0 & 0.381 & 0.391 & 0.383 & 0.369 & 0.434 & 0.478 & 0.492 & 0.482 \\
\hline 9 & 700.0 & 0.383 & 0.393 & 0.385 & 0.371 & 0.436 & 0.480 & 0.495 & 0.485 \\
\hline 10 & 800.0 & 0.386 & 0.396 & 0.388 & 0.374 & 0.439 & 0.484 & 0.498 & 0.489 \\
\hline 11 & 900.0 & 0.390 & 0.400 & 0.392 & 0.378 & 0.443 & 0.489 & 0.503 & 0.494 \\
\hline 12 & 1000.0 & 0.394 & 0.404 & 0.396 & 0.382 & 0.448 & 0.494 & 0.509 & 0.499 \\
\hline 13 & 1100.0 & 0.399 & 0.410 & 0.401 & 0.387 & 0.454 & 0.500 & 0.515 & 0.505 \\
\hline 14 & 1200.0 & 0.404 & 0.415 & 0.407 & 0.392 & 0.460 & 0.506 & 0.521 & 0.511 \\
\hline 15 & 1300.0 & 0.410 & 0.421 & 0.413 & 0.398 & 0.467 & 0.513 & 0.529 & 0.519 \\
\hline
\end{tabular}


Table 7.70. Problem 6.2.1 reference solution power peaking factors in element 5 for charge-pan II (2/2).

\begin{tabular}{|c|c|c|c|c|c|c|c|c|c|}
\hline \multirow[t]{2}{*}{ Step } & \multirow{2}{*}{$\begin{array}{l}\text { Burnup } \\
{\left[\frac{\mathrm{MWd}}{\mathrm{M} t \mathrm{II}}\right]}\end{array}$} & \multicolumn{8}{|c|}{ Shift Power Peaking Factors } \\
\hline & & $\mathrm{E}$ & $\mathrm{F}$ & G & $\mathrm{H}$ & $\mathrm{J}$ & K & $\mathrm{L}$ & M \\
\hline 0 & 0.0 & 0.508 & 0.511 & 0.509 & 0.506 & 0.473 & 0.458 & 0.461 & 0.478 \\
\hline 1 & 25.0 & 0.481 & 0.483 & 0.483 & 0.479 & 0.449 & 0.434 & 0.434 & 0.453 \\
\hline 2 & 50.0 & 0.481 & 0.484 & 0.484 & 0.480 & 0.450 & 0.435 & 0.435 & 0.452 \\
\hline 3 & 100.0 & 0.488 & 0.490 & 0.491 & 0.489 & 0.458 & 0.442 & 0.443 & 0.460 \\
\hline 4 & 200.0 & 0.503 & 0.505 & 0.504 & 0.500 & 0.468 & 0.453 & 0.455 & 0.470 \\
\hline 5 & 300.0 & 0.512 & 0.515 & 0.514 & 0.509 & 0.477 & 0.463 & 0.465 & 0.481 \\
\hline 6 & 400.0 & 0.518 & 0.521 & 0.520 & 0.515 & 0.483 & 0.467 & 0.469 & 0.485 \\
\hline 7 & 500.0 & 0.520 & 0.524 & 0.525 & 0.520 & 0.486 & 0.472 & 0.473 & 0.491 \\
\hline 8 & 600.0 & 0.524 & 0.527 & 0.526 & 0.521 & 0.491 & 0.475 & 0.477 & 0.494 \\
\hline 9 & 700.0 & 0.525 & 0.528 & 0.530 & 0.522 & 0.490 & 0.474 & 0.476 & 0.494 \\
\hline 10 & 800.0 & 0.522 & 0.525 & 0.525 & 0.519 & 0.487 & 0.474 & 0.477 & 0.493 \\
\hline 11 & 900.0 & 0.522 & 0.526 & 0.526 & 0.519 & 0.486 & 0.472 & 0.475 & 0.490 \\
\hline 12 & 1000.0 & 0.519 & 0.523 & 0.524 & 0.518 & 0.484 & 0.470 & 0.470 & 0.486 \\
\hline 13 & 1100.0 & 0.513 & 0.516 & 0.519 & 0.514 & 0.481 & 0.465 & 0.467 & 0.482 \\
\hline 14 & 1200.0 & 0.511 & 0.515 & 0.515 & 0.511 & 0.478 & 0.462 & 0.463 & 0.479 \\
\hline 15 & 1300.0 & 0.509 & 0.514 & 0.514 & 0.508 & 0.475 & 0.460 & 0.462 & 0.478 \\
\hline \multirow[t]{2}{*}{ Step } & Burnup & \multicolumn{8}{|c|}{ MPACT Power Peaking Factors } \\
\hline & $\frac{\mathrm{MWd}}{\mathrm{MtU}}$ & $\mathrm{E}$ & $\mathrm{F}$ & G & $\mathrm{H}$ & $\mathrm{J}$ & K & $\mathrm{L}$ & M \\
\hline 0 & 0.0 & 0.432 & 0.446 & 0.475 & 0.480 & 0.481 & 0.479 & 0.449 & 0.433 \\
\hline 1 & 25.0 & 0.467 & 0.482 & 0.513 & 0.518 & 0.520 & 0.517 & 0.486 & 0.469 \\
\hline 2 & 50.0 & 0.463 & 0.478 & 0.508 & 0.513 & 0.515 & 0.513 & 0.482 & 0.465 \\
\hline 3 & 100.0 & 0.453 & 0.467 & 0.497 & 0.502 & 0.504 & 0.501 & 0.471 & 0.455 \\
\hline 4 & 200.0 & 0.439 & 0.453 & 0.482 & 0.486 & 0.488 & 0.486 & 0.456 & 0.440 \\
\hline 5 & 300.0 & 0.430 & 0.444 & 0.472 & 0.477 & 0.478 & 0.476 & 0.447 & 0.431 \\
\hline 6 & 400.0 & 0.426 & 0.440 & 0.468 & 0.473 & 0.474 & 0.472 & 0.443 & 0.427 \\
\hline 7 & 500.0 & 0.424 & 0.438 & 0.467 & 0.471 & 0.472 & 0.470 & 0.441 & 0.426 \\
\hline 8 & 600.0 & 0.425 & 0.439 & 0.468 & 0.472 & 0.473 & 0.471 & 0.442 & 0.427 \\
\hline 9 & 700.0 & 0.428 & 0.441 & 0.470 & 0.475 & 0.476 & 0.473 & 0.445 & 0.429 \\
\hline 10 & 800.0 & 0.431 & 0.445 & 0.473 & 0.478 & 0.479 & 0.477 & 0.448 & 0.432 \\
\hline 11 & 900.0 & 0.435 & 0.449 & 0.478 & 0.483 & 0.484 & 0.482 & 0.453 & 0.436 \\
\hline 12 & 1000.0 & 0.440 & 0.454 & 0.483 & 0.488 & 0.489 & 0.487 & 0.457 & 0.441 \\
\hline 13 & 1100.0 & 0.445 & 0.460 & 0.489 & 0.494 & 0.495 & 0.493 & 0.463 & 0.447 \\
\hline 14 & 1200.0 & 0.451 & 0.466 & 0.496 & 0.500 & 0.502 & 0.499 & 0.470 & 0.452 \\
\hline 15 & 1300.0 & 0.458 & 0.473 & 0.503 & 0.508 & 0.509 & 0.507 & 0.476 & 0.459 \\
\hline
\end{tabular}


Table 7.71. Problem 6.2.1 reference solution power peaking factors in element 6 for charge-pan II (1/2).

\begin{tabular}{|c|c|c|c|c|c|c|c|c|c|}
\hline \multirow[t]{2}{*}{ Step } & \multirow{2}{*}{$\begin{array}{l}\text { Burnup } \\
{\left[\frac{\mathrm{MWd}}{\mathrm{MtU}}\right]}\end{array}$} & \multicolumn{8}{|c|}{ Shift Power Peaking Factors } \\
\hline & & A & B & $\mathrm{C}$ & D & $\mathrm{P}$ & Q & $\mathrm{R}$ & $S$ \\
\hline 0 & 0.0 & 0.185 & 0.179 & 0.171 & 0.179 & 0.228 & 0.249 & 0.244 & 0.201 \\
\hline 1 & 25.0 & 0.174 & 0.168 & 0.160 & 0.168 & 0.213 & 0.233 & 0.232 & 0.189 \\
\hline 2 & 50.0 & 0.174 & 0.168 & 0.161 & 0.168 & 0.213 & 0.234 & 0.231 & 0.188 \\
\hline 3 & 100.0 & 0.176 & 0.171 & 0.163 & 0.170 & 0.216 & 0.237 & 0.234 & 0.192 \\
\hline 4 & 200.0 & 0.182 & 0.175 & 0.167 & 0.176 & 0.222 & 0.243 & 0.241 & 0.195 \\
\hline 5 & 300.0 & 0.185 & 0.180 & 0.172 & 0.179 & 0.228 & 0.249 & 0.245 & 0.203 \\
\hline 6 & 400.0 & 0.188 & 0.182 & 0.174 & 0.182 & 0.230 & 0.251 & 0.250 & 0.204 \\
\hline 7 & 500.0 & 0.190 & 0.184 & 0.177 & 0.184 & 0.233 & 0.255 & 0.251 & 0.207 \\
\hline 8 & 600.0 & 0.190 & 0.186 & 0.177 & 0.184 & 0.234 & 0.255 & 0.252 & 0.208 \\
\hline 9 & 700.0 & 0.193 & 0.187 & 0.178 & 0.186 & 0.235 & 0.258 & 0.254 & 0.209 \\
\hline 10 & 800.0 & 0.191 & 0.185 & 0.177 & 0.186 & 0.235 & 0.256 & 0.252 & 0.208 \\
\hline 11 & 900.0 & 0.192 & 0.186 & 0.179 & 0.186 & 0.235 & 0.258 & 0.254 & 0.209 \\
\hline 12 & 1000.0 & 0.191 & 0.184 & 0.178 & 0.185 & 0.233 & 0.256 & 0.253 & 0.208 \\
\hline 13 & 1100.0 & 0.189 & 0.183 & 0.176 & 0.184 & 0.231 & 0.254 & 0.251 & 0.206 \\
\hline 14 & 1200.0 & 0.189 & 0.183 & 0.174 & 0.182 & 0.232 & 0.254 & 0.250 & 0.205 \\
\hline 15 & 1300.0 & 0.188 & 0.182 & 0.175 & 0.182 & 0.230 & 0.252 & 0.249 & 0.205 \\
\hline \multirow[t]{2}{*}{ Step } & Burnup & \multicolumn{8}{|c|}{ MPACT Power Peaking Factors } \\
\hline & $\frac{\mathrm{MWd}}{\mathrm{MtU}}$ & A & B & $\mathrm{C}$ & $\mathrm{D}$ & $\mathrm{P}$ & Q & $\mathrm{R}$ & S \\
\hline 0 & 0.0 & 0.162 & 0.168 & 0.162 & 0.155 & 0.182 & 0.223 & 0.225 & 0.205 \\
\hline 1 & 25.0 & 0.184 & 0.190 & 0.184 & 0.176 & 0.206 & 0.251 & 0.254 & 0.233 \\
\hline 2 & 50.0 & 0.182 & 0.188 & 0.182 & 0.174 & 0.204 & 0.248 & 0.251 & 0.230 \\
\hline 3 & 100.0 & 0.175 & 0.181 & 0.175 & 0.168 & 0.197 & 0.240 & 0.242 & 0.222 \\
\hline 4 & 200.0 & 0.167 & 0.172 & 0.166 & 0.159 & 0.187 & 0.228 & 0.230 & 0.211 \\
\hline 5 & 300.0 & 0.161 & 0.166 & 0.160 & 0.153 & 0.180 & 0.220 & 0.222 & 0.203 \\
\hline 6 & 400.0 & 0.158 & 0.163 & 0.157 & 0.150 & 0.177 & 0.216 & 0.218 & 0.199 \\
\hline 7 & 500.0 & 0.156 & 0.161 & 0.156 & 0.149 & 0.175 & 0.215 & 0.216 & 0.198 \\
\hline 8 & 600.0 & 0.156 & 0.161 & 0.155 & 0.149 & 0.175 & 0.214 & 0.216 & 0.197 \\
\hline 9 & 700.0 & 0.157 & 0.162 & 0.156 & 0.149 & 0.175 & 0.215 & 0.217 & 0.198 \\
\hline 10 & 800.0 & 0.158 & 0.163 & 0.157 & 0.150 & 0.176 & 0.217 & 0.218 & 0.199 \\
\hline 11 & 900.0 & 0.159 & 0.165 & 0.159 & 0.152 & 0.178 & 0.219 & 0.221 & 0.202 \\
\hline 12 & 1000.0 & 0.162 & 0.167 & 0.161 & 0.154 & 0.181 & 0.222 & 0.224 & 0.204 \\
\hline 13 & 1100.0 & 0.164 & 0.169 & 0.163 & 0.157 & 0.184 & 0.225 & 0.227 & 0.208 \\
\hline 14 & 1200.0 & 0.167 & 0.172 & 0.166 & 0.159 & 0.187 & 0.229 & 0.231 & 0.211 \\
\hline 15 & 1300.0 & 0.170 & 0.175 & 0.170 & 0.162 & 0.191 & 0.233 & 0.235 & 0.215 \\
\hline
\end{tabular}


Table 7.72. Problem 6.2.1 reference solution power peaking factors in element 6 for charge-pan II (2/2).

\begin{tabular}{|c|c|c|c|c|c|c|c|c|c|}
\hline \multirow[t]{2}{*}{ Step } & \multirow{2}{*}{$\begin{array}{l}\text { Burnup } \\
{\left[\frac{\mathrm{MWd}}{\mathrm{M} t \mathrm{II}}\right]}\end{array}$} & \multicolumn{8}{|c|}{ Shift Power Peaking Factors } \\
\hline & & $\mathrm{E}$ & $\mathrm{F}$ & G & $\mathrm{H}$ & $\mathrm{J}$ & K & $\mathrm{L}$ & M \\
\hline 0 & 0.0 & 0.222 & 0.225 & 0.244 & 0.240 & 0.205 & 0.197 & 0.198 & 0.206 \\
\hline 1 & 25.0 & 0.206 & 0.210 & 0.229 & 0.227 & 0.193 & 0.185 & 0.186 & 0.194 \\
\hline 2 & 50.0 & 0.208 & 0.211 & 0.229 & 0.227 & 0.193 & 0.185 & 0.186 & 0.195 \\
\hline 3 & 100.0 & 0.210 & 0.213 & 0.232 & 0.232 & 0.196 & 0.187 & 0.189 & 0.197 \\
\hline 4 & 200.0 & 0.216 & 0.220 & 0.239 & 0.236 & 0.201 & 0.192 & 0.195 & 0.202 \\
\hline 5 & 300.0 & 0.222 & 0.225 & 0.243 & 0.242 & 0.205 & 0.197 & 0.200 & 0.208 \\
\hline 6 & 400.0 & 0.225 & 0.228 & 0.248 & 0.246 & 0.209 & 0.200 & 0.202 & 0.211 \\
\hline 7 & 500.0 & 0.227 & 0.229 & 0.251 & 0.247 & 0.210 & 0.202 & 0.204 & 0.213 \\
\hline 8 & 600.0 & 0.228 & 0.231 & 0.251 & 0.250 & 0.211 & 0.202 & 0.205 & 0.213 \\
\hline 9 & 700.0 & 0.229 & 0.232 & 0.253 & 0.251 & 0.212 & 0.204 & 0.206 & 0.216 \\
\hline 10 & 800.0 & 0.228 & 0.230 & 0.251 & 0.250 & 0.212 & 0.202 & 0.206 & 0.214 \\
\hline 11 & 900.0 & 0.228 & 0.231 & 0.253 & 0.251 & 0.213 & 0.204 & 0.207 & 0.216 \\
\hline 12 & 1000.0 & 0.228 & 0.231 & 0.253 & 0.250 & 0.211 & 0.202 & 0.205 & 0.214 \\
\hline 13 & 1100.0 & 0.226 & 0.227 & 0.250 & 0.248 & 0.209 & 0.200 & 0.203 & 0.212 \\
\hline 14 & 1200.0 & 0.225 & 0.229 & 0.249 & 0.246 & 0.208 & 0.200 & 0.202 & 0.210 \\
\hline 15 & 1300.0 & 0.224 & 0.227 & 0.248 & 0.246 & 0.209 & 0.199 & 0.203 & 0.210 \\
\hline \multirow[t]{2}{*}{ Step } & Burnup & \multicolumn{8}{|c|}{ MPACT Power Peaking Factors } \\
\hline & $\frac{\mathrm{MWd}}{\mathrm{MtU}}$ & $\mathrm{E}$ & $\mathrm{F}$ & G & $\mathrm{H}$ & $\mathrm{J}$ & K & $\mathrm{L}$ & M \\
\hline 0 & 0.0 & 0.178 & 0.186 & 0.219 & 0.221 & 0.203 & 0.200 & 0.187 & 0.179 \\
\hline 1 & 25.0 & 0.202 & 0.210 & 0.247 & 0.249 & 0.230 & 0.227 & 0.212 & 0.204 \\
\hline 2 & 50.0 & 0.199 & 0.208 & 0.244 & 0.246 & 0.227 & 0.224 & 0.210 & 0.201 \\
\hline 3 & 100.0 & 0.192 & 0.201 & 0.236 & 0.238 & 0.219 & 0.217 & 0.203 & 0.194 \\
\hline 4 & 200.0 & 0.183 & 0.191 & 0.224 & 0.226 & 0.208 & 0.206 & 0.192 & 0.184 \\
\hline 5 & 300.0 & 0.176 & 0.184 & 0.217 & 0.218 & 0.201 & 0.198 & 0.185 & 0.178 \\
\hline 6 & 400.0 & 0.173 & 0.181 & 0.213 & 0.214 & 0.197 & 0.194 & 0.182 & 0.174 \\
\hline 7 & 500.0 & 0.171 & 0.179 & 0.211 & 0.213 & 0.195 & 0.193 & 0.180 & 0.173 \\
\hline 8 & 600.0 & 0.171 & 0.179 & 0.211 & 0.212 & 0.195 & 0.192 & 0.180 & 0.172 \\
\hline 9 & 700.0 & 0.171 & 0.179 & 0.211 & 0.213 & 0.195 & 0.193 & 0.180 & 0.173 \\
\hline 10 & 800.0 & 0.173 & 0.180 & 0.213 & 0.215 & 0.197 & 0.194 & 0.182 & 0.174 \\
\hline 11 & 900.0 & 0.175 & 0.183 & 0.215 & 0.217 & 0.199 & 0.197 & 0.184 & 0.176 \\
\hline 12 & 1000.0 & 0.177 & 0.185 & 0.218 & 0.220 & 0.201 & 0.199 & 0.186 & 0.178 \\
\hline 13 & 1100.0 & 0.180 & 0.188 & 0.222 & 0.223 & 0.205 & 0.202 & 0.189 & 0.181 \\
\hline 14 & 1200.0 & 0.183 & 0.191 & 0.225 & 0.227 & 0.208 & 0.206 & 0.192 & 0.184 \\
\hline 15 & 1300.0 & 0.186 & 0.195 & 0.229 & 0.231 & 0.212 & 0.210 & 0.196 & 0.188 \\
\hline
\end{tabular}




\section{Charge-Pan III}

Table 7.73. Problem 6.2.1 reference solution power peaking factors in element 1 for charge-pan III (1/2).

\begin{tabular}{|c|c|c|c|c|c|c|c|c|c|}
\hline \multirow[t]{2}{*}{ Step } & \multirow{2}{*}{$\begin{array}{l}\text { Burnup } \\
{\left[\frac{\mathrm{MWd}}{\mathrm{MtU}}\right]}\end{array}$} & \multicolumn{8}{|c|}{ Shift Power Peaking Factors } \\
\hline & & A & B & $\mathrm{C}$ & $\mathrm{D}$ & $\mathrm{P}$ & Q & $\mathrm{R}$ & $S$ \\
\hline 0 & 0.0 & 1.034 & 1.033 & 1.033 & 1.032 & 1.044 & 1.043 & 1.045 & 1.044 \\
\hline 1 & 25.0 & 1.060 & 1.063 & 1.064 & 1.058 & 1.071 & 1.076 & 1.082 & 1.075 \\
\hline 2 & 50.0 & 1.058 & 1.063 & 1.061 & 1.059 & 1.068 & 1.071 & 1.081 & 1.076 \\
\hline 3 & 100.0 & 1.056 & 1.056 & 1.053 & 1.054 & 1.063 & 1.069 & 1.071 & 1.070 \\
\hline 4 & 200.0 & 1.048 & 1.044 & 1.038 & 1.041 & 1.053 & 1.057 & 1.054 & 1.063 \\
\hline 5 & 300.0 & 1.043 & 1.035 & 1.025 & 1.033 & 1.043 & 1.046 & 1.041 & 1.054 \\
\hline 6 & 400.0 & 1.038 & 1.032 & 1.018 & 1.030 & 1.040 & 1.042 & 1.032 & 1.051 \\
\hline 7 & 500.0 & 1.035 & 1.026 & 1.013 & 1.025 & 1.037 & 1.039 & 1.030 & 1.052 \\
\hline 8 & 600.0 & 1.037 & 1.028 & 1.014 & 1.025 & 1.037 & 1.039 & 1.027 & 1.049 \\
\hline 9 & 700.0 & 1.034 & 1.025 & 1.011 & 1.025 & 1.036 & 1.038 & 1.027 & 1.048 \\
\hline 10 & 800.0 & 1.039 & 1.028 & 1.014 & 1.028 & 1.040 & 1.043 & 1.031 & 1.055 \\
\hline 11 & 900.0 & 1.039 & 1.031 & 1.015 & 1.033 & 1.042 & 1.045 & 1.028 & 1.055 \\
\hline 12 & 1000.0 & 1.045 & 1.039 & 1.024 & 1.036 & 1.050 & 1.051 & 1.039 & 1.061 \\
\hline 13 & 1100.0 & 1.043 & 1.042 & 1.022 & 1.038 & 1.048 & 1.051 & 1.042 & 1.061 \\
\hline 14 & 1200.0 & 1.049 & 1.046 & 1.026 & 1.043 & 1.052 & 1.058 & 1.047 & 1.066 \\
\hline 15 & 1300.0 & 1.050 & 1.050 & 1.032 & 1.047 & 1.056 & 1.064 & 1.048 & 1.070 \\
\hline \multirow[t]{2}{*}{ Step } & Burnup & \multicolumn{8}{|c|}{ MPACT Power Peaking Factors } \\
\hline & $\frac{\mathrm{MWd}}{\mathrm{MtU}}$ & A & B & C & D & $\mathrm{P}$ & Q & $\mathrm{R}$ & $S$ \\
\hline 0 & 0.0 & 1.046 & 1.046 & 1.046 & 1.046 & 1.058 & 1.057 & 1.058 & 1.058 \\
\hline 1 & 25.0 & 1.029 & 1.029 & 1.029 & 1.029 & 1.040 & 1.040 & 1.040 & 1.041 \\
\hline 2 & 50.0 & 1.030 & 1.030 & 1.030 & 1.031 & 1.041 & 1.041 & 1.041 & 1.042 \\
\hline 3 & 100.0 & 1.033 & 1.033 & 1.033 & 1.033 & 1.044 & 1.044 & 1.045 & 1.044 \\
\hline 4 & 200.0 & 1.036 & 1.036 & 1.036 & 1.036 & 1.047 & 1.047 & 1.047 & 1.048 \\
\hline 5 & 300.0 & 1.039 & 1.039 & 1.039 & 1.039 & 1.050 & 1.050 & 1.051 & 1.051 \\
\hline 6 & 400.0 & 1.040 & 1.040 & 1.040 & 1.040 & 1.051 & 1.052 & 1.052 & 1.052 \\
\hline 7 & 500.0 & 1.040 & 1.040 & 1.040 & 1.040 & 1.052 & 1.051 & 1.052 & 1.052 \\
\hline 8 & 600.0 & 1.040 & 1.040 & 1.040 & 1.040 & 1.051 & 1.052 & 1.052 & 1.052 \\
\hline 9 & 700.0 & 1.038 & 1.039 & 1.039 & 1.039 & 1.050 & 1.050 & 1.050 & 1.051 \\
\hline 10 & 800.0 & 1.037 & 1.038 & 1.038 & 1.038 & 1.049 & 1.049 & 1.049 & 1.050 \\
\hline 11 & 900.0 & 1.036 & 1.036 & 1.036 & 1.036 & 1.047 & 1.047 & 1.047 & 1.048 \\
\hline 12 & 1000.0 & 1.034 & 1.034 & 1.035 & 1.034 & 1.046 & 1.046 & 1.046 & 1.046 \\
\hline 13 & 1100.0 & 1.032 & 1.032 & 1.033 & 1.032 & 1.044 & 1.043 & 1.044 & 1.045 \\
\hline 14 & 1200.0 & 1.030 & 1.030 & 1.031 & 1.030 & 1.041 & 1.041 & 1.041 & 1.042 \\
\hline 15 & 1300.0 & 1.027 & 1.028 & 1.028 & 1.028 & 1.039 & 1.039 & 1.039 & 1.040 \\
\hline
\end{tabular}


Table 7.74. Problem 6.2.1 reference solution power peaking factors in element 1 for charge-pan III (2/2).

\begin{tabular}{|c|c|c|c|c|c|c|c|c|c|}
\hline \multirow[t]{2}{*}{ Step } & \multirow{2}{*}{$\begin{array}{l}\text { Burnup } \\
{\left[\frac{\mathrm{MWd}}{\mathrm{M} t \mathrm{II}}\right]}\end{array}$} & \multicolumn{8}{|c|}{ Shift Power Peaking Factors } \\
\hline & & $\mathrm{E}$ & $\mathrm{F}$ & G & $\mathrm{H}$ & $\mathrm{J}$ & K & $\mathrm{L}$ & M \\
\hline 0 & 0.0 & 1.044 & 1.041 & 1.041 & 1.043 & 1.040 & 1.043 & 1.040 & 1.042 \\
\hline 1 & 25.0 & 1.067 & 1.070 & 1.075 & 1.072 & 1.076 & 1.075 & 1.072 & 1.073 \\
\hline 2 & 50.0 & 1.066 & 1.068 & 1.070 & 1.075 & 1.077 & 1.072 & 1.068 & 1.067 \\
\hline 3 & 100.0 & 1.064 & 1.065 & 1.069 & 1.070 & 1.066 & 1.067 & 1.064 & 1.059 \\
\hline 4 & 200.0 & 1.058 & 1.057 & 1.060 & 1.062 & 1.051 & 1.054 & 1.054 & 1.046 \\
\hline 5 & 300.0 & 1.052 & 1.049 & 1.052 & 1.055 & 1.039 & 1.047 & 1.045 & 1.037 \\
\hline 6 & 400.0 & 1.047 & 1.046 & 1.049 & 1.054 & 1.033 & 1.041 & 1.040 & 1.031 \\
\hline 7 & 500.0 & 1.046 & 1.040 & 1.045 & 1.048 & 1.025 & 1.039 & 1.039 & 1.025 \\
\hline 8 & 600.0 & 1.045 & 1.040 & 1.048 & 1.053 & 1.025 & 1.037 & 1.038 & 1.025 \\
\hline 9 & 700.0 & 1.044 & 1.040 & 1.046 & 1.051 & 1.024 & 1.038 & 1.039 & 1.021 \\
\hline 10 & 800.0 & 1.048 & 1.045 & 1.049 & 1.054 & 1.027 & 1.044 & 1.041 & 1.026 \\
\hline 11 & 900.0 & 1.049 & 1.046 & 1.048 & 1.054 & 1.029 & 1.046 & 1.046 & 1.026 \\
\hline 12 & 1000.0 & 1.054 & 1.052 & 1.055 & 1.062 & 1.036 & 1.054 & 1.052 & 1.033 \\
\hline 13 & 1100.0 & 1.053 & 1.046 & 1.056 & 1.064 & 1.037 & 1.055 & 1.054 & 1.033 \\
\hline 14 & 1200.0 & 1.057 & 1.052 & 1.060 & 1.068 & 1.046 & 1.063 & 1.061 & 1.037 \\
\hline 15 & 1300.0 & 1.058 & 1.052 & 1.062 & 1.070 & 1.046 & 1.066 & 1.061 & 1.040 \\
\hline \multirow[t]{2}{*}{ Step } & Burnup & \multicolumn{8}{|c|}{ MPACT Power Peaking Factors } \\
\hline & $\frac{\mathrm{MWd}}{\mathrm{MtU}}$ & $\mathrm{E}$ & $\mathrm{F}$ & G & $\mathrm{H}$ & $\mathrm{J}$ & K & $\mathrm{L}$ & M \\
\hline 0 & 0.0 & 1.055 & 1.055 & 1.055 & 1.055 & 1.055 & 1.056 & 1.056 & 1.056 \\
\hline 1 & 25.0 & 1.039 & 1.039 & 1.039 & 1.039 & 1.039 & 1.039 & 1.039 & 1.039 \\
\hline 2 & 50.0 & 1.039 & 1.040 & 1.040 & 1.040 & 1.040 & 1.040 & 1.040 & 1.040 \\
\hline 3 & 100.0 & 1.043 & 1.043 & 1.042 & 1.042 & 1.042 & 1.042 & 1.043 & 1.042 \\
\hline 4 & 200.0 & 1.045 & 1.045 & 1.045 & 1.045 & 1.046 & 1.046 & 1.046 & 1.046 \\
\hline 5 & 300.0 & 1.049 & 1.049 & 1.048 & 1.048 & 1.049 & 1.049 & 1.049 & 1.049 \\
\hline 6 & 400.0 & 1.050 & 1.049 & 1.050 & 1.050 & 1.050 & 1.050 & 1.050 & 1.050 \\
\hline 7 & 500.0 & 1.050 & 1.050 & 1.050 & 1.050 & 1.050 & 1.050 & 1.050 & 1.050 \\
\hline 8 & 600.0 & 1.050 & 1.050 & 1.049 & 1.050 & 1.050 & 1.050 & 1.050 & 1.050 \\
\hline 9 & 700.0 & 1.049 & 1.048 & 1.048 & 1.048 & 1.049 & 1.049 & 1.048 & 1.048 \\
\hline 10 & 800.0 & 1.048 & 1.047 & 1.047 & 1.047 & 1.048 & 1.048 & 1.047 & 1.048 \\
\hline 11 & 900.0 & 1.046 & 1.046 & 1.045 & 1.045 & 1.046 & 1.046 & 1.045 & 1.046 \\
\hline 12 & 1000.0 & 1.044 & 1.044 & 1.044 & 1.044 & 1.044 & 1.045 & 1.044 & 1.044 \\
\hline 13 & 1100.0 & 1.042 & 1.042 & 1.042 & 1.042 & 1.042 & 1.042 & 1.042 & 1.042 \\
\hline 14 & 1200.0 & 1.040 & 1.040 & 1.040 & 1.040 & 1.040 & 1.040 & 1.040 & 1.040 \\
\hline 15 & 1300.0 & 1.037 & 1.037 & 1.037 & 1.037 & 1.037 & 1.038 & 1.037 & 1.037 \\
\hline
\end{tabular}


Table 7.75. Problem 6.2.1 reference solution power peaking factors in element 2 for charge-pan III (1/2).

\begin{tabular}{|c|c|c|c|c|c|c|c|c|c|}
\hline \multirow[t]{2}{*}{ Step } & \multirow{2}{*}{$\begin{array}{l}\text { Burnup } \\
{\left[\frac{\mathrm{MWd}}{\mathrm{M} t \mathrm{II}}\right]}\end{array}$} & \multicolumn{8}{|c|}{ Shift Power Peaking Factors } \\
\hline & & A & B & $\mathrm{C}$ & $\mathrm{D}$ & $\mathrm{P}$ & Q & $\mathrm{R}$ & S \\
\hline 0 & 0.0 & 1.553 & 1.553 & 1.554 & 1.556 & 1.576 & 1.575 & 1.572 & 1.574 \\
\hline 1 & 25.0 & 1.577 & 1.581 & 1.580 & 1.575 & 1.592 & 1.608 & 1.614 & 1.605 \\
\hline 2 & 50.0 & 1.580 & 1.581 & 1.580 & 1.577 & 1.592 & 1.607 & 1.611 & 1.604 \\
\hline 3 & 100.0 & 1.577 & 1.581 & 1.576 & 1.572 & 1.590 & 1.596 & 1.600 & 1.600 \\
\hline 4 & 200.0 & 1.569 & 1.577 & 1.565 & 1.572 & 1.583 & 1.578 & 1.582 & 1.590 \\
\hline 5 & 300.0 & 1.567 & 1.572 & 1.557 & 1.565 & 1.581 & 1.567 & 1.562 & 1.579 \\
\hline 6 & 400.0 & 1.562 & 1.567 & 1.550 & 1.569 & 1.580 & 1.556 & 1.548 & 1.576 \\
\hline 7 & 500.0 & 1.561 & 1.566 & 1.551 & 1.561 & 1.580 & 1.550 & 1.543 & 1.577 \\
\hline 8 & 600.0 & 1.561 & 1.566 & 1.550 & 1.562 & 1.582 & 1.551 & 1.536 & 1.574 \\
\hline 9 & 700.0 & 1.556 & 1.564 & 1.547 & 1.559 & 1.574 & 1.548 & 1.531 & 1.572 \\
\hline 10 & 800.0 & 1.560 & 1.568 & 1.549 & 1.565 & 1.583 & 1.551 & 1.532 & 1.580 \\
\hline 11 & 900.0 & 1.560 & 1.571 & 1.547 & 1.563 & 1.576 & 1.546 & 1.527 & 1.582 \\
\hline 12 & 1000.0 & 1.561 & 1.571 & 1.555 & 1.565 & 1.582 & 1.555 & 1.529 & 1.586 \\
\hline 13 & 1100.0 & 1.565 & 1.576 & 1.559 & 1.569 & 1.581 & 1.558 & 1.538 & 1.596 \\
\hline 14 & 1200.0 & 1.564 & 1.579 & 1.561 & 1.571 & 1.586 & 1.564 & 1.537 & 1.596 \\
\hline 15 & 1300.0 & 1.565 & 1.580 & 1.565 & 1.572 & 1.583 & 1.566 & 1.543 & 1.601 \\
\hline \multirow[t]{2}{*}{ Step } & Burnup & \multicolumn{8}{|c|}{ MPACT Power Peaking Factors } \\
\hline & $\frac{\mathrm{MWd}}{\mathrm{MtU}}$ & A & B & $\mathrm{C}$ & $\mathrm{D}$ & $\mathrm{P}$ & Q & $\mathrm{R}$ & $S$ \\
\hline 0 & 0.0 & 1.590 & 1.591 & 1.591 & 1.591 & 1.609 & 1.608 & 1.609 & 1.611 \\
\hline 1 & 25.0 & 1.551 & 1.551 & 1.552 & 1.552 & 1.569 & 1.569 & 1.569 & 1.571 \\
\hline 2 & 50.0 & 1.557 & 1.557 & 1.558 & 1.558 & 1.575 & 1.575 & 1.575 & 1.577 \\
\hline 3 & 100.0 & 1.569 & 1.569 & 1.570 & 1.569 & 1.588 & 1.587 & 1.588 & 1.589 \\
\hline 4 & 200.0 & 1.585 & 1.586 & 1.586 & 1.585 & 1.604 & 1.603 & 1.604 & 1.606 \\
\hline 5 & 300.0 & 1.596 & 1.595 & 1.595 & 1.595 & 1.613 & 1.613 & 1.614 & 1.615 \\
\hline 6 & 400.0 & 1.599 & 1.599 & 1.599 & 1.599 & 1.618 & 1.617 & 1.618 & 1.620 \\
\hline 7 & 500.0 & 1.599 & 1.600 & 1.600 & 1.600 & 1.619 & 1.618 & 1.618 & 1.620 \\
\hline 8 & 600.0 & 1.598 & 1.598 & 1.598 & 1.598 & 1.617 & 1.616 & 1.617 & 1.620 \\
\hline 9 & 700.0 & 1.593 & 1.594 & 1.595 & 1.594 & 1.613 & 1.612 & 1.612 & 1.615 \\
\hline 10 & 800.0 & 1.589 & 1.589 & 1.591 & 1.589 & 1.609 & 1.607 & 1.608 & 1.610 \\
\hline 11 & 900.0 & 1.583 & 1.583 & 1.585 & 1.583 & 1.602 & 1.601 & 1.601 & 1.604 \\
\hline 12 & 1000.0 & 1.576 & 1.577 & 1.579 & 1.577 & 1.596 & 1.595 & 1.595 & 1.598 \\
\hline 13 & 1100.0 & 1.569 & 1.570 & 1.572 & 1.570 & 1.589 & 1.587 & 1.588 & 1.591 \\
\hline 14 & 1200.0 & 1.562 & 1.563 & 1.564 & 1.562 & 1.581 & 1.580 & 1.580 & 1.583 \\
\hline 15 & 1300.0 & 1.553 & 1.554 & 1.556 & 1.554 & 1.573 & 1.571 & 1.571 & 1.575 \\
\hline
\end{tabular}


Table 7.76. Problem 6.2.1 reference solution power peaking factors in element 2 for charge-pan III (2/2).

\begin{tabular}{|c|c|c|c|c|c|c|c|c|c|}
\hline \multirow[t]{2}{*}{ Step } & \multirow{2}{*}{$\begin{array}{l}\text { Burnup } \\
{\left[\frac{\mathrm{MWd}}{\mathrm{MtU}}\right]}\end{array}$} & \multicolumn{8}{|c|}{ Shift Power Peaking Factors } \\
\hline & & $\mathrm{E}$ & $\mathrm{F}$ & G & $\mathrm{H}$ & $\mathrm{J}$ & K & $\mathrm{L}$ & M \\
\hline 0 & 0.0 & 1.572 & 1.570 & 1.571 & 1.568 & 1.569 & 1.568 & 1.569 & 1.574 \\
\hline 1 & 25.0 & 1.589 & 1.594 & 1.598 & 1.601 & 1.609 & 1.608 & 1.599 & 1.592 \\
\hline 2 & 50.0 & 1.591 & 1.592 & 1.599 & 1.604 & 1.609 & 1.604 & 1.597 & 1.590 \\
\hline 3 & 100.0 & 1.592 & 1.588 & 1.596 & 1.602 & 1.595 & 1.596 & 1.590 & 1.585 \\
\hline 4 & 200.0 & 1.583 & 1.577 & 1.585 & 1.596 & 1.576 & 1.578 & 1.573 & 1.579 \\
\hline 5 & 300.0 & 1.579 & 1.573 & 1.578 & 1.588 & 1.553 & 1.564 & 1.560 & 1.568 \\
\hline 6 & 400.0 & 1.577 & 1.564 & 1.570 & 1.586 & 1.541 & 1.556 & 1.553 & 1.566 \\
\hline 7 & 500.0 & 1.571 & 1.566 & 1.571 & 1.587 & 1.535 & 1.552 & 1.546 & 1.562 \\
\hline 8 & 600.0 & 1.572 & 1.565 & 1.569 & 1.588 & 1.528 & 1.552 & 1.547 & 1.561 \\
\hline 9 & 700.0 & 1.571 & 1.559 & 1.570 & 1.588 & 1.523 & 1.548 & 1.540 & 1.558 \\
\hline 10 & 800.0 & 1.575 & 1.562 & 1.572 & 1.587 & 1.522 & 1.551 & 1.543 & 1.560 \\
\hline 11 & 900.0 & 1.572 & 1.559 & 1.570 & 1.591 & 1.522 & 1.549 & 1.545 & 1.559 \\
\hline 12 & 1000.0 & 1.575 & 1.567 & 1.578 & 1.591 & 1.525 & 1.554 & 1.547 & 1.567 \\
\hline 13 & 1100.0 & 1.577 & 1.571 & 1.585 & 1.598 & 1.528 & 1.564 & 1.553 & 1.566 \\
\hline 14 & 1200.0 & 1.576 & 1.574 & 1.587 & 1.597 & 1.528 & 1.565 & 1.554 & 1.572 \\
\hline 15 & 1300.0 & 1.575 & 1.577 & 1.591 & 1.603 & 1.535 & 1.569 & 1.560 & 1.573 \\
\hline \multirow[t]{2}{*}{ Step } & Burnup & \multicolumn{8}{|c|}{ MPACT Power Peaking Factors } \\
\hline & $\frac{\mathrm{MWd}}{\mathrm{MtU}}$ & $\mathrm{E}$ & $\mathrm{F}$ & G & $\mathrm{H}$ & $\mathrm{J}$ & K & $\mathrm{L}$ & M \\
\hline 0 & 0.0 & 1.605 & 1.606 & 1.605 & 1.606 & 1.606 & 1.607 & 1.607 & 1.606 \\
\hline 1 & 25.0 & 1.567 & 1.566 & 1.567 & 1.567 & 1.568 & 1.568 & 1.568 & 1.568 \\
\hline 2 & 50.0 & 1.572 & 1.572 & 1.572 & 1.573 & 1.574 & 1.574 & 1.574 & 1.573 \\
\hline 3 & 100.0 & 1.585 & 1.585 & 1.584 & 1.584 & 1.585 & 1.586 & 1.586 & 1.586 \\
\hline 4 & 200.0 & 1.601 & 1.600 & 1.600 & 1.601 & 1.602 & 1.602 & 1.603 & 1.602 \\
\hline 5 & 300.0 & 1.611 & 1.610 & 1.610 & 1.610 & 1.612 & 1.612 & 1.613 & 1.611 \\
\hline 6 & 400.0 & 1.614 & 1.614 & 1.614 & 1.615 & 1.616 & 1.616 & 1.616 & 1.616 \\
\hline 7 & 500.0 & 1.616 & 1.615 & 1.615 & 1.616 & 1.616 & 1.617 & 1.617 & 1.616 \\
\hline 8 & 600.0 & 1.614 & 1.614 & 1.614 & 1.614 & 1.615 & 1.615 & 1.615 & 1.615 \\
\hline 9 & 700.0 & 1.610 & 1.610 & 1.609 & 1.609 & 1.610 & 1.611 & 1.611 & 1.611 \\
\hline 10 & 800.0 & 1.605 & 1.605 & 1.605 & 1.605 & 1.606 & 1.607 & 1.606 & 1.606 \\
\hline 11 & 900.0 & 1.599 & 1.599 & 1.598 & 1.598 & 1.600 & 1.601 & 1.600 & 1.600 \\
\hline 12 & 1000.0 & 1.593 & 1.593 & 1.592 & 1.592 & 1.593 & 1.594 & 1.594 & 1.593 \\
\hline 13 & 1100.0 & 1.585 & 1.586 & 1.585 & 1.585 & 1.586 & 1.587 & 1.586 & 1.586 \\
\hline 14 & 1200.0 & 1.578 & 1.578 & 1.577 & 1.577 & 1.578 & 1.580 & 1.579 & 1.578 \\
\hline 15 & 1300.0 & 1.569 & 1.570 & 1.569 & 1.569 & 1.570 & 1.571 & 1.570 & 1.570 \\
\hline
\end{tabular}


Table 7.77. Problem 6.2.1 reference solution power peaking factors in element 3 for charge-pan III (1/2).

\begin{tabular}{|c|c|c|c|c|c|c|c|c|c|}
\hline \multirow[t]{2}{*}{ Step } & \multirow{2}{*}{$\begin{array}{l}\text { Burnup } \\
{\left[\frac{\mathrm{MWd}}{\mathrm{MtU}}\right]}\end{array}$} & \multicolumn{8}{|c|}{ Shift Power Peaking Factors } \\
\hline & & A & B & $\mathrm{C}$ & D & $\mathrm{P}$ & Q & $\mathrm{R}$ & $\mathrm{S}$ \\
\hline 0 & 0.0 & 1.501 & 1.500 & 1.501 & 1.511 & 1.534 & 1.515 & 1.506 & 1.510 \\
\hline 1 & 25.0 & 1.491 & 1.485 & 1.487 & 1.497 & 1.516 & 1.500 & 1.499 & 1.506 \\
\hline 2 & 50.0 & 1.490 & 1.485 & 1.487 & 1.493 & 1.513 & 1.502 & 1.497 & 1.499 \\
\hline 3 & 100.0 & 1.492 & 1.489 & 1.493 & 1.498 & 1.519 & 1.502 & 1.494 & 1.496 \\
\hline 4 & 200.0 & 1.493 & 1.495 & 1.502 & 1.507 & 1.528 & 1.507 & 1.500 & 1.496 \\
\hline 5 & 300.0 & 1.498 & 1.505 & 1.511 & 1.512 & 1.535 & 1.512 & 1.506 & 1.496 \\
\hline 6 & 400.0 & 1.499 & 1.505 & 1.512 & 1.515 & 1.540 & 1.513 & 1.503 & 1.493 \\
\hline 7 & 500.0 & 1.502 & 1.510 & 1.519 & 1.521 & 1.546 & 1.518 & 1.509 & 1.495 \\
\hline 8 & 600.0 & 1.502 & 1.511 & 1.516 & 1.521 & 1.543 & 1.515 & 1.506 & 1.491 \\
\hline 9 & 700.0 & 1.504 & 1.513 & 1.519 & 1.522 & 1.542 & 1.516 & 1.509 & 1.493 \\
\hline 10 & 800.0 & 1.502 & 1.517 & 1.520 & 1.523 & 1.543 & 1.518 & 1.511 & 1.495 \\
\hline 11 & 900.0 & 1.500 & 1.510 & 1.517 & 1.518 & 1.543 & 1.516 & 1.507 & 1.490 \\
\hline 12 & 1000.0 & 1.499 & 1.512 & 1.515 & 1.519 & 1.537 & 1.513 & 1.504 & 1.488 \\
\hline 13 & 1100.0 & 1.501 & 1.511 & 1.519 & 1.519 & 1.540 & 1.518 & 1.510 & 1.492 \\
\hline 14 & 1200.0 & 1.501 & 1.506 & 1.512 & 1.517 & 1.537 & 1.514 & 1.506 & 1.489 \\
\hline 15 & 1300.0 & 1.498 & 1.505 & 1.511 & 1.514 & 1.534 & 1.511 & 1.506 & 1.491 \\
\hline \multirow[t]{2}{*}{ Step } & Burnup & \multicolumn{8}{|c|}{ MPACT Power Peaking Factors } \\
\hline & $\frac{\mathrm{MWd}}{\mathrm{MtU}}$ & A & B & $\mathrm{C}$ & $\mathrm{D}$ & $\mathrm{P}$ & Q & $\mathrm{R}$ & S \\
\hline 0 & 0.0 & 1.515 & 1.520 & 1.526 & 1.519 & 1.529 & 1.524 & 1.529 & 1.551 \\
\hline 1 & 25.0 & 1.493 & 1.497 & 1.503 & 1.497 & 1.506 & 1.502 & 1.506 & 1.527 \\
\hline 2 & 50.0 & 1.497 & 1.501 & 1.507 & 1.501 & 1.509 & 1.505 & 1.510 & 1.531 \\
\hline 3 & 100.0 & 1.504 & 1.509 & 1.515 & 1.508 & 1.518 & 1.513 & 1.517 & 1.539 \\
\hline 4 & 200.0 & 1.516 & 1.520 & 1.526 & 1.520 & 1.530 & 1.524 & 1.530 & 1.551 \\
\hline 5 & 300.0 & 1.523 & 1.526 & 1.532 & 1.526 & 1.535 & 1.531 & 1.536 & 1.557 \\
\hline 6 & 400.0 & 1.525 & 1.529 & 1.535 & 1.529 & 1.539 & 1.534 & 1.539 & 1.562 \\
\hline 7 & 500.0 & 1.526 & 1.531 & 1.537 & 1.530 & 1.541 & 1.535 & 1.541 & 1.562 \\
\hline 8 & 600.0 & 1.525 & 1.529 & 1.535 & 1.529 & 1.539 & 1.534 & 1.540 & 1.562 \\
\hline 9 & 700.0 & 1.524 & 1.529 & 1.535 & 1.528 & 1.539 & 1.533 & 1.538 & 1.561 \\
\hline 10 & 800.0 & 1.522 & 1.527 & 1.533 & 1.526 & 1.537 & 1.531 & 1.536 & 1.558 \\
\hline 11 & 900.0 & 1.519 & 1.524 & 1.530 & 1.523 & 1.534 & 1.528 & 1.533 & 1.555 \\
\hline 12 & 1000.0 & 1.515 & 1.520 & 1.526 & 1.519 & 1.530 & 1.524 & 1.529 & 1.551 \\
\hline 13 & 1100.0 & 1.511 & 1.517 & 1.523 & 1.516 & 1.526 & 1.520 & 1.525 & 1.547 \\
\hline 14 & 1200.0 & 1.507 & 1.513 & 1.518 & 1.511 & 1.522 & 1.516 & 1.521 & 1.543 \\
\hline 15 & 1300.0 & 1.503 & 1.508 & 1.514 & 1.507 & 1.517 & 1.511 & 1.516 & 1.538 \\
\hline
\end{tabular}


Table 7.78. Problem 6.2.1 reference solution power peaking factors in element 3 for charge-pan III (2/2).

\begin{tabular}{|c|c|c|c|c|c|c|c|c|c|}
\hline \multirow[t]{2}{*}{ Step } & \multirow{2}{*}{$\begin{array}{l}\text { Burnup } \\
{\left[\frac{\mathrm{MWd}}{\mathrm{MtU}}\right]}\end{array}$} & \multicolumn{8}{|c|}{ Shift Power Peaking Factors } \\
\hline & & $\mathrm{E}$ & $\mathrm{F}$ & G & $\mathrm{H}$ & $\mathrm{J}$ & K & $\mathrm{L}$ & M \\
\hline 0 & 0.0 & 1.528 & 1.523 & 1.510 & 1.505 & 1.506 & 1.503 & 1.518 & 1.526 \\
\hline 1 & 25.0 & 1.515 & 1.511 & 1.499 & 1.495 & 1.496 & 1.497 & 1.507 & 1.511 \\
\hline 2 & 50.0 & 1.511 & 1.507 & 1.497 & 1.497 & 1.494 & 1.495 & 1.505 & 1.510 \\
\hline 3 & 100.0 & 1.514 & 1.510 & 1.498 & 1.495 & 1.497 & 1.497 & 1.508 & 1.514 \\
\hline 4 & 200.0 & 1.518 & 1.502 & 1.493 & 1.497 & 1.496 & 1.499 & 1.509 & 1.525 \\
\hline 5 & 300.0 & 1.523 & 1.507 & 1.494 & 1.498 & 1.502 & 1.504 & 1.519 & 1.532 \\
\hline 6 & 400.0 & 1.526 & 1.502 & 1.489 & 1.503 & 1.500 & 1.504 & 1.518 & 1.535 \\
\hline 7 & 500.0 & 1.528 & 1.504 & 1.492 & 1.504 & 1.506 & 1.507 & 1.517 & 1.541 \\
\hline 8 & 600.0 & 1.527 & 1.499 & 1.490 & 1.503 & 1.505 & 1.505 & 1.519 & 1.540 \\
\hline 9 & 700.0 & 1.526 & 1.502 & 1.488 & 1.505 & 1.503 & 1.507 & 1.520 & 1.540 \\
\hline 10 & 800.0 & 1.527 & 1.502 & 1.487 & 1.508 & 1.508 & 1.509 & 1.521 & 1.541 \\
\hline 11 & 900.0 & 1.525 & 1.498 & 1.486 & 1.507 & 1.506 & 1.508 & 1.517 & 1.541 \\
\hline 12 & 1000.0 & 1.523 & 1.497 & 1.483 & 1.504 & 1.502 & 1.507 & 1.514 & 1.536 \\
\hline 13 & 1100.0 & 1.523 & 1.500 & 1.485 & 1.506 & 1.508 & 1.509 & 1.518 & 1.540 \\
\hline 14 & 1200.0 & 1.524 & 1.497 & 1.487 & 1.504 & 1.503 & 1.509 & 1.516 & 1.537 \\
\hline 15 & 1300.0 & 1.519 & 1.498 & 1.483 & 1.504 & 1.503 & 1.506 & 1.513 & 1.529 \\
\hline \multirow[t]{2}{*}{ Step } & Burnup & \multicolumn{8}{|c|}{ MPACT Power Peaking Factors } \\
\hline & $\frac{\mathrm{MWd}}{\mathrm{MtU}}$ & $\mathrm{E}$ & $\mathrm{F}$ & G & $\mathrm{H}$ & $\mathrm{J}$ & K & $\mathrm{L}$ & M \\
\hline 0 & 0.0 & 1.524 & 1.523 & 1.523 & 1.525 & 1.537 & 1.544 & 1.544 & 1.537 \\
\hline 1 & 25.0 & 1.502 & 1.500 & 1.501 & 1.502 & 1.514 & 1.521 & 1.522 & 1.514 \\
\hline 2 & 50.0 & 1.505 & 1.504 & 1.504 & 1.507 & 1.519 & 1.526 & 1.526 & 1.518 \\
\hline 3 & 100.0 & 1.514 & 1.512 & 1.512 & 1.513 & 1.526 & 1.533 & 1.533 & 1.526 \\
\hline 4 & 200.0 & 1.525 & 1.524 & 1.523 & 1.526 & 1.538 & 1.545 & 1.545 & 1.538 \\
\hline 5 & 300.0 & 1.532 & 1.531 & 1.530 & 1.531 & 1.545 & 1.552 & 1.551 & 1.544 \\
\hline 6 & 400.0 & 1.534 & 1.533 & 1.534 & 1.536 & 1.547 & 1.554 & 1.555 & 1.547 \\
\hline 7 & 500.0 & 1.536 & 1.534 & 1.535 & 1.537 & 1.549 & 1.556 & 1.557 & 1.549 \\
\hline 8 & 600.0 & 1.535 & 1.534 & 1.534 & 1.536 & 1.547 & 1.555 & 1.554 & 1.548 \\
\hline 9 & 700.0 & 1.535 & 1.533 & 1.533 & 1.534 & 1.547 & 1.554 & 1.554 & 1.547 \\
\hline 10 & 800.0 & 1.532 & 1.531 & 1.530 & 1.532 & 1.544 & 1.552 & 1.552 & 1.544 \\
\hline 11 & 900.0 & 1.529 & 1.528 & 1.527 & 1.529 & 1.541 & 1.549 & 1.548 & 1.541 \\
\hline 12 & 1000.0 & 1.526 & 1.524 & 1.524 & 1.525 & 1.538 & 1.545 & 1.545 & 1.538 \\
\hline 13 & 1100.0 & 1.522 & 1.520 & 1.520 & 1.521 & 1.534 & 1.541 & 1.541 & 1.533 \\
\hline 14 & 1200.0 & 1.518 & 1.516 & 1.516 & 1.517 & 1.530 & 1.537 & 1.537 & 1.529 \\
\hline 15 & 1300.0 & 1.513 & 1.512 & 1.511 & 1.512 & 1.525 & 1.532 & 1.532 & 1.524 \\
\hline
\end{tabular}


Table 7.79. Problem 6.2.1 reference solution power peaking factors in element 4 for charge-pan III (1/2).

\begin{tabular}{|c|c|c|c|c|c|c|c|c|c|}
\hline \multirow[t]{2}{*}{ Step } & \multirow{2}{*}{$\begin{array}{l}\text { Burnup } \\
{\left[\frac{\mathrm{MWd}}{\mathrm{M} t \mathrm{II}}\right]}\end{array}$} & \multicolumn{8}{|c|}{ Shift Power Peaking Factors } \\
\hline & & A & B & $\mathrm{C}$ & $\mathrm{D}$ & $\mathrm{P}$ & Q & $\mathrm{R}$ & S \\
\hline 0 & 0.0 & 1.026 & 1.015 & 1.026 & 1.046 & 1.077 & 1.011 & 0.992 & 1.008 \\
\hline 1 & 25.0 & 0.997 & 0.981 & 0.991 & 1.014 & 1.044 & 0.977 & 0.959 & 0.975 \\
\hline 2 & 50.0 & 0.993 & 0.978 & 0.991 & 1.008 & 1.038 & 0.975 & 0.958 & 0.974 \\
\hline 3 & 100.0 & 1.000 & 0.989 & 1.002 & 1.018 & 1.051 & 0.987 & 0.969 & 0.982 \\
\hline 4 & 200.0 & 1.008 & 0.998 & 1.019 & 1.030 & 1.064 & 1.003 & 0.984 & 0.993 \\
\hline 5 & 300.0 & 1.016 & 1.011 & 1.031 & 1.042 & 1.075 & 1.018 & 0.998 & 1.005 \\
\hline 6 & 400.0 & 1.021 & 1.017 & 1.037 & 1.047 & 1.080 & 1.024 & 1.005 & 1.011 \\
\hline 7 & 500.0 & 1.023 & 1.023 & 1.046 & 1.055 & 1.086 & 1.027 & 1.011 & 1.015 \\
\hline 8 & 600.0 & 1.023 & 1.024 & 1.047 & 1.058 & 1.091 & 1.031 & 1.011 & 1.018 \\
\hline 9 & 700.0 & 1.021 & 1.025 & 1.047 & 1.058 & 1.092 & 1.032 & 1.011 & 1.014 \\
\hline 10 & 800.0 & 1.021 & 1.021 & 1.044 & 1.053 & 1.087 & 1.032 & 1.010 & 1.016 \\
\hline 11 & 900.0 & 1.018 & 1.023 & 1.045 & 1.056 & 1.089 & 1.027 & 1.008 & 1.012 \\
\hline 12 & 1000.0 & 1.016 & 1.018 & 1.041 & 1.052 & 1.083 & 1.024 & 1.003 & 1.010 \\
\hline 13 & 1100.0 & 1.010 & 1.015 & 1.035 & 1.046 & 1.079 & 1.018 & 0.996 & 1.002 \\
\hline 14 & 1200.0 & 1.006 & 1.012 & 1.032 & 1.046 & 1.075 & 1.015 & 0.989 & 1.000 \\
\hline 15 & 1300.0 & 1.006 & 1.007 & 1.027 & 1.041 & 1.072 & 1.009 & 0.988 & 0.999 \\
\hline \multirow[t]{2}{*}{ Step } & Burnup & \multicolumn{8}{|c|}{ MPACT Power Peaking Factors } \\
\hline & $\frac{\mathrm{MWd}}{\mathrm{MtU}}$ & A & B & $\mathrm{C}$ & $\mathrm{D}$ & $\mathrm{P}$ & Q & $\mathrm{R}$ & S \\
\hline 0 & 0.0 & 0.994 & 1.007 & 1.025 & 1.007 & 0.990 & 0.973 & 0.990 & 1.056 \\
\hline 1 & 25.0 & 1.015 & 1.028 & 1.046 & 1.028 & 1.010 & 0.993 & 1.010 & 1.077 \\
\hline 2 & 50.0 & 1.011 & 1.025 & 1.043 & 1.024 & 1.006 & 0.990 & 1.007 & 1.074 \\
\hline 3 & 100.0 & 1.005 & 1.019 & 1.037 & 1.018 & 1.001 & 0.984 & 1.001 & 1.067 \\
\hline 4 & 200.0 & 0.998 & 1.011 & 1.029 & 1.010 & 0.994 & 0.977 & 0.994 & 1.060 \\
\hline 5 & 300.0 & 0.993 & 1.006 & 1.025 & 1.007 & 0.990 & 0.973 & 0.990 & 1.055 \\
\hline 6 & 400.0 & 0.993 & 1.006 & 1.023 & 1.005 & 0.989 & 0.972 & 0.989 & 1.055 \\
\hline 7 & 500.0 & 0.993 & 1.006 & 1.024 & 1.006 & 0.989 & 0.972 & 0.990 & 1.055 \\
\hline 8 & 600.0 & 0.995 & 1.007 & 1.025 & 1.007 & 0.991 & 0.974 & 0.991 & 1.056 \\
\hline 9 & 700.0 & 0.998 & 1.012 & 1.029 & 1.011 & 0.995 & 0.977 & 0.995 & 1.061 \\
\hline 10 & 800.0 & 1.002 & 1.015 & 1.033 & 1.014 & 0.998 & 0.981 & 0.998 & 1.064 \\
\hline 11 & 900.0 & 1.006 & 1.019 & 1.037 & 1.019 & 1.003 & 0.985 & 1.003 & 1.069 \\
\hline 12 & 1000.0 & 1.010 & 1.023 & 1.041 & 1.023 & 1.006 & 0.989 & 1.007 & 1.073 \\
\hline 13 & 1100.0 & 1.015 & 1.028 & 1.046 & 1.027 & 1.011 & 0.994 & 1.011 & 1.078 \\
\hline 14 & 1200.0 & 1.019 & 1.033 & 1.051 & 1.032 & 1.016 & 0.999 & 1.016 & 1.083 \\
\hline 15 & 1300.0 & 1.024 & 1.038 & 1.056 & 1.037 & 1.021 & 1.004 & 1.021 & 1.088 \\
\hline
\end{tabular}


Table 7.80. Problem 6.2.1 reference solution power peaking factors in element 4 for charge-pan III (2/2).

\begin{tabular}{|c|c|c|c|c|c|c|c|c|c|}
\hline \multirow[t]{2}{*}{ Step } & \multirow{2}{*}{$\begin{array}{l}\text { Burnup } \\
{\left[\frac{\mathrm{MWd}}{\mathrm{MtU}}\right]}\end{array}$} & \multicolumn{8}{|c|}{ Shift Power Peaking Factors } \\
\hline & & $\mathrm{E}$ & $\mathrm{F}$ & G & $\mathrm{H}$ & $\mathrm{J}$ & $\mathrm{K}$ & $\mathrm{L}$ & M \\
\hline 0 & 0.0 & 1.063 & 1.042 & 1.003 & 0.997 & 0.998 & 1.003 & 1.042 & 1.063 \\
\hline 1 & 25.0 & 1.031 & 1.012 & 0.970 & 0.967 & 0.963 & 0.967 & 1.005 & 1.028 \\
\hline 2 & 50.0 & 1.028 & 1.007 & 0.969 & 0.963 & 0.961 & 0.969 & 1.004 & 1.027 \\
\hline 3 & 100.0 & 1.039 & 1.018 & 0.977 & 0.969 & 0.973 & 0.977 & 1.015 & 1.040 \\
\hline 4 & 200.0 & 1.048 & 1.031 & 0.990 & 0.979 & 0.989 & 0.993 & 1.034 & 1.054 \\
\hline 5 & 300.0 & 1.054 & 1.044 & 0.999 & 0.986 & 1.000 & 1.007 & 1.048 & 1.071 \\
\hline 6 & 400.0 & 1.061 & 1.048 & 1.009 & 0.989 & 1.007 & 1.013 & 1.055 & 1.076 \\
\hline 7 & 500.0 & 1.063 & 1.054 & 1.012 & 0.992 & 1.015 & 1.018 & 1.060 & 1.084 \\
\hline 8 & 600.0 & 1.065 & 1.057 & 1.012 & 0.993 & 1.014 & 1.021 & 1.065 & 1.086 \\
\hline 9 & 700.0 & 1.065 & 1.056 & 1.013 & 0.990 & 1.015 & 1.020 & 1.065 & 1.086 \\
\hline 10 & 800.0 & 1.063 & 1.056 & 1.013 & 0.988 & 1.013 & 1.018 & 1.064 & 1.086 \\
\hline 11 & 900.0 & 1.058 & 1.053 & 1.010 & 0.986 & 1.011 & 1.015 & 1.062 & 1.085 \\
\hline 12 & 1000.0 & 1.056 & 1.050 & 1.005 & 0.984 & 1.006 & 1.012 & 1.055 & 1.081 \\
\hline 13 & 1100.0 & 1.051 & 1.046 & 1.003 & 0.981 & 1.000 & 1.004 & 1.050 & 1.072 \\
\hline 14 & 1200.0 & 1.049 & 1.042 & 0.999 & 0.976 & 0.995 & 0.998 & 1.047 & 1.072 \\
\hline 15 & 1300.0 & 1.046 & 1.040 & 0.996 & 0.974 & 0.990 & 0.996 & 1.045 & 1.068 \\
\hline \multirow[t]{2}{*}{ Step } & Burnup & \multicolumn{8}{|c|}{ MPACT Power Peaking Factors } \\
\hline & $\frac{\mathrm{MWd}}{\mathrm{MtU}}$ & $\mathrm{E}$ & $\mathrm{F}$ & G & $\mathrm{H}$ & $\mathrm{J}$ & K & $\mathrm{L}$ & M \\
\hline 0 & 0.0 & 0.982 & 0.977 & 0.977 & 0.982 & 1.023 & 1.044 & 1.044 & 1.023 \\
\hline 1 & 25.0 & 1.002 & 0.997 & 0.997 & 1.003 & 1.044 & 1.065 & 1.066 & 1.044 \\
\hline 2 & 50.0 & 0.999 & 0.994 & 0.994 & 1.000 & 1.042 & 1.062 & 1.063 & 1.041 \\
\hline 3 & 100.0 & 0.993 & 0.988 & 0.988 & 0.994 & 1.035 & 1.055 & 1.056 & 1.035 \\
\hline 4 & 200.0 & 0.986 & 0.981 & 0.981 & 0.986 & 1.027 & 1.048 & 1.048 & 1.027 \\
\hline 5 & 300.0 & 0.982 & 0.978 & 0.977 & 0.982 & 1.023 & 1.044 & 1.044 & 1.023 \\
\hline 6 & 400.0 & 0.981 & 0.976 & 0.976 & 0.982 & 1.022 & 1.043 & 1.043 & 1.022 \\
\hline 7 & 500.0 & 0.981 & 0.977 & 0.977 & 0.982 & 1.023 & 1.044 & 1.044 & 1.023 \\
\hline 8 & 600.0 & 0.983 & 0.978 & 0.978 & 0.984 & 1.024 & 1.045 & 1.045 & 1.025 \\
\hline 9 & 700.0 & 0.987 & 0.982 & 0.982 & 0.987 & 1.029 & 1.049 & 1.049 & 1.028 \\
\hline 10 & 800.0 & 0.991 & 0.985 & 0.986 & 0.990 & 1.032 & 1.053 & 1.053 & 1.032 \\
\hline 11 & 900.0 & 0.995 & 0.990 & 0.990 & 0.995 & 1.036 & 1.057 & 1.057 & 1.036 \\
\hline 12 & 1000.0 & 1.000 & 0.994 & 0.994 & 0.999 & 1.041 & 1.061 & 1.061 & 1.040 \\
\hline 13 & 1100.0 & 1.004 & 0.998 & 0.999 & 1.003 & 1.045 & 1.066 & 1.066 & 1.045 \\
\hline 14 & 1200.0 & 1.009 & 1.003 & 1.003 & 1.008 & 1.050 & 1.071 & 1.071 & 1.050 \\
\hline 15 & 1300.0 & 1.014 & 1.008 & 1.008 & 1.013 & 1.056 & 1.076 & 1.076 & 1.055 \\
\hline
\end{tabular}


Table 7.81. Problem 6.2.1 reference solution power peaking factors in element 5 for charge-pan III (1/2).

\begin{tabular}{|c|c|c|c|c|c|c|c|c|c|}
\hline \multirow[t]{2}{*}{ Step } & \multirow{2}{*}{$\begin{array}{l}\text { Burnup } \\
{\left[\frac{\mathrm{MWd}}{\mathrm{M} t \mathrm{II}}\right]}\end{array}$} & \multicolumn{8}{|c|}{ Shift Power Peaking Factors } \\
\hline & & A & B & $\mathrm{C}$ & $\mathrm{D}$ & $\mathrm{P}$ & Q & $\mathrm{R}$ & S \\
\hline 0 & 0.0 & 0.576 & 0.564 & 0.575 & 0.589 & 0.611 & 0.560 & 0.546 & 0.560 \\
\hline 1 & 25.0 & 0.545 & 0.535 & 0.545 & 0.559 & 0.582 & 0.531 & 0.516 & 0.529 \\
\hline 2 & 50.0 & 0.546 & 0.536 & 0.546 & 0.560 & 0.581 & 0.529 & 0.515 & 0.531 \\
\hline 3 & 100.0 & 0.552 & 0.540 & 0.551 & 0.564 & 0.588 & 0.537 & 0.523 & 0.536 \\
\hline 4 & 200.0 & 0.565 & 0.551 & 0.565 & 0.575 & 0.599 & 0.552 & 0.537 & 0.552 \\
\hline 5 & 300.0 & 0.575 & 0.560 & 0.574 & 0.584 & 0.607 & 0.563 & 0.548 & 0.562 \\
\hline 6 & 400.0 & 0.581 & 0.565 & 0.581 & 0.591 & 0.616 & 0.570 & 0.556 & 0.572 \\
\hline 7 & 500.0 & 0.585 & 0.567 & 0.583 & 0.593 & 0.617 & 0.573 & 0.558 & 0.573 \\
\hline 8 & 600.0 & 0.586 & 0.566 & 0.585 & 0.593 & 0.619 & 0.573 & 0.560 & 0.576 \\
\hline 9 & 700.0 & 0.587 & 0.568 & 0.587 & 0.593 & 0.618 & 0.574 & 0.562 & 0.577 \\
\hline 10 & 800.0 & 0.583 & 0.565 & 0.585 & 0.590 & 0.616 & 0.574 & 0.557 & 0.572 \\
\hline 11 & 900.0 & 0.586 & 0.566 & 0.585 & 0.592 & 0.615 & 0.573 & 0.559 & 0.573 \\
\hline 12 & 1000.0 & 0.581 & 0.560 & 0.582 & 0.586 & 0.614 & 0.571 & 0.555 & 0.566 \\
\hline 13 & 1100.0 & 0.577 & 0.554 & 0.576 & 0.580 & 0.606 & 0.564 & 0.547 & 0.560 \\
\hline 14 & 1200.0 & 0.574 & 0.555 & 0.575 & 0.582 & 0.606 & 0.562 & 0.545 & 0.559 \\
\hline 15 & 1300.0 & 0.572 & 0.551 & 0.573 & 0.577 & 0.605 & 0.559 & 0.545 & 0.554 \\
\hline \multirow[t]{2}{*}{ Step } & Burnup & \multicolumn{8}{|c|}{ MPACT Power Peaking Factors } \\
\hline & $\frac{\mathrm{MWd}}{\mathrm{MtU}}$ & A & B & $\mathrm{C}$ & $\mathrm{D}$ & $\mathrm{P}$ & Q & $\mathrm{R}$ & $S$ \\
\hline 0 & 0.0 & 0.532 & 0.541 & 0.555 & 0.541 & 0.527 & 0.513 & 0.527 & 0.577 \\
\hline 1 & 25.0 & 0.574 & 0.584 & 0.599 & 0.584 & 0.569 & 0.553 & 0.569 & 0.622 \\
\hline 2 & 50.0 & 0.568 & 0.579 & 0.593 & 0.579 & 0.563 & 0.548 & 0.563 & 0.616 \\
\hline 3 & 100.0 & 0.556 & 0.566 & 0.580 & 0.566 & 0.551 & 0.536 & 0.551 & 0.602 \\
\hline 4 & 200.0 & 0.538 & 0.548 & 0.562 & 0.548 & 0.534 & 0.520 & 0.534 & 0.584 \\
\hline 5 & 300.0 & 0.528 & 0.537 & 0.551 & 0.537 & 0.524 & 0.509 & 0.524 & 0.573 \\
\hline 6 & 400.0 & 0.523 & 0.533 & 0.546 & 0.533 & 0.519 & 0.505 & 0.519 & 0.567 \\
\hline 7 & 500.0 & 0.521 & 0.531 & 0.545 & 0.531 & 0.517 & 0.503 & 0.517 & 0.566 \\
\hline 8 & 600.0 & 0.522 & 0.532 & 0.546 & 0.532 & 0.518 & 0.504 & 0.518 & 0.566 \\
\hline 9 & 700.0 & 0.525 & 0.535 & 0.549 & 0.535 & 0.521 & 0.507 & 0.521 & 0.570 \\
\hline 10 & 800.0 & 0.529 & 0.539 & 0.552 & 0.538 & 0.524 & 0.511 & 0.524 & 0.574 \\
\hline 11 & 900.0 & 0.534 & 0.544 & 0.558 & 0.544 & 0.530 & 0.516 & 0.530 & 0.580 \\
\hline 12 & 1000.0 & 0.540 & 0.550 & 0.564 & 0.550 & 0.535 & 0.521 & 0.535 & 0.586 \\
\hline 13 & 1100.0 & 0.546 & 0.556 & 0.571 & 0.557 & 0.542 & 0.527 & 0.542 & 0.593 \\
\hline 14 & 1200.0 & 0.554 & 0.564 & 0.578 & 0.564 & 0.549 & 0.534 & 0.549 & 0.601 \\
\hline 15 & 1300.0 & 0.561 & 0.572 & 0.586 & 0.572 & 0.557 & 0.542 & 0.557 & 0.609 \\
\hline
\end{tabular}


Table 7.82. Problem 6.2.1 reference solution power peaking factors in element 5 for charge-pan III (2/2).

\begin{tabular}{|c|c|c|c|c|c|c|c|c|c|}
\hline \multirow[t]{2}{*}{ Step } & \multirow{2}{*}{$\begin{array}{l}\text { Burnup } \\
{\left[\frac{\mathrm{MWd}}{\mathrm{M} t \mathrm{II}}\right]}\end{array}$} & \multicolumn{8}{|c|}{ Shift Power Peaking Factors } \\
\hline & & $\mathrm{E}$ & $\mathrm{F}$ & G & $\mathrm{H}$ & $\mathrm{J}$ & K & $\mathrm{L}$ & M \\
\hline 0 & 0.0 & 0.603 & 0.587 & 0.556 & 0.549 & 0.550 & 0.553 & 0.586 & 0.603 \\
\hline 1 & 25.0 & 0.570 & 0.555 & 0.524 & 0.521 & 0.520 & 0.524 & 0.556 & 0.572 \\
\hline 2 & 50.0 & 0.573 & 0.555 & 0.523 & 0.520 & 0.521 & 0.526 & 0.555 & 0.570 \\
\hline 3 & 100.0 & 0.581 & 0.563 & 0.531 & 0.527 & 0.528 & 0.532 & 0.563 & 0.580 \\
\hline 4 & 200.0 & 0.592 & 0.577 & 0.547 & 0.539 & 0.542 & 0.547 & 0.578 & 0.592 \\
\hline 5 & 300.0 & 0.603 & 0.589 & 0.556 & 0.547 & 0.553 & 0.558 & 0.589 & 0.604 \\
\hline 6 & 400.0 & 0.611 & 0.598 & 0.566 & 0.555 & 0.560 & 0.565 & 0.598 & 0.612 \\
\hline 7 & 500.0 & 0.614 & 0.601 & 0.569 & 0.558 & 0.564 & 0.566 & 0.601 & 0.614 \\
\hline 8 & 600.0 & 0.615 & 0.602 & 0.571 & 0.559 & 0.566 & 0.571 & 0.606 & 0.616 \\
\hline 9 & 700.0 & 0.618 & 0.604 & 0.571 & 0.562 & 0.567 & 0.570 & 0.604 & 0.618 \\
\hline 10 & 800.0 & 0.615 & 0.602 & 0.567 & 0.557 & 0.565 & 0.568 & 0.601 & 0.615 \\
\hline 11 & 900.0 & 0.616 & 0.603 & 0.568 & 0.560 & 0.563 & 0.569 & 0.604 & 0.617 \\
\hline 12 & 1000.0 & 0.612 & 0.597 & 0.563 & 0.554 & 0.560 & 0.563 & 0.598 & 0.614 \\
\hline 13 & 1100.0 & 0.605 & 0.590 & 0.560 & 0.549 & 0.554 & 0.558 & 0.592 & 0.605 \\
\hline 14 & 1200.0 & 0.604 & 0.589 & 0.557 & 0.548 & 0.553 & 0.558 & 0.591 & 0.607 \\
\hline 15 & 1300.0 & 0.603 & 0.585 & 0.552 & 0.544 & 0.551 & 0.554 & 0.589 & 0.605 \\
\hline \multirow[t]{2}{*}{ Step } & Burnup & \multicolumn{8}{|c|}{ MPACT Power Peaking Factors } \\
\hline & $\frac{\mathrm{MWd}}{\mathrm{MtU}}$ & $\mathrm{E}$ & $\mathrm{F}$ & G & $\mathrm{H}$ & $\mathrm{J}$ & K & $\mathrm{L}$ & M \\
\hline 0 & 0.0 & 0.522 & 0.517 & 0.517 & 0.522 & 0.552 & 0.568 & 0.568 & 0.552 \\
\hline 1 & 25.0 & 0.563 & 0.559 & 0.558 & 0.563 & 0.595 & 0.612 & 0.613 & 0.595 \\
\hline 2 & 50.0 & 0.558 & 0.553 & 0.553 & 0.558 & 0.590 & 0.606 & 0.607 & 0.590 \\
\hline 3 & 100.0 & 0.545 & 0.541 & 0.541 & 0.546 & 0.577 & 0.593 & 0.593 & 0.577 \\
\hline 4 & 200.0 & 0.528 & 0.524 & 0.524 & 0.528 & 0.559 & 0.575 & 0.575 & 0.559 \\
\hline 5 & 300.0 & 0.518 & 0.514 & 0.513 & 0.518 & 0.548 & 0.564 & 0.564 & 0.548 \\
\hline 6 & 400.0 & 0.513 & 0.509 & 0.509 & 0.514 & 0.543 & 0.559 & 0.559 & 0.543 \\
\hline 7 & 500.0 & 0.511 & 0.508 & 0.508 & 0.512 & 0.542 & 0.557 & 0.557 & 0.541 \\
\hline 8 & 600.0 & 0.512 & 0.508 & 0.508 & 0.513 & 0.543 & 0.558 & 0.558 & 0.543 \\
\hline 9 & 700.0 & 0.516 & 0.511 & 0.511 & 0.516 & 0.545 & 0.561 & 0.561 & 0.545 \\
\hline 10 & 800.0 & 0.519 & 0.515 & 0.515 & 0.519 & 0.549 & 0.565 & 0.565 & 0.549 \\
\hline 11 & 900.0 & 0.524 & 0.520 & 0.520 & 0.524 & 0.555 & 0.571 & 0.571 & 0.555 \\
\hline 12 & 1000.0 & 0.530 & 0.526 & 0.525 & 0.530 & 0.561 & 0.577 & 0.577 & 0.561 \\
\hline 13 & 1100.0 & 0.537 & 0.532 & 0.532 & 0.537 & 0.567 & 0.584 & 0.584 & 0.568 \\
\hline 14 & 1200.0 & 0.544 & 0.539 & 0.539 & 0.543 & 0.575 & 0.591 & 0.592 & 0.575 \\
\hline 15 & 1300.0 & 0.551 & 0.547 & 0.547 & 0.552 & 0.583 & 0.600 & 0.600 & 0.583 \\
\hline
\end{tabular}


Table 7.83. Problem 6.2.1 reference solution power peaking factors in element 6 for charge-pan III (1/2).

\begin{tabular}{|c|c|c|c|c|c|c|c|c|c|}
\hline \multirow[t]{2}{*}{ Step } & \multirow{2}{*}{$\begin{array}{l}\text { Burnup } \\
{\left[\frac{\mathrm{MWd}}{\mathrm{MtU}}\right]}\end{array}$} & \multicolumn{8}{|c|}{ Shift Power Peaking Factors } \\
\hline & & A & B & $\mathrm{C}$ & $\mathrm{D}$ & $\mathrm{P}$ & Q & $\mathrm{R}$ & S \\
\hline 0 & 0.0 & 0.254 & 0.249 & 0.254 & 0.261 & 0.272 & 0.246 & 0.243 & 0.246 \\
\hline 1 & 25.0 & 0.238 & 0.233 & 0.238 & 0.245 & 0.255 & 0.231 & 0.228 & 0.230 \\
\hline 2 & 50.0 & 0.237 & 0.232 & 0.238 & 0.246 & 0.256 & 0.231 & 0.229 & 0.231 \\
\hline 3 & 100.0 & 0.241 & 0.236 & 0.241 & 0.248 & 0.256 & 0.233 & 0.230 & 0.235 \\
\hline 4 & 200.0 & 0.250 & 0.243 & 0.246 & 0.255 & 0.265 & 0.241 & 0.236 & 0.241 \\
\hline 5 & 300.0 & 0.254 & 0.248 & 0.251 & 0.261 & 0.271 & 0.245 & 0.242 & 0.249 \\
\hline 6 & 400.0 & 0.260 & 0.252 & 0.255 & 0.265 & 0.277 & 0.249 & 0.245 & 0.253 \\
\hline 7 & 500.0 & 0.261 & 0.254 & 0.255 & 0.266 & 0.277 & 0.250 & 0.248 & 0.254 \\
\hline 8 & 600.0 & 0.263 & 0.254 & 0.257 & 0.270 & 0.279 & 0.253 & 0.249 & 0.257 \\
\hline 9 & 700.0 & 0.264 & 0.256 & 0.258 & 0.269 & 0.281 & 0.254 & 0.251 & 0.257 \\
\hline 10 & 800.0 & 0.262 & 0.254 & 0.257 & 0.268 & 0.281 & 0.253 & 0.248 & 0.256 \\
\hline 11 & 900.0 & 0.263 & 0.256 & 0.257 & 0.269 & 0.280 & 0.253 & 0.250 & 0.256 \\
\hline 12 & 1000.0 & 0.262 & 0.253 & 0.254 & 0.267 & 0.279 & 0.252 & 0.249 & 0.254 \\
\hline 13 & 1100.0 & 0.259 & 0.251 & 0.252 & 0.264 & 0.277 & 0.249 & 0.246 & 0.252 \\
\hline 14 & 1200.0 & 0.259 & 0.252 & 0.252 & 0.265 & 0.276 & 0.248 & 0.246 & 0.252 \\
\hline 15 & 1300.0 & 0.255 & 0.249 & 0.251 & 0.262 & 0.273 & 0.247 & 0.244 & 0.249 \\
\hline \multirow[t]{2}{*}{ Step } & Burnup & \multicolumn{8}{|c|}{ MPACT Power Peaking Factors } \\
\hline & $\frac{\mathrm{MWd}}{\mathrm{MtU}}$ & A & B & $\mathrm{C}$ & $\mathrm{D}$ & $\mathrm{P}$ & Q & $\mathrm{R}$ & $\mathrm{S}$ \\
\hline 0 & 0.0 & 0.224 & 0.229 & 0.236 & 0.229 & 0.222 & 0.219 & 0.222 & 0.245 \\
\hline 1 & 25.0 & 0.254 & 0.260 & 0.267 & 0.260 & 0.252 & 0.248 & 0.252 & 0.279 \\
\hline 2 & 50.0 & 0.251 & 0.257 & 0.264 & 0.257 & 0.249 & 0.245 & 0.249 & 0.275 \\
\hline 3 & 100.0 & 0.243 & 0.248 & 0.255 & 0.248 & 0.240 & 0.237 & 0.240 & 0.266 \\
\hline 4 & 200.0 & 0.230 & 0.235 & 0.242 & 0.235 & 0.228 & 0.225 & 0.228 & 0.252 \\
\hline 5 & 300.0 & 0.222 & 0.226 & 0.233 & 0.226 & 0.220 & 0.217 & 0.220 & 0.243 \\
\hline 6 & 400.0 & 0.217 & 0.222 & 0.229 & 0.222 & 0.215 & 0.213 & 0.216 & 0.238 \\
\hline 7 & 500.0 & 0.216 & 0.220 & 0.227 & 0.220 & 0.214 & 0.211 & 0.213 & 0.236 \\
\hline 8 & 600.0 & 0.215 & 0.220 & 0.226 & 0.220 & 0.213 & 0.211 & 0.213 & 0.236 \\
\hline 9 & 700.0 & 0.216 & 0.220 & 0.227 & 0.221 & 0.214 & 0.211 & 0.214 & 0.236 \\
\hline 10 & 800.0 & 0.217 & 0.222 & 0.229 & 0.222 & 0.215 & 0.213 & 0.215 & 0.238 \\
\hline 11 & 900.0 & 0.220 & 0.225 & 0.231 & 0.225 & 0.218 & 0.216 & 0.218 & 0.241 \\
\hline 12 & 1000.0 & 0.223 & 0.228 & 0.234 & 0.228 & 0.221 & 0.218 & 0.221 & 0.244 \\
\hline 13 & 1100.0 & 0.226 & 0.231 & 0.238 & 0.232 & 0.225 & 0.222 & 0.224 & 0.248 \\
\hline 14 & 1200.0 & 0.230 & 0.236 & 0.242 & 0.236 & 0.228 & 0.226 & 0.228 & 0.253 \\
\hline 15 & 1300.0 & 0.235 & 0.240 & 0.247 & 0.240 & 0.233 & 0.230 & 0.233 & 0.258 \\
\hline
\end{tabular}


Table 7.84. Problem 6.2.1 reference solution power peaking factors in element 6 for charge-pan III (2/2).

\begin{tabular}{|c|c|c|c|c|c|c|c|c|c|}
\hline \multirow[t]{2}{*}{ Step } & \multirow{2}{*}{$\begin{array}{l}\text { Burnup } \\
{\left[\frac{\mathrm{MWd}}{\mathrm{MtU}}\right]}\end{array}$} & \multicolumn{8}{|c|}{ Shift Power Peaking Factors } \\
\hline & & $\mathrm{E}$ & $\mathrm{F}$ & G & $\mathrm{H}$ & $\mathrm{J}$ & K & $\mathrm{L}$ & M \\
\hline 0 & 0.0 & 0.269 & 0.260 & 0.243 & 0.241 & 0.242 & 0.243 & 0.258 & 0.268 \\
\hline 1 & 25.0 & 0.250 & 0.242 & 0.228 & 0.225 & 0.226 & 0.228 & 0.243 & 0.251 \\
\hline 2 & 50.0 & 0.250 & 0.243 & 0.228 & 0.226 & 0.226 & 0.229 & 0.243 & 0.252 \\
\hline 3 & 100.0 & 0.254 & 0.246 & 0.231 & 0.229 & 0.229 & 0.231 & 0.245 & 0.254 \\
\hline 4 & 200.0 & 0.262 & 0.253 & 0.238 & 0.237 & 0.233 & 0.238 & 0.253 & 0.260 \\
\hline 5 & 300.0 & 0.268 & 0.261 & 0.244 & 0.243 & 0.241 & 0.243 & 0.259 & 0.265 \\
\hline 6 & 400.0 & 0.274 & 0.264 & 0.249 & 0.246 & 0.244 & 0.246 & 0.265 & 0.269 \\
\hline 7 & 500.0 & 0.275 & 0.266 & 0.249 & 0.249 & 0.245 & 0.249 & 0.265 & 0.271 \\
\hline 8 & 600.0 & 0.278 & 0.267 & 0.252 & 0.251 & 0.247 & 0.249 & 0.267 & 0.272 \\
\hline 9 & 700.0 & 0.278 & 0.269 & 0.254 & 0.252 & 0.249 & 0.252 & 0.267 & 0.271 \\
\hline 10 & 800.0 & 0.277 & 0.267 & 0.251 & 0.248 & 0.246 & 0.249 & 0.265 & 0.270 \\
\hline 11 & 900.0 & 0.278 & 0.268 & 0.252 & 0.249 & 0.247 & 0.250 & 0.268 & 0.273 \\
\hline 12 & 1000.0 & 0.276 & 0.266 & 0.251 & 0.248 & 0.247 & 0.248 & 0.265 & 0.270 \\
\hline 13 & 1100.0 & 0.273 & 0.264 & 0.248 & 0.246 & 0.243 & 0.248 & 0.264 & 0.267 \\
\hline 14 & 1200.0 & 0.272 & 0.264 & 0.247 & 0.245 & 0.244 & 0.246 & 0.264 & 0.266 \\
\hline 15 & 1300.0 & 0.270 & 0.261 & 0.246 & 0.243 & 0.243 & 0.245 & 0.261 & 0.265 \\
\hline \multirow[t]{2}{*}{ Step } & Burnup & \multicolumn{8}{|c|}{ MPACT Power Peaking Factors } \\
\hline & $\frac{\mathrm{MWd}}{\mathrm{MtU}}$ & $\mathrm{E}$ & $\mathrm{F}$ & G & $\mathrm{H}$ & $\mathrm{J}$ & K & $\mathrm{L}$ & M \\
\hline 0 & 0.0 & 0.219 & 0.217 & 0.217 & 0.219 & 0.234 & 0.241 & 0.241 & 0.234 \\
\hline 1 & 25.0 & 0.249 & 0.247 & 0.247 & 0.249 & 0.265 & 0.274 & 0.274 & 0.265 \\
\hline 2 & 50.0 & 0.246 & 0.243 & 0.244 & 0.246 & 0.262 & 0.270 & 0.271 & 0.262 \\
\hline 3 & 100.0 & 0.237 & 0.235 & 0.235 & 0.238 & 0.253 & 0.261 & 0.261 & 0.253 \\
\hline 4 & 200.0 & 0.225 & 0.223 & 0.223 & 0.225 & 0.240 & 0.248 & 0.248 & 0.240 \\
\hline 5 & 300.0 & 0.217 & 0.215 & 0.215 & 0.217 & 0.231 & 0.239 & 0.239 & 0.231 \\
\hline 6 & 400.0 & 0.213 & 0.211 & 0.211 & 0.213 & 0.227 & 0.234 & 0.234 & 0.227 \\
\hline 7 & 500.0 & 0.211 & 0.209 & 0.209 & 0.211 & 0.225 & 0.232 & 0.232 & 0.225 \\
\hline 8 & 600.0 & 0.210 & 0.209 & 0.209 & 0.211 & 0.224 & 0.232 & 0.232 & 0.224 \\
\hline 9 & 700.0 & 0.211 & 0.210 & 0.209 & 0.211 & 0.225 & 0.232 & 0.232 & 0.225 \\
\hline 10 & 800.0 & 0.213 & 0.211 & 0.211 & 0.213 & 0.227 & 0.234 & 0.234 & 0.227 \\
\hline 11 & 900.0 & 0.215 & 0.213 & 0.213 & 0.215 & 0.230 & 0.237 & 0.237 & 0.229 \\
\hline 12 & 1000.0 & 0.218 & 0.216 & 0.216 & 0.218 & 0.233 & 0.240 & 0.240 & 0.233 \\
\hline 13 & 1100.0 & 0.222 & 0.220 & 0.220 & 0.222 & 0.236 & 0.244 & 0.244 & 0.236 \\
\hline 14 & 1200.0 & 0.226 & 0.224 & 0.224 & 0.225 & 0.240 & 0.248 & 0.248 & 0.240 \\
\hline 15 & 1300.0 & 0.230 & 0.228 & 0.228 & 0.230 & 0.245 & 0.253 & 0.253 & 0.245 \\
\hline
\end{tabular}


Table 7.85. Problem 6.2.1 reference solution power peaking factors in element 1 for charge-pan IV (1/2).

\begin{tabular}{|c|c|c|c|c|c|c|c|c|c|}
\hline \multirow[t]{2}{*}{ Step } & \multirow{2}{*}{$\begin{array}{l}\text { Burnup } \\
{\left[\frac{\mathrm{MWd}}{\mathrm{MtU}}\right]}\end{array}$} & \multicolumn{8}{|c|}{ Shift Power Peaking Factors } \\
\hline & & A & B & $\mathrm{C}$ & D & $\mathrm{P}$ & Q & $\mathrm{R}$ & $S$ \\
\hline 0 & 0.0 & 1.032 & 1.033 & 1.033 & 1.036 & 1.045 & 1.047 & 1.047 & 1.046 \\
\hline 1 & 25.0 & 1.075 & 1.073 & 1.074 & 1.075 & 1.083 & 1.088 & 1.082 & 1.080 \\
\hline 2 & 50.0 & 1.075 & 1.074 & 1.071 & 1.074 & 1.080 & 1.089 & 1.082 & 1.082 \\
\hline 3 & 100.0 & 1.066 & 1.063 & 1.063 & 1.065 & 1.073 & 1.080 & 1.072 & 1.073 \\
\hline 4 & 200.0 & 1.045 & 1.047 & 1.047 & 1.048 & 1.054 & 1.061 & 1.055 & 1.055 \\
\hline 5 & 300.0 & 1.035 & 1.032 & 1.033 & 1.032 & 1.040 & 1.046 & 1.042 & 1.045 \\
\hline 6 & 400.0 & 1.023 & 1.025 & 1.024 & 1.025 & 1.035 & 1.035 & 1.035 & 1.037 \\
\hline 7 & 500.0 & 1.023 & 1.023 & 1.022 & 1.020 & 1.028 & 1.035 & 1.032 & 1.032 \\
\hline 8 & 600.0 & 1.022 & 1.021 & 1.020 & 1.018 & 1.027 & 1.030 & 1.027 & 1.031 \\
\hline 9 & 700.0 & 1.019 & 1.019 & 1.018 & 1.018 & 1.027 & 1.027 & 1.028 & 1.029 \\
\hline 10 & 800.0 & 1.022 & 1.026 & 1.022 & 1.023 & 1.031 & 1.031 & 1.030 & 1.035 \\
\hline 11 & 900.0 & 1.026 & 1.028 & 1.023 & 1.025 & 1.033 & 1.036 & 1.034 & 1.034 \\
\hline 12 & 1000.0 & 1.035 & 1.032 & 1.029 & 1.032 & 1.037 & 1.046 & 1.039 & 1.043 \\
\hline 13 & 1100.0 & 1.035 & 1.037 & 1.036 & 1.036 & 1.040 & 1.047 & 1.043 & 1.045 \\
\hline 14 & 1200.0 & 1.044 & 1.041 & 1.038 & 1.038 & 1.046 & 1.052 & 1.047 & 1.049 \\
\hline 15 & 1300.0 & 1.047 & 1.048 & 1.043 & 1.042 & 1.049 & 1.059 & 1.052 & 1.055 \\
\hline \multirow[t]{2}{*}{ Step } & Burnup & \multicolumn{8}{|c|}{ MPACT Power Peaking Factors } \\
\hline & $\frac{\mathrm{MWd}}{\mathrm{MtU}}$ & A & B & $\mathrm{C}$ & $\mathrm{D}$ & $\mathrm{P}$ & Q & $\mathrm{R}$ & $\mathrm{S}$ \\
\hline 0 & 0.0 & 1.046 & 1.046 & 1.046 & 1.046 & 1.057 & 1.058 & 1.057 & 1.058 \\
\hline 1 & 25.0 & 1.029 & 1.029 & 1.029 & 1.029 & 1.040 & 1.040 & 1.040 & 1.040 \\
\hline 2 & 50.0 & 1.030 & 1.030 & 1.030 & 1.030 & 1.041 & 1.041 & 1.040 & 1.041 \\
\hline 3 & 100.0 & 1.033 & 1.033 & 1.032 & 1.032 & 1.044 & 1.044 & 1.043 & 1.044 \\
\hline 4 & 200.0 & 1.036 & 1.035 & 1.035 & 1.035 & 1.047 & 1.047 & 1.047 & 1.047 \\
\hline 5 & 300.0 & 1.039 & 1.038 & 1.039 & 1.039 & 1.050 & 1.050 & 1.050 & 1.051 \\
\hline 6 & 400.0 & 1.040 & 1.040 & 1.040 & 1.040 & 1.052 & 1.051 & 1.052 & 1.051 \\
\hline 7 & 500.0 & 1.040 & 1.040 & 1.039 & 1.040 & 1.052 & 1.052 & 1.051 & 1.051 \\
\hline 8 & 600.0 & 1.040 & 1.039 & 1.040 & 1.039 & 1.052 & 1.052 & 1.051 & 1.051 \\
\hline 9 & 700.0 & 1.038 & 1.038 & 1.038 & 1.039 & 1.050 & 1.050 & 1.050 & 1.050 \\
\hline 10 & 800.0 & 1.037 & 1.037 & 1.037 & 1.038 & 1.049 & 1.050 & 1.049 & 1.049 \\
\hline 11 & 900.0 & 1.035 & 1.035 & 1.035 & 1.036 & 1.048 & 1.047 & 1.047 & 1.047 \\
\hline 12 & 1000.0 & 1.034 & 1.034 & 1.034 & 1.034 & 1.046 & 1.046 & 1.045 & 1.046 \\
\hline 13 & 1100.0 & 1.032 & 1.032 & 1.032 & 1.033 & 1.044 & 1.044 & 1.043 & 1.044 \\
\hline 14 & 1200.0 & 1.030 & 1.029 & 1.030 & 1.030 & 1.042 & 1.042 & 1.041 & 1.041 \\
\hline 15 & 1300.0 & 1.027 & 1.027 & 1.027 & 1.028 & 1.039 & 1.039 & 1.038 & 1.039 \\
\hline
\end{tabular}


Table 7.86. Problem 6.2.1 reference solution power peaking factors in element 1 for charge-pan IV (2/2).

\begin{tabular}{|c|c|c|c|c|c|c|c|c|c|}
\hline \multirow[t]{2}{*}{ Step } & \multirow{2}{*}{$\begin{array}{l}\text { Burnup } \\
{\left[\frac{\mathrm{MWd}}{\mathrm{MtU}}\right]}\end{array}$} & \multicolumn{8}{|c|}{ Shift Power Peaking Factors } \\
\hline & & $\mathrm{E}$ & $\mathrm{F}$ & G & $\mathrm{H}$ & $\mathrm{J}$ & K & $\mathrm{L}$ & M \\
\hline 0 & 0.0 & 1.045 & 1.043 & 1.043 & 1.044 & 1.043 & 1.044 & 1.044 & 1.043 \\
\hline 1 & 25.0 & 1.083 & 1.086 & 1.087 & 1.084 & 1.078 & 1.080 & 1.081 & 1.081 \\
\hline 2 & 50.0 & 1.083 & 1.086 & 1.085 & 1.084 & 1.082 & 1.079 & 1.080 & 1.079 \\
\hline 3 & 100.0 & 1.074 & 1.078 & 1.076 & 1.075 & 1.071 & 1.072 & 1.071 & 1.072 \\
\hline 4 & 200.0 & 1.056 & 1.056 & 1.059 & 1.057 & 1.053 & 1.054 & 1.052 & 1.053 \\
\hline 5 & 300.0 & 1.043 & 1.046 & 1.045 & 1.044 & 1.041 & 1.042 & 1.040 & 1.037 \\
\hline 6 & 400.0 & 1.035 & 1.035 & 1.034 & 1.035 & 1.035 & 1.034 & 1.033 & 1.034 \\
\hline 7 & 500.0 & 1.032 & 1.034 & 1.030 & 1.032 & 1.030 & 1.031 & 1.028 & 1.026 \\
\hline 8 & 600.0 & 1.028 & 1.030 & 1.030 & 1.029 & 1.027 & 1.029 & 1.025 & 1.026 \\
\hline 9 & 700.0 & 1.028 & 1.030 & 1.028 & 1.030 & 1.026 & 1.027 & 1.024 & 1.024 \\
\hline 10 & 800.0 & 1.030 & 1.035 & 1.031 & 1.032 & 1.029 & 1.031 & 1.027 & 1.026 \\
\hline 11 & 900.0 & 1.033 & 1.036 & 1.032 & 1.036 & 1.033 & 1.033 & 1.031 & 1.027 \\
\hline 12 & 1000.0 & 1.041 & 1.044 & 1.043 & 1.042 & 1.037 & 1.038 & 1.035 & 1.034 \\
\hline 13 & 1100.0 & 1.042 & 1.046 & 1.045 & 1.046 & 1.042 & 1.042 & 1.038 & 1.036 \\
\hline 14 & 1200.0 & 1.050 & 1.053 & 1.050 & 1.051 & 1.044 & 1.047 & 1.043 & 1.041 \\
\hline 15 & 1300.0 & 1.054 & 1.057 & 1.058 & 1.057 & 1.052 & 1.054 & 1.048 & 1.047 \\
\hline \multirow[t]{2}{*}{ Step } & Burnup & \multicolumn{8}{|c|}{ MPACT Power Peaking Factors } \\
\hline & $\frac{\mathrm{MWd}}{\mathrm{MtU}}$ & $\mathrm{E}$ & $\mathrm{F}$ & G & $\mathrm{H}$ & $\mathrm{J}$ & K & $\mathrm{L}$ & M \\
\hline 0 & 0.0 & 1.055 & 1.055 & 1.055 & 1.055 & 1.055 & 1.055 & 1.055 & 1.055 \\
\hline 1 & 25.0 & 1.039 & 1.039 & 1.038 & 1.038 & 1.038 & 1.038 & 1.039 & 1.039 \\
\hline 2 & 50.0 & 1.039 & 1.040 & 1.039 & 1.039 & 1.039 & 1.039 & 1.040 & 1.040 \\
\hline 3 & 100.0 & 1.042 & 1.042 & 1.042 & 1.042 & 1.041 & 1.042 & 1.042 & 1.042 \\
\hline 4 & 200.0 & 1.045 & 1.045 & 1.045 & 1.045 & 1.045 & 1.045 & 1.045 & 1.046 \\
\hline 5 & 300.0 & 1.048 & 1.049 & 1.049 & 1.048 & 1.048 & 1.048 & 1.048 & 1.048 \\
\hline 6 & 400.0 & 1.050 & 1.050 & 1.050 & 1.049 & 1.049 & 1.050 & 1.050 & 1.050 \\
\hline 7 & 500.0 & 1.050 & 1.050 & 1.049 & 1.049 & 1.050 & 1.050 & 1.050 & 1.050 \\
\hline 8 & 600.0 & 1.050 & 1.050 & 1.049 & 1.049 & 1.049 & 1.050 & 1.050 & 1.050 \\
\hline 9 & 700.0 & 1.048 & 1.048 & 1.048 & 1.048 & 1.048 & 1.048 & 1.048 & 1.048 \\
\hline 10 & 800.0 & 1.047 & 1.047 & 1.047 & 1.047 & 1.047 & 1.047 & 1.047 & 1.047 \\
\hline 11 & 900.0 & 1.045 & 1.045 & 1.045 & 1.045 & 1.045 & 1.046 & 1.045 & 1.045 \\
\hline 12 & 1000.0 & 1.044 & 1.044 & 1.044 & 1.044 & 1.044 & 1.044 & 1.044 & 1.044 \\
\hline 13 & 1100.0 & 1.042 & 1.042 & 1.042 & 1.042 & 1.041 & 1.042 & 1.042 & 1.042 \\
\hline 14 & 1200.0 & 1.039 & 1.039 & 1.039 & 1.039 & 1.039 & 1.040 & 1.039 & 1.040 \\
\hline 15 & 1300.0 & 1.037 & 1.037 & 1.037 & 1.037 & 1.037 & 1.037 & 1.037 & 1.037 \\
\hline
\end{tabular}


Table 7.87. Problem 6.2.1 reference solution power peaking factors in element 2 for charge-pan IV (1/2).

\begin{tabular}{|c|c|c|c|c|c|c|c|c|c|}
\hline \multirow[t]{2}{*}{ Step } & \multirow{2}{*}{$\begin{array}{l}\text { Burnup } \\
{\left[\frac{\mathrm{MWd}}{\mathrm{MtU}}\right]}\end{array}$} & \multicolumn{8}{|c|}{ Shift Power Peaking Factors } \\
\hline & & A & B & $\mathrm{C}$ & D & $\mathrm{P}$ & Q & $\mathrm{R}$ & $\mathrm{S}$ \\
\hline 0 & 0.0 & 1.551 & 1.553 & 1.553 & 1.553 & 1.574 & 1.568 & 1.572 & 1.573 \\
\hline 1 & 25.0 & 1.602 & 1.602 & 1.599 & 1.602 & 1.615 & 1.623 & 1.616 & 1.618 \\
\hline 2 & 50.0 & 1.601 & 1.598 & 1.599 & 1.603 & 1.616 & 1.622 & 1.615 & 1.616 \\
\hline 3 & 100.0 & 1.589 & 1.589 & 1.587 & 1.589 & 1.607 & 1.614 & 1.602 & 1.603 \\
\hline 4 & 200.0 & 1.567 & 1.567 & 1.569 & 1.566 & 1.582 & 1.586 & 1.582 & 1.584 \\
\hline 5 & 300.0 & 1.547 & 1.545 & 1.547 & 1.548 & 1.566 & 1.569 & 1.562 & 1.565 \\
\hline 6 & 400.0 & 1.535 & 1.534 & 1.534 & 1.537 & 1.552 & 1.553 & 1.552 & 1.552 \\
\hline 7 & 500.0 & 1.526 & 1.527 & 1.528 & 1.526 & 1.542 & 1.545 & 1.542 & 1.544 \\
\hline 8 & 600.0 & 1.522 & 1.520 & 1.523 & 1.521 & 1.540 & 1.542 & 1.540 & 1.540 \\
\hline 9 & 700.0 & 1.518 & 1.518 & 1.518 & 1.516 & 1.532 & 1.536 & 1.533 & 1.536 \\
\hline 10 & 800.0 & 1.518 & 1.521 & 1.517 & 1.519 & 1.537 & 1.534 & 1.532 & 1.537 \\
\hline 11 & 900.0 & 1.511 & 1.520 & 1.518 & 1.518 & 1.532 & 1.534 & 1.532 & 1.537 \\
\hline 12 & 1000.0 & 1.517 & 1.519 & 1.521 & 1.522 & 1.535 & 1.535 & 1.533 & 1.540 \\
\hline 13 & 1100.0 & 1.524 & 1.526 & 1.527 & 1.525 & 1.539 & 1.547 & 1.539 & 1.543 \\
\hline 14 & 1200.0 & 1.526 & 1.529 & 1.524 & 1.528 & 1.539 & 1.545 & 1.544 & 1.546 \\
\hline 15 & 1300.0 & 1.530 & 1.531 & 1.531 & 1.531 & 1.544 & 1.549 & 1.546 & 1.553 \\
\hline \multirow[t]{2}{*}{ Step } & Burnup & \multicolumn{8}{|c|}{ MPACT Power Peaking Factors } \\
\hline & $\frac{\mathrm{MWd}}{\mathrm{MtU}}$ & A & B & $\mathrm{C}$ & $\mathrm{D}$ & $\mathrm{P}$ & Q & $\mathrm{R}$ & $\mathrm{S}$ \\
\hline 0 & 0.0 & 1.589 & 1.588 & 1.588 & 1.589 & 1.608 & 1.608 & 1.607 & 1.608 \\
\hline 1 & 25.0 & 1.549 & 1.549 & 1.549 & 1.549 & 1.568 & 1.568 & 1.566 & 1.568 \\
\hline 2 & 50.0 & 1.555 & 1.554 & 1.555 & 1.555 & 1.575 & 1.573 & 1.571 & 1.574 \\
\hline 3 & 100.0 & 1.567 & 1.566 & 1.567 & 1.567 & 1.587 & 1.586 & 1.583 & 1.586 \\
\hline 4 & 200.0 & 1.584 & 1.582 & 1.583 & 1.583 & 1.603 & 1.603 & 1.601 & 1.603 \\
\hline 5 & 300.0 & 1.593 & 1.592 & 1.593 & 1.593 & 1.613 & 1.613 & 1.610 & 1.613 \\
\hline 6 & 400.0 & 1.597 & 1.596 & 1.597 & 1.596 & 1.618 & 1.616 & 1.616 & 1.617 \\
\hline 7 & 500.0 & 1.597 & 1.597 & 1.597 & 1.597 & 1.618 & 1.617 & 1.615 & 1.617 \\
\hline 8 & 600.0 & 1.596 & 1.595 & 1.596 & 1.596 & 1.616 & 1.617 & 1.614 & 1.616 \\
\hline 9 & 700.0 & 1.592 & 1.591 & 1.591 & 1.592 & 1.612 & 1.612 & 1.610 & 1.611 \\
\hline 10 & 800.0 & 1.587 & 1.586 & 1.587 & 1.588 & 1.607 & 1.608 & 1.605 & 1.607 \\
\hline 11 & 900.0 & 1.581 & 1.580 & 1.581 & 1.582 & 1.601 & 1.601 & 1.599 & 1.600 \\
\hline 12 & 1000.0 & 1.575 & 1.574 & 1.574 & 1.576 & 1.595 & 1.595 & 1.593 & 1.594 \\
\hline 13 & 1100.0 & 1.568 & 1.567 & 1.567 & 1.569 & 1.588 & 1.588 & 1.585 & 1.587 \\
\hline 14 & 1200.0 & 1.560 & 1.559 & 1.560 & 1.561 & 1.580 & 1.580 & 1.578 & 1.579 \\
\hline 15 & 1300.0 & 1.552 & 1.551 & 1.552 & 1.553 & 1.572 & 1.572 & 1.569 & 1.570 \\
\hline
\end{tabular}


Table 7.88. Problem 6.2.1 reference solution power peaking factors in element 2 for charge-pan IV (2/2).

\begin{tabular}{|c|c|c|c|c|c|c|c|c|c|}
\hline \multirow[t]{2}{*}{ Step } & \multirow{2}{*}{$\begin{array}{l}\text { Burnup } \\
{\left[\frac{\mathrm{MWd}}{\mathrm{MtU}}\right]}\end{array}$} & \multicolumn{8}{|c|}{ Shift Power Peaking Factors } \\
\hline & & $\mathrm{E}$ & $\mathrm{F}$ & G & $\mathrm{H}$ & $\mathrm{J}$ & K & $\mathrm{L}$ & M \\
\hline 0 & 0.0 & 1.569 & 1.566 & 1.564 & 1.566 & 1.568 & 1.570 & 1.573 & 1.571 \\
\hline 1 & 25.0 & 1.618 & 1.618 & 1.617 & 1.616 & 1.612 & 1.615 & 1.613 & 1.611 \\
\hline 2 & 50.0 & 1.616 & 1.617 & 1.618 & 1.620 & 1.614 & 1.615 & 1.613 & 1.612 \\
\hline 3 & 100.0 & 1.608 & 1.608 & 1.610 & 1.602 & 1.604 & 1.603 & 1.601 & 1.603 \\
\hline 4 & 200.0 & 1.584 & 1.579 & 1.583 & 1.583 & 1.580 & 1.582 & 1.577 & 1.577 \\
\hline 5 & 300.0 & 1.567 & 1.564 & 1.565 & 1.560 & 1.558 & 1.562 & 1.560 & 1.561 \\
\hline 6 & 400.0 & 1.553 & 1.550 & 1.552 & 1.549 & 1.549 & 1.551 & 1.548 & 1.549 \\
\hline 7 & 500.0 & 1.541 & 1.541 & 1.541 & 1.542 & 1.541 & 1.541 & 1.537 & 1.539 \\
\hline 8 & 600.0 & 1.539 & 1.538 & 1.538 & 1.538 & 1.536 & 1.539 & 1.536 & 1.534 \\
\hline 9 & 700.0 & 1.531 & 1.534 & 1.534 & 1.537 & 1.531 & 1.534 & 1.531 & 1.529 \\
\hline 10 & 800.0 & 1.536 & 1.533 & 1.533 & 1.535 & 1.530 & 1.534 & 1.529 & 1.532 \\
\hline 11 & 900.0 & 1.532 & 1.526 & 1.531 & 1.532 & 1.533 & 1.533 & 1.528 & 1.526 \\
\hline 12 & 1000.0 & 1.540 & 1.533 & 1.536 & 1.539 & 1.532 & 1.537 & 1.531 & 1.527 \\
\hline 13 & 1100.0 & 1.542 & 1.541 & 1.542 & 1.546 & 1.538 & 1.541 & 1.535 & 1.533 \\
\hline 14 & 1200.0 & 1.543 & 1.542 & 1.546 & 1.545 & 1.541 & 1.545 & 1.536 & 1.533 \\
\hline 15 & 1300.0 & 1.546 & 1.544 & 1.548 & 1.547 & 1.544 & 1.546 & 1.539 & 1.537 \\
\hline \multirow[t]{2}{*}{ Step } & Burnup & \multicolumn{8}{|c|}{ MPACT Power Peaking Factors } \\
\hline & $\frac{\mathrm{MWd}}{\mathrm{MtU}}$ & $\mathrm{E}$ & $\mathrm{F}$ & G & $\mathrm{H}$ & $\mathrm{J}$ & K & $\mathrm{L}$ & M \\
\hline 0 & 0.0 & 1.605 & 1.605 & 1.604 & 1.604 & 1.604 & 1.604 & 1.605 & 1.605 \\
\hline 1 & 25.0 & 1.566 & 1.566 & 1.564 & 1.564 & 1.563 & 1.565 & 1.566 & 1.566 \\
\hline 2 & 50.0 & 1.572 & 1.571 & 1.570 & 1.569 & 1.569 & 1.570 & 1.572 & 1.571 \\
\hline 3 & 100.0 & 1.583 & 1.584 & 1.583 & 1.582 & 1.581 & 1.582 & 1.584 & 1.583 \\
\hline 4 & 200.0 & 1.600 & 1.600 & 1.599 & 1.598 & 1.599 & 1.599 & 1.600 & 1.600 \\
\hline 5 & 300.0 & 1.610 & 1.610 & 1.609 & 1.608 & 1.608 & 1.609 & 1.610 & 1.610 \\
\hline 6 & 400.0 & 1.614 & 1.614 & 1.613 & 1.612 & 1.612 & 1.613 & 1.614 & 1.614 \\
\hline 7 & 500.0 & 1.615 & 1.614 & 1.613 & 1.612 & 1.613 & 1.613 & 1.614 & 1.614 \\
\hline 8 & 600.0 & 1.613 & 1.613 & 1.613 & 1.612 & 1.612 & 1.613 & 1.613 & 1.613 \\
\hline 9 & 700.0 & 1.609 & 1.609 & 1.608 & 1.607 & 1.607 & 1.608 & 1.609 & 1.609 \\
\hline 10 & 800.0 & 1.604 & 1.604 & 1.603 & 1.602 & 1.602 & 1.604 & 1.604 & 1.605 \\
\hline 11 & 900.0 & 1.598 & 1.598 & 1.597 & 1.596 & 1.596 & 1.598 & 1.598 & 1.599 \\
\hline 12 & 1000.0 & 1.592 & 1.592 & 1.591 & 1.590 & 1.590 & 1.591 & 1.592 & 1.592 \\
\hline 13 & 1100.0 & 1.584 & 1.585 & 1.583 & 1.583 & 1.582 & 1.584 & 1.585 & 1.585 \\
\hline 14 & 1200.0 & 1.577 & 1.577 & 1.576 & 1.575 & 1.575 & 1.577 & 1.577 & 1.577 \\
\hline 15 & 1300.0 & 1.568 & 1.569 & 1.567 & 1.566 & 1.566 & 1.568 & 1.569 & 1.569 \\
\hline
\end{tabular}


Table 7.89. Problem 6.2.1 reference solution power peaking factors in element 3 for charge-pan IV (1/2).

\begin{tabular}{|c|c|c|c|c|c|c|c|c|c|}
\hline \multirow[t]{2}{*}{ Step } & \multirow{2}{*}{$\begin{array}{l}\text { Burnup } \\
{\left[\frac{\mathrm{MWd}}{\mathrm{MtU}}\right]}\end{array}$} & \multicolumn{8}{|c|}{ Shift Power Peaking Factors } \\
\hline & & A & B & $\mathrm{C}$ & $\mathrm{D}$ & $\mathrm{P}$ & Q & $\mathrm{R}$ & S \\
\hline 0 & 0.0 & 1.449 & 1.457 & 1.460 & 1.459 & 1.498 & 1.471 & 1.496 & 1.500 \\
\hline 1 & 25.0 & 1.446 & 1.454 & 1.454 & 1.452 & 1.489 & 1.468 & 1.495 & 1.497 \\
\hline 2 & 50.0 & 1.444 & 1.450 & 1.455 & 1.451 & 1.485 & 1.470 & 1.493 & 1.495 \\
\hline 3 & 100.0 & 1.447 & 1.453 & 1.455 & 1.449 & 1.488 & 1.469 & 1.494 & 1.495 \\
\hline 4 & 200.0 & 1.446 & 1.455 & 1.457 & 1.450 & 1.491 & 1.471 & 1.495 & 1.498 \\
\hline 5 & 300.0 & 1.449 & 1.455 & 1.457 & 1.456 & 1.496 & 1.474 & 1.494 & 1.500 \\
\hline 6 & 400.0 & 1.447 & 1.456 & 1.460 & 1.455 & 1.497 & 1.473 & 1.496 & 1.502 \\
\hline 7 & 500.0 & 1.451 & 1.457 & 1.460 & 1.458 & 1.501 & 1.472 & 1.501 & 1.504 \\
\hline 8 & 600.0 & 1.450 & 1.457 & 1.462 & 1.457 & 1.497 & 1.474 & 1.502 & 1.504 \\
\hline 9 & 700.0 & 1.453 & 1.460 & 1.461 & 1.460 & 1.499 & 1.477 & 1.503 & 1.505 \\
\hline 10 & 800.0 & 1.454 & 1.457 & 1.461 & 1.457 & 1.498 & 1.475 & 1.499 & 1.505 \\
\hline 11 & 900.0 & 1.449 & 1.454 & 1.462 & 1.455 & 1.498 & 1.474 & 1.501 & 1.505 \\
\hline 12 & 1000.0 & 1.450 & 1.454 & 1.459 & 1.457 & 1.497 & 1.474 & 1.500 & 1.504 \\
\hline 13 & 1100.0 & 1.452 & 1.460 & 1.463 & 1.459 & 1.499 & 1.476 & 1.506 & 1.503 \\
\hline 14 & 1200.0 & 1.449 & 1.456 & 1.459 & 1.454 & 1.498 & 1.476 & 1.503 & 1.504 \\
\hline 15 & 1300.0 & 1.448 & 1.456 & 1.459 & 1.458 & 1.495 & 1.473 & 1.504 & 1.502 \\
\hline \multirow[t]{2}{*}{ Step } & Burnup & \multicolumn{8}{|c|}{ MPACT Power Peaking Factors } \\
\hline & $\frac{\mathrm{MWd}}{\mathrm{MtU}}$ & A & B & $\mathrm{C}$ & $\mathrm{D}$ & $\mathrm{P}$ & Q & $\mathrm{R}$ & $S$ \\
\hline 0 & 0.0 & 1.476 & 1.470 & 1.476 & 1.480 & 1.519 & 1.515 & 1.493 & 1.515 \\
\hline 1 & 25.0 & 1.453 & 1.447 & 1.453 & 1.457 & 1.496 & 1.491 & 1.470 & 1.492 \\
\hline 2 & 50.0 & 1.457 & 1.451 & 1.457 & 1.461 & 1.501 & 1.495 & 1.474 & 1.496 \\
\hline 3 & 100.0 & 1.464 & 1.459 & 1.464 & 1.469 & 1.509 & 1.503 & 1.481 & 1.503 \\
\hline 4 & 200.0 & 1.476 & 1.470 & 1.476 & 1.480 & 1.520 & 1.515 & 1.494 & 1.515 \\
\hline 5 & 300.0 & 1.482 & 1.476 & 1.482 & 1.486 & 1.527 & 1.522 & 1.500 & 1.522 \\
\hline 6 & 400.0 & 1.486 & 1.479 & 1.485 & 1.489 & 1.530 & 1.525 & 1.504 & 1.524 \\
\hline 7 & 500.0 & 1.487 & 1.481 & 1.487 & 1.491 & 1.531 & 1.526 & 1.504 & 1.526 \\
\hline 8 & 600.0 & 1.487 & 1.480 & 1.486 & 1.490 & 1.530 & 1.526 & 1.505 & 1.526 \\
\hline 9 & 700.0 & 1.484 & 1.478 & 1.484 & 1.489 & 1.529 & 1.524 & 1.502 & 1.524 \\
\hline 10 & 800.0 & 1.482 & 1.476 & 1.482 & 1.486 & 1.527 & 1.522 & 1.500 & 1.522 \\
\hline 11 & 900.0 & 1.479 & 1.474 & 1.479 & 1.483 & 1.524 & 1.519 & 1.498 & 1.519 \\
\hline 12 & 1000.0 & 1.476 & 1.470 & 1.476 & 1.480 & 1.520 & 1.516 & 1.494 & 1.516 \\
\hline 13 & 1100.0 & 1.473 & 1.467 & 1.472 & 1.477 & 1.516 & 1.512 & 1.491 & 1.512 \\
\hline 14 & 1200.0 & 1.469 & 1.463 & 1.468 & 1.473 & 1.512 & 1.508 & 1.487 & 1.507 \\
\hline 15 & 1300.0 & 1.464 & 1.458 & 1.464 & 1.468 & 1.507 & 1.504 & 1.482 & 1.503 \\
\hline
\end{tabular}


Table 7.90. Problem 6.2.1 reference solution power peaking factors in element 3 for charge-pan IV (2/2).

\begin{tabular}{|c|c|c|c|c|c|c|c|c|c|}
\hline \multirow[t]{2}{*}{ Step } & \multirow{2}{*}{$\begin{array}{l}\text { Burnup } \\
{\left[\frac{\mathrm{MWd}}{\mathrm{MtU}}\right]}\end{array}$} & \multicolumn{8}{|c|}{ Shift Power Peaking Factors } \\
\hline & & $\mathrm{E}$ & $\mathrm{F}$ & G & $\mathrm{H}$ & $\mathrm{J}$ & K & $\mathrm{L}$ & M \\
\hline 0 & 0.0 & 1.481 & 1.471 & 1.470 & 1.478 & 1.491 & 1.495 & 1.492 & 1.493 \\
\hline 1 & 25.0 & 1.472 & 1.465 & 1.469 & 1.479 & 1.489 & 1.490 & 1.487 & 1.482 \\
\hline 2 & 50.0 & 1.476 & 1.464 & 1.468 & 1.475 & 1.487 & 1.488 & 1.484 & 1.482 \\
\hline 3 & 100.0 & 1.475 & 1.468 & 1.466 & 1.474 & 1.487 & 1.493 & 1.486 & 1.485 \\
\hline 4 & 200.0 & 1.474 & 1.469 & 1.468 & 1.479 & 1.491 & 1.491 & 1.487 & 1.488 \\
\hline 5 & 300.0 & 1.475 & 1.470 & 1.467 & 1.479 & 1.491 & 1.495 & 1.493 & 1.491 \\
\hline 6 & 400.0 & 1.480 & 1.471 & 1.469 & 1.478 & 1.495 & 1.494 & 1.492 & 1.494 \\
\hline 7 & 500.0 & 1.478 & 1.469 & 1.473 & 1.482 & 1.494 & 1.497 & 1.495 & 1.496 \\
\hline 8 & 600.0 & 1.479 & 1.472 & 1.473 & 1.480 & 1.495 & 1.497 & 1.491 & 1.495 \\
\hline 9 & 700.0 & 1.479 & 1.474 & 1.472 & 1.482 & 1.496 & 1.498 & 1.496 & 1.497 \\
\hline 10 & 800.0 & 1.479 & 1.475 & 1.475 & 1.481 & 1.497 & 1.500 & 1.499 & 1.494 \\
\hline 11 & 900.0 & 1.477 & 1.470 & 1.472 & 1.479 & 1.499 & 1.500 & 1.493 & 1.493 \\
\hline 12 & 1000.0 & 1.477 & 1.470 & 1.469 & 1.477 & 1.495 & 1.499 & 1.495 & 1.494 \\
\hline 13 & 1100.0 & 1.482 & 1.474 & 1.475 & 1.482 & 1.503 & 1.502 & 1.495 & 1.495 \\
\hline 14 & 1200.0 & 1.481 & 1.472 & 1.471 & 1.479 & 1.497 & 1.497 & 1.497 & 1.495 \\
\hline 15 & 1300.0 & 1.478 & 1.473 & 1.469 & 1.481 & 1.497 & 1.498 & 1.496 & 1.493 \\
\hline \multirow[t]{2}{*}{ Step } & Burnup & \multicolumn{8}{|c|}{ MPACT Power Peaking Factors } \\
\hline & $\frac{\mathrm{MWd}}{\mathrm{MtU}}$ & $\mathrm{E}$ & $\mathrm{F}$ & G & $\mathrm{H}$ & $\mathrm{J}$ & K & $\mathrm{L}$ & M \\
\hline 0 & 0.0 & 1.511 & 1.510 & 1.498 & 1.490 & 1.490 & 1.498 & 1.510 & 1.511 \\
\hline 1 & 25.0 & 1.489 & 1.488 & 1.475 & 1.468 & 1.468 & 1.476 & 1.487 & 1.489 \\
\hline 2 & 50.0 & 1.493 & 1.491 & 1.479 & 1.472 & 1.472 & 1.479 & 1.492 & 1.493 \\
\hline 3 & 100.0 & 1.500 & 1.499 & 1.487 & 1.479 & 1.480 & 1.487 & 1.500 & 1.500 \\
\hline 4 & 200.0 & 1.512 & 1.510 & 1.498 & 1.491 & 1.491 & 1.498 & 1.511 & 1.512 \\
\hline 5 & 300.0 & 1.518 & 1.517 & 1.505 & 1.498 & 1.498 & 1.505 & 1.518 & 1.518 \\
\hline 6 & 400.0 & 1.521 & 1.520 & 1.508 & 1.501 & 1.501 & 1.508 & 1.520 & 1.522 \\
\hline 7 & 500.0 & 1.523 & 1.521 & 1.509 & 1.501 & 1.502 & 1.509 & 1.521 & 1.523 \\
\hline 8 & 600.0 & 1.523 & 1.521 & 1.509 & 1.502 & 1.502 & 1.509 & 1.522 & 1.523 \\
\hline 9 & 700.0 & 1.521 & 1.520 & 1.508 & 1.500 & 1.500 & 1.508 & 1.520 & 1.522 \\
\hline 10 & 800.0 & 1.518 & 1.518 & 1.505 & 1.498 & 1.498 & 1.505 & 1.518 & 1.519 \\
\hline 11 & 900.0 & 1.515 & 1.515 & 1.503 & 1.495 & 1.495 & 1.503 & 1.515 & 1.516 \\
\hline 12 & 1000.0 & 1.512 & 1.512 & 1.499 & 1.492 & 1.492 & 1.499 & 1.512 & 1.513 \\
\hline 13 & 1100.0 & 1.508 & 1.508 & 1.496 & 1.488 & 1.488 & 1.496 & 1.508 & 1.509 \\
\hline 14 & 1200.0 & 1.504 & 1.504 & 1.492 & 1.484 & 1.484 & 1.492 & 1.504 & 1.505 \\
\hline 15 & 1300.0 & 1.499 & 1.499 & 1.487 & 1.479 & 1.479 & 1.487 & 1.500 & 1.501 \\
\hline
\end{tabular}


Table 7.91. Problem 6.2.1 reference solution power peaking factors in element 4 for charge-pan IV (1/2).

\begin{tabular}{|c|c|c|c|c|c|c|c|c|c|}
\hline \multirow[t]{2}{*}{ Step } & \multirow{2}{*}{$\begin{array}{l}\text { Burnup } \\
{\left[\frac{\mathrm{MWd}}{\mathrm{M} t \mathrm{II}}\right]}\end{array}$} & \multicolumn{8}{|c|}{ Shift Power Peaking Factors } \\
\hline & & A & B & $\mathrm{C}$ & $\mathrm{D}$ & $\mathrm{P}$ & Q & $\mathrm{R}$ & S \\
\hline 0 & 0.0 & 0.761 & 0.780 & 0.795 & 0.782 & 0.956 & 0.889 & 0.954 & 0.977 \\
\hline 1 & 25.0 & 0.737 & 0.755 & 0.769 & 0.754 & 0.925 & 0.858 & 0.928 & 0.943 \\
\hline 2 & 50.0 & 0.737 & 0.755 & 0.769 & 0.755 & 0.923 & 0.861 & 0.927 & 0.942 \\
\hline 3 & 100.0 & 0.744 & 0.763 & 0.775 & 0.763 & 0.934 & 0.867 & 0.935 & 0.952 \\
\hline 4 & 200.0 & 0.756 & 0.776 & 0.789 & 0.772 & 0.946 & 0.880 & 0.949 & 0.966 \\
\hline 5 & 300.0 & 0.765 & 0.784 & 0.800 & 0.785 & 0.961 & 0.892 & 0.962 & 0.978 \\
\hline 6 & 400.0 & 0.771 & 0.789 & 0.804 & 0.789 & 0.967 & 0.899 & 0.968 & 0.986 \\
\hline 7 & 500.0 & 0.773 & 0.793 & 0.807 & 0.795 & 0.973 & 0.903 & 0.974 & 0.988 \\
\hline 8 & 600.0 & 0.774 & 0.795 & 0.808 & 0.796 & 0.973 & 0.905 & 0.975 & 0.989 \\
\hline 9 & 700.0 & 0.777 & 0.798 & 0.811 & 0.794 & 0.974 & 0.905 & 0.977 & 0.991 \\
\hline 10 & 800.0 & 0.772 & 0.791 & 0.807 & 0.794 & 0.970 & 0.899 & 0.974 & 0.989 \\
\hline 11 & 900.0 & 0.772 & 0.793 & 0.805 & 0.790 & 0.966 & 0.899 & 0.973 & 0.985 \\
\hline 12 & 1000.0 & 0.768 & 0.785 & 0.799 & 0.786 & 0.963 & 0.893 & 0.970 & 0.983 \\
\hline 13 & 1100.0 & 0.765 & 0.784 & 0.797 & 0.784 & 0.958 & 0.888 & 0.962 & 0.976 \\
\hline 14 & 1200.0 & 0.758 & 0.780 & 0.791 & 0.780 & 0.953 & 0.886 & 0.961 & 0.973 \\
\hline 15 & 1300.0 & 0.759 & 0.775 & 0.791 & 0.777 & 0.949 & 0.882 & 0.956 & 0.965 \\
\hline \multirow[t]{2}{*}{ Step } & Burnup & \multicolumn{8}{|c|}{ MPACT Power Peaking Factors } \\
\hline & $\frac{\mathrm{MWd}}{\mathrm{MtU}}$ & A & B & $\mathrm{C}$ & $\mathrm{D}$ & $\mathrm{P}$ & Q & $\mathrm{R}$ & $S$ \\
\hline 0 & 0.0 & 0.765 & 0.746 & 0.765 & 0.778 & 0.954 & 0.937 & 0.871 & 0.938 \\
\hline 1 & 25.0 & 0.781 & 0.763 & 0.782 & 0.795 & 0.973 & 0.956 & 0.890 & 0.957 \\
\hline 2 & 50.0 & 0.779 & 0.761 & 0.780 & 0.793 & 0.971 & 0.953 & 0.887 & 0.954 \\
\hline 3 & 100.0 & 0.774 & 0.756 & 0.775 & 0.787 & 0.965 & 0.947 & 0.882 & 0.948 \\
\hline 4 & 200.0 & 0.768 & 0.750 & 0.769 & 0.781 & 0.958 & 0.940 & 0.876 & 0.942 \\
\hline 5 & 300.0 & 0.765 & 0.747 & 0.765 & 0.778 & 0.954 & 0.936 & 0.872 & 0.938 \\
\hline 6 & 400.0 & 0.764 & 0.746 & 0.764 & 0.777 & 0.952 & 0.936 & 0.871 & 0.936 \\
\hline 7 & 500.0 & 0.765 & 0.747 & 0.766 & 0.778 & 0.954 & 0.937 & 0.872 & 0.937 \\
\hline 8 & 600.0 & 0.766 & 0.749 & 0.767 & 0.780 & 0.956 & 0.938 & 0.873 & 0.939 \\
\hline 9 & 700.0 & 0.769 & 0.751 & 0.770 & 0.783 & 0.959 & 0.941 & 0.877 & 0.943 \\
\hline 10 & 800.0 & 0.772 & 0.754 & 0.773 & 0.785 & 0.962 & 0.945 & 0.880 & 0.946 \\
\hline 11 & 900.0 & 0.776 & 0.758 & 0.776 & 0.789 & 0.966 & 0.949 & 0.884 & 0.951 \\
\hline 12 & 1000.0 & 0.780 & 0.762 & 0.780 & 0.793 & 0.970 & 0.953 & 0.888 & 0.955 \\
\hline 13 & 1100.0 & 0.784 & 0.766 & 0.784 & 0.797 & 0.975 & 0.958 & 0.893 & 0.959 \\
\hline 14 & 1200.0 & 0.788 & 0.770 & 0.789 & 0.802 & 0.980 & 0.962 & 0.897 & 0.964 \\
\hline 15 & 1300.0 & 0.793 & 0.774 & 0.793 & 0.806 & 0.985 & 0.968 & 0.902 & 0.969 \\
\hline
\end{tabular}


Table 7.92. Problem 6.2.1 reference solution power peaking factors in element 4 for charge-pan IV (2/2).

\begin{tabular}{|c|c|c|c|c|c|c|c|c|c|}
\hline \multirow[t]{2}{*}{ Step } & \multirow{2}{*}{$\begin{array}{l}\text { Burnup } \\
{\left[\frac{\mathrm{MWd}}{\mathrm{MtU}}\right]}\end{array}$} & \multicolumn{8}{|c|}{ Shift Power Peaking Factors } \\
\hline & & $\mathrm{E}$ & $\mathrm{F}$ & G & $\mathrm{H}$ & $\mathrm{J}$ & K & $\mathrm{L}$ & M \\
\hline 0 & 0.0 & 0.894 & 0.874 & 0.872 & 0.895 & 0.937 & 0.941 & 0.943 & 0.935 \\
\hline 1 & 25.0 & 0.864 & 0.842 & 0.843 & 0.865 & 0.909 & 0.910 & 0.910 & 0.905 \\
\hline 2 & 50.0 & 0.865 & 0.844 & 0.844 & 0.865 & 0.909 & 0.911 & 0.911 & 0.905 \\
\hline 3 & 100.0 & 0.873 & 0.851 & 0.852 & 0.874 & 0.915 & 0.921 & 0.919 & 0.915 \\
\hline 4 & 200.0 & 0.885 & 0.864 & 0.865 & 0.887 & 0.932 & 0.935 & 0.932 & 0.929 \\
\hline 5 & 300.0 & 0.898 & 0.877 & 0.877 & 0.899 & 0.941 & 0.946 & 0.948 & 0.942 \\
\hline 6 & 400.0 & 0.906 & 0.883 & 0.882 & 0.904 & 0.949 & 0.954 & 0.954 & 0.948 \\
\hline 7 & 500.0 & 0.908 & 0.885 & 0.886 & 0.908 & 0.952 & 0.956 & 0.958 & 0.952 \\
\hline 8 & 600.0 & 0.911 & 0.887 & 0.887 & 0.910 & 0.952 & 0.956 & 0.959 & 0.953 \\
\hline 9 & 700.0 & 0.911 & 0.890 & 0.888 & 0.911 & 0.955 & 0.959 & 0.959 & 0.957 \\
\hline 10 & 800.0 & 0.905 & 0.885 & 0.885 & 0.908 & 0.953 & 0.958 & 0.959 & 0.955 \\
\hline 11 & 900.0 & 0.905 & 0.883 & 0.883 & 0.904 & 0.953 & 0.954 & 0.955 & 0.950 \\
\hline 12 & 1000.0 & 0.898 & 0.879 & 0.877 & 0.902 & 0.947 & 0.951 & 0.950 & 0.943 \\
\hline 13 & 1100.0 & 0.894 & 0.874 & 0.872 & 0.897 & 0.943 & 0.945 & 0.947 & 0.942 \\
\hline 14 & 1200.0 & 0.891 & 0.871 & 0.871 & 0.891 & 0.940 & 0.941 & 0.942 & 0.934 \\
\hline 15 & 1300.0 & 0.888 & 0.866 & 0.866 & 0.888 & 0.935 & 0.937 & 0.938 & 0.930 \\
\hline \multirow[t]{2}{*}{ Step } & Burnup & \multicolumn{8}{|c|}{ MPACT Power Peaking Factors } \\
\hline & $\frac{\mathrm{MWd}}{\mathrm{MtU}}$ & $\mathrm{E}$ & $\mathrm{F}$ & G & $\mathrm{H}$ & $\mathrm{J}$ & K & $\mathrm{L}$ & M \\
\hline 0 & 0.0 & 0.921 & 0.916 & 0.875 & 0.854 & 0.854 & 0.876 & 0.917 & 0.922 \\
\hline 1 & 25.0 & 0.941 & 0.936 & 0.894 & 0.873 & 0.874 & 0.895 & 0.937 & 0.942 \\
\hline 2 & 50.0 & 0.938 & 0.933 & 0.892 & 0.871 & 0.872 & 0.893 & 0.934 & 0.939 \\
\hline 3 & 100.0 & 0.932 & 0.927 & 0.886 & 0.865 & 0.866 & 0.887 & 0.929 & 0.933 \\
\hline 4 & 200.0 & 0.926 & 0.920 & 0.880 & 0.859 & 0.859 & 0.880 & 0.921 & 0.926 \\
\hline 5 & 300.0 & 0.922 & 0.916 & 0.876 & 0.856 & 0.856 & 0.876 & 0.918 & 0.922 \\
\hline 6 & 400.0 & 0.921 & 0.915 & 0.875 & 0.855 & 0.855 & 0.876 & 0.917 & 0.921 \\
\hline 7 & 500.0 & 0.922 & 0.917 & 0.876 & 0.855 & 0.856 & 0.877 & 0.917 & 0.923 \\
\hline 8 & 600.0 & 0.924 & 0.918 & 0.878 & 0.857 & 0.857 & 0.878 & 0.920 & 0.924 \\
\hline 9 & 700.0 & 0.927 & 0.921 & 0.881 & 0.860 & 0.861 & 0.881 & 0.923 & 0.927 \\
\hline 10 & 800.0 & 0.930 & 0.925 & 0.884 & 0.863 & 0.864 & 0.885 & 0.926 & 0.930 \\
\hline 11 & 900.0 & 0.934 & 0.929 & 0.888 & 0.868 & 0.868 & 0.889 & 0.930 & 0.935 \\
\hline 12 & 1000.0 & 0.938 & 0.933 & 0.892 & 0.871 & 0.872 & 0.893 & 0.935 & 0.939 \\
\hline 13 & 1100.0 & 0.943 & 0.938 & 0.897 & 0.876 & 0.877 & 0.897 & 0.939 & 0.943 \\
\hline 14 & 1200.0 & 0.947 & 0.942 & 0.902 & 0.880 & 0.881 & 0.902 & 0.944 & 0.948 \\
\hline 15 & 1300.0 & 0.952 & 0.947 & 0.907 & 0.885 & 0.885 & 0.907 & 0.949 & 0.953 \\
\hline
\end{tabular}


Table 7.93. Problem 6.2.1 reference solution power peaking factors in element 5 for charge-pan IV (1/2).

\begin{tabular}{|c|c|c|c|c|c|c|c|c|c|}
\hline \multirow[t]{2}{*}{ Step } & \multirow{2}{*}{$\begin{array}{l}\text { Burnup } \\
{\left[\frac{\mathrm{MWd}}{\mathrm{M} t \mathrm{II}}\right]}\end{array}$} & \multicolumn{8}{|c|}{ Shift Power Peaking Factors } \\
\hline & & A & B & $\mathrm{C}$ & $\mathrm{D}$ & $\mathrm{P}$ & Q & $\mathrm{R}$ & S \\
\hline 0 & 0.0 & 0.400 & 0.411 & 0.423 & 0.415 & 0.522 & 0.469 & 0.517 & 0.531 \\
\hline 1 & 25.0 & 0.376 & 0.391 & 0.399 & 0.392 & 0.492 & 0.441 & 0.491 & 0.502 \\
\hline 2 & 50.0 & 0.379 & 0.391 & 0.403 & 0.391 & 0.491 & 0.443 & 0.492 & 0.504 \\
\hline 3 & 100.0 & 0.383 & 0.396 & 0.407 & 0.397 & 0.500 & 0.449 & 0.498 & 0.511 \\
\hline 4 & 200.0 & 0.393 & 0.406 & 0.418 & 0.409 & 0.512 & 0.461 & 0.511 & 0.524 \\
\hline 5 & 300.0 & 0.402 & 0.414 & 0.426 & 0.416 & 0.523 & 0.469 & 0.519 & 0.535 \\
\hline 6 & 400.0 & 0.407 & 0.421 & 0.432 & 0.421 & 0.530 & 0.477 & 0.527 & 0.540 \\
\hline 7 & 500.0 & 0.410 & 0.423 & 0.435 & 0.424 & 0.535 & 0.481 & 0.531 & 0.545 \\
\hline 8 & 600.0 & 0.412 & 0.423 & 0.435 & 0.427 & 0.535 & 0.482 & 0.530 & 0.546 \\
\hline 9 & 700.0 & 0.413 & 0.427 & 0.437 & 0.430 & 0.536 & 0.485 & 0.534 & 0.548 \\
\hline 10 & 800.0 & 0.410 & 0.424 & 0.434 & 0.425 & 0.531 & 0.482 & 0.531 & 0.543 \\
\hline 11 & 900.0 & 0.411 & 0.424 & 0.434 & 0.424 & 0.532 & 0.480 & 0.534 & 0.545 \\
\hline 12 & 1000.0 & 0.408 & 0.422 & 0.431 & 0.423 & 0.530 & 0.480 & 0.530 & 0.541 \\
\hline 13 & 1100.0 & 0.405 & 0.417 & 0.428 & 0.418 & 0.524 & 0.474 & 0.523 & 0.535 \\
\hline 14 & 1200.0 & 0.403 & 0.414 & 0.425 & 0.417 & 0.522 & 0.471 & 0.523 & 0.535 \\
\hline 15 & 1300.0 & 0.401 & 0.414 & 0.423 & 0.415 & 0.519 & 0.470 & 0.520 & 0.530 \\
\hline \multirow[t]{2}{*}{ Step } & Burnup & \multicolumn{8}{|c|}{ MPACT Power Peaking Factors } \\
\hline & $\frac{\mathrm{MWd}}{\mathrm{MtU}}$ & A & B & $\mathrm{C}$ & $\mathrm{D}$ & $\mathrm{P}$ & Q & $\mathrm{R}$ & S \\
\hline 0 & 0.0 & 0.388 & 0.376 & 0.390 & 0.398 & 0.500 & 0.486 & 0.441 & 0.491 \\
\hline 1 & 25.0 & 0.419 & 0.406 & 0.422 & 0.430 & 0.540 & 0.524 & 0.476 & 0.530 \\
\hline 2 & 50.0 & 0.416 & 0.403 & 0.418 & 0.427 & 0.535 & 0.519 & 0.473 & 0.525 \\
\hline 3 & 100.0 & 0.406 & 0.394 & 0.408 & 0.417 & 0.523 & 0.508 & 0.462 & 0.513 \\
\hline 4 & 200.0 & 0.393 & 0.381 & 0.395 & 0.404 & 0.507 & 0.492 & 0.447 & 0.497 \\
\hline 5 & 300.0 & 0.385 & 0.373 & 0.387 & 0.396 & 0.497 & 0.482 & 0.438 & 0.487 \\
\hline 6 & 400.0 & 0.382 & 0.370 & 0.384 & 0.392 & 0.492 & 0.478 & 0.434 & 0.483 \\
\hline 7 & 500.0 & 0.381 & 0.369 & 0.383 & 0.391 & 0.491 & 0.477 & 0.433 & 0.481 \\
\hline 8 & 600.0 & 0.381 & 0.369 & 0.383 & 0.391 & 0.492 & 0.478 & 0.433 & 0.482 \\
\hline 9 & 700.0 & 0.383 & 0.371 & 0.385 & 0.393 & 0.495 & 0.480 & 0.436 & 0.485 \\
\hline 10 & 800.0 & 0.386 & 0.374 & 0.388 & 0.396 & 0.498 & 0.484 & 0.439 & 0.488 \\
\hline 11 & 900.0 & 0.390 & 0.378 & 0.392 & 0.400 & 0.503 & 0.489 & 0.443 & 0.493 \\
\hline 12 & 1000.0 & 0.394 & 0.382 & 0.396 & 0.404 & 0.508 & 0.494 & 0.448 & 0.499 \\
\hline 13 & 1100.0 & 0.399 & 0.387 & 0.401 & 0.410 & 0.515 & 0.500 & 0.454 & 0.505 \\
\hline 14 & 1200.0 & 0.404 & 0.392 & 0.407 & 0.415 & 0.521 & 0.506 & 0.460 & 0.511 \\
\hline 15 & 1300.0 & 0.410 & 0.398 & 0.412 & 0.421 & 0.529 & 0.514 & 0.467 & 0.519 \\
\hline
\end{tabular}


Table 7.94. Problem 6.2.1 reference solution power peaking factors in element 5 for charge-pan IV (2/2).

\begin{tabular}{|c|c|c|c|c|c|c|c|c|c|}
\hline \multirow[t]{2}{*}{ Step } & \multirow{2}{*}{$\begin{array}{l}\text { Burnup } \\
{\left[\frac{\mathrm{MWd}}{\mathrm{MtU}}\right]}\end{array}$} & \multicolumn{8}{|c|}{ Shift Power Peaking Factors } \\
\hline & & $\mathrm{E}$ & $\mathrm{F}$ & G & $\mathrm{H}$ & $\mathrm{J}$ & K & $\mathrm{L}$ & M \\
\hline 0 & 0.0 & 0.479 & 0.461 & 0.459 & 0.474 & 0.504 & 0.510 & 0.512 & 0.509 \\
\hline 1 & 25.0 & 0.451 & 0.435 & 0.435 & 0.449 & 0.479 & 0.484 & 0.484 & 0.480 \\
\hline 2 & 50.0 & 0.451 & 0.435 & 0.435 & 0.450 & 0.481 & 0.484 & 0.485 & 0.482 \\
\hline 3 & 100.0 & 0.460 & 0.442 & 0.441 & 0.456 & 0.487 & 0.490 & 0.490 & 0.488 \\
\hline 4 & 200.0 & 0.469 & 0.453 & 0.452 & 0.467 & 0.500 & 0.503 & 0.506 & 0.501 \\
\hline 5 & 300.0 & 0.481 & 0.463 & 0.461 & 0.478 & 0.509 & 0.512 & 0.514 & 0.512 \\
\hline 6 & 400.0 & 0.486 & 0.471 & 0.467 & 0.483 & 0.516 & 0.520 & 0.522 & 0.518 \\
\hline 7 & 500.0 & 0.491 & 0.472 & 0.472 & 0.488 & 0.517 & 0.521 & 0.526 & 0.523 \\
\hline 8 & 600.0 & 0.493 & 0.475 & 0.471 & 0.488 & 0.520 & 0.524 & 0.526 & 0.522 \\
\hline 9 & 700.0 & 0.494 & 0.478 & 0.476 & 0.490 & 0.522 & 0.526 & 0.528 & 0.523 \\
\hline 10 & 800.0 & 0.489 & 0.472 & 0.473 & 0.485 & 0.519 & 0.521 & 0.525 & 0.522 \\
\hline 11 & 900.0 & 0.492 & 0.472 & 0.473 & 0.488 & 0.522 & 0.522 & 0.523 & 0.522 \\
\hline 12 & 1000.0 & 0.488 & 0.472 & 0.469 & 0.483 & 0.515 & 0.520 & 0.521 & 0.518 \\
\hline 13 & 1100.0 & 0.482 & 0.467 & 0.464 & 0.480 & 0.512 & 0.515 & 0.517 & 0.513 \\
\hline 14 & 1200.0 & 0.480 & 0.466 & 0.463 & 0.478 & 0.509 & 0.513 & 0.514 & 0.511 \\
\hline 15 & 1300.0 & 0.477 & 0.463 & 0.460 & 0.474 & 0.507 & 0.511 & 0.512 & 0.509 \\
\hline \multirow[t]{2}{*}{ Step } & Burnup & \multicolumn{8}{|c|}{ MPACT Power Peaking Factors } \\
\hline & $\frac{\mathrm{MWd}}{\mathrm{MtU}}$ & $\mathrm{E}$ & $\mathrm{F}$ & G & $\mathrm{H}$ & $\mathrm{J}$ & K & $\mathrm{L}$ & M \\
\hline 0 & 0.0 & 0.480 & 0.475 & 0.446 & 0.432 & 0.433 & 0.449 & 0.478 & 0.481 \\
\hline 1 & 25.0 & 0.518 & 0.513 & 0.482 & 0.467 & 0.469 & 0.486 & 0.518 & 0.520 \\
\hline 2 & 50.0 & 0.513 & 0.508 & 0.478 & 0.463 & 0.465 & 0.482 & 0.513 & 0.515 \\
\hline 3 & 100.0 & 0.502 & 0.497 & 0.467 & 0.453 & 0.454 & 0.471 & 0.501 & 0.504 \\
\hline 4 & 200.0 & 0.486 & 0.482 & 0.453 & 0.439 & 0.440 & 0.457 & 0.486 & 0.488 \\
\hline 5 & 300.0 & 0.477 & 0.472 & 0.444 & 0.430 & 0.431 & 0.447 & 0.476 & 0.478 \\
\hline 6 & 400.0 & 0.473 & 0.468 & 0.440 & 0.426 & 0.427 & 0.443 & 0.471 & 0.474 \\
\hline 7 & 500.0 & 0.471 & 0.467 & 0.438 & 0.425 & 0.426 & 0.442 & 0.470 & 0.472 \\
\hline 8 & 600.0 & 0.472 & 0.467 & 0.439 & 0.425 & 0.427 & 0.442 & 0.471 & 0.473 \\
\hline 9 & 700.0 & 0.475 & 0.470 & 0.441 & 0.428 & 0.429 & 0.444 & 0.473 & 0.476 \\
\hline 10 & 800.0 & 0.478 & 0.473 & 0.445 & 0.431 & 0.432 & 0.448 & 0.477 & 0.479 \\
\hline 11 & 900.0 & 0.483 & 0.478 & 0.449 & 0.435 & 0.436 & 0.452 & 0.482 & 0.484 \\
\hline 12 & 1000.0 & 0.488 & 0.483 & 0.454 & 0.440 & 0.441 & 0.457 & 0.487 & 0.489 \\
\hline 13 & 1100.0 & 0.494 & 0.489 & 0.460 & 0.445 & 0.447 & 0.463 & 0.493 & 0.495 \\
\hline 14 & 1200.0 & 0.501 & 0.495 & 0.465 & 0.451 & 0.453 & 0.469 & 0.499 & 0.502 \\
\hline 15 & 1300.0 & 0.508 & 0.502 & 0.473 & 0.458 & 0.459 & 0.476 & 0.506 & 0.509 \\
\hline
\end{tabular}


Table 7.95. Problem 6.2.1 reference solution power peaking factors in element 6 for charge-pan IV (1/2).

\begin{tabular}{|c|c|c|c|c|c|c|c|c|c|}
\hline \multirow[t]{2}{*}{ Step } & \multirow{2}{*}{$\begin{array}{l}\text { Burnup } \\
{\left[\frac{\mathrm{MWd}}{\mathrm{MtU}}\right]}\end{array}$} & \multicolumn{8}{|c|}{ Shift Power Peaking Factors } \\
\hline & & A & B & $\mathrm{C}$ & $\mathrm{D}$ & $\mathrm{P}$ & Q & $\mathrm{R}$ & $\mathrm{S}$ \\
\hline 0 & 0.0 & 0.171 & 0.179 & 0.185 & 0.179 & 0.228 & 0.202 & 0.246 & 0.248 \\
\hline 1 & 25.0 & 0.162 & 0.168 & 0.174 & 0.169 & 0.214 & 0.190 & 0.232 & 0.234 \\
\hline 2 & 50.0 & 0.162 & 0.169 & 0.175 & 0.170 & 0.214 & 0.190 & 0.232 & 0.234 \\
\hline 3 & 100.0 & 0.163 & 0.170 & 0.176 & 0.171 & 0.216 & 0.191 & 0.235 & 0.237 \\
\hline 4 & 200.0 & 0.167 & 0.175 & 0.181 & 0.175 & 0.222 & 0.197 & 0.240 & 0.242 \\
\hline 5 & 300.0 & 0.170 & 0.179 & 0.185 & 0.179 & 0.226 & 0.201 & 0.246 & 0.248 \\
\hline 6 & 400.0 & 0.174 & 0.182 & 0.188 & 0.182 & 0.229 & 0.205 & 0.251 & 0.252 \\
\hline 7 & 500.0 & 0.176 & 0.183 & 0.189 & 0.183 & 0.232 & 0.206 & 0.253 & 0.254 \\
\hline 8 & 600.0 & 0.177 & 0.185 & 0.191 & 0.185 & 0.233 & 0.208 & 0.253 & 0.255 \\
\hline 9 & 700.0 & 0.178 & 0.186 & 0.192 & 0.185 & 0.235 & 0.210 & 0.255 & 0.257 \\
\hline 10 & 800.0 & 0.176 & 0.185 & 0.190 & 0.185 & 0.233 & 0.207 & 0.253 & 0.257 \\
\hline 11 & 900.0 & 0.178 & 0.186 & 0.192 & 0.186 & 0.234 & 0.208 & 0.255 & 0.256 \\
\hline 12 & 1000.0 & 0.177 & 0.185 & 0.191 & 0.185 & 0.233 & 0.208 & 0.253 & 0.256 \\
\hline 13 & 1100.0 & 0.175 & 0.183 & 0.190 & 0.183 & 0.231 & 0.206 & 0.251 & 0.254 \\
\hline 14 & 1200.0 & 0.176 & 0.183 & 0.189 & 0.183 & 0.231 & 0.205 & 0.251 & 0.252 \\
\hline 15 & 1300.0 & 0.176 & 0.183 & 0.188 & 0.183 & 0.229 & 0.206 & 0.250 & 0.252 \\
\hline \multirow[t]{2}{*}{ Step } & Burnup & \multicolumn{8}{|c|}{ MPACT Power Peaking Factors } \\
\hline & $\frac{\mathrm{MWd}}{\mathrm{MtU}}$ & A & B & $\mathrm{C}$ & $\mathrm{D}$ & $\mathrm{P}$ & Q & $\mathrm{R}$ & $S$ \\
\hline 0 & 0.0 & 0.162 & 0.155 & 0.162 & 0.168 & 0.225 & 0.223 & 0.182 & 0.205 \\
\hline 1 & 25.0 & 0.184 & 0.176 & 0.184 & 0.190 & 0.254 & 0.251 & 0.206 & 0.233 \\
\hline 2 & 50.0 & 0.182 & 0.174 & 0.182 & 0.188 & 0.251 & 0.248 & 0.204 & 0.230 \\
\hline 3 & 100.0 & 0.175 & 0.168 & 0.175 & 0.181 & 0.242 & 0.240 & 0.197 & 0.223 \\
\hline 4 & 200.0 & 0.167 & 0.159 & 0.166 & 0.172 & 0.230 & 0.228 & 0.187 & 0.211 \\
\hline 5 & 300.0 & 0.161 & 0.153 & 0.160 & 0.166 & 0.222 & 0.220 & 0.180 & 0.203 \\
\hline 6 & 400.0 & 0.158 & 0.151 & 0.157 & 0.163 & 0.218 & 0.217 & 0.177 & 0.200 \\
\hline 7 & 500.0 & 0.156 & 0.149 & 0.156 & 0.161 & 0.216 & 0.215 & 0.175 & 0.198 \\
\hline 8 & 600.0 & 0.156 & 0.149 & 0.155 & 0.161 & 0.216 & 0.214 & 0.175 & 0.197 \\
\hline 9 & 700.0 & 0.157 & 0.149 & 0.156 & 0.162 & 0.217 & 0.215 & 0.175 & 0.198 \\
\hline 10 & 800.0 & 0.158 & 0.150 & 0.157 & 0.163 & 0.218 & 0.217 & 0.176 & 0.199 \\
\hline 11 & 900.0 & 0.159 & 0.152 & 0.159 & 0.165 & 0.221 & 0.219 & 0.179 & 0.202 \\
\hline 12 & 1000.0 & 0.162 & 0.154 & 0.161 & 0.167 & 0.224 & 0.222 & 0.181 & 0.204 \\
\hline 13 & 1100.0 & 0.164 & 0.157 & 0.164 & 0.169 & 0.227 & 0.225 & 0.184 & 0.208 \\
\hline 14 & 1200.0 & 0.167 & 0.159 & 0.166 & 0.172 & 0.231 & 0.229 & 0.187 & 0.211 \\
\hline 15 & 1300.0 & 0.170 & 0.162 & 0.170 & 0.175 & 0.235 & 0.233 & 0.190 & 0.215 \\
\hline
\end{tabular}


Table 7.96. Problem 6.2.1 reference solution power peaking factors in element 6 for charge-pan IV (2/2).

\begin{tabular}{|c|c|c|c|c|c|c|c|c|c|}
\hline \multirow[t]{2}{*}{ Step } & \multirow{2}{*}{$\begin{array}{l}\text { Burnup } \\
{\left[\frac{\mathrm{MWd}}{\mathrm{M} t \mathrm{II}}\right]}\end{array}$} & \multicolumn{8}{|c|}{ Shift Power Peaking Factors } \\
\hline & & $\mathrm{E}$ & $\mathrm{F}$ & G & $\mathrm{H}$ & $\mathrm{J}$ & K & $\mathrm{L}$ & M \\
\hline 0 & 0.0 & 0.207 & 0.198 & 0.197 & 0.206 & 0.242 & 0.242 & 0.224 & 0.222 \\
\hline 1 & 25.0 & 0.194 & 0.187 & 0.186 & 0.193 & 0.227 & 0.230 & 0.211 & 0.208 \\
\hline 2 & 50.0 & 0.195 & 0.188 & 0.186 & 0.194 & 0.228 & 0.230 & 0.211 & 0.208 \\
\hline 3 & 100.0 & 0.197 & 0.190 & 0.186 & 0.196 & 0.229 & 0.233 & 0.213 & 0.210 \\
\hline 4 & 200.0 & 0.203 & 0.195 & 0.193 & 0.201 & 0.236 & 0.238 & 0.219 & 0.218 \\
\hline 5 & 300.0 & 0.207 & 0.199 & 0.195 & 0.206 & 0.242 & 0.244 & 0.222 & 0.221 \\
\hline 6 & 400.0 & 0.211 & 0.202 & 0.200 & 0.209 & 0.245 & 0.249 & 0.227 & 0.224 \\
\hline 7 & 500.0 & 0.212 & 0.203 & 0.201 & 0.212 & 0.248 & 0.250 & 0.227 & 0.225 \\
\hline 8 & 600.0 & 0.214 & 0.205 & 0.203 & 0.212 & 0.251 & 0.252 & 0.229 & 0.227 \\
\hline 9 & 700.0 & 0.216 & 0.207 & 0.204 & 0.213 & 0.251 & 0.253 & 0.231 & 0.228 \\
\hline 10 & 800.0 & 0.214 & 0.205 & 0.202 & 0.211 & 0.249 & 0.251 & 0.230 & 0.227 \\
\hline 11 & 900.0 & 0.215 & 0.204 & 0.203 & 0.212 & 0.250 & 0.253 & 0.231 & 0.228 \\
\hline 12 & 1000.0 & 0.214 & 0.205 & 0.203 & 0.211 & 0.250 & 0.252 & 0.230 & 0.227 \\
\hline 13 & 1100.0 & 0.213 & 0.204 & 0.201 & 0.208 & 0.247 & 0.248 & 0.229 & 0.225 \\
\hline 14 & 1200.0 & 0.211 & 0.204 & 0.201 & 0.210 & 0.247 & 0.249 & 0.227 & 0.225 \\
\hline 15 & 1300.0 & 0.211 & 0.203 & 0.201 & 0.208 & 0.246 & 0.247 & 0.226 & 0.223 \\
\hline \multirow[t]{2}{*}{ Step } & Burnup & \multicolumn{8}{|c|}{ MPACT Power Peaking Factors } \\
\hline & $\frac{\mathrm{MWd}}{\mathrm{MtU}}$ & $\mathrm{E}$ & $\mathrm{F}$ & G & $\mathrm{H}$ & $\mathrm{J}$ & K & $\mathrm{L}$ & M \\
\hline 0 & 0.0 & 0.221 & 0.219 & 0.186 & 0.178 & 0.179 & 0.187 & 0.200 & 0.202 \\
\hline 1 & 25.0 & 0.249 & 0.247 & 0.210 & 0.201 & 0.204 & 0.213 & 0.227 & 0.229 \\
\hline 2 & 50.0 & 0.246 & 0.244 & 0.208 & 0.199 & 0.201 & 0.210 & 0.224 & 0.227 \\
\hline 3 & 100.0 & 0.238 & 0.236 & 0.201 & 0.192 & 0.194 & 0.203 & 0.216 & 0.219 \\
\hline 4 & 200.0 & 0.226 & 0.224 & 0.191 & 0.183 & 0.184 & 0.192 & 0.206 & 0.208 \\
\hline 5 & 300.0 & 0.218 & 0.217 & 0.184 & 0.176 & 0.178 & 0.185 & 0.198 & 0.200 \\
\hline 6 & 400.0 & 0.215 & 0.213 & 0.181 & 0.173 & 0.174 & 0.182 & 0.194 & 0.197 \\
\hline 7 & 500.0 & 0.213 & 0.211 & 0.179 & 0.171 & 0.172 & 0.180 & 0.192 & 0.195 \\
\hline 8 & 600.0 & 0.212 & 0.211 & 0.179 & 0.171 & 0.172 & 0.180 & 0.192 & 0.194 \\
\hline 9 & 700.0 & 0.213 & 0.212 & 0.179 & 0.171 & 0.173 & 0.180 & 0.193 & 0.195 \\
\hline 10 & 800.0 & 0.215 & 0.213 & 0.180 & 0.173 & 0.174 & 0.182 & 0.194 & 0.197 \\
\hline 11 & 900.0 & 0.217 & 0.215 & 0.183 & 0.175 & 0.176 & 0.184 & 0.196 & 0.199 \\
\hline 12 & 1000.0 & 0.220 & 0.218 & 0.185 & 0.177 & 0.178 & 0.186 & 0.199 & 0.201 \\
\hline 13 & 1100.0 & 0.223 & 0.221 & 0.188 & 0.180 & 0.181 & 0.189 & 0.202 & 0.205 \\
\hline 14 & 1200.0 & 0.227 & 0.225 & 0.191 & 0.183 & 0.184 & 0.192 & 0.206 & 0.208 \\
\hline 15 & 1300.0 & 0.231 & 0.229 & 0.195 & 0.186 & 0.188 & 0.196 & 0.210 & 0.212 \\
\hline
\end{tabular}




\section{CONCLUSIONS}

This report documents progression problems for a graphite-moderated, gas-cooled reactor for two state-of-the-art neutronics codes. The configuration of all problems were modeled and simulated successfully in both the Shift Monte Carlo and MPACT codes. The parameters calculated are listed in detail and include: eigenvalue, peaking factors, and isotope concentrations. The values for these can be compared with those calculated by other neutron transport codes or other Magnox-style reactors. Despite being originally developed for LWR calculations, MPACT is suitable for modeling graphite-moderated reactors, especially with the newly developed 69-group library. Shift, a Monte Carlo code, can already model general geometries. With MPACT, pin-resolved neutron transport equations can be solved and sub-pin isotopics can be resolved.

The volume of data generated across all simulations is large. Not all information can be compared in this report. Tables 8.1 and 8.2 summarize the eigenvalue for the BOC and depletion simulations, respectively. The BOC values all compared well. For the depletion simulations, large deviations existed for $<$ $0.25 \mathrm{GWd} / \mathrm{t}$, but the differences tended to lessen with increasing burnup. It is speculated that at these low burnups, which correspond to weeks in operating time, that small differences in the solution result in relatively large differences in fission product poisons compared to later in the cycle.

Both codes allowed for flexible calculation methods and standard output in the form of HDF5 files, which facilitated comparison between the codes. Shift showed consistent performance that held constant with depletion steps, which is typical for Monte Carlo codes. Because the convergence speed of the MPACT solution relies more on the previous time step's source distribution, each depletion step past the zeroth takes between $30 \%$ and $60 \%$ less time depending on the geometry and solution convergence parameters. However, the MPACT timing was not a cure-all as finer convergence parameters sometimes resulted in the solution taking longer Shift (as in problem 5.1.1) or not finishing due to convergence issues. For the 3D cases, challenges in the convergence of the axial solution was experienced because of the lack of fuel in the end cap layers;

This report documents the success of tools originally developed for LWRs to model Magnox reactors. Shift and MPACT (neutronic solvers in the state-of-the-art SCALE and VERA codes, respectively) demonstrate good agreement in eigenvalue and power peaking factors. Tables 8.1 and 8.2 show that the BOC differences in eigenvalue range from 10 to $200 \mathrm{pcm}$; the root mean square (RMS) for the EOC differences range from around 100 to $300 \mathrm{pcm}$. 
Table 8.1. Summary of BOC cases of progression problems and eigenvalue comparisons.

\begin{tabular}{|c|c|c|c|c|c|}
\hline Geometry & $\begin{array}{c}\text { Progression } \\
\text { Problem } \\
\text { Number }\end{array}$ & $\begin{array}{c}\text { Temperature Set } \\
\text { Library (Tab. 2.1) } \\
\text { Operating (Tab. 2.4) }\end{array}$ & $\begin{array}{c}\text { Coolant } \\
\text { Channel } \\
\text { Radius }\end{array}$ & $\begin{array}{c}\text { Control } \\
\text { Rods }\end{array}$ & $\begin{array}{c}\text { MPACT - Shift } \\
\text { RMS (pcm) }\end{array}$ \\
\hline \multirow{3}{*}{ 2D Pin Cell } & 1.1 .1 & Library & ABC & - & 33 \\
\cline { 2 - 6 } & 1.1 .2 & Operating & ABC & - & 22 \\
\cline { 2 - 6 } 2D Charge Pan & 2.1 .1 & Operating & C & None & 28 \\
\cline { 2 - 6 } & 2.1 .2 & Operating & A & None & 58 \\
\hline \multirow{3}{*}{ 2D Quarter Core } & 3.1 .3 & Operating & A & Yes & 20 \\
\cline { 2 - 6 } & 3.1 .1 & Operating & - & None & 7 \\
\hline 3D Channel & 4.1 .1 & Operating & - & ARI & -61 \\
\hline \multirow{3}{*}{ 3D Charge Pan } & 5.1 .1 & Operating & B & - & -98 \\
\cline { 2 - 6 } & 5.1 .2 & Operating & C & None & -148 \\
\hline 3D Mini Core & 6.1 .1 & Operating & A & $50 \%$ & -214 \\
\hline
\end{tabular}

Table 8.2. Summary of depletion cases of progression problems and eigenvalue comparisons with root mean square (RMS) deviations.

\begin{tabular}{|c|c|c|c|c|c|}
\hline Geometry & $\begin{array}{c}\text { Progression } \\
\text { Problem } \\
\text { Number }\end{array}$ & $\begin{array}{c}\text { Temperature Set } \\
\text { Library (Tab. 2.1) } \\
\text { Operating (Tab. 2.4) }\end{array}$ & $\begin{array}{c}\text { Coolant } \\
\text { Channel } \\
\text { Radius }\end{array}$ & $\begin{array}{c}\text { Control } \\
\text { Rods }\end{array}$ & $\begin{array}{c}\text { MPACT - Shift } \\
\text { RMS (pcm) }\end{array}$ \\
\hline 2D Pin Cell & 1.2 .1 & Operating & B & - & 92 \\
\hline 2D Charge Pan & 2.2 .1 & Operating & A & None & 114 \\
\hline 2D Quarter Core & 3.2 .1 & Operating & - & Rods Out & 197 \\
& 3.2 .2 & Operating & - & Main Bank & 135 \\
\hline 3D Channel & 4.2 .1 & Operating & B & - & 118 \\
\hline 3D Charge Pan & 5.2 .1 & Operating & C & $50 \%$ & 154 \\
\hline 3D Mini Core & 6.2 .1 & Operating & - & $50 \%$ & 290 \\
\hline
\end{tabular}




\section{FUTURE WORK}

There are two main components of future work that have been identified.

First, one of the significant improved capabilities with MPACT is the improved capability to resolve the neutron transport equation and isotope concentrations at the sub-pin level. Further examination is still required to understand the accuracy and distributions of isotope concentrations within different radial regions of the pin. While some of the major actinides $(\mathrm{U}, \mathrm{Pu}, \mathrm{Cm}, \mathrm{Am})$ and major fission products $(\mathrm{Cs}, \mathrm{Nd}$, Sr-90) agreed well between Shift and MPACT, several isotopes did not agree well. These isotopes typically have concentrations $<10^{-9}$ atoms/barn-cm and include ${ }^{84,86,87} \mathrm{Sr}$, ${ }^{132,134,135} \mathrm{Ba}$, and ${ }^{85} \mathrm{Rb}$. Of those, the only two isotopes with non-negligible cumulative fission yields are ${ }^{135} \mathrm{Ba}$ and ${ }^{85} \mathrm{Rb}$. Further examination is needed on how MPACT handles these isotopes in depletion should they need to be considered in comparison to measured data.

Second, MPACT showed a higher discrepancy with 3D problems, which could be due to issues with the axial solver in the non-fueled regions. While improvements have been made in MPACT to deal with axial reflectors, i.e., bookending of fuel regions, non-fuel regions in between fuel elements were found to stress axial methods due to leakage from the fuel regions. Convergence criteria needed loosening to reach a solution. The capability to improve the solution for non-fueled regions would be desired for faster calculations.

Third, future work involves full-core, coupled thermal hydraulic calculations with sub-pin isotopics as previously mentioned in [1]. Calculating sub-pin isotopics in small thickness fueled regions requires fine ray spacing, e.g. on the order of the smallest fuel region thickness. This report shows that small ray spacing may slow down performance of MPACT to be comparable of that to Shift or other MC codes. Follow-on calculations will need to account for this 



\section{REFERENCES}

\section{References}

[1] Brian J. Ade, Nicholas P. Luciano, Cole Gentry, Shane Simpson, Ben Collins, and Robert Mills. Development of mpact for full-core simulations of MAGNOX gas-cooled nuclear reactors. In Proceedings of Physics of Reactors (PHYSOR) 2020: Transition to a Scalable Nuclear Future, University of Cambridge, United Kingdom, March 2020.

[2] Benjamin Collins, Shane Stimpson, Blake W. Kelley, Mitchell T. H. Young, Brendan Kochunas, Aaron Graham, Edward W. Larsen, Thomas Downar, and Andrew Godfrey. Stability and accuracy of 3D neutron transport simulations using the 2D/1D method in MPACT. Journal of Computational Physics, 326:612-628, 2016.

[3] Thomas M. Evans, Alissa S. Stafford, Rachel N. Slaybaugh, and Kevin T. Clarno. Denovo: A new three-dimensional parallel discrete ordinates code in SCALE. Nuclear Technology, 171(2):171-200, 2010 .

[4] I. C. Gauld, G. Radulescu, G. Ilas, B. D. Murphy, M. L. Williams, and D. Wiarda. Isotopic depletion and decay methods and analysis capabilities in SCALE. Nuclear Technology, 174(2):169-195, 2011. cited By 132.

[5] Andrew T. Godfrey. VERA core physics benchmark progression problem specifications. Technical Report CASL-U-2012-0131-004, Oak Ridge National Laboratory, Oak Ridge, Tennessee, August 2014.

[6] International Atomic Energy Agency. Thermophysical Properties of Materials for Nuclear Engineering: A Tutorial and Collection of Data. International Atomic Energy Agency, Vienna, 2008.

[7] S. E. Jensen and E. Nonbøl. Description of the Magnox Type of Gas-Cooled Reactor (MAGNOX). Technical Report NKS/RAK-2(97)TR-C5, Nordic Nuclear Safety Research (NKS), Risø National Laboratory, Roskilde, Denmark, November 1998.

[8] S. Johnson, T. Evans, G. Davidson, S. Hamilton, T. Pandya, K. Royston, and E. Biondo. Omnibus Users Manual. Technical Report ORNL/TM-2018/1073, Oak Ridge National Laboratory, August 2020 .

[9] Kang Seog Kim, Brian J. Ade, and Nicholas P. Luciano. Development of the MPACT 69-group library for the Magnox reactor analysis using CASL VERA. In Proceedings of Physics of Reactors (PHYSOR) 2020: Transition to a Scalable Nuclear Future, University of Cambridge, United Kingdom, March 2020.

[10] Brendan Kochunas, Benjamin Collins, Dan Jabaay, Thomas J. Downar, and William R. Martin. Overview of development and design of MPACT: Michigan parallel characteristics transport code. In Proceedings of the 2013 International Conference on Mathematics and Computational Methods Applied to Nuclear Science and Engineering, pages 42-53, Sun Valley, Idaho, 2013. American Nuclear Society.

[11] Jaakko Leppanen, Maria Pusa, Tuomas Viitanen, Ville Valtavirta, and Toni Kaltiaisenaho. The Serpent Monte Carlo code: Status, development and applications in 2013. Annals of Nuclear Energy, 
82:142-150, 2015. Joint International Conference on Supercomputing in Nuclear Applications and Monte Carlo 2013, SNA + MC 2013. Pluri- and Trans-disciplinarity, Towards New Modeling and Numerical Simulation Paradigms.

[12] N. Luciano, B. Ade, K. S. Kim, and A. Conant. MPACT verification with Magnox reactor neutronics progression problems. In Proceedings of Physics of Reactors (PHYSOR) 2020: Transition to a Scalable Nuclear Future, University of Cambridge, United Kingdom, March 2020.

[13] Ronald J. McConn, Christopher J. Gesh, Richard T. Pagh, Robert A. Rucker, and Robert Williams III. Compendium of material composition data for radiation transport modeling. Technical Report PNNL-15870 Rev. 1, Pacific Northwest National Laboratory, March 2011.

[14] B. D. Murphy. ORIGEN-ARP cross-section libraries for Magnox, advanced gas-cooled, and VVER reactor designs. Technical Report ORNL/TM-2003/263, Oak Ridge National Laboratory, February 2004.

[15] Tara M. Pandya, Seth R. Johnson, Thomas M. Evans, Gregory G. Davidson, Steven P. Hamilton, and Andrew T. Godfrey. Implementation, capabilities, and benchmarking of Shift, a massively parallel Monte Carlo radiation transport code. Journal of Computational Physics, 308:239-272, 2016.

[16] D. R. Poulter. The Design of Gas-Cooled Graphite-Moderated Reactors. Oxford University Press, Amen House, London, E.C. 4, 1963.

[17] W. Wieselquist, R. Lefebvre, and M. Jessee. SCALE Code System. Technical Report ORNL/TM-2005/39, Oak Ridge National Laboratory, April 2020.

[18] N. E. Woldman and J. P. Frick. Woldman's Engineering Alloys. Materials data series. ASM International, 2000. 